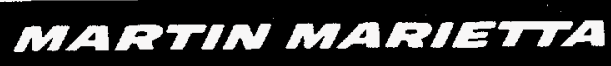

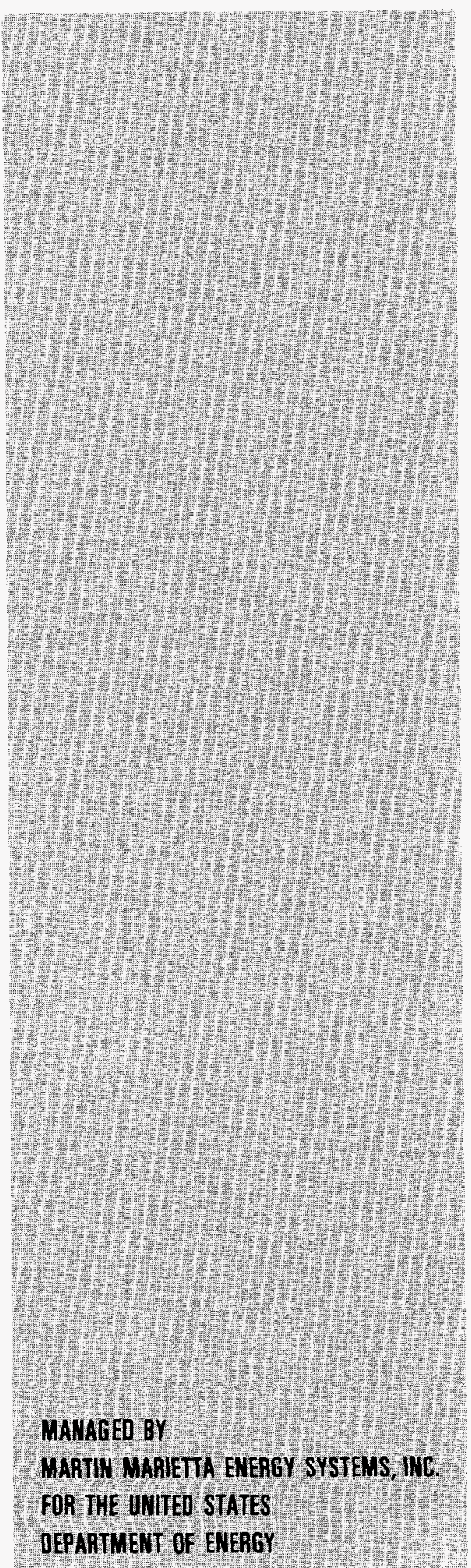

\section{Environmental Monitoring and Surveillance on the Oak Ridge Reservation: 1993 Data}




\section{DISCLAIMER}

Portions of this document may be illegible in electronic image products. Images are produced from the best available original document. 


\section{DISCLAIMER}

This report was prepared as an account of work sponsored by an agency of the United States Government. Neither the United States Government nor any agency thereof, nor any of their employees, makes any warranty, express or implied, or assumes any legal liability or responsibility for the accuracy, completeness, or usefulness of any information, apparatus, product, or process disclosed, or represents that its use would not infringe privately owned rights. Reference herein to any specific commercial product, process, or service by trade name, trademark, manufacturer, or otherwise does not necessarily constitute or imply its endorsement, recommendation, or favoring by the United States Government or any agency thereof. The views and opinions of authors expressed herein do not necessarily state or reflect those of the United States Government or any agency thereof.
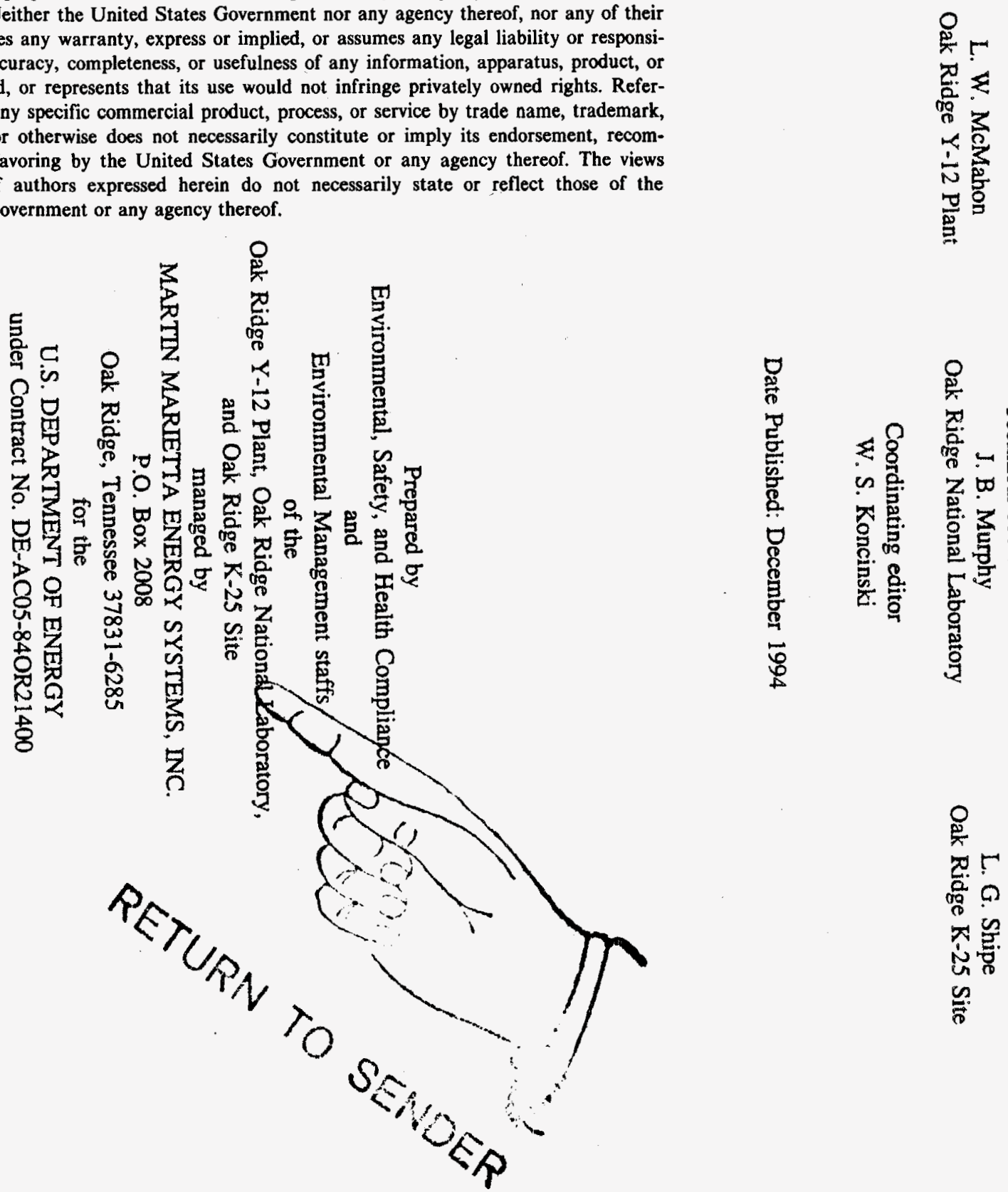
-<smiles>[I-]</smiles> 


\section{Contents}

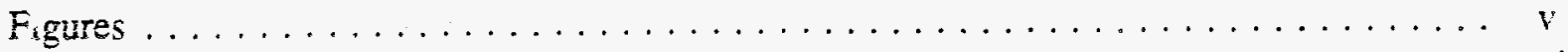

Tables $\ldots \ldots \ldots \ldots \ldots \ldots \ldots \ldots \ldots \ldots \ldots \ldots \ldots \ldots \ldots \ldots \ldots \ldots \ldots$ vii

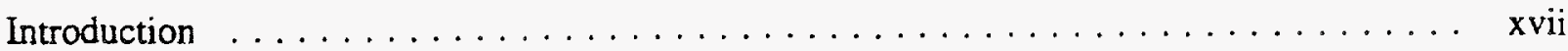

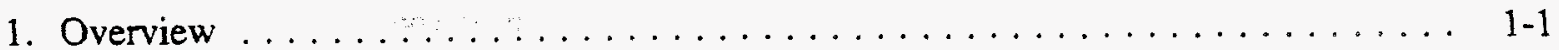

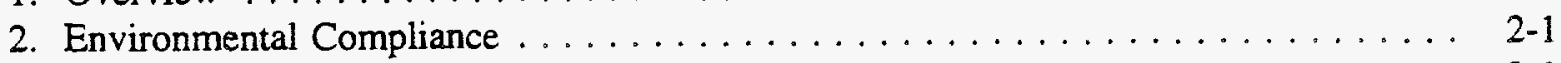

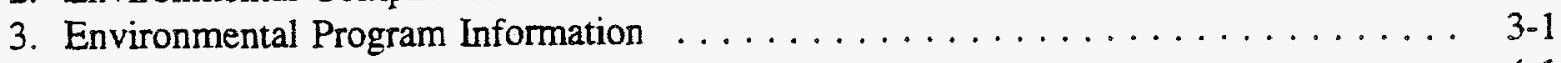

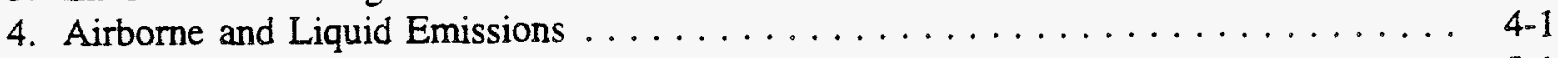

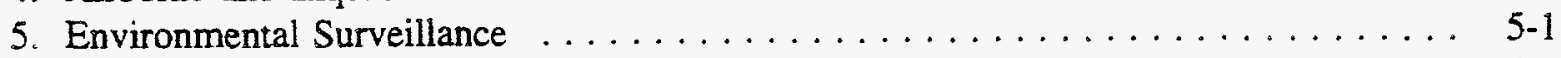

6. Dose ...................................... 6-1

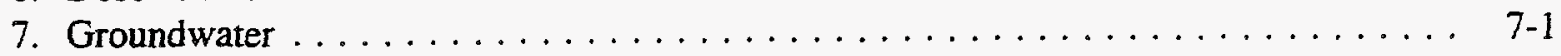

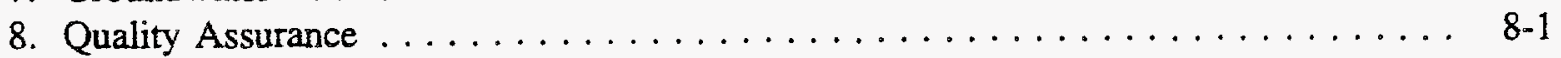

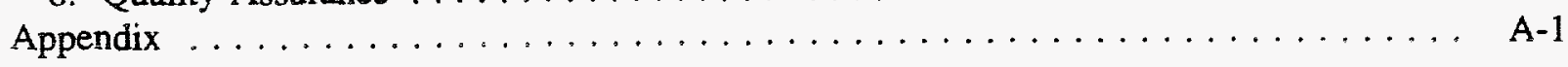


-

. 


\section{Figures}

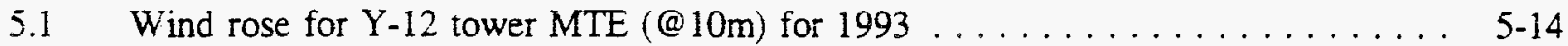

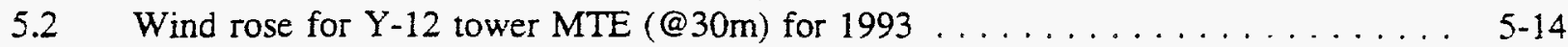

5.3 Wind rose for Y-12 tower MTE (@100m) for $1993 \ldots \ldots \ldots \ldots \ldots \ldots \ldots . \ldots .15$

$5.4 \quad$ Wind rose for $\mathrm{Y}-12$ tower MTW $(@ 10 \mathrm{~m})$ for $1993 \ldots \ldots \ldots \ldots \ldots \ldots \ldots . \ldots \ldots$

$5.5 \quad$ Wind rose for $\mathrm{Y}-12$ tower MTW $(@ 60 \mathrm{~m})$ for $1993 \ldots \ldots \ldots \ldots \ldots \ldots \ldots$

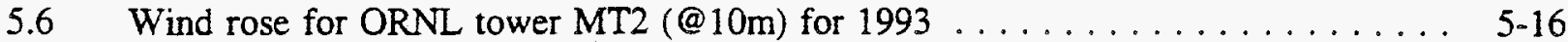

5.7 Wind rose for ORNL tower MT2 $(@ 30 \mathrm{~m})$ for $1993 \ldots \ldots \ldots \ldots \ldots \ldots \ldots$. . . . . . . . 5 .

$5.8 \quad$ Wind rose for ORNL tower MT2 $(@ 100 \mathrm{~m})$ for $1993 \ldots \ldots \ldots \ldots \ldots \ldots \ldots$. . . . . . . $5-17$

5.9 Wind rose for ORNL tower MT3 $(@ 10 \mathrm{~m})$ for $1993 \ldots \ldots \ldots \ldots \ldots \ldots \ldots$

5.10 Wind rose for ORNL tower MT3 $(@ 30 \mathrm{~m})$ for $1993 \ldots \ldots \ldots \ldots \ldots \ldots \ldots$

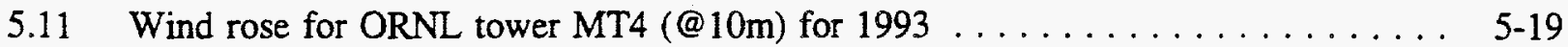

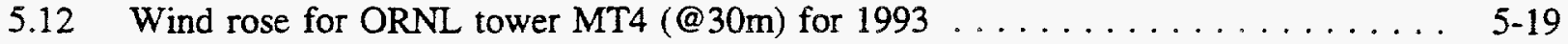

5.13 Wind rose for K-25 tower MT1 (@10m) for $1993 \ldots . . \ldots \ldots \ldots \ldots \ldots . . . . .55-20$

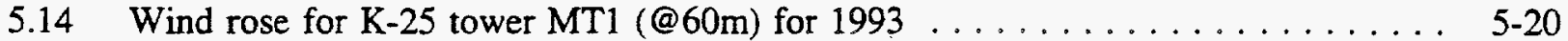

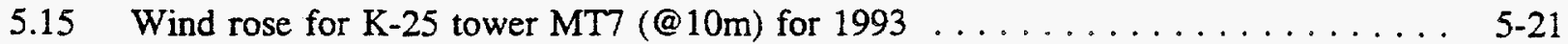

5.16 Wind rose for K-25 tower MT7 (@30m) for $1993 \ldots . . . . . . . . . . . . . .5-21$ 
-

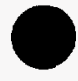

- 


\section{Tables}

1.1 Administrative units on the Oak Ridge Reservation $\ldots \ldots \ldots \ldots \ldots \ldots \ldots$

$2.1 \quad$ RCRA and CERCLA corrective action processes $\ldots \ldots \ldots \ldots \ldots \ldots \ldots \ldots$

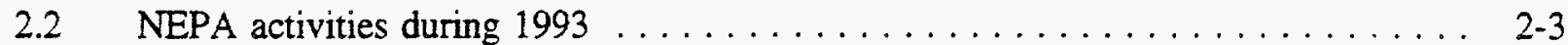

$2.3 \quad$ Oak Ridge Reservation recycling activities $\ldots \ldots \ldots \ldots \ldots \ldots \ldots \ldots$

2.4 Summary of environmental audits and assessments conducted

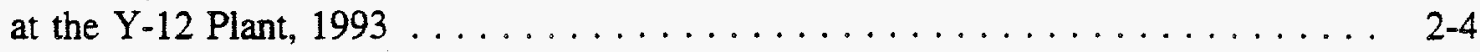

2.5 Summary of environmental audits and assessments conducted at ORNL, $1993 \ldots \ldots$. . $2-5$

2.6 Summary of environmental audits and assessments conducted at the

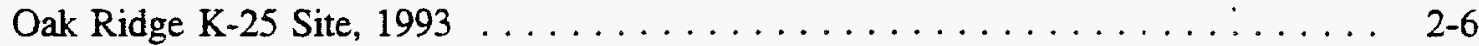

2.7 Summary of Tiger Team corrective actions . . . . . . . . . . . . . . . . 2-6

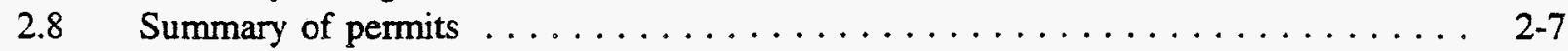

3.1 Underground storage tanks (USTs) at the $\mathrm{Y}-12$ Plant. . . . . . . . . . . . $3-3$

4.1 Y-12 Plant airborne uranium emission estimates, $1993 \ldots \ldots \ldots \ldots \ldots \ldots$. . . . .

4.2 Major sources of radiological airborne emissions (in curies) at ORNL, $1993 \ldots$. . . . 4-4

4.3 Minor sources of radiological airborne emissions (in curies) at ORNL, $1993 \ldots \ldots$. . . 4-5

4.4 Data sources for airborne radioactive emissions from ORNL, $1993 \ldots \ldots$. . . . . . . 4-6

$4.5 \quad$ K-25 Site radionuclide air emission totals (curies), $1993 \ldots \ldots \ldots \ldots$. . . . . . . 4-7

4.6 ORNL nonradiological airborne emissions, $1993 \ldots \ldots \ldots \ldots \ldots$. . . . . . . . . .

4.7 Potential emissions of criteria pollutants from the K-25 Site, 1992 and $1993 \ldots$. . . 4 4-9

4.8 Estimated K-25 Site emissions of ozone-depleting substances, $1993 \ldots \ldots$. . . . . . . 4-9

4.9 Estimated air emissions from the K-1501 Steam Plant at the K-25 Site, 1993 . . . . 4-9

4.10 Estimated air emissions from the TSCA Incinerator at

the $\mathrm{K}-25$ Site, $1993 \ldots \ldots \ldots \ldots \ldots \ldots \ldots \ldots \ldots \ldots \ldots \ldots$. . . . . . . . . . . . . . . . . .

4.11 Summary of $Y-12$ Plant radiological monitoring plan sample requirements . . . . 4-10

4.12 Release of uranium from the $Y-12$ Plant to the off-site environment

4.13 Radionuclide concentrations at K-25 Site surface water

effluent discharge points . . . . . . . . . . . . . . . . . . 4-11

4.14 Radionuclides released to off-site surface waters from the K-25 Site, 1993

Effluent discharge points are K-1203, K-1407-J, and K-1515-C . . . . . . . . 4-13

4.15 Summary of $Y-12$ Plant NPDES excursions, $1993 \ldots \ldots \ldots \ldots \ldots \ldots$. . . . . . . . . .

4.16 Y-12 Plant NPDES compliance monitoring requirements and record, $1993 \ldots \ldots$. . . 4-16

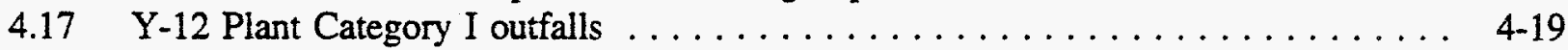

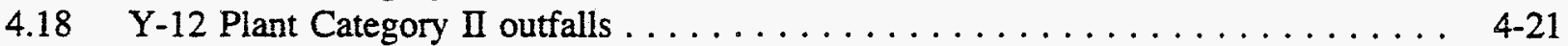

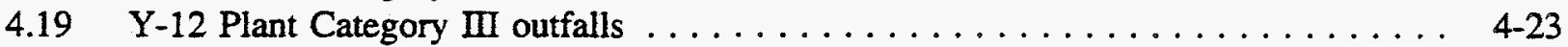

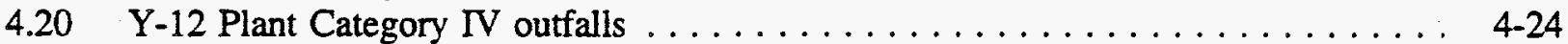

$4.21 \quad \mathrm{Y}-12$ Plant 300 -series NPDES outfalls-perimeter outfalls . . . . . . . . . . . 4-25

4.22 Y-12 Plant 500-series NPDES outfalls-Wastewater Treatment Facilities . . . . . . 4 4-25

4.23 Y-12 Plant outfalls not specifically enumerated on the current NPDES permit . . . . 4-26

4.24 Miscellaneous $Y-12$ Plant source discharges $\ldots \ldots \ldots \ldots \ldots \ldots \ldots \ldots \ldots \ldots$. . . . . . . . . . .

4.25 Permit status of miscellaneous $\mathrm{Y}-12$ Plant source discharges . . . . . . . . . . 4-28

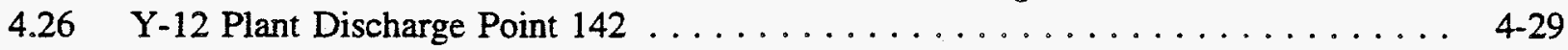

$4.27 \quad \mathrm{Y}-12$ Plant Station 8, radiological data summary $\ldots \ldots \ldots \ldots \ldots \ldots \ldots$. . . . . . . . .

$4.28 \quad \mathrm{Y}-12$ Plant Instream Monitoring Site, Upstream Drum Room . . . . . . . . . . . . . . 4-31

4.29 Y-12 Plant Discharge Point 301, Kerr Hollow Quarry . . . . . . . . . . . . . . . . . 4-32

$4.30 \quad Y-12$ Plant Discharge Point 301, radiological data summary . . . . . . . . . . . . 4-33

4.31 CY 1993 NPDES Permit Number TN 0002968

Y-12 Plant Discharge Point 302, Rogers Quarry (McCoy Branch) . . . . . . . . . 4-34 
4.32 CY 1993 radiological data summary

Y-12 Plant Discharge Point 302, Rogers Quarry (McCoy Branch) . . . . . . . . . 4-35

$4.33 \quad \mathrm{Y}-12$ Plant Discharge Point 304, Bear Creek . . . . . . . . . . . . . . . . 4-36

4.34 Y-12 Plant Discharge Point 304, radiologica! data summary . . . . . . . . . . . 4-37

$4.35 \quad$ Y-12 Plant Discharge Point 307, West Borrow Area . . . . . . . . . . . . . 4-38

$4.36 \quad \mathrm{Y}-12$ Plant Discharge Point 308, East Borrow Area . . . . . . . . . . . . . . . . 4-38

4.37 Y-12 Plant Discharge Point 309, Sanitary Landfill IV Sedimentation Basin . . . . . 4-39

4.38 CY 1993 NPDES Permit Number TN 0002968

Y-12 Plant Discharge Point 501, Central Pollution Control Facility . . . . . . . 4-40

4.39 CY 1993 radiological data summary

Y-12 Plant Discharge Point 501, Central Pollution Control Facility . . . . . . . 4 4-41

$4.40 \quad$ Y-12 Plant Discharge Point 502, West End Treatment Facility . . . . . . . . . . . . 4-42

$4.41 \quad \mathrm{Y}-12$ Plant Discharge Point 502, radiological data summary $\ldots \ldots \ldots \ldots \ldots . \ldots 43$

4.42 CY 1993 NPDES Permit Number TN 0002986

Y-12 Plant Discharge Point 503, Steam Plant Wastewater Treatment Facility . . . . 4-44

4.43 CY 1993 radiological data summary

Y-12 Plant Discharge Point 503, Steam Plant Wastewater Treatment Facility . . . . 4-45

$4.44 \quad \mathrm{Y}-12$ Discharge Point 504, Plating Rinsewater Facilities . . . . . . . . . . . . . 4-46

4.45 CY 1993 radiological data summary

Y-12 Plant Discharge Point 504, Plating Rinsewater Treatment Facilities . . . . . . 4-47

4.46 CY 1993 NPDES Permit Number TN 0002968

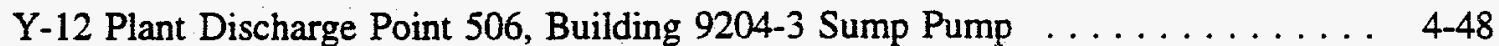

4.47 CY 1993 NPDES Permit Number TN 0002968

Discharge Point 512, Y-12 Plant, Groundwater Treatment Facility . . . . . . . . . . 4-49

4.48 CY 1993 radiological data summary

Y-12 Plant Discharge Point 512, Groundwater Treatment Facility . . . . . . . . . 4-50

4.49 CY 1993 NPDES Permit Number TN 0002968

Y-12 Plant Discharge Point 623 , Flyash Sluice . . . . . . . . . . . . . . . 4-51

4.501993 Y-12 Plant annual nonradiological data summary for Station $17 \ldots \ldots$. . . . 4-52

4.511993 Y-12 Plant annual radiological data summary for Station $17 \ldots \ldots$. . . . . 4-54

$4.52 \quad \begin{aligned} & \text { Y-12 Plant annual summary for Upper Bear Creek nonradioiogical } \\ & \text { data } \mathrm{km} 11.97 \ldots \ldots \ldots \ldots \ldots \ldots \ldots \ldots \ldots \ldots \ldots \ldots \ldots \ldots \ldots\end{aligned}$

4.53 Y-12 Plant annual summary for upper Bear Creek radiolngical

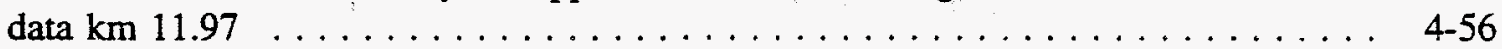

4.54 Y-12 Plant annual summary for upper Bear Creek nonradiological

4.55 Y-12 Plant annual radiological summary for upper Bear Creek

radiological data $\mathrm{km} 12.4 \ldots \ldots \ldots \ldots \ldots \ldots \ldots \ldots \ldots \ldots \ldots$

S.56 Surface water analytical results of polychlorinated biphenyls monitoring
plan for the Oak Ridge $Y-12$ Plant, CY $1993 \ldots \ldots \ldots \ldots \ldots \ldots \ldots \ldots$

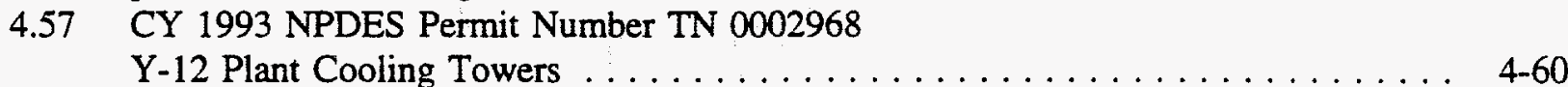

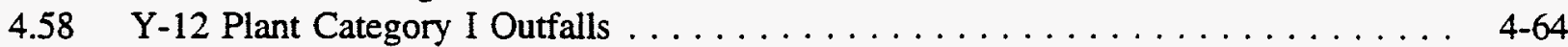

$4.59 \quad \mathrm{Y}-12$ Plant Category II Outfalls $\ldots \ldots \ldots \ldots \ldots \ldots \ldots \ldots \ldots \ldots \ldots \ldots$

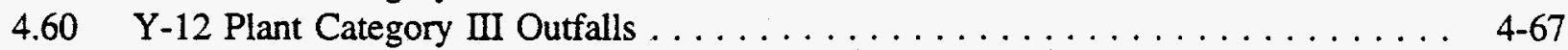

4.61 CY 1993 NPDES Permit Number TN 0002968 Y-12 Plant

4.62 CY 1993 Y-12 Plant data summary for outfall not specifically

enumerated on the NPDES permit . . . . . . . . . . . . . . . 4-69 
4.63 Surface water analytical results of polychlorinated biphenyls

monitoring plan for the $\mathrm{Y}-12$ Plant, $1993 \ldots \ldots \ldots \ldots \ldots$ 4-77

4.64 Permit limits for Y-12 Plant sanitary sewer dischar?e to City of

Oak Ridge Publicly Owned Treatment Works (POTW) . . . . . . . . . . . . . 4-78

4.65 City Flow Monitoring Station (northeast of $\mathrm{Y}-12$ Plant) $\ldots \ldots \ldots \ldots$. . . . . . 4-79

4.66 City Flow Monitoring Station (northeast of $\mathrm{Y}-12$ Plant) $\ldots \ldots \ldots \ldots \ldots$. . . . . . . 41

4.67 Union Valley Flow Monitoring Station (east of Y-12 Plant) $\ldots \ldots \ldots \ldots$. . . . . . 4-82

4.68 Union Valley Flow Monitoring Station (east of Y-12 Plant) . . . . . . . . . . . . 4-84

$4.69 \mathrm{Y}-12$ calculated sanitary sewer concentrations $\ldots \ldots \ldots \ldots \ldots \ldots \ldots \ldots \ldots$

$4.70 \quad \mathrm{Y}-12$ calculated sanitary sewer concentrations $\ldots \ldots \ldots \ldots \ldots \ldots \ldots \ldots$. . . . . . . . . . . .

4.711993 sampling and analysis plan for ORNL off-site treated water monitoring $\ldots . .4$ 4-88

4.721993 analyses for ORNL off-site treated water monitoring at

Gallaher water treatment plant . . . . . . . . . . . . . . . . . . . . . . . 4-89

4.731993 analyses for ORNL off-site treated water monitoring at

Kingston water treatment plant . . . . . . . . . . . . . . . . . . . 4-90

4.74 Calculated sanitary sewer compliance summary for Y-12 Plant, $1993 \ldots \ldots$. . . . . 4-91

4.75 NPDES compliance at the K-25 Site, $1993 \ldots \ldots \ldots \ldots \ldots \ldots \ldots$. . . . . . . . . . . . . . . . .

4.76 Y-12 Plant Toxicity Control and Monitoring Program (TCMP) summary information, $1993 \ldots \ldots \ldots \ldots \ldots \ldots \ldots \ldots \ldots$. . . . . . . . . . . . . . .

4.77 Toxicity test results of ORNL wastewaters and ambient waters, $1993 \ldots \ldots$. . . 4-95

4.78 Average water quality parameters measured during toxicity tests of

ORNL wastewaters and ambient waters, $1993 \ldots \ldots \ldots \ldots \ldots \ldots$ 4.96 . . . . . . . . . .

4.79 Toxicity test results of $\mathrm{K}-25$ Site wastewaters, $1993 \ldots \ldots \ldots \ldots \ldots$. . . . . . . . . 4-97

4.80 Average water quality parameters measured during toxicity tests of

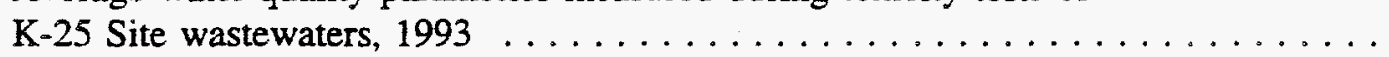

4-98

4.81 Changes in average concentrations of PCBs and fraction of fish exceeding

U.S. Food and Drug Administration (FDA) limit for channel catfish, 1986-93 . . . . 4-98

4.82 1993 Potable water data from DOE-owned water-treatment facility serving the

Y-12 Plant, ORNL, and city of Oak Ridge . . . . . . . . . . . . . 4-99

4.83 1993 potable water data-lead and copper rule data from DOE-owned

water-treatment facility serving the Y-12 Plant, ORNL. and the city

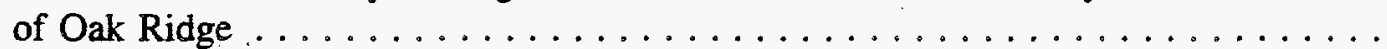

4-102

4.84 1993 sampling and analysis plan for ORNL surface waters receiving effiuents . . . . 4-102

4.851993 radionuclide concentrations at ORNL NPDES locations . . . . . . . . . . 4-103

4.861993 radionuclide concentrations in surface waters around ORNL . . . . . . . . 4 4-104

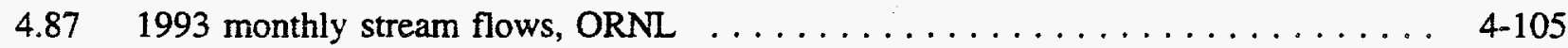

4.88 ORNL NPDES radionuclide sampling and analysis plan for the

4.891993 radionuclide concentrations at ORNL category outfalls . . . . . . . . . . 4-107

4.901993 sampling and analysis plan for ORNL reference surface waters:

Melton Hill Dam and White Oak Creek headwaters . . . . . . . . . . . . . . . 4-108

4.911993 analyses for ORNL reference surface waters . . . . . . . . . . . . . . . . . 4-109

4.921993 NPDES compliance at ORNL . . . . . . . . . . . . . . . . 4-111

4.93 ORNL NPDES sampling and analysis plan for the facility and

ambient locations . . . . . . . . . . . . . . . . . . . . . .

4.94 ORNL NPDES sampling and aralysis plan for the category outfalls

4.95 NPDES Permit Number TN 0002941,1993 , ORNL discharge point X01
(Sewage Treatment Plant) $\ldots \ldots \ldots \ldots \ldots \ldots \ldots \ldots \ldots \ldots \ldots \ldots \ldots \ldots \ldots \ldots$ 
4.96 NPDES Permit Number TN 0002941, 1993, ORNL discharge point X02

(Coal Yard Runoff Treatment Facility) . . . . . . . . . . . . . . . 4 4-117

4.97 NPDES Permit Number TN 0002941, 1993, ORNL discharge point X12

(Nonradiological Wastewater Treatment Facility) . . . . . . . . . . . . . 4-118

4.98 NPDES Permit Number TN 0002941, 1993, ORNL discharge point X13

(Melton Branch) . . . . . . . . . . . . . . . . . . . . . . . 4-119

4.99 NPDES Permit Number TN 0002941, 1993, ORNL discharge point X14

(White Oak Creek) . . . . . . . . . . . . . . . . . . . . 4-120

4.100 NPDES Permit Number TN 0002941, 1993, ORNL discharge point X15

(White Oak Dam) . . . . . . . . . . . . . . . . . . . . . . . 4-121

4.101 NPDES Permit Number TN 0002941, 1993, ORNL Category I outfalls . . . . . . 4-122

4.102 NPDES Permit Number TN 0002941, 1993, ORNL Category II outfalls . . . . . . . 4-123

4.103 NPDES Permit Number TN 0002941, 1993, ORNL Category III outfalls . . . . . . 4 4-123

4.104 NPDES Permit Number TN 0002941, 1993, ORNL Cooling Systems . . . . . . . . . 4-124

4.1051993 mercury concentrations in ORNL surface water $\ldots \ldots \ldots \ldots \ldots \ldots \ldots \ldots$. . . . . . . . . . . .

4.1061993 mercury concentrations in ORNL sediment $\ldots \ldots \ldots \ldots \ldots \ldots \ldots \ldots \ldots$. . . . . . . . . . . . . . . .

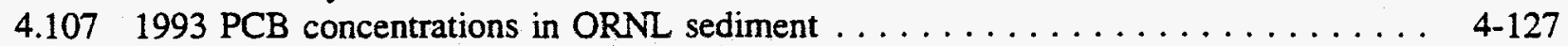

4.108 Radionuclide concentrations at K-25 Site discharges and surface water

monitoring locations . . . . . . . . . . . . . . . . . . . . . . . . . . 4 4-129

$4.109 \mathrm{~K}-25$ Site NPDES sampling and analysis frequencies . . . . . . . . . . . . . . . 4-134

4.1101993 NPDES Permit Number TN $0002950 \ldots \ldots \ldots \ldots \ldots \ldots \ldots \ldots . \ldots \ldots$. . . . . . . . . . . . . . . . . .

4.1111993 NPDES Permit Number TN 0002950

4.1121993 NPDES Permit Number TN 0002950

Discharge Point K-1407-J, Treatment Pond at K-25 Site . . . . . . . . . 4 4-157

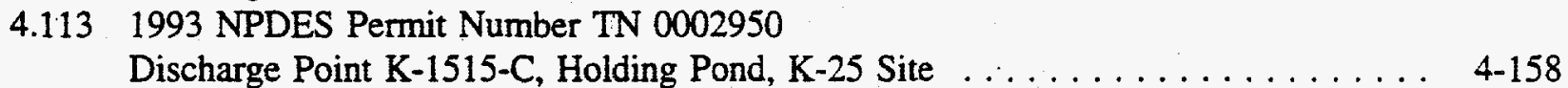

5.1 External gamma radiation measurements at $\mathrm{ORR}$ perimeter

5.2 Summary of collection and analysis frequencies for continuous

5.3 ORR radionuclide concentrations in air, $1993 \ldots \ldots \ldots \ldots \ldots \ldots \ldots \ldots \ldots$

5.4 Uranium in ambient air at the $\mathrm{Y}-12$ Plant, $1993 \ldots \ldots \ldots \ldots \ldots \ldots \ldots$

$5.5 \quad$ Fluorides in ambient air at the $Y-12$ Plant, $1993 \ldots \ldots \ldots \ldots \ldots \ldots$

5.6 Total suspended particulates in ambient air at the $Y-12$ Plant, $1993 \ldots \ldots$. . . . .

5.7 PM10 concentrations in ambient air at the $\mathrm{Y}-12$ Plant, $1993 \ldots \ldots \ldots$. . . . . .

5.8 Annual results of the $Y-12$ Plant ambient air mercury monitoring program, $1993 \ldots \ldots \ldots \ldots \ldots \ldots \ldots \ldots \ldots$

5.9 Summary of collection and analysis frequencies for low-volume samples at

ORNL ambient air monitoring stations $\ldots \ldots \ldots \ldots \ldots \ldots \ldots \ldots \ldots \ldots$

5.10 ORNL radionuclide concentrations in air, $1993 \ldots \ldots \ldots \ldots \ldots \ldots \ldots$. . . . . . .

5.11 Summary of ambient air pollutants measured by the K-25 Site network, $1993 \ldots \ldots$. . .

5.12 Results of ambient air sampling for lead at the K-25 Site, $1993 \ldots \ldots \ldots \ldots$. . . . . 5-8

5.13 Surface water sampling measurements exceeding Tennessee water quality criteria at the Y-12 Plant, $1993 \ldots \ldots \ldots \ldots \ldots \ldots \ldots$. . . . . . . . . . 5-9

5.14 Results of radiological analysis of ORR soil samples, $1993 \ldots \ldots \ldots \ldots \ldots$

5.15 Concentrations of radionuclides and fluoride in hay from the $\mathrm{ORR}, 1993 \ldots \ldots$

5.16 Results of radiological analysis of immatoes grown on the ORR, $1993 \ldots \ldots$. . . . . 5-10 
5.17 Results of radiclogical analysis of turnip greens grown on the ORR, 1993

5.18 Results of radiological analysis of turnips grown in private gardens on the ORR, 1993.

5.19 Concentrations of total radioactive strontium $\left({ }^{89} \mathrm{Sr}+{ }^{90} \mathrm{Sr}\right)$ in raw milk, $1993 \ldots \ldots$. . . $5-12$

5.20 Parameters detected in sunfish from Poplar Creek and Clinch River locations, 1993

5.21 Parameters detected in catfish from two Clinch River locations, $1993 \ldots \ldots$. . . . . 5-13

5.221993 Total uranium averages in ambient air at the $Y-12$ Plant . . . . . . . . . 5-22

$5.231993 \mathrm{Y}-12$ Plant ambient air \%U235 in total uranium . . . . . . . . . . . . 5-23

5.241993 Monthly fluoride averages in ambient air - Y-12 Plant . . . . . . . . . . 5-24

5.25 Total suspended particulates in air at the $\mathrm{Y}-12$ Plant $\ldots \ldots \ldots \ldots \ldots \ldots \ldots$

$5.261993 \mathrm{PM}-10$ concentrations in air at the Y-12 Plant $\ldots \ldots \ldots \ldots \ldots \ldots$.26

5.27 Annual results of the Y-12 Plant airborne mercury monitoring

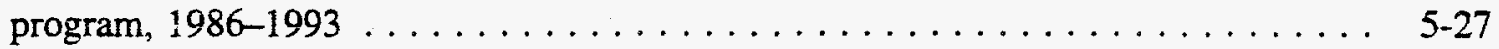

5.281993 EMP surface water sampling locations $\ldots \ldots \ldots \ldots \ldots \ldots \ldots \ldots \ldots \ldots$

5.291993 sampling and analysis plan for EMP surface water locations . . . . . . . . . 5-29

5.301993 concentrations at EMP surface water locations $\ldots \ldots \ldots \ldots \ldots \ldots \ldots \ldots$. . . . . . . . . . . . .

5.311993 K-25 Site surface water parameters detected at K-901-A . . . . . . . . . . . 5-50

5.321993 K-25 Site surface water parameters detected at K-716 . . . . . . . . . . 5-51

$5.331993 \mathrm{~K}-25$ Site surface water parameters detected at K-1007-B . . . . . . . . . 5 5-52

$5.341993 \mathrm{~K}-25$ Site surface water parameters detected at K-1700 . . . . . . . . . 5 5-53

$5.351993 \mathrm{~K}-25$ Site surface water parameters detected at $\mathrm{K}-1710 \ldots \ldots \ldots \ldots \ldots$. . . . . . . .

$5.361993 \mathrm{~K}-25$ Site surface water parameters detected at WFPC $\ldots \ldots \ldots \ldots \ldots \ldots$

5.37 Summary of constituents detected in off-site residential groundwater

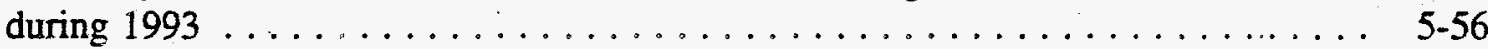

5.38 Concentration of radioactivity in soil samples taken in the vicinity

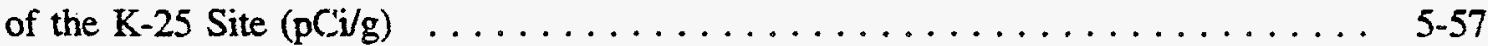

5.391993 EMP sediment sampling locations $\ldots \ldots \ldots \ldots \ldots \ldots \ldots \ldots \ldots \ldots$. . . . . . . . . . 58

5.401993 sampling and analysis plan for EMP sediment locations $\ldots \ldots \ldots \ldots \ldots$. . . . 5-59

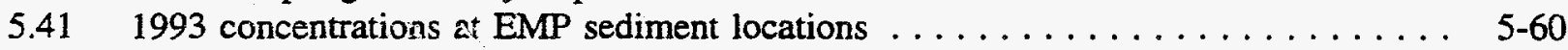

5.42 Concentration of radioactivity in sediment samples taken in the vicinity

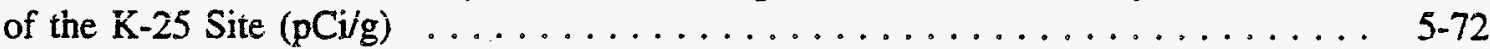

5.431993 selected results of sediment analysis at the $\mathrm{K}-25$ Site $\ldots \ldots \ldots \ldots \ldots$. . . . . . . . . . . .

5.441993 sampling and analysis plan for EMP fish $\ldots \ldots \ldots \ldots \ldots \ldots \ldots \ldots \ldots$. . . . . . . .

5.451993 concentrations in sunfish tissue $\ldots \ldots \ldots \ldots \ldots \ldots \ldots \ldots \ldots \ldots \ldots$

5.461993 concentrations in catfish tissue $\ldots \ldots \ldots \ldots \ldots \ldots \ldots \ldots \ldots \ldots \ldots$

6.1 Release point parameters and receptor locations used in the dose calculations . . . . . . . . . . . . . . . . . . . . . . $6-3$

6.2 Calculated radiation doses to maximally exposed off-site individuals from airborne releases during $1993 \ldots \ldots \ldots \ldots \ldots \ldots \ldots \ldots \ldots \ldots$

6.3 Calculated collective EDEs from airborne releases during $1993 \ldots \ldots \ldots \ldots$. . . . . . $6-4$

6.4 Potential maximum individual EDEs (mrem) from use of off-site waters . . . . . . 6-5

6.5 Average EDEs from ingesting vegetables grown at ORR ambient air monitoring stations $\ldots \ldots \ldots \ldots \ldots \ldots \ldots \ldots \ldots \ldots \ldots$. $6.6 \ldots$

6.61993 total dose rate for aquatic organisms (rad/day), ORNL $\ldots \ldots \ldots \ldots$. . . . . 6 6-6

6.71993 total dose rate for aquatic organisms (rad/day), Y-12 Plant

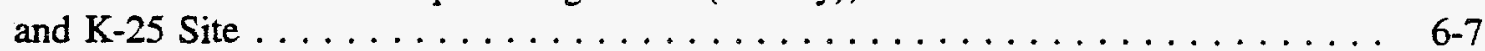

6.8 Summary of estimated radiation dose equivalents to an aduit during 1993 at locations of maximum exposure 
6.9 Trends in committed effective dose equivalent for selected pathways $\ldots \ldots \ldots$. $6-8$

6.10 Chemical reference doses and slope factors used in drinking water and fish intake analysis . . . . . . . . . . . . . . . . . . . . . 6-9

6.11 Chemical hazard quotients for drinking water $\ldots \ldots \ldots \ldots \ldots \ldots \ldots \ldots$

6.12 Chemical hazard quotient and I/CDI for fish intake $\ldots \ldots \ldots \ldots \ldots \ldots \ldots$

$7.1 \quad \mathrm{Y}-12$ Plant monitoring wells installed in $1993 \ldots \ldots \ldots \ldots \ldots \ldots$

7.2 Summary of the comprehensive groundwater monitoring program at the Y-12 Plant, $1993 \ldots \ldots \ldots \ldots \ldots \ldots \ldots \ldots \ldots \ldots$. . . . . . . . . . . . . . . . .

7.3 Description of surface-water and spring monitoring stations included in the Exit-Pathway Monitoring Program . . . . . . . . . . 7-6

7.4 Waste management sites, CERCLA operable units, and underground storage tanks included in the 1993 Groundwater Protection Program;

East Fork Hydrogeologic Regime . . . . . . . . . . . . . . . . . . . 7-7

7.5 Waste management sites and CERCLA operable units included in the 1993 Groundwater Protection Program; Bear Creek Hydrogeologic Regime . . . . . . 7-8

7.6 Waste management sites and CERCLA operable units included in the 1993 Groundwater Protection Program; Chestnut Ridge Hydrogeologic Regime . . . . . . . 7-9

7.7 Annual average summed VOC concentrations in groundwater at the Chestnut Ridge Security Pits, $1989-93 \ldots \ldots \ldots \ldots \ldots \ldots \ldots \ldots \ldots \ldots$ 7-10

7.8 Summary of ORNL waste area groupings $\ldots \ldots \ldots \ldots \ldots \ldots \ldots \ldots \ldots$

7.9 Sunumary of the groundwater surveillance program at ORNL, $1993 \ldots \ldots \ldots \ldots$. . . .

7.10 Summary of the plant perimeter surveillance

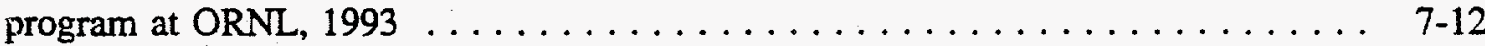

7.11 Reference standards for $\mathrm{Y}-12$ groundwater and surface water $\ldots \ldots \ldots \ldots \ldots$ 7-14

7.12 Constituents in groundwater at the $\mathrm{Y}-12$ Plant site $\mathrm{HGR}=\mathrm{BC}$ area=Background . . . . . . . . . . . . . . . . . . . 7-19

7.13 Constituents in groundwater at the $\mathrm{Y}-12$ Plant site $\mathrm{HGR}=\mathrm{BC}$ area=Above Ground Low-Level Storage Facility . . . . . . . . . . . 7-22

7.14 Constituents in groundwater at the $\mathrm{Y}-12$ Plant site HGR $=\mathrm{BC}$ area=Bear Creek Burial Grounds . . . . . . . . . . . . . . 7-2A

7.15 Constituents in groundwater at the Y-12 Plant site HGR $=B C$ area=exit Pathway

7.16 Constituents in groundwater at the $\mathrm{Y}-12$ Plant site $\mathrm{HGR}=\mathrm{BC}$ area=Oil Landfarm . . . . . . . . . . . . . . . . . .

7.17 Constituents in groundwater at the $\mathrm{Y}-12$ Plant site

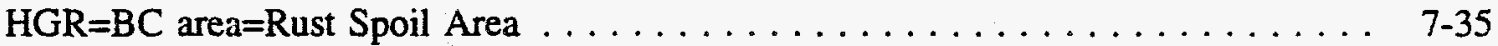

7.18 Constituents in groundwater at the $\mathrm{Y}-12$ Plant site

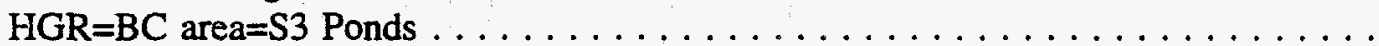

7.19 Constituents in groundwater at the $\mathrm{Y}-12$ Plant site

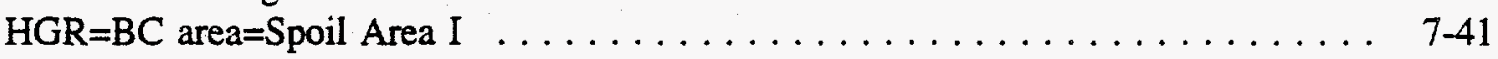

7.20 Constituents in groundwater at the $\mathrm{Y}-12$ Plant site

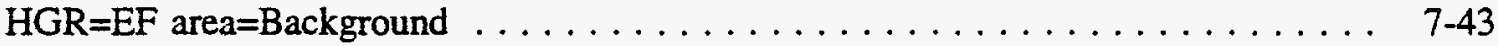

7.21 Constituents in groundwater at the Y-12 Plant site

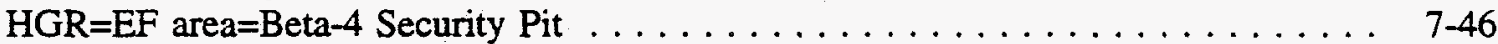

7.22 Constituents in groundwater at the Y-12 Plant site

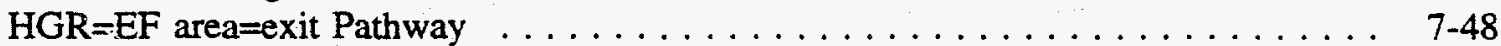

7.23 Constituents in groundwater at the Y-12 Plant site HGR $=E F$ area $=$ Fire Training Facility $\ldots \ldots \ldots \ldots \ldots \ldots \ldots \ldots$ 7-51

7.24 Constituents in groundwater at the Y-12 Plant site $\mathrm{HGK}=\mathrm{EF}$ area=Grid $\mathrm{C} 1$ 
7.25 Constituents in groundwater at the Y-12 Plant site

$\mathrm{HGR}=\mathrm{EF}$ area=Grid $\mathrm{E} 1$

7.26 Constituents in groundwater at the Y-12 Plant site

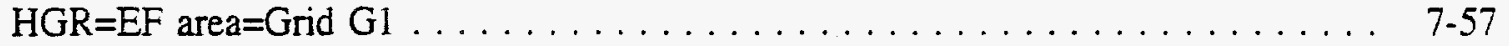

7.27 Constituents in groundwater at the $\mathrm{Y}-12$ Plant site

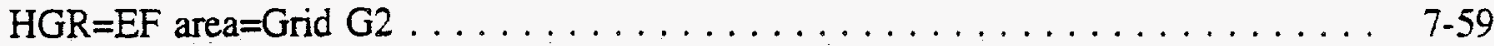

7.28 Constituents in groundwater at the $\mathrm{Y}-12$ Plant site

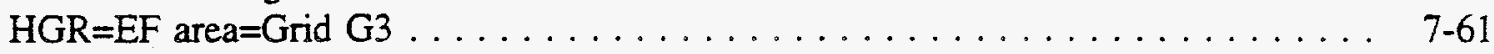

7.29 Constituents in groundwater at the Y-12 Plant site

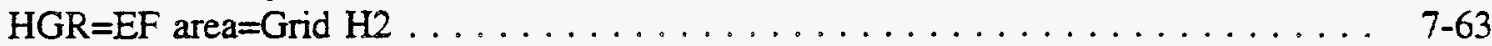

7.30 Constituents in groundwater at the Y-12 Plant site

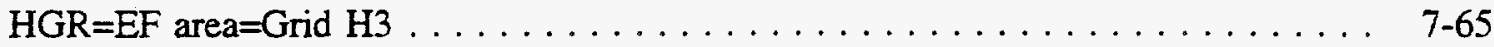

7.31 Constituents in groundwater at the $\mathrm{Y}-12$ Plant site

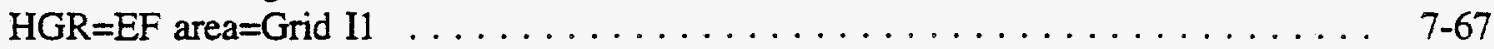

7.32 Constituents in groundwater at the $\mathrm{Y}-12$ Plant site

$\mathrm{HGR}=\mathrm{EF}$ area $=$ Grid $\mathrm{I} 2 \ldots \ldots \ldots \ldots \ldots \ldots \ldots \ldots \ldots \ldots$. . . . . . . . . . . . . . . . .

7.33 Constituents in groundwater at the Y-12 Plant site

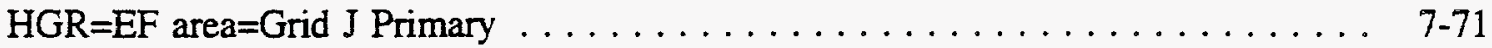

7.34 Constituents in groundwater at the $\mathrm{Y}-12$ Plant site

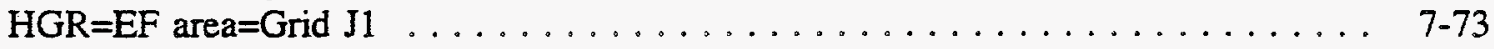

7.35 Constituents in groundwater at the $\mathrm{Y}-12$ Plant site

7.36 Constituents in groundwater at the $\mathrm{Y}-12$ Plant site

7.37 Constituents in groundwater at the $\mathrm{Y}-12$ Plant site

$\mathrm{HGR}=\mathrm{EF}$ area $=\mathrm{Grid} \mathrm{K1} \ldots \ldots \ldots \ldots \ldots \ldots \ldots \ldots \ldots \ldots$

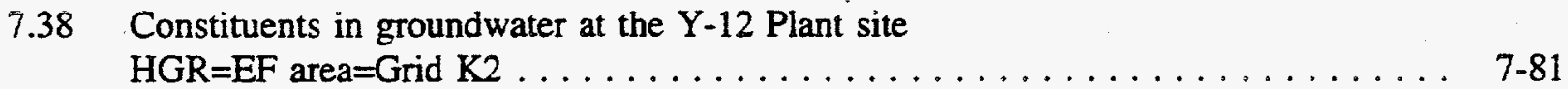

7.39 Constituents in groundwater at the $\mathrm{Y}-12$ Plant site
$\mathrm{HGR}=\mathrm{EF}$ area=New Hope Pond $\ldots \ldots \ldots \ldots \ldots \ldots \ldots \ldots \ldots$

7.40 Constituents in groundwater at the $\mathrm{Y}-12$ Plant site

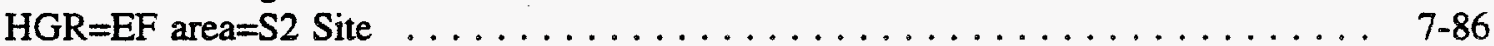

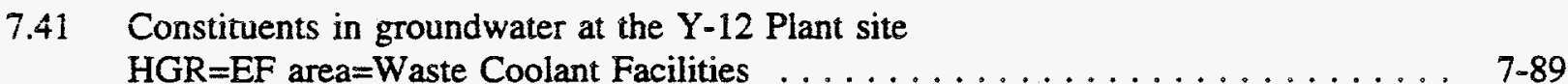

7.42 Constituents in groundwater at the Y-12 Plant site

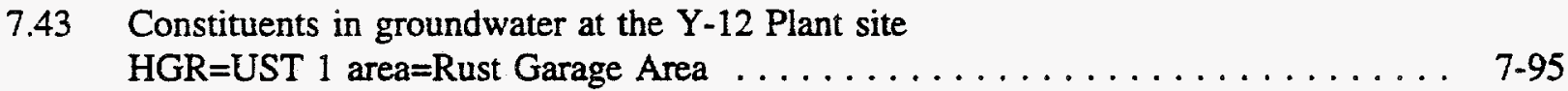

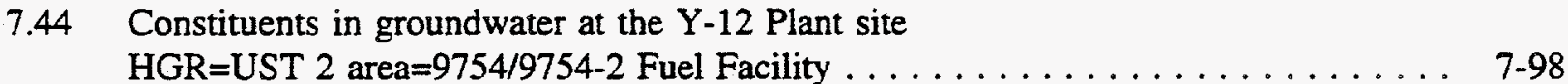

7.45 Constituents in groundwater at the Y-12 Plant site

7.46 Constituents in groundwater at the Y-12 Plant site

7.47 Constituents in groundwater at the Y-12 Plant site

HGR $=C R$ area $=$ Ash Disposal Basin . . . . . . . . . . . . . . 7-107

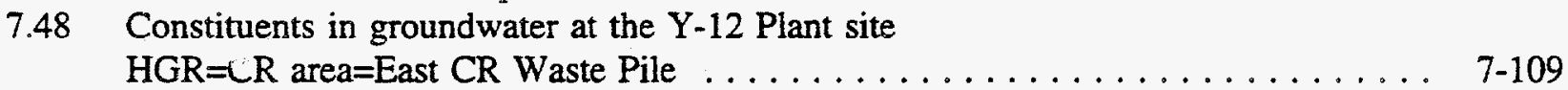

7.49 Constituents in groundwater at the Y-12 Plant site

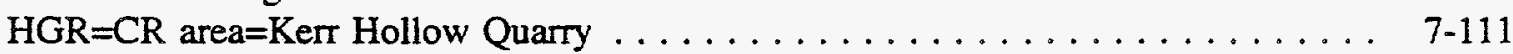


7.50 Constituents in groundwater at the $\mathrm{Y}-12$ Plant site

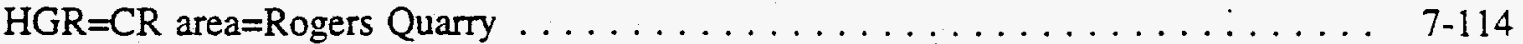

7.51 Constituents in groundwater at the $\mathrm{Y}-12$ Plant site

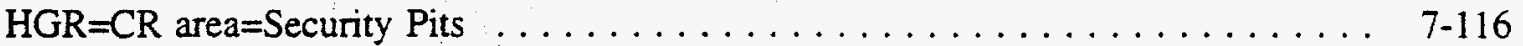

7.52 Constituents in groundwater at the Y-12 Plant site

HGR $=\mathrm{CR}$ area $=$ Sediment Disposal Basin . . . . . . . . . . . . . . 7-119

7.53 Constituents in groundwater at the Y-12 Plant site

HGR $=\mathrm{CR}$ area $=$ United Nuclear Site $\ldots \ldots \ldots \ldots \ldots \ldots \ldots \ldots \ldots \ldots \ldots \ldots \ldots$ 7-122

7.54 Constituents in groundwater at the Y-12 Plant site

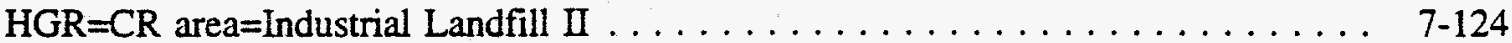

7.55 Constituents in groundwater at the $\mathrm{Y}-12$ Plant site

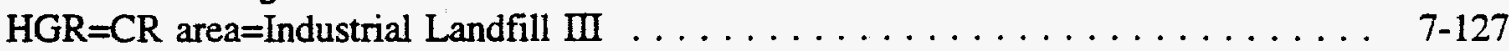

7.56 Constituents in groundwater at the Y-12 Plant site

$\mathrm{HGR}=\mathrm{CR}$ area $=$ Industrial Landfill $\mathrm{IV} \ldots \ldots \ldots \ldots \ldots \ldots \ldots \ldots \ldots$ 7-130

7.57 Constituents in groundwater at the $\mathrm{Y}-12$ Plant site

$\mathrm{HGR}=\mathrm{CR}$ area $=$ Industrial Landfill $\mathrm{V} \ldots \ldots \ldots \ldots \ldots \ldots \ldots \ldots \ldots \ldots \ldots$ 7-132

7.58 Constituents in groundwater at the Y-12 Plant site

HGR $=C R$ area $=$ Const. Debris Landfill VI . . . . . . . . . . . . . . . . . 7-135

7.59 Constituents in groundwater at the Y-12 Plant site

HGR $=\mathrm{CR}$ area $=$ Const. Debris Landfill VII . . . . . . . . . . . . . . . . . . 7-138

7.60 Constituents in Waste Area Grouping (WAG) 1 groundwater at ORNL,

May 19-June 25, $1993 \ldots \ldots \ldots \ldots \ldots \ldots \ldots \ldots \ldots \ldots \ldots \ldots$ 7-141

7.61 . Constituents in Waste Area Grouping (WAG) 2 groundwater at ORNL,

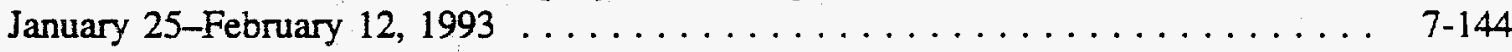

7.62 Constituents in Waste Area Grouping (WAG) 3 groundwater at ORNL,

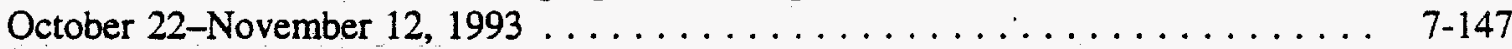

7.63 Constituents in Waste Area Grouping (WAG) 5 groundwater at ORNL, April 5-30, 1993

7.64 Constituents in Waste Area Grouping (WAG) 6 groundwater at ORNL,

February 18-March 29, May 6-14, August 9-September 24, and

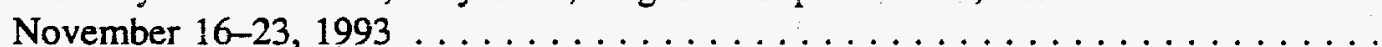

7.65 Constituents in Waste Area Grouping (WAG) 7 groundwater at ORNL,

July 14 -August $13,1993 \ldots \ldots \ldots \ldots \ldots \ldots \ldots \ldots$. . . . . . . . . . . . . . . . . .

7.66 Constituents in Waste Area Groupings (WAGs) $8 \& 9$ groundwater at ORNL,

September 28-October 15, $1993 \ldots \ldots \ldots \ldots \ldots$. . . . . . . . . . . . . . . . . . . . . .

7.67 Constituents in Waste Area Grouping (WAG) 11 groundwater at ORNL,

December 18, 1992-January 18,1993 . . . . . . . . . . . . . . 7-161

7.68 Constituents in Waste Area Grouping (WAG) 17 groundwater at ORNL, June 29-July 9, 1993

7.69 ORNL Plant Perimeter Monitoring summary statistics from

1993 sampling events . . . . . . . . . . . . . . . . . . . . . . 7-167

7.70 Constituents in the groundwater wells located at the K-1407 Operable Unit

at the K-25 Site, $1993 \ldots \ldots \ldots \ldots \ldots \ldots \ldots \ldots \ldots \ldots \ldots \ldots$. . . . . . . . . . . . . . . . . . . . . .

7.71 Constituents in the Exit Pathway Wells at the K-25 Site, $1993 \ldots \ldots \ldots \ldots$. . . . . 7-175

7.72 Constituents in the groundwater wells located at the

K-770 Operable Unit at the K-25 Site, $1993 \ldots \ldots \ldots \ldots \ldots \ldots \ldots$. . . . . . . . . . . . . . .

8.1 Number of measurements from external control programs,

Y-12 Analytical Services Organization, CY $1993 \ldots \ldots \ldots \ldots \ldots \ldots$. . . . . . . . .

8.2 ORNL Site Analytical Services Organization external performance

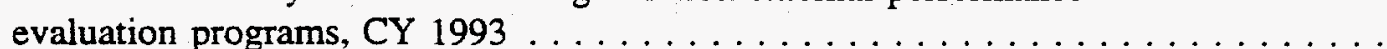


8.3 Number of measurements from external control programs, K-25 Site Analytical Services Organization, CY $1993 \ldots \ldots \ldots \ldots \ldots$. . . . . . . . . . . . . . . . . . . . . . . . . . . . . . . . . .

A.1 Reference standards for water $\ldots \ldots \ldots \ldots \ldots \ldots \ldots \ldots \ldots \ldots \ldots \ldots$

A.2 Air permits $\ldots \ldots \ldots \ldots \ldots \ldots \ldots \ldots \ldots \ldots \ldots \ldots \ldots \ldots$ 
-

○

- 


\section{Introduction}

Environmental monitoring and surveillance are conducted on the Oak Ridge Reservation and its environs throughout the year. Environmental monitoring ensures that (1) the reservation is a safe place to work, (2) activities on the reservation do not adversely affect the neighboring communities, and (3) compliance is made with federal and state regulations.

This document is a compilation of the monitoring and surveillance data for calendar year 1993. It is a tool for analysts in the fields of environmental monitoring and environmental restoration. The summary information found in the annual site environmental report was drawn from the contents of this document. 
Table 1.1. Administrative units on the Oak Ridge Reservation

\begin{tabular}{lcc}
\hline & \multicolumn{2}{c}{ Area } \\
\cline { 2 - 3 } Administering body & Hectares & Acres \\
\hline Oak Ridge Y-12 Plant & 1,769 & 4,370 \\
Oak Ridge National Laboratory & 9,879 & 24,400 \\
Oak Ridge K-25 Site & 1,954 & 4,825 \\
Oak Ridge Institute for & 274 & 680 \\
$\quad$ Science and Education & 93 & 230 \\
Johnson Controls World & & \\
Services, Inc. & 13,969 & 34,505 \\
$\quad$ Total & &
\end{tabular}


Table 2.1. RCRA and CERCLA corrective action processes

\begin{tabular}{lll}
\hline \multicolumn{1}{c}{ RCRA } & \multicolumn{1}{c}{ CERCLA } & \multicolumn{1}{c}{ Purpose } \\
\hline RCRA Facility Assessment & $\begin{array}{l}\text { Preliminary Assessment/Site } \\
\text { Investigation }\end{array}$ & $\begin{array}{c}\text { Identify reieases needing further } \\
\text { investigations }\end{array}$ \\
RCRA Facility Investigation & Remedial Investigation & $\begin{array}{c}\text { Characterize nature, extent, and } \\
\text { rate of contaminant releases }\end{array}$ \\
Corrective Measures Study & Feasibility Study & Evaluate and select remedy \\
$\begin{array}{l}\text { Corrective Measures } \\
\text { Implementation }\end{array}$ & $\begin{array}{l}\text { Remedial Design/Remedial } \\
\text { Action }\end{array}$ & $\begin{array}{c}\text { Design and implementation of } \\
\text { chosen remedy }\end{array}$ \\
\hline
\end{tabular}

Table 2.2. NEPA activities during 1993

\begin{tabular}{lccc}
\hline \multicolumn{1}{c}{ Types of NEPA documentation } & Y-12 Plant & ORNL & K-25 Site \\
\hline Categorical exclusion (CX) recommendation & 11 & 28 & 29 \\
CX granted & 11 & 27 & 16 \\
Approved under general CX documents & 332 & 97 & 71 \\
Environmental assessment & 8 & 10 & 2 \\
Special environmental analysis & 2 & 0 & 0 \\
Environmental impact statement & 0 & 1 & 0 \\
\hline
\end{tabular}

Table 2.3. Oak Ridge Reservation recycling activities

\begin{tabular}{lccc}
\hline \multicolumn{1}{c}{ Material } & $\begin{array}{c}1991 \\
\text { (tons) }\end{array}$ & $\begin{array}{c}1992 \\
\text { (tons) }\end{array}$ & $\begin{array}{c}1993 \\
\text { (tons) }\end{array}$ \\
\hline Aluminum cans & 15.7 & 24.8 & 28.7 \\
Cardboard & 85.5 & 315.4 & 428.5 \\
Paper & 302.4 & 552.8 & 786.6 \\
\hline
\end{tabular}


Table 2.4. Summary of environmental audits and assessments conducted at the Y-12 Plant, 1993

\begin{tabular}{|c|c|c|c|}
\hline Date & Reviewer & Subject & Findings \\
\hline $12 / 31-1 / 7 / 93$ & DOE-ORO & $\begin{array}{l}\text { DOE air pollution control program-airborne } \\
\text { effluent emission controls }\end{array}$ & 0 \\
\hline $1 / 13-2 / 4 / 93$ & DOE-ORO & Building $9201-1$ parking area and weir tank & 2 \\
\hline $1 / 19-29 / 93$ & DOE-ORO & $\begin{array}{l}\text { Best management practices plan } \\
\text { implementation }\end{array}$ & 2 \\
\hline $1 / 26-2 / 6 / 93$ & $\begin{array}{l}\text { DOE-ORO/Energy } \\
\text { Systems Environmental } \\
\text { Compliance }\end{array}$ & Review of NESHAP for radionuclides & 0 \\
\hline $2 / 18 / 93$ & DOE-ORO & Underground storage tank overfill protection & 0 \\
\hline $3 / 10 / 93$ & City of Oak Ridge & Sanitary sewer monitoring program & 1 \\
\hline $5 / 2 / 93$ & DOE-ORO & Herbicide use on East Fork Poplar Creek & 0 \\
\hline $5 / 5 / 93$ & EPA & $\begin{array}{l}\text { NPDES Compliance, Evaluation, and } \\
\text { Inspection }\end{array}$ & 3 \\
\hline $6 / 3 / 93$ & DOE-ORO & Y-12 Plant drain tie-in procedure & 0 \\
\hline $6 / 14 / 93$ & DOE-ORO & Groundwater well sampling & 0 \\
\hline $6 / 14-19 / 93$ & TDEC & $\begin{array}{l}\text { Annual RCRA inspection and groundwater } \\
\text { compliance evaluation inspection }\end{array}$ & 0 \\
\hline $6 / 18-7 / 2 / 93$ & DOE-ORO & Groundwater well installation & 0 \\
\hline $6 / 24-30 / 93$ & DOE-ORO & Laboratory and field quality control & 0 \\
\hline $6 / 24 / 93$ & DOE-ORO & Storm water monitoring program & 0 \\
\hline $7 / 7-9 / 93$ & TDEC & Air compliance & 0 \\
\hline $7 / 19-8 / 93$ & DOE-ORO & Environmental appraisal & 77 \\
\hline $7 / 27 / 93$ & DOE-ORO & $\begin{array}{l}\text { NPDES discharge-monitoring report } \\
\text { preparation }\end{array}$ & 0 \\
\hline $8 / 5 / 93$ & TDEC & Landfill V & 4 \\
\hline $9 / 23-24 / 93$ & EPA & Rad-NESHAP & 0 \\
\hline $10 / 1 / 93$ & DOE-ORO & Groundwater well plugging and abandonment & 0 \\
\hline $12 / 1 / 93$ & DOE-ORO & $\begin{array}{l}\text { Radiological monitoring for NPDES permit } \\
\text { compliance }\end{array}$ & 2 \\
\hline $12 / 1-3 / 93$ & $\begin{array}{l}\text { Defense Nuclear } \\
\text { Facilities Safety Board }\end{array}$ & $\begin{array}{l}\text { Review of liquid discharges and air } \\
\text { emissions at the Y-12 Plant }\end{array}$ & 0 \\
\hline $12 / 1-8 / 93$ & DOE-ORO & Groundwater Monitoring Program & 0 \\
\hline
\end{tabular}


Table 2.5. Summary of environmental audits and assessments conducted at ORNL, 1493

\begin{tabular}{|c|c|c|c|}
\hline Date & Reviewer & Subject & Fini'ings \\
\hline $2 / 11 / 93$ & DOE & $\begin{array}{l}\text { DOE inspector general audited records } \\
\text { management for Water Quality Control } \\
\text { Group and RCRA records }\end{array}$ & 0 \\
\hline $5 / 5 / 93$ & EPA Region IV & NPDES Compliance Evaluation Inspection & 2 \\
\hline $5 / 17-18 / 93$ & $\begin{array}{l}\text { TDECIDOE/Energy } \\
\text { Systems/ORNL }\end{array}$ & Hosted two-day permit renewal work session & 0 \\
\hline $6 / 6-7 / 93$ & TDEC & Inspection of ORNL air emission sources & 0 \\
\hline \multirow[t]{2}{*}{$6 / 14-16 / 93$} & TDEC & $\begin{array}{l}\text { Inspection of treatment, storage, and disposal } \\
\text { and generator areas, training. and } \\
\text { record keeping }\end{array}$ & 0 \\
\hline & & $\begin{array}{l}\text { Reviewed Pollution Prevention Program } \\
\text { reporting and planning requirements for } \\
\text { state of Tennessee }\end{array}$ & 0 \\
\hline $7 / 2 / 93$ & TDEC and DOE-ORO & $\begin{array}{l}\text { Surveillance of visible emissions from the } \\
\text { ORNL Steam Plant }\end{array}$ & 0 \\
\hline $8 / 9 / 93$ & $\begin{array}{l}\text { Tennessee Department } \\
\text { of Agriculture }\end{array}$ & Inspection of pesticide storage and use & 0 \\
\hline $8 / 9-10 / 93$ & TDEC & $\begin{array}{l}\text { Visit by RCRA Groundwater group to } \\
\text { observe the well-sampling activities at } \\
\text { SWSA-6 }\end{array}$ & 0 \\
\hline $8 / 12 / 93$ & EPA Region IV & $\begin{array}{l}\text { Visit by EPA Region IV regarding NPDES } \\
\text { issues }\end{array}$ & 0 \\
\hline $10 / 5 / 93$ & DOE-ORO & $\begin{array}{l}\text { Conduct of Operations-Liquid Waste } \\
\text { Solidification Project Readiness Review }\end{array}$ & 0 \\
\hline $10 / 7 / 93$ & DOE-ORO & $\begin{array}{l}\text { Conduct of Operations-Partial Assessment } \\
\text { of } 3608 \text { Nonradiological Wastewater } \\
\text { Treatment Plant }\end{array}$ & 0 \\
\hline $\begin{array}{l}10 / 8 / 93 \\
10 / 19 / 93, \text { and } \\
11 / 8-12 / 93\end{array}$ & $\begin{array}{l}\text { DOE Special Issue } \\
\text { Review }\end{array}$ & $\begin{array}{l}\text { Pollution prevention implementation across } \\
\text { ORNL }\end{array}$ & 3 \\
\hline $11 / 9 / 93$ & DOE & $\begin{array}{l}\text { Isotopes Shutdown Program involvement } \\
\text { with decontamination and decommissioning } \\
\text { activities associated with shutdown of } \\
\text { Isotopes Program }\end{array}$ & 0 \\
\hline
\end{tabular}


Table 2.6. Summary of environmental audits and assessments conducted at the Oak Ridge K-25 Site, 1993

\begin{tabular}{lllc}
\hline \multicolumn{1}{c}{ Date } & \multicolumn{1}{c}{ Reviewer } & \multicolumn{1}{c}{ Subject } & Findings \\
\hline $5 / 6 / 93$ & $\begin{array}{c}\text { EPA Regiun IV, TDEC, } \\
\text { DOE-ORO }\end{array}$ & NPDES compliance evaluation & 0 \\
$6 / 14-17 / 93$ & TDEC & RCRA compliance audit & 0 \\
$7 / 6-15 / 93$ & DOE-HQ & Quality assurance assessment of & 0 \\
& Oak Ridge facilities $:$ & \\
$7 / 26 / 93$ & TDEC & Air compliance & 0 \\
$9 / 23-24 / 93$ & EPA-HQ, EPA Region IV, & Rad-NESHAP & 0 \\
& TDEC-Tennessee & & \\
& Oversight Agreement & & \\
\hline
\end{tabular}

Table 2.7. Summary of Tiger Team corrective actions

\begin{tabular}{|c|c|c|c|}
\hline Date of review & Plant & $\begin{array}{l}\text { Environmental } \\
\text { findings }\end{array}$ & Status \\
\hline $\begin{array}{l}6 / 89 \\
2 / 10-21 / 92 \\
\text { (follow-up } \\
\text { visit) }\end{array}$ & $\mathrm{Y}-12$ & 62 & $\begin{array}{l}47 \text { have been closed; } 11 \text { are complete and } \\
\text { awaiting verification of closure; } \\
4 \text { remain open }\end{array}$ \\
\hline $10 / 22-11 / 30 / 90$ & ORNL & 69 & $\begin{array}{l}47 \text { have been closed: } 37 \text { of these have } \\
\text { been verifled as closed by DOE; } \\
22 \text { remain open }\end{array}$ \\
\hline $11 / 12-12 / 18 / 91$ & $\mathrm{~K}-25$ & 102 & $\begin{array}{l}38 \text { have peen closed; } 0 \text { of these have been } \\
\text { verified as closed by DOE; } 64 \text { remain } \\
\text { open }\end{array}$ \\
\hline
\end{tabular}


Table 2.8 Summary of permits

\begin{tabular}{|c|c|c|c|}
\hline & $\because 12$ Plant & ORNL & K-25 Site \\
\hline \multicolumn{4}{|c|}{ Resource Conservation and Recovery Act } \\
\hline Part B & 0 & 2 & 4 \\
\hline Part $B$ applications in process & $6^{a}$ & 3 & 0 \\
\hline Post-ciosure & 1 & 1 & 0 \\
\hline Permit-by-rule units & 10 & $173^{b}$ & 92 \\
\hline Solid waste landfills & $6^{c}$ & 0 & 0 \\
\hline Annual petroleum UST facility certificate & 2 & 1 & 1 \\
\hline \multicolumn{4}{|c|}{ Clean Water Act } \\
\hline NPDES & $1^{d}$ & $1^{6}$ & 1 \\
\hline Storm water & $1^{e}$ & $y^{s}$ & $1^{e}$ \\
\hline Aquatic resource alteration & 2 & 2 & 2 \\
\hline General storm water construction & $3^{8}$ & 0 & 0 \\
\hline \multicolumn{4}{|c|}{ Clean Air ACt } \\
\hline Operating air & 94 & 37 & 54 \\
\hline Construction & 38 & 0 & 4 \\
\hline Prevention of significant deterioration & 0 & 0 & 0 \\
\hline \multicolumn{4}{|c|}{ Sanitary Sewer } \\
\hline Sanitary sewer & 1 & 0 & 0 \\
\hline \multicolumn{4}{|c|}{ Toxic Substances Control Act } \\
\hline TSCA Incinerator & 0 & 0 & 1 \\
\hline$R \& D$ for alternative disposal methods & 1 & 2 & 0 \\
\hline
\end{tabular}

${ }^{a}$ Six applications have been submitted, representing 20 active units.

Tanks regulated by Permit-by-Rule.

Three landfills are operational, one (Spoil Area 1) is inactive, and one (Landfill VII) is under construction.

In renewal process.

TDEC has incorporated storm water into individual NPDES permit application.

TDEC is expected to incorporate storm water into the NPDES permit applications.

${ }^{8}$ Notice of intent that accesses a general NPDES permit. Notices of intent were filed for construction at landfills V, VI, VII, and the Walk-In Pits. 

Table 3.1 (contlinued)

\begin{tabular}{|c|c|c|c|c|c|c|c|c|c|}
\hline Location & $\begin{array}{c}\text { Tank } \\
\text { identification } \\
\text { number }\end{array}$ & $\begin{array}{l}\text { Installation } \\
\text { date }\end{array}$ & $\begin{array}{l}\text { Out-of- } \\
\text { service date }\end{array}$ & $\begin{array}{l}\text { Capacity } \\
\text { (gallons) }\end{array}$ & Contents & Status & $\begin{array}{c}\text { Preliminary } \\
\text { investigation(s) }\end{array}$ & $\begin{array}{l}\text { Environmental } \\
\text { assessment } \\
\text { () date to } \\
\text { regulatory agency }\end{array}$ & $\begin{array}{l}\text { Corrective } \\
\text { action }\end{array}$ \\
\hline $9754-1$ & 2082-U & 1981 & 1988 & 8,000 & Gasoline & Removed $12 / 89$ & EA & SIR (3/91) & $\begin{array}{l}\text { CAP (5/92), SRS (2/94), SRS } \\
\text { approval (3/94) }\end{array}$ \\
\hline PRW & $2310-U$ & 1975 & 1989 & 200 & Gasoline & Removed 11/89 & ISCR & SIR/CAP (7/91) & $\begin{array}{l}\text { EAR/CAP (3/93), CAP approval } \\
\text { (12/93), OE (4/94) }\end{array}$ \\
\hline $9201-1$ & $2331-U$ & 1973 & 1988 & 560 & Gasoline & Removed 12/88 & ISCR, FPRR & SIR $(3 / 92)$ & $\begin{array}{l}\text { EAR/CAP (7/92), CAP approval } \\
\text { (12/93), BMR (3/94). SRS (4/94) }\end{array}$ \\
\hline $9401-3$ & 0713-U & 1955 & 1988 & 10,500 & No. 2 fuel oil & Rernoved 11/88 & NI & NA & NA \\
\hline 9754 & $0836-U$ & 1944 & 1989 & 10,000 & Used oil & Removed $10 / 89$ & RCRA & RCRA & RCRA \\
\hline 9204-3 & $0928-U$ & 1966 & 1989 & 200 & Gasoline & Removed 5/89 & $\begin{array}{l}\text { RIR, closure } \\
\text { approved (8/92) }\end{array}$ & NA & NA \\
\hline 9995 & $2078-U$ & 1965 & 1979 & 110 & Gasoline & Inert filled 1979 & CERCLA & TBD & TBD \\
\hline 9995 & $2079 . U$ & 1965 & 1979 & 55 & Gasoline & Inert filled 1979 & CERCLA & TBD & TBD \\
\hline 9996 & $2080-U$ & 1971 & 1987 & 560 & Gasoline & Removed $12 / 88$ & RIR & NA & NA \\
\hline 9212 & 2081-U & 1958 & 1970 & 280 & Gasoline & Removed 4/91 & ISCR & NA & OE/CR (12/91) \\
\hline $9201-5$ & 2099-U & 1971 & 1989 & 560 & Gasoline & Removed 7/89 & $\begin{array}{l}\text { IAR, RIR, closure } \\
\text { approved (3/90) }\end{array}$ & NA & $\mathrm{NA}$ \\
\hline $9929-1$ & $2117-\mathrm{U}$ & 1971 & 1983 & 550 & No. 2 fuel oil & Removed 10/88 & $\mathrm{NI}$ & NA & $\mathrm{NA}$ \\
\hline $9204-4$ & $2130-U$ & 1960 & 1992 & 550 & Gasoline & Removed $12 / 92$ & RIR & NA & $\mathrm{NA}$ \\
\hline 9999 & 2293-U & 1954 & 1974 & 58 & Gasoline & Removed 1974 & NI & NA & NA \\
\hline 9999 & $2294-U$ & 1954 & 1974 & 58 & Gasoline & Removed 1974 & NI & NA & NA \\
\hline 9998 & $2305-U$ & 1956 & 1990 & 55 & Diesel & Remioved $10 / 90$ & RIR & $\mathrm{NA}$ & NA \\
\hline PRE & $2315-U$ & 1960 & 1988 & 64 & Gasoline & Removed I1/89 & ISCR & EAR/CAP $(2 / 91)$ & OE/CAR (12/92) \\
\hline 9769 & $2330-\mathrm{U}$ & 1949 & 1988 & 5,000 & No. 2 fuel oil & Inert filled 1988 & $\mathrm{NI}$ & NA & NA \\
\hline 0962 & $2336-\mathrm{U}$ & 1981 & 1991 & 550 & Gasoline & Removed 5/91 & RIR & $\mathrm{NA}$ & NA \\
\hline Buff. Mtn. & $2337-U$ & 1972 & 1990 & 250 & Gasoline & Removed 3/90 & $\begin{array}{l}\text { IAR, ISCR SIR, } \\
\text { SIR (1/92) }\end{array}$ & NA & $\mathrm{NA}$ \\
\hline $9720-13$ & $2338-U$ & 1970 & 1984 & 200 & Used oil & Removed 7/90 & RIR & $\mathrm{TBD}$ & TBD \\
\hline 9219 & $2395-U$ & 1964 & 1977 & 2,000 & No. 2 fuel oil & Removed $6 / 93$ & TBD & TBD & TBD \\
\hline
\end{tabular}


Table ä.1 (continued)

\begin{tabular}{|c|c|c|c|c|c|c|c|c|c|c|c|}
\hline Location & $\begin{array}{c}\text { Tank } \\
\text { identification } \\
\text { number }\end{array}$ & $\begin{array}{l}\text { Installation } \\
\text { date }\end{array}$ & $\begin{array}{l}\text { Out-of- } \\
\text { service date }\end{array}$ & $\begin{array}{l}\text { Capacity } \\
\text { (gallons) }\end{array}$ & & Contents & Status & $\begin{array}{c}\text { Preliminary } \\
\text { investigation(s) }\end{array}$ & $\begin{array}{r}\text { Envi } \\
\text { ass } \\
() \\
\text { regula }\end{array}$ & $\begin{array}{l}\text { ronmental } \\
\text { essment } \\
\text { date to } \\
\text { lory agency }\end{array}$ & $\begin{array}{l}\text { Corrective } \\
\text { action }\end{array}$ \\
\hline SYDD & $2063-\mathrm{U}$ & 1959 & 1989 & 130 & & il/solvent & Removed 7/89 & IAR, ISCR/FPRR & NA & & NA \\
\hline SYDD & $2328 \mathrm{U}$ & 1959 & 1989 & 475 & & il/solvent & Removed 7/89 & KAR, ISCR/FPRR & NA & & NA \\
\hline SYDD & $2329-U$ & 1959 & 1989 & 475 & & il/solvent & Removed 7/89 & IAR, ISCR/FPRR & NA & & NA \\
\hline \multicolumn{12}{|c|}{ Hazardous Substance USTs } \\
\hline $9767-13$ & $2102-U$ & 1987 & 1992 & 7,500 & & tethanol solid & Removed $1 / 93$ & $\mathrm{CR}$ & NA & & NA \\
\hline $9418-3$ & $2072-U$ & 1943 & 1960 & 45,000 & & $\begin{array}{l}\text { Iranium oxide } \\
\text { olid }\end{array}$ & Exemp! & $\mathrm{CR}$ & NA & & NA \\
\hline Chst. Ridge & $2129-U$ & 1984 & In use & 240,000 & & Iranium oxide & Exempi & NA & NA & & NA \\
\hline \multicolumn{12}{|l|}{ Notes } \\
\hline BMR & \multicolumn{4}{|c|}{$=$ baseline monitoring report } & \multicolumn{4}{|c|}{ FPRR = free product réncval report } & \multicolumn{3}{|c|}{ RIR = Release Investigation Report } \\
\hline CAP & \multicolumn{4}{|c|}{$=$ corrective action plan } & IAR & \multicolumn{3}{|c|}{ initial abatement report } & \multicolumn{3}{|c|}{$\mathrm{CBD}=$ to be determined } \\
\hline CAR & \multirow{2}{*}{\multicolumn{3}{|c|}{$\begin{array}{l}=\text { corrective action report } \\
=\text { conducted under CERCLA }\end{array}$}} & & \multirow{2}{*}{ ISCR } & \multicolumn{3}{|c|}{ initial site characterization report } & SIR & \multicolumn{2}{|c|}{$=$ site investigation report } \\
\hline CERCLA & & & & & & \multicolumn{3}{|c|}{ Not appîicable } & SRS & \multicolumn{2}{|c|}{$=$ site ranking system } \\
\hline $\mathrm{CR}$ & \multicolumn{3}{|c|}{ = closure report } & & N! & \multicolumn{3}{|c|}{ Not investigated } & SSSR & \multicolumn{2}{|c|}{$=$ site-specific standard request } \\
\hline EA & \multicolumn{3}{|c|}{$=$ environmental assessment } & & \multirow{2}{*}{$\begin{array}{l}\text { OE } \\
\text { RCRA }\end{array}$} & \multicolumn{2}{|c|}{ overexcavation } & & SYDD & \multirow[t]{2}{*}{$=$ Salvage } & Ird Drum Deheader \\
\hline EAR & \multicolumn{4}{|c|}{$=$ Environmental Assessment Report } & & \multicolumn{3}{|c|}{$\begin{array}{l}\text { conducied under Resource Conservation and } \\
\text { Recovery Act, Subtitle C }\end{array}$} & & & \\
\hline
\end{tabular}


Table 4.1. Y-12 Plant airborne uranium emission estimates, 1993

\begin{tabular}{|c|c|c|}
\hline \multirow{2}{*}{ Source of emissions } & \multicolumn{2}{|c|}{ Quantity emittec } \\
\hline & $(\mathrm{Ci})^{a}$ & $(\mathrm{~kg})$ \\
\hline \multicolumn{3}{|c|}{ Enriched uranium } \\
\hline Process exhaust (monitored) & 0.029 & 0.4 \\
\hline $\begin{array}{l}\text { Process and laboratory exhaust } \\
\text { (unmonitored) }\end{array}$ & 0.004 & 0.1 \\
\hline $\begin{array}{l}\text { Room exhaust (from health } \\
\text { physics data) }\end{array}$ & 0.017 & 0.3 \\
\hline \multicolumn{3}{|c|}{ Depleted uranium } \\
\hline Process exhaust (monitored) & 0.002 & 2.8 \\
\hline $\begin{array}{l}\text { Process and laboratory exhaust } \\
\text { (unmonitored) }\end{array}$ & 0.002 & 4.2 \\
\hline $\begin{array}{l}\text { Room exhaust (from health } \\
\text { physics data) }\end{array}$ & 0.001 & 1.2 \\
\hline Total & 0.055 & 9.0 \\
\hline
\end{tabular}

${ }^{a} 1 \mathrm{Ci}=3.7 \mathrm{E}+10 \mathrm{~Bq}$. 
Table 4.2. Major sources of radiological airborne emissions (in curies) ${ }^{a}$ at ORNL, 1993

\begin{tabular}{|c|c|c|c|c|}
\hline \multirow{2}{*}{ Isotope } & \multicolumn{4}{|c|}{ Group } \\
\hline & 2026 & 3020 & 3039 & 7911 \\
\hline${ }^{241} \mathrm{Am}$ & $4.05 E-06$ & $1.18 \mathrm{E}-07$ & $1.64 \mathrm{E}-07$ & $2.71 \mathrm{E}-07$ \\
\hline 4: $\mathrm{Ar}$ & & & & $1.80 \mathrm{E}+03$ \\
\hline${ }^{140} \mathrm{Ba}$ & $9.20 \mathrm{E}-0.05$ & & & $3.93 \mathrm{E}-04$ \\
\hline${ }^{7} \mathrm{Be}$ & & & $3.77 \mathrm{E}-0.4$ & \\
\hline${ }^{244} \mathrm{Cm}$ & $6.76 \mathrm{E}-05$ & $1.37 \mathrm{E}-06$ & $7.67 \mathrm{E}-08$ & $3.60 \mathrm{E}-06$ \\
\hline${ }^{60} \mathrm{Co}$ & $3.18 \mathrm{E}-07$ & & $2.20 \mathrm{E}-06$ & \\
\hline${ }^{134} \mathrm{Cs}$ & $2.67 \mathrm{E}-07$ & & $2.30 \mathrm{E}-07$ & $2.59 \mathrm{E}-08$ \\
\hline${ }^{137} \mathrm{Cs}$ & $3.53 \mathrm{E}-0.04$ & $3.40 \mathrm{E}-06$ & $1.43 \mathrm{E}-04$ & $1.26 \mathrm{E}-05$ \\
\hline${ }^{138} \mathrm{Cs}$ & & & & $7.1 \mathrm{E}+01$ \\
\hline${ }^{152} \mathrm{Eu}$ & $2.71 E-07$ & & $1.22 \mathrm{E}-06$ & $1.61 \mathrm{E}-07$ \\
\hline${ }^{154} \mathrm{Eu}$ & $2.35 \mathrm{E}-06$ & $1.39 \mathrm{E}-07$ & & \\
\hline${ }^{155} \mathrm{Eu}$ & $4.93 \mathrm{E}-06$ & $2.51 \mathrm{E}-07$ & & \\
\hline Gross alpha & & $1.55 \mathrm{E}-06$ & $1.48 \mathrm{E}-06$ & \\
\hline Gross beta & & $4.52 \mathrm{E}-06$ & $3.33 E-04$ & $2.16 \mathrm{E}-06$ \\
\hline${ }^{3} \mathrm{H}$ & & & $4.28 \mathrm{E}+01$ & $3.69 \mathrm{E}+01$ \\
\hline${ }^{129} \mathrm{I}$ & & & & $2.52 E-04$ \\
\hline${ }^{130} \mathrm{~T}$ & & & & $5.50 \mathrm{E}-05$ \\
\hline${ }^{131} I$ & $1.60 \mathrm{E}-04$ & & $1.75 \mathrm{E}-05$ & $5.30 \mathrm{E}-02$ \\
\hline${ }^{132} I$ & & & & $9.03 \mathrm{E}-01$ \\
\hline${ }^{133} I$ & & & $1.93 \mathrm{E}-05$ & $2.02 \mathrm{E}-01$ \\
\hline${ }^{1551}$ & $1.18 \mathrm{E}-04$ & $1.03 \mathrm{E}-04$ & $3.44 \mathrm{E}-0.4$ & $4.70 \mathrm{E}-01$ \\
\hline \multicolumn{5}{|l|}{${ }^{188} \mathrm{Ir}$} \\
\hline${ }^{191}$ Os & $1.80 \mathrm{E}-06$ & & $1.68 \mathrm{E}-01$ & $2.93 \mathrm{E}-07$ \\
\hline${ }^{212} \mathrm{~Pb}$ & $4.82 \mathrm{E}-02$ & $3.38 \mathrm{E}-02$ & $1.83 \mathrm{E}-01$ & $9.90 \mathrm{E}-02$ \\
\hline${ }^{238} \mathrm{Pu}$ & $2.46 \mathrm{E}-06$ & $5.22 \mathrm{E}-08$ & $5.31 \mathrm{E}-08$ & $1.93 \mathrm{E}-07$ \\
\hline${ }^{239} \mathrm{Pu}$ & $6.94 \mathrm{E}-06$ & $1.82 \mathrm{E}-07$ & $2.77 \mathrm{E}-07$ & $5.59 \mathrm{E}-07$ \\
\hline${ }^{188} \mathrm{Re}$ & & & $3.78 \mathrm{E}-01$ & \\
\hline${ }^{228} \mathrm{Th}$ & $1.31 \mathrm{E}-06$ & $2.81 \mathrm{E}-0.08$ & $6.39 \mathrm{E}-08$ & $1.44 \mathrm{E}-07$ \\
\hline${ }^{230} \mathrm{Th}$ & $7.00 \mathrm{E}-09$ & $5.08 \mathrm{E}-09$ & $2.69 \mathrm{E}-08$ & $1.75 \mathrm{E}-08$ \\
\hline${ }^{232} \mathrm{Th}$ & $3.40 \mathrm{E}-09$ & $4.86 \mathrm{E}-09$ & $1.42 \mathrm{E}-08$ & $9.95 \mathrm{E}-09$ \\
\hline Total Sr & $1.65 E-05$ & $8.13 E-07$ & $6.58 \mathrm{E}-06$ & $1.98 \mathrm{E}-05$ \\
\hline${ }^{234} \mathrm{U}$ & $7.17 \mathrm{E}-06$ & $2.25 \mathrm{E}-07$ & $3.50 \mathrm{E}-07$ & $8.00 \mathrm{E}-07$ \\
\hline${ }^{235} \mathrm{U}$ & $3.07 E-07$ & $9.54 \mathrm{E}-09$ & $6.25 \mathrm{E}-08$ & $7.67 \mathrm{E}-08$ \\
\hline${ }^{238} \mathrm{U}$ & $1.73 E-07$ & $1.36 \mathrm{E}-08$ & $3.56 \mathrm{E}-08$ & $2.02 \mathrm{E}-08$ \\
\hline${ }^{188} \mathrm{~W}$ & & & $4.30 \mathrm{E}-03$ & \\
\hline${ }^{135} \mathrm{Xe}$ & & & & $5.0 \mathrm{E}+01$ \\
\hline${ }^{138} \mathrm{Xe}$ & & & & $7.1 E+01$ \\
\hline
\end{tabular}

${ }^{a} 1 \mathrm{Ci}=3.7 \mathrm{E}+10 \mathrm{~Bq}$. 
Table 4.3. Minor sources of radiological airborne ernissions (in curles)" at ORNL, 1993

\begin{tabular}{|c|c|c|c|c|c|c|c|c|c|c|c|c|c|c|}
\hline \multirow{2}{*}{ Isotope } & \multicolumn{14}{|c|}{ Group } \\
\hline & 2000 & 2523 & 3018 & 3074 & 3544 & 7025 & 7512 & 7567 & 7569 & 7600 & 7830 & 7852 & 7860 & 7877 \\
\hline${ }^{241} \mathrm{Am}$ & & $2.71 \mathrm{E}-10$ & $5.70 \mathrm{E}-11$ & $1.50 \mathrm{E}-13$ & & & $3.70 \mathrm{E}-09$ & $8.78 \mathrm{E}-10$ & $8.78 \mathrm{E}-10$ & & $3.51 \mathrm{E}-09$ & $6.50 \mathrm{E}-12$ & $6.50 \mathrm{E}-12$ & \\
\hline${ }^{197} \mathrm{Ba}$ & & & & & & & & & & & & & $4.70 \mathrm{E}-09$ & \\
\hline${ }^{\prime} \mathrm{Be}$ & & & $9.20 \mathrm{E}-09$ & $1,40 \mathrm{E}-11$ & $9.64 \mathrm{E}-07$ & & & $8.15 \mathrm{E} .08$ & 8.15E-08 & & $3.26 \mathrm{E}-07$ & & & \\
\hline${ }^{251} \mathrm{Cm}$ & & & $1.00 \mathrm{E}-10$ & & & & & & & & & & & \\
\hline${ }^{214} \mathrm{Cm}$ & & & & $6.20 \mathrm{E}-12$ & $7.62 \mathrm{E}-10$ & & & $1.04 \mathrm{E}-08$ & $1.04 \mathrm{E}-08$ & & $4.17 \mathrm{E}-08$ & $6.71 \mathrm{E}-11$ & $6.70 \mathrm{E}-11$ & \\
\hline${ }^{61} \mathrm{Co}$ & & & & $1.20 \mathrm{E}-13$ & & & $1.26 \mathrm{E}-07$ & $6.40 \mathrm{E}-10$ & $6.40 \mathrm{E}-10$ & & $2.56 \mathrm{E}-09$ & $2.48 \mathrm{E}-11$ & $2.5015-11$ & \\
\hline${ }^{137} \mathrm{Cs}$ & & $8.59 \mathrm{E}-09$ & $6.50 \mathrm{E}-10$ & $3.00 \mathrm{E}-11$ & $1.09 \mathrm{E}-06$ & & & $.3 .63 \mathrm{E}-08$ & $3.63 \mathrm{E}-08$ & & $1.45 \mathrm{E}-07$ & $4.66 \mathrm{E}-09$ & $4.701-09$ & \\
\hline${ }^{152} \mathrm{Eu}$ & & & & $3.80 \mathrm{E}-13$ & & & & & & & & $2.08 \mathrm{E}-11$ & $2.10 \mathrm{~F}-11$ & \\
\hline${ }^{154} \mathrm{Eu}$ & & & & $2.80 \mathrm{E}-13$ & & & & & & & & $1.28 \mathrm{E}-11$ & $1.30 \mathrm{E}-11$ & \\
\hline${ }^{3} \mathrm{H}$ & $6.56 \mathrm{E}+01$ & & & & & $9.35 E+01$ & & $1.95 \mathrm{E}-01$ & $1.95 \mathrm{E}-01$ & & $7.80 \mathrm{E}-01$ & & & \\
\hline${ }^{129} \mathrm{~K}$ & & & & & & & & $1.45 \mathrm{E}-07$ & $1.45 \mathrm{E}-07$ & & $5.80 \mathrm{E}-07$ & & & \\
\hline 131 I & & $1.74 \mathrm{E}-07$ & & & & & & $7.50 \mathrm{E}-06$ & $7.50 \mathrm{E}-06$ & & $3.00 \mathrm{E}-05$ & & & \\
\hline${ }^{132} I$ & & & & & & & & $8.50 \mathrm{E}_{-05}$ & $8.50 \mathrm{E}-05$ & & $3.40 \mathrm{E}-04$ & & & \\
\hline${ }^{13} 3_{1}$ & & & & & & & & $4.25 \mathrm{E}-05$ & $4.25 \mathrm{E}-05$ & & $1.70 \mathrm{E}-04$ & & & 206509 \\
\hline $135 \mathrm{H}$ & & & & & $1.34 \mathrm{E}-07$ & & & $1.26 \mathrm{E}-04$ & $1.26 \mathrm{E}-04$ & & $5.03 \mathrm{E}-04$ & & & $5.50 \mathrm{E}-09$ \\
\hline${ }^{40} \mathrm{~K}$ & & & & 3.10E-12 & & & & & & & & & & \\
\hline${ }^{191} \mathrm{Os}$ & & & & & & & & $3.20 \mathrm{E}-07$ & $3.20 \mathrm{E}-07$ & & $1.28 \mathrm{E}-06$ & & & \\
\hline${ }^{21} \mathrm{~Pb}$ & & & & & 9.13E-08 & & $138 \mathrm{E}-04$ & $1.72 \mathrm{E}-03$ & $1.72 \mathrm{E}-03$ & & $6.86 \mathrm{E}-03$ & & & $1.21 \mathrm{E}-09$ \\
\hline${ }^{288} \mathrm{Pu}$ & & & & $3,90 \mathrm{E}-14$ & & & & $4.08 \mathrm{E}-10$ & $4.08 \mathrm{E}-10$ & & $1.63 \mathrm{E}-09$ & $3.23 \mathrm{E}-12$ & $3.20 \mathrm{E}-12$ & \\
\hline${ }^{279} \mathrm{Pu}$ & & & $3.10 \mathrm{E}-01$ & $2.50 \mathrm{E}-14$ & & & & $9.75 \mathrm{E}-10$ & $9.75 \mathrm{E}-10$ & & $3.90 \mathrm{E}-09$ & $1.69 \mathrm{E}-12$ & $1.70 \mathrm{E}-12$ & \\
\hline${ }^{228} \mathrm{Th}$ & & & $1,80 \mathrm{E}-11$ & $8.80 \mathrm{E}-14$ & $3.15 \mathrm{E}-10$ & & & $3.98 \mathrm{E}-10$ & $3.98 \mathrm{E}-10$ & & $1.59 \mathrm{E}-09$ & $1.59 \mathrm{E}-12$ & $1.600:-12$ & \\
\hline${ }^{210} \mathrm{Th}$ & & $2.11 \mathrm{E}-10$ & $4.20 \mathrm{E}-11$ & $7.00 \mathrm{E}-14$ & $2.19 \mathrm{E}-10$ & & & $7.83 \mathrm{E}-11$ & $7.83 \mathrm{E}-11$ & & $3.13 \mathrm{E}-10$ & $1.72 \mathrm{E}-13$ & $1.70 \mathrm{E}-13$ & \\
\hline${ }^{2 n} \mathrm{Th}$ & & $2.56 \mathrm{E}-10$ & $1.20 \mathrm{E}-10$ & $8.00 \mathrm{E}-14$ & & & & $7.98 \mathrm{E}-11$ & $7.98 \mathrm{E}-11$ & & $3.19 \mathrm{E}-10$ & $7.15 \mathrm{E}-14$ & $7.10 \mathrm{E}-14$ & \\
\hline Total Sr & & $8.74 \mathrm{E}-09$ & & $1.50 \mathrm{E}-11$ & $3.06 \mathrm{E}-07$ & & $1.87 \mathrm{E}-07$ & $5.53 \mathrm{E}-09$ & $5.53 \mathrm{E}-109$ & & $2.21 \mathrm{E}-08$ & $8.83 \mathrm{E}-10$ & $8.80 \mathrm{E}-10$ & \\
\hline${ }^{2 M} \mathrm{U}$ & $3.25 \mathrm{E}-09$ & $6.94 \mathrm{E}-09$ & $1.90 \mathrm{E}-10$ & $5.20 \mathrm{E}-13$ & $1.82 \mathrm{E}-08$ & & $6.17 \mathrm{E}-08$ & $2.46 \mathrm{E}-09$ & $2.46 \mathrm{E}-199$ & & 9.85E-09 & $4.74 \mathrm{E}-12$ & $4.70 \mathrm{E}-12$ & \\
\hline${ }^{2 n S} \mathrm{U}$ & & $1.51 \mathrm{E}-09$ & $3.20 \mathrm{E}-11$ & $3.60 \mathrm{E}-14$ & $1.23 \mathrm{E}-10$ & & $6.59 \mathrm{E}-09$ & $3.83 \mathrm{E}-10$ & $3.83 \mathrm{E}-10$ & & $1.53 \mathrm{E}-1) 9$ & $1.25 \mathrm{E}-13$ & $1.30 \mathrm{E}-13$ & \\
\hline${ }^{2 x} U$ & $1.67 E_{-09}$ & $1.18 \mathrm{E}-09$ & $4.70 \mathrm{E}-11$ & $2.10 \mathrm{E}-13$ & $4.76 \mathrm{E}-09$ & & $7.41 \mathrm{E}-09$ & $3.08 \mathrm{E}-10$ & $3.08 \mathrm{E}-10$ & $2.73 E-05$ & $1.23 \mathrm{E}-09$ & $2.56 \mathrm{E}-13$ & $2.60 E-13$ & \\
\hline
\end{tabular}

${ }^{\circ} \mathrm{Ci}=3.7 \mathrm{E}+10 \mathrm{~Bq}$ 
Table 4.4. Data sources for airborne radioactive emissions from ORNL, 1993

\begin{tabular}{|c|c|c|c|c|c|c|}
\hline Isotope & $\begin{array}{l}\text { Particulate } \\
\text { filter }\end{array}$ & $\begin{array}{l}\text { Probe } \\
\text { wash }\end{array}$ & $\begin{array}{l}\text { Charcoal } \\
\text { cartridge }\end{array}$ & $\begin{array}{c}\text { Silica } \\
\text { gel }\end{array}$ & $\begin{array}{c}\text { Real-time } \\
\text { monitor }\end{array}$ & $\begin{array}{c}\text { Grab } \\
\text { sampler }\end{array}$ \\
\hline${ }^{3} \mathrm{H}$ & & & & $x$ & & \\
\hline${ }^{7} \mathrm{Be}$ & $x$ & & & & & \\
\hline${ }^{60} \mathrm{Co}$ & $x$ & & & & & \\
\hline${ }^{82} \mathrm{Br}$ & & & $x$ & & & \\
\hline${ }^{90} \mathrm{Sr}$ & $x$ & $\mathrm{x}$ & & & & \\
\hline${ }^{129} \mathrm{I}$ & & & $\mathrm{x}$ & & & \\
\hline${ }^{131} I$ & $x$ & $x$ & $x$ & & & \\
\hline${ }^{132} \mathrm{I}$ & & & $x$ & & & \\
\hline${ }^{133} \mathbb{I}$ & & & $x$ & & & \\
\hline${ }^{134} \mathrm{I}$ & & & $x$ & & & \\
\hline${ }^{135} \mathrm{I}$ & & & $x$ & & & \\
\hline${ }^{134} \mathrm{Cs}$ & $x$ & $\mathrm{x}$ & & & & \\
\hline${ }^{137} \mathrm{Cs}$ & $x$ & $x$ & & & & \\
\hline${ }^{138} \mathrm{Cs}$ & & & & & $x$ & $x$ \\
\hline${ }^{140} \mathrm{Ba}$ & $x$ & $x$ & $x$ & & & \\
\hline${ }^{154} \mathrm{Eu}$ & $\mathrm{x}$ & $x$ & & & & \\
\hline${ }^{155} \mathrm{Eu}$ & $x$ & $\mathrm{x}$ & & & & \\
\hline${ }^{191} \mathrm{Os}$ & $\mathrm{x}$ & $x$ & $x$ & & & \\
\hline${ }^{194} \mathrm{Os}$ & $\mathrm{x}$ & $x$ & $x$ & & & \\
\hline${ }^{212} \mathrm{~Pb}$ & & & $x$ & & & \\
\hline${ }^{228} \mathrm{Th}$ & $\mathrm{x}$ & $x$ & & & & \\
\hline${ }^{230} \mathrm{Th}$ & $x$ & $\mathrm{x}$ & & & & \\
\hline${ }^{232} \mathrm{Th}$ & $x$ & & & & & \\
\hline${ }^{234} \mathrm{U}$ & $x$ & $x$ & & & & \\
\hline${ }^{235} \mathrm{U}$ & $\mathrm{x}$ & $\mathrm{x}$ & & & & \\
\hline${ }^{238} \mathrm{U}$ & $\mathrm{x}$ & $\mathrm{x}$ & & & & \\
\hline${ }^{238} \mathrm{Pu}$ & $\mathrm{x}$ & $x$ & & & & \\
\hline${ }^{239} \mathrm{Pu}$ & $x$ & $x$ & & & & \\
\hline${ }^{241} \mathrm{Am}$ & $\mathrm{x}$ & $x$ & & & & \\
\hline${ }^{244} \mathrm{Cm}$ & $\mathrm{x}$ & $x$ & & & & \\
\hline${ }^{41} \mathrm{Ar}$ & & & & & $x$ & $x$ \\
\hline${ }^{135} \mathrm{Xe}$ & & & & & $\mathrm{x}$ & $\mathrm{X}$ \\
\hline${ }^{135 m} \mathrm{Xe}$ & & & & & $x$ & \\
\hline${ }^{138} \mathrm{Xe}$ & & & & & $x$ & $x$ \\
\hline
\end{tabular}


Table 4.5. K-25 Site radionuclide air emission totals (curies), 1993

\begin{tabular}{ccc}
\hline Radionuclide & $\begin{array}{c}\text { TSCA } \\
\text { Incinerator }\end{array}$ & Minor sources \\
\hline${ }^{234} \mathrm{U}$ & $3.8 \mathrm{E}-03$ & $2.1 \mathrm{E}-04$ \\
${ }^{235} \mathrm{U}$ & $1.7 \mathrm{E}-04$ & $9.4 \mathrm{E}-06$ \\
${ }^{238} \mathrm{U}$ & $4.0 \mathrm{E}-03$ & $2.1 \mathrm{E}-04$ \\
${ }^{94} \mathrm{Tc}$ & $1.1 \mathrm{E}-01$ & $1.0 \mathrm{E}-02$ \\
${ }^{237} \mathrm{~Np}$ & $5.6 \mathrm{E}-04$ & $5.8 \mathrm{E}-06$ \\
${ }^{137} \mathrm{Cs}$ & $5.0 \mathrm{E}-03$ & $9.5 \mathrm{E}-08$ \\
${ }^{234 m} \mathrm{~Pa}$ & $2.2 \mathrm{E}-01$ & $1.5 \mathrm{E}-04$ \\
${ }^{238} \mathrm{Pu}$ & $2.5 \mathrm{E}-04$ & $4.2 \mathrm{E}-06$ \\
${ }^{239} \mathrm{Pu}$ & $-5.8 \mathrm{E}-05$ & $7.5 \mathrm{E}-07$ \\
${ }^{228} \mathrm{Th}$ & $3.8 \mathrm{E}-04$ & $3.7 \mathrm{E}-06$ \\
${ }^{230} \mathrm{Th}$ & $4.9 \mathrm{E}-05$ & $9.9 \mathrm{E}-06$ \\
${ }^{232} \mathrm{Th}$ & $1.1 \mathrm{E}-04$ & $2.3 \mathrm{E}-06$ \\
${ }^{234} \mathrm{Th}$ & $1.8 \mathrm{E}-02$ & $1.1 \mathrm{E}-04$ \\
${ }^{104} \mathrm{Cd}$ & $7.6 \mathrm{E}-03$ & 0.0 \\
${ }^{139} \mathrm{Ce}$ & $1.5 \mathrm{E}-07$ & 0.0 \\
${ }^{141} \mathrm{Ce}$ & $2.0 \mathrm{E}-04$ & 0.0 \\
${ }^{57} \mathrm{Co}$ & $1.2 \mathrm{E}-04$ & 0.0 \\
${ }^{60} \mathrm{Co}$ & $4.4 \mathrm{E}-03$ & 0.0 \\
${ }^{40} \mathrm{~K}$ & $4.0 \mathrm{E}-02$ & $1.7 \mathrm{E}-06$ \\
${ }^{106} \mathrm{Ru}$ & $4.5 \mathrm{E}-03$ & $-8.6 \mathrm{E}-07$ \\
${ }^{201} \mathrm{Tl}$ & $1.1 \mathrm{E}-06$ & 0.0 \\
${ }^{88} \mathrm{Y}$ & $3.6 \mathrm{E}-05$ & 0.0 \\
${ }^{28}$ & $4.2 \mathrm{E}-01$ & $1.1 \mathrm{E}-02$ \\
\hline${ }^{40} \mathrm{Cotal}$ & &
\end{tabular}

${ }^{a} \mathrm{Ci}=3.7 \mathrm{E}+10 \mathrm{~Bq}$. 
Table 4.6. ORNL nonradiological airborne emissions, 1993

\begin{tabular}{|c|c|c|c|c|}
\hline \multirow[b]{2}{*}{ Chemical } & \multicolumn{2}{|c|}{ Quantity released } & \multirow[b]{2}{*}{ Major release source } & \multirow[b]{2}{*}{ Basis of estimate } \\
\hline & $1 \mathrm{~b}$ & $\overline{\mathrm{kg}}$ & & \\
\hline \multicolumn{5}{|c|}{ SARA 313 chemicals $^{a}$} \\
\hline Nitric acid & 43 & 20 & Tank emissions & Engineering calculations \\
\hline Sulfuric acid & 0 & 0 & Tank emissions & Engineering calculations \\
\hline \multicolumn{5}{|c|}{ Other large-inventory chemicals ${ }^{b}$} \\
\hline Freon 11 & 15,600 & 7,090 & Refrigerant & Best engineering judgment \\
\hline Freon 12 & 3,073 & 1,397 & Refrigerant & Operating records \\
\hline Freon 22 & 3,545 & 1,611 & Refrigerant & Operating records \\
\hline Freon 113 & 4,700 & 2,136 & Refrigerant, laboratory uses & Inventory records \\
\hline \multicolumn{5}{|c|}{ Steam plant emissions (all calculated emissions) ${ }^{c}$} \\
\hline Particulates & 10,863 & 4,937 & Stack emission & $\begin{array}{l}\text { Engineering calculations } \\
\text { based on emission factors }\end{array}$ \\
\hline $\mathrm{SO}_{x}$ & $1,251,625$ & 568,863 & Stack emission & $\begin{array}{l}\text { Engineering calculations } \\
\text { based on emission factors }\end{array}$ \\
\hline Carbon monoxide & 88,075 & 40,030 & Stack emission & $\begin{array}{l}\text { Engineering calculations } \\
\text { based on emission factors }\end{array}$ \\
\hline $\begin{array}{l}\text { Volatile organic } \\
\text { compounds }\end{array}$ & 2,180 & 991 & Stack emission & $\begin{array}{l}\text { Engineering calculations } \\
\text { based on emission factors }\end{array}$ \\
\hline $\mathrm{NO}_{x}$ & 444,099 & 201,843 & Stack emission & $\begin{array}{l}\text { Engineering calculations } \\
\text { - based on emission factors }\end{array}$ \\
\hline
\end{tabular}

${ }^{a}$ Superfund Amendments and Reauthorization Act, Title III, Section 313.

${ }^{b}$ Fugitive emissions.

'Point-source emissions. 
Table 4.7. Potential emissions of criteria pollutants from the K-25 Site, 1992 and 1993

\begin{tabular}{lcc}
\hline \multirow{1}{1}{ Pollutant } & \multicolumn{2}{c}{$\begin{array}{c}\text { Potential to emit } \\
\text { (tons/year) }\end{array}$} \\
\cline { 2 - 3 } & 1992 & 1993 \\
\hline Particulate matter & 172 & 180 \\
Volatile organic & 262 & 166 \\
$\quad$ compounds & & \\
Sulfur dioxide & 429 & 429 \\
Nitrogen oxides & 226 & 226 \\
Carbon monoxide & 157 & 157 \\
Miscellaneous & 291 & 291 \\
$\quad$ Total & 1537 & 1449 \\
\hline
\end{tabular}

Table 4.8. Estimated K-25 Site emissions of ozonedepleting substances, 1993

\begin{tabular}{lr}
\hline $\begin{array}{c}\text { Ozone-depleting } \\
\text { substance }\end{array}$ & $\begin{array}{c}\text { Estimated emissions } \\
\text { (lb/year) }\end{array}$ \\
\hline CFC-12 & 295 \\
HCFC-22 & 2,175 \\
CFC-113 & $<50$ \\
CFC-114 & 28,500 \\
Halon-1301 & 72 \\
\hline
\end{tabular}

Table 4.9. Estimated air emissions from the K-1501 Steam Plant at the K-25 Site, 1993

\begin{tabular}{lcc}
\hline \multirow{2}{*}{ Pollutant } & \multicolumn{2}{c}{$\begin{array}{c}\text { Emissions } \\
\text { (tons/year) }\end{array}$} \\
\cline { 2 - 3 } & Estimated & Allowable \\
\hline Particulate matter & 1.63 & 18 \\
Sulfur dioxide & 3.97 & 390 \\
Nitrogen oxides & 19.03 & 205 \\
Organics & 1.14 & 8 \\
Carbon monoxide & 20.35 & 138 \\
\hline
\end{tabular}


Table 4.10. Estimated air emissions from the TSCA Incinerator at the K-25 Site, 1993

\begin{tabular}{|c|c|c|c|}
\hline \multirow{2}{*}{ Pollutant } & \multicolumn{2}{|c|}{$\begin{array}{l}\text { Emissions } \\
\text { (tons/year) }\end{array}$} & \multirow{2}{*}{$\begin{array}{c}\text { Percentage } \\
\text { of . } \\
\text { allowable }\end{array}$} \\
\hline & Estimated & Allowable & \\
\hline Lead & 0.00025 & 0.57 & 0.04 \\
\hline Beryllium & 0.000008 & 0.00037 & 2.33 \\
\hline Mercury & 0.0011 & 0.088 & 1.28 \\
\hline Fluorine & 0.00086 & 2.83 & 0.03 \\
\hline Chlorine & 0.054 & 16.12 & 0.33 \\
\hline Sulfur & 0.53 & 38.54 & 1.38 \\
\hline Particulate & 0.010 & 13.14 & 0.08 \\
\hline
\end{tabular}

Table 4.11. Summary of $Y-12$ Plant radioiogical monitoring plan sample requirements

\begin{tabular}{|c|c|c|c|c|}
\hline Outfall No. & Location & Sample frequen & Sample type & $\begin{array}{l}1993 \text { Sum } \\
\text { of DCG } \\
\text { percentage }\end{array}$ \\
\hline \multicolumn{5}{|c|}{ Y.12 Plant wastewater treatment facilities } \\
\hline 501 & Central Pollution Control Facility & $1 /$ week & $\begin{array}{c}\text { Composite during } \\
\text { batch operation }\end{array}$ & 4.72 \\
\hline 502 & West End Treatment Facility & 1/week & 24-hour composite & 2.28 \\
\hline 503 & Steam Plant Wastewater Treatment Facility & $1 /$ week & 24-hour composite & 1.46 \\
\hline 504 & Plating Rinsewater Treatment Facility & 1/week & 24-hour composite & 5.27 \\
\hline 512 & Groundwater Treatment Facility & $1 /$ week & 24-hour composite & 7.90 \\
\hline \multicolumn{5}{|c|}{ Other $Y-12$ Plant point and area source discharges } \\
\hline 142 & Isotope Separation Process & $1 /$ month $^{\alpha}$ & 24-hour composite & 3.29 \\
\hline 301 & Kerr Hollow Quarry & $1 /$ month & 24-hour composite & 1.09 \\
\hline 302 & Rogers Quarry & $1 /$ month & 24-hour composite & 1.04 \\
\hline \multicolumn{5}{|c|}{$Y-12$ Plant instream locations } \\
\hline 304 & Bear Creek, Plant Exit (west) & $1 /$ week & 7-day composite & 4.74 \\
\hline Station 17 & East Fork Poplar Creek, Plant Exit (east) & $1 /$ week & 7-day composite & 3.29 \\
\hline Station 8 & East Fork Poplar Creek, Plant Site & $1 /$ week & 7-day composite & 4.19 \\
\hline
\end{tabular}

${ }^{a}$ Only two samples were collected in 1993; there was no flow for 10 months of the year. 
Table 4.12. Release of uranium from

the Y-12 Plant to the off-site

environment as a liquid effiuent, 1989-93

\begin{tabular}{lrr}
\hline \multirow{2}{*}{ Year } & \multicolumn{2}{c}{ Quantity released } \\
\cline { 2 - 3 } & \multicolumn{1}{c}{$(\mathrm{Ci})^{a}$} & $(\mathrm{~kg})$ \\
\hline & Station 17 \\
1989 & 0.20 & 316 \\
1990 & 0.135 & 197 \\
1991 & 0.162 & 235 \\
1992 & 0.087 & 130 \\
1993 & 0.081 & 134 \\
& Outfall 304 & \\
1989 & 0.138 & 224 \\
1990 & 0.131 & 204 \\
1991 & 0.082 & 159 \\
1992 & 0.060 & 110 \\
1993 & 0.094 & 167 \\
\hline
\end{tabular}

${ }_{1} \mathrm{Ci}=3.7 \mathrm{E}+10 \mathrm{~Bq}$.

Table 4.13. Radionuclide concentrations at K-25 Site surface water effluent discharge points

\begin{tabular}{|c|c|c|c|c|c|c|c|c|}
\hline \multirow{2}{*}{ Isotope } & \multirow{2}{*}{$\begin{array}{l}\text { No. of } \\
\text { samples }\end{array}$} & \multicolumn{4}{|c|}{ Concentration $(\mathrm{pCi} / \mathrm{L})^{a}$} & \multirow{2}{*}{ DCG } & \multirow{2}{*}{$\begin{array}{l}\text { Percentage } \\
\text { of DCG }\end{array}$} & \multirow{2}{*}{$\begin{array}{l}\text { Sum of } \\
\text { fractions of } \\
\text { DCGs }\end{array}$} \\
\hline & & $\operatorname{Max}$ & Min & Median & Average & & & \\
\hline \multicolumn{9}{|c|}{ K-1203 Sewage Treatment Plant } \\
\hline${ }^{234} \mathrm{U}$ & 12 & $2.82 E+01$ & $4.54 \mathrm{E}+00$ & $1.55 \mathrm{E}+01$ & $1.63 E+01$ & $5.00 \mathrm{E}+02$ & $3.27+00$ & $b$ \\
\hline${ }^{235} \mathrm{U}$ & 12 & $4.80 \mathrm{E}+01$ & $-1.24 \mathrm{E}+01$ & $8.60 \mathrm{E}+00$ & $1.02 E+01$ & $6.00 E \div 02$ & $1.70+00^{c}$ & $b$ \\
\hline${ }^{238} \mathrm{U}$ & 12 & $3.54 \mathrm{E}+00$ & $0.00 \mathrm{E}+00$ & $1.35 \mathrm{E}+00$ & $1.74 \mathrm{E}+\infty 0$ & $6.00 \mathrm{E}+02$ & $2.91 \mathrm{E}-01$ & $b$ \\
\hline${ }^{137} \mathrm{Cs}$ & 12 & $7.30 \mathrm{E}+\infty 0$ & $-2.46 \mathrm{E}+01$ & $3.92 \mathrm{E}-01$ & $-2.64 E+00$ & $3.00 \mathrm{E}+03$ & $-8.81 \mathrm{E}-02^{\mathrm{r}}$ & $b$ \\
\hline${ }^{90} \mathrm{Tc}$ & 12 & $5.10 \mathrm{E}+02$ & $-3.24 \mathrm{E}+02$ & $-5.71 E+00$ & $3.66 \mathrm{E}+01$ & $1.00 \mathrm{E}+05$ & $3.66 \mathrm{E}-02$ & $b$ \\
\hline${ }^{237} \mathrm{~Np}$ & 12 & $5.85 \mathrm{E}+00$ & $-3.49 \mathrm{E}-01$ & $1.10 E+00$ & $1.57 \mathrm{E}+00$ & $3.00 E+01$ & $5.24 \mathrm{E}+00$ & $b$ \\
\hline${ }^{238} \mathrm{Pu}$ & 12 & $1.78 \mathrm{E}+\infty 0$ & $-7.30 \mathrm{E}-01$ & $0.00 \mathrm{E}+00$ & 4.43E-01 & $4.00 \mathrm{E}+01$ & $1.11 E+00$ & $b$ \\
\hline${ }^{239} \mathrm{Pu}$ & 12 & $1.05 E+\infty 0$ & $-3.56 \mathrm{E}+00$ & $0.00 \mathrm{E}+00$ & $-2.14 \mathrm{E}-01$ & $3.00 \mathrm{E}+01$ & $-7.13 \mathrm{E}-01$ & $b$ \\
\hline${ }^{228} \mathrm{Th}$ & 12 & $2.50 \mathrm{E}+03$ & $0.00 \mathrm{E}+00$ & $0.00 \mathrm{E}+00$ & $2.91 E+02$ & $4.00 \mathrm{E}+02$ & $7.27 \mathrm{E}+01^{c}$ & $b$ \\
\hline${ }^{234} \mathrm{Th}$ & 12 & $3.35 \mathrm{E}+02$ & $-1.08 E+03$ & $0.00 \mathrm{E}+00$ & $-1.99 \mathrm{E}+02$ & $1.00 \mathrm{E}+04$ & $-1.99 E+00^{\circ}$ & $b$ \\
\hline${ }^{234 m} \mathrm{~Pa}$ & 12 & $1.21 \mathrm{E}+04$ & $0.00 \mathrm{E}+00$ & $0.00 \mathrm{E}+00$ & $1.87 E+03$ & $7.00 \mathrm{E}+04$ & $2.67 \mathrm{E}+00$ & $b$ \\
\hline${ }^{106} \mathrm{Ru}$ & 12 & $1.21 \mathrm{E}+02$ & $0.00 \mathrm{E}+00$ & $0.00 \mathrm{E}+00$ & $1.01 E+01$ & $6.00 \mathrm{E}+03$ & $1.68 \mathrm{E}-01$ & $b$ \\
\hline${ }^{143} \mathrm{Ce}$ & 12 & $3.22 \mathrm{E}+03$ & $0.00 \mathrm{E}+00$ & $0.00 \mathrm{E}+00$ & $3.50 \mathrm{E}+02$ & $3.00 \mathrm{E}+04$ & $1.17 \mathrm{E}+00$ & $b$ \\
\hline${ }^{40} \mathrm{~K}$ & 12 & $4.76 \mathrm{E}+02$ & $0.00 \mathrm{E}+00$ & $0.00 \mathrm{E}+00$ & $3.97 \mathrm{E}+01$ & $7.00 \mathrm{E}+03$ & $5.67 \mathrm{E}-01^{c}$ & $b$ \\
\hline Gross alpha & 12 & $2.54 \mathrm{E}+01$ & $5.39 \mathrm{E}+00$ & $1.40 \mathrm{E}+01$ & $1.43 \mathrm{E}+01$ & $b$ & $b$ & $b$ \\
\hline Gross beta & 12 & $1.71 \mathrm{E}+01$ & $4.67 \mathrm{E}+00$ & $1.00 \mathrm{E}+01$ & $1.04 \mathrm{E}+01$ & $b$ & $b$ & $b$ \\
\hline All listed & & $b$ & $b$ & $b$ & $\dot{b}$ & $b$ & $b$ & $8.61 \mathrm{E}-01^{c}$ \\
\hline
\end{tabular}


Table 4.13 (continued)

\begin{tabular}{|c|c|c|c|c|c|c|c|c|}
\hline \multirow{2}{*}{ Isotope } & \multirow{2}{*}{$\begin{array}{l}\text { No. of } \\
\text { samples }\end{array}$} & \multicolumn{4}{|c|}{ Concentration $(\mathrm{pCi} / \mathrm{L})^{a}$} & \multirow{2}{*}{ DCG } & \multirow{2}{*}{$\begin{array}{l}\text { Percentage } \\
\text { of DCG }\end{array}$} & \multirow{2}{*}{$\begin{array}{c}\text { Sum of } \\
\text { fractions of } \\
\text { DCGs }\end{array}$} \\
\hline & & $\operatorname{Max}$ & $\operatorname{Min}$ & Median & Average & & & \\
\hline
\end{tabular}

K-1407.J treated ejfluents from Central Neutralization Facility and TSCA Incinerator

\begin{tabular}{|c|c|c|c|c|c|c|c|c|}
\hline \\
\hline${ }^{234} \mathrm{U}$ & 12 & $5.24 \mathrm{E}+01$ & $8.05 E+00$ & $2.38 \mathrm{E}+01$ & $2.63 E+01$ & $5.00 \mathrm{E}+02$ & $5.27 \mathrm{E}+00$ & $b$ \\
\hline${ }^{235} \mathrm{U}$ & 12 & $4.85 E+01$ & $-1.77 \mathrm{E}+01$ & $2.22 \mathrm{E}+01$ & $1.52 \mathrm{E}+01$ & $6.00 E+02$ & $2.54 \mathrm{E}+00^{c}$ & $b$ \\
\hline${ }^{236} \mathrm{U}$ & 12 & $1.17 \mathrm{E}+01$ & $0.00 \mathrm{E}+00$ & $3.94 \mathrm{E}+\infty 0$ & $4.07 \mathrm{E}+00$ & $5.00 E+02$ & $8.14 E-01$ & $b$ \\
\hline${ }^{238} \mathrm{U}$ & 12 & $1.70 \mathrm{E}+02$ & $5.84 \mathrm{E}+00$ & $2.78 E+01$ & $3.76 \mathrm{E}+01$ & $6.00 E+02$ & $6.27 \mathrm{E}+00$ & $b$ \\
\hline${ }^{137} \mathrm{Cs}$ & 12 & $2.88 \mathrm{E}+01$ & $-4.30 E+01$ & $5.41 E+00$ & $1.49 \mathrm{E}+00$ & $3.00 \mathrm{E}+03$ & $4.96 \mathrm{E}-02^{c}$ & $b$ \\
\hline${ }^{99} \mathrm{Tc}$ & 12 & $5.05 E+02$ & $-2.30 \mathrm{E}+02$ & $-6.20 E+00$ & $3.96 \mathrm{E}+01$ & $1.00 E+05$ & $3.96 \mathrm{E}-02$ & $b$ \\
\hline${ }^{237} \mathrm{~Np}$ & 12 & $1.33 E+01$ & $-5.51 \mathrm{E}-01$ & $1.29 E+00$ & $3.03 E+00$ & $3.00 E+01$ & $1.01 E+01$ & $b$ \\
\hline${ }^{238} \mathrm{Pu}$ & 12 & $1.45 E+00$ & $-2.19 E+00$ & $3.41 \mathrm{E}-01$ & $1.19 \mathrm{E}-02$ & $4.00 E+01$ & $2.98 \mathrm{E}-02$ & $b$ \\
\hline${ }^{239} \mathrm{Pu}$ & 12 & $7.49 \mathrm{E}-01$ & $-2.54 E+00$ & $0.00 \mathrm{E}+00$ & $-2.94 E-01$ & $3.00 E+01$ & $-9.81 \mathrm{E}-01$ & $b$ \\
\hline${ }^{228} \mathrm{Th}$ & 12 & $0.00 \mathrm{E}+00$ & $-1.01 E+00$ & $0.00 \mathrm{E}+\infty 0$ & $-8.42 \mathrm{E}-02$ & $4.00 \mathrm{E}+02$ & $-2.10 \mathrm{E}-02$ & $b$ \\
\hline${ }^{230} \mathrm{Th}$ & 12 & $2.53 E+\infty 0$ & $0.00 E+00$ & $0.00 \mathrm{E}+00$ & $2.11 \mathrm{E}-01$ & $3.00 \mathrm{E}+02$ & $7.03 E-02$ & $b$ \\
\hline${ }^{232} \mathrm{Th}$ & 12 & $0.00 \mathrm{E}+00$ & $-5.07 \mathrm{E}-01$ & $0.00 \mathrm{E}+00$ & $-4.23 E-02$ & $5.00 \mathrm{E}+01$ & $-8.45 \mathrm{E}-02$ & $b$ \\
\hline${ }^{234} \mathrm{Th}$ & 12 & $1.02 \mathrm{E}+03$ & $-2.52 E+02$ & $0.00 \mathrm{E}+00$ & $2.82 \mathrm{E}+02$ & $1.00 \mathrm{E}+04$ & $2.82 \mathrm{E}+00^{c}$ & $b$ \\
\hline${ }^{234 \mathrm{~m}} \mathrm{~Pa}$ & 12 & $3.26 \mathrm{E}+03$ & $-5.54 E+03$ & $0.00 E+00$ & $-9.83 E+02$ & $7.00 \mathrm{E}+04$ & $-1.40 \mathrm{E}+00^{c}$ & $b$ \\
\hline${ }^{106} \mathrm{Ru}$ & 12 & $1.98 \mathrm{E}+02$ & $0.00 \mathrm{E}+00$ & $0.00 \mathrm{E}+00$ & $1.65 \mathrm{E} \div 01$ & $6.00 \mathrm{E}+03$ & $2.75 E-01$ & $b$ \\
\hline Gross alpha & 12 & $1.19 \mathrm{E}+02$ & $9.81 E+00$ & $3.49 \mathrm{E}+01$ & $4.45 E+01$ & $b$ & $b$ & $b$ \\
\hline Gross beta & 12 & $1.37 \mathrm{E}+02$ & $5.45 \mathrm{E}+00$ & $4.44 E+01$ & $5.38 E+01$ & $b$ & $b$ & $b$ \\
\hline $\begin{array}{l}\text { All listed } \\
\text { isotopes }\end{array}$ & & $b$ & $b$ & $b$ & $b$ & $b$ & $b$ & $2.58 \mathrm{E}-01^{c}$ \\
\hline \multicolumn{9}{|c|}{$K \cdot 1515-C$ filter backwash from the Sanitary Water Treatment Facility } \\
\hline${ }^{234} \mathrm{U}$ & 12 & $8.63 \mathrm{E}+\infty 0$ & $0.00 \mathrm{E}+00$ & $7.38 \mathrm{E}-01$ & $1.64 E+00$ & $5.00 \mathrm{E}+02$ & $3.28 \mathrm{E}-01$ & $b$ \\
\hline${ }^{235} \mathrm{U}$ & 12 & $5.61 E+01$ & $-1.50+01$ & $1.27 \mathrm{E}+01$ & $1.58 \mathrm{E}+01$ & $6.00 \mathrm{E}+02$ & $2.63 \mathrm{E}+00^{c}$ & $b$ \\
\hline${ }^{238} \mathrm{U}$ & 12 & $2.25 E+00$ & $0.00 \mathrm{E}+00$ & $1.07 E+00$ & $8.83 E-01$ & $6.00 \mathrm{E}+02$ & $1.47 \mathrm{E}-01$ & $b$ \\
\hline${ }^{137} \mathrm{Cs}$ & 12 & $1.80 \mathrm{E}+01$ & $-2.11 E+01$ & $7.18 E+00$ & $5.01 \mathrm{E}+00$ & $3.00 \mathrm{E}+03$ & $1.67 \mathrm{E}-01^{c}$ & $b$ \\
\hline${ }^{99} \mathrm{TC}$ & 12 & $3.73 E+02$ & $-2.68 E+02$ & $-5.52 E+01$ & $-1.34 \mathrm{E}+01$ & $1.00 \mathrm{E}+05$ & $-1.34 \mathrm{E}-02$ & $b$ \\
\hline${ }^{237} \mathrm{~Np}$ & 12 & $1.17 \mathrm{E}+00$ & $-1.10 \mathrm{E}+00$ & $0.00 \mathrm{E}+00$ & $-3.22 \mathrm{E}-02$ & $3.00 \mathrm{E}+01$ & $-1.07 \mathrm{E}-0 \mathrm{i}$ & $b$ \\
\hline${ }^{238} \mathrm{Pu}$ & 12 & $2.99 E+00$ & $-2.09 E+00$ & $1.05 E-01$ & $5.61 \mathrm{E}-02$ & $4.00 \mathrm{E}+01$ & $1.40 \mathrm{E}-01$ & $b$ \\
\hline${ }^{239} \mathrm{Pu}$ & 12 & $7.49 \mathrm{E}-01$ & $-3.44 E+00$ & $0.00 \mathrm{E}+00$ & $-4.46 \mathrm{E}-01$ & $3.00 \mathrm{E}+01$ & $-1.49 E+00$ & $b$ \\
\hline${ }^{228} \mathrm{Th}$ & 12 & $1.62 E+03$ & $0.00 E+00$ & $0.00 E \div 00$ & $1.35 \mathrm{E}+02$ & $4.00 \mathrm{E}+02$ & $3.38 \mathrm{E}+01$ & $b$ \\
\hline${ }^{234} \mathrm{Th}$ & 12 & $1.53 E+03$ & $-8.63 E+02$ & $0.00 E+00$ & $1.90 \mathrm{E}+02$ & $1.00 \mathrm{E}+04$ & $1.90 \mathrm{E}+00^{c}$ & $b$ \\
\hline${ }^{234 m} \mathrm{~Pa}$ & 12 & $8.30 E+03$ & $0.00 \mathrm{E}+00$ & $0.00 \mathrm{E}+00$ & $1.42 E+03$ & $7.00 \mathrm{E}+04$ & $2.02 \mathrm{E}+00^{c}$ & $b$ \\
\hline${ }^{106} \mathrm{Ru}$ & 12 & $7.24 E+02$ & $0.00 E+00$ & $0.00 E+00$ & $7.28 \mathrm{E}+01$ & $6.00 \mathrm{E}+03$ & $1.21 \mathrm{E}+00$ & $b$ \\
\hline${ }^{143} \mathrm{Ce}$ & 12 & $1.22 E+03$ & $0.00 \mathrm{E}+00$ & $0.00 E+00$ & $1.02 \mathrm{E}+02$ & $3.00 \mathrm{E}+04$ & $3.39 \mathrm{E}-01$ & $b$ \\
\hline Gross alpha & 12 & $4.86 E+00$ & $-2.10 \mathrm{E}+00$ & $-7.81 E-01$ & $-4.46 \mathrm{E}-01$ & $b$ & $b$ & $b$ \\
\hline Gross beta & 12 & $5.70 E+00$ & $-4.21 E+00$ & $1.52 E+\infty$ & $1.42 \mathrm{E}+00$ & $b$ & $b$ & $b$ \\
\hline $\begin{array}{l}\text { All listed } \\
\text { isotopes }\end{array}$ & & $b$ & $b$ & $b$ & $b$ & $b$ & $b$ & $4.11 \mathrm{E}-01^{\circ}$ \\
\hline
\end{tabular}

${ }^{a} \mathrm{pCi} / \mathrm{L}=3.7 \mathrm{E}-2 \mathrm{~Bq} / \mathrm{L}$.

Not applicable.

This calculated value includes sampling results that are at or below the detection limits and/or below background activities. 
Table 4.14. Radionuclides released to off-site surface waters from the K-25 Site 1993

Effluent discharge points are K-120; K-1407-J, and K-1515-C

\begin{tabular}{ll||ll}
\hline Isotope & $\begin{array}{c}\text { Amount } \\
(\mathrm{Ci})^{a}\end{array}$ & Isotope & $\begin{array}{l}\text { Amount } \\
(\mathrm{Ci})^{a}\end{array}$ \\
\hline${ }^{137} \mathrm{Cs}$ & $1.24 \mathrm{E}-03$ & ${ }^{236} \mathrm{U}$ & $5.76 \mathrm{E}-04$ \\
${ }^{237} \mathrm{~Np}$ & $1.20 \mathrm{E}-03$ & ${ }^{238} \mathrm{U}$ & $6.05 \mathrm{E}-03$ \\
${ }^{238} \mathrm{Pu}$ & $1.62 \mathrm{E}-04$ & ${ }^{228} \mathrm{Th}$ & $2.03 \mathrm{E}-01$ \\
${ }^{234} \mathrm{Pu}$ & $-2.14 \mathrm{E}-04$ & ${ }^{230} \mathrm{Th}$ & $2.39 \mathrm{E}-05$ \\
${ }^{234 m \mathrm{~Pa}}$ & $1.14 \mathrm{E}+00$ & ${ }^{232} \mathrm{Th}$ & $-4.79 \mathrm{E}-06$ \\
${ }^{106} \mathrm{Ru}$ & $3.76 \mathrm{E}-02$ & ${ }^{234} \mathrm{Th}$ & $3.60 \mathrm{E}-02$ \\
${ }^{94} \mathrm{Tc}$ & $3.01 \mathrm{E}-02$ & ${ }^{40} \mathrm{~K}$ & $1.89 \mathrm{E}-02$ \\
${ }^{234} \mathrm{U}$ & $7.69 \mathrm{E}-03$ & ${ }^{143} \mathrm{Ce}$ & $2.01 \mathrm{E}-01$ \\
${ }^{235} \mathrm{U}$ & $1.44 \mathrm{E}-02$ & & \\
\hline
\end{tabular}

${ }^{a} \mathrm{Ci}=3.7 \mathrm{E}+10 \mathrm{Ba}$. 


\begin{tabular}{cccc}
\hline Date & Location & \multicolumn{1}{c}{ Excursion } & Explanation \\
\hline $1 / 7 / 93$ & $\begin{array}{l}\text { Outfall } 503 \text { (Steam } \\
\text { Plant Wastewater } \\
\text { Treatment Facility) }\end{array}$ & $\begin{array}{l}\text { Sample analysis } \\
\text { exceeded holding } \\
\text { time }\end{array}$ & $\begin{array}{l}\text { A provisional result was obtained and reported on the } \\
\text { January 1993 discharge monitoring report for total } \\
\text { suspended solids. }\end{array}$
\end{tabular}

1/13/93 Outfall 10

Unauthorized discharge

[/19/93

Outfall 21

\section{Unauthorized} discharge

2/3/93

Outfall 302 (Rogers Lost sample Quarry)

2/17/93 Outfall 503 (Steam

Plant Wastewater

Treatment Facility)

$3 / 3 / 93$

Outfall 503 (Steam

Plant Wastewater

Treatment Facility)

4/16/93 Outfall 109

Visible foam

$5 / 20 / 93 \quad$ Outfall 512

(Groundwater

Treatment Facility)

6/22/93 Outfall 503 (Steam

Plant Wastewater

Treatment Facility)
Sample concentration

(1.1 $\mathrm{mg} / \mathrm{L}$ iron)

limit

\section{Improper preservation}

of sample

The Plant Wastewater Treatment Facility, was improperly preserved prior to its being analyzed for metals.

A trace amount of foam was observed discharging to East Fork Poplar Creek. Investigations were immediately conducted in some of the buildings tied to Outfall 109, and dye tests were performed at the photo lab and at Medical. No source of the origin of the soapy solution was identified.

Sample concentration A pump located near the final holding tank failed and $(72 \mathrm{mg} / \mathrm{L}$ of oil and released oil into the treated effluent at the Groundwater grease) exceeded Treatment Facility. permit limit

Sample analysis exceeded holding time
The holding lime for total suspended solids is 7 days. One of the composite samples from 503 was not analyzed until the eighth day, resulting in a noncompliance.
Corrective action

Evidence of this noncompliance was not discovered until August 1993 while investigations were being conducted into the events leading to missed holding times. The lab has since improved internal computerized warnings to increase the efficiency of processing samples that have holding times.

The water was turned off immediately when the manhole staned overflowing. Sandbags will be placed around nearby storm drains prior to future sewer-line flushing.

The exact location of the sink responsible for the soapy discharge was not determined. A major effort is under way to reroute sinks and drains illicitly tied to the storm sewer system in buildings 9207 and 9208 to the sanitary sewer. Once the rerouting is completed. incidents of this nature are expected to cease.

The lab has since improved internal computerized warnings to increase the efficiency of processing samples that have holding times.

The facility was evaluated by a wastewater treatment consultant to improve the iron-removal efficiency of the current operation.

This noncompliance occurred because two bottles containing two different preservatives were stored together, looked the same, and were labeled similarly. Techinicians relocated the bottles to prevent confusion, and the lab color-coded the preservative labels to make them more distinctive.

Projects are under way to modify or reroute sink drains.

The facility was taken off line until the pump was replaced. Normal operations were resumed once the new pump was installed on June 4.

The computerized laboratory tracking system for holding times of composite samples has been modified to be more efficient at monitoring holding times. 
Table 4.15 (continued)

\begin{tabular}{|c|c|c|c|c|}
\hline Date & Location & Excursion & Explanation & Corrective action \\
\hline $6 / 29 / 93$ & Citfall 503 & $\begin{array}{l}\text { Sample concentration } \\
\text { ( } 1.3 \mathrm{mg} / \mathrm{L} \text { iron) } \\
\text { exceeded permit } \\
\text { limit }\end{array}$ & $\begin{array}{l}\text { Investigations indicated that corrosion of the sulfuric acid } \\
\text { tank and piping may contribute some iron during the } \\
\text { treatment process. It is unknown if this was the source of } \\
\text { elevated iron at the treatment facility. }\end{array}$ & $\begin{array}{l}\text { The corroded tank and piping were taken out of service and replaced } \\
\text { with a system of polyethylene tanks. }\end{array}$ \\
\hline $8 / 25 / 93$ & $\begin{array}{l}\text { Outfall } 302 \text { (Rogers } \\
\text { Quarry) }\end{array}$ & $\begin{array}{l}\text { Sample analyses } \\
\text { exceeded holding } \\
\text { time }\end{array}$ & $\begin{array}{l}\text { The 7-day holding time for the total suspended solids portion } \\
\text { of the chemical analysis for Outfall } 302 \text { was exceeded. } \\
\text { Incorrect analyses were requested at the laboratory. The } \\
\text { mistake was realized and a correction was made manually, } \\
\text { which risulied in the omission of the request for total } \\
\text { suspended solids. Anaiysis was eventually run for this } \\
\text { sample, but the vata were reported as provisional because } \\
\text { the sample had exceeded the 7-day holding time. }\end{array}$ & $\begin{array}{l}\text { Two unlikely errors in record keeping happened to the same sample, } \\
\text { resulting in an omission of the request for analysis of cotal suspended } \\
\text { solids, which happened to carry a 7-day holding time. Sampling } \\
\text { personnel have been instructed to double-check sample entry } \\
\text { information once it has been entered into the computer. Any changes } \\
\text { in lab requests must be verified by the appropriate personnel. Finally, } \\
\text { laboratory and sampling personnel have improved communication by } \\
\text { meeting weekly. }\end{array}$ \\
\hline $8 / 26 / 93$ & Outfall 21 & Visible oil sheen & $\begin{array}{l}\text { An oil sheen was observed entering the oil/water separator } \\
\text { on East Fork Poplar Creek. An investigation found that the } \\
\text { oil sheen was being emitted from Outfall } 21 \text {. Lab analyses } \\
\text { from samples taken at the outfall indicate that the active } \\
\text { ingredient of the substance is the same ingredient in many } \\
\text { cleaning agents. }\end{array}$ & $\begin{array}{l}\text { The oil skimmer located upstream of Lake Reality collected a large } \\
\text { portion of the visible sheen, and additional booms were set up near } \\
\text { the vicinity of Ouffall } 21 \text {. The oil sheen was containcd on site, and } \\
\text { none of the sheen was observed downstream of the plant. The source } \\
\text { of the sheen was not determined. }\end{array}$ \\
\hline 9/27/93 & Outfall 21 & Visible oil sheen & $\begin{array}{l}\text { A platform lift with a leaking hydraulic line was stationed in } \\
\text { a temporary dike in the Biology area near Building } 9207 . \\
\text { The equipment failure had occurred on the previous Friday } \\
(9 / 26) \text {, and the vehicle was moved to the diked area to } \\
\text { contain the leaking oil while the equipment was waiting to } \\
\text { be repaired. Heavy rain on September } 26 \text { flooded the } \\
\text { temporary dike around the lift, causing residue from the } \\
\text { oil leak to enter a storm drain. }\end{array}$ & $\begin{array}{l}\text { Spill response personnel contained the spill, and booms were placed at } \\
\text { the outfall to contain the sheen. The storm line leading to the outfall } \\
\text { was cleaned. Garage personnel were dispatched to replace the } \\
\text { hydraulic hose, and the equipment was repaired that moming. }\end{array}$ \\
\hline $12 / 17 / 93$ & Outfall 135 & $\begin{array}{l}\text { Unauthorized } \\
\text { discharge }\end{array}$ & $\begin{array}{l}\text { A tank and dike failure resulted in an estimated } 1000 \text { gal of } \\
\text { sodium hypochlorite solution being released from the } \\
\text { leaking dike to the nearby storm sewer. It is believed that } \\
\text { a portion of this solution reached East Fork Poplar Creek } \\
\text { through Outfall 135, resulting in an unauthorized } \\
\text { discharge. }\end{array}$ & $\begin{array}{l}\text { The storm sewer systern south of the leaking dike was plugged once } \\
\text { the release was discovered to limit the total volume of sodium } \\
\text { hypochlorite solution released to East Fork Poplar Creck. The } \\
\text { solution remaining in the dike was pumped into tankers to be } \\
\text { transferred to the K-25 Site for treatment and disposal. The north } \\
\text { storm basin was plugged as an extra precaution, and the dike was } \\
\text { cleaned to remove traces of the spill to prevent subsequent } \\
\text { contanination of rainwater collected in the area. }\end{array}$ \\
\hline
\end{tabular}


Table 4.16. Y-12 Plant NPDES compliance monitoring requirements and record, 1993

\begin{tabular}{|c|c|c|c|c|c|c|c|}
\hline \multirow[b]{2}{*}{$\begin{array}{l}\text { Discharge } \\
\text { point }\end{array}$} & \multirow[b]{2}{*}{$\begin{array}{l}\text { Effluent } \\
\text { parameter }\end{array}$} & \multicolumn{4}{|c|}{ Effluent limits } & \multirow[b]{2}{*}{$\begin{array}{c}\text { Percentage } \\
\text { of } \\
\text { compliance }\end{array}$} & \multirow[b]{2}{*}{$\begin{array}{l}\text { No. of } \\
\text { samples }\end{array}$} \\
\hline & & $\begin{array}{c}\text { Daily } \\
\text { av } \\
(\mathrm{kg} / \mathrm{d})\end{array}$ & $\begin{array}{c}\text { Daily } \\
\max \\
(\mathrm{kg} / \mathrm{d})\end{array}$ & $\begin{array}{c}\text { Daily av } \\
\text { (mg/L) }\end{array}$ & $\begin{array}{c}\text { Daily } \\
\max \\
(\mathrm{mg} / \mathrm{L})\end{array}$ & & \\
\hline \multirow[t]{5}{*}{301 (Kerr Hollow Quarry) } & Lithium & & & & 5.0 & 100 & 20 \\
\hline & pH, standard units & & & $a$ & 8.5 & 100 & 20 \\
\hline & Total suspended solids & & & 30.0 & 50.0 & 100 & 20 \\
\hline & Temperature, ${ }^{\circ} \mathrm{C}$ & & & & 30.5 & 100 & 20 \\
\hline & Zirconium & & & & 3.0 & 100 & 20 \\
\hline \multirow[t]{5}{*}{302 (Rogers Quarry) } & Oil and grease & & & 10.0 & 15.0 & 100 & 52 \\
\hline & pH, standard units & & & $a$ & 8.5 & 100 & 52 \\
\hline & Settleable solids, mL/ & & & & 0.5 & 100 & 52 \\
\hline & Total suspended solids & & & 30.0 & $50.0^{b}$ & $98^{c}$ & 52 \\
\hline & Temperature, ${ }^{\circ} \mathrm{C}$ & & & & 30.5 & 100 & 52 \\
\hline \multirow[t]{2}{*}{304 (Bear Creek) } & Oil and grease & & & 10.0 & 15.0 & 100 & 52 \\
\hline & pH, standard units & & & $a$ & 8.5 & 100 & 52 \\
\hline \multirow{4}{*}{307 (West Borrow Area) $^{d}$} & Temperature, ${ }^{\circ} \mathrm{C}$ & & & & & 100 & 2 \\
\hline & pH, standard units & & & & & 100 & 2 \\
\hline & Oil and grease & & & & & 100 & 2 \\
\hline & Total suspended solids & & & & & 100 & 2 \\
\hline \multirow[t]{4}{*}{308 (East Borrow Area) $^{c}$} & Temperature, ${ }^{\circ} \mathrm{C}$ & & & & & 100 & 4 \\
\hline & pH, standard units & & & & & 100 & 4 \\
\hline & Oil and grease & & & & & 100 & 4 \\
\hline & Total suspended solids & & & & & 100 & 4 \\
\hline \multirow{13}{*}{$\begin{array}{l}501 \text { [Central Pollution } \\
\text { Control Facility }(\mathrm{CPCF}-1)]\end{array}$} & Cadmium, total & 0.07 & 0.19 & 0.26 & 0.69 & 100 & 52 \\
\hline & Chromium total & 0.5 & 0.75 & 1.71 & 2.77 & 100 & 52 \\
\hline & Copper, total & 0.6 & 0.9 & 2.07 & 3.38 & 100 & 52 \\
\hline & Cyanide, total & 0.2 & 0.33 & 0.65 & 1.20 & 100 & 52 \\
\hline & Lead, total & 0.12 & 0.19 & 0.43 & 0.69 & 100 & 52 \\
\hline & Nickel, total & 0.65 & 1.1 & 2.38 & 3.98 & 100 & 52 \\
\hline & Oil and grease & 7.1 & 14.2 & 26.0 & 52.0 & 100 & 52 \\
\hline & $\mathrm{pH}$, standard units & & & $a$ & 9.0 & 100 & 52 \\
\hline & Silver, total & 0.07 & 0.12 & 0.24 & 0.43 & 100 & 52 \\
\hline & Temperature, ${ }^{\circ} \mathrm{C}$ & & & & 30.5 & $10 \hat{0}$ & 52 \\
\hline & Total suspended solids & 8.5 & 16.4 & 31.0 & 60.0 & 100 & 52 \\
\hline & Total toxic organics & & 0.6 & & 2.13 & 100 & 52 \\
\hline & Zinc, total & 0.4 & 0.7 & 1.48 & 2.61 & 100 & 52 \\
\hline \multirow{13}{*}{$\begin{array}{l}502 \text { [West End Treatment } \\
\text { Facility (WETF)] }\end{array}$} & Cadmium, total & 0.07 & 0.019 & 0.26 & 0.69 & 100 & 46 \\
\hline & Chromium, total & 0.5 & 0.75 & 1.71 & 2.77 & 100 & 46 \\
\hline & Copper, total & 0.6 & 0.92 & 2.07 & 3.38 & 100 & 46 \\
\hline & Cyanide, total & 0.2 & 0.33 & 0.65 & 1.20 & 100 & 47 \\
\hline & Lead, total & 0.12 & 0.19 & 0.43 & 0.69 & 100 & 46 \\
\hline & Nickel, total & 0.65 & 1.10 & 2.38 & 3.98 & 100 & 46 \\
\hline & Oil and grease & 7.1 & 14.2 & 26.0 & 52.0 & 100 & 47 \\
\hline & pH. standard units & & & $a$ & 9.0 & 100 & 47 \\
\hline & Silver, total & 0.07 & 0.12 & 0.24 & 0.43 & 100 & 46 \\
\hline & Temperature. ${ }^{\circ} \mathrm{C}$ & & & & 30.5 & 100 & 47 \\
\hline & Total suspended solids & 8.5 & 16.4 & 31.0 & 60.0 & 100 & 46 \\
\hline & Total toxic organics & & 0.6 & & 2.13 & 100 & 13 \\
\hline & Zinc, total & 0.4 & 0.7 & 1.48 & 2.61 & 100 & 46 \\
\hline
\end{tabular}


Table 4.16 (continued)

\begin{tabular}{|c|c|c|c|c|c|c|c|}
\hline \multirow{2}{*}{$\begin{array}{c}\text { Discharge } \\
\text { point }\end{array}$} & \multirow[b]{2}{*}{$\begin{array}{l}\text { Efĩuent } \\
\text { parameter }\end{array}$} & \multicolumn{4}{|c|}{ Effivent limits } & \multirow[b]{2}{*}{$\begin{array}{l}\text { Percentage } \\
\text { of } \\
\text { compliance }\end{array}$} & \multirow[b]{2}{*}{$\begin{array}{l}\text { No. of } \\
\text { samples }\end{array}$} \\
\hline & & $\begin{array}{l}\text { Daily } \\
\text { av } \\
(\mathrm{kg} / \mathrm{d})\end{array}$ & $\begin{array}{l}\text { Daily } \\
\max \\
(\mathrm{kg} / \mathrm{d})\end{array}$ & $\begin{array}{l}\text { Daily av } \\
(\mathrm{mg} / \mathrm{L})\end{array}$ & $\begin{array}{c}\text { Daily } \\
\max \\
(\mathrm{mg} / \mathrm{L})\end{array}$ & & \\
\hline \multirow{8}{*}{$\begin{array}{l}503 \text { (Steam Plant Wastewater } \\
\text { Treatment Facility) }\end{array}$} & Chromium, total & 0.38 & 0.38 & 0.20 & 0.20 & 99 & 156 \\
\hline & Copper, total & 1.89 & 1.89 & 1.0 & 1.0 & $99^{\circ}$ & 156 \\
\hline & lron, total & 1.89 & 1.89 & 1.0 & 1.0 & 98 & 156 \\
\hline & Zinc, total & 1.89 & 1.89 & 1.0 & 1.0 & 99 & 156 \\
\hline & Oil and grease & 28.4 & 37.9 & 15.0 & 20.0 & 100 & 155 \\
\hline & Total suspended solids & 57.0 & 189.0 & 30.0 & 100.0 & $98^{c}$ & 156 \\
\hline & Temperarure, ${ }^{\circ} \mathrm{C}$ & & & & 30.5 & 100 & 155 \\
\hline & $\mathrm{pH}$, standard units & & & $a$ & 9.0 & 100 & 155 \\
\hline $\begin{array}{l}\text { Category I outfalls } \\
\text { (precipitation runoff and } \\
\text { small amounts of } \\
\text { groundwater) }\end{array}$ & $\mathrm{pH}$, standard units & & & $a$ & 8.5 & 100 & 27 \\
\hline \multirow{2}{*}{$\begin{array}{l}\text { Category II outfalls } \\
\text { (cusling waters, condensate. } \\
\text { precipitation runoff, and } \\
\text { building, roof, and founda- } \\
\text { tion drains) }\end{array}$} & $\mathrm{pH}_{\text {。 standard units }}$ & & & $a$ & 8.5 & 100 & 91 \\
\hline & Temperature, ${ }^{\circ} \mathrm{C}$ & & & & & 100 & 91 \\
\hline $\begin{array}{l}\text { Category III outfalls } \\
\text { (process wastewaters) }\end{array}$ & $\mathrm{pH}$, standard units & & & $a$ & 8.5 & 100 & 39 \\
\hline $\begin{array}{l}\text { Category IV outfalls } \\
\text { (untreated process } \\
\text { wastewaters) }\end{array}$ & $\mathrm{pH}$, standard units & & & $a$ & 8.5 & 100 & 92 \\
\hline \multirow{13}{*}{$\begin{array}{l}504 \text { (Plating Rinsewater } \\
\text { Treatment Facility) }\end{array}$} & Cadmium, total & 0.07 & 0.019 & 0.26 & 0.69 & 100 & 3 \\
\hline & Chromium, total & 0.50 & 0.75 & 1.71 & 2.77 & 100 & 3 \\
\hline & Copper, total & 0.60 & 0.92 & 2.07 & 3.38 & 100 & 3 \\
\hline & Cyanide, total & 0.2 & 0.33 & 0.65 & 1.20 & 100 & 3 \\
\hline & Lead, total & 0.12 & 0.19 & 0.43 & 0.69 & 100 & 3 \\
\hline & Nickel, total & 0.65 & 1.10 & 2.38 & 3.98 & 100 & 3 \\
\hline & Oil and grease & 7.1 & 14.2 & 26.0 & 52.0 & 100 & 3 \\
\hline & $\mathrm{pH}$, standard units & & & $a$ & 9.0 & 100 & 3 \\
\hline & Silver, total & 0.07 & 0.12 & 0.24 & 0.43 & 100 & 3 \\
\hline & Temperature, ${ }^{\circ} \mathrm{C}$ & & & & 30.5 & 100 & 3 \\
\hline & Total suspended solids & 8.5 & 16.4 & 31.0 & 60.0 & 100 & 3 \\
\hline & Total toxic organics & & 0.6 & & 2.13 & 100 & 3 \\
\hline & Zinc, total & 0.4 & 0.7 & 1.48 & 2.61 & 100 & 3 \\
\hline \multirow{13}{*}{$\begin{array}{l}\text { 501/504 (combined discharge } \\
\text { from Central Pollution } \\
\text { Control Facility and Plating } \\
\text { Rinsewater Treatment } \\
\text { Facility) }\end{array}$} & Cadmium, total & 0.07 & 0.019 & 0.26 & 0.69 & 100 & 0 \\
\hline & Chromium, total & 0.50 & 0.75 & 1.71 & 2.77 & 100 & 0 \\
\hline & Copper, total & 0.60 & 0.92 & 2.07 & 3.38 & 100 & 0 \\
\hline & Cyanide, total & 0.2 & 0.33 & 0.65 & 1.20 & 100 & 0 \\
\hline & Lead, total & 0.12 & 0.19 & 0.43 & 0.69 & 100 & 0 \\
\hline & Nickel, total & 0.65 & 1.10 & 2.38 & 3.98 & 100 & 0 \\
\hline & Oil and grease & 7.1 & 14.2 & 26.0 & 52.0 & 100 & 0 \\
\hline & pH, standard units & & & $a$ & 9.0 & 100 & 0 \\
\hline & Silver, total & 0.07 & 0.12 & 0.24 & 0.43 & 100 & 0 \\
\hline & Temperature, ${ }^{\circ} \mathrm{C}$ & & & & 30.5 & 100 & 0 \\
\hline & Total suspended solids & 8.5 & 16.4 & 31.0 & 60.0 & 100 & 0 \\
\hline & Total toxic organics & & 0.6 & & 2.13 & 100 & 0 \\
\hline & Zinc, total & 0.4 & 0.7 & 1.48 & 2.61 & 100 & 0 \\
\hline
\end{tabular}




\begin{tabular}{|c|c|c|c|c|c|c|c|}
\hline \multirow[b]{2}{*}{$\begin{array}{l}\text { Discharge } \\
\text { point }\end{array}$} & \multirow[b]{2}{*}{$\begin{array}{l}\text { Effluent } \\
\text { parameter }\end{array}$} & \multicolumn{4}{|c|}{ Effluent limits } & \multirow{2}{*}{$\begin{array}{l}\text { Percentage } \\
\text { of } \\
\text { compliance }\end{array}$} & \multirow[b]{2}{*}{$\begin{array}{l}\text { No. of } \\
\text { samples }\end{array}$} \\
\hline & & $\begin{array}{c}\text { Daily } \\
\text { av } \\
(\mathrm{kg} / \mathrm{d})\end{array}$ & $\begin{array}{l}\text { Daily } \\
\max \\
(\mathrm{kg} / \mathrm{d})\end{array}$ & $\begin{array}{l}\text { Daily av } \\
\text { (mg/L) }\end{array}$ & $\begin{array}{c}\text { Daily } \\
\max \\
(\mathrm{mg} / \mathrm{L})\end{array}$ & & \\
\hline $\begin{array}{l}623 \text { (Steam Plant fly ash } \\
\text { sluce water) }\end{array}$ & $\mathrm{pH}$, standard units & & & $\bar{a}$ & 8.5 & 100 & 25 \\
\hline \multirow{3}{*}{$\begin{array}{l}1506 \text { (9204-3 sump } \\
\text { pump oil) }\end{array}$} & Temperature, ${ }^{\circ} \mathrm{C}$ & & & & 30.5 & 100 & 47 \\
\hline & Oil and grease & & & 10.0 & 15.0 & 100 & 47 \\
\hline & pH, standard units & & & $a$ & 8.5 & 100 & 47 \\
\hline \multirow{3}{*}{$\begin{array}{l}508 \text { (Experimental Mobile } \\
\text { Wastewater Treatment } \\
\text { Facility) }\end{array}$} & Mercury, total & & & 0.002 & 0.004 & $g$ & $a$ \\
\hline & pH, standard units & & & $a$ & 9.0 & $g$ & \\
\hline & Total suspended solids & & & 30.0 & 45.0 & $g$ & \\
\hline \multirow{5}{*}{$\begin{array}{l}510 \text { (Waste Coolant } \\
\text { Processing Facility) }\end{array}$} & Biochemical oxygen demand & 1.33 & 2.65 & & & $g$ & \\
\hline & Oil and grease & & & $15.0^{\circ}$ & 20.0 & $g$ & \\
\hline & $\mathrm{pH}$, standard units & & & $a$ & 9.0 & $g$ & \\
\hline & Temperature, ${ }^{\circ} \mathrm{C}$ & & & & 30.5 & $g$ & \\
\hline & Total suspended solids & & & 30.0 & 50.0 & $g$ & \\
\hline \multirow{4}{*}{$\begin{array}{l}512 \text { (Groundwater } \\
\text { Trearment Facility) }\end{array}$} & Oil and grease & & & $a$ & 15 & 99 & 168 \\
\hline & Iron, total & & & $a$ & 1.0 & 100 & 168 \\
\hline & pH, standard units & & & 6 & 9.0 & 100 & continuous \\
\hline & PCBs & & & & & 100 & 168 \\
\hline \multirow{6}{*}{$\begin{array}{l}\text { Miscellaneous discharges } \\
\text { (cooling tower blowdown) }\end{array}$} & Chromium, total & & & & 1.0 & 100 & 56 \\
\hline & Copper, total & & & 0.5 & 1.0 & 100 & 56 \\
\hline & Free available chlorine & & & 0.2 & 0.5 & 100 & 56 \\
\hline & $\mathrm{pH}$, standard units & & & $a$ & 8.5 & 100 & 56 \\
\hline & Temperature, ${ }^{\circ} \mathrm{C}$ & & & 35 & 38 & 100 & 56 \\
\hline & Zinc, total & & & 0.5 & 1.0 & 100 & 56 \\
\hline \multirow{2}{*}{$\begin{array}{l}\text { Miscellaneous discharges } \\
\text { (demineralizers) }\end{array}$} & pH, standard units & & & $a$ & 8.5 & $g$ & \\
\hline & Total suspended solids & & & 30 & 50 & $g$ & \\
\hline
\end{tabular}

${ }^{a}$ Not appiicable.

${ }^{b}$ Limit not applicable during periods of increased surface runoff resulting from precipitation.

'One analysis was not performed according to appropriate protocol: i.e.. improper presentation, holding-time violation, or lost sample.

Application submitted to add this outfall to the current permit. No timits have been set.

'Analytical hoiding times were exceeded twice (administrative error).

Temperature shall be controlled such that the stream temperature standards delineated in the General Water Quality Criteria for the Definition and Control of Pollution in the Waters of Tennessee, as amended, are not violated as a result of this discharge.

No discharge. 
Table 4.17. Y-12 Plant Category I outfalls

\begin{tabular}{|c|c|}
\hline Outfall No. & Discharge status \\
\hline 1 & Active (to be eliminated) \\
\hline 3 & Active \\
\hline 6 & Active \\
\hline 7 & Active \\
\hline 9 & Active \\
\hline 11 & Active \\
\hline 12 & Eliminated \\
\hline 15 & Active \\
\hline 17 & Active \\
\hline 18 & Active \\
\hline 19 & Active \\
\hline 31 & Manually operated potable water blowdown \\
\hline 32 & Active \\
\hline 41 & Active \\
\hline 44 & Active \\
\hline 45 & Active \\
\hline 57 & Active \\
\hline 62 & Active \\
\hline 86 & Active \\
\hline 101 & Eliminated \\
\hline 102 & No flow (to be eliminated) \\
\hline 108 & No flow (to be eliminated) \\
\hline 127 & Eliminated \\
\hline 134 & Active \\
\hline 136 & Eliminated \\
\hline 138 & Active \\
\hline 140 & Eliminated \\
\hline 145 & Eliminated \\
\hline 146 & Eliminated \\
\hline 149 & Eliminated \\
\hline 151 & Eliminated \\
\hline 152 & Eliminated \\
\hline 153 & Eliminated \\
\hline 155 & Eliminated \\
\hline 156 & Active \\
\hline 159 & Active \\
\hline 161 & $\begin{array}{l}\text { Eliminated because of construction; modified } \\
\text { sampling point established that combines } \\
\text { previous OF } 161 \text { and OF } 232 \text { effluent }\end{array}$ \\
\hline 164 & Eliminated \\
\hline 170 & Active \\
\hline 177 & Eliminated \\
\hline 178 & Active \\
\hline 179 & Eliminated \\
\hline 180 & Active \\
\hline 182 & Active \\
\hline 183 & Active \\
\hline 184 & Active \\
\hline
\end{tabular}


Table 4.17 (continued)

\begin{tabular}{|c|c|}
\hline Outfall No. & Discharge status \\
\hline 186 & Active \\
\hline 193 & Active \\
\hline 194 & Active \\
\hline 195 & Active \\
\hline 196 & Active \\
\hline 197 & Active \\
\hline 198 & Active \\
\hline 199 & Eliminated \\
\hline 200 & Eliminated \\
\hline 202 & Active \\
\hline 205 & Active \\
\hline 206 & Active \\
\hline 207 & Active \\
\hline 208 & $\begin{array}{l}\text { Eliminated because of construction; } \\
\text { modified sampling point established }\end{array}$ \\
\hline 209 & Eliminated \\
\hline 215 & $\begin{array}{l}\text { Eliminated because of construction; } \\
\text { modified sampling point established }\end{array}$ \\
\hline 221 & Active \\
\hline 223 & Active \\
\hline 224 & Active \\
\hline 228 & Eliminated \\
\hline 229 & Eliminated \\
\hline 230 & Eliminated \\
\hline 231 & Eliminated \\
\hline 232 & $\begin{array}{l}\text { Eliminated because of construction; } \\
\text { modified sampling point established that } \\
\text { combines previous OF } 161 \text { and OF } 232 \\
\text { effluent }\end{array}$ \\
\hline 233 & Active \\
\hline 234 & Active \\
\hline 235 & Active \\
\hline 236 & Active \\
\hline 237 & Active \\
\hline 247 & Eliminated \\
\hline 248 & Eliminated \\
\hline
\end{tabular}


Table 4.18. Y-12 Plant Category II outtalls

\begin{tabular}{|c|c|}
\hline Outfall No. & Discharge stanus \\
\hline 13 & Manually operated potable water line flushing \\
\hline 16 & Active \\
\hline 20 & Active \\
\hline 23 & Active \\
\hline 24 & Eliminated \\
\hline 25 & Eliminated \\
\hline 26 & Eliminated \\
\hline 27 & No flow (to be eliminated) \\
\hline 28 & Eliminated \\
\hline 29 & Eliminated \\
\hline 30 & Eliminated \\
\hline 35 & No flow (to be eliminated) \\
\hline 40 & Eliminated \\
\hline 43 & Eliminated \\
\hline 46 & Active \\
\hline 53 & No flow (to be eliminated) \\
\hline 54 & Active \\
\hline 58 & Active \\
\hline 59 & Eliminated \\
\hline 60 & Eliminated \\
\hline 66 & Active \\
\hline 68 & Active \\
\hline 69 & Eliminated \\
\hline 73 & Active \\
\hline 74 & Eliminated \\
\hline 75 & Eliminated \\
\hline 76 & Eliminated \\
\hline 77 & Active \\
\hline 78 & Eliminated \\
\hline 79 & Eliminated \\
\hline 80 & Eliminated \\
\hline 81 & Eliminated \\
\hline 84 & Eliminated \\
\hline 87 & Active \\
\hline 92 & Eliminated \\
\hline 93 & Eliminated \\
\hline 94 & Eliminated \\
\hline 95 & Eliminated \\
\hline 96 & Eliminated \\
\hline 98 & Active \\
\hline 100 & Eliminated \\
\hline 111 & No flow (to be eliminated) \\
\hline 112 & Eliminated \\
\hline 115 & Eliminated \\
\hline 117 & Active \\
\hline 118 & Eliminated \\
\hline 119 & Eliminated \\
\hline 120 & Eliminated \\
\hline
\end{tabular}


Table 4.18 (continued)

\begin{tabular}{|c|c|}
\hline Outfall No. & Discharge status \\
\hline 123 & Eliminated \\
\hline 124 & Eliminated \\
\hline 131 & Eliminated \\
\hline 133 & Active \\
\hline 137 & Eliminated \\
\hline 144 & Outfall inaccessible (security) \\
\hline 171 & Eliminated \\
\hline 172 & $\begin{array}{l}\text { Alternate sampling point established for } \\
\text { Building } 9720-5 \text { roof drain }\end{array}$ \\
\hline 173 & Eliminated \\
\hline 174 & Eliminated \\
\hline 175 & Eliminated \\
\hline 185 & Active \\
\hline 188 & Eliminated \\
\hline 201 & Active \\
\hline 203. & Active \\
\hline 204 & Active \\
\hline 210 & No flow (to be eliminated) \\
\hline 212 & Eliminated \\
\hline 213 & Active \\
\hline 214 & Eliminated \\
\hline 216 & Eliminated \\
\hline 217 & No flow (to be eliminated) \\
\hline 218 & Eliminated \\
\hline 219 & Eliminated \\
\hline 220 & Eliminated \\
\hline 226 & Eliminated \\
\hline 238 & Eliminated \\
\hline 239 & Eliminated \\
\hline 240 & Active \\
\hline 241 & Eliminated \\
\hline 243 & Eliminated \\
\hline 244 & Eliminated \\
\hline 245 & Eliminated \\
\hline 246 & Eliminated \\
\hline
\end{tabular}


Table 4.19. Y-12 Plant Category III outtalls

\begin{tabular}{cl}
\hline Outfall No. & L scharge status \\
\hline 2 & Active \\
71 & Active \\
135 & Active \\
147 & Active \\
150 & Active \\
157 & Active \\
160 & Active \\
162 & Eliminated \\
163 & Active \\
166 & Eliminated \\
168 & Eliminated \\
169 & Active \\
181 & Active \\
191 & Eliminated \\
192 & Active \\
\hline
\end{tabular}


Table 4.20. Y-12 Plant Category IV outtalls

\begin{tabular}{|c|c|c|c|}
\hline Outfall No. & Location/Building & Description & Permit status \\
\hline 401 & 92044 & Dye penetrant & Treated at on-site treatment facility \\
\hline 402 & $9204-2$ & Steam condensate & Actively discharging \\
\hline 403 & 9818 & $\begin{array}{l}\text { Overhead, steam condensate, } \\
\text { cooling water }\end{array}$ & Treated at on-site treatment facility \\
\hline 404 & $9201-5 N$ & $X$-ray & Routed to the sanitary sewer \\
\hline 405 & Sanitary landfill 2 & Leachate pond & Actively discharging \\
\hline 406 & $9201-1$ & Plasma torch wastewater & Treated at on-site treatment facility \\
\hline 407 & $9201-4$ & ECM sink rinses, Room 11 & Eliminated \\
\hline 408 & 9202 & Catch basin & Actively discharging \\
\hline 409 & $9201-5$ & Dye penetrant shower rinse & Treated at on-site treatment facility \\
\hline 410 & $9201-5$ & Dye penetrant sink rinse & Routed to the sanitary sewer \\
\hline 411 & $9204-2 E$ & $\mathrm{X}$-ray & Routed to the sanitary sewer \\
\hline 412 & $9204-4$ & $\mathrm{X}$-ray, Room 001B, east X-omat & Routed to the sanitary sewer \\
\hline 413 & $9204-4$ & X-ray, Room 001B, west X-omat & Routed to the sanitary sewer \\
\hline 414 & 9981 & X-ray & Routed to the sanitary sewer \\
\hline 415 & $9201-5$ & $X$-ray & Eliminated \\
\hline 416 & $9204-4$ & X-ray, hand developer & Routed to the sanitary sewer \\
\hline 417 & $9201-4$ & ECM sink rinses, Room 21 & Eliminated \\
\hline 418 & $9201-3$ & X-ray & Routed to the sanitary sewer \\
\hline 419 & 9737 & Planting shop rinsewater & Eliminated \\
\hline 420 & 9980 & $X$-ray & Routed to the sanitary sewer \\
\hline 421 & 9737 & Plating shop rinsewater & Eliminated \\
\hline 422 & 9737 & Plating shop rinsewater & Eliminated \\
\hline
\end{tabular}


Table 4.21. Y-12 Plant 300-series NPDES outfalls-perimeter outfalis

\begin{tabular}{|c|c|c|}
\hline Ou: fall No. & Description & Permit stans \\
\hline 301 & Kerr Hollow Quarty & $\begin{array}{l}\text { Undergoing RCRA Closure: } \\
\text { periodically discharges }\end{array}$ \\
\hline 302 & Rogers Quarry & Actively discharging \\
\hline 303 & New Hope Pond & Closed under RCRA \\
\hline 304 & Bear Creek at Highway 95 & Actively discharging \\
\hline 305 & Oil Retention Pond 1 & Closed under RCRA \\
\hline 305 & Oil Retention Pond 2 & Closed under RCRA \\
\hline
\end{tabular}

Tabie 4.22. Y-12 Plant 500-series NPDES outfalis-Wastewater Treatment Facilities

\begin{tabular}{cll}
\hline Ourfall No. & \multicolumn{1}{c}{ Description } & \multicolumn{1}{c}{ Permit status } \\
\hline 501 & Central Pollution Control Facility & Actively discharging \\
502 & West End Treatment Facility & Actively discharging \\
503 & Steam Plant Wastewater Treatment Facility & Actively discharging \\
504 & Plating Rinsewater Treatment Facility & Actively discharging \\
505 & ORNi Biology Wastewater Treatment Facility & Never constructed \\
506 & $9204-3$ ORNL OilWwater Separator & Eliminated; discharge collected \\
507 & S-3 Treatment Facility & No Ionger in operation \\
508 & Mobile Wastewater Treatment Unit & No longer in operation \\
510 & Waste Coolant Processing Facility & Pretreatment facility for OFs $501 \& 502$ \\
512 & Groundwater Treatment Facility & Actively discharging \\
\hline
\end{tabular}


Table 4.23. Y-12 Plant outfalls not specifically enumerated on the current NPDES permit

\begin{tabular}{|c|c|}
\hline Outfall No. & Discharge status \\
\hline 4 & Active \\
\hline 8 & Active \\
\hline 10 & Active \\
\hline 14 & Active \\
\hline 21 & Active \\
\hline 22 & No flow (alternate sampling at OF 21) \\
\hline 33 & Active \\
\hline 34 & Active \\
\hline 36 & No flow (to be eliminated) \\
\hline 39 & No flow (to be eliminated) \\
\hline 42 & Active \\
\hline 47 & Active \\
\hline 48 & Active \\
\hline 49 & Active \\
\hline 50 & Eliminated \\
\hline 51 & Active \\
\hline 52 & Eliminated \\
\hline 55 & Active \\
\hline 56 & Eliminated \\
\hline 63 & Active \\
\hline 64 & Active \\
\hline 65 & Eliminated \\
\hline 67 & Active \\
\hline 72 & Eliminated \\
\hline 82 & Eliminated \\
\hline 83 & Active \\
\hline 85 & Eliminated \\
\hline 88 & Active \\
\hline 89 & Eliminated \\
\hline 90 & Eliminated \\
\hline 91 & Eliminated \\
\hline 97 & No flow (to be eliminated) \\
\hline 99 & Active \\
\hline 103 & Eliminated \\
\hline 104 & Eliminated \\
\hline 105 & Eliminated \\
\hline 106 & Eliminated \\
\hline 107 & Eliminated \\
\hline 109 & Active \\
\hline 110 & Active \\
\hline 113 & Active \\
\hline 114 & Active \\
\hline 116 & Eliminated \\
\hline 121 & No flow (to be eliminated) \\
\hline 122 & Active \\
\hline 125 & Active \\
\hline 126 & Active \\
\hline
\end{tabular}


Table 4.23 (continued)

\begin{tabular}{ll}
\hline Outfall No. & \multicolumn{1}{c}{ Discharge status } \\
\hline 128 & Eliminated \\
132 & No flow (to be eliminated) \\
142 & Active \\
211 & No flow (to be eliminated) \\
$307^{b}$ (West & \\
Borrow Area) & \\
$308^{b}$ (East & \\
Borrow Area) & \\
$309^{b}$ (Y-12 Plant & \\
Industrial Waste & \\
Landfill) IV & \\
\hline
\end{tabular}

These outfalls were not specifically enumerated on the current NPDES permit. Sampling is being performed quarterly for flow, temperature, $\mathrm{pH}$, biological oxygen demand, chemical oxygen demand, total organic carbon. total suspended solids, and ammonia as nitrogen. These sample results will be attached to the monthly DMR for the month in which the samples were taken.

'These outfalls are monitored once per calendar guarter for flow', $\mathrm{pH}$, ammonia, total suspended solids, total organic carbon, chemical oxygen demand, biological oxygen demand, color, and oil and grease. Because of the inaccessibility of these outfalls, they are sometimes sampled while discharge is not taking place. These samples should represent discharge that has or will occur in that quarter. 
Table 4.24. Miscellaneous Y-12 Plant source discharges"

\begin{tabular}{cl}
\hline Ourfall No. & \multicolumn{1}{c}{ Location } \\
\hline 602 & Cooling Tower $9409-2$ \\
604 & Cooling Tower $9409-4$ \\
606 & Cooling Tower $9409-6$ \\
610 & Cooling Tower $9409-10$ \\
612 & Cooling Tower $9409-12$ \\
613 & Cooling Tower $9409-13$ \\
615 & Cooling Tower $9409-15$ \\
616 & Cooling Tower $9409-16$ \\
617 & Cooling Tower $9409-17$ \\
618 & Cooling Tower $9409-18 / 31$ \\
619 & Cooling Tower $9409-19$ \\
620 & Cooling Tower $9409-20$ \\
622 & Cooling Tower $9409-22$ \\
624 & Cooling Tower $9409-24$ \\
626 & Cooling Tower $9409-26$ \\
628 & Cooling Tower $9409-28 / 29$ \\
630 & Cooling Tower $9409-30$ \\
632 & Cooling Tower $9409-32$ \\
634 & Cooling Tower $9409-23$ \\
\hline
\end{tabular}

${ }^{a}$ Sources are recirculating cooling water, cooling tower blowdown, and cleaning wastes originating at space cooling facilities. Flows from these facilities are calculated using operational sampie resuits to determine the blowdown for that time period. Tower blowdown is valved directly from the retum line. Samples are taken at the tower water return line; therefore, results may be reported when there was no blowdown flow.

Table 4.25. Permit status of miscellaneous Y-12 Plant source discharges

\begin{tabular}{cll}
\hline Outfall No. & \multicolumn{1}{c}{ Location } & \multicolumn{1}{c}{ Permit status } \\
\hline 702 & Building 9201-2 demineralizer & Eliminated \\
703 & Building 9731 demineralizer & Eliminated \\
704 & Building 9204-1 vapor blaster & Routed to the sanitary sewer \\
\hline
\end{tabular}


Table 4.26. Y-12 Plant Discharge Point 142 (a)

(Summed DCG percentage $=3.29$.)

\begin{tabular}{|c|c|c|c|c|c|c|c|c|c|}
\hline \multirow[b]{2}{*}{ Parameter } & \multirow{2}{*}{$\begin{array}{c}\text { Number of } \\
\text { samples }\end{array}$} & \multicolumn{5}{|c|}{ Concentration } & & \multirow{2}{*}{$\begin{array}{c}\text { Standard } \\
\text { error }\end{array}$} & \multirow{2}{*}{$\begin{array}{c}\text { Percentage } \\
\text { of DCG }\end{array}$} \\
\hline & & $\operatorname{Max}$ & +1. & Min & $+1-$ & Median & $+\%$ & & \\
\hline Alpha activity (pCi/L) & 2 & 11 & 6.9 & -0.40 & 0.22 & 5.3 & $\mathrm{~b}$ & 5.7 & $b$ \\
\hline Americium-241 ( $\mathrm{pCi} / \mathrm{L})$ & 2 & 0.53 & 0.49 & 0.31 & 0.50 & 0.42 & b & 0.11 & 1.40 \\
\hline Beta activity (pCi/L) & 2 & 7.1 & 5.2 & -3.0 & 0.68 & 2.1 & o & 5.1 & $b$ \\
\hline Neptunium-237 (pCi/L) & 2 & 0.07 & 0.08 & 0.052 & 0.10 & 0.06 & $b$ & 0.01 & 0.2 \\
\hline Plutonium-238 (pCi/L) & 2 & 0.04 & 0.08 & -0.81 & 0.39 & -0.4 & $b$ & 0.4 & 0.0 \\
\hline Plutonium-239/240 (pCi/L) & 2 & 0.08 & 0.12 & -0.81 & 0.12 & -0.4 & $\mathrm{~b}$ & 0.4 & 0.0 \\
\hline Radium-226 ( $\mathrm{pCi} / \mathrm{L})$ & 2 & -0.14 & 0.028 & -1.6 & 0.31 & -0.87 & $\mathrm{~b}$ & 0.73 & 0.00 \\
\hline Radium-228 ( $\rho \mathrm{Ci} / \mathrm{L})$ & 2 & -0.17 & 0.017 & -1.8 & 0.20 & -0.99 & $b$ & 0.82 & 0.00 \\
\hline Strontium-90 (pCi/L) & 2 & 3.1 & 2.0 & -4.1 & 2.5 & -0.50 & $\mathrm{~b}$ & 3.6 & 0.00 \\
\hline Technetium-99(pCi/mL) & 2 & 0.026 & 0 & 0.01 & 0.01 & 0.02 & $b$ & 0.01 & 0.02 \\
\hline Thorium, total (mg $/$ ) & 2 & $<0.003$ & $b$ & $<0.003$ & $b$ & $<0.003$ & $b$ & $b$ & $b$ \\
\hline Thorium-228 (pCi/L) & 2 & 0.25 & 0.28 & 0.13 & 0.38 & 0.19 & $\mathrm{~b}$ & 0.060 & 0.048 \\
\hline Thorium-230 (pCi/L) & 2 & 0.24 & 0.21 & 0.13 & 0.18 & 0.19 & b & 0.055 & 0.062 \\
\hline Thorium-232 (pCi/L) & 2 & 0.13 & 0.18 & 0.05 & 0.12 & 0.09 & b & 0.04 & 0.2 \\
\hline Thorium-234 (pCi/L) & 2 & 0.99 & 0.38 & 0.16 & 0.18 & 0.58 & $b$ & 0.42 & 0.0058 \\
\hline Tritium (pCi/l) & 2 & 130 & 170 & 34 & 180 & 82 & $b$ & 48 & 0.0041 \\
\hline Uranium, total (mg/L) & 2 & 0.001 & $b$ & 0.001 & b & 0.001 & $b$ & $b$ & b \\
\hline Uranium-234 ( $\mathrm{pCi} / \mathrm{L})$ & 2 & 8.6 & 1.9 & 0.80 & 0.39 & 4.7 & b & 3.9 & 0.94 \\
\hline Uranium-235 (pCi/L) & 2 & 0.22 & 0.18 & 0.14 & 0.16 & 0.18 & b & 0.040 & 0.030 \\
\hline Uranium-235 (\%) & 1 & 1.9 & 3.4 & 0.39 & 3.4 & 0 & b & $b$ & $b$ \\
\hline Uranium-236 (pCi/L) & 0 & 0 & 0 & 0 & 0 & 0 & b & $b$ & 0 \\
\hline Uranium-238 (pCill) & 2 & 0.99 & 0.38 & 0.16 & 0.18 & 0.58 & $b$ & 0.42 & 0.10 \\
\hline
\end{tabular}

(a) There was no flow for 10 months of the year. Average flows for the 2 months that were sampled was 1902 gpd.

(b) Not applicable. 
Table 4.27. Y-12 Plant Station 8, radiological data summary (a)

(Summed DCG percentage $=4.19$ )

\begin{tabular}{|c|c|c|c|c|c|c|c|c|c|}
\hline \multirow[b]{2}{*}{ Parameter } & \multirow{2}{*}{$\begin{array}{l}\text { Number of } \\
\text { samples }\end{array}$} & \multicolumn{6}{|c|}{ Concentration } & \multirow{2}{*}{$\begin{array}{l}\text { Standard } \\
\text { error }\end{array}$} & \multirow{2}{*}{$\begin{array}{c}\text { Percentage } \\
\text { of DCG }\end{array}$} \\
\hline & & $\operatorname{Max}$ & $+\%$ & Min & +1 & Median & $+\%$ & & \\
\hline Alpha activity (pCi/L) & 50 & 45 & 13 & -1.6 & 17 & 14 & b & 1.6 & b \\
\hline Americium-24l (pCi/L) & 50 & 0.88 & 0.68 & -0.25 & 0.27 & 0.060 & b & 0.032 & 0.20 \\
\hline Beta activity (pCi/L) & 50 & 23 & 7.5 & -7.1 & 14 & 11 & b & 1.0 & b \\
\hline Neptunium-237 (pCi/L) & 50 & 26 & 2.4 & -0.22 & 0.15 & 0.082 & $b$ & 0.52 & 0.27 \\
\hline Plutonium-238 (pCi/L) & 50 & 0.40 & 0.55 & -0.32 & 0.42 & 0 & $b$ & 0.020 & 0 \\
\hline Plutonium-239/240 (pCi/) & 50 & 1.2 & 0.59 & -0.30 & 0.31 & 0 & $\mathrm{~b}$ & 0.030 & 0 \\
\hline Radium-226 (pCi/L) & 49 & 1.3 & 0.30 & -2.4 & 0.60 & 0.020 & $b$ & 0.090 & 0.020 \\
\hline Radium-228 (pCi/L) & 49 & 2.8 & 0.27 & -1.8 & 2.5 & -0.29 & b & 0.13 & 0.00 \\
\hline Strontium-90 (pCi/L) & 50 & 16 & 12 & -11 & 12 & -0.50 & $b$ & 0.74 & 0.00 \\
\hline Technetium- $99(\mathrm{pCi} / \mathrm{mL})$ & 50 & 0.46 & 0.01 & -0.068 & 0.01 & 0.017 & $b$ & 0.010 & 0.017 \\
\hline Thoriurn, total (mg/L) & 49 & 0.027 & b & $<0.003$ & $b$ & $<0.003$ & b & 0.001 & $b$ \\
\hline Thorium-228 (pCi几) & 50 & 2.8 & 2.4 & -0.49 & 0.63 & 0.37 & b & 0.086 & 0.093 \\
\hline Thorium-230 (pCi/L) & 50 & 3.6 & 2.3 & -0.34 & 0.58 & 0.30 & b & 0.092 & 0.10 \\
\hline Thorium-232 (pCi/L) & 50 & 0.66 & 0.94 & -0.075 & 0.15 & 0.078 & b & 0.019 & 0.16 \\
\hline Thorium-234 (pCi/L) & 50 & 31 & 4.8 & 2.1 & 0.75 & 8.5 & b & 0.94 & 0.85 \\
\hline Tritium (pCi/L) & 50 & 790 & 190 & -240 & 190 & 360 & b & 28 & 0.018 \\
\hline Uranium, total (mg/L) & 50 & 0.079 & $b$ & 0.008 & $b$ & 0.02 & $b$ & 0.003 & $b$ \\
\hline Uranium-234 (pCi/L) & 50 & 15 & 3.6 & 1.5 & 0.63 & 5.0 & $\mathrm{~b}$ & 0.36 & 1.0 \\
\hline Uranium-235 (\%) & 50 & 1.1 & $b$ & 0.26 & $b$ & 0.43 & $b$ & 0.021 & $b$ \\
\hline Uranium-235 (pCi/L) & 50 & 1.0 & 0.44 & -0.04 & 0.07 & 0.3 & $b$ & 0.03 & 0.05 \\
\hline Uranium-236 (pCi/L) & 47 & 0.88 & 0.48 & -0.043 & 0.16 & 0.13 & 0.15 & 0.027 & 0.026 \\
\hline Uranium-238 ( $\mathrm{pCi} / \mathrm{L})$ & 50 & 31 & 4.8 & 2.1 & 0.75 & 8.5 & $b$ & 0.94 & 1.4 \\
\hline
\end{tabular}

(a)Flow not available.

(b)Not applicable. 
Table 4.28. Y-12 Plant Instream Monitoring Site, Upstream Drum 30om

(Summed DCG percentage $=2.92$ )

\begin{tabular}{|c|c|c|c|c|c|c|c|c|c|}
\hline \multirow[b]{2}{*}{ Parameter } & \multirow{2}{*}{$\begin{array}{c}\text { Number of } \\
\text { samples }\end{array}$} & \multicolumn{6}{|c|}{ Concentration } & \multirow{2}{*}{$\begin{array}{c}\text { Standard } \\
\text { error }\end{array}$} & \multirow{2}{*}{$\begin{array}{c}\text { Percentage } \\
\text { of DCG }\end{array}$} \\
\hline & & $\operatorname{Max}$ & $+\%$ & Min & +1 & Median & +1 & & \\
\hline Alpha activity ( $\mathrm{pCi} / \mathrm{L}$ ) & 51 & 76 & 16 & -7.2 & 10 & 9.8 & 7.4 & 1.8 & a \\
\hline Americium-241 (pCi/L) & 51 & 0.66 & 0.70 & -0.26 & 0.45 & 0.050 & 0.38 & 0.026 & 0.17 \\
\hline Beta activity (pCi/L) & 51 & 260 & 37 & 0 & 0 & 8.7 & .6 .0 & 5.1 & a \\
\hline Neptunium-237 (pCi/L) & 51 & 0.93 & 0.45 & -0.33 & 0.20 & 0.10 & 0.12 & 0.028 & 0.34 \\
\hline Plutonium-238 (pCi/L) & 48 & 0.45 & 0.40 & -0.34 & 0.24 & -0.010 & a & 0.023 & 0.00 \\
\hline Plutonium-239/240 (pCi/L) & 48 & 0.51 & 0.32 & -0.16 & 0.18 & 0 & a & 0.015 & 0 \\
\hline Radium-226 (pCi/L) & 51 & 1.9 & 0.29 & -0.52 & 0.12 & 0.23 & 0.05 & 0.063 & 0.23 \\
\hline Radium-228 (pCi/L) & 51 & 2.7 & 0.28 & -2.8 & 0.34 & -0.020 & 0.06 & 0.14 & 0.00 \\
\hline Strontium-90 (pCi/L) & 51 & 6.7 & 1.7 & -6.1 & 2.1 & 0 & 0.06 & 0.40 & 0.023 \\
\hline Technetium-99 (pCi/mL) & 51 & 0.048 & 0.01 & -0.04 & 0.01 & 0.02 & a & 0.003 & 0.02 \\
\hline Thorium, total (mg $L$ ) & 46 & 0.012 & $\mathbf{a}$ & 0.002 & a & $<0.003$ & a & 0.0002 & a \\
\hline Thorium-228 (pCi/L) & 51 & 1.4 & 0.87 & -0.53 & 0.35 & 0.23 & 0.56 & 0.053 & 0.058 \\
\hline Thorium-230 (pCi/L) & 51 & 3.9 & 3.9 & -0.075 & 0.28 & 0.29 & 0.27 & 0.086 & 0.097 \\
\hline Thorium-232 (pCi/L) & 51 & 0.66 & 0.56 & -0.17 & 0.20 & 0.060 & 0.11 & 0.020 & 0.12 \\
\hline Thorium-234 (pCi/L) & 51 & 34 & 5.0 & 2.0 & 0.85 & 5 & 1.2 & 1 & 0.1 \\
\hline Tritium (pCi/L) & 51 & 1100 & 210 & -300 & 200 & 380 & 190 & 32 & 0.02 \\
\hline Uranium, total (mg/L) & 51 & 0.071 & a & 0.006 & a & 0.02 & a & 0.002 & $a$ \\
\hline Uranium-234 (pCi/L) & 51 & 100 & 14 & 1.9 & 0.63 & 3.6 & 0.92 & 2 & 0.7 \\
\hline Uranium-235 (pCi/L) & 51 & 1.7 & 0.51 & -0.08 & 0.11 & 0.2 & 0.22 & 0.04 & 0.04 \\
\hline Uranium-235 (\%) & 51 & 0.64 & a & 0.23 & a & 0.42 & $\mathbf{a}$ & 0.014 & a \\
\hline Uranium-236 (pCi/L) & 46 & 0.33 & 0.23 & -0.13 & 0.18 & 0.10 & $\mathbf{a}$ & 0.014 & 0.020 \\
\hline Uranium-238 (pCi/L) & 51 & 34 & 5.0 & 2.0 & 0.85 & 5 & 1.2 & i & 1 \\
\hline
\end{tabular}

(a)Not applicable. 
Table 4.29. Y-12 Plant Discharge Point 301, Kerr Hollow Quarry

\begin{tabular}{|c|c|c|c|c|c|c|}
\hline \multirow[b]{2}{*}{ Parameter } & \multirow{2}{*}{$\begin{array}{l}\text { Number of } \\
\text { samples }\end{array}$} & \multicolumn{3}{|c|}{ Concentration(a) } & \multirow{2}{*}{$\begin{array}{l}\text { Reference } \\
\text { value(b) }\end{array}$} & \multirow{2}{*}{$\begin{array}{l}\text { Number of values } \\
\text { exceeding reference }\end{array}$} \\
\hline & & $\operatorname{Max}$ & $\operatorname{Min}$ & Av & & \\
\hline Flow(c), gpm & 20 & 427.8 & 0.0222 & 93.5 & $d$ & d \\
\hline pH, standard units & 20 & 8.1 & 7.0 & $d$ & $6.5 / 8.5(e)$ & 0 \\
\hline Temperature, ${ }^{\circ} \mathrm{C}$ & 20 & 20.7 & 7.3 & 11 & 30.5 & 0 \\
\hline Mercury & 20 & 0.0003 & $<0.0002$ & $<0.0002$ & $\mathrm{~d}$ & d \\
\hline Total suspended solids & 20 & 10 & $<5$ & $<5$ & 50 & 0 \\
\hline Selenium & 20 & $<0.1$ & $<0.002$ & $<0.01$ & d & $d$ \\
\hline Arsenic & 20 & $<0.04$ & $<0.04$ & $<0.04$ & d & d \\
\hline Cadmium & 20 & $<0.004$ & $<0.004$ & $<0.004$ & d & $d$ \\
\hline Chromium & 20 & $<0.006$ & $<0.006$ & $<0.006$ & d & $\mathrm{d}$ \\
\hline Copper & 20 & 0.012 & $<0.006$ & $<0.007$ & d & $d$ \\
\hline Iron & 20 & 0.13 & $<0.06$ & $<0.07$ & d & d \\
\hline Lead & 20 & $<0.02$ & $<0.02$ & $<0.02$ & $d$ & $d$ \\
\hline Lithium & 20 & 0.25 & 0.03 & 0.11 & 5 & 0 \\
\hline Nickel & 20 & $<0.008$ & $<0.008$ & $<0.008$ & d & $d$ \\
\hline Potassium & 20 & 1.6 & 1.0 & 1.2 & d & $d$ \\
\hline Sodium & 20 & 0.62 & 0.58 & 0.60 & d & d \\
\hline Zinc & 20 & 0.04 & $<0.01$ & $<0.02$ & d & d \\
\hline Zirconium & 20 & $<0.004$ & $<0.004$ & $<0.004$ & 3 & 0 \\
\hline
\end{tabular}

(a)Units in $\mathrm{mg} / \mathrm{L}$ unless otherwise indicated.

(b)NPDES permit limits.

(c)Flow during operations and/or discharging.

(d)Not applicable.

(e)Minimum value/maximum value. 
Table 4.30, Y-12 Plant Discharge Point 301, radiological data summary (Summed DCG percentage $=1.09$ )

\begin{tabular}{|c|c|c|c|c|c|c|c|c|c|}
\hline \multirow[b]{2}{*}{ Parameter } & \multirow{2}{*}{$\begin{array}{l}\text { Number of } \\
\text { samples }\end{array}$} & \multicolumn{6}{|c|}{ Concentration } & \multirow{2}{*}{$\begin{array}{c}\text { Standard } \\
\text { error }\end{array}$} & \multirow{2}{*}{$\begin{array}{c}\text { Percentage } \\
\text { of DCG }\end{array}$} \\
\hline & & $\operatorname{Max}$ & $+\%$ & Min & $+1-$ & Median & $+/-$ & & \\
\hline Alpha activity (pCi/L) & 12 & 12 & 13 & -0.46 & 4.4 & 7.8 & a & 1.3 & a \\
\hline Americium-241 (pCi/L) & 12 & 2.1 & 0.77 & -0.48 & 0.64 & 0.020 & a & 0.19 & 0.065 \\
\hline Beta activity ( $\mathrm{pCi} / \mathrm{L}$ ) & 12 & 7.6 & 7.0 & -8.2 & 9.5 & 2.6 & a & 1.2 & a \\
\hline Neptunium-237 (pCi/L) & 12 & 0.22 & 0.18 & -0.10 & 0.31 & 0.03 & a & 0.03 & 0.08 \\
\hline Plutonium-238 (pCi/L) & 12 & 0.30 & 0.25 & -0.16 & 0.20 & 0 & a & 0.04 & 0 \\
\hline Plutonium-239/240 (pCi/L) & 12 & 0.11 & 0.12 & -0.14 & 0.14 & -0.0036 & a & 0.020 & 0.00 \\
\hline Radium-226 (pCi/L) & 12 & 2.4 & 0.41 & -1.4 & 0.27 & -0.075 & a & 0.26 & 0.00 \\
\hline Radium-228 (pCi/L) & 12 & 1.4 & 0.13 & -2.0 & 2.2 & -0.53 & a & 0.30 & 0.00 \\
\hline Strontium-90 (pCi/L) & 12 & 4.9 & 2.3 & -6.1 & 2.6 & 0.055 & a & 0.98 & 0.0055 \\
\hline Technetium-99 (pCi/mL) & 12 & 0.032 & 0.01 & -0.05 & 0.01 & 0.007 & a & 0.006 & 0.007 \\
\hline Thorium, total (mg/L) & 12 & 0.008 & a & $<0.003$ & a & $<0.003$ & a & 0.0004 & a \\
\hline Thorium-228 (pCi/L) & 12 & 1.6 & 0.95 & -0.22 & 1.2 & 0.25 & a & 0.16 & 0.063 \\
\hline Thorium-230 (pCiL) & 12 & 1.2 & 0.63 & 0.02 & 0.13 & 0.4 & a & 0.1 & 0.1 \\
\hline Thonum-232 (pCi/L) & 12 & 0.16 & 0.16 & -0.43 & 0.36 & 0.013 & a & 0.045 & 0.026 \\
\hline Thorium-234 (pCi/L) & 12 & 3.4 & 1.1 & 0.49 & 0.34 & 1.2 & a & 0.22 & 0.012 \\
\hline Tritium (pCi/L) & 12 & 330 & 160 & -260 & 170 & -13 & 2 & 44 & 0.00 \\
\hline Uranium, total (mg/L) & 12 & 0.007 & a & 0.002 & a & 0.003 & a & 0.001 & $\mathrm{a}$ \\
\hline Uranium-234 (pCi/L) & 12 & 6.1 & 1.6 & 1.5 & 0.60 & 2.6 & a & 0.43 & 0.51 \\
\hline Uranium-235 (\%) & 12 & 1.5 & a & 1.0 & a & 1.2 & a & 0.047 & a \\
\hline Uranium-235 ( $\mathrm{pCi} / \mathrm{L})$ & 12 & 0.25 & 0.32 & 0.015 & 0.12 & 0.13 & $\mathbf{a}$ & 0.022 & 0.021 \\
\hline Uranium-236 (pCi/L) & 11 & 0.24 & 0.25 & -0.091 & 0.11 & 0.020 & 0.11 & 0.025 & 0.0040 \\
\hline Uranium-238 ( $\mathrm{pCi} / \mathrm{L})$ & 12 & 3.4 & 1.1 & 0.49 & 0.34 & 1.2 & a & 0.22 & 0.20 \\
\hline
\end{tabular}

(a)Not appiicable. 
Table 4.31. CY 1993 NPDES Permit Number TN 0002968

Y-12 Plant Discharge Point 302, Rogers Quarry (McCoy Branch)

\begin{tabular}{|c|c|c|c|c|c|c|}
\hline \multirow[b]{2}{*}{ Parameter } & \multirow{2}{*}{$\begin{array}{c}\text { Number of } \\
\text { samples }\end{array}$} & \multicolumn{3}{|c|}{ Concentration(a) } & \multirow{2}{*}{$\begin{array}{c}\text { Reference } \\
\text { value(b) }\end{array}$} & \multirow{2}{*}{$\begin{array}{l}\text { Number of values } \\
\text { exceeding reference }\end{array}$} \\
\hline & & $\operatorname{Max}$ & Min & Av & & \\
\hline Total suspended solids & 51 & 5 & $<5.0$ & $<5.0$ & 50 & 0 \\
\hline Chemical oxygen demand (COD) & 52 & so & $<5.0$ & $<5.0$ & $c$ & $c$ \\
\hline Sulfate & 51 & 33 & $<10$ & $<19$ & $c$ & $c$ \\
\hline Oil and grease & 52 & 3.2 & $<1.4$ & 2.1 & 15 & 0 \\
\hline Settleable solids, $\mathrm{ml} / \mathrm{L}$ & 52 & $<0.1$ & $<0.1$ & $<0.1$ & 0.5 & 0 \\
\hline Selenium & 52 & 0.1 & $<0.002$ & $<0.043$ & 0.020 & 0 \\
\hline Mercury & 52 & 0.0006 & $<0.0002$ & $<0.0002$ & 0.00015 & $52(d)$ \\
\hline Arsenic & 52 & $<0.04$ & $<0.04$ & $<0.04$ & $c$ & $c$ \\
\hline Cadmium & 52 & $<0.004$ & $<0.004$ & $<0.004$ & 0.004 & 0 \\
\hline Chromium & 52 & $<0.01$ & $<0.01$ & $<0.01$ & 0.016 & 0 \\
\hline Copper & 52 & $<0.01$ & $<0.01$ & $<0.01$ & 0.018 & 0 \\
\hline Iron & 52 & 0.08 & $<0.06$ & $<0.06$ & c & $c$ \\
\hline Nickel & 52 & $<0.008$ & $<0.008$ & $<0.008$ & 1.400 & 0 \\
\hline Zinc & 52 & 0.07 & $<0.01$ & $<0.01$ & 0.117 & 0 \\
\hline Lead & 52 & $<0.02$ & $<0.02$ & $<0.02$ & 0.082 & 0 \\
\hline $\mathrm{pH}$, standard units & 52 & 8.2 & 7.3 & $c$ & $6.5 / 8.5(\mathrm{e})$ & $0 / 0$ \\
\hline Temperature, C & 52 & 21.0 & 7.7 & 11.4 & 30.5 & 0 \\
\hline Turbidity, NTU & 52 & 4.9 & 0.09 & 0.67 & $c$ & $c$ \\
\hline Flow, Mgd(f) & 357 & 1.390 & 0.078 & 0.293 & $c$ & $c$ \\
\hline
\end{tabular}

(a) Units in $\mathrm{mg} / \mathrm{L}$ unless otherwise indicated.

(b)NPDES permit limits and/or Tennessee Water Quality criteria.

(c)Not applicabie

(d)The analytical detection limit for this parameter is higher than the reference value.

(e)Minimum value/maximum value.

(f)Flow during operation and/or discharging. 
Table 4.32. CY 1993 radiological data summary

Y-12 Plant Discharge Point 302, Rogers Quarry (McCoy Branch)

(Summed DCG percentage $=1.04$ )

\begin{tabular}{|c|c|c|c|c|c|c|c|c|c|c|}
\hline \multirow[b]{2}{*}{ Parameter } & \multirow[b]{2}{*}{$\begin{array}{c}\text { Number of } \\
\text { samples }\end{array}$} & \multicolumn{5}{|c|}{ Concentration } & \multirow[b]{2}{*}{$+1-$} & \multirow[b]{2}{*}{ Standard error } & \multirow[b]{2}{*}{$\begin{array}{c}\text { Percentage of } \\
\text { DCG }\end{array}$} & \multirow[b]{2}{*}{ Total curies } \\
\hline & & Max & $+1-$ & $\operatorname{Min}$ & $+1-$ & Med & & & & \\
\hline Alpha activity (pCi/L) & 15 & 11 & 13 & -2.5 & 1.5 & 0.87 & 0.56 & 0.90 & $a$ & \\
\hline Americium-241 (pCi/L) & 15 & 0.86 & 0.53 & -0.44 & 0.28 & 0.054 & 0.11 & 0.070 & 0.18 & $3.2 \mathrm{E}-5$ \\
\hline Beta activity $(p C i / L)$ & 15 & 11 & 2.1 & -6.7 & 6.7 & -0.33 & 0.07 & 1.5 & $\mathbf{a}$ & \\
\hline Neptunium-237 (pCi/L) & 15 & 0.80 & 0.40 & -0.08 & 0.095 & 0.06 & 0.08 & 0.07 & 0.20 & $5.4 \mathrm{E}-5$ \\
\hline Plutonium-238 (pCi/L) & 12 & 0.69 & 0.97 & -0.08 & 0.20 & 0.041 & 0 & 0.08 & 0.1 & $5.8 \mathrm{E}-5$ \\
\hline Plutonium-239/240 (pCi/L) & 12 & 0.11 & 0.16 & -0.13 & 0.96 & 0.0095 & 0 & 0.018 & 0.032 & $4.7 \mathrm{E}-6$ \\
\hline Radium-226 (pCi/L) & 15 & 0.41 & 0.09 & -1.5 & 0.38 & .0 .064 & 0.014 & 0.15 & 0.00 & $-2.2 \mathrm{E}-4$ \\
\hline Radium-228 (pCi/L) & 15 & 1.9 & 0.19 & -2.2 & 0.27 & -0.8 & 0.08 & 0.30 & 0.00 & $-2.1 E-4$ \\
\hline Strontium-90 (pCi/L) & 15 & 3.6 & 2.2 & -4.6 & 2.0 & 0.68 & 2.1 & 0.64 & 0.068 & $-5.6 \mathrm{E}-5$ \\
\hline Technetium-99 (pCi/mL) & 15 & 0.046 & 0.01 & -0.05 & 0.01 & 0.007 & 0 & 0.006 & 0.007 & $3,3 \mathrm{E}-3$ \\
\hline Thorium-234 (pCi/L) & 12 & 0.69 & 0.36 & .0 .036 & 0.18 & 0.21 & 0 & 0.060 & 0.002 & $9.9 \mathrm{E}-5$ \\
\hline Thorium, total (mg/L) & 15 & 0.007 & a & $<0.003$ & a & $<0.003$ & a & 0.0003 & $\mathbf{a}$ & \\
\hline Thorium-228 (pCi/L) & 15 & 1.3 & 0.51 & -0.1 & 0.44 & 0.5 & 0.42 & 0.11 & 0.125 & $2.1 \mathrm{E}-4$ \\
\hline Thorium-230 (pCi/L) & 15 & 0.99 & 0.75 & 0.044 & 0.09 & 0.3 & 0.23 & 0.07 & 0.09 & $1.4 \mathrm{E}-4$ \\
\hline Thorium-232 (pCi/L) & 15 & 0.71 & 0.62 & 0.02 & 0.13 & 0.062 & 0.15 & 0.044 & 0.124 & $4.4 \mathrm{E}-5$ \\
\hline Tritium (pCi/L) & 15 & 420 & 180 & -210 & 170 & 200 & 170 & 38 & 0.01 & $6.7 \mathrm{E}-2$ \\
\hline Uranium, total (mg/L) & 15 & 0.029 & $\mathbf{a}$ & $<0.001$ & $\mathbf{a}$ & $<0.001$ & a & 0.002 & $\mathbf{a}$ & \\
\hline Uranium-234 (pCi/L) & 15 & 0.66 & 0.33 & 0.19 & 0.19 & 0.33 & 0.25 & 0.030 & 0.070 & $1.5 \mathrm{E}-4$ \\
\hline Uranium-235 (pCi/L) & 15 & 0.15 & 0.18 & -0.023 & 0.047 & 0 & 0 & 0.014 & 0 & $1.2 \mathrm{E}-5$ \\
\hline Uranium-235 (\%) & 1 & 0.35 & a & 0.35 & a & 0.35 & a & $\mathbf{a}$ & $\mathbf{a}$ & \\
\hline Uranium-236 (pCi/L) & 11 & 0.055 & 0.11 & -0.064 & 0.092 & 0 & a & 0.010 & 0 & $1.8 \mathrm{E}-7$ \\
\hline Uranium-238 (pCi/L) & 12 & 0.69 & 0.36 & -0.036 & 0.18 & 0.21 & a & 0.060 & 0.040 & $9.9 \mathrm{E}-5$ \\
\hline Garnma, total (pCi/L) & 3 & 59 & 16 & -3.3 & 16 & 18 & 16 & 18 & a & \\
\hline
\end{tabular}

(a) Not applicable. 
Table 4.33. Y-12 Plant Discharge Point 304, Bear Creek

\begin{tabular}{|c|c|c|c|c|c|c|}
\hline \multirow[b]{2}{*}{ Parameter } & \multirow{2}{*}{$\begin{array}{l}\text { Number of } \\
\text { samples }\end{array}$} & \multicolumn{3}{|c|}{ Concentration(a) } & \multirow{2}{*}{$\begin{array}{c}\text { Reference } \\
\text { value(b) }\end{array}$} & \multirow{2}{*}{$\begin{array}{l}\text { Number of values } \\
\text { exceeding reference }\end{array}$} \\
\hline & & $\operatorname{Max}$ & Min & $\overline{A v}$ & & \\
\hline Oil and grease & 52 & 4.0 & $<2$ & $<2$ & 15 & 0 \\
\hline Biochemical oxygen demand & 52 & 9.6 & $<5$ & $<5$ & $\mathfrak{c}$ & c \\
\hline Chemical oxygen demand & 52 & 38 & $<5$ & $<10$ & c & c \\
\hline Total dissolved solids & 52 & 460 & $<5$ & $<210$ & c & c \\
\hline Total suspended solids & 52 & 18 & $<5$ & $<6$ & c & c \\
\hline Nitrate as $\mathrm{N}$ & 52 & 15 & 0.28 & 4.2 & c & c \\
\hline Conductivity, mhos/cm & 52 & 380 & 115 & 250 & c & c \\
\hline Dissolved oxygen & 52 & 13 & 7.2 & 9.4 & 3 & 0 \\
\hline Turbidity, NTU & 52 & 84.0 & 0.36 & 5.9 & c & c \\
\hline $\mathrm{pH}$, standards units & 52 & 8.1 & 7.1 & c & $6.5 / 8.5(d)$ & 0 \\
\hline Flow, Mgd(e) & 365 & 145 & 0.151 & 4.47 & c & c \\
\hline
\end{tabular}

(a)Units in $\mathrm{mg} / \mathrm{L}$ unless otherwise indicated.

(b)NPDES Permit limits.

(c)Not applicable.

(d)Minimum value/maximum value.

(e)Flow during operations and/or discharging. 
Table 4.34. Y-12 Plant Discharge Point 304, radiological data summary

(Summed DCG percentage $=4.74$ )

\begin{tabular}{|c|c|c|c|c|c|c|c|c|c|c|}
\hline \multirow[b]{2}{*}{ Parameter } & \multirow{2}{*}{$\begin{array}{c}\text { Number of } \\
\text { samples }\end{array}$} & \multicolumn{6}{|c|}{ Concentration } & \multirow{2}{*}{$\begin{array}{l}\text { Standard } \\
\text { error }\end{array}$} & \multirow{2}{*}{$\begin{array}{c}\text { Percentage } \\
\text { of DCG }\end{array}$} & \multirow{2}{*}{$\begin{array}{l}\text { Total } \\
\text { curies }\end{array}$} \\
\hline & & $\operatorname{Max}$ & $+1-$ & Min & $+1-$ & Median & $+1 /$ & & & \\
\hline Alpha activity (pCiL) & 52 & 43 & 13 & -2.3 & 7.2 & 16 & a & 1.2 & a & \\
\hline Americium-241 (pCi/L) & 52 & 0.71 & 0.71 & -1.2 & 0.58 & 0.060 & a & 0.042 & 0.20 & $3.1 \mathrm{E}-4$ \\
\hline Beta activity $(\mathrm{pCi} / \mathrm{L})$ & 52 & 51 & 12 & 0.50 & 5.0 & 13 & a & 1.5 & a & \\
\hline Neptunium-237 (pCi/L) & 52 & 1.1 & 0.54 & -0.31 & 0.28 & 0.15 & a & 0.026 & 0.50 & $1.0 \mathrm{E}-3$ \\
\hline Plutonium-238 (pCi/L) & 52 & 2.0 & 2.2 & -0.29 & 0.36 & -0.0015 & a & 0.042 & 0.00 & $2.1 \mathrm{E}-4$ \\
\hline Plutonium-239/240 (pCi/L) & 52 & 2.0 & 2.2 & -0.31 & 0.24 & 0.015 & a & 0.041 & 0.050 & $3.6 E-4$ \\
\hline Radium-226 (pCi/L) & 51 & 2.7 & 0.27 & -1.9 & 0.43 & 0.050 & 0.27 & 0.10 & 0.050 & $4.7 E-4$ \\
\hline Radium-228 (pCi/L) & 51 & 2.2 & 0.22 & -2.0 & 1.5 & 0.020 & 0.040 & 0.11 & 0.020 & $-2.9 \mathrm{E}-4$ \\
\hline Strontium-90 (pCin) & 52 & 19 & 32 & -16 & 12 & 0.77 & a & 0.59 & 0.077 & $4.0 \mathrm{E}-3$ \\
\hline Technetium-99 (pCi/mL) & 52 & 0.15 & 0.01 & -0.067 & 0.01 & 0.020 & a & 0.0048 & 0.02 & $1.5 \mathrm{E}-1$ \\
\hline Thorium, total (mg/L) & 51 & 0.007 & a & $<0.003$ & a & $<0.003$ & a & 0.0001 & a & \\
\hline Thorium-228 (pCi/) & 52 & 2.6 & 1.2 & -0.34 & 0.47 & 0.43 & a & 0.082 & 0.11 & $3.5 \mathrm{E}-3$ \\
\hline Thorium-230 (pCi/L) & 52 & 2.9 & 2.2 & -0.17 & 0.35 & 0.31 & a & 0.061 & 0.10 & $2.5 \mathrm{E} \cdot 3$ \\
\hline Thorium-232 (pCi/L) & 52 & 0.38 & 0.35 & -0.065 & 0.13 & 0.088 & a & 0.014 & 0.18 & $5.9 \mathrm{E}-4$ \\
\hline Thorium-234 ( $\mathrm{pCi} / \mathrm{L})$ & 52 & 22 & 3.7 & 1.1 & 0.47 & 8.8 & a & 0.62 & 0.88 & $6.0 \mathrm{E}-2$ \\
\hline Tritium (pCi/L) & 52 & 570 & 280 & -380 & 180 & 170 & a & 28 & 0.0085 & $9.2 \mathrm{E}-1$ \\
\hline Uranium, total (mg/L) & 52 & 0.064 & $\mathbf{a}$ & $<0.001$ & a & $<0.02$ & $\mathbf{a}$ & 0.002 & $\mathbf{a}$ & \\
\hline Uranium-234 (pCi/L) & 52 & 11 & 2.3 & 0.56 & 0.31 & 5.0 & a & 0.32 & 1.0 & $3.1 \mathrm{E}-2$ \\
\hline Uranium-235 (\%) & 51 & 0.71 & $\mathbf{a}$ & 0.29 & a & 0.38 & a & 0.0086 & a & \\
\hline Uranium-235 (pCi/L) & 52 & 0.85 & 0.45 & -0.03 & 0.06 & 0.3 & a & 0.02 & 0.04 & $2.1 \mathrm{E}-3$ \\
\hline Uranium-236 $(p \mathrm{Ci} / \mathrm{L})$ & 48 & 0.43 & 0.28 & -0.10 & 0.14 & 0.11 & a & 0.017 & 0.022 & $7.6 \mathrm{E}-4$ \\
\hline Uranium-238 $(\mathrm{pCi} / L)$ & $\$ 2$ & 22 & 3.7 & 1.1 & 0.47 & 8.8 & a & 0.62 & 1.5 & $6.0 \mathrm{E}-2$ \\
\hline
\end{tabular}

(a)Not applicabie. 
Table 4.35. Y-12 Plant Discharge Point 307, West Borrow Area

\begin{tabular}{|c|c|c|c|c|c|c|}
\hline \multirow[b]{2}{*}{ Parameter } & \multirow{2}{*}{$\begin{array}{l}\text { Number of } \\
\text { samples }\end{array}$} & \multicolumn{3}{|c|}{ Concentration(a) } & \multirow{2}{*}{$\begin{array}{l}\text { Reference } \\
\text { value(b) }\end{array}$} & \multirow{2}{*}{$\begin{array}{l}\text { Number of values } \\
\text { exceeding reference }\end{array}$} \\
\hline & & $\operatorname{Max}$ & Min & Av & & \\
\hline Flow, gpd & 2 & 57142 & 45649 & 51396 & $c$ & $c$ \\
\hline $\mathrm{pH}$, standard units & 2 & 7.9 & 7.6 & $c$ & $6 / 9$ (d) & 0 \\
\hline Temperature, $C$ & 2 & 22.8 & 9.3 & 16 & 30.5 & $c$ \\
\hline Nitrogen (as ammonia) & 2 & $<0.2$ & $<0.2$ & $<0.2$ & $c$ & $c$ \\
\hline Biochemical oxygen demand & 2 & $<5$ & $<5$ & $<5$ & $c$ & $c$ \\
\hline Chemical oxygen demand & 2 & 5.6 & 5.0 & 5.3 & $c$ & $c$ \\
\hline Color, $\mathrm{ACU}$ & 2 & 125 & 10 & 68 & $c$ & $c$ \\
\hline Oil and grease & 2 & $<2$ & $<.0$ & $<2$ & $c$ & $c$ \\
\hline Total organic carbon & 2 & 8.0 & 4.1 & 6.1 & $c$ & $c$ \\
\hline Total suspended solids & 2 & 35 & $<5$ & $<20$ & $c$ & $c$ \\
\hline
\end{tabular}

(a)Units in $\mathrm{mg} / \mathrm{L}$ unless otherwise noted.

(b)Tennessee Water Quality criteria

(c)Not applicable

(d)Minimum value/maximum value.

Table 4.36. Y-12 Plant Discharge Point 308, East Borrow Area

\begin{tabular}{|c|c|c|c|c|c|c|}
\hline \multirow[b]{2}{*}{ Parameter } & \multirow{2}{*}{$\begin{array}{c}\text { Number of } \\
\text { samples }\end{array}$} & \multicolumn{3}{|c|}{ Concentration (a) } & \multirow{2}{*}{$\begin{array}{l}\text { Reference } \\
\text { value(b) }\end{array}$} & \multirow{2}{*}{$\begin{array}{l}\text { Number of values } \\
\text { exceeding reference }\end{array}$} \\
\hline & & $\operatorname{Max}$ & Min & Av & & \\
\hline Flow, gpd & 4 & 152380 & 2282.4 & 64166 & c & $c$ \\
\hline $\mathrm{pH}$, standard units & 4 & 8.2 & 7.9 & $e$ & $6 / 9(d)$ & 0 \\
\hline Temperature, $\mathrm{C}$ & 4 & 24.5 & 9.2 & 18 & 30.5 & $c$ \\
\hline Nitrogen (as ammonia) & 4 & $<0.2$ & $<0.2$ & $<0.2$ & $c$ & $c$ \\
\hline Biochemical oxygen demand & 4 & $<5$ & $<5.0$ & $<5$ & $c$ & $c$ \\
\hline Chemical oxygen demand & 4 & $<0$ & 5.7 & 10 & $c$ & $c$ \\
\hline Color, ACU & 4 & $12 j$ & 5 & 60 & $c$ & $c$ \\
\hline Oil and grease & 4 & $<$ & $<2.0$ & $<$ & $c$ & $c$ \\
\hline Total organic carbon & 4 & 8.9 & 4.6 & 6.2 & $c$ & $c$ \\
\hline Total suspended solids & 4 & 40 & $<5.0$ & $<18$ & $c$ & $c$ \\
\hline
\end{tabular}

(a)Units in $\mathrm{mg} / \mathrm{L}$ unless otherwise noted.

(b)Tennessee Water Quality criteria

(c)Not applicable

(d)Minimum value/maximum value. 
Table 4.37. Y-12 Plant Discharge Point 309, Sanitary Landfill IV Sedimentation Basin

\begin{tabular}{|c|c|c|c|c|c|c|}
\hline \multirow[b]{2}{*}{ Parameter } & \multirow{2}{*}{$\begin{array}{l}\text { Number of } \\
\text { samples }\end{array}$} & \multicolumn{3}{|c|}{ Concentration(a) } & \multirow{2}{*}{$\begin{array}{l}\text { Reference } \\
\text { value(b) }\end{array}$} & \multirow{2}{*}{$\begin{array}{l}\text { Number of values } \\
\text { exceeding reference }\end{array}$} \\
\hline & & $\operatorname{Max}$ & Min & Av & & \\
\hline Flow, gpd & 2 & 22824 & 1521 & 12173 & $c$ & $c$ \\
\hline $\mathrm{pH}$, standard units & 2 & 7.9 & 7.7 & $c$ & $6 / 9$ (d) & 0 \\
\hline Temperature, $\mathrm{C}$ & 2 & 9.7 & 8.6 & 9.2 & 30.5 & $c$ \\
\hline Nitrogen (as ammonia) & 2 & $<0.2$ & $<0.2$ & $<0.2$ & $c$ & $c$ \\
\hline Biochemical oxygen demand & 2 & $<5$ & $<5.0$ & $<5$ & $c$ & $c$ \\
\hline Chemical oxygen demand & 2 & $<20$ & $<5$ & $<13$ & $c$ & $c$ \\
\hline Color, ACU & 2 & 400 & 7 & 200 & $c$ & c \\
\hline Oil and grease & 2 & $<$ & $<2.0$ & $<2$ & $c$ & $c$ \\
\hline Total organic carbon & 2 & 10.0 & 5.9 & 8.0 & c & $c$ \\
\hline Total suspended solids & 2 & 24 & $<5$ & $<15$ & $c$ & $c$ \\
\hline
\end{tabular}

(a)Units in $\mathrm{mg} / \mathrm{L}$ unless otherwise noted.

(b)Tennessee Warer Quality criteria

(c)Not applicable

(d)Minimum value/maximum value. 
Table 4.38. CY 1993 NPDES Permit Number TN 0002968

Y-12 Plant Discharge Point 501, Central Pollution Control Facility

\begin{tabular}{|c|c|c|c|c|c|c|}
\hline \multirow[b]{2}{*}{ Parameter } & \multirow{2}{*}{$\begin{array}{l}\text { Number of } \\
\text { samples }\end{array}$} & \multicolumn{3}{|c|}{ Concentration(a) } & \multirow{2}{*}{$\begin{array}{l}\text { Reference } \\
\text { value(b) }\end{array}$} & \multirow{2}{*}{$\begin{array}{l}\text { Number of values } \\
\text { exceeding reference }\end{array}$} \\
\hline & & $\operatorname{Max}$ & Min & Av & & \\
\hline Flow(c), gpd & 52 & 14481 & 2050 & 10175 & $d$ & $d$ \\
\hline $\mathrm{pH}$, standard units & 52 & 8.7 & 7.0 & $d$ & $6.0 / 9.0(\mathrm{e})$ & 0 \\
\hline Temperature, $C$ & 52 & 30.4 & 8.4 & 22.0 & 30.5 & 0 \\
\hline Cyanide & 52 & 0.02 & $<0.002$ & $<0.010$ & 1.2 & 0 \\
\hline Oil and grease & 52 & 9.8 & $<2.0$ & $<2.4$ & 52 & 0 \\
\hline Phenols & 52 & 0.020 & $<0.001$ & $<0.004$ & $d$ & $d$ \\
\hline Total toxic organics & 52 & $<0.010$ & $<0.010$ & $<0.010$ & 2.13 & 0 \\
\hline Chloride & 52 & 890 & 15 & 170 & $d$ & $d$ \\
\hline Color & 52 & 40.0 & $<5.0$ & $<9.7$ & $d$ & $d$ \\
\hline Fluoride & 52 & 8.5 & 0.48 & 1.2 & $d$ & $d$ \\
\hline Mercury & 52 & $<0.0002$ & $<0.0002$ & $<0.0002$ & $d$ & $d$ \\
\hline Nitrate & 52 & 200 & $<0.10$ & $<13$ & $d$ & $d$ \\
\hline Total suspended solids & 52 & 23 & $<5 . \dot{0}$ & $<6.0$ & 60 & 0 \\
\hline Sulfate & 52 & 6900 & 1400 & 2488 & $d$ & $d$ \\
\hline Sufactants, (MBAS) & 52 & 0.06 & $<0.05$ & $<0.05$ & $d$ & $d$ \\
\hline Aluminum & 52 & 0.7 & $<0.2$ & $<0.3$ & $d$ & $d$ \\
\hline Beryllium & 52 & $<0.006$ & $<0.006$ & $<0.002$ & $d$ & $d$ \\
\hline Cadmium & 52 & 0.02 & $<0.001$ & $<0.002$ & 0.69 & 0 \\
\hline Chromium & 52 & $<0.09$ & $<0.03$ & $<0.03$ & 2.77 & 0 \\
\hline Copper & 52 & $<0.09$ & $<0.03$ & $<0.03$ & 3.38 & 0 \\
\hline Iron & 52 & 3.26 & $<0.3$ & $<0.9$ & $d$ & $d$ \\
\hline Lead & 52 & 0.1 & $<0.005$ & $<0.08$ & 0.69 & 0 \\
\hline Nickel & 52 & 0.94 & $<0.01$ & $<0.3$ & 3.98 & 0 \\
\hline Phosphorts & 52 & 0.81 & $<0.10$ & $<0.24$ & $d$ & $d$ \\
\hline Silver & 52 & $<0.09$ & $<0.03$ & $<0.03$ & 0.43 & 0 \\
\hline Sodium & 52 & 1880 & 31.7 & 350 & $d$ & $d$ \\
\hline Zinc & 52 & 0.20 & $<0.05$ & $<0.06$ & 2.61 & 0 \\
\hline
\end{tabular}

(a)Units in $\mathrm{mg} / \mathrm{L}$ unless otherwise noted.

(b)NPDES permit limits.

(c)Flow during operations and/or discharging.

(d)Not applicable

(e)Minimum value/maximum value. 
Table 4.39. CY 1993 radiological data summary

Y-12 Plant Discharge Point 501, Central Pollution Control Facility

(Summed DCG percentage $=4.72$ )

\begin{tabular}{|c|c|c|c|c|c|c|c|c|c|c|}
\hline \multirow[b]{2}{*}{ Parameter } & \multirow{2}{*}{$\begin{array}{l}\text { Number of } \\
\text { samples }\end{array}$} & \multicolumn{6}{|c|}{ Concentration } & \multirow{2}{*}{ Standard error } & \multirow{2}{*}{$\begin{array}{l}\text { Percentage of } \\
\text { DCG }\end{array}$} & \multirow{2}{*}{ Total curies } \\
\hline & & $\operatorname{Max}$ & $+1-$ & Min & $+1-$ & Med & +1 & & & \\
\hline Alpha activity (pCi/L) & 37 & 170 & 170 & -190 & 300 & 32 & 51 & 9.8 & $\bar{a}$ & \\
\hline Americium-241 (pCi/L) & 37 & 1.6 & 1.2 & -0.38 & 0.42 & 0.060 & 0.23 & 0.060 & 0.20 & $2.6 \mathrm{E}-7$ \\
\hline Beta activity (pCi/L) & 37 & 2800 & 320.0 & 2.2 & 6.7 & -6.7 & 0 & 100 & $\mathbf{a}$ & \\
\hline Neptunium-237 (pCi/L) & 37 & 1.50 & 0.84 & -0.11 & 0.24 & 0.11 & 0.14 & 0.050 & 0.37 & $3.7 \mathrm{E}-7$ \\
\hline Plutonium-238 (pCi/L) & 36 & 0.37 & 0.47 & -0.13 & 0.19 & 0 & $\mathbf{a}$ & 0.020 & 0 & $6.6 \mathrm{E}-8$ \\
\hline Plutonium-239/240 (pCi/L) & 36 & 0.29 & 0.34 & -0.1 & 0.14 & 0.01 & a & 0.01 & 0.05 & $3.2 \mathrm{E}-8$ \\
\hline Radium-226 (pCi/L) & 37 & 3.5 & 0.47 & -14 & 0.37 & 0.61 & 0.49 & 0.14 & 0.61 & $1.6 \mathrm{E}-6$ \\
\hline Radium-228 (pCi/L) & 37 & 2.6 & 2.1 & -2.3 & 1.4 & -0.020 & 0.82 & 0.20 & 0.00 & $-1.5 \mathrm{E}-7$ \\
\hline Strontium-90 (pCi/L) & 37 & 33 & 17 & -23 & 12 & 2.9 & 10 & 2.2 & 0.29 & $6.6 \mathrm{E}-6$ \\
\hline Technetium-99 (pCi/mL) & 37 & 2.838 & $\mathbf{0}$ & 0.01 & 0.01 & 0.1 & 0 & 0.1 & 0.10 & $9.2 \mathrm{E}-4$ \\
\hline Thorium-234 (pCi/L) & 37 & 47 & 8.1 & -0.02 & 0.24 & 8 & 2 & 2 & 0.08 & $2.2 \mathrm{E}-5$ \\
\hline Thorium, total (mg/L) & 37 & 0.016 & $\mathbf{a}$ & $<0.0003$ & $a$ & $<0.003$ & a & 0.0004 & $\mathbf{a}$ & \\
\hline Thorium-228 (pCi/L) & 37 & 21 & 60 & -0.92 & 1.1 & 0.39 & 0.47 & 0.64 & 0.098 & $3.4 \mathrm{E}-6$ \\
\hline Thorium-230 (pCi/L) & 37 & 3.5 & 2.5 & $\mathbf{0}$ & 0 & 0.41 & 0.28 & 0.10 & 0.14 & $1.1 \mathrm{E}-6$ \\
\hline Thorium-232 (pCi/L) & 37 & 18 & 3.7 & -27 & 46 & 0.060 & 0.14 & 0.90 & 0.12 & $-1.2 \mathrm{E}-6$ \\
\hline Tritium (pCi/L) & 37 & 4700 & 340 & -390 & 190 & 110 & 230 & 140 & 0.0055 & $7.1 \mathrm{E}-4$ \\
\hline Uranium, total (mg/L) & 37 & 0.137 & a & $<0.00 \mathrm{i}$ & $\mathbf{a}$ & $<0.02$ & a & 0.006 & $\mathbf{a}$ & \\
\hline Uranium-234 (pCi/L) & 37 & 79 & 13 & 0.15 & 0.23 & 8.0 & 1.5 & 3.4 & 1.6 & $2.8 \mathrm{E}-5$ \\
\hline Uranium-235 (pCi/L) & 37 & 4.8 & 1.4 & -0.08 & 0.16 & 0.5 & 0.70 & 0.2 & 0.08 & $1.4 \mathrm{E}-6$ \\
\hline Uranium-235 (\%) & 31 & 3.8 & $\mathbf{a}$ & 0.5 & a & 0.7 & a & 0.1 & a & \\
\hline Uranium-236 (pCi/L) & 35 & 4.9 & 1.4 & -0.03 & 0.06 & 0.4 & 0.3 & 0.2 & 0.08 & 1.7 E-6 \\
\hline Uranium-238 (pCi/L) & 37 & 47 & 8.1 & -0.02 & 0.24 & 8 & 2 & 2 & 1 & $2.2 \mathrm{E}-5$ \\
\hline
\end{tabular}

(a)Not applicable. 
Table 4.40. Y-12 Plant Discharge Point 502, West End Treatment Facility

\begin{tabular}{|c|c|c|c|c|c|c|}
\hline \multirow[b]{2}{*}{ Parameter } & \multirow{2}{*}{$\begin{array}{l}\text { Number of } \\
\text { samples }\end{array}$} & \multicolumn{3}{|c|}{ Concentration(a) } & \multirow{2}{*}{$\begin{array}{l}\text { Reference } \\
\text { value(b) }\end{array}$} & \multirow{2}{*}{$\begin{array}{l}\text { Number of values } \\
\text { exceeding reference }\end{array}$} \\
\hline & & $\operatorname{Max}$ & Min & Av & & \\
\hline Flow(c), gpd & 47 & 24874 & 1247 & 15923 & d & $d$ \\
\hline $\mathrm{pH}$, standard units & 47 & 8.3 & 6.6 & d & $6.0 / 9.0(\mathrm{e})$ & 0 \\
\hline Temperature, ${ }^{\circ} \mathrm{C}$ & 47 & 27.5 & 18.1 & 21.5 & 30.5 & 0 \\
\hline Residual chlorine & 47 & 0.08 & 0.01 & 0.03 & $d$ & d \\
\hline Cyanide & 47 & $<0.01$ & $<0.002$ & $<0.01$ & 1.2 & 0 \\
\hline Oil and grease & 47 & 8.0 & $<2$ & $<3$ & 52 & 0 \\
\hline Total toxic organics & 13 & $<0.01$ & $<0.01$ & $<0.01$ & 2.13 & 0 \\
\hline Chloride & 46 & 590 & 230 & 519 & $d$ & d \\
\hline Fluoride & 46 & 42 & 2.8 & 19 & d & d \\
\hline Mercury & 46 & 0.0045 & $<0.0002$ & $<0.003$ & d & d \\
\hline Nitrate & 46 & 110 & $<0.1$ & $<15.0$ & d & d \\
\hline Total suspended solids & 46 & 16 & $<5$ & $<7$ & 60 & 0 \\
\hline Sulfate & 46 & 11000 & 4100 & 8191 & d & d \\
\hline Aluminum & 46 & $<0.6$ & $<0.2$ & $<0.2$ & $d$ & d \\
\hline Arsenic & 46 & $<0.6$ & $<0.2$ & $<0.2$ & $d$ & d. \\
\hline Barium & 46 & 0.153 & $<0.004$ & $<0.037$ & d & d \\
\hline Beryllium & 46 & $<0.006$ & $<0.002$ & $<0.002$ & d & $d$ \\
\hline Calcium & 46 & 131 & 20.3 & 59.7 & d & $d$ \\
\hline Cadmium & 46 & $<0.06$ & $<0.02$ & $<0.02$ & 0.69 & 0 \\
\hline Chromium & 46 & $<0.09$ & $<0.03$ & $<0.03$ & 2.77 & 0 \\
\hline Cobalt & 46 & 0.03 & $<0.01$ & $<0.01$ & $d$ & $d$ \\
\hline Copper & 46 & 0.09 & $<0.03$ & $<0.04$ & 3.38 & 0 \\
\hline Iron & 46 & $<0.9$ & $<0.3$ & $<0.3$ & $d$ & d \\
\hline Lead. & 46 & $<0.3$ & $<0.1$ & $<0.1$ & 0.69 & 0 . \\
\hline Magnesium & 46 & 20.4 & 10.1 & 17.0 & $d$ & $d$ \\
\hline Manganese & 46 & 0.269 & 0.011 & 0.076 & d & d \\
\hline Molybdenum & 46 & 0.50 & $<0.03$ & $<0.21$ & d & d \\
\hline Nickel & 46 & 1.52 & $<0.04$ & $<0.27$ & 3.98 & 0 \\
\hline Phosphorus & 46 & 12 & $<0.1$ & $<6$ & $d$ & d \\
\hline Potassium & 46 & 144 & 112 & 130 & d & d \\
\hline Silver & 46 & $<0.09$ & $<0.03$ & $<0.03$ & 0.43 & 0 \\
\hline Sodium & 46 & 5660 & 3090 & 4270 & d & d \\
\hline Zinc & 46 & 0.55 & 0.09 & $<0.18$ & 2.61 & 0 \\
\hline
\end{tabular}

(a) Units in $\mathrm{mg} / \mathrm{L}$ unless otherwise indicated.

(b)NPDES permit limits.

(c)Flow during operations and/or discharging.

(d) Not applicable

(e)Minimum value/maximum value. 
Table 4.41. Y-12 Plant Dic:charge Point 502, radiological data summary

(Summe. DCG percentage $=2.28$ )

\begin{tabular}{|c|c|c|c|c|c|c|c|c|c|c|}
\hline \multirow[b]{2}{*}{ Parameter } & \multirow{2}{*}{$\begin{array}{c}\text { Number of } \\
\text { samples }\end{array}$} & \multicolumn{6}{|c|}{ Concentration } & \multirow{2}{*}{$\begin{array}{l}\text { Standard } \\
\text { ertor }\end{array}$} & \multirow{2}{*}{$\begin{array}{c}\text { Percentage } \\
\text { of } D C G\end{array}$} & \multirow{2}{*}{$\begin{array}{l}\text { Total } \\
\text { curies }\end{array}$} \\
\hline & & $\operatorname{Max}$ & $+\%$ & Min & $+i-$ & Median & $+\%$ & & & \\
\hline Alpha activity $(\mathrm{pCi} / \mathrm{L})$ & 14 & 150 & 120 & -230 & 230 & 27 & a & 28 & a & \\
\hline Americium-241 (pCi/L) & 14 & 0.24 & 0.28 & -0.57 & 0.49 & 0.034 & a & 0.051 & 0.11 & $-3.1 \mathrm{E}-8$ \\
\hline Beta activity (pCi/L) & 14 & 260 & 180 & -320 & 560 & 120 & a & 38 & a & \\
\hline Neptunium-237 (pCi/L) & 14 & 2.2 & 1.2 & -0.32 & 0.73 & 0.094 & a & 0.16 & 0.31 & $6.8 \mathrm{E}-7$ \\
\hline Plutonium-238 (pCi/L) & 14 & 0.72 & 0.90 & -0.19 & 0.30 & 0.0080 & a & 0.061 & 0.020 & $-1.4 \mathrm{E}-8$ \\
\hline Plutonium-239/240 (pCi/ $)$ & 14 & 0.21 & 0.28 & -0.14 & 0.19 & 0.0055 & a & 0.021 & 0.018 & $4.8 \mathrm{E}-8$ \\
\hline Radium-226 (pCi/L) & 14 & 2.5 & 0.91 & 0.17 & 0.59 & 0.58 & a & 0.18 & 0.58 & $2.9 \mathrm{E}-6$ \\
\hline Radium-228 (pCi/L) & 14 & 1.1 & 0.11 & -2.0 & 1.4 & -0.37 & a & 0.24 & 0.00 & $-1.0 \mathrm{E}-6$ \\
\hline Strontium-90 (pCi/L) & 14 & 21 & 9.9 & -35 & 18 & -2.5 & $\mathbf{a}$ & 4.0 & 0.00 & $4.3 \mathrm{E}-4$ \\
\hline Technetium-99 (pCi/mL) & 14 & 0.26 & 0.02 & 0.04 & 0.01 & 0.2 & a & 0.01 & 0.2 & $4.3 \mathrm{E}-4$ \\
\hline Thorium, total $(\mathrm{mg} / \mathrm{L})$ & 14 & 0.015 & a & $<0.003$ & a & $<0.003$ & a & 0.001 & $\mathbf{a}$ & \\
\hline Thorium-228 (pCilL) & 14 & 2.9 & 3.8 & 0.037 & 0.59 & 0.44 & a & 0.21 & 0.11 & $2.3 \mathrm{E}-6$ \\
\hline Thorium-230 (pCi/L) & 14 & 2.6 & 1.2 & -0.11 & 1.7 & 0.22 & a & 0.19 & 0.073 & $1,4 \mathrm{E}-6$ \\
\hline Thorium-232 (pCi/L) & 14 & 0.45 & 1.0 & -0.036 & 0.073 & 0.095 & a & 0.035 & 0.19 & $3.5 \mathrm{E}-7$ \\
\hline Thorium-234 $(\mathrm{pCi} / \mathrm{L})$ & 14 & 26 & 4.9 & 0.24 & 0.22 & 2.8 & a & 2.1 & 0.028 & $2.3 \mathrm{E}-5$ \\
\hline Tritium $(\mathrm{pCi} / \mathrm{L})$ & 14 & 950 & 220 & -49 & 210 & 380 & a & 75 & 0.019 & $1.2 \mathrm{E}-3$ \\
\hline Uranium, total $(\mathrm{mg} / \mathrm{L})$ & 14 & 0.065 & a & $<0.001$ & a & $<0.007$ & a & 0.005 & a & \\
\hline Uranium-234 $(\mathrm{pCi} / \mathrm{L})$ & 14 & 17 & 3.4 & 0.11 & 0.18 & 1.6 & $a$ & 1.4 & 0.32 & $1.5 \mathrm{E}-5$ \\
\hline Uranium-235 (\%) & 13 & 0.56 & a & 0.19 & a & 0.42 & a & 0.023 & a & \\
\hline Uranium-235 ( $\mathrm{pCi} / \mathrm{L})$ & 14 & 1.5 & 0.68 & 0 & 0 & 0.16 & a & 0.11 & 0.026 & $1.1 \mathrm{E}-6$ \\
\hline Uranium-236 $(\mathrm{pCi} / \mathrm{L})$ & 14 & 1.1 & 0.52 & -0.06 & 0.09 & 0.04 & a & 0.09 & 0.01 & $7.7 \mathrm{E}-7$ \\
\hline Uranium-238 $(\mathrm{pCi} / \mathrm{L})$ & 14 & 26 & 4.9 & 0.24 & 0.22 & 2.8 & a & 2.1 & 0.47 & $2.3 \mathrm{E}-5$ \\
\hline
\end{tabular}

(a)Not applicable. 
Table 4.42. CY 1993 NPDES Permit Number TN 0002986

Y-12 Plant Discharge Point 503, Steam Plant Wastewater

Treatment Facility

\begin{tabular}{|c|c|c|c|c|c|c|}
\hline \multirow[b]{2}{*}{ Parameter } & \multirow{2}{*}{$\begin{array}{l}\text { Number of } \\
\text { samples }\end{array}$} & \multicolumn{3}{|c|}{ Concentration(a) } & \multirow{2}{*}{$\begin{array}{l}\text { Reference } \\
\text { yalue(b) }\end{array}$} & \multirow{2}{*}{$\begin{array}{l}\text { Number of values } \\
\text { exceeding reference }\end{array}$} \\
\hline & & $\operatorname{Max}$ & Min & Av & & \\
\hline Flow(c), gpd & 365 & 274900 & 0 & 103486 & d & $\bar{d}$ \\
\hline $\mathrm{pH}$, standard units & 155 & 8.5 & 6.4 & d & $6.0 / 9.0(\mathrm{e})$ & 0 \\
\hline Temperature & 155 & 30.3 & 12.5 & 21.5 & 30.5 & 0 \\
\hline Oil and grease & 155 & 5.7 & 2.0 & 2.2 & 20 & 0 \\
\hline Phenols & 155 & 0.014 & $<0.0010$ & $<0.0036$ & $d$ & d \\
\hline Chloride & 156 & 800 & 0.76 & 300.62 & $d$ & $d$ \\
\hline Fluoride & 156 & 5.0 & 0.97 & 2.97 & $d$ & $d$ \\
\hline Mercury & 156 & 0.0003 & $<0.0002$ & $<0.0002$ & $d$ & $\mathrm{~d}$ \\
\hline Total suspended solids & 156 & 19 & $<5.0$ & $<6.3$ & 100 & 0 \\
\hline Selenium & 156 & 0.5 & $<0.002$ & $<0.194$ & $d$ & d \\
\hline Sulfate & 156 & 2300 & 500 & 1439 & d & $d$ \\
\hline Sulfide & 156 & $<1.0$ & $<1.0$ & $<1.0$ & d & $d$ \\
\hline Aluminum & 156 & 3.0 & $<0.2$ & $<0.5$ & $d$ & $d$ \\
\hline Arsenic & 156 & $<0.6$ & $<0.2$ & $<0.2$ & $d$ & $d$ \\
\hline Barium & 156 & 0.170 & 0.040 & 0.092 & $d$ & $d$ \\
\hline Beryllium & 156 & $<0.006$ & $<0.002$ & $<0.002$ & d & $d$ \\
\hline Boron & 156 & 0.22 & $<0.03$ & $<0.07$ & d & d \\
\hline Cadmium & 156 & $<0.06$ & $<0.02$ & $<0.02$ & $d$ & d \\
\hline Calcium & 156 & 861 & 214 & 550 & d & d \\
\hline Cerium & 156 & $<0.3$ & $<0.08$ & $<0.08$ & $d$ & d \\
\hline Chromium & 156 & $<0.09$ & $<0.03$ & $<0.03$ & 0.2 & 0 \\
\hline Cobalt & 156 & $<0.03$ & $<0.01$ & $<0.01$ & $\mathrm{~d}$ & d \\
\hline Copper & 156 & $<0.09$ & $<0.03$ & $<0.03$ & 1.0 & 0 \\
\hline Gallium & 156 & $<0.3$ & $<0.09$ & $<0.09$ & $d$ & d \\
\hline Iron & 156 & 1.37 & $<0.3$ & $<0.38$ & 1.0 & 2 \\
\hline Lead & 156 & $<0.3$ & $<0.1$ & $<0.1$ & $d$ & d \\
\hline Lithium & 156 & 6.55 & $<0.08$ & $<0.33$ & $d$ & d \\
\hline Magnesium & 156 & 40.0 & $<0.2$ & 5.9 & $d$ & $d$ \\
\hline Manganese & 156 & 0.04 & $<0.009$ & $<0.011$ & $d$ & d \\
\hline Molybdenum & 156 & $<0.09$ & $<0.03$ & $<0.03$ & d & d \\
\hline Nickel & 156 & $<0.20$ & $<0.04$ & $<0.04$ & $d$ & d \\
\hline Niobium & 156 & $<0.20$ & $<0.05$ & $<0.05$ & d & $d$ \\
\hline Phosphorus & 156 & 2.22 & $<0.04$ & $<0.14$ & d & $d$ \\
\hline Potassium & 156 & 13 & $<3$ & $<8$ & $d$ & d \\
\hline Scandium & 1 & $<0.002$ & $<0.002$ & $<0.002$ & $d$ & $d$ \\
\hline Silver & 156 & $<0.09$ & $<0.03$ & $<0.03$ & d & $d$ \\
\hline Sodium & 156 & 1000 & 132 & 281 & d & $d$ \\
\hline Strontium & 156 & 0.920 & 0.270 & 0.572 & d & $d$ \\
\hline Throrium & 156 & 0.20 & $<0.05$ & $<0.05$ & d & $d$ \\
\hline Titanium & 156 & $<0.20$ & $<0.06$ & $<0.06$ & $d$ & d \\
\hline Vanadium & 156 & $<0.06$ & $<0.02$ & $<0.02$ & $d$ & $d$ \\
\hline Zinc & 156 & 0.39 & $<0.05$ & $<0.05$ & 1.0 & 0 \\
\hline Zirconium & 156 & 0.06 & $<0.02$ & $<0.02$ & $d$ & d \\
\hline
\end{tabular}

(a) Units in mg/L unless otherwise noted.

(b)NPDES permit limits.

(c)Flow during operations and/or discharging.

(d) Not applicable

(e)Minimum value/maximum value. 


\section{Table 4.43. CY 1993 radiological data summary}

Y-12 Plant Discharge Point 503, Steam Plant Wastewater

Treatment Facility

\begin{tabular}{|c|c|c|c|c|c|c|c|c|c|c|}
\hline \multirow[b]{2}{*}{ Parameler } & \multirow{2}{*}{$\begin{array}{c}\text { Number of } \\
\text { samples }\end{array}$} & \multicolumn{6}{|c|}{ Concentration } & \multirow{2}{*}{ Standard etror } & \multirow{2}{*}{$\begin{array}{c}\text { Percentage of } \\
\text { DCG }\end{array}$} & \multirow{2}{*}{ Total curie } \\
\hline & & $\operatorname{Max}$ & +1 & Min & +1 & Med & $+/$ & & & \\
\hline Alpha activity (pCi/L) & 52 & 77 & 35 & -26 & 30 & 1.8 & 0 & 2.9 & $a$ & \\
\hline Americium-24I (pCi/L) & 52 & 0.72 & 0.45 & -0.34 & 0.49 & 0.070 & 0 & 0.030 & 0.22 & $1.5 \mathrm{E}-4$ \\
\hline Beta sctivity $(\mathrm{pCi} / \mathrm{L})$ & 52 & 47 & $\mathbf{0}$ & -26 & 0 & 5.8 & 14 & 1.4 & $a$ & \\
\hline Neptunium-237 (pCi/L) & 52 & 0.54 & 0.33 & -0.70 & 1 & 0.030 & $a$ & 0.030 & 0.23 & $1.1 \mathrm{E}-5$ \\
\hline Plutonium-238 (pCi/L) & 48 & 0.61 & 0.71 & -3.5 & 4.0 & -0.013 & 0 & 0.10 & 0.00 & $-3.3 \mathrm{E}-6$ \\
\hline Plutonium-239/240 (pCi/L) & 48 & 0.2 & 0.21 & -0.34 & 0.48 & 0 & 0 & 0.016 & $a$ & $2.6 \mathrm{E}-7$ \\
\hline Radium-226 (pCi/L) & 52 & 1.5 & 0.27 & -1.1 & 0.28 & 0.11 & 0 & 0.090 & 0.11 & $2.0 \mathrm{E}-5$ \\
\hline Radium-228 (pCi/L) & 52 & 2.4 & 0.23 & -2.5 & 1.6 & -0.19 & 0 & 0.10 & 0.00 & $-9.1 \mathrm{E}-6$ \\
\hline Strontium-90 (pCi/L) & 52 & 44 & 8.7 & -23 & 8.4 & 1.2 & 0 & 1.9 & 0.12 & $2.9 \mathrm{E}-4$ \\
\hline Technetium-99(pCi/mL) & 52 & 0.19 & 0.02 & -0.05 & 0.01 & 0.010 & 0.01 & 0.002 & 0.00001 & $1.9 \mathrm{E}-3$ \\
\hline Thorium-234 (pCi/L) & 52 & 0.37 & 0.27 & -0.15 & 0.29 & 0.10 & 0.10 & 0.020 & 0.00070 & $1.3 \mathrm{E}-5$ \\
\hline Thorium, total (mg/L) & 52 & 0.018 & $a$ & $<0.003$ & $a$ & $<0.003$ & $a$ & 0.0002 & $a$ & \\
\hline Thorium-228 (pCi/L) & 52 & 9.3 & 10 & -0.74 & 1.5 & 0.45 & 0.33 & 0.22 & 0.11 & $1.2 \mathrm{E}-4$ \\
\hline Thorium-230 (pCi/L) & 52 & 3.8 & 2.6 & 0.03 & 0.26 & 0.55 & 0 & 0.10 & 0.18 & $9.3 \mathrm{E}-5$ \\
\hline Thorium-232 (pCi/L) & 52 & 0.93 & 0.6 & -0.49 & 0.41 & 0.061 & 0 & 0.026 & 0.12 & $1.5 \mathrm{E}-5$ \\
\hline Tritium (pCi/L) & 52 & 1400 & 240 & -540 & 190 & 240 & 180 & 47 & 0.012 & $3.5 \mathrm{E}-2$ \\
\hline Uranium, total $(\mathrm{mg} / \mathrm{L})$ & 52 & $<0.001$ & $a$ & $<0.001$ & $a$ & $<0.001$ & $a$ & $a$ & $a$ & \\
\hline Uranium-234 $(\mathrm{pCi} / \mathrm{L})$ & 52 & 2.8 & 0.94 & -0.2 & 0.54 & 0.10 & $\mathbf{0}$ & 0.06 & 0.02 & $2.6 \mathrm{E}-5$ \\
\hline Uranium-235 (pCi/L) & 52 & 13 & 0.25 & -0.098 & 0.71 & 0 & 0 & 0.012 & $a$ & 8.4 E-6 \\
\hline Uranium-235 (\%) & 0 & $a$ & $a$ & $a$ & $a$ & $a$ & $a$ & $a$ & $a$ & \\
\hline Uranium-236 $(\mathrm{pCi} / \mathrm{L})$ & 48 & 0.42 & 0.08 & -0.08 & 0.11 & 0 & 0 & 0.01 & $a$ & $1.6 \mathrm{E}-6$ \\
\hline Uranium-238 (pCi/L) & 52 & 0.37 & 0.27 & -0.31 & 0.32 & 0.066 & 0 & 0.020 & 0.010 & $1.1 \mathrm{E}-5$ \\
\hline Gamma, total (pCi/L) & 4 & 40 & 17 & 4 & 16 & 32 & $\mathbf{0}$ & 8 & $a$ & \\
\hline
\end{tabular}

(a)Not applicable. 
Table 4.44. Y-12 Discharge Point 504, Plating Rinsewater Facilities

\begin{tabular}{|c|c|c|c|c|c|c|}
\hline \multirow[b]{2}{*}{ Parameter } & \multirow[b]{2}{*}{$\begin{array}{l}\text { Number of } \\
\text { samples }\end{array}$} & \multicolumn{3}{|c|}{ Concentration(a) } & \multirow[b]{2}{*}{$\begin{array}{c}\text { Reference } \\
\text { value(b) }\end{array}$} & \multirow[b]{2}{*}{$\begin{array}{l}\text { Number of values } \\
\text { exceeding reference }\end{array}$} \\
\hline & & $\operatorname{Max}$ & $\operatorname{Min}$ & $\mathrm{Av}$ & & \\
\hline Flow(c), gpd & 3 & 15588 & 6890 & 12051 & $d$ & $\mathrm{~d}$ \\
\hline pH, standard units & 3 & 8.3 & 7.5 & $d$ & $6.0 / 9.0(e)$ & 0 \\
\hline Temperature, $\mathrm{C}$ & 3 & 27.5 & 22.0 & 24.2 & 30.5 & 0 \\
\hline Cyanide & 3 & 0.01 & $<0.003$ & $<0.005$ & 1.2 & 0 \\
\hline Oil and grease & 3 & $<2.0$ & $<2.0$ & $<2.0$ & 52.0 & 0 \\
\hline Total toxic organics & 3 & $<0.010$ & $<0.010$ & $<0.010$ & 2.13 & 0 \\
\hline Chloride & 3 & 1100 & 8.7 & 370 & $d$ & $d$ \\
\hline Fluoride & 3 & 13 & 0.70 & 4.9 & $\mathrm{~d}$ & $d$ \\
\hline Mercury & 3 & $<0.0002$ & $<0.0002$ & $<0.0002$ & $d$ & $d$ \\
\hline Nitrate & 3 & 18 & 0.13 & 6.1 & $\mathrm{~d}$ & d \\
\hline Total suspended solids & 3 & $<5$ & $<$ & $<5$ & 60.0 & 0 \\
\hline Sulfate & 3 & 920 & 120 & 403 & $d$ & $d$ \\
\hline Aluminum & 3 & 0.4 & 0.2 & 0.3 & $d$ & d \\
\hline Beryllium & 3 & $<0.002$ & $<0.002$ & $<0.002$ & d & $d$ \\
\hline Cadmium & 3 & 0.02 & $<0.001$ & $<0.01$ & 0.69 & 0 \\
\hline Chromium & 3 & $<0.03$ & $<0.03$ & $<0.03$ & 2.77 & 0 \\
\hline Copper & 3 & $<0.03$ & $<0.03$ & $<0.03$ & 3.38 & 0 \\
\hline Iron & 3 & 3.6 & 0.7 & 3 & $d$ & d \\
\hline Lead & 3 & $<0.1$ & $<0.005$ & $<0.07$ & 0.69 & 0 \\
\hline Nickel & 3 & 0.78 & 0.5 & 0.6 & 3.98 & 0 \\
\hline Phosphorus & 3 & 0.3 & $<0.22$ & $<0.3$ & $d$ & $d$ \\
\hline Potassium & 3 & 111 & 30 & 70 & $d$ & $d$ \\
\hline Silver & 3 & $<0.03$ & $<0.03$ & $<0.03$ & 0.43 & 0 \\
\hline Sodium & 3 & 860 & 9.6 & 290 & d & d \\
\hline Zinc & 3 & 0.07 & 0.06 & 0.06 & 2.61 & d \\
\hline
\end{tabular}

(a) Units in $\mathrm{mg} / \mathrm{L}$ unless otherwise noted.

(b)NPDES permit limits.

(c)Flow during operations and/or discharging.

(d)Not applicabie

(e)Minimum value/maximum value. 


\section{Table 4.45. CY 1993 radlological data summary}

Y-12 Plant Discharge Point 504, Plating Ainsewater Treatment Facilities

(Summed DCG percentage $=5.27$ )

\begin{tabular}{|c|c|c|c|c|c|c|c|c|c|c|}
\hline \multirow[b]{2}{*}{ Parameter } & \multirow{2}{*}{$\begin{array}{l}\text { Number of } \\
\text { samples }\end{array}$} & \multicolumn{5}{|c|}{ Concentration } & \multirow[b]{2}{*}{$+1-$} & \multirow{2}{*}{ Standard error } & \multirow{2}{*}{ Percentage of DCG } & \multirow{2}{*}{ Total curies } \\
\hline & & $\operatorname{Max}$ & $+\%$ & Min & $+1-$ & Med & & & & \\
\hline Alpha activity (pCi/L) & 3 & 150 & 55 & 8.6 & 3.9 & 31 & 12 & 44 & a & \\
\hline Americium-24l (pCi/L) & 3 & 0.21 & 0.42 & $-0.05 i$ & 0.073 & 0.060 & 0.27 & 0.080 & 0.20 & $1.0 \mathrm{E}-8$ \\
\hline Beta activity (pCi/L) & 3 & 67 & 7.0 & 26 & 9.5 & 39 & 5.6 & 12 & a & \\
\hline Neptunium-237 (pCi/L) & 3 & 0.19 & 0.21 & 0.043 & 11 & 0.11 & 0.13 & 0.040 & 0.37 & $1.6 \mathrm{E}-8$ \\
\hline Plutonium-238 (pCi/L) & 2 & 0.21 & 0.30 & -0.032 & 0.16 & 0.089 & a & 0.12 & 0.22 & $1.2 \mathrm{E}-8$ \\
\hline Plutionium-239/240 (pCi/L) & 2 & -0.045 & 0.13 & -0.073 & 0.15 & -0.059 & a & 0.014 & 0.00 & $-8.1 \mathrm{E}-9$ \\
\hline Radium-226 (pCi/L) & 3 & 0.16 & 0.37 & -1.8 & 0.50 & -0.12 & 0.026 & 0.61 & 0.00 & $-8.0 \mathrm{E}-8$ \\
\hline Radium-228 (pCi/L) & 3 & 1.7 & 0.15 & -0.9 & 0.09 & 0.02 & 0.05 & 0.7 & 0.02 & $4.0 \mathrm{E}-8$ \\
\hline Strontium-90(pCi/L) & 3 & 2.4 & 11 & -14 & 11 & -9.1 & 9.7 & 4.9 & 0.00 & -9.4 E-7 \\
\hline Technetium-99 (pCi/mL) & 3 & 0.13 & 0 & 0.032 & 0.01 & 0.080 & 0.01 & 0.028 & 0.080 & 1.1 E-5 \\
\hline Thorium-234 (pCi/L) & 3 & 11 & 2.4 & 2.5 & 0.72 & 9.6 & 1.8 & 2.63 & 0.096 & $6.2 \mathrm{E}-7$ \\
\hline Thorium, total (mg/L) & 3 & $<0.003$ & a & $<0.003$ & a & $<0.003$ & a & a & a & \\
\hline Thorium-228 (pCi/L) & 3 & 0.87 & 0.63 & -0.06 & 0.44 & 0.15 & 0.53 & 0.28 & 0.038 & $6.2 \mathrm{E}-8$ \\
\hline Thorium-230 (pCi/L) & 3 & 0.35 & 0.29 & 0.091 & 0.13 & 0.29 & 0.34 & 0.078 & 0.097 & $2.0 \mathrm{E}-8$ \\
\hline Thorium-232 (pCi/L) & 3 & 0.14 & $\mathbf{0}$ & -0.07 & 0.13 & 0.06 & 0.12 & 0.06 & 0.1 & $4.4 \mathrm{E}-7$ \\
\hline Tritium (pCi/L) & 3 & -38 & 230 & -450 & 0 & -74 & 160 & 132 & 0.00 & $6.2 \mathrm{E}-7$ \\
\hline Uranium, total $(\mathrm{mg} / \mathrm{L})$ & 3 & 0.031 & a & 0.009 & $\mathbf{a}$ & 0.03 & $\mathbf{a}$ & 0.007 & a & \\
\hline Uranium-234 (pCi/L) & 3 & 22 & 3.5 & 2.8 & 0.80 & 12 & 2.6 & 5.5 & 2.4 & $1.7 \mathrm{E}-6$ \\
\hline Uranium-235 (pCi/L) & 3 & 1.2 & 0.52 & 0.21 & 0.19 & 0.65 & 0.40 & 0.286 & 0.11 & $9.4 \mathrm{E}-8$ \\
\hline Uranium-235 (\%) & 3 & 1.3 & a & 0.5 & $a$ & 0.72 & a & a & a & \\
\hline Uranium-236 (pCi/L) & 2 & 1.1 & 0.45 & 0.34 & 0.25 & 0.72 & a & 0.38 & 0.14 & \\
\hline Uranium-238 (pCi/L) & 3 & 11 & 2.4 & 2.5 & 0.72 & 9.6 & 1.8 & 2.6 & 1.6 & $9.2 \mathrm{E}-7$ \\
\hline
\end{tabular}

(a)Not applicable. 
Table 4.46. CY 1993 NPDES Permit Number TN 0002968

Y-12 Plant Discharge Point 506, Building 9204-3 Sump Pump

\begin{tabular}{|c|c|c|c|c|c|c|}
\hline \multirow[b]{2}{*}{ Parameter } & \multirow{2}{*}{$\begin{array}{l}\text { Number of } \\
\text { samples }\end{array}$} & \multicolumn{3}{|c|}{ Concentration } & \multirow{2}{*}{$\begin{array}{l}\text { Reference } \\
\text { value(a) }\end{array}$} & \multirow{2}{*}{$\begin{array}{l}\text { Number of values } \\
\text { exceeding reference }\end{array}$} \\
\hline & & $\operatorname{Max}$ & Min & Av & & \\
\hline Flow, gpm (b) & 46 & 50 & 50 & 50 & c & c \\
\hline pH, standard units & 47 & 7.9 & 6.5 & c & $6.5 / 8.5(d)$ & $0 / 0$ \\
\hline Temperature, $\mathrm{C}$ & 47 & 29.9 & 17.4 & 23.4 & 30.5 & 0 \\
\hline Oil and grease, mg/L & 47 & 8.1 & $<2.0$ & $<2.7$ & 15 & 0 \\
\hline PCB & 47 & $<0.0005$ & $<0.0005$ & $<0.0005$ & c & 0 \\
\hline
\end{tabular}
(a)NPDES permit limits.
(b)Flow during operation and/or discharging.
(c) Not applicable.
(d)Minimum value/maximum value. 
Table 4.47. CY 1993 NPDES Permit Number TN 0002968

Discharge Point 512, Y-12 Plant, Groundwater Treatment Facility

\begin{tabular}{|c|c|c|c|c|c|c|}
\hline \multirow[b]{2}{*}{ Parameter } & \multirow{2}{*}{$\begin{array}{l}\text { Number of } \\
\text { samples }\end{array}$} & \multicolumn{3}{|c|}{ Concentration(a) } & \multirow{2}{*}{$\begin{array}{l}\text { Reference } \\
\text { value(b) }\end{array}$} & \multirow{2}{*}{$\begin{array}{l}\text { Number of values } \\
\text { exceeding reference }\end{array}$} \\
\hline & & $\operatorname{Max}$ & Min & Av & & \\
\hline Flow(c), gpd & 172 & 40110 & 2260 & 16464 & $d$ & $d$ \\
\hline $\mathrm{pH}$, standard units & 172 & 7.1 & 8.3 & $d$ & $6.0 / 9.0(\mathrm{e})$ & 0 \\
\hline Temperature, $\mathrm{C}$ & 168 & 29.9 & 16.6 & 22.6 & 30.5 & $d$ \\
\hline Oil and grease & 168 & 72 & 2.0 & $<2.7$ & 15 & 1 \\
\hline Aluminum & 168 & 0.3 & $<0.2$ & $<0.2$ & $d$ & $d$ \\
\hline Arsenic & 168 & 0.2 & $<0.2$ & $<0.2$ & $d$ & $d$ \\
\hline Barium & 168 & 0.235 & 0.055 & 0.101 & $d$ & $d$ \\
\hline Beryllium & 168 & $<0.002$ & $<0.002$ & $<0.002$ & $d$ & $d$ \\
\hline Boron & 168 & 77.9 & 26.0 & 54.3 & $d$ & $d$ \\
\hline Cadmium & 168 & $<0.02$ & $<0.02$ & $<0.02$ & $d$ & $d$ \\
\hline Calcium & 168 & 103 & 75.6 & 90.7 & $d$ & $d$ \\
\hline Cerium & 168 & $<0.08$ & $<0.08$ & $<0.08$ & $d$ & $d$ \\
\hline Chromium & 168 & $<0.03$ & $<0.03$ & $<0.03$ & $d$ & $d$ \\
\hline Cobalt & 168 & $<0.01$ & $<0.01$ & $<0.01$ & $d$ & $d$ \\
\hline Copper & 168 & 0.35 & $<0.03$ & $<0.04$ & $d$ & $d$ \\
\hline Gallium & 168 & $<0.09$ & $<0.09$ & $<0.09$ & $d$ & $d$ \\
\hline Iron & 168 & 0.3 & $<0.3$ & $<0.3$ & 1.0 & 0 \\
\hline Lead & 168 & $<0.1$ & $<0.1$ & $<0.1$ & $d$ & $d$ \\
\hline Lithium & 168 & 18.4 & 7.9 & 13.6 & $d$ & $d$ \\
\hline Magnesium & 168 & 21.9 & 13.5 & 18.9 & $d$ & $d$ \\
\hline Manganese & 168 & 4.64 & $<0.009$ & $<0.515$ & $d$ & $d$ \\
\hline Molybdenum & 168 & 0.04 & $<0.03$ & $<0.03$ & $d$ & $d$ \\
\hline Nickel & 168 & 0.09 & $<0.04$ & $<0.04$ & $d$ & $d$ \\
\hline Niobium & 168 & $<0.05$ & $<0.05$ & $<0.05$ & $d$ & $d$ \\
\hline Phosphorus & 168 & 0.5 & $<0.2$ & $<0.3$ & $d$ & $d$ \\
\hline Potassium & 168 & 13 & 6 & 10 & $d$ & $d$ \\
\hline Silver & 168 & $<0.03$ & $<0.03$ & $<0.03$ & $d$ & $d$ \\
\hline Sodium & 168 & 25.8 & 8.6 & 20.4 & $d$ & $d$ \\
\hline Strontium & 168 & 0.400 & 0.273 & 0.315 & $d$ & $d$ \\
\hline Throrium & 168 & $<0.05$ & $<0.05$ & $<0.05$ & $d$ & $d$ \\
\hline Titanium & 168 & $<0.06$ & $<0.06$ & $<0.06$ & $d$ & $d$ \\
\hline Vanadium & 168 & $<0.02$ & $<0.02$ & $<0.02$ & $d$ & $d$ \\
\hline Zinc & 168 & 0.20 & $<0.05$ & $<0.05$ & $\dot{a}$ & $d$ \\
\hline Zirconium & 168 & $<0.02$ & $<0.02$ & $<0.02$ & $d$ & $d$ \\
\hline PCB & 168 & $<0.0005$ & $<0.0005$ & $<0.0005$ & 0.001 & 0 \\
\hline Methylene chloride & 168 & $<0.010$ & $<0.010$ & $<0.010$ & $d$ & $d$ \\
\hline Tetrachoroethylene & 168 & $<0.010$ & $<0.010$ & $<0.010$ & $d$ & $d$ \\
\hline Trichorethyiene & 168 & $<0.010$ & $<0.010$ & $<0.010$ & $d$ & $d$ \\
\hline
\end{tabular}

(a) Units in $\mathrm{mg} / \mathrm{L}$ unless otherwise noted.

(b)NPDES permit limits.

(c)Flow during operations and/or discharging.

(d)Not applicable

(e)Minimum value/maximum value. 
Table 4.48. CY 1993 radiological data summary

Y-12 Plant Discharge Point 512, Groundwater Treatment Facility

\begin{tabular}{|c|c|c|c|c|c|c|c|c|c|c|}
\hline \multirow[b]{2}{*}{ Parameter } & \multirow[b]{2}{*}{$\begin{array}{l}\text { Number of } \\
\text { samples }\end{array}$} & \multicolumn{6}{|c|}{ Concentration } & \multirow[b]{2}{*}{ Standard error } & \multirow[b]{2}{*}{$\begin{array}{c}\text { Percentage of } \\
\text { DCG }\end{array}$} & \multirow[b]{2}{*}{ Total curies } \\
\hline & & $\operatorname{Max}$ & $+\%$ & $\operatorname{Min}$ & $+/-$ & Med & +1 & & & \\
\hline Alpha activity (pCi/L) & 49 & 510 & 51 & 5.4 & 0 & 37 & 0 & 17.1 & $\mathbf{a}$ & \\
\hline Americium-241 (pCi/L) & 49 & 0.66 & 0.54 & -0.73 & 0.41 & 0.090 & 0 & 0.040 & 0.30 & $8.2 \mathrm{E}-7$ \\
\hline Beta activity (pCi/L) & 49 & 140 & 14.0 & -10 & $\mathbf{0}$ & 23 & 8.1 & 4.2 & $\mathbf{a}$ & \\
\hline Neptunium-237 (pCi/L) & 49 & 0.66 & 0.69 & -0.62 & $\mathbf{0}$ & 0.070 & 0.18 & 0.020 & 0.23 & $9.6 \mathrm{E}-7$ \\
\hline Plutonium-238 (pCi/L) & 45 & 0.47 & 0.32 & -0.35 & 0.50 & -0.020 & 0 & 0.020 & 0.00 & $-2.8 \mathrm{E}-7$ \\
\hline Plutonium $239 / 240(\mathrm{pCi} / \mathrm{L})$ & 45 & 0.36 & 0.43 & -0.27 & 0.54 & 0.028 & 0 & 0.018 & 0.093 & $4.4 \mathrm{E}-7$ \\
\hline Radium-226 (pCi/L) & 49 & 1.3 & 0.27 & -1.4 & 0.26 & 0.15 & 0.30 & 0.090 & 0.15 & $1.1 \mathrm{E}-6$ \\
\hline Radium-228 (pCi/L) & 49 & 1.8 & 0.19 & -2.3 & 0.27 & -0.23 & 0 & 0.20 & 0.00 & $4.4 \mathrm{E}-7$ \\
\hline Strontium-90 (pCi/L) & 49 & 71 & 14 & -35 & 11.0 & 0.60 & 0.40 & 2.6 & 0.058 & $4.7 \mathrm{E}-5$ \\
\hline Technetium-99(pCi/mL) & 49 & 0.201 & 0 & -0.07 & 0.01 & 0.02 & 0 & 0.008 & 0.02 & $3.7 \mathrm{E}-4$ \\
\hline Thorium-234 (pCi/L) & 49 & 370 & 73 & 0.04 & 0.08 & 30 & 6 & 11 & 0.3 & $1.2 \mathrm{E}-4$ \\
\hline Thorium, total (mg/L) & 49 & 0.01 & a & $<0.003$ & $\mathbf{a}$ & $<0.003$ & a & 0.0002 & a & \\
\hline Thorium-228 (pCi/L) & 49 & 4.5 & 3.1 & -0.32 & 0.34 & 0.58 & 0.40 & 0.14 & 0.15 & $8.3 \mathrm{E}-6$ \\
\hline Thorium-230 (pCi/L) & 49 & 3.3 & 2.2 & -0.08 & 0.88 & 0.41 & 0 & 0.1 & 0.1 & $1.4 \mathrm{E}-6$ \\
\hline Thorium-232 (pCi/L) & 49 & 1.6 & 1.5 & -0.6 & 0.12 & 0.07 & 0 & 0.05 & 0.1 & $1.1 \mathrm{E}-6$ \\
\hline Tritium (pCi/L) & 49 & 5291 & 334 & 2100 & 0 & 4300 & 310 & 66 & 0.22 & $4.5 \mathrm{E}-2$ \\
\hline Uranium, total (mg/L) & 49 & 1.01 & $\mathbf{a}$ & 0.043 & a & 0.078 & $\mathbf{a}$ & 0.039 & $\mathbf{a}$ & \\
\hline Uraniurn-234 (pCi/L) & 49 & 57 & 12 & 0.04 & 0.08 & 6 & 2 & 2 & 1 & $1.2 \mathrm{E}-4$ \\
\hline Uranium-235 ( $\mathrm{pCi} / \mathrm{L})$ & 49 & 6.5 & 0.6 & -0.03 & 0.06 & 0.7 & 0.5 & 0.2 & 0.1 & $1.2 \mathrm{E}-5$ \\
\hline Uranium-235 (\%) & 49 & 0.39 & $\mathbf{a}$ & 0.16 & $\mathbf{a}$ & 0.23 & a & 0.0040 & a & \\
\hline Uranium-236 (pCi/L) & 45 & 2.2 & 0.96 & -0.02 & 0.05 & 0.3 & 0.4 & 0.1 & 0.10 & 2.9 E-6 \\
\hline Uranium-238 (pCi/L) & 49 & 370 & 73 & 0.04 & 0.08 & 30 & 6 & 11 & 5 & $6.3 \mathrm{E}-4$ \\
\hline Gamma, total (pCi/L) & 4 & 89 & 17 & 39 & 16 & 49 & $\mathbf{a}$ & 11 & $\mathbf{a}$ & \\
\hline
\end{tabular}

(a)Not applicable. 
Table 4.49. CY 1993 NPDES Permit Ni:mber TN 0002968

Y-12 Plant Discharge Point 623, Fiyash Sluice

\begin{tabular}{lccccccc}
\hline & & \multicolumn{4}{c}{ Concentration } & & Number of \\
\cline { 3 - 7 } \multicolumn{1}{c}{ Parameter } & samples & Max & Min & Av & Reference & Number of values \\
value(a) & exceeding reference \\
\hline pH, standard units & 25 & 8.4 & 7.5 & $\mathrm{~b}$ & $6.5 / 8.5(\mathrm{c})$ & 0 \\
Flow, gpd(d) & 59 & 440,000 & 5,000 & 69.780 & $\mathrm{~b}$ & $\mathrm{~b}$ \\
\hline
\end{tabular}
(a)NPDES permit limits.
(b)Not applicable.
(c)Minimum valueimaximum value.
(d)Flow during operation and/or discharging. 
Table 4.50. 1993 Y-12 Plant annual nonradiological data summary for Station 17

\begin{tabular}{|c|c|c|c|c|c|c|}
\hline \multirow[b]{2}{*}{ Paranneter } & \multirow{2}{*}{$\begin{array}{c}\text { Number of } \\
\text { samples }\end{array}$} & \multicolumn{3}{|c|}{ Concentration(a) } & \multirow{2}{*}{$\begin{array}{l}\text { Reference } \\
\text { value(b) }\end{array}$} & \multirow{2}{*}{$\begin{array}{l}\text { Number of values } \\
\text { exceeding reference }\end{array}$} \\
\hline & & $\operatorname{Max}$ & Min & Av & & \\
\hline Acrolein & 203 & $<0.010$ & $<0.010$ & $<0.010$ & $c$ & $c$ \\
\hline Silver & 203 & $<0.006$ & $<0.006$ & $<0.006$ & $0.004(d)$ & 203(d) \\
\hline Aluminum & 203 & 2.22 & $<0.04$ & $<0.38$ & c & c \\
\hline Arsenic & 203 & 0.04 & $<0.04$ & $<0.04$ & $0.36(e)$ & 0 \\
\hline Tetrachlorethane & 203 & $<0.010$ & $<0.010$ & $<0.010$ & c & c \\
\hline Boron & 203 & 0.309 & $<0.006$ & $<0.108$ & c & c \\
\hline Barium & 203 & 0.0762 & $<0.0008$ & $<0.0550$ & c & c \\
\hline Bromodichloromethane & 203 & $<0.010$ & $<0.010$ & $<0.010$ & c & c \\
\hline Beryllium & 203 & $<0.0004$ & $<0.0004$ & $<0.0004$ & 0.0013 & 0 \\
\hline Benzene & 203 & $<0.010$ & $<0.010$ & $<0.010$ & 0.71 & 0 \\
\hline Bromoform & 203 & $<0.010$ & $<0.010$ & $<0.010$ & 4.7 & 0 \\
\hline Chlorobenzene & 203 & $<0.010$ & $<0.010$ & $<0.010$ & c & c \\
\hline Carbon tetrachloride & 203 & $<0.01$ & $<0.010$ & $<0.010$ & 0.044 & 0 \\
\hline 1,2 Dichloropropene & 203 & $<0.010$ & $<0.010$ & $<0.010$ & c & c \\
\hline Cerium & 203 & $<0.02$ & $<0.02$ & $<0.02$ & c & c \\
\hline Chioroethane & 203 & $<0.010$ & 0.010 & $<0.010$ & c & c \\
\hline Choroethylvinyl ether & 203 & $<0.010$ & $<0.010$ & $<0.010$ & c & c \\
\hline Bromomethane & 203 & $<0.010$ & $<0.010$ & $<0.010$ & c & c \\
\hline Chloromethane & 203 & $<0.010$ & $<0.010$ & $<0.010$ & c & c \\
\hline Chloroform & 203 & $<0.01$ & $<0.010$ & $<0.010$ & 4.7 & 0 \\
\hline Cobalt & 203 & $<0.002$ & $<0.002$ & $<0.002$ & c & c \\
\hline Dibromochloromethane & 203 & $<0.010$ & $<0.010$ & $<0.010$ & 4.7 & 0 \\
\hline Ethylbenzene & 203 & $<0.010$ & $<0.010$ & $<0.010$ & 29 & 0 \\
\hline Iron & 203 & 3.0 & $<0.06$ & $<0.51$ & c & c \\
\hline Trichlorofluoromethane & 203 & $<0.010$ & $<0.010$ & $<0.010$ & c & c \\
\hline Gallium & 203 & $<0.02$ & $<0.02$ & $<0.02$ & c & c \\
\hline Methylene chloride & 203 & $<0.01$ & $<0.010$ & $<0.010$ & 16 & 0 \\
\hline Methanol & 203 & $<0.010$ & $<0.010$ & $<0.010$ & c & c \\
\hline Manganese & 203 & 0.328 & $<0.002$ & $<0.094$ & c & c \\
\hline Niobium & 203 & $<0.01$ & $<0.01$ & $<0.01$ & c & c \\
\hline Tetrachoroethylene & 203 & $<0.01$ & $<0.010$ & $<0.010$ & 0.88 & 0 \\
\hline Strontium & 203 & 0.195 & $<0.001$ & $<0.130$ & c & c \\
\hline Trichloroethene & 203 & $<0.010$ & $<0.010$ & $<0.010$ & 0.807 & 0 \\
\hline Dichloroethene & 203 & $<0.010$ & $<0.010$ & $<0.010$ & 0.032 & 0 \\
\hline 1,3 Dichloropropene & 203 & $<0.010$ & $<0.010$ & $<0.010$ & c & c \\
\hline Thorium & 203 & 0.01 & $<0.01$ & $<0.01$ & c & c \\
\hline Titanium & 203 & 0.03 & $<0.01$ & $<0.02$ & c & c \\
\hline Thallium & 203 & 0.03 & $<0.03$ & $<0.03$ & $c$ & c \\
\hline Vanadium & 203 & 0.004 & $<0.004$ & $<0.004$ & $\therefore$ & $c$ \\
\hline Vinyl chloride & 203 & $<0.010$ & $<0.010$ & $<0.010$ & $\mathrm{c}$ & c \\
\hline Acrylonitrile & 203 & $<0.01$ & 0.010 & 0.010 & c & c \\
\hline Zirconium & 203 & 0.004 & $<0.004$ & $<0.004$ & $c$ & c \\
\hline Mercury & 203 & 0.0093 & $<0.0002$ & $<0.0016$ & 0.00015 & 203(d) \\
\hline
\end{tabular}


Table 4.50 (continued)

\begin{tabular}{|c|c|c|c|c|c|c|}
\hline \multirow[b]{2}{*}{ Parameter } & \multirow{2}{*}{$\begin{array}{l}\text { Number of } \\
\text { samples }\end{array}$} & \multicolumn{3}{|c|}{ Concentration(a) } & \multirow{2}{*}{$\begin{array}{c}\text { Reference } \\
\text { value(b) }\end{array}$} & \multirow{2}{*}{$\begin{array}{l}\text { Number of values } \\
\text { exceeding reference }\end{array}$} \\
\hline & & $\operatorname{Max}$ & Min & Av & & \\
\hline Nitrate & 203 & 7.8 & $<0.008$ & $<3.122$ & c & c \\
\hline Total phosphorus & 202 & 7.2 & 0.19 & 1.34 & $c$ & $c$ \\
\hline Copper & 203 & 0.017 & $<0.006$ & $<0.008$ & 0.018 & 0 \\
\hline Zine & 203 & 0.16 & $<0.01$ & $<0.07$ & 0.117 & 0 \\
\hline Chromium & $20 \dot{3}$ & 0.028 & $<0.006$ & $<0.006$ & $0.016(f)$ & 1 \\
\hline Toluene & 203 & $<0.010$ & $<0.010$ & $<0.010$ & 300 & 0 \\
\hline 1 Tricholoroethane & 203 & $<0.010$ & $<0.010$ & $<0.010$ & 170 & 0 \\
\hline 2 Tricholoroethane & 203 & $<0.010$ & $<0.010$ & $<0.010$ & 0.45 & 0 \\
\hline Molybdenum & 203 & 0.01 & $<0.006$ & $<0.006$ & c & c \\
\hline Lithium & 203 & 0.19 & $<0.02$ & $<0.06$ & c & c \\
\hline Selenium & 196 & $<0.1$ & $<0.002$ & $<0.03$ & 0.02 & 0 \\
\hline Cadmium & 203 & 0.004 & $<0.0005$ & $<0.004$ & 0.004 & 0 \\
\hline Lead & 202 & 0.02 & $<0.002$ & $<0.016$ & 0.082 & 0 \\
\hline Nickel & 203 & 0.012 & $<0.008$ & $<0.008$ & 1.4 & 0 \\
\hline Caleium & 203 & 84.3 & $<0.2$ & $<57.7$ & c & c \\
\hline Magnesium & 203 & 18.2 & $<0.04$ & $<11.0$ & c & $c$ \\
\hline Sodium & 203 & 47.7 & $<0.04$ & $<17.81$ & c & c \\
\hline Potassium & 203 & 4.0 & $<0.6$ & $<2.2$ & c & $c$ \\
\hline Sulfate & 203 & 160 & 27 & 71 & c & c \\
\hline Chioride & 203 & 42 & 9.1 & 18.7 & $\mathrm{c}$ & c \\
\hline Fluoride & 203 & 2.9 & 0.01 & 0.84 & c & c \\
\hline Total suspended solids & 203 & 82 & $<5$ & $<17$ & c & c \\
\hline Total dissolved solids & 203 & 500 & 10 & 304 & c & c \\
\hline Alkanlinity & 201 & 190 & 56 & 107 & c & c \\
\hline Total organic carbons & 202 & 37 & 2.6 & 19.1 & c & c \\
\hline Residual chlorine, total & 200 & 0.08 & -0.010 & 0.014 & $s$ & c \\
\hline Cadmium (AA) & 94 & 0.0082 & $<0.0005$ & $<0.0019$ & 0.004 & 1 \\
\hline Flow, MGD (g) & 242 & 105.4 & 0 & 4.7 & c & c \\
\hline Temperature, $\mathbf{F}$ & 245 & 84.8 & 51.0 & 68.3 & 86.9 & 0 \\
\hline $\mathrm{pH}$, standard units & 246 & 8.7 & 7.4 & c & $6 / 9(h)$ & 0 \\
\hline Dissolved oxygen & 231 & 10.5 & 5.2 & 8.2 & c & c \\
\hline Conductivity, mhos/cm & 245 & 70.6 & 31.8 & 50.1 & c & c \\
\hline
\end{tabular}

(a) Units are in $\mathrm{mg} / \mathrm{L}$ unless otherwise noted.

(b)Tennessee Water Quality Criteria mininum limits.

(c)Not applicable.

(d)The analytical detection level for this parameter is higher than the reference value.

(e)This limit applies to arsenic IIl only. The data represent all forms of arsenic.

(f)This limit applies to hexavalent chromium only. The data represent total chromium.

(g)Flow during operation and/or discharging.

(h)Minimum value/maximum value. 
Table 4.51. 1993 Y-12 Plant annual radlological data summary for Station 17

(EFPC 23.4 km, near junction of Bear Creek and Scarboro Road)

\begin{tabular}{|c|c|c|c|c|c|c|c|c|c|c|}
\hline \multirow[b]{2}{*}{ Parameter } & \multirow{2}{*}{$\begin{array}{c}\text { Number of } \\
\text { samples }\end{array}$} & \multicolumn{5}{|c|}{ Concentration } & \multirow[b]{2}{*}{$+1-$} & \multirow{2}{*}{ Standard error } & \multirow{2}{*}{$\begin{array}{c}\text { Percentage of } \\
\text { DCG }\end{array}$} & \multirow{2}{*}{ Total curies } \\
\hline & & $\operatorname{Max}$ & $+/-$ & Min & $+1-$ & Med & & & & \\
\hline Flow, MGD (a) & 242 & 1054 & b & 0 & b & $3.5(\mathrm{c})$ & b & b & b & \\
\hline Alpha activity (pCi/L) & 47 & 35 & 5 & -2.2 & 0 & 9.8 & 7.5 & 1.1 & $b$ & \\
\hline Americium-241 (pCi/L) & 46 & 0.84 & 0.79 & -0.82 & 0.57 & 0.090 & 0 & 0.040 & 0.29 & $6.7 \mathrm{E}-4$ \\
\hline Beta activity (pCi/L) & 47 & 43 & 14.0 & -4.1 & 0 & 9 & 5.6 & 1.0 & b & \\
\hline Neptunium-237 (pCi/L) & 47 & 1.6 & 0.60 & -0.25 & 0 & 0.11 & 0.22 & 0.040 & 0.37 & $1.1 \mathrm{E}-3$ \\
\hline Plutonium-238 (pCi/L) & 47 & 0.3 & 0.42 & -0.35 & 0.23 & 0.011 & 0 & 0.02 & 0.028 & $5.9 \mathrm{E}-5$ \\
\hline Plutonium-239/240 (pCi/L) & 47 & 0.31 & 0 & -0.27 & 0.54 & 0 & $\mathbf{0}$ & 0.012 & 0 & $8.4 \mathrm{E}-5$ \\
\hline Radium-226 (pCi/L) & 47 & 2.7 & 0.45 & -1.14 & 0.44 & 0.17 & 0.23 & 0.11 & 0.17 & $1.6 \mathrm{E}-3$ \\
\hline Radium-228 (pCi/L) & 47 & 4.5 & 0 & -1.8 & 0 & 0.11 & 0.015 & 0.10 & 0.11 & $2.2 \mathrm{E}-3$ \\
\hline Strontium-90 (pCi/L) & 47 & 6.4 & 2.6 & -5.9 & 0.0 & 0 & 0.38 & 0.10 & 0 & $1.2 \mathrm{E}-4$ \\
\hline Technetium-99 (pCi/mL) & 47 & 0.16 & 0.01 & -0.064 & 0.01 & 0.010 & 0.010 & 0.0050 & 0.02 & $9.7 \mathrm{E}-2$ \\
\hline Thorium, total (mg/L) & 47 & 0.009 & b & $<0.003$ & $\mathbf{b}$ & $<0.003$ & b & 0.0002 & b & \\
\hline Thorium-228 (pCi/L) & 47 & 3.3 & 0 & -0.51 & 0.63 & 0.33 & $\mathbf{0}$ & 0.080 & 0.083 & $3.2 \mathrm{E}-3$ \\
\hline Thorium-230 (pCi/L) & 47 & 3.0 & 2.1 & -0.01 & 0.21 & 0.3 & 0.24 & 0.08 & 0.1 & $3.2 \mathrm{E}-3$ \\
\hline Thorium-232 (pCi/L) & 47 & 0.77 & 0.39 & -0.081 & 0.16 & 0.049 & b & 0.022 & 0.098 & $5.3 \mathrm{E}-4$ \\
\hline Tritium (pCi/L) & 46 & 660 & $\mathbf{0}$ & -380 & 190 & 350 & b & 31 & 0.020 & $2.1 \mathrm{EO}$ \\
\hline Uranium, total (mg/L) & 47 & 0.051 & b & $<0.009$ & b & $<0.02$ & b & 0.001 & b & \\
\hline Uranium-234 (pCi/L) & 47 & 13 & 0 & 1.9 & 0.38 & 4.5 & 0 & 0.21 & 0.90 & $3.2 \mathrm{E}-2$ \\
\hline Uranium-235 (pCi/L) & 47 & 0.89 & 0.74 & -0.01 & 0.17 & 0.23 & 0.50 & 0.027 & 0.040 & $1.8 \mathrm{E}-3$ \\
\hline Uranium-235 (\%) & 47 & 1.8 & b & 0.31 & b & 0.51 & b & 0.038 & b & \\
\hline Uranium-236 (pCi/L) & 40 & 0.25 & 0.23 & -0.03 & 0.06 & 0.1 & $\mathbf{b}$ & 0.01 & 0.02 & $7.7 \mathrm{E}-4$ \\
\hline Uranium-238 (pCi/L) & 47 & 19 & 3.5 & 2.7 & 0.71 & 6.2 & 1.3 & 0.48 & 1.0 & $4.6 \mathrm{E}-2$ \\
\hline
\end{tabular}

(a)Flow during operation and/or discharging.

(b)Not applicable.

(c)Average flow, not median. 
Table 4.52. Y-12 Plant annual summary for Upper Bear Creek nonradiological data km 11.97

\begin{tabular}{|c|c|c|c|c|c|c|}
\hline \multirow[b]{2}{*}{ Parameter } & \multirow{2}{*}{$\begin{array}{l}\text { Number of } \\
\text { samples }\end{array}$} & \multicolumn{3}{|c|}{ Concentration(a) } & \multirow{2}{*}{$\begin{array}{c}\text { Reference } \\
\text { value(b) }\end{array}$} & \multirow{2}{*}{$\begin{array}{l}\text { Number of values } \\
\text { exceeding reference }\end{array}$} \\
\hline & & $\operatorname{Max}$ & Min & Av & & \\
\hline Arsenic & 52 & $<0.2$ & $<0.005$ & $<0.1$ & 0.360 & 0 \\
\hline Cadmium & 52 & $<0.02$ & $<0.0005$ & $<0.010$ & 0.004 & $33(c, d))$ \\
\hline Chromium & 52 & 0.03 & $<0.001$ & $<0.01$ & 0.016 & $2 l(d, e)$ \\
\hline Cyanide & 52 & $<0.2$ & $<0.002$ & $<0.01$ & 0.022 & 1 (d) \\
\hline Lead & 52 & $<0.1$ & $<0.004$ & $<0.04$ & 0.082 & $19(\mathrm{c}, \mathrm{d})$ \\
\hline Mercury & 52 & 0.0003 & $<0.0002$ & $<0.0002$ & 0.00015 & $52(d)$ \\
\hline Nitrate (as N) & 52 & 230 & 17 & 91 & $f$ & $\mathrm{f}$ \\
\hline Dissolved oxygen & 52 & 15.2 & 6.7 & 9.8 & $3(g)$ & 0 \\
\hline Phenols & 52 & $<0.005$ & $<0.001$ & $<0.003$ & $\mathrm{f}$ & $\mathrm{f}$ \\
\hline Total dissolved solids & 52 & 2600 & 530 & 1200 & $f$ & $f$ \\
\hline Total suspended solids & 52 & 410 & $<5$ & $<16$ & $f$ & $f$ \\
\hline Selenium & 52 & $<0.5$ & $<0.002$ & $<0.2$ & 0.02 & $22(d)$ \\
\hline Thallium & 52 & $<0.2$ & $<0.001$ & $<0.1$ & $\mathrm{f}$ & $f$ \\
\hline $\mathrm{pH}$, standard units & 52 & 8.3 & 7.4 & f & $6 / 9(h)$ & 0 \\
\hline Aluminum & 52 & 0.7 & $<0.04$ & $<0.3$ & $f$ & $f$ \\
\hline Barium & 52 & 0.6 & 0.112 & 0.3 & $f$ & $\mathrm{f}$ \\
\hline Beryllium & 52 & $<0.002$ & $<0.0004$ & $<0.001$ & 0.0013 & $20(d)$ \\
\hline Boron & 52 & 0.2 & 0.026 & 0.1 & $f$ & f \\
\hline Calcium & 52 & 334 & 68.1 & 195 & $f$ & $f$ \\
\hline Cerium & 52 & $<0.1$ & $<0.02$ & $<0.04$ & $f$ & f \\
\hline Cobalt & 52 & 0.01 & $<0.002$ & $<0.01$ & f & $\mathrm{f}$ \\
\hline Copper & 52 & $<0.03$ & $<0.006$ & $<0.02$ & 0.018 & $20(c, d)$ \\
\hline Gallium & 52 & $<0.1$ & $<0.02$ & $<0.05$ & $f$ & $\mathrm{f}$ \\
\hline Iron & 52 & 0.9 & $<0.06$ & $<0.3$ & $f$ & $\mathrm{f}$ \\
\hline Lithium & 52 & $<0.1$ & $<0.02$ & $<0.04$ & $f$ & $f$ \\
\hline Magnesium & 52 & 50.2 & 9.58 & 26.3 & $f$ & $f$ \\
\hline Mangariese & 52 & 2.3 & 0.02 & 0.8 & $f$ & $f$ \\
\hline Molydentin & 52 & $<0.03$ & $<0.006$ & $<0.02$ & f & $\mathrm{f}$ \\
\hline Nickel & 52 & 0.1 & $<0.008$ & $<0.03$ & 1.4 & 0 \\
\hline Niobium & 52 & $<0.1$ & $<0.01$ & $<0.03$ & f & $f$ \\
\hline Phosphorus & 17 & 0.3 & $<0.05$ & $<0.1$ & $f$ & $\mathrm{f}$ \\
\hline Potassium & 51 & 7.5 & $<2.3$ & $<4.4$ & $\tilde{f}$ & $\mathrm{f}$ \\
\hline Silver & 52 & $<0.030$ & $<0.006$ & $<0.015$ & 0.004 & $52(c, d)$ \\
\hline Sodium & 52 & 154 & 22.5 & 49.8 & $f$ & f \\
\hline Strctstium & 52 & 1.1 & 0.219 & 0.57 & $f$ & $\mathrm{f}$ \\
\hline Thurium & 52 & $<0.05$ & $<0.01$ & $<0.03$ & $\mathrm{f}$ & $f$ \\
\hline Titanium & 52 & $<0.1$ & $<0.02$ & $<0.04$ & $f$ & $f$ \\
\hline Vanadium & 52 & $<0.02$ & $<0.004$ & $<0.01$ & $\mathrm{f}$ & $\mathrm{f}$ \\
\hline Zinc & 52 & $<0.05$ & $<0.01$ & $<0.03$ & 0.117 & 0 \\
\hline Zirconium & 52 & $<0.02$ & $<0.004$ & $<0.01$ & $f$ & f \\
\hline PCB, total & 52 & $<0.0005$ & $<0.0005$ & $<0.0005$ & 0.000001 & $52(d)$ \\
\hline Volatile organics, total & 52 & $<0.01$ & $<0.01$ & $<0.01$ & 544 & 0 \\
\hline
\end{tabular}

(a)All units in $\mathrm{mg} / \mathrm{L}$ unless otherwise indicated.

(b)Tennessee Water Quality criteria.

(c) Reference value represents the dissolved form of this metal only. The actual data are representative of all forms.

(d)The analytical detection limit for this parameter is higher than the reference value.

(e)This limit applies to hexavalent Chromium only. The data represent total chromium.

(f)Not applicable.

(g)Minimum value.

(h) Minimum value/maximum value. 
Table 4.53. Y-12 Plant annual summary for upper Bear Creek radiological data $\mathrm{km} 11.97$

(Summed DCG percentage $=4.57$ )

\begin{tabular}{|c|c|c|c|c|c|c|c|c|c|}
\hline \multirow[b]{2}{*}{ Parameter } & \multirow{2}{*}{$\begin{array}{l}\text { Number of } \\
\text { samples }\end{array}$} & \multicolumn{6}{|c|}{ Concentration } & \multirow{2}{*}{$\begin{array}{l}\text { Standard } \\
\text { emor }\end{array}$} & \multirow{2}{*}{$\begin{array}{c}\text { Percentage } \\
\text { of DCG }\end{array}$} \\
\hline & & $\operatorname{Max}$ & +1. & $\operatorname{Min}$ & $+\%$ & Median & $+1 /$ & & \\
\hline Alpha Activity (pCi/L) & 52 & 210 & 18 & 4.6 & 8.9 & 110 & $\mathbf{a}$ & 6.9 & a \\
\hline Americium-241 (pCi/L) & 52 & 0.43 & 1.1 & -0.49 & 0.47 & 0.070 & $\mathbf{a}$ & 0.026 & 0.23 \\
\hline Beta activity $(\mathrm{pCi} / \mathrm{L})$ & 52 & 610 & 68 & 14 & 7.6 & 190 & $\mathbf{a}$ & 16 & $\mathbf{a}$ \\
\hline Neptunium-237 (pCi/L) & 52 & 3.1 & 0.67 & 0.14 & 0.12 & 1.2 & $\mathbf{a}$ & 0.093 & 3.8 \\
\hline Plutonium-238 (pCi/L) & 52 & 0.28 & 0.35 & -0.31 & 0 & 0.029 & $\mathbf{a}$ & 0.015 & 0.07 \\
\hline Plutonium-239/240 (pCi/L) & 52 & 0.29 & 0.25 & -0.17 & 0.16 & -0.0016 & $\mathbf{a}$ & 0.011 & 0.00 \\
\hline Technetium-99 (pCi/mL) & 52 & 1.3 & 0.03 & 0.098 & $\mathbf{a}$ & 0.47 & $\mathbf{a}$ & 0.0005 & 0.47 \\
\hline Uranium, total (mgl) & 52 & 0.63 & $\mathbf{a}$ & 0.32 & a & 0.37 & $\mathbf{a}$ & 0.006 & $\mathrm{a}$ \\
\hline Uranium-235 (\%) & 52 & 0.45 & a & 0.007 & $\mathbf{a}$ & 0.2 & $\mathbf{a}$ & 0.01 & a \\
\hline
\end{tabular}

(a)Not applicable. 
Table 4.54. Y-12 Plant annual summary for upper Bear Creek nonradiological data $\mathrm{km} \mathbf{1 2 . 4}$

\begin{tabular}{|c|c|c|c|c|c|c|}
\hline \multirow[b]{2}{*}{ Parameter } & \multirow{2}{*}{$\begin{array}{l}\text { Number of } \\
\text { samples }\end{array}$} & \multicolumn{3}{|c|}{ Concentration(a) } & \multirow{2}{*}{$\begin{array}{c}\text { Reference } \\
\text { value(b) }\end{array}$} & \multirow{2}{*}{$\begin{array}{l}\text { Number of values } \\
\text { exceeding reference }\end{array}$} \\
\hline & & $\operatorname{Max}$ & Min & Av & & \\
\hline Arsenic & 50 & $<0.2$ & $<0.005$ & $<0.1$ & 0.360 & 0 \\
\hline Cadmium & 50 & $<0.02$ & $<0.0003$ & $<0.01$ & 0.004 & $19(c, d)$ \\
\hline Chromium & 50 & 0.03 & $<0.001$ & $<0.01$ & 0.016 & $21(\mathrm{~d}, \mathrm{e})$ \\
\hline Cyanide & 50 & 0.02 & $<0.002$ & $<0.01$ & 0.022 & 0 \\
\hline Lead & 50 & 0.1 & $<0.004$ & $<0.04$ & 0.082 & $19(c, d)$ \\
\hline Mercury & 50 & 0.0004 & $<0.0002$ & $<0.0002$ & 0.00015 & $50(d)$ \\
\hline Nitrate (as N) & 50 & 35 & 4.2 & i8 & $f$ & $\mathrm{f}$ \\
\hline Dissolved oxygen & 50 & 12.3 & 4.9 & 7.3 & $3(g)$ & 0 \\
\hline Phenols & 50 & 0.005 & $<0.001$ & $<0.003$ & f & $\mathrm{f}$ \\
\hline Total dissolved solids & 50 & 1400 & 510 & 960 & $f$ & $\mathrm{f}$ \\
\hline Total suspended solids & 50 & 290 & $<5$ & $<13$ & f & f \\
\hline Selenium & 50 & 0.5 & $<0.002$ & $<0.2$ & 0.02 & $22(d)$ \\
\hline Thallium & 50 & $<0.2$ & $<0.001$ & $<0.1$ & f & f \\
\hline $\mathrm{pH}$, standard units & 50 & 7.9 & 7.1 & $f$ & 6/9(h) & 0 \\
\hline Aluminum & 50 & 1.8 & $<0.04$ & $<0.3$ & f & $f$ \\
\hline Barium & 50 & 0.12 & 0.058 & 0.092 & f & f \\
\hline Beryllium & 50 & $<0.002$ & $<0.0004$ & $<0.001$ & 0.0013 & $20(d)$ \\
\hline Boron & 50 & 0.11 & 0.027 & 0.073 & $\mathrm{f}$ & f \\
\hline Cajcium & 50 & 200 & 95.8 & 160 & f & $f$ \\
\hline Cerium & 50 & $<0.08$ & $<0.02$ & $<0.04$ & $\mathrm{f}$ & $\hat{\mathbf{i}}$ \\
\hline Cobalt & 50 & $<0.01$ & $<0.002$ & $<0.01$ & $f$ & $f$ \\
\hline Copper & 50 & $<0.03$ & $<0.006$ & $<0.02$ & 0.018 & $20(c, d)$ \\
\hline Gallium & 50 & $<0.09$ & $<0.02$ & $<0.05$ & f & f \\
\hline Iron & 50 & 3.2 & $<0.06$ & $<0.4$ & f & f \\
\hline Lithium & 50 & 0.08 & $<0.02$ & $<0.04$ & $\mathrm{f}$ & $\mathrm{f}$ \\
\hline Magnesium & 50 & 27 & 11.9 & 21 & f & f \\
\hline Manganese & 50 & 0.109 & 0.01 & 0.02 & $\tilde{\mathbf{i}}$ & $f$ \\
\hline Molydenum & 50 & $<0.03$ & $<0.006$ & $<0.02$ & $f$ & f \\
\hline Nickel & 50 & $<0.04$ & $<0.008$ & $<0.02$ & 1.4 & f \\
\hline Niobium & 50 & 0.05 & $<0.01$ & $<0.03$ & $\mathrm{f}$ & $f$ \\
\hline Phosphorus & 18 & 0.3 & $<0.05$ & $<0.1$ & f & f \\
\hline Potassium & 50 & 6 & 3 & 5 & $f$ & f \\
\hline Silver & 50 & $<0.03$ & $<0.006$ & $<0.02$ & 0.004 & $50(c, d)$ \\
\hline Sodium & 50 & 351 & 51.5 & 110 & $\mathrm{f}$ & f \\
\hline Strontium & 50 & 0.503 & 0.27 & 0.42 & f & f \\
\hline Thorium & 50 & $<0.05$ & $<0.01$ & $<0.03$ & r & f \\
\hline Titanium & 50 & $<0.06$ & $<0.02$ & $<0.04$ & f & $\mathrm{f}$ \\
\hline Vanadium & 50 & $<0.02$ & $<0.004$ & $<0.01$ & f & f \\
\hline Zinc & 50 & 0.05 & $<0.01$ & $<0.03$ & 0.117 & 0 \\
\hline Zirconium & 50 & $<0.02$ & $<0.004$ & $<0.01$ & f & $\mathrm{f}$ \\
\hline PCB, total & 50 & $<0.0060$ & $<0.0005$ & $<0.0006$ & 0.000001 & $50(d)$ \\
\hline Volatile Organics, total & 50 & $<0.01$ & $<0.01$ & $<0.01$ & 544 & 0 \\
\hline
\end{tabular}

(a)All units are $\mathrm{mg} / \mathrm{L}$ uniess otherwise indicated.

(b)Tennessee Water Quality criteria.

(c)Reference value represents the dissoived form of this metal only. The actual data are representative of all forms.

(d)The analytical detection limit for this parameter is higher than the reference value.

(e)This limit applies to hexavalent Chromium only. The data represent total Chromium.

(f) Not applicable.

(g)Minimum value.

(h)Minimum value/maximum value. 
Table 4.55. Y-12 Plant annual radiological summary for upper Bear Creek radiological data $\mathrm{km} \mathbf{1 2 . 4}$ (Summed DCG percentage $=2.64$ )

\begin{tabular}{|c|c|c|c|c|c|c|c|c|c|}
\hline \multirow{2}{*}{ Parameter } & \multirow{2}{*}{$\begin{array}{l}\text { Number of } \\
\text { samples }\end{array}$} & \multicolumn{5}{|c|}{ Concentration } & & \multirow{2}{*}{$\begin{array}{l}\text { Standard } \\
\text { ertor }\end{array}$} & \multirow{2}{*}{$\begin{array}{c}\text { Percentage } \\
\text { of DCG }\end{array}$} \\
\hline & & $\operatorname{Max}$ & $+1-$ & Min & $+1-$ & Median & $+/ 6$ & & \\
\hline Alpha Activity (pCi/L) & 50 & 550 & 86 & 110 & 23 & 260 & $\mathbf{a}$ & 13 & $\mathbf{a}$ \\
\hline Americium-241 (pCi/L) & 50 & 0.83 & 0.42 & -0.81 & 0.65 & 0.052 & $\mathbf{a}$ & 0.035 & 0.17 \\
\hline Beta activity (pCi/L) & 50 & 280 & 44 & 40 & 9.4 & 110 & $\mathbf{a}$ & 7.0 & a \\
\hline Neptunium-237 (pCi/L) & 50 & 1.9 & 0.59 & 0.0033 & 0.17 & 0.73 & $\mathbf{a}$ & 0.048 & 2.42 \\
\hline Plutonium-238 (pCi/L) & 50 & 0.44 & 0.39 & -0.34 & 0.50 & 0.018 & a & 0.018 & 0.045 \\
\hline Plutonium-239/240 (pCi/L) & 50 & 0.18 & 0.18 & -0.17 & 0.14 & 0 & a & 0.0095 & 0 \\
\hline Technetium-99(pCi/mL) & 50 & 0.61 & 0.01 & 0.011 & 0.01 & 0.081 & $\mathbf{a}$ & 0.012 & 0.081 \\
\hline Uranium, total (mg/L) & 50 & 0.91 & a & 0.272 & $\mathbf{a}$ & 0.58 & $\mathbf{a}$ & 0.020 & $\mathbf{a}$ \\
\hline Uranium-235 (\%) & 50 & 0.58 & $\mathbf{a}$ & 0.26 & a & 0.33 & a & 0.0075 & a \\
\hline
\end{tabular}

(a)Not applicable. 
Table 4.56. Surface water analytical results of polychlorinated biphenyls monitoring plan for the Oak Ridge Y-12 Plant, CY 1993

\begin{tabular}{|c|c|c|c|}
\hline $\begin{array}{c}\text { Site } \\
\text { number }\end{array}$ & Location & $\begin{array}{c}\text { Date } \\
\text { sampled }\end{array}$ & $\begin{array}{c}\mathrm{PCB} \\
\text { concentration } \\
(\mathrm{mg} / \mathrm{L})\end{array}$ \\
\hline \multirow[t]{4}{*}{ PCB-1 } & Outfall 301, Kerr Hollow Quarry & $2 / 3 / 93$ & $<0.0005$ \\
\hline & & $5 / 11 / 93$ & $<0.0005$ \\
\hline & & $9 / 15 / 93$ & $<0.0005$ \\
\hline & & $12 / 8 / 93$ & $<0.0005$ \\
\hline \multirow[t]{4}{*}{ PCB-2 } & Outfall 302, Rogers Quarry & $2 / 3 / 93$ & $<0.0005$ \\
\hline & & $5 / 11 / 93$ & $<0.0005$ \\
\hline & & $9 / 15 / 93$ & $<0.0005$ \\
\hline & & $12 / 8 / 93$ & $<0.0005$ \\
\hline PCB-3 & Outfall 303, New Hope Pond & a & \\
\hline PCB-5 & New Hope Pond Inlet & b & \\
\hline \multirow[t]{4}{*}{ PCB-6 } & Upstream of Outfall 135 & $2 / 3 / 93$ & $<0.0005$ \\
\hline & & $5 / 11 / 93$ & $<0.0005$ \\
\hline & & $9 / 15 / 93$ & $<0.0005$ \\
\hline & & $12 / 8 / 93$ & $<0.0005$ \\
\hline \multirow[t]{4}{*}{ РCB-7 } & Outfall 304, Bear Creek & $2 / 3 / 93$ & $<0.0005$ \\
\hline & & $5 / 11 / 93$ & $<0.0005$ \\
\hline & & $9 / 15 / 93$ & $<0.0005$ \\
\hline & & $12 / 8 / 93$ & $<0.0005$ \\
\hline
\end{tabular}

(a)This outlet was closed in April 1989.

(b)This inlet was closed in November 1988. 
Table 4.57. CY 1993 NPDES Permit Number TN 0002968

Y.12 Plant Cooling Towers

\begin{tabular}{|c|c|c|c|c|c|c|}
\hline \multirow[b]{2}{*}{ Parameter } & \multirow[b]{2}{*}{$\begin{array}{c}\text { Number of } \\
\text { sumples }\end{array}$} & \multicolumn{3}{|c|}{ Concentration(a) } & \multirow[b]{2}{*}{$\begin{array}{c}\text { Reference } \\
\text { value(b) }\end{array}$} & \multirow[b]{2}{*}{$\begin{array}{l}\text { Number of values } \\
\text { exceeding reference }\end{array}$} \\
\hline & & $\operatorname{Max}$ & Min & Av & & \\
\hline
\end{tabular}

Discharge point 602

Temperarure, $C$

$\mathrm{pH}$, standard units

Free chlorine

Chromium

Copper

Zinc

Flow, gpd(e)

Temperature, $\mathrm{C}$

$\mathrm{pH}$, standard units

Free chlorine

Chromium

Copper

Zinc

Flow, gpd(e)

Temperature, $C$

$\mathrm{pH}$, standard units

Free chlorine

Chromium

Copper

Zine

Flow, gpd(e)

21.2
7.9
0.01
$<0.01$
$<0.03$
$<0.05$
7263

Discharge point 604

$\begin{array}{cc}4 & 25.1 \\ 4 & 8.0 \\ 4 & 0.08 \\ 4 & <0.03 \\ 4 & <0.03 \\ 4 & 0.26 \\ 4 & 41725\end{array}$

41725
14.1

7.9

0.01

$<0.01$

$<0.03$

0.19

22889

\section{7}

c

0.04

$<0.02$

$<0.03$

$<0.05$

21162

Discharge point 606 (out of service)

33683

20.4
$c$
0.04
$<0.02$
$<0.03$
0.22
33683

)

$\begin{array}{cc}30.5 & 0 \\ 6.5 / 8.5(d) & 0 \\ 0.5 & 0 \\ 1 & 0 \\ 1 & 0 \\ 1 & 0 \\ c & c\end{array}$

30.5
$6.5 / 8.5(d)$
0.5
1
1
1
$c$

0
0
0
0
0
0
c

f
f
f
f
f

Temperarure, C

pH, standard units

Free chlorine

Chromium

Copper

Zine

Flow, gpd(e)

$\begin{array}{cc}4 & 27.8 \\ 4 & 7.9 \\ 4 & 0.08 \\ 4 & <0.03 \\ 4 & <0.03 \\ 4 & 0.09 \\ 4 & 97031\end{array}$

Discharge point 610

24.2
7.3
0.02
$<0.01$
$<0.03$
$<0.05$
20427

25.9

c

0.05

$<0.02$

$<0.03$

$<0.06$

43496

30.5
$6.5 / 8.5(d)$
0.5
1
1
1
$c$

0
0
0
0
0
0
c

Discharge point 612

Temperature, C

pH, standard units

Free chlorine

Chromium

Copper

Zinc

Flow, gpd(e)

$\begin{array}{cc}22.0 & 30.5 \\ c & 6.5 / 8.5(d) \\ 0.02 & 0.5 \\ <0.03 & 1 \\ <0.03 & 1 \\ <0.05 & 1 \\ & c\end{array}$

0
0
0
0
0
0
$c$


Table 4.57 (contir sed)

\begin{tabular}{|c|c|c|c|c|c|c|}
\hline \multirow[b]{2}{*}{ Parameter } & \multirow[b]{2}{*}{$\begin{array}{l}\text { Number of } \\
\text { samples }\end{array}$} & \multicolumn{3}{|c|}{ Concentration(a) } & \multirow[b]{2}{*}{$\begin{array}{l}\text { Reference } \\
\text { value(b) }\end{array}$} & \multirow[b]{2}{*}{$\begin{array}{l}\text { Number of values } \\
\text { exceeding reference }\end{array}$} \\
\hline & & $\operatorname{Max}$ & Min & $A V$ & & \\
\hline
\end{tabular}

Temperature, C

$\mathrm{pH}$, standard units

Free chlorine

Chromium

Copper

Zinc

Flow, gpd(e)

Temperature, $\mathrm{C}$

$\mathrm{pH}$, standard units

Free chlorine

Chromium

Copper

Zinc

Flow, gpd(e)

Temperature, C

$\mathrm{pH}$, standard units

Free chlorine

Chromium

Copper

Zine

Flow, gpd(e)

Discharge point 613

$\begin{array}{ccc}28.1 & 24.8 & 26.3 \\ 8.4 & 7.5 & c \\ 0.02 & 0.01 & 0.02 \\ <0.03 & <0.01 & <0.02 \\ <0.03 & <0.03 & <0.03 \\ <0.05 & <0.05 & <0.05 \\ 57500 & 35458 & 47554\end{array}$

30.5
$6.5 / 8.5(\mathrm{~d})$
0.5
1
1
1
$c$

Discharge point 615

$\begin{array}{cc}4 & 24.2 \\ 4 & 8.3 \\ 4 & 0.03 \\ 4 & <0.03 \\ 4 & 0.06 \\ 4 & 0.65 \\ 4 & 3677\end{array}$

21.8
8.0
0.01
$<0.01$
$<0.03$
0.27
1386

23.2

$$
30.5
$$

$6.5 / 8.5(\mathrm{~d})$

0.5

0.02

$<0.02$

$<0.04$

0.43

2502

Discharge point 616 (torn down)

f
f
$f$
$f$
f
f

Temperature, $C$

$\mathrm{pH}$, standard units

Free chiorine

Chromium

Copper

Zinc

Flow, gpd(e)

$\begin{array}{cc}4 & 25.8 \\ 4 & 8.1 \\ 4 & 0.04 \\ 4 & <0.03 \\ 4 & 0.06 \\ 4 & 0.71 \\ 4 & 36068\end{array}$

$$
18.5
$$

7.5

0.01

$<0.01$

$<0.03$

0.07

18670

20.9

30.5

$6.5 / 8.5(d)$

0

c

0.03

$<0.02$

$<0.04$

0.24

28765

Discharge point 618

Temperature, C

pH, standard units

Free chlorine

Chromium

Copper

Zine

Flow, gpd(e)

26.3

8.4

0.08

$<0.03$

$<0.03$

0.08

14671

23.1
7.7
0.01
$<0.01$
$<0.03$
$<0.05$
8558

23.1

7.7

$<0.01$

$<0.03$

$<0.05$

8558
24.8
$c$
0.05
$<0.02$
$<0.03$
$<0.06$
12030
30.5

$6.5 / 8.5$ (d)

0.5

1

1
0.5

1

1 
Table 4.57 (continued)

\begin{tabular}{|c|c|c|c|c|c|c|}
\hline \multirow[b]{2}{*}{ Parameter } & \multicolumn{4}{|c|}{ Concentration(a) } & \multirow[b]{2}{*}{$\begin{array}{l}\text { Reference } \\
\text { value(b) }\end{array}$} & \multirow[b]{2}{*}{$\begin{array}{l}\text { Number of values } \\
\text { exceeding reference }\end{array}$} \\
\hline & $\begin{array}{c}\text { Number of } \\
\text { samples }\end{array}$ & Max & Min & Av & & \\
\hline
\end{tabular}

Discharge point 619

Temperarure, C

pH. standard units

Free chlorine

Chromium

Copper

Zinc

Flow, gpd(e)

Temperature, $C$

pH, standard units

Free chlorine

Chromium

Copper

Zine

Flow, gpd(e)

Temperature, $C$

$\mathrm{pH}$, standard units

Free chlorine

Chromium

Copper

Zine

Flow, gpd(c)

Temperature, $C$

pH, standard units

Free chlorine

Chromium

Copper

Zinc

Flow, gpd(e)

Temperature, C

pH, standard units

Free chlorine

Chromium

Copper

Zinc

Flow, gpd(e)

20.9
7.8
0.02
$<0.01$
$<0.03$
0.07
4500

23.3

$c$

0.04

$<0.02$

$<0.03$

0.11

11850

17520

Discharge point 620

22.8
8.0
0.01
$<0.01$
$<0.03$
0.10
900

25.0

$c$

0.02

$<0.02$

$<0.03$

0.34

2866

6900

Discharge point 622

$\begin{array}{cc}30.1 & 25.8 \\ 8.3 & 7.5 \\ 0.04 & 0.01 \\ <0.03 & <0.01 \\ <0.03 & <0.03 \\ <0.05 & <0.05 \\ 82800 & 27000\end{array}$

$\begin{array}{cc}28.6 & 30.5 \\ c & 6.5 / 8.5(d) \\ 0.03 & 0.5 \\ <0.02 & 1 \\ <0.03 & 1 \\ <0.05 & 1 \\ 51600 & c\end{array}$

$\begin{array}{cc}30.5 & 0 \\ 6.5 / 8.5(d) & 0 \\ 0.5 & 0 \\ 1 & 0 \\ 1 & 0 \\ 1 & 0 \\ c & c\end{array}$

30.5

$6.5 / 8.5(d)$

0.5

1

1

1

c

Discharge point 624 (combined with 622)

$\mathrm{f}$
$\mathrm{f}$
$\mathrm{f}$
$\mathrm{f}$
$\mathrm{f}$

Discharge point 626

$\begin{array}{cccc}4 & 22.7 & 14.5 & 19.9 \\ 4 & 8.3 & 7.8 & c \\ 4 & 0.06 & 0.02 & 0.04 \\ 4 & <0.03 & <0.01 & <0.02 \\ 4 & <0.03 & <0.03 & <0.03 \\ 4 & 0.06 & <0.05 & <0.05 \\ 4 & 3593 & 862 & 1909\end{array}$

$\begin{array}{cc}30.5 & 0 \\ 6.5 / 8.5(d) & 0 \\ 0.5 & 0 \\ 1 & 0 \\ 1 & 0 \\ 1 & 0 \\ c & c\end{array}$


Table 4.57 (continued)

\begin{tabular}{|c|c|c|c|c|c|c|}
\hline \multirow[b]{2}{*}{ Parameter } & \multirow[b]{2}{*}{$\begin{array}{c}\text { Number of } \\
\text { samples }\end{array}$} & \multicolumn{3}{|c|}{ Concentration(a) } & \multirow[b]{2}{*}{$\begin{array}{c}\text { Reference } \\
\text { value(b) }\end{array}$} & \multirow{2}{*}{$\begin{array}{l}\text { Number of values } \\
\text { exceeding reference }\end{array}$} \\
\hline & & $\operatorname{Max}$ & Min & Av & & \\
\hline
\end{tabular}

\section{Temperature, $\mathrm{C}$}

$\mathrm{pH}$, standard units

Free chlorine

Chromium

Copper

Zinc

Flow, gpd(e)

Temperature, C

$\mathrm{pH}$, standard units

Free chlorine

Chromium

Copper

Zine

Flow, gpd(e)

$\begin{array}{lc}3 & 28.5 \\ 3 & 8.0 \\ 3 & 0.06 \\ 3 & <0.01 \\ 3 & <0.03 \\ 3 & 0.14 \\ 3 & 46000\end{array}$

28.5

8.0

0.06

$<0.01$

$<0.03$

0.14
46000

Discharge point 628

$\begin{array}{cc}16.4 & 24.1 \\ 7.7 & c \\ 0.01 & 0.04 \\ <0.01 & <0.01 \\ <0.03 & <0.03 \\ <0.05 & <0.09 \\ 14113 & 27878\end{array}$

30.5
$6.5 / 8.5(d)$
0.5
1
1
1
$c$

0

$c$

0.01

0.03

27878

Discharge point 630

24.0
7.9
0.02
$<0.03$
0.06
0.26
657

22.6

7.5

0.02

$<0.01$

$<0.03$

0.08

4200

23.3
$c$
0.02
$<0.02$
$<0.05$
0.17
5386

30.5

$6.5 / 8.5(d)$

0.5

1

1

1

c

Discharge point 632

Temperature, $\mathrm{C}$

$\mathrm{pH}$, standard units

Free chlorine

Chromium

24.2

8.2

0.04

$<0.01$

$<0.03$

$<0.05$

Zinc

Flow, gpd(e)

$\begin{array}{cc}25.9 & 24.2 \\ 8.4 & 8.2 \\ 0.08 & 0.04 \\ <0.01 & <0.01 \\ <0.03 & <0.03 \\ 0.08 & <0.05 \\ 14785 & 7137\end{array}$

24.9
$c$
0.06
$<0.01$
$<0.03$
$<0.06$
9686

30.5

6.5/8.5(d)

0.5

1

1

1

1137

9686

Discharge point 633 (out of service)

Temperature, $C$

$\mathrm{pH}$, standard units

Free chlorine

Chromium

Copper

Zinc

Flow, gpd(e) i

$\mathrm{f}$

f

f

$f$

f

Discharge point 634

Temperature, $C$

$\mathrm{pH}$, standard units

Free chlorine

Chromium

Copper

Zinc

Flow, gpd(e) (a) Units in mgil unless otherwise indicated

(b)NPDES permit limits.

(c)Not applicable.

24.4
8.0
0.01
$<0.01$
$<0.03$
0.10
6325

25.8

$c$

0.02

$<0.02$

$<0.03$

0.12

22770

(d)Minimum value/maximum value.

(e)Flow during operaxion and/or discharging.

(f) No flow. 
Table 4.58. Y-12 Plant Category I Outfalls

\begin{tabular}{|c|c|c|c|c|c|c|c|c|c|c|c|}
\hline \multirow{2}{*}{ Outfall } & \multirow{2}{*}{$\begin{array}{l}\text { Number } \\
\text { of samples }\end{array}$} & \multicolumn{3}{|c|}{$\mathrm{pH}$ (standard units) } & \multirow{2}{*}{$\begin{array}{l}\text { Min ref. } \\
\text { value(a) }\end{array}$} & \multirow{2}{*}{$\begin{array}{l}\text { No. of } \\
\text { values } \\
\text { exceeding } \\
\text { reference }\end{array}$} & \multirow{2}{*}{$\begin{array}{l}\text { Max ref. } \\
\text { value (a) }\end{array}$} & \multirow{2}{*}{$\begin{array}{l}\text { No. of } \\
\text { values } \\
\text { exceeding } \\
\text { reference }\end{array}$} & \multicolumn{3}{|c|}{ Flow (gpd)(b) } \\
\hline & & $\operatorname{Max}$ & Min & $\mathrm{Av}$ & & & & & $\operatorname{Max}$ & $\operatorname{Min}$ & Av \\
\hline 001 & 1 & 7.5 & 7.5 & $\mathrm{c}$ & 6.5 & 0 & 8.5 & 0 & 25800 & 25800 & 25800 \\
\hline 003 & 1 & 7.5 & 7.5 & $c$ & 6.5 & 0 & 8.5 & 0 & 15218 & 15218 & 15218 \\
\hline 006 & 1 & 8.0 & 8.0 & $c$ & 6.5 & 0 & 8.5 & 0 & 8369 & 8369 & 8369 \\
\hline 007 & 1 & 7.9 & 7.9 & $c$ & 6.5 & 0 & 8.5 & 0 & 6960 & 6960 & 6960 \\
\hline 009 & 0 & d & $d$ & $\mathrm{c}$ & 6.5 & 0 & 8.5 & 0 & $d$ & $d$ & $d$ \\
\hline 011 & 0 & d & d & c & 6.5 & 0 & 8.5 & 0 & d & d & d \\
\hline 012 & 0 & $d$ & $d$ & c & 6.5 & 0 & 8.5 & 0 & $d$ & $d$ & d \\
\hline 015 & 0 & $d$ & $d$ & c & 6.5 & 0 & 8.5 & 0 & d & d & $d$ \\
\hline 017 & 1 & 7.5 & 7.5 & $c$ & 6.5 & 0 & 8.5 & 0 & 22827 & 22827 & 22827 \\
\hline 018 & 1 & 8.0 & 8.0 & $\mathfrak{c}$ & 6.5 & 0 & 8.5 & 0 & 2282 & 2282 & 2282 \\
\hline 019 & 1 & 7.9 & 7.9 & c & 6.5 & 0 & 8.5 & 0 & 380 & 380 & 380 \\
\hline 031 & 1 & 7.7 & 7.7 & c & 6.5 & 0 & 8.5 & 0 & 6400 & 6400 & 6400 \\
\hline 032 & 0 & $d$ & $d$ & c & 6.5 & 0 & 8.5 & 0 & $\mathrm{~d}$ & $d$ & $d$ \\
\hline 041 & 0 & d & d & $c$ & 6.5 & 0 & 8.5 & 0 & d & d & $d$ \\
\hline 044 & 1 & 7.9 & 7.9 & $\mathfrak{c}$ & 6.5 & 0 & 8.5 & 0 & 6050 & 6050 & 6050 \\
\hline 045 & 1 & 8.1 & 8.1 & $c$ & 6.5 & 0 & 8.5 & 0 & 127 & 127 & 127 \\
\hline 057 & 1 & 8.1 & 8.1 & c & 6.5 & 0 & 8.5 & 0 & 1141 & 1141 & 1141 \\
\hline 062 & 0 & d & $d$ & c & 6.5 & 0 & 8.5 & 0 & $d$ & $d$ & d \\
\hline 108 & 1 & 8.4 & 8.4 & c & 6.5 & 0 & 8.5 & 0 & 15218 & 15218 & 15218 \\
\hline 134 & 0 & d & d & $c$ & 6.5 & 0 & 8.5 & 0 & d & d & $\mathrm{d}$ \\
\hline 156 & 1 & 8.4 & 8.4 & c & 6.5 & 0 & 8.5 & 0 & 1141 & 1141 & 1141 \\
\hline 159 & 1 & 8.4 & 8.4 & $c$ & 6.5 & 0 & 8.5 & 0 & 13316 & 13316 & 13316 \\
\hline 178 & 1 & 8.2 & 8.2 & c & 6.5 & 0 & 8.5 & 0 & 15218 & 15218 & 15218 \\
\hline 180 & 1 & 8.3 & 8.3 & c & 6.5 & 0 & 8.5 & 0 & 2282 & 2282 & 2282 \\
\hline 183 & 1 & 7.8 & 7.8 & c & 6.5 & 0 & 8.5 & 0 & 3044 & 3044 & 3044 \\
\hline 186 & 1 & 8.1 & 8.1 & c & 6.5 & 0 & 8.5 & 0 & 4504 & 4504 & 4504 \\
\hline 193 & 1 & 8.2 & 8.2 & c & 6.5 & 0 & 8.5 & 0 & 7609 & 7609 & 7609 \\
\hline 196 & 1 & 8.2 & 8.2 & c & 6.5 & 0 & 8.5 & 0 & 1522 & 1522 & 1522 \\
\hline 197 & 0 & $d$ & $d$ & $c$ & 6.5 & 0 & 8.5 & 0 & $d$ & $d$ & d \\
\hline 198 & 1 & 7.9 & 7.9 & c & 6.5 & 0 & 8.5 & 0 & 18216 & 18216 & 18216 \\
\hline 202 & 1 & 8.2 & 8.2 & $c$ & 6.5 & 0 & 8.5 & 0 & 1141 & 1141 & 1141 \\
\hline 205 & 1 & 7.2 & 7.2 & $c$ & 6.5 & 0 & 8.5 & 0 & 10652 & 10652 & 10652 \\
\hline 206 & 1 & 8.5 & 8.5 & c & 6.5 & 0 & 8.5 & 0 & 9511 & 9511 & 9511 \\
\hline 223 & 1 & 8.4 & 8.4 & $c$ & 6.5 & 0 & 8.5 & 0 & 13316 & 13316 & 13316 \\
\hline 235 & 1 & 8.4 & 8.4 & c & 6.5 & 0 & 8.5 & 0 & 1522 & 1522 & 1522 \\
\hline 236 & 1 & 8.1 & 8.1 & c & 6.5 & 0 & 8.5 & 0 & 17120 & 17120 & 17120 \\
\hline
\end{tabular}

(a)NPDES permit limits.

(b)Flow during operations and/or discharging. No reference value for flow rate.

(c)Not applicable.

(d) No flow. 
Table 4.59. Y-12 Plant Category II Outfalls

\begin{tabular}{|c|c|c|c|c|c|c|c|c|c|c|c|c|c|c|}
\hline \multirow{2}{*}{ Outfall } & \multirow{2}{*}{$\begin{array}{l}\text { Number } \\
\text { of samples }\end{array}$} & \multicolumn{3}{|c|}{ pH (standard units) } & \multirow{2}{*}{$\begin{array}{l}\text { Min ref. } \\
\text { value(a) }\end{array}$} & \multirow{2}{*}{$\begin{array}{l}\text { No. of } \\
\text { values } \\
\text { exceeding } \\
\text { reference }\end{array}$} & \multirow{2}{*}{$\begin{array}{l}\text { Max ref. } \\
\text { value (a) }\end{array}$} & \multirow{2}{*}{$\begin{array}{l}\text { No. of } \\
\text { values } \\
\text { exceeding } \\
\text { reference }\end{array}$} & \multicolumn{3}{|c|}{ Temperature $\left({ }^{\circ} \mathrm{C}\right)(\mathrm{b})$} & \multicolumn{3}{|c|}{ Flow (gpd)(c) } \\
\hline & & $\operatorname{Max}$ & Min & Av & & & & & Max & Min & Av & $\operatorname{Max}$ & Min & $\Delta \mathbf{v}$ \\
\hline 013 & 1 & 7.8 & 7.8 & d & 6.5 & 0 & 8.5 & 0 & 15.6 & 15.6 & 15.6 & 2525 & 2525 & 2525 \\
\hline 016 & 3 & 8.1 & 7.8 & d & 6.5 & 0 & 8.5 & 0 & 17.3 & 9.1 & 12.8 & 68700 & 320 & 23133 \\
\hline 020 & 4 & 8.3 & 7.7 & d & 6.5 & 0 & 8.5 & 0 & 26.2 & 9.3 & 15.4 & 76090 & 47 & 19365 \\
\hline 023 & 3 & 8.2 & 7.7 & d & 6.5 & $\mathbf{0}$ & 8.5 & 0 & 46.9 & 39.9 & 43.0 & 1350 & 190 & 597 \\
\hline 025 & 2 & 8.4 & 7.5 & d & 6.5 & 0 & 8.5 & 0 & 39.5 & 25.0 & 32.3 & 380 & 210 & 295 \\
\hline 026 & 0 & $\mathbf{e}$ & $\mathbf{e}$ & d & 6.5 & $\mathbf{0}$ & 8.5 & 0 & $\mathbf{e}$ & e & e & e & e & $\mathrm{e}$ \\
\hline 029 & 0 & e & e & d & 6.5 & 0 & 8.5 & 0 & e & e & e & e & e & e \\
\hline 035 & 3 & 8.5 & 7.4 & d & 6.5 & 0 & 8.5 & 0 & 19.2 & 15.4 & 17.4 & 1522 & 95 & 599 \\
\hline 043 & 0 & e & e & d & 6.5 & 0 & 8.5 & 0 & e & e & e & $\mathrm{e}$ & $\mathrm{e}$ & e \\
\hline 046 & 4 & 8.1 & 7.5 & d & 6.5 & 0 & 8.5 & 0 & 40.6 & 13.5 & 29.1 & 30436 & 180 & 8014 \\
\hline 053 & 1 & 8.0 & 8.0 & d & 6.5 & 0 & 8.5 & 0 & 80.0 & 80.0 & 80.0 & 130 & 130 & 130 \\
\hline 054 & 4 & 8.4 & 7.6 & d & 6.5 & 0 & 8.5 & 0 & 24.9 & 10.5 & 17.6 & 7609 & 129 & 2515 \\
\hline 058 & 4 & 7.6 & 7.3 & d & 6.5 & 0 & 8.5 & 0 & 23.5 & 11.0 & 15.4 & 761 & 95 & 293 \\
\hline 060 & 0 & e & e & d & 6.5 & 0 & 8.5 & 0 & e & e & e & e & e & e \\
\hline 066 & 3 & 7.7 & 7.5 & d & 6.5 & 0 & 8.5 & 0 & 28.9 & 23.9 & 26.9 & 190 & 95 & 137 \\
\hline 068 & 4 & 8.4 & 7.0 & d & 6.5 & 0 & 8.5 & 0 & 31.7 & 15.3 & 22.3 & 190 & 91 & 118 \\
\hline 073 & 4 & 8.3 & 7.5 & d & 6.5 & 0 & 8.5 & 0 & 29.7 & 20.1 & 23.6 & 28100 & 10652 & 18781 \\
\hline 075 & 0 & e & e & d & 6.5 & 0 & 8.5 & 0 & e & e & e & e & e & e \\
\hline 076 & 0 & e & e & d & 6.5 & 0 & 8.5 & 0 & e & e & e & e & e & e \\
\hline 077 & 1 & 7.5 & 7.5 & d & 6.5 & 0 & 8.5 & 0 & 16.9 & 16.9 & 16.9 & 34240 & 34240 & 34240 \\
\hline 078 & 0 & $\mathbf{e}$ & $\mathbf{e}$ & d & 6.5 & 0 & 8.5 & 0 & e & e & e & e & c & e \\
\hline 080 & 0 & e & e & d & 6.5 & 0 & 8.5 & 0 & e & $\mathrm{e}$ & e & e & e & c \\
\hline 081 & 0 & $e$ & e & d & 6.5 & 0 & 8.5 & 0 & e & e & e & e & c & c \\
\hline 087 & 4 & 7.4 & 6.9 & d & 6.5 & 0 & 8.5 & 0 & 22.7 & 12.5 & 16.2 & 7609 & 190 & 2187 \\
\hline 093 & 1 & 8.5 & 8.5 & d & 6.5 & 0 & 8.5 & 0 & 7.9 & 7.9 & 7.9 & 80 & 80 & 80 \\
\hline 094 & 0 & e & e & d & 6.5 & 0 & 8.5 & 0 & e & $\mathrm{e}$ & $\mathrm{e}$ & e & e & e \\
\hline 095 & 0 & e & e & d & 6.5 & 0 & 8.5 & 0 & e & e & e & e & c & e \\
\hline 096 & 0 & e & e & d & 6.5 & 0 & 8.5 & 0 & e & e & e & e & e & e \\
\hline 098 & 4 & 8.4 & 7.4 & d & 6.5 & 0 & 8.5 & 0 & 21.6 & 9.6 & 17.4 & 11413 & 1902 & 5802 \\
\hline 111 & 4 & 8.1 & 7.3 & d & 6.5 & 0 & 8.5 & 0 & 22.4 & 10.8 & 18.0 & 6860 & 190 & 2243 \\
\hline 112 & 1 & 7.7 & 7.7 & d & 6.5 & 0 & 8.5 & 0 & 13.5 & 13.5 & 13.5 & 195 & 195 & 195 \\
\hline 117 & 4 & 8.4 & 7.8 & d & 6.5 & 0 & 8.5 & 0 & 33.0 & 20.8 & 26.4 & 380 & 90 & 236 \\
\hline 131 & 0 & e & e & d & 6.5 & 0 & 8.5 & 0 & e & e & e & e & e & e \\
\hline 133 & 4 & 8.2 & 7.3 & d & 6.5 & 0 & 8.5 & 0 & 24.9 & 12.0 & 19.1 & 8520 & 780 & 3657 \\
\hline 137 & 3 & 8.0 & 7.3 & d & 6.5 & 0 & 8.5 & 0 & 20.3 & 9.8 & 15.9 & 34240 & 780 & 12941 \\
\hline 172 & 1 & 7.9 & 7.9 & d & 6.5 & 0 & 8.5 & 0 & 11.8 & 11.8 & 11.8 & 761 & 761 & 761 \\
\hline 185 & 4 & 8.1 & 7.3 & d & 6.5 & 0 & 8.5 & 0 & 32.1 & 19.2 & 25.3 & 7609 & 127 & 2505 \\
\hline
\end{tabular}


$\stackrel{+}{2}$

Table 4.59 (continued)

\begin{tabular}{|c|c|c|c|c|c|c|c|c|c|c|c|c|c|c|}
\hline \multirow{2}{*}{ Outfall } & \multirow{2}{*}{$\begin{array}{c}\text { Number } \\
\text { of samples }\end{array}$} & \multicolumn{3}{|c|}{ pH (standard units) } & \multirow{2}{*}{$\begin{array}{l}\text { Min ref. } \\
\text { value(a) }\end{array}$} & \multirow{2}{*}{$\begin{array}{l}\text { No. of } \\
\text { values } \\
\text { exceeding } \\
\text { reference }\end{array}$} & \multirow{2}{*}{$\begin{array}{l}\text { Max ref. } \\
\text { value (a) }\end{array}$} & \multirow{2}{*}{$\begin{array}{l}\text { No. of } \\
\text { values } \\
\text { exceeding } \\
\text { reference }\end{array}$} & \multicolumn{3}{|c|}{ Temperature $\left({ }^{\circ} \mathrm{C}\right)(\mathrm{b})$} & \multicolumn{3}{|c|}{ Flow (gpd)(c) } \\
\hline & & $\operatorname{Max}$ & Min & $A \mathbf{v}$ & & & & & $\operatorname{Max}$ & Min & $A \mathbf{v}$ & $\operatorname{Max}$ & Min & $\Lambda \mathbf{v}$ \\
\hline 201 & 4 & 8.2 & 7.3 & d & 6.5 & 0 & 8.5 & 0 & 24.9 & 12.4 & 18.5 & 8520 & 1522 & 5084 \\
\hline 203 & 4 & 7.8 & 6.8 & d & 6.5 & 0 & 8.5 & 0 & 20.6 & 8.9 & 15.3 & 34240 & 1522 & 11477 \\
\hline 204 & 4 & 8.1 & 7.5 & d & 6.5 & 0 & 8.5 & $\mathbf{0}$ & 25.3 & 11.4 & 18.6 & 780 & 360 & 475 \\
\hline 213 & 4 & 7.9 & 7.2 & d & 6.5 & 0 & 8.5 & 0 & 23.5 & 10.3 & 16.1 & 3044 & 95 & 862 \\
\hline 238 & 0 & e & e & d & 6.5 & 0 & 8.5 & 0 & e & e & $\mathbf{e}$ & $\mathbf{e}$ & e & e \\
\hline 239 & 0 & e & e & d & 6.5 & 0 & 8.5 & 0 & e & e & $\mathbf{e}$ & $\mathbf{e}$ & e & e \\
\hline 240 & 4 & 8.3 & 7.6 & d & 6.5 & 0 & 8.5 & 0 & 19.4 & 4.2 & 14.7 & 7609 & 380 & 2948 \\
\hline 241 & 0 & e & e & d & 6.5 & 0 & 8.5 & 0 & e & e & e & e & e & e \\
\hline
\end{tabular}

(a)NPDES permit limits

(b) No reference value.

(c)Flow during operations and/or discharging. No reference value for flow rate

(d) Not applicable.

(e)No flow. 
Table 4.60. Y-12 Plant Category III Outfalls

\begin{tabular}{|c|c|c|c|c|c|c|c|c|c|c|c|c|c|c|c|c|}
\hline \multirow{2}{*}{ Outfall } & \multirow{2}{*}{$\begin{array}{l}\text { Number } \\
\text { of samples }\end{array}$} & \multicolumn{3}{|c|}{ pH (standard units) } & \multirow{2}{*}{$\begin{array}{l}\text { Min ref. } \\
\text { value(a) }\end{array}$} & \multirow{2}{*}{$\begin{array}{l}\text { No. of } \\
\text { values } \\
\text { exceeding } \\
\text { reference } \\
\end{array}$} & \multirow{2}{*}{$\begin{array}{l}\text { Max ref. } \\
\text { value (a) }\end{array}$} & \multirow{2}{*}{$\begin{array}{l}\text { No. of } \\
\text { values } \\
\text { exceeding } \\
\text { reference } \\
\end{array}$} & \multicolumn{3}{|c|}{ Temperature $\left({ }^{\circ} \mathrm{C}\right)(\mathrm{b})$} & \multirow{2}{*}{$\begin{array}{c}\text { Ref. } \\
\text { value(b) }\end{array}$} & \multirow{2}{*}{$\begin{array}{l}\text { No. of } \\
\text { values } \\
\text { exceeding } \\
\text { reference }\end{array}$} & \multicolumn{3}{|c|}{ Flow (gpd)(b) } \\
\hline & & Max & Min & Av & & & & & $\operatorname{Max}$ & Min & Av & & & $\operatorname{Max}$ & Min & $\Lambda v$ \\
\hline 002 & 4 & 8.3 & 7.4 & c & 6.5 & 0 & 8.5 & 0 & 27.9 & 10.1 & 15.3 & 30.5 & 0 & 137960 & 30436 & 68871 \\
\hline 071 & 4 & 8.1 & 7.5 & c & 6.5 & 0 & 8.5 & 0 & 21.4 & 11.2 & 15.5 & 30.5 & 0 & 94250 & 13710 & 40317 \\
\hline 135 & 4 & 8.3 & 7.2 & c & 6.5 & 0 & 8.5 & 0 & 31.8 & 15.2 & 24.1 & 30.5 & 0 & 499680 & 432000 & 465420 \\
\hline 147 & 4 & 8.1 & 7.2 & c & 6.5 & 0 & 8.5 & 0 & 25.5 & 14.0 & 18.7 & 30.5 & 0 & 9891 & 380 & 3424 \\
\hline 150 & 4 & 8.0 & 7.8 & c & 6.5 & 0 & 8.5 & $\mathbf{0}$ & 27.7 & 22.8 & 25.9 & 30.5 & 0 & 777600 & 497511 & 630479 \\
\hline 157 & 3 & 7.7 & 7.5 & c & 6.5 & 0 & 8.5 & o & 20.2 & 15.7 & 18.1 & 30.5 & 0 & 6087 & 1141 & 2917 \\
\hline 160 & 4 & 8.1 & 7.9 & c & 6.5 & 0 & 8.5 & 0 & 31.1 & 21.1 & 25.2 & 30.5 & 0 & 250082 & 1256 & 89915 \\
\hline 163 & 4 & 8.2 & 8.0 & c & 6.5 & 0 & 8.5 & 0 & 27.8 & 19.2 & 23.0 & 30.5 & 0 & 272752 & 2983 & 160405 \\
\hline 169 & 4 & 8.4 & 7.7 & c & 6.5 & 0 & 8.5 & 0 & 24.4 & 14.5 & 20.7 & 30.5 & 0 & 380426 & 149112 & 279279 \\
\hline 181 & 0 & d & d & c & 6.5 & 0 & 8.5 & 0 & d & d & d & 30.5 & 0 & d & d & $d$ \\
\hline 192 & 4 & 8.1 & 6.7 & c & 6.5 & 0 & 8.5 & 0 & 23.8 & 15.3 & 18.7 & 30.5 & 0 & 9131 & 1141 & 3139 \\
\hline
\end{tabular}

(a)NPDES permit limits.

(b)Flow during operations and/or discharging. No reference value for flow rate.

(c)Not applicable.

(d)No flow. 
Table 4.61. CY 1993 NPDES Permit Number TN 0002968 Y-12 Plant Category IV Outfalls(a)

\begin{tabular}{|c|c|c|c|c|c|c|c|c|}
\hline \multirow[b]{2}{*}{ Outfall } & \multirow{2}{*}{$\begin{array}{l}\text { Number of } \\
\text { samples }\end{array}$} & \multicolumn{3}{|c|}{$\mathrm{pH}$ (standard units) } & \multirow{2}{*}{$\begin{array}{l}\text { Minimum } \\
\text { reference } \\
\text { value(b) }\end{array}$} & \multirow{2}{*}{$\begin{array}{l}\text { Number of values } \\
\text { below reference }\end{array}$} & \multirow{2}{*}{$\begin{array}{l}\text { Maximum } \\
\text { reference value }\end{array}$} & \multirow{2}{*}{$\begin{array}{l}\text { Number of } \\
\text { values } \\
\text { exceeding } \\
\text { reference }\end{array}$} \\
\hline & & $\operatorname{Max}$ & Min & $\mathrm{Av}$ & & & & \\
\hline 401 & $c$ & & & & 6.5 & $\bar{d}$ & 8.5 & $d$ \\
\hline 402 & 10 & 7.8 & 6.7 & $d$ & 6.5 & 0 & 8.5 & 0 \\
\hline 403 & $c$ & & & & 6.5 & 0 & 8.5 & 0 \\
\hline 404 & $c$ & & & & 6.5 & $d$ & 8.5 & $d$ \\
\hline 405 & 30 & 8.5 & 7.1 & $d$ & 6.5 & 0 & 8.5 & 0 \\
\hline 406 & $c$ & & & & 6.5 & $d$ & 8.5 & $d$ \\
\hline 407 & $c$ & & & & 6.5 & $d$ & 8.5 & $d$ \\
\hline 408 & 52 & 8.2 & 6.8 & $d$ & 6.5 & 0 & 8.5 & 0 \\
\hline 409 & c & & & & 6.5 & $d$ & 8.5 & $d$ \\
\hline 410 & $c$ & & & & 6.5 & $d$ & 8.5 & $d$ \\
\hline 411 & $c$ & & & & 6.5 & $d$ & 8.5 & $d$ \\
\hline 412 & $c$ & & & & 6.5 & $d$ & 8.5 & $d$ \\
\hline 413 & $c$ & & & & 6.5 & $d$ & 8.5 & $d$ \\
\hline 414 & $c$ & & & & 6.5 & $d$ & 8.5 & $d$ \\
\hline 415 & $c$ & & & & 6.5 & $d$ & 8.5 & $d$ \\
\hline 416 & $c$ & & & & 6.5 & $d$ & 8.5 & $d$ \\
\hline 417 & $c$ & & & & 6.5 & $d$ & 8.5 & $d$ \\
\hline 418 & $c$ & & & & 6.5 & $d$ & 8.5 & $d$ \\
\hline 419 & $c$ & & & & 6.5 & $d$ & 8.5 & $d$ \\
\hline 420 & $c$ & & & & 6.5 & $d$ & 8.5 & $d$ \\
\hline 421 & $c$ & & & & 6.5 & $d$ & 8.5 & $d$ \\
\hline 422 & $c$ & & & & 6.5 & $d$ & 8.5 & $d$ \\
\hline
\end{tabular}

(a)Flow during operation and/or discharging. (b)NPDES permit limits.

(c) No flow.

(d) Not applicable. 
Table 4.62. CY 1993 Y-12 Plant data summary for outfall rot specificaliy enumerated on the NPDES permit

\begin{tabular}{|c|c|c|c|c|c|c|}
\hline \multirow[b]{2}{*}{ Parameter } & \multirow{2}{*}{$\begin{array}{l}\text { Number of } \\
\text { samples }\end{array}$} & \multicolumn{3}{|c|}{ Concentration(a) } & \multirow{2}{*}{$\begin{array}{l}\text { Reference } \\
\text { value(b) }\end{array}$} & \multirow{2}{*}{$\begin{array}{l}\text { Number of values } \\
\text { exceeding reference }\end{array}$} \\
\hline & & $\operatorname{Max}$ & Min & $\mathrm{Av}$ & & \\
\hline
\end{tabular}

Discharge Point 4

Flow(c), gpd

$\mathrm{pH}$, standard units

Temperature, C

Ammonia

Biochemical oxygen demand Chemical oxygen demand

Total organic carbon

Total suspended solids

15218
8.1
22.8
0.22
$<5$
13
45
$<5$

1902

7.7

10.5

$<0.2$

$<5$

$<5.0$

$<5$

Discharge Point 8
Flow(c), gpd

$\mathrm{pH}$, standard units

Temperature, $\mathrm{C}$

Ammonia

Biochemical oxygen demand

Chemical oxygen dernand

Total organic carbon

Total suspended solids

9511
8.1
26.4
$<0.2$
6.6
6.8
27
5.0

Discharge Point 10

Flow(c), gpd

$\mathrm{pH}$, standard units

Temperature, $\mathrm{C}$

Ammonia

Biochemical oxygen demand

Chemical oxygen demand

Total organic carbon

Total suspended solids

18261
8.1
25.6
0.21
$<5$
6.5
18
$<5$

18261

2282

7.9

20.0

$<0.2$

$<5$

$<5$

2.9

$<5$
Discharge Point 14

Flow(c), gpd

$\mathrm{pH}$, standard units

Temperature, $\mathrm{C}$

Ammonia

Biochemical oxygen demand

Chemical oxygen demand

Total organic carbon

Total suspended solids
15979

8.3

28.3

0.70

$<5$

9.0

26

$<5$
2980

7.4

14.4

$<0.2$

$<5$

$<5$

4.5

$<5$
19

8845
$d$
16.1
$<0.21$
$<5$
$<7.6$
33
$<5$

$d$
$d$
$d$
$d$
$d$
$d$
$d$
$d$

$d$
$d$
$d$
$d$
$d$
$d$
$d$
$d$

$d$

$\begin{array}{lcll}761 & 3139 & d & d \\ 7.5 & d & d & d \\ 14.8 & 20.1 & d & d \\ <0.2 & <0.2 & d & d \\ <5 & <5.4 & d & d \\ <5 & <5.5 & d & d \\ 11 & 1.7 & d & d \\ <5 & <5 & d & d\end{array}$

$d$
$d$
$d$
$d$
$d$
$d$
$d$
$d$

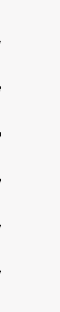


Table 4.62 (continued)

\begin{tabular}{|c|c|c|c|c|c|c|}
\hline \multirow[b]{2}{*}{ Parameter } & \multirow{2}{*}{$\begin{array}{c}\text { Number of } \\
\text { samples }\end{array}$} & \multicolumn{3}{|c|}{ Concentration(a) } & \multirow{2}{*}{$\begin{array}{c}\begin{array}{c}\text { Reference } \\
\text { value(b) }\end{array} \\
\end{array}$} & \multirow{2}{*}{$\begin{array}{l}\text { Number of values } \\
\text { exceeding reference }\end{array}$} \\
\hline & & $\operatorname{Max}$ & Min & Av & & \\
\hline \multicolumn{7}{|c|}{ Discharge Point 21} \\
\hline Flow(c), gpd & 4 & 152180 & 57070 & 118553 & $d$ & $d$ \\
\hline $\mathrm{pH}$, standard units & 4 & 8.1 & 7.5 & $d$ & $d$ & $d$ \\
\hline Temperature, $\mathrm{C}$ & 4 & 29.7 & 16.7 & 20.3 & $d$ & $d$ \\
\hline Ammonia & 4 & 0.3 & $<0.2$ & $<0.2$ & $d$ & $d$ \\
\hline Biochemical oxygen demand & 3 & $<5$ & $<5$ & $<5$ & $d$ & $d$ \\
\hline Chemical oxygen demand & 3 & $<5$ & $<5$ & $<5$ & $d$ & $d$ \\
\hline Total organic carbon & 4 & 21 & 4.8 & 13.5 & $d$ & $d$ \\
\hline Total suspended solids & 4 & $<5$ & $<5$ & $<5$ & $d$ & $d$ \\
\hline
\end{tabular}

Discharge Point 22

Flow(c), gpd

$\mathrm{pH}$, standard units

Temperature, $\mathrm{C}$

Ammonia

Biochemical oxygen demand

Chemical oxygen demand

Total organic carbon

Total suspended solids
3

3

3

3

3

3

3

3

20925
8.4
27.6
$<0.2$
$<5$
11
19
58

8.4

27.6

$<0.2$

$<5$

1

19

58

3424
7.6
12.6
$<0.2$
$<5$
$<5$
14
$<5$

13189
$d$
19.2
$<0.2$
$<5$
$<7$
16
$<23$

Discharge Point 33

Flow(c), gpd

$\mathrm{pH}$, standard units

Temperature, $\mathrm{C}$

Ammonia

Biochemical oxygen demand

Chemical oxygen demand

Total organic carbon

Total suspended solids

$\begin{array}{cc}4 & 22840 \\ 4 & 8.2 \\ 4 & 23.5 \\ 4 & <0.2 \\ 4 & <5.0 \\ 4 & 20 \\ 4 & 33 \\ 4 & 5.0\end{array}$

1522
6.8
10.5
$<0.2$
$<5$
$<5$
7.3
$<5$

8183
$d$
16.7
$<0.2$
$<5$
$<10$
20.8
$<5$

$d$
$d$
$d$
$d$
$d$
$d$
$d$
$d$

Discharge Point 34

Flow(c), gpd

$\mathrm{pH}$, standard units

Temperature, $\mathrm{C}$

Ammonia

Biochemical oxygen demand

Chemical oxygen demand

Total organic carbon

Total suspended solids

182611
8.3
32.4
$<0.2$
$<5$
9.8
24
$<5$

$\begin{array}{cc}41088 & 107500 \\ 7.4 & d \\ 21.7 & 25.6 \\ <0.2 & <0.2 \\ <5 & <5 \\ <5 & <6.6 \\ 13 & 18 \\ <5 & <5\end{array}$

$\begin{array}{ll}d & d \\ d & d \\ d & d \\ d & d \\ d & d \\ d & d \\ d & d \\ d & d\end{array}$


Table 4.62 (continued)

\begin{tabular}{|c|c|c|c|c|c|c|}
\hline \multirow[b]{2}{*}{ Parameter } & \multirow{2}{*}{$\begin{array}{l}\text { Number of } \\
\text { samples }\end{array}$} & \multicolumn{3}{|c|}{ Conce itration(a) } & \multirow{2}{*}{$\begin{array}{l}\text { Reference } \\
\text { value(b) }\end{array}$} & \multirow{2}{*}{$\begin{array}{l}\text { Number of values } \\
\text { exceeding reference }\end{array}$} \\
\hline & & $\operatorname{Max}$ & Min & Av & & \\
\hline
\end{tabular}

Discharge Point 36

Flow(c), gpd

$\mathrm{pH}$, standard units

Temperature, $\mathrm{C}$

Ammonia

Biochemical oxygen demand

Chemical oxygen demand

Total organic carbon

Total suspended solids

$\mathrm{pH}$, standard units

Temperature, $\mathrm{C}$

Ammonia

Biochemical oxygen demand

Chemical oxygen demand

Total organic carbon

Total suspended solius

$\begin{array}{cc}4 & 6100 \\ 4 & 8.4 \\ 4 & 24.0 \\ 4 & 0.21 \\ 4 & <5.0 \\ 4 & 48 \\ 4 & 35 \\ 4 & 10\end{array}$

Flow(c), gpd

$\mathrm{pH}$, standard units

Temperature, $\mathrm{C}$

Ammonia

Biochemical oxygen demand

Chemical oxygen demand

Total organic carbon

Total suspended solids

$\mathrm{pH}$, standard units

Temperature, $\mathrm{C}$

Ammonia

Biochemical oxygen demand

Chemical oxygen demand

Total organic carbon

Total suspended solids
10

Discharge Point 39

1310

8.3

15.4

$<0.2$

$<5$

42

19

$<5$

$\begin{array}{cc}190 & 2278 \\ 7.8 & d \\ 8.7 & 16.9 \\ <0.2 & <0.20 \\ <5 & <5 \\ 14 & 32 \\ 15 & 23 \\ <5 & <6\end{array}$

$\begin{array}{ll}d & d \\ d & d \\ d & d \\ d & d \\ d & d \\ d & d \\ d & d \\ d & d\end{array}$

780
7.7
8.8
$<0.2$
$<5$
7.0
18
$<5$

Discharge Point 42

30400
8.5
30.9
$<0.2$
$<5$
9.0
19
$<5$

5707
7.9
$i 2.5$
$<0.2$
$<5$
$<5$
12
$<5$

16158
$d$
20.4
$<0.2$
$<5$
$<6.3$
16
$<5$

Discharge Point 47

$\begin{array}{ccc}1045 & d & d \\ d & d & d \\ 12.1 & d & d \\ <0.2 & d & d \\ <5 & d & d \\ 25 & d & d \\ 19 & d & d \\ <5 & d & d\end{array}$


Table 4.62 (continued)

\begin{tabular}{|c|c|c|c|c|c|c|}
\hline \multirow[b]{2}{*}{ Parameter } & \multirow{2}{*}{$\begin{array}{c}\text { Number of } \\
\text { samples }\end{array}$} & \multicolumn{3}{|c|}{ Concentration(a) } & \multirow{2}{*}{$\begin{array}{c}\text { Reference } \\
\text { value(b) }\end{array}$} & \multirow{2}{*}{$\begin{array}{l}\text { Number of values } \\
\text { exceeding reference }\end{array}$} \\
\hline & & $\operatorname{Max}$ & Min & Av & & \\
\hline \multicolumn{7}{|c|}{ DischargePoint 48} \\
\hline Flow(c), gpd & 4 & 8640 & 2282 & 5145 & $d$ & $d$ \\
\hline $\mathrm{pH}$, standard units & 4 & 8.4 & 7.9 & $d$ & $d$ & $d$ \\
\hline Temperature, $\mathrm{C}$ & 4 & 26.0 & 19.4 & 21.5 & $d$ & $d$ \\
\hline Ammonia & 4 & $<0.2$ & $<0.2$ & $<0.2$ & $d$ & $d$ \\
\hline Biochemical oxygen demand & 2 & $<5$ & $<5$ & $<5$ & $d$ & $d$ \\
\hline Chemical oxygen demand & 2 & 19 & $<5$ & $<12$ & $d$ & $d$ \\
\hline Total organic carbon & 4 & 21 & 14 & 16 & $d$ & $d$ \\
\hline Total suspended solids & 4 & 25 & $<5$ & $<15$ & $d$ & $d$ \\
\hline
\end{tabular}

Discharge Point 49

Flow(c), gpd

$\mathrm{pH}$, standard units

Temperature, $\mathrm{C}$

Ammonia

Biochemical oxygen demand

Chemical oxygen demand

Total organic carbon

Total suspended solids

\section{Flow(c), gpd}

pH, standard units

Temperature, $\mathrm{C}$

Ammonia

Biochemical oxygen demand

Chemical oxygen demand

Totai organic carbon

Total suspended solids

21600
8.3
26.4
$<0.2$
$<5$
19
23
$<5$

1600

9250

7.7

16.7

$<0.2$

$<5$

$<5$

2.8

$<5$

Discharge Point 5 !

436159
7.8
20.0
$<0.2$
$<5$
8.7
49
$<5$

15920
6.6
14.7
$<0.2$
$<5$
$<5$
35
$<5$

Discharge Point 55

$\begin{array}{ccc}15321 & d & d \\ d & d & d \\ 19.8 & d & d \\ <0.2 & d & d \\ <5 & d & d \\ <12 & d & d \\ 14.7 & d & d \\ <5 & d & d\end{array}$

170070
$d$
16.9
$<0.2$
$<5$
$<6.9$
41
$<5$

$\begin{array}{ll}d & d \\ d & d \\ d & d \\ d & d \\ d & d \\ d & d \\ d & d \\ d & d\end{array}$

\section{Flow(c), gpd}

pH, standard units

Temperature, $\mathrm{C}$

Ammonia

Biochemical oxygen demand

Chemical oxygen demand

Total organic carbon

Total suspended solids
4

4

4

2

2

4

4

4
4
4
4
2
2
4
4

4

4

4

4

2

2

4

4

114135
7.7
27.3
$<0.2$
$<5$
$<5$
30
$<5$

82080
6.7
18.1
$<0.2$
$<5$
$<5$
3.0
$<5$

101711
$d$
22.3
$<0.2$
$<5$
$<5$
15.5
$<5$


Table 4.62 (continued)

\begin{tabular}{|c|c|c|c|c|c|c|}
\hline \multirow[b]{2}{*}{ Parameter. } & \multirow{2}{*}{$\begin{array}{l}\text { Number of } \\
\text { samples }\end{array}$} & \multicolumn{3}{|c|}{ Concentration(a) } & \multirow{2}{*}{$\begin{array}{l}\text { Reference } \\
\text { value(b) }\end{array}$} & \multirow{2}{*}{$\begin{array}{l}\text { Number of values } \\
\text { exceeding reference }\end{array}$} \\
\hline & & $\operatorname{Max}$ & Min & $A v$ & & \\
\hline
\end{tabular}

Discharge Point 63

Flow(c), gpd

$\mathrm{pH}$, standard units

Temperanure, C

Ammonia

Biochemical oxygen demand

Chemical oxygen demand

Total organic carbon

Total suspended solids

$\begin{array}{cc}4 & 3424 \\ 4 & 8.0 \\ 4 & 22.5 \\ 4 & <0.2 \\ 4 & <5 \\ 4 & 17 \\ 4 & 17 \\ 4 & 26\end{array}$

1522
7.7
12.1
$<0.2$
$<5$
$<5$
3.9
$<5$

2093
$d$
17.1
$<0.2$
$<5$
$<11$
12.7
$<11$

Discharge Point 64

Flow(c), gpd

$\mathrm{pH}$, standard units

Temperature, $\mathrm{C}$

Ammonia

Biochemical oxygen demand

Chemical oxygen demand

Total organic carbon

Total suspended solids

4

4

4

4

4

4

4

4

Flow(c), gpd

$\mathrm{pH}$, standard units

Temperature, $\mathrm{C}$

Ammonia

Biochemical oxygen demand

Chemical oxygen demand

Total organic carbon

Total suspended solids

23920
8.2
23.4
0.45
5.9
38
13
46

46

\section{Discharge Point 67}

190
7.8
7.3
$<0.2$
$<5$
$<5$
6.4
$<5$

6693
$d$
15.3
$<0.26$
$<5.2$
$<16$
9.9
$<15$

$\begin{array}{ll}d & d \\ d & d \\ d & d \\ d & d \\ d & d \\ d & d \\ d & d \\ d & d\end{array}$

$d$
$d$
$d$
$d$
$d$
$d$
$d$
$d$

$d$ 
Table 4.62 (continued)

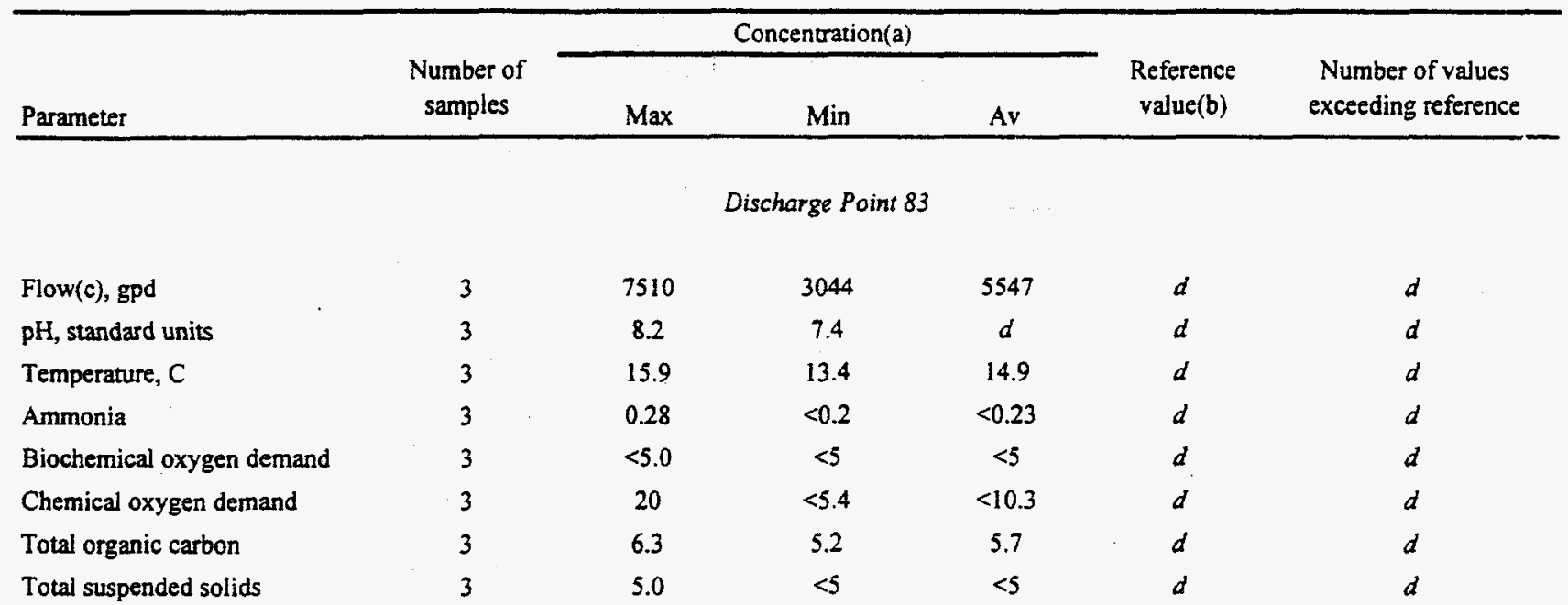

\section{Discharge Point 88}

Flow(c), gpd

$\mathrm{pH}$, standard units

Temperature, $\mathrm{C}$

Ammonia

Biochemical oxygen demand

Chemical oxygen demand

Total organic carbon
Total suspended solids

7609
8.1
28.4
0.45
$<5.0$
$<5$
21
$<5$

7609

8.1

28.4

0.45

5.0

$<5$

4

Flow(c), gpd

$\mathrm{pH}$, standard units

Temperature, $\mathrm{C}$

Ammonia

Biochemical oxygen demand

Chemical oxygen demand

Total organic carbon

Total suspended solids

5707
8.1
23.6
$<0.2$
$<5.0$
20
16
$<5$

560
7.7
13.8
$<0.2$
$<5$
$<5$
4
$<5$

Discharge Point 99

\section{Discharge Point 109}

$\begin{array}{ccc}3310 & d & d \\ d & d & d \\ 19.4 & d & d \\ <0.26 & d & d \\ <5 & d & d \\ <5 & d & d \\ 11 & d & d \\ <5 & d & d\end{array}$

3044
$d$
18.3
$<0.2$
$<5$
$<10$
9.2
$<5$

$\begin{array}{ll}d & d \\ d & d \\ d & d \\ d & d \\ d & d \\ d & d \\ d & d \\ d & d\end{array}$

Flow(c), gpd

$\mathrm{pH}$, standard units

Temperature, $\mathrm{C}$

Ammonia

Biochemical oxygen demand

Chemical oxygen demand

Total organic carbon

Total suspended solids

110500
7.3
13.7
$<0.2$
$<5$
$<5$
8.1
$<5$

$\begin{array}{cc}4 & 159790 \\ 4 & 8.2 \\ 4 & 23.8 \\ 4 & <0.2 \\ 3 & <5.0 \\ 3 & <5 \\ 4 & 21 \\ 4 & <5\end{array}$

138018
$d$
17.9
$<0.2$
$<5$
$<5$
14.6
$<5$


Table 4.62 (continued)

\begin{tabular}{|c|c|c|c|c|c|c|}
\hline \multirow[b]{2}{*}{ Parameter } & \multirow{2}{*}{$\begin{array}{l}\text { Number of } \\
\text { samples }\end{array}$} & \multicolumn{3}{|c|}{ Concentration(a) } & \multirow{2}{*}{$\begin{array}{c}\text { Reference } \\
\text { value(b) }\end{array}$} & \multirow{2}{*}{$\begin{array}{l}\text { Number of values } \\
\text { exceeding reference }\end{array}$} \\
\hline & & Max & Min & $\mathrm{Av}$ & & \\
\hline
\end{tabular}

Discharge Point 110

Flow(c), gpd

$\mathrm{pH}$, standard units

Temperature, $\mathrm{C}$

Ammonia

Biochemical oxygen demand

Chemical oxygen demand

Total organic carbon

Total suspended solids

3804
8.4
26.2
0.20
$<5.0$
19.0
15
$<5$

$<5$

Discharge Point $/ 13$

Flow(c), gpd

pH, standard units

Temperature, $\mathrm{C}$

Ammonia

Biochemical oxygen demand

Chemical oxygen demand

Totai organic carbon

Total suspended solids

8110
8.4
29.8
$<0.2$
$<5.0$
6.0
16
$<5$

$<5$

4565
7.9
15.6
$<0.2$
$<5$
$<5$
7.3
$<5$

\section{Discharge Point 114}

1141
7.8
19.5
$<0.2$
$<5$
17
7.3
$<5$

Flow(c), gpd

pH, standard units

Temperature, $\mathrm{C}$

Ammonia

Biochemical oxygen demand

Chemical oxygen demand

Total organic carbon

Total suspended solids

3044
8.4
27.5
$<0.2$
5.4
21
16
$<5$

$<5$

Discharge Point 122

Flow(c), gpd

$\mathrm{pH}$, standard units

Temperature, $\mathrm{C}$

Ammonia

Biochemical oxygen demand

Chemical oxy yen demand

Total organic carbon

Total suspended solids

$\begin{array}{cc}95 & 1450 \\ 7.8 & d \\ 8.5 & 18.7 \\ <0.2 & <0.20 \\ <5 & <5 \\ <5 & <10.4 \\ 5.5 & 8.8 \\ <5 & <5\end{array}$

6498

$d$

19.5

$<0.2$

$<5.0$

$<5.3$

11.9

$<5$

1739

$d$

23.3

$<0.2$

$<5.1$

18

11.9

$<5$

$\begin{array}{ll}d & d \\ d & d \\ d & d \\ d & d \\ d & d \\ d & d \\ d & d \\ d & d\end{array}$

$d$
$d$
$d$
$d$
$d$
$d$
$d$
$d$ 
Table 4.62 (continued)

\begin{tabular}{|c|c|c|c|c|c|c|}
\hline \multirow[b]{2}{*}{ Parameter } & \multirow{2}{*}{$\begin{array}{l}\text { Number of } \\
\text { samples }\end{array}$} & \multicolumn{3}{|c|}{ Concentration(a) } & \multirow{2}{*}{$\begin{array}{l}\text { Reference } \\
\text { value(b) }\end{array}$} & \multirow{2}{*}{$\begin{array}{l}\text { Number of values } \\
\text { exceeding reference }\end{array}$} \\
\hline & & $\operatorname{Max}$ & Min & $\mathrm{Av}$ & & \\
\hline
\end{tabular}

Discharge Point 125

$\begin{array}{llccccc}\text { Flow(c), gpd } & 4 & 366545 & 136960 & 252224 & d & d \\ \text { pH, standard units } & 4 & 8.0 & 7.5 & d & d & d \\ \text { Temperature, C } & 4 & 26.9 & 17.1 & 20 & d & d \\ \text { Ammonia } & 4 & <0.2 & <0.2 & <0.2 & d & d \\ \text { Biochemical oxygen demand } & 3 & <5.0 & <5 & <5 & d & d \\ \text { Chemical oxygen demand } & 3 & <5 & <5 & <5 & d & d \\ \text { Total organic carbon } & 4 & 20 & 2.0 & 14.0 & d & d \\ \text { Total suspended solids } & 4 & <5 & <5 & <5 & d & d\end{array}$

Discharge Point 135

Flow(c), gpd

pH, standard units

Temperature, $\mathrm{C}$

Ammonia

Biochemical oxygen demand

Chemical oxygen demand

Total organic carbon

Total suspended solids
3

3

3

3

2

2

3

3

499680
8.3
31.8
$<0.2$
$<5.0$
$<5.0$
19
$<5$

432000
7.7
22.9
$<0.2$
$<5$
$<5$
4.0
$<5$

460560
$d$
26.3
$<0.2$
$<5.0$
$<5.0$
12.0
$<5$

Discharge Point 142

Flow(c), gpd

$\mathrm{pH}$, standard units

Temperature, $\mathrm{C}$

Ammonia

Biochemical oxygen demand

Chemical oxygen demand

Total organic carbon

Total suspended solids

4

2282

8.4

22.0

0.21

$<5.0$

35

13

64

320
8.1
13.1
$<0.2$
$<$
13
3.
$<5$

320

8.1

13.1

$<0.2$

$<5$

13

3.1

$<5$

1316
$d$
18.1
$<0.20$
$<5.0$
22
8.7
$<24$

1316

$\begin{array}{ll}d & d \\ d & d \\ d & d \\ d & d \\ d & d \\ d & d \\ d & d \\ d & d\end{array}$

(a) Units in $\mathrm{mg} / \mathrm{L}$ uniess otherwise indicated

(b)NPDES permit limits.

(c)Flow during operation and/or discharging.

(d)Not applicable. 
Table 4.63. Surface water analytical results of polychlorinated biphenyls monitoring plan for the Y-12 Plant, 1993

\begin{tabular}{|c|c|c|c|}
\hline Site No. & Location & $\begin{array}{c}\text { Date } \\
\text { sampled }\end{array}$ & $\begin{array}{c}\text { PCB } \\
\text { concentration } \\
(\mathrm{mg} / \mathrm{L})\end{array}$ \\
\hline \multirow[t]{4}{*}{ PCB-1 } & Outfall 301, KerT & $2 / 3 / 93$ & $<0.0005$ \\
\hline & Hollow Quarry & $5 / 11 / 93$ & $<0.0005$ \\
\hline & & $9 / 15 / 93$ & $<0.0005$ \\
\hline & & $12 / 8 / 93$ & $<0.0005$ \\
\hline \multirow[t]{4}{*}{ PCB-2 } & Outfall 302 & $2 / 3 / 93$ & $<0.0005$ \\
\hline & Rogers Quarry & $5 / 11 / 93$ & $<0.0005$ \\
\hline & & $9 / 15 / 93$ & $<0.0005$ \\
\hline & & $12 / 8 / 93$ & $<0.0005$ \\
\hline PCB-3 & $\begin{array}{l}\text { Outfall } 303 \text {, New } \\
\text { Hope Pond }\end{array}$ & $a$ & \\
\hline PCB-5 & $\begin{array}{l}\text { New Hope Pond } \\
\text { Inlet }\end{array}$ & $b$ & \\
\hline \multirow[t]{4}{*}{ PCB-6 } & $\begin{array}{l}\text { Upstream of } \\
\text { Outfall } 135\end{array}$ & $2 / 3 / 93$ & $<0.0005$ \\
\hline & & $5 / 11 / 93$ & $<0.0005$ \\
\hline & & $9 / 15 i 93$ & $<0.0005$ \\
\hline & & $12 / 8 / 93$ & $<0.0005$ \\
\hline \multirow[t]{4}{*}{ PCB-7 } & Outfall 304, Bear & $2 / 3 / 93$ & $<0.0005$ \\
\hline & Creek & $5 / 11 / 93$ & $<0.0005$ \\
\hline & & $9 / 15 / 93$ & $<0.0005$ \\
\hline & & $12 / 8 / 93$ & $<0.0005$ \\
\hline
\end{tabular}

This outlet was closed in April 1989.

This inlet was closed in November 1988. 
Table 4.64. Permit limits for Y-12 Plant sanitary sewer discharge to City of Oak Ridge Publicly

Owned Treatment Works (POTW)

\begin{tabular}{lc}
\hline \multicolumn{1}{c}{ Parameter } & $\begin{array}{c}\text { City of Oax Ridge sanitary } \\
\text { sewer industrial discharge } \\
\text { permit limits }\end{array}$ \\
\hline pH & $6-9 \mathrm{pH}$ units \\
Cyanide & 0.007 \\
Oil and grease & 50 \\
Phenols & 5 \\
Biochemical oxygen demand & 300 \\
Hexavalent chromium & 0.002 \\
$\quad$ (chromium VI) & \\
Mercury & 0.1 \\
Total Kjeldahl nitrogen & 90 \\
Total suspended solids (TSS) & 300 \\
Arsenic & 0.1 \\
Cadmium & 0.000024 \\
Copper & 0.04 \\
Iron & 1.5 \\
Lead & 0.0016 \\
Manganese & 1 \\
Nickel & 0.1 \\
Silver & 0.1 \\
Zinc & 2 \\
\hline
\end{tabular}

${ }^{a}$ All values in $\mathrm{mg} / \mathrm{L}$ uniess noted otherwise.

${ }^{b} \mathrm{Maximum}$ value/minimum value. 
Table 4.65. City Flow Monitoring Station (northeast of Y-12 Plaiit)

\begin{tabular}{|c|c|c|c|c|c|c|}
\hline \multirow[b]{2}{*}{ Parameter } & \multirow{2}{*}{$\begin{array}{l}\text { Number of } \\
\text { samples }\end{array}$} & \multicolumn{3}{|c|}{ Concentration(a) } & \multirow{2}{*}{$\begin{array}{l}\text { Reference } \\
\text { value(b) }\end{array}$} & \multirow{2}{*}{$\begin{array}{l}\text { Number of values } \\
\text { exceeding reference }\end{array}$} \\
\hline & & $\operatorname{Max}$ & Min & Av & & \\
\hline pH, standard units & 45 & 8.6 & 6.9 & c & c & c \\
\hline Cyanide & 45 & 0.02 & $<0.002$ & $<0.01$ & c & $c$ \\
\hline Oil and Grease & 45 & 83 & $<2.0$ & $<11$ & c & $\mathfrak{c}$ \\
\hline Phenols & 45 & 0.064 & $<0.0013$ & $<0.018$ & c & c \\
\hline Total Volatile Organics & 45 & 0.034 & $<0.01$ & $<0.01$ & $c$ & $c$ \\
\hline Benzene & 45 & $<0.01$ & $<0.01$ & $<0.01$ & $c$ & c \\
\hline Metbylene Chloride & 45 & 0.021 & $<0.001$ & $<0.01$ & $c$ & c \\
\hline Tetrachloroethene & 45 & 8 & $<0.002$ & 2 & $c$ & c \\
\hline Trichloroethylene & 45 & $<0.01$ & $<0.01$ & $<0.01$ & $\varepsilon$ & c \\
\hline Toluene & 45 & 0.01 & $<0.004$ & $<0.01$ & c & $\mathrm{c}$ \\
\hline Total Chlorinated Hydrocarbons & 45 & 0.034 & $<0.01$ & $<0.01$ & $c$ & c \\
\hline Ammonia & 45 & 21 & 4.8 & 11 & c & c \\
\hline Biochemical Oxygen Demand & 45 & 160 & 23 & 55 & $c$ & c \\
\hline Chemical Oxygen Demand & 45 & 600 & 24 & 120 & $c$ & c \\
\hline Hexavalent Chromium & 45 & 0.05 & $<0.01$ & $<0.01$ & c & c \\
\hline Trivalent Chromium & 45 & 0.003 & -0.044 & -0.005 & $c$ & c \\
\hline Mercury & 45 & 0.0083 & $<0.0002$ & $<0.0008$ & c & c \\
\hline Total Kjeldahl Nitrogen & 45 & 42 & 7.0 & 16 & c & c \\
\hline Total Suspended Solids & 45 & 140 & 13 & 45 & c & c \\
\hline Selenium & 45 & $<0.1$ & $<0.002$ & $<0.05$ & $c$ & c \\
\hline Aluminum & 45 & 0.68 & 0.10 & 0.20 & c & c \\
\hline Arsenic & 45 & $<0.04$ & $<0.04$ & $<0.04$ & c. & $c$ \\
\hline Barium & 45 & 0.474 & 0.0348 & 0.0567 & c & $c$ \\
\hline Beryllium & 45 & 0.0005 & $<0.0004$ & $<0.0004$ & $\varsigma$ & c \\
\hline Boron & 45 & 0.098 & 0.024 & 0.045 & $c$ & $c$ \\
\hline Cadmium & 45 & $<0.004$ & $<0.004$ & $<0.004$ & c & c \\
\hline Calcium & 45 & 58.8 & 37.0 & 42.2 & c & c \\
\hline Cerium & 45 & $<0.02$ & $<0.02$ & $<0.02$ & c & $c$ \\
\hline Chromium, total & 45 & 0.016 & $<0.006$ & $<0.006$ & c & c \\
\hline Cobalt & 45 & 0.138 & $<0.002$ & $<0.006$ & c & c \\
\hline Copper & 45 & 0.031 & 0.009 & 0.02 & c & c \\
\hline Gallium & 45 & $<0.02$ & $<0.02$ & 0.02 & c & c \\
\hline Iron & 45 & 0.68 & 0.21 & 0.37 & c & c \\
\hline Lead & 45 & 0.05 & $<0.02$ & $<0.02$ & $c$ & c \\
\hline Lithium & 45 & 0.16 & $<0.02$ & $<0.03$ & $c$ & c \\
\hline Magnesium & 45 & 11.3 & 8.85 & 9.96 & $c$ & $c$ \\
\hline Manganese & 45 & 0.106 & 0.04 & 0.07 & c & c \\
\hline Molybdenum & 45 & 0.008 & $<0.006$ & $<0.006$ & $c$ & c \\
\hline Nickel & 45 & 0.009 & $<0.008$ & $<0.008$ & c & c \\
\hline Niobium & 45 & $<0.01$ & $<0.01$ & $<0.01$ & c & c \\
\hline Phosphorus & 6 & 3.77 & $<1.33$ & $<2.38$ & c & c \\
\hline Potassium & 45 & 11.3 & 5.1 & 7.2 & $c$ & $\approx$ \\
\hline Silver & 45 & 0.018 & $<0.006$ & $<0.007$ & c & $c$ \\
\hline Sodium & 45 & 49.1 & 13.0 & 17.5 & c & c \\
\hline
\end{tabular}


Table 4.65 (continued)

\begin{tabular}{|c|c|c|c|c|c|c|}
\hline \multirow[b]{2}{*}{ Parameter } & \multirow{2}{*}{$\begin{array}{l}\text { Number of } \\
\text { samples }\end{array}$} & \multicolumn{3}{|c|}{ Concentration(a) } & \multirow{2}{*}{$\begin{array}{l}\text { Reference } \\
\text { value(b) }\end{array}$} & \multirow{2}{*}{$\begin{array}{l}\text { Number of values } \\
\text { exceeding reference }\end{array}$} \\
\hline & & $\operatorname{Max}$ & Min & Av & & \\
\hline Strontium & 45 & 0.168 & 0.095 & 0.11 & c & $c$ \\
\hline Thorium & 45 & $<0.01$ & $<0.01$ & $<0.01$ & c & c \\
\hline Thallium & 45 & $<0.03$ & $<0.03$ & $<0.03$ & c & c \\
\hline Titanium & 45 & 1.64 & $<0.01$ & $<0.06$ & c & $c$ \\
\hline Vanadium & 45 & $<0.004$ & $<0.004$ & $<0.004$ & c & c \\
\hline Zinc & 45 & 0.26 & 0.07 & 0.1 & c & c \\
\hline Zirconium & 45 & $<0.004$ & $<0.004$ & $<0.004$ & c & c \\
\hline
\end{tabular}

(a)All units in $\mathrm{mg} / \mathrm{L}$ unless otherwise indicated.

(b)Sanitary Sewer Industrial Discharge Permit limits.

(c)Not applicable. 
Table 4.66. City Flow Monitoring Station (northeast of Y-12 Plant)

(Sumined DCG percentage $=1.88$ )

\begin{tabular}{|c|c|c|c|c|c|c|c|c|c|}
\hline \multirow[b]{2}{*}{ Parameter } & \multirow{2}{*}{$\begin{array}{c}\text { Number of } \\
\text { samples }\end{array}$} & \multicolumn{6}{|c|}{ Concentration } & \multirow{2}{*}{$\begin{array}{c}\text { Standard } \\
\text { error }\end{array}$} & \multirow{2}{*}{$\begin{array}{c}\text { Percentage } \\
\text { of DCG }\end{array}$} \\
\hline & & $\operatorname{Max}$ & $+1-$ & Min & +1 & Median & $+1-$ & & \\
\hline Alpha activity (pCiL) & 45 & 33 & 11 & -4.8 & 7.4 & 6.7 & 7.1 & 1.0 & a \\
\hline Beta activity ( $p C i L$ ) & 45 & 24 & 7.4 & -8.5 & 8.8 & 8.1 & 5.6 & 0.90 & a \\
\hline Plutonium-238 (pCi/L) & 45 & 0.54 & 0.40 & -0.44 & 0.84 & 0 & 0 & 0.029 & 0 \\
\hline Plutonium-239/240 (pCi/L) & 45 & 2.9 & 0.78 & -0.18 & 0.18 & 0.25 & 0 & 0.066 & 0.83 \\
\hline Uranium-234 (pCi/L) & 45 & 19 & 3.7 & 0.99 & 0.48 & 3.5 & 1.1 & 0.47 & 0.70 \\
\hline Uranium-235 (\%) & 45 & 10 & $a$ & 0.2 & a & 0.9 & a & 0.2 & $\mathbf{a}$ \\
\hline Uranium-235 $(\mathrm{pCi} / \mathrm{L})$ & 44 & 0.73 & 0.45 & -0.04 & 0.07 & 0.2 & a & 0.03 & 0.03 \\
\hline Uranium-236 (pCi/L) & 44 & 0.31 & 0.26 & -0.026 & 0.053 & 0.11 & $\mathbf{a}$ & 0.013 & 0.021 \\
\hline Uranium-238 $(\mathrm{pCi} / \mathrm{L})$ & 45 & 19 & 3.2 & 0.25 & 0.20 & 1.8 & 0.67 & 0.42 & 0.30 \\
\hline Uranium, total (mg/L) & 45 & 0.039 & a & 0.002 & $\mathbf{a}$ & 0.004 & a & a & a \\
\hline Gamma activity $(\mathrm{pCi} / \mathrm{L})$ & 40 & 78 & 16 & .14 & 16 & 28 & a & 3.7 & $a$ \\
\hline
\end{tabular}

(a)Not applicable. 
Table 4.67. Union Valley Flow Monitoring Station (east of Y-12 Plant)

\begin{tabular}{|c|c|c|c|c|c|c|}
\hline \multirow[b]{2}{*}{ Parameter } & \multirow{2}{*}{$\begin{array}{l}\text { Number of } \\
\text { samples }\end{array}$} & \multicolumn{3}{|c|}{ Concentration(a) } & \multirow{2}{*}{$\begin{array}{l}\text { Reference } \\
\text { value(b) }\end{array}$} & \multirow{2}{*}{$\begin{array}{l}\text { Number of values } \\
\text { exceeding reference }\end{array}$} \\
\hline & & $\operatorname{Max}$ & Min & Av & & \\
\hline pH, standard units & 45 & 8.8 & 6.4 & c & c & c \\
\hline Cyanide & 45 & 0.056 & $<0.0017$ & $<0.010$ & c & c \\
\hline Oil and Grease & 45 & 250 & 3.3 & 34 & $\mathrm{c}$ & c \\
\hline Phenols & 45 & 0.13 & $<0.0018$ & $<0.036$ & c & c \\
\hline Total Volatile Organics & 45 & 0.579 & $<0.01$ & $<0.04$ & $c$ & $c$ \\
\hline Benzene & 45 & $<0.01$ & $<0.01$ & $<0.01$ & c & c \\
\hline Methylene Chloride & 45 & 540 & $<0.01$ & $<20$ & c & c \\
\hline Tetrachloroethene & 45 & 2 & $<0.001$ & $<0.05$ & c & c \\
\hline Trichloroethylene & 45 & $<0.01$ & $<0.01$ & $<0.01$ & c & c \\
\hline Toluene & 45 & 22 & $<0.001$ & $<0.5$ & c & c \\
\hline Total Chlorinated Hydrocarbons & 45 & 0.579 & $<0.01$ & $<0.04$ & c & c \\
\hline Ammonia & 45 & 41 & 0.94 & 23 & c & c \\
\hline Biochemical Oxygen Demand & 45 & $>400$ & $<31$ & $<140$ & c & c \\
\hline Chemical Oxygen Demand & 45 & 1100 & $<5$ & $<350$ & c & c \\
\hline Hexavalent Chromium & 45 & 0.4 & $<0.01$ & $<0.02$ & c & c \\
\hline Trivalent Chromium & 45 & 0.15 & -0.004 & 0.007 & c & c \\
\hline Mercury & 45 & 0.015 & $<0.0002$ & $<0.001$ & c & c \\
\hline To:al Kjeldahl Nitrogen & 45 & 65 & 6.7 & 35 & c & c \\
\hline Total Suspended Solids & 45 & 900 & 6.0 & 157 & c & c \\
\hline Selenium & 45 & 0.1 & $<0.002$ & $<0.05$ & c & c \\
\hline Aluminum & 45 & 33 & 0.14 & 1.5 & c & c \\
\hline Arsenic & 45 & $<0.04$ & $<0.004$ & $<0.04$ & c & c \\
\hline Barium & 45 & 0.237 & 0.0424 & 0.0763 & c & c \\
\hline Beryllium & 45 & 0.0052 & $<0.0004$ & $<0.001$ & c & c \\
\hline Boron & 45 & 1.05 & 0.026 & 0.22 & c & c \\
\hline Cadmium & 45 & 0.006 & $<0.004$ & $<0.004$ & c & c \\
\hline Calcium & 45 & 72.9 & 45.7 & 56.6 & c & c \\
\hline Cerium & 45 & 0.02 & $<0.02$ & $<0.02$ & c & c \\
\hline Chromium, total & 45 & 0.55 & $<0.006$ & $<0.03$ & c & c \\
\hline Cobalt & 45 & 1.85 & $<0.002$ & $<0.06$ & c & c \\
\hline Copper & 45 & 0.212 & 0.014 & 0.061 & c & c \\
\hline Gallium & 45 & $<0.02$ & $<0.02$ & $<0.02$ & c & c \\
\hline Iron & 45 & 3.47 & 0.30 & 0.77 & c & c \\
\hline Lead & 45 & 0.12 & $<0.02$ & $<0.03$ & c & c \\
\hline Lithium & 45 & 1.11 & $<0.02$ & $<0.09$ & c & c \\
\hline Magnesium & 44 & 14.3 & 8.45 & 11.6 & c & $c$ \\
\hline Manganese & 45 & 0.349 & 0.079 & 0.22 & c & c \\
\hline Molybdenum & 45 & 0.031 & $<0.006$ & $<0.007$ & c & $c$ \\
\hline Nickel & 45 & 0.339 & $<0.008$ & $<0.02$ & c & $c$ \\
\hline Niobium & 45 & $<0.01$ & $<0.01$ & $<0.01$ & c & $c$ \\
\hline Phosphorus & 7 & 9.53 & $<2.02$ & $<4.05$ & c & c \\
\hline Potassium & 45 & 29.6 & 6.7 & 18 & c & c \\
\hline Silver & 45 & 0.015 & $<0.006$ & $<0.006$ & c & $c$ \\
\hline
\end{tabular}


Table 4.67 (continued)

\begin{tabular}{|c|c|c|c|c|c|c|}
\hline \multirow[b]{2}{*}{ Parameter } & \multirow{2}{*}{$\begin{array}{l}\text { Number of } \\
\text { samples }\end{array}$} & \multicolumn{3}{|c|}{ Concentration(a) } & \multirow{2}{*}{$\begin{array}{l}\text { Reference } \\
\text { value(b) }\end{array}$} & \multirow{2}{*}{$\begin{array}{l}\text { Number of values } \\
\text { exceeding reference }\end{array}$} \\
\hline & & $\operatorname{Max}$ & Min & Av & & \\
\hline & & - & & $\ldots$ & & \\
\hline Sodium & 45 & 64.5 & 11.5 & 28.0 & c & c \\
\hline Strontium & 45 & 0.209 & 0.111 & 0.138 & $c$ & c \\
\hline Thorium & 45 & $<0.03$ & $<0.03$ & $<0.03$ & c & c \\
\hline Thallium & 45 & $<0.01$ & $<0.01$ & $<0.01$ & c & $c$ \\
\hline Titanium & 45 & 11.6 & $<0.01$ & $<0.4$ & c & c \\
\hline Vanadium & 45 & 0.018 & $<0.004$ & $<0.004$ & c & c \\
\hline Zinc & 45 & 14 & 0.06 & 0.5 & c & c \\
\hline Zirconium & 45 & $<0.012$ & $<0.004$ & $<0.004$ & c & c \\
\hline
\end{tabular}

(a)All units in $\mathrm{mg} / \mathrm{L}$ unless otherwise indicated.

(b)Sanitary Sewer Industrial Discbarge Permit limits.

(c)Not applicable. 
Table 4.68. Union Valley Flow Monitoring Station (east of Y-12 Plant)

(Summed DCG percentage $=1.27$ )

\begin{tabular}{|c|c|c|c|c|c|c|c|c|c|}
\hline \multirow[b]{2}{*}{ Parameter } & \multirow{2}{*}{$\begin{array}{l}\text { Number of } \\
\text { samples }\end{array}$} & \multicolumn{6}{|c|}{ Concentration } & \multirow{2}{*}{$\begin{array}{l}\text { Standard } \\
\text { error }\end{array}$} & \multirow{2}{*}{$\begin{array}{c}\text { Percentage } \\
\text { of DCG }\end{array}$} \\
\hline & & $\operatorname{Max}$ & $+1-$ & Min & $+1-$ & Median & $+/ /$ & & \\
\hline Alpha activity (pCi/L) & 45 & 42 & 13 & -5.8 & 6.7 & 8.8 & 8.9 & 1.6 & a \\
\hline Beta activity (pCi/L) & 45 & 49 & 10 & 1.2 & 8.8 & 18 & 6.9 & 1.6 & $\mathbf{a}$ \\
\hline Plutonium-238 $(\mathrm{pCi} / \mathrm{L})$ & 45 & 0.61 & 0.95 & -0.27 & 0.23 & 0.013 & 0.10 & 0.025 & 0.033 \\
\hline Plutonium-239/240 (pCi $/ \mathrm{L})$ & 45 & 0.65 & 0.60 & -0.11 & 0.16 & 0 & 0 & 0.019 & 0 \\
\hline Uranium-234 (pCi/L) & 45 & 6.4 & 1.9 & -0.007 & 0.21 & 1 & 0.48 & 0.2 & 0.2 \\
\hline Uranium-235 (\%) & 45 & 0.78 & a & 0.12 & a & 0.22 & $\mathbf{a}$ & 0.017 & $\mathbf{a}$ \\
\hline Uranium-235 (pCi/L) & 44 & 0.76 & 0.57 & -0.09 & 0.18 & 0.2 & $\mathbf{a}$ & 0.03 & 0.03 \\
\hline Uranium-236 (pCi/L) & 44 & 0.36 & 0.33 & -0.06 & 0.09 & 0.05 & $\mathbf{a}$ & 0.01 & 0.01 \\
\hline Uranium-238 (pCi/L) & 45 & 45 & 9.8 & 0.03 & 0.17 & 8 & 1.7 & 2 & 1 \\
\hline Uranium, total (mg/L) & 45 & 0.092 & $\mathbf{a}$ & $<0.001$ & a & $<0.015$ & $\mathbf{a}$ & $\mathbf{a}$ & a \\
\hline Gamma activity (pCi/L) & 40 & 510 & 51 & -35 & 0 & 35 & $\mathbf{a}$ & 14 & $\mathbf{a}$ \\
\hline
\end{tabular}

(a)Not applicable. 
Table 4.69. Y-12 calculated sanitary sewer concentrations

\begin{tabular}{|c|c|c|c|c|c|}
\hline \multirow[b]{2}{*}{ Parameter } & \multirow{2}{*}{$\begin{array}{l}\text { Number of } \\
\text { samples }\end{array}$} & \multicolumn{3}{|c|}{ Concentration(a) } & \multirow{2}{*}{$\begin{array}{c}\text { Reference } \\
\text { value(b) }\end{array}$} \\
\hline & & Max & Min & Av & \\
\hline Flow, I & 45 & 38138761 & 1361488 & 3530860 & c \\
\hline Flow, gal & 45 & 10074720 & 359650 & 932711 & $c$ \\
\hline $\mathrm{pH}$, standard units & 45 & 8.7 & 6.9 & 7.5 & $6-9(d)$ \\
\hline Cyanide & 45 & $<0.020$ & $<0.002(e)$ & $<0.008$ & 0.007 \\
\hline Oil and Grease & 45 & 75 & $<2(\mathrm{e})$ & $<9$ & 50 \\
\hline Phenols & 45 & 0.059 & $<0.001(\mathrm{e})$ & $<0.016$ & 5.0 \\
\hline Total Volatile Organics & 45 & 0.04 & $<0.010(\mathrm{e})$ & $<0.012$ & c \\
\hline Benzene & 45 & $<0.01$ & $<0.010$ & $<0.010$ & c \\
\hline Methylene Chloride & 45 & 0.02 & $<0.010(\mathrm{e})$ & $<0.010(\mathrm{e})$ & c \\
\hline Tetrachloroethene & 45 & 10.57 & $<0.001$ & $<2.41$ & c \\
\hline Trichloroethylene & 45 & $<0.01$ & $<0.01$ & $<0.010$ & c \\
\hline Toluene & 45 & $<0.01$ & $<0.010(\mathrm{e})$ & $<0.010(\mathrm{e})$ & c \\
\hline Total Chlorinated Hydrocarbons & 45 & 0.04 & $<0.010(\mathrm{e})$ & $<0.012$ & c \\
\hline Ammonia & 45 & 20.1 & $<0.2(\mathrm{e})$ & 9.7 & c \\
\hline Biochemical Oxygen Demand & 45 & 176 & $<5(\mathrm{e})$ & $<43$ & 300 \\
\hline Chemical Oxygen Demand & 45 & 387 & $<5(\mathrm{e})$ & $<90$ & c \\
\hline Hexavalent Chromium & 45 & $<0.05$ & $<0.01(\mathrm{e})$ & $<0.01$ & 0.002 \\
\hline Trivalent Chromium & 45 & 0.004 & $<0.03(\mathrm{e})$ & $<0.03(e)$ & c \\
\hline Mercury & -45 & 0.0124 & $<0.0005$ & $<0.001$ & 0.1 \\
\hline Total Kjeldahl Nitrogen & 45 & 42.3 & $<0.2(\mathrm{e})$ & 13.1 & 90 \\
\hline Total Suspended Solids & 45 & 127.4 & $<5$ & 25.5 & 300 \\
\hline Selenium & 45 & $<0.100$ & $<0.002$ & $<0.05$ & $c$ \\
\hline Aluminum & 45 & 0.27 & $<0.2(\mathrm{e})$ & 0.073 & c \\
\hline Arsenic & 45 & $<0.04$ & $<0.04$ & $<0.04$ & 0.1 \\
\hline Barium & 45 & 0.508 & $<0.004(\mathrm{e})$ & 0.052 & $\dot{c}$ \\
\hline Beryllium & 45 & 0.0004 & $<0.002(\mathrm{e})$ & $<0.0003$ & c \\
\hline Boron & 45 & 0.059 & $<0.006(\mathrm{e})$ & 0.021 & $c$ \\
\hline Cadmium & 45 & $<0.004$ & $<0.004$ & $<0.004$ & 0.000024 \\
\hline Calcium & 45 & 58.2 & 26.5 & 40.3 & c \\
\hline Cerium & 45 & $<0.02$ & $<0.02$ & $<0.02$ & c \\
\hline Chromium, total & 45 & 0.014 & $<0.006(\mathrm{e})$ & $<0.004$ & c \\
\hline Cobalt & 45 & 0.008 & $<0.01(\mathrm{e})$ & $<0.01(\mathrm{e})$ & c \\
\hline Copper & 45 & 0.022 & $<0.006(\mathrm{e})$ & 0.010 & 0.04 \\
\hline Gallium & 45 & $<0.02$ & $<0.02$ & $<0.02$ & c \\
\hline Iron & 45 & 0.66 & $<0.06(\mathrm{e})$ & 0.31 & 1.5 \\
\hline Lead & 45 & $<0.05$ & $<0.01$ & $<0.02$ & 0.0016 \\
\hline Lithium & 45 & 0.07 & $<0.08(e)$ & $<0.01$ & c \\
\hline Magnesium & 45 & 11.6 & 8.7 & 9.8 & c \\
\hline Manganese & 45 & 0.103 & $<0.002(\mathrm{e})$ & 0.054 & 1.0 \\
\hline Molybdenum & 45 & 0.006 & $<0.002$ & $<0.006$ & c \\
\hline Nickel & 45 & 0.008 & $<0.008(e)$ & $<0.005$ & 0.10 \\
\hline Niobium & 45 & $<0.01$ & $<0.01$ & $<0.01$ & c \\
\hline
\end{tabular}


Table 4.69 (continued)

\begin{tabular}{|c|c|c|c|c|c|c|}
\hline \multirow{2}{*}{\multicolumn{2}{|c|}{ Parameter }} & \multirow{2}{*}{$\begin{array}{c}\text { Number of } \\
\text { samples }\end{array}$} & \multicolumn{3}{|c|}{ Concentration(a) } & \multirow{2}{*}{$\begin{array}{l}\text { Reference } \\
\text { value(b) }\end{array}$} \\
\hline & & & $\operatorname{Max}$ & Min & Av & \\
\hline Phosphorus & & .5 & 3.7 & 1.2 & 2.1 & c \\
\hline Potassium & & 45 & 10.5 & 1.2 & 5.9 & c \\
\hline Silver & & 45 & $<0.019$ & $<0.005$ & $<0.007$ & 0.1 \\
\hline Sodium & & 45 & 51.8 & 6.8 & 16.1 & c \\
\hline Strontium & & 45 & 0.17 & 0.05 & 0.11 & c \\
\hline Thorium & & 45 & $<0.03$ & $<0.03$ & $<0.03$ & c \\
\hline Thallium & & 45 & $<0.01$ & $<0.01$ & $<0.01$ & c \\
\hline Titanium & & 45 & 0.059 & $<0.06(\mathrm{e})$ & $<0.06(\mathrm{e})$ & c \\
\hline Vanadium & & 45 & $<0.004$ & $<0.02(\mathrm{e})$ & $<0.004$ & c \\
\hline Zinc & & 45 & 0.212 & $<0.01(\mathrm{e})$ & $<0.107$ & 2.0 \\
\hline Zirconium & & 45 & $<0.004$ & $<0.004$ & $<0.004$ & c \\
\hline
\end{tabular}

(a)All units in $\mathrm{mg} / \mathrm{L}$ unless otherwise indicated.

(b)Sanitary Sewer Industrial Discharge Permit limits.

(c)Not applicable.

(d)Minimum value/maximum value.

(e)Calculated value was below the detection limit. 
Table 4.70. Y-12 calculated sanitary sewer concentrations

(Summed DCG percentage $=1.03$ )

\begin{tabular}{|c|c|c|c|c|c|c|c|c|c|}
\hline \multirow[b]{2}{*}{ Parameter } & \multirow{2}{*}{$\begin{array}{c}\text { Number of } \\
\text { samples }\end{array}$} & \multicolumn{5}{|c|}{ Concentration } & & \multirow{2}{*}{$\begin{array}{l}\text { Standard } \\
\text { error }\end{array}$} & \multirow{2}{*}{$\begin{array}{c}\text { Percentage } \\
\text { of DCG }\end{array}$} \\
\hline & & $\operatorname{Max}$ & $+\%$ & Min & $+i-$ & Median & $+1-$ & & \\
\hline Alpha activity ( $\mathrm{pCi} / \mathrm{L}$ ) & 45 & 34.4 & 11 & -5.6 & 8 & 6.1 & 8 & 1.1 & a \\
\hline Beta activity (pCi/L) & 45 & 24.8 & 7 & -10.7 & 9 & 6.97 & 6 & 0.970 & a \\
\hline Plutonium-238 $(\mathrm{pCi} / \mathrm{L})$ & 44 & 0.61 & 0.41 & -0.48 & 0.39 & 0.012 & a & 0.033 & 0.029 \\
\hline Plutonium-239/240 (pCi/L) & 43 & 3.13 & 0.83 & -0.19 & 0.16 & 0.0023 & 0.0046 & 0.074 & 0.0058 \\
\hline Uranium-234 $(\mathrm{pCi} / \mathrm{L})$ & 45 & 20.27 & 3.92 & 0.99 & 0.48 & 3.8 & 1.2 & 0.50 & 0.75 \\
\hline Uranium-235 (\%) & 45 & 14.41 & a & -8.57 & a & 1.04 & a & 0.416 & $\mathbf{a}$ \\
\hline Uranium-235 (pCi/L) & 44 & 0.80 & 0.50 & -0.07 & 0.06 & 0.18 & $\mathbf{a}$ & 0.03 & 0.03 \\
\hline Uranium-236 (pCi/L) & 42 & 0.32 & 0.27 & -0.03 & 0.05 & 0.11 & $a$ & 0.01 & 0.02 \\
\hline Uranium-238 (pCil $)$ & 45 & 19.13 & 3.22 & -3.05 & 0.23 & 1.1 & 0.50 & 0.46 & 0.19 \\
\hline Uranium, total (mg/L) & 45 & 0.050 & a & -0.005 & $\mathbf{a}$ & $<0.004$ & a & 0.001 & a \\
\hline Gamma activity ( $p C i / L)$ & 40 & 81.8 & 16 & -20.2 & 13 & 25.5 & a & 4.08 & a \\
\hline
\end{tabular}

(a) Nớt applicable. 
Table 4.71. 1993 sampling and analysis plan for

oRNL off-site treated water monitoring

\begin{tabular}{|c|c|c|c|c|}
\hline Station & Analysis & $\begin{array}{l}\text { Collection } \\
\text { frequency }\end{array}$ & Sample type & $\begin{array}{l}\text { Analysis } \\
\text { frequency }\end{array}$ \\
\hline Gallaher & $\begin{array}{l}\text { Gamma scan, Gross alpha, } \\
\text { Gross beta, H-3, Pu-238, } \\
\text { Pu-239, Total rad Sr, } \\
\text { Total U }\end{array}$ & Weekly & $\begin{array}{l}\text { Time } \\
\text { proportional } \\
\text { composite }\end{array}$ & Quarterly \\
\hline Kingston & $\begin{array}{l}\text { Gamma scan, Gross alpha, } \\
\text { Gross beta, H-3, Pu-238, } \\
\text { Pu-239, Total rad Sr, } \\
\text { Total U }\end{array}$ & Weel:Iy & Grab & Quarterly \\
\hline
\end{tabular}


Teble 4.72. 1993 analyses for OFNL off-site treated water monttortng at Galleber we-er treatment plant

\begin{tabular}{|c|c|c|c|c|c|c|c|}
\hline \multirow[b]{2}{*}{ Parameter } & \multirow[b]{2}{*}{$\begin{array}{l}\text { det/ } \\
\text { total }\end{array}$} & \multicolumn{4}{|c|}{ Concentration } & \multirow[b]{2}{*}{ DWS ${ }^{d}$} & \multirow[b]{2}{*}{$\begin{array}{l}\text { Percent } \\
\text { of DWS }\end{array}$} \\
\hline & & $\operatorname{Max}{ }^{a}$ & $\operatorname{Min}^{a}$ & $A v^{b}$ & $\begin{array}{c}\text { Standard } \\
\text { error }\end{array}$ & & \\
\hline \multicolumn{8}{|l|}{ Metals (mg/L) } \\
\hline Uranium, totalf & $4 / 4$ & 0.00025 & 0.00010 & $0.00017 *$ & 0.000030 & $g$ & $g$ \\
\hline \multicolumn{8}{|c|}{ Radionuclides (pci/L) } \\
\hline$C 0-60$ & $0 / 4$ & 0.41 & -0.27 & 0.14 & 0.16 & 200 & $g$ \\
\hline$C s-137$ & $0 / 4$ & 0.49 & -0.081 & 0.21 & 0.13 & 120 & $g$ \\
\hline Gross alpha & $2 / 4$ & $0.70 *$ & 0.054 & $0.39 *$ & 0.15 & 15 & 2.6 \\
\hline Gross beta & $4 / 8$ & $3.8 *$ & $1.9 *$ & $2.6^{\star}$ & 0.42 & 50 & 5,3 \\
\hline $\mathrm{H}-3$ & $0 / 4$ & 810 & -650 & 150 & 300 & 20,000 & $g$ \\
\hline Pu -238 & $0 / 4$ & 0.011 & -0.011 & -0.00061 & 0.0051 & 1.6 & $g$ \\
\hline Pu-239 & $0 / 4$ & 0.0014 & -0.097 & -0.030 & 0.023 & 1.2 & $g$ \\
\hline Total rad sr & $4 / 4$ & $1.5^{\star}$ & $0.57 *$ & $1.1 *$ & 0.23 & 8 & 14 \\
\hline Total $u^{f, h}$ & $4 / 4$ & 0.16 & 0.066 & $0.11 *$ & 0.020 & 20 & 0.57 \\
\hline
\end{tabular}

Individual radionuclide concentrations significantly greater than zero are identified by an *。

b Average radionuclide concentrations significantly greater than zero are identified by an $\star$.

$c_{\text {Standard error of the mean. }}$.

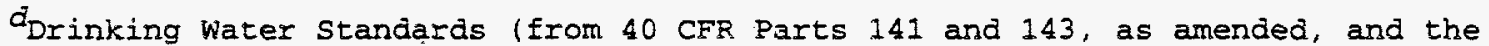
Tennessee General Water Quality Criteria for Domestic Water Supplies, as amended) . For radionuclides that do not have a drinking water standard, 48 of DCG for ingestion of water (from DOE order 5400.5) is used.

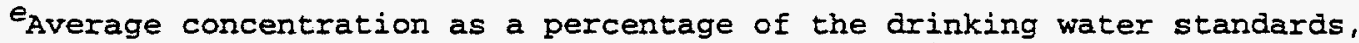
calculated when a reference exists and the parameter is a contaminant. For radionuclides, percentage of DWS is calculated only when a reference exists and the average concentration is significantly greater than zero.

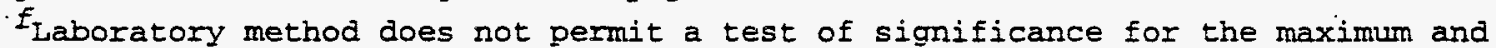
minimum values.

$g_{\text {Not applicable. }}$ $\mathrm{U}-238$.

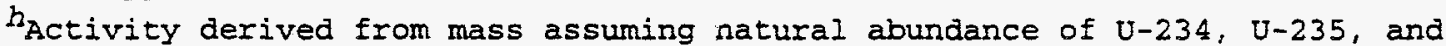


Table 4.73. 1993 analyses for oral off-site treated water monitoring at Ringston water treatment plant

\begin{tabular}{|c|c|c|c|c|c|c|c|}
\hline \multirow[b]{2}{*}{ Parameter } & \multirow[b]{2}{*}{$\begin{array}{l}\text { det } / \\
\text { total }\end{array}$} & \multicolumn{4}{|c|}{ Concentration } & \multirow[b]{2}{*}{ DWS $^{d}$} & \multirow[b]{2}{*}{$\begin{array}{l}\text { Percent } \\
\text { of DWS }\end{array}$} \\
\hline & & $\operatorname{Max}^{a}$ & $\operatorname{Min}^{a}$ & bV & $\begin{array}{l}\text { Standara } \\
\text { error }\end{array}$ & & \\
\hline \multicolumn{8}{|l|}{ Metals (mg/L) } \\
\hline Uranium, total $f$ & $4 / 4$ & 0.00014 & 0.00010 & $0.00012 *$ & 0.0000082 & $g$ & $g$ \\
\hline \multicolumn{8}{|c|}{ Radionuclides ( $\mathrm{pCi} / \mathrm{L}$ ) } \\
\hline$C 0-60$ & $0 / 4$ & 0.11 & -0.27 & -0.014 & 0.087 & 200 & $g$ \\
\hline Cs -137 & $2 / 4$ & $0.35 *$ & 0.027 & $0.18 *$ & 0.068 & 120 & 0.15 \\
\hline Gross alpha & $2 / 4$ & $0.95 *$ & 0.016 & 0.46 & 0.21 & 15 & $g$ \\
\hline Gross beta & $4 / 4$ & $2.2 \star$ & $1.0^{*}$ & $1.7 *$ & 0.26 & 50 & 3.4 \\
\hline $\mathrm{H}-3$ & $0 / 4$ & 110 & -350 & -88 & 100 & 20,000 & $g$ \\
\hline $\mathrm{Pu}-238$ & $0 / 4$ & 0.011 & -0.00019 & 0.0058 & 0.0026 & 1.6 & $g$ \\
\hline Pu-239 & $0 / 4$ & 0.0084 & 0.00051 & 0.0036 & 0.0018 & 1.2 & $g$ \\
\hline Total rad sr & $4 / 4$ & $0.70 *$ & $0.20 *$ & $0.38^{*}$ & 0.11 & 8 & 4.8 \\
\hline Tocal u $f, h$ & $4 / 4$ & 0.093 & 0.066 & $0.079 *$ & 0.0054 & 20 & 0.39 \\
\hline
\end{tabular}

\footnotetext{
andividual radionuclide concentrations significantly greater than zero are identified by an $\star$.

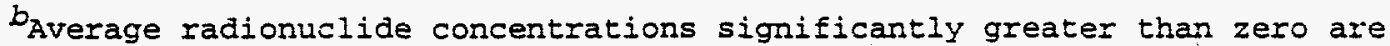
identified by an *

$c_{\text {Standard error of the mean. }}$

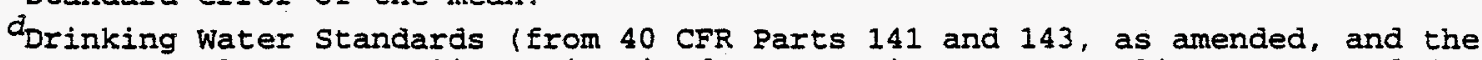
Tennessee General Water Quality Criteria for Domestic Water Supplies, as amended). For radionuclides that do not have a drinking water standard, $4 \%$ of DCG for ingestion of water (from DOE order 5400.5 ) is used.

e Average concentration as a percentage of the drinking water standards, calculated when a reference exists and the parameter is a contaminant. For radionuclides, percentage of DWS is calculated only when a reference exists and the average concentration is significantly greater than zero.

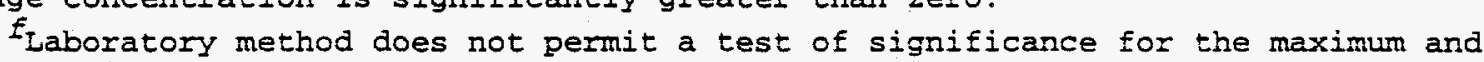
minimum values.

$g_{\text {Not applicable. }}$ $\mathrm{U}-238$.

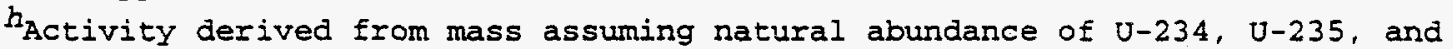


Talsle 4.74. Calculated sanitary sewer compliance summary for Y-12 Plant, 1993

\begin{tabular}{|c|c|c|c|c|c|c|}
\hline \multirow{2}{*}{ Parameter } & \multirow{2}{*}{$\begin{array}{l}\text { No. of } \\
\text { samples }\end{array}$} & \multicolumn{3}{|c|}{ Concentration $^{a}$} & \multirow{2}{*}{$\begin{array}{l}\text { Reference } \\
\text { value }^{b}\end{array}$} & \multirow{2}{*}{$\begin{array}{l}\text { No. of } \\
\text { values } \\
\text { exceeding } \\
\text { reference }\end{array}$} \\
\hline & & $\operatorname{Max}$ & Min & $\mathrm{Av}$ & & \\
\hline $\mathrm{pH}$, standard units & 45 & 8.7 & 6.9 & 7.5 & $6-9$ & 0 \\
\hline Cyanide & 45 & 0.020 & $<0.002^{d}$ & $<0.008$ & 0.007 & 0 \\
\hline Oil and grease & 45 & 75 & $<2^{d}$ & 9 & 50 & 0 \\
\hline Phenols & 45 & 0.059 & $<0.001^{d}$ & $<0.016$ & 5.0 & 0 \\
\hline Biochemical oxygen demand & 45 & 176 & $<5^{d}$ & $<43$ & 300 & 0 \\
\hline Mercury & 45 & 0.0124 & $<0.0005$ & $<0.001$ & 0.1 & 0 \\
\hline Total Kjeldahl nitrogen & 45 & 42.3 & $<0.2$ & 13.1 & 90 & 0 \\
\hline Total suspended solids & 45 & 127.4 & $<5$ & 25.5 & 300 & 0 \\
\hline Arsenic & 45 & $<0.04$ & $<0.04$ & $<0.04$ & 0.1 & 0 \\
\hline Cadmium & 45 & $<0.004$ & $<0.004$ & $<0.004$ & 0.000024 & 0 \\
\hline Copper & 45 & 0.022 & $<0.006^{d}$ & 0.10 & 0.04 & 0 \\
\hline Iron & 45 & 0.66 & $<0.06^{d}$ & 0.31 & 1.5 & 0 \\
\hline Lead & 45 & $<0.05$ & $<0.01$ & $<0.02$ & 0.0016 & 0 \\
\hline Manganese & 45 & 0.103 & $<0.002^{d}$ & 0.054 & 1.0 & 0 \\
\hline Nickel & 45 & 0.008 & $<0.008^{d}$ & $<0.005$ & 0.10 & 0 \\
\hline Silver & 45 & $<0.019$ & $<0.005$ & $<0.007$ & 0.1 & 0 \\
\hline Zinc & 45 & 0.212 & $<0.01^{\delta}$ & $<0.107$ & 2.0 & 0 \\
\hline
\end{tabular}

${ }^{a} \mathrm{All}$ units in $\mathrm{mg} / \mathrm{L}$ unless otherwise indicated.

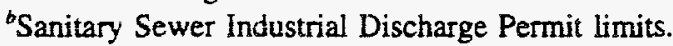

Minimum to maximum value.

${ }^{d}$ Calculated value was below the detection limit. 
Table 4.75. NPDES compliance at the K-25 Site, 1993

\begin{tabular}{|c|c|c|c|c|c|c|c|}
\hline \multirow[b]{2}{*}{$\begin{array}{c}\text { Discharge } \\
\text { point }\end{array}$} & \multirow[b]{2}{*}{ Effluent parameter } & \multicolumn{4}{|c|}{ Effluent limits } & \multirow{2}{*}{$\begin{array}{l}\text { No. of } \\
\text { noncom- } \\
\text { pliances }\end{array}$} & \multirow[b]{2}{*}{$\begin{array}{l}\text { Percentage } \\
\text { compliance }\end{array}$} \\
\hline & & $\begin{array}{c}\text { Monthly } \\
\mathrm{av}^{2}\end{array}$ & Daily $\max$ & $\begin{array}{l}\text { Monthily } \\
\text { av (kg/d) }\end{array}$ & $\begin{array}{c}\text { Daily } \\
\max \\
\text { (kg/d) }\end{array}$ & & \\
\hline \multirow{14}{*}{$\begin{array}{l}005 \text { (K-1203 } \\
\text { Sewage } \\
\text { Trearment } \\
\text { Facility) }\end{array}$} & Ammonia nitrogen & 5 & 7 & 12 & 17 & & 100 \\
\hline & Biochemical oxygen demand & 15 & 20 & 37 & 49 & & 100 \\
\hline & Chlorine, total residual & 0.14 & 0.24 & & & & 100 \\
\hline & Dissolved oxygen & & $5^{b}$ & & & & 100 \\
\hline & Fecal coliform, col $/ 100 \mathrm{ml}$ & $200^{x}$ & 400 & & & & 99.6 \\
\hline & Flow, Mgd & $d$ & $d$ & & & & 100 \\
\hline & $\mathrm{LC}_{50}$, Ceriodaphnia, $\%$ & & $14.6^{b}$ & & & & 100 \\
\hline & $\mathrm{LC}_{\text {son }}$ Pimephales, $\%$ & & $14.6^{b}$ & & & & 100 \\
\hline & NOEL, Ceriodaphnia, \% & & $4.2^{\circ}$ & & & & 83.3 \\
\hline & NOEL, Pimephales, \% & & $4.2^{b}$ & & & & 100 \\
\hline & $\mathrm{pH}$, standard units & & $6.0-9.0$ & & & & 100 \\
\hline & Settleable solids, mLL & & 0.5 & & & & 100 \\
\hline & Suspended solids & 30 & 45 & 74 & 111 & & 100 \\
\hline & Unpermitted discharge & $f$ & $f$ & $f$ & $f$ & & $f$ \\
\hline \multirow{7}{*}{$\begin{array}{l}009 \text { (K-1515-C } \\
\text { Sanitary } \\
\text { Water } \\
\text { Plant) }\end{array}$} & Aluminum & 1.0 & 2.0 & & & & \\
\hline & Chlorine, total residual & & 1.0 & & & & \\
\hline & Flow, Mgd & $d$ & $d$ & & & & \\
\hline & pH, standard units & & $6.0-9.0$ & & & & \\
\hline & Settleable solids, mL & & 0.5 & & & & \\
\hline & Suspended solids & 30 & 40 & & & & \\
\hline & Unpermitted discharge & $f$ & $f$ & $f$ & $f$ & & $f$ \\
\hline \multirow{33}{*}{$\begin{array}{l}011 \text { (K-1407-J } \\
\text { Central } \\
\text { Neutralization } \\
\text { Facility) }\end{array}$} & 1,1,1-Trichloroethane & $d$ & $d$ & & & & 100 \\
\hline & Acetone & $d$ & $d$ & & & & 100 \\
\hline & Acetonitrile & $d$ & $d$ & & & & 100 \\
\hline & Benzene & $d$ & $d$ & & & & 100 \\
\hline & Bromoform & $d$ & $d$ & & & & 100 \\
\hline & Cadmium & 0.18 & 0.69 & & & & 100 \\
\hline & Carbon tetrachloride & 0.5 & 0.5 & & & & 100 \\
\hline & Chemical oxygen demand & $d$ & $d$ & & & & 100 \\
\hline & Chloride, total & 9711 & 39,479 & & & & 100 \\
\hline & Chlorine, total residual & & 0.14 & & & & 100 \\
\hline & Chlorodibromomethane & $d$ & $d$ & & & & 100 \\
\hline & Chloroform & 0.5 & 0.5 & & & & 100 \\
\hline & Chromium & 1.71 & 2.77 & & & & 100 \\
\hline & Copper & 1.34 & 2.15 & & & & 100 \\
\hline & Dichlorobromomethane & $d$ & $d$ & & & & 100 \\
\hline & Flow, Mgd & $d$ & $d$ & & & & 100 \\
\hline & Ethylbenzene & $d$ & $d$ & & & & 100 \\
\hline & Gross alpha, pci/L & $d$ & $d$ & & & & 100 \\
\hline & Gross beta, pci/L & $d$ & $d$ & & & & 100 \\
\hline & $\mathrm{LC}_{\text {sor }}$ Ceriodaphnia, $\%$ & & $7.05^{\circ}$ & & & & 100 \\
\hline & $\mathrm{LC}_{\text {so }}$, Pimephales, $\%$ & & $7.05^{b}$ & & & & 100 \\
\hline & Lead & 0.38 & 0.69 & & & & 100 \\
\hline & Methyl ethyl ketone & $d$ & $d$ & & & & 100 \\
\hline & Methylene chloride & $d$ & $d$ & & & & 100 \\
\hline & Naphthalene & $d$ & $d$ & & & & 100 \\
\hline & Nickel & 2.38 & 3.98 & & & & 100 \\
\hline & NOEL', Ceriodaphnia, \% & & $2.11^{b}$ & & & & 100 \\
\hline & NOEL', Pimephales, \% & & $2.11^{b}$ & & & & 100 \\
\hline & Oil and grease & & 30 & & & & 100 \\
\hline & PCB & 0.00014 & 0.00014 & & & & 100 \\
\hline & pH, standard units & & $6.0-9.0$ & & & & 100 \\
\hline & Silver & 0.24 & 0.43 & & & & 100 \\
\hline & Suspended solids & & 40 & & & & 100 \\
\hline
\end{tabular}


Table 4.75 (continued)

\begin{tabular}{|c|c|c|c|c|c|c|c|}
\hline \multirow[b]{2}{*}{$\begin{array}{l}\text { Discharge } \\
\text { point }\end{array}$} & \multirow[b]{2}{*}{ Effivent parameter } & \multicolumn{4}{|c|}{ Effivent limits } & \multirow[b]{2}{*}{$\begin{array}{l}\text { No. of } \\
\text { noncom- } \\
\text { pliances }\end{array}$} & \multirow[b]{2}{*}{$\begin{array}{l}\text { Fercentage } \\
\text { compliance }\end{array}$} \\
\hline & & $\begin{array}{c}\text { Monthly } \\
\mathrm{av}^{2}\end{array}$ & Daily max ${ }^{\circ}$ & $\begin{array}{l}\text { Monthly } \\
\text { av (kg/d) }\end{array}$ & $\begin{array}{l}\text { Daily' } \\
\max \\
\text { (kg/d) }\end{array}$ & & \\
\hline \multirow{12}{*}{$\begin{array}{l}\text { Category I } \\
\text { storm drains }\end{array}$} & Temperatures & & & & & & \\
\hline & Tetrachloroethylene & & 0.7 & & & & 100 \\
\hline & Toluene & $d$ & $d$ & & & & 100 \\
\hline & Total toxic organics & & 2.13 & & & & 100 \\
\hline & Trichloroethylene & 0.5 & 0.5 & & & & 100 \\
\hline & Unpermitted discharge & $f$ & $f$ & $f$ & $f$ & & $f$ \\
\hline & Uranium, total & $d$ & $d$ & & & & 100 \\
\hline & Vinyl chloride & 0.2 & 0.2 & & & & 100 \\
\hline & Zinc & 1.48 & 2.61 & & & & 100 \\
\hline & Flow, Mgd & $d$ & $d$ & & & & 100 \\
\hline & $\mathrm{pH}$, standard units & & $4.0-9.0$ & & & & 100 \\
\hline & Unpermitted discharge & $f$ & $f$ & $f$ & $f$ & & $f$ \\
\hline \multirow{4}{*}{$\begin{array}{l}\text { Category II } \\
\text { storm drains }\end{array}$} & Flow, Mgd & $d$ & $d$ & & & & 100 \\
\hline & $\mathrm{pH}$, standard units & & $4.0-9.0$ & & & & 100 \\
\hline & Suspended solids & $d$ & $d$ & & & & 100 \\
\hline & Unpernitted discharge & $f$ & $f$ & $f$ & $f$ & 1 & $f$ \\
\hline \multirow{5}{*}{$\begin{array}{l}\text { Category III } \\
\text { storm drains }\end{array}$} & Flow, Mgd & $d$ & $d$ & & & & 100 \\
\hline & Oil and grease & $d$ & $d$ & & & & 100 \\
\hline & $\mathrm{pH}$, standard units & & $4.0-9.0$ & & & 1 & 99.7 \\
\hline & Suspended solids & $d$ & $d$ & & & & 100 \\
\hline & Unpermitted discharge & $f$ & $f$ & $f$ & $f$ & & $f$ \\
\hline \multirow{6}{*}{$\begin{array}{l}\text { Category IV } \\
\text { storm drains } \\
\text { (to Poplar } \\
\text { Creek) }\end{array}$} & Chlorine, total residual & & 0.14 & & & & 99 \\
\hline & Flow, Mgd & $d$ & $d$ & & & & 100 \\
\hline & Oil and grease & $d$ & $d$ & & & & 100 \\
\hline & $\mathrm{pH}_{8}$ standard units & & $6.0-9.0$ & & & 1 & 99.3 \\
\hline & Suspended solids & $d$ & $d$ & & & & 100 \\
\hline & Unpermitted discharge & $f$ & $f$ & $f$ & & & $f$ \\
\hline \multirow{6}{*}{$\begin{array}{l}\text { Category IV } \\
\text { storm drains } \\
\text { (to Mitchell } \\
\text { Branch) }\end{array}$} & Chlorine, total residual & & 0.019 & & & 1 & 99.5 \\
\hline & Flow, Mgd & $d$ & $d$ & & & & 100 \\
\hline & Oil and grease & $d$ & $d$ & & & & 100 \\
\hline & $\mathrm{pH}$, standard units & & $6.0-9.0$ & & & & 100 \\
\hline & Suspended solids & $d$ & $d$ & & & & 100 \\
\hline & Unpermitted discharge & $f$ & $f$ & $f$ & $f$ & 4 & $f$ \\
\hline
\end{tabular}

${ }^{a}$ Units are $\mathrm{mg} / \mathrm{L}$ unless otherwise stated.

Daily minimum.

'Geometric mean.

Nonlimited parameter.

No-observed-effect limit.

Not applicable.

'Effluent must not cause the temperature of the receiving stream to exceed $30.5^{\circ} \mathrm{C}$. 
Table 4.76. Y-12 Plant Toxicity Control and Monitoring Program (TCMP) summary information, $1993^{\circ}$

\begin{tabular}{cclcc}
\hline Outfall & Test date & \multicolumn{1}{c}{ Species } & $\begin{array}{c}\text { NOEC } \\
(\%)\end{array}$ & $\begin{array}{c}\text { IWC } \\
(\%)\end{array}$ \\
\hline Lithium evaporator process & $1 / 21 / 93$ & Fathead minnow & $<3$ & $<$ NOEC \\
condensate (Outfall 402) & $1 / 21 / 93$ & Ceriodaphnia & $<3$ & $<$ HOEC \\
Steam Plant Wastewater Treatment & $2 / 4 / 93$ & Fathead minnow & 100 & 13.3 \\
Facility (Outfall 503) & $2 / 4 / 93$ & Ceriodaphnia & 25 & 13.3 \\
OilWater Separator-9204-3 & $3 / 4 / 93$ & Fathead minnow & 50 & 0.3 \\
(Outfall 506) & $3 / 4 / 93$ & Ceriodaphnia & 100 & 0.3 \\
Central Pollution Control Facility & $5 / 13 / 93$ & Fathead minnow & 100 & 1.6 \\
(Outfall 501) & $5 / 13 / 93$ & Ceriodaphnia & 50 & 1.6 \\
Groundwater Treatment & $6 / 10 / 93$ & Fathead minnow & $<6$ & 2.2 \\
Facility (Outfall 512) & $6 / 10 / 93$ & Ceriodaphnia & $<6$ & 2.2 \\
Groundwater Treatment & $6 / 15 / 93$ & Fathead minnow & 3 & 2.2 \\
Facility (Outfall 512) & $6 / 15 / 93$ & Ceriodaphnia & 1.5 & 2.2 \\
West End Treatment Facility & $6 / 24 / 93$ & Fathead minnow & 30 & 0.9 \\
(Outfall 502) & $6 / 24 / 93$ & Ceriodaphnia & 15 & 0.9 \\
Proposed Outfall 201 & $9 / 9 / 93$ & Fathead minnowi & 100 & $c$ \\
Groundwater Treatment & $9 / 9 / 93$ & Ceriodaphnia & 50 & $c$ \\
Facility (Outfall 512) & $10 / 14 / 93$ & Fathead minnow & 3 & 2.2 \\
Cooling Tower No. 13 & $10 / 14 / 93$ & Ceriodaphnia & $<1$ & 2.2 \\
(Outfall 613) & $10 / 14 / 93$ & Fathead minnow & 100 & 2.0 \\
West End Treatment Facility & $10 / 14 / 93$ & Ceriodaphnio & 100 & 2.0 \\
(Outfall 502) & $10 / 28 / 93$ & Fathead minnow & 30 & 0.9 \\
Groundwater Treatment & $10 / 28 / 93$ & Cerioaaphnia & $<10$ & 0.9 \\
Facility (Outfall 512) & $11 / 11 / 93$ & Fathead minnow & 3 & 2.2 \\
\hline
\end{tabular}

These 7-day toxicity tests using fathead minnows and Ceriodaphnia were completed in 1993 as part of the TCMP conducted for the Y-12 Plant by Oak Ridge National Laboratory. Summarized are the effluents and their corresponding no-observed-effect concentrations (NOECs) and instream waste concentrations (TWCs). Note: Discharge from the treatment facilities is intermittent because of batch operations.

The instream waste concentration (IWC) is based on $3.9 \mathrm{cfs}$ at East Fork Poplar Creek, Station 8 (based on U.S. Geological Study data taken during drought conditions).

This is an instream point; therefore, an IWC is not applicable. 
Table 4.T7. Toxicity test results of ORNL wastewaters and ambient waters, 1993

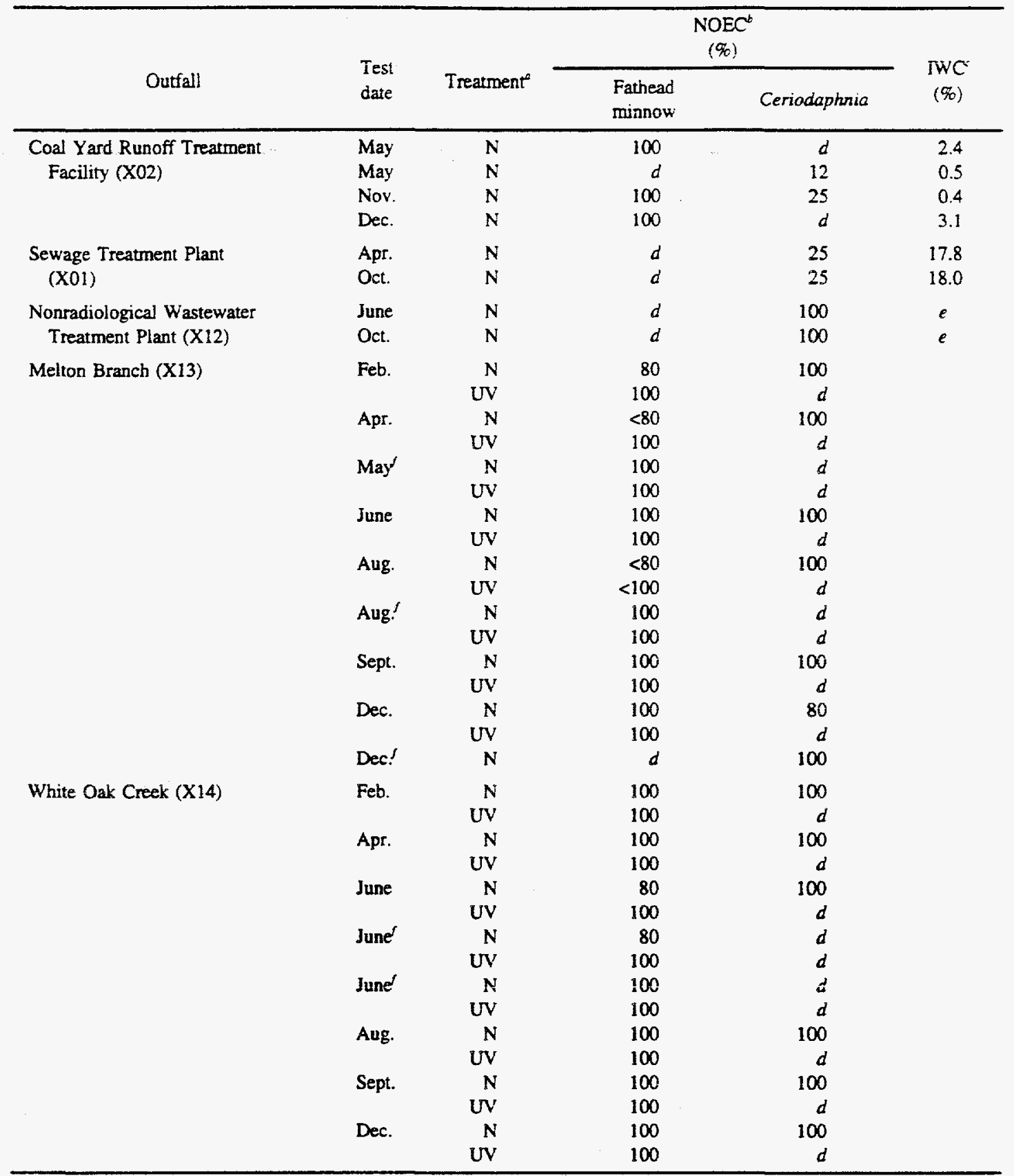

${ }^{\mathrm{N}} \mathrm{N}=$ no sample pretreatment; UV = ultraviolet light pretreatment.

No-observed-effect concentration.

Instream waste concentration (based on critical low flow of White Oak Creek).

Not tested.

Not calculated.

'Confirmatory test. 
Table 4.78. Average water quality parameters measured during toxicity tests

of ORNL wastewaters and ambient waters, 1993

Values are for full-strength wastewater for each test $(N=1)$ or averages of full-strength ambient water for each test $(N=7)$

\begin{tabular}{|c|c|c|c|c|c|}
\hline Outfall & Test date & $\begin{array}{c}\mathrm{pH} \\
\text { (standard units) }\end{array}$ & $\begin{array}{c}\text { Conductivity } \\
(\mu \mathrm{S} / \mathrm{cm})\end{array}$ & $\begin{array}{c}\text { Alkalinity } \\
\left.(\mathrm{mg} / \mathrm{L} \mathrm{CaCO})_{3}\right)\end{array}$ & $\begin{array}{c}\text { Hardness } \\
\left.(\mathrm{mg} / \mathrm{L} \mathrm{CaCO})_{3}\right)\end{array}$ \\
\hline $\begin{array}{l}\text { Coal Yard Runoff } \\
\text { Treatment Facility } \\
(\mathrm{X02})\end{array}$ & $\begin{array}{l}\text { May }^{2} \\
\text { May } \\
\text { Nov. } \\
\text { Dec. }\end{array}$ & $\begin{array}{l}7.45 \\
7.48 \\
7.44 \\
7.39\end{array}$ & $\begin{array}{l}1567 \\
1737 \\
2400 \\
4130\end{array}$ & $\begin{array}{l}16 \\
20 \\
27 \\
29\end{array}$ & $\begin{array}{r}880 \\
1128 \\
1600 \\
2000\end{array}$ \\
\hline $\begin{array}{l}\text { Sewage Treatment } \\
\text { Plant (X01) }\end{array}$ & $\begin{array}{l}\text { Apr. } \\
\text { Oct. }\end{array}$ & $\begin{array}{l}8.13 \\
7.83\end{array}$ & $\begin{array}{l}425 \\
403\end{array}$ & $\begin{array}{l}112 \\
100\end{array}$ & $\begin{array}{l}173 \\
171\end{array}$ \\
\hline $\begin{array}{l}\text { Nonradiological } \\
\text { Wastewater } \\
\text { Treatment } \\
\text { Facility (X12) }\end{array}$ & $\begin{array}{l}\text { June } \\
\text { Oct. }\end{array}$ & $\begin{array}{l}8.02 \\
7.78\end{array}$ & $\begin{array}{l}782 \\
764\end{array}$ & $\begin{array}{l}88 \\
89\end{array}$ & $\begin{array}{l}172 \\
172\end{array}$ \\
\hline $\begin{array}{l}\text { Meiton Branch } \\
\quad(\mathrm{X} 13)\end{array}$ & $\begin{array}{l}\text { Feb. } \\
\text { Apr. } \\
\text { May } \\
\text { June } \\
\text { Aug. } \\
\text { Aug. } \\
\text { Sept. } \\
\text { Dec. } \\
\text { Dec. }\end{array}$ & $\begin{array}{l}7.95 \\
8.04 \\
8.24 \\
8.02 \\
7.95 \\
7.78 \\
8.00 \\
7.99 \\
7.96\end{array}$ & $\begin{array}{l}527 \\
251 \\
389 \\
747 \\
568 \\
793 \\
390 \\
278 \\
357\end{array}$ & $\begin{array}{r}109 \\
113 \\
145 \\
102 \\
103 \\
65 \\
134 \\
92 \\
80\end{array}$ & $\begin{array}{l}237 \\
144 \\
203 \\
380 \\
292 \\
407 \\
190 \\
130 \\
169\end{array}$ \\
\hline $\begin{array}{l}\text { White Oak Creek } \\
\text { (X14) }\end{array}$ & $\begin{array}{l}\text { Feb. } \\
\text { Apr. } \\
\text { June } \\
\text { Junen } \\
\text { June } \\
\text { Aug. } \\
\text { Sept. } \\
\text { Dec. }\end{array}$ & $\begin{array}{l}7.98 \\
8.07 \\
8.14 \\
8.07 \\
8.11 \\
8.04 \\
8.07 \\
8.08\end{array}$ & $\begin{array}{l}380 \\
291 \\
372 \\
392 \\
343 \\
358 \\
373 \\
361\end{array}$ & $\begin{array}{l}113 \\
112 \\
118 \\
113 \\
113 \\
113 \\
119 \\
112\end{array}$ & $\begin{array}{r}169 \\
147 \\
171 \\
175 \\
154 \\
156 \\
164 \\
155\end{array}$ \\
\hline
\end{tabular}

aData for test conducted May 6-13, 1993.

${ }^{b}$ Data for test conducted May 19-26, 1993.

'Data for test conducted August 12-19, 1993.

'Data for test conducted August 27-September 3, 1993.

'Data for test conducted December 9-16, 1993.

Data for test conducted December 3G-January 6, 1994.

${ }^{8}$ Data for test conducted June 3-10, 1993.

"Data for test conducted June 10-17, 1993.

'Data for test conducted June 30-July $\%, 1993$. 
Table 4.79. Toxicity test results of K-25 Site wastewaters, 1993

\begin{tabular}{|c|c|c|c|c|}
\hline Outfall & Test date & Species & $\begin{array}{c}\text { MOEL } \\
(\because a)\end{array}$ & $\begin{array}{c}\mathrm{LC}_{50}{ }^{\circ} \\
(\%)\end{array}$ \\
\hline \multirow[t]{12}{*}{ K-1407-J (Outfall 011) } & January & Fathead minnows & 75 & $>75$ \\
\hline & & Ceriodaphnia & 25 & $>75$ \\
\hline & March ${ }^{6}$ & Fathead minnows & 25 & $>75$ \\
\hline & April & Ceriodaphnia & 75 & $>75$ \\
\hline & May & Fathead minnows & 75 & $>75$ \\
\hline & & Ceriodaphnia & 75 & $>75$ \\
\hline & July & Fathead minnows & 7.05 & $>7.05$ \\
\hline & & Ceriodaphnia & 25 & $>25$ \\
\hline & September & Fathead minnows & 75 & $>75$ \\
\hline & & Ceriodaphnia & 25 & $>75$ \\
\hline & November & Fathead minnows & 75 & $>75$ \\
\hline & & Ceriodaphnia & 75 & $>75$ \\
\hline \multirow[t]{12}{*}{ K-1203 (Outfall 005) } & January & Fathead minnows & 100 & $>100$ \\
\hline & & Ceriodaphnia & 100 & $>100$ \\
\hline & $\mathrm{March}^{c}$ & Fathead minnows & 100 & $>100$ \\
\hline & April & Ceriodaphnia & 100 & $>100$ \\
\hline & May & Fathead minnows & 100 & $>100$ \\
\hline & & Ceriodaphnia & 100 & $>100$ \\
\hline & July & Fathead minnows & 14.6 & $>14.6$ \\
\hline & & Ceriodaphnia & $<4.2$ & $>30$ \\
\hline & August & Ceriodaphnio & 30 & $>100$ \\
\hline & September & Fathead minnows & $\begin{array}{r}100 \\
30\end{array}$ & $>100$ \\
\hline & November & & & $>100$ \\
\hline & 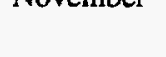 & Ceriodaphnia & 14.6 & $>100$ \\
\hline
\end{tabular}

No-observed-effect limit.

"96-hour lethal concentration for $50 \%$ of the test organisms.

Individual Ceriodaphnia test (unacceptable control survival); a retest w'as conducted in April 1993.

${ }^{d}$ Confirmatory test. 
Table 4.80. Average water quality parameters measured during toxicity tests of K-25 Site wastewaters, 1993

\begin{tabular}{clcccc}
\hline Outfall & Test date & $\begin{array}{c}\mathrm{pH} \\
\text { (standard } \\
\text { units })\end{array}$ & $\begin{array}{c}\text { Conductivity } \\
(\mu \mathrm{S} / \mathrm{cm})\end{array}$ & $\begin{array}{c}\text { Alkalinity } \\
\left(\mathrm{mg}_{\mathrm{L} \mathrm{CaCO}}\right)\end{array}$ & $\begin{array}{c}\text { Hardness } \\
\left(\mathrm{mg} / \mathrm{L} \mathrm{CaCO}_{3}\right)\end{array}$ \\
\hline K-1407-J & January & 7.80 & 2831 & 52 & 594 \\
(Outfall 011) & March & 7.69 & 1831 & 74 & 363 \\
& April & 7.73 & 1484 & 41 & 568 \\
& May & 7.76 & 1899 & 41 & 511 \\
& July & 7.92 & 3007 & 80 & 438 \\
& July & 7.57 & 4044 & 41 & 455 \\
& September & 7.65 & 1808 & 54 & 639 \\
K-1203 & November & 7.63 & 1615 & 64 & 680 \\
& January & 7.97 & 390 & 101 & 149 \\
& March & 8.09 & 380 & 101 & 172 \\
& April & 8.02 & 390 & 96 & 158 \\
& May & 7.93 & 402 & 85 & 147 \\
& July & 7.71 & 400 & 86 & 155 \\
& July & & 414 & 90 & 154 \\
& August & 7.88 & 389 & 81 & 143 \\
& September & 7.83 & 391 & 92 & 147 \\
& November & 7.89 & 435 & 107 & 151 \\
\hline
\end{tabular}

${ }^{a}$ Data are for test conducted July 8-15, 1993.

${ }^{b}$ Data are for test conducted July 29-August 5, 1993.

Table 4.81. Changes in average concentrations of PCBs and fraction of fish exceeding U.S. Food and Drug Administration (FDA) limit for channel cattish, 1986-93

\begin{tabular}{lcccccccc}
\hline \multicolumn{1}{c}{ Site $^{a}$} & 1986 & 1987 & \multicolumn{1}{c}{1988} & 1989 & 1990 & 1991 & 1992 & 1993 \\
\hline WCK 0.3 & 1.30 & 1.59 & 0.96 & 1.54 & 3.56 & 3.60 & 3.29 & 8.40 \\
CRK 32.2 & 1.01 & 1.61 & 0.58 & 1.20 & 0.31 & 1.38 & 0.36 & 0.67 \\
MHR & 0.46 & 0.81 & 0.52 & 0.28 & 0.41 & 0.29 & 0.34 & 0.62 \\
PCK 6.9 & $b$ & $b$ & 0.71 & 1.07 & 0.92 & 0.68 & 0.34 & 0.92 \\
CRK 15.0 & $b$ & $b$ & 0.50 & 0.79 & 0.88 & 1.08 & 1.27 & 0.63 \\
& & \multicolumn{7}{c}{ Fraction over FDA limit } \\
WCK 0.3 & $3 / 12$ & $2 / 8$ & $2 / 8$ & $4 / 8$ & $4 / 8$ & $6 / 8$ & $5 / 8$ & $4 / 4$ \\
CRK 32.2 & $0 / 8$ & $2 / 8$ & $1 / 8$ & $1 / 8$ & $0 / 8$ & $1 / 8$ & $0 / 8$ & $0 / 8$ \\
MHR & $0 / 6$ & $1 / 7$ & $0 / 10$ & $0 / 8$ & $0 / 8$ & $0 / 8$ & $0 / 8$ & $0 / 8$ \\
PCK 6.9 & $b$ & $b$ & $0 / 8$ & $1 / 8$ & $1 / 8$ & $0 / 8$ & $0 / 8$ & $0 / 8$ \\
CRK 15.0 & $b$ & $b$ & $0 / 9$ & $1 / 8$ & $1 / 8$ & $1 / 8$ & $2 / 8$ & $0 / 8$ \\
\hline
\end{tabular}

${ }^{a} \mathrm{WCK}=$ White Oak Creek Embayment kilometer, CRK = Clinch River kilometer, MHR = Melton Hill Reservoir, and PCK = Poplar Creek kilometer.

${ }^{b}$ Not sampled. 
Table 4.82. 1993 Potable water data from DOE-owned water-treatment facility serving the $Y-12$ Plant, ORNL, and city of Oak Ridge ${ }^{\circ}$

\begin{tabular}{|c|c|c|c|c|c|}
\hline Parameter & Max & Min & Avg & No. samples & Frequency \\
\hline Alachlor & $<0.0001$ & $<0.0001$ & $<0.0001$ & 1 & Annual \\
\hline Aldicarb & $<0.0001$ & $<0.0001$ & $<0.0001$ & 1 & Annual \\
\hline Aldicarb sulfoxide & $<0.0001$ & $<0.0001$ & $<0.0001$ & 1 & Annual \\
\hline Aldicarb sulfone & $<0.0001$ & $<0.0001$ & $<0.0001$ & 1 & Annual \\
\hline Atrazine & $<0.0001$ & $<0.0001$ & $<0.0001$ & 1 & Annual \\
\hline Carbofuran & $<0.0001$ & $<0.0001$ & $<0.0001$ & 1 & Annual \\
\hline Chlordane & $<0.0001$ & $<0.0001$ & $<0.0001$ & 1 & Annual \\
\hline Dalapon & $<0.0001$ & $<0.0001$ & $<0.0001$ & 1 & Annual \\
\hline Dinoseb & $<0.0001$ & $<0.0001$ & $<0.0001$ & 1 & Annual \\
\hline Diquat & $<0.0001$ & $<0.0001$ & $<0.0001$ & 1 & Annual \\
\hline Endothall & $<0.0001$ & $<0.0001$ & $<0.0001$ & 1 & Annual \\
\hline Glyphosate & $=0.0001$ & $<0.0001$ & $<0.0001$ & 1 & Annual \\
\hline Oxamyl (Vydate) & $<0.0001$ & $<0.0001$ & $<0.0001$ & 1 & Annual \\
\hline Picloram & $<0.0001$ & $<0.0001$ & $<0.0001$ & 1 & Annual \\
\hline Simazine & $<0.0001$ & $<0.0001$ & $<0.0001$ & 1 & Annual \\
\hline Toxaphene & $<0.002$ & $<0.002$ & $<0.002$ & 1 & Annual \\
\hline Methoxychlor & $<0.0002$ & $<0.0002$ & $<0.002$ & 1 & Annual \\
\hline Lindane & $<0.0004$ & $<0.0004$ & $<0.0004$ & 1 & Annual \\
\hline Endrin & $<0.0004$ & $<0.0004$ & $<0.0004$ & 1 & Annual \\
\hline $2,4-D$ & $<0.008$ & $<0.008$ & $<0.008$ & 1 & Annual \\
\hline $2,4,5,-\mathrm{TP}$ (silvex) & $<0.0008$ & $<0.0008$ & $<0.0008$ & 1 & Annual \\
\hline trans-1,2-Dichloroethylene & $<0.0005$ & $<0.0001$ & $<0.0001$ & 8 & Quarterly \\
\hline tert-Butylbenzene & $<0.0005$ & $<0.0002$ & $<0.0005$ & 8 & Quarterly \\
\hline sec-Butylbenzene & $<0.0005$ & $<0.0002$ & $<0.0005$ & 8 & Quarterly \\
\hline$p$-Xylene & $<0.0005$ & $<0.0002$ & $<0.0005$ & 8 & Quarterly \\
\hline$p$-Isopropyltoluene & $<0.0005$ & $<0.0001$ & $<0.0005$ & 8 & Quarterly \\
\hline$p$-Dichlorobenzene & $<0.005$ & $<0.0001$ & $<0.005$ & 8 & Quarterly \\
\hline$p$-Chlorotoluene & $<0.0005$ & $<0.0002$ & $<0.0005$ & 8 & Quarterly \\
\hline o-Xylene & $<0.006 .5$ & $<0.0001$ & $<0.0005$ & 8 & Quarterly \\
\hline o-Dichlorobenzene & $<0.0065$ & $<0.0001$ & $<0.0005$ & 8 & Quarterly \\
\hline o-Chlorotoluene & $<0.0005$ & $<0.0002$ & $<0.0005$ & 8 & Quarteriy \\
\hline n-Propylbenzene & $<0.0005$ & $<0.0001$ & $<0.0005$ & 8 & Quarterly \\
\hline$n$-Butylbenzene & $<0.0005$ & $<0.0002$ & $<0.0005$ & 8 & Quarterly \\
\hline$m$-Xylene & $<0.0005$ & $<0.0002$ & $<0.0005$ & 8 & Quarterly \\
\hline$m$-Dichlorobenzene & $<0.0005$ & $<0.0001$ & $<0.0005$ & 8 & Quarterly \\
\hline cis-1,2-Dichloroethylene & $<0.0005$ & $<0.0001$ & $<0.0005$ & 8 & Quarterly \\
\hline Xylenes (total) & $<0.0005$ & $<0.0001$ & $<0.0005$ & 8 & Quarterly \\
\hline Vinyl chloride & $<0.0005$ & $<0.0002$ & $<0.0005$ & 8 & Quarterly \\
\hline Trichloroethylene & $<0.0005$ & $<0.0001$ & $<0.0005$ & 8 & Quarterly \\
\hline Toluene & $<0.0005$ & $<0.0005$ & $<0.0005$ & 8 & Quarterly \\
\hline Tetrachloroethylene & $<0.0005$ & $<0.0002$ & $<0.0005$ & 8 & Quarterly \\
\hline Styrene & $<0.0005$ & $<0.0002$ & $<0.0005$ & 8 & Quarterly \\
\hline Naphthalene & $<0.0005$ & $<0.0002$ & $<0.0005$ & 8 & Quarterly \\
\hline Isopropylbenzene & $<0.0005$ & $<0.0001$ & $<0.0005$ & 8 & Quarterly \\
\hline Hexachlorobutadiene & $<0.0005$ & $<0.0002$ & $<0.0005$ & 8 & Quarterly \\
\hline Fluorotrichloromethane & $<0.0005$ & $<0.0005$ & $<0.0005$ & 8 & Quarterly \\
\hline
\end{tabular}


Table 4.82 (continued)

\begin{tabular}{|c|c|c|c|c|c|}
\hline Parameter & $\operatorname{Max}$ & Min & Avg & No. samples & Frequency \\
\hline Ethylene dibromide & $<0.00002$ & $<0.00001$ & $<0.00002$ & 8 & Quarteriy \\
\hline Ethylbenzene & $<0.0005$ & $<0.0001$ & $<0.0005$ & 8 & Quarterly \\
\hline Dichloromethane & $<0.0005$ & $<0.0005$ & $<0.0005$ & 8 & Quarterly \\
\hline Dichlorodifluoromethane & $<0.0005$ & $<0.0005$ & $<0.0005$ & 8 & Quarterly \\
\hline Dibromomethane & $<0.0005$ & $<0.0001$ & $<0.0005$ & 8 & Quarterly \\
\hline Chloromethane & $<0.0005$ & $<0.0005$ & $<0.0005$ & 8 & Quarterly \\
\hline Chloroform & 0.011 & $<0.0001$ & $<0.0044$ & 8 & Quarterly \\
\hline Chloroethane & $<0.0005$ & $<0.0005$ & $<0.0005$ & 8 & Quarterly \\
\hline Chlorodibromomethane & 0.0009 & $<0.0001$ & $<0.0002$ & 8 & Quarterly \\
\hline Chlorobenzene & $<0.0005$ & $<0.0002$ & $<0.0005$ & 8 & Quarterly \\
\hline Carbon tetrachloride & $<0.0005$ & $<0.0001$ & $<0.0005$ & 8 & Quarterly \\
\hline Bromomethene & $<0.0005$ & $<0.0005$ & $<0.0005$ & 8 & Quarterly \\
\hline Bromoform & $<0.0005$ & $<0.0001$ & $<0.0005$ & 8 & Quarterly \\
\hline Bromodichloromethane & 0.0058 & $<0.0001$ & 0.0012 & 8 & Quarterly \\
\hline Bromochloromethane & 0.0045 & $<0.0002$ & 0.0006 & 8 & Quarterly \\
\hline Bromobenzene & $<0.0005$ & $<0.0002$ & $<0.0005$ & 8 & Quarterly \\
\hline Benzene & $<0.0005$ & $<0.0005$ & $<0.0005$ & 8 & Quarterly \\
\hline 2,2-Dichloropropane & $<0.0005$ & $<0.0002$ & $<0.0005$ & 8 & Quarterly \\
\hline 1,3-Dichloropropene & $<0.0005$ & $<0.0001$ & $<0.0005$ & 8 & Quarterly \\
\hline 1,3-Dichloropropane & $<0.0005$ & $<0.0001$ & $<0.0005$ & 8 & Quarterly \\
\hline 1,3,5-Trimethylbenzene & $<0.0005$ & $<0.0001$ & $<0.0005$ & 8 & Quarterly \\
\hline 1,2-Dichloropropane & $<0.0005$ & $<0.0001$ & $<0.0005$ & 8 & Quarterly \\
\hline 1,2-Dichloroethane & $<0.005$ & $<0.0001$ & $<0.005$ & 8 & Quarterly \\
\hline 1,2-dibromo-3-chloropropane & $<0.00002$ & $<0.00002$ & $<0.00002$ & 8 & Quarterly \\
\hline 1,2,4-Trimethylbenzene & $<0.0005$ & $<0.0001$ & $<0.0005$ & 8 & Quarterly \\
\hline 1,2,4-Trichlorobenzene & $<0.0005$ & $<0.0002$ & $<0.0005$ & 8 & Quarterly \\
\hline 1,2,3-Trichloropropane & $<0.0005$ & $<0.0002$ & $<0.0005$ & 8 & Quarterly \\
\hline 1,2,3-Trichlorobenzene & $<0.0005$ & $<0.0002$ & $<0.0005$ & 8 & Quarterly \\
\hline 1,1-Dichloropropene & $<0.0005$ & $<0.0001$ & $<0.0005$ & 8 & Quarterly \\
\hline 1,1-Dichloroethylene & $<0.005$ & $<0.0002$ & $<0.005$ & 8 & Quarterly \\
\hline 1,1-Dichloroethane & $<0.0005$ & $<0.0001$ & $<0.0005$ & 8 & Quarteriy \\
\hline 1,1,2-Trichloroethane & $<0.0005$ & $<0.0001$ & $<0.0005$ & 8 & Quarteriy \\
\hline 1,1,2,2-Tetrachloroethane & $<0.0005$ & $<0.0001$ & $<0.0005$ & 8 & Quarterly \\
\hline 1,1,1-Trichloroethane & $<0.0005$ & $<0.0005$ & $<0.005$ & 8 & Quarterly \\
\hline 1,1,1,2-Tetrachloroethane & $<0.0005$ & $<0.0001$ & $<0.0005$ & 8 & Quarterly \\
\hline Trihalomethanes (total) & 0.043 & 0.01 & 0.025 & 16 & Quarterly \\
\hline Trichloromethane & 0.035 & 0.01 & 0.02 & 16 & Quarterly \\
\hline Tribromomethane & $<0.005$ & $<0.005$ & $<0.005$ & 16 & Quarterly \\
\hline Dibromochloromethane & $<0.005$ & $<0.005$ & $<0.005$ & 16 & Quarterly \\
\hline Bromodichloromethane & 0.009 & $<0.005$ & 0.005 & 16 & Quarterly \\
\hline P-PO4 & 0.432 & 0.21 & 0.358 & 9 & Monthly \\
\hline Ortho P & 0.075 & $<0.01$ & 0.037 & 4 & Monthly \\
\hline $\mathrm{pH}$ & 7.4 & 6.8 & 7.2 & 12 & Monthly \\
\hline Zinc & 0.177 & 0.042 & 0.119 & 12 & Monthly \\
\hline Volatile & 101 & 18.5 & 49.6 & 12 & Monthly \\
\hline Uranium & 0.003 & $<0.001$ & 0.0004 & 12 & Monthly \\
\hline Turbidity & $<2$ & $<2$ & $<2$ & 12 & Monthly \\
\hline Total solids & 198 & 121 & 163.7 & 12 & Monthly \\
\hline Total dissolved solids (TDS) & 182 & 100 & 156.2 & 12 & Monthly \\
\hline TOC & 1.36 & $<1$ & 0.113 & 12 & Monthly \\
\hline
\end{tabular}


Table 4.82 (continued)

\begin{tabular}{|c|c|c|c|c|c|}
\hline Parameter & $\operatorname{Max}$ & Min & Avg & No. samples & Frequency \\
\hline Thorium $(\mathrm{Ci} / \mathrm{L})$ & $<0.6$ & $<0.6$ & $<0.60$ & 12 & Monthly \\
\hline Suspended solids & 6 & $<2$ & 0.5 & 12 & Monthly \\
\hline Sodium & 7.2 & 4.48 & 5.8 & 12 & Monthiy \\
\hline $\mathrm{SO}_{4}$ & $<28.3$ & 19.6 & 23.7 & 12 & Monthly \\
\hline Silver & $<0.1$ & $<0.007$ & $<0.1$ & 12 & Monthly \\
\hline Selenium & 0.003 & $<0.002$ & 0.0004 & 12 & Monthly \\
\hline Potassium & 2.07 & 1.13 & 1.62 & 12 & Monthly \\
\hline Poly P & 0.879 & 0.034 & 0.37 & 12 & Monthly \\
\hline Odor (T.O.N.) & 0 & 0 & 0 & 12 & Monthiy \\
\hline Nitrate (AS N) & 0.712 & 0.106 & 0.439 & 12 & Monthly \\
\hline $\mathrm{N}$-total & 2.53 & 1.06 & 1.67 & 12 & Monthly \\
\hline N-Kjeldahl & 2.95 & $<0.5$ & 1.24 & 12 & Monthly \\
\hline Mercury & $<0.001$ & $<0.001$ & 0 & 12 & Monthly \\
\hline MBAS & 0.048 & 0.006 & 0.074 & 12 & Monthly \\
\hline Manganese & 0.03 & $<0.002$ & 0.003 & 12 & Monthly \\
\hline Magnesium & 11.6 & 8.9 & 10.2 & 12 & Monthly \\
\hline Lithium & $<0.002$ & $<0.002$ & $<0.002$ & 12 & Monthly \\
\hline Lead & $<0.002$ & $<0.001$ & $<0.002$ & 12 & Monthly \\
\hline Iron & 0.226 & $<0.007$ & 0.0563 & 12 & Monthly \\
\hline Hardness & 149 & 116 & 132 & 12 & Monthly \\
\hline Fluoride & 1.27 & 0.5 & 1.05 & 12 & Monthly \\
\hline $\mathrm{Cr}+6$ & $<0.004$ & $<0.004$ & $<0.004$ & 12 & Monthly \\
\hline Cyanide & $<0.001$ & $<0.001$ & $<0.004$ & 12 & Monthly \\
\hline Copper & 0.018 & $<0.006$ & 0.0015 & 12 & Monthly \\
\hline Conductance & 280 & 130 & 242 & 12 & Monthly \\
\hline Color (color units) & $<1$ & $<1$ & $<1$ & 12 & Monthly \\
\hline Cobalt $60(\mathrm{pCi} / \mathrm{L})$ & $<5$ & $<5$ & $<5$ & 12 & Monthly \\
\hline Chromium & 0.009 & $<0.007$ & 0.0008 & 12 & Monthly \\
\hline Chlorine & 1.28 & 0.9 & 1.10 & 12 & Monthly \\
\hline Chloride & 10 & 3.75 & 7.11 & 12 & Monthly \\
\hline Calcium nardress & 107 & 79.2 & 87.5 & 12 & Monthly \\
\hline Calcium & 43 & 31.7 & 36.0 & 12 & Monthiy \\
\hline Cadmium & $<0.004$ & $<0.002$ & $<0.004$ & 12 & Monthly \\
\hline Beta activity (pCi/L) & 32 & $<3$ & 8.2 & 12 & Monthly \\
\hline Barium & 0.09 & 0.027 & 0.024 & 12 & Monthly \\
\hline Arsenic & $<0.002$ & $<0.001$ & $<0.002$ & 12 & Monthly \\
\hline Aluminium & 0.71 & $<0.01$ & 0.15 & 12 & Monthly \\
\hline Alpha activiry (pCi/L) & 11 & $<2$ & 1.5 & 12 & Monthiy \\
\hline Alkalinity & 138 & 100 & 111.3 & 12 & Monthly \\
\hline Chlorine & 1.7 & 0.1 & 0.71 & 98 & 8/Month \\
\hline Coliform (per $100 \mathrm{~mL}$ ) & $<1$ & $<1$ & $<1$ & 96 & 8/Month \\
\hline Asbestos (MFL) & 0 & 0 & 0 & 0 & \\
\hline
\end{tabular}

${ }^{a}$ Operated by Johnson Controls World Services, Inc.

bUnits are $\mathrm{mg} / \mathrm{L}$ unless noted otherwise. 
Table 4.83. 1993 potable water data-lead and copper rule data from DOE-owned watertreatment facility serving the Y-12 Plant, ORNL, and the city of Oak Ridge

\begin{tabular}{lccc}
\hline Parameter & Unit & No. samples & $\begin{array}{c}90 \% \text { Percentile results } \\
\text { July } 1993\end{array}$ \\
\hline Lead & $\mathrm{mg} / \mathrm{L}$ & 40 & 0.004 \\
Copper & $\mathrm{mg} / \mathrm{L}$ & 40 & 0.374 \\
\hline
\end{tabular}

Table 4.84. 1993 sampling and anlysis plan for ORRI surface watera receiving effluents

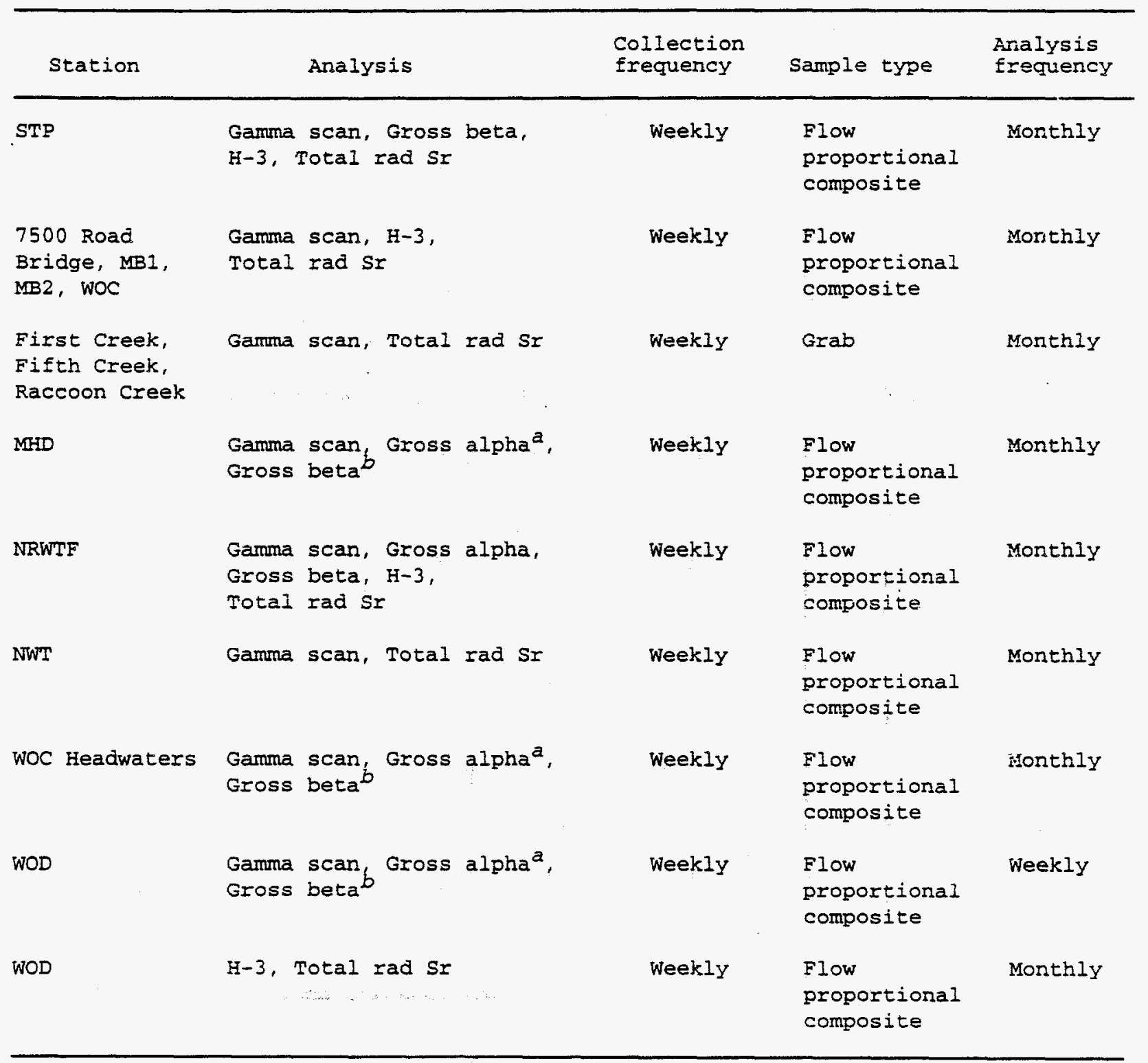

\footnotetext{
af gross alpha $>27$ pCi/L, analyze for Am-241, Cm-244, Pu-238, Pu-239, Th-228, $T h-230, T h-232, U-234, U-235$, and $U-238$.

Iff gross beta $>810 \mathrm{pCi} / \mathrm{L}$, analyze for total rad sx.
} 
Concentration (pci/L)

\begin{tabular}{|c|c|c|c|c|c|c|c|}
\hline & & & & & & & \\
\hline Radionuclide & $\begin{array}{l}N \text { det } / \\
N \text { total }\end{array}$ & $\operatorname{Max}^{a}$ & $\operatorname{Min}^{a}$ & $A v^{b}$ & $\begin{array}{c}\text { Standard } \\
\text { error }\end{array}$ & $D_{C G}{ }^{d}$ & $\begin{array}{l}\text { Percent } \\
\text { of DCG }\end{array}$ \\
\hline
\end{tabular}

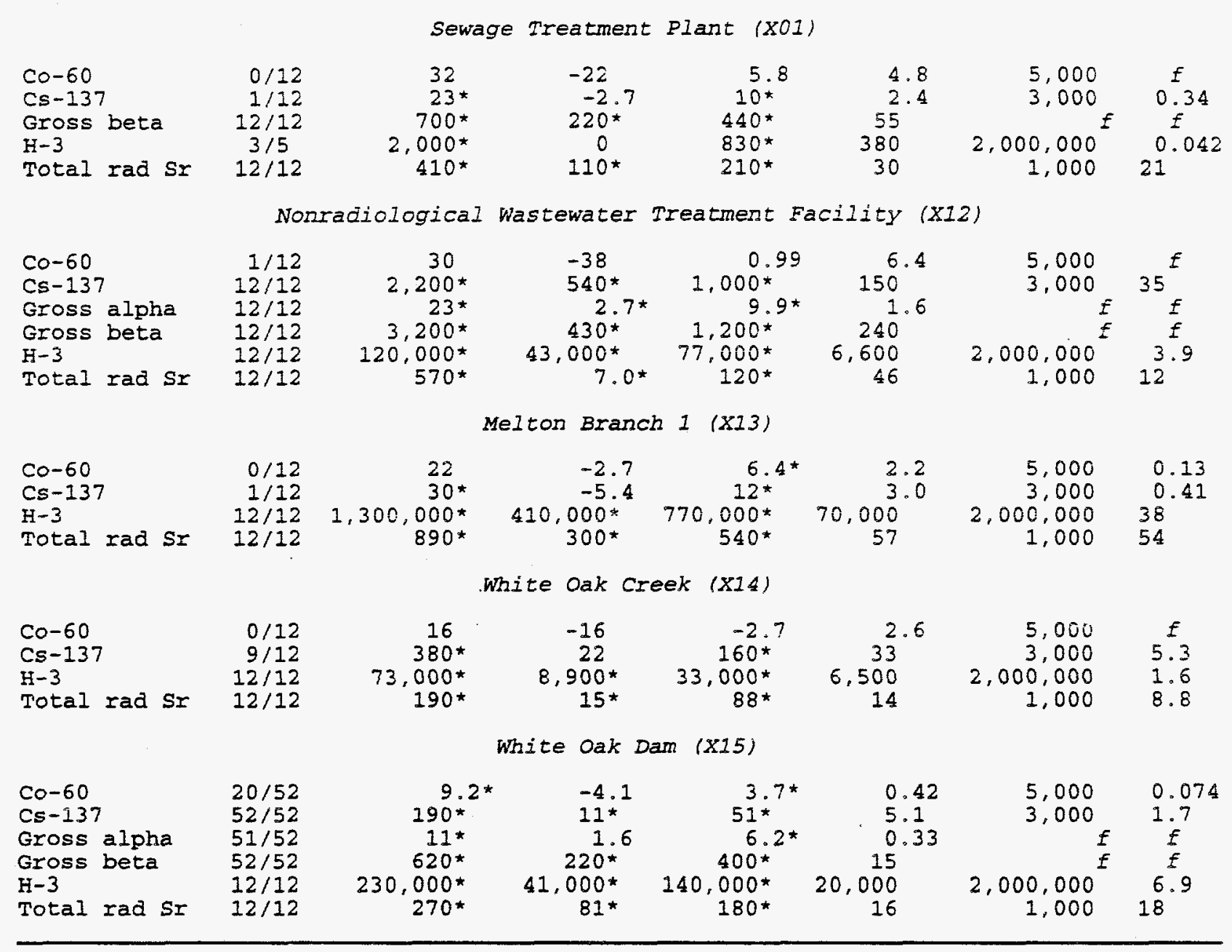

\footnotetext{
andividual radionuclide concentrations significantly greater than zero are identified by an *

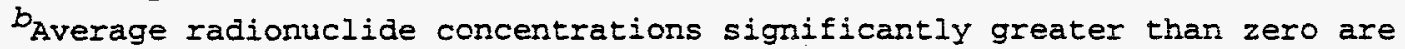
identified by an *.

$c_{\text {standard error of the mean. }}$

d Derived concentration guide for ingestion of water. From DOE order 5400.5.

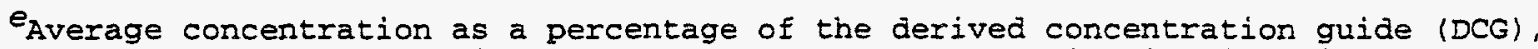
calculated only when a DCG exists and the average concentration is significantly greater than zero.

$f_{\text {Not applicable. }}$
} 
Table 4.86. 1993 radionuclide concentrations in aurface waters around oRkL

\begin{tabular}{|c|c|c|c|c|c|c|c|}
\hline \multirow[b]{2}{*}{ Radionuclide } & \multirow[b]{2}{*}{$\begin{array}{l}\mathrm{N} \text { det/ } \\
\mathrm{N} \text { total }\end{array}$} & \multicolumn{4}{|c|}{ Concentration ( $\mathrm{PCi} / \mathrm{I}$ ) } & \multirow[b]{2}{*}{$D C G^{d}$} & \multirow[b]{2}{*}{$\begin{array}{l}\text { Percent } \\
\text { of } D C G\end{array}$} \\
\hline & & $\operatorname{Max}^{a}$ & $\sin ^{a}$ & $A v^{b}$ & $\begin{array}{l}\text { Standard } \\
\text { error }\end{array}$ & & \\
\hline \multicolumn{8}{|c|}{ Melton Hill Dam } \\
\hline $\begin{array}{l}\text { Co- } 60 \\
\text { Cs }-137 \\
\text { Gross alpha } \\
\text { Gross beta }\end{array}$ & $\begin{array}{r}0 / 12 \\
0 / 12 \\
7 / 12 \\
12 / 12\end{array}$ & $\begin{array}{l}19 \\
16 \\
7.0^{\star} \\
24 *\end{array}$ & $\begin{array}{r}-14 \\
-32 \\
-0.27 \\
2.7^{\star}\end{array}$ & $\begin{array}{l}0.72 \\
-2.5 \\
2.1^{\star} \\
11^{\star}\end{array}$ & $\begin{array}{l}3.3 \\
4.2 \\
0.61 \\
1.9\end{array}$ & $\begin{array}{r}5,000 \\
3,000 \\
f \\
E\end{array}$ & $\begin{array}{r}f \\
f \\
-f \\
f\end{array}$ \\
\hline \multicolumn{8}{|c|}{ White Oak Creek Headwaters } \\
\hline $\begin{array}{l}\text { Co- } 60 \\
\text { Cs }-137 \\
\text { Gross alpha } \\
\text { Gross beta }\end{array}$ & $\begin{array}{r}1 / 12 \\
1 / 12 \\
8 / 12 \\
10 / 12\end{array}$ & $\begin{array}{l}51^{\star} \\
24 \\
8.9 * \\
22^{\star}\end{array}$ & $\begin{array}{c}-16 \\
-14 \\
0.81 \\
1.1\end{array}$ & $\begin{array}{l}9.0^{\star} \\
3.7 \\
2.4^{*} \\
8.0^{*}\end{array}$ & $\begin{array}{l}4.9 \\
3.6 \\
0.65 \\
1.5\end{array}$ & $\begin{array}{r}5,000 \\
3,000 \\
f \\
f\end{array}$ & $\begin{array}{l}0.18 \\
f \\
f \\
f\end{array}$ \\
\hline \multicolumn{8}{|c|}{7500 Road Bridge } \\
\hline $\begin{array}{l}\text { Co-60 } \\
\mathrm{Cs}-137 \\
\text { H-3 } \\
\text { Total rad } \mathrm{Sr}\end{array}$ & $\begin{array}{r}0 / 12 \\
12 / 12 \\
12 / 12 \\
12 / 12\end{array}$ & $\begin{array}{r}22 \\
430^{\star} \\
21,000^{\star} \\
120^{\star}\end{array}$ & $\begin{array}{r}-11 \\
38 \star \\
3,200 \star \\
35 \star\end{array}$ & $\begin{array}{c}5.4^{*} \\
140^{*} \\
8,800^{\star} \\
68^{\star}\end{array}$ & $\begin{array}{r}2.7 \\
29 \\
1.600 \\
7.3\end{array}$ & $\begin{array}{r}5,000 \\
3,000 \\
2,000,000 \\
1,000\end{array}$ & $\begin{array}{l}0.11 \\
4.7 \\
0.44 \\
6.8\end{array}$ \\
\hline \multicolumn{8}{|c|}{ First Creek } \\
\hline $\begin{array}{l}\text { Co- } 60 \\
\text { Cs }-137 \\
\text { Total rad sr }\end{array}$ & $\begin{array}{r}2 / 12 \\
3 / 12 \\
12 / 12\end{array}$ & $\begin{array}{r}38^{\star} \\
49^{\star} \\
490^{\star}\end{array}$ & $\begin{array}{l}-27 \\
-2.7 \\
86 \star\end{array}$ & $\begin{array}{c}5.7 \\
14^{\star} \\
220^{\star}\end{array}$ & $\begin{array}{l}6.1 \\
5.4 \\
34\end{array}$ & $\begin{array}{l}5,000 \\
3,000 \\
1,000\end{array}$ & $2^{f} 2^{\frac{f}{4}}$ \\
\hline \multicolumn{8}{|c|}{ Fifth creek } \\
\hline $\begin{array}{l}\text { co- } 60 \\
\text { Cs }-137 \\
\text { Total rad } s r\end{array}$ & $\begin{array}{r}1 / 12 \\
3 / 12 \\
12 / 12\end{array}$ & $\begin{array}{l}32 \\
51 \star \\
41 \star\end{array}$ & $\begin{array}{l}-30 \\
-22 \\
11\end{array}$ & $\begin{array}{l}5.5 \\
17{ }^{\star} \\
27 \star\end{array}$ & $\begin{array}{l}4.8 \\
7.0 \\
2.5\end{array}$ & $\begin{array}{l}5,000 \\
3,000 \\
1,000\end{array}$ & $\begin{array}{l}f \\
0.57 \\
2.7\end{array}$ \\
\hline \multicolumn{8}{|c|}{ Melton Branch 2} \\
\hline $\begin{array}{l}\text { Co-60 } \\
\text { Cs }-137 \\
\text { H-3 } \\
\text { Total rad } \mathrm{Sr}\end{array}$ & $\begin{array}{r}1 / 12 \\
0 / 12 \\
12 / 12 \\
6 / 12\end{array}$ & $\begin{array}{c}38 \star \\
32 \\
30,000^{\star} \\
5.7 *\end{array}$ & $\begin{array}{c}-16 \\
-16 \\
5,700^{\star} \\
0.38\end{array}$ & $\begin{array}{c}4.8 \\
1.1 \\
17.000^{\star} \\
2.4^{\star}\end{array}$ & $\begin{array}{c}4.7 \\
4.1 \\
2,300^{4} \\
0.44\end{array}$ & $\begin{array}{r}5,000 \\
3,000 \\
2,000,000 \\
1,000\end{array}$ & $\begin{array}{c}f \\
f \\
0.86 \\
0.24\end{array}$ \\
\hline \multicolumn{8}{|c|}{ Northwest Tributary } \\
\hline $\begin{array}{l}\text { Co- } 60 \\
\text { Cs }-137 \\
\text { Total rad sr }\end{array}$ & $\begin{array}{r}2 / 12 \\
4 / 12 \\
12 / 12\end{array}$ & $\begin{array}{l}51 \star \\
65 * \\
59 \star\end{array}$ & $\begin{array}{r}-30 \\
-22 \\
3.8 \star\end{array}$ & $\begin{array}{l}7.3 \\
19 * \\
34^{\star}\end{array}$ & $\begin{array}{l}6.2 \\
7.7 \\
6.2\end{array}$ & $\begin{array}{l}5,000 \\
3,000 \\
1,000\end{array}$ & $\begin{array}{l}f \\
0.63 \\
3.4\end{array}$ \\
\hline \multicolumn{8}{|c|}{ Raccoon Creek } \\
\hline $\begin{array}{l}\text { Co }-60 \\
\text { Cs }-137 \\
\text { Total rad } \mathrm{Sr}\end{array}$ & $\begin{array}{r}1 / 12 \\
1 / 12 \\
12 / 12\end{array}$ & $\begin{array}{l}46^{*} \\
32^{\star} \\
78^{*}\end{array}$ & $\begin{array}{r}-24 \\
-24 \\
4.6\end{array}$ & $\begin{array}{r}5.4 \\
4.3 \\
32 \star\end{array}$ & $\begin{array}{l}5.2 \\
5.1 \\
8.2\end{array}$ & $\begin{array}{l}5,000 \\
3,000 \\
1,000\end{array}$ & $\stackrel{f}{f}_{3.2}^{f}$ \\
\hline
\end{tabular}

\footnotetext{
Individual radionuclide concentrations significantly greater than zero are identified by an *.

baverage radionuclide concentrations significantly greater than zero are identified by an *.

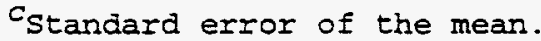

Derived concentration guide for ingestion of water. From DOE Order 5400.5 .

Average concentration as a percentage of the derived concentration guide (DCG), calculated only when a DCG exists and the average coicentration is significantly greater than zero.

$f_{\text {Not applicable. }}$
} 
Table 4.87. 1993 monthly stroam flows, ORNL

\begin{tabular}{|c|c|c|c|c|c|}
\hline \multirow[b]{2}{*}{ Month } & \multicolumn{4}{|c|}{ Fiow $\left(10^{9} \mathrm{~L}\right)$} & \multirow[b]{2}{*}{$\begin{array}{l}\text { Average } \\
\text { Ratiod }\end{array}$} \\
\hline & $\begin{array}{c}\text { Melton } \\
\text { Branch } 1\end{array}$ & $\begin{array}{l}\text { White Oak } \\
\text { Creek }\end{array}$ & $\begin{array}{l}\text { White } \mathrm{OaK} \\
\text { Damb }\end{array}$ & $\begin{array}{l}\text { Clinch } \\
\text { Riverc }\end{array}$ & \\
\hline January & 0.39 & 0.95 & 1.3 & 480 & 0.0057 \\
\hline February & 0.26 & 0.73 & 1.2 & 270 & 0.0083 \\
\hline March & 0.60 & 1.5 & 1.9 & 640 & 0.0054 \\
\hline April & 0.32 & 0.88 & 1.2 & 520 & 0.0046 \\
\hline May & 0.10 & 0.37 & 0.59 & 110 & 0.0059 \\
\hline June & 0.061 & 0.31 & 0.50 & 250 & 0.0024 \\
\hline July & 0.048 & 0.32 & 0.60 & 250 & 0.0029 \\
\hline August & 0.058 & 0.35 & 0.45 & 390 & 0.0012 \\
\hline September & 0.040 & 0.36 & 0.42 & 350 & 0.0013 \\
\hline October & 0.023 & 0.33 & 0.38 & 200 & 0.0043 \\
\hline November & 0.048 & 0.44 & 0.56 & 200 & 0.0065 \\
\hline December & 0.61 & 0.74 & 1.6 & 600 & 0.0097 \\
\hline
\end{tabular}

White Oak Creek above its confluence with Melton Branch.

White Oak Creek at White Oak Dam.

clinch River at Melton Hill Dam.

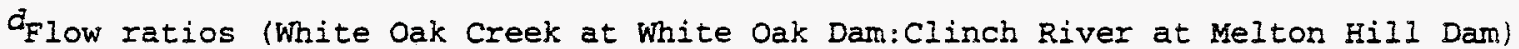
are calculated daily and averaged for the month. 
Table 4.88. ORWL NPDES radionuclide sampling and analysis plan for the category outfalls

\begin{tabular}{|c|c|c|c|}
\hline Radionuclide & $\begin{array}{l}\text { Collection } \\
\text { frequency }\end{array}$ & Sample type & $\begin{array}{l}\text { Analysis } \\
\text { frequency }\end{array}$ \\
\hline \multicolumn{4}{|c|}{ Category I outfalis } \\
\hline Gross beta ${ }^{a}$ & Yearly & Grab & Yearly \\
\hline \multicolumn{4}{|c|}{ Category II outfalIs } \\
\hline Gross beta ${ }^{a}$ & Quarterly & Grab & Quarterly \\
\hline \multicolumn{4}{|c|}{ Category III outfalis } \\
\hline $\begin{array}{l}\text { Co }-60 \\
\text { Cs }-137 \\
\text { Gross beta }\end{array}$ & $\begin{array}{l}\text { Quarterly } \\
\text { Quarterly } \\
\text { Quarterly }\end{array}$ & $\begin{array}{l}\text { Grab } \\
\text { Grab } \\
\text { Grab }\end{array}$ & $\begin{array}{l}\text { Quarterly } \\
\text { Quarterly } \\
\text { Quarterly }\end{array}$ \\
\hline
\end{tabular}

a If gross beta $>810 \mathrm{pCi} / \mathrm{L}$, analyze for total rad $\mathrm{Sr}$. 
Table 4.89. 1993 radionuclide concentrations at JRAL category outfalis

\begin{tabular}{|c|c|c|c|c|c|c|c|}
\hline \multirow[b]{2}{*}{ Radionuclide } & \multirow[b]{2}{*}{$\begin{array}{l}\mathbf{N} \text { det } / \\
\mathbf{N} \text { total }\end{array}$} & \multicolumn{4}{|c|}{ Concentration (pCi/L) } & \multirow[b]{2}{*}{$D C G^{d}$} & \multirow[b]{2}{*}{$\begin{array}{l}\text { Percent } \\
\text { of DCG }\end{array}$} \\
\hline & & $\operatorname{Max}^{a}$ & $\operatorname{Min}^{a}$ & $A v^{b}$ & $\begin{array}{l}\text { Standard } \\
\text { error }\end{array}$ & & \\
\hline \multicolumn{8}{|c|}{ Category I outfalis } \\
\hline $\begin{array}{l}\text { Gross beta } \\
\text { Total rad } \mathrm{Sr}^{g}\end{array}$ & $\begin{array}{c}20 / 21 \\
1 / 1\end{array}$ & $\begin{array}{l}2,100 * \\
1,300^{\star}\end{array}$ & $1.300^{2.7}$ & $\begin{array}{r}120 \\
1,300\end{array}$ & $98 f$ & 1,000 & $\begin{array}{l}f \\
E\end{array}$ \\
\hline \multicolumn{8}{|c|}{ Category II outfalis } \\
\hline $\begin{array}{l}\text { Gross beta } \\
\text { Total rad } \mathrm{Sr}^{g}\end{array}$ & $\begin{array}{c}149 / 163 \\
1 / 1\end{array}$ & $\begin{array}{l}1,900 * \\
1,100 *\end{array}$ & $1.100 *^{0.54}$ & $\begin{array}{l}46^{\star} \\
1,100^{\star}\end{array}$ & 14 & $\frac{f}{1,000}$ & $f$ \\
\hline \multicolumn{8}{|c|}{ Category III outfallsh } \\
\hline $\begin{array}{l}\text { Co }-60 \\
\text { Cs }-137 \\
\text { Gross beta } \\
\text { Total rad } \mathrm{Sr}^{g}\end{array}$ & $\begin{array}{l}15 / 20 \\
13 / 20 \\
20 / 20 \\
13 / 13\end{array}$ & $\begin{array}{r}65 * \\
190 * \\
9,700^{*} \\
4,900^{*}\end{array}$ & $\begin{array}{l}-0.81 \\
-1.1 \\
35 \star \\
380 \star\end{array}$ & $\begin{array}{r}11 * \\
29 * \\
3,100 * \\
2,000 *\end{array}$ & $\begin{array}{l}3.1 \\
12 \\
730 \\
360\end{array}$ & $\begin{array}{l}5,000 \\
3,000 \\
E \\
1,000\end{array}$ & $\begin{array}{c}0.22 \\
0.97 \\
200^{E}\end{array}$ \\
\hline
\end{tabular}

andividual radionuclide concentrations significantly greater than zero are identified by an *

$b_{\text {Average radionuclide concentrations significantly greater than zero are }}$ identified by an *

$c_{\text {Standard error of the mean. }}$ 5400.5 .

Derived concentration guide for ingestion of water. From DOE order

eAverage concentration as a percentage of the derived concentration guide (DCG), calculated only when a DCG exists and the average concentration is significantly greater than zero.

$f_{\text {Not applicable. }}$

ITotal rad Sr analyzed when gross beta $>810 \mathrm{pCi} / \mathrm{L}$.

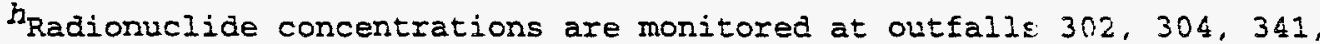
342 , and 368 . 
Table 4.90. 1993 sampling and analysis plan for ORAL reference surface waters: Melton Hill Dam and White Oak Creek headwaters

\begin{tabular}{|c|c|c|c|}
\hline Parameter & $\begin{array}{l}\text { Collection } \\
\text { frequency }\end{array}$ & Sample type & $\begin{array}{l}\text { Analysis } \\
\text { frequency }\end{array}$ \\
\hline $\begin{array}{l}\text { Anions } \\
\text { Fluoride } \\
\text { Nitrate, as } \mathrm{N} \\
\text { Sulfate, as } \mathrm{SO}_{4}\end{array}$ & $\begin{array}{l}\text { Monthly } \\
\text { Monthly } \\
\text { Monthly }\end{array}$ & $\begin{array}{l}\text { Flow proportional composite } \\
\text { Flow proportional composite } \\
\text { Flow proportional composite }\end{array}$ & $\begin{array}{l}\text { Monthly } \\
\text { Monthly } \\
\text { Monthly }\end{array}$ \\
\hline $\begin{array}{l}\text { Field Measurements } \\
\text { Conductivity } \\
\text { Dissolved oxygen } \\
\text { pH } \\
\text { Temperature } \\
\text { Turbidity }\end{array}$ & $\begin{array}{l}\text { Monthly } \\
\text { Monthly } \\
\text { Monthly } \\
\text { Monthly } \\
\text { Monthly }\end{array}$ & $\begin{array}{l}\text { Grab, instant read } \\
\text { Grab, instant read } \\
\text { Grab, instant read } \\
\text { Grab, instant read } \\
\text { Grab, instant read }\end{array}$ & $\begin{array}{l}\text { Monthly } \\
\text { Monthly } \\
\text { Monthly } \\
\text { Monthly } \\
\text { Monthly }\end{array}$ \\
\hline $\begin{array}{l}\text { Metals } \\
\text { Aluminum } \\
\text { Mrsenic } \\
\text { Cadmium } \\
\text { Chromium } \\
\text { Copper } \\
\text { Iron } \\
\text { Lead } \\
\text { Manganese } \\
\text { Nickel } \\
\text { Phosphorus } \\
\text { Silver } \\
\text { Zinc }\end{array}$ & $\begin{array}{l}\text { Monthly } \\
\text { Monthly } \\
\text { Monthly } \\
\text { Monthly } \\
\text { Monthly } \\
\text { Monthly } \\
\text { Monthly } \\
\text { Monthly } \\
\text { Monthly } \\
\text { Monthly } \\
\text { Monthly } \\
\text { Monthly }\end{array}$ & $\begin{array}{l}\text { Flow proportional composite } \\
\text { Flow proportional composite } \\
\text { Flow proportional composite } \\
\text { Flow proportional composite } \\
\text { Flow proportional composite } \\
\text { Flow proportional composite } \\
\text { Flow proportional composite } \\
\text { Flow proportional composite } \\
\text { Flow proportional composite } \\
\text { Flow proportional composite } \\
\text { Flow proportional composite } \\
\text { Flow proportional composite }\end{array}$ & $\begin{array}{l}\text { Monthly } \\
\text { Monthly } \\
\text { Monthly } \\
\text { Monthly } \\
\text { Monthly } \\
\text { Monthly } \\
\text { Monthly } \\
\text { Monthly } \\
\text { Monthly } \\
\text { Monthly } \\
\text { Monthly } \\
\text { Monthly }\end{array}$ \\
\hline $\begin{array}{l}\text { Others } \\
\text { Oil and grease } \\
\text { Total dissolved solids } \\
\text { Total organic carbon } \\
\text { Total suspended solids }\end{array}$ & $\begin{array}{l}\text { Monthly } \\
\text { Monthly } \\
\text { Monthly } \\
\text { Monthly }\end{array}$ & $\begin{array}{l}\text { Grab } \\
\text { Grab } \\
\text { Grab } \\
\text { Flow proportional composite }\end{array}$ & $\begin{array}{l}\text { Monthly } \\
\text { Monthly } \\
\text { Monthly } \\
\text { Monthly }\end{array}$ \\
\hline
\end{tabular}




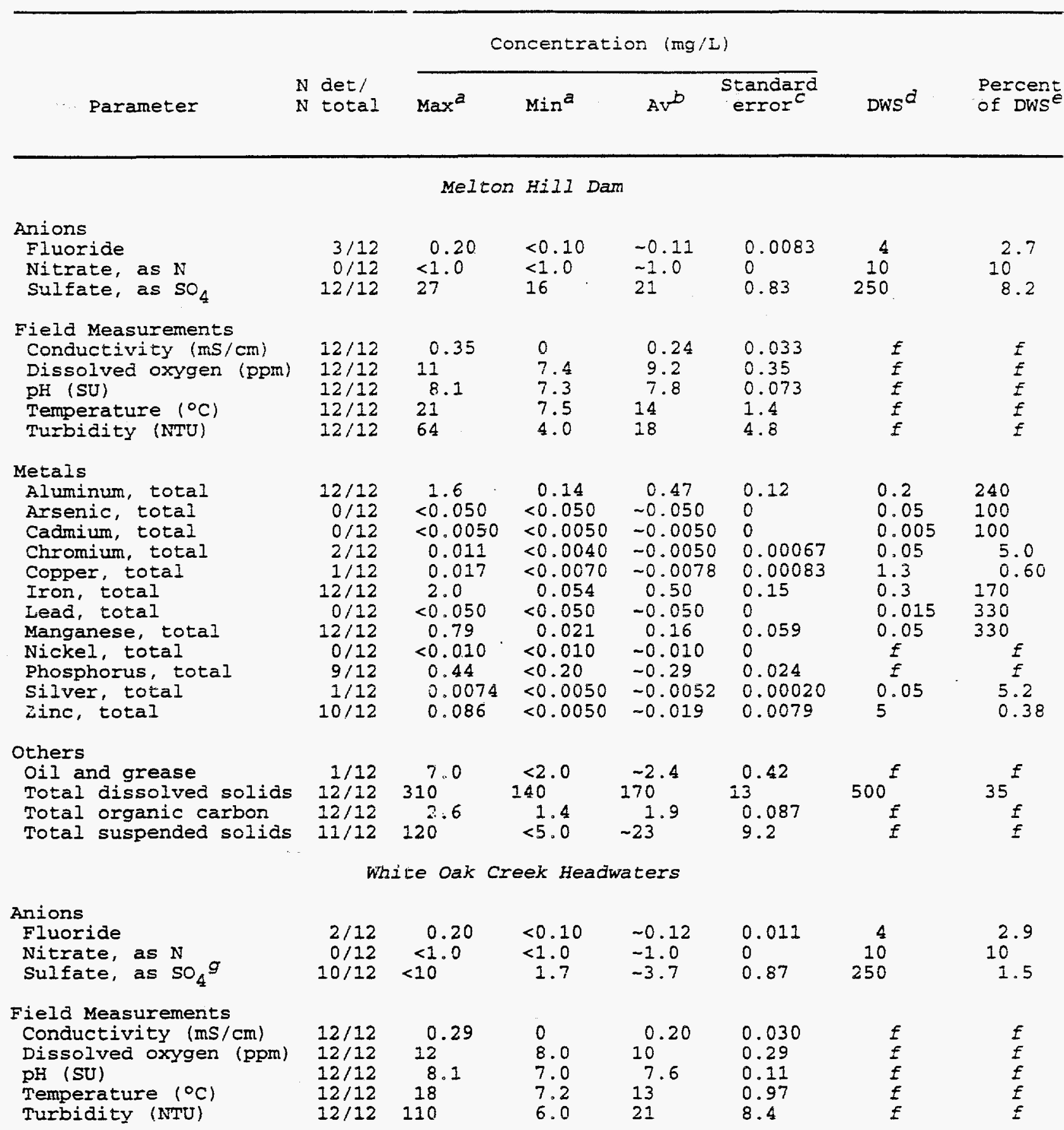


Table 4.91 (continued)

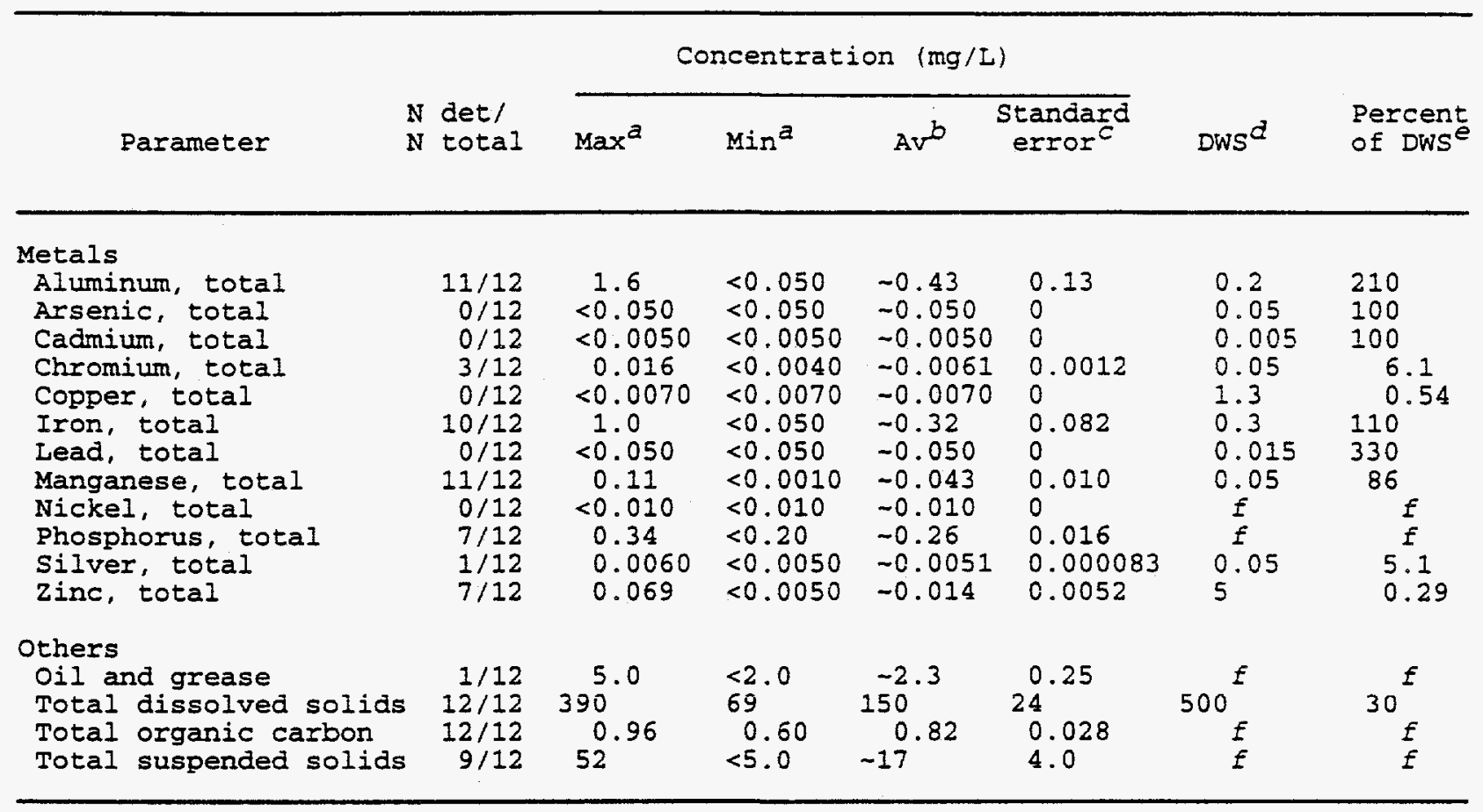

"Prefix "<" indicates the value of a parameter (excluding organics) was not guantifiable at the analytical detection limit.

$b_{A}$ tilde $(\sim)$ indicates that estimated values and/or detection limits were used in the calculation.

Cstandard error of the mean.

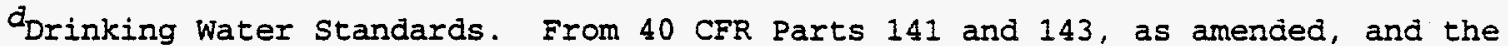
Tennessee General Water Quality Criteria for Domestic Water Supplies, as amended.

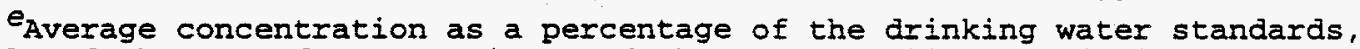
calculated when a reference exists and the parameter is a contaminant.

$f_{\text {Not applicable. }}$

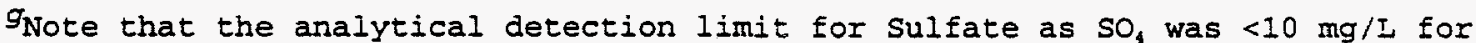
January and February. The detection limit for March through December was $<1 \mathrm{mg} / \mathrm{L}$. 
Table 4.92. 1993 NPDES compliance at ORNI

\begin{tabular}{|c|c|c|c|c|c|c|c|c|c|}
\hline \multirow[b]{2}{*}{ Discharge Point } & \multirow[b]{2}{*}{ Effluent parameters } & \multicolumn{5}{|c|}{ Permit Limits } & \multicolumn{3}{|c|}{ Permit Compliance } \\
\hline & & $\begin{array}{l}\text { Monthly } \\
\text { avg } \\
(\mathrm{kg} / \mathrm{d})\end{array}$ & $\begin{array}{l}\text { Daily } \\
\max \\
(\mathrm{kg} / \mathrm{d})\end{array}$ & $\begin{array}{l}\text { Monthly } \\
\text { avg } \\
(\mathrm{mg} / \mathrm{L})\end{array}$ & $\begin{array}{l}\text { Daily } \\
\max \\
(\mathrm{mg} / \mathrm{L})\end{array}$ & $\begin{array}{l}\text { Daily } \\
\text { min } \\
(\mathrm{mg} / \mathrm{L})\end{array}$ & $\begin{array}{l}\text { Number } \\
\text { of } \\
\text { noncompliances }\end{array}$ & $\begin{array}{l}\text { Number } \\
\text { of } \\
\text { samples }\end{array}$ & $\begin{array}{l}\text { Percent } \\
\text { of } \\
\text { compliance }^{a}\end{array}$ \\
\hline X01 & Ammonia, as $\mathrm{N}$ (summer) & 3.5 & 5.2 & 4.0 & 6.0 & & $\mathbf{0}$ & 91 & 100 \\
\hline (Sewage Treatment & Ammonia, as $\mathrm{N}$ (winter) & 7.8 & 11.8 & 9.0 & 13.5 & & 0 & 65 & 100 \\
\hline \multirow[t]{9}{*}{ Plant) } & $\begin{array}{l}\text { Biochemical oxygen demand } \\
\text { (summer) }\end{array}$ & 8.7 & 13.1 & 10 & 15 & & 0 & 91 & 100 \\
\hline & $\begin{array}{l}\text { Biochemical oxygen demand } \\
\text { (winter) }\end{array}$ & 17.4 & 26.2 & 20 & 30 & & 0 & 65 & 100 \\
\hline & Chlorine, total residual & & & & 0.5 & & 0 & 156 & 100 \\
\hline & Dissolved oxygen & & & & & 6.0 & 0 & 249 & 100 \\
\hline & Downstream pH (SU) & & & & 9.0 & 6.0 & 0 & 52 & 100 \\
\hline & Fecal coliform $(\mathrm{col} / 100 \mathrm{~mL})^{b}$ & & & 1000 & 5000 & & 0 & 156 & 100 \\
\hline & Oil and grease & 8.7 & 13.1 & 10 & 15 & & 0 & 156 & 100 \\
\hline & $\mathrm{pH}(\mathrm{SU})$ & & & & 9.0 & 6.0 & 0 & 52 & 100 \\
\hline & Total suspended solids & 26.2 & 39.2 & 30 & 45 & & 2 & 156 & 99 \\
\hline $\mathrm{X} 02$ & Chromium, total & & & 0.2 & 0.2 & & $\mathbf{0}$ & 47 & 100 \\
\hline (Coal Yard Runoff & Copper, total & & & 1.0 & 1.0 & & $\mathbf{0}$ & 47 & 100 \\
\hline \multirow[t]{8}{*}{ Treatment Facility) } & Downstream pH (SU) & & & & 9.0 & 6.0 & 0 & 248 & 100 \\
\hline & Iron, total & & & 1.0 & 1.0 & & 0 & 47 & 100 \\
\hline & Oil and grease & & & 15 & 20 & & 0 & 47 & 100 \\
\hline & $\mathrm{pH}(\mathrm{SU})$ & & & & 9.0 & 6.0 & 0 & 248 & 100 \\
\hline & Selenium, total & & & 0.22 & 0.95 & & 0 & 47 & 100 \\
\hline & Temperature $\left({ }^{\circ} \mathrm{C}\right)$ & & & & 30.5 & & 0 & 248 & 100 \\
\hline & Total suspended solids & & & & 50 & & 1 & 47 & 98 \\
\hline & Zinc & & & 1.0 & 1.0 & & 0 & 47 & 100 \\
\hline $\mathrm{X} 12$ & Cadmium, total & 0.79 & 2.09 & 0.26 & 0.69 & & 0 & 52 & 100 \\
\hline (Nonradiological & Chromium, total & 5.18 & 8.39 & 1.71 & 2.77 & & 0 & 52 & 100 \\
\hline Wastewater Treatment & Copper, total & 6.27 & 10.24 & 2.07 & 3.38 & & 0 & 52 & 100 \\
\hline \multirow[t]{5}{*}{ Facility) } & Cyanide, total & 1.97 & 3.64 & 0.65 & 1.20 & & 0 & 52 & 100 \\
\hline & Downstream pH (SU) & & & & 9.0 & 6.0 & 0 & 249 & 100 \\
\hline & Lead, total & 1.30 & 2.09 & 0.43 & 0.69 & & 0 & 52 & 100 \\
\hline & Nickel, total & 7.21 & 12.06 & 2.38 & 3.98 & & 0 & 52 & 100 \\
\hline & Oil and grease & 30.3 & 45.4 & 10 & 15 & & 0 & 52 & 100 \\
\hline
\end{tabular}


Table 4.92 (continued)

\begin{tabular}{|c|c|c|c|c|c|c|c|c|c|}
\hline \multirow[b]{2}{*}{ Discharge Point } & \multirow[b]{2}{*}{ Effluent parameters } & \multicolumn{5}{|c|}{ Permit Limits } & \multicolumn{3}{|c|}{ Permit Compliance } \\
\hline & & $\begin{array}{l}\text { Monthly } \\
\text { avg } \\
\text { (kg/d) }\end{array}$ & $\begin{array}{l}\text { Daily } \\
\max \\
(\mathrm{kg} / \mathrm{d})\end{array}$ & $\begin{array}{l}\text { Monthly } \\
\text { avg } \\
(\mathrm{mg} / \mathrm{L})\end{array}$ & $\begin{array}{c}\text { Daily } \\
\max \\
(\mathrm{mg} / \mathrm{L})\end{array}$ & $\begin{array}{l}\text { Daily } \\
\text { min } \\
\text { (mg/L) }\end{array}$ & $\begin{array}{l}\text { Number } \\
\text { of } \\
\text { noncompliances }\end{array}$ & $\begin{array}{l}\text { Number } \\
\text { of } \\
\text { samples }\end{array}$ & $\begin{array}{l}\text { Percent } \\
\text { of } \\
\text { compliance }^{a}\end{array}$ \\
\hline \multirow{6}{*}{$\begin{array}{l}\text { X12 } \\
\text { (Nonradiological } \\
\text { Wastewater Treatment } \\
\text { Facility) }\end{array}$} & $\mathrm{pH}(\mathrm{SU})$ & & & & 9.0 & \multirow[t]{6}{*}{6.0} & 0 & $c$ & 100 \\
\hline & Silver, total & 0.73 & 1.30 & 0.24 & 0.43 & & 0 & 52 & 100 \\
\hline & Temperature $\left({ }^{\circ} \mathrm{C}\right)$ & & & & 30.5 & & 0 & 249 & 100 \\
\hline & Total suspended solids & 93.9 & 182 & 31 & 60 & & 0 & 52 & 100 \\
\hline & Total toxic organics & & 6.45 & & 2.13 & & 0 & 52 & 100 \\
\hline & Zinc, total & 4.48 & 7.91 & 1.48 & 2.61 & & 0 & 52 & 100 \\
\hline \multirow[t]{5}{*}{ Category I outfalls ${ }^{d}$} & Downstream pH (SU) & & & & 9.0 & \multirow[t]{2}{*}{6.0} & 0 & 20 & 100 \\
\hline & Oil and grease & & & 10 & 15 & & 0 & 20 & 100 \\
\hline & $\mathrm{pH}(\mathrm{SU})$ & & & & 9.0 & \multirow[t]{3}{*}{6.0} & 0 & 20 & 100 \\
\hline & Temperature $\left({ }^{\circ} \mathrm{C}\right)$ & & & & 30.5 & & 0 & 20 & 100 \\
\hline & Total suspended solids & & & 30 & 50 & & 6 & 20 & 70 \\
\hline \multirow[t]{5}{*}{ Category II outfalls } & Downstream pH (SU) & & & & 9.0 & \multirow[t]{3}{*}{6.0} & 0 & 154 & 100 \\
\hline & Downstream temperature $\left({ }^{\circ} \mathrm{C}\right)^{e}$ & & & & 30.5 & & 0 & 39 & 100 \\
\hline & Oil and grease & & & 10 & 15 & & 4 & 154 & 97 \\
\hline & $\mathrm{pH}(\mathrm{SU})$ & & & & 9.0 & \multirow[t]{2}{*}{6.0} & 0 & 154 & 100 \\
\hline & Total suspended solids & & & 30 & 50 & & 9 & 154 & 94 \\
\hline \multirow[t]{7}{*}{ Cooling Systems } & Chlorine, total residual & & & & 0.2 & & 0 & 38 & 100 \\
\hline & Chromium, total & & & & 1.0 & & 0 & 38 & 100 \\
\hline & Copper, total & & & 0.5 & 1.0 & & 0 & 38 & 100 \\
\hline & Downstream pH (SU) & & & & 9.0 & 6.0 & 0 & 38 & 100 \\
\hline & $\mathrm{pH}(\mathrm{SU})$ & & & & 9.0 & 6.0 & 0 & 38 & 100 \\
\hline & Temperature $\left({ }^{\circ} \mathrm{C}\right)$ & & & 35 & 38 & & 0 & 38 & 100 \\
\hline & Zinc, total & & & 0.5 & 1.0 & & 0 & 38 & 100 \\
\hline
\end{tabular}

${ }^{a}$ Percent compliance $=100-[($ number of noncompliances/number of samples $) * 100]$

${ }^{\circ}$ Colonies per $100 \mathrm{~mL}$.

pill monitoring is continuous.

${ }^{d}$ Category I outfalls are monitored annually by the NPDES Permit year of April 1-March 31.

'Downstream temperature is monitored to check that the stream temperature standards stated in the General Water Quality Criteria for the Definition and Control of Pollution in the Waters of Tennessee, as amended, are not violated as a result of this discharge. 
Table 4.93. ORWL NPDES sampling and andysis plan for the Eacility and ambiant locations

$\begin{array}{lll} & \text { Collection } & \text { Analysis } \\ \text { Analysis } & \text { frequency } & \text { Sample type }\end{array}$

X01 (Sewage Treatment Plant)

Field Measurements

Chlorine, total residual

Dissolved oxygen

$3 /$ week

$5 /$ week

Downstream pH

Flow

$\mathrm{pH}$

Metals

weekly

Daily

weekly

others

Monthly

Ammonia, as $\mathrm{N}$

Biochemical oxygen demand

Cyanide, total

Fecal coliform

Oil and grease

Phenolics, total recoverable

Total suspended solids

$3 /$ week

3 /week

Monthly

$3 /$ week

$3 /$ week

Monthly

volatile organics

3 /week

Monthly

Grab, instant read
Grab, instant read
Grab, instant read
Continuous
Grab, instant read
$24-h$ composite
$24-h$ composite
$24-h$ composite
Grab
Grab
Grab
Grab
$24-h$ composite
Grab

$3 /$ week

$5 /$ week

Weekly

Daily

Weekly

Montily

$3 /$ week

$3 /$ week

Monthly

$3 /$ week

$3 /$ week

Monthly

3 /week

Monthly

X02 (Coal Yard Runoff Treatment Facility)

Arions

Field Measurements

Downstream pH

Flow

$\mathrm{pH}$

Temperature

Metals

Others

Oil and grease

Total suspended solids
NonthIy

Weekly

Daily

weekly

weekly

Week.y

weekly

Weekly 24-h composite

Grab, instant read

Continuous

Grab, instant read

Grab, instant read

24-h composite

Grab

24-h composite
Monthly

Weekly

Daily

weekly

Weekly

weekly

$3 /$ week

$3 /$ week

\section{XI2 (Nonradiological Wastewater Treatment Facility)}

Arions

Field Measurements

Downstream $\mathrm{pH}$

Flow

$\mathrm{pH}$

Temperature

Metals

Others

Biochemical oxygen demand

Cyanide, total

oil and grease

Phenolics, total recoverable

Total suspended solids

Total toxic organics

Volatile organics
WeekIy

Daily

Daily

Continuous

weekly

Weekly

Weekly

Weekly

weekly

weekly

weekly

weekly

weekly 24-h composite

Grab, instant read

Continuous

Continuous

Grab, instant read

24-h composite

24-h composite

Grab

Grab

Grab

24-h composite

Grab

Grab
Weekly

Daily

Daily

Continuous

weekly

weekly

Weekly

weekly

Weekly

weekly

weekly

Weekly

Weekly 
Table 4.93 (continued)

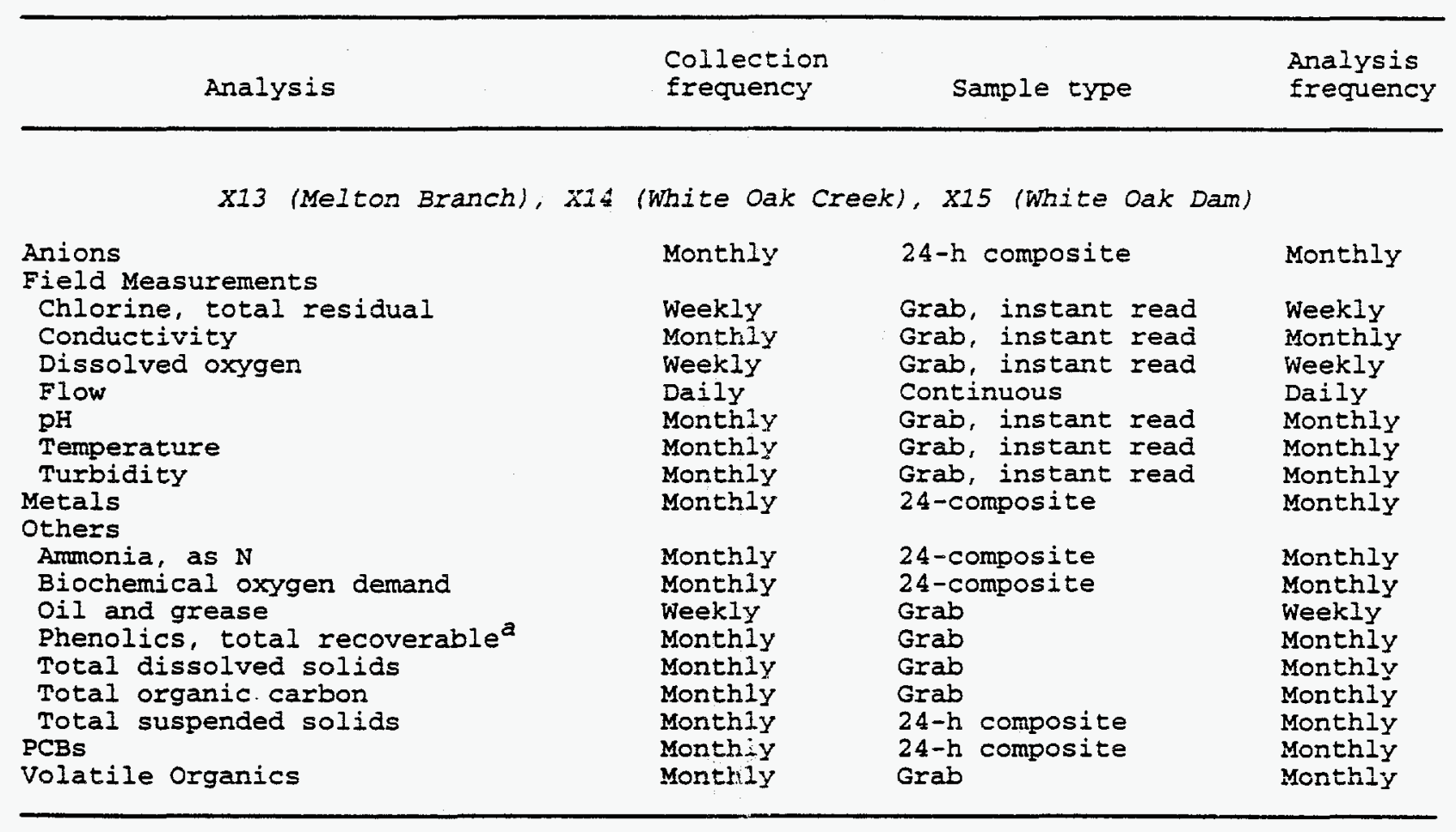

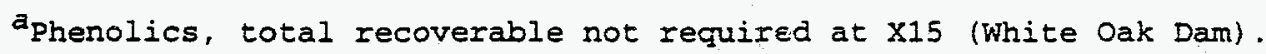


Table 4.94. ORAL NPDES sampling and analysis plan for the category outfalls and cooling systems

\begin{tabular}{|c|c|c|}
\hline Analysis & $\begin{array}{l}\text { Collection } \\
\text { frequency }\end{array}$ & Sample type \\
\hline
\end{tabular}

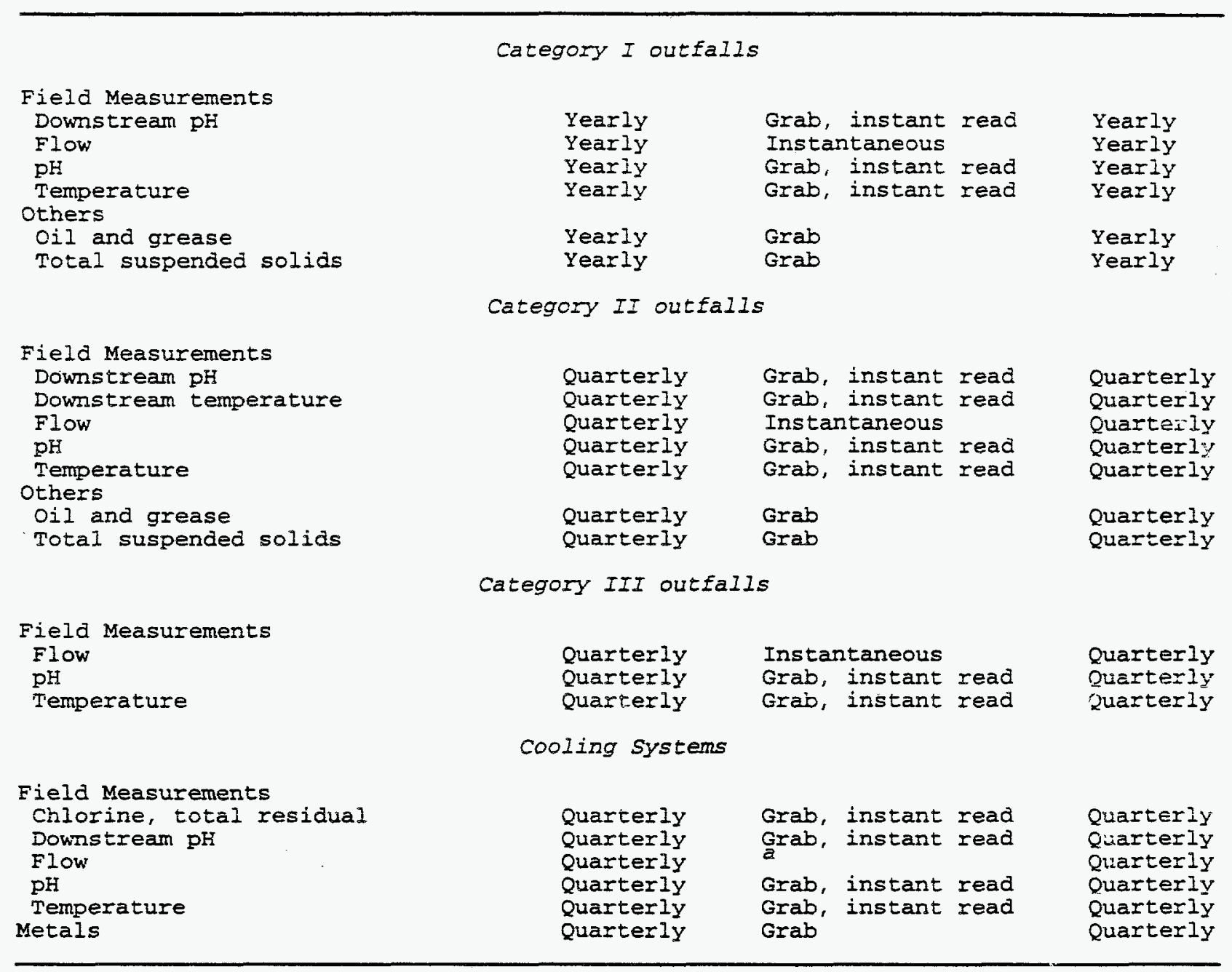

$a_{\text {Not applicable. }}$ 
Table 4.95. NPDES Permit Number TN 0002941, 1993,

ORNL discharge point XO1 (Sewage Treatment Plant)

Flow rates $\left(1 \times 10^{6} \mathrm{~L} / d\right)$-Max: 2.0 , Min: 0.051 , Avg: 0.92

\begin{tabular}{|c|c|c|c|c|c|}
\hline \multirow[b]{2}{*}{ Parameter } & \multirow[b]{2}{*}{$\begin{array}{l}N \text { det } \\
N \text { total }\end{array}$} & \multicolumn{4}{|c|}{ Concentration ( $\mathrm{mg} / \mathrm{L}$ ) } \\
\hline & & $\operatorname{Max}^{a}$ & $\operatorname{Min}^{2}$ & $A v^{b}$ & $\begin{array}{l}\text { Stancard } \\
\text { error }\end{array}$ \\
\hline $\begin{array}{l}\text { Field Measurements } \\
\text { Chlorine, total residual } \\
\text { Dissolved oxygen } \\
\text { Downstream pH (SU) } \\
\text { pH (SU) }\end{array}$ & $\begin{array}{c}155 / 156 \\
249 / 249 \\
52 / 52 \\
52 / 52\end{array}$ & $\begin{array}{r}0.39 \\
13 \\
8.0 \\
7.8\end{array}$ & $\begin{array}{l}0.050 \\
7.1 \\
7.2 \\
7.0\end{array}$ & $\begin{array}{l}0.19 \\
9.0 \\
7.6 \\
7.3\end{array}$ & $\begin{array}{l}0.0064 \\
0.070 \\
0.019 \\
0.022\end{array}$ \\
\hline $\begin{array}{l}\text { Metals } \\
\text { Copper, total } \\
\text { Mercury, total } \\
\text { Silver, total } \\
\text { Zinc, total }\end{array}$ & $\begin{array}{r}0 / 12 \\
2 / 12 \\
0 / 12 \\
12 / 12\end{array}$ & $\begin{array}{l}<0.0070 \\
0.000088 \\
<0.0050 \\
0.15\end{array}$ & $\begin{array}{l}<0.0070 \\
<0.000050 \\
<0.0050 \\
0.028\end{array}$ & $\begin{array}{l}\sim 0.0070 \\
-0.000056 \\
\sim 0.0050 \\
0.066\end{array}$ & $\begin{array}{l}0 \\
0.0000042 \\
0 \\
0.0085\end{array}$ \\
\hline $\begin{array}{l}\text { Others } \\
\text { Ammonia, as } \mathrm{N} \\
\text { Biochemical oxygen demand } \\
\text { Cyanide, total } \\
\text { Fecal coliform (col/100 mL) } \\
\text { Oil and grease } \\
\text { Phenolics, total recoverable } \\
\text { Total suspended solids }\end{array}$ & $\begin{array}{c}156 / 156 \\
3 / 156 \\
0 / 12 \\
101 / 156 \\
28 / 156 \\
0 / 12 \\
7 / 156\end{array}$ & $\begin{aligned} & 2.0 \\
& 6.0 \\
&<0.0020 \\
& 3,600 \\
& 9.0 \\
&<0.0010 \\
& 50\end{aligned}$ & $\begin{aligned} & 0.030 \\
&<5.0<0 \\
&<0.0020 \\
&<1.0 \\
&<2.0 \\
&<0.0010 \\
& 1.0\end{aligned}$ & $\begin{array}{l}0.26 \\
-5.0 \\
-0.0020 \\
-3.8 \\
-2.3 \\
\sim 0.0010 \\
-5.3\end{array}$ & $\begin{array}{l}0.023 \\
0.0090 \\
0 \\
1.2 \\
0.080 \\
0 \\
0.29\end{array}$ \\
\hline $\begin{array}{l}\text { Volatile organics } \\
\text { Bromodichloromethane } \\
\text { Trichloroethene }\end{array}$ & $\begin{array}{l}0 / 12 \\
0 / 12\end{array}$ & $\begin{array}{l}\text { U0.0050 } \\
\text { U0.0050 }\end{array}$ & $\begin{array}{l}\text { UO. } 0050 \\
\text { Uo. } 0050\end{array}$ & $\begin{array}{l}-0.0050 \\
-0.0050\end{array}$ & $\begin{array}{l}0 \\
0\end{array}$ \\
\hline
\end{tabular}

aprefix "<" indicates the value for a parameter (excluding organics) was not quantifiable at the analytical detection limit; and "U" indicates the value for an organic parameter was undetected at the analytical detection limit.

$b_{A}$ tilde $(\sim)$ indicates that estimated values and/or detection limits were used in the calculation.

$c_{\text {Standard error of the mean. }}$

The geometric mean is computed rather than the average. 
Table 4.96. NPDES Permit Nurber TN 0002941, 1993,

ORNL discharge point XO2 (COal Yard Runoff rreatment Facility)

Flow rates $\left(1 \times 10^{i} \mathrm{~L} / \bar{a}\right)$-Max: 3.3. Min: 0, Avg: 0.057

\begin{tabular}{|c|c|c|c|c|c|}
\hline \multirow[b]{2}{*}{ Parameter } & \multirow[b]{2}{*}{$\begin{array}{l}\mathrm{N} \text { det } / \\
\mathrm{N} \text { total }\end{array}$} & \multicolumn{4}{|c|}{ Concentration (mg/L) } \\
\hline & & $\operatorname{Max}^{a}$ & $\operatorname{Min}^{a}$ & $A v^{b}$ & $\begin{array}{l}\text { Standard } \\
\text { error }\end{array}$ \\
\hline $\begin{array}{l}\text { Anions } \\
\text { Sulfate, as } \mathrm{SO}_{4}\end{array}$ & $12 / 12$ & 2,100 & 67 & 1,300 & 190 \\
\hline $\begin{array}{l}\text { Field Measurements } \\
\text { Downstream pH (SU) } \\
\text { pHi (SU) } \\
\text { Temperature }\left({ }^{\circ} \mathrm{C}\right)\end{array}$ & $\begin{array}{l}248 / 248 \\
248 / 248 \\
248 / 248\end{array}$ & $\begin{array}{l}8.2 \\
8.9 \\
30\end{array}$ & $\begin{array}{l}7.1 \\
6.8 \\
2.9\end{array}$ & $\begin{array}{r}7.8 \\
7.6\end{array}$ & $\begin{array}{l}0.012 \\
0.028 \\
0.50\end{array}$ \\
\hline $\begin{array}{l}\text { Metals } \\
\text { Arsenic, total } \\
\text { Cadmium, total } \\
\text { Chromium, total } \\
\text { Copper, total } \\
\text { Iron, total } \\
\text { Lead, total } \\
\text { Manganese, total } \\
\text { Nickel, total } \\
\text { Selenium, total } \\
\text { Silver, total } \\
\text { Zinc, total }\end{array}$ & $\begin{array}{r}5 / 47 \\
0 / 47 \\
12 / 47 \\
5 / 47 \\
45 / 47 \\
1 / 47 \\
46 / 47 \\
1 / 47 \\
12 / 47 \\
0 / 47 \\
31 / 47\end{array}$ & $\begin{array}{l}0.29 \\
<0.0050 \\
0.020 \\
0.0095 \\
0.88 \\
0.051 \\
0.17 \\
0.016 \\
0.10 \\
<0.0050 \\
0.082\end{array}$ & $\begin{array}{l}<0.050 \\
<0.0050 \\
<0.0040 \\
<0.0070 \\
<0.050 \\
<0.050 \\
<0.0010 \\
<0.010 \\
<0.050 \\
<0.0050 \\
<0.0050\end{array}$ & $\begin{array}{l}-0.064 \\
-0.0050 \\
-0.0064 \\
-0.0071 \\
-0.29 \\
-0.050 \\
-0.039 \\
-0.010 \\
-0.057 \\
-0.0050 \\
-0.013\end{array}$ & $\begin{array}{l}0.0066 \\
0 \\
0.00071 \\
0.000069 \\
0.024 \\
0.000021 \\
0.0057 \\
0.00013 \\
0.0022 \\
0 \\
0.0020\end{array}$ \\
\hline $\begin{array}{l}\text { Others } \\
\text { Oil and grease } \\
\text { Total suspended solids }\end{array}$ & $\begin{array}{l}11 / 47 \\
24 / 47\end{array}$ & $\begin{array}{l}13 \\
69\end{array}$ & $\begin{array}{l}<2.0 \\
<5.0\end{array}$ & $\begin{array}{l}-3.1 \\
-7.6\end{array}$ & $\begin{array}{l}0.39 \\
1.4\end{array}$ \\
\hline
\end{tabular}

$a_{\text {prefix }}<$ " indicates the value for a parameter (excluding organios) was not quantifiable at the analytical detection limit.

$b_{\mathrm{A}}$ tilde $(-)$ indicates that estimated values and/or detection Iimits were used in the calculation.

$c_{\text {Standard error of the mean. }}$ 
Table 4.97. NPDES Permit Number TN 0002941, 1993, oRrL discharge point X12 (Nonradiological wastewater rreatment Facility)

Flow rates $\left(1 \times 10^{6} \mathrm{~L} / \mathrm{d}\right)-\operatorname{Max}: 2.9, \mathrm{Min}: 1.3$, Avg: 1.9

\begin{tabular}{|c|c|c|c|c|c|}
\hline \multirow[b]{2}{*}{ Parameter } & \multirow[b]{2}{*}{$\begin{array}{l}\mathrm{N} \text { det } / \\
\mathrm{N} \text { total }\end{array}$} & \multicolumn{4}{|c|}{ Concentration (mg/L) } \\
\hline & & $\operatorname{Max}^{a}$ & $\operatorname{Min}^{a}$ & $A v^{b}$ & $\begin{array}{c}\text { Standard } \\
\text { error }\end{array}$ \\
\hline $\begin{array}{l}\text { Anions } \\
\text { Fluoride } \\
\text { Nitrate, as } \mathrm{N} \\
\text { Sulfate, as } \mathrm{SO}_{4}\end{array}$ & $\begin{array}{l}52 / 52 \\
41 / 45 \\
52 / 52\end{array}$ & $\begin{array}{l}2.2 \\
19 \\
330\end{array}$ & $\begin{array}{l}0.50 \\
<1.0 \\
18\end{array}$ & $\begin{array}{r}1.3 \\
-4 \cdot 3 \\
200\end{array}$ & $\begin{array}{l}0.049 \\
0.58 \\
7.8\end{array}$ \\
\hline $\begin{array}{l}\text { Field Measurements } \\
\text { Downstream pH (SU) } \\
\text { pH (SU) } \\
\text { Temperature }\left({ }^{\circ} \mathrm{C}\right)\end{array}$ & $\begin{array}{c}249 / 249 \\
d \\
249 / 249\end{array}$ & $\begin{array}{r}8.4 \\
7.6 \\
27\end{array}$ & $\begin{array}{r}7.1 \\
7 \cdot 1 \\
10\end{array}$ & $\begin{array}{r}7.8 \\
7.4 \\
19\end{array}$ & $\begin{array}{l}0.014 \\
0.0064 \\
0.27\end{array}$ \\
\hline $\begin{array}{l}\text { Metals } \\
\text { Arsenic, total } \\
\text { Cadmium, total } \\
\text { Chromium, total } \\
\text { Copper, total } \\
\text { Iron, total } \\
\text { Lead, total } \\
\text { Mercury, total } \\
\text { Nickel, total } \\
\text { Phosphorus, total } \\
\text { Selenium, total } \\
\text { Silver, total } \\
\text { zinc, total }\end{array}$ & $\begin{array}{r}8 / 52 \\
2 / 52 \\
31 / 52 \\
29 / 52 \\
19 / 52 \\
16 / 52 \\
3 / 52 \\
17 / 52 \\
44 / 52 \\
11 / 52 \\
1 / 52 \\
51 / 52\end{array}$ & $\begin{array}{l}<0.050 \\
<0.0050 \\
0.018 \\
0.025 \\
0.26 \\
0.068 \\
0.00034 \\
<0.010 \\
0.48 \\
<0.050 \\
0.0090 \\
0.13\end{array}$ & $\begin{array}{l}<0.00050 \\
<0.00050 \\
<0.00050 \\
<0.00050 \\
<0.050 \\
<0.00050 \\
<0.000050 \\
<0.00050 \\
0.070 \\
<0.00050 \\
<0.00050 \\
0.0032\end{array}$ & $\begin{array}{l}\sim 0.033 \\
\sim 0.0035 \\
\sim 0.0058 \\
-0.0097 \\
\sim 0.068 \\
\sim 0.034 \\
\sim 0.000056 \\
\sim 0.0075 \\
\sim 0.28 \\
\sim 0.033 \\
-0.0035 \\
\sim 0.027\end{array}$ & $\begin{array}{l}0.0033 \\
0.00030 \\
0.00054 \\
0.00064 \\
0.0051 \\
0.0032 \\
0.0000056 \\
0.00051 \\
0.013 \\
0.0032 \\
0.00032 \\
0.0024\end{array}$ \\
\hline $\begin{array}{l}\text { Others } \\
\text { Biochemical oxygen demand } \\
\text { Cyanide, total } \\
\text { Oil and grease } \\
\text { Phenolics, total recoverable } \\
\text { Total suspended solids } \\
\text { Total toxic organics }\end{array}$ & $\begin{array}{r}0 / 52 \\
2 / 52 \\
10 / 52 \\
0 / 52 \\
0 / 52 \\
2 / 52\end{array}$ & $\begin{array}{l}<5.0 \\
0.0060 \\
6.0 \\
<0.0010 \\
<5.0 \\
\text { B0.041 }\end{array}$ & $\begin{array}{l}<5.0 \\
<0.0020 \\
<2.0 \\
<0.0010 \\
<5.0 \\
<0.010\end{array}$ & $\begin{array}{l}-5.0 \\
-0.0021 \\
-2.3 \\
-0.0010 \\
-5.0 \\
-0.011\end{array}$ & $\begin{array}{l}0 \\
0.000077 \\
0.12 \\
0 \\
0 \\
0.00060\end{array}$ \\
\hline $\begin{array}{l}\text { Volatile Organics } \\
\text { 1,1-Dichloroethane } \\
\text { Benzene } \\
\text { Bromodichloromethane } \\
\text { Chlorobenzene } \\
\text { Chloroform } \\
\text { Methylene chloride } \\
\text { Tetrachloroethene } \\
\text { Trichloroethene }\end{array}$ & $\begin{array}{l}0 / 52 \\
0 / 52 \\
0 / 52 \\
0 / 52 \\
1 / 52 \\
1 / 52 \\
0 / 52 \\
0 / 52\end{array}$ & $\begin{array}{l}\text { UO. } 0050 \\
\text { UO. } 0050 \\
\text { U0.0050 } \\
\text { UO. } 0050 \\
\text { UO. } 0050 \\
\text { UO. } 0050 \\
\text { UO. } 0050 \\
\text { UO. } 0050\end{array}$ & $\begin{array}{l}\text { UO. } 0050 \\
\text { U0.0050 } \\
\text { UO. } 0050 \\
\text { UO. } 0050 \\
\text { Jo.0024 } \\
\text { Jo.0024 } \\
\text { UO. } 0050 \\
\text { UO. } 0050\end{array}$ & $\begin{array}{l}\sim 0.0050 \\
\sim 0.0050 \\
\sim 0.0050 \\
\sim 0.0050 \\
\sim 0.0050 \\
\sim 0.0050 \\
\sim 0.0050 \\
\sim 0.0050\end{array}$ & $\begin{array}{l}0 \\
0 \\
0 \\
0 \\
0.000050 \\
0.000050 \\
0 \\
0\end{array}$ \\
\hline
\end{tabular}

aprefix "<" indicates the value for a parameter (excluding organics) was not quantifiable at the analytical detection limit; "B" indicates the compound was found in the laboratory blank; " $U$ " indicates the value for an organic parameter was undetected at the analytical detection limit; and "J" indicates the value was estimated at or below the analytical detection limit by the laboratory.

$b_{A}$ tilde ( ) indicates that estimated values and/or detection limits were used in the calculation.

$c_{\text {Standard error of the mean. }}$

$d_{\mathrm{pH}}$ monitoring is continuous. 
Table 4.98. NPDEs zermtt Number 2 0002941, 1993, ORfL discherge potat Xi3 (Melton Branch)

Elow rates $\left(1 \times 10^{6} \mathrm{~L} / \mathrm{d}\right)$-Max: 190, Min: 0.26 , Avg: 6.6

\begin{tabular}{|c|c|c|c|c|c|}
\hline \multirow[b]{2}{*}{ Parameter } & \multirow[b]{2}{*}{$\begin{array}{l}\operatorname{det} / \\
\text { total }\end{array}$} & \multicolumn{4}{|c|}{ Concentration (mg/I) } \\
\hline & & $\operatorname{Max}^{2}$ & $\operatorname{Min}^{a}$ & $A V^{b}$ & $\begin{array}{l}\text { Standard } \\
\text { error }\end{array}$ \\
\hline $\begin{array}{l}\text { Anions } \\
\text { Fluoride } \\
\text { Nitrate, as } \mathrm{N} \\
\text { Sulfate, as } \mathrm{SO}_{4}\end{array}$ & $\begin{array}{r}12 / 12 \\
2 / 12 \\
12 / 12\end{array}$ & $\begin{array}{r}2.7 \\
1.5 \\
310\end{array}$ & $\begin{array}{l}0.20 \\
<1.0 \\
18\end{array}$ & $\begin{array}{r}\frac{1}{1} \cdot 1 \\
110\end{array}$ & $\begin{array}{l}0.22 \\
0.047 \\
28\end{array}$ \\
\hline $\begin{array}{l}\text { Field Measurements } \\
\text { Chlorine, total residual } \\
\text { conductivity (ms/cm) } \\
\text { Dissolved oxygen } \\
\text { pH (SU) } \\
\text { Temperature (o }) \\
\text { Turbidity (NTU) }\end{array}$ & $\begin{array}{r}0 / 52 \\
12 / 12 \\
52 / 52 \\
13 / 13 \\
65 / 65 \\
12 / 12\end{array}$ & $\begin{array}{c}<0.010 \\
0.85 \\
14 \\
8.0 \\
25 \\
29\end{array}$ & $\begin{array}{l}<0.010 \\
0.17 \\
6.0 \\
7.1 \\
4.3 \\
2.0\end{array}$ & $\begin{array}{l}-0.010 \\
0.49 \\
9.2 \\
7.6 \\
14 \\
13\end{array}$ & $\begin{array}{l}0 \\
0.058 \\
0.27 \\
0.072 \\
0.83 \\
2.2\end{array}$ \\
\hline $\begin{array}{l}\text { Metals } \\
\text { Aluminum, total } \\
\text { Arsenic, total } \\
\text { Cacmium, total } \\
\text { Chromium, total } \\
\text { Copper, total } \\
\text { Iron, total } \\
\text { Lead, total } \\
\text { Manganese, total } \\
\text { Mercury, total } \\
\text { Nickel, total } \\
\text { Phosphorus, total } \\
\text { Silver, total } \\
\text { Zinc, total }\end{array}$ & $\begin{array}{r}12 / 12 \\
0 / 12 \\
0 / 12 \\
3 / 12 \\
4 / 12 \\
12 / 12 \\
0 / 12 \\
12 / 12 \\
0 / 12 \\
0 / 12 \\
11 / 12 \\
0 / 12 \\
12 / 12\end{array}$ & $\begin{array}{l}2.2 \\
<0.050 \\
<0.0050 \\
0.020 \\
0.017 \\
2.3 \\
<0.050 \\
0.25 \\
<0.000050 \\
<0.010 \\
1.2 \\
<0.0050 \\
0.11\end{array}$ & $\begin{array}{l}0.084 \\
<0.050 \\
<0.0020 \\
<0.0040 \\
<0.0070 \\
0.16 \\
<0.0040 \\
0.070 \\
<0.000050 \\
<0.010 \\
<0.20 \\
<0.0050 \\
0.016\end{array}$ & $\begin{array}{l}0.73 \\
-0.050 \\
-0.0048 \\
-0.0067 \\
-0.0082 \\
0.71 \\
-0.046 \\
0.15 \\
-0.000050 \\
-0.010 \\
-0.57 \\
-0.0050 \\
0.056\end{array}$ & $\begin{array}{l}0.15 \\
0 \\
0.00025 \\
0.0015 \\
0.00086 \\
0.17 \\
0.0038 \\
0.016 \\
0 \\
0 \\
0.097 \\
0 \\
0.0091\end{array}$ \\
\hline $\begin{array}{l}\text { Others } \\
\text { Ammonia, as } \mathrm{N} \\
\text { Biochemical oxygen demand } \\
\text { Oil and grease } \\
\text { Phenolics, total recoverable } \\
\text { Total dissolved solids } \\
\text { Total organic carbon } \\
\text { Total suspended solids }\end{array}$ & $\begin{array}{r}13 / 13 \\
0 / 12 \\
17 / 52 \\
0 / 12 \\
12 / 12 \\
12 / 12 \\
10 / 12\end{array}$ & $\begin{array}{l}0.11 \\
<5.0 \\
17 \\
<0.0010 \\
790 \\
4.0 \\
89\end{array}$ & $\begin{aligned} & 0.030 \\
< & 5.0 \\
< & 2.0 \\
< & 0.0010 \\
140 & \\
& 2.0 \\
< & 5.0\end{aligned}$ & $\begin{array}{l}0.052 \\
-5.0 \\
-3.2 \\
-0.0010 \\
-310 \\
2.8 \\
-30\end{array}$ & $\begin{array}{l}0.0060 \\
0 \\
0.43 \\
0 \\
54 \\
0.19 \\
7.5\end{array}$ \\
\hline $\begin{array}{l}\text { PCBs } \\
\text { Total Aroclors }\end{array}$ & $0 / 12$ & 00.0020 & 00.0020 & -0.0020 & 0 \\
\hline $\begin{array}{l}\text { Volatile organics } \\
\text { Chloroform } \\
\text { Trichloroethene }\end{array}$ & $\begin{array}{l}0 / 12 \\
0 / 12\end{array}$ & $\begin{array}{l}\text { U0.0050 } \\
\text { UO.0050 }\end{array}$ & $\begin{array}{l}\text { U0.0050 } \\
\text { U0.0050 }\end{array}$ & $\begin{array}{l}-0.0050 \\
-0.0050\end{array}$ & $\begin{array}{l}0 \\
0\end{array}$ \\
\hline
\end{tabular}

aprefix "<" indicates the value for a parameter (excluding organics) was not quantifiable at the analytical detection limit; and "U" indicates the value for an organic parameter was undetected at the analytical detection 1 imit.

$b_{A}$ tilde ( ) indicates that estimated values and/or detection limits were used in the calculation.

cstandard error of the mean. 
Table 4.99. NTPDES Permit Number TN 0002941, 1993, ORNL discharge point X14 (White oak Creek)

Flow rates $\left(1 \times 10^{6} \mathrm{~L} / \mathrm{d}\right)-\operatorname{Max}: 320$, Min: 7.9 , Avg: 20

Concentration (mg/L)

\begin{tabular}{|c|c|c|c|c|c|}
\hline \multirow[b]{2}{*}{ Parameter } & \multirow[b]{2}{*}{$\begin{array}{l}\mathrm{N} \text { det } / \\
\mathrm{N} \text { total }\end{array}$} & \\
\hline & & $\operatorname{Max}^{2}$ & $\operatorname{Min}^{a}$ & $A V^{b}$ & $\begin{array}{l}\text { Standard } \\
\text { error }\end{array}$ \\
\hline $\begin{array}{l}\text { Anions } \\
\text { Fluoride } \\
\text { Nitrate, as } \mathrm{N} \\
\text { Sulfate, as } \mathrm{SO}_{4}\end{array}$ & $\begin{array}{r}12 / 12 \\
7 / 12 \\
12 / 12\end{array}$ & $\begin{array}{r}1.2 \\
2.3 \\
87\end{array}$ & $\begin{array}{l}0.20 \\
<1.0 \\
17\end{array}$ & $\begin{array}{l}0.71 \\
-1.3 \\
51\end{array}$ & $\begin{array}{l}0.089 \\
0.11 \\
5.7\end{array}$ \\
\hline $\begin{array}{l}\text { Field Measurements } \\
\text { Chlorine, total residual } \\
\text { Conductivity (ms/cm) } \\
\text { Dissolved oxygen } \\
\text { pH (SU) } \\
\text { Temperature }\left({ }^{\circ} \mathrm{C}\right) \\
\text { Turbidity (NTU) }\end{array}$ & $\begin{array}{r}0 / 52 \\
12 / 12 \\
52 / 52 \\
13 / 13 \\
65 / 65 \\
12 / 12\end{array}$ & $\begin{array}{l}<0.010 \\
0.51 \\
12 \\
8.2 \\
25 \\
24\end{array}$ & $\begin{array}{c}<0.010 \\
0.20 \\
7.1 \\
7.3 \\
8.8 \\
2.0\end{array}$ & $\begin{array}{c}-0.010 \\
0.37 \\
9.2 \\
7.8 \\
16 \\
8.8\end{array}$ & $\begin{array}{l}0 \\
0.023 \\
0.18 \\
0.062 \\
0.63 \\
2.0\end{array}$ \\
\hline $\begin{array}{l}\text { Metals } \\
\text { Aluminum, total } \\
\text { Arsenic, total } \\
\text { Cadmium, total } \\
\text { Chromium, total } \\
\text { Copper, total } \\
\text { Iron, total } \\
\text { Lead, total } \\
\text { Manganese, total } \\
\text { Mercury, total } \\
\text { Nickel, total } \\
\text { Phosphorus, total } \\
\text { Silver, total } \\
\text { Zinc, total }\end{array}$ & $\begin{array}{r}10 / 12 \\
0 / 12 \\
0 / 12 \\
3 / 12 \\
2 / 12 \\
11 / 12 \\
0 / 12 \\
12 / 12 \\
0 / 12 \\
0 / 12 \\
9 / 12 \\
0 / 12 \\
12 / 12\end{array}$ & $\begin{aligned} & 1.2 \\
&<0.050 \\
&<0.0050 \\
& 0.013 \\
& 0.014 \\
& 1.3 \\
&<0.050 \\
& 0.093 \\
&<0.000050 \\
&<0.010 \\
& 0.50 \\
&<0.0050 \\
& 0.091\end{aligned}$ & $\begin{array}{l}<0.050 \\
<0.050 \\
<0.0020 \\
<0.0040 \\
<0.0070 \\
<0.050 \\
<0.0040 \\
0.0033 \\
<0.000050 \\
<0.010 \\
<0.20 \\
<0.0050 \\
0.019\end{array}$ & $\begin{array}{l}-0.26 \\
-0.050 \\
-0.0048 \\
-0.0059 \\
-0.0076 \\
-0.29 \\
-0.046 \\
0.035 \\
-0.000050 \\
-0.010 \\
-0.36 \\
-0.0050 \\
0.041\end{array}$ & $\begin{array}{l}0.10 \\
0 \\
0.00025 \\
0.0010 \\
0.00058 \\
0.12 \\
0.0038 \\
0.0080 \\
0 \\
0 \\
0.038 \\
0 \\
0.0058\end{array}$ \\
\hline $\begin{array}{l}\text { Others } \\
\text { Ammonia, as } \mathrm{N} \\
\text { Biochemical oxygen demand } \\
\text { Oil and grease } \\
\text { Phenolics, total recoverable } \\
\text { Total dissolved solids } \\
\text { Total organic carbon } \\
\text { Total suspended solids }\end{array}$ & $\begin{array}{r}13 / 13 \\
0 / 12 \\
14 / 52 \\
0 / 12 \\
12 / 12 \\
12 / 12 \\
4 / 12\end{array}$ & $\begin{array}{l}0.080 \\
<5.0 \\
11 \\
<0.0010 \\
310 \\
3.5 \\
43\end{array}$ & $\begin{aligned} & 0.020 \\
&<5.0 \\
&<2.0 \\
&<0.0010 \\
& 140 \\
& 1.3 \\
&<5.0\end{aligned}$ & $\begin{array}{l}0.050 \\
-5.0 \\
-2.7 \\
-0.0010 \\
-230 \\
-11.8\end{array}$ & $\begin{array}{l}0.0044 \\
0 \\
0.26 \\
0 \\
16 \\
0.17 \\
3.5\end{array}$ \\
\hline $\begin{array}{l}\text { PCBs } \\
\text { Total Aroclors }\end{array}$ & $0 / 12$ & 00.0020 & $\mathrm{UO} .0020$ & -0.0020 & 0 \\
\hline $\begin{array}{l}\text { Volatile organics } \\
\text { Chloroform } \\
\text { Trichloroethene }\end{array}$ & $\begin{array}{l}7 / 12 \\
0 / 12\end{array}$ & $\begin{array}{l}\text { Uo. } 0050 \\
\text { Uo.0050 }\end{array}$ & $\begin{array}{l}\text { Jo. } 0010 \\
\text { UO. } 0050\end{array}$ & $\begin{array}{l}-0.0030 \\
-0.0050\end{array}$ & $\begin{array}{l}0.00052 \\
0\end{array}$ \\
\hline
\end{tabular}

"Prefix " $<$ " indicates the value for a parameter (excluding organics) was not quantifiable at the analytical detection limit; "U" indicates the value for an organic parameter was undetected at the analytical detection limit; and " $J$ " indicates the value estimated at or below the analytical detection limit by the laboratory.

$b_{A}$ tilde (-) indicates that estimated values and/or detection limits were used in the calculation.

$c_{\text {Standard error of the mean. }}$ 
Table 4.100. NPDES Parmit Nember IN 0002941, 1993, ORNL discharge point X15 (White oak Dan)

Flow rates $\left(1 \times 10^{6} \mathrm{I} / \mathrm{d}\right)-\mathrm{Max}: 340$, Min: $9.4, \mathrm{Avg}: 29$

\begin{tabular}{|c|c|c|c|c|c|}
\hline \multirow[b]{2}{*}{ Parameter } & \multirow[b]{2}{*}{$\begin{array}{l}\mathrm{N} \text { det } / \\
\mathrm{N} \text { total }\end{array}$} & \multicolumn{4}{|c|}{ Concentration (mg/L) } \\
\hline & & $\operatorname{Max}^{a}$ & $\operatorname{Min}^{a}$ & $A V^{b}$ & $\begin{array}{c}\text { Standard } \\
\text { error }\end{array}$ \\
\hline $\begin{array}{l}\text { Anions } \\
\text { Fluoride } \\
\text { Nitrate, as } \mathrm{N} \\
\text { Sulfate, as } \mathrm{SO}_{4}\end{array}$ & $\begin{array}{r}12 / 12 \\
2 / 12 \\
12 / 12\end{array}$ & $\begin{array}{r}1.2 \\
1.2 \\
74\end{array}$ & $\begin{aligned} & 0.20 \\
&< 1.0 \\
& 20\end{aligned}$ & $\begin{aligned} & 0.65 \\
&- 1.0 \\
& 48\end{aligned}$ & $\begin{array}{l}0.087 \\
0.022 \\
5.6\end{array}$ \\
\hline $\begin{array}{l}\text { Field Measurements } \\
\text { Chlorine, total residual } \\
\text { Conductivity (ms/cm) } \\
\text { Dissolved oxygen } \\
\text { pH (SU) } \\
\left.\text { Temperature ( }{ }^{\circ} \mathrm{C}\right) \\
\text { Turbidity (NTU) }\end{array}$ & $\begin{array}{r}1 / 52 \\
12 / 12 \\
52 / 52 \\
12 / 12 \\
64 / 64 \\
12 / 12\end{array}$ & $\begin{array}{l}0.040 \\
0.42 \\
14 \\
8.8 \\
30 \\
32\end{array}$ & $\begin{array}{l}<0.010 \\
0.20 \\
6.3 \\
7.4 \\
5.8 \\
8.0\end{array}$ & $\begin{array}{l}-0.011 \\
0.35 \\
9.0 \\
8.0 \\
17 \\
17\end{array}$ & $\begin{array}{l}0.00058 \\
0.018 \\
0.25 \\
0.12 \\
0.99 \\
1.9\end{array}$ \\
\hline $\begin{array}{l}\text { Metals } \\
\text { Aluminum, total } \\
\text { Arsenic, total } \\
\text { Cadmium, total } \\
\text { Chromium, total } \\
\text { Copper, total } \\
\text { Iron, total } \\
\text { Lead, total } \\
\text { Manganese, total } \\
\text { Mercury, total } \\
\text { Nickel, total } \\
\text { Phosphorus, total } \\
\text { Silver, total } \\
\text { Zinc, total }\end{array}$ & $\begin{array}{r}12 / 12 \\
0 / 12 \\
0 / 12 \\
10 / 12 \\
1 / 12 \\
12 / 12 \\
0 / 12 \\
12 / 12 \\
1 / 12 \\
1 / 12 \\
8 / 12 \\
0 / 12 \\
11 / 12\end{array}$ & $\begin{array}{l}1.9 \\
<0.050 \\
<0.0050 \\
0.025 \\
0.0084 \\
2.0 \\
<0.050 \\
0.18 \\
0.00042 \\
0.010 \\
0.52 \\
<0.0050 \\
0.059\end{array}$ & $\begin{array}{l}0.22 \\
<0.050 \\
<0.0020 \\
<0.0040 \\
<0.0070 \\
0.30 \\
<0.0040 \\
0.035 \\
<0.000050 \\
<0.010 \\
<0.20 \\
<0.0050 \\
<0.0050\end{array}$ & $\begin{array}{l}0.85 \\
-0.050 \\
-0.0048 \\
-0.013 \\
-0.0071 \\
0.87 \\
-0.046 \\
0.10 \\
-0.000081 \\
-0.010 \\
-0.33 \\
-0.0050 \\
-0.027\end{array}$ & $\begin{array}{l}0.18 \\
0 \\
0.00025 \\
0.0022 \\
0.00012 \\
0.16 \\
0.0038 \\
0.015 \\
0.000031 \\
0 \\
0.032 \\
0 \\
0.0046\end{array}$ \\
\hline $\begin{array}{l}\text { Others } \\
\text { Ammonia, as } \mathrm{N} \\
\text { Biochemical oxygen demand } \\
\text { Oil and grease } \\
\text { Total dissolved solias } \\
\text { Total organic carbon } \\
\text { Total suspended solids }\end{array}$ & $\begin{array}{r}12 / 12 \\
2 / 12 \\
27 / 52 \\
12 / 12 \\
12 / 12 \\
12 / 12\end{array}$ & $\begin{array}{c}0.20 \\
6.0 \\
36 \\
730 \\
4.8 \\
71\end{array}$ & $\begin{array}{c}0.020 \\
<5.0 \\
<2.0 \\
140 \\
1.7 \\
5.0\end{array}$ & $\begin{array}{c}0.085 \\
-5.1 \\
-4.3 \\
-270 \\
3.0 \\
31\end{array}$ & $\begin{array}{c}0.015 \\
0.087 \\
0.79 \\
44 \\
0.27 \\
6.4\end{array}$ \\
\hline $\begin{array}{l}\text { PCBs } \\
\text { Total Aroclors }\end{array}$ & $0 / 14$ & U0.0020 & 00.0020 & -0.0020 & 0 \\
\hline $\begin{array}{l}\text { Volatile organics } \\
\text { Chloroform } \\
\text { Trichloroethene }\end{array}$ & $\begin{array}{l}0 / 12 \\
0 / 12\end{array}$ & $\begin{array}{l}\text { UO. } 0050 \\
\text { UO.0050 }\end{array}$ & $\begin{array}{l}\text { UO. } 0050 \\
\text { UO.0050 }\end{array}$ & $\begin{array}{l}-0.0050 \\
-0.0050\end{array}$ & $\begin{array}{l}0 \\
0\end{array}$ \\
\hline
\end{tabular}

aprefix " $<$ " indicates the value for a parameter (excluding organics) was not quantifiable at the analytical detection limit; and "U" indicates the value for an organic parameter was undetected at the analytical detection limit.

$b_{A}$ tilde ( ) indicates that estimated values and/or detection limits were used in the calculation.

$c_{\text {Standard error of the mean. }}$ 
Table 4.101. NPDEs Permit Number TN 0002941, 1993, ORAL Category I outEalls

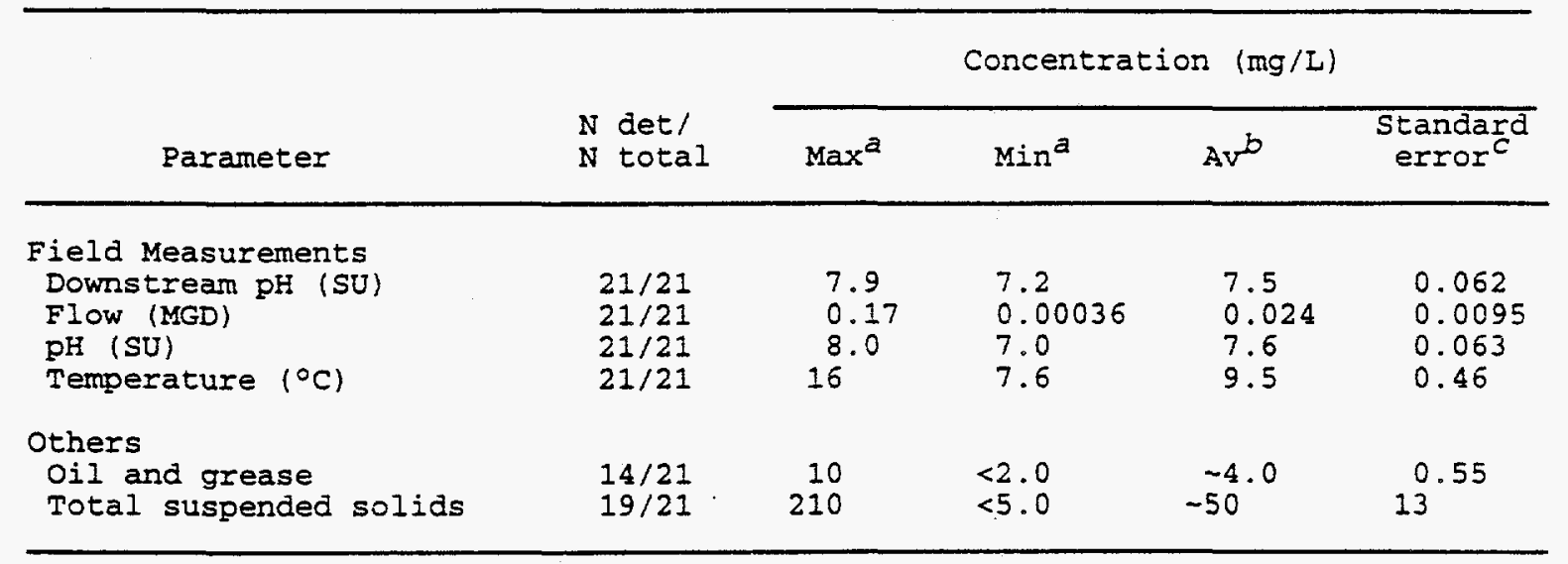

"Prefix " $<$ " indicates the value for a parameter (excluding organics) was not quantifiable at the analytical detection limit.

$b_{\mathrm{A}}$ tilde $(\sim)$ indicates that estimated values and/or detection limits were used in the calculation.

standard error of the mean. 
Table 4.102. NPDES Permit Nrmber TN 0002941, 1993, ORNL Category II outfalis

\begin{tabular}{|c|c|c|c|c|c|}
\hline \multirow[b]{2}{*}{ Parameter } & \multirow[b]{2}{*}{$\begin{array}{l}\mathrm{N} \text { det } / \\
\mathrm{N} \text { total }\end{array}$} & \multicolumn{4}{|c|}{ concentration (mg/L) } \\
\hline & & $\operatorname{Max}^{a}$ & $\operatorname{Min}^{a}$ & $A v^{b}$ & $\begin{array}{l}\text { Standard } \\
\text { erroI }\end{array}$ \\
\hline $\begin{array}{l}\text { Field Measurements } \\
\text { Downstream pH (SU) } \\
\text { Downstream temperature }\left({ }^{\circ} \mathrm{C}\right) \\
\text { Flow (MGD) } \\
\text { pH (SU) } \\
\text { Temperature }\left({ }^{\circ} \mathrm{C}\right)\end{array}$ & $\begin{array}{l}163 / 163 \\
39 / 39 \\
166 / 166 \\
163 / 163 \\
163 / 163\end{array}$ & $\begin{array}{l}8.4 \\
25 \\
0.12 \\
8.4 \\
60\end{array}$ & $\begin{array}{l}7.1 \\
8.0 \\
0.00014 \\
6.9 \\
6.4\end{array}$ & $\begin{array}{l}7.8 \\
17 \\
0.016 \\
7.7 \\
18\end{array}$ & $\begin{array}{l}0.016 \\
0.75 \\
0.0018 \\
0.022 \\
0.54\end{array}$ \\
\hline $\begin{array}{l}\text { Others } \\
\text { Oil and grease } \\
\text { Total suspended solids }\end{array}$ & $\begin{array}{l}64 / 163 \\
71 / 163\end{array}$ & $\begin{array}{r}34 \\
390\end{array}$ & $\begin{array}{l}<2.0 \\
<5.0\end{array}$ & $\begin{array}{l}-4.1 \\
-20\end{array}$ & $\begin{array}{l}0.38 \\
3.7\end{array}$ \\
\hline
\end{tabular}

$a_{\text {prefix }}<$ indicates the value for a parameter (excluding organics) was not quantifiable at the analytical detection limit.

$b_{A}$ tilae ( ) indicates that estimated values and/or detection limits were used in the calculation.

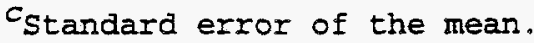

Table 4.103. NPDES Permit Number IN 0002941, 1993, ORAT Category III outfalls

\begin{tabular}{|c|c|c|c|c|c|}
\hline \multirow[b]{2}{*}{ Parameter } & \multirow[b]{2}{*}{$\begin{array}{l}\text { N det/ } \\
\text { N total }\end{array}$} & \multicolumn{4}{|c|}{ Concentration (mg/L) } \\
\hline & & $\operatorname{Max}$ & Min & Av & $\begin{array}{l}\text { Standard } \\
\text { error }\end{array}$ \\
\hline $\begin{array}{l}\text { Field Measurements } \\
\text { Flow (MGD) } \\
\text { pi (SU) } \\
\left.\text { Temperature ( }{ }^{\circ} \mathrm{C}\right)\end{array}$ & $\begin{array}{l}55 / 55 \\
56 / 56 \\
56 / 56\end{array}$ & $\begin{array}{l}0.14 \\
9.0 \\
33\end{array}$ & $\begin{array}{l}0.00022 \\
7.3 \\
6.9\end{array}$ & $\begin{array}{l}0.018 \\
7.8 \\
17\end{array}$ & $\begin{array}{l}0.0041 \\
0.041 \\
0.73\end{array}$ \\
\hline
\end{tabular}

${ }^{a}$ Standard error of the mean. 
Table 4.104. NPDES Rermit Number IN 0002941, 1993, ORAL Cooling Systems

\begin{tabular}{|c|c|c|c|c|c|}
\hline \multirow[b]{2}{*}{ Parameter } & \multirow[b]{2}{*}{$\begin{array}{l}\mathrm{N} \text { det } / \\
\mathrm{N} \text { total }\end{array}$} & \multicolumn{4}{|c|}{ Concentration (mg/L) } \\
\hline & & $\operatorname{Max}$ & $\operatorname{Min}^{a}$ & $A v^{b}$ & $\begin{array}{l}\text { Standard } \\
\text { error }\end{array}$ \\
\hline $\begin{array}{l}\text { Field Measurements } \\
\text { Chlorine, total residual } \\
\text { Downstream pH (SU) } \\
\text { Flow (MGD) } \\
\text { pH (SU) } \\
\text { Temperature }\left({ }^{\circ} \mathrm{C}\right)\end{array}$ & $\begin{array}{l}26 / 38 \\
38 / 38 \\
38 / 38 \\
38 / 38 \\
38 / 38\end{array}$ & $\begin{array}{l}0.18 \\
8.3 \\
0.19 \\
8.9 \\
33\end{array}$ & $\begin{array}{l}<0.010 \\
7.3 \\
0.0016 \\
7.0 \\
7.8\end{array}$ & $\begin{array}{l}-0.051 \\
7.9 \\
0.035 \\
8.2 \\
22\end{array}$ & $\begin{array}{l}0.0074 \\
0.049 \\
0.011 \\
0.080 \\
1.0\end{array}$ \\
\hline $\begin{array}{l}\text { Metals } \\
\text { Chromium, total } \\
\text { Copper, total } \\
\text { zinc, total }\end{array}$ & $\begin{array}{l}14 / 38 \\
28 / 38 \\
38 / 38\end{array}$ & $\begin{array}{l}0.19 \\
0.32 \\
0.94\end{array}$ & $\begin{array}{c}<0.0040 \\
<0.0070 \\
0.026\end{array}$ & $\begin{array}{c}-0.019 \\
-0.083 \\
0.20\end{array}$ & $\begin{array}{l}0.0060 \\
0.014 \\
0.034\end{array}$ \\
\hline
\end{tabular}

aprefix "<" indicates the value for a parameter (excluding organics) was not quantifiable at the analytical detection limit.

$b_{A}$ tilde ( ) indicates that estimated values and/or detection limits were used in the calculation.

$c_{\text {standard error of the mean. }}$ 
Table 4.105. 1993 mercury concentrations in ORNL surface water

\begin{tabular}{|c|c|c|c|c|c|c|}
\hline \multirow[b]{2}{*}{ Location } & \multirow[b]{2}{*}{$\begin{array}{l}N \text { det } / \\
N \text { total }\end{array}$} & \multicolumn{4}{|c|}{ Concentration $(\mu \mathrm{g} / \mathrm{L})$} & \multirow[b]{2}{*}{$\begin{array}{l}\text { Percent } \\
\text { of TwQ }\end{array}$} \\
\hline & & $\operatorname{Max}^{2}$ & $\operatorname{Min}^{2}$ & $A v^{b}$ & $\begin{array}{l}\text { Standard } \\
\text { error }\end{array}$ & \\
\hline \multicolumn{7}{|c|}{ First creek } \\
\hline Outfall 341 & $0 / 6$ & $<0.050$ & $<0.050$ & -0.050 & 0 & 2.1 \\
\hline \multicolumn{7}{|c|}{ Fifth Creek } \\
\hline $\begin{array}{ll}\text { Outfall } & 261 \\
\text { Outfall } & 363 \\
\text { Outfall } & 367\end{array}$ & $\begin{array}{l}0 / 6 \\
0 / 6 \\
2 / 6\end{array}$ & $\begin{array}{l}<0.050 \\
<0.050 \\
0.12\end{array}$ & $\begin{array}{l}<0.050 \\
<0.050 \\
<0.050\end{array}$ & $\begin{array}{l}-0.050 \\
-0.050 \\
-0.072\end{array}$ & $\begin{array}{l}0 \\
0 \\
0.014\end{array}$ & $\begin{array}{l}2.1 \\
2.1 \\
5.0\end{array}$ \\
\hline \multicolumn{7}{|c|}{ White Oak Creek } \\
\hline $\begin{array}{ll}\text { Outfall } & 106 \\
\text { Outfall } & 202 \\
\text { Outfall } & 207 \\
\text { Outfali } & 222 \\
\text { Outfall } & 301 \\
\text { Outfall } & 302 \\
\text { Outfall } & 304 \\
\text { Headwaters } \\
\text { Sewage Treatment Plant }\end{array}$ & $\begin{array}{l}6 / 6 \\
3 / 6 \\
3 / 6 \\
0 / 6 \\
4 / 6 \\
3 / 6 \\
6 / 6 \\
0 / 6 \\
1 / 6\end{array}$ & $\begin{array}{l}0.12 \\
0.10 \\
0.31 \\
<0.050 \\
0.10 \\
0.096 \\
0.26 \\
<0.050 \\
0.066\end{array}$ & $\begin{aligned} & 0.066 \\
&<0.050 \\
&<0.050 \\
&<0.050 \\
&<0.050 \\
&<0.050 \\
& 0.12 \\
&<0.050 \\
&<0.050\end{aligned}$ & $\begin{array}{rl} & 0.090 \\
- & 0.072 \\
- & 0.18 \\
- & 0.050 \\
- & 0.070 \\
-0.067 & 0.17 \\
& 0.050 \\
& 0.050 \\
- & 0.053\end{array}$ & $\begin{array}{l}0.0094 \\
0.010 \\
0.057 \\
0 \\
0.0092 \\
0.0082 \\
0.023 \\
0 \\
0.0027\end{array}$ & $\begin{array}{r}5.0 \\
4.2 \\
13 \\
2.1 \\
4.2 \\
4.0 \\
11 \\
2.1 \\
2.7\end{array}$ \\
\hline
\end{tabular}

$a_{\text {prefix }}<$ " indicates the value for a parameter (excluding organics) was not guantifiable at the analytical detection limit.

$b_{A}$ tilde $(\sim)$ indicates that estimated values and/or detection limits were used in the calculation.

$c_{\text {Standard error of the mean. }}$

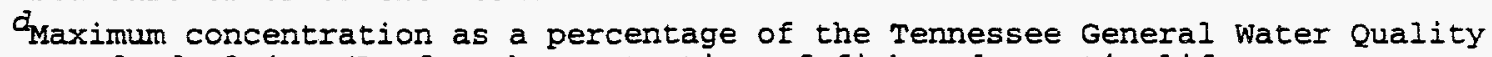
(TWQ) standard, $2.4 \mu \mathrm{g} / \mathrm{L}$, for the protection of fish and aquatic life. 
Table 4.106. 1993 mercury concentrations in ORNL sediment

\begin{tabular}{|c|c|c|c|c|c|}
\hline \multirow[b]{2}{*}{ Location } & \multirow[b]{2}{*}{$\begin{array}{l}\mathrm{N} \text { det } / \\
\mathrm{N} \text { total }\end{array}$} & \multicolumn{4}{|c|}{ Concentration $(\mu \mathrm{g} / \mathrm{g})$} \\
\hline & & $\operatorname{Max}$ & Min & Av & $\begin{array}{l}\text { Standard } \\
\text { errora }\end{array}$ \\
\hline \multicolumn{6}{|c|}{ First Creek } \\
\hline \multirow[t]{2}{*}{ Upstream Northwest Tributary } & $6 / 6$ & 0.085 & 0.029 & 0.060 & 0.0095 \\
\hline & \multicolumn{2}{|c|}{ Fifth Creek } & & & \\
\hline $\begin{array}{ll}\text { Outfall } & 261 \\
\text { Outfall } & 362\end{array}$ & $\begin{array}{l}6 / 6 \\
6 / 6\end{array}$ & $\begin{array}{l}57 \\
20\end{array}$ & $\begin{array}{l}5.5 \\
4.3\end{array}$ & $\begin{array}{l}27 \\
42\end{array}$ & $18^{9.1}$ \\
\hline \multicolumn{6}{|c|}{ Melton Branch } \\
\hline \multirow[t]{2}{*}{ Headwaters } & $6 / 6$ & 0.047 & 0.016 & 0.031 & 0.0055 \\
\hline & White & Creek & & & \\
\hline $\begin{array}{l}\text { Upstream Fifth Creek } \\
\text { Downstream First Creek } \\
\text { Headwaters } \\
\text { Upstream Melton Branch } \\
\text { Downstream White Oak Dam }\end{array}$ & $\begin{array}{l}6 / 6 \\
6 / 6 \\
6 / 6 \\
6 / 6 \\
6 / 6\end{array}$ & $\begin{array}{l}2.9 \\
3.2 \\
0.068 \\
0.20 \\
0.12\end{array}$ & $\begin{array}{l}0.64 \\
2.0 \\
0.049 \\
0.041 \\
0.020\end{array}$ & $\begin{array}{l}1.4 \\
2.4 \\
0.059 \\
0.10 \\
0.060\end{array}$ & $\begin{array}{l}0.33 \\
0.19 \\
0.0030 \\
0.026 \\
0.018\end{array}$ \\
\hline
\end{tabular}

astandard error of the mean. 
Table 4.107. 1993 PCB concentrations in ORNL sediment

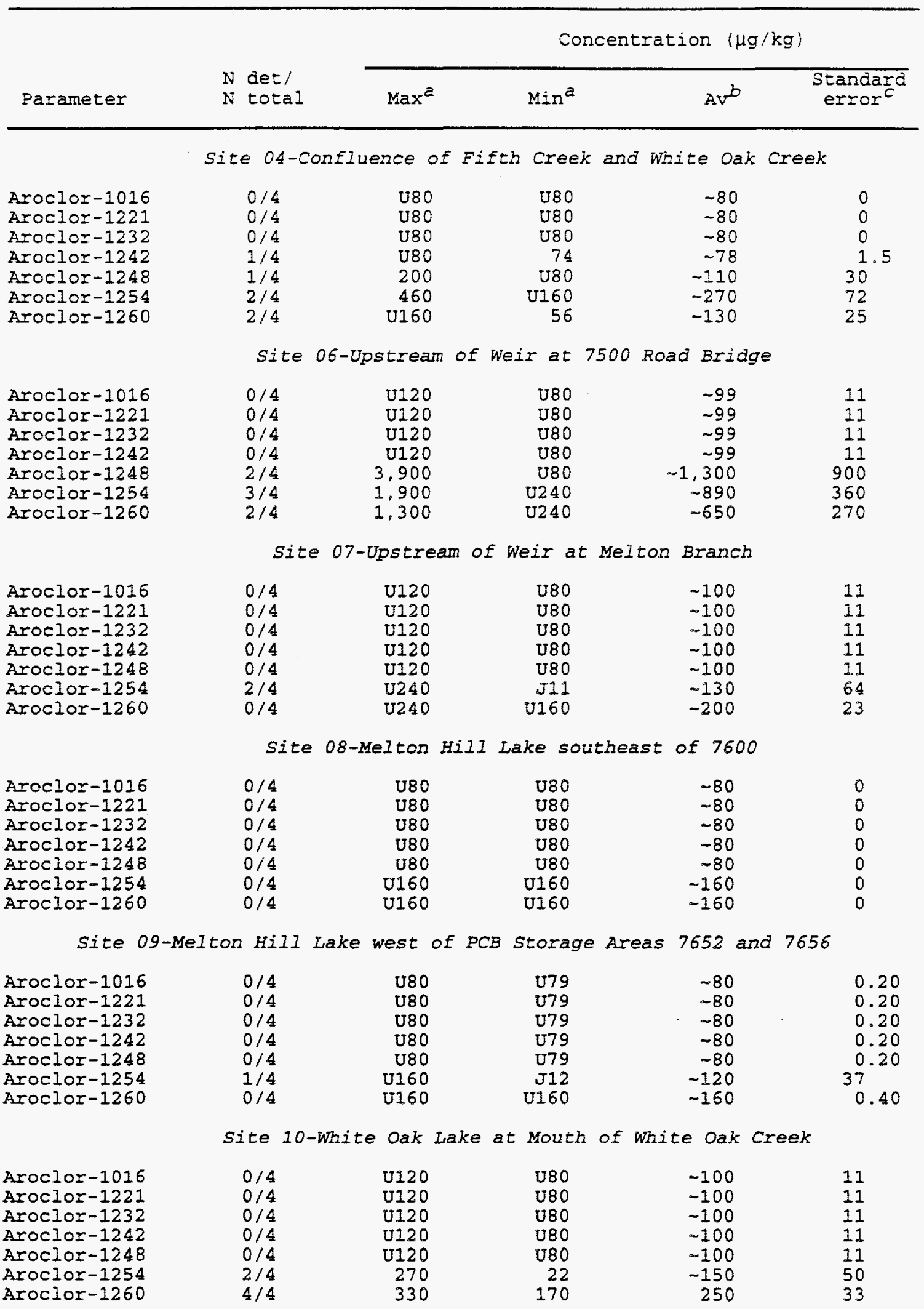


Table 4.107 (contimued)

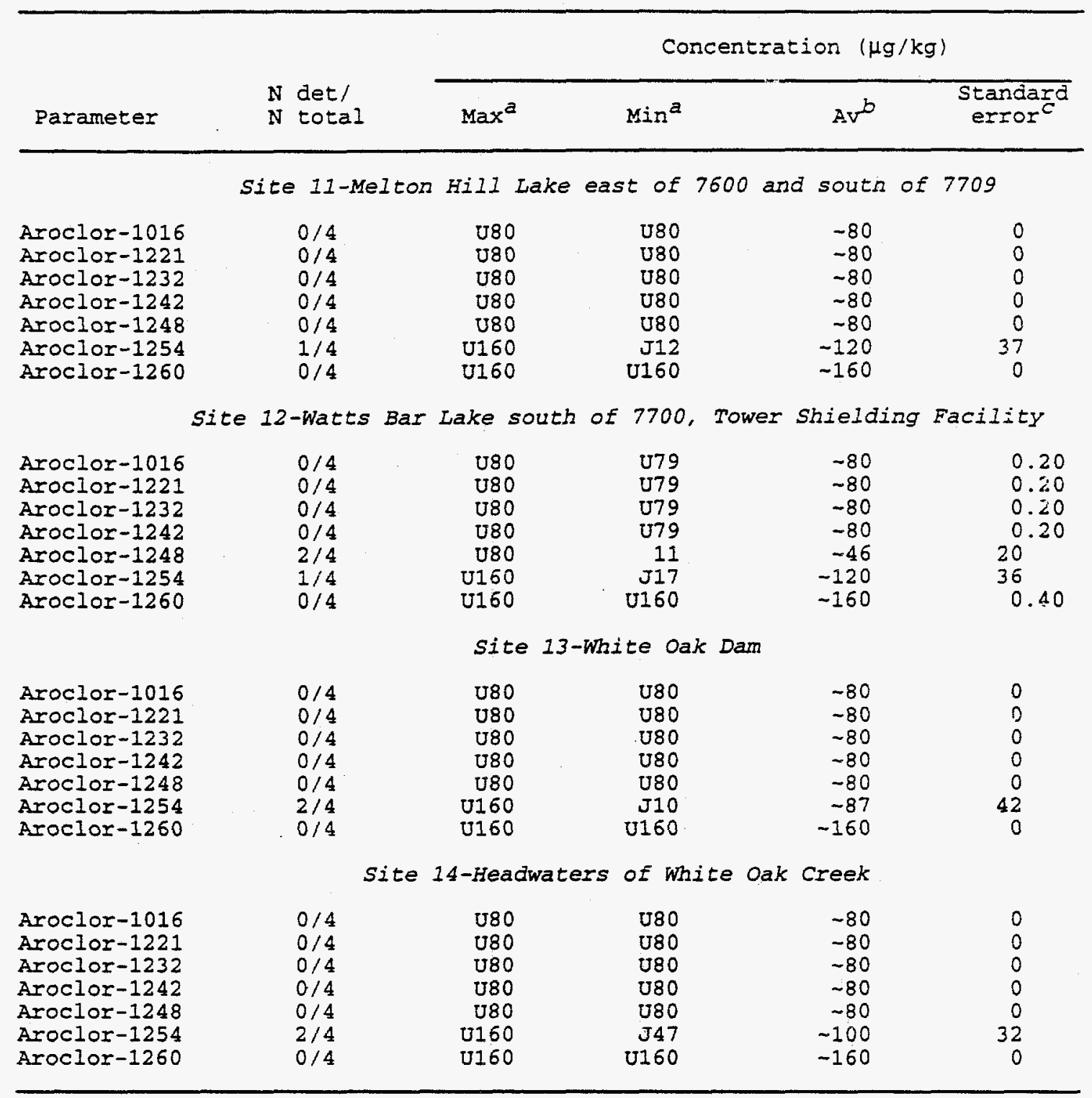

aprefix "U" indicates the value for an organic parameter was undetected at the analytical detection limit and " $\mathrm{J}$ " indicates the value was estimated at or below the analytical detection limit by the laboratory.

$b_{A}$ tilde (-) indicates that estimated values and/or detection limits were used in the calculation.

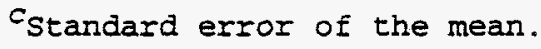


Table 4.108. Radlonuclide concentratlons at K-25 Site discharges and surface water monitoring locations

\begin{tabular}{|c|c|c|c|c|c|c|c|c|}
\hline \multirow{2}{*}{ Radionuclide } & \multirow{2}{*}{$\begin{array}{l}\text { Number of } \\
\text { samples }\end{array}$} & \multicolumn{4}{|c|}{ Concentration $(\mathrm{pCi} / \mathrm{L})^{a}$} & \multirow[b]{2}{*}{ DCG } & \multirow[b]{2}{*}{$\begin{array}{l}\text { Percent of } \\
\text { DCG }\end{array}$} & \multirow[b]{2}{*}{$\begin{array}{c}\text { Sum of the fractions } \\
\text { of the DCGis }\end{array}$} \\
\hline & & $\operatorname{Max}$ & Min & Median & Average & & & \\
\hline \multicolumn{9}{|c|}{ K-716 (Poplar Creek) } \\
\hline${ }^{234} \mathrm{U}$ & 12 & $5.80 \mathrm{E}+00$ & $0.00 \mathrm{E}+00$ & $7.74 \mathrm{E}-01$ & $1.45 \mathrm{E}+00$ & $5.00 \mathrm{E}+02$ & $2.91 \mathrm{E}-01$ & $b$ \\
\hline${ }^{235} \mathrm{U}$ & 12 & $7.62 \mathrm{E}+01$ & $0.00 \mathrm{E}+00$ & $1.35 \mathrm{E}+01$ & $2.77 \mathrm{E}+01$ & $6.00 \mathrm{E}+02$ & $4.61 \mathrm{E}+00^{\circ}$ & $b$ \\
\hline${ }^{238} \mathrm{U}$ & 12 & $2.96 \mathrm{E}+00$ & $-7.48 \mathrm{E}-01$ & $9.09 \mathrm{E}-01$ & $8.65 \mathrm{E}-01$ & $6.00 \mathrm{E}+02$ & $1.44 \mathrm{E}-01$ & $b$ \\
\hline${ }^{137} \mathrm{Cs}$ & 12 & $5.91 \mathrm{E}+01$ & $-1.66 \mathrm{E}+01$ & $1.12 \mathrm{E}+01$ & $1.35 \mathrm{E}+01$ & $3.00 \mathrm{E}+03$ & $4.48 \mathrm{E}-0 \mathrm{I}^{\mathrm{C}}$ & $b$ \\
\hline${ }^{99} \mathrm{Tc}$ & 12 & $3.92 \mathrm{E}+02$ & $-1.37 \mathrm{E}+03$ & $-8.50 \mathrm{E}+01$ & $-1.65 \mathrm{E}+02$ & $1.00 \mathrm{E}+05$ & $-1.65 \mathrm{E}-01$ & $b$ \\
\hline${ }^{237} \mathrm{~Np}$ & 12 & $2.08 \mathrm{E}+00$ & $-7.59 \mathrm{E}-01$ & $0.00 \mathrm{E}+00$ & $2.14 \mathrm{E}-01$ & $3.00 \mathrm{E}+01$ & $7.12 \mathrm{E}-01$ & $b$ \\
\hline${ }^{238} \mathrm{Pu}$ & 12 & $1.04 \mathrm{E}+00$ & $-2.28 \mathrm{E}+00$ & $0.00 \mathrm{E}+00$ & $-3.23 \mathrm{E}-01$ & $4.00 \mathrm{E}+01$ & $-8.06 \mathrm{E}-01$ & $b$ \\
\hline${ }^{239} \mathrm{Pu}$ & 12 & $7.59 \mathrm{E}-01$ & $-7.54 \mathrm{E}-01$ & $0.00 \mathrm{E}+00$ & $-1.13 \mathrm{E}-01$ & $3.00 \mathrm{E}+01$ & $-3.78 \mathrm{E}-01$ & $b$ \\
\hline${ }^{234} \mathrm{Th}$ & 12 & $1.51 \mathrm{E}+03$ & $-3.99 \mathrm{E}+02$ & $0.00 \mathrm{E}+00$ & $2.31 E+02$ & $1.00 \mathrm{E}+04$ & $2.31 \mathrm{E}+00^{c}$ & $b$ \\
\hline${ }^{234 m} \mathrm{~Pa}$ & 12 & $8.03 \mathrm{E}+03$ & $0.00 \mathrm{E}+00$ & $0.00 \mathrm{E}+00$ & $2.51 \mathrm{E}+03$ & $7.00 \mathrm{E}+04$ & $3.59 \mathrm{E}+00^{c}$ & $b$ \\
\hline${ }^{106} \mathrm{Ru}$ & 12 & $1.68 \mathrm{E}+02$ & $0.00 \mathrm{E}+00$ & $0.00 \mathrm{E}+00$ & $1.40 \mathrm{E}+01$ & $6.00 \mathrm{E}+03$ & $2.33 \mathrm{E}-01^{c}$ & $b$ \\
\hline${ }^{40} K$ & 12 & $6.08 \mathrm{E}+02$ & $0.00 \mathrm{E}+00$ & $0.00 \mathrm{E}+00$ & $9.81 \mathrm{E}+01$ & $7.00 \mathrm{E}+03$ & $1.40 \mathrm{E}+00^{c}$ & $b$ \\
\hline Gross alpha & 12 & $2.46 \mathrm{E}+00$ & $-1.52 \mathrm{E}+00$ & $-9.20 \mathrm{E}-02$ & $1.57 \mathrm{E}-01$ & $b$ & $b$ & $b$ \\
\hline Gross beta & 12 & $8.80 \mathrm{E}+00$ & $-3.55 \mathrm{E}+00$ & $2.14 \mathrm{E}+00$ & $2.50 \mathrm{E}+00$ & $b$ & $b$ & $b$ \\
\hline All listed isotopes & & $b$ & $b$ & $b$ & $b$ & $b$ & $b$ & $1.24 \mathrm{E}-01^{c}$ \\
\hline \multicolumn{9}{|c|}{$K-901-A$ (settling basin for laboratory and surface wrer runoff) } \\
\hline${ }^{234} \mathrm{U}$ & 12 & $7.26 \mathrm{E}+00$ & $0.00 \mathrm{E}+00$ & $2.38 \mathrm{E}+00$ & $2.36 \mathrm{E}+00$ & $5.00 \mathrm{E}+02$ & $4.71 \mathrm{E}-01$ & $b$ \\
\hline${ }^{235} \mathrm{U}$ & 12 & $5.55 \mathrm{E}+01$ & $-5.00 \mathrm{E}+01$ & $1.44 \mathrm{E}+00$ & $7.09 \mathrm{E}+00$ & $6.00 \mathrm{E}+02$ & $1.18 \mathrm{E}+00^{r}$ & $b$ \\
\hline${ }^{238} \mathrm{U}$ & 12 & $2.11 \mathrm{E}+01$ & $0.00 \mathrm{E}+00$ & $1.33 \mathrm{E}+00$ & $3.16 \mathrm{E}+02$ & $6.00 \mathrm{E}+02$ & $5.27 \mathrm{E}-01$ & $b$ \\
\hline${ }^{137} \mathrm{Cs}$ & 12 & $1.17 \mathrm{E}+01$ & $-1.70 \mathrm{E}+01$ & $-8.35 \mathrm{E}-01$ & $-3.22 \mathrm{E}+100$ & $3.00 \mathrm{E}+03$ & $-1.07 \mathrm{E}-01^{\mathrm{c}}$ & $b$ \\
\hline${ }^{99} \mathrm{Tc}$ & 12 & $4.12 \mathrm{E}+02$ & $-4.37 \mathrm{E}+02$ & $3.04 \mathrm{E}+01$ & $-3.39 \mathrm{E}+01$ & $1.00 \mathrm{E}+05$ & $-3.39 E-02$ & $b$ \\
\hline${ }^{237} \mathrm{~Np}$ & 12 & $7.30 \mathrm{E}-01$ & $-6.68 \mathrm{E}-01$ & $0.00 \mathrm{E}+00$ & $-4.27 \mathrm{E}-02$ & $3.00 \mathrm{E}+01$ & $-1.42 \mathrm{E}-01$ & $b$ \\
\hline${ }^{238} \mathrm{Pu}$ & 12 & $4.49 \mathrm{E}+00$ & $-2.00 \mathrm{E}+00$ & $0.00 E+00$ & $6.88 \mathrm{E}-01$ & $4.00 \mathrm{E}+01$ & $1.72 \mathrm{E}+00$ & $b$ \\
\hline
\end{tabular}


Table 4.108 (contlnued)

$\frac{t}{\omega}$

\begin{tabular}{|c|c|c|c|c|c|c|c|c|}
\hline \multirow{2}{*}{ Radionuclide } & \multirow{2}{*}{$\begin{array}{l}\text { Number of } \\
\text { samples }\end{array}$} & \multicolumn{4}{|c|}{ Concentration $(\mathrm{pCi} / \mathrm{L})^{a}$} & \multirow[b]{2}{*}{ DCG } & \multirow[b]{2}{*}{$\begin{array}{l}\text { Percent of } \\
\text { DCG }\end{array}$} & \multirow[b]{2}{*}{$\begin{array}{c}\text { Sum of the fractions } \\
\text { of the DCGs }\end{array}$} \\
\hline & & $\operatorname{Max}$ & Min & Median & Average & & & \\
\hline${ }^{239} \mathrm{Pu}$ & 12 & $3.49 \mathrm{E}-01$ & $-1.59 \mathrm{E}+00$ & $0.00 \mathrm{E}+00$ & $-2.62 \mathrm{E}-01$ & $3.00 \mathrm{E}+01$ & $-8.73 E-01$ & $b$ \\
\hline${ }^{228} \mathrm{Th}$ & 12 & $1.04 \mathrm{E}+03$ & $0.00 \mathrm{E}+00$ & $0.00 \mathrm{E}+00$ & $8.67 \mathrm{E}+01$ & $4.00 \mathrm{E}+02$ & $2.17 \mathrm{E}+01$ & $b$ \\
\hline${ }^{234} \mathrm{Th}$ & 12 & $1.61 \mathrm{E}+03$ & $-2.22 \mathrm{E}+02$ & $0.00 \mathrm{E}+00$ & $2.92 \mathrm{E}+02$ & $1.00 \mathrm{E}+04$ & $2.92 \mathrm{E}+00^{c}$ & $b$ \\
\hline${ }^{234 m} \mathrm{~Pa}$ & 12 & $4.63 E+03$ & $-4.71 \mathrm{E}+02$ & $0.00 \mathrm{E}+00$ & $7.17 \mathrm{E}+02$ & $7.00 \mathrm{E}+04$ & $1.02 \mathrm{E}+00^{\circ}$ & $b$ \\
\hline${ }^{106} \mathrm{Ru}$ & 12 & $5.49 \mathrm{E}+02$ & $0.00 \mathrm{E}+00$ & $0.00 \mathrm{E}+00$ & $9.73 \mathrm{E}+01$ & $6.00 \mathrm{E}+03$ & $1.62 \mathrm{E}+00$ & $b$ \\
\hline${ }^{143} \mathrm{Ce}$ & 12 & $1.06 \mathrm{E}+03$ & $0.00 \mathrm{E}+00$ & $0.00 \mathrm{E}+00$ & $8.83 E+01$ & $3.00 \mathrm{E}+04$ & $2.94 \mathrm{E}-01$ & $b$ \\
\hline${ }^{51} \mathrm{Cr}$ & 12 & $2.41 E+03$ & $0.00 \mathrm{E}+00$ & $0.00 \mathrm{E}+00$ & $2.01 \mathrm{E}+02$ & $1.00 \mathrm{E}+06$ & $2.01 \mathrm{E}-02$ & $b$ \\
\hline${ }^{331} I$ & 12 & $1.25 \mathrm{E}+02$ & $0.00 \mathrm{E}+00$ & $0.00 \mathrm{E}+00$ & $1.04 \mathrm{E}+01$ & $3.00 \mathrm{E}+03$ & $3.47 \mathrm{E}-01$ & $b$ \\
\hline Gross alpha & 12 & $3.98 \mathrm{E}+00$ & $-9.02 \mathrm{E}-01$ & $1.01 \mathrm{E}+00$ & $1.29 \mathrm{E}+00$ & $b$ & $b$ & $b$ \\
\hline Gross beta & 12 & $2.47 E+01$ & $-1.65 \mathrm{E}-01$ & $5.54 \mathrm{E}+00$ & $9.03 \mathrm{E}+00$ & $b$ & $b$ & $b$ \\
\hline All listed isotopes & & $b$ & $b$ & $b$ & $b$ & $b$ & $b$ & $3.07 E-01^{\mathrm{C}}$ \\
\hline
\end{tabular}

$K-1007-B$ (settling basin for laboratory and surface water run $)$ f)

$\begin{array}{lllllllll}{ }^{234} \mathrm{U} & 12 & 1.32 \mathrm{E}+01 & -4.94 \mathrm{E}-01 & 1.64 \mathrm{E}+00 & 2.64 \mathrm{E}+00 & 5.00 \mathrm{E}+02 & 5.28 \mathrm{E}-01 & b \\ { }^{235} \mathrm{U} & 12 & 3.78 \mathrm{E}+01 & -9.25 \mathrm{E}+01 & 1.26 \mathrm{E}+01 & 5.63 \mathrm{E}+00 & 6.00 \mathrm{E}+02 & 9.38 \mathrm{E}-01^{c} & b \\ { }^{238} \mathrm{U} & 12 & 1.77 \mathrm{E}+01 & 0.00 \mathrm{E}+00 & 1.14 \mathrm{E}+00 & 2.27 \mathrm{E}+00 & 6.00 \mathrm{E}+02 & 3.79 \mathrm{E}-01 & b \\ { }^{137} \mathrm{Cs} & 12 & 1.88 \mathrm{E}+01 & -2.04 \mathrm{E}+01 & 4.52 \mathrm{E}+00 & 2.88 \mathrm{E}+00 & 3.00 \mathrm{E}+03 & 9.59 \mathrm{E}-02^{r} & b \\ { }^{99} \mathrm{Tc} & 12 & 4.96 \mathrm{E}+02 & -4.18 \mathrm{E}+02 & -5.38 \mathrm{E}+01 & -1.73 \mathrm{E}+01 & 1.00 \mathrm{E}+05 & -1.73 \mathrm{E}-02 & b \\ { }^{237} \mathrm{~Np} & 12 & 6.95 \mathrm{E}-01 & -1.10 \mathrm{E}+00 & 0.00 \mathrm{E}+00 & 4.42 \mathrm{E}-02 & 3.00 \mathrm{E}+01 & 1.47 \mathrm{E}-01 & b \\ { }^{238} \mathrm{Pu} & 12 & 1.37 \mathrm{E}+00 & -2.08 \mathrm{E}+00 & 0.00 \mathrm{E}+00 & -3.90 \mathrm{E}-02 & 4.00 \mathrm{E}+01 & -9.75 \mathrm{E}-02 & b \\ { }^{239} \mathrm{Pu} & 12 & 3.28 \mathrm{E}-01 & -3.60 \mathrm{E}+00 & 0.00 \mathrm{E}+00 & -5.16 \mathrm{E}-01 & 3.00 \mathrm{E}+01 & -1.72 \mathrm{E}+00 & b \\ { }^{228} \mathrm{Th} & 12 & 1.22 \mathrm{E}+03 & 0.00 \mathrm{E}+00 & 0.00 \mathrm{E}+00 & 1.02 \mathrm{E}+02 & 4.00 \mathrm{E}+02 & 2.54 \mathrm{E}+01 & b \\ { }^{234} \mathrm{Th} & 12 & 1.25 \mathrm{E}+03 & -1.06 \mathrm{E}+03 & 0.00 \mathrm{E}+00 & 1.30 \mathrm{E}+02 & 1.00 \mathrm{E}+04 & 1.30 \mathrm{E}+00^{c} & b \\ { }^{234 m} \mathrm{~Pa} & 12 & 4.86 \mathrm{E}+03 & 0.00 \mathrm{E}+00 & 0.00 \mathrm{E}+00 & 1.06 \mathrm{E}+03 & 7.00 \mathrm{E}+04 & 1.52 \mathrm{E}+00^{c} & b \\ { }^{106} \mathrm{Ru} & 12 & 1.67 \mathrm{E}+02 & 0.00 \mathrm{E}+00 & 0.00 \mathrm{E}+00 & 1.39 \mathrm{E}+01 & 6.00 \mathrm{E}+03 & 2.32 \mathrm{E}-01 & b\end{array}$


Table 4.108 (continued)

\begin{tabular}{|c|c|c|c|c|c|c|c|c|}
\hline \multirow{2}{*}{ Radionuclide } & \multirow{2}{*}{$\begin{array}{c}\text { Number of } \\
\text { samples }\end{array}$} & \multicolumn{4}{|c|}{ Concentration $(\mathrm{pCi} / \mathrm{L})^{a}$} & \multirow[b]{2}{*}{ DCG } & \multirow{2}{*}{$\begin{array}{l}\text { Percent of } \\
\text { DCG }\end{array}$} & \multirow{2}{*}{$\begin{array}{l}\text { Sum of the fractions } \\
\text { of the DCGs }\end{array}$} \\
\hline & & $\operatorname{Max}$ & Min & Median & Average & & & \\
\hline Gross alpha & 12 & $2.09 \mathrm{E}+00$ & $-6.01 \mathrm{E}-01$ & $3.77 \mathrm{E}-01$ & $3.94 \mathrm{E}-01$ & $b$ & $b$ & $b$ \\
\hline Gross beta & 12 & $9.73 \mathrm{E}+00$ & $2.06 \mathrm{E}+00$ & $5.15 E+00$ & $5.17 \mathrm{E}+00$ & $b$ & $b$ & $b$ \\
\hline All listed isotopes & & $b$ & $\boldsymbol{b}$ & $b$ & $b$ & $b$ & $b$ & $2.78 \mathrm{E}-01^{c}$ \\
\hline
\end{tabular}

$K-1700$ (coal pile runoff and once-through cooling water)

\begin{tabular}{|c|c|c|c|c|c|c|c|c|}
\hline${ }^{234} \mathrm{U}$ & 12 & $1.38 \mathrm{E}+01$ & $5.84 \mathrm{E}-01$ & $7.61 \mathrm{E}+00$ & $8.18 E+00$ & $5.00 \mathrm{E}+02$ & $1.64 \mathrm{E}+00$ & $b$ \\
\hline${ }^{239} \mathrm{U}$ & 12 & $6.65 \mathrm{E}+01$ & $-3.89 E+01$ & $1.69 \mathrm{E}-01$ & $7.19 \mathrm{E}+00$ & $6.00 \mathrm{E}+02$ & $1.20 \mathrm{E}+00^{c}$ & $b$ \\
\hline${ }^{238} \mathrm{U}$ & 12 & $1.16 \mathrm{E}+01$ & $8.34 \mathrm{E}-01$ & $4.55 \mathrm{E}+00$ & $4.68 \mathrm{E}+00$ & $6.00 \mathrm{E}+02$ & $7.81 \mathrm{E}-01$ & $b$ \\
\hline${ }^{137} \mathrm{Cs}$ & 12 & $3.57 \mathrm{E}+01$ & $-3.90 \mathrm{E}+01$ & $2.36 \mathrm{E}+\infty 0$ & $2.94 \mathrm{E}+00$ & $3.00 \mathrm{E}+03$ & $9.79 \mathrm{E}-02^{c}$ & $b$ \\
\hline${ }^{99} \mathrm{Tc}$ & 12 & $4.94 E+02$ & $-3.78 \mathrm{E}+02$ & $-4.52 \mathrm{E}+01$ & $1.22 \mathrm{E}+00$ & $1.00 \mathrm{E}+05$ & $1.22 \mathrm{E}-03$ & $b$ \\
\hline${ }^{237} \mathrm{~Np}$ & 12 & $1.90 \mathrm{E}+00$ & $-6.57 \mathrm{E}-01$ & $8.74 \mathrm{E}-01$ & $7.89 \mathrm{E}-01$ & $3.00 \mathrm{E}+01$ & $2.63 E+00$ & $b$ \\
\hline${ }^{238} \mathrm{Pu}$ & 12 & $7.24 \mathrm{E}-01$ & $-2.22 \mathrm{E}+00$ & $0.00 \mathrm{E}+00$ & $-4.24 \mathrm{E}-01$ & $4.00 \mathrm{E}+01$ & $-1.06 \mathrm{E}+00$ & $b$ \\
\hline${ }^{239} \mathrm{Pu}$ & 12 & $1.48 \mathrm{E}+00$ & $-3.54 E+00$ & $0.00 \mathrm{E}+00$ & $-1.40 \mathrm{E}-01$ & $3.00 \mathrm{E}+01$ & $-4.67 \mathrm{E}-01$ & $b$ \\
\hline${ }^{228} \mathrm{Th}$ & 12 & $1.48 \mathrm{E}+03$ & $0.00 E+00$ & $0.00 \mathrm{E}+00$ & $1.28 \mathrm{E}+02$ & $4.00 \mathrm{E}+02$ & $3.20 \mathrm{E}+0 \mathrm{I}^{c}$ & $b$ \\
\hline${ }^{234} \mathrm{Th}$ & 12 & $1.04 E+03$ & $-2.63 E+02$ & $0.00 \mathrm{E}+00$ & $2.27 \mathrm{E}+02$ & $1.00 \mathrm{E}+04$ & $2.27 \mathrm{E}+00^{r}$ & $b$ \\
\hline${ }^{234 m} \mathrm{~Pa}$ & 12 & $4.24 \mathrm{E}+03$ & $0.00 \mathrm{E}+00$ & $0.00 \mathrm{E}+00$ & $9.98 \mathrm{E}+02$ & $7.00 \mathrm{E}+04$ & $1.43 \mathrm{E}+00 \sigma^{r}$ & $b$ \\
\hline${ }^{40} \mathrm{~K}$ & 12 & $4.63 \mathrm{E}+02$ & $0.00 \mathrm{E}+00$ & $0.00 \mathrm{E}+00$ & $3.86 \mathrm{E}+01$ & $7.00 \mathrm{E}+03$ & $5.51 \mathrm{E}-01^{c}$ & $b$ \\
\hline Gross alpha & 12 & $1.31 \mathrm{E}+01$ & $4.93 \mathrm{E}+00$ & $9.31 \mathrm{E}+00$ & $8.81 E+00$ & $b$ & $b$ & $b$ \\
\hline Gross beta & 12 & $2.26 \mathrm{E}+01$ & $1.03 E+01$ & $1.79 \mathrm{E}+01$ & $1.71 \mathrm{E}+01$ & $b$ & $b$ & $b$ \\
\hline All listed isotopes & & $b$ & $b$ & $b$ & $b$ & $b$ & $b$ & $4.11 \mathrm{E}-01^{\circ}$ \\
\hline \multicolumn{9}{|c|}{$K-1710$ (Poplar Creek upstream of the K-25 Site) } \\
\hline${ }^{234} \mathrm{U}$ & 12 & $3.21 \mathrm{E}+00$ & $0.00 \mathrm{E}+00$ & $4.97 \mathrm{E}-01$ & $1.03 \mathrm{E}+00$ & $5.00 \mathrm{E}+02$ & $2.06 \mathrm{E}-01$ & $b$ \\
\hline${ }^{235} \mathrm{U}$ & 12 & $5.84 \mathrm{E}+01$ & $-3.46 \mathrm{E}+01$ & $1.10 \mathrm{E}+01$ & $1.16 \mathrm{E}+01$ & $6.00 \mathrm{E}+02$ & $1.94 \mathrm{E}+00^{c}$ & $b$ \\
\hline${ }^{238} \mathrm{U}$ & 12 & 4.35E+00 & $0.00 \mathrm{E}+00$ & $9.36 \mathrm{E}-01$ & $1.31 \mathrm{E}+00$ & $6.00 \mathrm{E}+02$ & $2.19 \mathrm{E}-01$ & $b$ \\
\hline${ }^{137} \mathrm{Cs}$ & 12 & $6.01 E+01$ & $-2.89 \mathrm{E}+01$ & $5.36 \mathrm{E}+00$ & $1.15 \mathrm{E}+01$ & $3.00 \mathrm{E}+03$ & $3.83 \mathrm{E}-01^{c}$ & $b$ \\
\hline
\end{tabular}


Table 4.108 (continued)

\begin{tabular}{|c|c|c|c|c|c|c|c|c|}
\hline \multirow{2}{*}{ Radionuclide } & \multirow{2}{*}{$\begin{array}{l}\text { Number of } \\
\text { samples }\end{array}$} & \multicolumn{4}{|c|}{ Concentration $(\mathrm{pCi} / \mathrm{L})^{a}$} & \multirow[b]{2}{*}{ DCG } & \multirow[b]{2}{*}{$\begin{array}{l}\text { Percent of } \\
\text { DCG }\end{array}$} & \multirow{2}{*}{$\begin{array}{c}\text { Sum of the fractions } \\
\text { of the DCGs }\end{array}$} \\
\hline & & Max & Min & Median & Average & & & \\
\hline${ }^{99} \mathrm{Tc}$ & 12 & $4.58 \mathrm{E}+02$ & $-1.31 \mathrm{E}+03$ & $-1.39 \mathrm{E}+02$ & $-2.30 \mathrm{E}+02$ & $1.00 \mathrm{E}+05$ & $-2.30 \mathrm{E}-01$ & $b$ \\
\hline${ }^{237} \mathrm{~Np}$ & 12 & $1.78 \mathrm{E}+00$ & $-7.30 \mathrm{E}-01$ & $0.00 \mathrm{E}+00$ & $2.03 \mathrm{E}-01$ & $3.00 \mathrm{E}+01$ & $6.76 \mathrm{E}-01$ & $b$ \\
\hline${ }^{238} \mathrm{Pu}$ & 12 & $1.50 \mathrm{E}+00$ & $-2.19 \mathrm{E}+00$ & $0.00 \mathrm{E}+00$ & $9.10 \mathrm{E}-02$ & $4.00 \mathrm{E}+01$ & $2.28 \mathrm{E}-01$ & $b$ \\
\hline${ }^{2 m n} \mathrm{Pu}$ & 12 & $7.30 \mathrm{E}-01$ & $-1.50 \mathrm{E}+00$ & $0.00 \mathrm{E}+00$ & $-1.19 \mathrm{E}-01$ & $3.00 E+01$ & $-3.98 E-01$ & $b$ \\
\hline${ }^{234} \mathrm{Th}$ & 12 & $9.33 \mathrm{E}+02$ & $-3.08 \mathrm{E}+02$ & $0.00 \mathrm{E}+00$ & $1.10 \mathrm{E}+02$ & $1.00 \mathrm{E}+04$ & $1.10 \mathrm{E}+00^{c}$ & $b$ \\
\hline${ }^{234 m} \mathrm{~Pa}$ & 12 & $8.34 E+03$ & $-2.83 E+02$ & $0.00 \mathrm{E}+00$ & $1.73 \mathrm{E}+03$ & $7.00 \mathrm{E}+04$ & $2.47 \mathrm{E}+00^{c}$ & $b$ \\
\hline${ }^{106} \mathrm{Ru}$ & 12 & $1.69 \mathrm{E}+02$ & $0.00 \mathrm{E}+00$ & $0.00 \mathrm{E}+00$ & $2.73 \mathrm{E}+01$ & $6.00 \mathrm{E}+03$ & $4.54 \mathrm{E}-01^{c}$ & $b$ \\
\hline${ }^{143} \mathrm{Ce}$ & 12 & $8.25 E+03$ & $0.00 \mathrm{E}+10$ & $0.00 \mathrm{E}+00$ & $6.88 \mathrm{E}+02$ & $3.00 \mathrm{E}+04$ & $2.29 \mathrm{E}+00$ & $b$ \\
\hline Gross alpha & 12 & $2.52 \mathrm{E}+00$ & $-1.15 \mathrm{E}+00$ & $7.62 \mathrm{E}-01$ & $8.41 \mathrm{E}-01$ & $b$ & $b$ & $b$ \\
\hline Gross beta & 12 & $8.73 E+00$ & $-1.75 \mathrm{E}+00$ & $2.40 \mathrm{E}+00$ & $2.87 \mathrm{E}+00$ & $\boldsymbol{b}$ & $b$ & $b$ \\
\hline \multirow[t]{2}{*}{ All listed isotopes } & & $b$ & $\boldsymbol{b}$ & $b$ & $b$ & $b$ & $b$ & $9: 34 \mathrm{E}-02^{c}$ \\
\hline & \multicolumn{7}{|c|}{ West Fork Poplar Creek (upstream of the K-25 Site) } & . \\
\hline${ }^{234} \mathrm{U}$ & 12 & $4.21 \mathrm{E}+00$ & $-6.90 \mathrm{E}-01$ & $7.65 \mathrm{E}-01$ & $1.10 \mathrm{E}+00$ & $5.00 \mathrm{E}+02$ & $2.20 \mathrm{E}-01$ & $b$ \\
\hline${ }^{235} \mathrm{U}$ & 12 & $4.61 \mathrm{E}+01$ & $-1.73 E+02$ & $5.26 \mathrm{E}+00$ & $-2.05 \mathrm{E}+00$ & $6.00 \mathrm{E}+02$ & $-3.41 \mathrm{E}-01^{c}$ & $b$ \\
\hline${ }^{238} \mathrm{U}$ & 12 & $1.40 \mathrm{E}+00$ & $-5.83 \mathrm{E}-01$ & $4.20 \mathrm{E}-01$ & $4.76 \mathrm{E}-01$ & $6.00 \mathrm{E}+02$ & $7.94 \mathrm{E}-02$ & $b$ \\
\hline${ }^{137} \mathrm{Cs}$ & 12 & $2.27 \mathrm{E}+01$ & $-2.11 \mathrm{E}+01$ & $7.11 \mathrm{E}+00$ & $3.95 \mathrm{E}+00$ & $3.00 \mathrm{E}+03$ & $1.32 \mathrm{E}-01^{\mathrm{c}}$ & $b$ \\
\hline${ }^{99} \mathrm{Tc}$ & 12 & $4.51 \mathrm{E}+02$ & $-1.09 \mathrm{E}+03$ & $-2.19 \mathrm{E}+02$ & $-2.30 \mathrm{E}+02$ & $1.00 \mathrm{E}+05$ & $-2.30 \mathrm{E}-01$ & $b$ \\
\hline${ }^{237} \mathrm{~Np}$ & 12 & $6.57 \mathrm{E}-01$ & $-6.68 \mathrm{E}-01$ & $0.00 \mathrm{E}+00$ & $7.21 \mathrm{E}-02$ & $3.00 \mathrm{E}+01$ & $2.40 \mathrm{E}-01$ & $b$ \\
\hline${ }^{238} \mathrm{Pu}$ & 12 & $2.99 \mathrm{E}+00$ & $-2.00 \mathrm{E}+00$ & $0.00 \mathrm{E}+00$ & $1.81 \mathrm{E}-01$ & $4.00 \mathrm{E}+01$ & $4.51 \mathrm{E}-01$ & $b$ \\
\hline${ }^{239} \mathrm{Pu}$ & 12 & $7.54 \mathrm{E}-01$ & $-1.62 \mathrm{E}+00$ & $0.00 \mathrm{E}+00$ & $-5.07 \mathrm{E}-02$ & $3.00 \mathrm{E}+01$ & $-1.69 \mathrm{E}-01$ & $b$ \\
\hline${ }^{228} \mathrm{Th}$ & 12 & $9.54 \mathrm{E}+02$ & $0.00 \mathrm{E}+00$ & $0.00 \mathrm{E}+00$ & $7.95 \mathrm{E}+01$ & $4.00 \mathrm{E}+02$ & $1.99 \mathrm{E}+0 \mathrm{I}^{c}$ & $b$ \\
\hline${ }^{234} \mathrm{Th}$ & 12 & $7.12 \mathrm{E}+02$ & $-1.10 \mathrm{E}+03$ & $0.00 \mathrm{E}+00$ & $-7.72 \mathrm{E}+01$ & $1.00 \mathrm{E}+04$ & $-7.72 \mathrm{E}-01^{c}$ & $b$ \\
\hline${ }^{234 m} \mathrm{~Pa}$ & 12 & $6.13 E+03$ & $-2.61 \mathrm{E}+02$ & $0.00 \mathrm{E}+00$ & $1.05 \mathrm{E}+03$ & $7.00 \mathrm{E}+04$ & $1.49 \mathrm{E}+00^{c}$ & $b$ \\
\hline${ }^{106} \mathbf{R u}$ & 12 & $2.85 \mathrm{E}+02$ & $0.00 \mathrm{E}+00$ & $0.00 \mathrm{E}+00$ & $4.50 \mathrm{E}+01$ & $6.00 E+03$ & $7.50 \mathrm{E}-01$ & $b$ \\
\hline
\end{tabular}


Table 4.108 (continued)

\begin{tabular}{|c|c|c|c|c|c|c|c|c|}
\hline \multirow{2}{*}{ Radionuclide } & \multirow{2}{*}{$\begin{array}{l}\text { Number of } \\
\text { samples }\end{array}$} & \multicolumn{4}{|c|}{ Concentration $(\mathrm{pCi} / \mathrm{L})^{n}$} & \multirow[b]{2}{*}{ DCG } & \multirow[b]{2}{*}{$\begin{array}{l}\text { Percent of } \\
\text { DCG }\end{array}$} & \multirow[b]{2}{*}{$\begin{array}{c}\text { Sum of the fractions } \\
\text { of the DCGs }\end{array}$} \\
\hline & & Max & Min & Median & Average & & & \\
\hline${ }^{40} \mathrm{~K}$ & 12 & $4.09 \mathrm{E}+02$ & $0.00 \mathrm{E}+00$ & $0.00 \mathrm{E}+00$ & $5.83 \mathrm{E}+01$ & $7.00 \mathrm{E}+03$ & $8.32 \mathrm{E}-01$ & $b$ \\
\hline Gross alpha & 12 & $5.36 \mathrm{E}+00$ & $-2.51 \mathrm{E}+00$ & $-8.64 \mathrm{E}-01$ & $-1.94 \mathrm{E}-01$ & $b$ & $b$ & $b$ \\
\hline Gross beta & 12 & $8.25 E+00$ & $-3.49 \mathrm{E}+00$ & $1.36 \mathrm{E}+00$ & $1.57 \mathrm{E}+00$ & $b$ & $b$ & $b$ \\
\hline All listed isotopes & & $b$ & $b$ & $b$ & $b$ & $b$ & $b$ & $2.26 \mathrm{~F} 01^{r}$ \\
\hline
\end{tabular}

.

Not applicable.

This calculated value includes sampling results that are at or below the detection limits and/or below background activities. 
Table 4.109. K-25 Site NPDES sampling and analysis frequencles

\begin{tabular}{|c|c|c|c|c|}
\hline $\begin{array}{c}\text { Discharge } \\
\text { point }\end{array}$ & $\begin{array}{c}\text { Effluent } \\
\text { parameters }\end{array}$ & $\begin{array}{c}\text { Sampla } \\
\text { type }\end{array}$ & $\begin{array}{c}\text { Sample } \\
\text { Irequency }\end{array}$ & $\begin{array}{c}\text { Analysis } \\
\text { froquency a }\end{array}$ \\
\hline \multicolumn{5}{|l|}{005} \\
\hline (K-1203 Sewage & Ammonia nitrogon & grab & 3/woek & \\
\hline \multirow{12}{*}{ Treatment Facility) } & Biochemicel oxvgen demand & 24 hour composite b & 3/week & \\
\hline & Chlorine, total residual & grab & daily & \\
\hline & Dissolved oxygen & grab & daily & \\
\hline & Focel coliform & grab & 3/wook & \\
\hline & Flow & rocorder & continuous & \\
\hline & LC-50, Coriodaphnia & $7 \cdot 24$ hour composites & bimonthly & \\
\hline & LC-50. Pimophales & $7 \cdot 24$ hour composites & bimonthly & \\
\hline & NOEL, Ceriodaphnia & $7-24$ hour composites & bimonthly & \\
\hline & NOEL. Pimophalos & 7.24 hour composites & bimonthly & \\
\hline & $\mathrm{pH}$ & grab & daily. & \\
\hline & Sottleable solids & grab & $5 /$ week & \\
\hline & Suspended solids & composito & $3 /$ week b & \\
\hline \multicolumn{5}{|l|}{009} \\
\hline (K-1515-C Sanitary & Aluminum & grab & woekly & \\
\hline \multirow[t]{5}{*}{ Wator Plant) } & Chlorine, total residual & grab & weekly & \\
\hline & Flow & instantaneous & daily & \\
\hline & $\mathrm{pH}$, standard units & grab & weekly & \\
\hline & $\mathrm{pH}$ & grab & woekly & \\
\hline & Settleable solids & grab & wookly & \\
\hline \multicolumn{5}{|l|}{011} \\
\hline (K-1407-J Contral & 1,1,1-Trichloroethane & grab & monthly & \\
\hline \multirow[t]{16}{*}{ Neutralization Facility) } & Acetone & grab & quarterly & \\
\hline & Acetonitrile & grab & quarterly & \\
\hline & Benzene & grab & monthly & \\
\hline & Bromotarm & grab & monthly & \\
\hline & Cadmium & composito & quarterly & \\
\hline & Carbon tetrachloride & grab & monthly & \\
\hline & Chemical oxygen demand & composite & weekly & \\
\hline & Chloride, total & composite & $4 /$ week & \\
\hline & Chlorine, total residual & grab & $2 /$ week & \\
\hline & Chlorodibromomethane & grab & monthly & \\
\hline & Chlorolorm & grab & monthly & \\
\hline & Chromium & composite & quarterly & \\
\hline & Copper & composite & quarterly & \\
\hline & Dichlorobromomethane & grab & monthly & \\
\hline & Ethylbenzene & grab & quarteriv & \\
\hline & Flow & recorder & continuous & \\
\hline
\end{tabular}


Tah. 109 (continued)

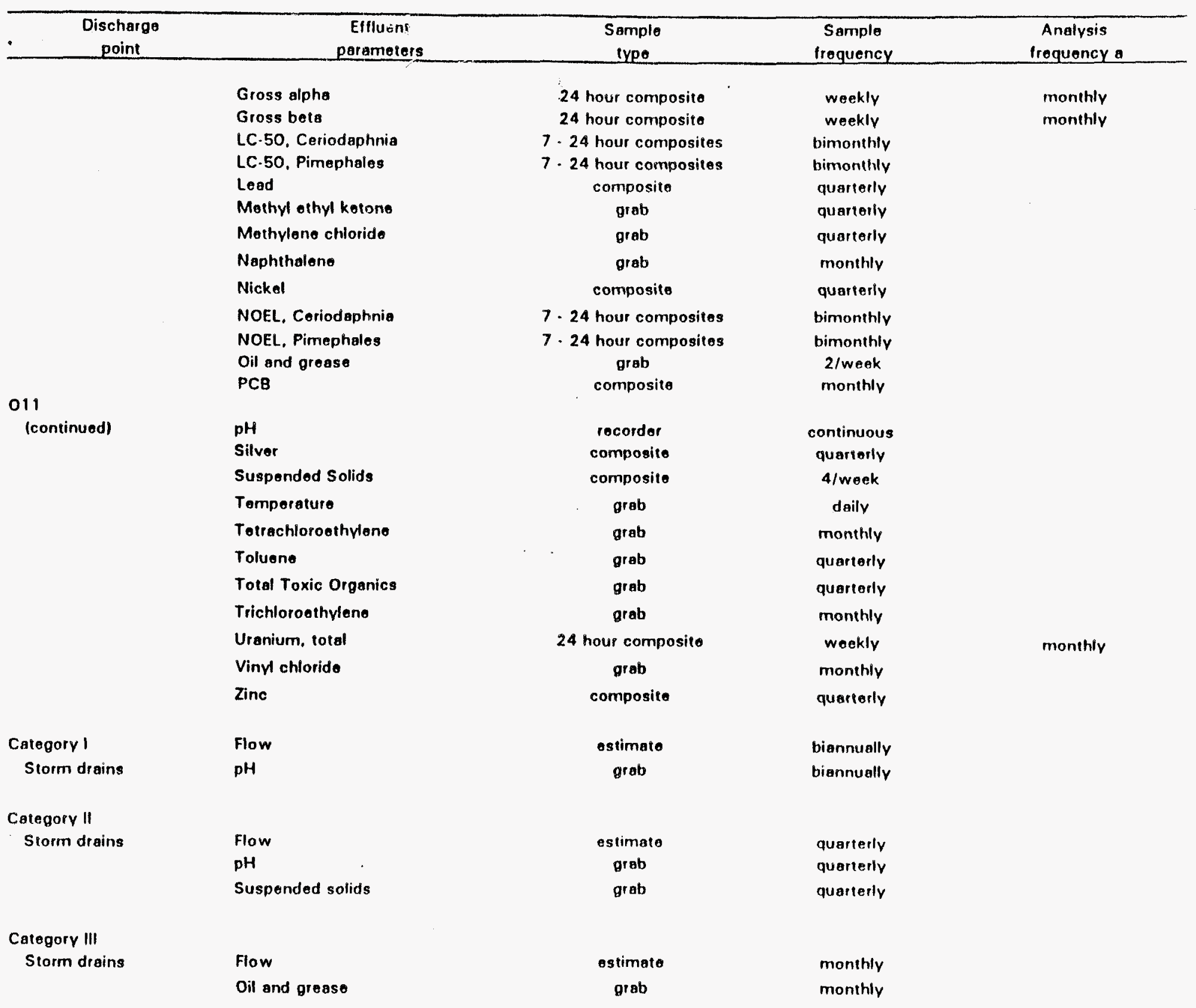


Table 4.109 (continued)

\begin{tabular}{|c|c|c|c|c|}
\hline $\begin{array}{c}\text { Discharge } \\
\text { point }\end{array}$ & $\begin{array}{c}\text { Effluent } \\
\text { parameters }\end{array}$ & $\begin{array}{c}\text { Sarnplo } \\
\text { type }\end{array}$ & $\begin{array}{c}\text { Sample } \\
\text { Irequency }\end{array}$ & $\begin{array}{c}\text { Analysis } \\
\text { froquency a }\end{array}$ \\
\hline & $\begin{array}{l}\mathrm{pH} \\
\text { Suspended solids }\end{array}$ & $\begin{array}{l}\text { grab } \\
\text { grab }\end{array}$ & $\begin{array}{l}\text { monthly } \\
\text { monthly }\end{array}$ & \\
\hline $\begin{array}{l}\text { Category IV } \\
\text { Storm drains }\end{array}$ & $\begin{array}{l}\text { Chlorine, total residual } \\
\text { Flow } \\
\text { Oil and grease } \\
\text { pH } \\
\text { Suspended solids }\end{array}$ & $\begin{array}{c}\text { grab } \\
\text { estimate } \\
\text { grab } \\
\text { grab } \\
\text { grab }\end{array}$ & $\begin{array}{l}\text { wookly } \\
\text { weekly } \\
\text { weekly } \\
\text { weekly } \\
\text { weekly }\end{array}$ & \\
\hline
\end{tabular}

a Analysis frequency is identical to sample frequency unless otherwise noted.

b Sampled at influent and effluent locations. Influent sampling data aro used for operational purposes only. 
Table 4.110. 1993 NPDES Permit Number TN 0002950

Peremeter
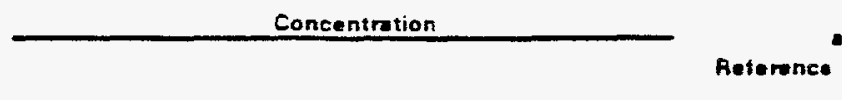

Number of vatuer

Numbar of

eamples

$\operatorname{Max}$

Min

Value

excending

pterence

Direharge Paint SD 05A

Flow, MGD

Oil and Gresese, mo/L

Totat Suspended Salids, mg/L

pH, Standard Unit:

Chbrine, Tatal Aesidual, mg/h

Flow. MGD

Oil and Groese, $\mathrm{ma} / \mathrm{L}$

Total Suspended Solide. mg/L

pH. Stenderd Unite

Flow. MGD

Tatal Sukponded Solids, mo/t

Standerd Unite

\section{Flow. MGD}

Oil and Greese, mo/L

Total Suepended Solide, $\mathrm{mg} / \mathrm{L}$

oH. Standard Units

Chborine, Total Rasidual, mg/L

Flow, GPD

Oil and Greese. mg/L

Total Suapended Solids, mo/L

pH, Standard Units

Chlorine, Total Residual, mo/l

Flow. MGD

Oil and Greese, mg/L

Total Suspended Solids, ma/L

p' Sndard Units

$\begin{array}{ll}11 & 0.0750 \\ 11 & 2.3 \\ 11 & 35 \\ 11 & 8.1\end{array}$

Discharge Podnt SD 100

$\begin{array}{ll}119 & 1.27 \\ 52 & 32.3160 \\ 52 & 5.5 \\ 52 & 114 \\ 52 & 9.2\end{array}$

Diseharge Paint SO 710

$\begin{array}{ll}3 & 0.0782 \\ 3 & 158 \\ 3 & 7.7\end{array}$

Diseherge Point 50720

$\begin{array}{ll}10 & 0.7756 \\ 10 & 2.4 \\ 10 & 25 \\ 10 & 7.7\end{array}$

Discherge Poin: SO 124

$\begin{array}{llll}47 & 0.31 & <0.05 & <0.07 \\ 46 & 45654 & 54 & 3409 \\ 46 & 2.6 & <2.0 & <2.1 \\ 46 & 17 & <1 & <3 \\ 46 & 8.8 & 7.0 & b\end{array}$

Diseharge Paint 50730

$\begin{array}{llcl}52 & 0.20 & <0.05 & <0.06 \\ 52 & 5.2261 & 0.0055 & 0.5320 \\ 52 & 2.6 & <2.0 & <2.0 \\ 52 & 89 & 2 & 13 \\ 52 & 8.2 & 7.0 & 0\end{array}$

$\begin{array}{ll}<0.05 & <0.34 \\ 0.0533 & \\ <2.0 & <1.1214 \\ <1 & <6 \\ 7.4 & b\end{array}$

0.001
8
7.5

0.0243

60

b

$\begin{array}{cc}b & b \\ b & b \\ 4.0 / 6.0 & 0\end{array}$


Table 4.110 (continued)

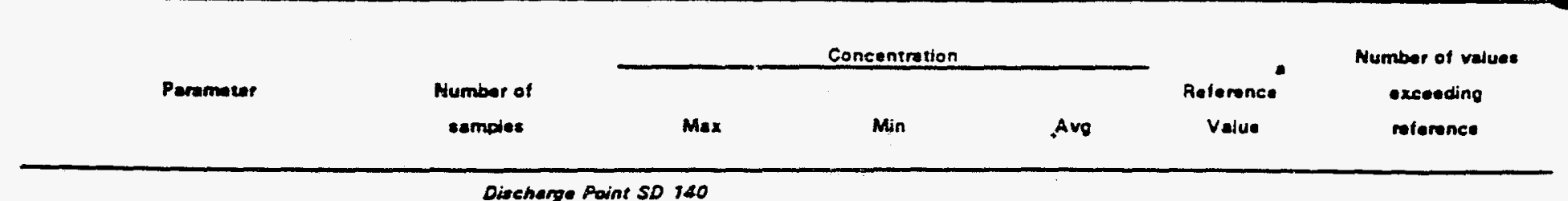

Dischange Point so 140

Flow, GPO

Total Suspended Solids, mo/l pH, Standard Units

$\begin{array}{ll}4 & 10963 \\ 4 & 34 \\ 4 & 8.0\end{array}$

Dtecherge Palat SD 142

Flow. MGD

Oil and Grosea. mo/L

Total Susponded Salids, mo/l

pH. Standard Unite

$\begin{array}{ll}12 & 0.2517 \\ 12 & 7.3 \\ 12 & 17 \\ 12 & 8.5\end{array}$

Discherge Polnt So 144

Flow. MGD

Oil and Graese. mo/L

Total Sueponded Solids, mo/h pH. Standard Unit:

$\begin{array}{ll}10 & 0.4226 \\ 10 & 3.3 \\ 10 & 17 \\ 10 & 8.0\end{array}$

Diecharge Point so 746

Flow, MGo

Oil and Greese, mg/L

Totel Suspended Solids, mo/L pH. Standerd Units

\section{Flow, GPD}

Oil and Grease, mo/L

Total Suspended Solids. mo/L ph. Standerd Urits

$\begin{array}{ll}9 & 0.5007 \\ 9 & <2.0 \\ 9 & 57 \\ 9 & 8.8\end{array}$

Dheharge Point SO 148

\begin{tabular}{|c|c|c|}
\hline 0.0015 & & 0.1610 \\
\hline$<2.0$ & $<$ & 2.2 \\
\hline$<1$ & $<$ & 7 \\
\hline 6.9 & & $b$ \\
\hline
\end{tabular}

\begin{tabular}{|c|c|c|}
\hline 0.0002 & & 0.0356 \\
\hline 2.0 & $<$ & 2.6 \\
\hline 1 & $<$ & 4 \\
\hline 6.4 & & $b$ \\
\hline
\end{tabular}

\section{b}

b

b

4.019.0

$\begin{array}{cc}b & b \\ b & b \\ 40 / 9.0 & 0\end{array}$


Table 4.110 (continued)

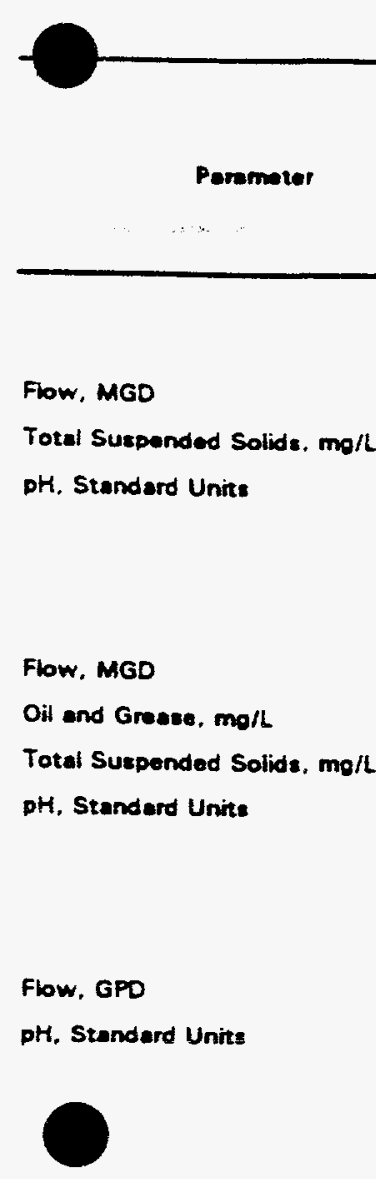

Fiow. GPD

Total Suspended Solids, mo/l pH. Standard Units

Flow. GPD

Total Surpended Solids, mo/h pH, Standard Units

Flow, MGO

Oil and Grease, $m g / h$

Totel Suspended Solids, $\mathbf{m g} / \mathrm{h}$

Flow, GPD
Number of

sampios

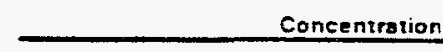

$\max$
Concentration

$\operatorname{Min}$

Discharge Paint SD 152

$\begin{array}{ll}4 & 0.2074 \\ 4 & 138 \\ 4 & 8.1\end{array}$

Dkeharge Paint SD 154

$\begin{array}{llll}10 & 0.3623 & 0.0190 & 0.0893 \\ 11 & 4.1 & <2.0 & <2.3 \\ 11 & 12 & 1 & 5 \\ 11 & 8.1 & 6.7 & 0\end{array}$

Okeherge Point SO 156

$\begin{array}{ll}1 & 190 \\ 1 & 7.3\end{array}$

190

190

7.3

b
Number of velues:

exeneding

moterence

\section{Diseharge Point SD 158}

$\begin{array}{llcccc}4 & 16878 & 380 & 6685 & b & b \\ 4 & 2 & <1 & <2 & b & b \\ 4 & 7.9 & 7.0 & b & 4.0 / 9.0 & 0\end{array}$

\section{Dlecherge Paint SD 160}

$\begin{array}{llcccc}3 & 11520 & 495 & 4697 & b & b \\ 3 & 2 & <1 & < & 1 & b \\ 3 & 7.8 & 7.4 & 0 & 4.019 .0 & 0\end{array}$

\section{Dheharge Paint SD 162}

$\begin{array}{ll}9 & 0.4955 \\ 9 & 2.0 \\ 9 & 16 \\ 9 & 8.0\end{array}$

0.0300

0.1890

$b$

4.019 .0

b

$b$

$4.0 / 5.0$

$b$

pH, Standard Unit:

Total \$ssponded Solids, mg/L

pH. Yard Units

\section{Diseherge Paint SD 168}

$\begin{array}{ll}2 & 760 \\ 2 & 1 \\ 2 & 8.5\end{array}$

2.0
1
6.8

6.8

$<2.0$

5

$b$

$\begin{array}{cc}b & b \\ 4.019 .0 & b\end{array}$

(1)


Table 4.110 (continued)

\begin{tabular}{|c|c|c|c|c|c|c|c|c|}
\hline \multirow{2}{*}{ Paramitor } & \multirow{2}{*}{$\begin{array}{l}\text { Number of } \\
\text { samples }\end{array}$} & \multicolumn{5}{|c|}{ Concentration } & \multirow{2}{*}{$\begin{array}{c}\text { Reterence } \\
\text { Value }\end{array}$} & \multirow{2}{*}{$\begin{array}{l}\text { Number of valuat } \\
\text { exceeding } \\
\text { meterence }\end{array}$} \\
\hline & & Max & & Min & & Avo & & \\
\hline \multicolumn{9}{|c|}{ Discherge Point SO 170} \\
\hline Chlorine. Total Rosidual, mo/l & 65 & 0.09 & $<$ & 0.05 & $<$ & 0.05 & $0.019 \mathrm{c}$ & 1 \\
\hline Flow. MGD & 52 & 0.4440 & & 0.1008 & & 0.1671 & $b$ & 6 \\
\hline Oil and Grease. mo/l & 52 & 3.2 & $<$ & 2.0 & & 2.1 & b & b \\
\hline Total Susponded Solids, mo/l & 52 & 17 & $<$ & 1 & $<$ & 2 & $b$ & 0 \\
\hline pH. Standerd Units & 53 & 8.8 & & 7.4 & & b & $6.0 / 9.0$ & 0 \\
\hline \multicolumn{9}{|c|}{ Discharge Palne SO 780} \\
\hline Chiorine, Total Rosiduel, mo/l & 67 & 0.16 & $<$ & 0.05 & $<$ & 0.06 & $0.019 c$ & 0 \\
\hline Flow, MGD & 53 & 0.1584 & & 0.0288 & & 0.0545 & $b$ & $b$ \\
\hline Oil and Gresese. mo/l & 53 & 3.7 & $<$ & 2.0 & $<$ & 2.1 & $b$ & $b$ \\
\hline Total Suspended Solids, ma/l & 53 & 26 & $<$ & 1 & $<$ & 2 & $b$ & $b$ \\
\hline pH. Standerd Units & 53 & 8.7 & & 7.5 & & $b$ & 6.019 .0 & 0 \\
\hline \multicolumn{9}{|c|}{ Dbeherge Paint SO 190} \\
\hline Charine, Total Aesidual, $\mathrm{mo} / \mathrm{L}$ & 65 & 0.22 & $<$ & 0.05 & $<$ & 0.06 & $0.019 e$ & b \\
\hline Flow. MGD & 53 & 0.6959 & & 0.000043 & & 0.1302 & $b$ & $b$ \\
\hline Oil and Grense. mo/l & 52 & 4.6 & $<$ & 2.0 & $<$ & 2.1 & $b$ & b \\
\hline Total Susponded Solids. ma/t & 52 & 176 & $<$ & 1 & $<$ & 7 & $b$ & $b$ \\
\hline sh. Standerd Unit: & 52 & 8.4 & & 7.9 & & b & 6.019 .0 & 0 \\
\hline
\end{tabular}

Dhecharge Point SO 792

Wow, GPD

H. Standerd Units

bw, GPO

$H$. Standard Units

ow. GPD

1. Standard Units
1

1

380

8.0

380

8.0

Oheharge Palat so 7 se

2

2

Obehorge Paint SD 196
76

7.9
1141
7.0

2853

b

76

\section{6}

b
380

b

4.019 .0
0
7.9

1
4.0/9.0 b

0 
Table 4.110 (continued)

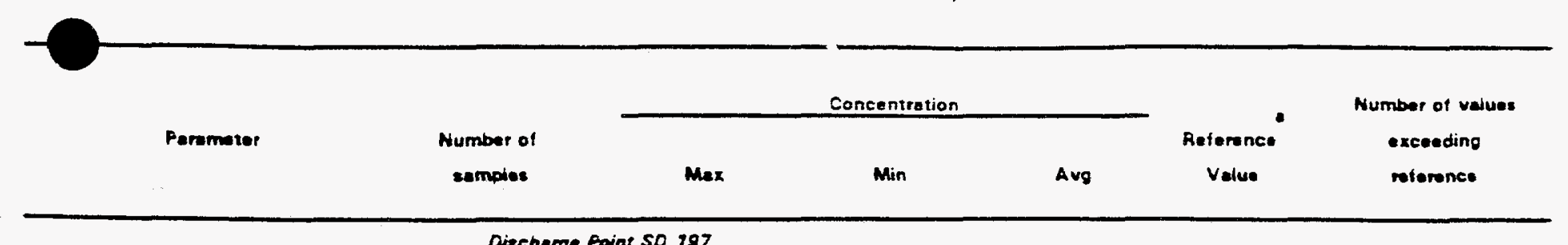

Discherge Point SO 797

Flow, MGD

Oil and Grease, mo/L

Total Suspended Solids, mo/h pH. Stenderd Unit:

Flow, MOD

Total Suspended Solids, mo/L pH, Standand Unite

Oil and Gresese. $m g / L$

Tatal Suspended Solids, mo/L pH gderd Unit:

Flow. MGD

Totel Suspended Solids, $\mathrm{mg} / \mathrm{L}$ pH. Standard Units

Flow, MGD

Oil and Greses, $\mathrm{mg} / \mathrm{L}$

Total Suspended Solids, mo/L

pH, Standard Units

Flow, MGD

Oil and Grease, mo/L

Total Suspended Solids, mo/L

oH, Standard Units

$\begin{array}{ll}11 & 0.3501 \\ 11 & 2.4 \\ 11 & 82 \\ 11 & 8.4\end{array}$

Discherge Point SO 108

$\begin{array}{ll}6 & 0.0457 \\ 6 & 35 \\ 6 & 8.8\end{array}$

Dbehere Point so 200

$\begin{array}{cccc}10 & 1.4184 & 0.0004 & 0.1901 \\ 10 & 2.0 & <2.0 & <2.0 \\ 10 & 102 & <1 & <23 \\ 10 & 8.2 & 6.9 & 0\end{array}$

Dischage Paint SD 210

$\begin{array}{llll}4 & 2.7894 & 0.0014 & 0.7379 \\ 4 & 68 & 13 & 43 \\ 4 & 7.9 & 6.6 & 0\end{array}$

Diecherge Paint SO 220

$\begin{array}{ll}7 & 0.6832 \\ 7 & 3.6 \\ 7 & 462 \\ 7 & 8.6\end{array}$

0.0008
$<2.0$
7
7.2

0.1289
$<\quad 2.4$
78
b

$b$
$b$
4019

b

b

b

0

Dischange Point SO 230

$\begin{array}{llllll}12 & 30.6664 & 0.0008 & 2.6548 & b & b \\ 12 & 3.5 & <2.0 & <2.9 & b & b \\ 12 & 16 & <1 & <3 & b & b \\ 12 & 8.6 & 7.0 & b & 4.019 .0 & 0\end{array}$


Table 4.110 (continued)

\begin{tabular}{|c|c|c|c|c|c|c|c|}
\hline \multirow{2}{*}{ Paramatar } & \multirow{2}{*}{$\begin{array}{c}\text { Number of } \\
\text { comples }\end{array}$} & \multicolumn{4}{|c|}{ Concentration } & \multirow{2}{*}{$\begin{array}{c}\text { Reference } \\
\text { Value }\end{array}$} & \multirow{2}{*}{$\begin{array}{l}\text { Number of values } \\
\text { exceeding } \\
\text { moterence }\end{array}$} \\
\hline & & Max & & $\operatorname{Min}$ & Avg & & \\
\hline \multicolumn{8}{|c|}{ Discherpe Point SO 240} \\
\hline Flow. MOD & 12 & 4.9123 & & 0.0038 & 0.4686 & $b$ & $b$ \\
\hline Oil and Graces. mo/L & 12 & 4.3 & $<$ & 2.0 & $<2.2$ & $b$ & $b$ \\
\hline Total Suspended Solids, mg/h & 12 & 41 & $<$ & 1 & $<\mathbf{8}$ & b & 0 \\
\hline oH. Standard Unit: & 12 & 8.4 & & 7.0 & b & $4.0 / 9.0$ & 0 \\
\hline
\end{tabular}

Dlecharge Paint so 242

Flow, GPD

pH. Standard Unite

Flow. MGD

Total Suspended Solids, mo/L pH. Standard Units

Totul Suspended Solids, mg/l pH, Standard Unite

Flow, MGD

pH. Standard Units

Flow, MGD

oH. Standard Units

How. GPD

H. Standand Units

\section{0}

$1 \quad 7.5$

Obeherge Paint SO 250

$\begin{array}{ll}2 & 1.7122 \\ 2 & 33 \\ 2 & 8.2\end{array}$

Dischargo Paint SO 254

$$
1
$$

1

1

\begin{abstract}
432
23

7.7
\end{abstract}

Dischergo Point SO 282

10.0256

7.0

Discherge Point SO 264

2

0.6900

7.7

Otscharge Paint SO 270

$1 \quad 673$

, 7.9
673
7.9

18540
7.5

7.5

0.0216
30
6.6

432

23

7.7

0.0256
7.0

0.0256

$b$

18540

b

b

4.0/9.0

b 
Table 4.110 (continued)

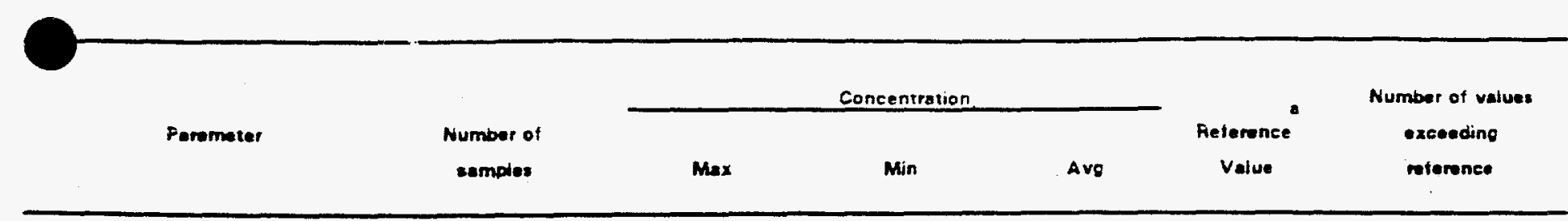

Discharge Point SO 280

Flow. GPD

pH. Standard Unit:

Flow, SFo

pH. Standerd Units

Fiow, GPD

pH. Stenderd Units

Flow, GPD

F Gandard Unit:

Flow. GFD

pH. Standard Unite

Flow, MGD

pH. Stendard Unite

Flow, GPD

pH. Standard Unit:

Flow, GPD

pH. Standard Units

$\begin{array}{ll}1 & 993 \\ 1 & 8.1\end{array}$

8. 1

Discharpe Point SO 290

14320

18.1

Dircharge Paint SO 292

295

27.8

Discharge Point SO 294

$\begin{array}{ll}1 & 12371 \\ 1 & 7.8\end{array}$

Diecharge Point SD 296

1

?

69369

7.6

Olscherge Paine so 297

2

2

Discherge Point SD 300

2

2

288

8.0

0.0457

8.1

0.000038

7.2

12379
7.8

92371

b

4320

b

79

$b$

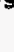

$4.0 / 9.0$ $b$

$4.0 / 9.0$ b

0

Discharge Point so 310

1141

17.4

$\begin{array}{ll}1141 & 1141 \\ 7.4 & b\end{array}$

$b$

0 
Table 4.110 (continued)

\begin{tabular}{|c|c|c|c|c|c|c|}
\hline \multirow{2}{*}{ Porematet } & \multirow{2}{*}{$\begin{array}{l}\text { Number of } \\
\text { semples }\end{array}$} & \multicolumn{3}{|c|}{ Concentration } & \multirow{2}{*}{$\begin{array}{l}\text { Reference } \\
\text { Velue }\end{array}$} & \multirow{2}{*}{$\begin{array}{c}\text { Numbet of values } \\
\text { exceeding } \\
\text { meterence }\end{array}$} \\
\hline & & Max & Min & Avg & & \\
\hline \multicolumn{7}{|c|}{ Diseherge Aaint SO 320} \\
\hline Flow. MGO & 2 & 0.5273 & 0.3176 & 0.4225 & $b$ & $b$ \\
\hline \multirow[t]{2}{*}{ pH. Standerd Units } & 2 & 7.7 & 7.4 & $b$ & 4.019 .0 & 0 \\
\hline & \multicolumn{2}{|c|}{ Diecherpe Aoint SD 322} & & & & \\
\hline Flow, GPD & 2 & 25885 & 4120 & 15003 & $b$ & $b$ \\
\hline \multirow[t]{2}{*}{ pH, Standard Unita } & 2 & 7.7 & 7.2 & $b$ & $4.0 / 9.0$ & 0 \\
\hline & \multicolumn{2}{|c|}{ Diseherge Paint SD 326} & & & & \\
\hline Flow, GPD & 2 & 176300 & 360 & 88330 & b & b \\
\hline \multirow[t]{2}{*}{ PH, Stendard Unite } & 2 & 7.8 & 7.6 & b & 4.019 .0 & 0 \\
\hline & \multicolumn{2}{|c|}{ Dineharge Paint SO 330} & & & & \\
\hline Flow. MGD & 3 & 1.7540 & 0.0031 & 0.6356 & $b$ & $b$ \\
\hline Total Suspended Solids. mg/t & 3 & 5 & $<1$ & $<4$ & $b$ & $b$ \\
\hline \multirow[t]{2}{*}{ ph. Standard Unita } & 3 & 7.9 & 7.6 & b & 4.019 .0 & 0 \\
\hline & \multicolumn{2}{|c|}{ Dbeharge Paint SO 332} & & & & \\
\hline Flow. MGD & 2 & 0.1553 & 0.1526 & 0.1540 & b & $b$ \\
\hline pH. Standard Units & 2 & 7.6 & 7.2 & $b$ & 4.019 .0 & 0 \\
\hline
\end{tabular}

\section{Discherge Paint SO 334}

Fiow, GPD

PH, Standard Units

Flow, MGO

pH. Standard Units

Flow, GPO

pH. Standard Units

$\begin{array}{ll}2 & 177400 \\ 2 & 7.3\end{array}$

2

Discherge Point SO 340

$\begin{array}{ll}3 & 0.5848 \\ 3 & 8.0\end{array}$

Dlecharge Point SO 350

144
7.3

7.3

0.0004
7.6

88772

b

0

4.019 .0

0

0
4

4

6575

7.9
432

7.3
0.2999

b
0
4.019 .0

$b$

0 
Table 4.110 (continued)

\begin{tabular}{|c|c|c|c|c|c|c|}
\hline \multirow{2}{*}{ Parmmeter } & \multirow{2}{*}{$\begin{array}{l}\text { Number of } \\
\text { enmpies }\end{array}$} & \multicolumn{3}{|c|}{ Concentretion } & \multirow{2}{*}{$\begin{array}{c}\text { Reference } \\
\text { Value }\end{array}$} & \multirow{2}{*}{$\begin{array}{l}\text { Number of values } \\
\text { exceeding } \\
\text { eference }\end{array}$} \\
\hline & & $\operatorname{Max}$ & Min & Avo & & \\
\hline \multicolumn{7}{|c|}{ Dbecherge Point SO 352} \\
\hline Flow. GPO & 2 & 495 & 144 & 320 & $\mathbf{b}$ & b \\
\hline Total Suspended Solids. mo/L & 2 & 4 & 3 & 4 & $\phi$ & $b$ \\
\hline oH. Standerd Unit: & 2 & 8.2 & 7.0 & $b$ & $4.0 / 9.0$ & 0 \\
\hline
\end{tabular}

Diecharge Point SD 360

Flow. MGD

pH. Standard Units

Fow, GFD

pH, Standard Units

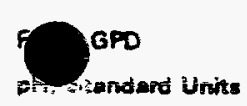

Flow. MGD

Dit and Grease, mo/L

Total Suspended Solids, mg/t

phi. Standerd Units

Flow. GPD

ph. Standerd Units

Flow. MGD

Oil and Gresee, $\mathrm{mo} / \mathrm{L}$

Total Suspended Solids, $\mathrm{mg} / \mathrm{h}$

pH. Standard Unit:

$\begin{array}{lll}0.1430 & 0.1430 & 0.1430 \\ 7.5 & 7.5 & b\end{array}$

1

i

Dirchage Paint sD 362

$\begin{array}{ll}2 & 2880 \\ 2 & 7.5\end{array}$

Dheherge Paint 50370

$\begin{array}{ll}1 & 31216 \\ 1 & 7.7\end{array}$

31216

7.7

31216

b

2525

7.4 - b

b

$\$ .0 / 9.0$

b

0

Dtseharge Point SO 380

$\begin{array}{ll}10 & 3.2631 \\ 10 & 2.0 \\ 10 & 8 \\ 10 & 8.5\end{array}$

Diseharge Point so 382

2

380

2

7.9

48

7.8

0.0038

$<2.0$

$<1$

6.4

.4

$<3$

b

214

b

4.019 .0

b

0

Diecharge Paint SO 390

$\begin{array}{llllll}6 & 0.8104 & 0.0014 & 0.2714 & b & b \\ 6 & 2.9 & <2.0 & <2.2 & b \\ 6 & 29 & 3 & 16 & b & b \\ 6 & 7.7 & 6.9 & b & 4.0 / 9.0 & 0\end{array}$


Table 4.110 (continued)

\begin{tabular}{|c|c|c|c|c|c|c|}
\hline \multirow{2}{*}{ Paramater } & \multirow{2}{*}{$\begin{array}{c}\text { Number of } \\
\text { samples }\end{array}$} & \multicolumn{3}{|c|}{ Concentration } & \multirow{2}{*}{$\begin{array}{c}\text { Reference } \\
\text { Value }\end{array}$} & \multirow{2}{*}{$\begin{array}{l}\text { Number of veiues } \\
\text { exceeding } \\
\text { noference }\end{array}$} \\
\hline & & Max & Min & Avg & & \\
\hline \multicolumn{7}{|c|}{ Discherge Paint SO 400} \\
\hline Flow. GPD & 3 & 761 & 360 & 614 & $\bullet$ & $b$ \\
\hline DH. Standard Unite & 3 & 7.9 & 7.2 & $b$ & 4.019 .0 & 0 \\
\hline \multicolumn{7}{|c|}{ Diseharge Paint SD 470} \\
\hline Flow. GPD & 3 & 3600 & 720 & 2400 & b & b \\
\hline PH. Standard Units & 3 & 7.8 & 7.4 & b & 4.019 .0 & 0 \\
\hline
\end{tabular}

PH. Stendard Uniti

Fiow, GPD

pH. Standard Unite

Fiow, MGD

Oil and Grease, $\mathrm{mg} / \mathrm{L}$

Tozal Suapended Solids, mo/h

pH. Standerd Units

Oil and Greese, mo/L

Total Suepended Salids. mo/L

pH. Standerd Units

Flow, MGD

pH. Standerd Units

Fiow. MGD

pH. Standard Units

pH. Standerd Units
3

7.8

7.4

Diseharge Point SO 420

$1 \quad 128970$

2

8.0

360

7.1

645030

$b$

4.0/9.0

$b$

0

Discharge Point SD 430

$\begin{array}{llll}12 & 12.1173 & 0.0023 & 1.2230 \\ 12 & 3.5 & <2.0 & <2.2 \\ 12 & 22 & <1 & <5 \\ 12 & 8.2 & 6.9 & b\end{array}$

\section{b}

b

$b$

4.0/9.0

\section{b}

$b$

b

0

Diecharge Paint SO 440

$\begin{array}{llllll}10 & 12.8190 & 0.0011 & 2.6405 & b & b \\ 10 & 2.9 & <2.0 & <2.1 & b & b \\ 10 & 131 & 1 & 19 & b & b \\ 10 & 7.6 & 7.0 & b & 4.0 / 9.0 & 0\end{array}$

Discherge Point SO 450

$\begin{array}{llllll}4 & 0.0404 & 0.0007 & 0.0177 & b & b \\ 4 & 8.0 & 7.1 & b & 4.019 .0 & 0\end{array}$

Direharge Poins SD 460

$\begin{array}{llllll}2 & 0.0573 & 0.0010 & 0.0291 & b & b \\ 2 & 7.7 & 7.1 & b & 4.0 / 9.0 & 0\end{array}$

Diseharge Paint SO 470

32283

38.0

144

7.6

929

b

$4.0 / 9.0$

b

b

0 
Table 4.110 (continued)

\begin{tabular}{|c|c|c|c|c|c|c|}
\hline \multirow[b]{2}{*}{ Parmeter } & & \multicolumn{3}{|c|}{ Concentration } & & \multirow{2}{*}{$\begin{array}{c}\text { Number of values } \\
\text { exceeding }\end{array}$} \\
\hline & Number of & & & & & \\
\hline & semples & $\operatorname{Max}$ & Min & Avg & Value & reference \\
\hline
\end{tabular}

Diecherge Point SO 480

Flow, MGD

Oil and Grease, $\mathrm{mg} / \mathrm{h}$

Total Suspended Solids, mo/L pH. Stenderd Units

Flow, MGD

Oil and Graese, $\mathrm{mg} / \mathrm{L}$

Total Suspended Solids, mo/L

pH. Standard Unite

Flow, GPD

nd Greese. $m o / L$

Surpended Solids, moht

pH. Stendard Units

Flow. MGD

pH. Stendard Units

\section{Fiow. MGD}

pH, Standard Units

Ftow, GPD

pH, Standard Units

Flow. GFO

pH. Standerd Units
11

11

11

11

Diacherge Aoint so 490

$\begin{array}{ll}11 & 11.9590 \\ 11 & 11.5 \\ 11 & 43 \\ 11 & 7.9\end{array}$

Discherpe Point 50510

$\begin{array}{ll}12 & 37598 \\ 12 & 5.1 \\ 12 & 11 \\ 12 & 8.2\end{array}$

Discharge Point SD 520

$\begin{array}{ll}1 & 2.4454 \\ 1 & 7.4\end{array}$

Diacharge Point SO 522

$\begin{array}{ll}1 & 0.0784 \\ 1 & 7.8\end{array}$

Discharge Point SO 532

$\begin{array}{ll}2 & 2880 \\ 2 & 8.0\end{array}$

Discharge Point SD 540

$\begin{array}{ll}3 & 87710 \\ 3 & 7.4\end{array}$

2.4454

7.4

\begin{tabular}{|c|c|c|}
\hline 0.0874 & & \\
\hline$<2.0$ & $<$ & \\
\hline$<1$ & $<$ & \\
\hline 6.8 & & \\
\hline
\end{tabular}

$\begin{array}{ll}0.0874 & 1.5479 \\ 2.0 & 3.0 \\ 1 & 11 \\ 6.8 & b\end{array}$

$\begin{array}{ll}3504 & 3135 \\ <2.0 & <2.4 \\ <1 & <5 \\ 6.7 & 0\end{array}$

3135

2.4454

$b$

4.0.9.0

b

o 
Table 4.110 (continued)

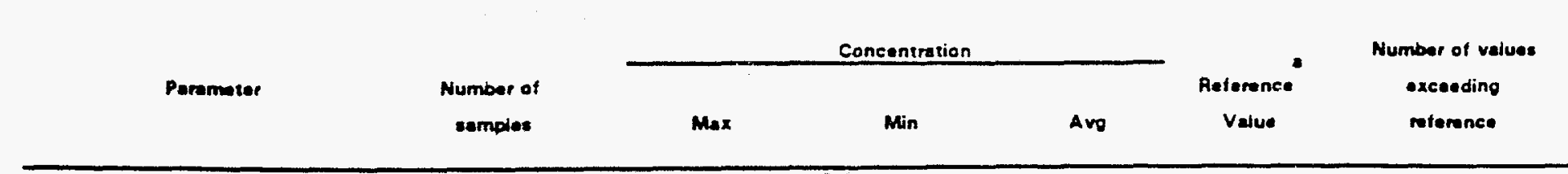

Olecherge Paint SO 550

Flow, GPD

pH. Standard Unit:

Flow. MGO

Oit and Gresce, ma/L

Total Suspended Solida, morh

pH. Standard Unita

Flow, MGO

pH, Standard Unite

Flow, MGD

pH. Standerd Units

Flow, GPD

pH. Standerd Units

Flow. MGD

Oil and Gresee. mo/L

Total Suspended Solids. $\mathrm{mo} / \mathrm{L}$

pH. Standard Unite

Flow. MGD

pH, Standard Units

Flow, GPC

pH. Standand Units

$\begin{array}{ll}3 & 35648 \\ 3 & 7.4\end{array}$

3

Obeherge Point so 560

$\begin{array}{ll}10 & 3.0309 \\ 10 & 2.8 \\ 10 & 15 \\ 10 & 8.1\end{array}$

Dtecharge Palnt SD 570

2

2

0.2046

8.0

Discharge Point SO 580

$\begin{array}{ll}2 & 0.0214 \\ 2 & 7.7\end{array}$

Diecherge Point SD 590

$1 \quad 576$

576

7.4

0.0043

7.4

7.4

Diseherge Paint SD 670

$\begin{array}{ll}7 & 1.8450 \\ 7 & 4.8 \\ 7 & 11 \\ 7 & 8.3\end{array}$

Dlseherge Aaint 50620

$\begin{array}{ll}1 & 0.2705 \\ 1 & 7.5 \\ \text { Diecherge Paint so } 630\end{array}$

1

2880

7.8
432

7.3
0.5416

< 2.2

$<6$

$b$
13947

b $b$

4.019 .0 b

0

$0.0058 \quad 0.1552$

7.4

b

4.019.0

b

0

4.019.0 0


Table 4.110 (continued)

Parminter

Parametor
Number of

sampias
Coneeneration

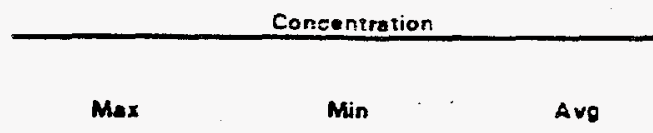

Number of values

exceeding

reterence
Flow. MGD

Totel Suspended Solids, $\mathrm{mo} / \mathrm{L}$ pH. Stendard Unin:

Flow. MGO

oH. Stendard Units

Flow. GPO

Total Suepended Solide, $\mathrm{mg} / \mathrm{L}$ pH. Standard Units

\section{Flow. GPD}

Total Suspended Solide, $m g / h$ pH. Standard Unite

\section{Flow. GPD}

Total Suspanded Salids, mo/h pH, Standard Unit:

\section{Discharge Paint SD 640}

$\begin{array}{ll}2 & 0.0345 \\ 2 & 15 \\ 2 & 8.0\end{array}$

\section{Dicchare Paint SD 550}

$\begin{array}{ll}1 & 0.0114 \\ 1 & 7.3\end{array}$

Discherge Paint 50660

$\begin{array}{ll}2 & 2064 \\ 2 & 350 \\ 2 & 8.2\end{array}$

\section{Discharge Point SO 670}

$\begin{array}{ll}2 & 1240 \\ 2 & 41 \\ 2 & 7.8\end{array}$

Dircherge Paint SD 680

$\begin{array}{ll}3 & 4421 \\ 3 & 8 \\ 3 & 7.7\end{array}$

Diseherg* Point SO 690

Flow, MGD

Oil and Grease, moll

Toral Suspended Solidt. mo/t pH. Standard Units

Flow, MGD

pH. Standard Units

Discharge Paint SO 692

$\begin{array}{lll}14.3177 & 0.0004 & 1.3543 \\ 3.1 & <2.0 & <2.1 \\ 20 & <1 & <3 \\ 8.1 & 6.7 & b\end{array}$

6.7
1001

30

b

0.0190
10
$b$

$b$

$\begin{array}{ll}3240 & 4027 \\ 1 & < \\ 7.4 & 5\end{array}$

b

b

$\begin{array}{cc}b & b \\ b & b \\ 0 & b \\ 4.019 .0 & 0\end{array}$

$\begin{array}{llllll}2 & 0.6472 & 0.0024 & 0.3248 & b & r \\ 2 & 7.7 & 6.9 & b & 4.0 / 9.0 & 0\end{array}$


Table 4.110 (continued)

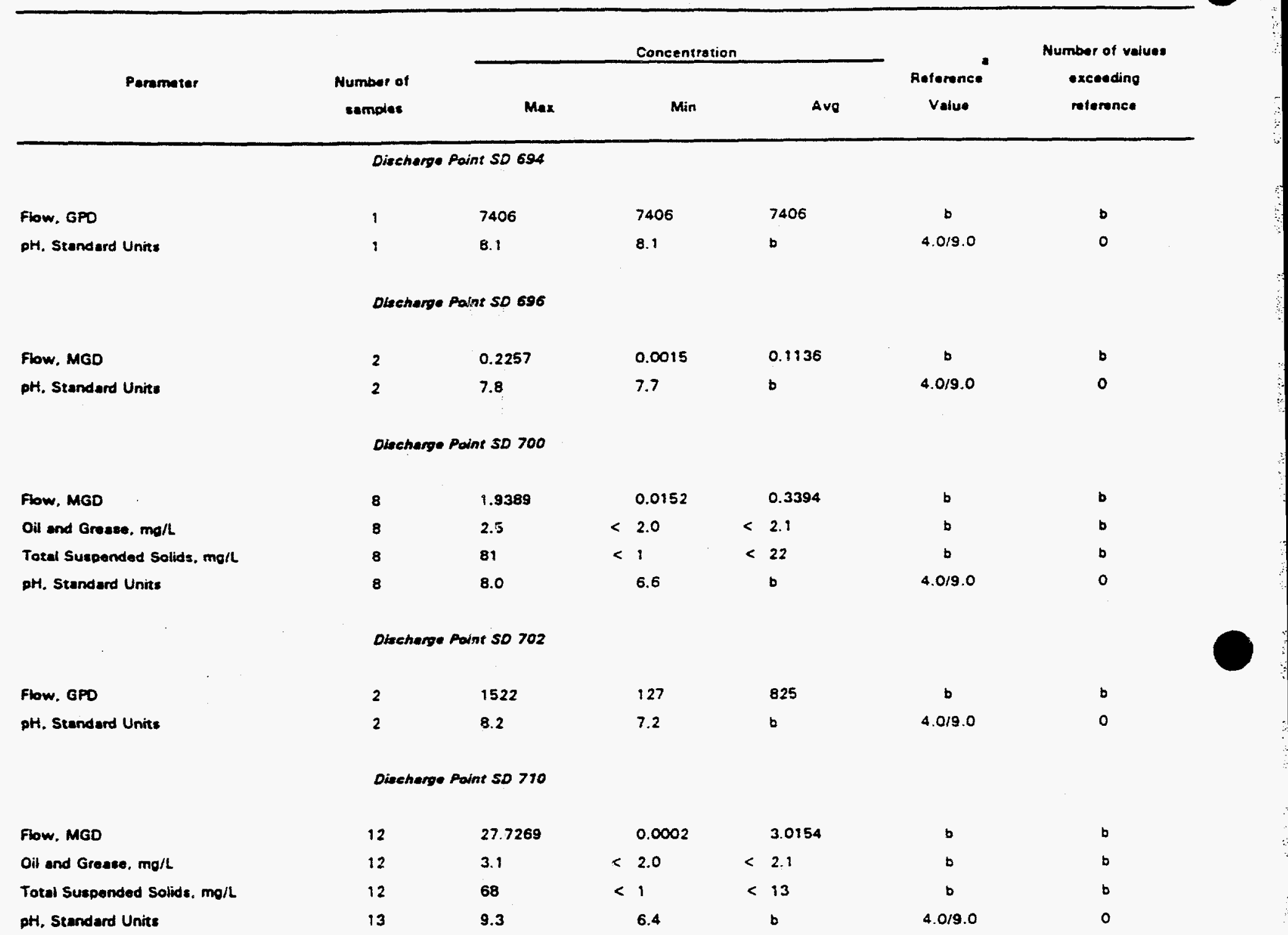

Direherge Point SD 712

Flow, MGD

Oil and Gresse. mo/L

Total Suspended Solids. morl

pH. Standard Units

$\begin{array}{ll}9 & 0.1837 \\ 9 & 3.5 \\ 9 & 15 \\ 9 & 7.9\end{array}$

$\begin{array}{ll}0.0014 & 0.0225 \\ 2.0 & 2.2 \\ 1 & 5 \\ 6.9 & b\end{array}$

b

b

b

4.019 .0

$b$
$b$
0

Discherge Point SD 720

Fow, MGD

Dil and Grease. mo/L

Total Suspended Solids. $\mathrm{mg} / \mathrm{h}$ pH. Standard Units

$\begin{array}{llcccc}8 & 1.4542 & 0.0004 & 0.2366 & b & b \\ 8 & 2.2 & <2.0 & <2.0 & b & b \\ 8 & 12 & <1 & <7 & b & b \\ 8 & 8.5 & 6.8 & b & 4.019 .0 & 0\end{array}$


Table iv.110 (continued)

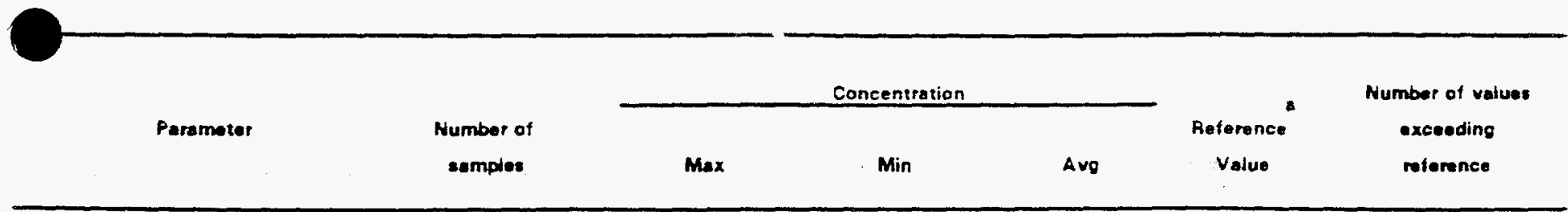

Discharge Foint SD 724

Flow, MGD

pH, Standard Units

pH. Standard Units

Flow. GPD

pta, Standard Units

Flow, GPD

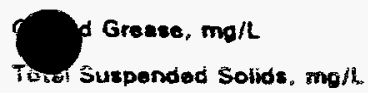

pH. Standard Units

$\begin{array}{ll}2 & 0.2859 \\ 2 & 8.0\end{array}$

Discharge Paint SO 730
457

8. 1
0.0582

7.7

23

7.5

Discharge Point SO 740

$3 \quad 15218$

3

\section{2}

7.2
0.1720

b

$4.0 / 9.0$

b

0

Discharge Point SD 750

$\begin{array}{lccc}5 & 14400 & 761 & 4706 \\ 5 & 2.0 & < & <.0 \\ 5 & 4 & 1 & 3.0 \\ 5 & 7.9 & 7.2 & b\end{array}$

$b$
$b$
$b$

Discharge Paint SD 760

Flow. MGD

Oil and Grease, mo/L

Total Suspended Solids. $\mathrm{mg} / \mathrm{L}$ pH. Standerd Units

Flow, GPD

Oil and Grease, mo/L

Total Suspended Solids. mo/L pH, Standard Units

0.0173
$<\quad 2.0$
3
8.1

8.1

$\begin{array}{ll} & 0.0057 \\ < & 0.0108 \\ < & <2.0 \\ 7.5 & <2 \\ 7.5 & b\end{array}$

7.5 b

o

Discharge Point SO 770

$\begin{array}{llll}3 & 285 & 76 & 215 \\ 3 & 2.0 & <2.0 & <2.0 \\ 3 & 8 & <1 & <3 \\ 3 & 8.3 & 7.5 & b\end{array}$

$\begin{array}{cc}b & b \\ b & b \\ b & b \\ 4.019 .0 & 0\end{array}$

Discharge Point SO 780

Fiow, MGD

Total Suspended Solids, $\mathrm{mg} / \mathrm{L}$ pH, Standard Units

0.0304
20
8.2

0.0304

4

4

4
8.2

$\begin{array}{ll}0.0001 & 0.0142 \\ 1 & 7 \\ 7.5 & \text { b }\end{array}$

$\begin{array}{cc}b & b \\ b & b \\ 4.019 .0 & 0\end{array}$


Table 4.110 (continued)

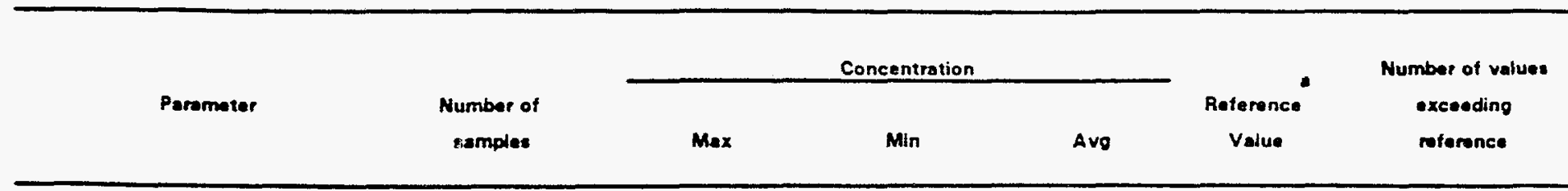

Discharge Paint SD 800

Flow, GPD

Total Suspended Solids, mo/L

pH. Standard Units

Flow, MGD

Total Suspended Solids, ma/l pH. Standard Unit:

Flow. GPD

Total Suspended Solids, mg/L pH. Standard Units

Flow. MGD

Total Suspended Solids, $\mathrm{mg} / \mathrm{h}$ pH. Standard Units

Flow, GPD

Total Suspended Solids, mo/L pH. Standard Units

Flow, GPD

Total Suspended Solids, mo/l pH. Standard Units

Flow, GPD

Total Suspended Solids, mg/L pH. Standard Units

$\begin{array}{ll}4 & 11413 \\ 4 & 28 \\ 4 & 8.2\end{array}$

Discherge Palnt SO 870

$\begin{array}{ll}3 & 0.0173 \\ 3 & 13 \\ 3 & 7.9\end{array}$

\section{Discharge Faint SD 820}

$\begin{array}{ll}3 & 11413 \\ 3 & 12 \\ 3 & 8.4\end{array}$

\section{Dlschenge Point SD 830}

$\begin{array}{llcc}3 & 0.3390 & 0.0685 & 0.2371 \\ 3 & 16 & <1 & <6 \\ 3 & 7.9 & 7.4 & b\end{array}$

\section{Olechare Point SO 850}

$\begin{array}{ll}4 & 9511 \\ 4 & 5 \\ 4 & 8.1\end{array}$

\section{Dbeherge Point SO 860}

$\begin{array}{ll}2 & 380 \\ 2 & 15 \\ 2 & 8.0\end{array}$

Discharge Point so 870

$\begin{array}{ll}2 & 8640 \\ 2 & 4 \\ 2 & 7.8\end{array}$

3804
3
7.2

6222

4

b

253

9

b

6594

8

b

b

b

0 
Table 4.110 (continued)

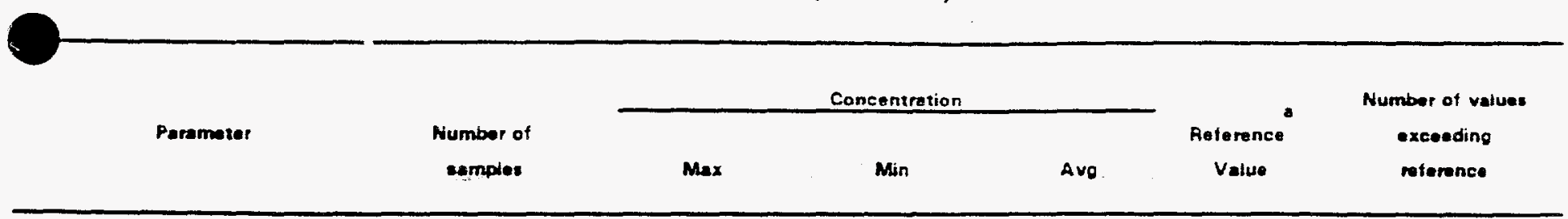

Discherge Point SD 880

Flow, MGD

Total Suxpanded Solide, $\mathbf{m g} / \mathrm{L}$ pH. Standard Units:

$\begin{array}{ll}3 & 0.0216 \\ 3 & 10 \\ 3 & 8.0\end{array}$

Discherge Point SO 890

Flow. MGD

Total Sunpended Solide, mo/l pH, Stenderd Unit:

$\begin{array}{ll}3 & 0.0230 \\ 3 & 6 \\ 3 & 7.8\end{array}$

3
Diacharpe fount SD 900

\section{Flow, GPD}

Totel Suepended Solide, mo/L pH, Standard Units

\section{Fiow. GPD}

pH, Standard Units.

Flew, Geo

pH. Standard Units

Flow, GPD

pH, Stenderd Unit:

Flow, GPD

pH. Standard Units

Flow, GPD

nderd Units
3

3

3

Otscharge Paint SD 970

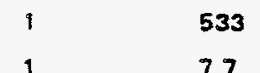

Discharge Point so 920

$\begin{array}{ll}\hat{1} & 5760 \\ 1 & 7.2\end{array}$

Discherge Point SO 930

2

2

12240

6. 1

Discherge Palnt 50934
8.2
1522

7.9
5760

7.2

266

7.8
0.0046

2

7.3

751

2

7.2

533

7.7

$b$

5760

$b$

3170

3

$b$
0.0119

4

$b$

$\begin{array}{cc}b & b \\ b & b\end{array}$

4.0/9.0 
Table 4.110 (continued)

\begin{tabular}{|c|c|c|c|c|c|c|}
\hline \multirow{2}{*}{ Paramater } & \multirow{2}{*}{$\begin{array}{l}\text { Number of } \\
\text { semples }\end{array}$} & \multicolumn{3}{|c|}{ Concentration } & \multirow{2}{*}{$\begin{array}{c}\text { Reference } \\
\text { Value }\end{array}$} & \multirow{2}{*}{$\begin{array}{l}\text { Number of values } \\
\text { exceeding } \\
\text { meference }\end{array}$} \\
\hline & & $\operatorname{Max}$ & Min & Avg & & \\
\hline \multicolumn{7}{|c|}{ Discharge Paint SO 960} \\
\hline Flow, GPD & 2 & 17280 & 2160 & 9720 & $b$ & b \\
\hline pH. Standard Unit: & 2 & 7.7 & 7.5 & $b$ & $4.0 / 9.0$ & 0 \\
\hline \multicolumn{7}{|c|}{ Dircharge Point so 970} \\
\hline Fow, GPD & 2 & 419400 & 720 & 210060 & b & $b$ \\
\hline pHt, Standard Units & 2 & 7.9 & 6.6 & b & 4.019 .0 & 0 \\
\hline \multicolumn{7}{|c|}{ Diseherge Paint SO 980} \\
\hline Flow; GPD & 2 & 266900 & 720 & 133810 & b & $b$ \\
\hline pHt, Standard Units & 2 & 7.6 & 6.6 & b & $4.0 / 9.0$ & 0 \\
\hline
\end{tabular}

\section{Discherge Point SD 982}

Frow, GPD

pht, Standand Units

$2 \quad 226300 \quad 360$

113330

b

4.0/9.0

$b$

2

7.6

7.3

b

o

Direharge Paint SO 990

Flow, GPO

pH. Standard Units

Flow, MGD

ail and Greese, $m$ g/L

Totat Suspended Solids. mo/L

pH. Standerd Units

2

2

2164

8.0

761

7.8

1463

b

$b$

4.019 .0

Discharge Point SD S92

$\begin{array}{ll}10 & 1.2479 \\ 10 & 2.1 \\ 10 & 299 \\ 10 & 7.7\end{array}$

0.0011

0.4201

$<2.0$

$<2.0$

10

62

5.0

b

Discherge Point so $994^{\circ}$

Flow, GPD

Oil and Grease. $\mathrm{mg} / \mathrm{L}$

Total Suspended Solids, ma/h

pht. Standard Units

\begin{tabular}{|c|c|c|c|c|c|c|}
\hline 6 & & 6087 & & 114 & & 1361 \\
\hline 6 & $<$ & 2.0 & $<$ & 2.0 & $<$ & 2.0 \\
\hline 6 & & 3 & $<$ & 1 & $<$ & 2 \\
\hline 6 & & 8.2 & & 6.5 & & \\
\hline
\end{tabular}

b

$\begin{array}{cc}b & b \\ b & b \\ b & b \\ 4.019 .0 & 0\end{array}$

4.019 .0 
Table 4.110 (continued)

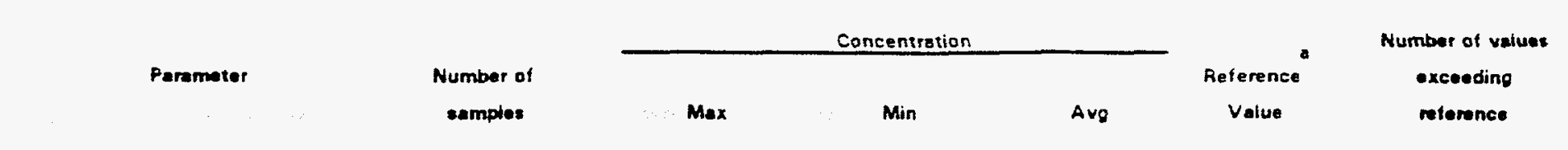

Discherge Point SO 996

Flow, GPD

pH. Standard Units
2

\begin{abstract}
11520
\end{abstract}
8.0
5707

7.9
8614

b b

$4.0 / 9.0$ b

0

$a=$ NPDES permit limit

$b=$ not applicable

$c=$ NPOES permit fimit became effective $10 / 1 / 93$

$\checkmark=$ storm drain was removed from permit during 1993

$e=$ stom drain was sealed during 1993 
Table 4.111. 1993 NPDES Permit Number TN 0002950

Discharge Point K-1203, Sewage Treatment Plant, K-25 Site

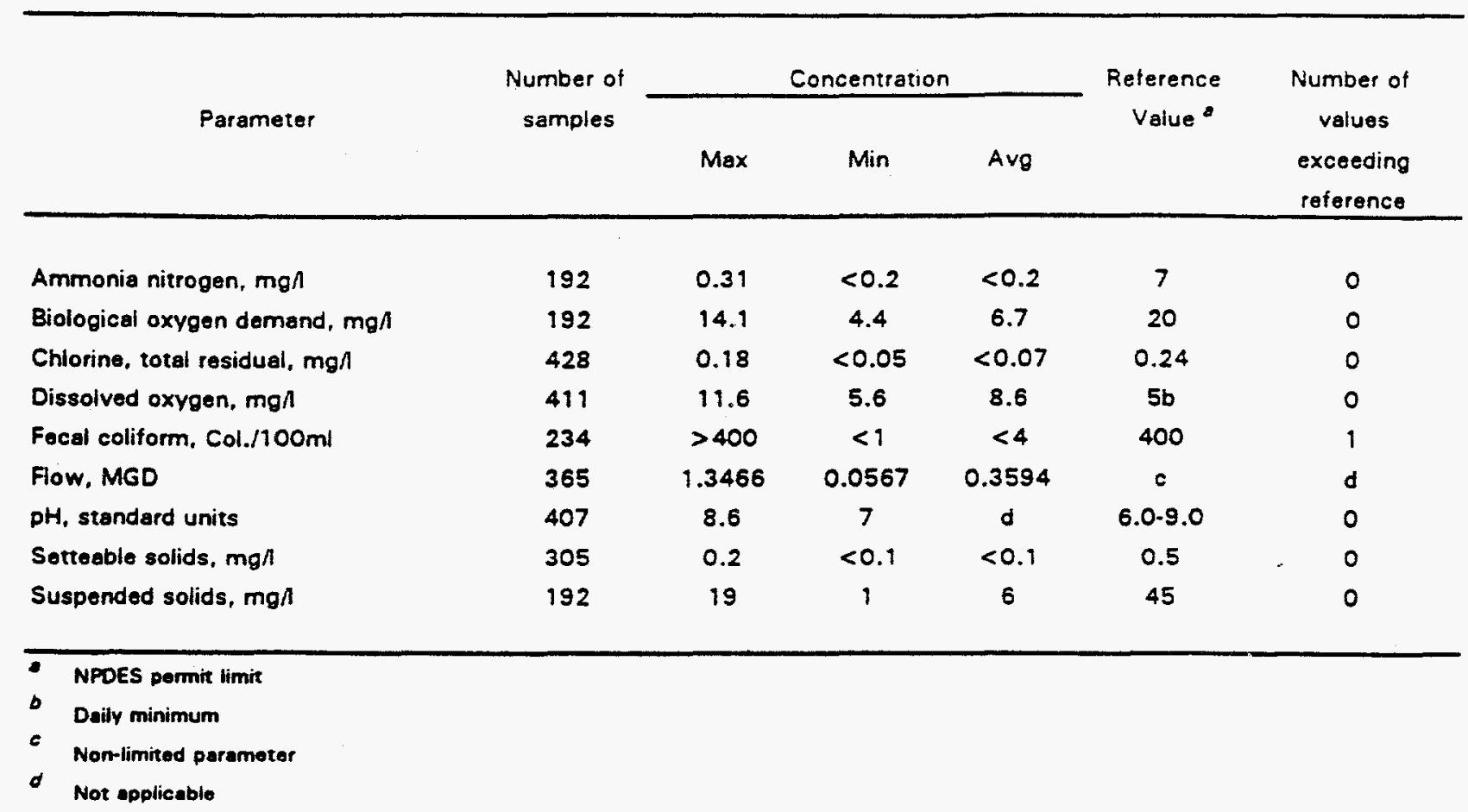


Table 4.112. 1993 NPDES Permit Number TN 0002950

Discharge Point K-1407-J, Treatment Pond at K-25 Site

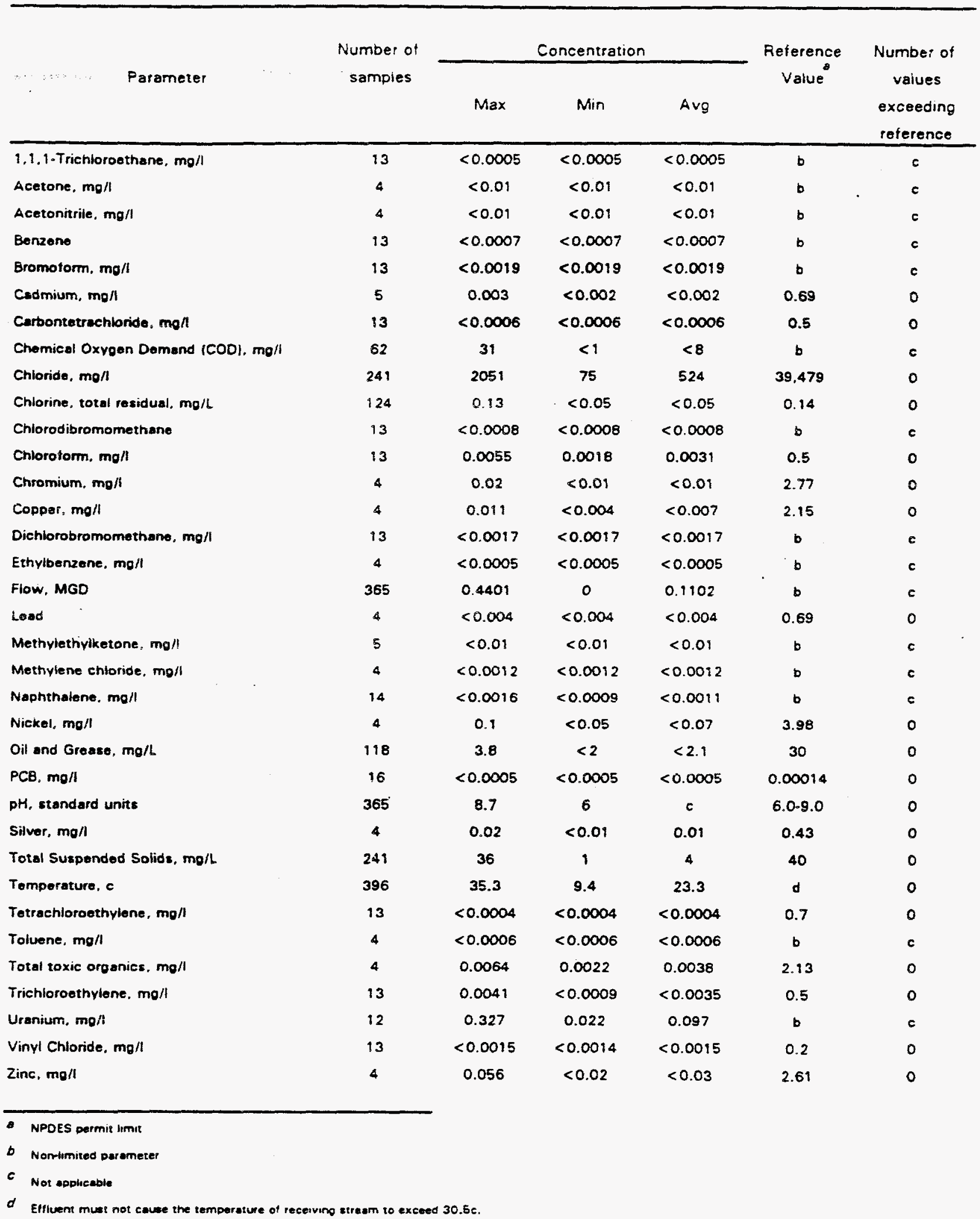


Table 4.113. 1993 NPDES Permit Number TN 0002950

Discharge Point K-1515-C, Holding Pond, K-25 Site

\begin{tabular}{|c|c|c|c|c|c|c|}
\hline \multirow{2}{*}{ Parameter } & \multirow{2}{*}{$\begin{array}{c}\text { Number of } \\
\text { samples }\end{array}$} & \multicolumn{3}{|c|}{ Concentration } & \multirow{2}{*}{$\begin{array}{c}\text { Reference } \\
\text { Value }\end{array}$} & \multirow{2}{*}{$\begin{array}{c}\text { Number of } \\
\text { values } \\
\text { exceeding } \\
\text { reference }\end{array}$} \\
\hline & & Max & Min & Avg & & \\
\hline Aluminum, $\mathrm{mg} /$ & 69 & 1.7 & 0.21 & 0.67 & 2.0 & 0 \\
\hline Chlorine, total residual, $\mathrm{mg} / \mathrm{h}$ & 70 & 0.25 & $<0.05$ & $<0.09$ & 1.0 & 0 \\
\hline Flow, MGD & 365 & 2.0685 & 0.0361 & 0.2336 & b & c \\
\hline pH, standard units & 70 & 8.5 & 6.1 & c & 6.0 .9 .0 & 0 \\
\hline Setteable solids, mg/ & 69 & 0.2 & $<0.1$ & $<0.1$ & 0.5 & 0 \\
\hline Suspended solids, $m g n$ & 69 & 32 & 2 & 8 & 40 & 0 \\
\hline $\begin{array}{l}\text { - NPDES permit limit } \\
\text { - Non-limited parameter } \\
\therefore \text { Not applicable }\end{array}$ & & & & & & \\
\hline
\end{tabular}


Table 5.1. External gamma radiation measurements at ORR perimeter air monitoring stations, 1993

\begin{tabular}{cccccc}
\hline & & \multicolumn{5}{c}{$\begin{array}{c}\text { Exposure rate } \\
(\mu \mathrm{R} / \mathrm{hr})\end{array}$} \\
\cline { 3 - 6 } $\begin{array}{c}\text { Station } \\
\text { No. }\end{array}$ & $\begin{array}{c}\text { No. of } \\
\text { measurements }\end{array}$ & Max & Min & Av & $\begin{array}{c}\text { Standard } \\
\text { error }\end{array}$ \\
\hline 35 & 32 & 11.9 & 0.4 & 7.4 & 0.3 \\
39 & 44 & 12.6 & 7.0 & 9.9 & 0.2 \\
40 & 44 & 9.4 & 4.1 & 7.6 & 0.1 \\
41 & 50 & 11.0 & 0.5 & 4.6 & 0.2 \\
42 & 48 & 21.8 & 1.0 & 7.4 & 0.4 \\
46 & 52 & 13.5 & 2.9 & 8.5 & 0.2 \\
48 & 50 & 11.0 & 3.4 & 7.0 & 0.2 \\
Average & 46 & 13.0 & 2.8 & 7.5 & 0.2 \\
\hline
\end{tabular}

${ }^{a}$ Standard error of the mean.

Table 5.2. Summary of collection and anaiysis frequencies for continuous high- and lowvolume samples at ORR ambient air monitoring stations

\begin{tabular}{lll}
\hline \multicolumn{1}{c}{ Parameter } & $\begin{array}{c}\text { Collection } \\
\text { frequency }\end{array}$ & $\begin{array}{c}\text { Analysis } \\
\text { frequency }\end{array}$ \\
\hline U, Pu, Th, Be, Am, Cm & Weekly & Monthly \\
Total rad Sr & Weekly & Monthly \\
Gamma scan (filter) & Weekly & Monthly \\
Tritium & Biweekly & Monthly \\
\hline
\end{tabular}

${ }^{a}$ All parameters are checked at all locations ( 35 , $37,38,39,40,42,46$, and remote locations 51 and 52). 
Table 5.3. ORA radionuclide concentrations in air, 1993

\begin{tabular}{|c|c|c|c|c|c|c|}
\hline \multirow[b]{2}{*}{ Determination } & \multirow{2}{*}{$\begin{array}{l}\text { No. detected/ } \\
\text { No. sampled }\end{array}$} & \multicolumn{4}{|c|}{$\begin{array}{l}\text { Concentration } \\
\left(10^{-15} \mu \mathrm{Ci} / \mathrm{mL}\right)^{a}\end{array}$} & \multirow[b]{2}{*}{ DCG \% } \\
\hline & & Average $^{b}$ & $\operatorname{Max}$ & Min & $\begin{array}{l}\text { Standard } \\
\text { error }\end{array}$ & \\
\hline \multicolumn{7}{|c|}{ PAMs } \\
\hline${ }^{241} \mathrm{Am}$ & $32 / 72$ & $1.37 \mathrm{E}-03$ & $1.90 \mathrm{E}-03$ & $6.77 \mathrm{E}-04$ & $1.51 \mathrm{E}-04$ & 0.0069 \\
\hline${ }^{7} \mathrm{Be}$ & $72 / 72$ & $8.18 E+01$ & $8.99 \mathrm{E}+01$ & $7.40 \mathrm{E}+01$ & $2.03 \mathrm{E}+00$ & 0.00016 \\
\hline${ }^{244} \mathrm{Cm}$ & $9 / 72$ & $3.05 \mathrm{E}-03$ & $9.51 \mathrm{E}-03$ & $1.34 \mathrm{E}-04$ & $1.40 \mathrm{E}-03$ & 0.0076 \\
\hline${ }^{60} \mathrm{Co}$ & $12 / 72$ & $8.53 E-02$ & $8.53 \mathrm{E}-02$ & $2.93 \mathrm{E}-02$ & $7.78 \mathrm{E}-03$ & 0.00007 \\
\hline${ }^{137} \mathrm{Cs}$ & $8 / 72$ & $3.82 \mathrm{E}-02$ & $5.51 \mathrm{E}-02$ & $1.40 \mathrm{E}-03$ & $8.21 \mathrm{E}-03$ & 0.00001 \\
\hline${ }^{238} \mathrm{Pu}$ & $15 / 72$ & $1.77 \mathrm{E}-03$ & $2.84 \mathrm{E}-03$ & $4.00 \mathrm{E}-04$ & $3.45 \mathrm{E}-04$ & 0.0059 \\
\hline${ }^{239} \mathrm{Pu}$ & $11 / 72$ & $9.66 \mathrm{E}-04$ & $1.32 \mathrm{E}-03$ & $3.59 \mathrm{E}-04$ & $1.51 \mathrm{E}-04$ & 0.0048 \\
\hline${ }^{228} \mathrm{Th}$ & $69 / 72$ & $7.86 E-03$ & $1.05 \mathrm{E}-02$ & $5.17 \mathrm{E}-03$ & $5.58 \mathrm{E}-04$ & 0.01964 \\
\hline${ }^{230} \mathrm{Th}$ & $68 / 72$ & $5.41 E-03$ & $6.71 E-03$ & $4.15 E-03$ & $3.34 \mathrm{E}-04$ & 0.01353 \\
\hline${ }^{232} \mathrm{Th}$ & $67 / 72$ & $6.40 \mathrm{E}-03$ & $8.10 \mathrm{E}-03$ & $3.72 \mathrm{E}-03$ & $5.18 \mathrm{E}-04$ & 0.0914 \\
\hline Total Sr & $35 / 72$ & $4.79 E-02$ & $6.53 \mathrm{E}-02$ & $3.00 \mathrm{E}-02$ & $3.69 \mathrm{E}-03$ & 0.00053 \\
\hline${ }^{3} \mathrm{H}$ & $49 / 99$ & $4.53 E+04$ & $7.38 \mathrm{E}+05$ & $1.56 \mathrm{E}+03$ & $1.58 \mathrm{E}+04$ & 0.0453 \\
\hline${ }^{234} \mathrm{U}$ & $71 / 72$ & $5.75 \mathrm{E}-02$ & $1.09 \mathrm{E}-01$ & $2.48 \mathrm{E}-02$ & $1.08 \mathrm{E}-02$ & 0.064 \\
\hline${ }^{235} \mathrm{U}$ & $55 / 72$ & $9.63 \mathrm{E}-03$ & $1.48 \mathrm{E}-02$ & $3.29 \mathrm{E}-03$ & $1.21 \mathrm{E}-03$ & 0.0096 \\
\hline${ }^{238} \mathrm{U}$ & $67 / 72$ & $1.93 \mathrm{E}-02$ & $2.24 \mathrm{E}-02$ & $1.61 \mathrm{E}-02$ & $8.53 \mathrm{E}-04$ & 0.0193 \\
\hline \multicolumn{7}{|c|}{$R A M s$} \\
\hline${ }^{241} \mathrm{Am}$ & $6 / 18$ & $3.09 \mathrm{E}-03$ & $4.25 \mathrm{E}-03$ & $1.92 \mathrm{E}-03$ & $1.17 \mathrm{E}-03$ & 0.01544 \\
\hline${ }^{7} \mathrm{Be}$ & $18 / 18$ & $9.11 E+01$ & $9.78 \mathrm{E}+01$ & $8.44 \mathrm{E}+01$ & $6.68 \mathrm{E}+00$ & 0.00018 \\
\hline${ }^{244} \mathrm{Cm}$ & $3 / 18$ & $2.82 \mathrm{E}-03$ & $3.56 \mathrm{E}-03$ & $2.05 \mathrm{E}-03$ & $7.68 \mathrm{E}-04$ & 0.0074 \\
\hline${ }^{60} \mathrm{Co}$ & $1 / 18$ & $7.09 \mathrm{E}-03$ & $7.09 \mathrm{E}-03$ & $7.09 \mathrm{E}-03$ & $0.00 E+00$ & 0.00001 \\
\hline${ }^{137} \mathrm{Cs}$ & $4 / 18$ & $3.15 E-02$ & $4.14 \mathrm{E}-02$ & $2.18 \mathrm{E}-02$ & $9.79 \mathrm{E}-03$ & 0.00001 \\
\hline${ }^{238} \mathrm{Pu}$ & $4 / 18$ & $1.36 \mathrm{E}-03$ & $1.37 \mathrm{E}-03$ & $1.32 \mathrm{E}-03$ & $2.25 \mathrm{E}-05$ & 0.0045 \\
\hline${ }^{228} \mathrm{Th}$ & $16 / 18$ & $7.99 \mathrm{E}-03$ & $8.08 \mathrm{E}-03$ & $7.88 \mathrm{E}-03$ & $9.96 \mathrm{E}-05$ & 0.02 \\
\hline${ }^{230} \mathrm{Th}$ & $18 / 18$ & $5.52 \mathrm{E}-03$ & $5.82 E-03$ & $5.22 \mathrm{E}-03$ & $3.00 \mathrm{E}-04$ & 0.0138 \\
\hline${ }^{232} \mathrm{Th}$ & $17 / 18$ & $6.74 \mathrm{E}-03$ & $7.06 \mathrm{E}-03$ & $6.41 E-03$ & $3.23 \mathrm{E}-04$ & 0.096 \\
\hline Total Sr & $8 / 18$ & $5.85 \mathrm{E}-02$ & $7.14 \mathrm{E}-02$ & $4.57 \mathrm{E}-09$ & $1.28 \mathrm{E}-02$ & 0.00065 \\
\hline${ }^{3} \mathrm{H}$ & $12 / 26$ & $2.36 \mathrm{E}+04$ & $8.19 E+04$ & $5.73 E+03$ & $6.81 E+03$ & 0.0236 \\
\hline${ }^{234} \mathrm{U}$ & $17 / 18$ & $3.80 \mathrm{E}-02$ & $4.33 \mathrm{E}-02$ & $3.28 \mathrm{E}-02$ & $5.24 \mathrm{E}-03$ & 0.0422 \\
\hline${ }^{235} \mathrm{U}$ & $11 / 18$ & $8.08 \mathrm{E}-03$ & $9.15 \mathrm{E}-03$ & $7.00 \mathrm{E}-03$ & $1.07 \mathrm{E}-03$ & 0.0081 \\
\hline${ }^{238} \mathrm{U}$ & $15 / 18$ & $1.41 \mathrm{E}-02$ & $1.56 \mathrm{E}-02$ & $1.40 \mathrm{E}-02$ & $7.80 \mathrm{E}-04$ & 0.01481 \\
\hline
\end{tabular}

${ }^{a} 1 \mu \mathrm{Ci}=3.7 \mathrm{E}+4 \mathrm{~Bq}$.

${ }^{b}$ Average concentration is the average of significant values only; this average is divided by the derived concentration guide (DCG) for inhalation of that isotope, multiplied by 100 , and presented in the table as the percentage of the DCG, unless the percentage is less than 0.01 ; in that case, the percentage is reported as less than 0.01 . 
Table 5.4. Uranium in ambient air at the $v_{-12}$ Plant, 1993

\begin{tabular}{ccccc}
\hline \multirow{2}{*}{$\begin{array}{c}\text { Station } \\
\text { No. }\end{array}$} & $\begin{array}{c}\text { No. of } \\
\text { samples }\end{array}$ & \multicolumn{3}{c}{$\begin{array}{c}\text { 7-(iay concentration } \\
\left(\mu \mathrm{g} / \mathrm{m}^{3}\right)^{a}\end{array}$} \\
\cline { 3 - 5 } & 51 & 0.00028 & $<0.00001$ & $\mathrm{Mv}$ \\
\hline 1 & 51 & 0.00019 & $<0.00001$ & 0.00008 \\
2 & 43 & 0.00058 & 0.00003 & 0.00006 \\
3 & 51 & 0.06333 & $<0.00001$ & 0.00151 \\
4 & 51 & 0.00033 & $<0.00001$ & 0.00007 \\
5 & 51 & 0.00017 & $<0.00001$ & 0.00006 \\
6 & 51 & 0.0002 & $<0.00001$ & 0.00007 \\
7 & 51 & 0.01621 & $<0.00001$ & 0.00039 \\
8 & 51 & 0.00042 & $<0.00001$ & 0.00008 \\
9 & 50 & 0.00015 & $<0.00001$ & 0.00006 \\
10 & 51 & 0.00047 & $<0.00001$ & 0.00009 \\
11 & 51 & 0.00015 & $<0.00001$ & 0.00006 \\
12 & 51 & & & \\
\hline
\end{tabular}

${ }^{\sigma}$ Because of the low level of uranium on the filters, the ${ }^{235} \mathrm{U}$ measurements were not always attainable. When they were, the results varied such that activity values could not be assigned.

Table 5.5. Fluorides in ambient air at the $\mathrm{Y}-12$ Plant, 1993

\begin{tabular}{|c|c|c|c|c|c|c|}
\hline \multirow{2}{*}{$\begin{array}{l}\text { Station } \\
\text { No. }\end{array}$} & \multirow{2}{*}{$\begin{array}{l}\text { No. of } \\
\text { samples }\end{array}$} & \multicolumn{4}{|c|}{$\begin{array}{l}\text { 7-day concentration } \\
\left(\mu \mathrm{g} / \mathrm{m}^{3}\right)^{a}\end{array}$} & \multirow{2}{*}{$\begin{array}{l}\text { Percentage of } \\
\text { standard } d^{b}\end{array}$} \\
\hline & & Max & Min & $\mathrm{Av}$ & Tenn. std ${ }^{a}$ & \\
\hline 1 & 46 & 0.0186 & 0.0072 & 0.0094 & 1.6 & $<2$ \\
\hline 2 & 51 & 0.0236 & 0.0066 & 0.0104 & 1.6 & $<2$ \\
\hline 3 & 47 & 0.0148 & 0.0041 & 0.0081 & 1.6 & $<1$ \\
\hline 4 & 51 & 0.0168 & 0.0058 & 0.0102 & 1.6 & $<2$ \\
\hline 5 & 51 & 0.0127 & 0.0055 & 0.0070 & 1.6 & $<1$ \\
\hline 6 & 51 & 0.0376 & 0.0042 & 0.0089 & 1.6 & $<3$ \\
\hline 7 & 51 & 0.0217 & 0.0047 & 0.0094 & 1.6 & $<2$ \\
\hline 8 & 51 & 0.0144 & 0.0040 & 0.0084 & 1.6 & $<1$ \\
\hline 9 & 51 & 0.0264 & 0.0047 & 0.0083 & 1.6 & $<2$ \\
\hline 10 & 51 & 0.0210 & 0.0042 & 0.0087 & 1.6 & $<2$ \\
\hline 11 & 51 & 0.0219 & 0.0044 & 0.0091 & 1.6 & $<2$ \\
\hline
\end{tabular}

${ }^{\circ}$ Tennessee standard 7-day average $=1.6 \mu \mathrm{g} / \mathrm{m}^{3}$.

'Percentage of standard calculated using the annual 7-day average fluoride concentration. 
Table 5.6. Total suspended particulates in ambient air at the Y-12 Plant, 1993

\begin{tabular}{|c|c|c|c|c|c|c|}
\hline \multirow{2}{*}{$\begin{array}{l}\text { TSP } \\
\text { station }\end{array}$} & \multirow{2}{*}{$\begin{array}{c}\text { No. of } \\
\text { samples }\end{array}$} & \multicolumn{4}{|c|}{$\begin{array}{c}\text { Concentration } \\
\left(\mu \mathrm{g} / \mathrm{m}^{3}\right)\end{array}$} & \multirow{2}{*}{$\begin{array}{l}\operatorname{Max} \% \text { of } \\
\operatorname{std}^{a}\end{array}$} \\
\hline & & Max & Min & $\mathrm{Av}$ & $\begin{array}{l}\text { Tenn. } \\
\text { std }\end{array}$ & \\
\hline East & 53 & 128.25 & 3.15 & 38.14 & 260 & 49.33 \\
\hline West & 39 & 57.14 & $7.79^{\circ}$ & 25.09 & 260 & 21.98 \\
\hline
\end{tabular}

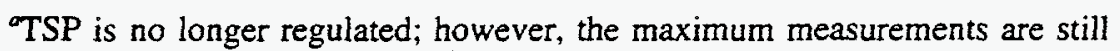
compared with the previous Tennessee 24-hour primary air quality standard of $260 \mu \mathrm{g} / \mathrm{m}^{3}$. There were no exceedences at either TSP station.

Table 5.7. PM10 concentrations in ambient air at the Y-12 Plant, 1993

\begin{tabular}{|c|c|c|c|c|c|c|}
\hline \multirow{2}{*}{$\begin{array}{l}\text { PM10 } \\
\text { station }\end{array}$} & \multirow{2}{*}{$\begin{array}{l}\text { No. of } \\
\text { samples }\end{array}$} & \multicolumn{4}{|c|}{$\begin{array}{c}\text { Concentration } \\
\left(\mu \mathrm{g} / \mathrm{m}^{3}\right)\end{array}$} & \multirow{2}{*}{$\begin{array}{c}\text { Max \%o } \\
\text { std }\end{array}$} \\
\hline & & $\operatorname{Max}^{a}$ & Min & Av & $\begin{array}{c}\text { Tenn. } \\
\text { std }\end{array}$ & \\
\hline West & 54 & 64.74 & 1.39 & 18.09 & 150 & 43.16 \\
\hline East & 58 & 58.91 & 0.30 & 17.88 & 150 & 39.27 \\
\hline East collocated & 60 & 64.92 & 0.59 & 21.30 & 150 & 43.28 \\
\hline
\end{tabular}

${ }^{\circ}$ Maximum measurements are compared with the Tennessee primary air quality standard of $150 \mu \mathrm{g} / \mathrm{m}^{3}$ per 24 hours. There were no exceedences at any of the PM10 stations.

Tabie 5.8. Annual results of the $Y-12$ Plant ambient air mercury monitoring program, 1993

\begin{tabular}{lcccc}
\hline \multirow{2}{*}{ Site } & \multirow{2}{*}{$\begin{array}{c}\text { No. of } \\
\text { samples }\end{array}$} & \multicolumn{3}{c}{$\begin{array}{c}\text { Mercury vapor concentration } \\
\left(\mu \mathrm{g} / \mathrm{m}^{3}\right)\end{array}$} \\
\cline { 3 - 5 } & & $\mathrm{Max}$ & $\mathrm{Min}$ & $\mathrm{Av}^{a}$ \\
\hline Station No. 2 (east end of Y-12 Plant) & 45 & 0.026 & 0.003 & 0.008 \\
Station No. 8 (west end of Y-12 Plant) & 45 & 0.031 & 0.004 & 0.012 \\
Bldg. 9404-13 (SW of Bldg. 9201-4) & 45 & 0.250 & 0.017 & 0.078 \\
Bldg. 9805-1 (SE of Bldg. 9201-4) & 45 & 0.314 & 0.010 & 0.088 \\
Reference site, Rain Gage No. 2 & 47 & 0.016 & 0.002 & 0.006 \\
(Chestnut Ridge) $^{b}$ & 47 & 0.015 & $<0.001$ & 0.005 \\
\hline
\end{tabular}

National Emission Standards for Hazardous Air Pollutants 30-day average standard = $1 \mu \mathrm{g} / \mathrm{m}^{3}$. American Conference of Governmental Industrial Hygienists 8-hour day, 40-hour work week standard $=50 \mu \mathrm{g} / \mathrm{m}^{3}$.

${ }^{b}$ Data for this site are for February-December 1988 (first line) and January-October 1989 (second line); monitoring was discontinued on October 31, 1989. 
Table 5.9. Summary of collection and analysis trequencies for low-volume samples ${ }^{\hat{t}}$ at $\mathrm{ORNL}$ ambient air monitoring stations

\begin{tabular}{lrl}
\hline \multicolumn{1}{c}{ Parameter } & $\begin{array}{r}\text { Collection } \\
\text { frequency }\end{array}$ & $\begin{array}{c}\text { Analysis } \\
\text { frequency }\end{array}$ \\
\hline & Stations $1,2,3$, and 7 & \\
$\begin{array}{l}\text { U, Pu, Th, Be, Am, } \\
\text { and Cm }\end{array}$ & Biweekly & Annual \\
Total rad Sr & Biweekly & Annual \\
Gamma scan (filter) & Biweekly & Annual \\
$\begin{array}{l}\text { Gamma scan } \\
\text { (charcoal) }\end{array}$ & Biweekly & Biweekly \\
& Station 3 only & \\
Tritium & Biweekly & Monthly \\
\hline
\end{tabular}

Type of sampling = continuous.

Table 5.10. ORNL radionuclide concentrations in air, 1993

\begin{tabular}{|c|c|c|c|c|c|c|}
\hline \multirow[b]{2}{*}{ Determination } & \multirow{2}{*}{$\begin{array}{l}\text { No. detected/ } \\
\text { No. sampled }\end{array}$} & \multicolumn{4}{|c|}{$\begin{array}{c}\text { Concentration } \\
\left(10^{-15} \mu \mathrm{C} \mathrm{i} / \mathrm{mL}\right)^{a} \\
\end{array}$} & \multirow[b]{2}{*}{ DCG $\%$} \\
\hline & & Average $^{b}$ & $\operatorname{Max}$ & Min & $\begin{array}{l}\text { Standard } \\
\text { error }\end{array}$ & \\
\hline${ }^{241} \mathrm{Am}$ & $2 / 4$ & $3.57 \mathrm{E}-03$ & $3.88 E-03$ & $3.29 \mathrm{E}-03$ & $2.82 E-04$ & 0.01787 \\
\hline${ }^{7} \mathrm{Be}$ & $4 / 4$ & $2.60 E+01$ & $3.53 E+01$ & $1.80 \mathrm{E}+01$ & $3.73 E+00$ & 0.00005 \\
\hline${ }^{24} \mathrm{Cm}$ & $3 / 4$ & $4.58 \mathrm{E}-03$ & $6.15 E-03$ & $3.24 \mathrm{E}-03$ & $8.48 \mathrm{E}-04$ & 0.001145 \\
\hline${ }^{137} \mathrm{Cs}$ & $2 / 4$ & $9.15 \mathrm{E}-02$ & $1.04 \mathrm{E}-01$ & $7.87 \mathrm{E}-02$ & $1.28 \mathrm{E}-02$ & 0.00002 \\
\hline${ }^{131} \mathrm{I}$ & $2 / 103$ & $5.26 \mathrm{E}+\infty 0$ & $5.16 \mathrm{E} \div 00$ & $5.16 \mathrm{E}+00$ & $0.00 E+00$ & 0.0013 \\
\hline${ }^{133} \mathrm{I}$ & $7 / 103$ & $7.81 \mathrm{E}+00$ & $9.70 E+00$ & $6.09 \mathrm{E}+00$ & $8.17 \mathrm{E}-01$ & 0.00039 \\
\hline${ }^{135} \mathrm{I}$ & $4 / 103$ & $6.48 \mathrm{E}+00$ & $8.78 \mathrm{E}+00$ & $4.89 E+00$ & $1.18 E+\infty 0$ & 0.00065 \\
\hline${ }^{19 i} \mathrm{Os}$ & $1 / 103$ & $3.10 \mathrm{E}-01$ & $3.10 \mathrm{E}-01$ & $3.10 \mathrm{E}-01$ & $0.00 \mathrm{E}+00$ & 0.001 \\
\hline${ }^{212} \mathrm{~Pb}$ & $12 / 103$ & $3.68 \mathrm{E}+01$ & $7.16 E+01$ & $1.77 \mathrm{E}+01$ & $1.20 \mathrm{E}+01$ & 0.046 \\
\hline${ }^{238} \mathrm{Pu}$ & $2 / 4$ & $4.05 E-03$ & $6.97 \mathrm{E}-03$ & $1.14 \mathrm{E}-03$ & $2.91 \mathrm{E}-03$ & 0.0136 \\
\hline${ }^{228} \mathrm{Th}$ & $4 / 4$ & $1.23 \mathrm{E}-02$ & $1.69 \mathrm{E}-02$ & $8.24 \mathrm{E}-03$ & $1.93 \mathrm{E}-03$ & 0.0309 \\
\hline${ }^{230} \mathrm{Th}$ & $4 / 4$ & $8.85 \mathrm{E}-03$ & $1.23 \mathrm{E}-02$ & $5.11 \mathrm{E}-03$ & $1.47 \mathrm{E}-03$ & 0.02212 \\
\hline${ }^{232} \mathrm{Th}$ & $4 / 4$ & $1.09 \mathrm{E}-02$ & $1.46 \mathrm{E}-02$ & $8.45 \mathrm{E}-03$ & $1.33 \mathrm{E}-03$ & 0.1554 \\
\hline Total St & $2 / 4$ & $3.68 \mathrm{E}-02$ & $3.76 \mathrm{E}-02$ & $3.60 \mathrm{E}-02$ & $8.01 \mathrm{E}-04$ & 0.00041 \\
\hline${ }^{3} \mathrm{H}$ & $11 / 13$ & $2.84 \mathrm{E}+03$ & $1.36 \mathrm{E}+04$ & $1.12 \mathrm{E}+02$ & $1.27 E+03$ & 0.00284 \\
\hline${ }^{234} \mathrm{U}$ & $4 / 4$ & $7.91 \mathrm{E}-02$ & $9.53 \mathrm{E}-02$ & $6.97 \mathrm{E}-02$ & $5.61 E-03$ & 0.088 \\
\hline${ }^{235} \mathrm{U}$ & $4 / 4$ & $2.15 E-02$ & $2.82 \mathrm{E}-02$ & $1.25 \mathrm{E}-02$ & $3.30 \mathrm{E}-03$ & 0.0215 \\
\hline${ }^{238} \mathrm{U}$ & $4 / 4$ & $4.61 \mathrm{E}-02$ & $5.52 \mathrm{E}-02$ & $3.96 \mathrm{E}-02$ & $3.81 \mathrm{E}-03$ & 0.0461 \\
\hline
\end{tabular}

${ }^{a} \mu \mathrm{Ci}=3.7 \mathrm{E}+4 \mathrm{~Bq}$.

${ }^{b}$ Average concentration is the werage of significant values only; this average is divided by the derived concentration guide (DCG) for inhalation of that isotope, multiplied by 100 , and presented in the table as the percentage of the DCG, unless the percentage is less than 0.01 ; in that case, the percentage is reported as less than 0.01 . 
Table 5.11. Summary of ambient air pollutants measured by the K-25 Site network, 1993

\begin{tabular}{lccccccc}
\hline \multirow{2}{*}{ Parameter $^{\circ}$} & \multicolumn{7}{c}{ Station No. } \\
\cline { 2 - 7 } & $\mathrm{K} 1$ & $\mathrm{~K} 2$ & $\mathrm{~K} 3$ & $\mathrm{~K} 4$ & $\mathrm{~K} 5$ & $\mathrm{TSCA}$ 1 & TSCA2 \\
\hline TSP & $\mathrm{X}$ & $\mathrm{X}$ & $\mathrm{X}$ & $\mathrm{X}$ & $\mathrm{X}$ & & \\
PM10 & $\mathrm{X}$ & $\mathrm{X}$ & $\mathrm{X}$ & $\mathrm{X}$ & $\mathrm{X}$ & & \\
Arsenic $^{b}$ & $\mathrm{X}$ & $\mathrm{X}$ & $\mathrm{X}$ & $\mathrm{X}$ & $\mathrm{X}$ & & \\
Beryllium $^{b}$ & $\mathrm{X}$ & $\mathrm{X}$ & $\mathrm{X}$ & $\mathrm{X}$ & $\mathrm{X}$ & & \\
Cadmium $^{b}$ & $\mathrm{X}$ & $\mathrm{X}$ & $\mathrm{X}$ & $\mathrm{X}$ & $\mathrm{X}$ & & \\
Chromium (total) $^{\text {Lead }}$ & $\mathrm{X}$ & $\mathrm{X}$ & $\mathrm{X}$ & $\mathrm{X}$ & $\mathrm{X}$ & & \\
Nickel & $\mathrm{X}$ & $\mathrm{X}$ & $\mathrm{X}$ & $\mathrm{X}$ & $\mathrm{X}$ & & \\
Uranium (total) $^{c}$ & $\mathrm{X}$ & $\mathrm{X}$ & $\mathrm{X}$ & $\mathrm{X}$ & $\mathrm{X}$ & $\mathrm{X}$ & $\mathrm{X}$ \\
PCBs $_{\text {Furan }}$ & & & & & & $\mathrm{X}$ & $\mathrm{X}$ \\
Hexachlorobenzene & & & & & & $\mathrm{X}$ & $\mathrm{X}$ \\
Dioxin & & & & & & $\mathrm{X}$ & $\mathrm{X}$ \\
\hline
\end{tabular}

${ }^{a}$ All parameters are reported as mass per unit volume of air.

${ }^{b}$ Measurement of these pollutants began on October 1, 1993.

Measurement of this pollutant was discontinued on September 30, 1993.

Table 5.12. Results of ambient air sampling for lead at the K-25 Site, 1993

\begin{tabular}{|c|c|c|c|c|c|c|c|c|}
\hline \multirow[t]{2}{*}{$\begin{array}{l}\text { Station } \\
\text { No. }\end{array}$} & \multirow[t]{2}{*}{$\begin{array}{l}\text { No. of } \\
\text { samples }\end{array}$} & \multicolumn{4}{|c|}{$\begin{array}{l}\text { Average concentrations } \\
\qquad\left(\mu g / \mathrm{m}^{3}\right)\end{array}$} & \multicolumn{2}{|c|}{$\begin{array}{c}\text { Individual } \\
\text { measurements } \\
\left(\mu g / \mathrm{m}^{3}\right)\end{array}$} & \multirow{2}{*}{$\begin{array}{c}\text { Percentage } \\
\text { of standard } \\
\text { per qtr. }\end{array}$} \\
\hline & & Qtr. 1 & Qtr. 2 & Qtr. 3 & Qtr. 4 & Min & $\operatorname{Max}$ & \\
\hline $\mathrm{K} 1$ & 59 & 0.02482 & 0.01297 & 0.00502 & 0.00710 & 0.00357 & 0.04023 & 1.7 \\
\hline $\mathrm{K} 2$ & 56 & 0.02411 & 0.01186 & 0.00441 & 0.00874 & 0.00304 & 0.03641 & 1.6 \\
\hline $\mathrm{K} 3$ & 55 & 0.02360 & 0.00997 & 0.00397 & 0.00441 & 0.00289 & 0.03019 & 1.6 \\
\hline K4 & 59 & 0.02414 & 0.01304 & 0.00567 & 0.00714 & 0.00317 & 0.03652 & 1.6 \\
\hline K5 & 56 & 0.02242 & 0.01562 & 0.00421 & 0.00546 & 0.00317 & 0.02997 & 1.5 \\
\hline
\end{tabular}

${ }^{a}$ Lead concentration averages are the quarterly arithmetic mean of 24 -hour results for the first quarter and of monthly composite results for the second, third, and fourth quarters.

${ }^{b}$ Based on the maximum quarterly average compared with the standard for lead equal to $1.5 \mu \mathrm{g} / \mathrm{m}^{3}$ quarterly arithmetic mean. 
Table 5.13. Surface water sampling measurements exceeding Tennessee water quality criteria at the Y-12 Plant, 1993

\begin{tabular}{|c|c|c|c|c|c|c|c|}
\hline \multirow{2}{*}{ Parameter } & \multirow{2}{*}{ Location } & \multirow{2}{*}{$\begin{array}{l}\text { No. of } \\
\text { samples }\end{array}$} & \multicolumn{3}{|c|}{$\begin{array}{l}\text { Concentration } \\
(\mathrm{mg} / \mathrm{L})\end{array}$} & \multirow{2}{*}{$\begin{array}{l}\text { Water } \\
\text { quality } \\
\text { criteria } \\
\text { (mg/L) }\end{array}$} & \multirow{2}{*}{$\begin{array}{l}\text { No. of } \\
\text { measurements } \\
\text { exceeding } \\
\text { criteria }\end{array}$} \\
\hline & & & $\begin{array}{l}\text { Detection } \\
\text { limit }\end{array}$ & $\operatorname{Max}$ & Av & & \\
\hline Silver & Station 17 & 203 & 0.006 & $<0.006$ & $<0.006$ & 0.004 & 203 \\
\hline Mercury & Station 17 & 203 & 0.0002 & 0.0093 & $<0.0016$ & $0.00015^{\circ}$ & 203 \\
\hline Mercury & $\begin{array}{l}\text { Rogers Quarty } \\
\text { (Outfall 302) }\end{array}$ & 52 & 0.0002 & 0.0006 & $<0.0002$ & 0.00015 & 52 \\
\hline Chromium & Station 17 & 203 & 0.006 & 0.028 & $<0.006$ & 0.016 & 1 \\
\hline Cadmium & Station 17 & 94 & 0.0005 & 0.0082 & $<0.0019$ & 0.004 & 1 \\
\hline
\end{tabular}

The Tennessee water quality standard for recreation is $0.00015 \mathrm{mg} / \mathrm{L}$. The freshwater fish and aquatic life standards are 0.0024 and $0.000012 \mathrm{mg} / \mathrm{L}$ for the maximum and continuous concentrations, respectively.

Table 5.14. Results of radiological analysis of ORR soil samples, 1993

\begin{tabular}{|c|c|c|c|c|c|c|c|c|c|}
\hline \multirow[t]{2}{*}{ Parameter } & \multicolumn{9}{|c|}{$\begin{array}{c}\text { Station } \\
\text { Concentration }(\mathrm{pCi} / \mathrm{g})^{a}\end{array}$} \\
\hline & 35 & 37 & 38 & 39 & 40 & 42 & 46 & 48 & 51 \\
\hline${ }^{241} \mathrm{AM}$ & & 0.01 & & 0.01 & & & & & \\
\hline${ }^{60} \mathrm{Co}$ & & & & & & 0.06 & & & \\
\hline${ }^{137} \mathrm{Cs}$ & & 0.09 & & & & & & & \\
\hline Gross alpha & 1.27 & 1.06 & 1.04 & 1.18 & 0.81 & 1.19 & 0.80 & 1.16 & 0.95 \\
\hline Gross beta & 3.1 & 2.35 & 2.28 & 2.22 & 2.39 & 2.5 & 2.19 & 2.42 & 2.38 \\
\hline${ }^{238} \mathrm{Pu}$ & & & & & & & & 0.01 & \\
\hline${ }^{228} \mathrm{Th}$ & 0.02 & 0.03 & 0.02 & 0.03 & 0.02 & 0.03 & 0.02 & 0.04 & 0.03 \\
\hline${ }^{230} \mathrm{Th}$ & 0.04 & 0.03 & 0.03 & 0.03 & 0.02 & 0.37 & 0.01 & 0.04 & 0.03 \\
\hline${ }^{232} \mathrm{Th}$ & 0.03 & 0.03 & 0.02 & 0.02 & 0.02 & 0.03 & 0.01 & 0.04 & \\
\hline Total Rad Sr & 0.35 & 0.45 & 0.42 & 0.46 & 0.65 & 0.62 & 0.45 & 0.62 & \\
\hline${ }^{234} \mathrm{U}$ & 0.05 & 0.05 & 0.05 & 0.05 & 0.05 & 0.04 & 0.03 & 0.06 & \\
\hline${ }^{235} \mathrm{U}$ & 0.01 & 0.01 & 0.01 & 0.01 & 0.004 & 0.01 & 0.01 & 0.01 & \\
\hline${ }^{238} \mathrm{U}$ & 0.04 & 0.03 & 0.02 & 0.04 & 0.04 & 0.03 & 0.02 & 0.03 & \\
\hline
\end{tabular}

${ }^{a} 1 \mathrm{pCi}=3.7 \mathrm{E}-02 \mathrm{~Bq}$. 
Table 5.15. Concentrations of radionuclides and fluoride in hay from the ORR, 1993

\begin{tabular}{lcccccc}
\hline \multirow{2}{*}{ Area } & \multicolumn{5}{c}{$\begin{array}{c}\text { Isotope } \\
(\mathrm{Ci} / \mathrm{kg})^{d}\end{array}$} & $\begin{array}{c}\text { Fluoride } \\
(\mu \mathrm{g} / \mathrm{g})\end{array}$ \\
\cline { 2 - 6 } & ${ }^{7} \mathrm{Be}$ & ${ }^{60} \mathrm{Co}$ & ${ }^{40} \mathrm{~K}$ & Gross alpha & Gross beta & \\
\hline 1,2 , and 3 & $3.1 \mathrm{E}-09$ & $3.0 \mathrm{E}-11$ & $1.3 \mathrm{E}-08$ & $2.8 \mathrm{E}-10$ & $7.0 \mathrm{E}-09$ & 1.1 \\
2,4 , and 5 & $5.5 \mathrm{E}-09$ & $3.6 \mathrm{E}-11$ & $1.4 \mathrm{E}-08$ & $1.0 \mathrm{E}-09$ & $6.4 \mathrm{E}-09$ & 1.6 \\
6 & $1.1 \mathrm{E}-08$ & $b$ & $8.5 \mathrm{E}-09$ & $3.4 \mathrm{E}-10$ & $1.3 \mathrm{E}-08$ & 1.1 \\
\hline
\end{tabular}

${ }^{a} 1 \mathrm{Ci}=3.7 \mathrm{E}+10 \mathrm{~Bq}$.

${ }^{b}$ Not significant.

Table 5.16. Results of radiological analysis of tomatoes grown on the ORR, 1993

\begin{tabular}{|c|c|c|c|c|c|c|c|c|c|}
\hline \multirow{2}{*}{ Parameter } & \multicolumn{9}{|c|}{$\begin{array}{c}\text { Station } \\
\text { Concentration }(\mathrm{pCi} / \mathrm{g})^{a}\end{array}$} \\
\hline & 35 & 37 & 38 & 39 & 40 & 42 & 46 & 48 & 51 \\
\hline${ }^{60} \mathrm{Co}$ & & & & & & & & $4.9 \mathrm{E}-03$ & \\
\hline Gross alpha & $8.7 \mathrm{E}-01$ & & & & & & & & \\
\hline Gross beta & & $1.8 \mathrm{E}+00$ & $2.0 E+00$ & $1.7 \mathrm{E}+00$ & $1.3 E+\infty 0$ & $8.7 E-01$ & $1.3 E+\infty 0$ & $1.5 E+\infty$ & $1.1 E+\infty 0$ \\
\hline${ }^{239} \mathrm{Pu}$ & & $1.6 \mathrm{E}-04$ & & $1.7 \mathrm{E}-04$ & & & & $1.4 \mathrm{E}-04$ & $9.0 \mathrm{E}-0 \mathrm{~S}$ \\
\hline${ }^{228} \mathrm{Th}$ & $1.3 \mathrm{E}-04$ & $1.5 \mathrm{E}-04$ & & & & 1.3E-04 & & & \\
\hline${ }^{230} \mathrm{Th}$ & $1.5 \mathrm{E}-04$ & $1.7 \mathrm{E}-04$ & & $1.3 \mathrm{E}-04$ & & & $1.3 \mathrm{E}-04$ & $1.5 \mathrm{E}-04$ & $1.9 \mathrm{E}-04$ \\
\hline${ }^{232} \mathrm{Th}$ & & & & & $1.1 \mathrm{E}-03$ & & & & \\
\hline Total rad Sr & & & & $2.3 \mathrm{E}-02$ & $1.8 \mathrm{E}-02$ & & & & \\
\hline${ }^{234} \mathrm{U}$ & $3.0 \mathrm{E}-04$ & $6.4 \mathrm{E}-04$ & $1.1 \mathrm{E}-03$ & $1.1 \mathrm{E}-03$ & $5.3 \mathrm{E}-04$ & $4.0 \mathrm{E}-0.4$ & $3.4 \mathrm{E}-04$ & $1.0 \mathrm{E}-03$ & $9.5 \mathrm{E}-04$ \\
\hline${ }^{235} \mathrm{U}$ & & $2.7 \mathrm{E}-04$ & & & & $1.8 \mathrm{E}-04$ & & $2.6 \mathrm{E}-0.04$ & $2.8 \mathrm{E}-04$ \\
\hline${ }^{238} \mathrm{U}$ & 4.5E-04 & 4.1E-04 & $4.3 \mathrm{E}-04$ & & $2.4 \mathrm{E}-04$ & $2.4 \mathrm{E}-04$ & & $3.6 \mathrm{E}-04$ & $6.8 \mathrm{E}-04$ \\
\hline
\end{tabular}

${ }^{a} 1 \mathrm{pCi}=3.7 \mathrm{E}-02 \mathrm{~Bq}$. 
Table 5.17. Results of radiological analysis of turnip greens grown on the ORR, 1993

\begin{tabular}{|c|c|c|c|c|c|c|c|c|c|}
\hline \multirow[t]{2}{*}{ Parameter } & \multicolumn{9}{|c|}{$\begin{array}{c}\text { Station } \\
\text { Concentration }(\mathrm{pCi} / \mathrm{g})^{a}\end{array}$} \\
\hline & 35 & 37 & 38 & 39 & 40 & 42 & 46 & 48 & $5 \mathrm{i}$ \\
\hline${ }^{241} \mathrm{Am}$ & & $2.8 \mathrm{E}-03$ & $6.5 \mathrm{E}-03$ & & $1.6 \mathrm{E}-03$ & & $2.8 \mathrm{E}-03$ & $2.3 \mathrm{E}-03$ & $2.8 \mathrm{E}-03$ \\
\hline${ }^{3} \mathrm{Be}$ & $8.2 \mathrm{E}-01$ & $1.4 E+00$ & $7.0 \mathrm{E}-01$ & $6.4 \mathrm{E}-01$ & $6.6 \mathrm{E}-01$ & $6.4 \mathrm{E}-01$ & $9.3 \mathrm{E}-01$ & $8.9 \mathrm{E}-01$ & $6.1 \mathrm{E}-01$ \\
\hline${ }^{137} \mathrm{Cs}$ & & & & & $1.2 \mathrm{E}-02$ & & $2.1 E-02$ & & \\
\hline Gross alpha & $2.1 \mathrm{E}-01$ & $2.6 \mathrm{E}-01$ & $3.1 \mathrm{E}-01$ & $1.1 \mathrm{E}-01$ & $6.5 \mathrm{E}-01$ & $1.1 E-01$ & $4.6 \mathrm{E}-01$ & $2.2 E-01$ & $1.6 \mathrm{E}-01$ \\
\hline Gross beta & $\begin{array}{l}3.8 E+0 \\
0\end{array}$ & $4.1 E+\infty$ & $4.7 \mathrm{E}+\infty$ & $3.6 E+00$ & $3.9 E+00$ & $4.1 E+00$ & $4.2 E+00$ & $3.7 \mathrm{E}+00$ & $4.5 E+00$ \\
\hline${ }^{40} \mathrm{~K}$ & $\begin{array}{l}5.1 E+0 \\
0\end{array}$ & $5.0 \mathrm{E}+00$ & $5.0 \mathrm{E}+00$ & $4.6 \mathrm{E}+00$ & $4.5 \mathrm{E}+00$ & $5.4 \mathrm{E}+00$ & $5.5 \mathrm{E}+00$ & $5.4 \mathrm{E}+00$ & $4.2 \mathrm{E}+00$ \\
\hline${ }^{239} \mathrm{Pu}$ & & & & & & & $3.7 \mathrm{E}-03$ & & \\
\hline${ }^{228} \mathrm{Th}$ & $1.0 \mathrm{E}-02$ & & $6.4 \mathrm{E}-03$ & & $8.9 \mathrm{E}-03$ & $5.1 \mathrm{E}-03$ & $1.2 \mathrm{E}-02$ & $8.5 \mathrm{E}-03$ & $3.8 \mathrm{E}-03$ \\
\hline${ }^{230} \mathrm{Th}$ & $1.3 \mathrm{E}-02$ & $1.1 \mathrm{E}-02$ & $8.1 \mathrm{E}-03$ & $3.1 \mathrm{E}-03$ & $1.0 \mathrm{E}-02$ & $1.8 \mathrm{E}-03$ & $1.5 \mathrm{E}-02$ & $1.3 \mathrm{E}-02$ & $5.3 \mathrm{E}-03$ \\
\hline${ }^{232} \mathrm{Th}$ & $6.3 \mathrm{E}-0.4$ & & $3.5 \mathrm{E}-03$ & & $7.3 \mathrm{E}-03$ & $2.4 \mathrm{E}-03$ & $1.3 \mathrm{E}-02$ & $4.9 \mathrm{E}-03$ & $2.7 \mathrm{E}-0.3$ \\
\hline Total rad Sr & $2.1 \mathrm{E}-01$ & $2.3 \mathrm{E}-01$ & $1.7 \mathrm{E}-01$ & & $2.1 \mathrm{E}-0 \mathrm{i}$ & & $3.1 \mathrm{E}-01$ & $2.0 \mathrm{E}-01$ & $2.2 E-01$ \\
\hline${ }^{234} \mathrm{U}$ & $2.7 \mathrm{E}-02$ & $1.6 \mathrm{E}-02$ & $1.4 \mathrm{E}-02$ & $7.6 \mathrm{E}-03$ & $2.2 \mathrm{E}-0.2$ & $7.6 \mathrm{E}-03$ & $1.8 \mathrm{E}-02$ & $1.3 \mathrm{E}-02$ & $5.6 \mathrm{E}-03$ \\
\hline${ }^{235} \mathrm{U}$ & $3.0 \mathrm{E}-03$ & $3.2 \mathrm{E}-03$ & $3.7 \mathrm{E}-03$ & $2.8 \mathrm{E}-03$ & & & $1.8 \mathrm{E}-03$ & $1.5 \mathrm{E}-03$ & $2.4 \mathrm{E}-03$ \\
\hline${ }^{238} \mathrm{U}$. & $1.2 \mathrm{E}-02$ & $9.2 \mathrm{E}-03$ & $9.7 \mathrm{E}-03$ & 2.7E-03 & $8.8 \mathrm{E}-03$ & $2.2 \mathrm{E}-03$ & $1.1 \mathrm{E}-02$ & $1.0 \mathrm{E}-02$ & $6.0 \mathrm{E}-03$ \\
\hline
\end{tabular}

${ }^{\circ} 1 \mathrm{pCi}=3.7 \mathrm{E}-02 \mathrm{~Bq}$.

Table 5.18. Results of radiological analysis of turnips grown in private gardens on the ORR, 1993

\begin{tabular}{lccc}
\hline \multirow{2}{*}{ Parameter } & \multicolumn{3}{c}{$\begin{array}{c}\text { Station } \\
\text { Concentration }(\mathrm{pCi} / \mathrm{g})^{a}\end{array}$} \\
\cline { 2 - 4 } & \multicolumn{2}{c}{39} & \multicolumn{1}{c}{40} \\
\hline${ }^{7} \mathrm{Be}$ & $8.7 \mathrm{E}-02$ & $5.0 \mathrm{E}-02$ & \\
Gross alpha & $8.2 \mathrm{E}-02$ & $7.2 \mathrm{E}-02$ & \\
Gross beta & $2.3 \mathrm{E}+00$ & $2.0 \mathrm{E}+00$ & $2.1 \mathrm{E}+00$ \\
${ }^{40} \mathrm{~K}$ & $2.8 \mathrm{E}+00$ & $2.7 \mathrm{E}+00$ & $3.5 \mathrm{E}+00$ \\
${ }^{230} \mathrm{Th}$ & $3.1 \mathrm{E}-03$ & $1.4 \mathrm{E}-02$ & $1.2 \mathrm{E}-02$ \\
${ }^{232} \mathrm{Th}$ & $3.1 \mathrm{E}-03$ & & \\
${ }^{234} \mathrm{U}$ & $1.3 \mathrm{E}-02$ & $2.1 \mathrm{E}-02$ & $1.1 \mathrm{E}-02$ \\
${ }^{235} \mathrm{U}$ & & $1.3 \mathrm{E}-02$ & \\
${ }^{238} \mathrm{U}$ & & $8.4 \mathrm{E}-03$ & $5.0 \mathrm{E}-03$ \\
\hline
\end{tabular}

${ }^{a} 1 \mathrm{pCi}=3.7 \mathrm{E}-02 \mathrm{~Bq}$. 
Table 5.19. Concentrations of total radioactive strontium $\left({ }^{89} \mathrm{Sr}+{ }^{90} \mathrm{Sr}\right)$ in raw milk, 1993

\begin{tabular}{|c|c|c|c|c|c|}
\hline \multirow{2}{*}{ Station } & \multirow{2}{*}{$\begin{array}{l}\text { No. detected/ } \\
\text { No. of samples }\end{array}$} & \multicolumn{3}{|c|}{$\begin{array}{l}\text { Detected concentration } \\
\qquad(\mathrm{pCi} / \mathcal{L})^{a}\end{array}$} & \multirow{2}{*}{$\begin{array}{l}\text { Standard } \\
\text { error }\end{array}$} \\
\hline & & $\operatorname{Max}$ & Min & $\mathrm{Av}$ & \\
\hline Buttermilk Road & $9 / 12$ & 2.03 & 0.68 & $1.16^{c}$ & 0.14 \\
\hline Powell & $11 / 12$ & 2.54 & 0.70 & $1.41^{c}$ & 0.18 \\
\hline Clinton & $11 / 11$ & 3.78 & 0.59 & $1.55^{\circ}$ & 0.28 \\
\hline Frost Bottom & $12 / 12$ & 3.24 & 1.05 & $1.79^{c}$ & 0.17 \\
\hline Solway & $6 / 6$ & 5.67 & 2.11 & $3.64^{c}$ & 0.39 \\
\hline Network summary & $49 / 53$ & 5.67 & 0.59 & 1.76 & 0.15 \\
\hline
\end{tabular}

${ }^{a} 1 \mathrm{pCi}=3.7 \mathrm{E}-2 \mathrm{~Bq}$.

${ }^{b}$ Standard error of the mean.

${ }^{c}$ Average is significantly greater than zero at the $95 \%$ confidence level. The average value for EPA Region IV is 1.8 pCilL (U.S. EPA 1993a).

Table 5.20. Parameters detected in sunfish from Poplar Creek and Clinch River locations, 1993

\begin{tabular}{|c|c|c|c|c|c|c|}
\hline & PCK 2.2 & CRK 16 & CRK 32 & CRK 66 & CRK 80 & CRK 84 \\
\hline \multicolumn{7}{|c|}{ Metals } \\
\hline Arsenic & $\mathrm{X}$ & $\mathrm{X}$ & & & & \\
\hline Chromium & $\mathrm{X}$ & $X$ & $X$ & $\mathrm{X}$ & $\mathrm{X}$ & $\mathrm{X}$ \\
\hline Copper & $\mathrm{X}$ & $\mathrm{X}$ & $X$ & $\mathrm{X}$ & $\mathrm{X}$ & $\mathrm{X}$ \\
\hline Lead & $X$ & & & & & \\
\hline Mercury & $\mathrm{X}$ & $X$ & $\mathrm{X}$ & $X$ & $\mathrm{x}$ & $\mathrm{X}$ \\
\hline Selenium & $\mathrm{X}$ & $\mathrm{X}$ & $\mathrm{X}$ & $\mathrm{X}$ & $\mathrm{x}$ & $X$ \\
\hline Silver & $X$ & & & & & $X$ \\
\hline Uranium & $\mathrm{X}$ & $\mathrm{X}$ & $\mathrm{X}$ & $\mathrm{X}$ & $\mathrm{X}$ & $\mathrm{X}$ \\
\hline Zinc & $\mathrm{X}$ & $\mathrm{X}$ & $\mathrm{X}$ & $\mathrm{X}$ & $\mathrm{X}$ & $\mathrm{X}$ \\
\hline \multicolumn{7}{|c|}{ Pesticides } \\
\hline $4,4^{\prime}-\mathrm{DDE}$ & $\mathrm{X}$ & & $X$ & & & \\
\hline \multicolumn{7}{|c|}{$P C B s$} \\
\hline Aroclor- 1254 & $\mathrm{X}$ & $\mathrm{X}$ & $\mathrm{X}$ & & & \\
\hline \multicolumn{7}{|c|}{ Radionuclides } \\
\hline${ }^{137} \mathrm{Cs}$ & $\mathrm{X}$ & $\mathrm{X}$ & $\mathrm{X}$ & & & $\mathrm{X}$ \\
\hline${ }^{60} \mathrm{Co}$ & & & $x$ & & & \\
\hline Total rad Sr & $\mathrm{X}$ & $\mathrm{X}$ & $\mathrm{X}$ & $\mathrm{X}$ & $\mathrm{X}$ & $\mathrm{X}$ \\
\hline
\end{tabular}


Table 5.21. Parameters detected ir. cattish from two Clinch River locations, 1993

\begin{tabular}{lcc}
\hline & CRK 16 & CRi 32 \\
\hline \multicolumn{3}{c}{ Metals } \\
Arsenic & $\mathrm{X}$ & \\
Mercury & $\mathrm{X}$ & $\mathrm{X}$ \\
Nickel & & $\mathrm{X}$ \\
Thallium & $\mathrm{X}$ & \\
Uranium & $\mathrm{X}$ & \\
Zinc & & $\mathrm{X}$ \\
& & \\
$4,4^{\prime}$-DDD & Pesticides & $\mathrm{X}$ \\
$4,4^{\prime}$-DDE & $\mathrm{X}$ & $\mathrm{X}$ \\
Alpha-Chlordane & $\mathrm{X}$ & $\mathrm{X}$ \\
& Radionuclides & \\
${ }^{137}$ Cs & $\mathrm{X}$ & $\mathrm{X}$ \\
Total rad Sr & & $\mathrm{X}$ \\
\hline
\end{tabular}




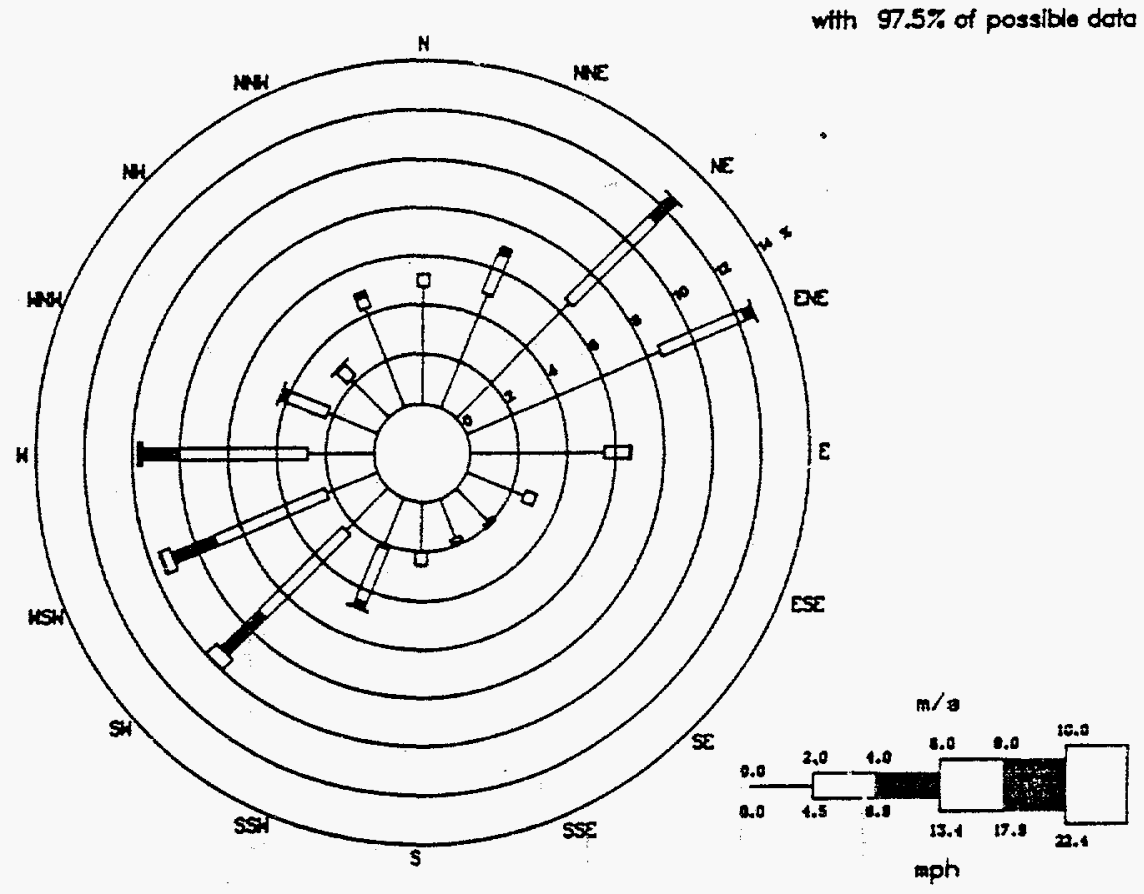

Fig. 5.1. Wind rose for $Y-12$ tower MTE (@10m) for 1993.

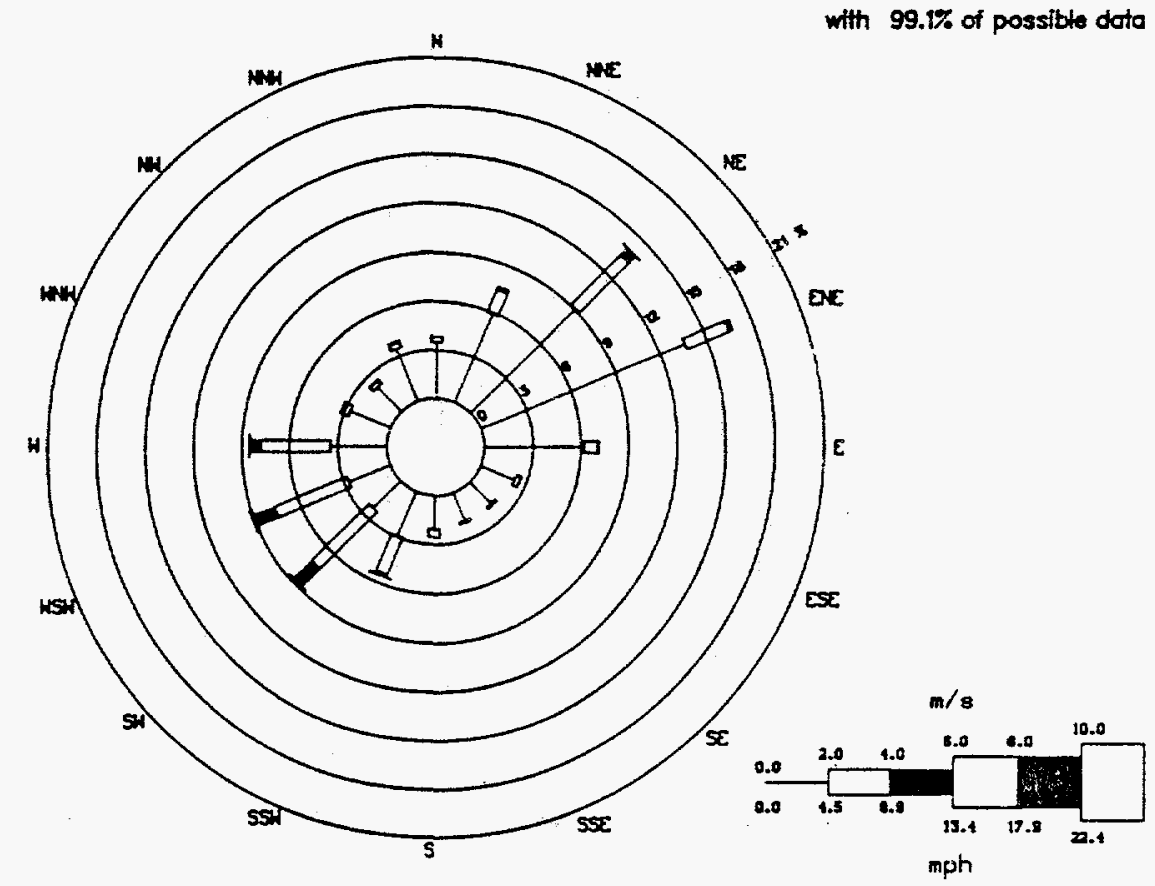

Fig. 5.2. Wind rose for $Y-12$ tower MTE (@30m) for 1993 


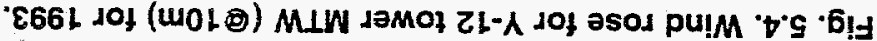

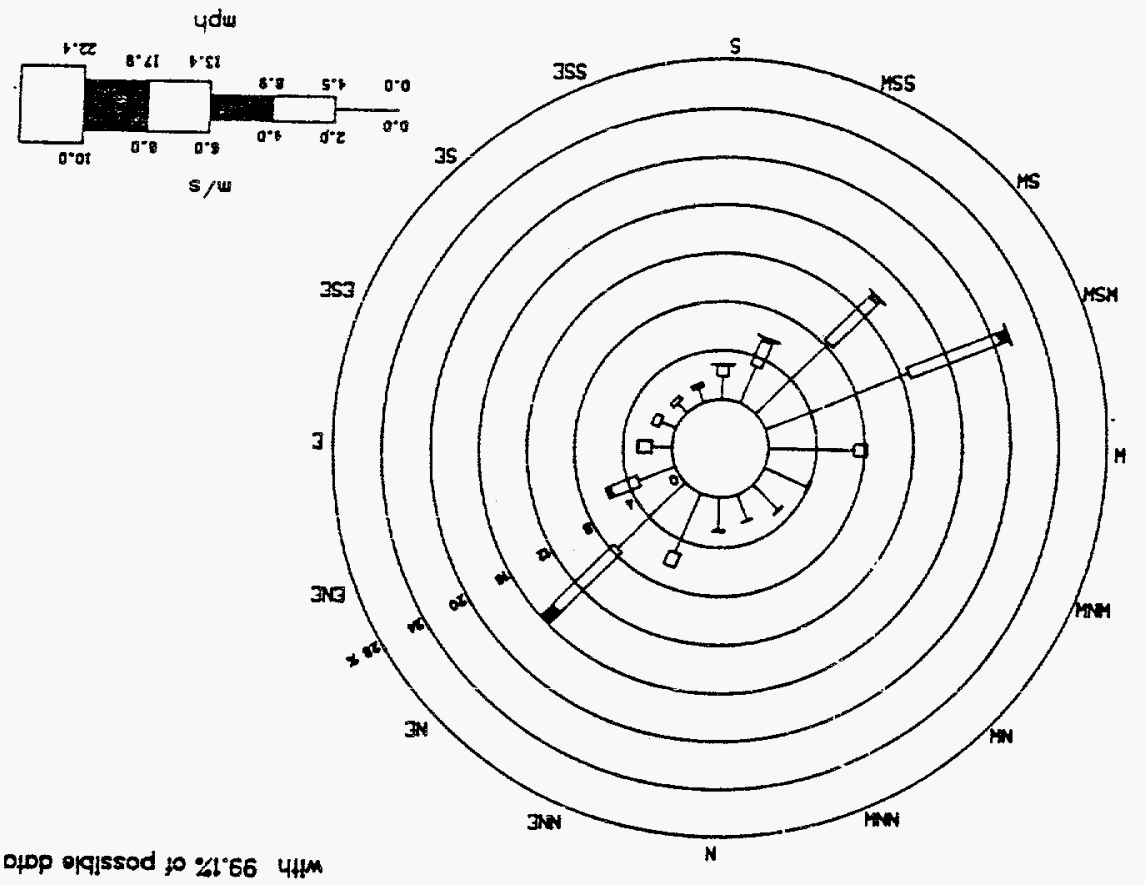

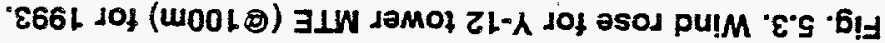

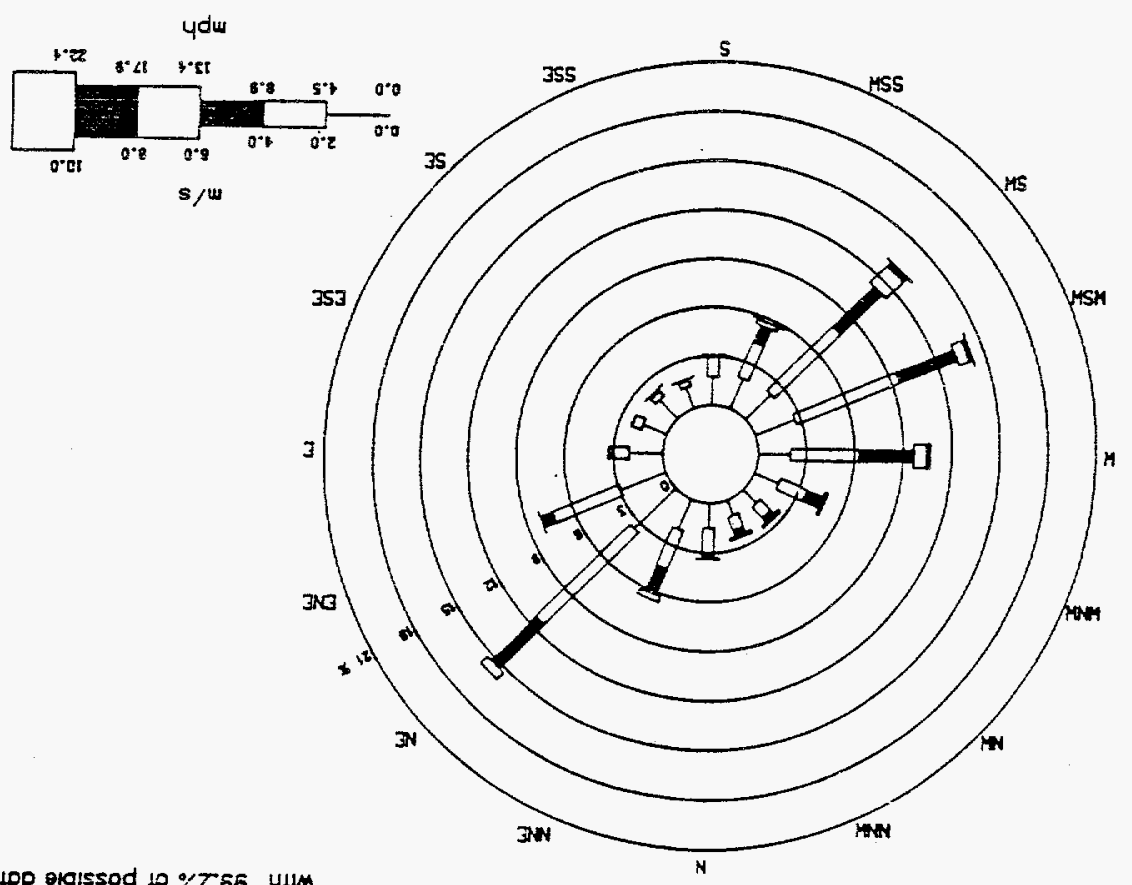

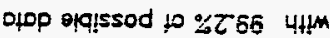




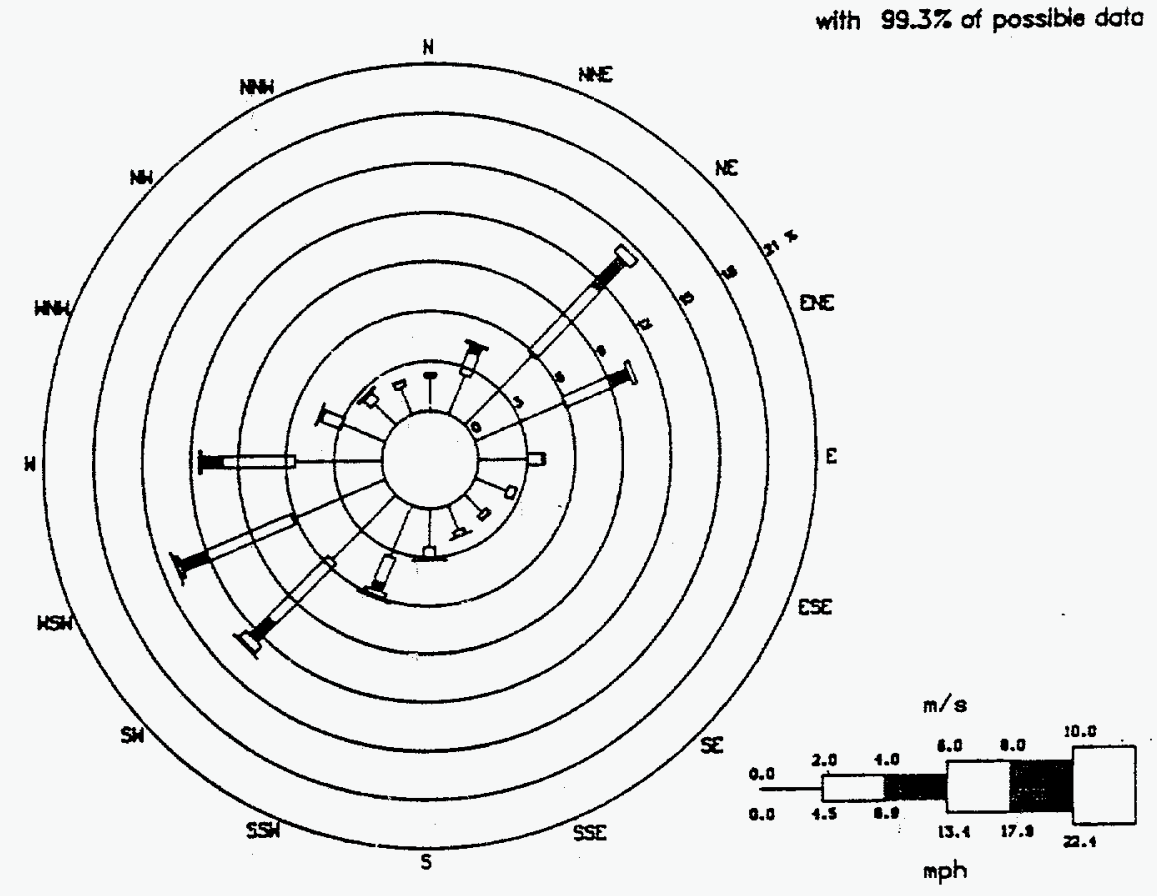

Fig. 5.5. Wind rose for Y-12 tower MTW (@60m) for 1993.

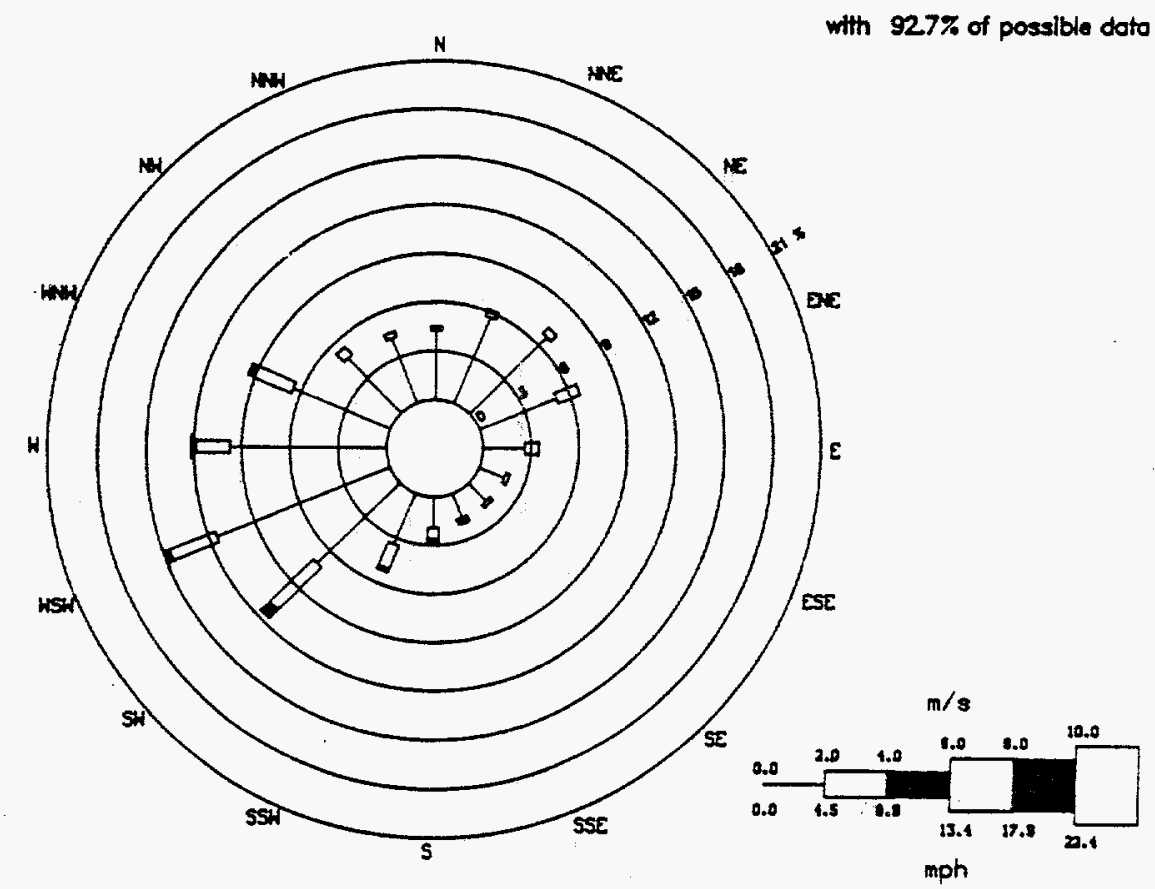

Fig. 5.6. Wind rose f.3r CRNL tower MT2 $(\$ 10 \mathrm{~m})$ for 1993. 


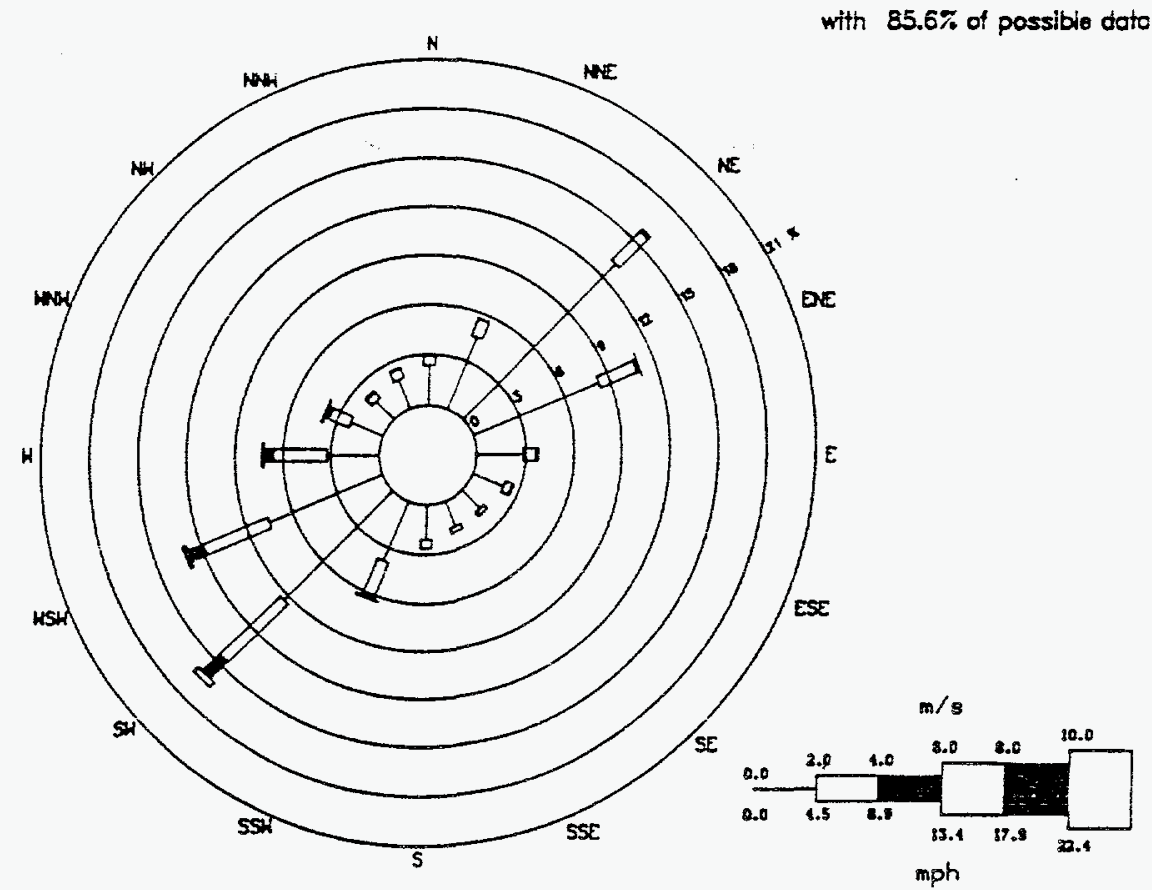

Fig. 5.7. Wind rose for ORNL tower MT2 (@30m) for 1993.

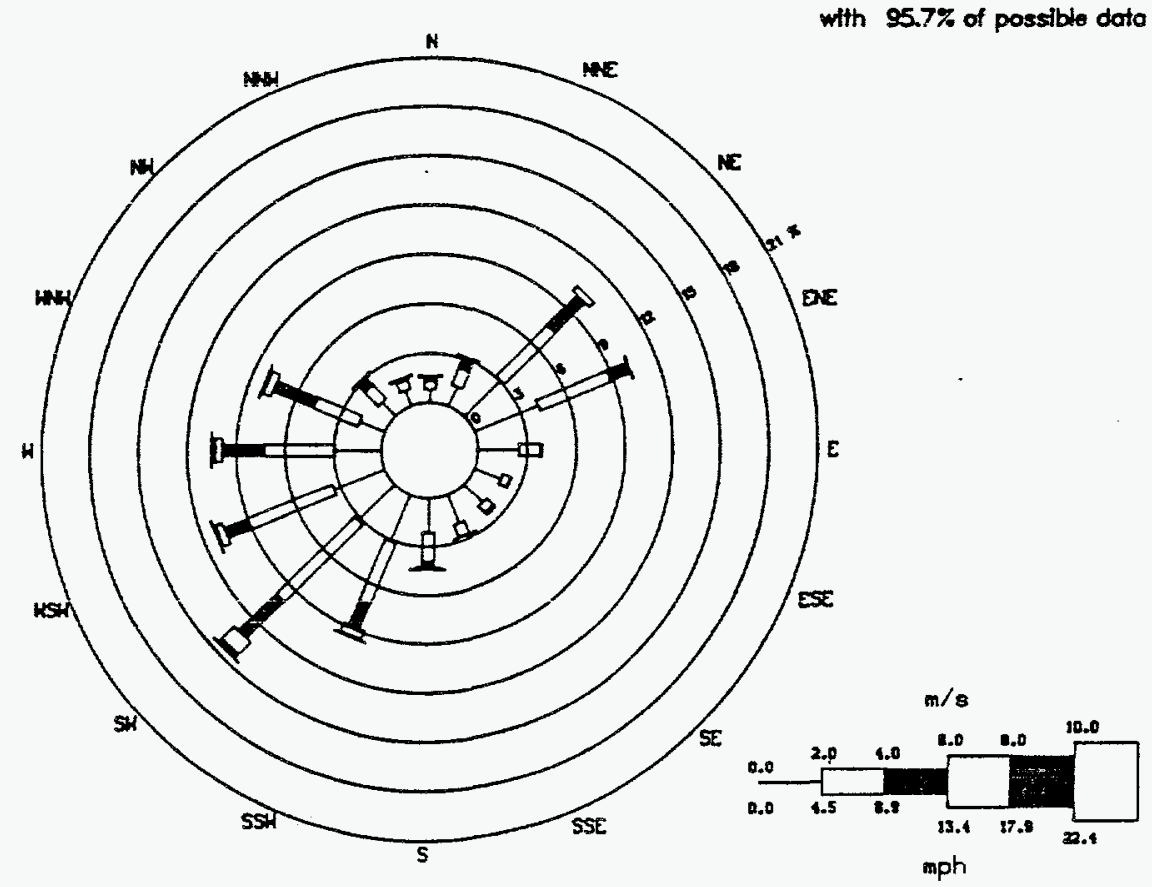

Fig. 5.8. Wind rose for ORNL tower MT2 (@100m) for 1993 . 


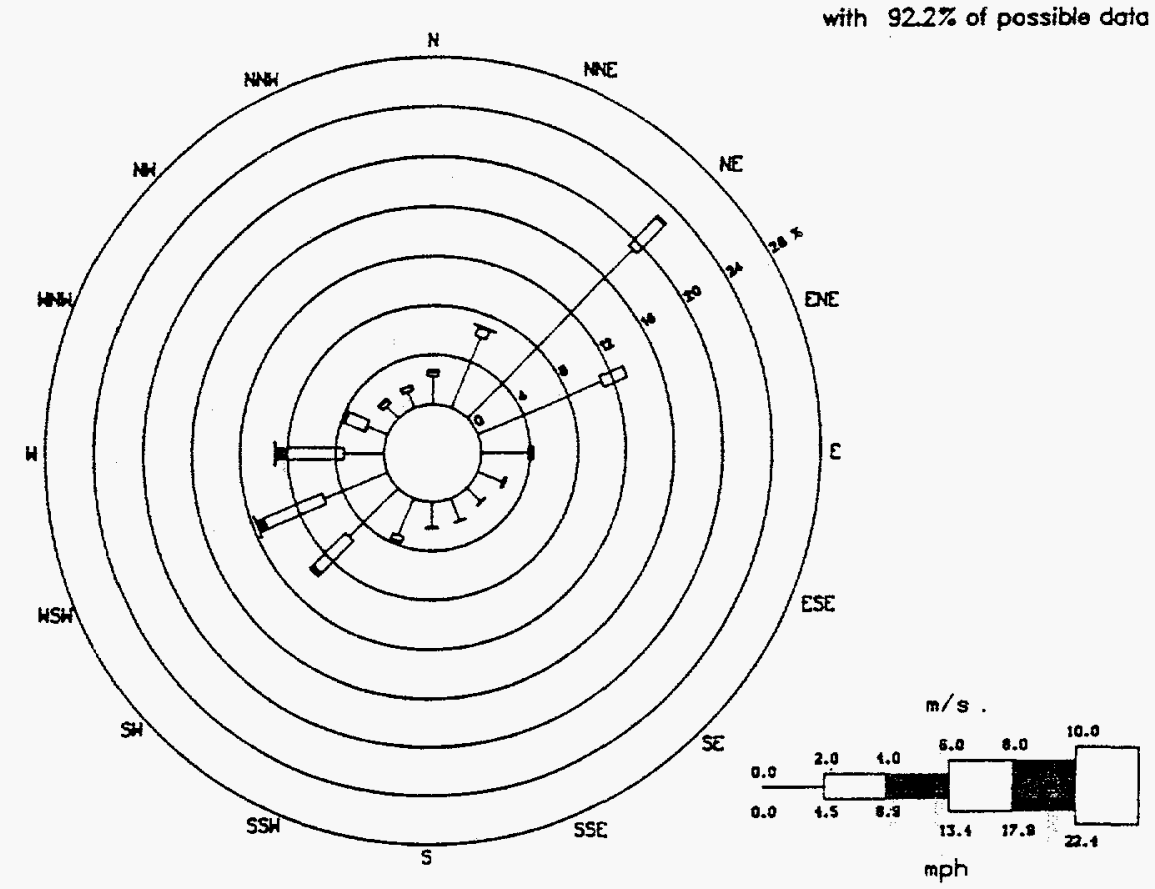

Fig. 5.9. Wind rose for ORNL tower MT3 (@10m) for 1993.

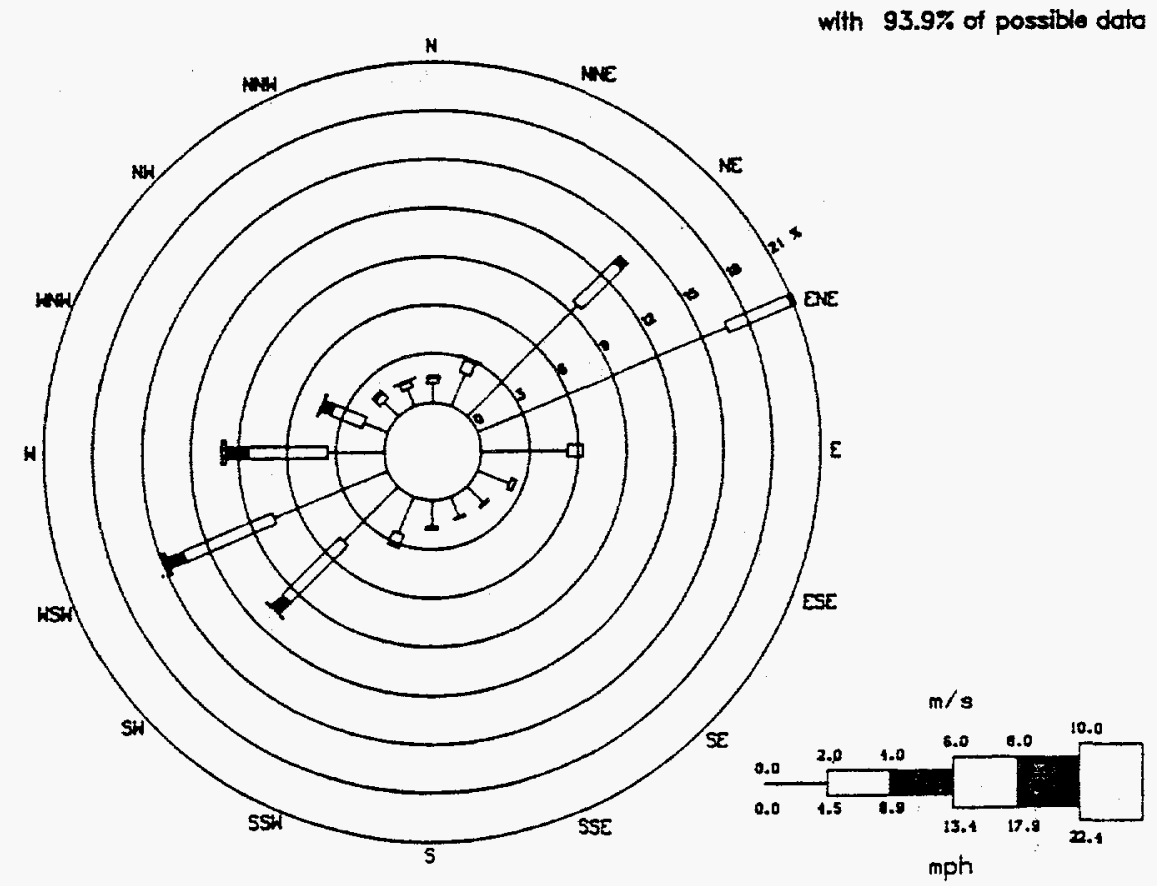

Fig. 5.10. Wind rose for OFNL tower MT3 (@30m) for 1993. 


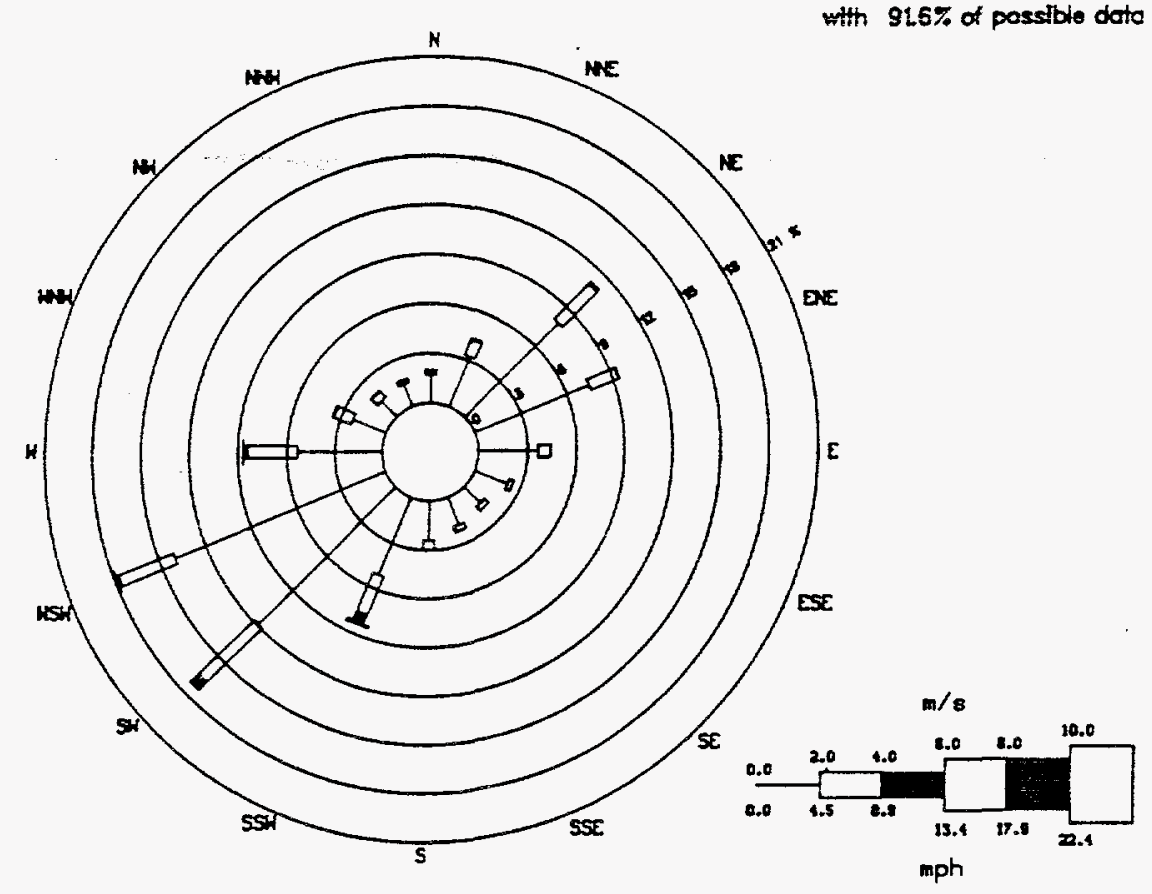

Fig. 5.11. Wind rose for ORNL tower MT4 (@10m) for 1993.

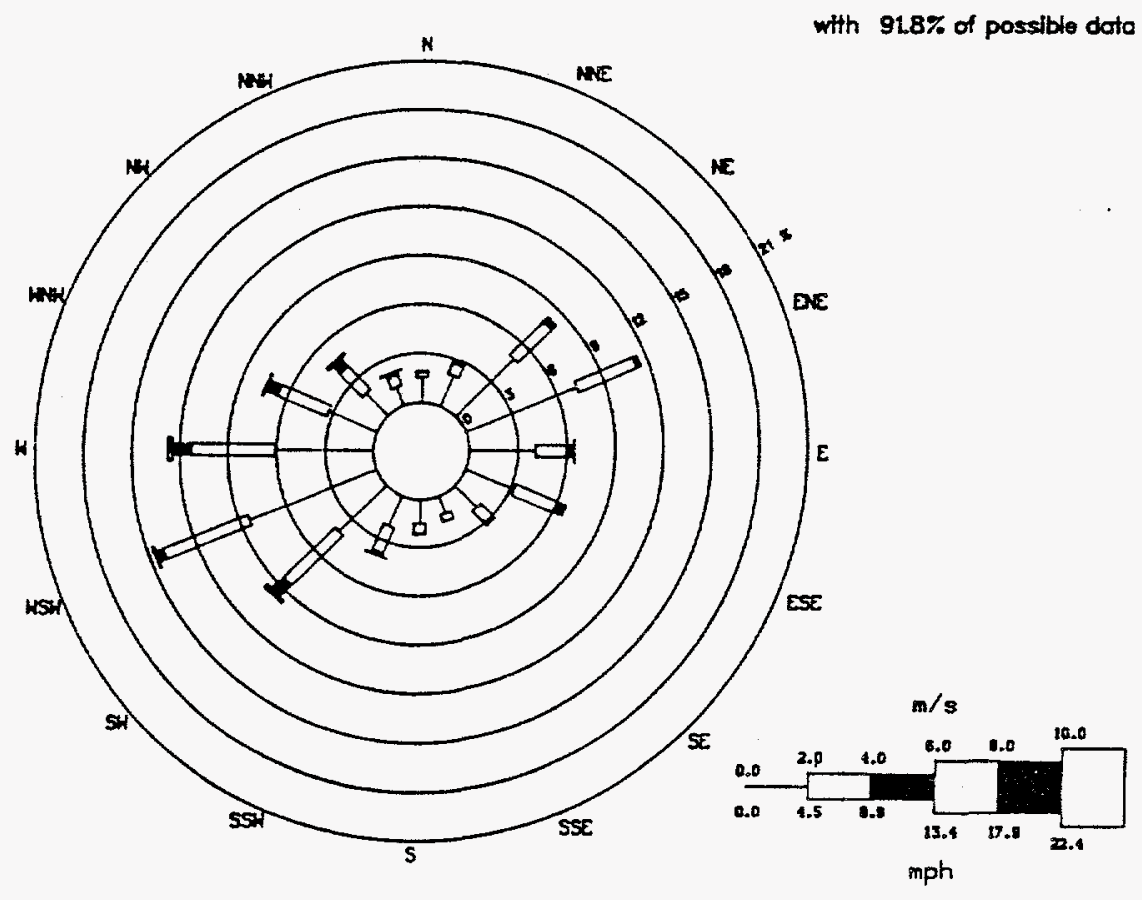

Fig. 5.12. Wind rose for ORNL tower MT4 (@30m) for 1993. 


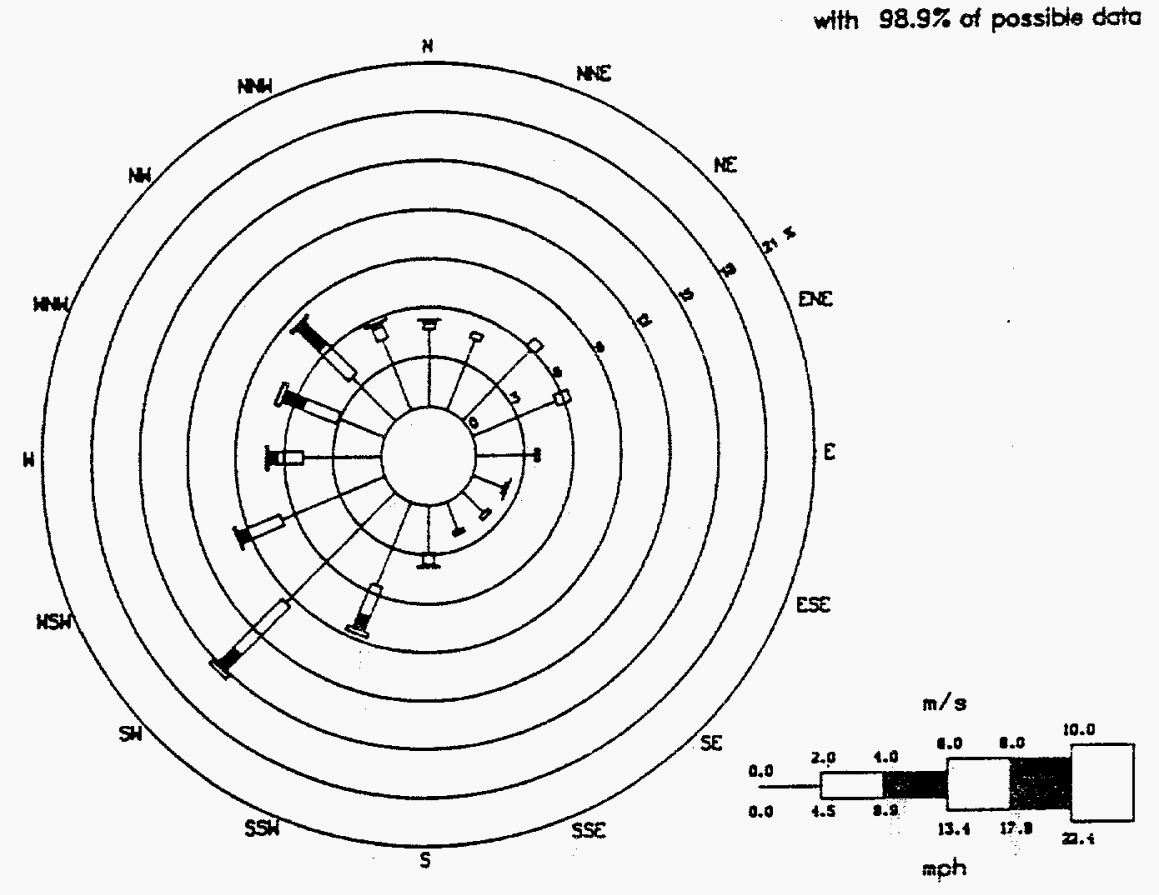

Fig. 5.13. Wind rose for K-25 tower MT1 (@10m) for 1993.

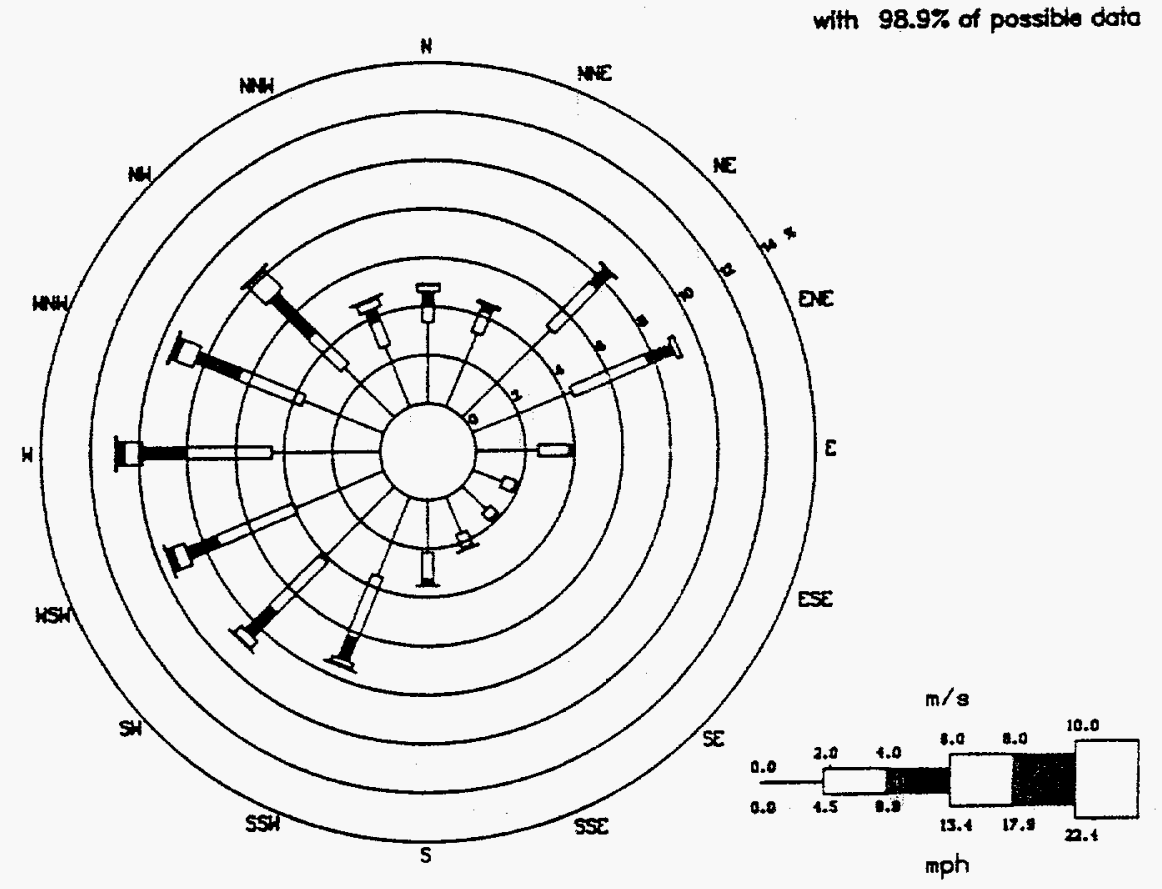

Fig. 5.14. Wind rose for K-25 tower MT1 ( $\Theta 60 \mathrm{~m})$ for 1993. 


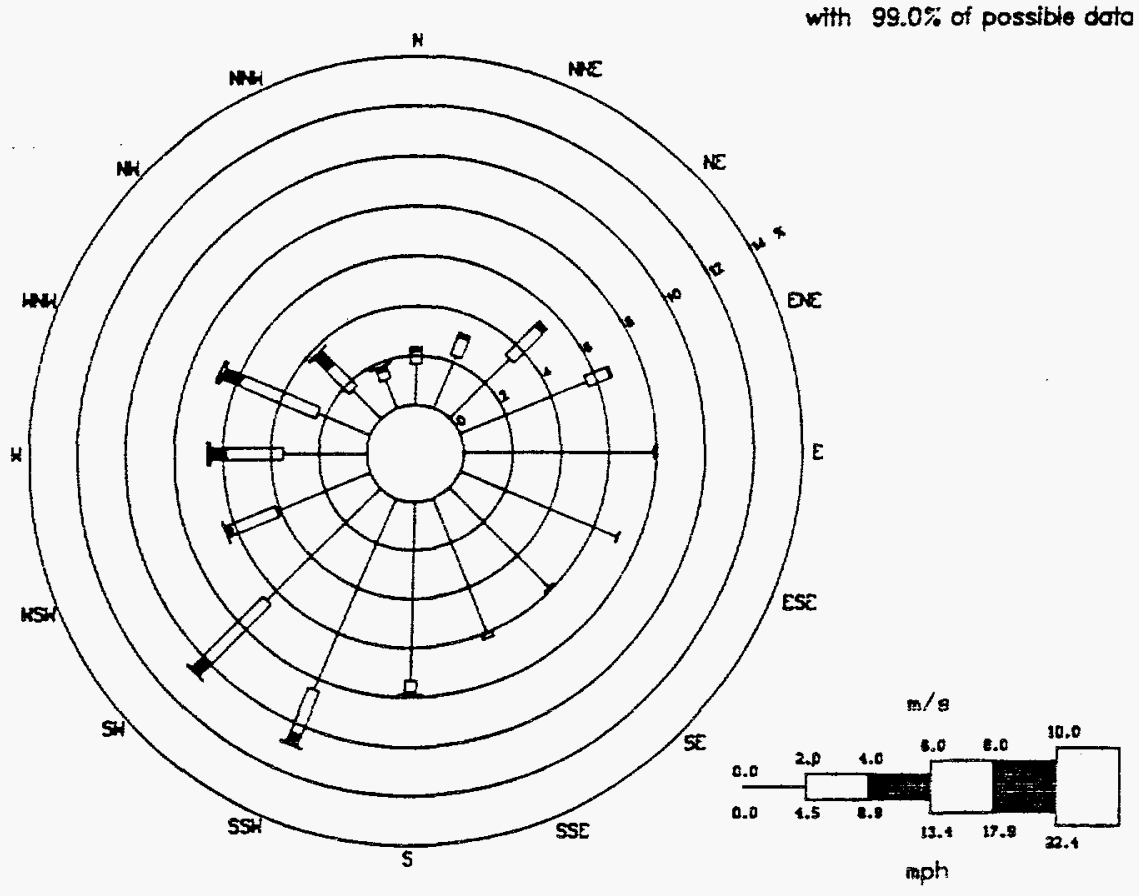

Fig. 5.15. Wind rose for K-25 tower MT7 (@10m) for 1993.

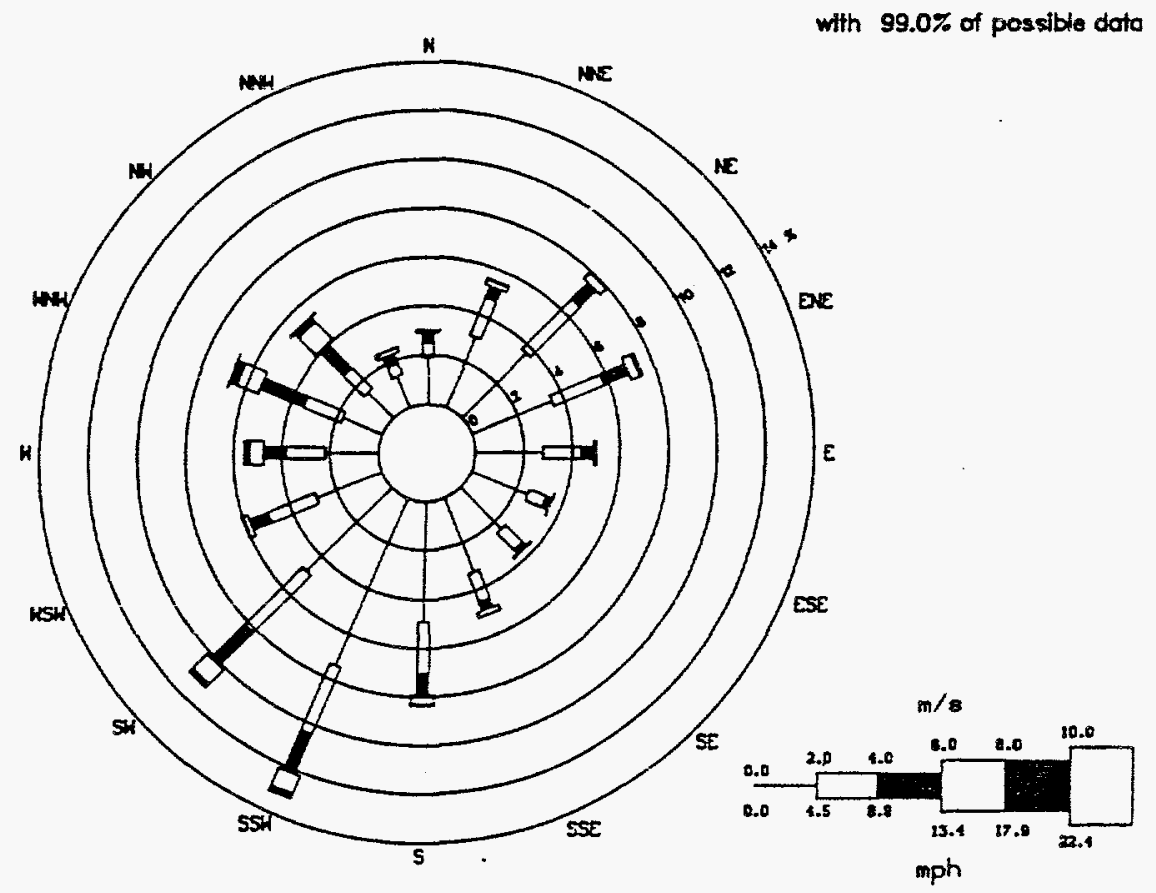

Fig. 5.16. Wind rose for K-25 tower MT7 (@30m) for 1993. 
Table 5.22. 1993 Total uranium averages in ambient air at the Y-12 Plant

\begin{tabular}{|c|c|c|c|c|c|c|}
\hline \multirow{2}{*}{ Station } & \multicolumn{5}{|c|}{$\begin{array}{l}\text { Average monthly concentrations } \\
\text { (ug/m3) }\end{array}$} & \multirow[b]{2}{*}{ Jun } \\
\hline & $\operatorname{Jan}$ & Feb & Mar & Apr & May & \\
\hline 1 & $*<0.00008$ & $<0.00008$ & $<0.00011$ & $<0.00007$ & 0.00018 & $<0.00006$ \\
\hline 2 & $<0.00007$ & $<0.00007$ & $<0.00009$ & 0.00006 & 0.00005 & $<0.00008$ \\
\hline 3 & $<0.00008$ & $<0.00008$ & $<0.00009$ & 0.00006 & 0.00008 & $<0.00005$ \\
\hline 4 & $<0.00023$ & $<0.00024$ & $<0.00023$ & $<0.00018$ & 0.00008 & 0.00009 \\
\hline 5 & $<0.00007$ & $<0.00007$ & $<0.00008$ & 0.00014 & 0.00008 & 0.00006 \\
\hline 6 & $<0.00080$ & $<0.00008$ & $<0.00010$ & $<0.00006$ & 0.00012 & $<0.00006$ \\
\hline 7 & $<0.00009$ & $<0.00009$ & $<0.00010$ & 0.00009 & 0.00011 & $<0.00006$ \\
\hline 8 & $<0.00008$ & $<0.00015$ & $<0.00333$ & $<0.00010$ & 0.00009 & $<0.00007$ \\
\hline 9 & $<0.00008$ & $<0.00022$ & $<0.00011$ & $<0.00004$ & 0.00009 & $<0.00005$ \\
\hline 10 & $<0.00007$ & $<0.00007$ & $<0.00009$ & $<0.00004$ & 0.00009 & $<0.00004$ \\
\hline 11 & $<0.00007$ & $<0.00007$ & $<0.00009$ & 0.00009 & 0.00034 & $<0.00007$ \\
\hline \multirow[t]{2}{*}{12} & $<0.00008$ & $<0.00007$ & $<0.00009$ & $<0.00006$ & 0.00007 & $<0.00005$ \\
\hline & JuI & Aug & Sept & Oct & Nov & Dec \\
\hline 1 & 0.00013 & 0.00006 & $<0.00005$ & $<0.00004$ & $<0.00006$ & a \\
\hline 2 & 0.00006 & 0.00003 & 0.00004 & $<0.00007$ & 0.00007 & 0.00005 \\
\hline 3 & 0.00025 & a & 0.00007 & $<0.00004$ & 0.00006 & 0.00004 \\
\hline 4 & 0.00017 & 0.00006 & $<0.00005$ & 0.00006 & 0.00162 & 0.00005 \\
\hline 5 & 0.00008 & 0.00004 & 0.00007 & $<0.00004$ & 0.00007 & 0.00006 \\
\hline 6 & 0.00003 & 0.00005 & $<0.00004$ & $<0.00004$ & $<0.00005$ & $<0.00004$ \\
\hline 7 & 0.00005 & 0.00005 & $<0.00007$ & $<0.00003$ & $<0.00005$ & $<0.00005$ \\
\hline 8 & $<0.00006$ & 0.00006 & 0.00006 & $<0.00005$ & $<0.00006$ & $<0.00006$ \\
\hline 9 & 0.00005 & 0.00004 & 0.00006 & $<0.00006$ & 0.00008 & $<0.00009$ \\
\hline 10 & $<0.00005$ & 0.00004 & $<0.00005$ & $<0.00004$ & 0.00006 & $<0.00004$ \\
\hline 11 & 0.00004 & 0.00008 & $<0.00009$ & $<0.00004$ & $<0.00004$ & 0.00005 \\
\hline 12 & 0.00005 & 0.00003 & $<0.00005$ & $<0.00004$ & $<0.00006$ & 0.00006 \\
\hline
\end{tabular}

* < Indicates that total U concentration for the samples was less than detectable. For those values the detection limit is used to calculate the concentration in air. In March the analytical sample size increased which resulted in more data for uranium.

(a) Indicates invalid sample or no sample (downtime). 


\begin{tabular}{|c|c|c|c|c|c|c|}
\hline \multirow{2}{*}{ Station } & \multicolumn{6}{|c|}{ Monthly Average } \\
\hline & $\operatorname{Jan}$ & Feb & Mar & Apr & May & Jun \\
\hline 1 & * NA & NA & 2.32 & 2.03 & 3.83 & 1.72 \\
\hline 2 & 1.28 & 0.73 & 7.14 & 6.18 & 3.05 & 4.18 \\
\hline 3 & 2.50 & NA & 8.30 & 21.00 & 10.10 & 8.38 \\
\hline 4 & $\mathrm{NA}$ & NA & 3.00 & 9.20 & 11.75 & 14.80 \\
\hline 5 & NA & NA & 14.26 & 21.03 & 20.28 & 16.00 \\
\hline 6 & $\mathrm{NA}$ & NA & 3.22 & 12.80 & 7.03 & 2.78 \\
\hline 7 & $\mathrm{NA}$ & NA & 5.00 & 11.55 & 8.43 & 2.66 \\
\hline 8 & $\mathrm{NA}$ & 2.33 & 4.79 & 2.28 & 2.50 & 1.74 \\
\hline 9 & $\mathrm{NA}$ & 1.90 & 4.44 & 2.90 & 4.13 & 1.58 \\
\hline 10 & $\mathrm{NA}$ & $\mathrm{NA}$ & 1.10 & 3.58 & 2.43 & 0.24 \\
\hline 11 & $\mathrm{NA}$ & NA & 2.30 & 3.35 & 1.72 & 0.68 \\
\hline \multirow[t]{2}{*}{12} & NA & 1.27 & 4.32 & 4.55 & 2.50 & 1.76 \\
\hline & Jul & Aug & Sept & Oct & Nov & $\mathrm{Dec}$ \\
\hline 1 & 3.18 & 2.80 & 1.33 & $\mathrm{NA}$ & 6.73 & $\overline{N A}$ \\
\hline 2 & 4.00 & 5.06 & 4.13 & 3.00 & 4.64 & 5.18 \\
\hline 3 & 9.03 & a & 15.95 & 1.75 & 8.48 & 7.75 \\
\hline 4 & 14.80 & 20.80 & 5.03 & 11.98 & 8.00 & 12.65 \\
\hline 5 & 15.08 & 28.80 & 20.50 & 19.75 & 10.38 & 8.43 \\
\hline 6 & 3.20 & 25.80 & 7.88 & 27.00 & 7.28 & 4.93 \\
\hline 7 & 3.08 & 13.40 & 3.95 & 13.25 & 7.12 & 2.75 \\
\hline 8 & 1.45 & 3.44 & 3.28 & 2.58 & 1.80 & 0.55 \\
\hline 9 & 2.73 & 4.56 & 2.78 & 7.33 & 4.52 & 1.40 \\
\hline 10 & 1.00 & 0.76 & 1.50 & $\mathrm{NA}$ & 2.82 & 0.82 \\
\hline 11 & 1.68 & 3.12 & 1.05 & NA & 2.72 & 2.45 \\
\hline 12 & 3.28 & 2.80 & 2.45 & NA & 5.00 & 3.08 \\
\hline
\end{tabular}

* NA $=$ Not analyzed. Total $U$ concentration for these samples were less than detectable. In March the analytical sample size increased which resulted in more data for uranium.

(a)- Indicates invalid sample or no sample (downtime). 
Table 5.24. 1993 Monthly fluoride averages in ambient air - Y-12 Plant (a)

\begin{tabular}{|c|c|c|c|c|c|c|}
\hline \multirow{2}{*}{ Station } & \multicolumn{5}{|c|}{$\begin{array}{l}\text { Average concentrations } \\
\qquad(\mathrm{ug} / \mathrm{m} \hat{\mathrm{3}})\end{array}$} & \multirow[b]{2}{*}{ Jun } \\
\hline & Jan & Feb & Mar & Apr & May & \\
\hline 1 & $<0.0085$ & $<0.0097$ & $<0.0086$ & $<0.0095$ & 0.0100 & $<0.0095$ \\
\hline 2 & $<0.0103$ & $<0.0118$ & $<0.0097$ & $<0.0108$ & $<0.0128$ & $<0.0090$ \\
\hline 3 & $<0.0082$ & $<0.0076$ & $<0.0068$ & $<0.0079$ & $<0.0107$ & $<0.0081$ \\
\hline 4 & $<0.0132$ & $<0.0129$ & $<0.0106$ & $<0.0123$ & $<0.0091$ & $<0.0091$ \\
\hline 5 & $<0.0064$ & $<0.0066$ & $<0.0070$ & $<0.0064$ & $<0.0071$ & $<0.0061$ \\
\hline 6 & $<0.0079$ & $<0.0076$ & $<0.0068$ & $<0.0076$ & $<0.0095$ & $<0.0079$ \\
\hline 7 & $<0.0087$ & $<0.0088$ & $<0.0074$ & $<0.0095$ & $<0.0133$ & $<0.0093$ \\
\hline 8 & $<0.0080$ & $<0.0070$ & $<0.0068$ & $<0.0083$ & $<0.0085$ & $<0.0099$ \\
\hline 9 & $<0.0073$ & $<0.0075$ & $<0.0068$ & $<0.0073$ & $<0.0097$ & $<0.0079$ \\
\hline 10 & $<0.0072$ & $<0.0074$ & $<0.0071$ & $<0.0081$ & $<0.0089$ & $<0.0077$ \\
\hline \multirow[t]{2}{*}{11} & $<0.0070$ & $<0.0079$ & $<0.0077$ & $<0.0086$ & $<0.0122$ & $<0.0081$ \\
\hline & Jul & Aug & Sept & Oct & Nov & Dec \\
\hline 1 & $<0.0099$ & $<0.0097$ & $<0.0090$ & $<0.0075$ & $<0.0099$ & $\mathrm{~b}$ \\
\hline 2 & $<0.0108$ & $<0.0099$ & $<0.0117$ & $<0.0095$ & $<0.0109$ & $<0.0089$ \\
\hline 3 & $<0.0086$ & b & $<0.0087$ & $<0.0071$ & $<0.0082$ & $<0.0071$ \\
\hline 4 & $<0.0107$ & $<0.0087$ & 0.0128 & $<0.0079$ & $<0.0088$ & $<0.0081$ \\
\hline 5 & $<0.0067$ & $<0.0064$ & $<0.0105$ & $<0.0066$ & $<0.0076$ & $<0.0067$ \\
\hline 6 & $<0.0100$ & $<0.0107$ & $<0.0155$ & $<0.0076$ & $<0.0085$ & $<0.0073$ \\
\hline 7 & $<0.0097$ & $<0.0083$ & $<0.0106$ & $<0.0108$ & $<0.0083$ & $<0.0091$ \\
\hline 8 & $<0.0094$ & $<0.0083$ & $<0.0099$ & $<0.0080$ & $<0.0082$ & $<0.0075$ \\
\hline 9 & $<0.0086$ & $<0.0080$ & $<0.0075$ & $<0.0086$ & $<0.0129$ & $<0.0073$ \\
\hline 10 & $<0.0086$ & $<0.0114$ & $<0.0107$ & $<0.0082$ & $<0.0108$ & $<0.0080$ \\
\hline 11 & $<0.0082$ & $<0.0094$ & $<0.0110$ & $<0.0086$ & $<0.0118$ & $<0.0080$ \\
\hline
\end{tabular}

(a) Tennessee standard for 30-day average $=1.2 \mathrm{ug} / \mathrm{m} 3$

(b) Indicates no sample or downtime 
Table 5.25. Total suspended particulates in air at the $Y-12$ Plant

\begin{tabular}{|c|c|c|c|c|c|}
\hline \multirow[b]{2}{*}{$\begin{array}{l}\text { Sample } \\
\text { Date }\end{array}$} & \multicolumn{2}{|c|}{$\begin{array}{c}\text { Concentration (a) } \\
\text { (ug/m3) }\end{array}$} & \multirow[b]{2}{*}{$\begin{array}{l}\text { Sample } \\
\text { date }\end{array}$} & \multicolumn{2}{|c|}{$\begin{array}{c}\text { Concentration (a) } \\
(\mathrm{ug} / \mathrm{m} 3)\end{array}$} \\
\hline & East & West & & East & West \\
\hline $01 / 02 / 93$ & 16.12 & $10.41 \mathrm{c}$ & $07 / 06 / 93$ & $\overline{66.96}$ & 53.39 \\
\hline $01 / 08 / 93$ & 9.48 & 8.10 & $07 / 12 / 93$ & 41.63 & $26.17 \mathrm{c}$ \\
\hline $01 / 14 / 93$ & 22.99 & $14.53 \mathrm{c}$ & $07 / 18 / 93$ & 48.78 & $39.26 c$ \\
\hline $01 / 20 / 93$ & 31.25 & $20.88 c$ & $07 / 24 / 93$ & 55.14 & $32.61 \mathrm{c}$ \\
\hline $01 / 26 / 93$ & 24.07 & $11.43 \mathrm{c}$ & $07 / 30 / 93$ & 47.07 & 30.06 \\
\hline $02 / 01 / 93$ & 20.60 & $b$ & $08 / 05 / 93$ & 3.15 & 24.54 \\
\hline $02 / 07 / 93$ & 38.34 & $22.71 \mathrm{c}$ & $08 / 11 / 93$ & 38.85 & $b$ \\
\hline $02 / 13 / 93$ & 12.03 & 10.62 & $08 / 17 / 93$ & 67.96 & 51.03 \\
\hline $02 / 19 / 93$ & 18.19 & 15.16 & $08 / 23 / 93$ & 65.96 & 57.14 \\
\hline $02 / 25 / 93$ & 15.21 & 15.30 & $08 / 29 / 93$ & 50.17 & 43.15 \\
\hline $03 / 03 / 93$ & 24.76 & $19.91 \mathrm{c}$ & $09 / 04 / 93$ & 16.48 & 17.67 \\
\hline $03 / 09 / 93$ & 25.98 & $\mathrm{~b}$ & $09 / 10 / 93$ & 40.13 & $b$ \\
\hline $03 / 15 / 93$ & 27.04 & $b$ & $09 / 16 / 93$ & 13.17 & b \\
\hline $03 / 21 / 93$ & 41.02 & b & $09 / 22 / 93$ & 80.85 & $b$ \\
\hline$* 03 / 26 / 93$ & 24.58 & b & $09 / 28 / 93$ & $b$ & 14.47 \\
\hline $04 / 01 / 93$ & 9.76 & b & $10 / 04 / 93$ & 26.85 & $b$ \\
\hline $04 / 07 / 93$ & 29.77 & b & $10 / 10 / 93$ & b & $b$ \\
\hline $04 / 13 / 93$ & 50.36 & b & $10 / 16 / 93$ & b & 28.12 \\
\hline $04 / 19 / 93$ & 68.43 & b & $10 / 22 / 93$ & 22.64 & $12.19 \mathrm{c}$ \\
\hline $04 / 25 / 93$ & 35.19 & b & $10 / 28 / 93$ & 61.17 & $18.34 \mathrm{c}$ \\
\hline $05 / 01 / 93$ & 66.06 & b & $11 / 03 / 93$ & 27.43 & 24.61 \\
\hline $05 / 07 / 93$ & 74.12 & b & $11 / 09 / 93$ & 128.25 & $29.56 \mathrm{c}$ \\
\hline $05 / 13 / 93$ & 38.90 & b & $11 / 15 / 93$ & b & 7.79 \\
\hline $05 / 19 / 93$ & 35.75 & b & $11 / 21 / 93$ & b & $8.88 c$ \\
\hline $05 / 25 / 93$ & 55.58 & b & $11 / 27 / 93$ & b & $2.87 \mathfrak{c}$ \\
\hline $05 / 31 / 93$ & 26.72 & 20.09 & $12 / 03 / 93$ & 42.59 & 36.01 \\
\hline $06 / 06 / 93$ & 27.44 & 26.51 & $12 / 09 / 93$ & 34.39 & 23.67 \\
\hline $06 / 12 / 93$ & 35.76 & 32.22 & $12 / 15 / 93$ & b & $5.33 \mathrm{c}$ \\
\hline $06 / 18 / 93$ & $b$ & 44.43 & $12 / 21 / 93$ & 11.24 & 8.03 \\
\hline $06 / 24 / 93$ & 54.94 & $b$ & $12 / 27 / 93$ & $18.04 \mathrm{c}$ & 14.82 \\
\hline $06 / 30 / 93$ & 31.98 & 23.43 & & & \\
\hline
\end{tabular}

* Schedule changed to match Tennessee Dept. of Environment and Conservation run dates.

a - Previous Tennessee primary air quality standard $=260 \mathrm{ug} /(\mathrm{m} 3 / 24 \mathrm{~h})$.

Previous Tennessee secondary air quality standard $=150 \mathrm{ug} /(\mathrm{m} 3 / 24 \mathrm{~h})$.

b - Invalid sample or no sample (downtime)

c - Sampler exceeded $60 \mathrm{cfm}$ 
Table 5.26. $1993 \mathrm{PM}-10$ concentrations in air at the $\mathrm{Y}-12$ Plant

\begin{tabular}{|c|c|c|c|c|c|c|c|}
\hline \multirow{3}{*}{$\begin{array}{l}\text { Sample } \\
\text { Date }\end{array}$} & \multicolumn{3}{|c|}{$\begin{array}{l}\text { Concentration (a) } \\
(u g / \mathrm{m} 3)\end{array}$} & \multirow{3}{*}{$\begin{array}{l}\text { Sample } \\
\text { Date }\end{array}$} & \multicolumn{3}{|c|}{$\begin{array}{l}\text { Concentration (a) } \\
\text { (ug/m3) }\end{array}$} \\
\hline & & & East & & & & East \\
\hline & West & East & Collected & & West & East & Collocated \\
\hline $01 / 02 / 93$ & 9.55 & 9.75 & $4.19 c$ & $07 / 06 / 93$ & 44.36 & 45.76 & 48.39 \\
\hline $01 / 08 / 93$ & 5.24 & $b$ & $1.21 \mathrm{c}$ & $07 / 12 / 93$ & 23.59 & 24.17 & 25.39 \\
\hline $01 / 14 / 93$ & 9.99 & 12.17 & $4.59 \mathrm{c}$ & $07 / 18 / 93$ & 44.25 & 31.30 & 40.23 \\
\hline $01 / 20 / 93$ & 15.66 & 18.29 & $5.80 \mathrm{c}$ & $07 / 24 / 93$ & 32.44 & 33.25 & 35.27 \\
\hline $01 / 26 / 93$ & 5.13 & 8.00 & $3.84 c$ & $07 / 30 / 93$ & 19.14 & 20.55 & 21.42 \\
\hline $02 / 01 / 93$ & 5.71 & 6.26 & $2.23 \mathrm{c}$ & $08 / 05 / 93$ & 26.64 & 27.08 & 28.50 \\
\hline $02 / 07 / 93$ & 18.66 & 20.39 & $9.13 \mathrm{c}$ & $08 / 11 / 93$ & 35.00 & 34.69 & 36.25 \\
\hline $02 / 13 / 93$ & 6.45 & 6.34 & $2.16 \mathrm{c}$ & $08 / 17 / 93$ & 64.74 & 58.91 & 64.92 \\
\hline $02 / 19 / 93$ & $\mathrm{~b}$ & 7.77 & $3.04 \mathrm{c}$ & $08 / 23 / 93$ & 49.09 & 48.08 & 45.77 \\
\hline $02 / 25 / 93$ & $b$ & 8.39 & $2.64 \mathrm{c}$ & $08 / 29 / 93$ & 37.09 & 37.24 & 40.86 \\
\hline $03 / 03 / 93$ & b & 17.98 & $8.32 \mathrm{c}$ & $09 / 04 / 93$ & 12.06 & 11.07 & 12.07 \\
\hline $03 / 09 / 93$ & 7.02 & 10.07 & $5.90 \mathrm{c}$ & $09 / 10 / 93$ & 13.83 & 17.10 & 17.29 \\
\hline $03 / 15 / 93$ & 11.36 & 15.25 & $7.12 \mathrm{c}$ & $09 / 16 / 93$ & 11.34 & 9.03 & 9.44 \\
\hline $03 / 21 / 93$ & 22.13 & 23.05 & $10.64 \mathrm{c}$ & $09 / 22 / 93$ & 17.88 & 25.15 & 26.80 \\
\hline $03 / 26 / 93^{*}$ & 14.82 & 14.52 & $5.68 \mathrm{c}$ & $09 / 28 / 93$ & 10.13 & 11.56 & 12.29 \\
\hline $04 / 01 / 93$ & 6.85 & 7.14 & $3.10 \mathrm{c}$ & $10 / 04 / 93$ & $b$ & 10.07 & 10.47 \\
\hline $04 / 07 / 93$ & 15.74 & 17.52 & $8.08 c$ & $10 / 10 / 93$ & $b$ & 4.06 & 3.59 \\
\hline $04 / 13 / 93$ & 19.51 & 23.73 & $11.92 \mathrm{c}$ & $10 / 16 / 93$ & $b$ & 16.00 & 16.43 \\
\hline $04 / 19 / 93$ & 19.17 & 26.62 & $12.57 \mathrm{c}$ & $10 / 22 / 93$ & 4.27 & 7.05 & 8.03 \\
\hline $04 / 25 / 93$ & 14.01 & 3.85 & $0.46 \mathrm{c}$ & $10 / 28 / 93$ & 8.95 & 17.60 & 17.09 \\
\hline $05 / 01 / 93$ & 23.03 & 25.26 & 25.74 & $11 / 03 / 93$ & 9.79 & 11.79 & 13.58 \\
\hline $05 / 07 / 93$ & 31.20 & 31.20 & 27.45 & $11 / 09 / 93$ & 14.98 & 27.81 & 27.96 \\
\hline $05 / 13 / 93$ & 15.78 & 17.19 & 16.91 & $11 / 15 / 93$ & 2.89 & 0.64 & 0,91 \\
\hline $05 / 19 / 93$ & 16.81 & 15.93 & $b$ & $11 / 21 / 93$ & 2.77 & 4.59 & 5.18 \\
\hline $05 / 25 / 93$ & 22.22 & 28.31 & 27.01 & $11 / 27 / 93$ & $b$ & 0.30 & 0.59 \\
\hline $05 / 31 / 93$ & 14.60 & 17.04 & 16.33 & $12 / 03 / 93$ & 17.11 & 18.01 & 17.72 \\
\hline $06 / 06 / 93$ & 20.46 & 19.71 & 19.22 & $12 / 09 / 93$ & 8.64 & 10.50 & 9.16 \\
\hline $06 / 12 / 93$ & 21.86 & b & 22.61 & $12 / 15 / 93$ & 1.57 & 2.69 & 0.71 \\
\hline $06 / 18 / 93$ & 35.91 & b & 37.88 & $12 / 21 / 93$ & 1.39 & 2.66 & 2.06 \\
\hline $06 / 24 / 93$ & 31.02 & 30.95 & 32.76 & $12 / 27 / 93$ & 3.27 & 4.25 & 3.77 \\
\hline $06 / 30 / 93$ & 19.89 & 21.63 & 23.86 & & & & \\
\hline
\end{tabular}

* Schedule changed to match Tennessee Dept. of Environment and Conservation run dates.

a - Tennessee primary air quality standard $=150 \mathrm{ug} / \mathrm{m} 3 / 24 \mathrm{~h}$

b - Invalid sample or no sample (down time)

c - Evasive monitor malfunction on collocated sampler from Jan. thru April, solved April 30, 1993. 
Table 5.27. Annual results of the Y-12 Plant airborne mercury monitoring program, 1986-1993.

\begin{tabular}{|c|c|c|c|c|c|}
\hline \multirow[b]{2}{*}{ Site } & \multirow[b]{2}{*}{ Year } & \multirow{2}{*}{$\begin{array}{c}\text { Number of } \\
\text { samples }\end{array}$} & \multicolumn{3}{|c|}{$\begin{array}{l}\text { Mercury vapor concentration } \\
\qquad\left(\mu \mathrm{g} / \mathrm{m}^{3}\right)\end{array}$} \\
\hline & & & $\operatorname{Max}$ & Min & $A v^{a}$ \\
\hline Ambient No. 2 & 1986 & 34 & 0.058 & 0.003 & 0.011 \\
\hline \multirow[t]{7}{*}{ (east end of Y-12 Plant) } & 1987 & 52 & 0.033 & 0.001 & 0.009 \\
\hline & 1988 & 52 & 0.036 & 0.003 & 0.010 \\
\hline & 1989 & 52 & 0.012 & 0.003 & 0.006 \\
\hline & 1990 & 52 & 0.018 & $<.001$ & 0.006 \\
\hline & 1991 & 51 & 0.073 & $<.001$ & 0.008 \\
\hline & 1992 & 36 & 0.015 & 0.002 & 0.005 \\
\hline & 1993 & 45 & 0.026 & 0.003 & 0.008 \\
\hline Ambient No. 8 & 1986 & 27 & 0.034 & $<.001$ & 0.017 \\
\hline \multirow[t]{7}{*}{ (west end of Y-12 Plant) } & 1987 & 52 & 0.067 & 0.007 & 0.032 \\
\hline & 1988 & 52 & 0.407 & 0.007 & 0.041 \\
\hline & 1989 & 52 & 1.187 & 0.006 & 0.143 \\
\hline & 1990 & 51 & 0.025 & 0.002 & 0.011 \\
\hline & 1991 & 51 & 0.067 & 0.005 & 0.016 \\
\hline & 1992 & 49 & 0.022 & 0.001 & 0.007 \\
\hline & 1993 & 45 & 0.031 & 0.004 & 0.012 \\
\hline Bldg. 9404-13 & 1986 & 31 & 0.197 & 0.033 & 0.108 \\
\hline \multirow[t]{7}{*}{ (SW of Bldg. 9201-4) } & 1987 & 52 & 0.465 & 0.044 & 0.174 \\
\hline & 1988 & 51 & 0.340 & 0.028 & 0.137 \\
\hline & 1989 & 52 & 0.250 & 0.024 & 0.101 \\
\hline & 1990 & 52 & 0.277 & 0.001 & 0.067 \\
\hline & 1991 & 51 & 0.181 & 0.018 & 0.070 \\
\hline & 1992 & 45 & 0.137 & 0.010 & 0.056 \\
\hline & 1993 & 45 & 0.250 & 0.017 & 0.078 \\
\hline Bldg. 9805-1 & 1986 & 15 & 0.137 & 0.026 & 0.070 \\
\hline \multirow[t]{7}{*}{ (SE of Bldg. 9201-4) } & 1987 & 52 & 0.226 & 0.036 & 0.109 \\
\hline & 1988 & 52 & 0.384 & 0.017 & 0.097 \\
\hline & 1989 & 51 & 0.206 & 0.017 & 0.072 \\
\hline & 1990 & 52 & 0.162 & 0.018 & 0.070 \\
\hline & 1991 & 48 & 0.275 & 0.003 & 0.058 \\
\hline & 1992 & 49 & 0.125 & 0.001 & 0.038 \\
\hline & 1993 & 45 & 0.314 & 0.010 & 0.088 \\
\hline \multirow{3}{*}{ New Hope Pond ${ }^{b}$} & 1987 & 20 & 0.039 & 0.006 & 0.016 \\
\hline & 1988 & 52 & 0.412 & 0.004 & 0.046 \\
\hline & 1989 & 37 & 0.009 & 0.002 & 0.004 \\
\hline \multirow{2}{*}{$\begin{array}{l}\text { Rain Gage No. } 2^{c} \\
\text { (Chestnut Ridge) }\end{array}$} & 1988 & 47 & 0.016 & 0.002 & 0.006 \\
\hline & 1989 & 47 & 0.015 & $<.001$ & 0.005 \\
\hline
\end{tabular}

a NESHAP 30-day average standard $=1 \mu \mathrm{g} / \mathrm{m}^{3}$

ACGIH 8-hour day, 40-hour work week standard $=50 \mu \mathrm{g} / \mathrm{m}^{3}$

b Site discontinued September 19, 1989.

' Site discontinued October 31, 1989. 
BCK 0.6

BCK 9.4

CRK 16

CRK 23

CRK 32

CRK 58

CRK 66

CRK 80

CRK 84

EFK 5.4

EFK 23.4

HC

MEK 0.2

MEK 2.1

MIK 0.1

MIK 1.4

PCK 2.2

PCK 22

TRK 915

WCK 1.0

WCK 2.6

WCK 6.8
Bear Creek downstream from all DOE inputs

Bear Creek downstream from the Y-12 Plant burial grounds

Clinch River downstream from all DOE inputs

Water supply intake for the K-25 Site

Clinch River downstream from ORNL

Water supply intake for Knox County

Melton Hill Reservoir above City of Oak Ridge water intake

Melton Hill Reservoir-Oak Ridge Marina

Melton Hill Reservoir above all DOE inputs-Anderson County Filtration Plant

East Fork Poplar Creek downstream from floodplain

East Fork Poplar Creek downstream from the Y-12 Plant

Hinds Creek (reference site for East Fork Poplar Creek)

Meiton Branch downstream from ORNL

Melton Branch upstream from ORNL

Mitchell Branch downstream from the K-25 Site

Mitchell Branch upstream from the K-25 Site

Poplar Creek downstream from the K-25 Site

Poplar Creek upstream from the K-25 Site and East Fork Poplar Creek

Water supply intake for City of Kingston

White Oak Lake at White Oak Dam

White Oak Creek downstream from ORNL

White Oak Creek upstream from ORNL 


\begin{tabular}{|c|c|c|}
\hline \multicolumn{3}{|l|}{ Parameters: } \\
\hline Anions & Others & Volatile Organics \\
\hline Chloride & Alkalinity & 1,1,1-Trichloroethane \\
\hline Fluoride & Ammonia, as $\mathrm{N}$ & 1,1,2,2-Tetrachloroethane \\
\hline Nitrate & Biochemical oxygen demand & 1,1,2-Trichloroethane \\
\hline Sulfate, as $\mathrm{SO}_{4}$ & Chemical oxygen demand & 1.1-Dichloroethane \\
\hline & Color & 1,1-Dichloroethene \\
\hline Metals & Cyanide, total & 1,2-Dichloroethane \\
\hline$\overline{\text { Aluminum }}$ & Total dissolved solids & 1,2-Dichloroethene, total \\
\hline Antimony & Total hardness & 1,2-Dichloropropane \\
\hline Arsenic & Total suspended solids & 2-Butanone \\
\hline Barium & & 2-Hexanone \\
\hline Beryllium & Radionuclides & 4-Methyl-2-pentanone \\
\hline Cadmium & $\mathrm{Co}-60$ & Acetone \\
\hline Calcium & Cs-137 & Benzene \\
\hline Chromium & Gross alpha $^{a}$ & Bromodichloromethane \\
\hline Cobalt & Gross beta & Bromoform \\
\hline Copper & $\mathrm{H}-3$ & Bromomethane \\
\hline Iron & Tc-99 & Carbon disulfide \\
\hline Lead & Total Uranium & Carbon tetrachloride \\
\hline Magnesium & Total rad Sr & Chlorobenzene \\
\hline Manganese & & Chloroethane \\
\hline Mercury & Field Measurements & Chloroform \\
\hline Nickel & Chlorine, total residual & Chloromethane \\
\hline Phosphorus & Conductivity & cis-1,3-Dichloropropene \\
\hline Potassium & Dissolved oxygen & Dibromochloromethane \\
\hline Selenium & $\mathrm{pH}$ & Ethylbenzere \\
\hline Silver & Temperature & Methylene chloride \\
\hline Sodium & & Styrene \\
\hline Thallium & & Tetrachloroethene \\
\hline Uranium & & Toluene \\
\hline Vanadium & & trans-1,3-Dichloropropene \\
\hline Zinc & & Trichloroethene \\
\hline & & Vinyl chloride \\
\hline & & Xylene, total \\
\hline
\end{tabular}

\section{Sample type:}

Field Measurements - Grab, instant read

Anions, Metals, Radionuclides, Others, and Volatile Organics - Grab

Collection frequency:

Bi-monthly

Analysis frequency:

Bi-monthly

If Gross alpha $>3 \mathrm{pCi} / \mathrm{L}$ ( $20 \%$ of EPA drinking water limit), report U-234, U-235, and U-238 from the Total Uranium sample results and do isotopic analysis for Th-228, Th-230, Th-232, Pu-238, Pu-239, Np-237, Am-241, and $\mathrm{Cm}-244$, as needed to identify cause. 
Table 5.30. 1993 concentrations at EMP surface water locations

\begin{tabular}{|c|c|c|c|c|c|c|}
\hline \multirow[b]{2}{*}{ Parameter } & \multirow[b]{2}{*}{$\begin{array}{l}\text { N det } / \\
\mathrm{N} \text { total }\end{array}$} & \multicolumn{4}{|c|}{ Concentration } & \multirow[b]{2}{*}{ TWQC } \\
\hline & & $\operatorname{Max}$ & $\operatorname{Min}^{b}$ & $A V^{C}$ & $\begin{array}{l}\text { Standard } \\
\text { error }^{d}\end{array}$ & \\
\hline Bear Cre & eek dor & stream from & al1 DOE inputs & $(B C K \quad 0.6)$ & & \\
\hline \multicolumn{7}{|l|}{ Anions (mg/L) } \\
\hline Chloride & $5 / 5$ & 9.3 & 4.5 & 5.9 & 0.87 & $f$ \\
\hline Fluoride & $2 / 5$ & 0.30 & $<0.10$ & -0.16 & 0.040 & $f$ \\
\hline Nitrate & $5 / 5$ & 23 & 3.4 & 9.5 & 3.5 & $f$ \\
\hline Sulfate, as $\mathrm{SO}_{4}$ & $5 / 5$ & 51 & 15 & 32 & 6.4 & $f$ \\
\hline \multicolumn{7}{|l|}{ Field Measurements } \\
\hline Conductivity (ms/cm) & $5 / 5$ & 0.45 & 0.30 & 0.37 & 0.025 & $f$ \\
\hline Dissolved oxygen (ppm) & $5 / 5$ & 11 & 7.4 & 8.9 & 0.62 & 5 \\
\hline $\mathrm{pH}$ (SU) & $5 / 5$ & 8.1 & 7.6 & 7.8 & 0.097 & $f$ \\
\hline Temperature $\left({ }^{\circ} \mathrm{C}\right)$ & $5 / 5$ & 21 & 9.4 & 16 & 2.1 & $f$ \\
\hline \multicolumn{7}{|l|}{ Metals (mg/L) } \\
\hline Aluminum, total & $5 / 5$ & 0.48 & 0.061 & 0.20 & 0.076 & $f$ \\
\hline Barium, tocal & $5 / 5$ & 0.14 & 0.059 & 0.087 & 0.014 & $f$ \\
\hline Calcium, total & $5 / 5$ & 57 & 30 & 46 & 4.8 & $f$ \\
\hline Chromium, total & $2 / 5$ & 0.014 & $<0.0040$ & -0.0078 & 0.0023 & 0.016 \\
\hline Iron, total & $5 / 5$ & 0.37 & 0.082 & 0.17 & 0.052 & $f$ \\
\hline Magnesium, total & $5 / 5$ & 18 & 8.5 & 14 & 1.6 & $f$ \\
\hline Manganese, total & $5 / 5$ & 0.054 & 0.0078 & 0.026 & 0.0080 & $f$ \\
\hline Phosphorus, total & $2 / 5$ & 0.50 & $<0.20$ & -0.26 & 0.060 & $f$ \\
\hline Potassiun, total & $3 / 4$ & 2.4 & 1.8 & -2.1 & 0.13 & \pm \\
\hline Sodium, total & $5 / 5$ & 5.4 & 2.8 & 4.0 & 0.41 & $f$ \\
\hline Uranium, total & $3 / 4$ & 0.027 & $<0.00010$ & $\sim 0.015$ & 0.0056 & $f$ \\
\hline zinc, total & $2 / 5$ & 0.060 & $<0.0050$ & -0.022 & 0.011 & 0.117 \\
\hline \multicolumn{7}{|l|}{ Others } \\
\hline Alkalinity (mg $\mathrm{CaCO}_{3} / \mathrm{L}$ ) & $5 / 5$ & 160 & 98 & 140 & 12 & $f$ \\
\hline Ammonia, as $\mathrm{N}$ (mg/L) & $5 / 5$ & 0.080 & 0.030 & 0.048 & 0.0097 & $E$ \\
\hline Biochemical oxygen demand (mg/L) & $1 / 5$ & 5.0 & $<5.0$ & -5.0 & 0 & E \\
\hline Chemical oxygen demand $(\mathrm{mg} / \mathrm{L})$ & $1 / 5$ & 5.0 & $<5.0$ & -5.0 & 0 & $f$ \\
\hline Color (CPU) & $4 / 5$ & 11 & $<1.0$ & -5.6 & 1.7 & $f$ \\
\hline Total dissolved solids (mg/L) & $5 / 5$ & 290 & 170 & 240 & 20 & $f$ \\
\hline Total hardness (mg/L) & $5 / 5$ & 210 & 120 & 170 & 18 & $f$ \\
\hline \multicolumn{7}{|l|}{ Radionuclides $(\mathrm{pCi} / L)^{g}$} \\
\hline Co-60 & $1 / 5$ & $5.1 *$ & 0.27 & 1.8 & 0.87 & $f$ \\
\hline Gross alpha & $5 / 5$ & $12 *$ & $7.8^{*}$ & $10^{*}$ & 0.75 & $f$ \\
\hline Gross beta & $5 / 5$ & $19 *$ & $6.2^{*}$ & 13* & 2.5 & $f$ \\
\hline $\mathrm{B}-3$ & $1 / 5$ & $1,300 *$ & 0 & 370 & 230 & $f$ \\
\hline Pu-238 & $1 / 2$ & $0.54 *$ & -0.11 & 0.22 & 0.32 & $f$ \\
\hline Tc-99 & $5 / 5$ & $25 *$ & $6.2^{*}$ & $13^{\circ}$ & 3.4 & $E$ \\
\hline Total rad Sr & $3 / 5$ & $3.0 *$ & -0.68 & 1.4 & 0.67 & $E$ \\
\hline Total uranium & $5 / 5$ & $12^{\circ}$ & $8.1 *$ & $9.4^{*}$ & 0.66 & $f$ \\
\hline $0-234$ & $4 / 4$ & $4.6 *$ & $3.2^{*}$ & $3.6^{\star}$ & 0.32 & $f$ \\
\hline $\mathrm{u}-235$ & $3 / 4$ & $0.59 *$ & 0.027 & $0.29 *$ & 0.12 & $f$ \\
\hline $\mathrm{U}-238$ & $4 / 4$ & $7.0 *$ & $4.6^{*}$ & $5.4^{*}$ & 0.57 & $f$ \\
\hline Volatile organics $(\mu \mathrm{g} / \mathrm{L})$ & & & & & & \\
\hline Methylene chloride & $1 / 5$ & U5. 0 & 582.6 & -4.5 & 0.48 & $\bar{f}$ \\
\hline
\end{tabular}


Table 5.30 (continued)

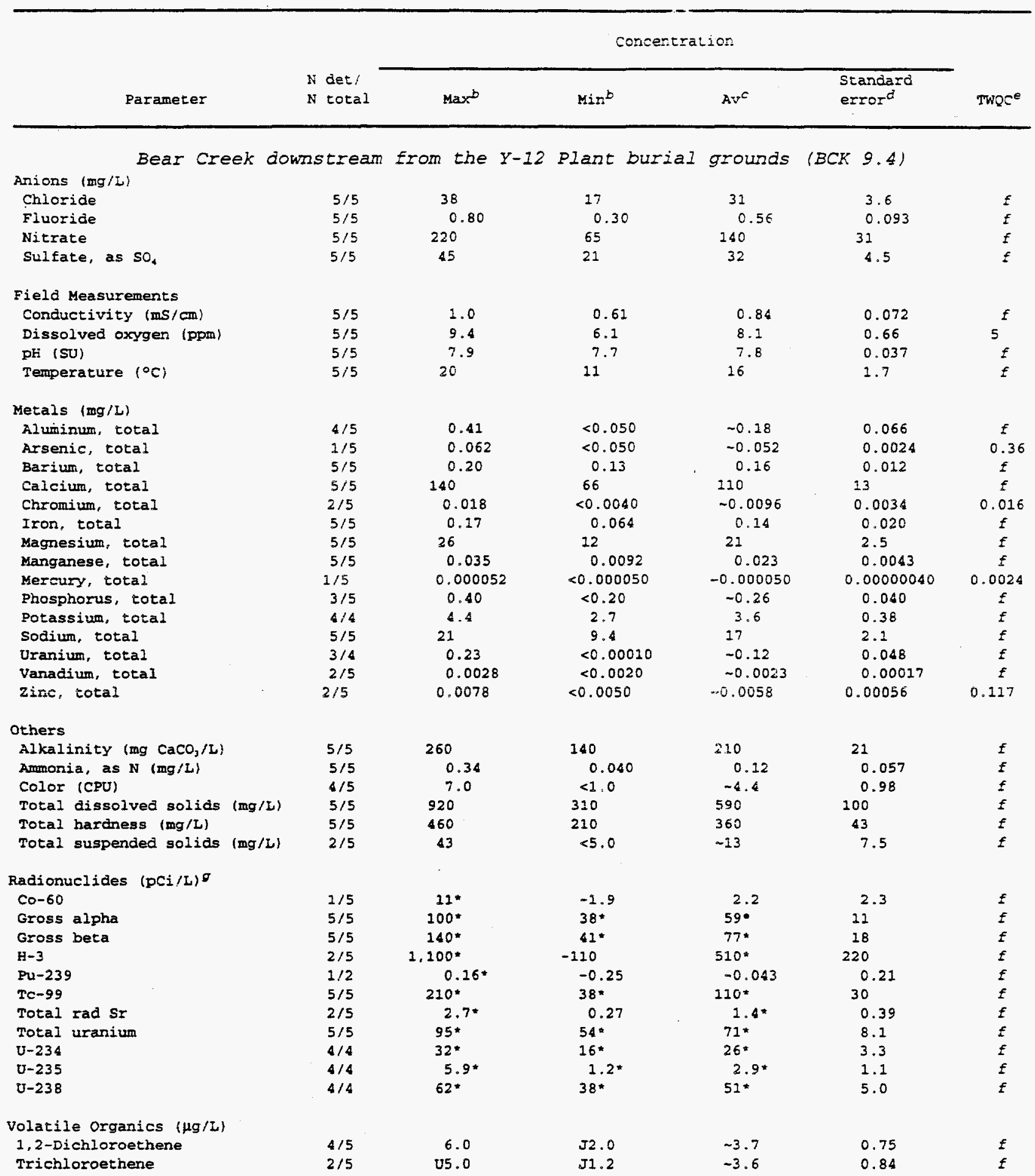


Table 5.30 (continued)

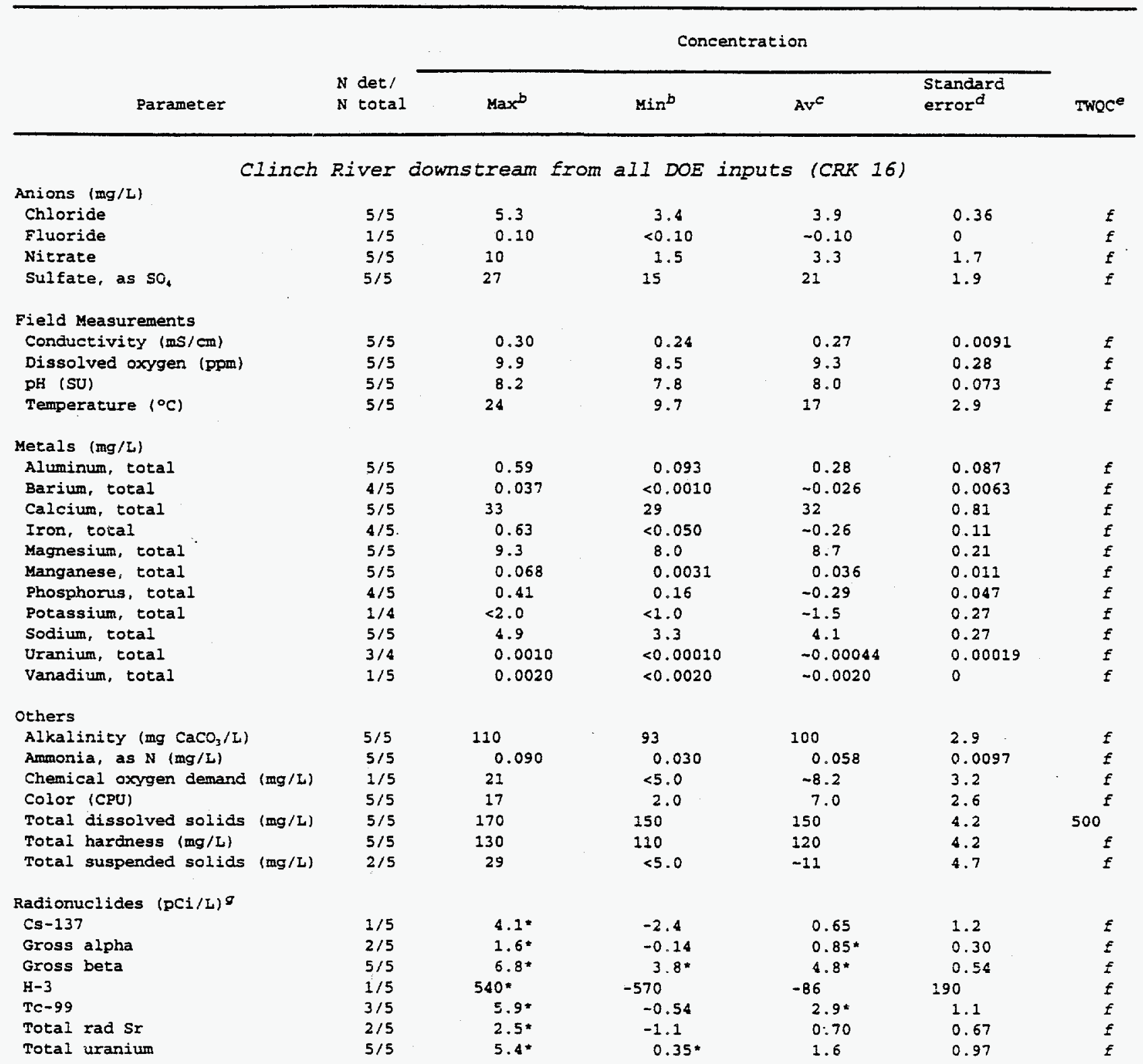

Water supply intake for the $K-25$ Site (CRK 23)

Anions $(\mathrm{mg} / \mathrm{L})$

Chloride

Nitrate

sulfate, as so,

Field Measurements

Conductivity (mS/ cm)

Dissolved oxygen (Dpm)

$\mathrm{pH}$ (SU)

Temperature $\left({ }^{\circ} \mathrm{C}\right)$
5.1

$5 / 5$

$5 / 5$

$5 / 5$

$5 / 5$
$5 / 5$
$5 / 5$

$5 / 5$
3.3

1.0

20

0.36
10
8.1
21

0.28

7.0

7.8

7.7
3.9

2.7

22

0.31

8.7

8.0

15
0.32

0.71

1.1

0.015
0.66
0.055
2.7 $f$
$f$
$f$

$f$
$f$
$f$
$f$ 


\begin{tabular}{|c|c|c|c|c|c|c|}
\hline \multirow[b]{2}{*}{ Parameter } & \multirow[b]{2}{*}{$\begin{array}{l}N \text { det; } \\
N \text { cozal }\end{array}$} & \multicolumn{4}{|c|}{ Concertration. } & \multirow[b]{2}{*}{ TwQ ${ }^{e}$} \\
\hline & & $\operatorname{Max}$ & $\operatorname{Min}^{b}$ & $\mathrm{Av}^{\mathrm{C}}$ & $\begin{array}{l}\text { Standard } \\
\text { error }\end{array}$ & \\
\hline \multicolumn{7}{|l|}{ Metals $(\mathrm{mg} / \mathrm{L})$} \\
\hline Aluminum, total & $5 / 5$ & 0.41 & 0.19 & 0.27 & 0.037 & $f$ \\
\hline Barium, total & $4 / 5$ & 0.031 & $<0.0010$ & -0.024 & 0.0058 & $f$ \\
\hline Calcium, rotal & $5 / 5$ & 36 & 31 & 33 & 1.1 & $f$ \\
\hline Iron, total & $4 / 5$ & 0.52 & $<0.050$ & -0.28 & 0.076 & $f$ \\
\hline Magnesium, total & $5 / 5$ & 9.9 & 8.3 & 9.1 & 0.33 & $\varepsilon$ \\
\hline Manganese, total & $5 / 5$ & 0.11 & 0.020 & 0.054 & 0.015 & $\varepsilon$ \\
\hline Phosphorus, total & $3 / 5$ & 0.40 & $<0.20$ & -0.28 & 0.043 & $E$ \\
\hline Potassium, total & $1 / 4$ & $<2.0$ & $<1.0$ & -1.5 & 0.25 & $f$ \\
\hline Sodium, total & $5 / 5$ & 4.9 & 3.8 & 4.4 & 0.20 & $f$ \\
\hline Uranium, total & $2 / 4$ & 0.00020 & $<0.00010$ & -0.00015 & 0.000029 & $f$ \\
\hline Vanadium, total & $1 / 5$ & 0.0024 & $<0.0020$ & -0.0021 & 0.000080 & $f$ \\
\hline zine, cotal & $1 / 5$ & 0.0056 & $<0.0050$ & -0.0051 & 0,00012 & $f$ \\
\hline \multicolumn{7}{|l|}{ Others } \\
\hline Alkalinity (mg $\mathrm{CaCO}_{3} / L$ ) & $5 / 5$ & 120 & 98 & 110 & 3.2 & $f$ \\
\hline Ammonia, as $N(m g / L)$ & $5 / 5$ & 0.090 & 0.020 & 0.048 & 0.012 & 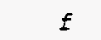 \\
\hline Chemical oxyges demand (mg/L) & $3 / 5$ & 6.0 & $<5.0$ & -5. & 0.24 & $f$ \\
\hline Color (CPU) & $4 / 5$ & 7.0 & $<1.0$ & -3.6 & 1.0 & $f$ \\
\hline Total dissolved solids (mg/L) & $5 / 5$ & 170 & 140 & 150 & 4.4 & 500 \\
\hline Total hardness (mg/L) & $5 / 5$ & 130 & 120 & 120 & 2.2 & $f$ \\
\hline Totai suspended solids $(\mathrm{mg} / \mathrm{L})$ & $4 / 5$ & 40 & $<5.0$ & -14 & 6.5 & $f$ \\
\hline \multicolumn{7}{|l|}{ Radionuclides $(p C i / L)^{g}$} \\
\hline$c s-137$ & $2 / 5$ & $5.7 *$ & 0.27 & $3.1 *$ & 0.96 & $E$ \\
\hline Gross alpha & $2 / 5$ & $2.7 *$ & -0.027 & $1.1 *$ & 0.48 & $f$ \\
\hline Gross beta & $4 / 5$ & $6.2 *$ & 2.4 & $4.2 *$ & 0.64 & $f$ \\
\hline $\mathrm{H}-3$ & $1 / 5$ & $620^{*}$ & 54 & $360^{*}$ & 93 & $f$ \\
\hline TC-99 & $1 / 5$ & 3.8 & -1.9 & 1.5 & 0.93 & $f$ \\
\hline Sotal rad SI & $3 / 5$ & $3.0^{*}$ & -0.32 & $1,3 *$ & 0.61 & $E$ \\
\hline Total urasium & $4 / 5$ & $1.6^{*}$ & 0.14 & $0.76^{*}$ & 0.24 & $f$ \\
\hline $\begin{array}{l}\text { Volatile Organics }(\mu \mathrm{g} / \mathrm{L}) \\
\text { Carbon disulfide }\end{array}$ & $1 / 5$ & 10 & 05.0 & -6.0 & 1.0 & $f$ \\
\hline
\end{tabular}

Clinch River downstream from ORNL (CRK 32)

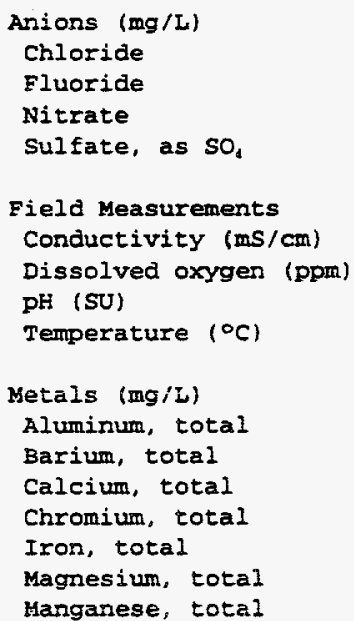

$\begin{array}{ll}5 / 5 & 5.3 \\ 1 / 5 & 0.10 \\ 5 / 5 & 4.3 \\ 5 / 5 & 27\end{array}$

$5 / 5$

$5 / 5$

$5 / 5$

$5 / 5$

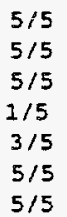

$5 / 5$

$5 / 5$

$1 / 5$

$5 / 5$

$5 / 5$
0.10
4.3

27

0.35
10
8.0
20

1.2

0.031

38

0.032

1.1

9.8

0.091

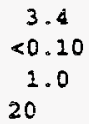

20

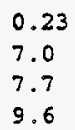

0.11

0.029

30

$<0.0040$

$<0.050$

8.7

0.0098
4.0

$-0.10$

2.5

22

0.36

0

0.57

1.3

0.022

0.61

0.058

7.9

2.2

0.21

0.00039

1.2

34

$-0.0096$

$-0.29$

0.0056

0.20

0.19

0.014 
Table 5.30 (continued)

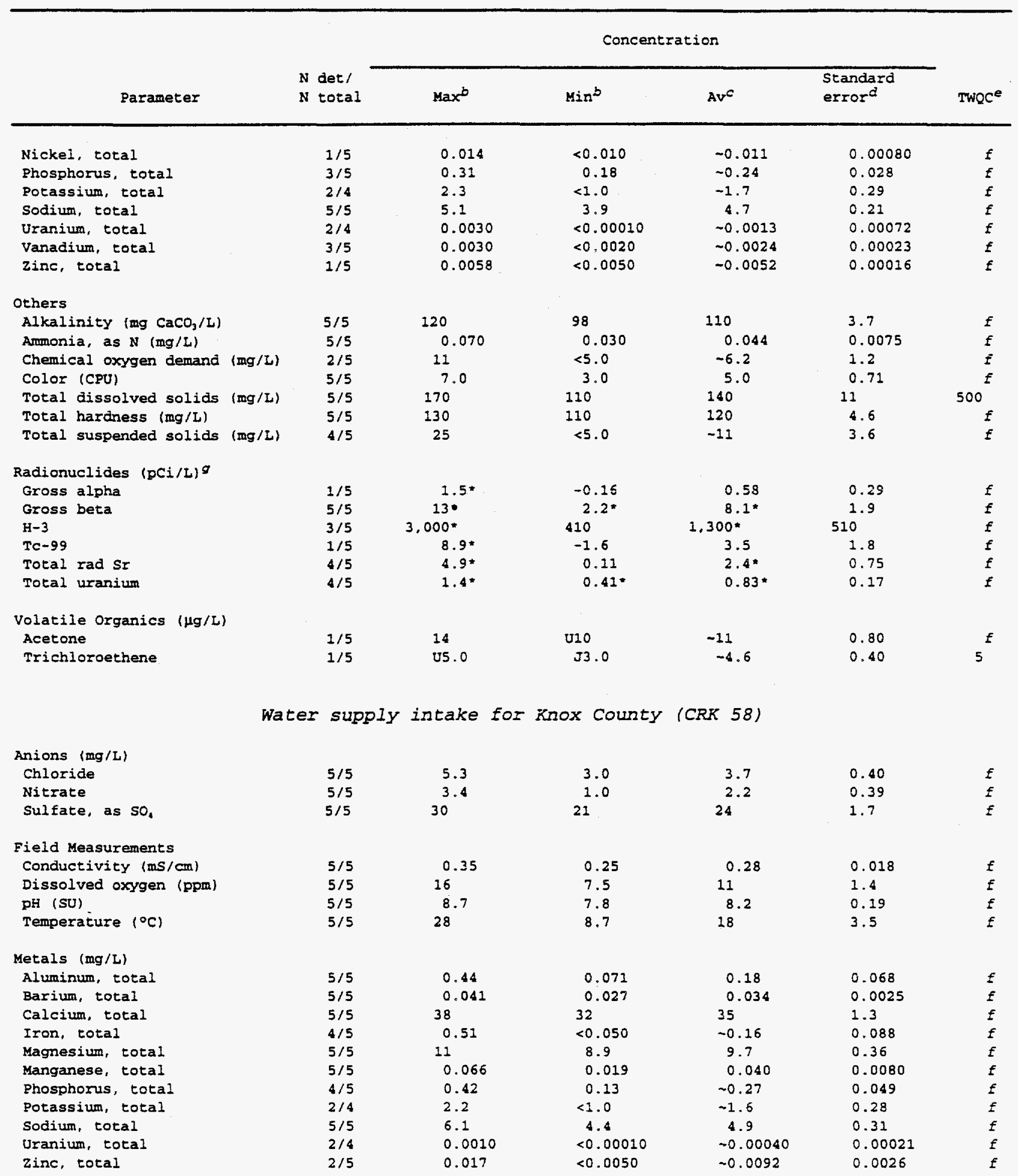


Table 5.30 (continued)

\begin{tabular}{|c|c|c|c|c|c|c|}
\hline \multirow[b]{2}{*}{ Parameter } & \multirow[b]{2}{*}{$\begin{array}{l}N \text { det } / \\
N \text { total }\end{array}$} & \multicolumn{4}{|c|}{ Concentration } & \multirow[b]{2}{*}{ TWQC } \\
\hline & & $\operatorname{Max}^{b}$ & $\operatorname{Min}^{b}$ & $\mathrm{AV}^{e}$ & $\begin{array}{l}\text { Standard } \\
\text { error }{ }^{d}\end{array}$ & \\
\hline \multicolumn{7}{|l|}{ others } \\
\hline Alkalinity (mg $\mathrm{CaCO}_{3} / \mathrm{L}$ ) & $5 / 5$ & 120 & 100 & 110 & 2.7 & $f$ \\
\hline Ammonia, as $\mathrm{N}(\mathrm{mg} / \mathrm{L})$ & $4 / 5$ & 0.060 & $<0.030$ & -0.038 & 0.0058 & $f$ \\
\hline Chemical oxygen demand (mg/L) & $1 / 5$ & 12 & $<5.0$ & -6.4 & 1.4 & $f$ \\
\hline Color $(C P U)$ & $4 / 5$ & 7.0 & $<1.0$ & -4.0 & 1.1 & $f$ \\
\hline Total dissolved solids (mg/h) & $5 / 5$ & 160 & 150 & 150 & 2.0 & 500 \\
\hline Total hardness (mg/L) & $5 / 5$ & 130 & 120 & 120 & 2.4 & $f$ \\
\hline Total suspended solids (mg/L) & $2 / 5$ & 10 & $<5.0$ & -6.6 & 1.0 & $f$ \\
\hline \multicolumn{7}{|l|}{ Radionuclides $(\mathrm{pCi} / \mathrm{L})^{g}$} \\
\hline $\operatorname{co-} 60$ & $1 / 5$ & $15 *$ & -1.4 & 3.0 & 3.0 & $f$ \\
\hline Gross alpha & $3 / 5$ & $3.0^{*}$ & 0.35 & $1.3 *$ & 0.47 & $f$ \\
\hline Gross beta & $4 / 5$ & $4.6 *$ & $1.7 *$ & $2.7 *$ & 0.51 & $f$ \\
\hline $\mathrm{H}-3$ & $1 / 5$ & $620^{*}$ & -460 & 110 & 170 & $f$ \\
\hline TC-99 & $2 / 5$ & $7.6 *$ & -0.65 & 3.1 & $1: 6$ & \pm \\
\hline Total rad SI & $1 / 5$ & $1.5 *$ & 0.35 & $0.98^{*}$ & 0.22 & E \\
\hline Total uranium & $3 / 5$ & $1.1 *$ & 0.32 & $0.64^{*}$ & 0.14 & $f$ \\
\hline
\end{tabular}

Melton Hill Reservoir above City of Oak Ridge water intake (CRK 66)

\begin{tabular}{|c|c|c|c|}
\hline \multicolumn{4}{|l|}{ Anions (mg/L) } \\
\hline Chloride & $5 / 5$ & 5.0 & 3.2 \\
\hline Nitrate & $5 / 5$ & 3.9 & 1.9 \\
\hline Suifate, as so, & $5 / 5$ & 26 & 21 \\
\hline Field measurements & & & \\
\hline Conductivity (ms/cm) & $5 / 5$ & 0.33 & 0.25 \\
\hline Dissolved oxygen (ppm) & $5 / 5$ & 11 & 7.0 \\
\hline $\mathrm{pH}$ (SU) & $5 / 5$ & 8.5 & 7.9 \\
\hline Temperatiure $\left({ }^{\circ} \mathrm{C}\right)$ & $5 / 5$ & 28 & 8.6 \\
\hline Metals $(m g / \ddot{n})$ & & & \\
\hline Aluminum, total & $5 / 5$ & 0.79 & 0.073 \\
\hline Barium, total & $5 / 5$ & 0.040 & 0.030 \\
\hline Calciun, total & $5 / 5$ & 39 & 33 \\
\hline Chromium, total & $1 / 5$ & 0.0053 & $<0.0040$ \\
\hline Iron, total & $3 / 5$ & 0.93 & $<0.050$ \\
\hline Magnesium, total & $5 / 5$ & 9.9 & 8.6 \\
\hline Manganese, total & $4 / 5$ & 0.073 & $<0.0010$ \\
\hline Mercury, total & $1 / 5$ & 0.000083 & $<0.00005$ \\
\hline Phosphorus, total & $4 / 5$ & 0.43 & 0.17 \\
\hline Potassium, total & $2 / 4$ & 2.5 & $<1.0$ \\
\hline Sodiun, total & $5 / 5$ & 5.4 & 4.0 \\
\hline Uraniun, total & $3 / 4$ & 0.0010 & $<0.0001$ \\
\hline Vanadium, total & $1 / 5$ & 0.0028 & $<0.0020$ \\
\hline zinc, total & $1 / 5$ & 0.0059 & $<0.0050$ \\
\hline Others & & & \\
\hline Alkalinity (mg $\mathrm{CaCO}_{3} / \mathrm{L}$ ) & $5 / 5$ & 120 & 95 \\
\hline Ammonia, as $N$ (mg/L) & $5 / 5$ & 0.080 & 0.030 \\
\hline Color (CPU) & $4 / 5$ & 9.0 & $<1.0$ \\
\hline Total dissolved solids (mg/L) & $5 / 5$ & 190 & 150 \\
\hline Total hardness $(\mathrm{mg} / \mathrm{L})$ & $5 / 5$ & 130 & 120 \\
\hline Total suspended solids (mg/L) & $2 / 5$ & 14 & $<5.0$ \\
\hline
\end{tabular}

$\begin{array}{rl}3.9 & 0.32 \\ 2.7 & 0.39 \\ 24 & 0.93\end{array}$

0.32
0.39

0.93

$\begin{array}{ll}0.28 & 0.015 \\ 9.2 & 0.64 \\ 8.0 & 0.12 \\ 18 & 3.3\end{array}$

$f$
$f$
$f$

Metals (mg/:)

0.25

.9

0.64

3.3 
Table 5.30 (continued)

\begin{tabular}{|c|c|c|c|c|c|c|}
\hline \multirow[b]{2}{*}{ Parameter } & \multirow[b]{2}{*}{$\begin{array}{l}\text { N det } / \\
\text { N total }\end{array}$} & \multicolumn{4}{|c|}{ Concentration } & \multirow[b]{2}{*}{ TWQC } \\
\hline & & $\operatorname{Max}^{b}$ & $\operatorname{Min}^{b}$ & $A v^{2}$ & $\begin{array}{l}\text { Standard } \\
\text { errord }\end{array}$ & \\
\hline \multicolumn{7}{|l|}{ Radionuclides $(\mathrm{pCi} / \mathrm{L})^{g}$} \\
\hline $00-60$ & $1 / 5$ & $4.3 *$ & -0.27 & 1.3 & 0.82 & $f$ \\
\hline Gross alpha & $2 / 5$ & $1.6^{*}$ & 0.27 & $0.83 *$ & 0.26 & $f$ \\
\hline Gross beta & $5 / 5$ & $5.1^{*}$ & $2.1 *$ & $4.0=$ & 0.56 & $f$ \\
\hline $\mathrm{H}-3$ & $1 / 5$ & $3,000^{*}$ & -430 & 560 & 620 & $E$ \\
\hline$T C-99$ & $2 / 5$ & $23 *$ & 0.19 & 5.6 & 4.3 & $f$ \\
\hline Total rad Sx & $1 / 5$ & $2.7 *$ & -0.41 & $1.1 *$ & 0.49 & $f$ \\
\hline Total uranium & $3 / 5$ & 2.1* & 0.081 & $1.1 *$ & 0.39 & $f$ \\
\hline
\end{tabular}

Melton Hill Reservoir-Oak Ridge Marina (CRK 80)

\begin{tabular}{|c|c|c|c|c|c|c|}
\hline \multicolumn{7}{|l|}{ Anions (mg/L) } \\
\hline Chloride & $5 / 5$ & 3.8 & 3.2 & 3.4 & 0.11 & $E$ \\
\hline Nitrate & $5 / 5$ & 25 & 1.6 & 6.9 & 4.5 & $E$ \\
\hline Sulfate, as so, & $5 / 5$ & 24 & 16 & 21 & 1.3 & $f$ \\
\hline \multicolumn{7}{|l|}{ Field Measurements } \\
\hline Conductivity $(\mathrm{ms} / \mathrm{cm})$ & $5 / 5$ & 0.35 & 0.26 & 0.29 & 0.017 & $E$ \\
\hline Dissolved oxygen (ppm) & $5 / 5$ & 11 & 7.5 & 9.4 & 0.78 & $\boldsymbol{E}$ \\
\hline $\mathrm{pH}$ (50) & $5 / 5$ & 8.1 & 7.8 & 7.9 & 0.058 & $E$ \\
\hline Temperature $\left.\mid{ }^{\circ} \mathrm{C}\right\}$ & $5 / 5$ & 23 & 8.0 & 15 & 2.3 & $\xi$ \\
\hline \multicolumn{7}{|l|}{ Metals (mg/L) } \\
\hline Aluminum, total & $4 / 5$ & 0.35 & $<0.050$ & -0.14 & 0.054 & $f$ \\
\hline Barium, total & $5 / 5$ & 0.092 & 0.028 & 0.045 & 0.012 & 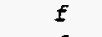 \\
\hline Calcium, total & $5 / 5$ & 36 & 31 & 34 & 0.89 & $f$ \\
\hline Chromium, total & $2 / 5$ & 0.012 & $<0.0040$ & -0.0066 & 0.0017 & 0.05 \\
\hline Iron, total & $5 / 5$ & 0.32 & 0.073 & 0.14 & 0.046 & $E$ \\
\hline Magnesium, total & $5 / 5$ & 10 & 8.8 & 9.5 & 0.26 & $f$ \\
\hline Manganese, total & $5 / 5$ & 0.10 & 0.026 & 0.066 & 0.014 & $f$ \\
\hline Phosphorus, total & $4 / 5$ & 0.40 & $<0.20$ & -0.28 & 0.038 & $f$ \\
\hline Potassium, total & $2 / 4$ & $<2.0$ & $<1.0$ & -1.6 & 0.22 & $f$ \\
\hline Sodium, total & $5 / 5$ & 5.6 & 4.0 & 4.8 & 0.31 & $f$ \\
\hline Uranium, total & $3 / 4$ & 0.00040 & $<0.00010$ & -0.00023 & 0.000063 & 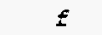 \\
\hline Vanadium, total & $1 / 5$ & 0.0027 & $<0.0020$ & -0.0021 & 0.00014 & $f$ \\
\hline Zinc, total & $3 / 5$ & 0.020 & $<0.0050$ & -0.0084 & 0.0029 & $f$ \\
\hline \multicolumn{7}{|l|}{ Others } \\
\hline Alkalinity (mg $\mathrm{CaCO}_{3} / \mathrm{L}$ ) & $5 / 5$ & 110 & 100 & 110 & 1.4 & $f$ \\
\hline Ammonia, as $\mathrm{N}(\mathrm{mg} / \mathrm{L})$ & $5 / 5$ & 0.090 & 0.030 & 0.063 & 0.011 & $f$ \\
\hline Chemical oxygen demand (mg/L) & $1 / 5$ & 7.0 & $<5.0$ & -5.4 & 0.40 & $E$ \\
\hline Color (CPU) & $4 / 5$ & 8.0 & $<1.0$ & -4.2 & 1.2 & $f$ \\
\hline Total dissolved solids (mg/L) & $5 / 5$ & 180 & 150 & 170 & 4.7 & 500 \\
\hline Total hardness (mg/L) & $5 / 5$ & 130 & 120 & 130 & 2.2 & $f$ \\
\hline Total suspended solids (mg/t) & $2 / 5$ & 10 & $<5.0$ & -6.2 & 0.97 & $E$ \\
\hline \multicolumn{7}{|l|}{ Radionuclides $(\mathrm{pCi} / \mathrm{L})^{g}$} \\
\hline$C 0-60$ & $1 / 5$ & $3.2 \star$ & -1.4 & 1.8 & 0.87 & $f$ \\
\hline Gross alpina & $2 / 5$ & $1.3^{*}$ & -0.65 & 0.67 & 0.37 & $f$ \\
\hline Gross beta & $4 / 5$ & $7.6^{*}$ & 0.81 & $4.4^{*}$ & 1.2 & $f$ \\
\hline TC-99 & $1 / 5$ & $4.9=$ & -3.8 & 2.2 & 1.6 & $f$ \\
\hline Total rad Sr & $2 / 5$ & $3.2 *$ & 0.73 & $1.4 *$ & 0.46 & $f$ \\
\hline Total uranium & $4 / 5$ & $3.5^{*}$ & 0.46 & $1.3^{*}$ & 0.57 & $f$ \\
\hline
\end{tabular}


Table 5.30 (continued)

\begin{tabular}{|c|c|c|c|c|c|c|}
\hline \multirow[b]{2}{*}{ Parameter } & \multirow[b]{2}{*}{$\begin{array}{l}\text { N det } / \\
\mathbf{N} \text { total }\end{array}$} & \multicolumn{4}{|c|}{ Concentration } & \multirow[b]{2}{*}{ TwQ $c^{e}$} \\
\hline & & $\operatorname{Max}$ & $\operatorname{Min}^{b}$ & $A V^{e}$ & $\begin{array}{l}\text { Standard } \\
\text { error }\end{array}$ & \\
\hline Melton Hill Reservoir & above all & DOE inputs & -Anderson C & CountyFiltration & Plant ICRK & $84)$ \\
\hline \multicolumn{7}{|l|}{ Anions (mg/L) } \\
\hline chloride & $5 / 5$ & 3.8 & 3.1 & 3.4 & 0.12 & $f$ \\
\hline Nitrate & $5 / 5$ & 3.8 & 1.3 & 2.4 & 0.43 & $f$ \\
\hline Sulfate, as So, & $5 / 5$ & 25 & 20 & 22 & 0.81 & $\varepsilon$ \\
\hline \multicolumn{7}{|l|}{ Field Measurements } \\
\hline Conductivity $(\mathrm{ms} / \mathrm{cm})$ & $5 / 5$ & 0.35 & 0.26 & 0.28 & 0.017 & $f$ \\
\hline Dissolved oxygen (ppm) & $5 / 5$ & 12 & 7.9 & 10 & 0.58 & $E$ \\
\hline $\mathrm{pH}$ (SU) & $5 / 5$ & 8.1 & 7.9 & 8.0 & 0.037 & $f$ \\
\hline Temperature $\left({ }^{\circ} \mathrm{C}\right)$ & $5 / 5$ & 18 & 9.8 & 15 & 1.4 & $f$ \\
\hline \multicolumn{7}{|l|}{ Metals (mg/L) } \\
\hline Aluminum, total & $5 / 5$ & 0.34 & 0.20 & 0.27 & 0.023 & $f$ \\
\hline Arsenic, total & $1 / 5$ & 0.050 & $<0.050$ & -0.050 & 0 & 0.05 \\
\hline Barium, total & $5 / 5$ & 0.39 & 0.027 & 0.070 & 0.030 & \pm \\
\hline Calcium, total & $5 / 5$ & 36 & 32 & 34 & 0.67 & 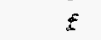 \\
\hline Chromium, rotal & $2 / 5$ & 0.012 & $<0.0040$ & -0.0067 & 0.0017 & 0.05 \\
\hline Iron, total & $5 / 5$ & 0.41 & 0.21 & 0.30 & 0.032 & $f$ \\
\hline Magnesium, total & $5 / 5$ & 10 & 8.9 & 9.6 & 0.23 & $E$ \\
\hline Nanganese, total & $5 / 5$ & 0.16 & 0.041 & 0.091 & 0.020 & \pm \\
\hline Phosphorus, total & $4 / 5$ & 0.40 & $<0.20$ & -0.28 & 0.037 & $f$ \\
\hline Potassium, total & $2 / 4$ & $<2.0$ & $<1.0$ & -1.5 & 0.21 & f \\
\hline Sodium, total & $5 / 5$ & 5.5 & 3.9 & 4.8 & 0.32 & $\varepsilon$ \\
\hline uranius, cotal & $3 / 4$ & 0.00058 & $<0.00010$ & -0.00025 & 0.00011 & $E$ \\
\hline vanadium, total & $1 / 5$ & 0.0028 & $<0.0020$ & -0.0022 & 0.00016 & $f$ \\
\hline zinc, total & $2 / 5$ & 0.038 & $<0.0050$ & -0.012 & 0.0063 & $f$ \\
\hline \multicolumn{7}{|l|}{ Others } \\
\hline Alkalinity (mg $\mathrm{CaCO}_{3} / \mathrm{L}$ ) & $5 / 5$ & 110 & 100 & 110 & 2.5 & 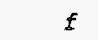 \\
\hline Amonia, as $\mathrm{N}(\mathrm{mg} / \mathrm{L})$ & $5 / 5$ & 0.080 & 0.040 & 0.059 & 0.0088 & $\varepsilon$ \\
\hline Color (CPU) & $4 / 5$ & 10 & $<1.0$ & -4.8 & 1.7 & $\xi$ \\
\hline Total dissolved solids (mg/L) & $5 / 5$ & 180 & 150 & 170 & 5.6 & 500 \\
\hline Total hardness $(\mathrm{mg} / \mathrm{L})$ & $5 / 5$ & 130 & 120 & 120 & 2.8 & $f$ \\
\hline Total suspended solids (mg/L) & $3 / 5$ & 11 & $<5.0$ & -6.6 & 1.2 & $E$ \\
\hline \multicolumn{7}{|l|}{ Radionuclides $(p C i / L) \sigma$} \\
\hline Gross alpha & $4 / 5$ & $2.7 *$ & 0.43 & $1.9 *$ & 0.46 & $f$ \\
\hline Gross beta & $4 / 5$ & $4.1 *$ & 2.2 & $2.9 *$ & 0.32 & $f$ \\
\hline Total uranium & $3 / 5$ & $3.0^{*}$ & 0.32 & 1.0 & 0.50 & $f$ \\
\hline
\end{tabular}

East Fork Poplar Creek downstream from floodplain (EFK 5.4)

Anions (mg/L)

$\begin{array}{lcc}\text { Chlozide } & 5 / 5 & 23 \\ \text { Fluoride } & 5 / 5 & 19 \\ \text { Nitrate } & 5 / 5 & 37 \\ \text { Sulfate, as so } & 5 / 5 & 41 \\ & & \\ \text { Field Measurements } & & \\ \text { Conductivity (ms/cm) } & 5 / 5 & 0.43 \\ \text { Dissolved oxygen (ppm) } & 5 / 5 & 9.5 \\ \text { pH (SU) } & 5 / 5 & 7.9 \\ \text { Temperature (' }{ }^{\circ} \text { ) } & 5 / 5 & 22\end{array}$

0.30

7.0

20
0.34

7.8

7.5

31

16
4.3
21
33

0.39

8.7

9.7

16
2.8

3.7

5.3

4.4

$\begin{array}{lc}0.019 & f \\ 0.32 & 5 \\ 0.071 & f \\ 2.4 & f\end{array}$ 
Table 5.30 (continued)

\begin{tabular}{|c|c|c|c|c|c|c|}
\hline \multirow[b]{2}{*}{ Parameter } & \multirow[b]{2}{*}{$\begin{array}{l}\text { det/ } \\
\text { total }\end{array}$} & \multicolumn{4}{|c|}{ Concentration } & \multirow[b]{2}{*}{ TWQC } \\
\hline & & $\operatorname{Max}^{b}$ & $\operatorname{Min}{ }^{b}$ & $A v^{C}$ & $\begin{array}{l}\text { Standard } \\
\text { errord }\end{array}$ & \\
\hline \multicolumn{7}{|l|}{ Metals $(\mathrm{mg} / \mathrm{L})$} \\
\hline Aluminum, total & $4 / 5$ & 0.71 & $<0.050$ & -0.28 & 0.12 & $\boldsymbol{f}$ \\
\hline Arsenic, total & $1 / 5$ & 0.055 & $<0.050$ & -0.051 & 0.00090 & 0.36 \\
\hline Barium, total & $4 / 5$ & 0.036 & $<0.0010$ & -0.026 & 0.0064 & $f$ \\
\hline Calcium, total & $5 / 5$ & 50 & 38 & 45 & 1.9 & $E$ \\
\hline Iron, total & $4 / 5$ & 0.61 & $<0.050$ & -0.20 & 0.10 & $E$ \\
\hline Magnesium, total & $5 / 5$ & 11 & 6.0 & 8.7 & 0.96 & $E$ \\
\hline Manganese, total & $4 / 5$ & 0.065 & $<0.0010$ & -0.021 & 0.011 & $f$ \\
\hline Mercury, total & $3 / 5$ & 0.00012 & $<0.000050$ & -0.000074 & 0.000013 & 0.0024 \\
\hline Phosphorus, total & $4 / 5$ & 0.92 & $<0.20$ & -0.48 & 0.14 & $f$ \\
\hline Sodium, cotal & $5 / 5$ & $22^{\circ}$ & 6.3 & 15 & 3.2 & $f$ \\
\hline Uranium, total & $3 / 4$ & 0.015 & $<0.00010$ & -0.0073 & 0.0031 & $f$ \\
\hline Vanadium, total & $1 / 5$ & 0.0020 & $<0.0020$ & -0.0020 & 0 & $f$ \\
\hline zinc, total & $4 / 5$ & 0.021 & $<0.0050$ & -0.014 & 0.0028 & 0.117 \\
\hline \multicolumn{7}{|l|}{ others } \\
\hline Alkalinity (mg $\left.\mathrm{CaCO}_{3} / \mathrm{L}\right)$ & $5 / 5$ & 130 & 98 & 120 & 4.9 & $f$ \\
\hline Ammonia, as $N(m g / L)$ & $5 / 5$ & 0.060 & 0.040 & 0.048 & 0.0045 & $E$ \\
\hline Biochemical oxygen demand $(\mathrm{mg} / \mathrm{L})$ & $1 / 5$ & 8.0 & $<5.0$ & -5.6 & 0.60 & $f$ \\
\hline Chemical oxygen demand (mg/L) & $2 / 5$ & 12 & $<5.0$ & -7.4 & 1.5 & $E$ \\
\hline Color (CPU) & $5 / 5$ & 25 & 3.0 & 9.0 & 2.2 & $f$ \\
\hline Total dissolved solidis (mg/L) & $5 / 5$ & 280 & 250 & 230 & 24 & $f$ \\
\hline Total hardness $(\mathrm{mg} / \mathrm{L})$ & $5 / 5$ & 170 & 110 & 150 & 9.5 & $f$ \\
\hline Total suspended solids (mg/L) & $3 / 5$ & 33 & $<5.0$ & -11 & 5.5 & $f$ \\
\hline \multicolumn{7}{|l|}{ Radionuclides $(\mathrm{pCi} / \mathrm{L})^{g}$} \\
\hline$A m-241$ & $1 / 1$ & $0.38^{*}$ & $0.38 *$ & 0.38 & $f$ & $f$ \\
\hline Gross beta & $4 / 5$ & a. $6^{*}$ & 0.81 & $6.6^{*}$ & 1.5 & $E$ \\
\hline $\mathrm{H}-3$ & $2 / 5$ & $3,000^{*}$ & -320 & 630 & 610 & $f$ \\
\hline Tc-99 & $4 / 5$ & $10^{*}$ & 0.54 & $5.9 *$ & 1.8 & $E$ \\
\hline Total rad Sr & $2 / 5$ & $2.2 *$ & 0.54 & $1.6^{*}$ & 0.30 & $E$ \\
\hline Total uranium & $4 / 5$ & $5.4^{*}$ & 0.00059 & $3.6 *$ & 0.93 & $f$ \\
\hline$U-234$ & $4 / 4$ & $2.2^{*}$ & $1.5^{*}$ & $1.9 *$ & 0.17 & $f$ \\
\hline $\mathrm{U}-235$ & $2 / 4$ & $0.43 *$ & 0.059 & $0.24 *$ & 0.077 & $f$ \\
\hline$v-238$ & $4 / 4$ & $2.7^{*}$ & $2.2 \star$ & $2.5 *$ & 0.11 & $f$ \\
\hline
\end{tabular}

East Fork Poplar Creek downstream from the Y-12 Plant (EFK 23.4)

Anions (mg/t)

Chloride
Fluoride
Nitrate
Sulfate, as so.

Sulfate, as so.

Field Measurements

Conductivity $(\mathrm{mS} / \mathrm{cm})$

Dissolved oxygen (ppm)

pH (SU)

Temperature $\left({ }^{\circ} \mathrm{C}\right)$

Mecals (mg/L)

Aluminum, total

Barium, total

Calcium. Eotal"
$5 / 5$

$5 / 5$

$5 / 5$

$5 / 5$

$5 / 5$

$5 / 5$

$5 / 5$

$5 / 5$

$5 / 5$

$5 / 5$

$5 / 5$
32

1.7

26

150
14

75

0.46

8.3

8.9

27

0.27

0.060

70
0.068

0.043
24

1.3

19

98

0.57

9.0

21

0.33

0.20

2.4

2.2
0.12
2.0

$f$
$f$
$f$

5

$f$

f

$\begin{array}{cl}0.16 & 0.040 \\ 0.051 & 0.0030 \\ 62 & 3.4\end{array}$


Table 5.30 (continued)

\begin{tabular}{|c|c|c|c|c|c|c|}
\hline \multirow[b]{2}{*}{ Parameter } & \multirow[b]{2}{*}{$\begin{array}{l}N \text { det } / \\
N \text { total }\end{array}$} & \multicolumn{4}{|c|}{ Concentration } & \multirow[b]{2}{*}{ TWQCE } \\
\hline & & Maxt & $\operatorname{Min}^{b}$ & Ave & $\begin{array}{l}\text { Standard } \\
\text { erros }\end{array}$ & \\
\hline Chromium, total & $2 / 5$ & 0.013 & $<0.0040$ & -0.0070 & 0.0019 & 0.016 \\
\hline Iros, total & $5 / 5$ & 0.18 & 0.20 & 0.14 & 0.014 & $f$ \\
\hline Magnesium, total & $5 / 5$ & 13 & 11 & 11 & 0.41 & $f$ \\
\hline Manganese, total & $5 / 5$ & 0.082 & 0.025 & 0.059 & 0.011 & 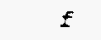 \\
\hline Mercury, total & $5 / 5$ & 0.00092 & 0.00030 & 0.00058 & 0.00010 & 0.0024 \\
\hline Phosphorus, total & $5 / 5$ & 0.50 & 0.20 & 0.35 & 0.050 & $f$ \\
\hline Potassium, total & $3 / 4$ & 2.5 & $<1.0$ & -1.9 & 0.31 & $f$ \\
\hline Sodium, total & $5 / 5$ & 46 & 14 & 22 & 6.0 & $f$ \\
\hline Uranium, total & $4 / 4$ & 0.020 & 0.00020 & 0.012 & 0.0047 & $f$ \\
\hline Vanadium, total & $1 / 5$ & 0.0047 & $<0.0020$ & -0.0025 & 0.00054 & $\mathrm{f}$ \\
\hline zine, total & $5 / 5$ & 0.048 & 0.031 & 0.041 & 0.0029 & 0.117 \\
\hline \multicolumn{7}{|l|}{ Others } \\
\hline Alkalinity (mg $\mathrm{CaCO}_{3} / \mathrm{L}$ ) & $5 / 5$ & 120 & 95 & 110 & 3.9 & $E$ \\
\hline Ammonia, as $\mathrm{N}(\mathrm{mg} / \mathrm{L})$ & $5 / 5$ & 1.2 & 0.060 & 0.30 & 0.22 & \pm \\
\hline Biochenical oxygen demand (mg/L) & $2 / 5$ & 6.0 & $<5.0$ & -5.2 & 0.20 & $f$ \\
\hline Chemical oxygen demand (mg/L) & $1 / 5$ & 7.0 & $<5.0$ & $\sim 5.4$ & 0.40 & 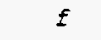 \\
\hline Color $(\mathrm{CPO})$ & $4 / 5$ & 11 & $<1.0$ & -6.4 & 1.7 & $f$ \\
\hline Total dissolved solids (mg/L) & $5 / 5$ & 410 & 280 & 340 & 22 & $f$ \\
\hline Tatal hardness (mg/L) & $5 / 5$ & 230 & 180 & 210 & 7.4 & $f$ \\
\hline Total suspended solids (mg/L) & $1 / 5$ & 5.0 & $<5.0$ & -5.0 & 0 & \pm \\
\hline \multicolumn{7}{|l|}{ Radionuclides $(\mathrm{PC} / \mathrm{L} /)^{\sigma}$} \\
\hline$A m-241$ & $1 / 1$ & $0.86^{*}$ & $0.86^{*}$ & 0.86 & $f$ & 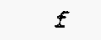 \\
\hline Gross alpha & $5 / 5$ & $16^{*}$ & $5.4^{*}$ & $8.7 *$ & 2.0 & $\xi$ \\
\hline Gross beta & $5 / 5$ & $18 *$ & $6.5^{*}$ & $11 *$ & 2.2 & $f$ \\
\hline $\mathrm{H}-3$ & $1 / 5$ & 620 & 110 & $340=$ & 87 & $\underline{f}$ \\
\hline Pu-238 & $1 / 2$ & 0.22 & 0.18 & $0.20^{*}$ & 0.019 & $\underline{f}$ \\
\hline -Pu-239 & $1 / 2$ & $0.84 *$ & 0.19 & 0.51 & 0.32 & $f$ \\
\hline Te-99 & $4 / 5$ & $19 *$ & 1.6 & $8.4 *$ & 2.9 & $f$ \\
\hline Total rad Sr & $2 / 5$ & $8.9 *$ & -0.65 & 2.6 & 1.6 & $E$ \\
\hline Total uranium & $5 / 5$ & $17 *$ & $5.7 *$ & $9.4^{*}$ & 2.0 & $f$ \\
\hline$U-234$ & $4 / 4$ & $3.5^{*}$ & $2.7 *$ & $3.2 *$ & 0.19 & $f$ \\
\hline$v-235$ & $3 / 4$ & $0.46 *$ & -0.054 & 0.23 & 0.11 & $f$ \\
\hline $\mathrm{v}-238$ & $4 / 4$ & $5.1 *$ & $3.0 *$ & $3.7^{*}$ & 0.51 & $f$ \\
\hline \multicolumn{7}{|l|}{ volatile organics ( $\mu \mathrm{g} / \mathrm{L})$} \\
\hline Chloroform & $1 / 5$ & U5.0 & $\mathrm{J1.2}$ & -4.2 & 0.76 & $f$ \\
\hline
\end{tabular}

Hinds Creek (reference site for East Fork Poplar Creek) (HC)

Anions (mg/L)

Chloride

$5 / 5$

Fluoride

Nitrate

Sulfate, as so,

$2 / 5$

$5 / 5$

0.30

3.0

$5 / 5$

21

$<0.10$

1.4

0.16

2.3

14

0.040

0.29

Field Measurements

Conductivity $(\mathrm{ms} / \mathrm{cm})$

Dissolved axygen (ppm)

$5 / 5$

0.37

12

$\mathrm{pH}$ (SU)

Temperature $\left({ }^{\circ} \mathrm{C}\right)$

$5 / 5 \quad 8.1$

$5 / 5$

25

0.30

5.6

7.6

8.0

0.34

9.2

7.9

Metals (mg/L)

Aluminum, total

Barium, total

Calcium, total

Chromium, total

$\begin{array}{ccc}5 / 5 & 0.49 & 0.13 \\ 5 / 5 & 0.054 & 0.034 \\ 5 / 5 & 49 & 42 \\ 2 / 5 & 0.014 & <0.0040\end{array}$

0.13

$<0.0040$

0.046

45

$-0.0074$

\pm
\pm
$f$

$\begin{array}{ll}0.014 & f \\ 1.1 & 5 \\ 0.097 & f \\ 3.3 & f\end{array}$

0.071

0.0037

1.2

0.0021 
Table 5.30 (continued)

\begin{tabular}{|c|c|c|c|c|c|c|}
\hline \multirow[b]{2}{*}{ Parameter } & \multirow[b]{2}{*}{$\begin{array}{l}N \text { det } / \\
N \text { total }\end{array}$} & \multicolumn{4}{|c|}{ Concentration } & \multirow[b]{2}{*}{ TWQC } \\
\hline & & $\operatorname{Max}{ }^{b}$ & $\operatorname{Min}^{b}$ & $A v^{e}$ & $\begin{array}{l}\text { Standard } \\
\text { error }\end{array}$ & \\
\hline Iron, total & $5 / 5$ & 0.59 & 0.16 & 0.34 & 0.075 & $f$ \\
\hline Magnesium, cocal & $5 / 5$ & 15 & 9.3 & 13 & 0.97 & $E$ \\
\hline Manganese, total & $4 / 5$ & 0.064 & $<0.0010$ & -0.039 & 0.012 & $f$ \\
\hline Phosphorus, total & $4 / 5$ & 0.40 & $<0.20$ & -0.29 & 0.039 & $f$ \\
\hline Potassium, total & $3 / 4$ & 3.4 & $<1.0$ & -2.2 & 0.49 & $f$ \\
\hline Sodium, total & $5 / 5$ & 3.3 & 2.0 & 2.6 & 0.23 & $E$ \\
\hline Uraniun, total & $4 / 4$ & 0.00057 & 0.00020 & 0.00034 & 0.000079 & 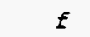 \\
\hline Vanadium, total & $2 / 5$ & 0.0029 & $<0.0020$ & -0.0022 & 0.00017 & $f$ \\
\hline zinc, total & $3 / 5$ & 0.026 & $<0.0050$ & -0.0097 & 0.0042 & 0.117 \\
\hline \multicolumn{7}{|l|}{ others } \\
\hline Alkalinity (mg $\mathrm{CaCO}_{3} / \mathrm{L}$ ) & $5 / 5$ & 180 & 130 & 150 & 8.2 & $E$ \\
\hline Ammonia, as $\mathrm{N}(\mathrm{mg} / \mathrm{L})$ & $5 / 5$ & 0.090 & 0.040 & 0.059 & 0.0093 & $f$ \\
\hline Chemical oxygen demand (mg/L) & $2 / 5$ & 8.0 & $<5.0$ & -6.0 & 0.63 & $f$ \\
\hline Color (CPU) & $5 / 5$ & 11 & 5.0 & 8.6 & 1.0 & $f$ \\
\hline Total dissolved solids (mg/L) & $5 / 5$ & 220 & 170 & 200 & 9.7 & $f$ \\
\hline Total hardness (mg/L) & $5 / 5$ & 180 & 140 & 170 & 7.2 & $f$ \\
\hline Total suspended solids (mg/L) & $4 / 5$ & 23 & $<5.0$ & -11 & 3.3 & $E$ \\
\hline \multicolumn{7}{|l|}{ Radionuclides $(\mathrm{pCi} / \mathrm{L})^{g}$} \\
\hline $\mathrm{Co}-60$ & $1 / 5$ & $4.9 *$ & 0.27 & 2.1* & 0.78 & 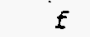 \\
\hline Gross alpha & $1 / 5$ & $1.6^{*}$ & -0.14 & 0.57 & 0.29 & $f$ \\
\hline Gross beta & $3 / 5$ & $6.5^{*}$ & 0.27 & $2.9 *$ & 1.0 & f \\
\hline $\mathrm{H}-3$ & $1 / 5$ & $1,000^{*}$ & -190 & 86 & 230 & $\mathbf{f}$ \\
\hline Total rad SI & $1 / 5$ & $1.1 *$ & -0.70 & 0.027 & 0.31 & $E$ \\
\hline Total uranium & $5 / 5$ & $3.2 *$ & $0.84^{*}$ & $1.4 *$ & 0.46 & $f$ \\
\hline
\end{tabular}

Melton Branch downstream from ORNL (MEK 0.2)

Anions (mg/L)

Chloride

Fluoride

Nitrate

Sulfate, as so.

Field Measurements

Conductivity (ms/cm)

Dissolved oxygen (ppm)

pH (SU)

Temperature $\left({ }^{\circ} \mathrm{C}\right)$

Metals (mg/L)

Aluminum, total

Arsenic, total

Barium, total

Calcium, total

Chromium, total

Copper, total

Iron, total

Magnesium, total

Manganese, total

Phosphorus, total

Potassium, total

Sodium, total

Uranium, total

vanadium, total

zine, total
$5 / 5 \quad 16$

$5 / 5 \quad 1.9$

$5 / 5 \quad 210^{5}$

$5 / 5$

$5 / 5$

$5 / 5$

$5 / 5$

$5 / 5$

$1 / 5$

$5 / 5$

$5 / 5$

$2 / 5$

$1 / 5$

$5 / 5$

$5 / 5$

$5 / 5$

$3 / 4$

$5 / 5$

$4 / 4$

$2 / 5$

$4 / 5$

0.71
12
8.0
23

4.1

0.059

1.1

93

0.023

0.0081

0.46

20

0.27

0.90

4.0

17

0.0016

0.0024

1.1
8.5
0.50
$<1.0$
37

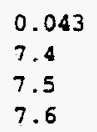

0.30

\section{$<0.050$}

0.043

47

$<0.0040$

$<0.0070$

0.32

9.0

0.060

0.20

$<2.0$

6.3

0.00030

$<0.0020$

$<0.0050$

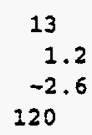

120

$$
\begin{aligned}
& 0.45 \\
& 9.3 \\
& 7.8 \\
& 16
\end{aligned}
$$

1.1
-0.052
0.26
69
-0.0089
-0.0072
0.42
14
0.17
0.60
-2.9
10
0.00078
-0.0021
-0.24

1.3
0.27
0.80
28

$E$
$E$
$E$

$\begin{array}{lr}0.12 & E \\ 1.1 & 5 \\ 0.086 & E \\ 3.1 & E\end{array}$

0.75

0.0016

0.21

8.2

0.0037

0.00022

0.025

2.0

0.037

0.14

0.41

1.9

0.00030

0.000087

0.22
${ }^{f}$

f

0.36

$f$

0.016

0.018

$\pm$

$f$
0.117 


\begin{tabular}{|c|c|c|c|c|c|c|}
\hline \multirow[b]{2}{*}{ Parameter } & \multirow[b]{2}{*}{$\begin{array}{l}\mathrm{N} \text { det } / \\
\mathrm{N} \text { total }\end{array}$} & \multicolumn{4}{|c|}{ Con:entration } & \multirow[b]{2}{*}{ Twoce } \\
\hline & & $\max$ & $\operatorname{Min}^{b}$ & $A V^{c}$ & $\begin{array}{l}\text { Standard } \\
\text { error }\end{array}$ & \\
\hline \multicolumn{7}{|l|}{ Others } \\
\hline Alkalinity (mg $\mathrm{CaCO}_{3} / \mathrm{L}$ ) & $5 / 5$ & 140 & 110 & 120 & 5.5 & $f$ \\
\hline Ammonia, as $\mathrm{N}(\mathrm{mg} / \mathrm{L})$ & $5 / 5$ & 0.080 & 0.030 & 0.053 & 0.0086 & $f$ \\
\hline Chemical oxygen demand (mg/L) & $1 / 5$ & 6.0 & $<5.0$ & -5.2 & 0.20 & $\varepsilon$ \\
\hline Color (CPU) & $4 / 5$ & 11 & $<1.0$ & -6.6 & 1.7 & \pm \\
\hline Total dissolved solids (mg/L) & $5 / 5$ & 500 & 220 & 360 & 54 & $f$ \\
\hline Total hardness $(\mathrm{mg} / \mathrm{L})$ & $5 / 5$ & 330 & 170 & 260 & 26 & $E$ \\
\hline Total suspended solids (mg/L) & $5 / 5$ & 15 & 6.0 & 11 & 1.7 & $f$ \\
\hline \multicolumn{7}{|l|}{ Radionuclides $(p C i / L)^{g}$} \\
\hline Cs-137 & $1 / 5$ & $3.0 *$ & -0.27 & $1.2 *$ & 0.56 & $E$ \\
\hline Gross alpha & $5 / 5$ & $6.2 *$ & $1.5^{*}$ & $3.5 *$ & 0.84 & $E$ \\
\hline Gross beta & $5 / 5$ & $2,500 *$ & $1,100^{*}$ & $1,900 *$ & 260 & $f$ \\
\hline $\mathrm{H}-3$ & $5 / 5$ & $3,000,000 *$ & $1,000,000^{*}$ & $1,600,000^{*}$ & 350,000 & $f$ \\
\hline Pu-238 & $1 / 1$ & $4.6^{*}$ & $4.6 *$ & 4.6 & $E$ & $E$ \\
\hline TC-99 & $1 / 5$ & $2.1 *$ & -3.8 & -0.82 & 1.0 & $f$ \\
\hline Total rad $S=$ & $5 / 5$ & 1,200 & $540^{*}$ & $830 *$ & 110 & $f$ \\
\hline Totel urenium & $5 / 5$ & $1.6^{*}$ & $0.59 *$ & $2.2 *$ & 0.20 & $\hat{f}$ \\
\hline$U-234$ & $1 / 1$ & $0.95^{*}$ & $0.95 *$ & 0.95 & $f$ & 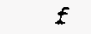 \\
\hline U-235 & $1 / 1$ & $0.35^{*}$ & $0.35 *$ & 0.35 & $f$ & $E$ \\
\hline $0-238$ & $1 / 1$ & $0.32 *$ & $0.32 \%$ & 0.32 & $f$ & $E$ \\
\hline \multicolumn{7}{|l|}{ volatile organics ( $\mu \mathrm{g} / \mathrm{L}$ ) } \\
\hline 1,2-Dichloroethene & $1 / 5$ & 05.0 & $\mathrm{~J} 1.4$ & -4.3 & 0.72 & $E$ \\
\hline Trichloroethere & $1 / 5$ & U5.0 & $\mathrm{J1} .2$ & -4.2 & 0.76 & $f$ \\
\hline
\end{tabular}

Melton Branch upstream from ORNL (MEK 2.1)

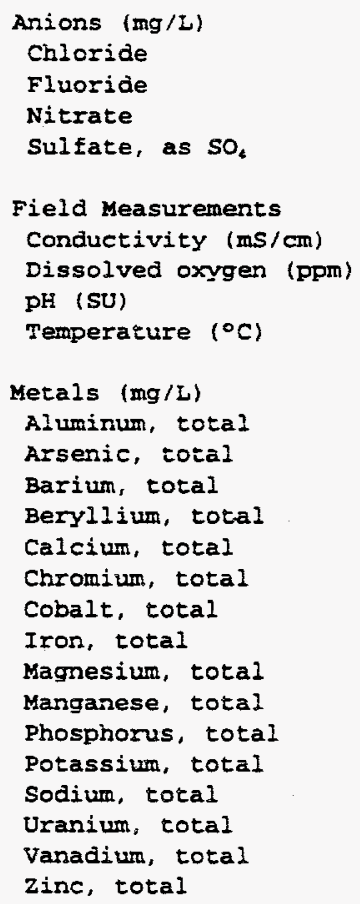

$5 / 5$

$4 / 5$

$$
\begin{aligned}
& 2.4 \\
& 0.20 \\
& 2.1
\end{aligned}
$$

35
1.1
$<0.10$
$<1.0$
6.2

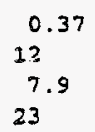

1.7

0.056

0.15

0.0010

61

0.012

0.0040

1.5

6.5

0.13

0.40

2.5

6.4

0.0020

0.0043

0.018

\subsection{9 \\ 6.1 \\ 7.5 \\ 11}

0.63
$<0.050$
0.029
$<0.0010$
28

$2 \mathrm{~B}$

$<0.0040$

$<0.0040$

0.39

$$
3.2
$$

0.014

$<0.20$

1.6

1.3

$<0.00010$

$<0.0020$

$<0.0050$
1.6

$-0.12$

$-1.2$

20

0.31
8.0
7.8
17

1.0

$-0.053$

0.065

$-0.0010$

48

$-0.0068$

$-0.0080$

0.82

$$
5.2
$$

0.059

$-0.27$

$-1.9$

3.1

$\sim 0.00068$

$-0.0025$

$-0.013$
0.24
0.020
0.22
6.2

$\begin{array}{ll}0.034 & f \\ 0.99 & 5 \\ 0.080 & f \\ 2.0 & f\end{array}$

0.23

0.0032

0.021

0

6.2

0.0015

0

0.21

0.58

0.020

0.037

0.19

0.93

0.00045

0.00045

0.0025 $f$
$f$
$f$

5

$f$
0.36
$f$
$f$
$f$
0.016
$f$
$f$
$f$
$f$
$f$
$f$
$f$
$f$
$f$
0.117 
Table 5.30 (continued)

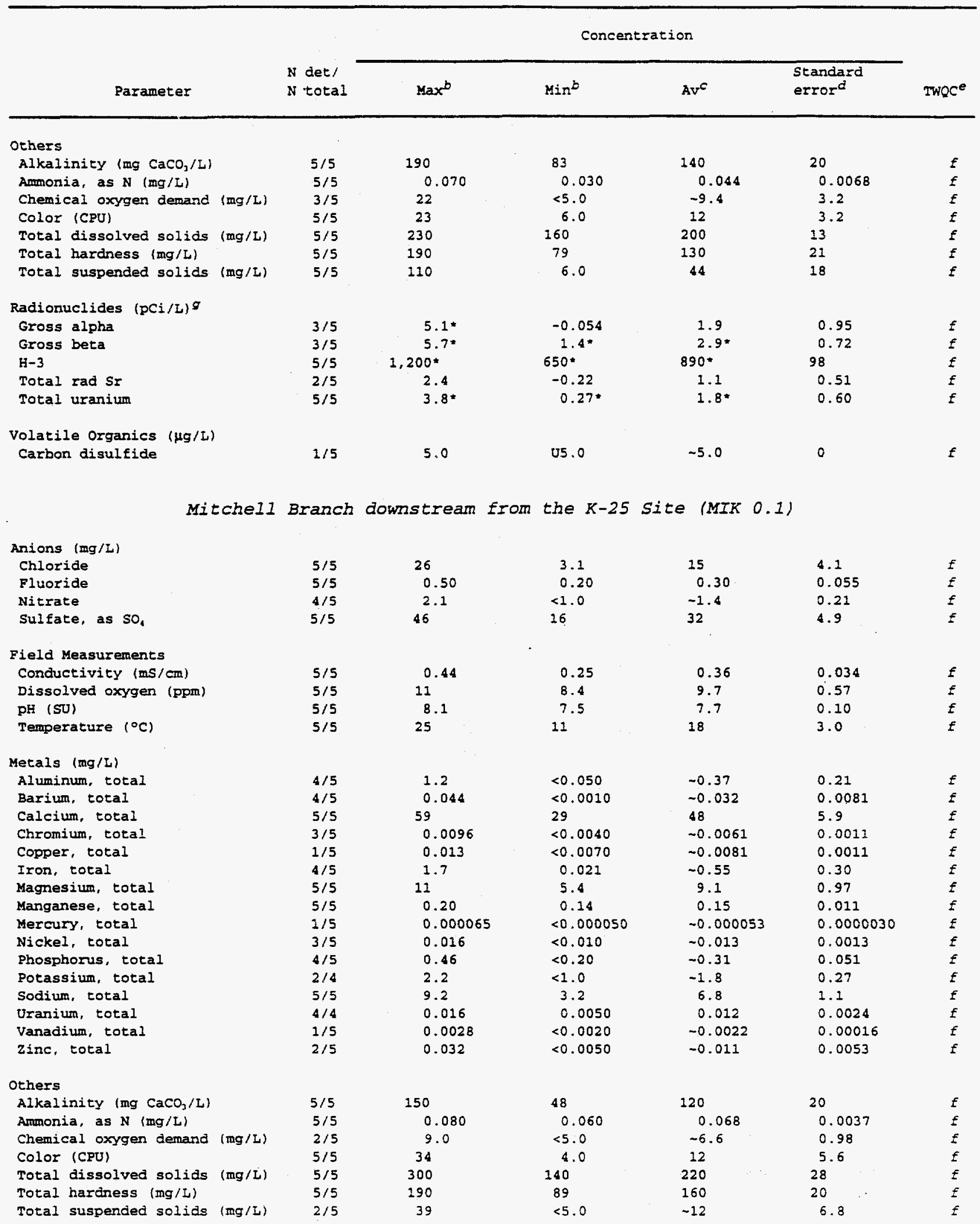


Table 5.30 (continued)

\begin{tabular}{|c|c|c|c|c|c|c|}
\hline \multirow[b]{2}{*}{ Parameter } & \multirow[b]{2}{*}{$\begin{array}{l}N \text { det } \\
N \text { total }\end{array}$} & \multicolumn{4}{|c|}{ concentrazion } & \multirow[b]{2}{*}{${ }^{T W Q C^{e}}$} \\
\hline & & $\operatorname{Max}{ }^{6}$ & $\operatorname{Min}^{b}$ & $A v^{C}$ & $\begin{array}{l}\text { Standard } \\
\text { error }{ }^{d}\end{array}$ & \\
\hline \multicolumn{7}{|l|}{ Radionuclides $(\mathrm{pCi} / L)^{g}$} \\
\hline Gross alpha & $5 / 5$ & $15^{*}$ & $10^{*}$ & $12 *$ & 1.1 & $E$ \\
\hline Gross beta & $5 / 5$ & $35 *$ & $13^{*}$ & 23 * & 3.6 & $f$ \\
\hline $\mathrm{H}-3$ & $2 / 5$ & $540^{*}$ & -240 & 140 & 140 & $f$ \\
\hline TC-99 & $5 / 5$ & $32 *$ & $15 *$ & $23 *$ & 3.1 & $f$ \\
\hline Total rad Sr & $1 / 5$ & $1.7^{\star}$ & -0.11 & $0.95^{*}$ & 0.31 & $\varepsilon$ \\
\hline Total uranium & $5 / 5$ & $14^{*}$ & $4.1 *$ & $11 *$ & 1.9 & $f$ \\
\hline $\mathrm{U}-234$ & $4 / 4$ & $10^{*}$ & $7.3 *$ & $8.5^{*}$ & 0.60 & $f$ \\
\hline$u-235$ & $3 / 4$ & $0.65 *$ & 0.12 & $0.37 *$ & 0.11 & $\mathrm{f}$ \\
\hline $0-238$ & $4 / 4$ & $4.6 *$ & $3.0^{*}$ & $3.7 *$ & 0.37 & \pm \\
\hline \multicolumn{7}{|l|}{ Volatile organies $(\mu g / L)$} \\
\hline 1,I-Dichloroethane & $2 / 5$ & U5.0 & Ji.2 & -3.5 & 0.93 & $E$ \\
\hline 1,2-Dichloroethene & $5 / 5$ & 40 & 6.0 & 28 & 6.6 & 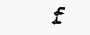 \\
\hline Chloroform & $2 / 5$ & U5.0 & 52.9 & -4.2 & 0.48 & $f$ \\
\hline Tetrachloroethene & $2 / 5$ & $\mathrm{v} 5.0$ & $\mathrm{~J} 1.2$ & -3.5 & 0.92 & $f$ \\
\hline Trichloroethene & $4 / 5$ & 50 & 05.0 & -31 & 9.6 & 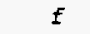 \\
\hline vinyi chioride & $1 / 5$ & U10 & 54.9 & -9.0 & 1.0 & \pm \\
\hline cis-1,3-Dichloropropene & $1 / 5$ & 25 & 05.0 & -9.0 & 4.0 & $\underline{f}$ \\
\hline
\end{tabular}

MitcheII Branch upstream from the K-25 Site (MIK 1.4)

$\begin{array}{ll}\text { Anions (mg/L) } & \\ \text { Chloride } & 5 / 5 \\ \text { Fluoride } & 4 / 5 \\ \text { Nitrate } & 3 / 5 \\ \text { Sulfate, as so, } & 5 / 5 \\ & \\ \text { Field Measurements } & \\ \text { Conductivity (ms/cm) } & 5 / 5 \\ \text { Dissolved oxygen (ppm) } & 5 / 5 \\ \text { pH (SU) } & 5 / 5 \\ \text { Temperature (o) } & 5 / 5 \\ & \\ \text { Metals (mg/L) } & \\ \text { Aluminum, total } & 5 / 5 \\ \text { Barium, total } & 4 / 5 \\ \text { Calcium, total } & 5 / 5 \\ \text { Chromium, total } & 2 / 5 \\ \text { Cobalt, total } & 1 / 5 \\ \text { Copper, total } & 2 / 5 \\ \text { Iron, total } & 5 / 5 \\ \text { Magnesium, total } & 5 / 5 \\ \text { Manganese, total } & 5 / 5 \\ \text { Nickel, total } & 1 / 5 \\ \text { Phosphorus, total } & 4 / 5 \\ \text { Potassium, total } & 3 / 4 \\ \text { Sodium, total } & 5 / 5 \\ \text { Uranium, total } & 1 / 4 \\ \text { Vanadium, total } & 2 / 5 \\ \text { Zinc, total } & 2 / 5 \\ & \\ \text { Others } & 5 / 5 \\ \text { Alkalinity (mg CaCO, } / \mathrm{L} \text { ) } & 4 / 5 \\ \text { Ammonia, as } \mathrm{N} \text { (mg/L) } & 3 / 5 \\ \text { Chemical oxyger: demand (mg/L) } & 5 / 5 \\ \text { Color (CPU) } & 5 / 5 \\ \text { Total dissolved solids (mg/L) } & \\ & \end{array}$

$\begin{array}{ll}5 / 5 & 2.5 \\ 4 / 5 & 0.40 \\ 3 / 5 & 9.5 \\ 5 / 5 & 40\end{array}$

$5 / 5$

$5 / 5$

$5 / 5$

$\begin{array}{ll}5 / 5 & 11 \\ 4 / 5 & 0.28 \\ 5 / 5 & 34 \\ 2 / 5 & 0.019 \\ 1 / 5 & 0.015 \\ 2 / 5 & 0.020 \\ 5 / 5 & 17 \\ 5 / 5 & 11 \\ 5 / 5 & 1.7 \\ 1 / 5 & 0.064 \\ 4 / 5 & 0.43 \\ 3 / 4 & 5.8 \\ 5 / 5 & 8.5 \\ 1 / 4 & 0.0020 \\ 2 / 5 & 0.019 \\ 2 / 5 & 0.048\end{array}$

$$
\begin{aligned}
& 0.29 \\
& 9.2 \\
& 7.6 \\
& 22
\end{aligned}
$$

29
.2
.6

1

0.019

0.019

0.020

1.7

064

5.8

8.5

0.019

0.048

100
0.070
15
25
180

$\begin{array}{ll}.5 & 1.5 \\ .40 & <0.10 \\ 5 & <1.0 \\ & 16\end{array}$

2.0

$\sim 0.24$

$-3.1$

26

0.17
5.3
7.0
8.8

0.24

$<0.0010$

18

$<0.0040$

$<0.0040$

$<0.0070$

0.37

5.1

0.047

$<0.010$

$<0.20$

$<1.0$

2.7

$<0.00010$

$<0.0020$

$<0.0050$

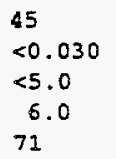

45

$<0.030$

5.0

71

0.22
7.3
7.6
7.5

2.7

$-0.064$

25

$-0.0072$

$-0.0063$

$-0.011$

4.1

7.7

0.46

$-0.021$

$-0.31$

$-3.0$

5.5

$-0.00058$

$-0.0056$

$\sim 0.014$

$\begin{array}{ll}74 & 11 \\ -0.050 & 0.0089 \\ -8.4 & 1.8 \\ 24 & 3.3 \\ 130 & 20\end{array}$

0.38

0.060

1.6

5.0

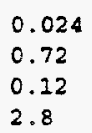

2.8

2.1

0.030

3.1

0.0030

0.0023

0.0026

3.2

1.1

0.32

0.011

0.037

1.0

1.1

0.00048

0.0033

0.0084

20 
Table 5.30 (continued)

\begin{tabular}{|c|c|c|c|c|c|c|}
\hline \multirow[b]{2}{*}{ Parameter } & \multirow[b]{2}{*}{$\begin{array}{l}\mathbf{N} \text { det } / \\
\mathbf{N} \text { total }\end{array}$} & \multicolumn{4}{|c|}{ Concentration } & \multirow[b]{2}{*}{ TWQC } \\
\hline & & $\operatorname{Max}{ }^{b}$ & $\operatorname{Min}^{b}$ & Ave & $\begin{array}{l}\text { Standard } \\
\text { errord }\end{array}$ & \\
\hline Total hardness (mg/L) & $5 / 5$ & 180 & 63 & 110 & 21 & $E$ \\
\hline Total suspended solids (mg/L) & $2 / 5$ & 420 & $<5.0$ & -100 & 80 & $f$ \\
\hline \multicolumn{7}{|l|}{ Radionuclides $(\mathrm{pCi} / \mathrm{L})$ g } \\
\hline $\mathrm{Cg}-137$ & $2 / 5$ & $4.3 *$ & 0.27 & $2.5 *$ & 0.87 & $f$ \\
\hline Gross alpha & $3 / 5$ & $2.5^{\star}$ & 0.054 & $1.4^{*}$ & 0.50 & $f$ \\
\hline Gross beta & $5 / 5$ & $7.8 *$ & $3.2 *$ & $5.5 *$ & 0.94 & $f$ \\
\hline$H-3$ & $1 / 5$ & 270 & -810 & -100 & 220 & $f$ \\
\hline TC-99 & $2 / 5$ & $8.1^{*}$ & 0.73 & $3.8 *$ & 1.5 & $f$ \\
\hline rotal rad sr & $1 / 5$ & 1.8 & 0.054 & $1.0^{*}$ & 0.31 & $\varepsilon$ \\
\hline
\end{tabular}

Poplar Creek downstream from the $K-25$ Site (PCK 2.2)

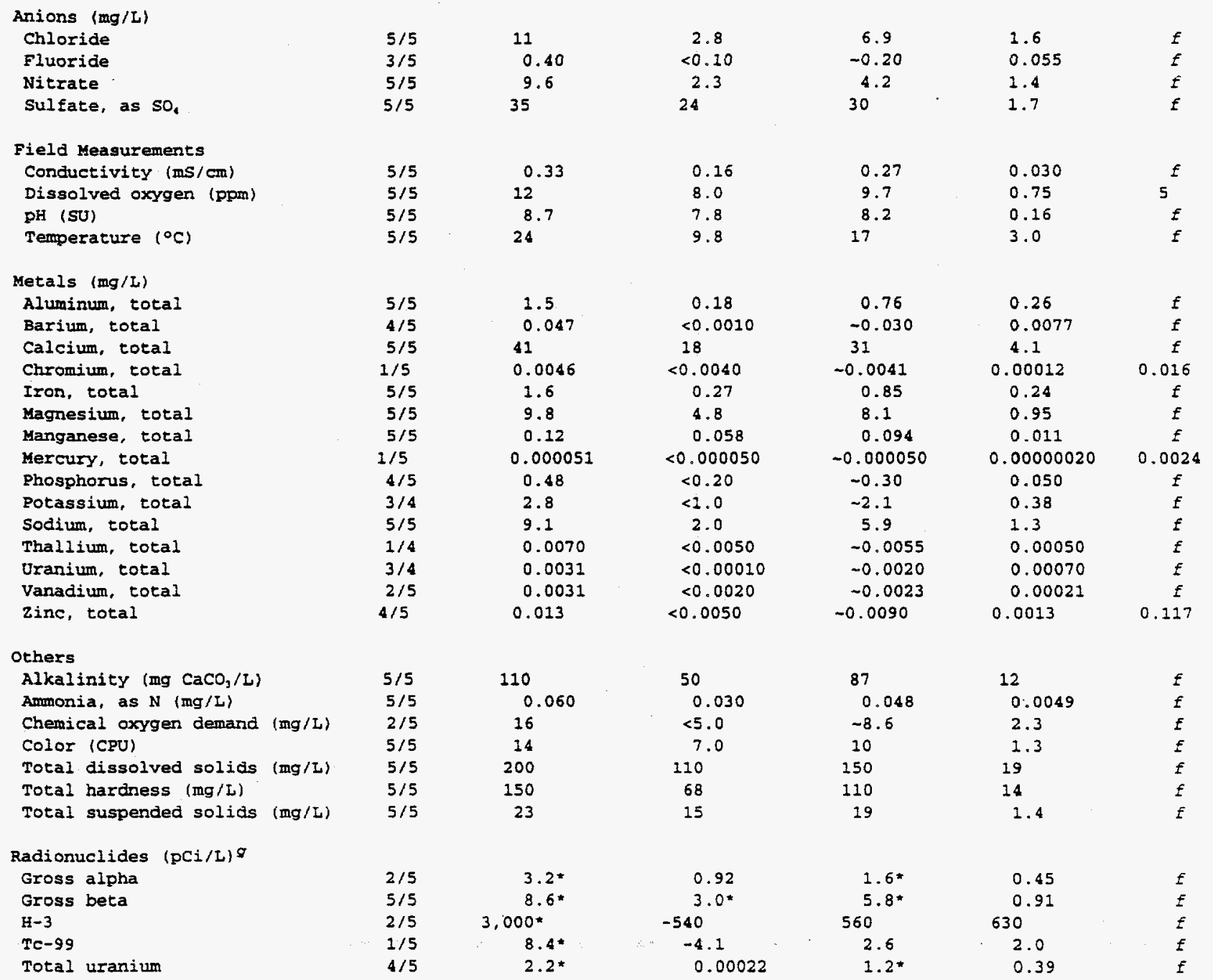


Table 5.30 (continued)

\begin{tabular}{|c|c|c|c|c|c|c|}
\hline \multirow[b]{2}{*}{ Earameter } & \multirow[b]{2}{*}{$\begin{array}{l}N \text { det } / \\
N \text { cotal }\end{array}$} & \multicolumn{4}{|c|}{ Concentration } & \multirow[b]{2}{*}{ TWQC } \\
\hline & & $\operatorname{Max}^{b}$ & $\operatorname{Min}{ }^{b}$ & $A v^{C}$ & $\begin{array}{l}\text { Standard } \\
\text { errord }\end{array}$ & \\
\hline Total rad Sr & $1 / 5$ & $2.2 *$ & -0.81 & 0.92 & 0.51 & $\varepsilon$ \\
\hline$U-234$ & $1 / 1$ & $1.9 *$ & $1.9 *$ & 1.9 & $f$ & $f$ \\
\hline$U-238$ & $1 / 1$ & $1.6^{*}$ & $1.6 *$ & 1.6 & $f$ & $f$ \\
\hline $\begin{array}{l}\text { Volatile organics ( } \mathrm{\mu g} / \\
\text { Carbor disulfide }\end{array}$ & $1 / 5$ & $\mathrm{U} 5.0$ & 54.0 & -4.8 & 0.20 & $f$ \\
\hline
\end{tabular}

Poplar Creek upstream from the K-25 Site and East Fork Poplar Creek (PCK

22)

Anions (mg/L)

Chioride

Fiuoride

$5 / 5$

Nitrace

Sulfate, as $\mathrm{SO}$

$1 / 5$

$5 / 5$

$5 / 5$

5.9

0.20

2.0

3.3

$<0.10$

1.1

Field Measurements

Conductivity (ms/em)

Dissolved oxygen (ppm)

pH (SU)

Temperature $\left({ }^{\circ} \mathrm{C}\right)$

$\begin{array}{ccc}5 / 5 & 0.34 & 0.22 \\ 5 / 5 & 11 & 4.2 \\ 5 / 5 & 8.0 & 7.4 \\ 5 / 5 & 22 & 10\end{array}$

0.22

4.2

20

7.7

7.6

4.0

2.2

0.72

Metals (mg/L)

Aluminum, total

Arsenic, total

Barium, total

Calcium, total

Chromium, total

Iron, total

Magnesium, total

Manganese, total

Nickel. total

Phospharus, total

Potassitm, total

Sociium, total

Uranium, total

zinc, total

$4 / 5$

0.46

$<0.050$

0.053

0.058

40

$<0.050$

$5 / 5$

$5 / 5$

$1 / 5$

$4 / 5$

0.010

0.62

12

0.28

0.010

0.37

2.6

5.7

0.0020

0.011

0.031

20

$<0.0040$

$<0.050$

7.0

0.13

$<0.010$

$<0.20$

$<\hat{1} .0$

2.5

$5 / 5$
$1 / 8$

$<0.00010$

$2 / 5$

$<0.0050$

16

38

0.45

3.1

Other's

Alkalinity (mg $\mathrm{CaCO}_{3} / \mathrm{L}$ )

Ancionia, as $\mathrm{N}$ (mg/L)

Chemical oxygen demand (mg/L) COlor (CPU)

Total dissolved solids (mg/L)

Total hardness (mg/L)

Total suspended solias (mg/L)

Radionuclides $(\mathrm{pCi} / \mathrm{L}) \sigma$

Co-60

Gross alpha

Gross beta

$\mathrm{kH}-3$

TC-99

Total rad Sr

Total uranium

Volatile organics $(\mu g / L)$

Acetone

Carbon disulfide
$5 / 5$

$5 / 5$

$2 / 5$

$4 / 5$

$5 / 5$

$5 / 5$

$4 / 5$

140
0.28
6.0
15
240
160
24

$2 / 5$

$3 / 5$

$5 / 5$

$2 / 5$

$1 / 5$

$1 / 5$

$3 / 5$

$2 / 5$

$1 / 5$

$5.1^{*}$
$1.5^{*}$
$5.9^{*}$
$2.700^{*}$
$8.4^{*}$
$1.7^{*}$
$1.2^{*}$

45

24
50

0.050

$<5.0$

$<1.0$

97

$7 B$

$<5.0$

$-0.81$

0.27

3.2 *

$-54$

$-2.7$

$-0.89$

0.22

010

U5. 0
$-0.31$

$-0.051$

0.046

32

$-0.0052$

$-0.36$

10

0.16

$-0.010$

$-0.26$

$-1.9$

4.4

$\sim 0.00058$

$-0.0069$

94
0.15
$\sim 5.2$
-8.8
180
130
-13

1.5

$1.1 \%$

4.2 "

840

2.0

0.78

0.54 *

$-17$

$-8.8$

$\begin{array}{lc}0.023 & E \\ 1.1 & 5 \\ 0.10 & E \\ 2.5 & E\end{array}$

0.072

0.00062

0.0055

3.9

0.0012

0.11

1.1

0.029

0

0.034

0.32

0.63

0.00048

0.0012

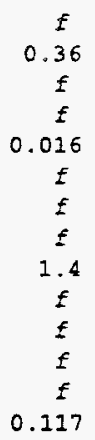

0.117 
Table 5.30 (continued)

\begin{tabular}{|c|c|c|c|c|c|c|}
\hline \multirow[b]{2}{*}{ Parameter } & \multirow[b]{2}{*}{$\begin{array}{l}\text { N det/ } \\
\text { N total }\end{array}$} & \multicolumn{4}{|c|}{ Concentration } & \multirow[b]{2}{*}{ TWQC $^{e}$} \\
\hline & & $\operatorname{Max}{ }^{b}$ & $\operatorname{Min}^{b}$ & $\mathrm{Av}^{\mathrm{C}}$ & $\begin{array}{l}\text { Standard } \\
\text { errord }\end{array}$ & \\
\hline Water & supply & take for & of Kings & ITRK 915 & & \\
\hline \multicolumn{7}{|l|}{ Arions (mg/L) } \\
\hline Chloride & $5 / 5$ & 11 & 4.0 & 7.6 & 1.4 & $f$ \\
\hline Fluoride & $4 / 5$ & 0.40 & $<0.10$ & -0.28 & 0.058 & 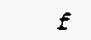 \\
\hline Nitrate & $5 / 5$ & 9.6 & 1.0 & 3.7 & 1.6 & $E$ \\
\hline Sulfate, as so, & $5 / 5$ & 18 & 9.0 & 13 & 1.6 & $f$ \\
\hline \multicolumn{7}{|l|}{ Field Measurements } \\
\hline Conductivity (ms/cm) & $5 / 5$ & 0.20 & 0.14 & 0.18 & 0.011 & $f$ \\
\hline Dissolved oxygen (ppm) & $5 / 5$ & 11 & 6.6 & 8.6 & 0.74 & $f$ \\
\hline $\mathrm{pH} \quad(\mathrm{SU})$ & $5 / 5$ & 7.9 & 7.7 & 7.8 & 0.037 & $f$ \\
\hline Temperature $\left({ }^{\circ} \mathrm{C}\right)$ & $5 / 5$ & 25 & 10 & 18 & 3.1 & $f$ \\
\hline \multicolumn{7}{|l|}{ Metals $(\mathrm{mg} / \mathrm{L})$} \\
\hline Aluminum, total & $5 / 5$ & 0.42 & 0.21 & 0.28 & 0.037 & $E$ \\
\hline Barium, total & $5 / 5$ & 0.036 & 0.021 & 0.025 & 0.0026 & $E$ \\
\hline Calcium, total & $5 / 5$ & 23 & 16 & 19 & 1.3 & $E$ \\
\hline Chromium, total & $1 / 5$ & 0.0058 & $<0.0040$ & -0.0044 & 0.00036 & 0.05 \\
\hline Iron, total & $4 / 5$ & 0.58 & $<0.050$ & -0.28 & $0 \quad 384$ & $f$ \\
\hline Magnesium, total & $5 / 5$ & 5.2 & 3.6 & 4.3 & 0.28 & $E$ \\
\hline Manganese, total & $5 / 5$ & 0.053 & 0.011 & 0.043 & 0.0081 & $f$ \\
\hline Phosphorus, total & $4 / 5$ & 0.39 & 0.17 & -0.28 & 0.040 & $f$ \\
\hline Potassium, total & $1 / 4$ & $<2.0$ & $<1.0$ & -1.6 & 0.24 & $f$ \\
\hline Sodium, cotal & $5 / 5$ & 12 & 4.3 & 7.2 & 1.4 & $f$ \\
\hline Uranium, total & $2 / 4$ & 0.0010 & $<0.00010$ & -0.00035 & 0.00022 & $f$ \\
\hline \multicolumn{7}{|l|}{ Others } \\
\hline Alkalinity (mg $\mathrm{CaCO}_{3} / \mathrm{L}$ ) & $5 / 5$ & 80 & 48 & 58 & 6.0 & 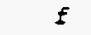 \\
\hline Ammonia, as $N(\mathrm{mg} / \mathrm{L})$ & $5 / 5$ & 0.070 & 0.040 & 0.050 & 0.0055 & $f$ \\
\hline Chemical oxygen demand (mg/L) & $1 / 5$ & 18 & $<5.0$ & -7.6 & 2.6 & $E$ \\
\hline Color (CPU) & $5 / 5$ & 20 & 3.0 & 10 & 3.0 & $f$ \\
\hline Total dissolved solids (mg/L) & $5 / 5$ & 140 & 77 & 100 & 10 & 500 \\
\hline Total hardness (mg/L) & $5 / 5$ & 84 & 55 & 64 & 5.5 & $\mathbf{f}$ \\
\hline Total suspended solids $(\mathrm{mg} / \mathrm{L})$ & $4 / 5$ & 12 & $<5.0$ & -7.2 & 1.2 & $f$ \\
\hline \multicolumn{7}{|l|}{ Radionuclides $(\mathrm{pC} i / L)^{\circ}$} \\
\hline Cs-137 & $1 / 5$ & $3.0^{*}$ & -0.27 & 0.70 & 0.60 & 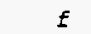 \\
\hline Gross alpha & $2 / 5$ & $1.6 *$ & -0.46 & 0.73 & 0.35 & $E$ \\
\hline Gross beta & $5 / 5$ & $7.0^{*}$ & $2.4^{*}$ & $3.9 *$ & 0.82 & $E$ \\
\hline$H-3$ & $1 / 5$ & $2,700 *$ & -380 & 530 & 560 & $E$ \\
\hline TC-99 & $1 / 5$ & $7.3 *$ & -1.9 & 2.4 & 1.6 & $f$ \\
\hline Total uranium & $5 / 5$ & $5.9 *$ & $0.30^{*}$ & 1.9 & 1.1 & $E$ \\
\hline
\end{tabular}

White Dak Lake at White Oak Dam (WCK 1.0)

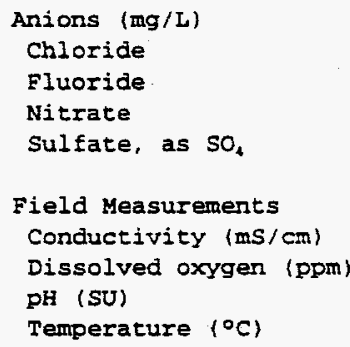

Anions (mg/L)

chloride

Sulfate, as $\mathrm{SO}_{+}$

Conductivity (ms/cm)

pH (SU)

Temperature $\left({ }^{\circ} \mathrm{C}\right\rangle$

$\begin{array}{lrl}5 / 5 & 11 & 2.5 \\ 5 / 5 & 1.0 & 0.40 \\ 4 / 5 & 2.6 & <1.0 \\ 5 / 5 & 62 & 25\end{array}$

$5 / 5$

$5 / 5$

$5 / 5$

$5 / 5$

0.44
11
8.4
27

\subsection{0}

25

0.32
7.0
7.5
6.0

7.0

7.5
6.0
1.6

0.12

0.34

7.1

$\begin{array}{lr}0.022 & f \\ 0.74 & 5 \\ 0.20 & f \\ 3.8 & f\end{array}$


Table 5.30 (continued)

\begin{tabular}{|c|c|c|c|c|c|c|}
\hline \multirow[b]{2}{*}{ Parameter } & \multirow[b]{2}{*}{$\begin{array}{l}\text { Net } / \\
\text { total }\end{array}$} & \multicolumn{4}{|c|}{ concentration } & \multirow[b]{2}{*}{ TwoCe } \\
\hline & & $\operatorname{sax}{ }^{b}$ & $\operatorname{Min}^{b}$ & $A v^{C}$ & $\begin{array}{l}\text { Standard } \\
\text { errord }\end{array}$ & \\
\hline \multicolumn{7}{|l|}{ Metals (mg/L) } \\
\hline Aluminum, total & $5 / 5$ & 2.2 & 0.31 & 1.1 & 0.35 & $f$ \\
\hline Barium, total & $5 / 5$ & 0.17 & 0.032 & 0.067 & 0.027 & $f$ \\
\hline Calcium, total & $5 / 5$ & 50 & 36 & 41 & 2,4 & $f$ \\
\hline Chromium, total & $5 / 5$ & 0.023 & 0.0068 & 0.015 & 0.0032 & 0.016 \\
\hline Cobalt, total & $1 / 5$ & 0.0077 & $<0.0040$ & -0.0047 & 0.00074 & $f$ \\
\hline Iron, total & $5 / 5$ & 1.4 & 0.37 & 0.88 & 0.20 & $f$ \\
\hline Magnesium, total & $5 / 5$ & 11 & 6.5 & 8.6 & 0.89 & $f$ \\
\hline Manganese, total & $5 / 5$ & 0.22 & 0.069 & 0.14 & 0.025 & $E$ \\
\hline Mercury, total & $1 / 5$ & 0.000095 & $<0.000050$ & -0.000059 & 0.0000090 & 0.0024 \\
\hline Phosphorus, tocal & $4 / 5$ & 0.50 & $<0.20$ & $\sim 0.36$ & 0.051 & $f$ \\
\hline Fotassium, total & $3 / 4$ & 2.7 & $<1.0$ & -2.0 & 0.38 & $E$ \\
\hline Sodium, total & $5 / 5$ & 19 & 6.8 & 23 & 2.6 & $f$ \\
\hline Uranium, total & $4 / 4$ & 0.010 & 0.00093 & 0.0041 & 0.0020 & $f$ \\
\hline Vanadium, total & $4 / 5$ & 0.0037 & $<0.0020$ & -0.0029 & 0.00032 & $E$ \\
\hline zinc, total & $5 / 5$ & 0.030 & 0.017 & 0.024 & 0.0024 & 0.117 \\
\hline \multicolumn{7}{|l|}{ Others } \\
\hline Alkalinicy (mg $\mathrm{CaCO}_{3} / \mathrm{L}$ ) & $5 / 5$ & 120 & 95 & 220 & 4.6 & $f$ \\
\hline Ammonia, as $\mathrm{N}(\mathrm{mg} / \mathrm{I})$ & $5 / 5$ & 0.11 & 0.051 & 0.074 & 0.010 & $f$ \\
\hline Biochemical oxygen demand (mg/L) & $1 / 5$ & 7.0 & $<5.0$ & -5.4 & 0.40 & $f$ \\
\hline Chemical oxygen demand (mg/L) & $3 / 5$ & 10 & $<5.0$ & -7.0 & 0.95 & $f$ \\
\hline CoIor $|C P U|$ & $5 / 5$ & 17 & 4.0 & -11 & 2.5 & $E$ \\
\hline Total dissolved solids (mg/L) & $5 / 5$ & 270 & 160 & 220 & 19 & $\varepsilon$ \\
\hline Total hardness (mg/L) & $5 / 5$ & 170 & 120 & 140 & 8.5 & $\hat{f}$ \\
\hline Total suspended solids (mg/L) & $5 / 5$ & 38 & 6.0 & 18 & 5.5 & $f$ \\
\hline \multicolumn{7}{|l|}{ Radionuclides $(\mathrm{pCi} / L)^{g}$} \\
\hline Gross alpha & $5 / 5$ & $12^{*}$ & $3.2 *$ & $6.1^{*}$ & 1.6 & $f$ \\
\hline Gross beta & $5 / 5$ & $510^{*}$ & $320^{*}$ & $410^{*}$ & 33 & $f$ \\
\hline $\mathrm{B}-3$ & $5 / 5$ & $160,000^{*}$ & $38,000^{\star}$ & 100,000 & 26,000 & $f$ \\
\hline$P u-239$ & $1 / 2$ & $0.27 *$ & 0.14 & 0.20 & 0.068 & $f$ \\
\hline$T c-99$ & $5 / 5$ & $35 *$ & $14 *$ & $20 *$ & 3.7 & $f$ \\
\hline Total rad $\mathrm{Sr}$ & $5 / 5$ & $200 \times$ & $110 *$ & $170 *$ & 17 & $f$ \\
\hline Total uranium & $5 / 5$ & $5.7 *$ & $3.2 *$ & $4.5 *$ & 0.45 & $f$ \\
\hline$v-234$ & $4 / 4$ & $3.8 *$ & $3.0 *$ & $3.3 *$ & 0.17 & $f$ \\
\hline$v-235$ & $2 / 4$ & $0.32 *$ & 0.057 & 0.20 & 0.067 & $E$ \\
\hline$v-238$ & $4 / 4$ & $1.1 *$ & $0.54^{*}$ & $0.83 *$ & 0.15 & $E$ \\
\hline \multicolumn{7}{|l|}{ Volatile organics $(\mu g / L)$} \\
\hline Acetone & $1 / 6$ & U10 & 57.8 & -9.6 & 0.37 & $f$ \\
\hline
\end{tabular}

White oak Creek downstream from ORNL (WCK 2.6)

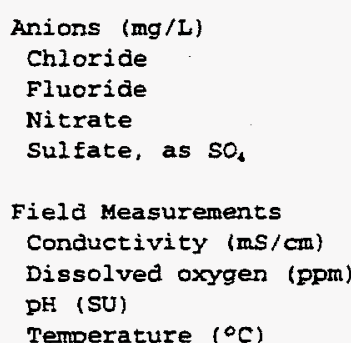

anions (mg/L)

Chloride

luoride

Sulfate, as so

Conductivity (ms/can)

$\mathrm{pH}$ (SU)

Temperature $\left({ }^{\circ} \mathrm{C}\right)$

$\begin{array}{lcc}5 / 5 & 15 & 4.7 \\ 5 / 5 & 1.2 & 0.50 \\ 5 / 5 & 11 & 3.0 \\ 5 / 5 & 62 & 28\end{array}$

$5 / 5$

$5 / 5$

$5 / 5$

$5 / 5$

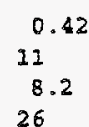

3.0

0.21

8.0

7.6

11
11

0.88

44

0.34
9.1
8.0

19
1.7

0.16

1.4

5.6

0.037

0.57

0.11

2.7 
Table 5.30 (continued)

\begin{tabular}{|c|c|c|c|c|c|c|}
\hline \multirow[b]{2}{*}{ Parameter } & \multirow[b]{2}{*}{$\begin{array}{l}\mathrm{N} \text { det } \\
\mathrm{N} \text { total }\end{array}$} & \multicolumn{4}{|c|}{ Concentration } & \multirow[b]{2}{*}{ TWQC } \\
\hline & & $\operatorname{Max}{ }^{b}$ & $\operatorname{Min}^{b}$ & $\mathrm{Av} C$ & $\begin{array}{l}\text { Standard } \\
\text { error }\end{array}$ & \\
\hline \multicolumn{7}{|l|}{ Metals (mg/L) } \\
\hline Aluminum, total & $5 / 5$ & 0.96 & 0.069 & 0.30 & 0.17 & $f$ \\
\hline Barium, total & $5 / 5$ & 0.039 & 0.031 & 0.033 & 0.0016 & $E$ \\
\hline Calcium, total & $5 / 5$ & 47 & 26 & 38 & 3.5 & $E$ \\
\hline Chromium, total & $2 / 5$ & 0.011 & $<0.0040$ & -0.0063 & 0.0014 & 0.016 \\
\hline Copper, total & $2 / 5$ & 0.017 & $<0.0070$ & -0.0090 & 0.0019 & 0.018 \\
\hline Iron, total & $5 / 5$ & 1.2 & 0.070 & 0.34 & 0.22 & $f$ \\
\hline Magnesium, total & $5 / 5$ & 9.8 & 4.9 & 8.3 & 0.89 & $f$ \\
\hline Manganese, total & $5 / 5$ & 0.17 & 0.0035 & 0.055 & 0.030 & $f$ \\
\hline Mercury, total & $1 / 5$ & 0.00017 & $<0.000050$ & -0.000074 & 0.000024 & 0.0024 \\
\hline Phosphorus, total & $4 / 5$ & 0.50 & $<0.20$ & $\sim 0.38$ & 0.057 & $f$ \\
\hline Potassium, total & $3 / 4$ & 2.2 & $<1.0$ & -1.9 & 0.30 & $\underline{f}$ \\
\hline Sodium, total & $5 / 5$ & 20 & 8.4 & 15 & 2.0 & $f$ \\
\hline Uranium, total & $4 / 4$ & 0.0016 & 0.00030 & 0.00093 & 0.00031 & $f$ \\
\hline Vanadium, total & $1 / 5$ & 0.0021 & $<0.0020$ & -0.0020 & 0.000020 & $f$ \\
\hline zinc, total & $5 / 5$ & 0.14 & 0.015 & 0.045 & 0.024 & 0.117 \\
\hline \multicolumn{7}{|l|}{ Others } \\
\hline Alkalinfty (mg $\left.\mathrm{CaCO}_{3} / \mathrm{L}\right)$ & $5 / 5$ & 120 & 55 & 100 & 11 & $f$ \\
\hline Ammonia, as $\mathrm{N}$ (mg/L) & $5 / 5$ & 0.17 & 0.030 & 0.076 & 0.025 & $f$ \\
\hline Chemical oxygen demand (mg/L) & $1 / 5$ & 21 & $<5.0$ & -8.2 & 3.2 & $f$ \\
\hline Color (CPU) & $4 / 5$ & 13 & $<1.0$ & $\sim 5.4$ & 2.1 & $f$ \\
\hline Total dissolved solids (mg/L) & $5 / 5$ & 260 & 150 & 210 & 19 & $f$ \\
\hline Total hardness (mg/L) & $5 / 5$ & 150 & 79 & 130 & 13 & $f$ \\
\hline Total suspended solids (mg/L) & $2 / 5$ & 53 & $<5.0$ & -17 & 9.3 & $f$ \\
\hline \multicolumn{7}{|l|}{ Radionuclides $(\mathrm{pCi} / L)^{g}$} \\
\hline$A m-2 \$ 1$ & $1 / 1$ & $0.30^{*}$ & $0.30 *$ & 0.30 & $\epsilon$ & 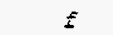 \\
\hline$C 0-60$ & $1 / 5$ & $5.7^{*}$ & 0.27 & $2.7 *$ & 0.90 & $f$ \\
\hline $\mathrm{Cs}-137$. & $5 / 5$ & $490 *$ & $51^{*}$ & 150 & 85 & $f$ \\
\hline Gross alpha & $5 / 5$ & $9.5 *$ & $1.3 *$ & $4.3 *$ & 1.4 & $f$ \\
\hline Gross beta & $5 / 5$ & $320 *$ & $160 *$ & $230 *$ & 30 & $f$ \\
\hline H-3 & $5 / 5$ & $43,000 *$ & $2,700^{*}$ & $16,000^{*}$ & 7,100 & $f$ \\
\hline Te-99 & $2 / 5$ & $7.0^{*}$ & -2.2 & 2.4 & 1.6 & $f$ \\
\hline Total rad Sx & $5 / 5$ & $140 *$ & $35^{*}$ & $70 *$ & 19 & $f$ \\
\hline Tocal uranium & $5 / 5$ & $4.3 *$ & $2.3 *$ & $3.3^{*}$ & 0.37 & $f$ \\
\hline $\mathrm{U}-234$ & $2 / 2$ & $3.2^{*}$ & $2.4^{*}$ & $2.8^{*}$ & 0.41 & 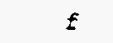 \\
\hline $0-238$ & $2 / 2$ & $1.1 *$ & $0.46^{*}$ & 0.78 & 0.32 & $f$ \\
\hline Volatile organics ( $\mu g / L)$ & & & & & & \\
\hline Chloroform & $3 / 5$ & U5. 0 & $\mathrm{~J} 1.7$ & -3.1 & 0.75 & $f$ \\
\hline
\end{tabular}

White Oak Creek upstream from ORNL (WCK 6.8)

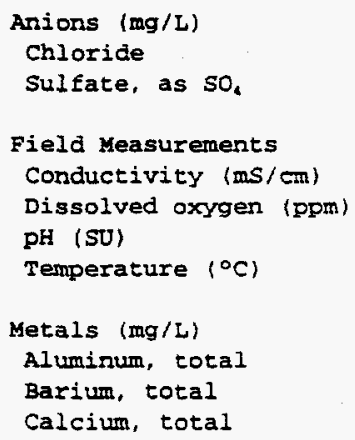

$\begin{array}{lcc}3 / 5 & 1.2 & <0.10 \\ 5 / 5 & 4.1 & 1.8 \\ & & \\ 5 / 5 & 0.29 & 0.15 \\ 5 / 5 & 11 & 8.9 \\ 5 / 5 & 8.1 & 13 \\ 5 / 5 & 21 & \\ & & <0.050 \\ 3 / 5 & 0.30 & 0.033 \\ 5 / 5 & 0.10 & 14 \\ 5 / 5 & 32 & \end{array}$

14

0.033

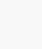
$-0.14$ 0.075

26

$\begin{array}{llll}0.10 & -0.68 & 0.24 & f \\ 1.8 & 3.0 & 0.47 & f \\ & & & \\ 0.15 & 0.24 & 0.024 & f \\ 8.9 & 9.7 & 0.40 & 5 \\ 7.5 & 7.9 & 0.13 & f \\ 3 & 17 & 1.6 & f\end{array}$

0.051

0.013

3.2 
Table 5.30 (continued)

\begin{tabular}{|c|c|c|c|c|c|c|}
\hline \multirow[b]{2}{*}{ Parameter } & \multirow[b]{2}{*}{$\begin{array}{l}\mathrm{N} \text { det } \\
\mathrm{N} \text { total }\end{array}$} & \multicolumn{4}{|c|}{ Concertzation } & \multirow[b]{2}{*}{ TWQC } \\
\hline & & $\operatorname{Max}^{6}$ & $\operatorname{Min}^{b}$ & $\mathrm{Av}^{\mathrm{C}}$ & $\begin{array}{l}\text { Standard } \\
\text { error }\end{array}$ & \\
\hline Chromium, total & $2 / 5$ & 0.014 & $<0.0040$ & -0.0076 & 0.0022 & 0.016 \\
\hline Iron, total & $3 / 5$ & 0.22 & $<0.050$ & -0.11 & 0.034 & 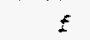 \\
\hline Magnesium, total & $5 / 5$ & 16 & 6.1 & 13 & 1.8 & $\mathrm{f}$ \\
\hline Manganese, total & $5 / 5$ & 0.029 & 0.0058 & 0.012 & 0.0044 & $f$ \\
\hline Phosphorus, total & $3 / 5$ & 0.30 & $<0,20$ & -0.23 & 0.022 & 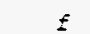 \\
\hline Sodium, total & $5 / 5$ & 0.51 & 0.37 & 0.46 & 0.025 & $f$ \\
\hline Uranium, total & $4 / 4$ & 0.00040 & 0.00020 & 0.00033 & 0.000048 & $\mathrm{f}$ \\
\hline Vanadium, total & $1 / 5$ & 0.0051 & $<0.0020$ & -0.0026 & 0.00052 & $f$ \\
\hline zinc, total & $4 / 5$ & 0.023 & $<0.0050$ & -0.015 & 0.0035 & 0.117 \\
\hline \multicolumn{7}{|l|}{ Others } \\
\hline Alkalinity (mo $\left.\mathrm{CaCO}_{3} / \mathrm{L}\right)$ & $5 / 5$ & 150 & 63 & 120 & 16 & $f$ \\
\hline Ammonia, as $\mathrm{N}(\mathrm{mg} / \mathrm{L})$ & $5 / 5$ & 0.060 & 0.020 & 0.035 & 0.0067 & \pm \\
\hline Chemical oxygen demand (mg/L) & $1 / 5$ & 5.0 & $<5.0$ & -5.0 & 0 & $f$ \\
\hline Color (CPU) & $4 / 5$ & 7.0 & $<1.0$ & -3.8 & 1.2 & $f$ \\
\hline Total dissolved solids (mg/L) & $5 / 5$ & 170 & 67 & 130 & 17 & 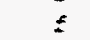 \\
\hline Total hardness (mg/L) & $5 / 5$ & 150 & 63 & 120 & 15 & 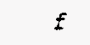 \\
\hline \multicolumn{7}{|l|}{ Radionuclides $(p C i / L)^{\circ}$} \\
\hline $\operatorname{co-} 60$ & $1 / 5$ & $8.9 *$ & -2.7 & 2.6 & 1.9 & $\mathbf{E}$ \\
\hline Gross alpha & $3 / 5$ & $2.7 *$ & -0.84 & 1.1 & 0.70 & $\mathrm{f}$ \\
\hline Gross beta & $2 / 5$ & $8.1 *$ & -1.6 & 2.3 & 1.6 & 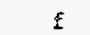 \\
\hline Tc-99 & $1 / 5$ & 3.2 & $-0.8 I$ & $1.7 \%$ & 0.73 & $\mathrm{f}$ \\
\hline Total rad sr & $1 / 5$ & $3.8^{*}$ & 0.97 & $1.8 *$ & 0.51 & $E$ \\
\hline Total uranium & $5 / 5$ & $2.2 *$ & $0.38 *$ & $1.2 *$ & 0.32 & $\mathrm{f}$ \\
\hline
\end{tabular}

"All values were included in the calculations. Only parameters that have detections in one or more samples are listed in the table. The sampling and analysis plan contains a complete list of analyses performed.

"Prefix "<" indicates the value for a parameter (excluding organics) was not quantifiable at the analytical detection limit; "U" incicates the the value for an organic parameter was undetected at the analytical detection limit; "JB" indicates the value was estimated at or below the analytical detection limit and was found in the laboracory blank; and " $\mathrm{J}$ " indicates the value was estimated at or below the analytical detection limit by the iaboratory.

A tilde ( ) indicates that estimated values and/or detection limits were used in the calculation.

'Standard error of the mean.

Tennessee General Water Quality Criteria (TWQC) for Domestic Water Supplies, as amended, are shown at locations CRK 16, CRK 23, CRK 32, CRK 58, CRK 66, CRK 80, CRK 84, and TRK 915. Tennessee General Water Quality Criteria (TWQC) for Freshwater Fish and Aguatic Life, as amended, are shown at locations BCK 0.6, BCK 9.4, EFK 23.4, EFK 5.4, HC, MEK 0.2، MEK 2.1, PCK 2.2, PCK 22, WCK 1.0, WCK 2.6, and WCK 6.8 .

Not applicable.

Individual and average radionuclide concentrations significantly greater than zero are identified by an *. 
Table 5.31. 1993 K-25 Site surface water parameters detected at K-901-A

\begin{tabular}{|c|c|c|c|c|c|c|}
\hline \multirow{2}{*}{ Parameter } & \multirow{2}{*}{$\begin{array}{l}\text { Number } \\
\text { detected/ } \\
\text { number of } \\
\text { samples }\end{array}$} & \multicolumn{3}{|c|}{ Detected results ${ }^{b}$} & \multirow{2}{*}{$\begin{array}{l}\text { Reference } \\
\text { value }^{a}\end{array}$} & \multirow{2}{*}{$\begin{array}{l}\text { Number of } \\
\text { values } \\
\text { exceeding } \\
\text { reference }\end{array}$} \\
\hline & & $\operatorname{Max}$ & Min & Avg & & \\
\hline Alkalinity & $\mathrm{c} / 4$ & 140 & 110 & 120 & $c$ & c \\
\hline Calcium hardness & $\mathrm{c} / 4$ & 97 & 78 & 85 & $c$ & $c$ \\
\hline $\begin{array}{l}\text { Chemical oxygen } \\
\text { demand }\end{array}$ & $3 / 4$ & 14 & $<5$ & $<10$ & c & $c$ \\
\hline Chloride & $4 / 4$ & 6.4 & 1.9 & 3.2 & $c$ & $c$ \\
\hline Chlorine & $3 / 3$ & 0.1 & 0.06 & 0.087 & $c$ & $c$ \\
\hline Color hue & $c / 4$ & 500 & 490 & 500 & $c$ & $c$ \\
\hline $\begin{array}{l}\text { Conductivity } \\
\mu \mathrm{mho} / \mathrm{cm}\end{array}$ & ci4 & 270 & 250 & 260 & $c$ & $c$ \\
\hline Dissolved oxygen & $c / 4$ & 9.8 & 3.4 & 5.8 & $5.0 \mathrm{~min}$ & 2 \\
\hline Dissolved solids & $4 / 4$ & 200 & 120 & 160 & $c$ & $c$ \\
\hline Fluoride & $4 / 4$ & 0.2 & 0.1 & 0.15 & $c$ & $c$ \\
\hline Iron & $4 / 4$ & 1.6 & 0.24 & 1.1 & $c$ & c \\
\hline Manganese & $4 / 4$ & 0.13 & 0.031 & 0.084 & $c$ & $c$ \\
\hline Mercury & $1 / 4$ & 0.0002 & $<0.00020$ & $<0.00020$ & 0.0024 & 0 \\
\hline Nitrate & $2 / 4$ & 0.41 & $<0.1$ & $<0.23$ & $c$ & $c$ \\
\hline $\begin{array}{l}\mathrm{pH} \text { (standard } \\
\text { units) }\end{array}$ & $c / 4$ & 8.7 & 7.2 & 7.9 & $c$ & 1 \\
\hline Potassium & $4 / 4$ & 2.9 & 1.2 & 2.3 & $c$ & $c$ \\
\hline Sodium & $4 / 4$ & 1.7 & 1.1 & 1.3 & $c$ & $c$ \\
\hline Sulfate & $4 / 4$ & 19 & 6.1 & 10 & $c$ & $c$ \\
\hline Suspended solids & $4 / 4$ & 32 & 7 & 18 & $c$ & $c$ \\
\hline Temperature ${ }^{\circ} \mathrm{C}$ & $\mathrm{c} / 4$ & 29 & 5.3 & 18 & 30.5 & 0 \\
\hline $\begin{array}{l}\text { Total phosphate } \\
\text { as phosphorus }\end{array}$ & $4 / 4$ & 0.22 & 0.06 & 0.11 & $c$ & $c$ \\
\hline
\end{tabular}

${ }^{a}$ Reference values are Tennessee Water Quality Criteria for Fish and Aquatic Life.

Units in $\mathrm{mg} / \mathrm{L}$ unless otherwise noted.

Not applicable. 
Table 5.32. 1993 K-25 Site sufface waier parameters detected at K-716

\begin{tabular}{|c|c|c|c|c|c|c|}
\hline \multirow{2}{*}{ Parameter } & \multirow{2}{*}{$\begin{array}{l}\text { Number } \\
\text { detected } \\
\text { number of } \\
\text { samples }\end{array}$} & \multicolumn{3}{|c|}{ Detected results ${ }^{b}$} & \multirow{2}{*}{$\begin{array}{l}\text { Reference } \\
\text { value }^{a}\end{array}$} & \multirow{2}{*}{$\begin{array}{l}\text { Number of } \\
\text { values } \\
\text { exceeding } \\
\text { reference }\end{array}$} \\
\hline & & Max & Min & Avg & & \\
\hline Alkalinity & $c / 4$ & 130 & 51 & 97 & $c$ & $c$ \\
\hline $\begin{array}{l}\text { Biological oxygen } \\
\text { demand }\end{array}$ & $c / 4$ & 5.3 & 5 & 5.1 & $c$ & $c$ \\
\hline Calcium hardness & $\mathrm{c} / 4$ & 91 & 49 & 73 & $c$ & $c$ \\
\hline $\begin{array}{l}\text { Chemical oxygen } \\
\text { demand }\end{array}$ & $2 / 4$ & 14 & $<5$ & $<7.8$ & $c$ & $c$ \\
\hline Chloride & $4 / 4$ & 10 & 7 & 8.7 & $c$ & $c$ \\
\hline Chlorine & $3 / 3$ & 0.09 & 0.02 & 0.067 & $c$ & $c$ \\
\hline Color hue & $\mathrm{c} / 4$ & 590 & 490 & 520 & $c$ & $c$ \\
\hline $\begin{array}{l}\text { Conductivity } \\
\mu \mathrm{mho} / \mathrm{cm}\end{array}$ & $c / 4$ & 300 & 170 & 260 & $c$ & $c$ \\
\hline Dissolved oxygen & $c / 4$ & 12 & 9.3 & 10 & $5.0 \mathrm{~min}$ & 0 \\
\hline Dissolved solids & $4 / 4$ & 210 & 110 & 170 & $c$ & $c$ \\
\hline Fluoride & $2 / 4$ & 0.2 & $<0.1$ & $<0.15$ & $c$ & $c$ \\
\hline Iron & $4 / 4$ & 1.7 & 0.46 & 1.1 & $c$ & $c$ \\
\hline Manganese & $4 / 4$ & 0.11 & 0.048 & 0.086 & $c$ & $c$ \\
\hline Mercury & $2 / 4$ & 0.00025 & $<0.0002$ & $<0.0002$ & 0.0024 & 0 \\
\hline Nitrate & $4 / 4$ & 0.9 & $<0.64$ & $<0.80$ & $c$ & $c$ \\
\hline $\begin{array}{l}\text { pH (standard } \\
\text { units) }\end{array}$ & $c / 4$ & 8.9 & 7 & 8.1 & $c$ & $c$ \\
\hline Potassium & $4 / 4$ & 3.1 & 1.6 & 2.2 & $c$ & $c$ \\
\hline Sodium & $4 / 4$ & 7.7 & 4.5 & 6.7 & $c$ & $c$ \\
\hline Sulfate & $4 / 4$ & 44 & 23 & 30 & $c$ & $c$ \\
\hline Suspended solids & $4 / 4$ & 49 & 12 & 29 & $c$ & $c$ \\
\hline Temperature ${ }^{\circ} \mathrm{C}$ & $\mathrm{c} / 4$ & 25 & 5 & 17 & 30.5 & 0 \\
\hline $\begin{array}{l}\text { Total phosphate } \\
\text { as phosphorus }\end{array}$ & $4 / 4$ & 0.22 & 0.12 & 0.16 & $c$ & $c$ \\
\hline Zine & $1 / 4$ & $<0.02$ & 0.011 & $<0.018$ & 0.117 & 0 \\
\hline
\end{tabular}

${ }^{\circ}$ Reference values are Tennessee Water Quality Criteria for Fish and Aquatic Life.

Units in $\mathrm{mg} / \mathrm{L}$ unless otherwise noted.

Not applicable. 
Table 5.33. 1993 K-25 Site surface water parameters detected at K-1007-B

\begin{tabular}{|c|c|c|c|c|c|c|}
\hline \multirow{2}{*}{ Parameter } & \multirow{2}{*}{$\begin{array}{l}\text { Number } \\
\text { detected } \\
\text { number of } \\
\text { samples }\end{array}$} & \multicolumn{3}{|c|}{ Detected results ${ }^{b}$} & \multirow{2}{*}{$\begin{array}{l}\text { Reference } \\
\text { value }^{a}\end{array}$} & \multirow{2}{*}{$\begin{array}{l}\text { Number of } \\
\text { values } \\
\text { exceeding } \\
\text { reference }\end{array}$} \\
\hline & & $\operatorname{Max}$ & Min & Avg & & \\
\hline Alkalinity & $c / 4$ & 108 & 75 & 96 & $c$ & $c$ \\
\hline Calcium hardness & $c / 4$ & 91 & 48 & 74 & $c$ & $c$ \\
\hline $\begin{array}{l}\text { Chemical oxygen } \\
\text { demand }\end{array}$ & $3 / 4$ & 40 & $<5$ & $<17$ & $c$ & $c$ \\
\hline Chloride & $4 / 4$ & 11 & 9.4 & 10 & $c$ & $c$ \\
\hline Chlorine & $3 / 3$ & 0.12 & 0.04 & 0.08 & $c$ & $c$ \\
\hline Color hue & $\mathrm{c} / 4$ & 500 & 490 & 500 & $c$ & $c$ \\
\hline $\begin{array}{l}\text { Conductivity } \\
\mu \mathrm{mho} / \mathrm{cm}\end{array}$ & $c / 4$ & 590 & 220 & 340 & $c$ & $c$ \\
\hline Dissolved oxygen & $4 / 4$ & 12 & 7.5 & 9.9 & $5.0 \mathrm{~min}$ & 0 \\
\hline Dissolved solids & $4 / 4$ & 190 & 140 & 160 & $c$ & $c$ \\
\hline Fluoride & $4 / 4$ & 0.10 & 0.10 & 0.10 & $c$ & $c$ \\
\hline Iron & $4 / 4$ & 0.61 & 0.12 & 0.33 & $c$ & $c$ \\
\hline Manganese & $4 / 4$ & 0.11 & 0.04 & 0.06 & $c$ & $c$ \\
\hline Nickel & $1 / 4$ & $<0.05$ & 0.012 & $<0.04$ & 1.4 & 0 \\
\hline Nitrate & $3 / 4$ & 0.45 & $<0.10$ & $<0.23$ & $c$ & $c$ \\
\hline $\begin{array}{l}\text { pH (standard } \\
\text { units) }\end{array}$ & $c / 4$ & 9.00 & 7.80 & 8.6 & $c$ & $c$ \\
\hline Potassium & $4 / 4$ & 2.90 & 1.90 & 2.2 & $c$ & $c$ \\
\hline Sodium & $4 / 4$ & 4.9 & 4 & 4.6 & $c$ & $c$ \\
\hline Sulfate & $4 / 4$ & 28 & 20 & 23 & $\mathfrak{c}$ & $c$ \\
\hline Suspended solids & $4 / 4$ & 24 & 5 & 13 & $c$ & $c$ \\
\hline Temperature ${ }^{\circ} \mathrm{C}$ & $\mathrm{c} / 4$ & 30 & 6.30 & 19 & 30.5 & 0 \\
\hline $\begin{array}{r}\text { Total phosphate } \\
\text { as phosphorus }\end{array}$ & $4 / 4$ & 0.17 & 0.06 & 0.12 & $c$ & $c$ \\
\hline Zinc & $2 / 4$ & 0.03 & $<0.02$ & $<0.02$ & 0.12 & 0 \\
\hline
\end{tabular}

${ }^{a}$ Reference values are Tennessee Water Quality Criteria for Fish and Aquatic Life.

${ }^{b}$ Units in $\mathrm{mg} / \mathrm{L}$ unless otherwise noted.

Not applicable. 
Table 5.34. 1993 K-25 Site surface water parameters detected at K-1700

\begin{tabular}{|c|c|c|c|c|c|c|}
\hline \multirow{2}{*}{ Parameter } & \multirow{2}{*}{$\begin{array}{l}\text { Nimber } \\
\text { detected } \\
\text { number of } \\
\text { samples }\end{array}$} & \multicolumn{3}{|c|}{ Detected results ${ }^{b}$} & \multirow{2}{*}{$\begin{array}{l}\text { Reference } \\
\text { value }^{\alpha}\end{array}$} & \multirow{2}{*}{$\begin{array}{c}\text { Number of } \\
\text { values } \\
\text { exceeding } \\
\text { reference }\end{array}$} \\
\hline & & $\operatorname{Max}$ & Min & Avg & & \\
\hline 1,2 Dichloroethene & $4 / 4$ & 0.022 & 0.006 & 0.011 & $c$ & $c$ \\
\hline Alkalinity & $c / 4$ & 160 & 64 & 92 & $c$ & $c$ \\
\hline Calcium hardness & $\mathrm{c} / 4$ & 130 & 59 & 82 & $c$ & $c$ \\
\hline $\begin{array}{l}\text { Chemical oxygen } \\
\text { demand }\end{array}$ & $2 / 4$ & 14 & $<5$ & $<8.5$ & $c$ & $c$ \\
\hline Chloride & $4 / 4$ & 39 & 4 & 17 & $c$ & $c$ \\
\hline Chlorine & $4 / 4$ & 0.1 & 0.01 & 0.045 & $c$ & $c$ \\
\hline Color hue & $c / 4$ & 500 & 490 & 500 & $c$ & $c$ \\
\hline $\begin{array}{l}\text { Conductivity } \\
\mu \mathrm{mho} / \mathrm{cm}\end{array}$ & $c / 4$ & 400 & 180 & 270 & $c$ & $c$ \\
\hline Dissolved oxygen & $\mathrm{c} / 5$ & 12 & 7.6 & 8.8 & $5.0 \mathrm{~min}$ & 0 \\
\hline Dissolved solids & $4 / 4$ & 260 & 130 & 200 & $c$ & $c$ \\
\hline Fluoride & $4 / 4$ & 0.2 & 0.1 & 0.15 & $c$ & $c$ \\
\hline Iron & $4 / 4$ & 0.82 & 0.39 & 0.68 & $c$ & $c$ \\
\hline Lead & $2 / 4$ & 0.011 & $<0.004$ & $<0.0058$ & 0.082 & 0 \\
\hline Manganese & $4 / 4$ & 0.27 & 0.11 & 0.19 & $c$ & $c$ \\
\hline Mercury & $1 / 4$ & 0.00022 & $<0.00020$ & $<0.00020$ & 0.0024 & 0 \\
\hline Nickel & $1 / 4$ & $<0.05$ & 0.028 & $<0.045$ & 1.4 & 0 \\
\hline Nirrate & $4 / 4$ & 0.62 & 0.29 & 0.48 & $c$ & $c$ \\
\hline $\begin{array}{l}\mathrm{pH} \text { (standard } \\
\text { units) }\end{array}$ & $₫ / 5$ & 8 & 7.3 & 7.7 & $c$ & $c$ \\
\hline Phosphate & $4 / 4$ & 0.17 & 0.1 & 0.14 & $c$ & $c$ \\
\hline Potassium & $4 / 4$ & 2.4 & 1.5 & 2.0 & $c$ & $c$ \\
\hline Sodium & $4 / 4$ & 40 & 2.8 & 14 & $c$ & $c$ \\
\hline Sulfate & $4 / 4$ & 39 & 16 & 25 & $c$ & $c$ \\
\hline Suspended solids & $3 / 4$ & 12 & $<1$ & $<7.8$ & $c$ & $c$ \\
\hline Temperature ${ }^{\circ} \mathrm{C}$ & $c / 4$ & 23 & 8.2 & 16 & 30.5 & 0 \\
\hline Trichl Jroethene & $4 / 4$ & 0.027 & 0.007 & 0.014 & 0.807 & 0 \\
\hline Zinc & $3 / 4$ & 0.056 & $<0.02$ & $<0.035$ & 0.117 & 0 \\
\hline
\end{tabular}

${ }^{\circ}$ Reference values are Tennessee Water Quality Criteria for Fish and Aquatic Life.

${ }^{b}$ Units in $\mathrm{mg} / \mathrm{L}$ unless otherwise noted.

Not applicable. 
Table 5.35. 1993 K-25 Site surface water parameters detected at K-1710

\begin{tabular}{|c|c|c|c|c|c|c|}
\hline \multirow{2}{*}{ Parameter } & \multirow{2}{*}{$\begin{array}{l}\text { Number } \\
\text { detected/ } \\
\text { number of } \\
\text { samples }\end{array}$} & \multicolumn{3}{|c|}{ Detected results ${ }^{b}$} & \multirow{2}{*}{$\begin{array}{l}\text { Reference } \\
\text { value }^{a}\end{array}$} & \multirow{2}{*}{$\begin{array}{l}\text { Number of } \\
\text { values } \\
\text { cxceeding } \\
\text { reference }\end{array}$} \\
\hline & & $\operatorname{Max}$ & Min & Avg & & \\
\hline Alkalinity & $c / 4$ & 130 & 47 & 87 & c & $c$ \\
\hline Calcium hardness & $c / 4$ & 92 & 48 & 73 & $c$ & c \\
\hline $\begin{array}{l}\text { Chemical oxygen } \\
\text { demand }\end{array}$ & $1 / 4$ & 7 & $<5$ & $<5.5$ & $c$ & $c$ \\
\hline Chloride & $4 / 4$ & 10 & 5 & 8.2 & $c$ & $c$ \\
\hline Chlorine & $3 / 3$ & 0.1 & 0.03 & 0.077 & $c$ & $c$ \\
\hline Color hue & $c / 4$ & 590 & 490 & 520 & $c$ & $c$ \\
\hline $\begin{array}{l}\text { Conductivity } \\
\mu \mathrm{mho} / \mathrm{cm}\end{array}$ & $c / 4$ & 340 & 170 & 260 & $c$ & $c$ \\
\hline Dissolved oxygen & $c / 4$ & 12 & 4.6 & 7.7 & $5.0 \mathrm{~min}$ & 1 \\
\hline Dissolved solids & $4 / 4$ & 200 & 110 & 170 & $c$ & $c$ \\
\hline Fluoride & $4 / 4$ & 0.3 & 0.1 & 0.2 & $c$ & $c$ \\
\hline Iron & $4 / 4$ & 2.8 & 0.38 & 1.1 & $c$ & $c$ \\
\hline Lead & $1 / 4$ & 0.0063 & $<0.004$ & $<0.0046$ & 0.082 & 0 \\
\hline Manganese & $4 / 4$ & 0.17 & 0.075 & 0.11 & $c$ & $c$ \\
\hline Mercury & $2 / 4$ & 0.00034 & $<0.0002$ & $<0.00024$ & 0.0024 & 0 \\
\hline Nitrate & $4 / 4$ & 1.6 & 0.59 & 1.1 & $c$ & $c$ \\
\hline $\begin{array}{l}\text { pH (standard } \\
\text { units) }\end{array}$ & $c / 4$ & 8 & 7 & 7.5 & $c$ & $c$ \\
\hline Potassium & $4 / 4$ & 3.9 & 1.6 & 2.8 & $c$ & $c$ \\
\hline Sodium & $4 / 4$ & 8.4 & 4.8 & 6.5 & $c$ & $c$ \\
\hline Sulfate & $4 / 4$ & 49 & 22 & 31 & $c$ & $c$ \\
\hline Suspended solids & $4 / 4$ & 36 & 7 & 19 & $c$ & $c$ \\
\hline Temperature ${ }^{\circ} \mathrm{C}$ & $\mathrm{c} / 4$ & 26 & 7.2 & 18 & 30.5 & 0 \\
\hline $\begin{array}{l}\text { Total phosphate } \\
\text { as phosphorus }\end{array}$ & $4 / 4$ & 0.26 & 0.12 & 0.18 & $c$ & $c$ \\
\hline Zinc & $1 / 4$ & $<0.02$ & 0.01 & $<0.018$ & 0.117 & 0 \\
\hline
\end{tabular}

${ }^{a}$ Reference values are Tennessee Water Quality Criteria for Fish and Aquatic Life.

'Units in $\mathrm{mg} / \mathrm{L}$ unless otherwise noted.

Not applicable. 
Table 5.36. 1993 K-25 Site surface water parameters detected at WFPC

\begin{tabular}{|c|c|c|c|c|c|c|}
\hline \multirow{2}{*}{ Parameter } & \multirow{2}{*}{$\begin{array}{c}\text { Number } \\
\text { detected } \\
\text { number of } \\
\text { samples }\end{array}$} & \multicolumn{3}{|c|}{ Detected results ${ }^{b}$} & \multirow{2}{*}{$\begin{array}{l}\text { Reference } \\
\text { value }^{a}\end{array}$} & \multirow{2}{*}{$\begin{array}{c}\text { Number o } \\
\text { values } \\
\text { exceeding } \\
\text { reference } \\
\end{array}$} \\
\hline & & $\operatorname{Max}$ & $\operatorname{Min}$ & Avg & & \\
\hline Alkalinity & $c / 4$ & 140 & 27 & 81 & $c$ & $c$ \\
\hline Calcium hardness & $c / 4$ & 90 & 30 & 66 & $c$ & $c$ \\
\hline $\begin{array}{l}\text { Chemical oxygen } \\
\text { demand }\end{array}$ & $2 / 4$ & 7 & $<5$ & $<6$ & $c$ & $c$ \\
\hline Chloride & $4 / 4$ & 6.9 & 2 & 4.8 & $c$ & $c$ \\
\hline Chlorine & $3 / 3$ & 0.12 & 0.05 & 0.077 & $c$ & $c$ \\
\hline Color hue & $c / 4$ & 590 & 490 & 520 & $c$ & $c$ \\
\hline $\begin{array}{l}\text { Conductivity } \\
\mu \mathrm{mho} / \mathrm{cm}\end{array}$ & $c / 4$ & 320 & 120 & 240 & $c$ & $c$ \\
\hline Dissoived oxygen & $c / 4$ & 12 & 4.3 & 7.4 & $5.0 \mathrm{~min}$ & 1 \\
\hline Dissolved solids & $4 / 4$ & 190 & 82 & 160 & $c$ & $c$ \\
\hline Fluoride & $2 / 4$ & 0.2 & $<0.1$ & $<0.13$ & $c$ & $c$ \\
\hline Iron & $4 / 4$ & 2.4 & 0.22 & 1.1 & $c$ & $c$ \\
\hline Manganese & $4 / 4$ & 0.22 & 0.11 & 0.16 & $c$ & $c$ \\
\hline Nitrate & $4 / 4$ & 0.62 & 0.28 & 0.43 & $c$ & $c$ \\
\hline $\begin{array}{l}\mathrm{pH} \text { (standard } \\
\text { units) }\end{array}$ & $c / 4$ & 7.8 & 7 & 7.4 & $c$ & $c$ \\
\hline Potassium & $4 / 4$ & 3.6 & 1.3 & 2.5 & $c$ & $c$ \\
\hline Sodium & $4 / 4$ & 5.7 & 2.1 & 4.1 & $c$ & $c$ \\
\hline Sulfate & $4 / 4$ & 60 & 18 & 32 & $c$ & $c$ \\
\hline Suspended solids & $4 / 4$ & 41 & 10 & 31 & $c$ & $c$ \\
\hline Temperature ${ }^{\circ} \mathrm{C}$ & $c / 4$ & 26 & 6.2 & 17 & 30.5 & 0 \\
\hline $\begin{array}{l}\text { Total phosphate } \\
\text { as phosphorus }\end{array}$ & $4 / 4$ & 0.12 & 0.06 & 0.1 & $c$ & $c$ \\
\hline Zinc & $1 / 4$ & $<0.02$ & 0.0097 & $<0.017$ & 0.117 & 0 \\
\hline
\end{tabular}

${ }^{a}$ Reference values are Tennessee Water Quality Criteria for Fish and Aquatic Life.

bUnits in $\mathrm{mg} / \mathrm{L}$ unless otherwise noted.

Not applicable. 
Table 5.37. Summary of constituents detected in off-site residential groundwater during 1993

\begin{tabular}{|c|c|c|c|c|c|c|}
\hline \multirow{2}{*}{ Parameter } & \multirow{2}{*}{$\begin{array}{l}\text { Number } \\
\text { detected/ } \\
\text { number } \\
\text { total }\end{array}$} & \multicolumn{3}{|c|}{ Detected results } & \multirow{2}{*}{$\begin{array}{l}\text { Reference } \\
\text { value }\end{array}$} & \multirow{2}{*}{$\begin{array}{l}\text { Number of } \\
\text { values } \\
\text { exceeding } \\
\text { reference } \\
\text { [ref] }\end{array}$} \\
\hline & & $\operatorname{Max}$ & $\operatorname{Min}^{3}$ & $\mathrm{Avg}^{B}$ & & \\
\hline \multicolumn{7}{|l|}{ Anions, Unfiltered (mg/L) } \\
\hline Chloride & $35 / 35$ & 68 & 1.0 & 7.7 & 250 & $0[3]$ \\
\hline Fluoride & $15 / 35$ & 7.0 & 0.10 & 1.3 & 4 & $2[2]$ \\
\hline Nitrate & $23 / 35$ & 14 & 1.1 & 3.9 & 10 & $2[2]$ \\
\hline Sulfate, as $\mathrm{SO}_{4}$ & $34 / 35$ & 62 & 1.0 & 12 & 250 & $0[3]$ \\
\hline \multicolumn{7}{|c|}{ Field measurements, unfiltered } \\
\hline Conductivity (mS/cm) & $35 / 35$ & 1.9 & 0.12 & 0.48 & $d$ & {$[d]$} \\
\hline Temperature $\left({ }^{\circ} \mathrm{C}\right)$ & $35 / 35$ & 25 & 7.1 & 16 & 30.5 & $0[1]$ \\
\hline $\mathrm{pH}(\mathrm{SU})$ & $35 / 35$ & 8.6 & 6.9 & 7.4 & $(6.5,8.5)$ & $1[3]$ \\
\hline \multicolumn{7}{|l|}{ Metals, unfiltered (mg/L) } \\
\hline Barium total & $35 / 35$ & 0.14 & 0.0025 & 0.072 & 2 & $0[2]$ \\
\hline Calcium, total & $35 / 35$ & 110 & 1.0 & 45 & $d$ & {$[d]$} \\
\hline Chromium, total & $1 / 35$ & 0.011 & 0.011 & 0.011 & 0.05 & $0[1]$ \\
\hline Cobalt, total & $1 / 35$ & 0.0044 & 0.0044 & 0.0044 & $d$ & {$[d]$} \\
\hline Copper, total & $22 / 35$ & 0.071 & 0.0081 & 0.020 & 1.3 & $0[2]$ \\
\hline Iron, total & $10 / 35$ & 0.34 & 0.052 & 0.17 & 0.3 & $1[3]$ \\
\hline Lead, total & $4 / 35$ & 0.025 & 0.0043 & 0.011 & 0.015 & $1[2]$ \\
\hline Magnesium, total & $35 / 35$ & 35 & 0.42 & 13 & $d$ & {$[d]$} \\
\hline Manganese, total & $13 / 35$ & 0.31 & 0.0013 & 0.031 & 0.05 & $1[3]$ \\
\hline Mercury, total & $3 / 35$ & 0.000055 & 0.000051 & 0.000053 & 0.002 & $0[1]$ \\
\hline Sodium, total & $35 / 35$ & 470 & 0.50 & 41 & $d$ & {$[d]$} \\
\hline Uranium, total & $24 / 35$ & 0.0015 & 0.00010 & 0.00044 & $d$ & [d] \\
\hline Vanadium, total & $3 / 35$ & 0.0023 & 0.0020 & 0.0021 & $d$ & [d] \\
\hline Zinc, total & $28 / 35$ & 0.66 & 0.0050 & 0.14 & 5 & $0[3]$ \\
\hline \multicolumn{7}{|c|}{ Radionuclides, unfiltered $(\mathrm{pCi} / \mathrm{L})^{e}$} \\
\hline${ }^{50} \mathrm{Co}$ & $2 / 35$ & $4.3^{*}$ & $2.3^{*}$ & 3.3 & 200 & $0[4]$ \\
\hline${ }^{137} \mathrm{Cs}$ & $2 / 35$ & $4.3^{*}$ & $3.5^{*}$ & 3.9 & 120 & $0[4]$ \\
\hline Gross alpha & $20 / 35$ & $18^{*}$ & $1.2^{*}$ & 3.3 & 15 & $1[2]$ \\
\hline Gross beta & $27 / 35$ & $17^{*}$ & $1.8^{*}$ & 6.3 & 50 & $0[2]$ \\
\hline${ }^{3} \mathrm{H}$ & $1 / 35$ & $1,200^{*}$ & $1,200^{*}$ & 1,200 & 20,000 & $0[2]$ \\
\hline${ }^{99} \mathrm{TC}$ & $9 / 35$ & $12^{*}$ & $4.1 *$ & 6.4 & 4,000 & $0[4]$ \\
\hline Total rad Sr & $10 / 35$ & $2.7^{*}$ & $1.4^{*}$ & 2.0 & 8 & $0[2]$ \\
\hline \multicolumn{7}{|c|}{ Volatile organics, unfiltered ( $\mu \mathrm{g} / \mathrm{L})$} \\
\hline Bromodichloromethane & $1 / 35$ & 5.6 & 5.6 & 5.6 & 100 & $0[2]$ \\
\hline Chloroform & $3 / 35$ & 13 & B6.0 & -10 & 100 & $0[2]$ \\
\hline
\end{tabular}

"Prefix " $\mathrm{B}$ " indicates the compound was found in the laboratory blank.

${ }^{b} \mathrm{~A}$ tilde $(\sim)$ indicates that estimated values and/or detection limits were used in the calculation of the average.

If a reference limit exists, the source is coded as: (1) Rules of Tennessee Deparment of Environment and Conservation, Division of Water Pollution Control, Chapter 1200-4-3, General Water Quality Criteria, Domestic Water Supply, as amended. (2) 40 CFR Part 141-National Primary Drinking Water Regulations, Subparts B and G, as amended. (3) 40 CFR Parr 143-National Secondary Drinking

Water Regulations, as amended. (4) DOE Order 5400.5, Chapter III, Derived Concentration Guides for Air and Water.

Not applicable.

Tndividual radionuclide concentrations significantly greater than zero are identified by an *. 
Table 5.38. Concentration of radioactivity In soll samples taken in the vicinity of the K-25 Site (pCl/g)

\begin{tabular}{|c|c|c|c|c|c|c|c|c|c|c|c|}
\hline Parameter & SI & $\$ 2$ & S3 & \$4 & S5 & \$6 & 57 & $\operatorname{Max}$ & Min & Median & Avernge \\
\hline Alpha & $2.14 \mathrm{E}+00$ & $4.13 \mathrm{E}+00$ & $3.41 \mathrm{E}-01$ & $1.74 \mathrm{E}+00$ & $3.98 \mathrm{E}+00$ & $1.20 \mathrm{E}+00$ & $2.69 \mathrm{E}+00$ & $4.13 E+00$ & $3.41 \mathrm{E}-01$ & $2.14 \mathrm{E}+00$ & $2.32 \mathrm{E}+100$ \\
\hline Beta & $5.28 \mathrm{E}+00$ & $-3.52 E+00$ & $-6.20 \mathrm{E}+00$ & $-4.93 \mathrm{E}+00$ & $-2.67 \mathrm{E}+00$ & $-5.15 E+00$ & $-3.42 \mathrm{E}+00$ & $5.28 \mathrm{E}+00$ & $-6.20 \mathrm{E}+00$ & $-3.52 \mathrm{E}+00$ & $-2.94 \mathrm{E}+00$ \\
\hline${ }^{137} \mathrm{Cs}$ & $1.67 \mathrm{E}+00$ & $-9.62 E+00$ & $1.08 \mathrm{E}+00$ & $-3.79 E+00$ & $3.40 \mathrm{E}+00$ & $-1.63 \mathrm{E}+00$ & $3.63 E+00$ & $3.63 \mathrm{E}+\infty 0$ & $-9.62 \mathrm{E}+00$ & $1.08 \mathrm{E}+00$ & $-7.51 \mathrm{E}-01$ \\
\hline${ }^{237} \mathrm{~Np}$ & $6.07 \mathrm{E}-01$ & $1.64 \mathrm{E}+01$ & $0.00 \mathrm{E}+00$ & $0.00 \mathrm{E}+00$ & $6.21 \mathrm{E}-01$ & $1.56 \mathrm{E}+00$ & $0.00 \mathrm{E}+00$ & $1.64 \mathrm{E}+01$ & $0.00 \mathrm{E}+00$ & $6.07 E_{-01}$ & $2.74 \mathrm{E}+00$ \\
\hline${ }^{238} \mathrm{Pu}$ & $0.00 \mathrm{E}+00$ & $-1.23 \mathrm{E}-01$ & $-1.23 \mathrm{E}-01$ & $1.40 \mathrm{E}-01$ & $2.61 \mathrm{E}-01$ & $-1.05 \mathrm{E}-01$ & $-1.21 \mathrm{E}-01$ & $2.61 \mathrm{E}-01$ & $-1.23 \mathrm{E}-01$ & $-1.05 \mathrm{E}-01$ & $-1.01 \mathrm{E}-02$ \\
\hline${ }^{239} \mathrm{Pu}$ & $1.29 \mathrm{E}-01$ & $-1.23 \mathrm{E}-01$ & $0.00 \mathrm{E}+00$ & $4.20 \mathrm{E}-01$ & $1.31 \mathrm{E}-01$ & $-1.05 \mathrm{E}-01$ & $1.21 \mathrm{E}-01$ & $4.20 \mathrm{E}-01$ & $-1.23 \mathrm{E}-01$ & $1.21 \mathrm{E}-01$ & $8.19 \mathrm{E}-02$ \\
\hline${ }^{23+m} \mathrm{~Pa}$ & $b$ & $b$ & $b$ & $b$ & $b$ & $1.06 E+03$ & $b$ & $1.06 \mathrm{E}+03$ & $0.00 E+00$ & $0.00 \mathrm{E}+00$ & $1.51 E+02$ \\
\hline${ }^{99} \mathrm{Tc}$ & $-3.27 \mathrm{E}-02$ & $4.80 \mathrm{E}+00$ & $-1.74 \mathrm{E}+00$ & $-4.70 \mathrm{E}-01$ & $4.86 \mathrm{E}+00$ & $8.16 \mathrm{E}+00$ & $4.49 \mathrm{E}+00$ & $8.16 \mathrm{E}+00$ & $-1.74 E+00$ & $4.49 \mathrm{E}+00$ & $2.87 \mathrm{E}+() 0$ \\
\hline${ }^{234} \mathrm{Th}$ & $b$ & $b$ & $7.62 \mathrm{E}+01$ & $b$ & $b$ & $b$ & $b$ & $7.62 \mathrm{E}+01$ & $0.00 \mathrm{E}+00$ & $0.00 \mathrm{E}+00$ & $1.09 \mathrm{E}+01$ \\
\hline${ }^{234} \mathrm{U}$ & $-2.45 \mathrm{E}-01$ & $1.93 \mathrm{E}+01$ & $b$ & $2.33 \mathrm{E}-01$ & $2.24 \mathrm{E}-01$ & $8.84 \mathrm{E}-01$ & $0.00 \mathrm{E}+00$ & $1.93 \mathrm{E}+01$ & $-2.45 \mathrm{E}-01$ & $2.24 \mathrm{E}-01$ & $2.91 \mathrm{E}+(\mathrm{X})$ \\
\hline${ }^{233} \mathrm{U}$ & $2.01 \mathrm{E}+\infty 0$ & $1.70 \mathrm{E}+00$ & $7.62 \mathrm{E}+01$ & $-9.00 \mathrm{E}-01$ & $5.00 \mathrm{E}+00$ & $-3.43 \mathrm{E}-01$ & $8.60 \mathrm{E}-01$ & $7.62 \mathrm{E}+01$ & $-9.00 \mathrm{E}-01$ & $1.70 \mathrm{E}+00$ & $1.21 \mathrm{E}+(01$ \\
\hline${ }^{238} \mathrm{U}$ & $2.45 \mathrm{E}-01$ & $1.78 \mathrm{E}+01$ & $2.08 \mathrm{E}-01$ & $2.33 \mathrm{E}-01$ & $1.12 \mathrm{E}+00$ & $5.30 \mathrm{E}-01$ & $1.94 \mathrm{E}-01$ & $1.78 \mathrm{E}+01$ & $1.94 \mathrm{E}-01$ & $2.45 \mathrm{E}-01$ & $2.90 \mathrm{E}+00$ \\
\hline${ }^{106} \mathrm{Ru}$ & $b$ & $b$ & $b$ & $2.30 \mathrm{E}+01$ & $b$ & $3.11 \mathrm{E}+01$ & $b$ & $3.11 E+01$ & $0.00 \mathrm{E}+00$ & $0.00 \mathrm{E}+00$ & $1.17 E+01$ \\
\hline${ }^{40} \mathrm{~K}$ & $6.74 E+01$ & $b$ & $b$ & $b$ & $b$ & $b$ & $4.53 \mathrm{E}+01$ & $6.74 \mathrm{E}+01$ & $0.00 \mathrm{E}+00$ & $0.00 \mathrm{E}+00$ & $1.61 \mathrm{E}+01$ \\
\hline
\end{tabular}

${ }^{a} 1 \mathrm{pCi} / \mathrm{g}=3.7 \mathrm{E}-02 \mathrm{~Bq} / \mathrm{g}$.

${ }^{b}$ Not detected. 
BCK 0.6

BCK 9.4

CRK 16

CRK 32

CRK 80

CRK 84

EFK 5.4

EFK 23.4

HC

MEK 2.1

MIK 0.1

MIK 1.4

PCK 2.2

PCK 22

WCK 1.0

WCK 6.8
Bear Creek downstream from all DOE inputs

Bear Creek downstream from Y-12 Plant burial grounds

Clinch River downstream from all DOE inputs

Clinch River downstream from ORNL

Melton Hill Reservoir-Oak Ridge Marina

Melton Hill Reservoir above all DOE inputs-Anderson County Filtration Plant

East Fork Poplar Creek downstream from floodplain

East Fork Poplar Creek downstream from the Y-12 Plant

Hinds Creek (reference site for East Fork Poplar Creek)

Melton Branch upstream from ORNL

Mitchell Branch downstream from the K-25 Site

Mitchell Branch upstream from the K-25 Site

Poplar Creek downstream from the K-25 Site

Poplar Creek upstream from the K-25 Site and East Fork Poplar Creek

White Oak Lake at White Oak Dam

White Oak Creek upstream from ORNL 
Collection frequency: Arnually

Sample Type: Grab

Analysis frequency: Annually

\begin{tabular}{l} 
Metals/nnor \\
\hline Aluminum \\
Antimony \\
Arsenic \\
Barium \\
Beryllium \\
Cadmium \\
Calcium \\
Chromium \\
Cobalt \\
Copper \\
lron \\
Lead \\
Magnesium \\
Manganese \\
Mercury \\
Nickel \\
Potassium \\
Selenium \\
Silver \\
Sodium \\
Thallium \\
Uranium \\
Vanadium \\
Zinc
\end{tabular}

Zinc

\section{Pesticides/PCBs \\ Aldrin \\ Alpha-BHC \\ Beta-BHC}

Delta-BHC

Gamma-BHC (Lindane)

Chlordane (technical)

4,4'-DDD

4,4'-DDE

4,4'-DDT

Dieldrin

Endosulfan I

Endosulfan II

Endosulfan sulfate

Endrin

Endrin aldehyde

Heptachlor

Heptachlor epoxide

Methoxychlor

Toxaphene

PCB-1016

PCB-1221

PCB-1232

PCB-1242

PCB-i248

PCB-1254

PCB-1260

2,4-D

2,4,5-TP (Silvex)

Semi-Volatile Organics 1.2-Dichlorobenzene

1,2,4-Trichlorobenzene

1,3-Dichlorobenzene

1,4-Dichlorobenzene

2-Chloronaphthalene

2-Chlorophenol

2-Methyinaphthalene

2-Methyiphenol

2-Nitroaniline

2-Nitrophenol

2,4-Dimethyphenol

2,4-Dinitrophenol

2,4-Dinitrotoluene

2,4,5-Trichlorophenol

2,4,6-Trichiorophenol

2,6-Dinitrotoluene

3-Nitroaniline

3,3'-Dichlorobenzidine

4-Bromophenyl-phenylether

4-Chloroaniline

4-Chlorophenyl-phenylether
4-Methylphenol

4-Nitroaniline

4-Nitrophenol

4-Chloro-3-methylphenol

4,6-Dinitro-2-methylphenol

Acenaphthene

Acenaphthylene

Anthracene

Benzo(a)anthracene

Benzo(a)pyrene

Benzo(b)fluoranthene

Benzo(g,h,i)perylene

Benzo(k)fluoranthene

bis(2-Chloroethyl)ether

bis (2-Chloroisopropyl)ether

bis(2-Ethylhexyl)phthalate

Butylbenzylphthalate

Carbazole

Chrysene

Di-n-octylphthalate

Dibenz(a,h)anthracene
Radionuclides

Am-241

Cm-244

Gross alpha

Gross beta

Co-60

Cs 137

$\mathrm{K}-40$

Np. 237

Pu-238

Pu-239

Sr-90

Te-99

Th-230

Th-232

U-234

U.235

U-238
Dibenzofuran

Diethylphthalate

Dimethylphthalate

Fiuoranthene

Fluorene

Hexachiorobenzene

Hexachiorobutadiene

Hexachlorocyclopentadiene

Hexachloroethane

Indeno(1,2,3-cd)pyrene

Isoporone

$\mathrm{N}$-nitroso-di-n-propylamine

$\mathrm{N}$-Nitrosodiphenylamine

Naphthalene

Nitrobenzene

Pentachlorophenol

Phenanthrene

Phenol

Pyrene 
Table 5.41. 1993 concentrations at EMP sediment locations ${ }^{a}$

\begin{tabular}{ll}
\hline Parameter & Concentration $^{b}$ \\
\hline
\end{tabular}

Bear Creek downstream from all DOE inputs (BCK 0.6)

Metais $(\mathrm{g} / \mathrm{kg})$

Aluminum, total

Arsenic, total

8.7

Barium, total

0.011

Beryllium, total

0.050

Calcium, total

0.00060

Chromium, total

1.6

Cobalt, total

0.015

Copper, total

Iron, total

0.0069

0.0064

Lead, total

Magnesium, total

Manganese, total

12

Mercury, total

Nickel, total

0.010

Potassium, total

Selenium, total

1.4

0.42

0.000069

0.010

Sodium, total

1.6

0.0015

0.024

Uranium, total

0.0058

Vanadium, total

0.018

Zinc, total

0.021

Radionuclides ( $\mathrm{pCi} / \mathrm{g}$ )

Gross alpha

$5.4^{*}$

Gross beta

$8.4^{*}$

$\mathrm{K}-40$

$\mathrm{Np}-237$

$13^{*}$

Tc-99

$0.020^{*}$

Th-230

$2.0^{*}$

Th-232

$0.073 *$

U-234

U. 235

$0.14^{*}$

$1.3^{*}$

$0.12^{*}$

U-238

$2.1^{*}$

Bear Creek downstream from Y.12 Plant burial grounds (BCX 9.4)

Base Neutral/Acid Extractable Organic ( $\mu \mathrm{g} / \mathrm{kg}$ )

Diethyl phthalate

$\mathrm{J} 180$

Metals $(\mathrm{g} / \mathrm{kg})$

Aluminum, total

7.0

Arsenic, total

0.014

Barium, total

0.087

Beryllium, total

0.00059

Cadmium, total

0.00049

Calcium, total

2.6

Chromium, total

0.013

Cobalt, total

0.012

Copper, total

0.0070

Iron, total 
Table 5.41 (continued)

\begin{tabular}{ll}
\hline Parameter & Concenrration \\
\hline & \\
Lead, total & 0.015 \\
Magnesium, total & 0.80 \\
Manganese, total & 1.2 \\
Mercury, total & 0.00026 \\
Nickel, total & 0.014 \\
Potassium, total & 0.82 \\
Selenium, total & 0.0018 \\
Sodium, total & 0.026 \\
Uranium, total & 0.0089 \\
Vanadium, total & 0.015 \\
Zinc, total & 0.024 \\
PCBs ( $\mu$ gg/kg) & \\
Aroclor-1254 & \\
Aroclor-1260 & $\mathrm{J13}$ \\
Radionuclides (pCi/g) & $\mathrm{J} 5.0$ \\
Cs-137 & \\
Gross alpha & \\
Gross beta & \\
K-40 & $0.11^{*}$ \\
Np-237 & $4.6^{*}$ \\
Tc-99 & $9.5^{*}$ \\
Th-230 & $6.8^{*}$ \\
Th-232 & $0.032^{*}$ \\
U-234 & $1.0^{*}$ \\
U-235 & $0.089^{*}$ \\
U-238 & $0.19^{*}$ \\
& $1.9^{*}$ \\
& $0.15^{*}$ \\
& $2.5^{*}$ \\
&
\end{tabular}

Clinch River downstream from all DOE inputs (CRK 16)

Metals $(\mathrm{g} / \mathrm{kg})$

Aluminum, total

Barium, total

Beryllium, total

Calcium, total

0.090

Chromium, total

0.00056

Cobalt, total

0.95

Copper, total

0.017

0.0087

Iron, total

0.0095

Lead, total

18

Magnesium, total

0.013

Manganese, total

1.2

Mercury, total

Nickel, total

Potassium, total

0.88

0.000090

0.011

Selenium, total

1.4

Sodium, tota?

Uranium, total

Vanadium, total

0.0011

0.037

0.00076

Zinc, total

0.027

0.038 
Table 5.41 (continued)

\begin{tabular}{ll}
\hline Parameter & Concentration $^{b}$ \\
\hline & \\
Radionuclides (pCi/g) & \\
Am-241 & $0.19^{*}$ \\
Cs-137 & $0.043^{*}$ \\
Gross aipha & $3.2^{*}$ \\
Gross beta & $4.1^{*}$ \\
K-40 & $7.3^{*}$ \\
Np-237 & $0.0062^{*}$ \\
Tc-99 & $0.092^{*}$ \\
Th-230 & $0.17^{*}$ \\
Th-232 & $0.22^{*}$ \\
U-234 & $0.35^{*}$ \\
U-235 & $0.022^{*}$ \\
U-238 & $0.12^{*}$
\end{tabular}

Clinch River downstream from ORNL (CRK 32)

Metals ( $g / \mathrm{kg}$ )

Aluminum, total

23

Arsenic, total

0.0070

Barium, tocal

0.094

Beryllium, total

0.00061

Calcium, total

0.38

Chromium, total

0.017

Cobalt, total

0.010

Copper, total

0.011

Iron, total

Lead, total

18

Magnesium, total

0.026

Manganese, total

1.4

Mercury, total

Nickel, total

Potassium, total

1.9

0.000066

0.014

Selenium, total

1.6

Sodium, total

0.0016

Uranium, total

0.053

Vanadium, total

0.00072

0.029

Zinc, total

0.046

Radionuclides $(\mathrm{pCi} / \mathrm{g})^{c}$

Am-241

Co-60

Cs-137

$0.043^{*}$

Gross alpha

$\mathrm{K}-40$

Pu-238

Pu-239

Sr-90

Tc-99

$1.4 *$

$3.5^{*}$

$16^{*}$

Th-230

Th-232

U-234

$0.0024^{*}$

$0.011^{*}$

$0.18^{*}$

$0.089^{*}$

$0.12 *$

$0.38 *$

$0.21 *$

U-235

$0.0078^{*}$

U-238

$0.15^{*}$ 
Table 5.41 (continued)

F arameter

Concentration:

Melton Hill Reservair-Oak Ridge Marina (CRK 80)

Metals $(g / k g)$

Aluminum, total

Barium, total

Beryllium, total

Calcium, total

Chromium, total

Cobalt, total

Copper, total

Iron, total

Lead, total

Magnesium, total

Manganese, total

Mercury, total

Nickel, total

Potassium, total

Selenium, total

Sodium, total

Uranium, total

Vanadium, total

Zinc, total

24

0.090

0.0011

4.5

0.021

0.0073

0.010

22

0.013

4.0

0.85

0.000051

0.015

3.9

0.0026

0.095

0.00041

0.028

0.043

Radionuclides ( $\mathrm{pCi} / \mathrm{g})^{c}$

Gross alpha

Gross beta

K.40

Tc-99

$4.3^{*}$

$6.2 *$

$24 *$

$0.12 *$

$0.073 *$

$0.17 *$

$0.14^{*}$

$0.035^{*}$

$0.12 *$

Melton Hill Reservoir above all DOE inputs (CRK 84)

Metals (g/kg)

Aluminum, total

17

Arsenic, total

Barium, total

0.14

Beryllium, total

0.0012

Calcium, total

2.5

Chromium, total

0.018

Cobalt, total

0.011

Copper, total

0.014

Iron, total

21

Lead, total

0.016

Magnesium, total

2.1

Manganese, total

Mercury, total

1.0

Nickel, total

0.000055

0.019

Potassium, total

1.9

Selenium, total

0.0032 
Table 5.41 (continued)

\begin{tabular}{lc}
\hline Parameter & Concentration $^{b}$ \\
\hline & \\
Sodium, total & 0.039 \\
Uranium, total & 0.00054 \\
Vanadium, total & 0.025 \\
Zinc, total & 0.061 \\
& \\
Radionuclides (pCi/g) & \\
Gross alpha & \\
Gross beta & $3.5^{*}$ \\
K-40 & $4.9^{*}$ \\
Th-230 & $12^{*}$ \\
Th-232 & $0.12^{*}$ \\
U-234 & $0.21^{*}$ \\
U-238 & $0.30^{*}$ \\
\end{tabular}

East Fork Poplar Creek downstream from floodplain (EFK 5.4)

Metals ( $/ \mathrm{kg}$ )

Aluminum, total

7.8

Barium, total

Beryllium, total

0.052

Calcium, total

0.00037

Chromium, total

2.5

Cobalt, total

0.014

Copper, total

0.0048

Iron, total

0.016

Lead, total

7.6

Magnesium, total

0.013

Manganese, total

1.1

Mercury, total

0.35

Nickel, total

0.010

Potassium, total

0.0095

Selenium, total

Silver, total

1.1

Sodium, total

0.0017

0.00068

0.049

Uranium, total

0.0031

Vanadium, total

0.011

Zinc, total

0.070

PCBs $(\mu \mathrm{g} / \mathrm{kg})$

Aroclor-1254

$\mathrm{J} 24$

Aroclor-1260

$\mathrm{J} 22$

Radionuclides $(\mathrm{pCi} / \mathrm{g})^{c}$

Co-60

$0.043^{*}$

Cs-137

$0.38 *$

Gross alpha

$3.8^{*}$

Gross beta

$5.4^{*}$

$\mathrm{K}-40$

$5.7 *$

Tc-99

$0.43^{*}$

Th-230

Th-232

0.49 *

U-234

0.12 *

U-235

$1.2^{*}$

U.238

$0.095 *$

$1.1^{*}$ 
Table 5.41 (continued)

Parameter

Concentration

East Fork Poplar Creek downstream from the Y.12 Plant (EFK 23.4)

Metals $(g / k g)$

Aluminum, total

5.8

Arsenic, total

0.0078

Barium, total

0.050

Beryliium, total

0.00031

Cadmium, total

0.0015

Calcium, total

6.5

Chromium, total

0.014

Cobalt, total

0.0050

Copper, total

0.040

Iron, total

7.9

Lead, total

0.014

Magnesium, total

1.5

Manganese, total

0.31

Mercury, total

0.061

Nickel, total

0.015

Potassium, total

0.90

Silver, total

0.00056

Sodium, total

0.030

Uranium, total

0.0081

Vanadium, total

0.0088

Zinc, total

0.10

Pesticides $(\mu \mathrm{g} / \mathrm{kg})$

Gamma-BHC (Lindane)

J0.81

PCBs $(\mu \mathrm{g} / \mathrm{kg})$

Aroclor-1260

330

Radionuclides (pCi/g)

Am-241

Cs-137

Gross alpha

Gross beta

K-40

Np-237

Pu-238

Pu-239

Tc-99

Th-230

Th-232

U-234

U-235

U-238

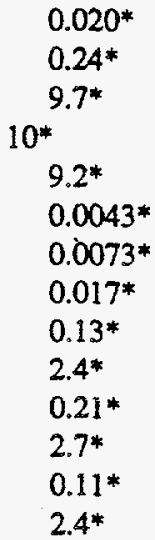

Hinds Creek (reference site for East Fork Poplar Creek) (HC)

Metals (g/kg)

Aluminum, total

11

Barium, total

0.074

Beryllium, total

0.00053 
Table 5.41 (continued)

\begin{tabular}{|c|c|}
\hline Parameter & Concentration ${ }^{b}$ \\
\hline Calcium, total & 4.1 \\
\hline Chromium, total & 0.013 \\
\hline Cobalt, total & 0.0073 \\
\hline Copper, total & 0.0080 \\
\hline Iron, total & 13 \\
\hline Lead, total & 0.011 \\
\hline Magnesium, total & 1.7 \\
\hline Manganese, total & 0.85 \\
\hline Mercury, total & 0.000017 \\
\hline Nickel, total & 0.0094 \\
\hline Potassium, total & 1.5 \\
\hline Selenium, total & 0.0016 \\
\hline Sodium, total & 0.030 \\
\hline Uranium, total & 0.00030 \\
\hline Vanadium, total & 0.017 \\
\hline Zinc, total & 0.039 \\
\hline \multicolumn{2}{|c|}{ Radionuclides $(\mathrm{pCi} / \mathrm{g})^{c}$} \\
\hline Am-241 & $0.022 *$ \\
\hline Cs- 137 & $0.084^{*}$ \\
\hline Gross alpha & $3.0^{*}$ \\
\hline Gross beta & $4.6^{*}$ \\
\hline$K-40$ & $8.9^{*}$ \\
\hline Th-230 & $0.10^{*}$ \\
\hline Th-232 & $0.16^{*}$ \\
\hline U-234 & $0.18^{*}$ \\
\hline U-238 & $0.078^{*}$ \\
\hline
\end{tabular}

Melton Branch upstream from ORNL (MEK 2.I)

Metals (g/kg)

Aluminum, total

9.0

Arsenic, total

0.0040

Barium, total

0.079

Beryllium, total

Calcium, total

0.00063

Chromium, total

1.8

Cobalt, total

0.015

Copper, total

0.0080

Iron, total

0.0071

Lead, total

13

Magnesium, total

0.011

Manganese, total

1.3

Mercury, total

0.40

Nickel, total

Potassium, total

0.000058

Sodium, total

0.013

Uranium, total

1.3

Vanadium, total

0.024

0.011

Zinc, total

0.016

0.026 
Tabie 5.41 (continued)

\begin{tabular}{ll}
\hline Parameter & Concentration \\
\hline Radionuclides $(\mathrm{pC} / \mathrm{g})^{c}$ & \\
Cm-244 & \\
Cs-137 & $0.0038^{*}$ \\
Gross alpha & $0.15^{*}$ \\
K-40 & $3.0^{*}$ \\
Sr-90 & $7.0^{*}$ \\
Th-230 & $0.25^{*}$ \\
Th-232 & $0.038^{*}$ \\
U-234 & $0.17^{*}$ \\
U-235 & $0.092^{*}$ \\
U-238 & $0.0078^{*}$
\end{tabular}

Mitchell Branch downstream from the K-25 Site (MIK 0.I)

\section{Metals $(g / \mathrm{kg})$}

Aluminum, total

Arsenic, total

Barium, total

Beryllium, total

Calcium, total

Chromium, total

Cobalt, total

Copper, total

Iron, total

Lead, total

Magnesium, total

Manganese, total

Mercury, total

Nickel, total

Potassium, total

Selenium, total

Sodium, total

Uranium, total

Vanadium, total

Zinc, total

$$
\begin{aligned}
& 8.5 \\
& 0.031 \\
& 0.061 \\
& 0.00045 \\
& 8.6 \\
& 0.027 \\
& 0.0057 \\
& 0.090 \\
& 14 \\
& 0.029 \\
& 2.2 \\
& 0.65 \\
& 0.0015 \\
& 0.15 \\
& 1.0 \\
& 0.0017 \\
& 0.046 \\
& 0.00053 \\
& 0.014 \\
& 0.12
\end{aligned}
$$

\section{PCBs $(\mu \mathrm{g} / \mathrm{kg})$ \\ Aroclor-1254 \\ Aroclor-1260}

$\mathrm{J} 180$

Radionuclides $(\mathrm{pCi} / \mathrm{g})^{c}$

\section{Am-241}

Cs-137

Gross alpha

Gross beta

K-40

Np-237

Pu-239

Tc-99

Th-230

Th-232

U-234

U-235

U-238

$0.026^{*}$
$0.30^{*}$
$43^{*}$
$150^{*}$
$6.2^{*}$
$0.17^{*}$
$0.20^{*}$
$54^{*}$
$1.2^{*}$
$1.6^{*}$
$24^{*}$
$1.1^{*}$
$14^{*}$


Table 5.41 (continued)

\begin{tabular}{lc}
\hline Parameter & Concentration $^{b}$ \\
\hline
\end{tabular}

Mitchell Branch upstream from the K.25 Site (MIK I.4)

Metals $(\mathrm{g} / \mathrm{kg})$

Aluminum, total

Arsenic, total

Barium, total

Beryllium, total

Calcium, total

Chromium, total

Cobalt, total

Copper, total

Iron, total

Lead, total

Magnesium, total

Manganese, total

Mercury, total

Nickel, total

Potassium, total

Selenium, total

Sodium, total

Uranium, total

Vanadium, total

12

Zinc, total

0.15

0.00074

1.6

0.022

0.018

0.014

26

0.0091

3.3

2.1

0.000034

0.056

1.7

0.0033

0.045

0.00051

0.021

0.037

Radionuclides ( $\mathrm{pCi} / \mathrm{g})^{c}$

Am-241

$0.0027 *$

Cs-137

Gross alpha

$0.032^{*}$

Gross beta

$4.1^{*}$

$\mathrm{K}-40$

$7.3^{*}$

Th-230

$15^{*}$

Th-232

$0.21 *$

U-234

U-235

$0.049^{*}$

$0.21^{*}$

$0.0049 *$

U-238

$0.13^{*}$

Poplar Creek downstream from the $K \cdot 25$ Site (PCK 2.2)

Metals $(\mathrm{g} / \mathrm{kg})$

Aluminum, total

Arsenic, total

0.0050

Barium, total

Beryllium, total

0.028

Calcium, total

0.00025

Chromium, total

1.1

Cobalt, total

0.0089

Copper, total

0.0044

Iron, total

0.0097

Lead, total

5.1

Magnesium, total

0.0052

Manganese, total

0.48

Mercury, total

0.14

0.0024

Nickel, total

0.010 
Table 5.41 (continued)

\begin{tabular}{|c|c|}
\hline Paramete: & Concentratiun ${ }^{b}$ \\
\hline Potassium, total & 0.42 \\
\hline Sodium, total & 0.012 \\
\hline Uranium, total & 0.0014 \\
\hline Vanadium, total & 0.0051 \\
\hline Zinc, total & 0.029 \\
\hline \multicolumn{2}{|l|}{ PCBs $(\mu \mathrm{g} / \mathrm{kg})$} \\
\hline Aroclor -1260 & $\mathrm{~J} 16$ \\
\hline \multicolumn{2}{|c|}{ Radionuclides $(\mathrm{pCi} / \mathrm{g})^{c}$} \\
\hline Am-241 & $0.0086^{*}$ \\
\hline $\mathrm{Cm}-244$ & $0.013^{*}$ \\
\hline Cs-137 & $0.15^{*}$ \\
\hline Gross alpha & $1.6^{*}$ \\
\hline Gross beta & $7.6^{*}$ \\
\hline$K-40$ & $4.1^{*}$ \\
\hline $\mathrm{Pu}-239$ & $0.023^{*}$ \\
\hline Tc-99 & $1.0^{*}$ \\
\hline Th-230 & $0.46^{*}$ \\
\hline Th-232 & $0.11^{*}$ \\
\hline U-234 & $0.62^{*}$ \\
\hline $\mathrm{U}=235$ & $0.030^{*}$ \\
\hline U-238 & $0.51^{*}$ \\
\hline \multicolumn{2}{|c|}{ Poplar Creek upstream from the K-25 Site and East Fork (PCK 22) } \\
\hline \multicolumn{2}{|l|}{ Metals $(\mathrm{g} / \mathrm{kg}$ ) } \\
\hline Aluminum, total & 10 \\
\hline Arsenic, total & 0.012 \\
\hline Barium, total & 0.068 \\
\hline Beryllium, total & 0.00076 \\
\hline Calcium, total & 0.86 \\
\hline Chromium, total & 0.011 \\
\hline Cobalt, total & 0.013 \\
\hline Copper, total & 0.011 \\
\hline Iron, total & 12 \\
\hline Lead, total & 0.011 \\
\hline Magnesium, total & 1.1 \\
\hline Manganese, total & 0.50 \\
\hline Mercury, total & 0.000044 \\
\hline Nickel, total & 0.020 \\
\hline Potassium, total & 0.94 \\
\hline Sodium, total & 0.024 \\
\hline Uranium, total & 0.00056 \\
\hline Vanadium, total & 0.015 \\
\hline Zinc, total & 0.054 \\
\hline \multicolumn{2}{|c|}{ Radionuclides $(\mathrm{pCi} / \mathrm{g})^{c}$} \\
\hline Gross alpha & $5.7^{*}$ \\
\hline $\mathrm{K}-40$ & $6.8^{*}$ \\
\hline Sr-90 & $0.11^{*}$ \\
\hline Tc-99 & $0.10^{*}$ \\
\hline Th-230 & $0.11^{*}$ \\
\hline
\end{tabular}


Table 5.41 (continued)

\begin{tabular}{ll}
\hline Parameter & Concentration \\
& \\
\hline & \\
Th-232 & \\
U-234 & $0.19^{*}$ \\
U-235 & $0.17^{*}$ \\
U-238 & $0.0059^{*}$
\end{tabular}

White Oak Lake at White Oak Dam (WCK 1.0)

Metals $(\mathrm{g} / \mathrm{kg})$

Aluminum, total

12

Arsenic, total

0.013

Barium, total

0.045

Beryllium, total

Calcium, total

Chromium, total

0.00049

0.91

Cobalt, total

0.016

Copper, total

0.0087

Iron, total

Lead, total

0.0071

Magnesium, total

12

Manganese, total

0.0085

1.2

0.21

Mercury, total

0.00016

Nickel, total

Potassium, total

0.0095

Sodium, total

1.3

Uranium, total

0.038

Vanadium, total

0.00033

0.016

Zinc, total

0.020

PCBs $(\mu \mathrm{g} / \mathrm{kg})$

Aroclor-1254

J14

Radionuclides $(\mathrm{pCi} / \mathrm{g})^{c}$

$\begin{array}{lc}\text { Am-241 } & 0.026^{*} \\ \text { Cm-244 } & 0.049^{*} \\ \text { Co-60 } & 0.81^{*} \\ \text { Cs-137 } & 35^{*} \\ \text { Gross alpha } & 3.0^{*} \\ \text { Gross beta } & 20^{*} \\ \text { K-40 } & 8.6^{*} \\ \text { Pu-238 } & 0.016^{*} \\ \text { Pu-239 } & 0.032^{*} \\ \text { Sr-90 } & 3.5^{*} \\ \text { Tc-99 } & 0.19^{*} \\ \text { Th-230 } & 0.14^{*} \\ \text { Th-232 } & 0.24^{*} \\ \text { U-234 } & 0.15^{*} \\ \text { U-235 } & 0.0041^{*} \\ \text { U-238 } & 0.068^{*}\end{array}$


Table 5.41 (continued)

\begin{tabular}{|c|c|}
\hline Parameter & Concentration ${ }^{b}$ \\
\hline \multicolumn{2}{|c|}{ White Oak Creek upstream from ORNL (WCK 6.8) } \\
\hline \multicolumn{2}{|l|}{ Metals $(g / k g)$} \\
\hline Aluminum, total & 3.6 \\
\hline Arsenic, total & 0.0036 \\
\hline Barium, total & 0.042 \\
\hline Beryllium, total & 0.00031 \\
\hline Calcium, total & 7.8 \\
\hline Chromium, total & 0.0051 \\
\hline Cobalt, total & 0.0039 \\
\hline Copper, total & 0.0028 \\
\hline Iron, total & 3.7 \\
\hline Lead, total & 0.0068 \\
\hline Magnesium, total & 2.0 \\
\hline Manganese, total & 0.12 \\
\hline Mercury, total & 0.00012 \\
\hline Nickel, total & 0.0043 \\
\hline Potassium, total & 0.34 \\
\hline Sodium, total & 0.010 \\
\hline Thallium, total & 0.0068 \\
\hline Uranium, total & 0.00039 \\
\hline Vanadium, total & 0.0084 \\
\hline Zinc, total & 0.021 \\
\hline \multicolumn{2}{|c|}{ Radionuclides $(\mathrm{pCi} / \mathrm{g})^{c}$} \\
\hline Am-241 & $0.027^{*}$ \\
\hline Cs- 137 & $0.12 *$ \\
\hline Gross alpha & $1.7^{*}$ \\
\hline Gross beta & $2.6^{*}$ \\
\hline$K-40$ & $3.2 *$ \\
\hline Th-230 & $0.10^{*}$ \\
\hline Th-232 & $0.12 *$ \\
\hline U.234 & $0.43^{*}$ \\
\hline U-235 & $0.011 *$ \\
\hline U.238 & $0.16^{*}$ \\
\hline
\end{tabular}

${ }^{a}$ All values were included in the calculations. Only parameters that have detections in one or more samples are listed in the table. The sampling and analysis plan contains a complete list of analyses performed.

${ }^{\circ}$ Prefix " $\mathrm{J}$ " indicates the value was estimated at or below the analytical detection limit by the laboratory.

Individual radionuclide concentrations significantly greater than zero are identified by an *. 
Table 5.42. Concentration of radioactivity in sediment samples taken in the vicinity of the K-25 Site (pCl/g)

\begin{tabular}{lccccccccccc}
\hline Parameter & SS1 & \multicolumn{1}{c}{ SS2 } & \multicolumn{1}{c}{ SS3 } & \multicolumn{1}{c}{ SS4 } & \multicolumn{1}{c}{ SS5 } & SS6 & SS7 & Max & Min & Median & Averagc \\
\hline Alpha & $-8.01 \mathrm{E}-01$ & $-1.64 \mathrm{E}-02$ & $1.04 \mathrm{E}+02$ & $-3.59 \mathrm{E}+00$ & $-3.02 \mathrm{E}+00$ & $-1.10 \mathrm{E}+00$ & $-1.90 \mathrm{E}+00$ & $1.04 \mathrm{E}+02$ & $-3.59 \mathrm{E}+00$ & $-1.10 \mathrm{E}+00$ & $1.34 \mathrm{E}+01$ \\
Beta & $4.25 \mathrm{E}-01$ & $-5.33 \mathrm{E}-01$ & $2.89 \mathrm{E}+02$ & $-1.93 \mathrm{E}+00$ & $-9.43 \mathrm{E}+00$ & $-1.31 \mathrm{E}+00$ & $-3.53 \mathrm{E}+00$ & $2.89 \mathrm{E}+02$ & $-9.43 \mathrm{E}+00$ & $-1.31 \mathrm{E}+00$ & $3.90 \mathrm{E}+01$ \\
${ }^{133} \mathrm{Cs}$ & $5.53 \mathrm{E}+00$ & $3.31 \mathrm{E}+00$ & $-1.61 \mathrm{E}+00$ & $-7.30 \mathrm{E}-01$ & $2.18 \mathrm{E}+00$ & $1.60 \mathrm{E}+00$ & $5.65 \mathrm{E}+00$ & $5.65 \mathrm{E}+00$ & $-1.61 \mathrm{E}+00$ & $2.18 \mathrm{E}+00$ & $2.28 \mathrm{E}+00$ \\
${ }^{237} \mathrm{~Np}$ & $-2.11 \mathrm{E}-01$ & $1.77 \mathrm{E}-01$ & $1.28 \mathrm{E}+00$ & $3.62 \mathrm{E}-01$ & $-1.66 \mathrm{E}-01$ & $-2.28 \mathrm{E}-01$ & $4.17 \mathrm{E}-02$ & $1.28 \mathrm{E}+00$ & $-2.28 \mathrm{E}-01$ & $4.17 \mathrm{E}-02$ & $1.79 \mathrm{E}-01$ \\
${ }^{238} \mathrm{Pu}$ & $-1.89 \mathrm{E}-01$ & $-1.66 \mathrm{E}-01$ & $-4.66 \mathrm{E}-02$ & $-2.01 \mathrm{E}-01$ & $-2.06 \mathrm{E}-01$ & $-2.27 \mathrm{E}-01$ & $-1.56 \mathrm{E}-01$ & $-4.66 \mathrm{E}-02$ & $-2.27 \mathrm{E}-01$ & $-1.89 \mathrm{E}-01$ & $-1.70 \mathrm{E}-01$ \\
${ }^{239} \mathrm{Pu}$ & $-1.86 \mathrm{E}-01$ & $-5.53 \mathrm{E}-02$ & $1.40 \mathrm{E}-01$ & $-1.00 \mathrm{E}-01$ & $-2.06 \mathrm{E}-01$ & $-1.70 \mathrm{E}-01$ & $-5.19 \mathrm{E}-02$ & $1.40 \mathrm{E}-01$ & $-2.06 \mathrm{E}-01$ & $-1.00 \mathrm{E}-01$ & $-8.99 \mathrm{E}-02$ \\
${ }^{234 m} \mathrm{~Pa}$ & $-2.40 \mathrm{E}+02$ & $1.35 \mathrm{E}+03$ & $-5.37 \mathrm{E}+02$ & $2.87 \mathrm{E}+02$ & $5.20 \mathrm{E}+02$ & $6.43 \mathrm{E}+02$ & $7.27 \mathrm{E}+02$ & $1.35 \mathrm{E}+03$ & $-5.37 \mathrm{E}+02$ & $5.20 \mathrm{E}+02$ & $3.93 \mathrm{E}+02$ \\
${ }^{99} \mathrm{Tc}$ & $9.27 \mathrm{E}+00$ & $1.21 \mathrm{E}+00$ & $2.34 \mathrm{E}+02$ & $-1.10 \mathrm{E}+00$ & $5.27 \mathrm{E}+00$ & $3.21 \mathrm{E}+00$ & $4.83 \mathrm{E}+00$ & $2.34 \mathrm{E}+02$ & $-1.10 \mathrm{E}+00$ & $4.83 \mathrm{E}+00$ & $3.67 \mathrm{E}+01$ \\
${ }^{234} \mathrm{Th}$ & $-5.17 \mathrm{E}+00$ & $9.92 \mathrm{E}+00$ & $8.22 \mathrm{E}+01$ & $6.78 \mathrm{E}+00$ & $5.03 \mathrm{E}+00$ & $-2.00 \mathrm{E}+01$ & $2.23 \mathrm{E}+01$ & $8.22 \mathrm{E}+01$ & $-2.00 \mathrm{E}+01$ & $6.78 \mathrm{E}+00$ & $1.44 \mathrm{E}+01$ \\
${ }^{234} \mathrm{U}$ & $-3.14 \mathrm{E}-01$ & $0.00 \mathrm{E}+00$ & $6.52 \mathrm{E}+01$ & $0.00 \mathrm{E}+00$ & $6.24 \mathrm{E}-01$ & $2.18 \mathrm{E}-01$ & $2.33 \mathrm{E}-01$ & $6.52 \mathrm{E}+01$ & $-3.14 \mathrm{E}-01$ & $2.18 \mathrm{E}-01$ & $9.42 \mathrm{E}+00$ \\
${ }^{235} \mathrm{U}$ & $3.11 \mathrm{E}+00$ & $-4.24 \mathrm{E}+00$ & $-8.27 \mathrm{E}-01$ & $2.37 \mathrm{E}+00$ & $-1.41 \mathrm{E}+00$ & $7.23 \mathrm{E}-02$ & $3.07 \mathrm{E}+00$ & $3.11 \mathrm{E}+00$ & $-4.24 \mathrm{E}+00$ & $7.23 \mathrm{E}-02$ & $3.06 \mathrm{E}-01$ \\
${ }^{238} \mathrm{U}$ & $9.42 \mathrm{E}-01$ & $3.35 \mathrm{E}-01$ & $3.96 \mathrm{E}+01$ & $0.00 \mathrm{E}+00$ & $0.00 \mathrm{E}+00$ & $2.18 \mathrm{E}-01$ & $0.00 \mathrm{E}+00$ & $3.96 \mathrm{E}+01$ & $0.00 \mathrm{E}+00$ & $2.18 \mathrm{E}-01$ & $5.87 \mathrm{E}+00$ \\
${ }^{106} \mathrm{Ru}$ & $b$ & $b$ & $2.52 \mathrm{E}+01$ & $b$ & $b$ & $b$ & $b$ & $2.52 \mathrm{E}+01$ & $0.00 \mathrm{E}+00$ & $0.00 \mathrm{E}+00$ & $3.60 \mathrm{E}+00$ \\
\hline
\end{tabular}

${ }^{a} 1 \mathrm{pCi} / \mathrm{g}=3.7 \mathrm{E}-02 \mathrm{~Bq} / \mathrm{g}$.

Not detected. 
Table 5.43. 1993 selected results of sediment analysis at the K-25 Site

\begin{tabular}{lccccccc}
\hline Parameter & SS-1 & SS-2 & SS-3 & SS-4 & SS- 5 & SS-6 & SS-7 \\
\hline Arclor-1254 & $<120$ & $48^{c}$ & 460 & $<170$ & $<120$ & $<130$ & $<210$ \\
Arsenic & 25 & $<4.9$ & 12 & 13 & $<5.0$ & $<4.9$ & 13 \\
Cadmium & 4.1 & 2.9 & 3.6 & 1.3 & 2.4 & 2.6 & 4.9 \\
Chromium & 15 & 16 & 20 & 12 & 8.3 & 12 & 12 \\
Cobalt & 14 & 15 & 4.3 & 6.3 & 9.1 & 9.1 & 8.2 \\
Copper & 16 & 9.2 & 25 & 5.1 & 5.7 & 9.3 & 26 \\
Lead & 84 & 21 & 31 & 23 & 12 & 17 & 40 \\
Magnesium & 1900 & 940 & 510 & 450 & 740 & 710 & 310 \\
Mercury & 0.13 & 0.22 & 0.24 & 0.044 & 0.044 & 0.14 & 0.058 \\
Molybdenum & 4.4 & 1.1 & 2.2 & 1.1 & $<0.99$ & $<0.98$ & 2.1 \\
Nickel & 14 & 12 & 41 & 6 & 8.7 & 9.2 & 12 \\
Phosphorus & 180 & 430 & 330 & 120 & 130 & 230 & 430 \\
Uranium & 4 & 3 & 120 & 2 & 3 & 3 & 9 \\
Vandium & 39 & 17 & 33 & 15 & 12 & 15 & 34 \\
\hline
\end{tabular}

Units are ug/g unless otherwise indicated.

'Units are ug/kg.

Estimated value. 
Table 5.44. 1993 sampling and analysis plan for EMP fish

Location and species:

PCK 2.2

CRK 16

CRK 32

CRK 66

CRK 80

CRK 84
Poplar Creek downstream from the K-25 Site Clinch River downstream from all DOE inputs Clinch River downstream from ORNL

Melton Hill Reservoir above City of Oak Ridge water intake

Melton Hill Reservoir-Oak Ridge Marina

Melton Hill Reservoir above all DOE inputs -Anderson County Filtration Plant
Sunfish

Sunfish, Catfish

Sunfish, Catfish

Sunfisin

Sunfish

Sunfish

Sample type:

Sunfish-Individual and Composite

Catfish-Composite

Collection frequency:

Annual

Analysis frequency:

Annual

Parameters:

Metals

Antimony

Arsenic

Beryllium

Cadmium

Chromium

Copper

Lead

Mercury

Nickel

Selenium

Silver

Thallium

Uranium

Zinc

\begin{tabular}{l}
\multicolumn{1}{c}{ Pesticides } \\
4,4'-DDD \\
$4,4^{\prime}$-DDE \\
$4,4^{\prime}$-DDT \\
Aldrin \\
Alpha-BHC \\
Alpha-Chlordane \\
Beta-BHC \\
Delta-BHC \\
Dieldrin \\
Endosulfan I \\
Endosulfan II \\
Endosulfan sulfate \\
Endrin \\
Endrin ketone \\
Gamma-BHC (Lindane) \\
Gamma-Chlordane \\
Heptachlor \\
Heptachlor epoxide \\
Methoxychlor \\
Toxaphene
\end{tabular}

PCBs

Aroclor-1016

Aroclor-1221

Aroclor-1232

Aroclor- 1242

Aroclor-1248

Aroclor-1254

Aroclor- 1260
Radionuclides

Co-60

Cs- 137

Total rad Sr 
Table 5.45. 1993 concentrations in sunfish tissue"

Concentration

\begin{tabular}{llllll} 
& $N$ det & & & Standard \\
Parameter & $N$ total & $\operatorname{Max}^{b}$ & Min $^{b}$ & ev $^{c}$ & \\
\hline
\end{tabular}

Poplar Creek downstream from the $K-25$ Site ( $P C K$ 2.2)

Metals (mg/kg wet wt)

Arsenic, total

Chromium, total

Copper, total

Lead, total

Mercury, total

Selenium, total

Silver, total

Uranium, total

Zinc, total

$\begin{array}{cl}2 / 6 & 1.3 \\ 6 / 6 & 0.24 \\ 6 / 6 & 0.31 \\ 1 / 6 & 0.69 \\ 6 / 6 & 0.33 \\ 2 / 6 & 0.83 \\ 1 / 6 & 0.051 \\ 1 / 6 & 0.0060 \\ 6 / 6 & 16\end{array}$

Pesticides $(\mu \mathrm{g} / \mathrm{kg}$ wet $w \mathrm{t})$

4,4'-DDE

$1 / 6$

U190

$1 / 6$

U1,900

Aroclor-1254
$<0.45$

0.18

0.17

$<0.45$

0.036

$<0.45$

$<0.045$

$<0.0010$

8.3

$\mathbf{J 1 . 9}$

$\sim 82$

$-820$

$-0.61$

0.21

0.22

$-0.51$

0.16

$-0.54$

$-0.048$

$-0.0018$

11

J28

$$
\begin{aligned}
& 1.4^{*} \\
& 1.6 *
\end{aligned}
$$

$1.2 *$

$0.46^{*}$

1.3*

0.91

0.055

$3 / 3$

$0.016 *$

$0.018 *$

$0.015^{*}$

$0.0055^{*}$
0.14

0.0087

0.022

0.037

0.046

0.061

0.00089

0.00083

1.1

25

250

Clinch River downstream from all DOE inputs (CRK 16)

Metals (mg/kg wet wt)

Arsenic, total

$1 / 6$

$6 / 6$

$6 / 6$

Copper, total

$6 / 6$

$1 / 6$

$1 / 6$

$6 / 6$

$<0.60$

0.25

0.51

0.27

$<0.60$

Uranium, total

0.0010

11

$<0.45$

$\sim 0.50$

0.21

0.24

0.22

$-0.50$

$-0.0010$

9.7

0.023

0.011

0.061

0.026

$<0.43$

$<0.0010$

0.027

8.5

0.42

PCBs $(\mu \mathrm{g} / \mathrm{kg}$ wet $w t)$

Aroclor-1254

$1 / 6$

U670

$\mathrm{J} 38$

$-480$

91

Radionuclides (pCi/g ash wt)

Cs-137

Total rad Sr
$3 / 3$

$3 / 3$
$1.5^{*}$

$1.0 *$
$1.2 *$

0.54 *
$1.4^{*}$

$0.75 *$
0.078

0.13 


\begin{tabular}{|c|c|c|c|c|c|}
\hline Parameter & $\begin{array}{l}\mathrm{N} \text { det } \\
\mathrm{N} \text { total }\end{array}$ & $\operatorname{Max}^{b}$ & $\operatorname{Min}^{b}$ & $A v^{c}$ & $\begin{array}{c}\text { Standard } \\
\text { error }\end{array}$ \\
\hline \multicolumn{6}{|c|}{ Radionuclides (pCi/g wet wt) ${ }^{e}$} \\
\hline Cs-137 & $3 / 3$ & $0.015 *$ & $0.013 *$ & $0.015^{*}$ & 0.00091 \\
\hline Total rad Sr & $3 / 3$ & $0.011^{*}$ & $0.0055^{*}$ & $0.0080^{*}$ & 0.0017 \\
\hline
\end{tabular}

Clinch River downstream from ORNL (CRK 32)

Metals (mg/kg wet wt)

Chromium, total

Copper, total

Mercury, total

Selenium, total

Uranium, total

Zinc, total

$\begin{array}{ll}6 / 6 & 0.27 \\ 6 / 6 & 0.22 \\ 6 / 6 & 0.17 \\ 1 / 6 & 0.69 \\ 2 / 6 & 0.0010 \\ 6 / 6 & 16\end{array}$

Pesticides $(\mu \mathrm{g} / \mathrm{kg}$ wet $w \mathrm{t})$

4,4'-DDE

PCBs $(\mu \mathrm{g} / \mathrm{kg}$ wet $w t)$

Aroclor-1254
$2 / 6$

U140

$1 / 6 \quad \mathrm{U} 1,400$

$11^{*}$

$2.7^{*}$

$0.13 *$

$0.032 *$

$3 / 3$

0.19
0.18
0.016
$<0.45$
$<0.0010$
8.0

$\mathrm{J} 1.9$

J68

$-670$

0.22

0.20

0.12

$\sim 0.51$

$\sim 0.0010$

13

$-55$

170

Radionuclides (pCi/g ash wt)
Cs-137

$3 / 3$

$3 / 3$

\section{$5.4^{*}$}

$1.8^{*}$

8.9*

$2.4^{*}$

Radionuclides (pCi/g wet $\mathrm{wt})^{e}$

Cs-137

Total rad Sr
$0.058^{*}$

ט. $021 *$
$0.10^{*}$

$0.027 *$

Melton Hill Reservoir above City of Oak Ridge water intake (CRK 66)

Metals (mg/kg wet wt)

Chromium, total

Copper, total

Mercury, total

Selenium, total

Uranium, total

Zinc, total

$\begin{array}{lll}6 / 6 & 0.24 & 0.14 \\ 6 / 6 & 0.24 & 0.12 \\ 6 / 6 & 0.047 & 0.022 \\ 6 / 6 & 1.2 & 0.70 \\ 1 / 6 & 0.0037 & <0.0030 \\ 6 / 6 & 11 & 8.9\end{array}$

$1 / 3$

$1 / 3$

Co-60
Total rad Sr

Radionuclides (pCi/g wet $w t)^{e}$

Co-60

Total rad Sr
$1 / 3$

$1 / 3$

\section{$0.24^{*}$}

$0.12 *$

$0.0032^{*}$

$0.0015^{*}$
21

1.8

0.27

0.011

0.0062

0.028

0.038

0

1.1

0.023

0.0031 


\section{Concentration}

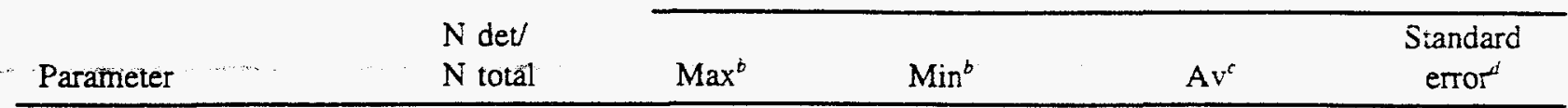

Melton Hill Reservoir - Oak Ridge Marina (CRK 80)

Metals (mg/kg wet wt)

Chromium, total

$6 / 6$

Copper, total

$5 / 6$

0.22

0.16

0.19

0.021

Mercury, total

$6 / 6$

$<0.14$

$\sim 0.18$

0.013

Selenium, total

$5 / 6$

0.075

0.015

0.030

0.0092

Uranium, total

$<0.98$

0.53

$-0.78$

0.071

Zinc, total

$3 / 6$

0.0050

$<0.0030$

$-0.0037$

0.00036

$6 / 6$

13

8.6

11

0.72

Radionuclides ( $\mathrm{pCi} / \mathrm{g}$ ash $w t)^{e}$

Total rad Sr

$1 / 3$

$0.16^{*}$

0.073

$0.12 *$

0.025

Radionuclides (pCi/g wet $\mathrm{wt})^{e}$

Total rad Sr

$1 / 3$

$0.0018^{*}$

0.00081

$0.0014^{*}$

0.00029

Melton Hill Reservoir above all DOE inputs - Anderson County

Filtration Plant (CRK 84)

Metals ( $\mathrm{mg} / \mathrm{kg}$ wet $\mathrm{wt}$ )

Chromium, total

Copper, total

Mercury, total

$6 / 6$

0.18

0.17

0.18

0.0022

Selenium, total

$6 / 6$

0.24

0.14

0.17

0.015

Silver, total

0.083

0.010

0.033

0.012

$6 / 6$

1.0

0.68

0.79

0.049

Uranium, total

$<0.21$

0.063

$3 / 6$

0.014

$<0.0030$

$\sim 0.15$

0.029

Zinc, total

$6 / 6$

17

10

$\sim 0.0054$

0.0018

13

0.95

Radionuclides ( $\mathrm{pCi} / \mathrm{g}$ ash $\mathrm{wt})^{e}$

Cs-137

$1 / 3$

$0.35 *$

0.027

0.14

$0.16^{*}$

$-0.038$

0.050

0.11

Total rad $\mathrm{Sr}$

$1 / 3$

$0.0039 *$

0.00029

$0.0019 *$

$-0.00041$

0.0015

0.0012

Total rad Sr

$1 / 3$

all values were included in the calculations. Only parameters that have detections in one or more samples are listed in the table. The sampling and analysis plan contains a complete list of analyses performed.

"Prefix " $<$ " indicates the value for a parameter (excluding organics) was not quantifiable at the analytical detection limit; " $U$ " indicates the value for an organic parameter was undetected at the analytical detection limit; and " $\mathrm{J}$ " indicates the value was estimated at or below the analytical detection limit by the laboratory.

'A tilde ( $\sim$ ) indicates that estimated values and/or detection limits were used in the calculation.

${ }^{d}$ Standard error of the mean.

Tndividual and average radionuclide concentrations significantly greater than zero are identified by an *. 
Table 5.46. 1993 concentrations in catfish tissue

Metals (mg/kg wet wt)

Arsenic, total

Mercury, total

Thallium, total

1.1

Uranium, total

Pesticides $(\mu g / \mathrm{kg}$ wet $w t)$

4, 4'-DDE

Alpha-Chlordane

$\mathrm{J} 34$

J7. 6

PCBs $(\mu \mathrm{g} / \mathrm{kg}$ wet $w t)$

Aroclor-1254

650

Arocior-1260

780

Radionuclides (pCi/g ash wt) ${ }^{c}$ Cs -137

$3.0 *$

Radionuclides $(p C i / g \text { wet } w t)^{e}$

Cs -137

0.034 *

Metals (mg/kg wet wt)

Mercury, total

0.16

Nickel, total

0.14

zinc, total

3.6

Pesticides ( $\mu \mathrm{g} / \mathrm{kg}$ wet wt)

$4,4^{\prime}-\mathrm{DDD}$

J14

4, 4' -DDE

80

Alpha-Chlordane

587

PCBs ( $\mu \mathrm{g} / \mathrm{kg}$ wet wt)

Aroclor -1254

770

Aroclor -1260

810

Radionuclides (pci/g ash wt) ${ }^{c}$

Cs -137

Total rad $\mathrm{Sr}$

$4.9 *$

$0.81 *$

Radionuclides ( $\mathrm{pci} / \mathrm{g}$ wet $\mathrm{wt})^{c}$

Cs -137

Total rad $\mathrm{Sr}$

$0.048 *$

$0.0080 *$

Anly parameters that have detections in one or more samples are listed in the table. The sampling and analysis plan contains a complete list of analyses performed.

Drefix "J" indicates the value was estimated at or below the analytical detection limit by the laboratory.

Individual radionuclide concentrations significantly greater

than zero are identified by an *. 
Table 6.1. Release point parameters and receptor locations used in the dose calculations

\begin{tabular}{|c|c|c|c|c|c|c|c|}
\hline \multirow{2}{*}{$\begin{array}{l}\text { Source } \\
\text { name }\end{array}$} & \multirow{2}{*}{ Type } & \multirow{2}{*}{$\begin{array}{l}\text { Release } \\
\text { height } \\
\text { (m) }\end{array}$} & \multirow{2}{*}{$\begin{array}{l}\text { Inner } \\
\text { diameter } \\
(\mathrm{m})\end{array}$} & \multirow{2}{*}{$\begin{array}{c}\text { Gas exit } \\
\text { velocity } \\
(\mathrm{m} / \mathrm{s})\end{array}$} & \multirow{2}{*}{$\begin{array}{c}\text { Gas exit } \\
\text { temperature } \\
\left({ }^{\circ} \mathrm{C}\right)\end{array}$} & \multicolumn{2}{|c|}{$\begin{array}{l}\text { Distance }(\mathrm{m}) \text { and direction to } \\
\text { maximally exposed individual }\end{array}$} \\
\hline & & & & & & Plant & ORR \\
\hline \multicolumn{8}{|c|}{ Y.12 Plant } \\
\hline All & Point & 20 & 0 & 0 & Ambient & $1080 \mathrm{NNE}$ & 1080 NNE \\
\hline \multicolumn{8}{|c|}{ ORNL } \\
\hline 2026 & Point & 22.9 & 1.07 & 10.1 & Ambient & $5450 \mathrm{E}$ & $9300 \mathrm{NE}$ \\
\hline 3020 & Point & 61.0 & 1.52 & 5.5 & Ambient & $5450 \mathrm{E}$ & $9300 \mathrm{NE}$ \\
\hline 3039 & Point & 76.2 & 2.44 & 2.0 & Ambient & $5450 \mathrm{E}$ & $9300 \mathrm{NE}$ \\
\hline 7025 & Point & 4.0 & 0.31 & 13.6 & Ambient & $3500 \mathrm{E}$ & 7550 NNE \\
\hline 7512 & Point & 30.5 & 0.91 & 8.4 & Ambient & $4540 \mathrm{ENE}$ & 9640 NNE \\
\hline 7911 & Point & 76.2 & 1.52 & 2.4 & Ambient & 4540 ENE & 9640 NNE \\
\hline 7830 & Point & 4.6 & 0.22 & 8.0 & Ambient & 5810 ENE & $10990 \mathrm{NNE}$ \\
\hline 7877 & Point & 13.9 & 0.51 & 8.6 & Ambient & 5810 ENE & $10990 \mathrm{NNE}$ \\
\hline 2000 & Point & 15.2 & 0.66 & 8.9 & Ambient & $5450 \mathrm{E}$ & $9300 \mathrm{NE}$ \\
\hline 3018 & Point & 61.0 & 4.11 & 0.2 & Ambient & $5450 \mathrm{E}$ & $9300 \mathrm{NE}$ \\
\hline 3074 & Point & 4.0 & 0.26 & 10.2 & Ambient & $5450 \mathrm{E}$ & $9300 \mathrm{NE}$ \\
\hline 3544 & Point & 9.5 & 0.27 & 18.0 & Ambient & $5450 \mathrm{E}$ & $9300 \mathrm{NE}$ \\
\hline 2523 & Point & 7.0 & 0.3 & 7.8 & 57.2 & $5450 \mathrm{E}$ & $9300 \mathrm{NE}$ \\
\hline \multicolumn{8}{|c|}{ K.25 Site } \\
\hline K-1435 & Point & 30.5 & 1.37 & 5.6 & 79.1 & 5180 SWS & $13000 \mathrm{ENE}$ \\
\hline K-1015 & Point & 3.7 & 0 & 0 & Ambient & 4340 WSW & 14000 ENE \\
\hline K-31/K-33 & Point & 25.9 & 0 & 0 & Ambient & & \\
\hline
\end{tabular}


Table 6.2. Calculated radiation doses to maximally exposed off-site individuals from airborne releases during 1993

\begin{tabular}{|c|c|c|}
\hline \multirow[t]{2}{*}{ Plant } & \multicolumn{2}{|c|}{$\begin{array}{l}\text { Total effective dose equivalents } \\
{[\text { mrem (mSv)] }}\end{array}$} \\
\hline & Plant max & ORR max \\
\hline ORNL & $0.1(1 E-3)^{a}$ & $3 E-2(3 E-4)$ \\
\hline K-25 Site & $0.1(1 \mathrm{E}-3)^{b}$ & $4 E-2(4 E-4)$ \\
\hline Y-12 Plant & $1.3(1.3 \mathrm{E}-2)^{c}$ & $1.3(1.3 \mathrm{E}-2)$ \\
\hline Entire ORR & $\mathrm{NA}^{d}$ & $1.4(1.4 \mathrm{E}-2)$ \\
\hline \multicolumn{3}{|c|}{$\begin{array}{l}\text { The maximally exposed individual is located } 5450 \mathrm{~m} \text { ( } 3.4 \text { miles) } \mathrm{E} \\
\text { of the } 3039 \text { stack and } 4540 \mathrm{~m} \text { ( } 2.8 \text { miles) ENE of the } 7911 \text { stack. } \\
\text { 'The maximally exposed individual is located } 5180 \mathrm{~m} \text { ( } 3.2 \text { miles) } \\
\text { WSW of the K-1435 stack. } \\
\text { The maximally exposed individual is located } 1080 \mathrm{~m} \text { ( } 0.7 \text { miles) } \\
\text { NNE of the Y-12 Plant release point. } \\
\text { The maximally exposed individual for the entire ORR is the Y-12 } \\
\text { Plant maximally exposed individual. }\end{array}$} \\
\hline \multicolumn{3}{|c|}{$\begin{array}{l}\text { Table 6.3. Calculated collective EDEs from } \\
\text { airborne releases during } 1993 \\
\end{array}$} \\
\hline \multirow[t]{2}{*}{ Plant } & \multicolumn{2}{|c|}{$\begin{array}{c}\text { Effective } \\
\text { dose equivalents }\end{array}$} \\
\hline & Person-rem ${ }^{a}$ & Person-Sv \\
\hline ORNL & 6 & $6 \mathrm{E}-2$ \\
\hline K-25 Site & 8 & $8 \mathrm{E}-2$ \\
\hline Y-12 Plant & 12 & $1.2 \mathrm{E}-1$ \\
\hline ORR & 26 & $2.6 \mathrm{E}-1$ \\
\hline
\end{tabular}

The collective effective dose equivalents to the 879,546 persons residing within $80 \mathrm{~km}$ ( 50 miles) of the ORR. 
Table 6.4. Potential maximum individual EDEs (mrem)" from use of ott-site waters

\begin{tabular}{|c|c|c|c|c|c|}
\hline Locution & Eating fish & Swimming & Boating & Using shoreline & Totai \\
\hline $\begin{array}{l}\text { CRK 84: Clinch River } \\
\text { above all DOE input }\end{array}$ & 0.03 & $6 E-9$ & $3 \mathrm{E}-9$ & $2 E-4$ & 0.03 \\
\hline $\begin{array}{l}\text { CRK 80: Clinch River at } \\
\text { Oak Ridge Marina }\end{array}$ & 0.02 & $6 E-4$ & $9 E-5$ & 0.2 & 0.2 \\
\hline $\begin{array}{l}\text { CRK 66: Clinch River } \\
\text { above Oak Ridge city } \\
\text { water intake }\end{array}$ & 0.02 & $2 E-4$ & $6 \mathrm{E}-5$ & 0.1 & 0.1 \\
\hline $\begin{array}{l}\text { CRK 58: Clinch River at } \\
\text { Knox County water } \\
\text { intake }\end{array}$ & $b$ & $3 E-4$ & $2 E-4$ & 0.2 & 0.2 \\
\hline $\begin{array}{l}\text { CRK 32: Clinch River } \\
\text { below ORNL }\end{array}$ & 0.2 & $8 E-5$ & $2 \mathrm{E}-7$ & $2 \mathrm{E}-3$ & 0.2 \\
\hline $\begin{array}{l}\text { CRK 23: Clinch River at } \\
\text { K-25 Site water intake }\end{array}$ & $b$ & $9 \mathrm{E}-5$ & $4 \mathrm{E}-5$ & 0.2 & 0.2 \\
\hline $\begin{array}{l}\text { CRK 16: Clinch River } \\
\text { below all DOE inpurs }\end{array}$ & 0.04 & $2 \mathrm{E}-5$ & $7 \mathrm{E}-6$ & $5 E-2$ & 0.1 \\
\hline $\begin{array}{l}\text { TRK 915: Tennessee } \\
\text { River at Kingston } \\
\text { Water Plant intake }\end{array}$ & $b$ & $5 E-5$ & $8 \mathrm{E}-6$ & $6 E-2$ & 0.06 \\
\hline $\begin{array}{l}\text { PCK 22: Poplar Creek } \\
\text { above union with East } \\
\text { Fork Poplar Creek }\end{array}$ & $b$ & $2 \mathrm{E}-4$ & $7 \mathrm{E}-5$ & 0.1 & 0.1 \\
\hline $\begin{array}{l}\text { PCK 2.2: Poplar Creek } \\
\text { below the K-25 Site }\end{array}$ & 0.06 & $3 \mathrm{E}-5$ & $7 \mathrm{E}-8$ & $1 E-3$ & 0.06 \\
\hline
\end{tabular}

'To convert mrem to $\mathrm{mSv}$, divide the given values by 100 .

${ }^{b}$ Not sampled. 
Table 6.5. Average EDEs from ingesting vegetables grown at ORR ambient air monitoring stations

\begin{tabular}{|c|c|c|}
\hline \multirow{2}{*}{ Vegetable } & \multicolumn{2}{|c|}{$\begin{array}{c}\text { EDE } \\
{[\mathrm{mrem}(\mathrm{mSv})]}\end{array}$} \\
\hline & $\begin{array}{l}\text { All reported } \\
\text { radionuclides }\end{array}$ & Excluding ${ }^{40} \mathrm{~K}$ \\
\hline Tomatoes & $1 E-2(1 E-4)$ & $1 E-2(1 E-4)^{a}$ \\
\hline Turnip greens & $2 \quad(2 E-2)$ & $0.7 \quad(7 \mathrm{E}-3)$ \\
\hline Turnips & $4 \quad(4 \mathrm{E}-2)$ & $0.8 \quad(8 \mathrm{E}-3)$ \\
\hline Total & $6 \quad(6 E-2)$ & $2 \quad(2 \mathrm{E}-2)$ \\
\hline
\end{tabular}

${ }^{\mathrm{N}} \mathrm{No}{ }^{40} \mathrm{~K}$ concentrations were reported in tomatoes.

Table 6.6. 1993 total dose rate for aquatic organisms (rad/day), ${ }^{8.0}$ ORNL

\begin{tabular}{lcccccc}
\hline $\begin{array}{c}\text { Measurement } \\
\text { location }\end{array}$ & $\begin{array}{c}\text { Fish } \\
\text { average }\end{array}$ & $\begin{array}{c}\text { Fish } \\
\text { maximum }\end{array}$ & $\begin{array}{c}\text { Crustacea } \\
\text { average }\end{array}$ & $\begin{array}{c}\text { Crustacea } \\
\text { maximum }\end{array}$ & $\begin{array}{c}\text { Muskrat } \\
\text { average }\end{array}$ & $\begin{array}{c}\text { Muskrat } \\
\text { maximum }\end{array}$ \\
\hline $\begin{array}{l}\text { Melton Branch } \\
(\mathrm{X}-13)\end{array}$ & $3.1 \mathrm{E}-3$ & $5.1 \mathrm{E}-3$ & $2.7 \mathrm{E}-2$ & $4.4 \mathrm{E}-2$ & $7.4 \mathrm{E}-3$ & $1.2 \mathrm{E}-2$ \\
$\begin{array}{l}\text { White Oak Creek } \\
\text { (X14) }\end{array}$ & $2.1 \mathrm{E}-3$ & $4.9 \mathrm{E}-3$ & $5.6 \mathrm{E}-3$ & $1.2 \mathrm{E}-2$ & $1.9 \mathrm{E}-3$ & $4.3 \mathrm{E}-3$ \\
$\begin{array}{l}\text { White Oak Dam } \\
(\mathrm{X} 15)\end{array}$ & $1.5 \mathrm{E}-3$ & $3.5 \mathrm{E}-3$ & $9.3 \mathrm{E}-3$ & $1.5 \mathrm{E}-2$ & $2.6 \mathrm{E}-3$ & $4.5 \mathrm{E}-3$ \\
$\begin{array}{l}7500 \text { Road Bridge } \\
\text { First Creek }\end{array}$ & $1.9 \mathrm{E}-3$ & $5.0 \mathrm{E}-3$ & $4.6 \mathrm{E}-3$ & $9.3 \mathrm{E}-3$ & $1.6 \mathrm{E}-3$ & $3.6 \mathrm{E}-3$ \\
Fifth Creek & $1.2 \mathrm{E}-3$ & $3.5 \mathrm{E}-3$ & $1.1 \mathrm{E}-2$ & $2.5 \mathrm{E}-2$ & $2.9 \mathrm{E}-3$ & $6.8 \mathrm{E}-3$ \\
Melton Branch 2 & $3.1 \mathrm{E}-4$ & $7.3 \mathrm{E}-4$ & $1.5 \mathrm{E}-3$ & $2.4 \mathrm{E}-3$ & $4.3 \mathrm{E}-4$ & $7.7 \mathrm{E}-4$ \\
Northwest Tributary & $1.7 \mathrm{E}-5$ & $6.3 \mathrm{E}-4$ & $1.2 \mathrm{E}-4$ & $1.4 \mathrm{E}-3$ & $3.8 \mathrm{E}-5$ & $3.3 \mathrm{E}-4$ \\
Raccoon Creek & $3.6 \mathrm{E}-4$ & $1.8 \mathrm{E}-3$ & $1.8 \mathrm{E}-3$ & $4.9 \mathrm{E}-3$ & $5.3 \mathrm{E}-4$ & $1.4 \mathrm{E}-3$ \\
\hline
\end{tabular}

Total dose rate includes the contribution of internally deposited radionuclides, sediment exposure (derived from water concentrations), and water immersion.

To convert from rad/day to gray/day divide by 100 . 
Table 6.7. 1993 total dose rate for aquatic organisms (rad/day), Y-12 Plant and K-25 Site

\begin{tabular}{|c|c|c|c|c|c|c|}
\hline $\begin{array}{l}\text { Measurement } \\
\text { location }\end{array}$ & $\begin{array}{c}\text { Fish } \\
\text { average }\end{array}$ & $\begin{array}{c}\text { Fish } \\
\text { maximum }\end{array}$ & $\begin{array}{l}\text { Crustacea } \\
\text { average }\end{array}$ & $\begin{array}{l}\text { Crustaceà } \\
\text { maximum }\end{array}$ & $\begin{array}{l}\text { Muskrat } \\
\text { average }\end{array}$ & $\begin{array}{l}\text { Muskrat } \\
\text { maximum }\end{array}$ \\
\hline \multicolumn{7}{|c|}{ Y-12 Plant } \\
\hline $\begin{array}{l}\text { East Fork Poplar } \\
\text { Creek (Station 17) }\end{array}$ & $6.9 \mathrm{E}-5$ & $4.3 \mathrm{E}-4$ & $3.3 \mathrm{E}-4$ & $2.3 \mathrm{E}-3$ & $4.7 \mathrm{E}-3$ & $6.1 E-2$ \\
\hline $\begin{array}{l}\text { Bear Creek } \\
\text { (Outfall 304) }\end{array}$ & $6.8 \mathrm{E}-5$ & $3.5 \mathrm{E}-4$ & $3.3 \mathrm{E}-4$ & $1.9 \mathrm{E}-3$ & $1.1 \mathrm{E}-3$ & 4.7E-2 \\
\hline $\begin{array}{l}\text { Rogers Quarry } \\
\text { Outfall } 302\end{array}$ & $3.0 \mathrm{E}-5$ & $1.1 E-4$ & $2.7 \mathrm{E}-4$ & $1.0 \mathrm{E}-3$ & $1.5 \mathrm{E}-5$ & $1.2 \mathrm{E}-4$ \\
\hline \multicolumn{7}{|c|}{$K \cdot 25$ Site } \\
\hline $\begin{array}{l}\text { Mitchell Branch } \\
(\mathrm{K}-1700)\end{array}$ & $3.8 \mathrm{E}-3$ & $4.3 \mathrm{E}-2$ & $3.7 \mathrm{E}-2$ & $4.3 E-1$ & $6.8 \mathrm{E}-4$ & $6.5 \mathrm{E}-3$ \\
\hline $\begin{array}{l}\text { Poplar Creek } \\
\text { (Outfall 005) }\end{array}$ & $8.7 \mathrm{E}-3$ & $7.5 \mathrm{E}-2$ & $8.4 \mathrm{E}-2$ & $7.3 \mathrm{E}-1$ & $1.4 \mathrm{E}-3$ & $1.0 \mathrm{E}-2$ \\
\hline $\begin{array}{l}\text { Clinch River } \\
\text { (Holding Pond, } \\
\text { K-901-A) }\end{array}$ & $2.7 \mathrm{E}-3$ & $3.2 \mathrm{E}-2$ & $2.9 \mathrm{E}-2$ & $3.3 \mathrm{E}-1$ & $2.3 \mathrm{E}-3$ & $2.5 \mathrm{E}-2$ \\
\hline
\end{tabular}

"Total dose rate includes the contribution of internally deposited radionuclides, sediment exposure (derived from water concentrations), and water immersion.

${ }^{b}$ To convert from rad/day to Gy/day divide by 100 . 
Table 6.8. Summary of estimated radiation dose equivalents

to an adult during 1993 at locations of maximum exposure

\begin{tabular}{|c|c|c|c|}
\hline \multirow{2}{*}{ Pathway } & \multirow{2}{*}{ Location } & \multicolumn{2}{|c|}{$\begin{array}{l}\text { Effective dose } \\
\text { equivalent }\end{array}$} \\
\hline & & mrem & $\mathrm{mSv}$ \\
\hline $\begin{array}{l}\text { Gaseous effluents } \\
\text { Inhalation plus direct } \\
\text { radiation from air, } \\
\text { ground, and food } \\
\text { chains }\end{array}$ & $\begin{array}{l}\text { Maximally exposed resident to } \\
\text { Y-12 Plant } \\
\text { ORNL } \\
\text { K-25 Site } \\
\text { ORR }\end{array}$ & $\begin{array}{l}1.3 \\
0.1 \\
0.1 \\
1.4\end{array}$ & $\begin{array}{l}0.013 \\
0.001 \\
0.001 \\
0.014\end{array}$ \\
\hline $\begin{array}{l}\text { Liquid effluents } \\
\text { Drinking water } \\
\text { Eating fish } \\
\text { Other activities }\end{array}$ & $\begin{array}{l}\text { Gallaher Water Plant } \\
\text { Clinch River, CRK } 32 \\
\text { Clinch River }\end{array}$ & $\begin{array}{l}0.2 \\
0.2 \\
0.2\end{array}$ & $\begin{array}{l}0.002 \\
0.016 \\
0.002\end{array}$ \\
\hline Direct radiation & $\begin{array}{l}\text { Clinch River shoreline } \\
\text { Poplar Creek (K-25 Site) }\end{array}$ & $\begin{array}{l}1^{a} \\
1\end{array}$ & $\begin{array}{l}0.01 \\
0.11\end{array}$ \\
\hline
\end{tabular}

${ }^{a}$ This is an overestimate of the potential dose because the source of direct radiation was remediated during 1993.

Table 6.9. Trends in committed effective dose equivalent for selected pathways

\begin{tabular}{lccccc}
\hline \multirow{2}{*}{ Pathway } & \multicolumn{5}{c}{$\begin{array}{c}\text { Effective dose equivalent } \\
\text { (mrem) }\end{array}$} \\
\cline { 2 - 6 } & 1989 & 1990 & 1991 & 1992 & 1993 \\
\hline All air & 1 & 2 & 2 & 1.3 & 1.4 \\
Fish consumption & 0.2 & 0.3 & 0.3 & 0.4 & 0.2 \\
Drinking water (Kingston) & $<0.3$ & 0.04 & 0.1 & 0.05 & 0.07 \\
Direct radiation (Clinch River) & $1^{b}$ & $1^{b}$ & $1^{b}$ & $1^{b}$ & $1^{c}$ \\
Direct radiation (Poplar Creek) & & & $11^{b}$ & $11^{b}$ & 1 \\
\hline
\end{tabular}

To convert mrem to $\mathrm{mSv}$, divide by 100 .

bThese values have been corrected by removing the contribution of natural background radiation and by using International Commission on Radiological Protection recommendations for converting external exposure to effective dose equivalent.

This is an overestimate of the potential dose because the source of the direct radiation was remediated during 1993. 
Table 6.10. Chemical reference doses and slope factors used in drinking water and fish intake analysis

\begin{tabular}{|c|c|c|}
\hline Chemical & $\begin{array}{l}\text { Reference dose } \\
\text { or slope factor }\end{array}$ & Reference ${ }^{a}$ \\
\hline $4,4^{\prime}-\mathrm{DDD}$ & $2.4 \mathrm{E}-1$ & SF \\
\hline $4,4^{\circ}-\mathrm{DDE}$ & $3.4 \mathrm{E}-1$ & SF \\
\hline Alpha chlordane & 1.3 & $\mathrm{SF}$ \\
\hline Aluminum & $6 \mathrm{E}-3$ & SMCL \\
\hline Arsenic & $3 E-4$ & $\mathrm{RfD}$ \\
\hline Barium & $7 \mathrm{E}-4$ & RfD \\
\hline Beryllium & $5 E-3$ & RfD \\
\hline Boron & $9 E-2$ & RfD \\
\hline Cadmium & $5 E-4$ & RfD \\
\hline Chloride & 7.14 & SMCL \\
\hline Chromium (VI) & $5 E-3$ & RfD \\
\hline Copper & 0.04 & SMCL \\
\hline Cyanide & $2 \mathrm{E}-2$ & $\mathrm{RfD}$ \\
\hline Fluoride & $6 \mathrm{E}-2$ & $\mathrm{RfD}$ \\
\hline Iron & $9 \mathrm{E}-3$ & SMCL \\
\hline Lead & $4 \mathrm{E}-4$ & $\mathrm{MCL}$ \\
\hline Manganese & $5 E-3$ & $\mathrm{RfD}$ \\
\hline Mercury & $5.7 \mathrm{E}-5$ & $\mathrm{MCL}$ \\
\hline Methylene chloride & $7.5 \mathrm{E}-3$ & SF \\
\hline Molybdenum & $5 E-3$ & RfD \\
\hline Nickel & $2 E-2$ & $\mathrm{RfD}$ \\
\hline Nitrate & 1.6 & RfD \\
\hline PCBs & 7.7 & $\mathrm{SF}$ \\
\hline Phenois & $6 \mathrm{E}-1$ & $\mathrm{RfD}$ \\
\hline Selenium & $5 E-3$ & RfD \\
\hline Silver & $5 E-3$ & $\mathrm{RfD}$ \\
\hline Strontium & $6 \mathrm{E}-1$ & $\mathrm{RfD}$ \\
\hline Sulfate & 7.14 & SMCL \\
\hline Thallium & $8 E-3$ & RfD \\
\hline Trichloroethane & $6 \mathrm{E}-3$ & MCL \\
\hline Uranium & $3 E-3$ & RfD \\
\hline Vanadium & $9 \mathrm{E}-3$ & $\mathrm{RfD}$ \\
\hline Zinc & $3 E-1$ & RfD \\
\hline Methyl isobutyl ketone & $8 \mathrm{E}-2$ & $\mathrm{RfD}$ \\
\hline
\end{tabular}

'SMCL: secondary maximum contaminant level; RfD: reference dose; MCL: maximum contaminant level; SF: slope factor. 
Table 6.11. Chemical hazard quotients for drinking water

\begin{tabular}{|c|c|}
\hline Chemical & Hazard quotient \\
\hline \multicolumn{2}{|c|}{ Melton Hill Reservoir above all DOE inputs (CRK 84) } \\
\hline \multicolumn{2}{|c|}{ Metals } \\
\hline Aluminium & $1 E+0$ \\
\hline Arsenic & $-5 E+0$ \\
\hline Barium & $3 E-2$ \\
\hline Chromium & $-4 E-2$ \\
\hline Iron & $1 E+0$ \\
\hline Manganese & $5 E-1$ \\
\hline Uranium & $-2 E-3$ \\
\hline Vanadium & $-7 \mathrm{E}-3$ \\
\hline Zinc & $-1 E-3$ \\
\hline \multicolumn{2}{|l|}{ Anions } \\
\hline Chloride & $1 \mathrm{E}-2$ \\
\hline Nitrate & $4 \mathrm{E}-2$ \\
\hline Sulfate & $9 E-2$ \\
\hline \multicolumn{2}{|c|}{ Water supply intake for Knox County (CRK 58) } \\
\hline \multicolumn{2}{|c|}{ Metals } \\
\hline Aluminium & $9 \mathrm{E}-1$ \\
\hline Barium & IE-2 \\
\hline Iron & $-5 E-1$ \\
\hline Manganese & $2 E-1$ \\
\hline Uranium & $-4 E-3$ \\
\hline Zine & $-9 E-4$ \\
\hline \multicolumn{2}{|l|}{ Anions } \\
\hline Chloride & $2 \mathrm{E}-2$ \\
\hline Nitrate & $4 E-2$ \\
\hline Sulfate & $1 E-1$ \\
\hline \multicolumn{2}{|c|}{ Water supply intake for the $K-25$ Site (CRK 23) } \\
\hline \multicolumn{2}{|c|}{ Metals } \\
\hline Aluminium & $1 E+0$ \\
\hline Barium & $-1 E-2$ \\
\hline Iron & $-9 E-1$ \\
\hline Manganese & $3 E-1$ \\
\hline Uranium & $-1 E-3$ \\
\hline Vanadium & $-7 E-3$ \\
\hline Zinc & $-5 E-4$ \\
\hline \multicolumn{2}{|l|}{ Anions } \\
\hline Chloride & $-2 \mathrm{E}-2$ \\
\hline Nitrate & SE-2 \\
\hline Suifate & $9 \mathrm{E}-2$ \\
\hline \multicolumn{2}{|c|}{ Clinch River downstream of all DOE inputs (CRK 16) } \\
\hline \multicolumn{2}{|l|}{ Metals } \\
\hline Aluminum & $1 E+0$ \\
\hline Barium & $-1 E-2$ \\
\hline Iron & $-8 \mathrm{E}-1$ \\
\hline Manganese & $2 E-1$ \\
\hline Uranium & $-4 E-3$ \\
\hline Vanadium & $-6 E-3$ \\
\hline \multicolumn{2}{|l|}{ Anions } \\
\hline Chloride & $2 E-2$ \\
\hline Fluoride & $-5 E-2$ \\
\hline Nitrate & $6 \mathrm{E}-2$ \\
\hline Sulfate & $8 \mathrm{E}-2$ \\
\hline
\end{tabular}


Table 6.12. Chemical hazard quotient and VCCi for fish intake

\begin{tabular}{|c|c|c|}
\hline Chemical & Hazard quotient & VCDI \\
\hline \multicolumn{3}{|c|}{$\begin{array}{l}\text { Melton Hill Resenoir-above all DOE inpu. } \\
\text { Anderson Count Filtration Plant (CRK \&4) }\end{array}$} \\
\hline \multicolumn{3}{|c|}{ Sunfish } \\
\hline \multicolumn{3}{|l|}{ Metals } \\
\hline Chromium & $3 E-2$ & \\
\hline Copper & $4 E-3$ & \\
\hline Mercury & $5 E-1$ & \\
\hline Selenium & IE-1 & \\
\hline Silver & $-3 \mathrm{E}-2$ & \\
\hline Uranium & $-2 \mathrm{E}-3$ & \\
\hline Zinc & $-4 \mathrm{E}-2$ & \\
\hline \multicolumn{3}{|c|}{ Melton Hill Reservoir-Oak Ridge Marina (above ORNL) (CRK 80) } \\
\hline & infish & $\cdot$ \\
\hline \multicolumn{3}{|l|}{ Metals } \\
\hline Chromium & $3 E-2$ & \\
\hline Copper & $-4 E-3$ & \\
\hline Mercury & $4 \mathrm{E}-1$ & \\
\hline Selenium & $-1 E-1$ & \\
\hline Uranium & $-1 \mathrm{E}-3$ & \\
\hline Zinc & $3 E-2$ & \\
\hline \multicolumn{3}{|c|}{ Melton Hill Reservoir above city of Oak Ridge water intake (CRK 66) } \\
\hline \multicolumn{3}{|c|}{ Sunfish } \\
\hline \multicolumn{3}{|l|}{ Metals } \\
\hline Chromium & $3 \mathrm{E}-2$ & \\
\hline Copper & $4 E-3$ & \\
\hline Mercury & $5 E-1$ & \\
\hline Selenium & $2 \mathrm{E}-1$ & \\
\hline Uranium & $-9 \mathrm{E}-4$ & \\
\hline Zinc & $3 \mathrm{E}-2$ & \\
\hline \multicolumn{3}{|c|}{ Clinch River downstream from ORNL (CRK 32) } \\
\hline \multicolumn{3}{|c|}{ Sunfish } \\
\hline \multicolumn{3}{|l|}{ Metals } \\
\hline Chromium & $4 E-2$ & \\
\hline Copper & $4 \mathrm{E}-3$ & \\
\hline Mercury & $2 \mathrm{E}+0$ & \\
\hline Selenium & $9 \mathrm{E}-2$ & \\
\hline Uranium & $3 E-4$ & \\
\hline Zine & $4 \mathrm{E}-2$ & \\
\hline \multicolumn{3}{|l|}{ Pesticides } \\
\hline 4,4'-DDE & & $-2 E+0$ \\
\hline \multicolumn{3}{|l|}{ PCB } \\
\hline Arocior- 1254 & & $4 E+2$ \\
\hline \multicolumn{3}{|c|}{ Carfish } \\
\hline \multicolumn{3}{|l|}{ Metals } \\
\hline Mercury & $2 \mathrm{E} \div 0$ & \\
\hline Nickel & $6 \mathrm{E}-3$ & \\
\hline Zinc & 1E-2 & \\
\hline
\end{tabular}


Table 6.12 (continued)

\begin{tabular}{|c|c|c|}
\hline Chemical & Hazard quotient & I/CDI \\
\hline \multicolumn{3}{|l|}{ Pesticides } \\
\hline $4,4^{\prime}-\mathrm{DDD}$ & & $-3 E-1$ \\
\hline $4,4^{\prime}-\mathrm{DDE}$ & & $-2 \mathrm{E}+0$ \\
\hline Alpha-chlordane & & $9 E+0$ \\
\hline \multicolumn{3}{|l|}{ PCB } \\
\hline Aroclor- 1254 & & $5 \mathrm{E}+2$ \\
\hline Aroclor- 1260 & & $5 E+2$ \\
\hline \multicolumn{3}{|c|}{ Clinch River downstream from all DOE inputs (CRK 16) } \\
\hline & Sunfish & \\
\hline \multicolumn{3}{|l|}{ Metals } \\
\hline Arsenic & $-1 E+0$ & \\
\hline Chromium & $4 \mathrm{E}-2$ & \\
\hline Copper & $5 E-3$ & \\
\hline Mercury & $3 E+0$ & \\
\hline Selenium & $-8 \mathrm{E}-2$ & \\
\hline Uranium & $-3 E-4$ & \\
\hline Zinc & $3 E-2$ & \\
\hline \multicolumn{3}{|l|}{ PCB } \\
\hline \multirow[t]{2}{*}{ Aroclor-1254 } & & $-3 E+2$ \\
\hline & Catfish & \\
\hline \multicolumn{3}{|l|}{ Metals } \\
\hline Arsenic & $3 \mathrm{E}+0$ & \\
\hline Mercury & $7 E+0$ & \\
\hline Thallium & $6 E-2$ & \\
\hline Uranium & $4 \mathrm{E}-4$ & \\
\hline \multicolumn{3}{|l|}{ Pesticide } \\
\hline $4,4^{\prime}-\mathrm{DDE}$ & & $1 E+0$ \\
\hline Alpha-chlordane & & 8E-1 \\
\hline \multicolumn{3}{|l|}{ PCBs } \\
\hline Aroclor-1254 & & $4 \mathrm{E}+2$ \\
\hline Aroclor- 1260 & & $5 E+2$ \\
\hline \multicolumn{3}{|c|}{ Poplar Creek downstream from the $K-25$ Site ( $P C K$ 2.2) } \\
\hline & Sunfish & \\
\hline \multicolumn{3}{|l|}{ Metals } \\
\hline Arsenic & $-2 \mathrm{E}+0$ & \\
\hline Chromium & $4 E-2$ & \\
\hline Copper & $5 E-3$ & \\
\hline Lead & $-1 E+0$ & \\
\hline Mercury & $2 \mathrm{E}+0$ & \\
\hline Selenium & $-9 \mathrm{E}-2$ & \\
\hline Silver & $\sim 8 \mathrm{E}-3$ & \\
\hline Uranium & $5 E-4$ & \\
\hline Zine & $3 \mathrm{E}-2$ & \\
\hline \multicolumn{3}{|l|}{ Pesticide } \\
\hline $4,4^{\prime}-\mathrm{DDE}$ & & $-2 E+0$ \\
\hline \multicolumn{3}{|l|}{ PCB } \\
\hline Aroclor-125a & & $-5 E+2$ \\
\hline
\end{tabular}


Table 7.1. Y-12 Plant monitoring wells installed in 1993

\begin{tabular}{lccc}
\hline $\begin{array}{c}\text { Monitoring } \\
\text { objective }\end{array}$ & $\begin{array}{c}\text { Bear Creek } \\
\text { regine }\end{array}$ & $\begin{array}{c}\text { East Fork } \\
\text { regime }\end{array}$ & $\begin{array}{c}\text { Chestnut Ridge } \\
\text { regime }\end{array}$ \\
\hline Category I & 0 & 0 & 0 \\
Category II & 0 & 0 & 0 \\
Category III & 1 & 9 & 5 \\
Category IV' & 5 & 0 & 0 \\
\hline
\end{tabular}

${ }^{a}$ Category $1-$ Wells added to define extent of groundwater contamination. Category II-Wells to monitor potential exit pathways. Category III-New or replacement wells for compliance monitoring. Category IV-Wells installed by Environmental Restoration Program.

Includes one replacement well at the Oil Landfarm, nine wells to monitor corrective actions at underground storage tanks, and five wells at Industrial Landfill $\mathrm{V}$ and Construction/Demolition Landfill VII.

Includes five wells installed as part of the Bear Creek OU 2 RI. 
Table 7.2. Summary of the comprehensive groundwater monitoring program at the Y-12 Plant, 1993

\begin{tabular}{|c|c|c|c|}
\hline $\begin{array}{l}\text { Hydrogeologic regime/waste } \\
\text { disposal site }\end{array}$ & Requirements $^{a}$ & $\begin{array}{l}\text { Number of } \\
\text { wells }\end{array}$ & Analytical parameters ${ }^{b}$ \\
\hline \multicolumn{4}{|c|}{ Bear Creek Hydrogeologic Regime } \\
\hline Background ${ }^{c}$ & BMP & 16 & Standard + CMP \\
\hline Bear Creek Springs & EXP & 5 & Standard + (beta for SS-1) \\
\hline Bear Creek surface water & EXP & 5 & Standard $+($ beta for NT-1) \\
\hline Exit pathway-Traverse A & EXP & 3 & Standard \\
\hline Exit pathway-Traverse B & EXP & 5 & $\begin{array}{l}\text { Standard + (beta for GW-694 and } \\
\text { GW-706) }\end{array}$ \\
\hline Exit pathway-Traverse $C$ & EXP & 8 & Standard \\
\hline Exit pathway-Traverse W & EXP & 6 & Standard \\
\hline Oil Landfarm & RCRA-AM/SMP & 18 & $\begin{array}{l}\text { Standard + (beta at GW-537 and } \\
\text { CMP at GW-40, GW-43, and } \\
\text { GW- } 44 \text { only) }\end{array}$ \\
\hline Rust Spoil Area & RCRA-AM & 2 & Standard \\
\hline S-3 Site & $\begin{array}{l}\text { RCRA-AM/RCRA- } \\
\text { CM/SMP }\end{array}$ & 9 & $\begin{array}{l}\text { Standard + (CMP for GW-115, } \\
\text { GW-324, GW-325, GW-613, and } \\
\text { GW-614) }\end{array}$ \\
\hline Spoil Area I & RCRA-AM/SMP & 4 & Standard \\
\hline Y-12 Burial Grounds & RCRA-AM/SMP & 31 & $\begin{array}{l}\text { Standard + (CMP for GW-40, } \\
\text { GW-42, GW-79, GW-80, GW-162, } \\
\text { GW-342, GW-372, GW-373, and } \\
\text { GW-642) }\end{array}$ \\
\hline $\begin{array}{l}\text { Above Grade Low-Level } \\
\text { Storage Facility }\end{array}$ & BMP & 3 & $\begin{array}{l}\text { Standard }+\left({ }^{234} \mathrm{U},{ }^{235} \mathrm{U},{ }^{238} \mathrm{U} \text { for }\right. \\
\text { GW-794 and } \mathrm{GW}-795)\end{array}$ \\
\hline \multicolumn{4}{|c|}{ East Fork Poplar Creek Hydrogeologic Regime } \\
\hline Background & BMP & 7 & Standard \\
\hline Beta-4 Security Pit & GRID & 4 & Standard \\
\hline Exit pathway-Traverse $\mathfrak{J}$ & EXP & 2 & Standard \\
\hline Grid C-1 & GRID & 2 & Standard \\
\hline Grid E-1 & GRID & 2 & Standard \\
\hline Grid G-1 & GRID & 2 & Standard \\
\hline Grid G-2 & GRID & 2 & Standard \\
\hline Grid G-3 & GRID & 2 & Standard \\
\hline Grid $\mathrm{H}-2$ & GRID & 2 & Standard \\
\hline Grid $\mathrm{H}-3$ & GRID & 2 & Standard \\
\hline Grid I-1 & GRID & 2 & Standard \\
\hline Grid I-2 & GRID & 2 & Standard + TPH \\
\hline Grid J-1 & GRID & 2 & Standard \\
\hline Grid J-2 & GRID & 2 & Standard \\
\hline Grid J-3 & GRID & 2 & Standard \\
\hline Grid K-1 & GRID & 3 & Standard \\
\hline Grid K-2 & GRID & 3 & Standard \\
\hline Grid K-3 & GRID & 1 & Standard \\
\hline J-Primary & GRID & 2 & Standard \\
\hline New Hope Pond & RCRA-AM & 11 & Standard \\
\hline
\end{tabular}


Table 7.2 (continued)

\begin{tabular}{|c|c|c|c|}
\hline $\begin{array}{l}\text { Hydrogeologic regime/waste } \\
\text { disposal site }\end{array}$ & Requirements $^{a}$ & $\begin{array}{l}\text { Number of } \\
\text { wells }\end{array}$ & Analytical parameters ${ }^{b}$ \\
\hline $\begin{array}{l}\text { U.S. Geological Survey } \\
\text { Sites/exit pathway }\end{array}$ & EXP & 12 & Standard \\
\hline UST Program & UST & 13 & $\begin{array}{l}\text { Standard + (TPH for GW-656, } \\
\text { GW-657, GW-658, GW-659, } \\
\text { GW-707, and GW-708) }\end{array}$ \\
\hline $\begin{array}{l}\text { Waste Coolant Facilities/ } \\
\text { Salvage Yard/Fire Training } \\
\text { Facility }\end{array}$ & GRID & 8 & Standard \\
\hline \multicolumn{4}{|c|}{ Chestnut Ridge Hydrogeologic Regime } \\
\hline Ash Disposal Basin & BMP & 4 & Standard + TOX + TOC \\
\hline Chestnut Ridge Security Pits & RCRA-AM & 11 & Standard \\
\hline $\begin{array}{l}\text { East Chestnut Ridge Waste } \\
\text { Pile }\end{array}$ & BMP & 4 & Standard \\
\hline Kerr Hollow Quarry & RCRA-DM & 7 & Standard + REP + PHEN \\
\hline Landfill II & SWDF & 3 & Standard + AOC + ORP \\
\hline $\begin{array}{l}\text { Landfill III (Chestnut Ridge } \\
\text { Borrow Area Waste Pile) }\end{array}$ & SWDF & 7 & $\begin{array}{l}\text { Standard + AOC + ORP + }(U+\text { beta } \\
\text { for GW-295 only })\end{array}$ \\
\hline Landfill IV & SWDF & 5 & Standard + AOC + ORP \\
\hline Landfill V & SWDF & 5 & $\begin{array}{l}\text { Standard + AOC + ORP + U + OMP } \\
+(\text { TPH for GW-799 only) }\end{array}$ \\
\hline Landfill VI & SWDF & 7 & Standard + AOC + ORP \\
\hline Landfill VII & SWDF & 4 & $\begin{array}{l}\text { Standard + AOC + ORP + OMP + } \\
(\mathrm{U}+\mathrm{TPH} \text { for GW-560, GW-562, } \\
\text { and GW-564) }\end{array}$ \\
\hline Rogers Quarry & SWDF & 4 & Standard + BNA \\
\hline Sediment Disposal Basin & RCRA-DM & 8 & Standard + REP + BNA \\
\hline United Nuclear Site & ROD & 6 & Standard + U + Ra \\
\hline
\end{tabular}

${ }^{a} \mathrm{BMP}=$ best management practices monitoring; EXP = exit-pathway monitoring under DOE Order 5400.1;

RCRA-AM = RCRA Assessment Monitoring at interim status units; RCRA-DM = RCRA Detection Monitoring at interim status units; RCRA-CM = RCRA post-closure compliance monitoring; SMP = Y-12 Plant Environmental Restoration Program's Surveillance and Maintenance Program; GRID = grid well monitoring locations under DOE Order 5400.1; UST = petroleum underground storage tank locations; SWDF = monitoring for solid waste disposal facilities under TDEC Rule 1200-1-7-.04; ROD = CERCLA record of decision post-closure monitoring.

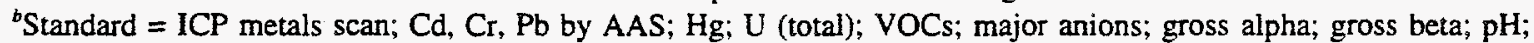
conductance; TSS; TDS; turbidity; standard field parameters, including dissolved oxygen, water level, pH, temperature, conductance, and redox potential. $\mathrm{CMP}=\mathrm{RCRA}$ compliance monitoring parameters, including ${ }^{241} \mathrm{Am},{ }^{129} \mathrm{l},{ }^{237} \mathrm{~Np},{ }^{238} \mathrm{Pu}$, total radium, total strontium, ${ }^{99} \mathrm{Tc},{ }^{3} \mathrm{H},{ }^{234} \mathrm{U},{ }^{235} \mathrm{U}$, and ${ }^{238} \mathrm{U}$. Beta = beta-emitting isotopes, including total strontium, ${ }^{99} \mathrm{Tc}$, and ${ }^{3} \mathrm{H}$. TPH = total petroleum hydrocarbons. REP = four replicate analyses for $\mathrm{pH}$, conductance, TOC, and TOX. PHEN $=$ phenois. TOX $=$ total organic halides. $T O C=$ total organic carbon. ORP $=$ other parameters required by TDEC Rule 1200-1-7-.04, including chemical oxygen demand, cyanide, TOC, and TOX. $U=$ isotopic uranium analysis, including ${ }^{234} \mathrm{U},{ }^{235} \mathrm{U}$, and ${ }^{238} \mathrm{U}$. OMP = other miscellaneous permit-required parameters including ammonia (as $\mathrm{N}$ ), gamma activity, and trans-1,2-dichloroethene. $\mathrm{Ra}=$ total radium. $\mathrm{BNA}=$ base/neutral/acid extractable organic compounds (semivolatile organics). $A O C=$ additional VOC list required by TDEC Rule 1200-1-7-.04.

'Background monitoring wells are illustrated separately only for comparative purposes. Background wells are associated with individual sites. 
Table 7.3. Description of surface-water and spring monitoring stations included in the Exit-Pathway Monitoring Program

\begin{tabular}{|c|c|}
\hline Locations & Description \\
\hline NT-13 (background) & $\begin{array}{l}\text { Tributary that enters Bear Creek at BCK } 6.76 \text { and represents drainage from a } \\
\text { relatively undisturbed catchment that has not been impacted by waste-disposal } \\
\text { activities in Bear Creek Valley. }\end{array}$ \\
\hline BCK 0.63 & $\begin{array}{l}\text { Upstream of the confluence with East Fork Poplar Creek. Represents essentially all } \\
\text { surface-water discharge from the Bear Creek watershed. }\end{array}$ \\
\hline BCK 4.55 & $\begin{array}{l}\text { Location of NPDES monitoring site } 304 \text {. Site represents surface-water discharge } \\
\text { from at least one area of the Bear Creek floodplain known to be contaminated } \\
\text { with uranium and PCBs. Formal perimeter monitoring location for the ORR. }\end{array}$ \\
\hline BCK 9.40 & $\begin{array}{l}\text { Represents surface-water discharge from area of Bear Creek watershed impacted by } \\
\text { waste-disposal activities. }\end{array}$ \\
\hline NT-1 & $\begin{array}{l}\text { North Tributary (NT)-1 to Bear Creek, which probably receives groundwater inputs } \\
\text { from S-3 Site contamination. }\end{array}$ \\
\hline SS-1 & $\begin{array}{l}\text { Located on south side of Bear Creek at the confluence with NT-1, near headwaters } \\
\text { of Bear Creek }\end{array}$ \\
\hline SS-4 & $\begin{array}{l}\text { Discharges on southside of Bear Creek Road at contact between the Knox Group } \\
\text { and the Maynardville Limestone. Location is about } 500 \mathrm{ft} \text { west of exit-pathway } \\
\text { Picket B. }\end{array}$ \\
\hline SS-5 & $\begin{array}{l}\text { Large spring located on south side of Bear Creek Road near contact between the } \\
\text { Knox Group and the Maynardville Limestone. Location is coincident with exit- } \\
\text { pathway Picket A. }\end{array}$ \\
\hline SS-6 & $\begin{array}{l}\text { Discharges on north side of Bear Creek Road; location is within the Maynardville } \\
\text { Limestone about } 500 \mathrm{ft} \text { west of exit-pathway Picket W. }\end{array}$ \\
\hline SS-8 & $\begin{array}{l}\text { Large spring located at junction of Bear Creek Road and TN } 95 \text { near Station } \\
\text { BCK } 4.55 \text {, within the Maynardville Limestone. Westernmost spring monitored } \\
\text { under the exit-pathway program. }\end{array}$ \\
\hline
\end{tabular}


Table 7.4. Waste management sites, CERCLA operable units, and underground storage tanks included in the 1993 Groundwater Protection Program; East Fork Hydrogeologic Regime

\begin{tabular}{|c|c|c|}
\hline \multirow{2}{*}{ Site name } & \multicolumn{2}{|c|}{ Regulatory classification } \\
\hline & Historical $^{a}$ & Current $^{b}$ \\
\hline New Hope Pond & TSD Unit & TSD Unit \\
\hline Mercury Process Spill Areas & SWMU & UEFPC OU 01 \\
\hline Abandoned Nitric Acid Pipeline & SWMU & UEFPC OU 02 \\
\hline Salvage Yard Scrap Metal Storage Area & SWMU & UEFPC OU 03 \\
\hline Salvage Yard Oil/Solvent Drum Storage Area & SWMU & UEFPC OU 03 \\
\hline Salvage Yard Oil Storage & SWMU & UEFPC OU 03 \\
\hline Salvage Yard Drum Deheader & SWMU & UEFPC OU 03 \\
\hline Tank 2063-U & SWMU & UEFPC OU 03 \\
\hline S-2 Site & SWMU & UEFPC OU 03 \\
\hline Waste Coolant Processing Area & SWMU & UEFPC OU 03 \\
\hline Tank 2328-U & SWMU & Y-12 SA \\
\hline Tank 2329-U & SWMU & $Y-12 \mathrm{SA}$ \\
\hline Interim Drum Yard & SWMU & $\mathrm{Y}-12 \mathrm{SA}$ \\
\hline Beta-4 Security Pits & SWMU & $\mathrm{Y}-12 \mathrm{SA}$ \\
\hline Tank 2331-U & UST & UST \\
\hline Tank 0134-U & UST & UST \\
\hline Building 9754-2 Fuel Facility & UST & UST \\
\hline Garage Underground Tanks & SMU/UST & Y-12 SAUST \\
\hline Rust Garage Area & SWMU/UST & Y-12 SA/UST \\
\hline
\end{tabular}

Tegulatory status before 1992 Federal Facility Agreement: TSD unit-RCRA-regulated land-based treatment, storage, or disposal unit; SWMU-RCRA-regulated solid waste management unit; and UST-Non-RCRA UST.

Modified from Oak Ridge Reservation Site Management Plan for the Environmental Restoration Program. (U.S. Department of Energy 1992). UEFPC OU 01 = East Fork. Poplar Creek Operable Unit 01 (integrator); UEFPC OU $02=$ East Fork Poplar Creek Operable Unit 02 (source control); UEFPC OU 03 = East Fork Poplar Creek Operable Unit 03 (source control); and Y-12 SA = Y-12 Plant Study Area. 
Table 7.5. Waste management sites and CERCLA operable units included in the 1993 Groundwater Protection Program; Bear Creek Hydrogeologic Regime

\begin{tabular}{lll}
\hline \multicolumn{1}{c}{ Site name } & \multicolumn{2}{c}{ Regulatory classification } \\
\cline { 2 - 3 } & \multicolumn{1}{c}{ Historical $^{u}$} & Current \\
\hline S-3 Site & TSD Unit & BC OU 01 \\
Oil Landfarm Waste Management Area & & BC OU 01 \\
Oil Landfarm & TSD Unit & BC OU 01 \\
Burnyard, Boneyard, and Hazardous Chemical Storage Area & SWMU & BC OU 01 \\
Sanitary Landfill 1 & SWMU & BC OU 01 \\
Bear Creek Burial Grounds Waste Management Area & & BC OU 01 \\
Burial Ground A (North and South) & TSD Unit & BC OU 01 \\
Burial Ground C & TSD Unit & BC OU 01 \\
Burial Grounds B, D, E, and J & SWMUs & BC OU 01 \\
Oil Retention Pond No. 1 & SWMU & BC OU 02 \\
Oil Retention Pond No. 2 & SWMU & BC OU 02 \\
Spoil Area I & SWMU & BC OU 02 \\
SY-200 Yard & SWMU & BC OU 03 \\
Rust Spoil Area & SWMU & BC OU 04 \\
Bear Creek floodplain soils & N/A & N/A \\
Bear Creek groundwater, surface water, and creek sediments & N/A & Stave Grade Low Level Storage Facility \\
\hline
\end{tabular}

${ }^{\top}$ Regulatory status before 1992 federal facility agreement: TSD Unit-RCRA-regulated land-based treatment, storage, or disposal unit and SWMU-RCRA-regulated solid waste management unit.

${ }^{b}$ Modified from Oak Ridge Reservation Site Management Plan for the Environmental Restoration Program (U.S. Department of Energy 1992): BC OU 01 = Bear Creek Operable Unit 01 (source control OU); $B C$ OU 02 = Bear Creek Operable Unit 02 (source control OU); BC OU $03=$ Bear Creek Operable Unit 03 (Source Control OU); and BC OU 04 = Bear Creek Operable Unit 04 (integrator OU).

N/A = Not applicable (not previously regulated as a separate unit or not currently regulated. 
Table 7.6. Waste management sites and CERCLA operable units included in the 1993 Groundwater Protection Program; Chestnut Ridge Hutrogeologic Regime

\begin{tabular}{|c|c|c|}
\hline \multirow{2}{*}{ Site } & \multicolumn{2}{|c|}{ Regu'atory classification } \\
\hline & Historical $^{a}$ & Current \\
\hline Chestnut Ridge Sediment Disposal Basin & TSD urit & TSD unit \\
\hline East Chestnut Ridge Waste Pile & TSD unit & TSD unit \\
\hline Kerr Hollow Quarry & TSD unit & TSD unit \\
\hline Chestnut Ridge Security Pits & TSD unit & $\mathrm{CR}$ OU 01 \\
\hline Ash Disposal Basin & SWMU & $\mathrm{CR}$ OU 02 \\
\hline United Nuclear Corporation Site & SWMU & CR OU 03 \\
\hline Rogers Quarry & SWMU & CR OU 04 \\
\hline Industrial Landfill II & SWDF & SWDF \\
\hline Industrial Landfill III & SWDF & SWDF \\
\hline Incustrial Landfill IV & SWDF & SWDF \\
\hline Industrial Landfill V & $N / A^{c}$ & SWDF \\
\hline Construction Debris Landfill VI & N/A & SWDF \\
\hline Construction Debris Landfill VII & N/A & SWDF \\
\hline
\end{tabular}

${ }^{a}$ Regulatory classification before the 1992 Federal Facility Agreement: TSD Unit-RCRA-regulated landbased treatment, storage, or disposal facility; SWDF-solid waste disposal facility (nonhazardous waste); and SWMU-RCRA regulated solid waste management unit.

${ }^{b}$ Modified from Oak Ridge Reservation Site Management Plan for the Environmenial Restoration Program (U.S. Department of Energy 1992): CR OU 01_-Chestnut Ridge Operable Unit 01 (source control and groundwater OU); CR OU 02-Chestnut Ridge Operable Unit 02 (source control and Groundwater OU); CR OU 03-Chestnut Ridge Operable Unit 03 (source control and groundwater OU); and CR OU 04-Chestnut Ridge Operable Unit 04 (source control and groundwater OU).

'N/A-Not applicable (new facility). 
Table 7.7. Annual average summed VOC concentrations in groundwater at the Chestnut Ridge Security Pits, 1989-93

\begin{tabular}{|c|c|c|c|c|c|c|}
\hline \multirow{2}{*}{$\begin{array}{l}\text { Well } \\
\text { No. }\end{array}$} & \multicolumn{5}{|c|}{$\begin{array}{l}\text { Summed average VOCs } \\
(\mu g / L)\end{array}$} & \multirow{2}{*}{$\begin{array}{c}\text { Percentage } \\
\text { decrease }\end{array}$} \\
\hline & 1989 & 1990 & 1991 & 1992 & 1993 & \\
\hline GW-173 & 17 & 13.5 & 11.8 & 11.7 & $\mathrm{NS}^{a}$ & 31 \\
\hline GW-174 & 47.8 & 48.5 & 43.7 & 34 & NS & 29 \\
\hline GW-175 & 31.8 & 38.5 & 31 & 29.5 & 17 & 47 \\
\hline GW-176 & 285.3 & 233.5 & 170.5 & 139.7 & NS & 51 \\
\hline GW-177 & 66.7 & 18.8 & 26.3 & 25.5 & 33.7 & 49 \\
\hline GW-178 & 43.4 & 40 & 34 & 29 & NS & 32 \\
\hline GW-179 & 838 & 455 & 328.3 & 262.3 & NS & 69 \\
\hline$G W-180$ & 145.8 & 99.5 & 74.2 & 52.3 & NS & 64 \\
\hline GW-322 & 696 & 730.3 & 633 & 538.3 & NS & 23 \\
\hline GW-607 & NS & 16.9 & $\mathrm{ND}^{b}$ & ND & ND & 100 \\
\hline GW-608 & NS & 14.8 & 15.5 & 4.5 & 5.8 & 61 \\
\hline GW-609 & NS & 78 & 67.5 & 35.5 & 30.9 & 55 \\
\hline GW-610 & NS & 1 & 0.5 & ND & ND & 100 \\
\hline GW-611 & NS & 16 & 9 & 13.5 & 15 & 6 \\
\hline GW-612 & NS & 505.8 & 451.3 & 358.3 & NS & 29 \\
\hline
\end{tabular}

${ }^{a} \mathrm{NS}=$ not sampled.

${ }^{b} \mathrm{ND}=$ not detected. 
Table 7.8. Summary of ORNL waste area groupings

\begin{tabular}{|c|c|c|}
\hline WAG & Description & $\begin{array}{c}\text { Number of } \\
\text { sites }^{a}\end{array}$ \\
\hline 1 & Main plant area & 117 \\
\hline 2 & White Oak Creek/White Oak Lake & 2 \\
\hline 3 & SWSA 3 & 3 \\
\hline 4 & SWSA 4 & 3 \\
\hline 5 & SWSA 5 & 28 \\
\hline 6 & SWSA 6 & 3 \\
\hline 7 & Low-level waste pits and trenches area & 19 \\
\hline 8 & Melton Valley area & 35 \\
\hline 9 & Hornogeneous reactor experiment area & 13 \\
\hline 10 & Hydrofracture injection wells and grout sheets & $4^{b}$ \\
\hline 11 & White Wing scrapyard & 1 \\
\hline 12 & Closed contractors' landfill & 1 \\
\hline 13 & Environmental researcin areas & 2 \\
\hline 14 & Tower Shielding Facility & 2 \\
\hline 15 & ORNL facilities at $Y-12$ Plant & 14 \\
\hline 16 & Health Physics Research Reactor area & 5 \\
\hline 17 & ORNL services area & 8 \\
\hline 18 & Consolidated fuel reprocessing area & 10 \\
\hline 19 & Hazardous waste treatment and storage facility & 8 \\
\hline \multirow[t]{2}{*}{20} & Oak Ridge Landfarm & 1 \\
\hline & Total & 279 \\
\hline \multicolumn{3}{|c|}{ Additional sites outside of WAGs } \\
\hline$c$ & Surplus contaminated facilities & 29 \\
\hline
\end{tabular}

'Source: July 18, 1991, latter from Lanny Bates, Director of Environmental Restoration, to Robert Sleeman, DOE-ORO.

${ }^{\circ}$ Principal sites are locared underground, beneath WAG 5.

Not applicable. 
Table 7.9. Summary of the groundwater surveillance program at ORNL, 1993

\begin{tabular}{|c|c|c|c|c|}
\hline WAG & Regulatory status & $\begin{array}{l}\text { Upgradienv } \\
\text { downgradient } \\
\text { wells }\end{array}$ & Parameters monitored & $\begin{array}{l}\text { Frequency and last } \\
\text { date sampled }\end{array}$ \\
\hline \multicolumn{5}{|c|}{ Bethel Valley Operable Unit } \\
\hline 1 & $\begin{array}{l}\text { DOE Orders } 5400.1 \\
\text { and } 5400.5\end{array}$ & 24 & Standard & $\begin{array}{l}\text { Rotation } \\
\text { June } 1993\end{array}$ \\
\hline 3 & $\begin{array}{l}\text { DOE Orders } 5400.1 \\
\text { and } 5400.5\end{array}$ & 12 & Standard & $\begin{array}{l}\text { Rotation } \\
\text { November } 1993\end{array}$ \\
\hline 17 & $\begin{array}{l}\text { DOE Orders } 5400.1 \\
\text { and } 5400.5\end{array}$ & 4 & Standard & $\begin{array}{l}\text { Rotation } \\
\text { July } 1993\end{array}$ \\
\hline \multicolumn{5}{|c|}{ Melton Valley Operable Unit } \\
\hline 2 & $\begin{array}{l}\text { DOE Orders } 5400.1 \\
\text { and } 5400.5\end{array}$ & 12 & Standard & $\begin{array}{l}\text { Rotation } \\
\text { February } 1993\end{array}$ \\
\hline 4 & $\begin{array}{l}\text { DOE Orders } 5400.1 \\
\text { and } 5400.5\end{array}$ & 11 & Standard & $\begin{array}{l}\text { Rotation } \\
\text { January } 1994^{h}\end{array}$ \\
\hline 5 & $\begin{array}{l}\text { DOE Orders } 5400.1 \\
\text { and } 5400.5\end{array}$ & 20 & Standard & $\begin{array}{l}\text { Rotation } \\
\text { April } 1993\end{array}$ \\
\hline 6 & $\begin{array}{l}\mathrm{RCRA}^{\mathrm{c}} \text { assessment } \\
\text { monitoring and DOE } \\
\text { Orders } 5400.1 \text { and } \\
5400.5\end{array}$ & 17 & $\begin{array}{l}\text { Volatile organics, } \\
\text { gross alpha, gross } \\
\text { beta, }{ }^{3} \mathrm{H},{ }^{137} \mathrm{Cs},{ }^{60} \mathrm{Co} \text {, } \\
\text { total rad } \mathrm{Sr}+\text { standard } \\
\text { field measurements }\end{array}$ & $\begin{array}{l}8 \text { wells quarterly; } \\
16 \text { wells } \\
\text { semiannually }\end{array}$ \\
\hline 7 & $\begin{array}{l}\text { DOE Orders } 5400.1 \\
\text { and } 5400.5\end{array}$ & 14 & Standard & $\begin{array}{l}\text { Rotation } \\
\text { August } 1993\end{array}$ \\
\hline 8 and 9 & $\begin{array}{l}\text { DOE Orders } 5400.1 \\
\text { and } 5400.5\end{array}$ & 2 & Standard & $\begin{array}{l}\text { Rotation } \\
\text { October } 1993\end{array}$ \\
\hline \multicolumn{5}{|c|}{ White Wing Scrapyard } \\
\hline 11 & $\begin{array}{l}\text { DOE Orders } 5400.1 \\
\text { and } 5400.5\end{array}$ & 5 & Standard & $\begin{array}{l}\text { Rotation } \\
\text { January } 1993\end{array}$ \\
\hline
\end{tabular}

aStandard: volatile and semivolatile organics, total organic carbon, total organic halides, metals, anions, total phenolics, total suspended solids, alkalinity, gross alpha and beta, ${ }^{3} \mathrm{H},{ }^{137} \mathrm{Cs},{ }^{60} \mathrm{Co}$, and total radioactive strontium. Standard field measurements: $\mathrm{pH}$, conductivity, turbidity, oxidation/reduction potential, temperiture, and dissolved oxygen.

'Initiated in December 1993 and completed in January 1994. Results will be repored in the 1994 report.

The appropriate regulatory authority at SWSA 6 in WAG 6 is RCRA. The regulatory authority is expected to change to CERCLA, with RCRA as an applicable and appropriate requirement.

Table 7.10. Summary of the plant perimeter surveillance program at OANL, 1993"

\begin{tabular}{lccl}
\multicolumn{1}{c}{ Exit pathway } & WAG & $\begin{array}{c}\text { Number of } \\
\text { weils }\end{array}$ & Surface water locations \\
\hline White Oak Creek/Melton Valley & $6 \& 2^{b}$ & 10 & White Oak Creek at White Oak Darn \\
West Bethel Valley & 3 & 3 & Raccoon Creek \\
East Bethel Valley & 17 & 4 & Bearden Creek \\
White Wing Scrapyard & 11 & 3 & Bear Creek \\
\hline
\end{tabular}

${ }^{9}$ All locations are monitored for volatile organics, tritium, total radioactive strontium, gross alpha and beta, ${ }^{60} \mathrm{Co}$, and ${ }^{137} \mathrm{Cs}$.

'Four wells are part of the ORNL WAG 6 perimeter network, and four wells are part of the ORNL WAG 2 perimeter network. Two other wells were not sampled in 1993, pending a decision regarding installing dedicated pumps in them. 


\section{Key to Y-12 Plant Groundwater Tables}

GENERAL FOOTNOTES:

* Duplicate analysis outside control limits.

** Indicates those standards that were not effective until January 17, 1994. All other values are currently applicable MCLs.

\section{VOA FOOTNOTES:}

B Analyte found in blank as well as sample.

D Compounds identified in an analysis at a secondary dilution factor.

E Concentrations exceed calibrated range of the CG/MS instrument.

$\mathrm{J} \quad$ Indicates an estimated value.

RAD FOOTNOTES:

D Spike control limits do not apply; sample activity exceeds the activity of the spike.

E Result of analysis is less than the MDA; confidence level is less than $95 \%$.

F Result is less than background.

G Gamma photopeak near MDA<, resulting in a poor curve fit.

I Insufficient amount of sample to meet customer's MDA requirements.

J Chemical tracer recovery is outside of control limits.

S Spike recovery not within limits.
ABBREVIATIONS:
NR no reference
NÁ not appiicable
HGR hydrogeologic regime
EF East Fork Poplar Creek
CR Chestnut Ridge
BC Bear Creek 


\begin{tabular}{|c|c|c|c|c|c|c|c|c|c|c|}
\hline \multirow[b]{2}{*}{ Parameter } & \multicolumn{7}{|c|}{ Groundwater reference standards } & \multicolumn{3}{|c|}{$\begin{array}{l}\text { Surface water reference stds. (to } \\
\text { supplement NPDES for creeks/streams) }\end{array}$} \\
\hline & $\begin{array}{c}\text { TDEC stds. for } \\
\text { hazardous } \\
\text { waste TSD } \\
\text { facilities } \\
1200-1-11-.06 \\
\end{array}$ & $\begin{array}{l}\text { TDEC stds. } \\
\text { for landfills } \\
\text { rule } 1200-1-7 \\
\text { Appendix } 1 \\
\end{array}$ & 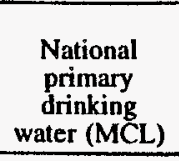 & $\begin{array}{c}\begin{array}{c}\text { National } \\
\text { secondary } \\
\text { drinking water } \\
(\mathrm{MCL})\end{array} \\
\end{array}$ & $\begin{array}{c}\text { Tennessee } \\
\text { primary } \\
\text { drinking water } \\
\text { (MCL) }\end{array}$ & $\begin{array}{c}\text { Tennessee } \\
\text { secondary } \\
\text { drinking water } \\
\text { (MCL) }\end{array}$ & $\begin{array}{l}4 \% \text { of } \\
\text { DOE } \\
\text { DCG }\end{array}$ & $\begin{array}{c}\text { DOE } \\
\text { Order } \\
5400.5 \\
\text { DCG } \\
\end{array}$ & $\begin{array}{l}\text { Tenn. water } \\
\text { quality fish \& } \\
\text { aquatic life } \\
\text { criterion max. } \\
\text { conc. (CMC) }\end{array}$ & $\begin{array}{c}\text { Tennessee } \\
\text { water quality } \\
\text { recreation }\end{array}$ \\
\hline \multicolumn{11}{|c|}{ Anions (mg/L) } \\
\hline Chloride & & & & 250 & & 250 & & & & \\
\hline Fluoride & $1.4-2.4$ & 4 & 4 & 2 & 4 & 2 & & & & \\
\hline Nitrate as nitrogen & 10 & 10 & 10 & & 10 & & & & & \\
\hline Nitrite as nitrogen & & & 1 & & 1 & & & & & \\
\hline Nitrate and nitrite & & & & & 10 & & & & & \\
\hline Sulfate & & & & 250 & & 250 & & & & \\
\hline \multicolumn{11}{|c|}{ Metals, (mg/L) } \\
\hline Aluminum & & & & $0.05-0.2$ & & 0.2 & & & & \\
\hline Antimony & & $0.006^{*}$ & $0.006^{*}$ & & 0.006 & & & & & 4.31 \\
\hline Arsenic, total & 0.05 & 0.05 & 0.05 & & 0.05 & & & & & \\
\hline Arsenic (III) & & & & & & & & & 0.36 & \\
\hline Barium & 1 & $2^{*}$ & 2 & & 2 & & & & & \\
\hline Beryllium & & 0.004 & $0.004^{*}$ & & 0.004 & & & & & 0.0013 \\
\hline Cadmium & 0.01 & 0.005 & 0.005 & & 0.005 & & & & & \\
\hline Cadmium, dissolved & & & & & & & & & 0.004 & \\
\hline Chromium, total & 0.05 & & 0.1 & & 0.1 & & & & & \\
\hline Chromium (III) & & & & & & & & & & 670 \\
\hline Chromium (VI) & & 0.1 & & & & & & & 0.016 & \\
\hline Copper & & & $1.3(1)$ & 1 & $1.3(1)$ & 1 & & & & \\
\hline Copper, dissolved & & & & & & & & & 0.018 & \\
\hline Iron & & & & 0.3 & & 0.3 & & & & \\
\hline Lead & 0.05 & 0.05 & $0.015(1)$ & & $0.015(1)$ & & & & & \\
\hline Lead, dissolved & & & & & & & & & 0.082 & \\
\hline Manganese & & & & 0.05 & & 0.05 & & & & \\
\hline Mercury & 0.002 & 0.002 & 0.002 & & 0.002 & . & & & 0.0024 & 0.00015 \\
\hline Nickel & & 0.1 & 0.1 & & 0.1 & & & & 1.4 & 4.6 \\
\hline Selenium & 0.01 & 0.05 & 0.05 & & 0.5 & & & & 0.02 & \\
\hline Silver & 0.05 & 0.01 & & 0.1 & & 0.1 & & & & \\
\hline Silver, dissolved & & & & & & & & & 0.004 & \\
\hline Thallium & & $0.002^{*}$ & $0.002 *$ & & 0.002 & & & & & \\
\hline Uranium & & & $0.020(2)$ & & & & & & & \\
\hline Zinc & & & & 5 & & 5 & & & & \\
\hline Zinc, dissolved & & & & & & & & & 0.117 & \\
\hline
\end{tabular}


Table 7.11 (continued)

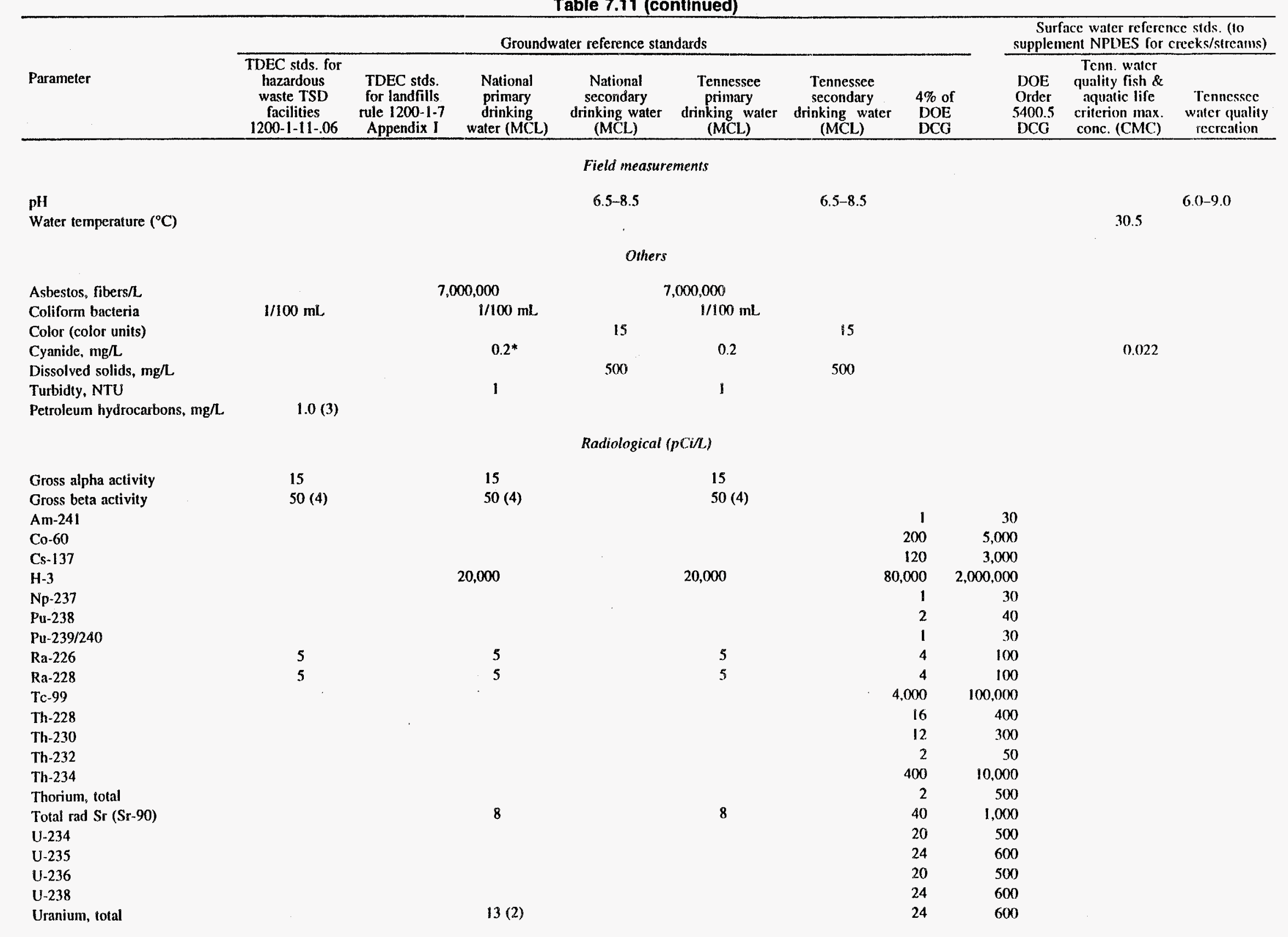


Table 7.11 (continued)

\begin{tabular}{|c|c|c|c|c|c|c|c|c|c|c|}
\hline \multirow[b]{2}{*}{ Parameter } & \multicolumn{7}{|c|}{ Groundwater reference standards } & \multicolumn{3}{|c|}{$\begin{array}{l}\text { Surface water reference stds. (to } \\
\text { supplement NPDES for creeks/streams) }\end{array}$} \\
\hline & $\begin{array}{l}\text { TDEC stds. for } \\
\text { hazardous } \\
\text { waste TSD } \\
\text { facilities } \\
1200 \cdot 1-11-.06 \\
\end{array}$ & $\begin{array}{c}\text { TDEC stds. } \\
\text { for landfills } \\
\text { rule } 1200-1-7 \\
\text { Appendix I }\end{array}$ & $\begin{array}{c}\text { National } \\
\text { primary } \\
\text { drinking } \\
\text { water (MCL) }\end{array}$ & $\begin{array}{c}\text { National } \\
\text { secondary } \\
\text { drinking water } \\
\text { (MCL) }\end{array}$ & $\begin{array}{c}\text { Tennessee } \\
\text { primary } \\
\text { drinking water } \\
\text { (MCL) }\end{array}$ & $\begin{array}{c}\text { Tennessee } \\
\text { secondary } \\
\text { drinking water } \\
\text { (MCL) }\end{array}$ & $\begin{array}{l}4 \% \text { of } \\
\text { DOE } \\
\text { DCG }\end{array}$ & $\begin{array}{c}\text { DOE } \\
\text { Order } \\
5400.5 \\
\text { DCG }\end{array}$ & $\begin{array}{l}\text { Tenn. water } \\
\text { quality fish \& } \\
\text { aquatic life } \\
\text { criterion max. } \\
\text { conc. (CMC) }\end{array}$ & $\begin{array}{c}\text { Tennessee } \\
\text { water quality } \\
\text { recreation }\end{array}$ \\
\hline
\end{tabular}

Volatile organics $(u g / L)$

Acrolein

Acrylonitrile

Benzene

Bromodichloromethane (THM)

Bromoform (THM)

Carbon tetrachloride

Chlorobenzene

Chloroform (THM)

Dibromochloromethane (THM)

1,1-Dichloroethene

1,2-Dichloroethane

cis-1,2-Dichloroethene

trans-1,2-Dichloroethene

1,2-Dichloropropane

cis-1,3-Dichloropropene

trans-1,3-Dichloropropene

Ethylbenzene

Methylene chloride

Styrene

1,1,2,2-Tetrachloroethane

Tetrachloroethene

Total trihalomethanes (THM)

Toluene

1,1,1-Trichloroethane

1,1,2-Trichloroethane

Trichloroethyene

Vinyl chloride

Xylene

$\begin{array}{rr}5 & 5 \\ & 100 \\ 5 & 100 \\ 100 & 5 \\ & 100 \\ & 100 \\ 7 & 100 \\ 5 & 7 \\ 70 & 5 \\ 100 & 70 \\ 5 & 100 \\ & 5 \\ & \\ 700 & 700 \\ 5 * & 5 \\ 100 & 100 \\ & \\ 5 & 5 \\ 100 & 100 \\ 1,000 & 1,000 \\ 200 & 200 \\ 5^{*} & 5 \\ 5 & 5 \\ 2 & 2 \\ 10,000 & 10,000 \\ & \end{array}$

780

6.7

710

4,700

44

4,700

4,700

32
990

-

70

100
5

1,700

1,700

29,000

16,000

5
100

110

5
100

100
1,000

88

100

30,0000

200

170,000

420

5,250

2

Base/neutraVacid extractable organics $(\mu g / L)$

Acenaphthylene

Anthracene

Benzo(a)anthracene

Benzo(a)pyrene

3,4-Benzofluoranthene

Benzo(k)fluoranthene

Di-n-butyl phthalate

bis-(2-chloroethyl)ether 
Table 7.11 (continued)

\begin{tabular}{|c|c|c|c|c|c|c|c|c|c|c|}
\hline \multirow[b]{2}{*}{ Parameter } & \multicolumn{7}{|c|}{ Groundwater reference standards } & \multicolumn{3}{|c|}{$\begin{array}{l}\text { Surface water reference stds. (10 } \\
\text { supplement NPDES for creeks/streams) }\end{array}$} \\
\hline & $\begin{array}{c}\text { TDEC stds. for } \\
\text { hazardous } \\
\text { waste TSD } \\
\text { facilities } \\
1200-1-11-.06 \\
\end{array}$ & $\begin{array}{l}\text { TDEC stds. } \\
\text { for landfills } \\
\text { rule } 1200-1.7 \\
\text { Appendix I }\end{array}$ & $\begin{array}{c}\text { National } \\
\text { primary } \\
\text { drinking } \\
\text { water (MCL) } \\
\end{array}$ & $\begin{array}{c}\text { National } \\
\text { secondary } \\
\text { drinking water } \\
\text { (MCL) }\end{array}$ & $\begin{array}{c}\text { Tennessee } \\
\text { primary } \\
\text { drinking water } \\
\text { (MCL) }\end{array}$ & $\begin{array}{c}\text { Tennessee } \\
\text { secondary } \\
\text { drinking water } \\
\text { (MCL) }\end{array}$ & $\begin{array}{l}4 \% \text { of } \\
\text { DOE } \\
\text { DCG }\end{array}$ & $\begin{array}{c}\text { DOE } \\
\text { Order } \\
5400.5 \\
\text { DCG } \\
\end{array}$ & $\begin{array}{l}\text { Tenn. water } \\
\text { quality fish \& } \\
\text { aquatic life } \\
\text { criterion max. } \\
\text { conc. (CMC) } \\
\end{array}$ & $\begin{array}{c}\text { Tennessec } \\
\text { water quality } \\
\text { recreation }\end{array}$ \\
\hline 1,2-Dichlorobenzene & & 600 & 600 & & 600 & & & \multicolumn{3}{|c|}{17,040} \\
\hline 1,3-Dichlorobenzene & & & & & & & & \multicolumn{3}{|r|}{2.600} \\
\hline 1,4-Dichlorobenzene & & 75 & 75 & & 75 & & & \multicolumn{3}{|r|}{2,600} \\
\hline bis-(2-Ethylhexyl)adipate & & $40^{*}$ & $40^{*}$ & & & & & & & \\
\hline bis-(2-Ethylhexyl)phthalate & & $6^{*}$ & $6^{*}$ & & 6 & & & \multicolumn{3}{|c|}{59} \\
\hline Diethyl phthalate & & & & & & & & \multicolumn{3}{|c|}{120,000} \\
\hline Dimethyl phthalate & & & & & & & & \multicolumn{3}{|c|}{$2,900.000$} \\
\hline 2,4-Dinitrophenol & & & & & & & & \multicolumn{3}{|r|}{1.400} \\
\hline 2,4-Dinitrotoluene & & & & & & & & \multicolumn{3}{|r|}{42} \\
\hline Fluoranthene & & & & & & & & \multicolumn{3}{|r|}{54} \\
\hline Fluorene & . & & & & & & & \multicolumn{3}{|r|}{0.03} \\
\hline Hexachlorobenzene & & $\mathbf{1}^{*}$ & $\mathbf{l}^{*}$ & & 1 & & & & & 0.007 \\
\hline Hexachlorobutadiene & & & & & & & & \multicolumn{3}{|r|}{500} \\
\hline Hexachlorocyclopentadiene & & $50^{*}$ & $50^{*}$ & & & & & \\
\hline Hexachloroethane & & & & & & & & \multicolumn{3}{|r|}{89} \\
\hline 2-Methyl-4,6-dinitrophenol & & & & & & & & \multicolumn{3}{|r|}{765} \\
\hline Nitrobenzene & & & & & & & & \multicolumn{3}{|r|}{1,900} \\
\hline Phenathrene & & & & & & & & \multicolumn{3}{|r|}{0.03} \\
\hline Pentachlorophenol & & 1 & 1 & & 1 & & & \multicolumn{3}{|c|}{20} \\
\hline Pyrene & & & & & & & & \multirow{2}{*}{\multicolumn{3}{|c|}{0.03}} \\
\hline 1,2,4-Trichlorobenzene & & $70^{*}$ & $70^{*}$ & & 70 & & & & & \\
\hline 2,4,6-Trichlorophenol & & & & & & & & \multicolumn{3}{|r|}{6.5} \\
\hline \multicolumn{11}{|c|}{ Pesticides/herbicides/PCBs $(\mu g /)$} \\
\hline Alachlor & & 2 & 2 & & 2 & & & & & \\
\hline Aldicarb & & 3 & & & & & & & & \\
\hline Aldicarb sulfoxide & & 4 & 4 & & & & & & & \\
\hline Aldicarb sulfone & & 2 & 2 & & & & & & & \\
\hline Aldrin & & & & & & & & & 3 & 0.0014 \\
\hline Atrazine & & 3 & 3 & & 3 & & & & & \\
\hline Carbofuran & & 40 & 40 & & 40 & & & & & \\
\hline Chlorodane & & 2 & 2 & & 2 & & & & 2.4 & 0.006 \\
\hline $2,4-10$ & 100 & 70 & 70 & & 70 & & & & & \\
\hline $4,4^{\prime}-\mathrm{DDT}$ & & & & & & & & & 1.1 & 0.1006 \\
\hline $4,4^{\prime}-\mathrm{DDE}$ & & & & & & . & & & & 0.066 \\
\hline $4,4^{\prime}-\mathrm{DDD}$ & & & & & & & & & & 0.008 \\
\hline Dalapon & & 200 & 200 & & 200 & & & & & \\
\hline 1,2-Dibromo-3-chloropropane & & & 0.2 & & 0.2 & & & & & \\
\hline Dieldren & & & & & & & & & 2.5 & 0.0014 \\
\hline Dinoseb & & $7^{*}$ & $7^{*}$ & & 7 & & & & & \\
\hline Diquat & & $20^{*}$ & $20^{*}$ & & 20 & & & & & \\
\hline
\end{tabular}


Table 7.11 (continued)

\begin{tabular}{|c|c|c|c|c|c|c|c|c|c|c|}
\hline \multirow[b]{2}{*}{ Parameter } & \multicolumn{7}{|c|}{ Groundwater reference standards } & \multicolumn{3}{|c|}{$\begin{array}{c}\text { Surface water reference } \text { stds. (to } \\
\text { supplement NPDES for creeks/streams) }\end{array}$} \\
\hline & $\begin{array}{l}\text { TDEC stds. for } \\
\text { hazardous } \\
\text { waste TSD } \\
\text { facilities } \\
1200-1-11-.06 \\
\end{array}$ & $\begin{array}{c}\text { TDEC stds. } \\
\text { for landfills } \\
\text { rule } 1200-1-7 \\
\text { Appendix I } \\
\end{array}$ & $\begin{array}{c}\text { National } \\
\text { primary } \\
\text { drinking } \\
\text { water (MCL) }\end{array}$ & $\begin{array}{c}\text { National } \\
\text { secondary } \\
\text { drinking water } \\
(\mathrm{MCL})\end{array}$ & $\begin{array}{c}\text { Tennessee } \\
\text { primary } \\
\text { drinking water } \\
(\mathrm{MCL}) \\
\end{array}$ & $\begin{array}{c}\text { Tennessee } \\
\text { secondary } \\
\text { drinking water } \\
\text { (MCL) }\end{array}$ & $\begin{array}{l}4 \% \text { of } \\
\text { DOE } \\
\text { DCG }\end{array}$ & $\begin{array}{c}\text { DOE } \\
\text { Order } \\
5400.5 \\
\text { DCG } \\
\end{array}$ & $\begin{array}{l}\text { Tenn. water } \\
\text { quality fish \& } \\
\text { aquatic life } \\
\text { criterion max. } \\
\text { conc. (CMC) } \\
\end{array}$ & $\begin{array}{c}\text { Tennessee } \\
\text { water quality } \\
\text { recreation }\end{array}$ \\
\hline Endothall & & $100^{*}$ & $100^{*}$ & & 100 & & & & & \\
\hline a-Endosulfan & 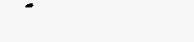 & & & & & & & & 0.22 & 2 \\
\hline b-Endosulfan & & & & & & & & & 0.22 & 2 \\
\hline Endrin & 0.2 & $2^{*}$ & $2^{*}$ & & 2 & & & & 0.18 & \\
\hline Ethylene dibromide & & 0.05 & 0.05 & & 0.05 & & & & & \\
\hline Glyghosate & & $700^{*}$ & $700^{*}$ & & 700 & & & & & \\
\hline Heptachlor & & 0.4 & 0.4 & & 0.4 & & & & 0.52 & 0.002 \\
\hline Heptachlor epoxide & & 0.2 & 0.2 & & 0.2 & & & & 2 & 0.001 \\
\hline g-BHC (Lindane) & 4 & 0.2 & 0.2 & & 0.2 & & & & 2 & 0.63 \\
\hline Methoxychlor & 100 & 40 & 40 & & 40 & & & & & \\
\hline Oxamyl (Vydate) & & $200^{*}$ & $200^{*}$ & & 200 & & & & & \\
\hline Picloram & & $500^{*}$ & $500^{*}$ & & 500 & & & & & \\
\hline PCB-1242 & & & & & & & & & & 0.000 .5 \\
\hline PCB-1254 & & · & & & & & & & & 0.0005 \\
\hline PCB-1221 & & & & & & & & & & 0.0005 \\
\hline PCB-1232 & & & & & & & & & & 0.0005 \\
\hline РCB-1248 & & & & & & & & & & 0.0005 \\
\hline PCB- 1260 & & & & & & & & & & 0.0005 \\
\hline PCB-1016 & & & & & & & & & & 0.0005 \\
\hline PCB total & & 0.5 & 0.5 & & 0.5 & & & & & \\
\hline Simazine & & $4^{*}$ & $4^{*}$ & & 3 & & & & & \\
\hline 2,3,7,8-TCDD (Dioxin) & & & $0.00003^{*}$ & & 0.00003 & & & & & 0.000001 \\
\hline 2,4,5-TP (Silvex) & 10 & 50 & 50 & & & & & & & \\
\hline Toxaphene & 5 & 3 & 3 & & 3 & & & & 0.73 & 0.008 \\
\hline
\end{tabular}

Toxaphene

Notes:

TDEC standards for hazardous waste facilities-TDEC rule 1200-1-11-.05, Appendix B.

National Primary Drinking Water MCLs-enforceable health-based maximum containment levels for water supply systems.

National Secondary Drinking Water MCLs-nonenforceable taste, odor, or appearance guidelines.

4\% of DCG-represents DOE criteria of 4 mrem effective dose equivalent.

Fish and aquatic life criteria maximum concentration-TDEC general water quality criteria and use classification of streams; TDEC rule 1200-4-3 and 1200-4-4.

The items marked with* indicate those standards that are not effective until January 17, 1994. All other values are currently applicable MCLs.

(1) Treatment-based standard.

(2) Proposed by EPA.

(3) TDEC UST regulation for groundwater.

(4) Regulatory standard is $4 \mathrm{mrem} / y e a r$ effective dose equivalent. $50 \mathrm{pCi} / \mathrm{L}$ is recommended level for screening of beta activity. 
Table 7.12. Constlluents in groundwater at the $Y-12$ Plant site $\mathrm{HGR}=\mathrm{BC}$ area $=$ Background

\begin{tabular}{|c|c|c|c|c|c|c|c|c|c|c|}
\hline & VARIABLE & & & $\begin{array}{c}\# \\
\text { SAMPLES }\end{array}$ & $\begin{array}{c}\# \\
\text { DETECTED }\end{array}$ & $\begin{array}{c}\text { MAXIMUM } \\
\text { DETECTED } \\
\text { MMT. }\end{array}$ & $\begin{array}{l}\text { MINIMUM } \\
\text { DETECTED } \\
\text { MMT. }\end{array}$ & $\begin{array}{c}\text { AVERAGE } \\
\text { DETECTED } \\
\text { MMTS. }\end{array}$ & $\begin{array}{c}\text { REFERENCE } \\
\text { VALUE }\end{array}$ & $\begin{array}{l}\text { \# MMTS. > } \\
\text { REFERENCE }\end{array}$ \\
\hline CHLORIDE & & & (MG/L) & 43 & 38 & 4.1 & 1.0 & 1.8 & 250.000 & 0 \\
\hline FLUORIDE & & & $(M G / L)$ & 43 & 28 & 1.7 & 0.10 & 0.35 & 2.000 & 0 \\
\hline SULFATE & & & (MG/L) & 43 & 43 & 33 & 3.0 & 15 & 250.000 & 0 \\
\hline ALUMINUM & & ICAP & (MG/L) & 43 & 35 & 44 & 0.027 & 3.7 & 0.200 & 23 \\
\hline ALUMINUM & FILTERED & ICAP & (MG/L) & 43 & 11 & 0.27 & 0.022 & 0.057 & 0.200 & 1 \\
\hline ARSENIC & & ICAP & (MG/L) & 43 & 2 & 0.062 & 0.057 & 0.060 & 0.050 & 2 \\
\hline BARIUM & & ICAP & (MG/L) & 43 & 43 & 0.35 & 0.015 & 0.15 & 1.000 & 0 \\
\hline BARIUM & FILTERED & ICAP & $(M G / L)$ & 43 & 43 & 0.24 & 0.013 & $0: 12$ & 1.000 & 0 \\
\hline BERYLLIUM & & ICAP & (MG/L) & 43 & 7 & 0.0018 & 0.0003 & 0.0008 & $0.004 * \star$ & 0 \\
\hline BERYLLIUM & FILTERED & ICAP & $(M G / L)$ & 43 & 1 & 0.0007 & 0.0007 & 0.0007 & $0.004 * *$ & 0 \\
\hline BORON & & ICAP & (MG/L) & 43 & 42 & 0.18 & 0.0041 & 0.032 & NR & $\mathrm{NA}$ \\
\hline BORON & FILTERED & ICAP & (MG/L) & 43 & 43 & 0.17 & 0.0048 & 0.031 & NR & NA \\
\hline CADMIUM & & AAS & (MG/L) & 43 & 4 & 0.066 & 0.0020 & 0.027 & 0.005 & 3 \\
\hline CADMIUM & & ICAP & $(M G / L)$ & 43 & 6 & 0.062 & 0.0038 & 0.019 & 0.005 & 3 \\
\hline CADMIUM & FILTERED & AAS & $(M G / L)$ & 43 & 5 & 0.077 & 0.0021 & 0.019 & 0.005 & 3 \\
\hline CALCIUM & & ICAP & $(M G / L)$ & 43 & 43 & 74 & 1.3 & 31 & NR & NA \\
\hline CALCIUM & FILTERED & ICAP & $(M G / L)$ & 43 & 43 & 72 & 1.1 & 30 & NR & NA \\
\hline CHROMIUM & & AAS & (MG/L) & 43 & 8 & 0.22 & 0.011 & 0.066 & 0.050 & 3 \\
\hline CHROMIUM & & ICAP & (MG/L) & 43 & 8 & 0.098 & 0.010 & 0.044 & 0.050 & 3 \\
\hline COBALT & & ICAP & (MG/L) & 43 & 7 & 0.021 & 0.0052 & 0.012 & NR & NA \\
\hline COBALT & FILTERJDD & ICAP & (MG/L) & 43 & 2 & 0.0065 & 0.0062 & 0.0064 & NR & NA \\
\hline COPPER & & ICAP & (MG/L) & 43 & 24 & 0.10 & 0.0042 & 0.018 & 1.000 & 0 \\
\hline COPPF $R$ & FILTERED & ICAP & (MG/L) & 43 & 10 & 0.037 & 0.0040 & 0.012 & 1.000 & 0 \\
\hline IRON & & ICAP & $(\mathrm{MG} / \mathrm{L})$ & 43 & 43 & 46 & 0.0050 & 4.3 & 0.300 & 28 \\
\hline IRON & FILTERID & ICAP & (MG/L) & 43 & 31 & 1.2 & 0.0069 & 0.16 & 0.300 & 5 \\
\hline LEAD & & AAS & (MG/L) & 43 & 9 & 0.042 & 0.0047 & 0.022 & 0.050 & 0 \\
\hline MAGNESIUM & & ICAP & (MG/L) & 43 & 43 & 12 & 0.14 & 5.6 & NR & $\mathrm{N} \Lambda$ \\
\hline MAGNESIUM & FILTERIED & ICAP & (MG/L) & 43 & 43 & 12 & 0.11 & 5.0 & NR & $\mathrm{NA}$ \\
\hline MANGANESE & & ICAP & (MG/L) & 43 & 43 & 1.4 & 0.0034 & 0.19 & 0.050 & 1.8 \\
\hline
\end{tabular}


Table 7.12 (continued)

\begin{tabular}{|c|c|c|c|c|c|c|c|c|}
\hline VARIABLE & & $\begin{array}{c}\# \\
\text { SAMPLES }\end{array}$ & $\begin{array}{c}\# \\
\text { DETECTED }\end{array}$ & $\begin{array}{c}\text { MAXIMUM } \\
\text { DETECTED } \\
\text { MMT. }\end{array}$ & $\begin{array}{l}\text { MINIMUM } \\
\text { DETECTED } \\
\text { MMT. }\end{array}$ & $\begin{array}{l}\text { AVERAGE } \\
\text { DETECTED } \\
\text { MMTS. }\end{array}$ & $\begin{array}{l}\text { REFERENCE } \\
\text { VALUE }\end{array}$ & $\begin{array}{l}\text { \# MMTS. > } \\
\text { REFERENCE }\end{array}$ \\
\hline MANGANESE & $\operatorname{ICAP}(\mathrm{MG} / \mathrm{L})$ & 43 & 40 & 1.3 & 0.0014 & 0.16 & 0.050 & 13 \\
\hline MERCURY & CVAA (MG/L) & 43 & 2 & 0.0003 & 0.0002 & 0.0002 & 0.002 & 0 \\
\hline FILTERED & CVAA (MG/L) & 43 & 2 & 0.0003 & 0.0002 & 0.0002 & 0.002 & 0 \\
\hline POTASSIUM & ICAP (MG/L) & 43 & 39 & 8.6 & 0.60 & 2.0 & $\mathrm{NR}$ & NA \\
\hline POTASSIUM & ICAP $(M G / L)$ & 43 & 37 & 3.1 & 0.60 & 1.4 & NR & $\mathrm{NA}$ \\
\hline SILVER & ICAP (MG/L) & 43 & 1 & 0.0074 & 0.0074 & 0.0074 & 0.100 & 0 \\
\hline SODIUM & ICAP $(\mathrm{MG} / \mathrm{L})$ & 43 & 43 & 110 & 1.4 & 16 & NR & NA \\
\hline FILTERED & ICAP (MG/L) & 43 & 43 & 110 & 1.4 & 16 & NR & NA \\
\hline STRONTIUM & ICAP (MG/L) & 43 & 43 & 0.66 & 0.010 & 0.11 & NR & NA \\
\hline ZINC & ICAP (MG/L) & 43 & 41 & 1.6 & 0.0024 & 0.063 & 5.000 & 0 \\
\hline FILTERED & ICAP (MG $/ L)$ & 43 & 38 & 0.17 & 0.0023 & 0.023 & 5.000 & 0 \\
\hline CONDUCTIVITY, FIELD MMT & T (UMHOS/CM) & 43 & NA & 480 & 35 & 250 & NR & NA \\
\hline DISSOLVED OXYGEN, FIELD & D MMT. (PPM) & 43 & NA & 12 & 0.30 & 3.9 & NR & NA \\
\hline PH, FIELD MMT. & (PH UNITS) & 43 & NA & 9.2 & 5.2 & 7.2 & $6.5 / 8.5$ & 14 \\
\hline REDOX, FIELD MMT . & (MV) & 43 & NA & 260 & -230 & 110 & NR & $\mathrm{NA}$ \\
\hline WATER TEMP, FIELD MMT । & (DEG. CENT.) & 43 & NA & 19 & 12 & 15 & NR & 0 \\
\hline ALKALINITY-CO3 & (MG/L) & 43 & 3 & 26 & 24 & 25 & NR & NA \\
\hline ALKALINI TY-HCO3 & (MG /L) & 43 & 43 & 240 & 8.0 & 120 & NR & $\mathrm{NA}$ \\
\hline CONDUCTIVITY & (UMHOS /CM) & 43 & 43 & 490 & 36 & 260 & $\mathrm{NR}$ & $\mathrm{NA}$ \\
\hline DISSOLVED SOLIDS & (MG/L) & 43 & 43 & 360 & 50 & 200 & 500.000 & 0 \\
\hline PH & (PH UNITS) & 43 & NA & 9.1 & 5.7 & 7.5 & $6.5 / 8.5$ & 11 \\
\hline TOTAL SUSPENDED SOLIDS & $(M G / L)$ & 43 & 32 & 730 & 1.0 & 63 & NR & NA \\
\hline
\end{tabular}


Table 7.12 (continued)

\begin{tabular}{|c|c|c|c|c|c|c|c|c|c|c|c|c|}
\hline VARIABLE & & $\begin{array}{c}\# \\
\text { SAMPLES }\end{array}$ & $\begin{array}{c}\# \\
\text { DETECTED }\end{array}$ & $\begin{array}{c}\text { MAXIMUM } \\
\text { DETECTED } \\
\text { MMT. }\end{array}$ & & & $\begin{array}{l}\text { MINIMUM } \\
\text { DETECTED } \\
\text { MMT. }\end{array}$ & & & $\begin{array}{c}\text { AVERAGE } \\
\text { DETECTED } \\
\text { MMTS. }\end{array}$ & $\begin{array}{c}\text { REFERENCE } \\
\text { VALUE }\end{array}$ & $\begin{array}{l}\text { \# MMTS. > } \\
\text { REFERENCE }\end{array}$ \\
\hline 234-URANIUM & $(\mathrm{PCI} / \mathrm{L})$ & 16 & 16 & 9.1 & & $\mathrm{~J}$ & -7.2 & & & 2.9 & 20.000 & 0 \\
\hline 235-URANIUM & $(\mathrm{PCI} / \mathrm{L})$ & 16 & 16 & 200 & & & -43 & & $F$ & 29 & 24.000 & 4 \\
\hline 237-NEPTUNIUM & (PCI/L) & 16 & 16 & 1.8 & & & -12 & & & -0.21 & 1.000 & 2 \\
\hline 238-URANIUM & $(\mathrm{PCI} / \mathrm{L})$ & 16 & 16 & 22 & & & -0.19 & & & 4.9 & 24.000 & 0 \\
\hline 241-AMERICIUM & $(\mathrm{PCI} / \mathrm{L})$ & 16 & 16 & 10 & & $\mathfrak{J}$ & -5.2 & & & 0.61 & 1.000 & 4 \\
\hline 99-TECHNETIUM & $(\mathrm{PCI} / \mathrm{L})$ & 16 & 16 & 110 & & & 11 & & & 54 & 4000.00 & 0 \\
\hline CESIUM-137 & $(\mathrm{PCI} / \mathrm{L})$ & 6 & 6 & 29 & & & -7.5 & & $F$ & 9.5 & 120 & NA \\
\hline GROSS ALPHA & $(\mathrm{PCI} / \mathrm{L})$ & 43 & 43 & 11 & & $s$ & -2.5 & & & 0.82 & 15.000 & 0 \\
\hline GROSS BETA & $(\mathrm{PCI} / \mathrm{L})$ & 43 & 43 & 23 & & & -14 & & & -0.54 & 50.000 & 0 \\
\hline PLUTONIUM-238 & $(\mathrm{PCT} / \mathrm{L})$ & 16 & 16 & 14 & & & -28 & & & -0.56 & 2.000 & 2 \\
\hline PLUTONIUM-239 & $(\mathrm{PCT} / \mathrm{L})$ & 16 & 16 & 15 & & & -7.4 & & & 1.1 & 1.000 & 2 \\
\hline PROTACTINIUM-234 & (PCI/L) & 5 & 5 & 2400 & & $\mathbf{E}$ & -780 & & $\mathbf{E}$ & 1200 & 2800 & NA \\
\hline RADIUM & $(\mathrm{PCI} / \mathrm{L})$ & 16 & 16 & 0.24 & & & 0,0000 & & & 0.055 & 4.000 & 0 \\
\hline RUTHENIUM-106 & $(\mathrm{PCI} / \mathrm{L})$ & 2 & 2 & 360 & & & 0.38 & & I & 180 & 240 & NA \\
\hline STRONTIUM & $(\mathrm{PCI} / L)$ & 16 & 1.6 & 61 & & & 0.075 & & & 38 & 8.000 & 1.4 \\
\hline THORIUM-228 & $(\mathrm{PCI} / \mathrm{L})$ & 2 & 2 & 1400 & & $\mathbf{E}$ & 7.4 & & $\mathrm{I}$ & 700 & 16 & NA \\
\hline TRITIUM & $\left(\mathrm{PCI} / \mathrm{I}_{3}\right)$ & 1.5 & 1.5 & 700 & & & -190 & & & 180 & 20000.0 & 0 \\
\hline 2-BUTANONE & $(U G / L)$ & 43 & 9 & 12 & B & & 7.0 & $\mathrm{BJ}$ & & 8.9 & NR & NA \\
\hline 4-METHYL-2-PENTANONE & (UG/L) & 43 & 7 & 3.0 & BJ & & 1.0 & $\mathbf{B J}$ & & 1.6 & NR & NA \\
\hline ACETONE & (UG/L) & 43 & 10 & 3.0 & $\mathrm{BJ}$ & & 1.0 & $\mathbf{B J}$ & & 2.1 & NR & $\mathrm{NA}$ \\
\hline CHLOROBENZENE & $(U G / L)$ & 43 & 1 & 0.60 & $\checkmark$ & & 0.60 & $\mathfrak{J}$ & & 0.60 & 100.000 & 0 \\
\hline METHYLENE CHLORIDE & (UG/L) & 43 & 20 & 3.0 & $\mathrm{BJ}$ & & 0.70 & $\mathrm{~J}$ & & 1.8 & NR & NA \\
\hline
\end{tabular}


Table 7.13. Constituents In groundwater at the $Y-12$ Plant site

$H G R=B C$ area=Above Ground Low-Level Storage Facility

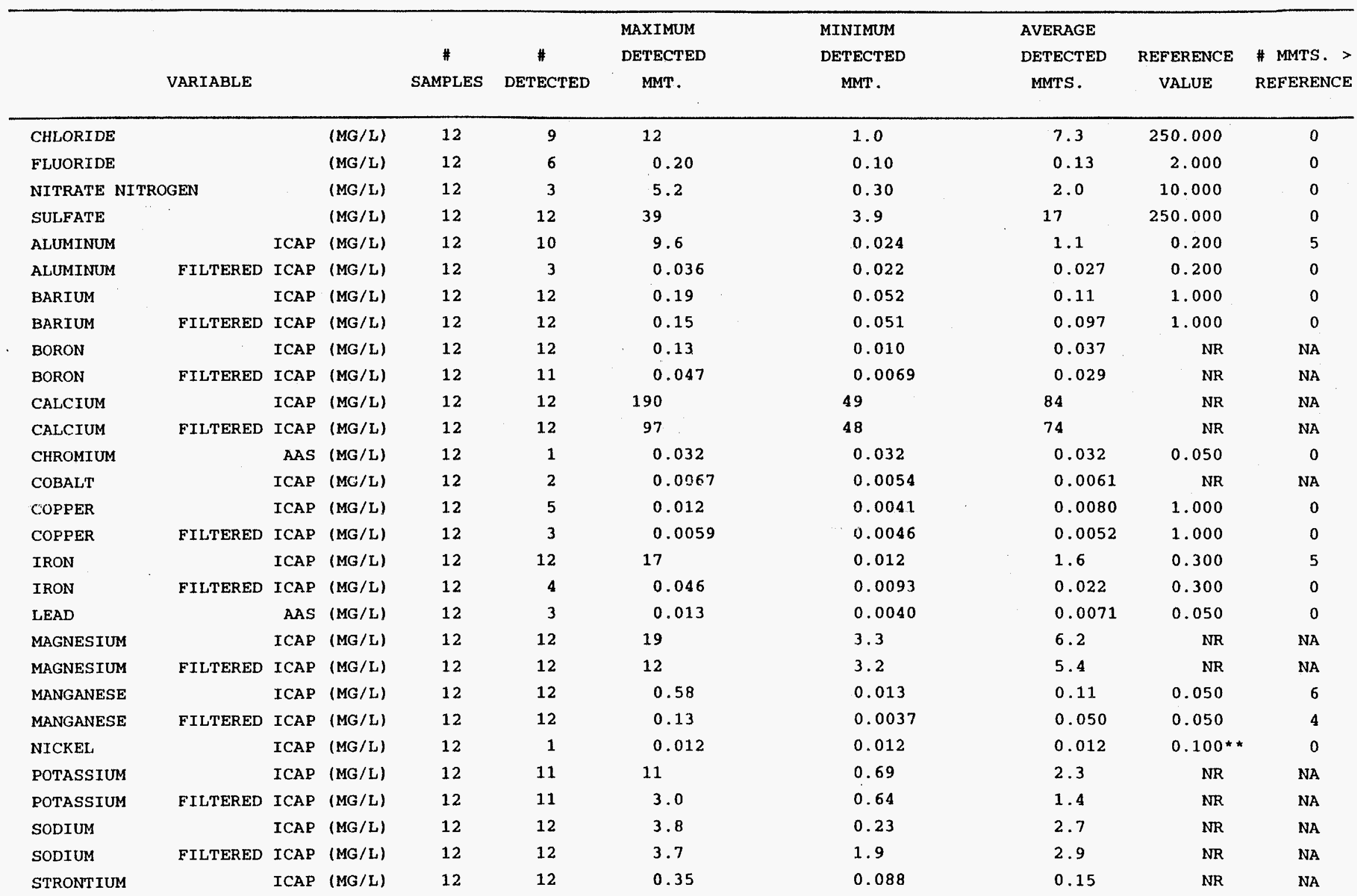


Table 7.13 (continued)

\begin{tabular}{|c|c|c|c|c|c|c|c|c|c|}
\hline VARIABLE & $\begin{array}{c}\# \\
\text { SAMPLES }\end{array}$ & $\begin{array}{c}\# \\
\text { DETECTED }\end{array}$ & $\begin{array}{c}\text { MAXIMUM } \\
\text { DETECTED } \\
\text { MMT. }\end{array}$ & & $\begin{array}{l}\text { MINIMUM } \\
\text { DETEC KED } \\
\text { MMT. }\end{array}$ & & $\begin{array}{l}\text { AVERAGE } \\
\text { DETECTED } \\
\text { MMTS. }\end{array}$ & $\begin{array}{c}\text { REFERENCE } \\
\text { VALUE }\end{array}$ & $\begin{array}{l}\# \text { MMTS. > } \\
\text { REFERENCE }\end{array}$ \\
\hline FILTERED ICAP (MG/L) & 12 & 12 & 0.16 & & 0.085 & & 0.13 & NR & NA \\
\hline FLUORIMETRIC (MG/L) & 12 & 10 & 0.0030 & & 0.001 .0 & & 0.001 .3 & 13.000 & 0 \\
\hline URANIUM FILT. FLUORIMETRIC (MG/L) & 12 & 8 & 0.0030 & & 0.001 .0 & & 0.0013 & 13.000 & 0 \\
\hline ICAP (MG/L) & 12 & 12 & 0.069 & & 0.0068 & & 0.017 & 5.000 & 0 \\
\hline FILTERED ICAP (MG/L) & 12 & 12 & 0.022 & & 0.0060 & & 0.011 & 5.000 & 0 \\
\hline CONDUCTIVITY, FIELD MMT (UMHOS/CM) & 12 & $\mathrm{NA}$ & 440 & & 240 & & 360 & NR & $\mathrm{NA}$ \\
\hline DISSOLVED OXYGEN, FIELD MMT. (PPM) & 12 & NA & 9.0 & & 0.40 & & 3.2 & NR & NA \\
\hline (PH UNITS) & 12 & NA & 7.7 & & 6.7 & & 7.1 & $6.5 / 8.5$ & 0 \\
\hline REDOX, FIELD MMT. & 12 & NA & 260 & & 89 & & 160 & NR & NA \\
\hline WATER TEMP, FIELD MMT (DEG. CENT.) & 12 & NA & 18 & & 12 & & 15 & NR & 0 \\
\hline ALKALINITY-HCO3 & 12 & 12 & 230 & & 150 & & 190 & NR & NA \\
\hline CONDUCTIVITY & 12 & 12 & 520 & & 290 & & 410 & NR & NA \\
\hline DISSOLVED SOLIDS & 12 & 12 & 370 & & 190 & & 260 & 500.000 & 0 \\
\hline (PH UNITS) & 12 & NA & 7.8 & & 7.3 & & 7.5 & $6.5 / 8.5$ & 0 \\
\hline TOTAL SUSPENDED SOLIDS & 12 & 8 & 220 & & 1.0 & & 36 & NR & NA \\
\hline TURBIDITY & 12 & 12 & 110 & & 1.0 & & 14 & 1.000 & 11 \\
\hline 234-URANIUM & 12 & 12 & 280 & & -11 & & 34 & 20.000 & 3 \\
\hline 235-URANIUM & 12 & 12 & 68 & & -24 & F & 28 & 24.000 & 6 \\
\hline 238-URANIUM & 12 & 12 & 74 & & -18 & & 15 & 24.000 & 4 \\
\hline CESIUM-137 & 1 & $\mathbb{1}$ & 33 & $\mathrm{E}$ & 33 & $\mathbf{E}$ & 33 & 120.000 & 0 \\
\hline GROSS ALPHA & 12 & 12 & 3.2 & & -6.2 & & -1.5 & 15.000 & 0 \\
\hline GROSS BETA & 12 & 12 & 10 & & -8.5 & & 1.9 & 50.000 & 0 \\
\hline POTASSIUM- 40 & 1 & 1 & 560 & I & 560 & I & 560 & NR & NA \\
\hline PROTACTINIUM-234 & 1 & 1 & 9600 & & 9600 & & 9600 & 2800 & 1 \\
\hline 2-BUTANONE & 12 & 5 & 10 & $\mathrm{~B}$ & 3.0 & $\mathrm{BJ}$ & 7.0 & NR & NA \\
\hline 4-METHYL-2-PENTANONE & 12 & 3 & 2.0 & BJ & 1.0 & $\mathrm{BJ}$ & 1.7 & NR & NA \\
\hline ACETONE & 12 & 4 & 2.0 & $\mathrm{BJ}$ & 1.0 & $\mathfrak{J}$ & 1.5 & $\mathrm{NR}$ & NA \\
\hline METHYLENE CHLORIDE & 12 & 4 & 3.0 & $\mathrm{BJ}$ & 1.0 & $\mathrm{BJ}$ & 2.0 & NR & NA \\
\hline
\end{tabular}


Table 7.14. Constltuents in groundwater at the $Y-12$ Plant site HGR $=B C$ area=Bear Creek Burial Grounds

\begin{tabular}{|c|c|c|c|c|c|c|c|c|c|c|}
\hline \multicolumn{4}{|c|}{ VARIABLE } & \multirow{2}{*}{$\begin{array}{c}\# \\
\text { SAMPLES } \\
95\end{array}$} & \multirow{2}{*}{$\begin{array}{c}\# \\
\text { DETECTED } \\
84\end{array}$} & \multirow{2}{*}{$\begin{array}{c}\text { MAXIMUM } \\
\text { DETECTED } \\
\text { MMT }\end{array}$} & \multirow{2}{*}{$\begin{array}{c}\begin{array}{c}\text { MINIMUM } \\
\text { DETECTED } \\
\text { MMT } .\end{array} \\
1.0\end{array}$} & \multirow{2}{*}{$\begin{array}{c}\text { AVERAGE } \\
\text { DETECTED } \\
\text { MMTS. } \\
170\end{array}$} & \multirow{2}{*}{$\begin{array}{c}\text { REFERENCE } \\
\text { VALUE } \\
250.000\end{array}$} & \multirow{2}{*}{$\begin{array}{c}\text { \# MMTS. > } \\
\text { REFERENCE } \\
4\end{array}$} \\
\hline CHLORIDE & & & (MG/L) & & & & & & & \\
\hline FLUORIDE & & & (MG/L) & 95 & 66 & 6.0 & 0.10 & 0.84 & 2.000 & 8 \\
\hline NIMKATE NI & GEEN & & (MG/L) & 95 & 6 & 12 & 0.20 & 3.3 & 10.000 & 1 \\
\hline SULFATE & & & (MG/L) & 95 & 88 & 40 & 1.8 & 14 & 250.000 & 0 \\
\hline ALUMINUMM & & ICAP & (MG/L) & 95 & 71 & 23 & 0.020 & 2.2 & 0.200 & 48 \\
\hline ALUMINUM & FILTERED & ICAP & (MG/L) & 95 & 23 & 0.27 & 0.022 & 0.053 & 0.200 & 1 \\
\hline ARSENIC & & ICAP & (MG/L) & 95 & 3 & 0.062 & 0.053 & 0.057 & 0.050 & 3 \\
\hline ARSENIC & FILTERED & ICAP & (MG/L) & 95 & 1 & 0.060 & 0.060 & 0.060 & 0.050 & 1 \\
\hline BARIUM & & ICAP & (MG/L) & 95 & 94 & 0.96 & 0.0058 & 0.15 & 1.000 & 0 \\
\hline BARIUM & FILTERED & ICAP & (MG/L) & 95 & 94 & 0.41 & 0.017 & 0.12 & 1.000 & 0 \\
\hline BERYLLIUM & & ICAP & (MG/L) & 95 & 17 & 0.0038 & 0.0003 & 0.0011 & $0.004 * *$ & 0 \\
\hline BERYLLIUM & FILTERED & ICAP & (MG/L) & 95 & 5 & 0.0008 & 0.0004 & 0.0005 & $0.004 * *$ & 0 \\
\hline BORON & & ICAP & (MG/L) & 95 & 92 & 0.65 & 0.0056 & 0.085 & NR & NA \\
\hline BORON & FILTERED & ICAP & (MG/L) & 95 & 93 & 0.59 & 0.0043 & 0.080 & NR & NA \\
\hline CADMIUM & & AAS & (MG/L) & 95 & 6 & 0.066 & 0.0045 & 0.024 & 0.005 & 3 \\
\hline CADMIUM & & ICAP & (MG/L) & 95 & 10 & 0.052 & 0.0036 & 0.016 & 0.005 & 6 \\
\hline CADMIUM & FILTERED & AAS & (MG /L) & 95 & 6 & 0.077 & 0.0021 & 0.016 & 0.005 & 2 \\
\hline CALCIUM & & ICAP & (MG/L) & 95 & 95 & 82 & 0.85 & 26 & NR & NA \\
\hline CALCIUM & FILTERED & ICAP & (MG/L) & 95 & 95 & 81 & 0.75 & 26 & NR & $\mathrm{NA}$ \\
\hline CHROMIUM & & AAS & (MG/L) & 95 & 13 & 0.29 & 0.011 & 0.067 & 0.050 & 3 \\
\hline CHROMIUM & & ICAP & (MG/L) & 95 & 13 & 0.18 & 0.010 & 0.043 & 0.050 & 3 \\
\hline COBALT & & ICAP & $(M G / L)$ & 95 & 10 & 0.023 & 0.0051 & 0.011 & NR & NA \\
\hline COBALT & FILTERED & ICAP & (MG/L) & 95 & 2 & 0.0065 & 0.0062 & 0.0064 & NR & NA \\
\hline COPPER & & ICAP & (MG/L) & 95 & 39 & 0.054 & 0.0040 & 0.011 & 1.000 & 0 \\
\hline COPPER & FILTERED & ICAP & (MG/L) & 95 & 19 & 0.078 & 0.0043 & 0.012 & 1.000 & 0 \\
\hline IRON & & ICAP & (MG/L) & 95 & 94 & 93 & 0.0094 & 4.2 & 0.300 & 62 \\
\hline IRON & FILTERED & ICAP & (MG/L) & 95 & 64 & 2.8 & 0.0050 & 0.25 & 0.300 & 12 \\
\hline LEAD & & AAS & (MG/L) & 95 & 21 & 0.040 & 0.0040 & 0.015 & 0.050 & 0 \\
\hline LEAD & & ICAP & (MG/L) & 49 & 1 & 0.062 & 0.062 & 0.062 & 0.050 & 1 \\
\hline
\end{tabular}


Table 7.14 (continued)

\begin{tabular}{|c|c|c|c|c|c|c|c|c|c|}
\hline VARIABLE & & & $\begin{array}{c}\# \\
\text { SAMPLES }\end{array}$ & $\begin{array}{c}\# \\
\text { DETECTED }\end{array}$ & $\begin{array}{c}\text { MAXIMUM } \\
\text { DETECTED } \\
\text { MMT. }\end{array}$ & $\begin{array}{l}\text { MINIMUM } \\
\text { DETECTED } \\
\text { MMT. }\end{array}$ & $\begin{array}{l}\text { AVERAGE } \\
\text { DETECTED } \\
\text { MMTS. }\end{array}$ & $\begin{array}{c}\text { REFERENCE } \\
\text { VALUE }\end{array}$ & $\begin{array}{l}\# \text { MMTS. > } \\
\text { REFERENCE }\end{array}$ \\
\hline MAGNESIUM & ICAP & (MG/L) & 95 & 95 & 21 & 0.041 & 5.3 & NR & NA \\
\hline MAGNESIUM & ICAP & $(M G / L)$ & 95 & 95 & 24 & 0.035 & 5.1 & NR & NA \\
\hline MANGANESE & ICAP & $(M G / L)$ & 95 & 91 & 2.4 & 0.0012 & 0.21 & 0.050 & 36 \\
\hline MANGANESE & ICAP & (MG/L) & 95 & 83 & 1.3 & 0.0010 & 0.15 & 0.050 & 21 \\
\hline MERCURY & CVAA & $(M G / L)$ & 95 & 3 & 0.0003 & 0.0002 & 0.0002 & 0.002 & 0 \\
\hline FILTERED & CVAA & (MG/L) & 95 & 7 & 0.0003 & 0.0002 & 0.0002 & 0.002 & 0 \\
\hline MOLYBDEINUM & ICAP & (MG/L) & 95 & 1 & 0.054 & 0.054 & 0.054 & NR & NA \\
\hline NICKEL & ICAP & (MG/L) & $95^{\circ}$ & 19 & 0.12 & 0.012 & 0.032 & $0.100^{* *}$ & 1 \\
\hline FILTERED & ICAP & $(M G / L)$ & 95 & 14 & 0.043 & 0.010 & 0.020 & $0.100 * *$ & 0 \\
\hline POTASSIUM & ICAP & (MG/L) & 95 & 90 & 31 & 0.62 & 3.3 & NR & NA \\
\hline POTASSIUM & ICAP & $(M G / L)$ & 95 & 86 & 34 & 0.62 & 3.0 & NR & NA \\
\hline SILVER & ICAP & $(M G / L)$ & 95 & 2 & 0.023 & 0.017 & 0.020 & 0.100 & 0 \\
\hline FILTERED & ICAP & (MG/L) & 95 & 3 & 0.0083 & 0.0065 & 0.0075 & 0.100 & 0 \\
\hline SODIUM & ICAP & $(M G / L)$ & 95 & 95 & 2100 & 0.92 & 120 & NR & NA \\
\hline FILTERED & ICAP & $(M G / L)$ & 95 & 95 & 2000 & 0.87 & 110 & NR & NA \\
\hline STRONTIUM & ICAP & (MG/L) & 95 & 95 & 1.6 & 0.014 & 0.22 & NR & NA \\
\hline STRONTIUM & ICAP & (MG/L) & 95 & 95 & 1.7 & 0.0095 & 0.22 & NR & NA \\
\hline FLUORIME & ETRIC & $(M G / L)$ & 95 & 22 & 0.064 & 0.0010 & 0.0065 & 13.000 & 0 \\
\hline URANIUM FILT. FLUORIME & ETRIC & (MG/L) & 95 & 21 & 0.046 & 0.0010 & 0.0054 & 13.000 & 0 \\
\hline VANADIUM & ICAP & $(M G / L)$ & 95 & 12 & 0.058 & 0.0056 & 0.020 & NR & NA \\
\hline ZINC & ICAP & $(M G / L)$ & 95 & 92 & 1.6 & 0.0024 & 0.038 & 5.000 & 0 \\
\hline FILTERED & ICAP & $(M G / L)$ & 95 & 87 & 0.12 & 0.0025 & 0.015 & 5.000 & 0 \\
\hline CONDUCTIVITY, FIELD MMT & $T$ IUMI & IOS/CM) & 95 & NA & 9260 & 28 & 660 & NR & NA \\
\hline DISSOLVED OXYGEN, FIELD & D MMT. & (PPM) & 95 & NA & 12 & 0.40 & 4.4 & NR & NA \\
\hline PH, FIELD MMT. & IPH & UNITSI & 95 & NA & 11 & 4.7 & 7.3 & $6.5 / 8.5$ & 48 \\
\hline REDOX, FIELD MMT. & & (MV) & 95 & NA & 310 & -160 & 120 & NR & NA \\
\hline WATER TEMP, FIELD MMT & (DEG. & CENT . I & 95 & NA & 20 & 4.5 & 15 & NR & 0 \\
\hline ALKALINITY-CO3 & & $(M G / L)$ & 95 & 18 & 190 & 6.0 & 74 & NR & NA \\
\hline ALKALINITY - HCO3 & & (MG/L) & 95 & 95 & 540 & 12 & 140 & NR & NA \\
\hline CONDUCTIVITY & IUMH & $10 S / C M)$ & 95 & 95 & 9400 & 32 & 700 & NR & NA \\
\hline
\end{tabular}


Table 7.14 (continued)

\begin{tabular}{|c|c|c|c|c|c|c|c|c|c|c|c|}
\hline VARIABLE & & $\begin{array}{c}\# \\
\text { SAMPLES }\end{array}$ & $\begin{array}{c}\# \\
\text { DETECTED }\end{array}$ & $\begin{array}{l}\text { MAXIMUM } \\
\text { DETECTED } \\
\text { MMT. }\end{array}$ & & $\begin{array}{l}\text { MINIMUM } \\
\text { DETECTED } \\
\text { MMT . }\end{array}$ & & & $\begin{array}{l}\text { AVERAGE } \\
\text { DETECTED } \\
\text { MMTS. }\end{array}$ & $\begin{array}{c}\text { REFERENCE } \\
\text { VALUE }\end{array}$ & $\begin{array}{l}\text { \# MMTS. > } \\
\text { REFERENCE }\end{array}$ \\
\hline DISSOLVED SOLIDS & $(M G / L)$ & 95 & 95 & 5100 & & 36 & & & 440 & 500.000 & 12 \\
\hline PH & (PH UNITS) & 95 & NA & 10 & & 5.7 & & & 7.6 & $6.5 / 8.5$ & 33 \\
\hline TOTAL SUSPENDED SOLIDS & $(M G / L)$ & 95 & 75 & 730 & & 1.0 & & & 66 & NR & NA \\
\hline TURBIDITY & $(\mathrm{NTU})$ & 95 & 95 & 1000 & & 0.30 & & & 34 & 1.000 & 82 \\
\hline 234-URANIUM & $(\mathrm{PCI} / \mathrm{L})$ & 9 & 9 & 8.3 & $\mathbf{J}$ & 0.0000 & & & 2.4 & 20.000 & 0 \\
\hline 235-URANIUM & $(\mathrm{PCI} / \mathrm{L})$ & 9 & 9 & 200 & & -43 & & $\mathbf{F}$ & 23 & 24.000 & 1 \\
\hline 237-NEPTUNIUM & $(\mathrm{PCI} / \mathrm{L})$ & 9 & 9 & 1.1 & & -12 & & & -1.7 & 1.000 & 1 \\
\hline 238-URANIUM & $(\mathrm{PCI} / \mathrm{L})$ & 9 & 9 & 17 & $\mathrm{~J}$ & -0.19 & & & 3.5 & 24.000 & 0 \\
\hline 241-AMERICIUM & $(\mathrm{PCI} / \mathrm{L})$ & 9 & 9 & 3.9 & & -5.2 & & & -1.4 & 1.000 & 1 \\
\hline 99-TECHNETIUM & $(\mathrm{PCI} / \mathrm{L})$ & 9 & 9 & 110 & & 11 & & & 51 & 4000.00 & 0 \\
\hline CESIUM-137 & $(\mathrm{PCI} / \mathrm{L})$ & 3 & 3 & 29 & & 0.075 & & & 11 & 120.00 & 0 \\
\hline GROSS ALPHA & $(P C I / L)$ & 95 & 95 & 33 & & -52 & & & 0.84 & 15.000 & 2 \\
\hline GROSS BETA & $(\mathrm{PCI} / \mathrm{L})$ & 95 & 95 & 44 & & -21 & & & 1.6 & 50.000 & 0 \\
\hline PLUTONIUM-238 & $(\mathrm{PCI} / \mathrm{L})$ & 9 & 9 & 6.9 & & -1.5 & & & 0.55 & 2.000 & 1 \\
\hline PLUTONIUM-239 & $(\mathrm{PCI} / \mathrm{L})$ & 9 & 9 & 0.74 & & -7.4 & & & -0.58 & 1.000 & 0 \\
\hline PROTACTINIUM-234 & $(\mathrm{PCI} / \mathrm{L})$ & 2 & 2 & 2400 & $\mathbf{E}$ & -780 & & $\mathbf{E}$ & 790 & 2800 & 0 \\
\hline RADIUM & $(\mathrm{PCI} / \mathrm{L})$ & 9 & 9 & 0.24 & & 0.023 & & & 0.083 & 4.000 & 0 \\
\hline RUTHENIUM-106 & $(\mathrm{PCI} / \mathrm{L})$ & 2 & 2 & 360 & & 0.38 & & I & 180 & 240 & 1 \\
\hline STRONTIUM & (PCI/L) & 9 & 9 & 61 & & 5.5 & & & 37 & 8.000 & 8 \\
\hline THORIUM-228 & (PCI/L) & 2 & 2 & 1400 & $\mathbf{E}$ & 7.4 & & I & 700 & 16.00 & 1 \\
\hline TRITIUM & $(\mathrm{PCI} / \mathrm{L})$ & 9 & 9 & 700 & & -27 & & & 290 & 20000.0 & 0 \\
\hline $1,1,1$-TRICHLOROETHANE & (UG/L) & 95 & 4 & 17 & $\mathbf{J}$ & 4.0 & $J$ & & 8.3 & 200.000 & 0 \\
\hline 1,1 -DICHLOROETHANE & (UG/L) & 95 & 8 & 51 & & 3.0 & $\mathbf{J}$ & & 16 & NR & NA. \\
\hline 1, 1-DICHLOROETHENE & (UG/L) & 95 & 5 & 28 & $J$ & 1.0 & $\mathbf{J}$ & & 11 & 7.000 & 3 \\
\hline 1,2-DICHLOROETHANE & (UG/L) & 95 & 2 & 4.0 & $J$ & 3.0 & $\mathbf{J}$ & & 3.5 & 5.000 & 0 \\
\hline 1,2-DICHLOROETHENE & (UG/L) & 95 & 11 & 1200 & & 3.0 & $\mathbf{J}$ & & 210 & 70.000 & 4 \\
\hline 2-BUTANONE & (UG/L) & 95 & 17 & 23 & B & 3.0 & $\mathrm{BJ}$ & & 9.4 & $\mathrm{NR}$ & NA \\
\hline 4-METHYL - 2-PENTANONE & (UG/L) & 95 & 8 & 3.0 & $\mathrm{BJ}$ & 1.0 & $\mathbf{J}$ & & 1.8 & NR & NA \\
\hline ACETONE & (UG/L) & 95 & 18 & 7.0 & BJ & 1.0 & $\mathrm{BJ}$ & & 3.3 & NR & NA \\
\hline
\end{tabular}


Table 7.14 (continued)

\begin{tabular}{|c|c|c|c|c|c|c|c|c|c|c|}
\hline VARIABLE & & $\begin{array}{c}\# \\
\text { SAMPLES }\end{array}$ & $\begin{array}{c}\# \\
\text { DETECTED }\end{array}$ & $\begin{array}{l}\text { MAXIMUM } \\
\text { DETECTED } \\
\text { MMT. }\end{array}$ & & $\begin{array}{c}\text { MINIMUM } \\
\text { DETECTED } \\
\text { MMT. }\end{array}$ & & $\begin{array}{l}\text { AVERAGE } \\
\text { DETECTED } \\
\text { MMTS. }\end{array}$ & $\begin{array}{c}\text { REFERENCE } \\
\text { VALUE }\end{array}$ & $\begin{array}{l}\text { \# MMTS. > } \\
\text { REFERENCE }\end{array}$ \\
\hline BENZENE & $(U G / L)$ & 95 & 2 & 2.0 & $J$ & 1.0 & $\mathrm{~J}$ & 1.5 & 5.000 & 0 \\
\hline CHLOROFORM & (UG/L) & 95 & 1 & 0.60 & $\mathrm{~J}$ & 0.60 & $\mathrm{~J}$ & 0.60 & 100.000 & 0 \\
\hline METHYLENE CHLORIDE & $(U G / L)$ & 95 & 35 & 7.0 & BJ & 0.70 & $\mathrm{~J}$ & 2.0 & NR & $\mathrm{NA}$ \\
\hline TETRACHLOROETHENE & (UG/L) & 95 & 12 & 140 & & 0.70 & $J$ & 36 & 5.000 & 8 \\
\hline TOLUENE & (UG/L) & 95 & 2 & 2.0 & $\mathcal{J}$ & 2.0 & $J$ & 2.0 & 1000.00 & 0 \\
\hline TRICHLOROETHENE & (UG/L) & 95 & 10 & 110 & & 3.0 & $J$ & 26 & 5.000 & 8 \\
\hline VINYL CHLORIDE & (UG/L) & 95 & 7 & 72 & $\mathfrak{J}$ & 0.80 & $J$ & 22 & 2.000 & 5 \\
\hline XYLENES & (UG/L) & 95 & 1 & 2.0 & 3 & 2.0 & $J$ & 2.0 & 10000.0 & 0 \\
\hline
\end{tabular}


Table 7.15. Constituents In groundwater at the Y-12 Plant site HGR $=B C$ area=exit Pathway

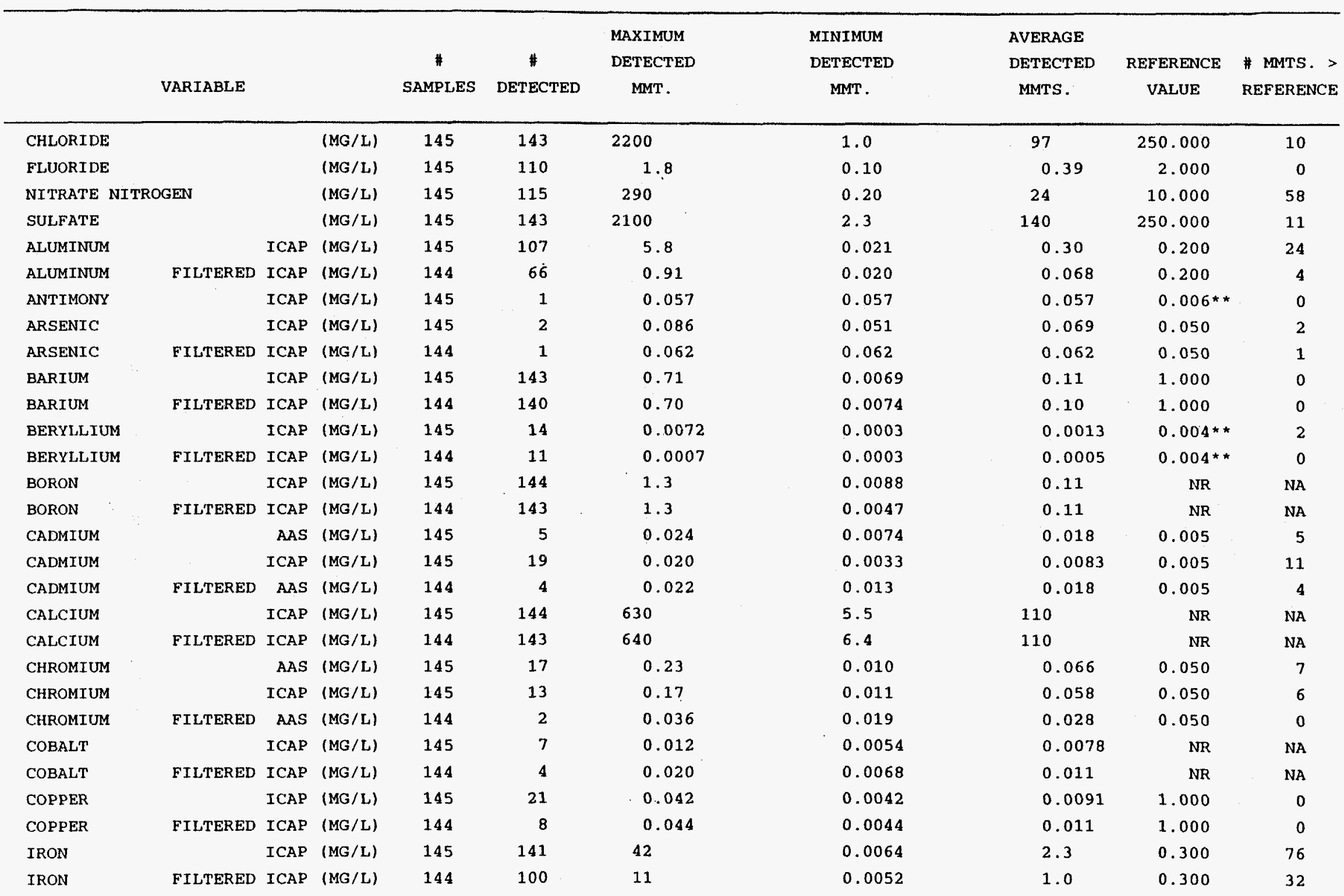


Table 7.15 (continued)

\begin{tabular}{|c|c|c|c|c|c|c|c|c|c|}
\hline VARIABLE & & & $\begin{array}{c}\# \\
\text { SAMPLES }\end{array}$ & $\begin{array}{c}\# \\
\text { DETECTED }\end{array}$ & $\begin{array}{l}\text { MAXIMUM } \\
\text { DETECTED } \\
\text { MMT. }\end{array}$ & $\begin{array}{l}\text { MINTMUM } \\
\text { DETECTED } \\
\text { MMT. }\end{array}$ & $\begin{array}{l}\text { AVERAGE } \\
\text { DETECTED } \\
\text { MMTS. }\end{array}$ & $\begin{array}{c}\text { REFERENCE } \\
\text { VAL_UE }\end{array}$ & $\begin{array}{l}\# \text { MMTS. > } \\
\text { REFERENCE }\end{array}$ \\
\hline LEAD & AAS & $(M G / L)$ & 145 & 5 & 0.0073 & 0.0046 & 0.0063 & 0.050 & 0 \\
\hline FILTERED & AAS & $(M G / L)$ & 144 & 1 & 0.018 & 0.018 & 0.018 & 0.050 & 0 \\
\hline MAGNESIUM & ICAP & (MG/L) & 145 & 144 & 250 & 1.3 & 33 & NR & $\mathrm{NA}$ \\
\hline MAGNESIUM & ICAP & (MG/L) & 144 & 143 & 260 & 1.4 & 33 & NR & NA \\
\hline MANGANESE & ICAP & (MG/L) & 145 & 142 & 4.0 & 0.0015 & 0.24 & 0.050 & 72 \\
\hline MANGANESE & ICAP & (MG/L) & 144 & 133 & 3.7 & 0.0010 & 0.22 & 0.050 & 56 \\
\hline MERCURY & CVAA & (MG/L) & 145 & 2 & 0.0002 & 0.0002 & 0.0002 & 0.002 & 0 \\
\hline FILTERED & CVAA & (MG/L) & 144 & 2 & 0.0002 & 0.0002 & 0.0002 & 0.002 & 0 \\
\hline MOLYBDENUM & ICAP & (MG/L) & 144 & 1 & 0.011 & 0.011 & 0.011 & NR & NA \\
\hline NICKEL & ICAP & (MG/L) & 145 & 37 & 0.22 & 0.010 & 0.040 & $0.100 \star \star$ & 3 \\
\hline FILTERED & ICAP & $(M G / L)$ & 144 & 32 & 0.24 & 0.011 & 0.044 & $0.100 * *$ & 3 \\
\hline POTASSIUM & ICAP & $(M G / L)$ & 145 & 140 & 26 & 0.67 & 3.8 & NR & NA \\
\hline POTASSIUM & ICAP & $(M G / L)$ & 144 & 142 & 31 & 0.60 & 3.7 & NR & NA \\
\hline FILTERED & ICAP & $(M G / L)$ & 144 & 2 & 0.060 & 0.057 & 0.059 & 0.050 & 2 \\
\hline SILVER & ICAP & (MG/L) & 145 & 2 & 0.015 & 0.0061 & 0.011 & 0.100 & 0 \\
\hline FILTERED & ICAP & (MG/I) & 144 & 1 & 0.046 & 0.046 & 0.046 & 0.100 & 0 \\
\hline SODIUM & ICAP & $(M G / L)$ & 145 & 144 & 1300 & .0 .45 & 56 & NR & NA \\
\hline FILTERED & ICAP & $(M G / L)$ & 144 & 143 & 1200 & 0.48 & 56 & NR & NA \\
\hline STRONTYUM & ICAP & $(M G / L)$ & 145 & 144 & 15 & 0.016 & 0.99 & NR & NA \\
\hline STRONTIUM & ICAP & $(M G / L)$ & 144 & 143 & 15 & 0.016 & 0.99 & NR & NA \\
\hline FLUORIME & ETRIC & (MG /L) & 145 & 113 & 0.30 & 0.0010 & 0.033 & 13.000 & 0 \\
\hline URANIUM FILT. FLUORIME & ETRIC & $(M G / L)$ & 144 & 122 & 0.31 & 0.0010 & 0.028 & 13.000 & 0 \\
\hline VANADIUM & ICAP & (MG/L) & 145 & 4 & 0.010 & 0.0056 & 0.0072 & NR & NA \\
\hline ZINC & ICAP & (MG/L) & 1.44 & 122 & 0.084 & 0.0032 & 0.013 & 5.000 & 0 \\
\hline FILTERED & ICAP & $(M G / L)$ & 145 & 116 & 0.12 & 0.0020 & 0.012 & 5.000 & 0 \\
\hline CONDUCTIVITY, FIELD MMT & I (UMH & IOS/CM) & 144 & NA & 7600 & 30 & 870 & NR & NA \\
\hline DISSOLVED OXYGEN, FIELD & MMT. & (PPM) & 145 & NA & 12 & 0.30 & 5.2 & NR & NA \\
\hline PH, FIELD MMT. & $(\mathrm{PH}$ & UNITS) & 144 & NA & 8.4 & 5.2 & 7.4 & $6.5 / 8.5$ & 3 \\
\hline REDOX, FIELD MMT. & & (MV) & 104 & NA & 280 & -140 & 82 & NR & NA \\
\hline WATER TEMP, FIELD MMT । & (DEG. & CENT. I & 144 & NA & 26 & 5.7 & 14 & NR & 0 \\
\hline
\end{tabular}


Table 7.15 (continued)

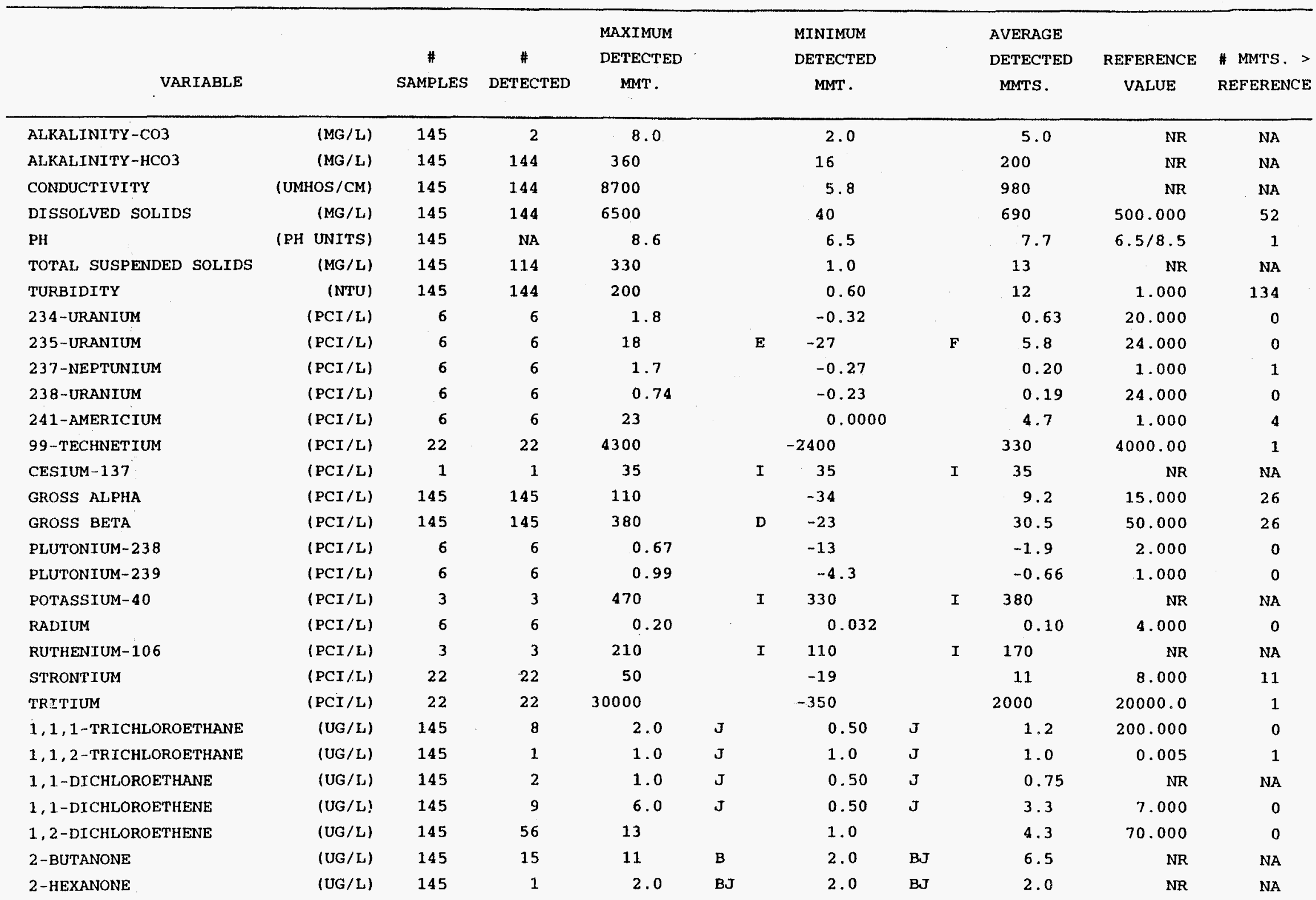


Table 7.15 (continued)

\begin{tabular}{|c|c|c|c|c|c|c|c|c|c|c|}
\hline VARIABLE & & $\begin{array}{c}\# \\
\text { SAMPLES }\end{array}$ & $\begin{array}{c}\# \\
\text { DETECTED }\end{array}$ & $\begin{array}{l}\text { MAXIMUM } \\
\text { DETECTED } \\
\text { MMT. }\end{array}$ & & $\begin{array}{l}\text { MINIMUM } \\
\text { DETECTED } \\
\text { MMT. }\end{array}$ & & $\begin{array}{l}\text { AVERAGE } \\
\text { DETECTED } \\
\text { MMTS. }\end{array}$ & $\begin{array}{c}\text { REFERENCE } \\
\text { VALUE }\end{array}$ & $\begin{array}{l}\text { \# MMTS. > } \\
\text { REFERENCE }\end{array}$ \\
\hline 4-METHYL-2-PENTANONE & (UG/L) & 145 & 29 & 6.0 & $\mathrm{BJ}$ & 1.0 & $\mathrm{BJ}$ & 1.8 & NR & NA \\
\hline ACETONE & (UG/L) & 145 & 17 & 7.0 & $\mathrm{BJ}$ & 1.0 & $J$ & 2.7 & NR & $\mathrm{NA}$ \\
\hline BENZENE & (UG/L) & 145 & 2 & 1.0 & $\mathcal{J}$ & 0.30 & $\mathrm{~J}$ & 0.65 & 5.000 & 0 \\
\hline CARBON DISULFIDE & $(U G / L)$ & 145 & 1 & 2.0 & $\mathfrak{J}$ & 2.0 & $\mathrm{~J}$ & 2.0 & $\mathrm{NR}$ & NA \\
\hline CARBON TETRACHLORIDE & $(U G / L)$ & 145 & 12 & 2.0 & $J$ & 0.40 & $J$ & 1.3 & 5.000 & 0 \\
\hline CHLOROBENZENE & (UG/L) & 145 & 1 & 1.0 & J & 1.0 & $\mathrm{~J}$ & 1.0 & 100.000 & 0 \\
\hline CHLOROFORM & $(U G / L)$ & 145 & 18 & 7.0 & & 0.40 & $\mathrm{~J}$ & 1.7 & 100.000 & 0 \\
\hline ETHXLBENZENE & (UG/L) & 145 & 1 & $2.0^{\circ}$ & J & 2.0 & $\mathrm{~J}$ & 2.0 & 700.000 & 0 \\
\hline METHYLENE CHLORIDE & (UG/L) & 145 & 69 & 3.0 & $\mathrm{BJ}$ & 0.60 & JB & 1.6 & NR & NA \\
\hline STYRENE & $(U G / L)$ & 145 & 1 & 1.0 & $\mathfrak{J}$ & 1.0 & $\mathrm{~J}$ & 1,0 & 100.000 & 0 \\
\hline TETRACHLOROETHENE & (UG/L) & 145 & 24 & 7.0 & & 0.50 & $J$ & 2.2 & 5.000 & 1 \\
\hline TOLUENE & (UG/L) & 145 & 1 & 2.0 & $J$ & 2.0 & $J$ & 2.0 & 1000.00 & 0 \\
\hline TRICHLOROETHENE & (UG/L) & 145 & 59 & 140 & & 0.70 & & 28 & 5.000 & 42 \\
\hline VINYL ACETÃTE & $(U G / L)$ & 145 & 1 & 1.0 & $\mathrm{~d}$ & 1.0 & $\mathrm{~J}$ & 1.0 & NR & $\mathrm{NA}$ \\
\hline XYLENES & (UG/L) & 145 & 1 & 5.0 & & 5.0 & & 5.0 & 10000.0 & 0 \\
\hline
\end{tabular}


Table 7.16. Constituents in groundwater at the Y-12 Plant site

$\mathrm{HGR}=\mathrm{BC}$ area $=\mathrm{O}$ il Landfarm

\begin{tabular}{|c|c|c|c|c|c|c|c|c|c|c|}
\hline & VARIABLE & & & $\begin{array}{c}\# \\
\text { SAMPLES }\end{array}$ & $\begin{array}{c}\# \\
\text { DETECTED }\end{array}$ & $\begin{array}{c}\text { MAXIMUM } \\
\text { DETECTED } \\
\text { MMT. }\end{array}$ & $\begin{array}{l}\text { MINIMUM } \\
\text { DETECTED } \\
\text { MMT. }\end{array}$ & $\begin{array}{l}\text { AVERAGE } \\
\text { DETECTED } \\
\text { MMTS. }\end{array}$ & $\begin{array}{c}\text { REFERENCE } \\
\text { VALUE }\end{array}$ & $\begin{array}{l}\text { \# MMTS. > } \\
\text { REFERENCE }\end{array}$ \\
\hline CHLORIDE & & & $(M G / L)$ & 65 & 61 & 130 & 1.0 & 20 & 250.000 & 0 \\
\hline FLUORIDE & & & $(M G / L)$ & 65 & 24 & 0.40 & 0.10 & 0.18 & 2.000 & 0 \\
\hline NITRATE NI & ITROGEN & & (MG/L) & 65 & 33 & 2100 & 0.23 & 210 & 10.000 & 14 \\
\hline SULFATE & & & (MG/L) & 65 & 60 & 180 & 2.1 & 20 & 250.000 & 0 \\
\hline ALUMINUM & & ICAP & $(M G / L)$ & 65 & 51 & 28 & 0.024 & 3.1 & 0.200 & 30 \\
\hline ALUMINUM & FILTERED & ICAP & (MG/L) & 64 & 21 & 0.16 & 0.021 & 0.051 & 0.200 & 0 \\
\hline ARSENIC & & ICAP & (MG/L) & 65 & 1 & 0.058 & 0.058 & 0.058 & 0.050 & 1 \\
\hline ARSENIC & FILTERED & ICAP & $(\mathrm{MG} / \mathrm{L})$ & 64 & 1 & 0.072 & 0.072 & 0.072 & 0.050 & 1 \\
\hline BARIUM & & ICAP & (MG/L) & 65 & 65 & 2.5 & 0.015 & 0.43 & 1.000 & 8 \\
\hline BARIUM & FILTERED & ICAP & $(M G / L)$ & 64 & 64 & 2.5 & 0.013 & 0.36 & 1.000 & 7 \\
\hline BERYLLIUM & & ICAP & $(M G / L)$ & 65 & 10 & 0.0019 & 0.0003 & 0.0010 & $0.004 * *$ & 0 \\
\hline BERYLLIUM & FILTERED & ICAP & (MG/L) & 64 & 1 & 0.0007 & 0.0007 & 0.0007 & $0.004^{\star \star}$ & 0 \\
\hline BORON & & ICAP & $(M G / L)$ & 65 & 61 & 0.42 & 0.0046 & 0.069 & NR & NA \\
\hline BORON & FILTERED & ICAP & $(M G / L)$ & 64 & 60 & 0.40 & 0.0060 & 0.065 & $\mathrm{NR}$ & NA \\
\hline CADMIUM & & AAS & (MG/L) & 65 & 2 & 0.0078 & 0.0020 & 0.0049 & 0.005 & 1 \\
\hline CADMIUM & & ICAP & (MG/L) & 65 & 7 & 0.0094 & 0.0030 & 0.0068 & 0.005 & 6 \\
\hline CADMIUM & FILTERED & AAS & (MG/L) & 64 & 3 & 0.0069 & 0.0021 & 0.0051 & 0.005 & 2 \\
\hline CALCIUM & & ICAP & $(M G / L)$ & 65 & 65 & 1200 & 7.7 & 170 & $\mathrm{NR}$ & NA \\
\hline CALCIUM & FILTERED & ICAP & (MG/L) & 64 & 64 & 1100 & 7.4 & 150 & NR & NA \\
\hline CHROMIUM & & AAS & (MG/L) & 65 & 8 & 0.067 & 0.012 & 0.039 & 0.050 & 2 \\
\hline CHROMIUM & & ICAP & $(M G / L)$ & 65 & 10 & 0.15 & 0.010 & 0.064 & 0.050 & 4 \\
\hline COBALT & " & ICAP & $(M G / L)$ & 65 & 7 & 0.026 & 0.0072 & 0.014 & $\mathrm{NR}$ & NA \\
\hline COPPER & & ICAP & $(M G / L)$ & 65 & 25 & 0.10 & 0.0040 & 0.021 & 1.000 & 0 \\
\hline COPPER & FILTERED & ICAP & $(M G / L)$ & 64 & 12 & 0.046 & 0.0040 & 0.010 & 1.000 & 0 \\
\hline IRON & & ICAP & $(M G / L)$ & 65 & 63 & 49. & 0.0050 & 3.5 & 0.300 & 41 \\
\hline IRON & FILTERED & ICAP & $(M G / L)$ & 64 & 33 & 1.4 & 0.0057 & 0.28 & 0.300 & 8 \\
\hline LEAD & & AAS & $(M G / L)$ & 65 & 12 & 0.031 & 0.0050 & 0.013 & 0.050 & 0 \\
\hline MAGNESIUM & & ICAP & $(M G / L)$ & 65 & 65 & 77 & 1.5 & 18 & $\mathrm{NR}$ & NA \\
\hline MAGNESIUM & FILTERED & ICAP & (MG/L) & 64 & 64 & 74 & 1.5 & 16 & NR & NA. \\
\hline
\end{tabular}


Table 7.16 (continued)

\begin{tabular}{|c|c|c|c|c|c|c|c|c|c|}
\hline VARIABLE & & & $\begin{array}{c}\# \\
\text { SAMPLES }\end{array}$ & $\begin{array}{c}\# \\
\text { DETECTED }\end{array}$ & $\begin{array}{c}\text { MAXIMUM } \\
\text { DETECTED } \\
\text { MMT: }\end{array}$ & $\begin{array}{l}\text { MINIMUM } \\
\text { DETECTED } \\
\text { MMT. }\end{array}$ & $\begin{array}{l}\text { AVERAGE } \\
\text { DETECTED } \\
\text { MMTS. }\end{array}$ & $\begin{array}{l}\text { REFERENCE } \\
\text { VALUE }\end{array}$ & $\begin{array}{l}\# \text { MMTS. > } \\
\text { REFERENCE }\end{array}$ \\
\hline MANGANESE & ICAP & $(M G / L)$ & 65 & 61 & 1.3 & 0.0019 & 0.17 & 0.050 & 18 \\
\hline MANGANESE & ICAP & (MG/L) & 64 & 53 & 1.3 & 0.0012 & 0.095 & 0.050 & 5 \\
\hline MERCURY & CVAA & (MG/L) & 65 & 1 & 0.0003 & 0.0003 & 0.0003 & 0.002 & 0 \\
\hline NICKEL & ICAP & (MG/L) & 65 & 13 & 0.15 & 0.011 & 0.042 & $0.100 * *$ & 1 \\
\hline FILTERED & ICAP & $(M G / L)$ & 64 & 11 & 0.037 & 0.011 & 0.022 & $0.100 * *$ & 0 \\
\hline POTASSIUM & ICAP & (MG/L) & 65 & 60 & 9.5 & 0.60 & 2.8 & NR & NA \\
\hline POTASSIUM & ICAP & (MG/L) & 64 & 57 & 5.3 & 0.60 & 2.2 & NR & $\mathrm{NA}$ \\
\hline SELENIUM & ICAP & (MG/L) & 65 & 3 & 0.074 & 0.058 & 0.066 & 0.050 & 3 \\
\hline FILTERED & ICAP & $(M G / L)$ & 64 & 3 & 0.26 & 0.089 & 0.15 & 0.050 & 3 \\
\hline SILVER & ICAP & (MG/L) & 65 & 1 & 0.0074 & 0.0074 & 0.0074 & 0.100 & is \\
\hline SODIUM & TCAP & (MG/L) & 65 & 65 & 78 & 0.81 & 14 & NR & NA \\
\hline FILTERED & ICAP & $(M G / L)$ & 64 & 64 & 75 & 0.84 & 14 & NR & NA \\
\hline STRONTIUM & ICAP & (MG/L) & 65 & 65 & 3.0 & 0.025 & 0.56 & $\mathrm{NR}$ & NA \\
\hline STRONTIUM & ICAP & $(M G / L)$ & 64 & 64 & 3.0 & 0.026 & 0.51 & NR & NA \\
\hline FLUORIME & ETRIC & $(M G / L)$ & 65 & 31 & 0.018 & 0.0010 & 0.0031 & 13.000 & 0 \\
\hline URANIUM FILT. FLUORIME & ETRIC & $(M G / L)$ & 61 & 31 & 0.018 & 0.0010 & 0.0029 & 13.000 & 0 \\
\hline VANADIUM & ICAP & $(M G / L)$ & 65 & 11 & 0.071 & 0.0051 & 0.021 & NR & NA \\
\hline ZINC & ICAP & (MG/L) & 65 & 58 & 0.19 & 0.0023 & 0.032 & 5.000 & 0 \\
\hline FILTERED & ICAP & $(M G / L)$ & 64 & 59 & 0.11 & 0.0027 & 0.020 & 5.000 & 0 \\
\hline CONDUCTIVITY, FIELD MMT & IUMH & HOS/CM) & 65 & NA & 5400 & 120 & 930 & NR & NA \\
\hline DISSOLVED OXYGEN, FIELD & MMT. & (PPM) & 65 & NA & 10 & 0.30 & 3.9 & $\mathrm{NR}$ & $\mathrm{NA}$ \\
\hline PH, FIELD MMT. & (PH & (UNITS) & 65 & NA & 8.4 & 6.0 & 7.1 & $6.5 / 8.5$ & 8 \\
\hline REDOX, FIELD MMT. & & (MV) & 65 & NA & 310 & -80 & 170 & $\mathrm{NR}$ & NA \\
\hline WATER TEMP, FIELD MMT । & (DEG. & CENT. . & 65 & NA & 20 & 9.8 & 15 & NR & 0 \\
\hline ALKALINITY-CO3 & & (MG/L) & 65 & 5 & 6.0 & 1.0 & 2.8 & NR & NA \\
\hline ALKALINITY-HCO3 & & (MG/L) & 65 & 65 & 350. & 52 & 190 & NR & NA \\
\hline CONDUCTIVITY & IUMH & HOS/CM) & 65 & 65 & 6100 & 120 & 990 & NR & NA \\
\hline DISSOLVED SOLIDS & & (MG/L) & 65 & 65 & 4700 & 110 & 730 & 500.000 & 19 \\
\hline PH & (PH & UNITS) & 65 & NA & 8.5 & 6.3 & 7.4 & $6.5 / 8.5$ & 3 \\
\hline TOTAL SUSPENDED SOLIDS & & (MG/L) & 65 & 57 & 5400 & 1.0 & 180 & $\mathrm{NR}$ & NA \\
\hline
\end{tabular}


Table 7.16 (continued)

\begin{tabular}{|c|c|c|c|c|c|c|c|c|c|c|c|c|}
\hline VARIABLE & & $\begin{array}{c}\# \\
\text { SAMPLES }\end{array}$ & $\begin{array}{c}\# \\
\text { DETECTED }\end{array}$ & $\begin{array}{c}\text { MAXIMUM } \\
\text { DETECTED } \\
\text { MMT. }\end{array}$ & & & $\begin{array}{c}\text { MINIMUM } \\
\text { DETECTED } \\
\text { MMT. }\end{array}$ & & & $\begin{array}{l}\text { AVERAGE } \\
\text { DETECTED } \\
\text { MMTS. }\end{array}$ & $\begin{array}{c}\text { REFERENCE } \\
\text { VALUE }\end{array}$ & $\begin{array}{l}\# \text { MMTS. > } \\
\text { REFERENCE }\end{array}$ \\
\hline TURBIDITY & (NTU) & 65 & 65 & 3100 & & & 0.50 & & & 98 & 1.000 & 59 \\
\hline 234-URANIUM & $(\mathrm{PCI} / \mathrm{L})$ & 3 & 3 & 9.1 & & 了 & -7.2 & & & 2.0 & 20.000 & 0 \\
\hline 235-URANIUM & (PCI/L) & 3 & 3 & 43 & & E & -1.9 & & $\mathbf{F}$ & 19 & 24.000 & 1 \\
\hline 237-NEPTUNIUM & (PCI/L) & 3 & 3 & 18 & & & -5.5 & & & 4.1 & 1.000 & 1 \\
\hline 238-URANIUM & $(\mathrm{PCI} / \mathrm{L})$ & 3 & 3 & 22 & & & 5.8 & & & 12 & 24.000 & 0 \\
\hline 99-TECHNETIUM & (PCI/L) & 8 & 8 & 1300 & & & 18 & & & 740 & 4000.00 & 0 \\
\hline CESIUM-137 & (PCI/L) & 2 & 2 & 19 & & $\mathbf{E}$ & 13 & & $\mathbf{E}$ & 16 & NR & NA \\
\hline GROSS ALPHA & $(\mathrm{PCT} / \mathrm{L})$ & 65 & 65 & 40 & & & -3.5 & & & 2.7 & 15.000 & 5 \\
\hline PLUTONIUM-238 & (PCI/L) & 3 & 3 & 14 & & & 0.0000 & & & 4.5 & 2.000 & 1 \\
\hline PLUTONIUM-239 & (PCI/L) & 3 & 3 & 15 & & & -6.8 & & & 2.7 & 1.000 & 1 \\
\hline PROTACTINIUM-234 & (PCI/L) & 2 & 2 & 2100 & & $\mathbf{E}$ & 2000 & & $\mathbf{E}$ & 2000 & NR & NA \\
\hline RADTUM & (PCI/L) & 3 & 3 & 0.025 & & & 0.0040 & & & 0.013 & 4.000 & 0 \\
\hline STRONTIUM & $(\mathrm{PCI} / \mathrm{L})$ & 8 & 8 & 58 & & & -3.7 & & & 30 & 8.000 & 6 \\
\hline TRITIUM & (PCI/L) & 8 & 8 & 3200 & & & -140 & & & 560 & 20000.0 & 0 \\
\hline $1,1,1$-TRICHLOROETHANE & $(\mathrm{UG} / \mathrm{L})$ & 64 & 11 & 11 & & & 0.50 & $\mathrm{~J}$ & & 2.4 & 200.000 & 0 \\
\hline 1,1-DICHLOROETHANE & $(\mathrm{UG} / \mathrm{L})$ & 64 & 6 & 5.0 & & & 2.0 & $\mathbf{J}$ & & 3.3 & NR & NA \\
\hline 1,1-DICHLOROETHENE & (UG/L) & 64 & 8 & 21 & & & 0.70 & $J$ & & 9.6 & 7.000 & 4 \\
\hline 1,2-DICHLOROETHENE & (UG/L) & 64 & 14 & 23 & & & 0.90 & $\mathbf{J}$ & & 8.4 & 70.000 & 0 \\
\hline 2-BUTANONE & $(\mathrm{UG} / \mathrm{L})$ & 64 & 11 & 12 & B & & 4.0 & $\mathrm{BJ}$ & & 8.8 & NR & $\mathrm{NA}$ \\
\hline 4-METHYL-2-PENTANONE & $(\mathrm{UG} / \mathrm{L})$ & 64 & 10 & 3.0 & BJ & & 1.0 & $\mathrm{BJ}$ & & 1.6 & NR & $\mathrm{NA}$ \\
\hline ACETONE & (UG/L) & 64 & 7 & 5.0 & $\mathrm{~J}$ & & 2.0 & BJ & & 3.3 & NR & $\mathrm{NA}$ \\
\hline CARBON TETRACHLORIDE & $(\mathrm{UG} / \mathrm{L})$ & 64 & 7 & 4.0 & J & & 2.0 & $\mathrm{~J}$ & & 2.7 & 5.000 & 0 \\
\hline CHLOROFORM & (UG/L) & 64 & 11 & 3.0 & J & & 0.60 & $\mathbf{J}$ & & 1.3 & 100.000 & 0 \\
\hline METHYLENE CHLORIDE & (UG/L) & 64 & 25 & 3.0 & BJ & & 0.60 & $\mathrm{JB}$ & & 1.4 & NR & $\mathrm{NA}$ \\
\hline TETRACHLOROETHENE & (UG/L) & 64 & 10 & 2.0 & $\mathrm{~J}$ & & 0.30 & $\mathbf{J}$ & & 1.0 & 5.000 & 0 \\
\hline TRICHLOROETHENE & (UG/L) & 64 & 17 & 140 & & & 6.0 & & & 70 & 5.000 & 17 \\
\hline VINYL ACETATE & (UG/L) & 64 & 1 & 3.0 & $\mathrm{~J}$ & & 3.0 & $\mathbf{J}$ & & 3.0 & NR & NA \\
\hline
\end{tabular}


Table 7.17. Constituents in groundwater at the $Y-12$ Plant site

$H G R=B C$ area=Aust Spoil Area

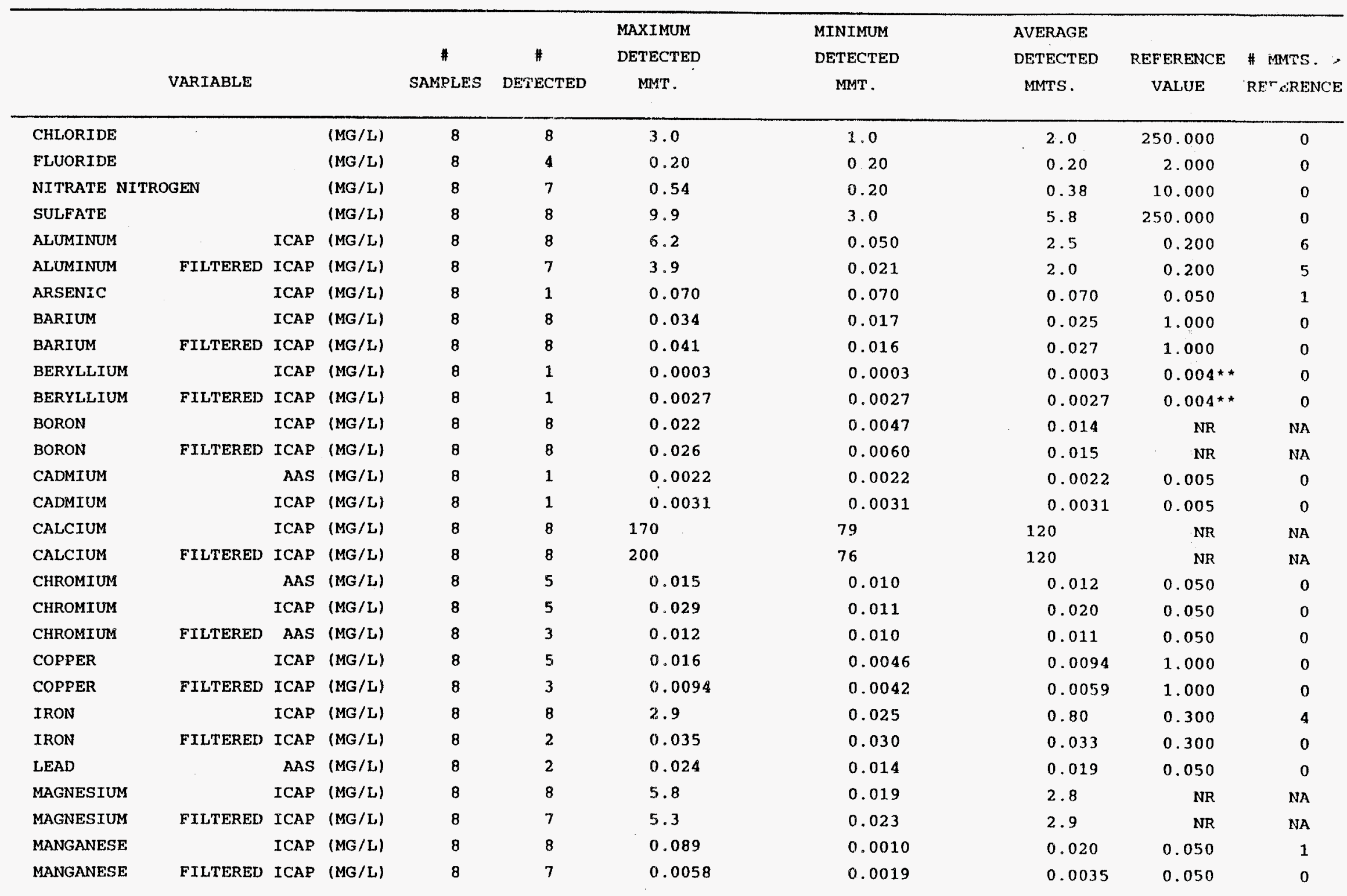


Table 7.17 (continued)

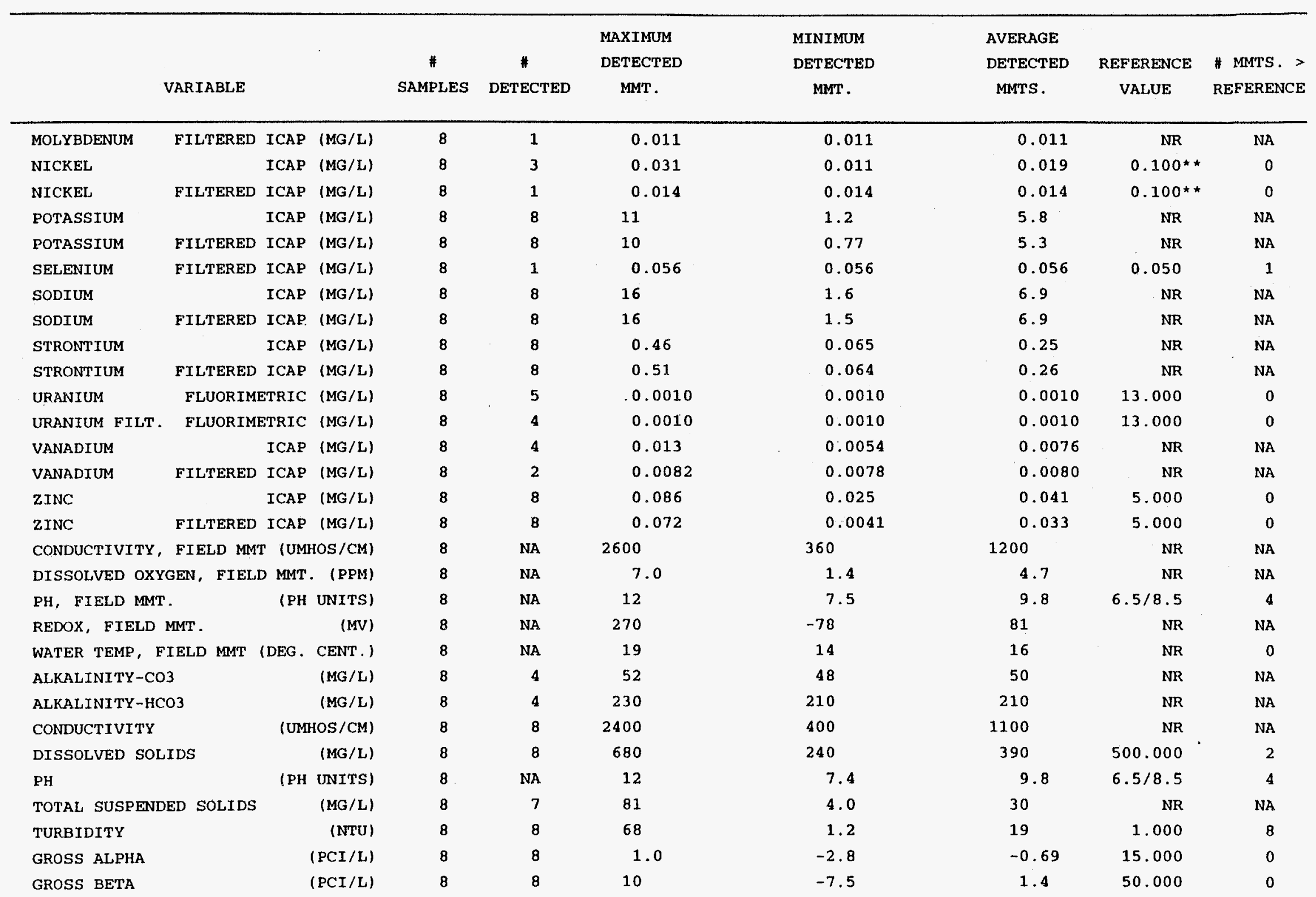


Table 7.17 (continued)

\begin{tabular}{|c|c|c|c|c|c|c|c|c|c|c|}
\hline VARIABLE & & $\begin{array}{c}\# \\
\text { SAMPLES }\end{array}$ & $\begin{array}{c}\# \\
\text { DETECTED }\end{array}$ & $\begin{array}{l}\text { MAXIMUM } \\
\text { DETECTED } \\
\text { MMT. }\end{array}$ & & $\begin{array}{c}\text { MINIMUM } \\
\text { DETECTED } \\
\text { MMT. }\end{array}$ & & $\begin{array}{c}\text { AVERAGE } \\
\text { DETECTED } \\
\text { MMTS. }\end{array}$ & $\begin{array}{c}\text { REFERENCE } \\
\text { VALUE }\end{array}$ & $\begin{array}{l}\text { \# MMTS. > } \\
\text { REFERENCE }\end{array}$ \\
\hline 2-BUTANONE & (UG/L) & 8 & 1 & 10 & B & 10 & B & 10 & NR & NA \\
\hline 4-METHYL-2-PENTANONE & (UG/L) & 8 & 1 & 1.0 & BJ & 1.0 & BJ & 1.0 & NR & NA \\
\hline ACETONE & $(U G / L)$ & 8 & 1 & 3.0 & BJ & 3.0 & $\mathrm{BJ}$ & 3.0 & NR & NA \\
\hline CARBON TETRACHLORIDE & (UG/L) & 8 & 4 & 3.0 & $\mathrm{~J}$ & 0.90 & $\mathrm{~J}$ & 1.7 & 5.000 & 0 \\
\hline CHLOROFORM & $(U G / L)$ & 8 & 5 & 1.0 & $\mathrm{~J}$ & 0.80 & $\mathrm{~J}$ & 0.96 & 100.000 & 0 \\
\hline METHYLENE CHLORIDE & $(U G / L)$ & 8 & 2 & 3.0 & $\mathrm{BJ}$ & 1.0 & $\mathrm{JB}$ & 2.0 & NR & NA \\
\hline TRICHLOROETHENE & $(U G / L)$ & 8 & 8 & 83 & & 10 & & 34 & 5.000 & 8 \\
\hline
\end{tabular}


Tablle 7.18. Constituents in groundwater at the Y-12 Plant site

$\mathrm{HGR}=\mathrm{BC}$ area=S3 Ponds

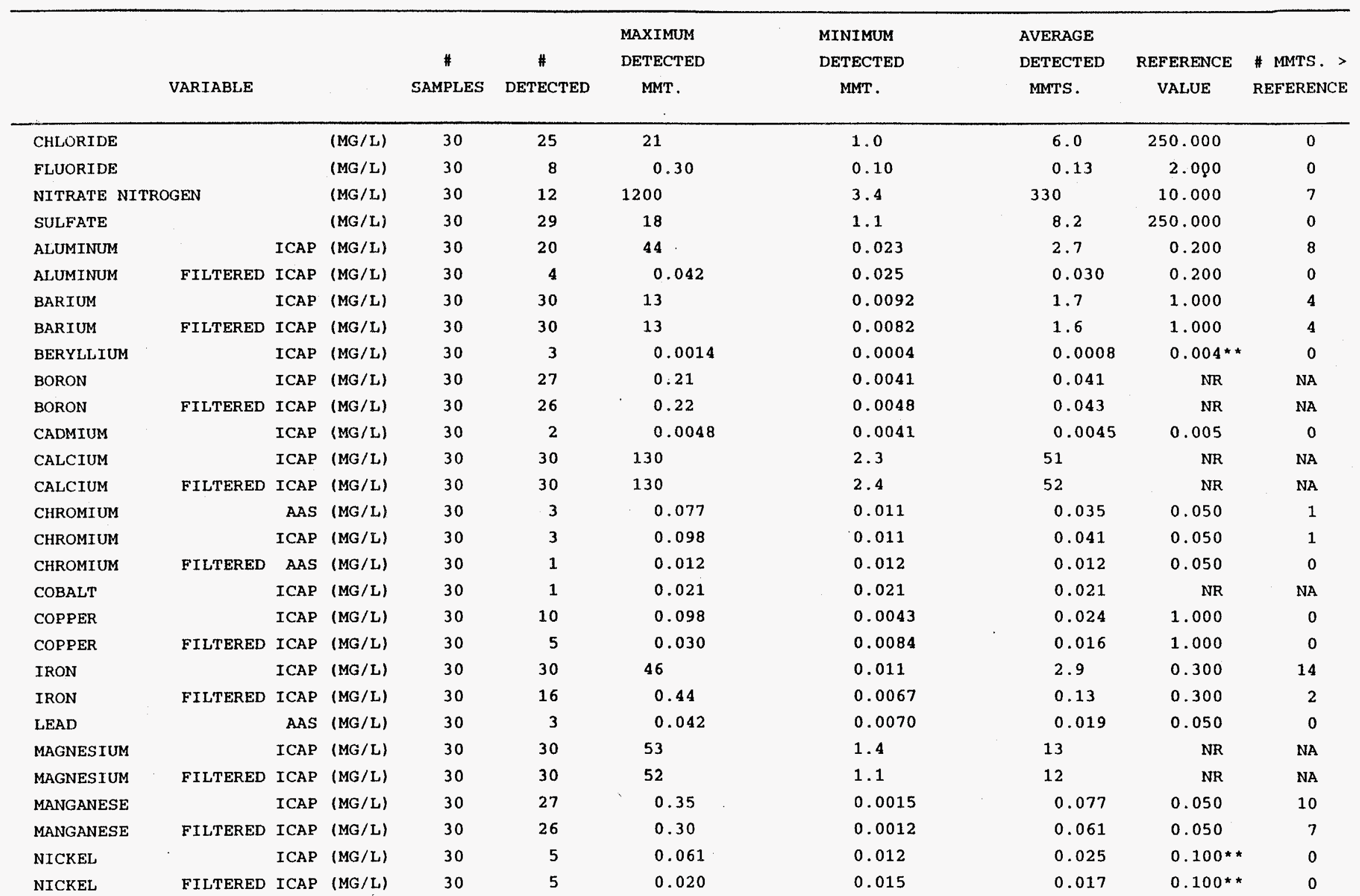


Table 7.18 (continued)

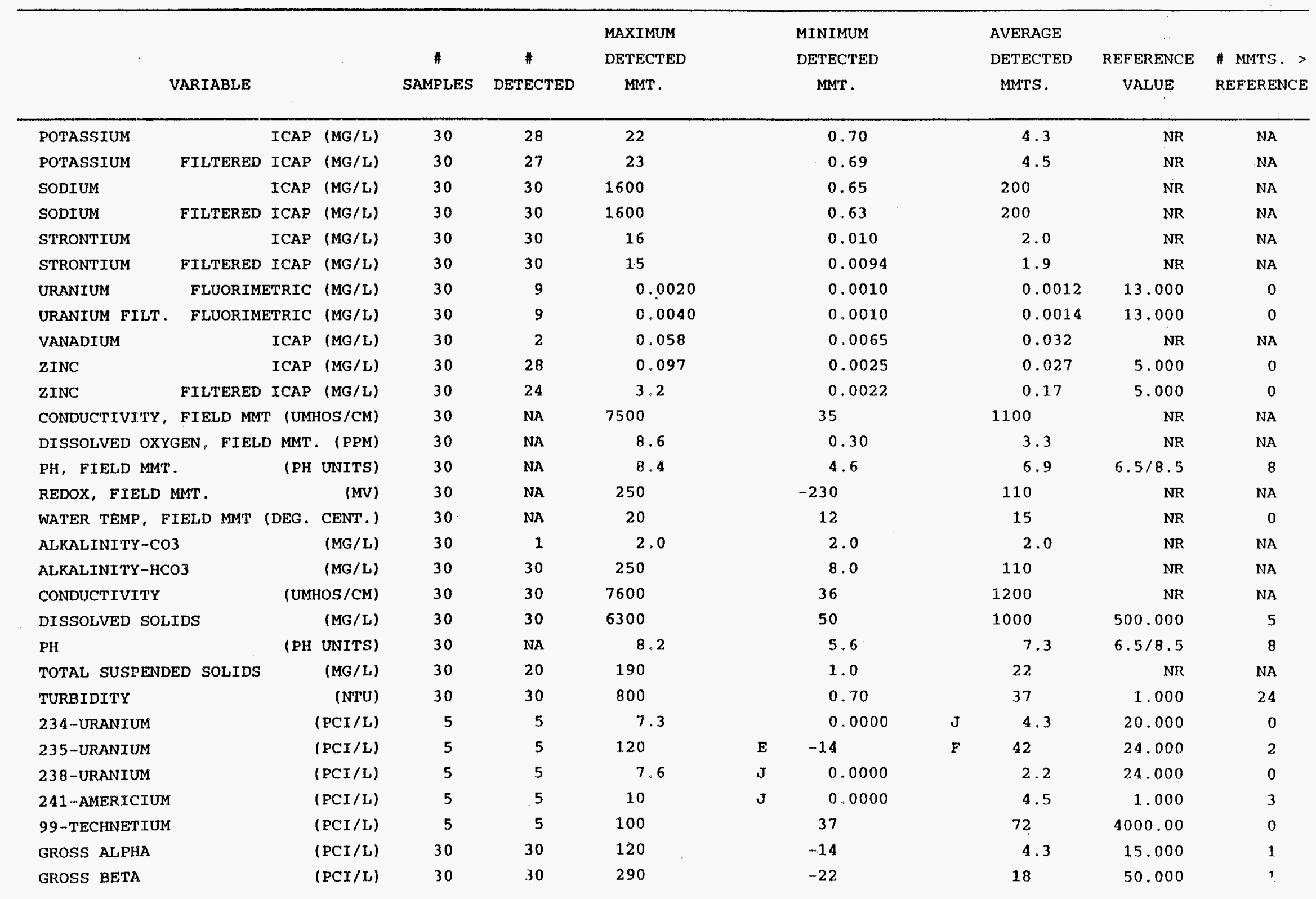


Table 7.18 (continued)

\begin{tabular}{|c|c|c|c|c|c|c|c|c|c|c|}
\hline VARIABLE & & $\begin{array}{c}\# \\
\text { SAMPLES }\end{array}$ & $\begin{array}{c}\# \\
\text { DETECTED }\end{array}$ & $\begin{array}{l}\text { MAXIMUM } \\
\text { DETECTED } \\
\text { MMT. }\end{array}$ & & $\begin{array}{l}\text { MINIMUM } \\
\text { DETECTED } \\
\text { MMT. }\end{array}$ & & $\begin{array}{l}\text { AVERAGE } \\
\text { DETECTED } \\
\text { MMTS. }\end{array}$ & $\begin{array}{c}\text { REF ERENCE } \\
\text { VALUE }\end{array}$ & $\begin{array}{l}\text { \# MMTS. > } \\
\text { REFERENCE }\end{array}$ \\
\hline PLUTONIUM-239 & $(\mathrm{PCI} / \mathrm{L})$ & 5 & 5 & 14 & & 0.0000 & & 3.0 & 1.000 & 1 \\
\hline PROTACTINIUM-234 & $(\mathrm{PCI} / \mathrm{L})$ & 1 & 1 & 580 & $\mathbf{E}$ & 580 & $\mathbf{E}$ & 580 & NR & NA \\
\hline RADIUM & $(\mathrm{PCI} / \mathrm{L})$ & 5 & 5 & 0.055 & & 0.0000 & & 0.030 & 4.000 & 0 \\
\hline STRONTIUM & (PCI/L) & 5 & 5 & 61 & & 0.075 & & 27 & 8.000 & 3 \\
\hline TRITIUM & $(\mathrm{PCI} / \mathrm{L})$ & 4 & 4 & 300 & & -190 & & 14 & 20000.0 & 0 \\
\hline 2-BUTANONE & (UG/L) & 30 & 7 & 9.0 & BJ & 6.0 & BJ & 7.6 & NR & $\mathrm{NA}$ \\
\hline 4-METHYL-2-PENTANONE & (UG/L) & 30 & 6 & 2.0 & BJ & 1.0 & $\mathrm{BJ}$ & 1.8 & NR & $\mathrm{NA}$ \\
\hline ACETONE & (UG/L) & 30 & 7 & 4.0 & $\mathrm{~J}$ & 1.0 & BJ & 2.1 & NR & $\mathrm{NA}$ \\
\hline CHLOROBENZENE & (UG/L) & 30 & 1 & 0.60 & $\mathbf{J}$ & 0.60 & $\mathrm{~J}$ & 0.60 & 100.000 & 0 \\
\hline CHLOROFORM & (UG/L) & 30 & 2 & 2.0 & $J$ & 2.0 & $\mathrm{~J}$ & 2.0 & 100.000 & 0 \\
\hline METHYLENE CHLORIDE & (UG/L) & 30 & 13 & 5.0 & $\mathbf{J}$ & 1.0 & $J$ & 2.4 & NR & NA \\
\hline
\end{tabular}


Table 7.19. Constituents in groundwater at the $Y-12$ Plant site

$H G R=B C$ area $=S p o i l$ Area I

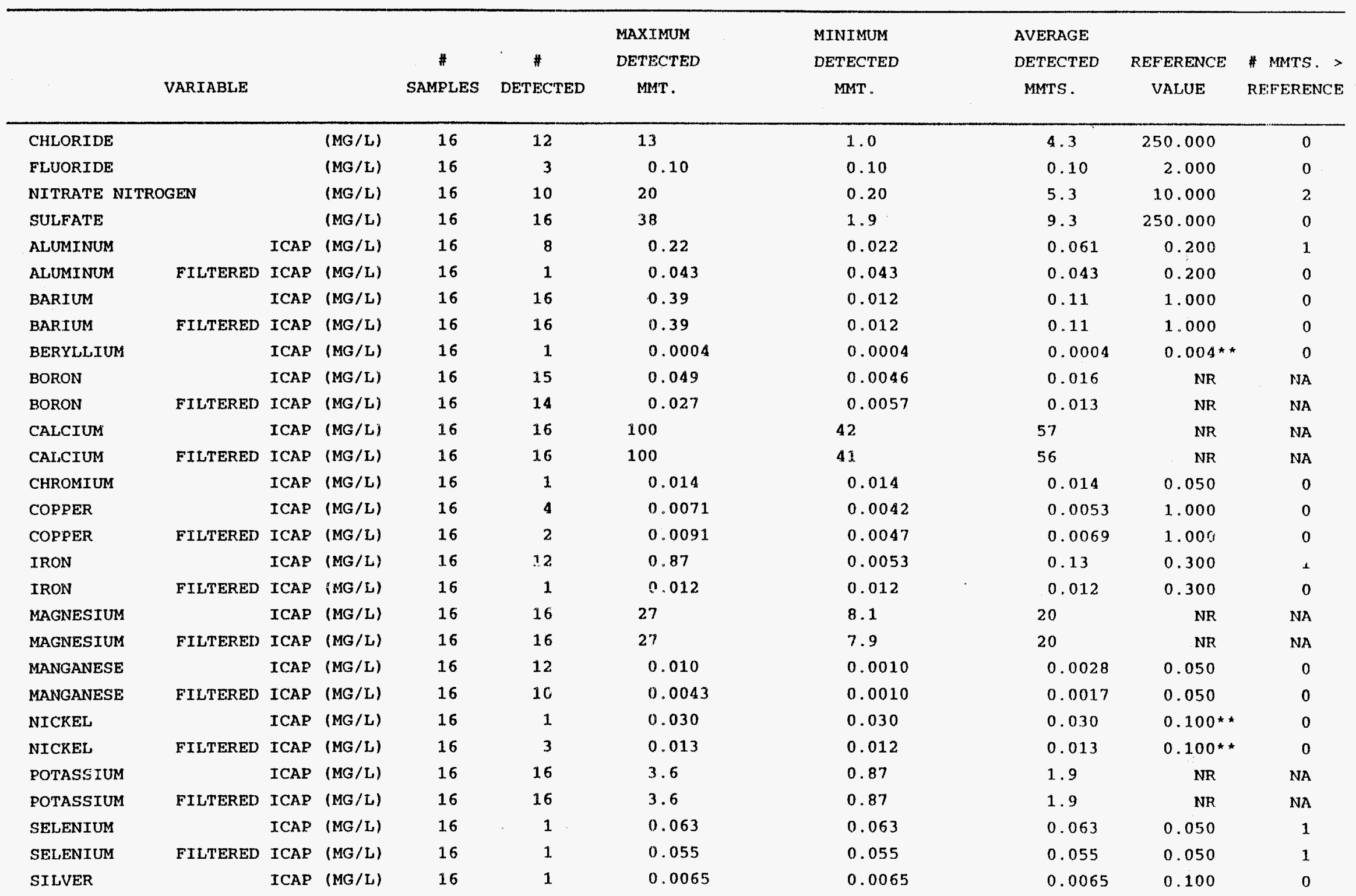


Table 7.19 (continued)

\begin{tabular}{|c|c|c|c|c|c|c|c|c|c|c|}
\hline VARIABLE & & $\begin{array}{c}\# \\
\text { SAMPLES }\end{array}$ & $\begin{array}{c}\# \\
\text { DETECTED }\end{array}$ & $\begin{array}{c}\text { MAXIMUM } \\
\text { DETECTED } \\
\text { MMT }\end{array}$ & & $\begin{array}{c}\text { MINIMUM } \\
\text { DETECTED } \\
\text { MMT. }\end{array}$ & & $\begin{array}{l}\text { AVERAGE } \\
\text { DETECTED } \\
\text { MMTS. }\end{array}$ & $\begin{array}{c}\text { REFERENCE } \\
\text { VALUE }\end{array}$ & $\begin{array}{l}\text { \# MMTS. > } \\
\text { REFERENCE }\end{array}$ \\
\hline SODIUM & ICAP (MG/L) & 16 & 16 & 7.1 & & 0.90 & & 3.4 & NR & NA \\
\hline FILTERED & ICAP (MG/L) & 16 & 16 & 6.9 & & 1.0 & & 3.4 & NR & NA \\
\hline STRONTIUM & ICAP (MG/L) & 16 & 16 & 0.17 & & $0: 018$ & & 0.058 & NR & NA \\
\hline STRONTIUM & ICAP (MG/L) & 16 & 16 & 0.17 & & 0.018 & & 0.059 & NR & NA \\
\hline FLUORIME & ETRIC (MG/L) & 16 & 9 & 0.0060 & & 0.0010 & & 0.0017 & 13.000 & 0 \\
\hline URANIUM FILT. FLUORIME & OTRIC (MG/L) & 16 & 8 & 0.0010 & & 0.0010 & & 0.0010 & 13.000 & 0 \\
\hline ZINC & ICAP (MG/L) & 16 & 16 & 0.049 & & 0.0034 & & 0.015 & 5.000 & 0 \\
\hline FILTERED & ICAP (MG/L) & 16 & 16 & 0.054 & & 0.0047 & & 0.016 & 5.000 & 0 \\
\hline CONDUCTIVITY, FIELD MMT & (UMHOS/CM) & 16 & $\mathrm{NA}$ & 580 & & 320 & & 400 & NR & NA \\
\hline DISSOLVED OXYGEN, FIELD & MMT. (PPM) & 16 & NA & 8.9 & & 2.5 & & 7.1 & NR & NA \\
\hline PH, FIELD MMT. & (PH UNITS) & 16 & NA & 8.1 & & 6.5 & & 7.5 & $6.5 / 8.5$ & 0 \\
\hline REDOX, FIELD MMT. & (MV) & 16 & NA & 230 & & 14 & & 180 & NR & NA \\
\hline WATER TEMP, FIELD MAT & (DEG. CENT.) & 16 & NA & 19 & & 12 & & 15 & NR & 0 \\
\hline ALKALINITY $-\mathrm{HCO} 3$ & (MG/L) & 16 & 16 & 230 & & 180 & & 210 & $\mathrm{NR}$ & $\mathrm{NA}$ \\
\hline CONDUCTIVITY & (UMHOS/CM) & 16 & 16 & 630 & & 360 & & 440 & NR & NA \\
\hline DISSOLVED SOLIDS & $(M G / L)$ & 16 & 16 & 410 & & 180 & & 250 & 500.000 & 0 \\
\hline PH & (PH UNITS) & 16 & NA & 8.0 & & 7.1 & & 7.8 & $6.5 / 8.5$ & 0 \\
\hline TOTAL SUSPENDED SOLIDS & (MG/L) & 16 & 3 & 4.0 & & 1.0 & & 2.7 & NR & NA \\
\hline TURBIDITY & (NTU) & 16 & 16 & 8. 0 & & 0.40 & & 1.8 & 1.000 & 7 \\
\hline GROSS ALPHA & $(\mathrm{PCI} / \mathrm{L})$ & 16 & 16 & 5.4 & & -2.5 & & 1.1 & 15.000 & 0 \\
\hline GROSS BETA & $(\mathrm{PCI} / \mathrm{L})$ & 16 & 16 & 54 & & -1.4 & & 9.0 & 50.000 & 1 \\
\hline 1,2-DICHLOROETHENE & $(U G / L)$ & 16 & 4 & 7.0 & & 2.0 & $\mathrm{~J}$ & 4.8 & 70.000 & 0 \\
\hline 2-BUTANONE & (UG/L) & 16 & 6 & 8.0 & BJ & 3.0 & $\mathrm{BJ}$ & 5.7 & NR & NA \\
\hline 4-METHYL-2-PENTANONE & (UG/L) & 16 & 4 & 2.0 & BJ & 2.0 & BJ & 2.0 & NR & NA \\
\hline ACETONE & (UG/L) & 16 & 5 & 3.0 & $\mathrm{BJ}$ & 1.0 & BJ & 2.2 & NR & NA \\
\hline BROMODICHLOROMETHANE & $(\mathrm{UG} / \mathrm{L})$ & 16 & 4 & 2.0 & $\mathfrak{J}$ & 1.0 & $J$ & 1.8 & 100.000 & 0 \\
\hline CHLOROFORM & (UG/L) & 16 & 4 & 9.0 & & 6.0 & & 7.5 & 100.000 & 0 \\
\hline METHYLENE CHLORIDE & (UG/L) & 16 & 4 & 2.0 & $\mathrm{BJ}$ & 1.0 & JB & 1.5 & NR & NA \\
\hline TETRACHLOROETHENE & $(U G / L)$ & 16 & 4 & 10 & & 5.0 & & 7.5 & 5.000 & 3 \\
\hline TRICHLOROETHENE & (UG/L) & 16 & 4 & 5.0 & & 2.0 & $J$ & 3.8 & 5.000 & 0 \\
\hline
\end{tabular}


Table 7.20. Constituents in groundwater at the Y-12. Plant site $\mathrm{HGR}=\mathrm{EF}$ area=Background

\begin{tabular}{|c|c|c|c|c|c|c|c|c|c|c|}
\hline & VARIABL,E & & & $\begin{array}{c}\# \\
\text { SAMPLES }\end{array}$ & $\begin{array}{c}\# \\
\text { DETECTED }\end{array}$ & $\begin{array}{l}\text { MAXIMUM } \\
\text { DETECTED } \\
\text { MMT. }\end{array}$ & $\begin{array}{l}\text { MINLMUM } \\
\text { DETECTED } \\
\text { MMT. }\end{array}$ & $\begin{array}{c}\text { AVERAGE } \\
\text { DETECTED } \\
\text { MMTS. }\end{array}$ & $\begin{array}{l}\text { REFERENCE } \\
\text { VALUE }\end{array}$ & $\begin{array}{l}\text { \# MMTS. > } \\
\text { REFERENCE }\end{array}$ \\
\hline \multicolumn{3}{|l|}{ CHLORIDE } & (MG/L) & 24 & 22 & 59 & 1.0 & 16 & 250.000 & 0 \\
\hline \multicolumn{3}{|l|}{ FLUORIDE } & (MG/L) & 24 & 20 & 0.30 & 0.10 & 0.16 & 2.000 & 0 \\
\hline \multicolumn{3}{|c|}{ NITRATE NITROGEN } & $(M G / L)$ & 24 & 4 & 0.60 & 0.27 & 0.45 & 10.000 & 0 \\
\hline \multicolumn{3}{|l|}{ SULFATE } & (MG/L) & 24 & 24 & 140 & 2.0 & 35 & 250.000 & 0 \\
\hline \multicolumn{2}{|l|}{ ALUMINUM } & ICAP & (MG/L) & 24 & 24 & 42 & 0.038 & 3.1 & 0.200 & $i 7$ \\
\hline ALUMINUM & FILTERED & ICAP & (MG/L) & 24 & 14 & 0.16 & 0.021 & 0.046 & 0.200 & $r$ \\
\hline \multicolumn{2}{|l|}{ BARIUM } & ICAP & (MG/L) & 24 & 24 & 1.1 & 0.024 & 0.22 & 1.000 & 1 \\
\hline BARIUM & FILTERED & ICAP & (MG/L) & 24 & 24 & 0.32 & 0.026 & 0.16 & 1.000 & 0 \\
\hline \multicolumn{2}{|l|}{ BERYLLIUM } & ICAP & (MG/L) & 24 & 3 & 0.0021 & 0.0004 & 0.0010 & $0.004^{* k}$ & 0 \\
\hline \multicolumn{2}{|l|}{ BORON } & ICAP & (MG/L) & 24 & 24 & 0.18 & 0.010 & 0.039 & NR & NA \\
\hline BORON & FILTERED & ICAP & $(M G / L)$ & 24 & 24 & 0.075 & 0.010 & 0.030 & NR & NA \\
\hline \multicolumn{2}{|l|}{ CADMIUM } & AAS & (MG/L) & 24 & 3 & 0.0067 & 0.0030 & 0.0045 & 0.005 & 1 \\
\hline \multicolumn{2}{|l|}{ CADMIUM } & ICAP & (MG/L) & 24 & 3 & 0.0062 & 0.0035 & 0.0049 & 0.005 & 1 \\
\hline CADMIUM & FILTERED & AAS & $(M G / L)$ & 24 & 3 & 0.0066 & 0.0025 & 0.0040 & 0.005 & 1. \\
\hline \multicolumn{2}{|l|}{ CALCIUM } & ICAP & (MG/L) & 24 & 24 & 220 & 46 & 90 & NR & NA \\
\hline CALCIUM & FILTERED & ICAP & (MG/L) & 24 & 24 & 150 & 46 & 84 & NR & NA \\
\hline \multicolumn{2}{|l|}{ CHROMIUM } & AAS & $(M G / L)$ & 24 & 6 & 5.8 & 0.016 & 1.0 & 0.050 & 4 \\
\hline \multicolumn{2}{|l|}{ CHROMIUM } & ICAP & (MG/L) & 24 & 5 & 5.0 & 0.036 & 1.1 & 0.050 & 4 \\
\hline CHROMIUM & FILTERED & AAS & (MG/L) & 24 & 1 & 0.026 & 0.026 & 0.026 & 0.050 & 0 \\
\hline \multicolumn{2}{|l|}{ COBALT } & ICAP & (MG/L) & 24 & 5 & 0.048 & 0.0054 & 0.020 & NR & NA \\
\hline COBALT & FILTERED & ICAP & (MG/L) & 24 & 2 & 0.0091 & 0.0066 & 0.0079 & NR & NA \\
\hline \multicolumn{2}{|l|}{ COPPER } & ICAP & (MG/L) & 24 & 12 & 0.20 & 0.0041 & 0.025 & 1.000 & 0 \\
\hline \multirow{2}{*}{\multicolumn{2}{|c|}{$\begin{array}{l}\text { COPPER } \\
\text { IRON }\end{array}$}} & ICAP & $(M G / L)$ & 24 & 2 & 0.034 & 0.0046 & 0.019 & 1.000 & 0 \\
\hline & & ICAP & $(M G / L)$ & 24 & 24 & 56 & 0.011 & 5.8 & 0.300 & 19 \\
\hline FILTERED & FILTERED & ICAP & (MG/L) & 24 & 17 & 4.6 & 0.0083 & 0.84 & 0.300 & 5 \\
\hline \multicolumn{2}{|l|}{ LEAD } & AÂS & (MG/L) & 24 & 3 & 0.11 & 0.0066 & 0.048 & 0.050 & 1 \\
\hline \multicolumn{2}{|l|}{ MAGNESIUM } & ICAP & $(M G / L)$ & 24 & 24 & 42 & 5.9 & 17 & NR & NA \\
\hline MAGNESIUM & FILTERED & ICAP & (MG/L) & 24 & 24 & 41 & 5.9 & 16 & NR & NA \\
\hline \multicolumn{2}{|l|}{ MANGANESE } & ICAP & $(M G / L)$ & 24 & 24 & 2.1 & 0.011 & 0.46 & 0.050 & 13 \\
\hline
\end{tabular}


Table 7.20 (continued)

\begin{tabular}{|c|c|c|c|c|c|c|c|c|}
\hline VARIABLE & & $\begin{array}{c}\# \\
\text { SAMPLES }\end{array}$ & $\begin{array}{c}\# \\
\text { DETECTED }\end{array}$ & $\begin{array}{l}\text { MAXIMUM } \\
\text { DETECTED } \\
\text { MMT. }\end{array}$ & $\begin{array}{l}\text { MINIMUM } \\
\text { DETECTED } \\
\text { MMT. }\end{array}$ & $\begin{array}{l}\text { AVERAGE } \\
\text { DETECTED } \\
\text { MMTS. }\end{array}$ & $\begin{array}{l}\text { REFERENCE } \\
\text { VALUE }\end{array}$ & $\begin{array}{l}\# \text { MMTS. > } \\
\text { REFERENCE }\end{array}$ \\
\hline MANGANESE & ICAP (MG/L) & 24 & 24 & 2.2 & 0.0027 & 0.38 & 0.050 & 9 \\
\hline MOLYBDENUM & ICAP (MG/L) & 24 & 1 & 0.051 & 0.051 & 0.051 & NR & NA \\
\hline NICKEL & $\operatorname{ICAP}(M G / L)$ & 24 & 7 & 2.1 & 0.011 & 0.45 & $0.100 * *$ & 4 \\
\hline FILTERED & ICAP $(M G / L)$ & 24 & 7 & 0.96 & 0.012 & 0.25 & $0.100 * *$ & 4 \\
\hline POTASSIUM & $\operatorname{ICAP}(M G / L)$ & 24 & 24 & 13 & 1.2 & 2.7 & NR & NA \\
\hline POTASSIUM & $\operatorname{ICAP}(\mathrm{MG} / \mathrm{L})$ & 24 & 24 & 3.8 & 0.91 & 2.1 & NR & NA \\
\hline SODIUM & $\operatorname{ICAP}(M G / L)$ & 24 & 24 & 29 & 6.8 & 15 & NR & NA \\
\hline FILTERED & $\operatorname{ICAP}(\mathrm{MG} / \mathrm{L})$ & 24 & 24 & 30 & 6.8 & 15 & NR & $\mathrm{NA}$ \\
\hline STRONTIUM & $\operatorname{ICAP}(\mathrm{MG} / \mathrm{L})$ & 24 & 24 & 0.96 & 0.087 & 0.25 & NR & NA \\
\hline STRONTIUM & ICAP (MG/L) & 24 & 24 & 0.53 & 0.087 & 0.23 & NR & NA \\
\hline URANIUM & ETRIC (MG/L) & 24 & 9 & 0.0020 & 0.0010 & 0.0011 & 13.000 & 0 \\
\hline URANIUM FILT. FLUORIME & ETRIC (MG/L) & 24 & 9 & 0.0010 & 0.0010 & 0.0010 & 13.000 & 0 \\
\hline VANADIUM & ICAP $(M G / L)$ & 24 & 4 & 0.060 & 0.0057 & 0.027 & NR & $\mathrm{NA}$ \\
\hline ZINC & ICAP (MG/L) & 24 & 24 & 0.20 & 0.0042 & 0.030 & 5.000 & 0 \\
\hline FILTERED & ICAP (MG/L) & 24 & 22 & 0.063 & 0.0024 & 0.017 & 5.000 & 0 \\
\hline CONDUCTIVITY, FIELD MMT & T (UMHOS/CM) & 24 & NA & 1000 & 300 & 550 & $\mathrm{NR}$ & NA \\
\hline DISSOLVED OXYGEN, FIELD & D MMT. (PPM) & 24 & $\mathrm{NA}$ & 8.4 & 1.2 & 5.6 & NR & NA \\
\hline PH， FIELD MMT. & (PH UNITS) & 24 & NA & 8.1 & 6.4 & 7.2 & $6.5 / 8.5$ & 2 \\
\hline REDOX, FIELD MMT. & (MV) & 24 & NA & 220 & -160 & 91 & NR & NA \\
\hline WATER TEMP, FIELD MMT & (DEG. CENT.) & 24 & NA & 29 & 12 & 18 & NR & 0 \\
\hline ALKALINITY-HCO3 & $(M G / L)$ & 24 & 24 & 450 & 150 & 250 & NR & NA \\
\hline CONDUCTIVITY & (UMHOS/CM) & 24 & 24 & 1100 & 3.60 & 570 & NR & NA \\
\hline CONDUCTIVITY, REP. 2 & (UMHOS/CM) & 1 & 1 & 460 & 460 & 460 & NR & NA \\
\hline DISSOLVED SOLIDS & $(\mathrm{MG} / \mathrm{L})$ & 24 & 24 & 740 & 220 & 370 & 500.000 & 4 \\
\hline PH & (PH UNITS) & 24 & NA & 8.3 & 6.7 & 7.6 & $6.5 / 8.5$ & 0 \\
\hline PH， REP. 2 & (PH UNITS) & 1 & NA & 7.9 & 7.9 & 7.9 & $6.5 / 8.5$ & 0 \\
\hline TOTAL SUSPENDED SOLIDS & (MG/L) & 24 & 23 & 2200 & 1.0 & 130 & $\mathrm{NR}$ & NA \\
\hline TURBIDITY & (NTU) & 24 & 24 & 1700 & 1.0 & 130 & 1.000 & 23 \\
\hline GROSS ALPHA & $(\mathrm{PCI} / \mathrm{L})$ & 24 & 24 & 28 & -4.3 & 1.3 & 15.000 & 1 \\
\hline
\end{tabular}


Table 7.20 (continued)

\begin{tabular}{|c|c|c|c|c|c|c|c|c|c|c|}
\hline VARIABLE & & $\begin{array}{c}\# \\
\text { SAMPLES }\end{array}$ & $\begin{array}{c}\# \\
\text { DETECTED }\end{array}$ & $\begin{array}{c}\text { MAXIMUM } \\
\text { DETECTED } \\
\text { MMT. }\end{array}$ & & $\begin{array}{l}\text { MINIMUM } \\
\text { DETECTED } \\
\text { MMT. }\end{array}$ & & $\begin{array}{c}\text { AVERAGE } \\
\text { DETECTED } \\
\text { MMTS. }\end{array}$ & $\begin{array}{c}\text { REFERENCE } \\
\text { VALUE }\end{array}$ & $\begin{array}{l}\text { \# MMTS. > } \\
\text { REFERENCE }\end{array}$ \\
\hline & & & & . & & & & & & \\
\hline GROSS BETA & (PCI/L) & 24 & 24 & 81 & & -8.5 & & 6.9 & 50.000 & 1 \\
\hline 1,1-DICHLOROETHANE & (UG/Is) & 24 & 2 & 1.0 & $\mathbf{J}$ & 1.0 & $\mathrm{~J}$ & 1.0 & $\mathrm{NR}$ & NA \\
\hline 1,2-DICHLOROETHENE & (UG/L) & 24 & 4 & 19 & & 9.0 & & 16 & 70.000 & 0 \\
\hline 2-BUTANONE & (UG/L) & 24 & 2 & 9.0 & $J B$ & 8.0 & $\mathrm{JB}$ & 8.5 & NR & NA \\
\hline 4-METHXL-2-PENTANONE & (UG/L) & 24 & 5 & 2.0 & $\mathrm{BJ}$ & 1.0 & $\mathrm{JB}$ & 1.6 & $\mathrm{NR}$ & NA \\
\hline ACETONE & (UG/L) & 24 & 3 & 32 & & 4.0 & JB & 13 & NR & NA \\
\hline METHYLENE CHLORIDE & (UG/L) & 24 & 8 & 2.0 & $\mathrm{JB}$ & 1.0 & $\mathrm{~J}$ & 1.4 & NR & NA \\
\hline TETRACHLOROETHENE & (UG/L) & 24 & 3 & 1.0 & $\mathrm{~J}$ & 1.0 & $\mathfrak{J}$ & 1.0 & 5.000 & 0 \\
\hline TRICHLOROETHENE & (UG/L) & 24 & 3 & 3.0 & $\mathbf{J}$ & 3.0 & $\mathrm{~J}$ & 3.0 & 5.000 & 0 \\
\hline
\end{tabular}


Table 7.21. Constituents in groundwater at the Y-12 Plant site HGR=EF area=Beta-4 Security Pit

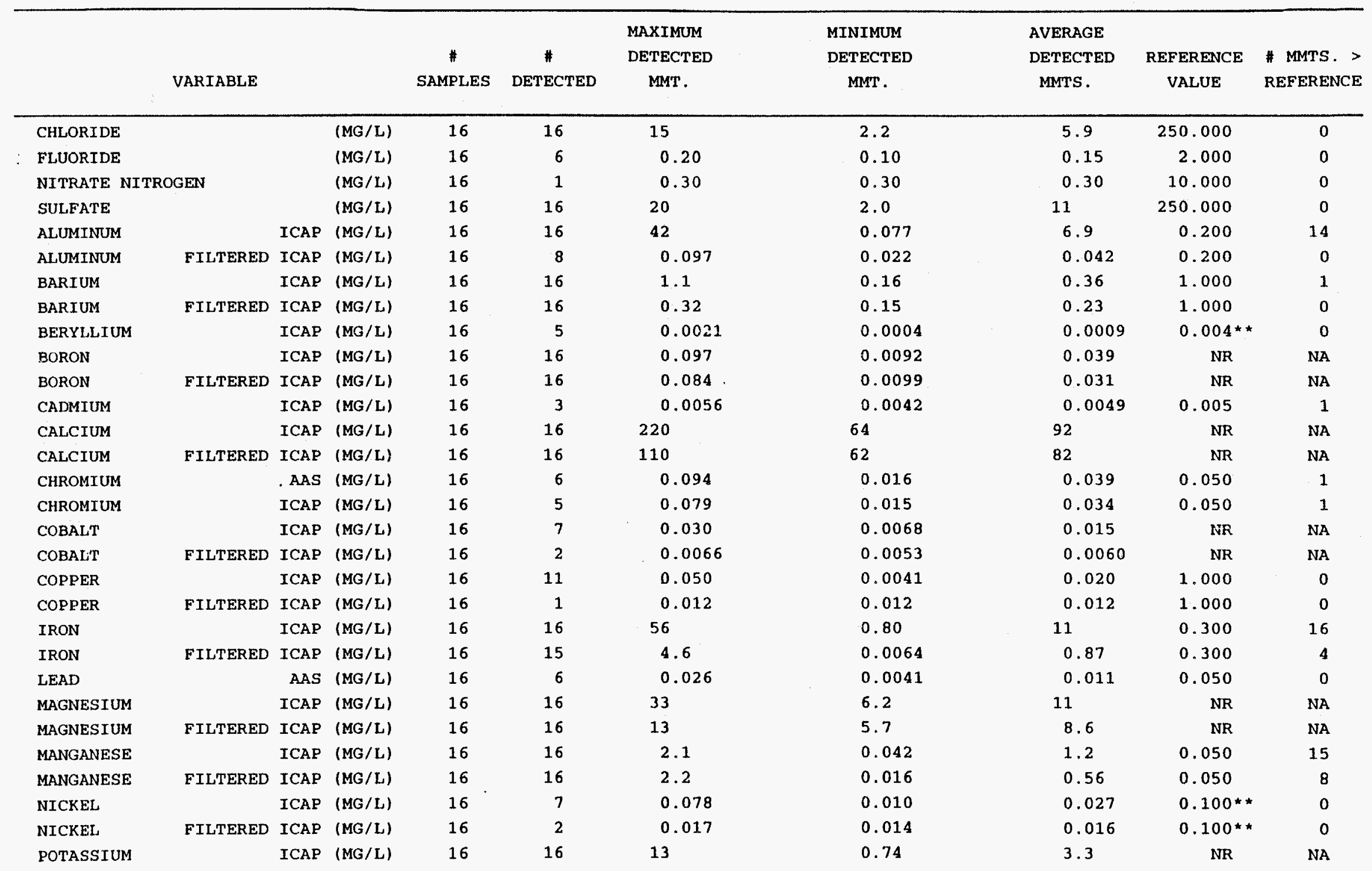


Table 7.21 (continued)

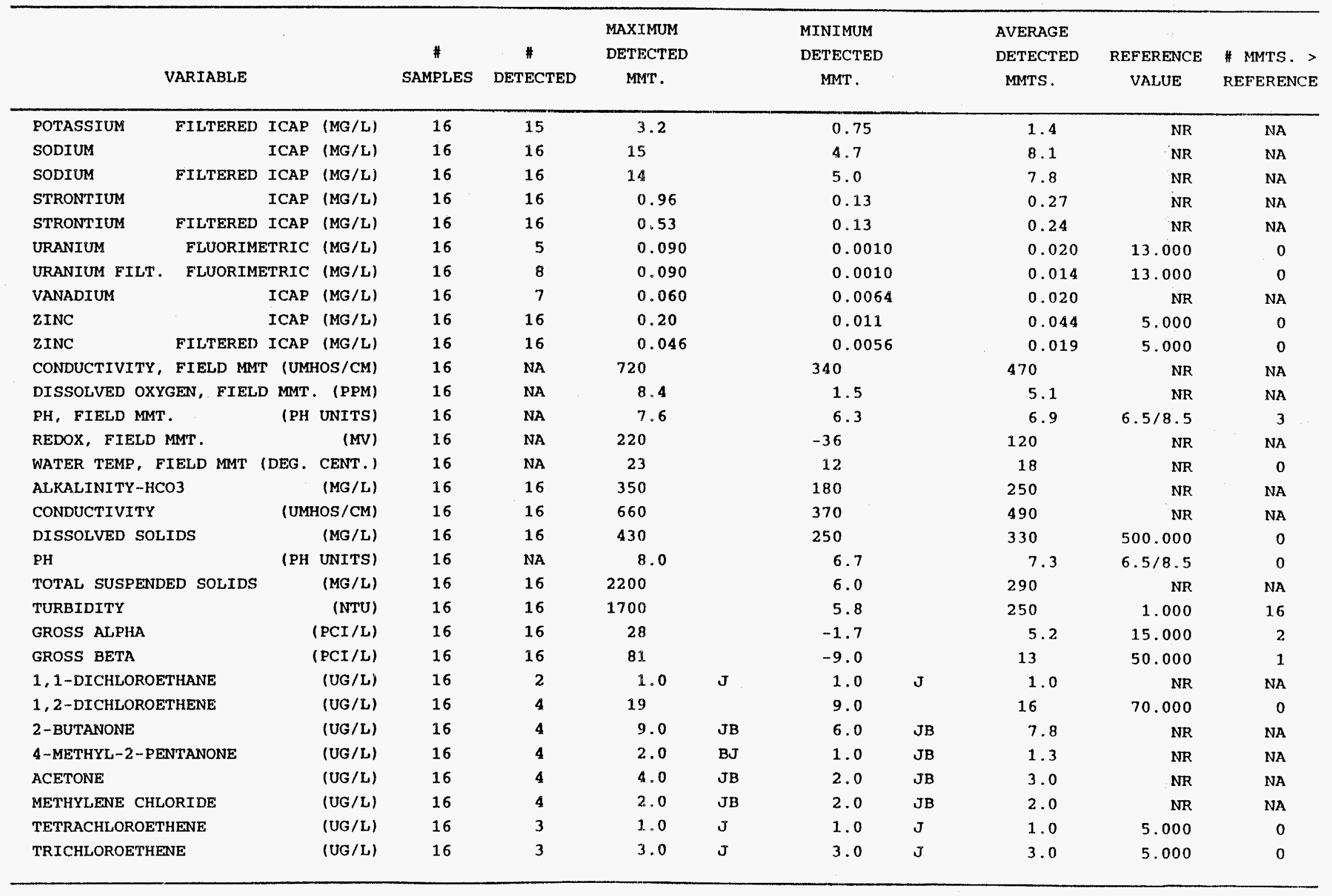


Table 7.22. Constituents in groundwater at the Y-12 Plant site

\begin{tabular}{|c|c|c|c|c|c|c|c|c|c|c|}
\hline & VARIABLE & & & $\begin{array}{c}\# \\
\text { SAMPLES }\end{array}$ & $\begin{array}{c}* \\
\text { DETECTED }\end{array}$ & $\begin{array}{l}\text { MAXIMUM } \\
\text { DETECTED } \\
\text { MMT. }\end{array}$ & $\begin{array}{l}\text { MINIMUM } \\
\text { DETECTED } \\
\text { MMT. }\end{array}$ & $\begin{array}{c}\text { AVERAGE } \\
\text { DETECTED } \\
\text { MMTS }\end{array}$ & $\begin{array}{c}\text { REFERENCE } \\
\text { VALUE }\end{array}$ & $\begin{array}{l}\# \text { MMTS, > } \\
\text { REFERENCE }\end{array}$ \\
\hline CHLORIDE & & & $(\mathrm{MG} / \mathrm{L})$ & 64 & 64 & 280 & 1.0 & 29 & 250.000 & 3 \\
\hline FLUORIDE & & & $(M G / L)$ & 64 & 43 & 3.6 & 0.10 & 0.63 & 2.000 & 6 \\
\hline NITRATE NI & DGEN & & $(\mathrm{MG} / \mathrm{L})$ & 64 & 35 & 2.4 & 0.20 & 0.82 & 10.000 & 0 \\
\hline SULFATE & & & $(M G / L)$ & 64 & 64 & 110 & 4.5 & 27 & 250.000 & 0 \\
\hline ALUMINUM & & ICAP & $(M G / L)$ & 64 & 45 & 75 & 0.020 & 7.2 & 0.200 & 20 \\
\hline ALUMINUM & FILTERED & ICAP & $(M G / L)$ & 64 & 17 & 2.3 & 0.023 & 0.28 & 0.200 & 4 \\
\hline ANTIMONY & FILTERED & ICAP & (MG/L) & 64 & 1 & 0.097 & 0.097 & 0.097 & $0.006 * \star$ & 0 \\
\hline ARSENIC & & ICAP & $(M G / L)$ & 64 & 1 & 0.063 & 0.063 & 0.063 & 0.050 & 1 \\
\hline ARSENIC & FILTERED & ICAP & $(M G / L)$ & 64 & 2 & 0.071 & 0.050 & 0.061 & 0.050 & 1 \\
\hline BARIUM & & ICAP & (MG/L) & 64 & 64 & 0.88 & 0.012 & 0.12 & 1.000 & 0 \\
\hline BARIUM & FILTERED & ICAP & (MG/L) & 64 & 64 & 0.60 & 0.010 & 0.091 & 1.000 & 0 \\
\hline BERYLLIUM & & ICAP & $(M G / L)$ & 64 & 9 & 0.0092 & 0.0006 & 0.0039 & $0.004 * *$ & 3 \\
\hline BERYLLIUM & FILTERED & ICAP & $(M G / L)$ & 64 & 1 & 0.0011 & 0.0011 & 0.0011 & $0.004^{* *}$ & 0 \\
\hline BORON & & ICAP & $(M G / L)$ & 64 & 63 & 1.6 & 0.0064 & 0.25 & NR & $\mathrm{NA}$ \\
\hline BORON & FILTERED & ICAP & (MG /L) & 64 & 63 & 1.6 & 0.0049 & 0.25 & NR & NA \\
\hline CADMIUM & & AAS & $(M G / L)$ & 64 & 7 & 0.030 & 0.0021 & 0.014 & 0.005 & 5 \\
\hline CADMIUM & & ICAP & $(M G / L)$ & 64 & 11 & 0.027 & 0.0034 & 0.015 & 0.005 & 9 \\
\hline CADMIUM & FILTERED & AAS & $(M G / L)$ & 64 & 4 & 0.022 & 0.0040 & 0.0089 & 0.005 & 2 \\
\hline CALCIUM & & ICAP & $(M G / L)$ & 64 & 64 & 150 & 1.2 & 62 & NR & NA \\
\hline CALCIUM & FILTERED & ICAP & $(M G / L)$ & 64 & 64 & 120 & 1.1 & 59 & NR & NA \\
\hline CHROMIUM & & AAS & (MG/L) & 64 & 12 & 0.20 & 0.010 & 0.071 & 0.050 & 8 \\
\hline CHROMIUM & & ICAP & $(M G / L)$ & 64. & 11 & 0.12 & 0.010 & 0.059 & 0.050 & 6 \\
\hline COBALT & & ICAP & (MG/L) & 64 & 13 & 0.089 & 0.0054 & 0.033 & NR & NA \\
\hline COBALT & FILTERED & ICAP & $(M G / L)$ & 64 & 6 & 0.025 & 0.0051 & 0.010 & $\mathrm{NR}$ & NA \\
\hline COPPER & & ICAP & $\left(M G / L_{1}\right)$ & 64 & 27 & 0.11 & 0.0040 & 0.023 & 1.000 & 0 \\
\hline COPPER & FILTERED & ICAP & $(M G / L)$ & 64 & 9 & 0.017 & 0.0040 & 0.0084 & 1.000 & 0 \\
\hline IRON & & ICAP & (MG/L) & 64 & 64 & 130 & 0.017 & 10 & 0.300 & 41 \\
\hline IRON & FILTERED & ICAP & $(M G / L)$ & 64 & 44 & 47 & 0.0071 & 1.9 & 0.300 & 13 \\
\hline
\end{tabular}


Table 7.22 (continued)

\begin{tabular}{|c|c|c|c|c|c|c|c|c|c|c|}
\hline & VARIABLE & & & $\begin{array}{c}\# \\
\text { SAMPLES }\end{array}$ & $\begin{array}{c}\# \\
\text { DETECTED }\end{array}$ & $\begin{array}{l}\text { MAXYMUM } \\
\text { DETECTED } \\
\text { MMT. }\end{array}$ & $\begin{array}{l}\text { MINIMUM } \\
\text { DETECTED } \\
\text { MMT. }\end{array}$ & $\begin{array}{l}\text { AVERAGE } \\
\text { DETECTED } \\
\text { MMTS. }\end{array}$ & $\begin{array}{c}\text { REFERENCE } \\
\text { VALUE }\end{array}$ & $\begin{array}{l}\text { \# MMTS. } \\
\text { REFERENCE }\end{array}$ \\
\hline LEAD & & AAS & (MG/L) & 64 & 11 & 0.18 & 0.0045 & 0.081 & 0.050 & 6 \\
\hline LEAD & & ICAP & (MG/L) & 32 & 4 & 0.16 & 0.0000 & 0.090 & 0.050 & 3 \\
\hline LEAD & FILTERED & AAS & (MG/L) & 64 & 1 & 0.064 & 0.064 & 0.064 & 0.050 & 1 \\
\hline MAGNESIUM & & ICAP & (MG/L) & 64 & 64 & 79 & 0.43 & 19 & NR & NA \\
\hline MAGNESIUM & FILTERED & ICAP & (MG/L) & 64 & 64 & 53 & 0.46 & 17 & NR & NA \\
\hline MANGANESE & & ICAP & (MG/L) & 64 & 61 & 7.8 & 0.0010 & 0.83 & 0.050 & 29 \\
\hline MANGANESE & FILTERED & ICAP & (MG/L) & 64 & 56 & 5.7 & 0.0012 & 0.49 & 0.050 & 22 \\
\hline MERCURY & & CVAA & (MG/L) & 64 & 1 & 0.0003 & 0.0003 & 0.0003 & 0.002 & 0 \\
\hline NICKEL & & ICAP & (MG/L) & 64 & 16 & 0.37 & 0.012 & 0.097 & $0.100 * *$ & 6 \\
\hline NICKEL & FILTERED & ICAP & $(M G / L)$ & 64 & 12 & 0.30 & 0.010 & 0.075 & $0.100 * *$ & 3 \\
\hline POTASSIUM & & ICAP & (MG/L) & 64 & 63 & 19 & 1.0 & 4.1 & $\mathrm{NR}$ & NA \\
\hline POTASSIUM & FILTERED & ICAP & (MG/L) & 64 & 63 & 7.5 & 0.92 & 3.0 & NR & $\mathrm{NA}$ \\
\hline SELENIUM & & ICAP & (MG/L) & 64 & 3 & 0.056 & 0.053 & 0.054 & 0.050 & 3 \\
\hline SELENIUM & FILTERED & ICAP & $(M G / L)$ & 64 & 1 & 0.061 & 0.061 & 0.061 & 0.050 & 1 \\
\hline SILVER & & ICAP & (MG/L) & 64 & 1 & 0.012 & 0.012 & 0.012 & 0.100 & 0 \\
\hline SODIUM & & ICAP & (MG /L) & 64 & 64 & 430 & 1.3 & 46 & NR & NA \\
\hline SODIUM & FILTERED & ICAP & (MG/L) & 65 & 64 & 430 & 1.2 & 46 & NR & NA \\
\hline STRONTIUM & & ICAP & (MG/L) & 65 & 64 & 1.5 & 0.036 & 0.31 & NR & NA \\
\hline STRONTIUM & FILTERED & ICAP & (MG/L) & 65 & 64 & 1.5 & 0.033 & 0.31 & $\mathrm{NR}$ & $\mathrm{NA}$ \\
\hline URANIUM & FLUORIME & ETRIC & (MG/L) & 65 & 20 & 0.19 & 0.0010 & 0.028 & 13.000 & 0 \\
\hline URANIUM FILT 。 & FLUORTME & ETRIC & (MG/L) & 65 & 17 & 0.19 & 0.0010 & 0.038 & 13.000 & 0 \\
\hline VANADIUM & & ICAP & $(M G / L)$ & 65 & 10 & 0.13 & 0.0061 & 0.062 & NR & $\mathrm{NA}$ \\
\hline VANADIUM & FILTERED & ICAP & (MG/L) & 65 & 1 & 0.021 & 0.021 & 0.021 & NR & NA \\
\hline ZINC & & ICAP & (MG/L) & 65 & 60 & 2.0 & 0.0037 & 0.16 & 5.000 & 0 \\
\hline ZINC & FILTERED & ICAP & (MG/L) & 65 & 61 & 1.8 & 0.0027 & 0.10 & 5.000 & 0 \\
\hline CONDUCTIVITY, & FIELD MMT & IUMH & $\mathrm{IOS} / \mathrm{CM})$ & 55 & NA & 1800 & 150 & 570 & NR & NA \\
\hline DISSOLVED OXYG & GEN， FIELD & MMT. & (PPM) & 65 & iNA & 9.3 & 0.20 & 2.8 & NR & NA \\
\hline PH, FIELD MMT. & & (PH & UNITS) & 65 & NA & 9.0 & 4.8 & 7.3 & $6.5 / 8.5$ & 13 \\
\hline REDOX，FIELD & MMT . & & (MV) & 65 & NA & 240 & -320 & 73 & NR & NA \\
\hline
\end{tabular}


Table 7.22 (continued)

\begin{tabular}{|c|c|c|c|c|c|c|c|c|c|c|}
\hline VARIABLE & & $\begin{array}{c}\# \\
\text { SAMPLES }\end{array}$ & $\begin{array}{c}\# \\
\text { DETECTED }\end{array}$ & $\begin{array}{l}\text { MAXIMUM } \\
\text { DETECTED } \\
\text { MMT. }\end{array}$ & & $\begin{array}{c}\text { MINIMUM } \\
\text { DETECTED } \\
\text { MMT. }\end{array}$ & & $\begin{array}{l}\text { AVERAGE } \\
\text { DETECTED } \\
\text { MMTS. }\end{array}$ & $\begin{array}{l}\text { REFERENCE } \\
\text { VALUE }\end{array}$ & $\begin{array}{l}\text { \# MMTS. > } \\
\text { REFERENCE }\end{array}$ \\
\hline WATER TEMP, FIELD MMT & (DEG. CENT.) & 65 & $\mathrm{NA}$ & 22 & & 12 & & 15 & NR & 0 \\
\hline ALKALINITY-CO3 & (MG/L) & 65 & 6 & 82 & & 40 & & 57 & NR & NA \\
\hline ALKALTNITY-HCO3 & (MG/L) & 65 & 64 & 510 & & 34 & & 250 & NR & NA \\
\hline CONDUCTIVITY & (UMHOS/CM) & 65 & 64 & 1900 & & 170 & & 610 & NR & $\mathrm{NA}$ \\
\hline DISSOLVED SOLIDS & (MG/L) & 65 & 64 & 1100 & & 110 & & 380 & 500.000 & 8 \\
\hline PH & (PH UNITS) & 65 & NA & 9.3 & & 5.7 & & 7.6 & $6.5 / 8.5$ & 12 \\
\hline TOTAL SUSPENDED SOLIDS & (MG/L) & 65 & 45 & 2800 & & 1.0 & & 250 & NR & NA \\
\hline TURBIDITY & (NTU) & 65 & 64 & 2300 & & 0.70 & & 120 & 1.000 & 59 \\
\hline GROSS ALPHA & (PCI/L) & 65 & 65 & 83 & & -11 & & 7.9 & 15.000 & 9 \\
\hline GROSS BETA & $(\mathrm{PCI} / \mathrm{L})$ & 65 & 65 & 200 & & -4.0 & & 14.3 & 50.000 & 5 \\
\hline 1,1-DICHLOROETHANE & (UG/L) & 65 & 3 & 3.0 & $J$ & 3.0 & $\mathrm{~J}$ & 3.0 & NR & $\mathrm{NA}$ \\
\hline 1,1-DICHLOROETHENE & (UG/L) & 65 & 3 & 2.0 & $\mathrm{~J}$ & 1.0 & $\mathrm{~J}$ & 1.3 & 7.000 & 0 \\
\hline 1,2-DICHLOROETHENE & (UG/L) & 65 & 14 & 41 & & 1.0 & $\mathrm{~J}$ & 22.7 & 70.000 & 0 \\
\hline 2-BUTANONE & (UG/L) & 65 & 4 & 8.0 & $\mathrm{BJ}$ & 3.0 & BJ & 6.3 & NR & NA \\
\hline 4-METHYL-2-PENTANONE & (UG/L) & 65 & 13 & 11 & BJ & 1.0 & BJ & 2.2 & $\mathrm{NR}$ & NA \\
\hline ACETONE & (UG/L) & 65 & 8 & 29 & $\mathrm{~J}$ & 1.0 & $\mathrm{BJ}$ & 6.3 & $\mathrm{NR}$ & $\mathrm{NA}$ \\
\hline CARBON TETRACHLORIDE & (UG/L) & 65 & 15 & 1100 & $\mathbf{E}$ & 1.0 & $\mathbf{J}$ & 260 & 5.000 & 14 \\
\hline CHLOROFORM & (UG/L) & 65 & 19 & 310 & & 1.0 & $\mathrm{~J}$ & 73 & 100.000 & 4 \\
\hline METHYLENE CHLORIDE & (UG/L) & 65 & 21 & 17 & BJ & 0.80 & $\mathrm{~J}$ & 3.2 & NR & NA \\
\hline TETRACHLOROETHENE & (UG/L) & 65 & 26 & 43 & & 1.0 & $\mathrm{~J}$ & 10.8 & 5.000 & 16 \\
\hline TRICHLOROETHENE & (UG/L) & 65 & 22 & 41 & & 0.90 & $\mathbf{J}$ & 9.1 & 5.000 & 9 \\
\hline VINYL ACETATE & (UG/L) & 65 & 1 & 10 & $\mathbf{J}$ & 10 & $J$ & 10 & NR & NA \\
\hline VINYL CHLORIDE & (UG/L) & 65 & 1 & 2.0 & $\mathbf{J}$ & 2.0 & $\mathrm{~J}$ & 2.0 & 2.000 & 0 \\
\hline
\end{tabular}


Table 7.23. Constituents In groundwater at the $\mathrm{Y}-12$ Plant site $H G R=E F$ area=Fire Training Facility

\begin{tabular}{|c|c|c|c|c|c|c|c|c|c|c|}
\hline & VARIABLE & & & $\begin{array}{c}\# \\
\text { SAMPLES }\end{array}$ & $\begin{array}{c}\# \\
\text { DETECTED }\end{array}$ & $\begin{array}{l}\text { MAXIMUM } \\
\text { DETECTED } \\
\text { MMT. }\end{array}$ & $\begin{array}{l}\text { MINIMUM } \\
\text { DETECTED } \\
\text { MMT. }\end{array}$ & $\begin{array}{l}\text { AVERAGE } \\
\text { DETECTED } \\
\text { MMTS. }\end{array}$ & $\begin{array}{c}\text { REFERENCE } \\
\text { VALUE }\end{array}$ & $\begin{array}{l}\text { \# MMTS. > } \\
\text { REFERENCE }\end{array}$ \\
\hline CHLORIDE & & & (MG/L) & 8 & 8 & 3.2 & 1.7 & 2.6 & 250.000 & 0 \\
\hline FLUORIDE & & & $(M G / L)$ & 8 & 4 & 0.20 & 0.10 & 0.15 & 2.000 & 0 \\
\hline NXTRATE NI & DGEN & & (MG/L) & 8 & 8 & 2.3 & 0.49 & 1.3 & 10.000 & 0 \\
\hline SULFATE & & & $(M G / L)$ & 8 & 8 & 9.1 & 2.8 & 5.1 & 250.000 & 0 \\
\hline ALUMINUM & & ICAP & (MG/L) & 8 & 8 & 3.7. & 0.14 & 1.2 & 0.200 & 5 \\
\hline ALUMINUM & FILTERED & ICAP & (MG/L) & 8 & 8 & 3.3 & 0.032 & 1.1 & 0.200 & 6 \\
\hline ARSENIC & FILTERED & ICAP & (MG/L) & 8 & 1 & 0.057 & 0.057 & 0.057 & 0.050 & 1 \\
\hline BARIUM & & ICAP & (MG/L) & 8 & 8 & 0.061 & 0.0069 & 0.024 & 1.000 & 0 \\
\hline BARIUM & FILTERED & ICAP & (MG/L) & 8 & 8 & 0.055 & 0.0067 & $0: 021$ & 1.000 & 0 \\
\hline BORON & & ICAP & (MG/L) & 8 & 8 & 0.084 & 0.019 & 0.042 & NR & NA \\
\hline BORON & FILTERED & ICAP & (MG/L) & 8 & 8 & 0.080 & 0.019 & 0.042 & NR & NA \\
\hline CALCIUM & & ICAP & (MG/L) & 8 & 8 & 200 & 44 & 74 & NR & NA \\
\hline CALCIUM & FILTERED & ICAP & $(M G / L)$ & 8 & 8 & 180 & 36 & 69 & NR. & NA \\
\hline CHROMIUM & & AAS & (MG/L) & B & 1 & 0.013 & 0.013 & 0.013 & 0.050 & 0 \\
\hline CHROMIUM & & ICAP & $(M G / L)$ & 8 & 1 & 0.011 & 0.011 & 0.011 & 0.050 & 0 \\
\hline CHROMIUM & FILTERED & AAS & (MG/L) & 8 & 1 & 0.011 & 0.011 & 0.011 & 0.050 & 0 \\
\hline COPPER & & ICAP & (MG/L) & 8 & 4 & 0.0067 & 0.0044 & 0.0057 & 1.000 & 0 \\
\hline COPPER & FILTERED & ICAP & (MG/L) & 8 & 1 & 0.0046 & 0.0046 & 0.0046 & 1.000 & 0 \\
\hline IRON & & ICAP & $(M G / L)$ & 8 & 7 & 0.11 & 0.013 & 0.042 & 0.300 & 0 \\
\hline IRON & FILTERED & ICAP & $(M G / L)$ & 8 & 2 & 0.012 & 0.0051 & 0.0086 & 0.300 & 0 \\
\hline MAGNESIUM & & ICAP & $(M G / L)$ & 8 & 8 & 9.0 & 0.68 & 3.7 & NR & NA \\
\hline MAGNESIUM & FILTERED & ICAP & (MG/L) & 8 & 8 & 8.5 & 0.030 & 2.5 & NR & NA \\
\hline MANGANESE & & ICAP & (MG/L) & 8 & 4 & 0.0052 & 0.0014 & 0.0030 & 0.050 & 0 \\
\hline MANGANESE & FILTERED & ICAP & (MG/L) & 8 & 2 & 0.0033 & 0.0014 & 0.0024 & 0.050 & 0 \\
\hline NICKEL & & ICAP & $(M G / L)$ & 8 & 1 & 0.010 & 0.010 & 0.010 & $0.100 * *$ & 0 \\
\hline POTASSIUM & & ICAP & $(M G / L)$ & 8 & 8 & 32 & 4.4 & 17 & NR & NA \\
\hline POTASSIUM & FILTERED & ICAP & (MG/L) & 8 & 8 & 32 & 4.9 & 19 & NR & NA \\
\hline SODIUM & & ICAP & (MG/L) & 8 & 8 & 6.0 & 2.5 & 3.6 & NR & NA \\
\hline SODIUM & FILTERED & ICAP & $(M G / L)$ & 8 & 8 & 5.9 & 2.5 & 3.9 & NR & NA \\
\hline
\end{tabular}


Table 7.23 (continued)

\begin{tabular}{|c|c|c|c|c|c|c|c|c|c|}
\hline VARIABLE & $\begin{array}{c}\# \\
\text { SAMPLES }\end{array}$ & $\begin{array}{c}\# \\
\text { DETECTED }\end{array}$ & $\begin{array}{l}\text { MAXIMUM } \\
\text { DETECTED } \\
\text { MMT. }\end{array}$ & & $\begin{array}{c}\text { MINIMUM } \\
\text { DETECTED } \\
\text { MMT. }\end{array}$ & & $\begin{array}{l}\text { AVERAGE } \\
\text { DETECTED } \\
\text { MMTS. }\end{array}$ & $\begin{array}{c}\text { REFERENCE } \\
\text { VALUE }\end{array}$ & $\begin{array}{l}\text { \# MMTS. > } \\
\text { REFERENCE }\end{array}$ \\
\hline ICAP (MG/L) & 8 & 8 & 0.60 & & 0.13 & & 0.23 & NR & NA \\
\hline FILTERED ICAP (MG/L) & 8 & 8 & 0.57 & & 0.11 & & 0.25 & NR & NA \\
\hline FLUORIMETRIC (MG/L) & 8 & 4 & 0.0010 & & 0.0010 & & 0.0010 & 13.000 & 0 \\
\hline URANIUM FILT. FLUORIMETRIC (MG/L) & 8 & 3 & 0.0010 & & 0.0010 & & 0.0010 & 13.000 & 0 \\
\hline ICAP (MG/L) & 8 & 3 & 0.0063 & & 0.0051 & & 0.0056 & NR & NA \\
\hline FILTERED ICAP (MG/L) & 8 & 1 & 0.0052 & & 0.0052 & & 0.0052 & NR & NA \\
\hline ICAP $(M G / L)$ & 8 & 6 & 0.022 & & 0.0022 & & 0.010 & 5.000 & 0 \\
\hline FILTERED ICAP (MG/L) & 8 & 6 & 0.0078 & & 0.0025 & & 0.0052 & 5.000 & 0 \\
\hline CONDUCTIVITY, FIELD MMT (UMHOS/CM) & 8 & NA & 2100 & & 240 & & 590 & NR & NA \\
\hline DISSOLVED OXYGEN, FIELD MMT. (PPM) & 8 & NA & 6.3 & & 1.9 & & 4.0 & NR & $\mathrm{NA}$ \\
\hline PH, FIELD MMT. & 8 & NA & 12 & & 9.1 & & 10 & $6.5 / 8.5$ & 8 \\
\hline REDOX, FIELD MMT. & 8 & NA & 84 & & -37 & & 23 & NR & NA \\
\hline WATER TEMP, FIELD MMT (DEG. CENT.) & 8 & NA & 20 & & 11 & & 16 & NR & 0 \\
\hline ALKALINITY-CO3 & 8 & 7 & 240 & & 8.0 & & 49 & NR & NA \\
\hline ALKALINITY-HCO3 & 8 & 4 & 140 & & 37 & & 74 & NR & NA \\
\hline CONDUCTIVITY & 8 & 8 & 2200 & & 120 & & 490 & NR & NA \\
\hline DISSOLVED SOLIDS & 8 & 8 & 560 & & 72 & & 190 & 500.000 & 1 \\
\hline (PH UNITS) & 8 & NA & 12 & & 8.2 & & 9.9 & $6.5 / 8.5$ & 7 \\
\hline TOTAL SUSPENDED SOLIDS & 8 & 7 & 78 & & 30 & & 53 & NR & NA \\
\hline TURBIDTTY & 8 & 8 & 36 & & 2.9 & & 19 & 1.000 & 8 \\
\hline GROSS ALPHA & 8 & 8 & 3.1 & & -0.67 & & 0.81 & 15.000 & 0 \\
\hline GROSS BETA & 8 & 8 & 20 & & 7.3 & & 13 & 50.000 & 0 \\
\hline 1,2-DICHLOROETHENE & 8 & 8 & 620 & & 61 & & 320 & 70.000 & 7 \\
\hline 2-BUTANONE & 8 & 1 & 41 & B & 41 & B & 41 & NR & NA \\
\hline 4-METHYL-2-PENTANONE & 8 & 1 & 4.0 & JB & 4.0 & JB & 4.0 & NR & NA \\
\hline ACETONE & 8 & 2 & 60 & & 20 & JB & 40 & NR & NA \\
\hline METHYLENE CHLORIDE & 8 & 2 & 9.0 & JB & 8.0 & $J B$ & 8.5 & NR & NA \\
\hline TETRACHLOROETHENE & 8 & 8 & 780 & & 95 & & 360 & 5.000 & 8 \\
\hline TRICHLOROETHENE & 8 & 8 & 230 & & 35 & & 120 & 5.000 & 8 \\
\hline
\end{tabular}


Table 7.24. Constituents In groundwater at the Y-12 Plant site

HGA $=E F$ area=Grid $C 1$

\begin{tabular}{|c|c|c|c|c|c|c|c|c|c|c|}
\hline & VARIABLE & & . & $\begin{array}{c}\# \\
\text { SAMPLES }\end{array}$ & $\begin{array}{c}\# \\
\text { DETECTED }\end{array}$ & $\begin{array}{c}\text { MAXIMUM } \\
\text { DETECTED } \\
\text { MMT. }\end{array}$ & $\begin{array}{l}\text { MINIMUM } \\
\text { DETECTED } \\
\text { MMT. }\end{array}$ & $\begin{array}{c}\text { AVERAGE } \\
\text { DETECTED } \\
\text { MMTS. }\end{array}$ & $\begin{array}{c}\text { REFERENCE } \\
\text { VALUE }\end{array}$ & $\begin{array}{l}\text { \# MMTS. > } \\
\text { REFERENCE }\end{array}$ \\
\hline CHLORIDE & & & $(M G / L)$ & 8 & 8 & 56 & 9.0 & 24 & 250.000 & 0 \\
\hline FLUORIDE & & & (MG/L) & 8 & 7 & 0.10 & 0.10 & 0.10 & 2.000 & 0 \\
\hline SULFATE & & & (MG/L) & 8 & 8 & 16 & 2.0 & 9.1 & 250.000 & 0 \\
\hline ALUMINUM & & ICAP & (MG/L) & 8 & 6 & 0.58 & 0.024 & 0.16 & 0.200 & 1 \\
\hline ALUMINUM & FILTERED & ICAP & (MG/L) & 8 & 4 & 0.048 & 0.023 & 0.036 & 0.200 & 0 \\
\hline BARIUM & & ICAP & (MG/L) & 8 & 8 & 0.17 & 0.084 & 0.12 & 1.000 & 0 \\
\hline BARIUM & FILTERED & ICAP & (MG/L) & 8 & 8 & 0.16 & 0.083 & 0.12 & 1.000 & 0 \\
\hline BERYLLIUM & & ICAP & (MG/L) & 8 & 4 & 0.0079 & 0.0005 & 0.0052 & $0.004 \star \star$ & 3 \\
\hline BERYLLIUM & FILTERED & ICAP & (MG/L) & 8 & 1 & 0.0051 & 0.0051 & 0.0051 & $0.004 * \star$ & 1 \\
\hline BORON & & ICAP & (MG/L) & 8 & 7 & 0.076 & 0.017 & 0.042 & NR & NA \\
\hline BORON & FILTERED & ICAP & (MG/L) & 8 & 7 & 0.11 & 0.0095 & 0.045 & NR & NA \\
\hline CADMIOM & & ICAP & (MG/L) & 8 & 2 & 0.010 & 0.0046 & 0.0073 & 0.005 & 1 \\
\hline CALCIUM & . & ICAP & (MG/L) & 8 & 8 & 77 & 11 & 42 & NR & NA \\
\hline CALCIUM & FILTERED & ICAP & (MG/L) & 8 & 8 & 77 & 11 & 42 & NR & NA \\
\hline CHROMIUM & & ICAP & (MG/L) & 8 & 2 & 0.038 & 0.011 & 0.025 & 0.050 & 0 \\
\hline COBALT & & ICAP & (MG/L) & 8 & 4 & 0.069 & 0.056 & 0.059 & NR & NA \\
\hline COBALT & FILTERED & ICAP & (MG/L) & 8 & 5 & 0.063 & 0.0054 & 0.047 & NR & NA \\
\hline COPPER & & ICAP & (MG/L) & 8 & 1 & 0.012 & 0.012 & 0.012 & 1.000 & 0 \\
\hline COPPER & FILTERED & ICAP & (MG/L) & 8 & 1 & 0.013 & 0.013 & 0.013 & 1.000 & 0 \\
\hline IRON & & ICAP & (MG/L) & 8 & 8 & 37 & 0.15 & 18 & 0.300 & 6 \\
\hline IRON & FILTEREI & ICAP & (MG/L) & 8 & 8 & 37 & 0.019 & 17 & 0.300 & 4 \\
\hline LEAD & & AAS & (MG/L) & 8 & 1 & 0.015 & 0.015 & 0.015 & 0.050 & 0 \\
\hline LEAD & FILTERED & AAS & (MG/L) & 8 & 1 & 0.0042 & 0.0042 & 0.0042 & 0.050 & 0 \\
\hline MAGNESIUM & & ICAP & (MG/L) & 8 & 8 & 7.5 & 5.0 & 6.2 & $\mathrm{NR}$ & NA \\
\hline MAGNESIUM & FILTERED & ICAP & (MG/L) & 8 & 8 & 7.5 & 5.0 & 6.3 & NR & NA \\
\hline MANGANESE & & ICAP & (MG/L) & 8 & 8 & 13 & 0.20 & 6.4 & 0.050 & 8 \\
\hline MANGANESE & FILTERED & ICAP & (MG/L) & 8 & 8 & 13 & 0.21 & 6.4 & 0.050 & 8 \\
\hline NICKEL & & ICAP & (MG/L) & 8 & 2 & 0.013 & 0.011 & 0.012 & $0.100 * *$ & 0 \\
\hline
\end{tabular}


Table 7.24 (continued)

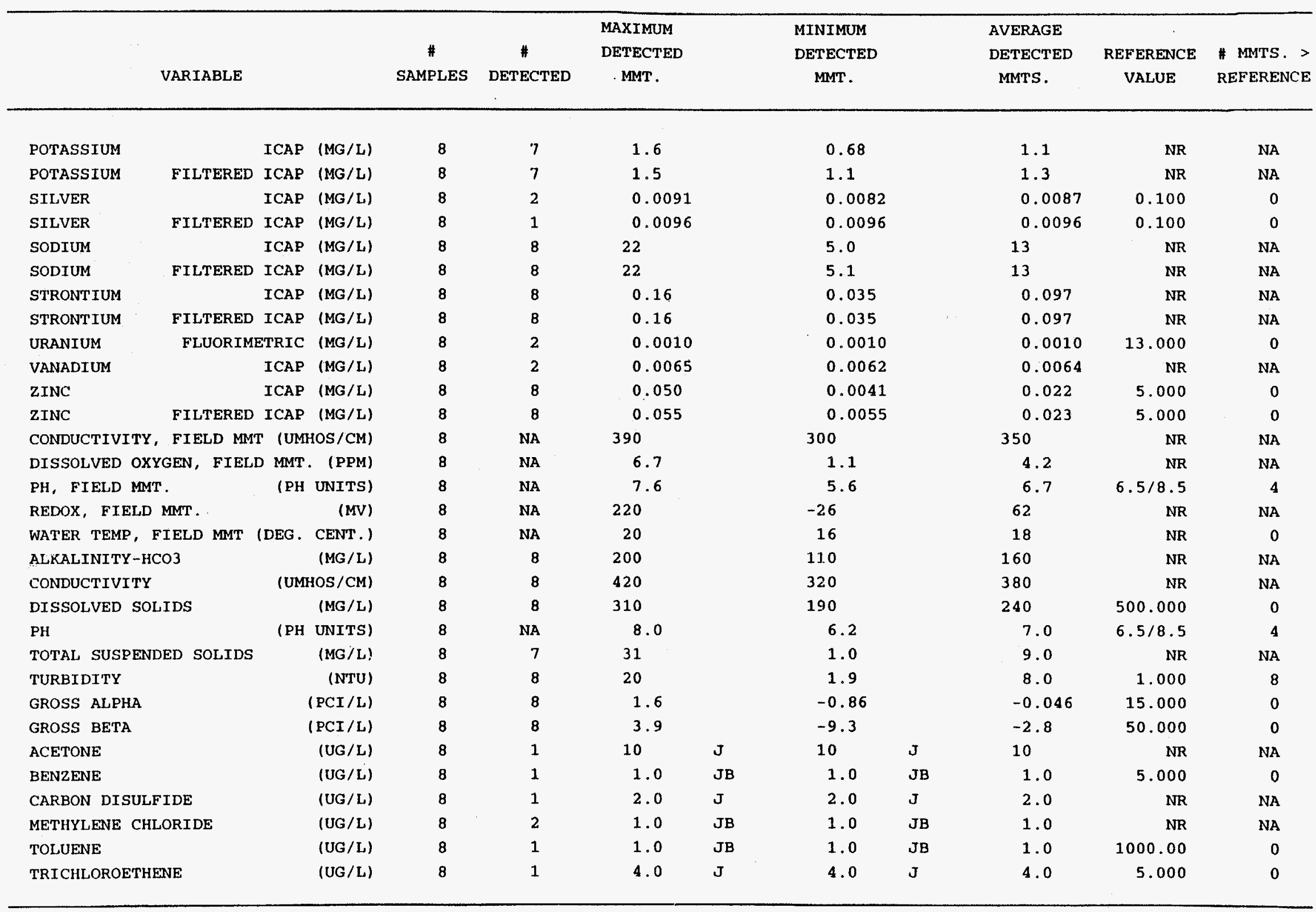


Table 7.25. Constituents in groundwater at the $Y-12$ Plant site $H G R=E F$ area $=$ Grid $E 1$

\begin{tabular}{|c|c|c|c|c|c|c|c|c|c|c|}
\hline & VARIABLE & & & $\begin{array}{c}\# \\
\text { SAMPLES }\end{array}$ & $\begin{array}{c}\# \\
\text { DETEC'TED }\end{array}$ & $\begin{array}{l}\text { MAXIMUA } \\
\text { DETECTED } \\
\text { MMT. }\end{array}$ & $\begin{array}{l}\text { MINIMUM } \\
\text { DETECTED } \\
\text { MMT. }\end{array}$ & $\begin{array}{l}\text { AVERAGE } \\
\text { DETECTED } \\
\text { MMTS. }\end{array}$ & $\begin{array}{c}\text { REFERENCE } \\
\text { VALUE }\end{array}$ & $\begin{array}{l}\text { \# MMTS. > } \\
\text { REFERENCE }\end{array}$ \\
\hline CHLORIDE & & & (MG/L) & 8 & 8 & 9.1 & 2.8 & 5.6 & 250.000 & 0 \\
\hline FLUORIDE & & & $(\mathrm{MG} / \mathrm{L})$ & 8 & 8 & 0.10 & 0.10 & 0.10 & 2.000 & 0 \\
\hline SULFATE & & & (MG/L) & 8 & 8 & 13 & 9.7 & 11 & 250.000 & 0 \\
\hline ALUMINUM & & ICAP & (MG/L) & 8 & 8 & 21 & 0.022 & 5.8 & 0.200 & 5 \\
\hline ALUMINUM & FILTERED & ICAP & (MG/L) & 8 & 6 & 0.19 & 0.022 & 0.073 & 0.200 & 0 \\
\hline BARIUM & & ICAP & $(M G / L)$ & 8 & 8 & 0.40 & 0.18 & 0.30 & 1.000 & 0 \\
\hline BARIUM & FILTERED & ICAP & (MG/L) & 8 & 8 & 0.31 & 0.056 & 0.21 & 1.000 & 0 \\
\hline BERYLLIUM & & ICAP & (MG/L) & 8 & 3 & 0.0008 & 0.0003 & 0.0006 & $0.004^{* *}$ & 0 \\
\hline BORON & & ICAP & (MG/L) & 8 & 8 & 0.087 & 0.020 & 0.056 & NR & NA \\
\hline BORON & FILTERED & ICAP & (MG/L) & 8 & 8 & 0.079 & 0.012 & 0.049 & NR & NA \\
\hline CADMIUM & & ICAP & (MG/L) & 8 & 1 & 0.0050 & 0.0050 & 0.0050 & 0.005 & 0 \\
\hline CALCIUM & & ICAP & (MG/L) & 8 & 8 & 100 & 43 & 75 & NR & NA \\
\hline CALCIUM & FILTERED & ICAP & $(M G / L)$ & 8 & 8 & 100 & 45 & 67 & NR & NA \\
\hline CHROMIUM & & AAS & (MG/L) & 8 & 3 & 0.068 & 0.022 & 0.038 & 0.050 & 1 \\
\hline CHROMIUM & & ICAP & (MG/L) & 8 & 4 & 0.047 & 0.013 & 0.025 & 0.050 & 0 \\
\hline COBALT & & ICAP & (MG/L) & 8 & 2 & 0.012 & 0.011 & 0.012 & NR & NA \\
\hline CORPER & & ICAP & $(\mathrm{MG} / \mathrm{L})$ & 8 & 2 & 0.015 & 0.0044 & 0.0097 & 1.000 & 0 \\
\hline COPPER & FILTERED & ICAP & (MG/L) & 8 & 1 & 0.013 & 0.013 & 0.013 & 1.000 & 0 \\
\hline IRON & & ICAP & (MG/L) & 8 & 8 & 27 & 0.11 & 8.5 & 0.300 & 6 \\
\hline IRON & FILTERED & ICAP & (MG/L) & 8 & 7 & 0.22 & 0.012 & 0.13 & 0.300 & 0 \\
\hline LEAD & & AAS & (MG/L) & 8. & 3 & 0.020 & 0.0041 & 0.011 & 0.050 & 0 \\
\hline MAGNESIUM & & ICAP & $(M G / L)$ & $B$ & 8 & 12 & 5.4 & 8.7 & NR & NA \\
\hline MAGNESIUM & FILTERED & ICAP & (MG/L) & 8 & 8 & 10 & 3.5 & 7.0 & NR & NA \\
\hline MANGANESE & & ICAP & (MG/L) & 8 & 8 & 0.82 & 0.069 & 0.32 & 0.050 & 8 \\
\hline MANGANESE & FILTERED & ICAP & (MG/L) & 8 & 8 & 0.37 & 0.027 & 0.14 & 0.050 & 7 \\
\hline NICKEL & & ICAP & (MG/L) & 8 & 2 & 0.031 & 0.020 & 0.026 & $0.100^{\star *}$ & 0 \\
\hline POTASSIUM & & ICAP & (MG/L) & 8 & 8 & 6.4 & 1.7 & 3.1 & NR & $\mathrm{NA}$ \\
\hline POTASSIUM & FILTERED & ICAP & (MG/L) & 8 & 6 & 2.2 & 1.1 & 1.9 & NR & NA \\
\hline SELENIUM & & ICAP & (MG/L) & 8 & 1 & 0.061 & 0.061 & 0.061 & 0.050 & 1 \\
\hline
\end{tabular}


Table 7.25 (continued)

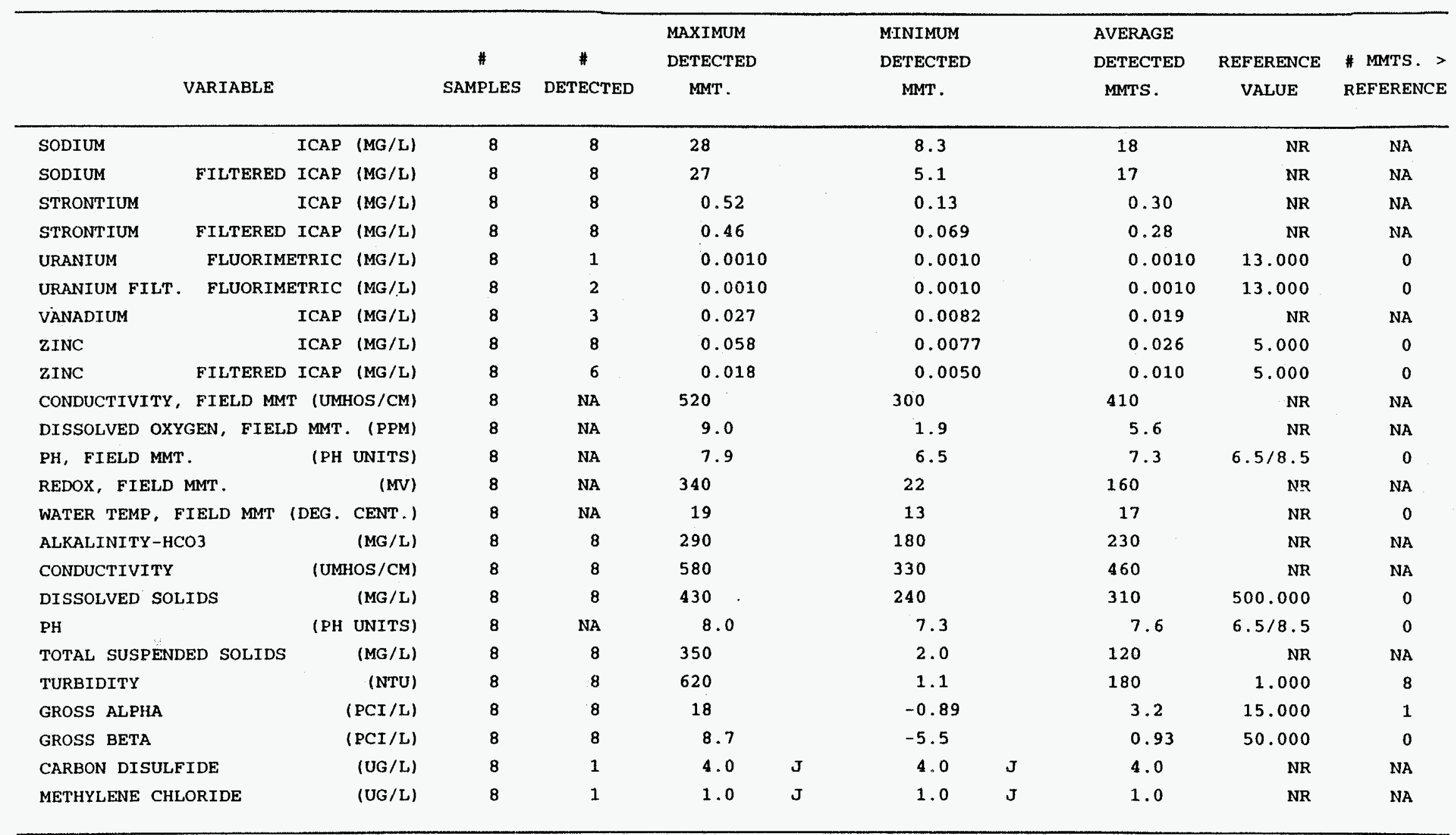


Table 7.25. Constituents in groundwater at the $\mathrm{Y}-12$ Plant site

$H G R=E F$ area $=$ Grid $G 1$

\begin{tabular}{|c|c|c|c|c|c|c|c|c|c|c|}
\hline & VARIABLE & & & $\begin{array}{c}\# \\
\text { SAMPLES }\end{array}$ & $\begin{array}{c}\# \\
\text { DETECTED }\end{array}$ & $\begin{array}{l}\text { MAXIMUM } \\
\text { DETECTED } \\
\text { MMT. }\end{array}$ & $\begin{array}{l}\text { MINIMUM } \\
\text { DETECTED } \\
\text { MMT. }\end{array}$ & $\begin{array}{l}\text { AVERAGE } \\
\text { DETECTED } \\
\text { MMTS. }\end{array}$ & $\begin{array}{c}\text { REFERENCE } \\
\text { VALUE }\end{array}$ & $\begin{array}{l}\text { \# MMTS. > } \\
\text { REFERENCE }\end{array}$ \\
\hline CHLORIDE & & & $(M G / L)$ & 8 & 8 & 67 & 14 & 24 & 250.000 & 0 \\
\hline FLUORIDE & & & (MG/L) & 8 & 7 & 0.20 & 0.10 & 0.11 & 2.000 & 0 \\
\hline SULFATE & & & (MG/L) & 8 & 8 & 21 & 16 & 19 & 250.000 & 0 \\
\hline ALUMINUM & & ICAP & (MG /L) & 8 & 7 & 12 & 0.023 & 2.1 & 0.200 & 3 \\
\hline ALUMINUM & FILTERED & ICAP & $(M G / L)$ & 8 & 3 & 0.050 & 0.030 & 0.037 & 0.200 & 0 \\
\hline ARSENIC & & ICAP & $(\mathrm{MG} / \mathrm{L})$ & 8 & 1 & 0.080 & 0.080 & 0.080 & 0.050 & 1 \\
\hline BARIUM & & ICAP & (MG/L) & 8 & 8 & 0.29 & 0.10 & 0.14 & 1.000 & 0 \\
\hline BARIUM & FILTERED & ICAP & (MG/L) & 8 & 8 & 0.18 & 0.072 & 0.12 & 1.000 & 0 \\
\hline BERYLLIUM & & ICAP & (MG/L) & 8 & 2 & 0.0007 & 0.0006 & 0.0007 & $0.004 * *$ & 0 \\
\hline BORON & & ICAP & (MG/L) & 8 & 8 & 0.085 & 0.020 & 0.042 & NR & NA \\
\hline BORON & FILTERED & ICAP & (MG/L) & 8 & 8 & 0.071 & 0.032 & 0.045 & NR & NA \\
\hline CADMIUM & & ICAP & (MG/L) & 8 & 1 & 0.0046 & 0.0046 & 0.0046 & 0.005 & 0 \\
\hline CALCTUM & & ICAP & (MG/L) & 8 & 8 & 96 & 63 & 74 & NR & NA \\
\hline CALCIUM & FILTERED & ICAP & (MG/L) & 8 & 8 & 75 & 32 & 65 & NR & NA \\
\hline CHROMIUM & & AAS & (MG/L) & 8 & 1 & 0.036 & 0.036 & 0.036 & 0.050 & 0 \\
\hline CHROMIUM & & ICAP & $(M G / L)$ & 8 & 2 & 0.052 & 0.010 & 0.031 & 0.050 & 1 \\
\hline COBALT & & ICAP & (MG/L) & 8 & 1 & 0.012 & 0.012 & 0.012 & NR & NA \\
\hline COPPER & & ICAP & $(M G / L)$ & 8 & 1 & 0.038 & 0.038 & 0.038 & 1.000 & 0 \\
\hline IRON & & ICAP & (MG/L) & 8 & 8 & 24 & 0.020 & 3.6 & 0.300 & 5 \\
\hline IRON & FILTERED & ICAP & (MG/L) & 8 & 7 & 0.34 & 0.055 & 0.17 & 0.300 & 1 \\
\hline LEAD & & AAS & (MG/L) & 8 & 1 & 0.014 & 0.014 & 0.014 & 0.050 & 0 \\
\hline MAGNESIUM & & ICAP & (MG/L) & 8 & 8 & 16 & 8.0 & 10 & NR & NA \\
\hline MAGNESIUM & FILTERED & ICAP & (MG/L) & 8 & 8 & 10 & 2.9 & 8.3 & NR & NA \\
\hline MANGANESE & & ICAP & (MG/L) & 8 & 8 & 1.1 & 0.18 & 0.33 & 0.050 & 8 \\
\hline MANGANESE & FILTERED & ICAP & (MG/L) & 8 & 8 & 0.35 & 0.0029 & 0.20 & 0.050 & 7 \\
\hline NICKEL & & ICAP & $(M G / L)$ & 8 & 2 & 0.061 & 0.024 & 0.043 & $0.100 * *$ & 0 \\
\hline NICKEL & FILTERED & ICAP & (MG/L) & 8 & 4 & 0.013 & 0.011 & 0.012 & $0.100^{*} *$ & 0 \\
\hline POTASSIUM & & ICAP & (MG/L) & 8 & 8 & 4.7 & 1.0 & 2.3 & NR & NA \\
\hline POTASSIUM & FILTEREI & ICAP & (MG/L) & 8 & 7 & 2.7 & 1.4 & 2.2 & NR & NA \\
\hline
\end{tabular}


Table 7.26 (continued)

\begin{tabular}{|c|c|c|c|c|c|c|c|c|}
\hline VARIABLE & $\begin{array}{c}\# \\
\text { SAMPLES }\end{array}$ & $\begin{array}{c}\# \\
\text { DETECTED }\end{array}$ & $\begin{array}{l}\text { MAXIMUM } \\
\text { DETECTED } \\
\text { MMT. }\end{array}$ & $\begin{array}{c}\text { MINIMUM } \\
\text { DETECTED } \\
\text { MMT }\end{array}$ & & $\begin{array}{l}\text { AVERAGE } \\
\text { DETECTED } \\
\text { MMTS. }\end{array}$ & $\begin{array}{c}\text { REFERENCE } \\
\text { VALUE }\end{array}$ & $\begin{array}{l}\text { \# MMTS. > } \\
\text { REFERENCE }\end{array}$ \\
\hline ICAP (MG/L) & 8 & 8 & 15 & 8.5 & & 12 & NR & NA \\
\hline FILTERED ICAP (MG/L) & 8 & 8 & 15 & 6.5 & & 12 & NR & NA \\
\hline ICAP (MG/L) & 8 & B & 0.49 & 0.14 & & 0.29 & NR & NA \\
\hline FILTERED ICAP (MG/L) & 8 & B & 0.51 & 0.052 & & 0.31 & NR & NA \\
\hline FLUORIMETRIC (MG/L) & 8 & 1 & 0.0010 & 0.0010 & & 0.0010 & 13.000 & 0 \\
\hline URANIUM FILT. FLUORIMETRIC (MG/L) & 8 & 1 & 0.0010 & 0.0010 & & 0.0010 & 13.000 & $\mathbf{0}$ \\
\hline ICAP (MG/L) & 8 & 1 & 0.032 & 0.032 & & 0.032 & NR & NA \\
\hline $\operatorname{ICAP}(M G / L)$ & 8 & 8 & 0.058 & 0.0022 & & 0.015 & 5.000 & 0 \\
\hline FILTERED ICAP (MG/L) & 8 & 6 & 0.022 & 0.0033 & & 0.0078 & 5.000 & 0 \\
\hline CONDUCTIVITY, FIELD MMT (UMHOS/CM) & 8 & NA & 460 & 370 & & 410 & NR & NA \\
\hline DISSOLVED OXYGEN, FIELD MMT. (PPM) & 8 & NA & 8.1 & 0.50 & & 4.6 & NR & NA \\
\hline PH, FIELD MMT. & 8 & NA & 7.6 & 6.9 & & 7.4 & $6.5 / 8.5$ & 0 \\
\hline REDOX, FIELD MMT. & 8 & NA & 290 & -24 & & 110 & NR & NA \\
\hline WATER TEMP, FIELD MMT (DEG. CENT.) & 8 & NA & 20 & 9.5 & & 16 & NR & 0 \\
\hline ALKALINITY- $\mathrm{HCO} 3$ & 8 & B & 200 & 190 & & 190 & NR & NA \\
\hline CONDUCTIVITY & 8 & 8 & 480 & 440 & & 460 & NR & NA \\
\hline DISSOLVED SOLIDS & 8 & 8 & 300 & 270 & & 280 & 500.000 & 0 \\
\hline (PH UNITS) & 8 & NA & $7: 8$ & 7.5 & & 7.7 & $6.5 / 8.5$ & 0 \\
\hline TOTAL SUSPENDED SOLIDS & 8 & B & 590 & 1.0 & & 86 & NR & NA \\
\hline TURBIDITY & 8 & 8 & 80 & 1.7 & & 20 & 1.000 & 8 \\
\hline GROSS ALPHA & 8 & 8 & 8.5 & -1.8 & & 0.94 & 15.000 & 0 \\
\hline GROSS BETA & 8 & 8 & 15 & -8.1 & & 0.44 & 50.000 & 0 \\
\hline ACETONE & 8 & 1 & 15 & 15 & & 15 & NR & NA \\
\hline METHYLENE CHLORIDE & 8 & 1. & 1.0 & 1.0 & $J$ & 1.0 & NR & NA \\
\hline
\end{tabular}


Table 7.27. Constituents in groundwater at the $Y-12$ Plant site

HGR=EF area $=$ Grid $G 2$

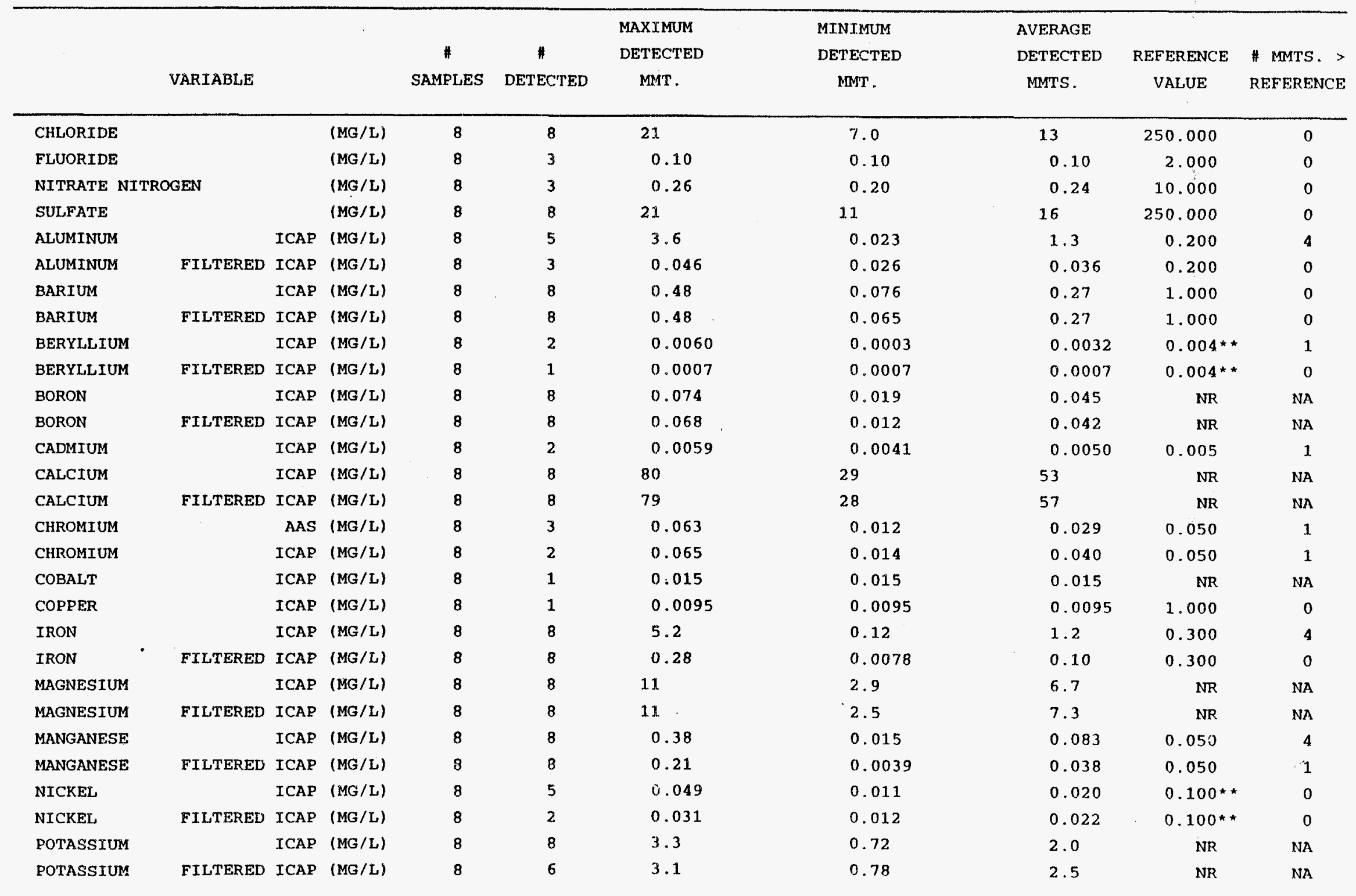


Table 7.27 (continued)

\begin{tabular}{|c|c|c|c|c|c|c|c|c|c|c|}
\hline VARIABLE & & $\begin{array}{c}\# \\
\text { SAMPLES }\end{array}$ & $\begin{array}{c}\# \\
\text { DETECTED }\end{array}$ & $\begin{array}{l}\text { MAXIMUM } \\
\text { DETECTED } \\
\text { MMT. }\end{array}$ & & $\begin{array}{l}\text { MINIMUM } \\
\text { DETECTED } \\
\text { MMT. }\end{array}$ & & $\begin{array}{l}\text { AVERAGE } \\
\text { DETECTED } \\
\text { MMTS. }\end{array}$ & $\begin{array}{c}\text { REFERENCE } \\
\text { VALUE }\end{array}$ & $\begin{array}{l}\text { \# MMTS. > } \\
\text { REFERENCE }\end{array}$ \\
\hline SILVER & ICAP (MG/L) & 8 & 1 & 0.0082 & & 0.0082 & & 0.0082 & 0.100 & 0 \\
\hline SODIUM & $\operatorname{ICAP}$ (MG/L) & 8 & 8 & 6.9 & & 5.9 & & 6.3 & NR & NA \\
\hline FILTERED & $\operatorname{ICAP}(\mathrm{MG} / \mathrm{L})$ & 8 & 8 & 15 & & 5.7 & & 7.4 & NR & NA \\
\hline STRONTIUM & ICAP (MG/L) & 8 & 8 & 0.44 & & 0.045 & & 0.24 & NR & NA \\
\hline STRONTIUM & ICAP (MG/L) & 8 & 8 & 0.46 & & 0.043 & & 0.29 & NR & NA \\
\hline FLUORIME & ETRIC (MG/L) & 8 & 1 & 0.0010 & & 0.0010 & & 0.0010 & 13.000 & 0 \\
\hline VANADIUM & $\operatorname{ICAP}(\mathrm{MG} / \mathrm{L})$ & 8 & 1 & 0.014 & & 0.014 & & 0.014 & NR & NA \\
\hline ZINC & ICAP $(M G / L)$ & 8 & 7 & 0.036 & & 0.0051 & & 0.018 & 5.000 & 0 \\
\hline FILTERED & ICAP (MG/L) & 8 & 8 & 0.026 & & 0.0035 & & 0.011 & 5.000 & 0 \\
\hline CONDUCTIVITY, FIELD MMT & I (UMHOS/CM) & 8 & NA & 470 & & 170 & & 300 & NR & NA \\
\hline DISSOLVED OXYGEN, FIELD & MMT. (PPM) & 8 & NA & 7.5 & & 0.50 & & 3.9 & NR & NA \\
\hline PH, FIELD MMT. & (PH UNITS) & 8 & NA & 7.5 & & 6.6 & & 7.0 & $6.5 / 8.5$ & 0 \\
\hline REDOX, FIELD MMT. & (MV) & 8 & NA & 430 & & 36 & & 180 & NR & NA. \\
\hline WATER TEMP, FIELD MMT & (DEG. CENT.) & 8 & NA & 21 & & 12 & & 17 & NR & 0 \\
\hline ALKALINITY-HCO3 & (MG/L) & 8 & 8 & 210 & & 72 & & 140 & NR & NA \\
\hline CONDUCTIVITY & (UMHOS/CM) & 8 & 8 & 480 & & 200 & & 340 & NR & NA \\
\hline DISSOLVED SOLIDS & (MG/L) & 8 & 8 & 300 & & 120 & & 210 & 500.000 & 0 \\
\hline PH & (PH UNITS) & 8 & NA & 7.9 & & 7.1 & & 7.5 & $6.5 / 8.5$ & 0 \\
\hline TOTAL SUSPENDED SOLIDS & (MG/L) & 8 & 5 & 41 & & 1.0 & & 17 & NR & NA \\
\hline TURBIDITY & (NTU) & 8 & 8 & 520 & & 0.90 & & 71 & 1.000 & 6 \\
\hline GROSS ALPHA & $(\mathrm{PCI} / \mathrm{L})$ & 8 & 8 & 3.3 & & -0.96 & & 0.49 & 15.000 & 0 \\
\hline GROSS BETA & $(\mathrm{PCI} / \mathrm{L})$ & 8 & 8 & 5.3 & & -2.8 & & 1.4 & 50.000 & 0 \\
\hline $1,1,1$-TRICHLOROETHANE & (UG/L) & 8 & 2 & 0.80 & $\mathfrak{J}$ & 0.40 & $\mathrm{~J}$ & 0.60 & 200.000 & 0 \\
\hline 1,1-DICHLOROETHANE & (UG/L) & 8 & 2 & 1.0 & $J$ & 1.0 & $\mathbf{J}$ & 1.0 & NR & NA \\
\hline 1,1-DICHLOROETHENE & (UG/L) & 8 & 2 & 1.0 & $J$ & 1.0 & $\mathbf{J}$ & 1.0 & 7.000 & 0 \\
\hline 1,2-DICHLOROETHENE & $(U G / L)$ & 8 & 1 & 1.0 & $J$ & 1.0 & $\mathbf{J}$ & 1.0 & 70.000 & 0 \\
\hline 4-METHYL-2-PENTANONE & (UG/L) & 8 & 1 & 2.0 & $\mathrm{BJ}$ & 2.0 & BJ & 2.0 & NR & NA \\
\hline CARBON TETRACHLORIDE & (UG/L) & 8 & 4 & 9.0 & & 5.0 & $J$ & 6.3 & 5.000 & 2 \\
\hline CHLOROFORM & (UG/L) & 8 & 4 & 1.0 & $\mathbf{J}$ & 0.70 & $\mathbf{J}$ & 0.90 & 100.000 & 0 \\
\hline TETRACHLOROETHENE & (UG/L) & 8 & 4 & 4.0 & $\mathbf{J}$ & 2.0 & $\mathbf{J}$ & 3.3 & 5.000 & 0 \\
\hline TRICHLOROETHENE & (UG/L) & 8 & 4 & 1.0 & $\mathbf{J}$ & 0.70 & $\mathbf{J}$ & 0.93 & 5.000 & 0 \\
\hline
\end{tabular}


Table 7.28. Constituents in groundwater at the $Y-12$ Plant site $\mathrm{HGA}=\mathrm{EF}$ area $=$ Grid $\mathrm{G3}$

\begin{tabular}{|c|c|c|c|c|c|c|c|c|c|c|}
\hline . & \multicolumn{3}{|l|}{ VARIABLE } & \multirow{2}{*}{$\begin{array}{c}\# \\
\text { SAMPLES } \\
8\end{array}$} & \multirow{2}{*}{$\frac{\begin{array}{l}\# \\
\text { DETECTED }\end{array}}{8}$} & \multirow{2}{*}{$\begin{array}{l}\text { MAXIMUM } \\
\text { DETECTED } \\
\text { MMT. } \\
170\end{array}$} & \multirow{2}{*}{$\begin{array}{l}\text { MINIMUM } \\
\text { DETECTED } \\
\text { MMT. } \\
4.4\end{array}$} & \multirow{2}{*}{$\begin{array}{l}\text { AVERAGE } \\
\text { DETECTED } \\
\text { MMTS. } \\
64\end{array}$} & \multirow{2}{*}{$\begin{array}{c}\text { REFERENCE } \\
\text { VALUE } \\
250.000\end{array}$} & \multirow{2}{*}{$\begin{array}{l}\text { \# MMTS. > } \\
\text { REFERENCE } \\
0\end{array}$} \\
\hline CHLORIDE & & & (MG/L) & & & & & & & \\
\hline FLUORIDE & & & $(M G / L)$ & 8 & 4 & 0.20 & 0.10 & 0.18 & 2.000 & 0 \\
\hline NITRATE NI & DGEN & & (MG/L) & 8 & 7 & 0.77 & 0.38 & 0.52 & 10.000 & 0 \\
\hline SULFATE & & & $(M G / L)$ & 8 & 8 & 25 & 18 & 21 & 250.000 & 0 \\
\hline ALUMINUM & & ICAP & $(M G / L)$ & 8 & 7 & 0.90 & 0.021 & 0.24 & 0.200 & 2 \\
\hline ALUMINUM & FILTERED & ICAP & (MG/L) & 8 & 2 & 0.11 & 0.065 & 0.088 & 0.200 & 0 \\
\hline BARIUM & & ICAP & (MG/L) & 8 & 8 & 0.47 & 0.050 & 0.24 & 1.000 & 0 \\
\hline BARIUM & FILTERED & ICAP & (MG/L) & 8 & 8 & 0.47 & 0.042 & 0.25 & 1.000 & 0 \\
\hline BERYLLIUM & & ICAP & (MG/L) & 8 & 1 & 0.0008 & 0.0008 & 0.0008 & $0.004 * \star$ & 0 \\
\hline BORON & & ICAP & (MG/L) & 8 & 8 & 0.074 & 0.017 & 0.036 & NR & NA \\
\hline BORON & FILTERED & ICAP & (MG/L) & 8 & 8 & 0.039 & 0.014 & 0.031 & NR & $\mathrm{NA}$ \\
\hline CADMIUM & & AAS & $(M G / L)$ & B & 2 & 0.020 & 0.0087 & 0.014 & 0.005 & 2 \\
\hline CADMIUM & & ICAP & (MG/L) & 8 & 2 & 0.020 & 0.0069 & 0.013 & 0.005 & 2 \\
\hline CADMIUM & FILTERED & AAS & (MG/L) & 8 & 2 & 0.014 & 0.013 & 0.014 & 0.005 & 2 \\
\hline CALCIUM & & ICAP & (MG/L) & 8 & 8 & 130 & 44 & 85 & NR & $\mathrm{NA}$ \\
\hline CALCIUM & FILTERED & ICAP & (MG/L) & 8 & 8 & 130 & 43 & 85 & NR & NA \\
\hline CHROMIUM & & AAS & $(M G / L)$ & 8 & 3 & 0.15 & 0.015 & 0.072 & 0.050 & 2 \\
\hline CHROMIUM & & ICAP & $(M G / L)$ & 8 & 3 & 0.081 & 0.014 & 0.052 & 0.050 & 2 \\
\hline COPPER & & ICAP & (MG/L) & 8 & 2 & 0.16 & 0.0047 & 0.082 & 1.000 & 0 \\
\hline IRON & & ICAP & (MG/L) & 8 & 7 & 1.0 & 0.058 & 0.42 & 0.300 & 4 \\
\hline IRON & FILTERED & ICAP & (MG/L) & 8 & 3 & 0.19 & 0.0078 & 0.070 & 0.300 & 0 \\
\hline MAGNESIUM & & ICAP & (MG/L) & 8 & 8 & 14 & 3.3 & 8.1 & NR & NA \\
\hline MAGNESIUM & FILTERED & ICAP & $(M G / L)$ & 8 & 8 & 14 & 3.2 & 8.7 & NR & NA \\
\hline MANGANESE & & ICAP & (MG/L) & 8 & 7 & 0.072 & 0.0024 & 0.035 & 0.050 & 2 \\
\hline MANGANESE & FILTERED & ICAP & $(M G / L)$ & 8 & 5 & 0.21 & 0.015 & 0.070 & 0.050 & 2 \\
\hline NICKEL & & ICAP & $(M G / L)$ & 8 & 4 & 0.080 & 0.024 & 0.052 & $0.100 * *$ & 0 \\
\hline NICKEL & FILTERED & ICAP & (MG/L) & 8 & 4 & 0.052 & 0.025 & 0.038 & $0.100 * *$ & 0 \\
\hline POTASSIUM & & ICAP & $(M G / L)$ & 8 & 8 & 2.9 & 1.3 & 2.0 & NR & NA \\
\hline POTASSIUM & FILTERED & ICAP & (MG/L) & 8 & 8 & $2: 3$ & 1.3 & 1.8 & NR & $\mathrm{NA}$ \\
\hline
\end{tabular}


Table 7.28 (continued)

홍

\begin{tabular}{|c|c|c|c|c|c|c|c|c|c|c|}
\hline VARIABLE & & $\begin{array}{c}\# \\
\text { SAMPLES }\end{array}$ & $\begin{array}{c}\# \\
\text { DETECTED }\end{array}$ & $\begin{array}{l}\text { MAXIMUM } \\
\text { DETECTED } \\
\text { MMT. }\end{array}$ & & $\begin{array}{l}\text { MINIMUM } \\
\text { DETECTED } \\
\text { MMT. }\end{array}$ & & $\begin{array}{l}\text { AVERAGE } \\
\text { DETECTED } \\
\text { MMTS. }\end{array}$ & $\begin{array}{l}\text { REFERENCE } \\
\text { VALUE }\end{array}$ & $\begin{array}{l}\# \text { MMTS. > } \\
\text { REFERENCE }\end{array}$ \\
\hline SODIUM & ICAP (MG/L) & 8 & 8 & 11 & & 4.5 & & 7.8 & NR & $\mathrm{NA}$ \\
\hline FILTERED & ICAP (MG /L) & 8 & 8 & 12 & & 4.5 & & 8.6 & NR & NA \\
\hline STRONTIUM & ICAP (MG/L) & 8 & 8 & 0.27 & & 0.061 & & 0.16 & NR & $\mathrm{NA}$ \\
\hline STRONTIUM & ICAP (MG/L) & 8 & 8 & 0.26 & & 0.060 & & 0.17 & NR & NA \\
\hline URANIUM FILT. FLUORIME & ETRIC (MG/L) & 8 & 2 & 0.0020 & & 0.0010 & & 0.0015 & 13.000 & 0 \\
\hline zINC & ICAP $(M G / L)$ & 8 & 8 & 0.029 & & 0.0061 & & 0.014 & 5.000 & 0 \\
\hline FILTERED & ICAP (MG/L) & 8 & 8 & 0.019 & & 0.0046 & & 0.011 & 5.000 & 0 \\
\hline CONDUCTIVITY, FIELD MMT & I (UMHOS/CM) & 8 & $\mathrm{NA}$ & 820 & & 230 & & 470 & NR & NA \\
\hline DISSOLVED OXYGEN, FIELD & D MMT. (PPM) & 8 & $\mathrm{NA}$ & 8.1 & & 3.6 & & 6.4 & NR & NA \\
\hline PH, FIELD MMT. & (PH UNITS) & 8 & NA & 8.0 & & 6.0 & & 7.0 & $6.5 / 8.5$ & 1 \\
\hline REDOX, FIELD MMT. & (MV) & 8 & $\mathrm{NA}$ & 360 & & 120 & & 220 & NR & NA \\
\hline WATER TEMP, FIELD MMT & (DEG. CENT.) & 8 & NA & 23 & & 15 & & 18 & NR & 0 \\
\hline ALKALINITY-HCO3 & (MG/L) & 8 & 8 & 170 & & 110 & & 140 & NR & NA \\
\hline CONDUCTIVITY & (UMHOS/CM) & 8 & 8 & 830 & & 290 & & 530 & NR & NA \\
\hline DISSOLVED SOLIDS & (MG/L) & 8 & 8 & 570 & & 190 & & 340 & 500.000 & 1 \\
\hline PH & (PH UNITS) & 8 & NA & 8.1 & & 7.3 & & 7.6 & $6.5 / 8.5$ & 0 \\
\hline TOTAL SUSPENDED SOLIDS & (MG/L) & 8 & 7 & 41 & & 2.0 & & 11 & NR & NA \\
\hline TURBIDITY & (NTU) & 8 & 8 & 24 & & 2.5 & & 9.5 & 1.000 & 8 \\
\hline GROSS ALPHA & $(\mathrm{PCI} / \mathrm{L})$ & 8 & 8 & 4.5 & & -2.6 & & 0.94 & 15.000 & 0 \\
\hline GROSS BETA & $(\mathrm{PCI} / \mathrm{L})$ & 8 & 8 & 3.5 & & -2.0 & & 1.6 & 50.000 & 0 \\
\hline 4-METHYL-2-PENTANONE & (UG/L) & 8 & 1 & 2.0 & BJ & 2.0 & $\mathrm{BJ}$ & 2.0 & NR & NA \\
\hline CHLOROFORM & (UG/L) & 8 & 4 & 9.0 & & 4.0 & $\mathrm{~J}$ & 5.5 & 100.000 & 0 \\
\hline METHYLENE CHLORIDE & (UG/L) & 8 & 1 & 0.90 & JB & 0.90 & JB & 0.90 & NR & NA \\
\hline
\end{tabular}


Table 7.29. Constlituents in groundwater at the Y-12 Plant site

$H G R=E F$ area=Grid $H 2$

\begin{tabular}{|c|c|c|c|c|c|c|c|c|c|c|}
\hline & VARIABLE & & & $\begin{array}{c}\# \\
\text { SAMPLES }\end{array}$ & $\begin{array}{c}\# \\
\text { DETECTED }\end{array}$ & $\begin{array}{c}\text { MAXIMUM } \\
\text { DETECTED } \\
\text { MMT. }\end{array}$ & $\begin{array}{c}\text { MINIMUM } \\
\text { DETECTED } \\
\text { MMT. }\end{array}$ & $\begin{array}{l}\text { AVERAGE } \\
\text { DETECTED } \\
\text { MMTS. }\end{array}$ & $\begin{array}{c}\text { REFERENCE } \\
\text { VAL.UE }\end{array}$ & $\begin{array}{l}\text { \# MMTS. > } \\
\text { REFERENCE }\end{array}$ \\
\hline CHLORIDE & & & (MG/L) & 8 & 8 & 4.1 & 2.8 & 3.5 & 250.000 & 0 \\
\hline FLUORIDE & & & $(M G / L)$ & 8 & 4 & 0.10 & 0.10 & 0.10 & 2.000 & 0 \\
\hline SULFATE & & & $(M G / L)$ & 8 & 8 & 13 & 5.0 & 8.6 & 250.000 & 0 \\
\hline ALUMINUM & & ICAP & (MG/L) & 8 & 6 & 24 & 0.023 & 4.2 & 0.200 & 2 \\
\hline ALUMINUM & FILTERED & ICAP & (MG/L) & 8 & 3 & 0.27 & 0.020 & 0.10 & 0.200 & 1 \\
\hline BARIUM & & ICAP & (MG/L) & 8 & 8 & 1.1 & 0.36 & 0.48 & 1.000 & 1 \\
\hline BARIUM & FILTERED & ICAP & (MG/L) & 8 & 8 & 0.42 & 0.36 & 0.39 & 1.000 & 0 \\
\hline BERYLLIUM & & ICAP & (MG/L) & 8 & 1 & 0.0016 & 0.0016 & 0.0016 & $0.004 * *$ & 0 \\
\hline BERYLILIUM & FIITERED & ICAP & (MG/L) & 8 & 2 & 0.0015 & 0.0005 & 0.0010 & $0.004 * *$ & 0 \\
\hline BORON & & ICAP & $(M G / L)$ & 8 & 8 & 0.056 & 0.010 & 0.022 & NR & NA \\
\hline BORON & FILTERED & ICAP & (MG/L) & 8 & 8 & 0.041 & 0.0076 & 0.019 & NR & NA \\
\hline CALCIUM & & ICAP & $(M G / L)$ & 8 & 8 & 74 & 51 & 61 & NR & NA \\
\hline CALCIUM & FILTERED & ICAP & $(M G / L)$ & 8 & 8 & 69 & 52 & 60 & NR & NA \\
\hline CHROMIUM & & AAS & (MG/L) & 8 & 1 & 0.089 & 0.089 & 0.089 & 0.050 & 1 \\
\hline CHROMIUM & & ICAP & (MG/L) & 8 & 1 & 0.067 & 0.067 & 0.067 & 0.050 & 1 \\
\hline COBALT & & ICAP & $(M G / L)$ & 8 & 2 & 0.023 & 0.0077 & 0.015 & NR & $\mathrm{NA}$ \\
\hline COPPER & & ICAP & $(M G / L)$ & 8 & 4 & 0.051 & 0.0044 & 0.019 & 1.000 & 0 \\
\hline COPPER & FILTERED & ICAP & (MG/L) & 8 & 2 & 0.0074 & 0.0054 & 0.0064 & 1.000 & 0 \\
\hline IRON & & ICAP & (MG/L) & 8 & 8 & 32 & 0.048 & 4.4 & 0.300 & 2 \\
\hline IRON & FILTERED & ICAP & (MG/L) & 8 & 5 & 0.34 & 0.036 & 0.11 & 0.300 & 1 \\
\hline LEAD & & AAS & (MG/L) & 8 & 1 & 0.021 & 0.021 & 0.021 & 0.050 & 0 \\
\hline MAGNESIUM & & ICAP & (MG/L) & 8 & 8 & 13 & 5.8 & 7.3 & $\mathrm{NR}$ & NA \\
\hline MAGNESIUM & FILTERED & ICAP & (MG/L) & 8 & 8 & 7.2 & 5.7 & 6.4 & $\mathrm{NR}$ & $\mathrm{NA}$ \\
\hline MANGANESE & & ICAP & (MG/L) & 8 & 8 & 1.8 & 0.027 & 0.26 & 0.050 & 2 \\
\hline MANGANESE & FILTERED & ICAP & (MG/L) & 8 & 8 & 0.054 & 0.017 & 0.030 & 0.050 & 1 \\
\hline NICKEL & & ICAP & (MG/L) & 8 & 1 & 0.031 & 0.031 & 0.031 & $0.100^{\star *}$ & 0 \\
\hline POTASSIUM & & ICAP & (MG/L) & 8 & 8 & 8.4 & 0.87 & 2.5 & NR & $\mathrm{NA}$ \\
\hline POTASSIUM & FILTERED & ICAP & (MG/L) & 8 & 7 & 3.0 & 1.1 & 1.8 & NR & $\mathrm{NA}$ \\
\hline SODIUM & & ICAP & (MG/L) & 8 & 8 & 4.9 & 4.1 & 4.5 & NR & NA \\
\hline
\end{tabular}


Table 7.29 (continued)

\begin{tabular}{|c|c|c|c|c|c|c|c|c|c|}
\hline VARIABLE & $\begin{array}{c}\# \\
\text { SAMPLES }\end{array}$ & $\begin{array}{c}\# \\
\text { DETECTED }\end{array}$ & $\begin{array}{c}\text { MAXIMUM } \\
\text { DETECTED } \\
\text { MMT. }\end{array}$ & & $\begin{array}{l}\text { MINIMUM } \\
\text { DETECTED } \\
\text { MMT. }\end{array}$ & & $\begin{array}{l}\text { AVERAGE } \\
\text { DETECTED } \\
\text { MMTS. }\end{array}$ & $\begin{array}{c}\text { REFERENCE } \\
\text { VALUE }\end{array}$ & $\begin{array}{l}\# \text { MMTS. > } \\
\text { REFERENCEE }\end{array}$ \\
\hline FILTERED ICAP (MG/L) & 8 & 8 & 4.6 & & 4.2 & & 4.4 & NR & NA \\
\hline ICAP (MG $/ \mathrm{L})$ & 8 & 8 & 0.14 & & 0.10 & & 0.13 & NR & NA \\
\hline FILTERED ICAP (MG/L) & 8 & 8 & 0.15 & & 0.10 & & 0.13 & NR & NA \\
\hline FLUORIMETRIC (MG/L) & 8 & 2 & 0.0010 & & 0.0010 & & 0.0010 & 13.000 & 0 \\
\hline ICAP (MG/L) & 8 & 1 & 0.038 & & 0.038 & & 0.038 & NR & NA \\
\hline ICAP (MG/L) & 8 & 8 & 0.23 & & 0.0028 & & 0.050 & 5.000 & 0 \\
\hline FILTERED ICAP (MG/L) & 8 & 8 & 0.022 & & 0.0028 & & 0.011 & 5.000 & 0 \\
\hline CONDUCTIVITY, FIELD MMT (UMHOS/CM) & 8 & NA & 380 & & 290 & & 340 & NR & NA \\
\hline DISSOLVED OXYGEN, FIELD MMT. (PPM) & 8 & NA & 5.3 & & 0.40 & & 1.9 & NR & NA \\
\hline PH， FIELD MMT. & 8 & NA & 7.9 & & 7.1 & & 7.4 & $6.5 / 8.5$ & 0 \\
\hline REDOX, FIELD MMT. & 8 & NA & 220 & & 12 & & 110 & NR & NA \\
\hline WATER TEMP, FIELD MMT (DEG. CENT.) & 8 & NA & 23 & & 15 & & 17 & NR & 0 \\
\hline ALKALINITY-HCO3 (MG/L) & 8 & 8 & 200 & & 160 & & 180 & NR & NA \\
\hline CONDUCTIVITY & 8 & 8 & 420 & & 320 & & 360 & NR & NA \\
\hline DISSOLVED SOLIDS & 8 & 8 & $32 \dot{0}$ & & 200 & & 240 & 500.000 & 0 \\
\hline (PH UNITS) & 8 & NA & 8.2 & & 7.3 & & 7.7 & $6.5 / 8.5$ & 0 \\
\hline TOTAL SUSPENDED SOLIDS & 8 & 6 & 22 & & 2.0 & & 10 & $\cdot \mathrm{NR}$ & NA \\
\hline TURBIDITY & 8 & 8 & 150 & & 0.90 & & 40 & 1.000 & 7 \\
\hline GROSS ALPHA & 8 & 8 & 1.2 & & -2.0 & & -0.27 & 15.000 & 0 \\
\hline GROSS BETA & 8 & 8 & 2.7 & & -3.0 & & -0.26 & 50.000 & 0 \\
\hline 4-METHYL-2-PENTANONE & 8 & 3 & 2.0 & BJ & 2.0 & $\mathrm{BJ}$ & 2.0 & NR & $\mathrm{NA}$ \\
\hline METHYLENE CHLORIDE & 8 & 1 & 2.0 & BJ & 2.0 & BJ & 2.0 & NR & $\mathrm{NA}$ \\
\hline
\end{tabular}


Table 7.30. Constituents in groundwater at the $\mathrm{Y}-12$ Plant site

$H G R=E F$ area=Grid $H 3$

\begin{tabular}{|c|c|c|c|c|c|c|c|c|c|c|}
\hline$\therefore$ & VARIABLE & & & $\begin{array}{c}\# \\
\text { SAMPLES }\end{array}$ & $\begin{array}{c}\# \\
\text { DETECTED }\end{array}$ & $\begin{array}{c}\text { MAXIMUM } \\
\text { DETECTED } \\
\text { MMT. }\end{array}$ & $\begin{array}{c}\text { MINIMUM } \\
\text { DETECTED } \\
\text { MMT . }\end{array}$ & $\begin{array}{l}\text { AVERAGE } \\
\text { DETECTED } \\
\text { MMTS. }\end{array}$ & $\begin{array}{c}\text { REFERENCE } \\
\text { VAL,UE }\end{array}$ & $\begin{array}{l}\text { \# MMTS. > } \\
\text { REFERENCE }\end{array}$ \\
\hline CHLORIDE & & & (MG/L) & 8 & 8 & 4.1. & 22 & 31 & 250.000 & 0 \\
\hline NITRATE NIT & DGEN & & $(M G / L)$ & 8 & 8 & 1.4 & 0.35 & 0.81 & 10.000 & 0 \\
\hline SULFATE & & & $(M G / L)$ & 8 & 8 & 40 & 25 & 33 & 250.000 & 0 \\
\hline ALUMINUM & & ICAP & (MG/L) & 8 & 7 & 0.91 & 0.025 & 0.31 & 0.200 & 3 \\
\hline ALUMINUM & FILTERED & ICAP & (MG/L) & 8 & 3 & 0.057 & 0.027 & 0.040 & 0.200 & 0 \\
\hline BARIUM & & ICAP & (MG/L) & 8 & 8 & 0.17 & 0.073 & 0.12 & 1.000 & 0 \\
\hline BARIUM & FILTERED & ICAP & (MG/L) & 8 & 8 & 0.16 & 0.066 & 0.11 & 1.000 & 0 \\
\hline BERYLLIUM & & ICAP & (MG/L) & 8 & 1 & 0.0006 & 0.0006 & 0.0006 & $0.004 * *$ & 0 \\
\hline BERYLLIUM & FILTERED & ICAP & (MG/L) & 8 & 1 & 0.0005 & 0.0005 & 0.0005 & $0.004 *$ & 0 \\
\hline BORON & & ICAP & (MG/L) & 8 & 8 & 0.047 & 0.014 & 0.027 & NR & NA \\
\hline BORON & FILTERED & ICAP & (MG/L) & 8 & 8 & 0.040 & 0.011 & 0.026 & NR & NA \\
\hline CALCIUM & & ICAP & $(M G / L)$ & 8 & 8 & 94 & 70 & 82 & NR & NA \\
\hline CALCIUM & FILTERED & ICAP & $(M G / L)$ & 8 & 8 & 92 & 68 & 80 & NR & NA \\
\hline CHROMIUM & & AAS & (MG/L) & 8 & 3 & 0.31 & 0.018 & 0.16 & 0.050 & 2 \\
\hline CHROMIUM & & ICAP & (MG/L) & 8 & 3 & 0.31 & 0.019 & 0.15 & 0.050 & 2 \\
\hline COBALT & & ICAP & (MG/L) & 8 & 3 & 0.0089 & 0.0060 & 0.0073 & NR & NA \\
\hline COBALT & FILTERED & ICAP & (MG/L) & 8 & 1 & 0.0083 & 0.0083 & 0.0083 & $\mathrm{NR}$ & NA \\
\hline COPPER & & ICAP & (MG/L) & 8 & 4 & 0.019 & 0.0063 & 0.011 & 1.000 & 0 \\
\hline COPPER & FILTERED & ICAP & (MG/L) & 8 & 2 & 0.013 & 0.0072 & 0.010 & 1.000 & 0 \\
\hline IRON & & ICAP & (MG/L) & 8 & 8 & 2.8 & 0.018 & 0.84 & 0.300 & 4 \\
\hline IRON & FILTERED & ICAP & (MG/L) & 8 & 4 & 0.23 & 0.0075 & 0.075 & 0.300 & 0 \\
\hline MAGNESIUM & & ICAP & (MG/L) & 8 & 8 & 6.7 & 4.6 & 5.7 & NR & NA \\
\hline MAGNESIUM & FILTERED & ICAP & $(M G / L)$ & 8 & 8 & 6.6 & 4,4 & 5.5 & NR & $\mathrm{NA}$ \\
\hline MANGANESE & & ICAP & (MG/L) & 8 & 8 & 0.099 & 0.0033 & 0.033 & 0.050 & 2 \\
\hline MANGANESE & FILTERED & ICAP & (MG/L) & 8 & 8 & 0.021 & 0.0025 & 0.012 & 0.050 & 0 \\
\hline MOLYBDENUM & FILTERED & ICAP & (MG/L) & 8 & 2 & 0.012 & 0.011 & 0.012 & NR & NA \\
\hline NICKEL & & ICAP & (MG/L) & 8 & 4 & 0.33 & 0.15 & 0.23 & $0.100 * \star$ & 4 \\
\hline NICKEL & FILTERED & ICAP & $(M G / L)$ & 8 & 4 & 0.32 & 0.13 & 0.21 & $0.100 * *$ & 4 \\
\hline POTASSIUM & & ICAP & $(M G / L)$ & 8 & 8 & 3.8 & 2.2 & 3.0 & NR & NA \\
\hline
\end{tabular}


Table 7.30 (continued)

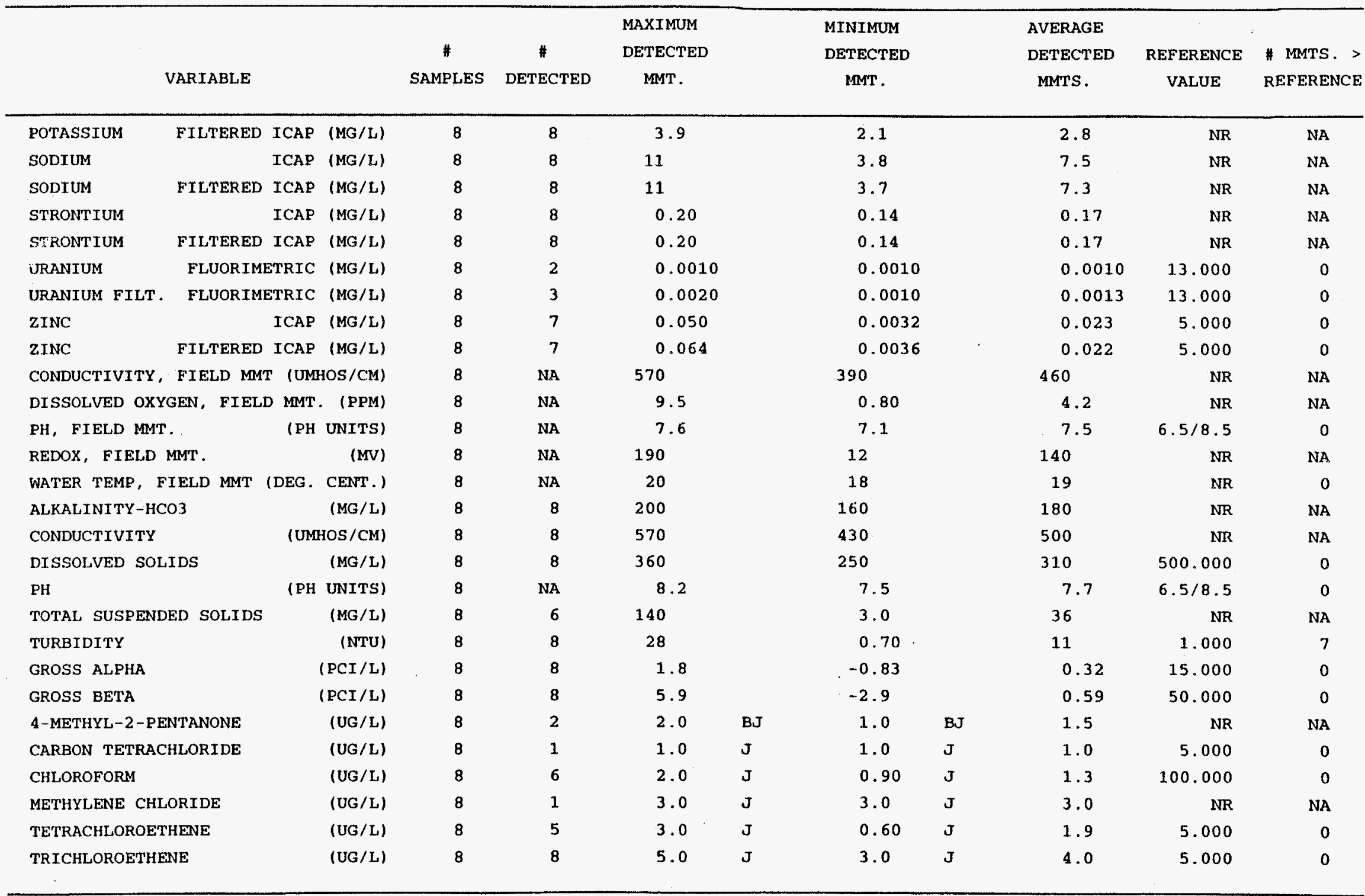


Table 7.31. Constlituents in groundwater at the Y-12 Plant site

$H G R=E F$ area $=$ Grid 11

\begin{tabular}{|c|c|c|c|c|c|c|c|c|c|c|}
\hline & VARIABLE & & & $\begin{array}{c}\# \\
\text { SAMPLES }\end{array}$ & $\begin{array}{c}\# \\
\text { DETECTED }\end{array}$ & $\begin{array}{c}\text { MAXIMUM } \\
\text { DETECTED } \\
\text { MMT. }\end{array}$ & $\begin{array}{c}\text { MINIMUM } \\
\text { DETECTED } \\
\text { MMT. }\end{array}$ & $\begin{array}{l}\text { AVERAGE } \\
\text { DETECTED } \\
\text { MMTS. }\end{array}$ & $\begin{array}{c}\text { REFERENCE } \\
\text { VAI,UE }\end{array}$ & $\begin{array}{l}\text { \# MMTS. > } \\
\text { REFERENCE }\end{array}$ \\
\hline CHLORIDE & & & (MG/L) & 8 & 8 & 21 & 2.9 & 8.2 & 250.000 & 0 \\
\hline FLUORIDE & & & $\langle M G / L\rangle$ & 8 & 8 & 0.40 & 0.20 & 0.29 & 2.000 & 0 \\
\hline NITRATE NI & DGEN & & (MG/L) & 8 & 4 & 0.40 & 0.23 & 0.30 & 10.000 & 0 \\
\hline SULFATE & & & (MG/L) & 8 & 8 & 31 & 12 & 21 & 250.000 & 0 \\
\hline ALUMINUM & & ICAP & (MG/L) & 8 & 8 & 0.75 & 0.021 & 0.27 & 0.200 & 4 \\
\hline ALUMINUM & FILTERED & ICAP & (MG/L) & 8 & 3 & 0.031 & 0.027 & 0.030 & 0.200 & 0 \\
\hline BARIUM & & ICAP & (MG/L) & 8 & 8 & 0.28 & 0.15 & 0.22 & 1.000 & 0 \\
\hline BARIUM & FILTERED & ICAP & (MG/L) & 8 & 8 & 0.27 & 0.10 & 0.19 & 1.000 & 0 \\
\hline BERYLLIUM & & ICAP & (MG/L) & 8 & 1 & 0.0009 & 0.0009 & 0.0009 & 0.004 ** & 0 \\
\hline BERYLLIUM & FILTERED & ICAP & $(M G / L)$ & 8 & 1 & 0.0015 & 0.0015 & 0.0015 & $0.004 * \star$ & 0 \\
\hline BORON & & ICAP & $(M G / L)$ & 8 & 8 & 0.11 & 0.050 & 0.082 & NR & $\mathrm{NA}$ \\
\hline BORON & FILTERED & ICAP & (MG/L) & 8 & 8 & 0.10 & 0.048 & 0.075 & NR & NA \\
\hline CALCIUM & & ICAP & (MG/L) & 8 & 8 & 58 & 36 & 46 & NR & NA \\
\hline CALCIUM & FILTERED & ICAP & $(M G / L)$ & 8 & 8 & 59 & 36 & 46 & NR & $\mathrm{NA}$ \\
\hline CHROMIUM & & AAS & $(M G / L)$ & 8 & 1 & 0.010 & 0.010 & 0.010 & 0.050 & 0 \\
\hline COBALT & & ICAP & $(M G / L)$ & 8 & 1 & 0.0063 & 0.0063 & 0.0063 & NR & NA \\
\hline COPPER & & ICAP & $(M G / L)$ & 8 & 3 & 0.0067 & 0.0050 & 0.0061 & 1.000 & 0 \\
\hline COPPER & FILTERED & ICAP & (MG/L) & 8 & 1 & 0.0061 & 0.0061 & 0.0061 & 1.000 & 0 \\
\hline IRON & & ICAP & $(M G / L)$ & 8 & 8 & 7.2 & 0.060 & 2.1 & 0.300 & 4 \\
\hline IRON & FILTERED & ICAP & (MG/L) & 8 & 6 & 0.20 & 0.0051 & 0.055 & 0.300 & 0 \\
\hline LEAD & & AAS & $(M G / L)$ & 8 & 1 & 0.034 & 0.034 & 0.034 & 0.050 & 0 \\
\hline MAGNESIUM & & ICAP & (MG/L) & 8 & 8 & 15 & 6.5 & 10 & NR & NA \\
\hline MAGNESIUM & FILTERED & ICAP & (MG/L) & 8 & 8 & 15 & 6.6 & 10 & NR & NA \\
\hline MANGANESE & & ICAP & $(M G / L)$ & 8 & 8 & 4.0 & 0.016 & 1.4 & 0.050 & 4 \\
\hline MANGANESE & FILTERED & ICAP & (MG/L) & 8 & 8 & 1.8 & 0.012 & 0.54 & 0.050 & 4 \\
\hline NICKEL & & ICAP & $(M G / L)$ & 8 & 1 & 0.012 & 0.012 & 0.012 & $0.100 * *$ & 0 \\
\hline NICKEL & FILTERED & ICAP & (MG/L) & 8 & 1 & 0.017 & 0.017 & 0.017 & $0.100^{* \star}$ & 0 \\
\hline POTASSIUM & & ICAP & (MG/L) & 8 & 8 & 2.9 & 2.1 & 2.6 & NR & NA \\
\hline POTASSTUM & FILTERED & ICAP & (MG/L) & 8 & 8 & 3.0 & 2.1 & 2.6 & NR & NA \\
\hline
\end{tabular}


Table 7.31 (continued)

\begin{tabular}{|c|c|c|c|c|c|c|c|c|c|c|}
\hline VARIABLE & & $\begin{array}{c}\# \\
\text { SAMPLES }\end{array}$ & $\begin{array}{c}\# \\
\text { DETECTED }\end{array}$ & $\begin{array}{l}\text { MAXIMUM } \\
\text { DETECTED } \\
\text { MMT . }\end{array}$ & & $\begin{array}{l}\text { MINIMUM } \\
\text { DETECTED } \\
\text { MMT. }\end{array}$ & & $\begin{array}{l}\text { AVERAGE } \\
\text { DETECTED } \\
\text { MMTS. }\end{array}$ & $\begin{array}{c}\text { REFERENCE } \\
\text { VALUE }\end{array}$ & $\begin{array}{l}\text { \# MMTS. > } \\
\text { REFERENCE }\end{array}$ \\
\hline SODIUM & ICAP (MG/L) & 8 & 8 & 71 & & 6.2 & & 37 & NR & NA \\
\hline FILTERED & ICAP (MG/L) & 8 & 8 & 69 & & 6.1 & . & 37 & NR & NA \\
\hline STRONTIUM & ICAP (MG/L) & 8 & 8 & .0 .68 & & 0.13 & & 0.40 & NR & NA \\
\hline STRONTIUM & ICAP (MG/L) & 8 & 8 & 0.67 & & 0.13 & & 0.40 & NR & NA \\
\hline FLUORIME & ETRIC (MG/L) & 8 & 4 & 0.0010 & & 0.0010 & & 0.0010 & 13.000 & 0 \\
\hline URANIUM FILT. FLUORIME & ETRIC (MG/L) & 8 & 3 & 0.0010 & & 0.0010 & & 0.0010 & 13.000 & 0 \\
\hline ZINC & ICAP $(M G / L)$ & 8 & 8 & 0.17 & & 0.0030 & & 0.036 & 5.000 & 0 \\
\hline FILTERED & ICAP (MG $/ L)$ & 8 & 7 & 0.18 & & 0.0034 & & 0.041 & 5.000 & 0 \\
\hline CONDUCTIVITY, FIELD MMT & T (UMHOS/CM) & 8 & NA & 600 & & 320 & & 460 & NR & NA \\
\hline DISSOLVED OXYGEN, FIELD & D MMT. (РPM) & 8 & NA & 8.9 & & 1.3 & & 3.8 & NR & NA \\
\hline PH, FIELD MMT. & (PH UNITS) & 8 & $\mathrm{NA}$ & 8.3 & & 6.1 & & 7.0 & $6.5 / 8.5$ & 3 \\
\hline REDOX, FIELD MMT. & (MV) & 8 & NA & 200 & & 16 & & 130 & NR & NA \\
\hline WATER TEMP, FIELD MMT & (DEG. CENT.) & 8 & NA & 19 & & 12 & & 15 & NR & 0 \\
\hline ALKALINITY-HCO3 & $(M G / L)$ & 8 & 8 & 260 & & 150 & & 210 & NR & NA \\
\hline CONDUCTIVITY & (UMHOS/CM) & 8 & 8 & 510 & & 380 & & 450 & NR & NA \\
\hline DISSOLVED SOLIDS & $(\mathrm{MG} / \mathrm{L})$ & 8 & 8 & 340 & & 220 & & 300 & 500.000 & 0 \\
\hline PH & (PH UNITS) & 8 & NA & 8.0 & & 6.5 & & 7.3 & $6.5 / 8.5$ & 0 \\
\hline TOTAL SUSPENDED SOLIDS & $(M G / L)$ & 8 & 6 & 630 & & 2.0 & & 120 & NR & NA \\
\hline TURBIDITY & (NTU) & 8 & 8 & 3900 & & 1.0 & & 490 & 1.000 & 7 \\
\hline GROSS ALPHA & $(\mathrm{PCI} / \mathrm{L})$ & 8 & 8 & 6.9 & & -3.4 & & 0.81 & 15.000 & 0 \\
\hline GROSS BETA & $(\mathrm{PCI} / \mathrm{L})$ & 8 & 8 & 15 & & -7.4 & & 4.9 & 50.000 & 0 \\
\hline METHYLENE CHLORIDE & $(U G / L)$ & 8 & 1 & 0.90 & JB & 0.90 & JB & 0.90 & NR & NA \\
\hline
\end{tabular}


Table 7.32. Constltuents in groundwater at the $Y-12$ Plant slie

$\mathrm{HGA}=\mathrm{EF}$ area $=$ Grid $\mathrm{I2}$

\begin{tabular}{|c|c|c|c|c|c|c|c|c|c|c|}
\hline \multicolumn{4}{|c|}{ VARIABLE } & \multirow{2}{*}{$\begin{array}{c}\text { \# } \\
\text { SAMPLES } \\
8\end{array}$} & \multirow{2}{*}{$\frac{\stackrel{\#}{\text { DETECTED }}}{8}$} & $\begin{array}{l}\text { MAXIMUM } \\
\text { DETECTED } \\
\text { MMT. }\end{array}$ & $\begin{array}{l}\text { MINJMUM } \\
\text { DETECTED } \\
\text { MMT. }\end{array}$ & $\begin{array}{l}\text { AVERAGE } \\
\text { DETECTED } \\
\text { MMTS. }\end{array}$ & $\begin{array}{c}\text { REFERENCE } \\
\text { VAL,UE }\end{array}$ & \multirow{2}{*}{$\begin{array}{c}\text { \# MMTS. > } \\
\text { REFERENCE } \\
0\end{array}$} \\
\hline CHLORIDE & & & (MG/L) & & & 5.4 & 2.3 & 3.5 & 250.000 & \\
\hline FLUORIDE & & & (MG/L) & 8 & 2 & 0.10 & 0.10 & 0.10 & 2.000 & 0 \\
\hline NITRATE NI & DGEN & & $(M G / L)$ & 8 & 1 & 0.25 & 0.25 & 0.25 & 10.000 & 0 \\
\hline SULFATE & & & $(M G / L)$ & 8 & 8 & 14 & 3.0 & 7.7 & 250.000 & 0 \\
\hline ALUMINUM & & ICAP & $(M G / L)$ & 8 & 8 & 0.46 & 0.027 & 0.13 & 0.200 & 2 \\
\hline ALUMINUM & FILTERED & ICAP & $(M G / L)$ & $B$ & 6 & 0.092 & 0.025 & 0.050 & 0.200 & 0 \\
\hline BARIUM & & ICAP & $(M G / L)$ & 8 & 8 & 0.25 & 0.048 & 0.16 & 1.000 & 0 \\
\hline BARIUM & FILTERED & ICAP & $(M G / L)$ & 8 & 8 & 0.23 & 0.047 & 0.14 & 1.000 & 0 \\
\hline BORON & & ICAP & (MG/L) & 8 & 8 & 0.044 & 0.0086 & 0.026 & NR & NA \\
\hline BORON & FILTERED & ICAP & (MG/L) & 8 & 8 & 0.040 & 0.014 & 0.026 & NR & NA \\
\hline CALCIUM & & ICAP & (MG/L) & 8 & 8 & 46 & 2.3 & 20 & NR & NA \\
\hline CALCIUM & FILTERED & ICAP & (MG/L) & 8 & 8 & 42 & 2.4 & 18 & NR & NA \\
\hline CHROMIUM & & AAS & $(M G / L)$ & 8 & 2 & 0.047 & 0.017 & 0.032 & 0.050 & 0 \\
\hline COBALT & & ICAP & $(M G / L)$ & 8 & 1 & 0.0065 & 0.0065 & 0.0065 & NR & NA \\
\hline COPPER & & ICAP & (MG/L) & 8 & 4 & 0.0080 & 0.0050 & 0.0066 & 1.000 & 0 \\
\hline COPPER & FILTERED & ICAP & (MG/L) & 8 & 1 & 0.014 & 0.014 & 0.014 & 1.000 & 0 \\
\hline IRON & & ICAP & (MG/L) & 8 & 8 & 1.3 & 0.017 & 0.26 & 0.300 & 2 \\
\hline IRON & FILTERED & ICAP & (MG/L) & 8 & 6 & 0.028 & 0.0074 & 0.015 & 0.300 & o \\
\hline MAGNESIUM & & ICAP & (MG/L) & 8 & 8 & 7.3 & 1,0 & 3.9 & NR & NA \\
\hline MAGNESIUM & FILTERED & ICAP & $(M G / L)$ & 8 & 8 & 7.1 & 1.1 & 3.7 & NK & NA \\
\hline MANGANESE & & ICAP & $(M G / L)$ & 8 & 8 & 0.15 & 0.0049 & 0.052 & 0.050 & 3 \\
\hline MANGANESE & FILTERED & ICAP & $(M G / L)$ & 8 & 7 & 0.099 & 0.0032 & 0.047 & 0.050 & 3 \\
\hline NICKEL & & ICAP & $(M G / L)$ & 8 & 3 & 0.016 & 0.011 & 0.013 & $0.100 * *$ & 0 \\
\hline NICKEL & FILTERED & ICAP & $(M G / L)$ & 8 & 4 & 0.028 & 0.013 & 0.018 & $0.100^{\star *}$ & 0 \\
\hline POTASSIUM & & ICAP & $(M G / L)$ & 8 & 7 & 4.6 & 0.80 & 2.8 & NR & NA \\
\hline POTASSIUM & FILTERED & ICAP & $(M G / L)$ & 8 & 7 & 5.4 & 0.85 & 3.0 & NR & NA \\
\hline SODIUM & & ICAP & (MG /L) & 8 & 8 & 12 & 2.4 & 7.1 & NR & NA \\
\hline SODIUM & FILTERED & ICAP & $(M G / L)$ & 8 & 8 & 13 & 2.3 & 7.3 & NR & NA \\
\hline STRONT LUM & & ICAP & $(M G / L)$ & 8 & 8 & 0.53 & 0.011 & 0.23 & NR & NA \\
\hline
\end{tabular}


Table 7.32 (continued)

\begin{tabular}{|c|c|c|c|c|c|c|c|c|c|}
\hline VARIABLE & $\begin{array}{c}\# \\
\text { SAMPLES }\end{array}$ & $\begin{array}{c}\# \\
\text { DETECTED }\end{array}$ & $\begin{array}{l}\text { MAXIMUM } \\
\text { DETECTED } \\
\text { MMT. }\end{array}$ & & $\begin{array}{l}\text { MINIMUM } \\
\text { DETECTED } \\
\text { MMT. }\end{array}$ & & $\begin{array}{l}\text { AVERAGE } \\
\text { DETECTED } \\
\text { MMTS. }\end{array}$ & $\begin{array}{l}\text { REFERENCE } \\
\text { VALUE }\end{array}$ & $\begin{array}{l}\# \text { MMTS. > } \\
\text { REFERENCE }\end{array}$ \\
\hline FILTERED ICAP (MG/L) & 8 & 8 & 0.50 & & 0.011 & & 0.23 & NR & NA \\
\hline URANIUM FILT. FLUORIMETRIC (MG/L) & 8 & 2 & 0.0010 & & 0.0010 & & 0.0010 & 13.000 & 0 \\
\hline ICAP (MG/L) & 8 & 7 & 0.16 & & 0.0043 & & 0.048 & 5.000 & 0 \\
\hline FILTERED ICAP (MG/L) & 8 & 7 & 0.20 & & 0.0024 & & 0.066 & 5.000 & 0 \\
\hline CONDUCTIVITY, FIELD MMT (UMHOS/CM) & 8 & NA & 270 & & 33 & & 150 & NR & NA \\
\hline DISSOLVED OXYGEN, FIELD MMT. (PPM) & 8 & NA & 6.7 & & 1.5 & & 4.1 & NR & NA \\
\hline PH, FIELD MMT. & 8 & NA & 10 & & 4.8 & & 7.2 & $6.5 / 8.5$ & 7 \\
\hline REDOX, FIELD MMT. & 8 & NA & 290 & & 11 & & 170 & NR & NA \\
\hline WATER TEMP, FIELD MMT (DEG. CENT.) & 8 & NA & 22 & & 12 & & 18 & NR & 0 \\
\hline ALKALINITY-CO3 & 8 & 4 & 26 & & 4.0 & & 14 & NR & NA \\
\hline ALKALINITY-HCO3 & 8 & 8 & 130 & & 7.0 & & 56 & NR & NA \\
\hline CONDUCTIVITY & 8 & 8 & 280 & & 39 & & 140 & NR & $\mathrm{NA}$ \\
\hline DISSOLVED SOLIDS & 8 & 8 & 200 & & 32 & & 110 & 500.000 & 0 \\
\hline (PH UNITS) & 8 & NA & 9.4 & & 5.5 & & 7.3 & $6.5 / 8.5$ & 7 \\
\hline TOT. PETROLEUM HYDROCARBONS (MG/L) & 8 & 2 & 0.0070 & & 0.0038 & & 0.0054 & 1.000 & 0 \\
\hline TOTAL SUSPENDED SOLIDS & 8 & 7 & 64 & & 1.0 & & 16 & NR & $\mathrm{NA}$ \\
\hline TURBIDITY & 8 & 8 & 7.4 & & 0.50 & & 2.4 & 1.000 & 7 \\
\hline GROSS ALPHA & 8 & 8 & 4.0 & & -2.3 & & 0.52 & 15.000 & 0 \\
\hline GROSS BETA & 8 & 8 & 6.3 & & -6.9 & & 1.7 & 50.000 & 0 \\
\hline 2-BUTANONE & 8 & 1 & 4.0 & BJ & 4.0 & $\mathrm{BJ}$ & 4.0 & NR & $\mathrm{NA}$ \\
\hline 4-METHYL-2-PENTANONE & 8 & 2 & 2.0 & BJ & 2.0 & BJ & 2.0 & NR & NA \\
\hline ACETONE & 8 & 2 & 2.0 & BJ & 1.0 & BJ & 1.5 & NR & NA \\
\hline METHYLENE CHLORIDE & 8 & 1 & 1.0 & JB & 1.0 & JB & 1.0 & NR & NA \\
\hline
\end{tabular}


Table 7.33. Constituents in groundwater at the $\mathrm{Y}-12$ Plant site HGR=EF area=Grid J Primary

\begin{tabular}{|c|c|c|c|c|c|c|c|c|c|c|}
\hline & VARIABLE & & & $\begin{array}{c}\# \\
\text { SAMPLES }\end{array}$ & $\begin{array}{c}\text { \# } \\
\text { DETECTED }\end{array}$ & $\begin{array}{c}\text { MAXIMUM } \\
\text { DETECTED } \\
\text { MMT. }\end{array}$ & $\begin{array}{c}\text { MINIMUM } \\
\text { DETECTED } \\
\text { MMT. }\end{array}$ & $\begin{array}{l}\text { AVERAGE } \\
\text { DETECTED } \\
\text { MMTS. }\end{array}$ & $\begin{array}{c}\text { REFERENCE } \\
\text { VALUE }\end{array}$ & $\begin{array}{l}\text { \# MMTS. > } \\
\text { REFERENCE }\end{array}$ \\
\hline CHLORIDE & & & (MG/L) & 8 & 8 & 47 & 24 & 33 & 250.000 & 0 \\
\hline FLUORIDE & & & $(M G / L)$ & 8 & 5 & 0.30 & 0.10 & 0.20 & 2.000 & 0 \\
\hline SULFATE & & & (MG/L) & 8 & 7 & 18 & 2.0 & 11 & 250.000 & 0 \\
\hline ALUMINUM & & ICAP & (MG/L) & 8 & 7 & 0.95 & 0.24 & 0.60 & 0.200 & 7 \\
\hline ALUMINUM & FILTERED & ICAP & (MG/L) & 8 & 4 & 0.13 & 0.021 & 0.051 & 0.200 & 0 \\
\hline BARIUM & & ICAP & (MG/L) & 8 & 8 & 0.64 & 0.065 & 0.35 & 1.000 & 0 \\
\hline BARIUM & FILTERED & ICAP & (MG/L) & 8 & 8 & 0.64 & 0.066 & 0.34 & 1.000 & 0 \\
\hline BERYLLIUM & & ICAP & (MG/L) & 8 & 1 & 0.0003 & 0.0003 & 0.0003 & $0.004^{\star \star}$ & 0 \\
\hline BORON & & ICAP & (MG/L) & 8 & 8 & 0.11 & 0.010 & 0.064 & NR & NA \\
\hline BORON & FILTERED & ICAP & (MG/L) & 8 & 8 & 0.12 & 0.014 & 0.064 & NR & NA \\
\hline CALCIUM & & ICAP & $(M G / L)$ & 8 & 8 & 120 & 73 & 93 & NR & NA \\
\hline CALCIUM & FILTERED & ICAP & (MG/L) & 8 & 8 & 120 & 69 & 92 & NR & NA \\
\hline COPPER & & ICAP & $(M G / L)$ & 8 & 1 & 0.011 & 0.011 & 0.011 & 1.000 & 0 \\
\hline COPPER & FILTERED & ICAP & $(M G / L)$ & 8 & 3 & 0.023 & 0.0047 & 0.012 & 1.000 & 0 \\
\hline IRON & & ICAP & (MG/L) & 8 & 8 & 22 & 0.58 & 9.9 & 0.300 & 8 \\
\hline IRON & FILTERED & ICAP & (MG/L) & 8 & 8 & 23 & 0.041 & 9.1 & 0.300 & 4 \\
\hline LEAD & & AAS & (MG/L) & 8 & 2 & 0.0043 & 0.0040 & 0.0042 & 0.050 & 0 \\
\hline MAGNESIUM & & ICAP & (MG/L) & 8 & 8 & 25 & 11 & 18 & NR & NA \\
\hline MAGNESIUM & FILTERED & ICAP & $(M G / L)$ & 8 & 8 & 26 & 12 & 18 & NR & NA \\
\hline MANGANESE & & ICAP & $(M G / L)$ & 8 & 8 & 1.4 & 0.20 & 0.80 & 0.050 & 8 \\
\hline MANGANESE & FILTERED & ICAP & (MG /L) & 8 & 8 & 1.4 & 0.15 & 0.75 & 0.050 & 8 \\
\hline POTASSIUM & & ICAP & $(M G / L)$ & 8 & 7 & 4.9 & 0.94 & 3.0 & NR & NA \\
\hline POTASSIUM & FILTERED & ICAP & (MG/L) & 8 & 8 & 4.7 & 0.73 & 2.5 & NR & NA \\
\hline SODIUM & & ICAP & (MG/L) & 8 & 8 & 11 & 8.0 & 9.1 & NR & NA \\
\hline SODIUM & FILTERED & ICAP & $(M G / L)$ & 8 & 8 & 11 & 8.0 & 9.2 & NR & NA \\
\hline STRONTIUM & & ICAP & $(M G / L)$ & 8 & 8 & 0.82 & 0.20 & 0.49 & NR & NA \\
\hline STRONTIUM & FILTERED & ICAP & (MG/L) & 8 & 8 & 0.83 & 0.20 & 0.48 & NR & NA \\
\hline ZINC & & ICAP & $(\mathrm{MG} / \mathrm{L})$ & 8 & 7 & 0.035 & 0.0052 & 0.014 & 5.000 & 0 \\
\hline ZINC & FILTERED & ICAP & $(M G / L)$ & 8 & 7 & 0.028 & 0.0076 & 0.013 & 5.000 & 0 \\
\hline
\end{tabular}


Table 7.33 (continued)

\begin{tabular}{|c|c|c|c|c|c|c|c|c|c|}
\hline VARTABLE & $\begin{array}{c}\# \\
\text { SAMPLES }\end{array}$ & $\begin{array}{c}\# \\
\text { DETECTED }\end{array}$ & $\begin{array}{l}\text { MAXIMUM } \\
\text { DETECTED } \\
\text { MMT . }\end{array}$ & & $\begin{array}{l}\text { MINIMUM } \\
\text { DETECTED } \\
\text { MMT. }\end{array}$ & & $\begin{array}{l}\text { AVERAGE } \\
\text { DETECTED } \\
\text { MMTS. }\end{array}$ & $\begin{array}{c}\text { REFERENCE } \\
\text { VALUE }\end{array}$ & $\begin{array}{l}\text { \# MMTS. > } \\
\text { REFERENCE }\end{array}$ \\
\hline CONDUCTIVITY, FIELD MMT (UMHOS/CM) & 8 & NA & 670 & & 500 & & 600 & NR & NA \\
\hline DISSOLVED OXYGEN, FIELD MMT. (PPM) & 8 & NA & 2.9 & & 0.40 & & 1.6 & NR & NA \\
\hline PH, FIELD MMT. & 8 & NA & 7.3 & & 6.3 & & 6.9 & $6.5 / 8.5$ & 1 \\
\hline REDOX, FIELD MMT. & 8 & NA & 110 & & -70 & & -4.8 & NR & NA \\
\hline WATER TEMP, FIELD MMT (DEG. CENT.) & 8 & NA & 22 & & 7.9 & & 17 & NR & 0 \\
\hline ALKALINITY-HCO3 & 8 & 8 & 320 & & 260 & & 300 & NR & NA \\
\hline CONDUCTIVITY & 8 & 8 & 720 & & 590 & & 660 & NR & $\mathrm{NA}$ \\
\hline DISSOLVED SOLIDS & 8 & 8 & 430 & & 330 & & 380 & 500.000 & 0 \\
\hline (PH UNITS) & 8 & NA & 7.6 & & 6.9 & & 7.3 & $6.5 / 8.5$ & 0 \\
\hline TOTAL SUSPENDED SOLIDS & 8 & 8 & 53 & & 8.0 & & 28 & NR & NA \\
\hline TURBIDITY & 8 & 8 & 46 & & 7.5 & & 26 & 1.000 & 8 \\
\hline GROSS ALPHA & 8 & 8 & 3.9 & & -3.4 & & -0.082 & 15.000 & 0 \\
\hline GROSS BETA & 8 & 8 & 6.1 & & -4.2 & & 2.4 & 50.000 & 0 \\
\hline 1,1-DICHLOROETHENE & 8 & 2 & 12 & $J$ & 2.0 & $\mathrm{~J}$ & 7.0 & 7.000 & 1 \\
\hline 1,2-DICHLOROETHENE & 8 & 7 & 120 & & 42 & & 79 & 70.000 & 5 \\
\hline 4-METHYL-2-PENTANONE & 8 & 1 & 24 & BJ & 24 & BJ & 24 & NR & NA \\
\hline METHYLENE CHLORIDE & 8 & 2 & 11 & $J$ & 1.0 & $\mathrm{JB}$ & 6.0 & NR & NA \\
\hline TETRACHLOROETHENE & 8 & 8 & 1000 & & 5.0 & & 490 & 5.000 & 7 \\
\hline TRICHLOROETHENE & 8 & 7 & 46 & $\mathrm{~J}$ & 1.0 & $J$ & 20 & 5.000 & 4 \\
\hline VINYL CHLORIDE & 8 & 4 & 59 & & 35 & & 47 & 2.000 & 4 \\
\hline
\end{tabular}


Table 7.34. Constltuents in groundwater at the $Y-12$ Plant site

$H G R=E F$ area=Grid $J 1$

\begin{tabular}{|c|c|c|c|c|c|c|c|c|c|c|}
\hline & VARIABLE & & & $\begin{array}{c}\# \\
\text { SAMPLES }\end{array}$ & $\begin{array}{c}\# \\
\text { DETECTED }\end{array}$ & $\begin{array}{c}\text { MAXIMUM } \\
\text { DETECTED } \\
\text { MMT. }\end{array}$ & $\begin{array}{c}\text { MINIMUM } \\
\text { DETECTED } \\
\text { MMT . }\end{array}$ & $\begin{array}{l}\text { AVERAGE } \\
\text { DETECTED } \\
\text { MMTS. }\end{array}$ & $\begin{array}{c}\text { REFERENCE } \\
\text { VALUE }\end{array}$ & $\begin{array}{l}\# \text { MMTS. > } \\
\text { REFERENCE }\end{array}$ \\
\hline CHLORIDE & & & $(M G / L)$ & 8 & 8 & 45 & 6.0 & 24 & 250.000 & 0 \\
\hline FLUORIDE & & & (MG/L) & 8 & 5 & 0.70 & 0.10 & 0.46 & 2.000 & 0 \\
\hline SULFATE & & & (MG/L) & 8 & 8 & 36 & 16 & 27 & 250.000 & 0 \\
\hline ALUMINUM & & ICAP & (MG/L) & 8 & 7 & 47 & 0.35 & 11 & 0.200 & 7 \\
\hline ALUMINUM & FILTERED & ICAP & $(M G / L)$ & 8 & 2 & 0.10 & 0.028 & 0.064 & 0.200 & 0 \\
\hline BARIUM & & ICAP & (MG/L) & 8 & 8 & 0.55 & 0.14 & 0.23 & 1.000 & 0 \\
\hline BARIUM & FILTERED & ICAP & (MG/L) & 8 & 8 & 0.20 & 0.075 & 0.12 & 1.000 & 0 \\
\hline BERYLLIUM & & ICAP & (MG/L) & 8 & 5 & 0.0026 & 0.0003 & 0.0009 & $0.004 * *$ & 0 \\
\hline BORON & & ICAP & (MG/L) & 8 & B & 0.13 & 0.023 & 0.080 & NR & NA \\
\hline BORON & FILTERED & ICAP & (MG/L) & 8 & 8 & 0.12 & 0.018 & 0.071 & NR & NA \\
\hline CADMIUM & & ICAP & $(M G / L)$ & 8 & 2 & 0.019 & 0.0063 & 0.013 & 0.005 & 2 \\
\hline CALCIUM & & ICAP & (MG/L) & 8 & 8 & 54 & 22 & 31 & NR & NA \\
\hline CALCIUM & FILTERED & ICAP & (MG/L) & $B$ & 8 & 29 & 19 & 26 & NR & NA \\
\hline CHROMIUM & & AAS & (MG/L) & 8 & 4 & 0.12 & $0: 012$ & 0.042 & 0.050 & 1 \\
\hline CHROMIUM & & ICAP & (MG/L) & 8 & 2 & 0.093 & 0.051 & 0.072 & 0.050 & 2 \\
\hline COBALT & & ICAP & $(M G / L)$ & 8 & 4 & 0.048 & 0.0061 & 0.023 & NR & NA \\
\hline COBALT & FILTERED & ICAP & (MG/L) & 8 & 2 & 0.015 & 0.0093 & 0.012 & NR & NA \\
\hline COPPER & & ICAP & (MG/L) & 8 & 6 & 0.061 & 0.0073 & 0.020 & 1.000 & 0 \\
\hline COPPER & FILTERED & ICAP & (MG/L) & 8 & 2 & 0.011 & 0.011 & 0.011 & 1.000 & 0 \\
\hline IRON & & ICAP & (MG/L) & 8 & 8 & 110 & 0.049 & 22 & 0.300 & 7 \\
\hline IRON & FILTERED & ICAP & (MG/L) & 8 & 6 & 1.3 & 0.012 & 0.34 & 0.300 & 2 \\
\hline LEAD & & AAS & (MG/L) & B & 4 & 0.042 & 0.0045 & 0.015 & 0.050 & 0 \\
\hline MAGNESIUM & & ICAP & (MG/L) & 8 & 8 & 14 & 4.3 & 7.4 & $\mathrm{NR}$ & NA \\
\hline MAGNESIUM & FILTERED & ICAP & (MG/L) & 8 & 8 & 6.3 & 4.0 & 5.4 & NR & NA \\
\hline MANGANESE & & ICAP & (MG/L) & 8 & 8 & 3.0 & 0.020 & 0.98 & 0.050 & 6 \\
\hline MANGANESE & FILTERED & ICAP & (MG/L) & 8 & 8 & 3.0 & 0.0060 & 0.89 & 0.050 & 4 \\
\hline NICKEL & & ICAP & (MG/L) & 8 & 3 & 0.079 & 0.018 & 0.056 & $0.100 * *$ & 0 \\
\hline NICKEL & FILTERED & ICAP & (MG/L) & 8 & 1 & 0.019 & 0.019 & 0.019 & $0.100 * *$ & 0 \\
\hline POTASSIUM & & ICAP & (MG/L) & 8 & 8 & 9.2 & 2.7 & 4.2 & NR & NA \\
\hline
\end{tabular}


Table 7.34 (continued)

\begin{tabular}{|c|c|c|c|c|c|c|c|c|c|c|}
\hline VARIABLE & & $\begin{array}{c}\# \\
\text { SAMPLES }\end{array}$ & $\begin{array}{c}\# \\
\text { DETECTED }\end{array}$ & $\begin{array}{l}\text { MAXIMUM } \\
\text { DETECTED } \\
\text { MMT. }\end{array}$ & & $\begin{array}{l}\text { MINIMUM } \\
\text { DETECTED } \\
\text { MMT. }\end{array}$ & & $\begin{array}{l}\text { AVERAGE } \\
\text { DETECTED } \\
\text { MMTS. }\end{array}$ & $\begin{array}{c}\text { REFERENCE } \\
\text { VALUE }\end{array}$ & $\begin{array}{l}\text { \# MMTS. > } \\
\text { REFERENCE }\end{array}$ \\
\hline POTASSIUM & ICAP (MG/L) & 8 & 8 & 3.5 & & 1.0 & & 2.1 & NR & NA \\
\hline SODIUM & ICAP (MG/L) & 8 & B & 81 & & 16 & & 47 & NR & NA \\
\hline FILTERED & ICAP (MG /L) & 8 & 8 & 79 & & 16 & & 47 & NR & NA \\
\hline STRONTIUM & ICAP $(M G / L)$ & 8 & 8 & 0.91 & & 0.090 & & 0.43 & NR & NA \\
\hline STRONTIUM & ICAP (MG/L) & 8 & 8 & 0.93 & & 0.080 & & 0.39 & $\mathrm{NR}$ & NA \\
\hline FLUORIME & ETRIC (MG/L) & 8 & 2 & 0.0020 & & 0.0010 & & 0.0015 & 13.000 & 0 \\
\hline URANIUM FILT. FLUORIME & ETRIC (MG/L) & 8 & 2 & 0.0010 & & 0.0010 & & 0.0010 & 13.000 & 0 \\
\hline VANADIUM & ICAP (MG/L) & 8 & 4 & 0.087 & & 0.0095 & & 0.034 & NR & NA \\
\hline ZINC & ICAP (MG/L) & 8 & 8 & 0.15 & & 0.014 & & 0.046 & 5.000 & 0 \\
\hline FILTERED & ICAP (MG/L) & 8 & 8 & 0.019 & & 0.0042 & & 0.011 & 5.000 & 0 \\
\hline CONDUCTIVITY, FIELD MMT & T (UMHOS/CM) & 8 & NA & 430 & & 250 & & 350 & NR & NA \\
\hline DISSOLVED OXYGEN, FIELD & D MMT. (PPM) & 8 & NA & 7.9 & & 0.90 & & 4.0 & NR & NA \\
\hline PH, FIELD MMT. & (PH UNITS) & 8 & NA & 8.2 & & 5.4 & & 6.8 & $6.5 / 8.5$ & 4 \\
\hline REDOX, FIELD MMT. & (MV) & 8 & NA & 200 & & 23 & & 110 & NR & NA \\
\hline WATER TEMP, FIELD MMT & (DEG. CENT.) & 8 & NA & 20 & & 13 & & 16 & NR & 0 \\
\hline ALKALINITY-CO3 & (MG/L) & 8 & 1 & 2.0 & & 2.0 & & 2.0 & NR & NA \\
\hline ALKALINITY - $\mathrm{HCO} 3$ & $(M G / L)$ & 8 & 8 & 220 & & 54 & & 140 & NR & NA \\
\hline CONDUCTIVITY & (UMHOS/CM) & 8 & 8 & 480 & & 280 & & 390 & NR & NA \\
\hline DISSOLVED SOLIDS & $(M G / L)$ & 8 & 8 & 310 & & 170 & & 250 & 500.000 & 0 \\
\hline PH & (PH UNITS) & 8 & NA & 8.4 & & 5.9 & & 7.1 & $6.5 / 8.5$ & 4 \\
\hline TOTAL SUSPENDED SOLIDS & (MG/L) & 8 & 7 & 1700 & & 12 & & 530 & NR & NA \\
\hline TURBIDITY & (NTU) & 8 & 8 & 1500 & & 2.7 & & 330 & 1.000 & 8 \\
\hline GROSS ALPHA & $(\mathrm{PCI} / \mathrm{L})$ & 8 & 8 & 18 & & -3.7 & & 2.1 & 15.000 & 1 \\
\hline GROSS BETA & $(\mathrm{PCI} / \mathrm{L})$ & 8 & 8 & 39 & & -8.9 & & 5.9 & 50.000 & 0 \\
\hline 2-BUTANONE & $(U G / L)$ & 8 & 1 & 8.0 & $\mathrm{BJ}$ & 8.0 & $\mathrm{BJ}$ & 8.0 & NR & $\mathrm{NA}$ \\
\hline 4-METHYL-2-PENTANONE & $(U G / L)$ & 8 & 1 & 1.0 & $\mathrm{BJ}$ & 1.0 & $\mathrm{BJ}$ & 1.0 & NR & NA \\
\hline ACETONE & (UG/L) & 8 & 1 & 2.0 & BJ & 2.0 & $\mathrm{BJ}$ & 2.0 & NR & $\mathrm{NA}$ \\
\hline METHYLENE CHLORIDE & $(U G / L)$ & 8 & 1 & 3.0 & BJ & 3.0 & $\mathrm{BJ}$ & 3.0 & NR & $\mathrm{NA}$ \\
\hline
\end{tabular}


Table 7.35. Constituents in groundwater at the $Y-12$ Plant site

$H G R=E F$ area $=$ Grid $\mathrm{J} 2$

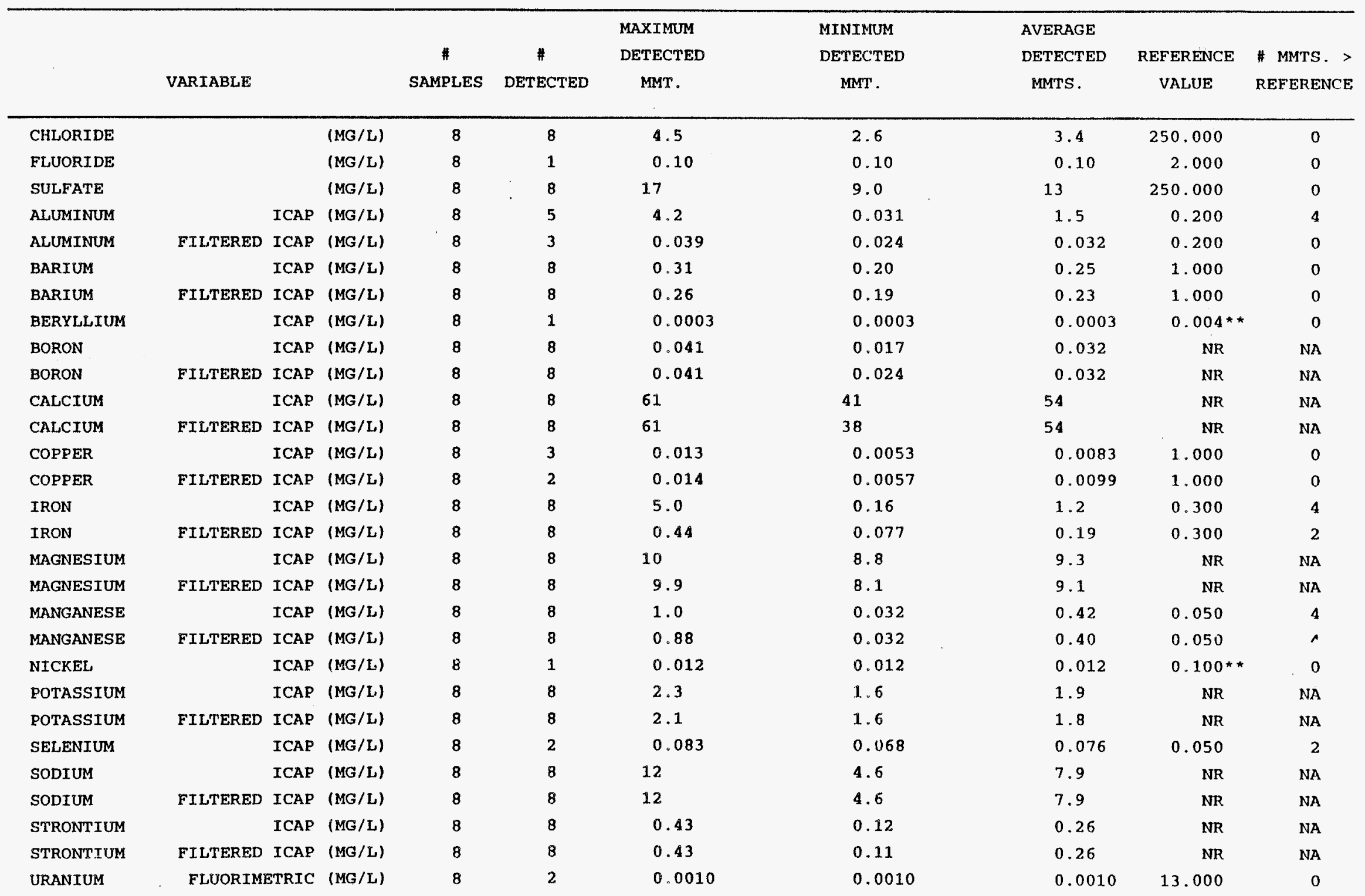


Table 7.35 (continued)

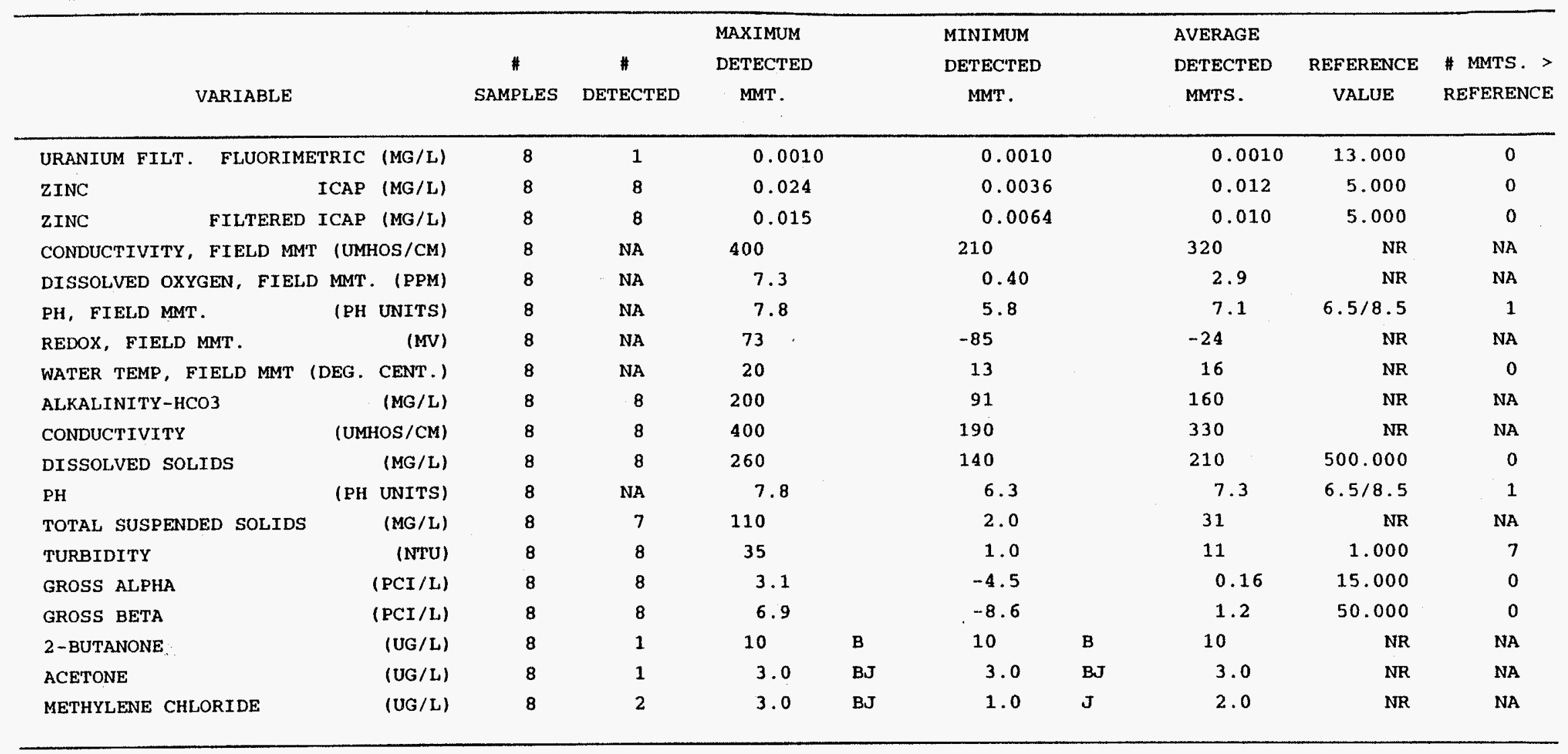


Table 7.36. Constituents in groundwater at the Y-12 Plant site

$H G R=E F$ area $=$ Grid $J 3$

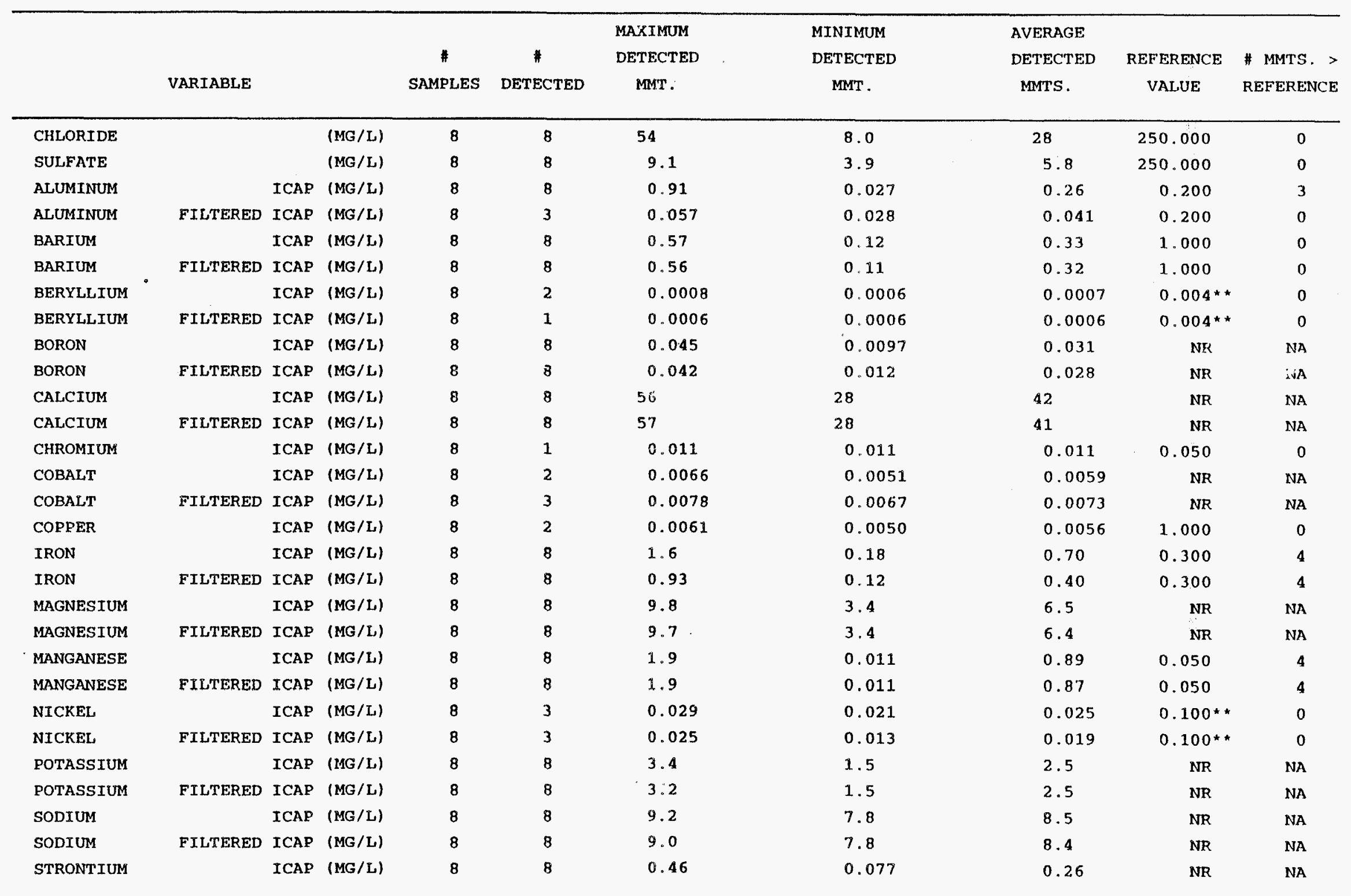


Table 7.36 (continued)

\begin{tabular}{|c|c|c|c|c|c|c|c|c|}
\hline VARIABLE & $\begin{array}{c}\# \\
\text { SAMPLES }\end{array}$ & $\begin{array}{c}\# \\
\text { DETECTED }\end{array}$ & $\begin{array}{l}\text { MAXIMUM } \\
\text { DETECTED } \\
\text { MMT. }\end{array}$ & $\begin{array}{l}\text { MINIMUM } \\
\text { DETECTED } \\
\text { MMT. }\end{array}$ & & $\begin{array}{l}\text { AVERAGE } \\
\text { DETECTED } \\
\text { MMTS. }\end{array}$ & $\begin{array}{c}\text { REFERENCE } \\
\text { VALUE }\end{array}$ & $\begin{array}{l}\text { \# MMTS. > } \\
\text { REFERENCE }\end{array}$ \\
\hline FILTERED ICAP (MG/L) & 8 & 8 & 0.47 & 0.075 & & 0.26 & NR & NA \\
\hline FLUORIMETRIC (MG/L) & 8 & 1 & 0.0010 & 0.0010 & & 0.0010 & 13.000 & 0 \\
\hline URANIUM FILT. FLUORIMETRIC (MG/L) & 8 & 2 & 0.0010 & 0.0010 & & 0.0010 & 13.000 & 0 \\
\hline ICAP $(M G / L)$ & 8 & 7 & 0.029 & 0.0055 & & 0.013 & 5.000 & 0 \\
\hline FILTERED ICAP (MG/L) & 8 & 7 & 0.014 & 0.0048 & & 0.0096 & 5.000 & 0 \\
\hline CONDUCTIVITY, FIELD MMT (UMHOS/CM) & 8 & NA & 370 & 230 & & 320 & NR & NA \\
\hline DISSOLVED OXYGEN, FIELD MMT. (PPM) & 8 & NA & 5.0 & 0.30 & & 1.7 & NR & NA \\
\hline PH, FIELD MMT. & 8 & NA & $7: 5$ & 4.7 & & 6.3 & $6.5 / 8.5$ & 4 \\
\hline REDOX，FIELD MMT. & 8 & NA & 140 & -76 & & 80 & NR & NA \\
\hline WATER TEMP, FIELD MMT (DEG. CENT.) & 8 & NA & 21 & 13 & & 16 & NR & 0 \\
\hline ALKALINITY-HCO3 & 8 & 8 & 180 & 45 & & 120 & NR & NA \\
\hline CONDUCTIVITY & 8 & 8 & 390 & 250 & & 330 & NR & NA \\
\hline DISSOLVED SOLIDS & 8 & 8 & 250 & 160 & & 200 & 500.000 & 0 \\
\hline (PH UNITS) & 8 & NA & 8.0 & 5.8 & & 6.9 & $6.5 / 8.5$ & 4 \\
\hline TOTAL SUSPENDED SOLIDS & 8 & 7 & 19 & 2.0 & & 9.1 & NR & NA \\
\hline TURBIDITY & 8 & 8 & 14 & 1.6 & & 5.7 & 1.000 & 8 \\
\hline GROSS ALPHA & 8 & 8 & 2.4 & -1.5 & & 0.35 & 15.000 & 0 \\
\hline GROSS BETA & 8 & 8 & 3.4 & -6.1 & & 0.38 & 50.000 & 0 \\
\hline ACETONE & 8 & 1 & 42 & 42 & & 42 & NR & NA \\
\hline METHYLENE CHLORIDE & 8 & 1 & 1.0 & 1.0 & $\mathfrak{J}$ & 1.0 & NR & NA \\
\hline
\end{tabular}


Table 7.37. Constlituents in groundwater at the Y-12 Plant site

HGR $=E F$ area $=$ Grid $K 1$

\begin{tabular}{|c|c|c|c|c|c|c|c|c|c|c|}
\hline & VARIABLE & & & $\begin{array}{c}\# \\
\text { SAMPLES }\end{array}$ & $\begin{array}{c}\# \\
\text { DETECTED }\end{array}$ & $\begin{array}{c}\text { MAXIMUM } \\
\text { DETECTED } \\
\text { MMT. }\end{array}$ & $\begin{array}{c}\text { MINIMUM } \\
\text { DETECTED } \\
\text { MMT'. }\end{array}$ & $\begin{array}{l}\text { AVERAGE } \\
\text { DETECTED } \\
\text { MMTS. }\end{array}$ & $\begin{array}{c}\text { REF ERENCE } \\
\text { VALUE }\end{array}$ & $\begin{array}{l}\text { \# MMTS. > } \\
\text { REFERENCF }\end{array}$ \\
\hline CHLORIDE & & & $(M G / L)$ & 12 & 1.2 & 16 & 3.2 & 7.7 & 250.000 & 0 \\
\hline FLUORIDE & & & $(M G / L)$ & 12 & 6 & 0.20 & 0.10 & 0.12 & 2.000 & 0 \\
\hline NITRATE NITI & DGEN & & (MG/L) & 12 & 4 & 0.61 & 0.40 & 0.49 & 10.000 & 0 \\
\hline SULFATE & & & (MG/L) & 12 & 12 & 30 & 18 & 25 & 250.000 & 0 \\
\hline ALUMINUM & & ICAP & (MG/L) & 12 & 10 & 20 & 0.021 & 2.4 & 0.200 & 5 \\
\hline ALUMTNUM & FILTERED & ICAP & $(M G / L)$ & 12 & 5 & 1.3 & 0.021 & 0.28 & 0.200 & 1 \\
\hline BARTUM & & ICAP & (MG/L) & 12 & 12 & 0.22 & 0.079 & 0.15 & 1.000 & 0 \\
\hline BARIUM & FILTERED & ICAP & (MG/L) & 12 & 12 & 0.20 & 0.079 & 0.14 & 1.000 & 0 \\
\hline BERYLLIUM & & ICAP & $(M G / L)$ & 12 & 2 & 0.0010 & 0.0005 & 0.0007 & $0.004^{\star \star}$ & 0 \\
\hline BORON & & ICAP & $(M G / L)$ & 12 & 12 & 0.094 & 0.0086 & 0.048 & NR & NA \\
\hline BORON & FILTERED & ICAP & (MG/L) & 12 & 12 & 0.072 & 0.017 & 0.047 & $\mathrm{NR}$ & NA \\
\hline CADMIUM & & ICAP & (MG/L) & 12 & 2 & 0.018 & 0.0076 & 0.013 & 0.005 & 2 \\
\hline CALCIUM & & ICAP & (MG/L) & 12 & 12 & 60 & 10 & 41 & NR & NA \\
\hline CALCIUM & FILTERED & ICAP & (MG/L) & 12 & 12 & 59 & 12 & 40 & NR & NA \\
\hline CHROMIUM & & AAS & (MG/L) & 12 & 3 & $0: 20$ & 0.013 & 0.084 & 0.050 & 1 \\
\hline CHROMIUM & & ICAP & (MG/L) & 12 & 2 & 0.18 & 0.036 & 0.11 & 0.050 & 1 \\
\hline COBALT & & ICAP & $\langle M G / L)$ & 12 & 2 & 0.019 & 0.014 & 0.017 & NR & NA \\
\hline COPPER & & ICAP & $(M G / L)$ & 12 & 7 & 0.048 & 0.0052 & 0.015 & 1.000 & 0 \\
\hline COPPER & FILTERED & ICAP & (MG/L) & 12 & 3 & 0.014 & 0.0044 & 0.0089 & 1.000 & 0 \\
\hline IRON & & ICAP & (MG/L) & 12 & 12 & 18 & 0.11 & 2.2 & 0.300 & 4 \\
\hline IRON & FILTERED & ICAP & (MG /L) & 12 & 10 & 0.86 & 0.011 & 0.21 & 0.300 & 1 \\
\hline LEAD & & AAS & $(M G / L)$ & 12 & 1 & 0.010 & 0.010 & 0.010 & 0.050 & 0 \\
\hline MAGNESIUM & & ICAP & (MG/L) & 12 & 12 & 10 & 3.8 & 7.9 & NR & NA \\
\hline MAGNESIUM & FILTERED & ICAP & $(M G / L)$ & 12 & 12 & 10 & 4.1 & 7.4 & NR & NA \\
\hline MANGANESE & & ICAP & (MG/L) & 12 & 12 & 0.78 & 0.033 & 0.24 & 0.050 & 10 \\
\hline MANGANESE & FILTERED & ICAP & (MG/L) & 12 & 12 & 0.78 & 0.029 & 0.24 & 0.050 & 8 \\
\hline MOLYBDENUM & & ICAP & (MG/L) & 12 & 1 & 0.019 & 0.019 & 0.019 & NR & NA \\
\hline NICKEL & & ICAP & (MG/L) & 12 & 3 & 0.044 & 0.013 & 0.030 & $0.100 * *$ & 0 \\
\hline NICKEL & FILTERED & ICAP & $(M G / L)$ & 12 & 2 & 0.028 & 0.028 & 0.028 & $0.100 * *$ & 0 \\
\hline
\end{tabular}


Table 7.37 (continued)

\begin{tabular}{|c|c|c|c|c|c|c|c|c|c|c|}
\hline VARIABLE & & $\begin{array}{c}\# \\
\text { SAMPLES }\end{array}$ & $\begin{array}{c}\# \\
\text { DETECTED }\end{array}$ & $\begin{array}{l}\text { MAXIMUM } \\
\text { DETECTED } \\
\text { MMT. }\end{array}$ & & $\begin{array}{c}\text { MINIMUM } \\
\text { DETECTED } \\
\text { MMT. }\end{array}$ & & $\begin{array}{l}\text { AVERAGE } \\
\text { DETECTED } \\
\text { MMTS. }\end{array}$ & $\begin{array}{c}\text { REFERENCE } \\
\text { VALUE }\end{array}$ & $\begin{array}{l}\# \text { MMTS. > } \\
\text { REFERENCE }\end{array}$ \\
\hline POTASSIUM & ICAP (MG/L) & 12 & 12 & 3.7 & & 1.2 & & 2.8 & NR & NA \\
\hline POTASSIUM & ICAP (MG/L) & 12 & 12 & 3.6 & & 1.1 & & 2.5 & $\mathrm{NR}$ & NA \\
\hline SELENIUM & $\operatorname{ICAP}(\mathrm{MG} / \mathrm{L})$ & 12 & 2 & 0.072 & & 0.070 & & 0.071 & 0.050 & 2 \\
\hline SODIUM & ICAP (MG/L) & 12 & 12 & 30 & & 5.8 & & 18 & NR & NA \\
\hline FILTERED & ICAP (MG/L) & 12 & 12 & 30 & & 5.8 & & 19 & NR & NA \\
\hline STRONTIUM & ICAP (MG/L) & 12 & 12 & 1.1 & & 0.046 & & 0.55 & NR & NA \\
\hline STRONT IUM & ICAP (MG/L) & 12 & 12 & 1.1 & & 0.050 & & 0.54 & NR & NA \\
\hline FLUORIME & ETRIC (MG/L) & 12 & 3 & 0.0020 & & 0.0010 & & 0.0013 & 13.000 & 0 \\
\hline URANIUM FILT. FLUORIME & ETRIC (MG/L) & 12 & 2 & 0.0010 & & 0.0010 & & 0.0010 & 13.000 & 0 \\
\hline VANADIUM & ICAP (MG/L) & 12 & 2 & 0.029 & & 0.010 & & 0.020 & NR & NA \\
\hline ZINC & ICAP (MG/L) & 12 & 12 & 0.099 & & 0.0040 & & 0.041 & 5.000 & 0 \\
\hline FILTERED & ICAP (MG/L) & 12 & 12 & 0.083 & & 0.0025 & & 0.031 & 5.000 & 0 \\
\hline CONDUCTIVITY, FIELD MMT & T (UMHOS/CM) & 12 & NA & 410 & & 120 & & 310 & NR & NA \\
\hline DISSOLVED OXYGEN, FIELD & D MMT. (PPM) & 12 & NA & 8.7 & & 0.30 & & 3.7 & NR & NA \\
\hline PH, FIELD MMT. & (PH UNITS) & 12 & NA & 7.6 & & 5.5 & & 6.9 & $6.5 / 8.5$ & 3 \\
\hline REDOX, FIELD MMT. & (MV) & 12 & NA & 230 & & -97 & & 74 & NR & NA \\
\hline WATER TEMP, FIELD MMT । & (DEG. CENT.) & 12 & NA & 21 & & 12 & & 16 & NR & 0 \\
\hline ALKALINITY-HCO3 & (MG/L) & 12 & 12 & 200 & & 32 & & 140 & NR & NA \\
\hline CONDUCTIVITY & (UMHOS/CM) & 12 & 12 & 450 & & 140 & & 340 & NR & NA \\
\hline DISSOLVED SOLIDS & (MG/L) & 12 & 12 & 300 & & 100 & & 220 & 500.000 & 0 \\
\hline PH & (PH UNITS) & 12 & NA & 8.1 & & 5.9 & & 7.1 & $6.5 / 8.5$ & 3 \\
\hline TOTAL SUSPENDED SOLIDS & $(M G / L)$ & 12 & 6 & 410 & & 2.0 & & 75 & NR & NA \\
\hline TURBIDITY & (NTU) & 12 & 12 & 230 & & 1.0 & & 49 & 1.000 & 11 \\
\hline GROSS ALPHA & $(\mathrm{PCI} / \mathrm{L})$ & 12 & 12 & 4.7 & & -3.6 & & 0.39 & 15.000 & 0 \\
\hline GROSS BETA & $(P C I / L)$ & 12 & 12 & 6.4 & & -1.4 & & 2.1 & 50.000 & 0 \\
\hline 2-BUTANONE & (UG/L) & 12 & 1 & 10 & B & 10 & $\mathbf{B}$ & 10 & $\mathrm{NR}$ & NA \\
\hline 4-METHYL - 2-PENTANONE & (UG/L) & 12 & 1 & 2.0 & $\mathrm{BJ}$ & 2.0 & $\mathrm{BJ}$ & 2.0 & $\mathrm{NR}$ & NA \\
\hline ACETONE & (UG/L) & 12 & 1 & 5.0 & $\mathrm{BJ}$ & 5.0 & $\mathrm{BJ}$ & 5.0 & NR & NA \\
\hline METHYLENE CHLORIDE & (UG/L) & 12 & 7 & 3.0 & $\mathrm{BJ}$ & 0.60 & $\mathbf{J}$ & 1.5 & $\mathrm{NR}$ & NA \\
\hline
\end{tabular}


Table 7.38. Constituents in groundwater at the Y-12 Plant site

$\mathrm{HGR}=\mathrm{EF}$ area $=$ Grid $\mathrm{K} 2$

\begin{tabular}{|c|c|c|c|c|c|c|c|c|c|c|}
\hline & \multicolumn{3}{|l|}{ VARIABLE } & $\begin{array}{c}\# \\
\text { SAMPLES } \\
16\end{array}$ & $\begin{array}{c}\stackrel{\#}{\text { DETECTED }} \\
16\end{array}$ & $\begin{array}{c}\text { MAXIMUM } \\
\text { DETECTED } \\
\text { MMT. }\end{array}$ & $\begin{array}{c}\text { MINIMUM } \\
\text { DETECTED } \\
\text { MMT. }\end{array}$ & $\begin{array}{c}\text { AVERAGE } \\
\begin{array}{c}\text { DETECTED } \\
\text { MMTS. }\end{array} \\
12\end{array}$ & $\begin{array}{c}\text { REFERENCE } \\
\text { VALUE }\end{array}$ & $\begin{array}{c}\text { \# MMTS. > } \\
\text { REFERENCE } \\
0\end{array}$ \\
\hline FLUORIDE & & & (MG/L) & 16 & 8 & 0.20 & 0.10 & 0.15 & 2.000 & 0 \\
\hline NITRATE NI & DGEN & & (MG/L) & 16 & 2 & 7.1 & 0.45 & 3.8 & 10.000 & 0 \\
\hline SULFATE & & & (MG/L) & 16 & 16 & 81 & 17 & 33 & 250.000 & 0 \\
\hline ALUMINUM & FILTERED & ICAP & (MG/L) & 16 & 5 & 0.034 & 0.022 & 0.026 & 0.200 & 0 \\
\hline BARIUM & & ICAP & (MG/L) & 16 & 16 & 0.81 & 0.12 & 0.31 & 1.000 & 0 \\
\hline BARIUM & FILTERED & ICAP & (MG/L) & 16 & 16 & 0.79 & 0.11 & 0.30 & 1.000 & 0 \\
\hline BORON & & ICAP & (MG/L) & 16 & 16 & 0.18 & 0.019 & 0.056 & NR & NA \\
\hline BORON & FILTERED & ICAP & (MG/L) & 16 & 16 & 0.10 & 0.010 & 0.057 & NR & NA \\
\hline CALCIUM & & ICAP & (MG/L) & 16 & 16 & 110 & 51 & 77 & $\mathrm{NR}$ & NA \\
\hline CALCIUM & FILTERED & ICAP & (MG/L) & 16 & 16 & 110 & 53 & 77 & NR & NA \\
\hline CHROMIUM & & AAS & (MG/L) & 16 & 1 & 0.014 & 0.014 & 0.014 & 0.050 & 0 \\
\hline CHROMIUM & & ICAP & (MG/L) & 16 & 1 & 0.014 & 0.014 & 0.014 & 0.050 & 0 \\
\hline COBALT & & ICAP & (MG/L) & 16 & 2 & 0.0060 & 0.0058 & 0.0059 & NR & NA \\
\hline COPPER & & ICAP & (MG/L) & 16 & 8 & 0.041 & 0.0060 & 0.013 & 1.000 & 0 \\
\hline COPPER & FILTERED & ICAP & (MG/L) & 16 & 6 & 0.018 & 0.0048 & 0.0083 & 1.000 & 0 \\
\hline IRON & & ICAP & (MG/L) & 16 & 16 & 5.1 & 0.11 & 0.99 & 0.300 & 9 \\
\hline IRON & FILTERED & ICAP & (MG/L) & 16 & 11 & 0.26 & 0.012 & 0.14 & 0.300 & 0 \\
\hline MAGNESIUM & & ICAP & (MG/L) & 16 & 16 & 12 & 7.7 & 10 & NR & NA \\
\hline MAGNESIUM & FILTERED & ICAP & (MG/L) & 16 & 16 & 13 & 7.6 & 10 & NR & NA \\
\hline MANGANESE & & ICAP & (MG/L) & 16 & 16 & 1.4 & 0.013 & 0.27 & 0.050 & 9 \\
\hline MANGANESE & FILTERED & ICAP & (MG/L) & 16 & 16 & 0.93 & 0.012 & 0.20 & 0.050 & 6 \\
\hline POTASSIUM & & ICAP & (MG/L) & 16 & 16 & 5.5 & 0.95 & 2.8 & NR & $\mathrm{NA}$ \\
\hline POTASSIUM & FILTERED & ICAP & (MG/L) & 16 & 16 & 5.7 & 0.74 & 2.6 & NR & NA \\
\hline SELENIUM & & ICAP & (MG/L) & 16 & 2 & 0.056 & 0.053 & 0.055 & 0.050 & 2 \\
\hline SELENIUM & FILTERED & ICAP & (MG/L) & 16 & 2 & 0.067 & 0.053 & 0.060 & 0.050 & 2 \\
\hline SODIUM & & ICAP & (MG/L) & 16 & 16 & 30 & 4.6 & 13 & NR & NA \\
\hline SODIUM & FILTERED & ICAP & (MG/L) & 16 & 16 & 30 & 4.6 & 13 & $\mathrm{NR}$ & NA \\
\hline
\end{tabular}


Table 7.38 (continued)

$\begin{array}{ccccc}\text { MAXIMUM } & \text { MINIMUM } & \text { AVERAGE } & & \\ \text { DETECTED } & \text { DETECTED } & \text { DETECTED } & \text { REFERENCE } & \text { \# MMTS. > } \\ \text { MMT. } & \text { MMT. } & \text { MMTS. } & \text { VALUE } & \text { REFERENCE }\end{array}$

VARIABLE

$\begin{array}{ccc}* & & \text { MAXIMUM } \\ * & \# & \text { DETECTED } \\ \text { SAMPLES } & \text { DETECTED } & \text { MMT. }\end{array}$

MMTS.

.

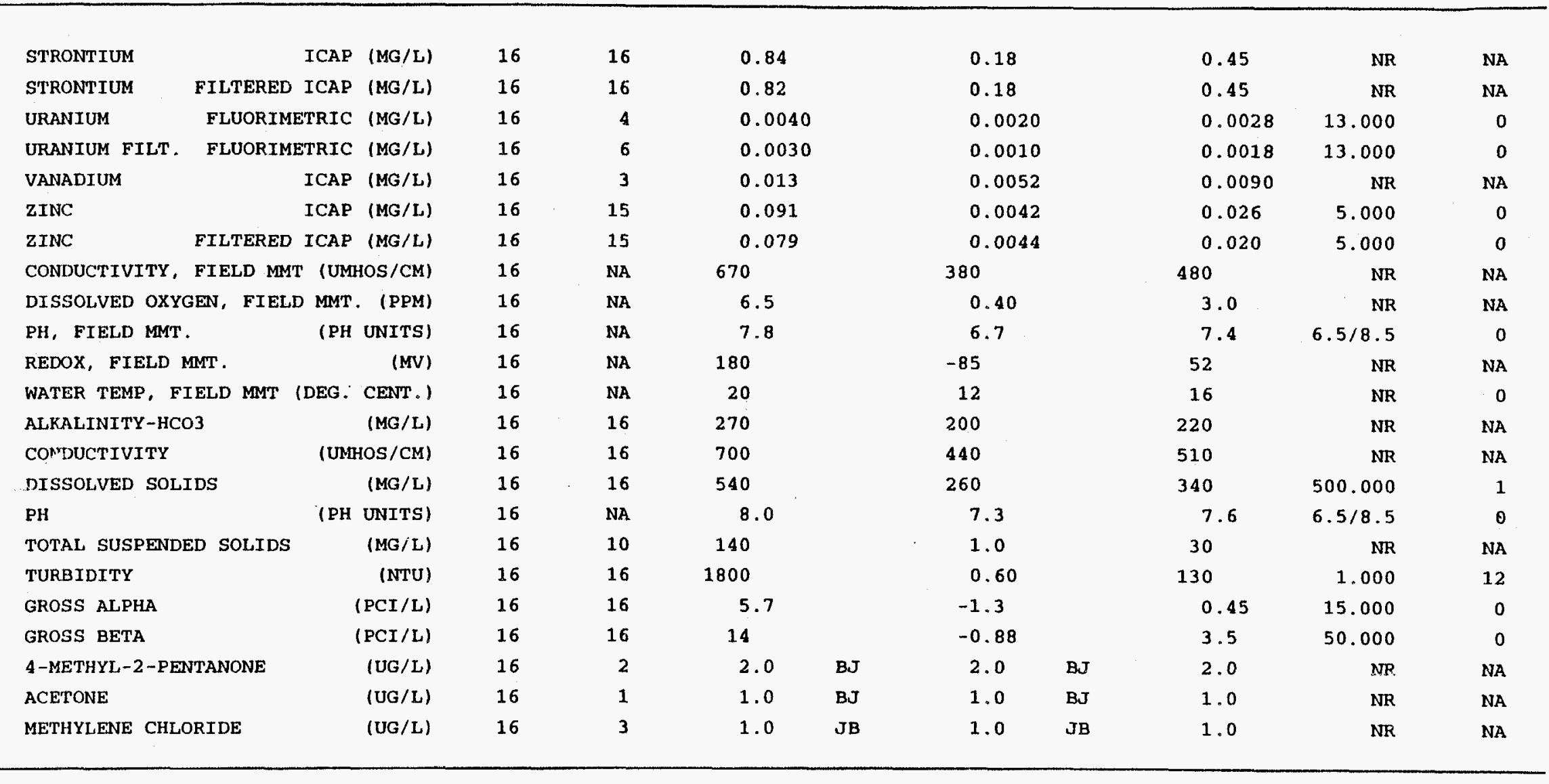


Table 7.39. Constituents In groundwater at the Y-12 Plant site HGR=EF area $=$ New Hope Pond

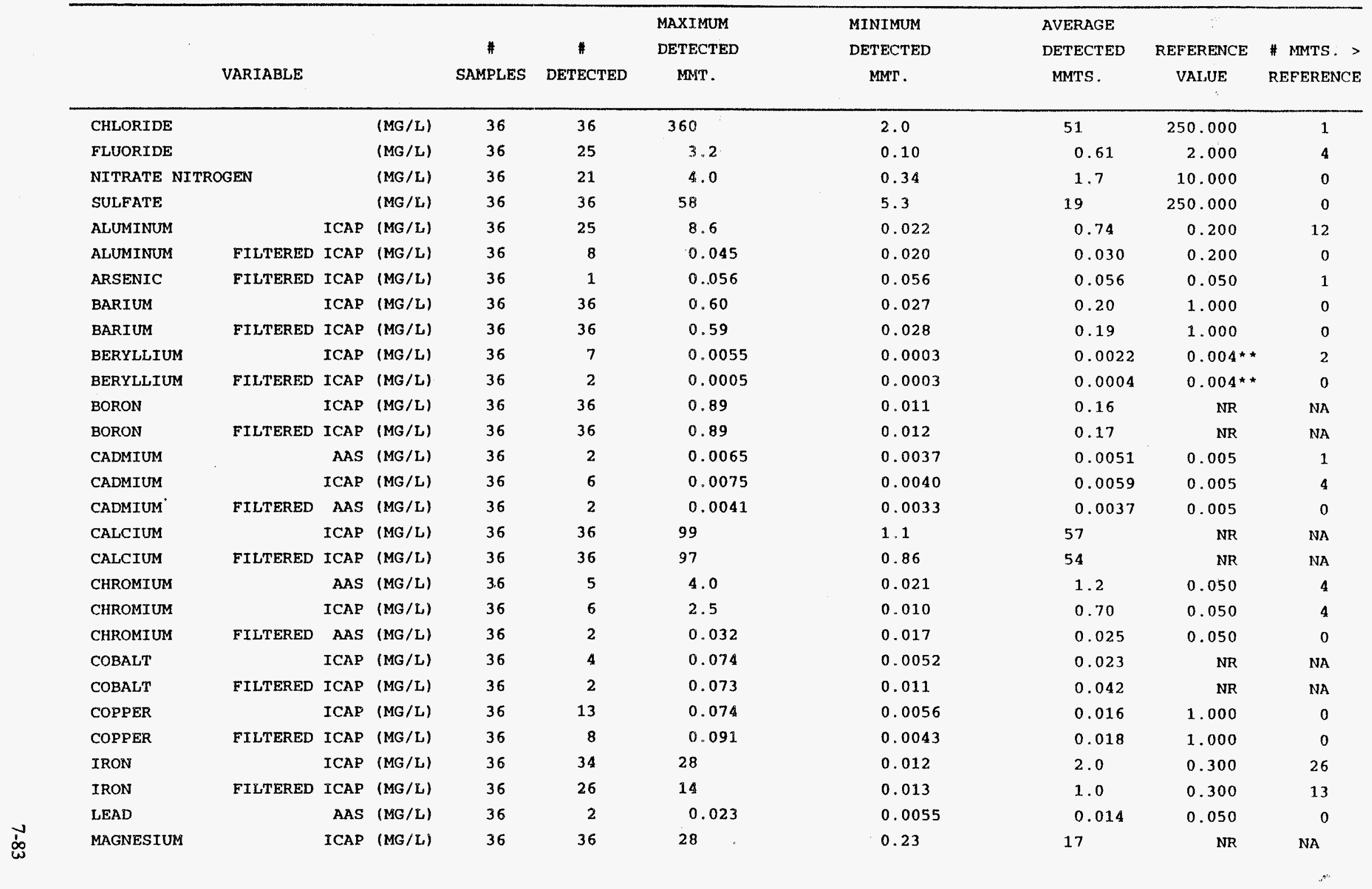


Table 7.39 (continued)

\begin{tabular}{|c|c|c|c|c|c|c|c|c|c|c|}
\hline & VARIABLE & & & $\begin{array}{c}\# \\
\text { SAMPLES }\end{array}$ & $\begin{array}{c}* \\
\text { DETECTED }\end{array}$ & $\begin{array}{l}\text { MAXIMUM } \\
\text { DETECTED } \\
\text { MMT. }\end{array}$ & $\begin{array}{l}\text { MINIMUM } \\
\text { DETECTED } \\
\text { MMT. }\end{array}$ & $\begin{array}{l}\text { AVERAGE } \\
\text { DETECTED } \\
\text { MMTS. }\end{array}$ & $\begin{array}{l}\text { REFERENCE } \\
\text { VALUE }\end{array}$ & $\begin{array}{l}\text { \# MMTS. > } \\
\text { REFERENCE }\end{array}$ \\
\hline MAGNESIUM & FILTERED & ICAP & $(M G / L)$ & 36 & 36 & 28 & 0.20 & 16 & $\mathrm{NR}$ & $\mathrm{NA}$ \\
\hline MANGANESE & & ICAP & $(M G / L)$ & 36 & 33 & 0.81 & 0.0017 & 0.15 & 0.050 & 17 \\
\hline MANGANESE & FILTERED & ICAP & $(M G / L)$ & 36 & 31 & 1.0 & 0.0011 & 0.15 & 0.050 & 14 \\
\hline MER.CURY & & CVAA & $(M G / L)$ & 36 & 1 & 0.0004 & 0.0004 & 0.0004 & 0.002 & 0 \\
\hline MOLYBDENUM & & ICAP & (MG/L) & 36 & 2 & 0.011 & 0.010 & 0.011 & NR & NA \\
\hline NICKEL & & ICAP & $(M G / L)$ & 36 & 6 & 3.9 & 0.017 & 0.82 & $0.100^{\star \star}$ & 5 \\
\hline NICKEL & FILTERED & ICAP & $(M G / L)$ & 36 & 6 & 4.0 & 0.015 & 0.80 & $0.100 * *$ & 3 \\
\hline POTASSIUM & & ICAP & $(M G / L)$ & 36 & 36 & 5.2 & 0.86 & 2.3 & NR & NA \\
\hline POTASSIUM & FILTERED & ICAP & $(M G / L)$ & 36 & 36 & 11 & 0.70 & 2.3 & NR & NA \\
\hline SELENIUM & FILTERED & ICAP & $(M G / L)$ & 36 & 2 & 0.072 & 0.063 & 0.068 & 0.050 & 2 \\
\hline SODIUM & & ICAP & $(M G / L)$ & 36 & 36 & 180 & 3.7 & 43 & $\mathrm{NR}$ & NA \\
\hline SODIUM & FILTERED & ICAP & $(M G / L)$ & 36 & 36 & 270 & 3.5 & 51 & NR & NA \\
\hline STRONTIUM & & ICAP & $(M G / L)$ & 36 & 36 & 0.58 & 0.032 & 0.29 & NR & NA \\
\hline STRONTIUM & FILTERED & ICAP & $(M G / L)$ & 36 & 36 & 0.58 & 0.029 & 0.27 & NR & NA \\
\hline URANIUM & FLUORIME & ETRIC & $(M G / L)$ & 36 & 14 & 0.0070 & 0.0010 & 0.0023 & 13.000 & 0 \\
\hline URANIUM FILT. & FLUORIME & ETRIC & (MG/L) & 36 & 15 & $0: 0050$ & 0.0010 & 0.0019 & 13.000 & 0 \\
\hline VANADIUM & & ICAP & $(M G / L)$ & 36 & 4 & 0.011 & 0.0067 & 0.0080 & NR & $\mathrm{NA}$ \\
\hline zINC & & ICAP & $(M G / L)$ & 36 & 35 & 0.031 & 0.0021 & 0.0099 & 5.000 & 0 \\
\hline ZINC & FILTERED & ICAP & (MG/L) & 36 & 31 & 0.18 & 0.0023 & 0.015 & 5.000 & 0 \\
\hline CONDUCTIVITY, & FIELD MMT & T (UMH & HOS/CM) & 36 & NA & 940 & 400 & 540 & NR & NA \\
\hline DISSOLVED OXY & YGEN, FIELI & D мMт. & . (PPM) & 36 & NA & 11 & 0.20 & 2.9 & NR & NA \\
\hline PH， FIELD MMT & & $(\mathrm{PH}$ & UNITS) & 36 & $\mathrm{NA}$ & 9.1 & 5.6 & 7.4 & $6.5 / 8.5$ & 5 \\
\hline REDOX, FIELD & MMT. & & (MV) & 36 & NA & 210 & -110 & 61 & NR & NA \\
\hline WATER TEMP, $F$ & IELD MMT & (DEG. & (ENT.) & 36 & $\mathrm{NA}$ & 23 & 13 & 16 & $\mathrm{NR}$ & 0 \\
\hline ALKALINITY-CO & & & $(M G / L)$ & 36 & 4 & 54 & 46 & 50 & NR & NA \\
\hline ALKALINITY-HC & & & $(M G / L)$ & 36 & 36 & 330 & 78 & 220 & NR & NA \\
\hline CONDUCTIVITY & & (UMH & HOS/CM) & 36 & 36 & 1200 & 480 & 610 & NR & NA \\
\hline DISSOLVED SOL & IDS & & $(\mathrm{MG} / \mathrm{L})$ & 36 & 36 & 590 & 260 & 360 & 500.000 & 1 \\
\hline $\mathrm{PH}$ & & $(\mathrm{PH}$ & UNITS) & 36 & $\mathrm{NA}$ & 9.2 & 6.7 & 7.7 & $6.5 / 8.5$ & 4 \\
\hline TOTAL SUSPEND & DED SOLIDS & & (MG/L) & 36 & 30 & 410 & 1.0 & 26 & NR & NA \\
\hline
\end{tabular}


Table 7.39 (continued)

\begin{tabular}{|c|c|c|c|c|c|c|c|c|c|c|}
\hline VARIABLE & & $\begin{array}{c}\# \\
\text { SAMPLES }\end{array}$ & $\begin{array}{c}\# \\
\text { DETECTED }\end{array}$ & $\begin{array}{l}\text { MAXIMUM } \\
\text { DETECTED } \\
\text { MMT. }\end{array}$ & & $\begin{array}{l}\text { MINIMUM } \\
\text { DETECTED } \\
\text { MMT. }\end{array}$ & & $\begin{array}{l}\text { AVERAGE } \\
\text { DETECTED } \\
\text { MMTS. }\end{array}$ & $\begin{array}{c}\text { REFERENCE } \\
\text { VALUE }\end{array}$ & $\begin{array}{l}\# \text { MMTS. > } \\
\text { REFERENCE }\end{array}$ \\
\hline TURBIDITY & (NTU) & 36 & 36 & 280 & & 0.50 & & 18 & 1.000 & 33 \\
\hline GROSS ALPHA & (PCI/L) & 36 & 36 & 3.9 & & -4.9 & & 0.59 & 15.000 & 0 \\
\hline GROSS BETA & (PCI/L) & 36 & 36 & 19 & & -8.5 & & 2.0 & 50.000 & 0 \\
\hline 1,2-DICHLOROETHENE & (UG/L) & 36 & 5 & 84 & & 7.0 & & 61 & 70.000 & 4 \\
\hline 4-METHYL-2-PENTANONE & $(U G / L)$ & 36 & 6 & 78 & $\mathrm{BJ}$ & 2.0 & $\mathrm{BJ}$ & 15 & NR & NA \\
\hline ACETONE & (UG/L) & 36 & 4 & 52 & $\mathbf{J}$ & 1.0 & $\mathbf{J}$ & 14 & NR & NA \\
\hline CARBON TETRACHLORIDE & $(U G / L)$ & 36 & 22 & 7000 & & 3.0 & $\mathrm{~J}$ & 2100 & 5.000 & 20 \\
\hline CHLOROFORM & $(U G / L)$ & 36 & 21 & 950 & & 0.80 & $\mathrm{~J}$ & 190 & 100.000 & 8 \\
\hline METHYLENE CHLORIDE & (UG/L) & 36 & 7 & 80 & JB & 1.0 & $\mathrm{JB}$ & 17 & NR & NA \\
\hline TETRACHLOROETHENE & (UG/L) & 36 & 18 & 400 & & 0.60 & $\mathrm{~J}$ & 120 & 5.000 & 16 \\
\hline TRICHLOROETHENE & (UG/L) & 36 & 13 & 130 & & 2.0 & $J$ & 35 & 5.000 & 8 \\
\hline
\end{tabular}


Table 7.40. Constituents in groundwater at the $\mathrm{Y}-12$ Plant site

HGR=EF area=S2 Site

\begin{tabular}{|c|c|c|c|c|c|c|c|c|c|c|}
\hline \multicolumn{4}{|c|}{ VARIABLE } & \multirow{2}{*}{$\begin{array}{c}\# \\
\text { SAMPLES } \\
12\end{array}$} & \multirow{2}{*}{$\begin{array}{c}\# \\
\text { DETECTED } \\
12\end{array}$} & \multirow{2}{*}{$\begin{array}{c}\text { MAXIMUM } \\
\text { DETECTED } \\
\text { MMT. }\end{array}$} & \multirow{2}{*}{$\begin{array}{c}\begin{array}{c}\text { MINIMUM } \\
\text { DETECTED } \\
\text { MMT. }\end{array} \\
1.0\end{array}$} & \multirow{2}{*}{$\begin{array}{c}\begin{array}{c}\text { AVERAGE } \\
\text { DETECTED } \\
\text { MMTS. }\end{array} \\
5.6\end{array}$} & \multirow{2}{*}{$\begin{array}{c}\text { REFERENCE } \\
\text { VALUE } \\
250.000\end{array}$} & \multirow{2}{*}{$\begin{array}{c}\text { \# MMTS. > } \\
\text { REFERENCE } \\
0\end{array}$} \\
\hline CHLORIDE & & & (MG/L) & & & & & & & \\
\hline FLUORIDE & & & (MG/L) & 12 & 6 & 1.6 & 0.20 & 1.0 & 2.000 & 0 \\
\hline NITRATE NI & OGEN & & $(M G / L)$ & 12 & 12 & 240 & 0.47 & 39 & 10.000 & 4 \\
\hline SULFATE & & & (MG/L) & 12 & 12 & 22 & 1.0 & 11 & 250.000 & 0 \\
\hline ALUMINUM & & ICAP & (MG/L) & 12 & 12 & 29 & 0.27 & 7.6 & 0.200 & 12 \\
\hline ALUMINUM & FILTERED & ICAP & (MG/L) & 12 & 6 & 0.43 & 0.021 & 0.14 & 0.200 & 2 \\
\hline BARIUM & & ICAP & (MG/L) & 12 & 12 & 0.22 & 0.010 & 0.082 & 1.000 & 0 \\
\hline BARIUM & FILTERED & ICAP & (MG/L) & 12 & 12 & 0.15 & 0.0094 & 0.052 & 1.000 & 0 \\
\hline BERYLLIUM & & ICAP & $(M G / L)$ & 12 & 8 & 0.0033 & 0.0003 & 0.0014 & $0.004^{\star \star}$ & 0 \\
\hline BERYLLIUM & FILTERED & ICAP & (MG/L) & 12 & 2 & 0.0019 & 0.0005 & 0.0012 & $0.004^{* *}$ & 0 \\
\hline BORON & & ICAP & (MG/L) & 12 & 12 & 0.084 & 0.012 & 0.031 & NR & NA \\
\hline BORON & FILTERED & ICAP & $(M G / L)$ & 12 & 12 & 0.084 & 0.0045 & 0.027 & NR & NA \\
\hline CADMIUM & & AAS & (MG/L) & 12 & 6 & 0.20 & 0.0023 & 0.088 & 0.005 & 4 \\
\hline CADMIUM & & ICAP & (MG/L) & 12 & 5 & 0.18 & 0.0077 & 0.10 & 0.005 & 5 \\
\hline CADMIUM & FILTERED & AAS & (MG/L) & 12 & 4 & 0.20 & 0.072 & 0.12 & 0.005 & 4 \\
\hline CALCIUM & & ICAP & (MG/L) & 12 & 12 & 120 & 7.2 & 62 & NR & NA \\
\hline CALCTUM & FILTERED & ICAP & (MG/L) & 12 & 12 & 130 & 5.0 & 62 & NR & NA \\
\hline CHROMIUM & & AAS & $(M G / L)$ & 12 & 9 & 0.050 & 0.011 & 0.025 & 0.050 & 0 \\
\hline CHROMIUM & & ICAP & (MG/L) & 12 & 7 & 0.042 & 0.011 & 0.024 & 0.050 & 0 \\
\hline CHROMIUM & FILTERED & AAS & (MG/L) & 12 & 1 & 0.010 & 0.010 & 0.010 & 0.050 & 0 \\
\hline COBALT & & ICAP & $(M G / L)$ & 12 & 7 & 0.039 & 0.0055 & 0.022 & $\mathrm{NR}$ & NA \\
\hline COBALT & FILTERED & ICAP & $(M G / L)$ & 12 & 4 & 0.036 & 0.0063 & 0.022 & NR & $\mathrm{NA}$ \\
\hline COPPER & & ICAP & (MG/L) & 12 & 9 & 0.95 & 0.0059 & 0.31 & 1.000 & 0 \\
\hline COPPER & FILTERED & ICAP & (MG/L) & 12 & 5 & 0.46 & 0.0044 & 0.24 & 1.000 & 0 \\
\hline IRON & & ICAP & (MG/L) & 12 & 12 & 39 & 0.40 & 9.7 & 0.300 & 12 \\
\hline IRON & FILTERED & ICAP & (MG/L) & 12 & 5 & 0.26 & 0.0055 & 0.089 & 0.300 & 0 \\
\hline LEAD & & AAS & $(M G / L)$ & 12 & 10 & 0.077 & 0.0056 & 0.033 & 0.050 & 3 \\
\hline LEAD & & ICAP & (MG/L) & 6 & 3 & 0.075 & 0.051 & 0.060 & 0.050 & 3 \\
\hline MAGNESIUM & & ICAP & $(M G / L)$ & 12 & 12 & 43 & 3.9 & 22 & NR & NA \\
\hline
\end{tabular}


Table 7.40 (continued)

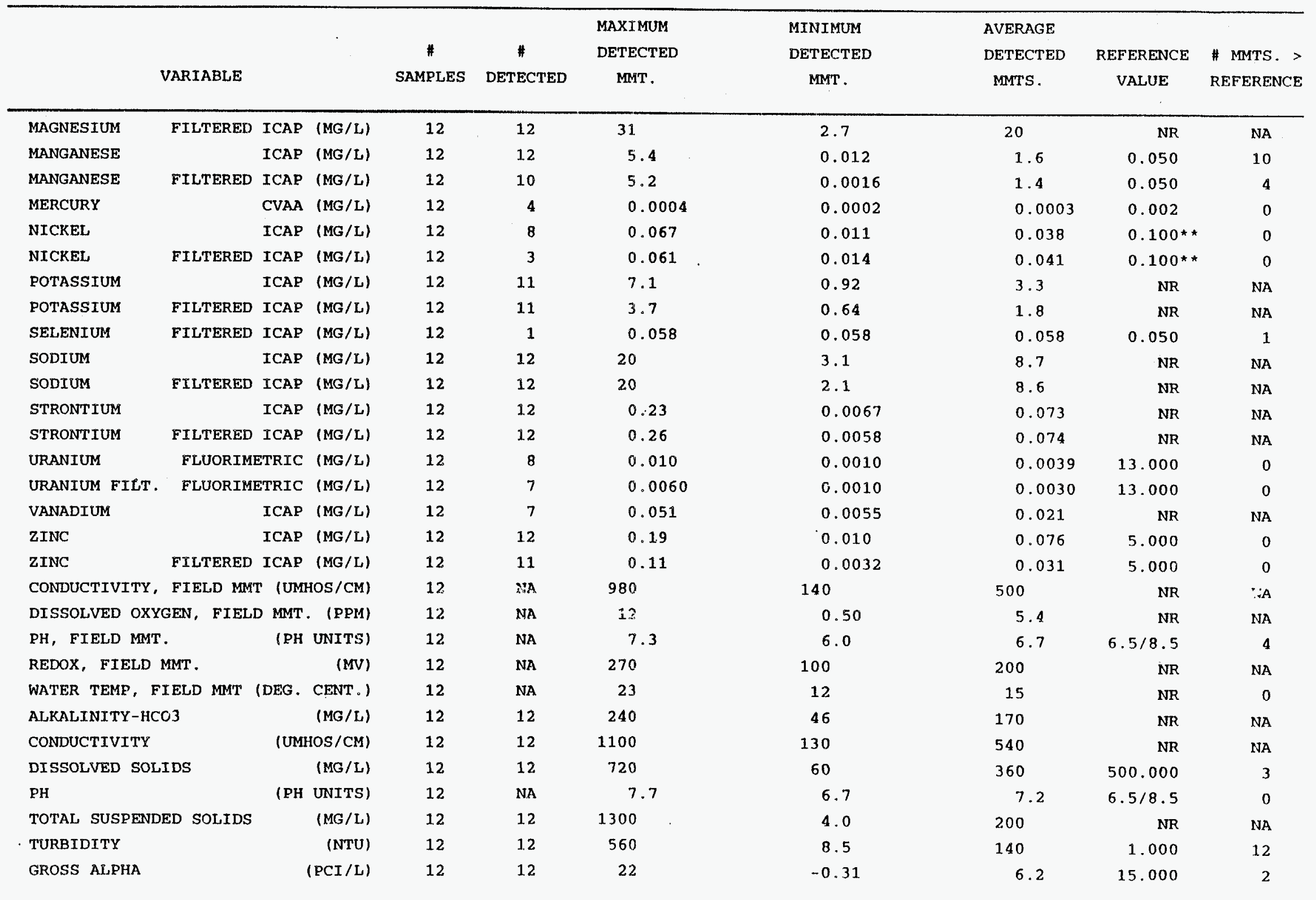


Table 7.40 (continued)

$\vec{d}$

\begin{tabular}{|c|c|c|c|c|c|c|c|c|c|c|}
\hline VARIABLE & & $\begin{array}{c}\# \\
\text { SAMPLES }\end{array}$ & $\begin{array}{c}\# \\
\text { DETECTED }\end{array}$ & $\begin{array}{l}\text { MAXIMUM } \\
\text { DETECTED } \\
\text { MMT. }\end{array}$ & & $\begin{array}{l}\text { MINIMUM } \\
\text { DETECTED } \\
\text { MMT. }\end{array}$ & & $\begin{array}{l}\text { AVERAGE } \\
\text { DETECTED } \\
\text { MMTS. }\end{array}$ & $\begin{array}{c}\text { REFERENCE } \\
\text { VALUE }\end{array}$ & $\begin{array}{l}\text { \# MMTS. > } \\
\text { REFERENCE }\end{array}$ \\
\hline GROSS BETA & $(\mathrm{PCI} / \mathrm{L})$ & 12 & 12 & 19 & & -1.1 & & 5.3 & 50.000 & 0 \\
\hline 1,2-DICHLOROETHENE & (UG/L) & 12 & 1 & 7.0 & & 7.0 & & 7.0 & 70.000 & 0 \\
\hline 2-BUTANONE & (UG/L) & 12 & 1 & 8.0 & $\mathrm{BJ}$ & 8.0 & $\mathrm{BJ}$ & 8.0 & NR & $\mathrm{NA}$ \\
\hline 4-METHYL-2-PENTANONE & (UG/L) & 12 & 3 & 1.0 & $\mathrm{BJ}$ & 1.0 & $\mathrm{BJ}$ & 1.0 & NR & NA \\
\hline ACETONE & (UG/L) & 12 & 2 & 11 & & 6.0 & $\mathrm{BJ}$ & 8.5 & NR & NA \\
\hline CARBON TETRACHLORIDE & (UG/L) & 12 & 3 & 11 & & 1.0 & $\mathfrak{J}$ & 4.7 & 5.000 & 1 \\
\hline CHLOROFORM & (UG/L) & 12 & 8 & 8.0 & & 1.0 & $\mathrm{~J}$ & 3.0 & 100.000 & 0 \\
\hline METHYLENE CHLORIDE & (UG/L) & 12 & 4 & 2.0 & JB & 1.0 & $J B$ & 1.8 & NR & NA \\
\hline TETRACHLOROETHENE & (UG/L) & 12 & 4 & 210 & $\mathbf{E}$ & 24 & & 78 & 5.000 & 4 \\
\hline TRICHLOROETHENE & (UG/L) & 12 & 4 & 82 & & 7.0 & & 29 & 5.000 & 4 \\
\hline
\end{tabular}


Table 7.41. Constituents In groundwater at the $Y-12$ Plant slte HGR $=E F$ area=Waste Coolant Facilities

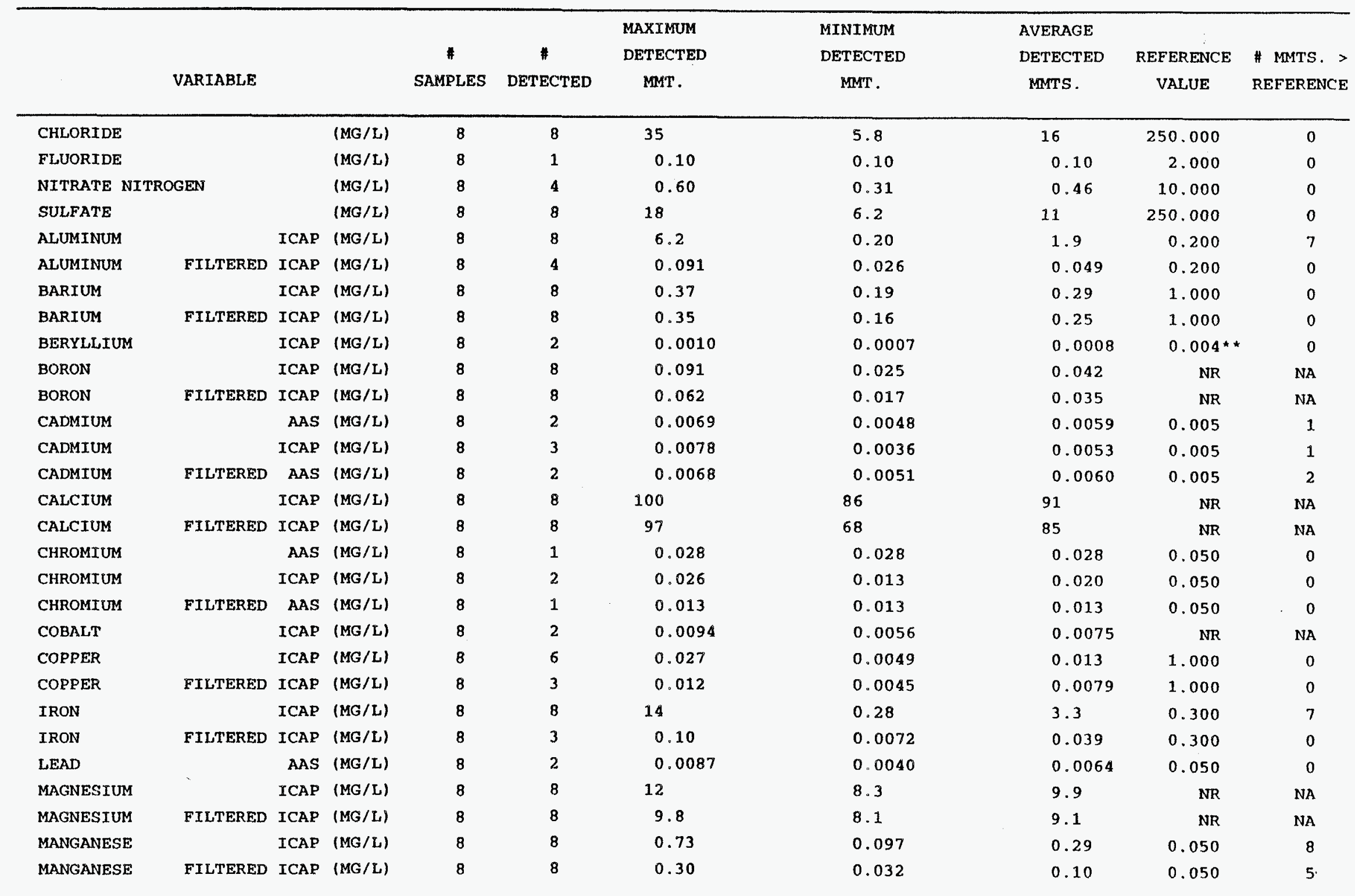


Table 7.41 (continued)

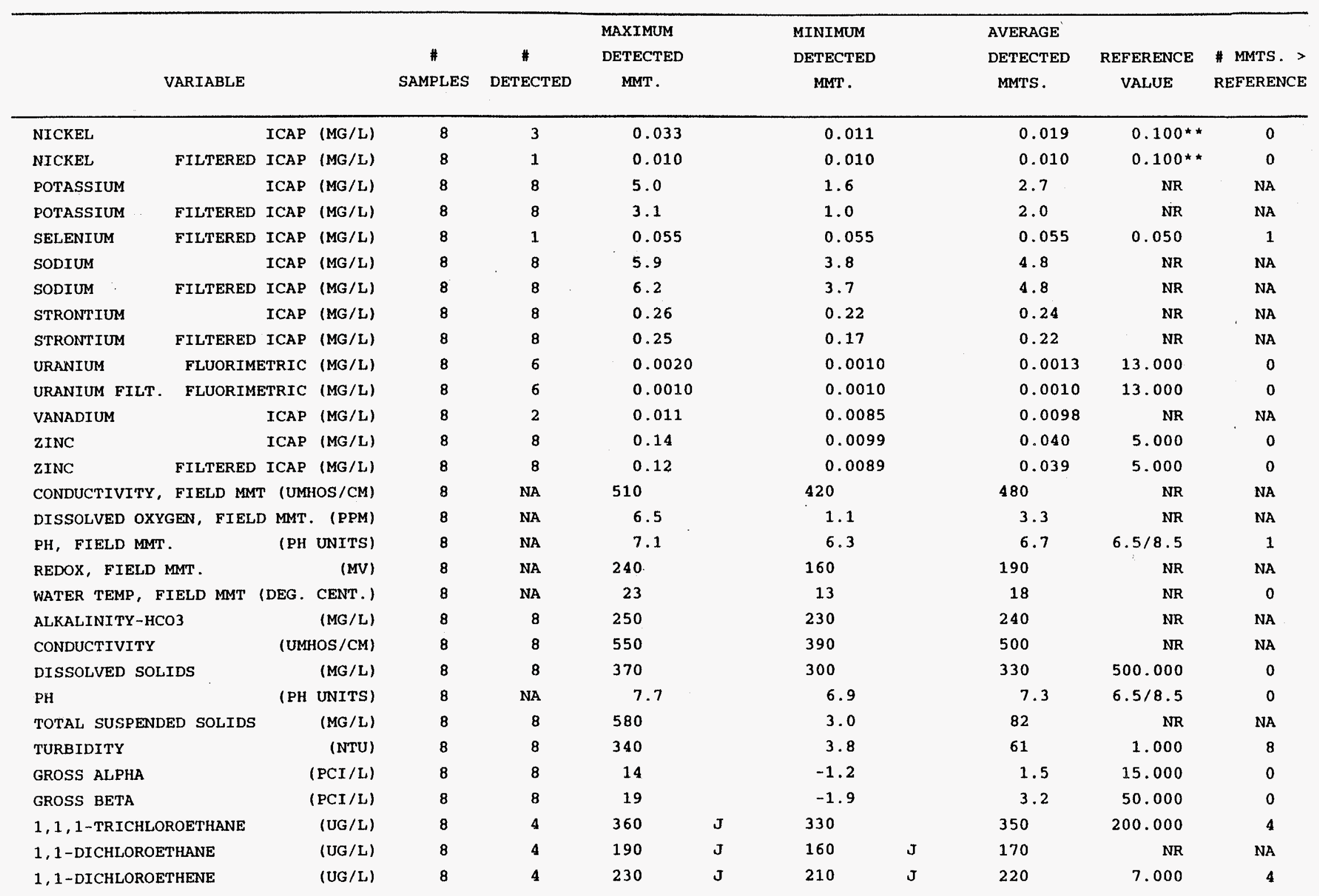




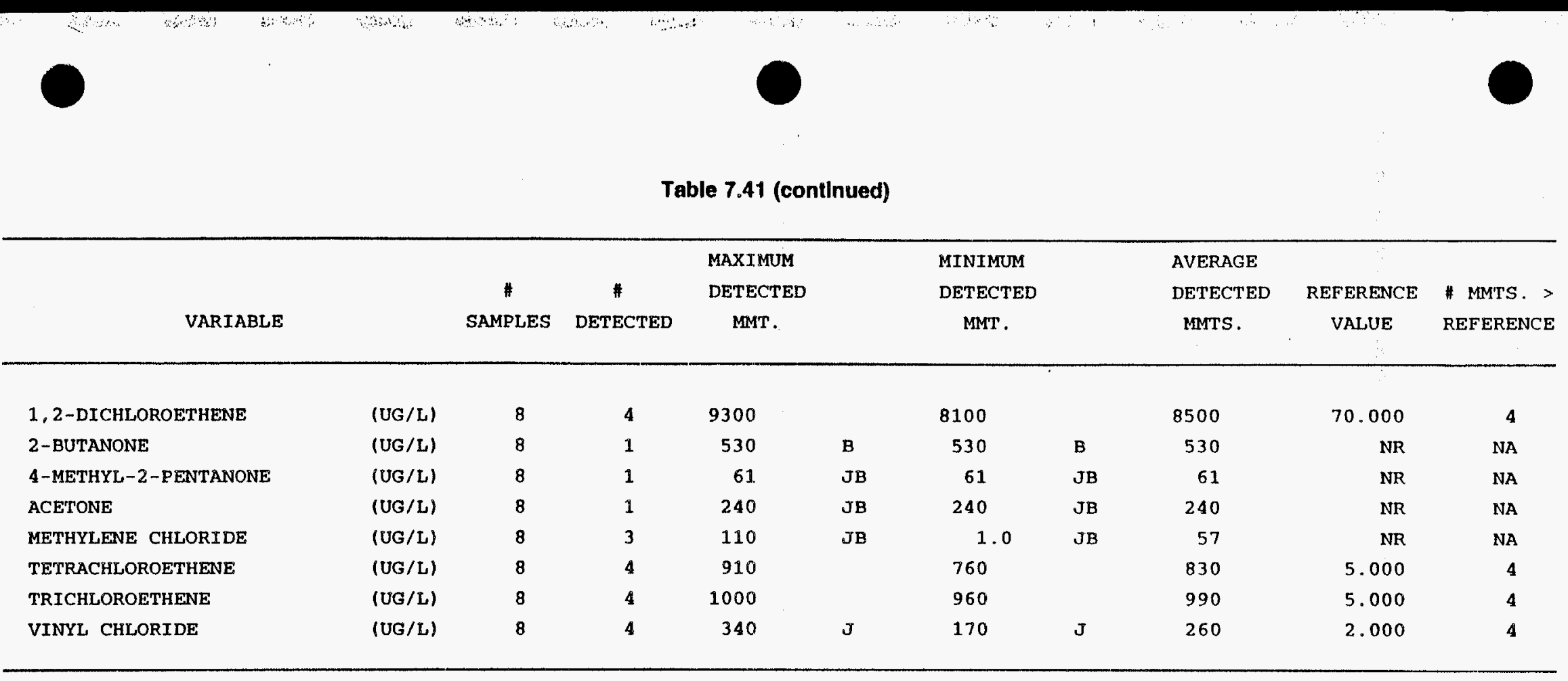


Table 7.42. Constltuents in groundwater at the $Y-12$ Plant site

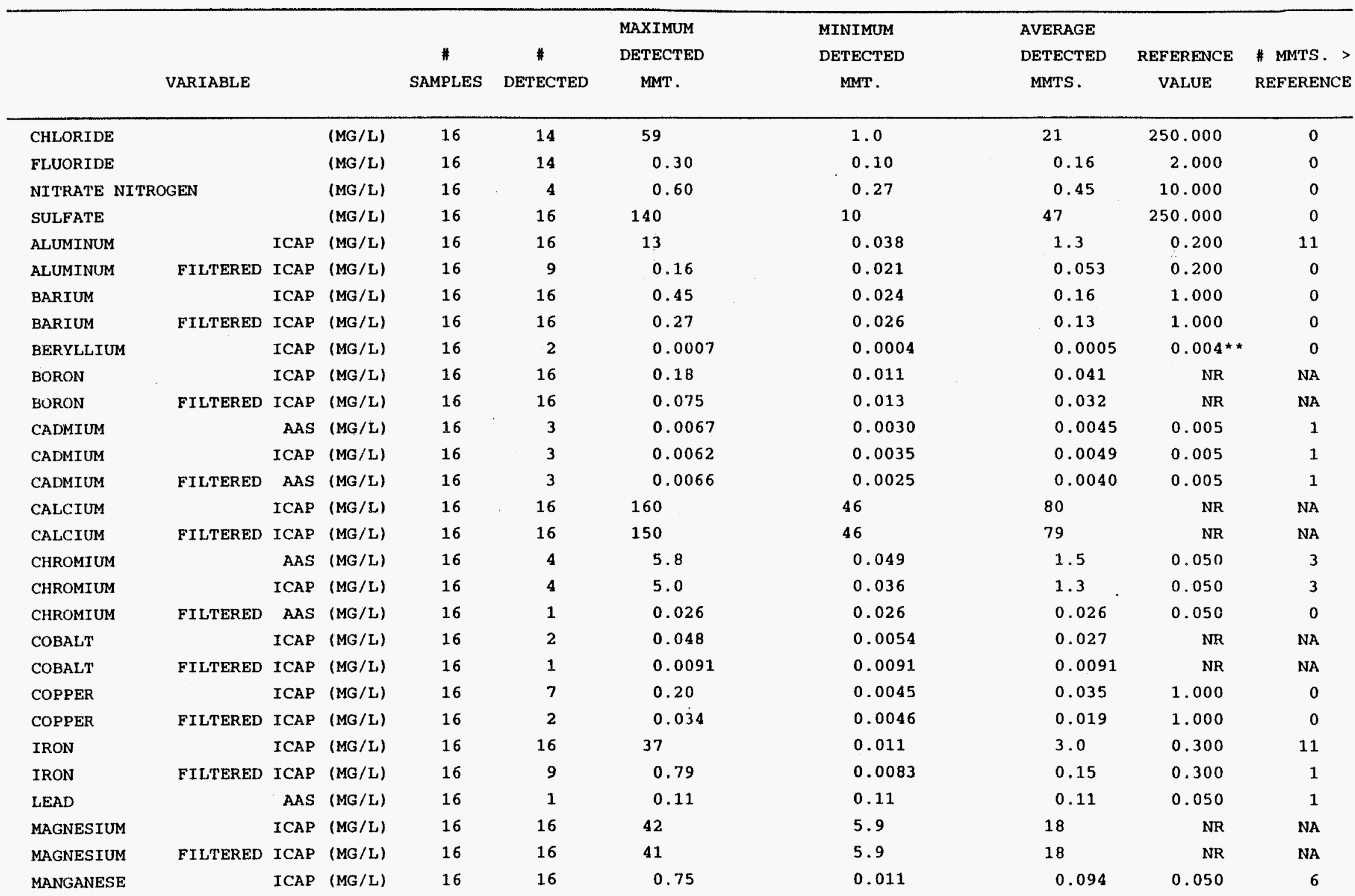


Table 7.42 (continued)

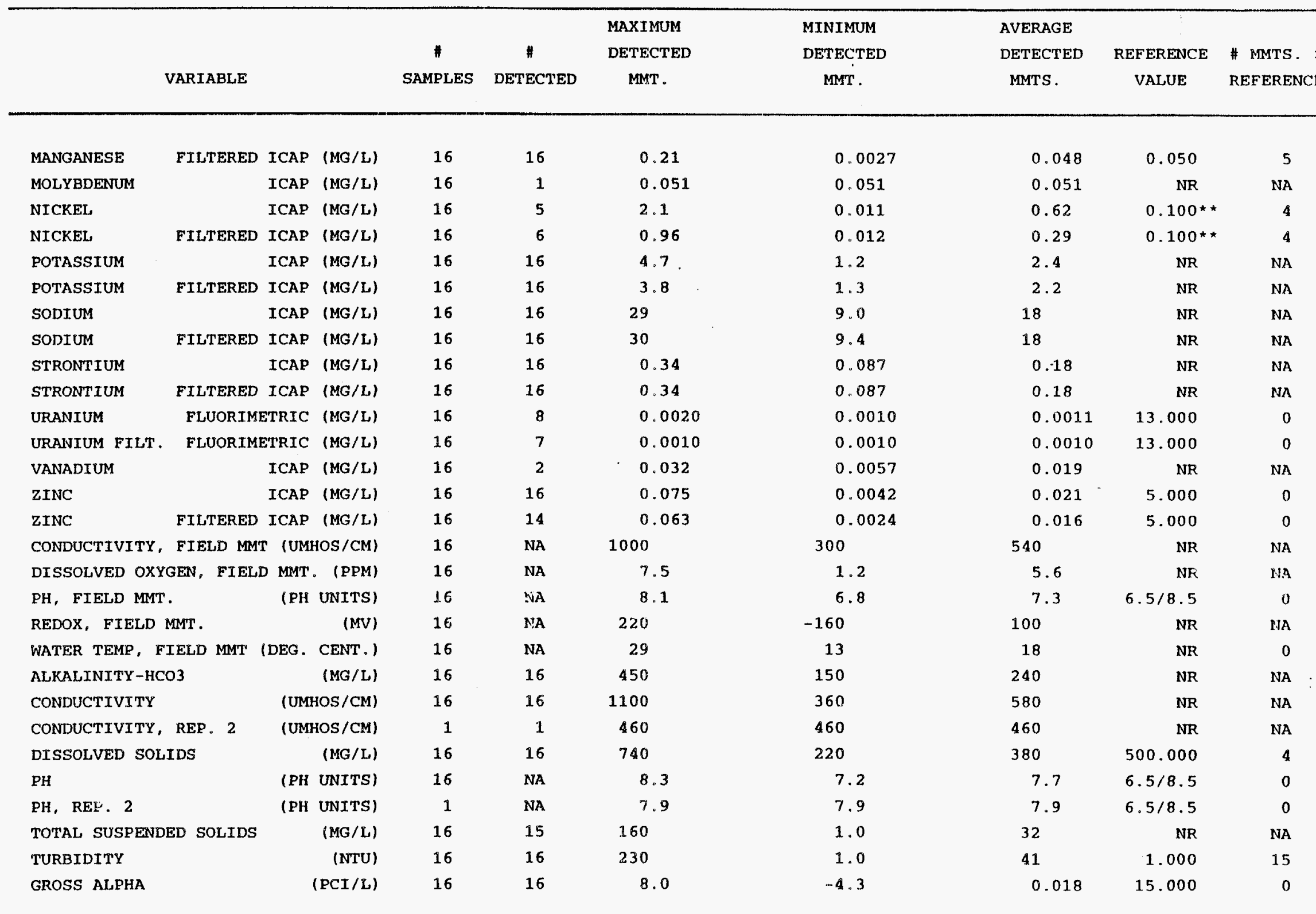


Table 7.42 (contlnued)

\begin{tabular}{|c|c|c|c|c|c|c|c|c|c|c|}
\hline VARIABLE & & $\begin{array}{c}\# \\
\text { SAMPLES }\end{array}$ & $\begin{array}{c}\# \\
\text { DETECTED }\end{array}$ & $\begin{array}{l}\text { MAXIMUM } \\
\text { DETECTED } \\
\text { MMT. }\end{array}$ & & $\begin{array}{l}\text { MINIMUM } \\
\text { DETECTED } \\
\text { MMT. }\end{array}$ & & $\begin{array}{l}\text { AVERAGE } \\
\text { DETECTED } \\
\text { MMTS. }\end{array}$ & $\begin{array}{l}\text { REFERENCE } \\
\text { VALUE }\end{array}$ & $\begin{array}{l}\text { \# MMTS. > } \\
\text { REFERENCE }\end{array}$ \\
\hline GROSS BETA & $(\mathrm{PCI} / \mathrm{L})$ & 16 & 16 & 39 & & -4.9 & & 5.1 & 50.000 & 0 \\
\hline 4-METHYL-2-PENTANONE & $(\mathrm{UG} / \mathrm{L})$ & 16 & 2 & 2.0 & $\mathrm{BJ}$ & 2.0 & BJ & 2.0 & NR & NA \\
\hline ACETONE & (UG/L) & 16 & 1 & 32 & & 32 & & 32 & NR & NA \\
\hline METHYLENE CHLORIDE & (UG/L) & 16 & 6 & 2.0 & $\mathrm{JB}$ & 1.0 & $\mathrm{~s}$ & 1.2 & NR & NA \\
\hline
\end{tabular}


Table 7.43. Constltuents II groundwater at the Y-12 Plant site HGR=UST 1 area=Rust Garage Area

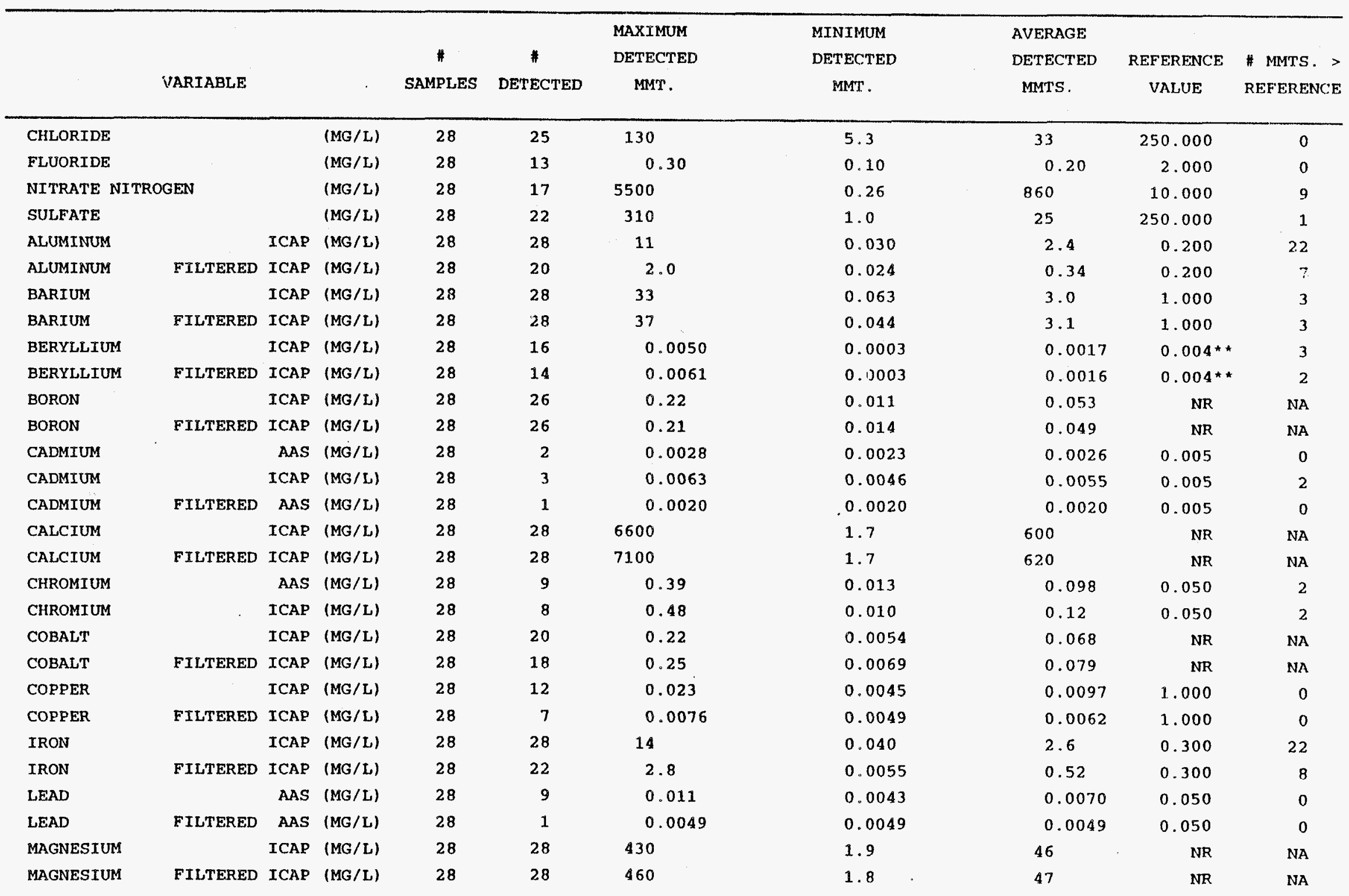


Table 7.43 (continued)

\begin{tabular}{|c|c|c|c|c|c|c|c|c|c|}
\hline VARIABLE & & & $\begin{array}{c}\# \\
\text { SAMPLES }\end{array}$ & $\begin{array}{c}\# \\
\text { DETECTED }\end{array}$ & $\begin{array}{l}\text { MAXIMUM } \\
\text { DETECTED } \\
\text { MMT. }\end{array}$ & $\begin{array}{l}\text { MINIMUM } \\
\text { DETECTED } \\
\text { MMT. }\end{array}$ & $\begin{array}{l}\text { AVERAGE } \\
\text { DETECTED } \\
\text { MMTS. }\end{array}$ & $\begin{array}{c}\text { REFERENCE } \\
\text { VALUE }\end{array}$ & $\begin{array}{l}\text { " MMTS. } \\
\text { REFERENC }\end{array}$ \\
\hline MANGANESE & ICAP & (MG/L) & 28 & 28 & 11 & 0.27 & 3.2 & 0.050 & 28 \\
\hline MANGANESE & ICAP & $(M G / L)$ & 28 & 28 & 13 & 0.022 & 3.2 & 0.050 & 26 \\
\hline MERCURY & CVAA & $(M G / L)$ & 28 & 3 & 0.0002 & 0.0002 & 0.0002 & 0.002 & 0 \\
\hline MOLYBDENUM & ICAP & (MG/L) & 28 & 2 & 0.017 & 0.016 & 0.017 & NR & NA \\
\hline NICKEL & ICAP & (MG/L) & 28 & 21 & 0.12 & 0.012 & 0.047 & $0.100^{* \star}$ & 2 \\
\hline FILTERED & ICAP & (MG/L) & 28 & 23 & 0.28 & 0.010 & 0.049 & $0.100^{* *}$ & 3 \\
\hline POTASSIUM & ICAP & (MG/L) & 28 & 24 & 5.0 & 0.64 & 1.9 & NR & NA \\
\hline POTASSIUM & ICAP & (MG/L) & 28 & 26 & 14 & 0.65 & 2.0 & $\mathrm{NR}$ & NA \\
\hline SILVER & ICAP & (MG/L) & 28 & 2 & 0.0090 & 0.0075 & 0.0083 & 0.100 & 0 \\
\hline FILTERED & ICAP & $(M G / L)$ & 28 & 1 & 0.0072 & 0.0072 & 0.0072 & 0.100 & 0 \\
\hline SODIUM & ICAP & $(M G / L)$ & 28 & 28 & 63 & 3.5 & 15 & $\mathrm{NR}$ & $\mathrm{NA}$ \\
\hline FILTERED & ICAP & $(M G / L)$ & 28 & 28 & 72 & 3.4 & 16 & NR & NA \\
\hline STRONTIUM & ICAP & (MG/L) & 28 & 28 & 15 & 0.011 & 1.4 & NR & $\mathrm{NA}$ \\
\hline STRONTIUM & ICAP & (MG/L) & 28 & 28 & 17 & 0.011 & 1.5 & NR & NA \\
\hline FLUORIME & ETRIC & $(M G / L)$ & 28 & 11 & 0.014 & 0.0010 & 0.0051 & 13.000 & 0 \\
\hline URANIUM FILT. FLUORIME & ETRIC & $(M G / L)$ & 28 & 13 & 0.014 & 0.0010 & 0.0032 & 13.000 & 0 \\
\hline VANADIUM & ICAP & $(M G / L)$ & 28 & 6 & 0.015 & 0.0051 & 0.0086 & NR & NA \\
\hline ZINC & ICAP & $(M G / L)$ & 28 & 26 & 0.23 & 0.016 & 0.063 & 5.000 & 0 \\
\hline FILTERED & ICAP & $(M G / L)$ & 28 & 27 & 0.26 & 0.0031 & 0.067 & 5.000 & 0 \\
\hline CONDUCTIVITY, FIELD MMT & T IUMH & HOS/CM) & 36 & NA & 27000 & 68 & 1800 & NR & NA \\
\hline DISSOLVED OXYGEN, FIELD & D MMT. & . (РPM) & 36 & NA & 7.3 & 0.90 & 3.8 & NR & $\mathrm{NA}$ \\
\hline PH, FIELD MMT. & (PH & UNITS) & 36 & NA & 9.8 & 3.9 & 5.5 & $6.5 / 8.5$ & 33 \\
\hline REDOX, FIELD MMT . & & (MV) & 36 & NA & 380 & 41 & 210 & NR & $\mathrm{NA}$ \\
\hline WATER TEMP，FIELD MMT （ & (DEG. & CENT.) & 36 & NA & 25 & 13 & 19 & $\mathrm{NR}$ & 0 \\
\hline ALKALINITY-HCO3 & & $(M G / L)$ & 28 & 28 & 270 & 2.0 & 73 & NR & $\mathrm{NA}$ \\
\hline CONDUCTIVITY & (UMH & HOS/CM) & 28 & 28 & 24000 & 71 & 2000 & NR & $\mathrm{NA}$ \\
\hline DISSOLVED SOLIDS & & $(\mathrm{MG} / \mathrm{L})$ & 28 & 28 & 26000 & 46 & 2700 & 500.000 & 4 \\
\hline PH & (PH & UNITS) & 28 & NA & 7.5 & 4.5 & 5.7 & $6.5 / 8.5$ & 23 \\
\hline TOT. PETROLEUM HYDROCAR & RBONS & $(M G / L)$ & 28 & 22 & 1400 & 0.0000 & 72 & 1.000 & 12 \\
\hline
\end{tabular}


Table 7.43 (continued)

\begin{tabular}{|c|c|c|c|c|c|c|c|c|c|c|}
\hline VARIABLE & & $\begin{array}{c}\# \\
\text { SAMPLES }\end{array}$ & $\stackrel{*}{\text { DETECTED }}$ & $\begin{array}{l}\text { MAXIMUM } \\
\text { DETECTED } \\
\text { MMT. }\end{array}$ & & $\begin{array}{c}\text { MINIMUM } \\
\text { DETECTED } \\
\text { MMT. }\end{array}$ & & $\begin{array}{l}\text { AVERAGE } \\
\text { DETECT'ED } \\
\text { MMTS. }\end{array}$ & $\begin{array}{c}\text { REFERENCE } \\
\text { VALUE }\end{array}$ & $\begin{array}{l}\# \text { MMTS. > } \\
\text { REFERENCE }\end{array}$ \\
\hline TOTAL SUSPENDED SOLIDS & (MG/L) & 28 & 26 & 220 & & 1.0 & & 41 & NR & NA \\
\hline TURBIDITY & (NTU) & 28 & 28 & 220 & & 1.0 & & 28 & 1.000 & 27 \\
\hline GROSS ALPHA & $(\mathrm{PCI} / \mathrm{L})$ & 28 & 28 & 19 & & -44 & & 2.7 & 15.000 & 3 \\
\hline GROSS BETA & (PCI/L) & 28 & 28 & 580 & & -4.7 & & 42 & 50.000 & 3 \\
\hline 1,1-DTCHLOROETHANE & (UG/L) & 28 & 3 & 170 & & 2.0 & $\mathbf{J}$ & 59 & NR & NA \\
\hline 1,1-DICHLOROETHENE & (UG/L) & 28 & 5 & 5.0 & $\mathbf{J}$ & 0.80 & $\mathfrak{J}$ & 2.0 & 7.000 & 0 \\
\hline 1,2-DICHLOROETHENE & (UG/L) & 28 & 9 & 220 & $\mathbf{E}$ & 1.0 & $\mathfrak{J}$ & 28 & 70.000 & 1 \\
\hline 2-BUTANONE & (UG/L) & 28 & 1 & 7.0 & $\mathrm{JB}$ & 7.0 & $J B$ & 7.0 & NR & NA \\
\hline 4-METHYL-2-PENTANONE & $(U G / L)$ & 28 & 2 & 71 & $\mathbf{J}$ & 2.0 & $\mathrm{~J}$ & 37 & NR & NA \\
\hline BENZENE & (UG/L) & 28 & 20 & 11000 & $\mathbf{E}$ & 1.0 & $\mathrm{~J}$ & 3400 & 5.000 & 15 \\
\hline BROMOFORM & (UG/L) & 28 & 2 & 2.0 & $\mathfrak{J}$ & 1.0 & $\mathrm{~J}$ & 1.5 & 100.000 & 0 \\
\hline CHLOROMETHANE & (UG/L) & 28 & 2 & 160 & $\mathfrak{J}$ & 130 & $\mathfrak{J}$ & 150 & NR & NA \\
\hline ETHYLBENZENE & (UG/L) & 28 & 13 & 3100 & & 1.0 & $\mathfrak{J}$ & 1200 & 700.000 & 6 \\
\hline METHYLENE CHLORIDE & (UG/L) & 28 & 9 & 350 & JB & 1.0 & JB & 89 & NR & NA \\
\hline STYRENE & (UG/L) & 28 & 1 & 53 & $\mathbf{J}$ & 53 & $J$ & 53 & 100.000 & 0 \\
\hline TETRACHLOROETHENE & (UG/L) & 28 & 14 & 110 & & 0.80 & $\mathrm{~J}$ & 41 & 5.000 & 8 \\
\hline TOLUENE & (UG/L) & 28 & 17 & 32000 & & 0.80 & $\mathfrak{J}$ & 7800 & 1000.00 & 7 \\
\hline TRICHLOROETHENE & (UG/L) & 28 & 9 & 67 & & 1.0 & 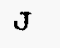 & 17 & 5.000 & 7 \\
\hline VINYL ACETATE & (UG/L) & 28 & 1 & 1000 & & 1000 & & 1000 & NR & NA \\
\hline VINYL CHLORIDE & (UG/L) & 28 & 3 & 57 & & 2.0 & $\mathfrak{J}$ & 36 & 2.000 & 2 \\
\hline XYLENES & (UG/L) & 28 & 17 & 18000 & & 1.0 & $\mathrm{~J}$ & 5200 & 10000.0 & 4 \\
\hline
\end{tabular}


Table 7.44. Constituents in groundwater at the Y-12 Plant site HGR=UST 2 area $=9754 / 9754-2$ Fuel Facility

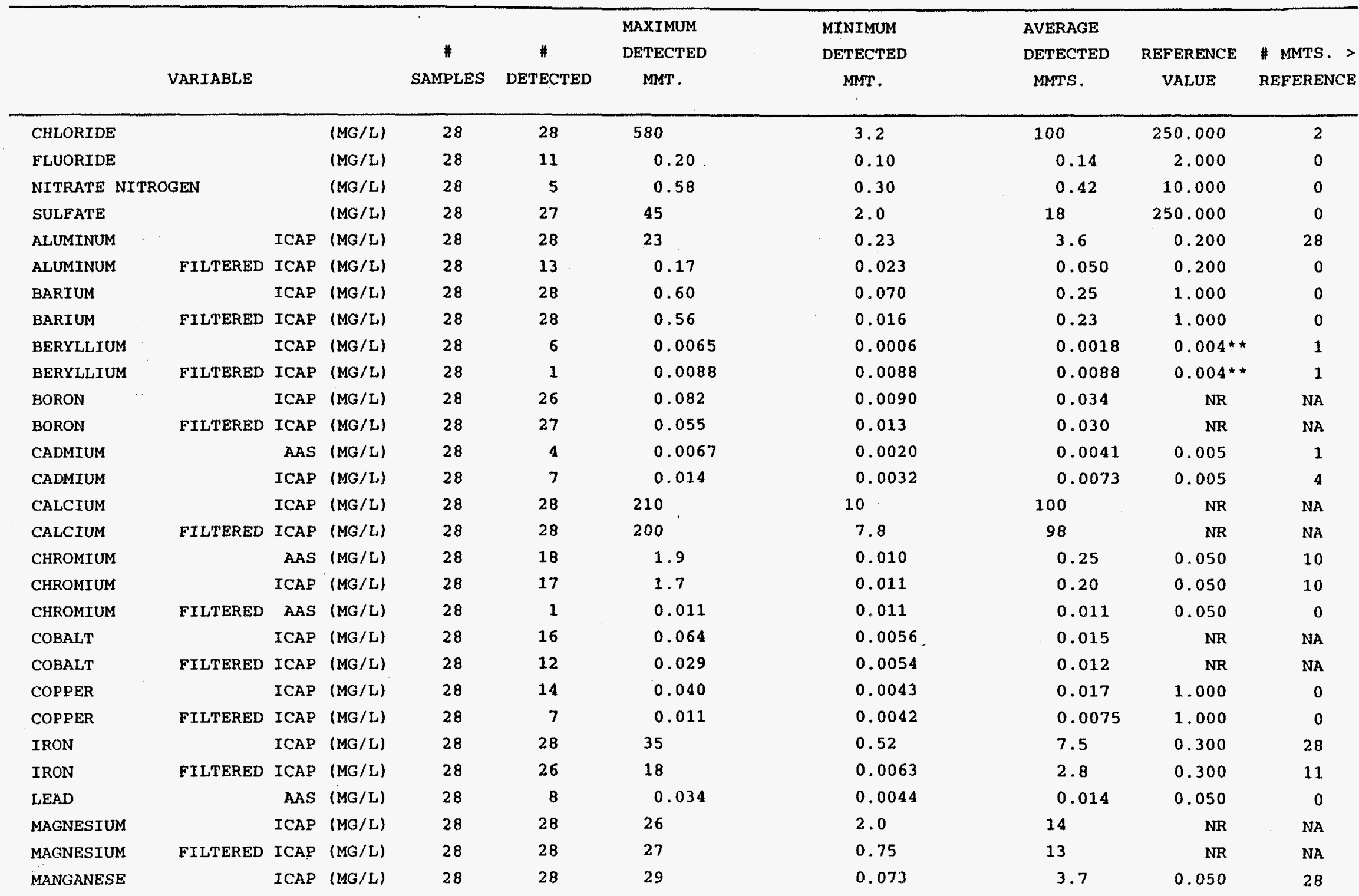




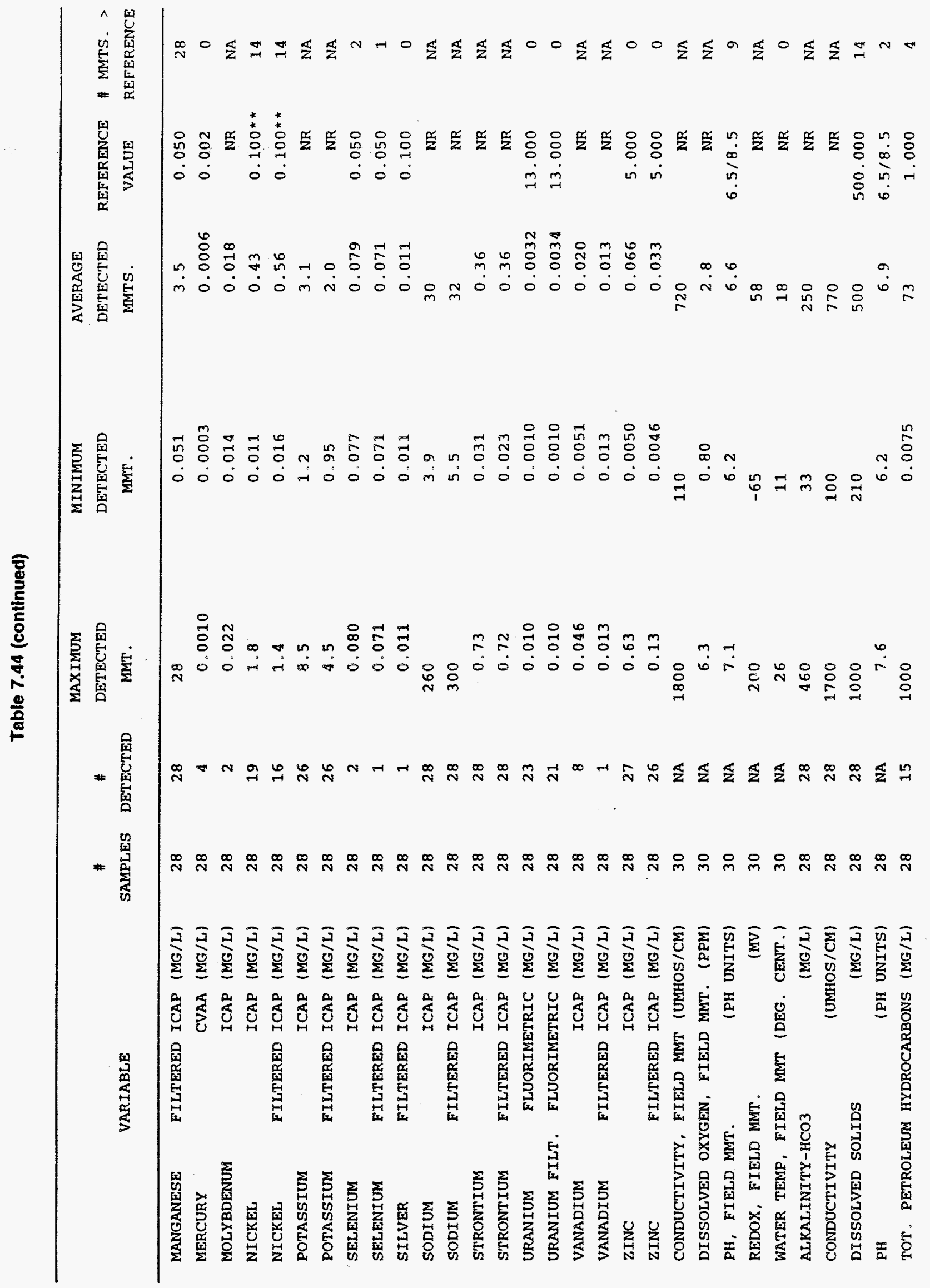


Table 7.44 (continued)

\begin{tabular}{|c|c|c|c|c|c|c|c|c|c|c|}
\hline VARIABLE & & $\begin{array}{c}* \\
\text { SAMPLES }\end{array}$ & $\begin{array}{c}\# \\
\text { DETECTED }\end{array}$ & $\begin{array}{c}\text { MAXIMUM } \\
\text { DETECTED } \\
\text { MMT. }\end{array}$ & & $\begin{array}{l}\text { MINIMUM } \\
\text { DETECTED } \\
\text { MMT. }\end{array}$ & & $\begin{array}{l}\text { AVERAGE } \\
\text { DETECTED } \\
\text { MMTS. }\end{array}$ & $\begin{array}{c}\text { REFERENCE } \\
\text { VALUE }\end{array}$ & $\begin{array}{l}\| \text { MMTS. > } \\
\text { REFERENCE }\end{array}$ \\
\hline TOTAL SUSPENDED SOLIDS & (MG/L) & 28 & 28 & 1100 & & 3.0 & & 140 & NR & $\mathrm{NA}$ \\
\hline TURBIDITY & (NTU) & 28 & 28 & 750 & & 2.5 & & 130 & 1.000 & 28 \\
\hline GROSS ALPHA & $(\mathrm{PCI} / \mathrm{L})$ & 28 & 28 & 11 & & -1.8 & & 2.5 & 15.000 & 0 \\
\hline GROSS BETA & $(\mathrm{PCI} / \mathrm{L})$ & 28 & 28 & 25 & & -1.6 & & 5.0 & 50.000 & 0 \\
\hline 1,2-DICHLOROETHANE & (UG/L) & 29 & 5 & 1000 & & 7.0 & & 550 & 5.000 & 5 \\
\hline 1,2-DICHLOROETHENE & (UG/L) & 29 & 1 & $1.0^{\circ}$ & $\mathrm{J}$ & 1.0 & $J$ & 1.0 & 70.000 & 0 \\
\hline 2-BUTANONE: & (UG/L) & 29 & 6 & 10 & B & 4.0 & BJ & 6.8 & NR & NA \\
\hline 4-METHYL-2-PENTANONE & (UG/L) & 29 & 5 & 2.0 & BJ & 2.0 & BJ & 2.0 & NR & $\mathrm{NA}$ \\
\hline ACETONE & (UG/L) & 29 & 7 & 20 & B & 1.0 & Bu & 4.6 & NR & NA \\
\hline BENZENE & (UG/L) & 29 & 13 & 14000 & & 2.0 & J & 3300 & 5.000 & 12 \\
\hline ETHYLBENZENE & (UG/L) & 29 & 11 & 1500 & & 1.0 & $\mathrm{~J}$ & 440 & 700.000 & 4 \\
\hline METHYLENE CHLORIDE & (UG/L) & 29 & 10 & 120 & JB & 0.90 & $\mathfrak{J}$ & 14 & NR & NA \\
\hline STYRENE & (UG/L) & 29 & 1 & 1.0 & $\mathfrak{J}$ & 1.0 & $\mathrm{~J}$ & 1.0 & 100.000 & 0 \\
\hline TOLUENE & (UG/L) & 29 & 9 & 9300 & & 6.0 & & 3000 & 1000.00 & 4 \\
\hline TRICHLOROETHENE & (UG/L) & 29 & 2 & 2.0 & J & 2.0 & $\mathbf{J}$ & 2.0 & 5.000 & 0 \\
\hline VINYL ACETATE & (UG/L) & 29 & 2 & 63 & & 38 & D & 51 & $\mathrm{NR}$ & $\mathrm{NA}$ \\
\hline XYLENES & (UG/L) & 29 & 13 & 7500 & & 1.0 & $\mathrm{~J}$ & 1900 & 10000.0 & 0 \\
\hline
\end{tabular}


Table 7.45. Constituents in groundwater at the $Y-12$ Plant site HGR=UST 2 area=Tank 0134-U

\begin{tabular}{|c|c|c|c|c|c|c|c|c|c|c|}
\hline & VARIABLE & & & $\begin{array}{c}\# \\
\text { SAMPLES }\end{array}$ & $\begin{array}{c}\# \\
\text { DETECTED }\end{array}$ & $\begin{array}{c}\text { MAXIMUM } \\
\text { DETECTED } \\
\text { MMT. }\end{array}$ & $\begin{array}{c}\text { MINIMUM } \\
\text { DETECTED } \\
\text { MMT. }\end{array}$ & $\begin{array}{l}\text { AVERAGE } \\
\text { DETECTED } \\
\text { MMTS. }\end{array}$ & $\begin{array}{c}\text { REFERENCE } \\
\text { VALUE }\end{array}$ & $\begin{array}{l}\text { \# MMTS. > } \\
\text { REFERENCE }\end{array}$ \\
\hline CHLORIDE & & & (MG/L) & 8 & 8 & 70 & 3.4 & 35 & 250.000 & 0 \\
\hline FLUORIDE & & & $(M G / L)$ & 8 & 5 & 1.9 & 0.10 & 1.1 & 2.000 & 0 \\
\hline NITRATE NIT & GEN & & (MG/L) & 8 & 3 & 0.33 & 0.26 & 0.29 & 10.000 & 0 \\
\hline SULFATE & & & (MG/L) & 8 & 8 & 67 & 4.3 & 48 & 250.000 & 0 \\
\hline ALUMINUM & & ICAP & (MG/L) & 8 & 8 & 14 & 0.067 & 3.0 & 0.200 & 7 \\
\hline ALUMINUM & FILTERED & ICAP & $(M G / L)$ & 8 & 5 & 0.045 & 0.022 & 0.030 & 0.200 & 0 \\
\hline BARIUM & & ICAP & (MG/L) & 8 & 8 & 0.37 & 0.12 & 0.19 & 1.000 & 0 \\
\hline BARIUM & FILTERED & ICAP & (MG/L) & 8 & 8 & 0.19 & 0.11 & 0.16 & 1.000 & 0 \\
\hline BERYLLIUM & & ICAP & (MG/L) & 8 & 2 & 0.0013 & 0.0004 & 0.0009 & $0.004^{* *}$ & 0 \\
\hline BORON & & ICAP & (MG/L) & 8 & 8 & 0.092 & 0.017 & 0.060 & NR & NA \\
\hline BORON & FILTERED & ICAP & $(M G / L)$ & 8 & 8 & 0.096 & 0.011 & 0.050 & NR & NA \\
\hline CADMIUM & & AAS & (MG/L) & 8 & 1 & 0.0021 & 0.0021 & 0.0021 & 0.005 & 0 \\
\hline CADMIUM & FILTERED & AAS & (MG/L) & 8 & 1 & 0.0022 & 0.0022 & 0.0022 & 0.005 & 0 \\
\hline CALCIUM & & ICAP & $(M G / L)$ & 8 & 8 & 120 & 40 & 83 & NR & NA \\
\hline CALCIUM & FILTERED & ICAP & (MG/L) & 8 & 8 & 120 & 39 & 80 & NR & NA \\
\hline CHROMIUM & & AAS & $(M G / L)$ & 8 & 4 & 0.072 & 0.011 & 0.043 & 0.050 & 2 \\
\hline CHROMIUM & & ICAP & (MG/L) & 8 & 3 & 0.069 & 0.016 & 0.049 & 0.050 & 2 \\
\hline COBALT & & ICAP & (MG/L) & 8 & 1 & 0.022 & 0.022 & 0.022 & NR & NA \\
\hline COBALT & FILTERED & ICAP & (MG/L) & 8 & 1 & 0.0055 & 0.0055 & 0.0055 & NR & NA \\
\hline COPPER & & ICAP & $(M G / L)$ & 8 & 6 & 0.035 & 0.0054 & 0.012 & 1.000 & 0 \\
\hline COPPER & FILTERED & ICAP & (MG/L) & 8 & 2 & 0.027 & 0.0062 & 0.017 & 1.000 & 0 \\
\hline IRON & & ICAP & (MG /L) & 8 & 8 & 41 & 0.28 & 7.7 & 0.300 & 7 \\
\hline IRON & FILTERED & ICAP & (MG/L) & 8 & 8 & 1.2 & 0.010 & 0.43 & 0.300 & 3 \\
\hline LEAD & & AAS & (MG/L) & 8 & 3 & 0.013 & 0.0043 & 0.0077 & 0.050 & c \\
\hline LEAD & FILTERED & AAS & (MG/t $)$ & 8 & 1 & 0.0055 & 0.0055 & 0.0055 & 0.050 & 0 \\
\hline MAGNESIUM & & ICAP & $(M G / L)$ & 8 & 8 & 10 & 6.4 & 7.8 & NR & NA \\
\hline MAGNESIUM & FILTERED & ICAP & (MG/L) & 8 & 8 & 9.3 & 5.9 & 6.9 & NR & NA \\
\hline MANGANESE & & ICAP & $(M G / L)$ & 8 & 8 & 2.0 & 0.33 & 1.0 & 0.050 & 8 \\
\hline MANGANESE & FILTERED & ICAP & (MG/L) & 8 & 8 & 1.9 & 0.18 & 0.81 & 0.050 & 8 \\
\hline
\end{tabular}


Table 7.45 (continued)

\begin{tabular}{|c|c|c|c|c|c|c|c|c|c|c|}
\hline & VARIABLE & & & $\begin{array}{c}\# \\
\text { SAMPLES }\end{array}$ & $\begin{array}{c}\# \\
\text { DETECTED }\end{array}$ & $\begin{array}{c}\text { MAXIMUM } \\
\text { DETECTED } \\
\text { MMT. }\end{array}$ & $\begin{array}{l}\text { MINIMUM } \\
\text { DETECTED } \\
\text { MMT. }\end{array}$ & $\begin{array}{l}\text { AVERAGE } \\
\text { DETECTED } \\
\text { MMTS. }\end{array}$ & $\begin{array}{c}\text { REFERENCE } \\
\text { VALUE }\end{array}$ & $\begin{array}{l}\text { \# MMTS. > } \\
\text { REFERENCE }\end{array}$ \\
\hline NICKEL & & ICAP & (MG/L) & 8 & 5 & 0.078 & 0.013 & 0.043 & $0.100^{\star \star}$ & 0 \\
\hline NICKEL & FILTERED & ICAP & $(M G / L)$ & 8 & 4 & 0.029 & 0.017 & 0.022 & $0.100 * \star$ & 0 \\
\hline POTASSIUM & & ICAP & (MG/L) & 8 & 8 & 8.2 & 2.2 & 3.7 & NR & NA \\
\hline POTASSIUM & FILTERED & ICAP & (MG/L) & 8 & 8 & 3.6 & 1.8 & 2.6 & NR & NA \\
\hline SILVER & FILTERED & ICAP & (MG/L) & 8 & 1 & 0.0075 & 0.0075 & 0.0075 & 0.100 & 0 \\
\hline SODIUM & & ICAP & $(M G / L)$ & 8 & 8 & 51 & 7.2 & 18 & NR & NA \\
\hline SODIUM & FILTERED & ICAP & $(M G / L)$ & 8 & 8 & 49 & 7.0 & 18 & NR & NA \\
\hline STRONTIUM & & ICAP & (MG/L) & 8 & 8 & 0.23 & 0.16 & 0.20 & NR & NA \\
\hline SRRONTIUM & FILTERED & ICAP & (MG/L) & 8 & $B$ & 0.23 & 0.15 & 0.19 & NR & NA \\
\hline URANIUM & FLUORIMI & ETRIC & (MG/L) & 8 & 5 & 0.21 & 0.0040 & 0.10 & 13.000 & 0 \\
\hline URANIUM FILT. & FLUORIMI & ETRIC & $(M G / L)$ & 8 & 5 & 0.19 & 0.0040 & 0.097 & 13.000 & 0 \\
\hline VANADIUM & & ICAP & $(M G / L)$ & 8 & 2 & 0.025 & 0.0058 & 0.015 & NR & NA \\
\hline ZINC & & ICAP & $(M G / L)$ & 8 & 8 & 0.25 & 0.021 & 0.083 & 5.000 & 0 \\
\hline $\operatorname{zINC}$ & FILTERED & ICAP & (MG/L) & 8 & 8 & 0.20 & 0.0085 & 0.065 & 5.000 & 0 \\
\hline CONDUCTIVITY, & FIELD MM? & T (UMH & HOS/CM) & 8 & NA & 650 & 370 & 510 & NR & NA \\
\hline DISSOLVED OXY & YGEN， FIELI & D MMT. & . (PPM) & 8 & NA & 8.5 & 1.1 & 3.7 & NR & NA \\
\hline PH, FIELD MMT & & $(\mathrm{PH}$ & UNITS) & 8 & NA & 7.6 & 6.5 & 7.0 & $6.5 / 8.5$ & 0 \\
\hline REDOX， FIELD & MMT . & & (MV) & 8 & NA & 240 & -29 & 89 & NR & NA \\
\hline WATER TEMP, $F$ & FIELD MMT & (DEG. & CENT.) & 8 & NA & 23 & 11 & 19 & $\mathrm{NR}$ & 0 \\
\hline ALKALINITY-HC & & & (MG/L) & 8 & 8 & 240 & 160 & 180 & NR & NA \\
\hline CONDUCTIVITY & & IUMH & HOS /CM) & 8 & 8 & 670 & 390 & 540 & NR & NA \\
\hline DISSOLVED SOL & LIDS & & (MG/L) & 8 & 8 & 480 & 190 & 360 & 500.000 & $\mathbf{0}$ \\
\hline PH & & (PH & (UNITS) & 8 & NA & 7.7 & 6.9 & 7.4 & $6.5 / 8.5$ & 0 \\
\hline TOT. PETROLEU & JM MYDROCAF & RBONS & (MG/L) & 8 & 5 & 0.44 & 0.0020 & 0.11 & 1.000 & 0 \\
\hline TOTAL SUSPENDI & DED SOLIDS & & $(M G / L)$ & 8 & 8 & 750 & 3.0 & 160 & NR & NA \\
\hline TURBIDITY & & & (NTU) & 8 & 8 & 380 & 3.3 & 100 & 1.000 & 8 \\
\hline GROSS ALPHA & & & $(\mathrm{PCI} / \mathrm{L})$ & 8 & 8 & 100 & -1.3 & 32 & 15.000 & 4 \\
\hline GROSS BETA & & & $(\mathrm{PCI} / \mathrm{L})$ & 8 & 8 & 39 & 2.6 & 19 & 50.000 & 0 \\
\hline 1,1-DICHLOROE & ETHENE & & $(U G / L)$ & 8 & 4 & 1700 & 1500 & 1600 & 7.000 & 4 \\
\hline 1,2-DICHLOROE & ETHENE & & $(U G / L)$ & 8 & 4 & 340 & 230 & 280 & 70.000 & 4 \\
\hline
\end{tabular}


Table 7.45 (continued)

\begin{tabular}{|c|c|c|c|c|c|c|c|c|c|c|}
\hline VARIABLE & & $\begin{array}{c}\# \\
\text { SAMPLES }\end{array}$ & $\begin{array}{c}\# \\
\text { DETECTED }\end{array}$ & $\begin{array}{c}\text { MAXIMUM } \\
\text { DETECTED } \\
\text { MMT. }\end{array}$ & & $\begin{array}{c}\text { MINIMUM } \\
\text { DETECTED } \\
\text { MMT. }\end{array}$ & & $\begin{array}{l}\text { AVERAGE } \\
\text { DETECTED } \\
\text { MMTS. }\end{array}$ & $\begin{array}{c}\text { REFERENCE } \\
\text { VALUE }\end{array}$ & $\begin{array}{l}\text { \# MMTS. > } \\
\text { REFERENCE }\end{array}$ \\
\hline ETHYLBENZENE & $(U G / L)$ & 8 & 2 & 2.0 & $\mathbf{J}$ & 2.0 & $\mathbf{J}$ & 2.0 & 700.000 & 0 \\
\hline METHYLENE CHLORIDE & (UG/L) & 8 & 2 & 1.0 & $J$ & 1.0 & $\mathbf{J}$ & 1.0 & NR & NA \\
\hline TETRACHLOROETHENE & (UG/L) & 8 & 2 & 99 & $\boldsymbol{J}$ & 81 & $\mathfrak{J}$ & 90 & 5.000 & 2 \\
\hline TRICHLOROETHENE & (UG/L) & 8 & 4 & 11000 & & 8600 & & 10000 & 5.000 & 4 \\
\hline XYLENES & (UG/L) & 8 & 1 & 2.0 & J & 2.0 & $\mathfrak{J}$ & 2.0 & 10000.0 & 0 \\
\hline
\end{tabular}


Table 7.46. Constituents in groundwater at the Y-12 Plant site HGR=UST 2 area=Tank $2331-U$

\begin{tabular}{|c|c|c|c|c|c|c|c|c|c|c|}
\hline \multicolumn{4}{|c|}{ VARIABLE } & $\begin{array}{c}\# \\
\text { SAMPLES }\end{array}$ & $\begin{array}{c}\# \\
\text { DETECTED }\end{array}$ & $\begin{array}{l}\text { MAXIMUM } \\
\text { DETECTED } \\
\text { MMT. }\end{array}$ & $\begin{array}{l}\text { MINIMUM } \\
\text { DETECTED } \\
\text { MMT. }\end{array}$ & $\begin{array}{l}\text { AVERAGE } \\
\text { DETECTED } \\
\text { MMTS. }\end{array}$ & $\begin{array}{c}\text { REFERENCE } \\
\text { VALUE }\end{array}$ & $\begin{array}{l}\text { \# MMTS. > } \\
\text { REFERENCE }\end{array}$ \\
\hline CHLORIDE & & & (MG/L) & 16 & 16 & 200 & 3.3 & 29 & 250.000 & 0 \\
\hline FLUORIDE & & & (MG/L) & 16 & 16 & 0.60 & 0.20 & 0.44 & 2.000 & 0 \\
\hline NITRATE NI? & DGEN & & (MG/L) & 16 & 5 & 1.3 & 0.23 & 0.48 & 10.000 & 0 \\
\hline SULFATE & & & (MG/L) & 16 & 15 & 45 & 3.7 & 20 & 250.000 & 0 \\
\hline ALUMINUM & & ICAP & (MG/L) & 16 & 12 & 9.3 & 0.033 & 0.95 & 0.200 & 4 \\
\hline ALUMINUM & FILTERED & ICAP & (MG/L) & 16 & 7 & 0.053 & 0.023 & 0.032 & 0.200 & 0 \\
\hline BARIUM & & ICAP & (MG/L) & 16 & 16 & 0.60 & 0.036 & 0.18 & 1.000 & 0 \\
\hline BARIUM & FILTERED & ICAP & (MG/L) & 16 & 16 & 0.58 & 0.014 & 0.17 & 1.000 & 0 \\
\hline BERYLLIUM & & ICAP & (MG/L) & 16 & 3 & 0.0047 & 0.0004 & 0.0019 & $0.004^{\star \star}$ & 1 \\
\hline BERYLLIUM & FILTERED & ICAP & (MG/L) & 16 & 1 & 0.0005 & 0.0005 & 0.0005 & $0.004^{\star *}$ & 0 \\
\hline BORON & & ICAP & (MG/L) & 16 & 16 & 0.15 & 0.011 & 0.063 & NR & $\mathrm{NA}$ \\
\hline BORON & FILTERED & ICAP & (MG/L) & 16 & 16 & 0.15 & 0.0079 & 0.066 & NR & NA \\
\hline CADMIUM & & AAS & (MG/L) & 16 & 1 & 0.0061 & 0.0061 & 0.0061 & 0.005 & 1 \\
\hline CADMIUM & & ICAP & (MG/L) & 16 & 4 & 0.0065 & 0.0032 & 0.0044 & 0.005 & 1 \\
\hline CALCIUM & & ICAP & $(M G / L)$ & 16 & 16 & 140 & 41 & 94 & NR & $\mathrm{NA}$ \\
\hline CALCIUM & FILTERED & ICAP & $(M G / L)$ & 16 & 16 & 140 & 26 & 93 & NR & NA \\
\hline CHROMIUM & & AAS & (MG/L) & 16 & 1 & 0.028 & 0.028 & 0.028 & 0.050 & 0 \\
\hline CHROMIUM & & ICAP & (MG/L) & 16 & 1 & 0.023 & 0.023 & 0.023 & 0.050 & 0 \\
\hline COBALT & & ICAP & $(M G / L)$ & 16 & 1 & 0.0056 & 0.0056 & 0.0056 & NR & NA \\
\hline COPPER & & ICAP & (MG/L) & 16 & 5 & 0.011 & 0.0052 & 0.0083 & 1.000 & 0 \\
\hline COPPER & FILTERED & ICAP & (MG/L) & 16 & 1 & 0.0099 & 0.0099 & 0.0099 & 1.000 & 0 \\
\hline IRON & & ICAP & $(M G / L)$ & 16 & 16 & 13 & 0.11 & 2.9 & 0.300 & 12 \\
\hline IRON & FILTERED & ICAP & (MG/L) & 16 & 16 & 7.7 & $0: 011$ & 1.8 & 0.300 & 10 \\
\hline LEAD & & AAS & (MG/L) & 16 & 1 & 0.0080 & 0.0080 & 0.0080 & 0.050 & 0 \\
\hline MAGNESIUM & & ICAP & $(M G / L)$ & 16 & 16 & 22 & 5.0 & 12 & NR & NA \\
\hline MAGNESIUM & FILTERED & ICAP & $(M G / L)$ & 16 & 16 & 23 & 2.2 & 12 & NR & NA \\
\hline MANGANESE & & ICAP & (MG/L) & 16 & 16 & 1.1 & 0.097 & 0.63 & 0.050 & 16 \\
\hline MANGANESE & FILTERED & ICAP & (MG/L) & 16 & 16 & 1.2 & 0.039 & 0.64 & 0.050 & 15 \\
\hline MOLYBDENUM & & ICAP & (MG/L) & 16 & 3 & 0.028 & 0.013 & 0.020 & NR & NA \\
\hline
\end{tabular}


Table 7.46 (continued)

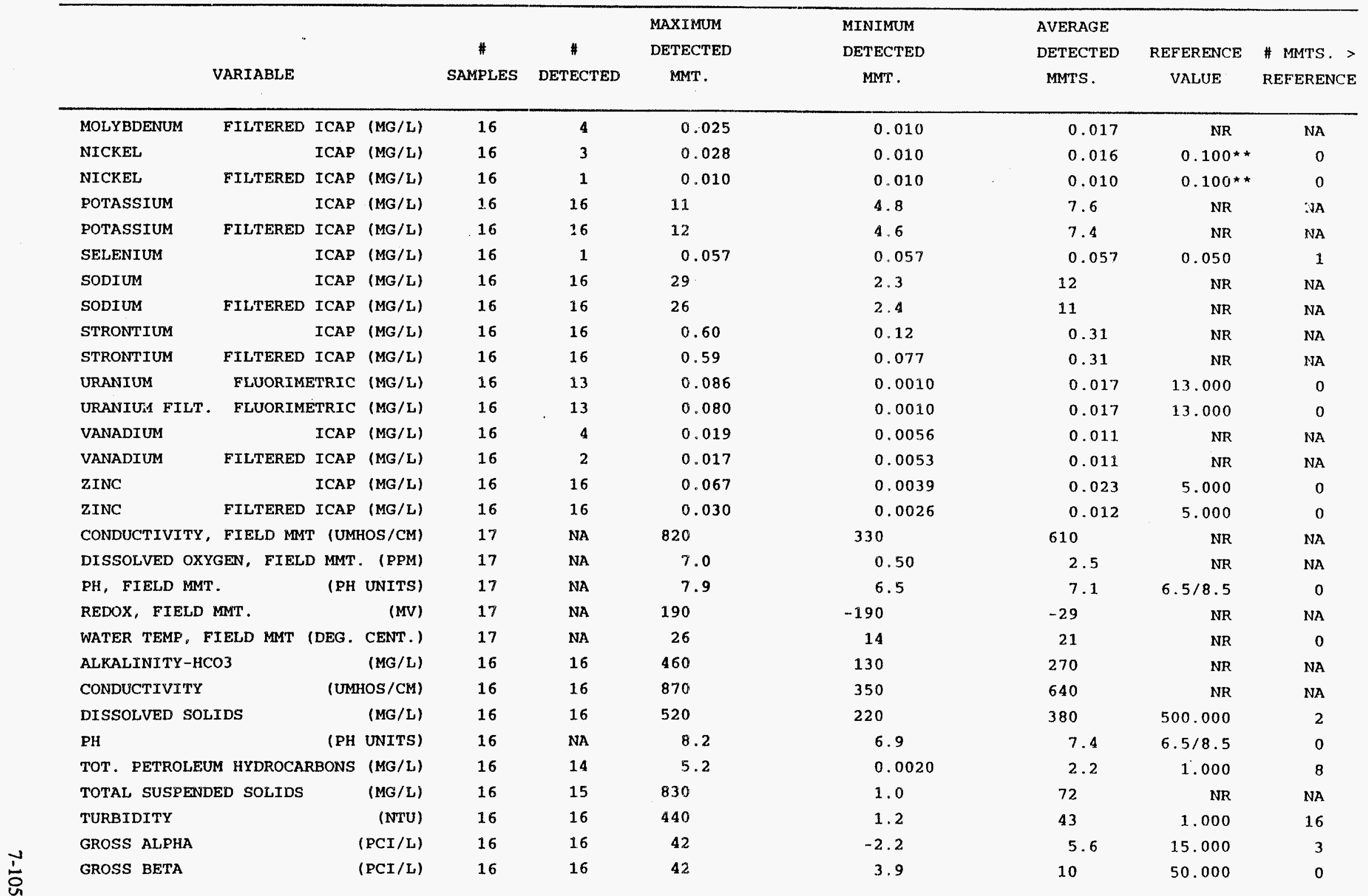


Table 7.46 (continued)

\begin{tabular}{|c|c|c|c|c|c|c|c|c|c|c|}
\hline VARIABLE & & $\begin{array}{c}\# \\
\text { SAMPLES }\end{array}$ & $\begin{array}{c}\# \\
\text { DETECTED }\end{array}$ & $\begin{array}{l}\text { MAXIMUM } \\
\text { DETECTED } \\
\text { MMT. }\end{array}$ & & $\begin{array}{l}\text { MINIMUM } \\
\text { DETECTED } \\
\text { MMT. }\end{array}$ & & $\begin{array}{l}\text { AVERAGE } \\
\text { DETECTED } \\
\text { MMTS. }\end{array}$ & $\begin{array}{c}\text { REFERENCE } \\
\text { VALUE }\end{array}$ & $\begin{array}{l}\# \text { MMTS. > } \\
\text { REFERENCE }\end{array}$ \\
\hline 4-METHYL-2-PENTANONE & (UG/L) & 16 & 2 & 57 & BJ & 2.0 & $\mathbf{B J}$ & 30 & NR & NA \\
\hline BENZENE & $(U G / L)$ & 16 & 8 & 2800 & $\mathbf{E}$ & 780 & & 1400 & 5.000 & 8 \\
\hline ETHYLBENZENE & (UG/L) & 16 & 8 & 790 & & 300 & & 600 & 700.000 & 3 \\
\hline METHYLENE CHLORIDE & $(U G / L)$ & 16 & 4 & 63 & BJ & 2.0 & BJ & 33 & NR & NA \\
\hline TOLUENE & (UG/L) & 16 & 8 & 700 & & 75 & $\mathrm{~J}$ & 220 & 1000.00 & 0 \\
\hline TRICHLOROETHENE & (UG/L) & 16 & 1 & 1.0 & $\mathrm{~J}$ & 1.0 & $\mathrm{~J}$ & 1.0 & 5.000 & 0 \\
\hline VINYL ACETATE & (UG/L) & 16 & 1 & 2.0 & $\mathfrak{J}$ & 2.0 & $\mathrm{~J}$ & 2.0 & NR & NA \\
\hline XYLENES & (UG/L) & 16 & 8 & 2600 & & 300 & & 1400 & 10000.0 & 0 \\
\hline
\end{tabular}




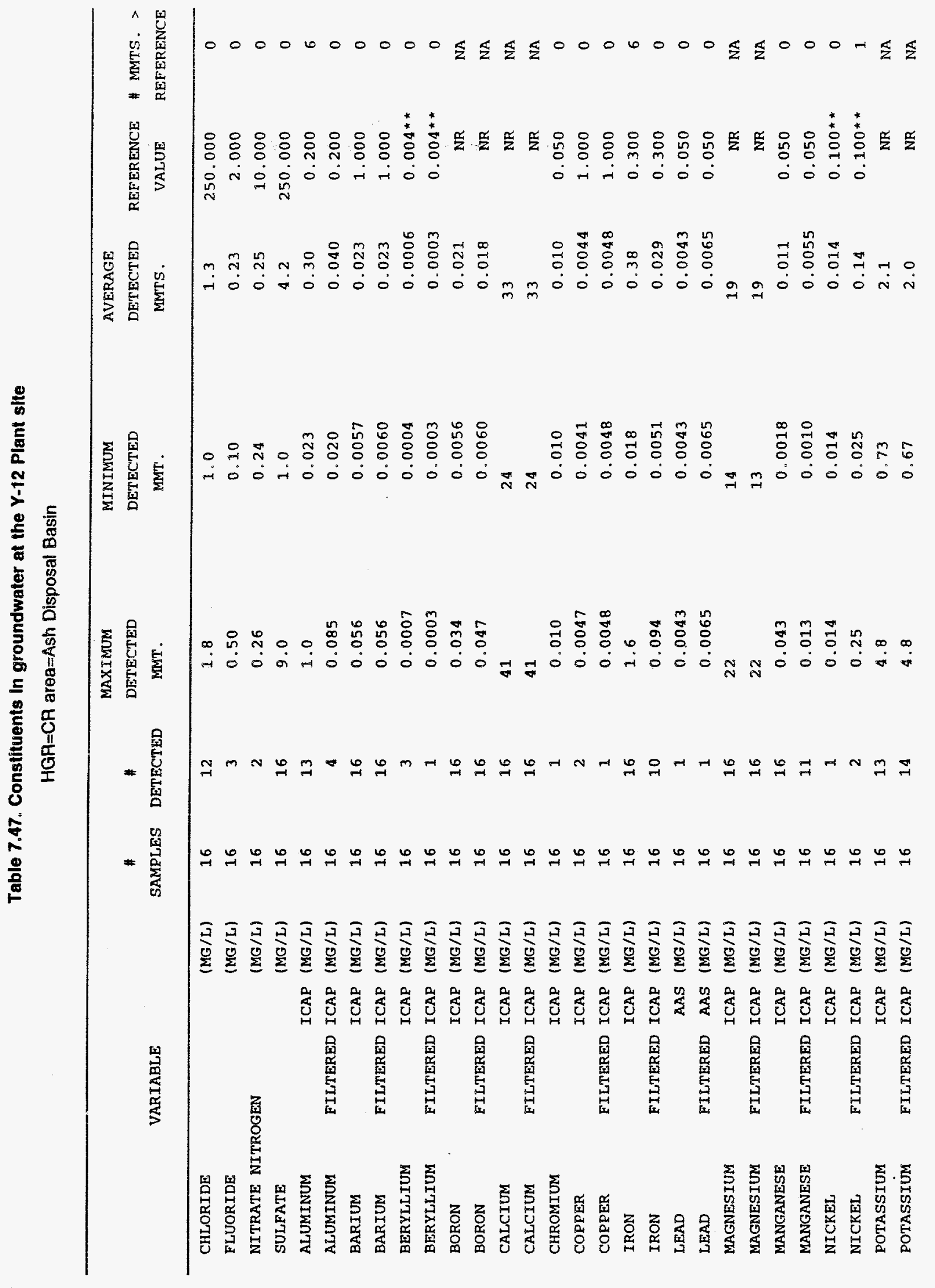


Table 7.47 (continued)

\begin{tabular}{|c|c|c|c|c|c|c|c|c|c|c|}
\hline VARIABLE & & $\begin{array}{c}\# \\
\text { SAMPLES }\end{array}$ & $\begin{array}{c}\# \\
\text { DETECTED }\end{array}$ & $\begin{array}{l}\text { MAXIMUM } \\
\text { DETECTED } \\
\text { MMT. }\end{array}$ & & $\begin{array}{l}\text { MINIMUM } \\
\text { DETECTED } \\
\text { MMT. }\end{array}$ & & $\begin{array}{l}\text { AVERAGE } \\
\text { DETECTED } \\
\text { MMTS. }\end{array}$ & $\begin{array}{l}\text { REFERENCE } \\
\text { VALUE }\end{array}$ & $\begin{array}{l}\text { \# MMTS. > } \\
\text { REFERENCE }\end{array}$ \\
\hline SODIUM & $\operatorname{ICAP}(M G / L)$ & 16 & 16 & 1.8 & & 0.37 & & 0.82 & NR & NA \\
\hline FILTERED & ICAP (MG/L) & 16 & 16 & 1.9 & & 0.36 & & 0.81 & NR & NA \\
\hline STRONTIUM & $\operatorname{ICAP}(M G / L)$ & 16 & 16 & 0.029 & & 0.017 & & 0.021 & NR & NA \\
\hline STRONTIUM & ICAP (MG/L) & 16 & 16 & 0.023 & & 0.016 & & 0.020 & NR & NA \\
\hline URANIUM FILT. FLUORIME & ETRIC $(M G / L)$ & 16 & 2 & 0.0030 & & 0.0010 & & 0.0020 & 13.000 & 0 \\
\hline VANADIUM & ICAP (MG/L) & 16 & 1 & 0.0065 & & 0.0065 & & 0.0065 & NR & $\mathrm{NA}$ \\
\hline ZINC & ICAP (MG/L) & 16 & 15 & 0.11 & & 0.0022 & & 0.021 & 5.000 & 0 \\
\hline FILTERED & $\operatorname{ICAP}(M G / L)$ & 16 & 15 & 0.10 & & 0.0031 & & 0.023 & 5.000 & 0 \\
\hline CONDUCTIVITY, FIELD MMT & (UMHOS/CM) & 16 & NA & 360 & & 220 & & 290 & NR & NA \\
\hline DISSOLVED OXYGEN, FIELD & MMT. (PPM) & 16 & $\mathrm{NA}$ & 9.2 & & 2.5 & & 6.4 & NR & NA \\
\hline PH, FIELD MMT. & (PH UNITS) & 16 & NA & B. 2 & & 6.7 & & 7.6 & $6.5 / 8.5$ & 0 \\
\hline REDOX, FIELD MMT . & (MV) & 16 & NA & 320 & & 130 & & 220 & NR & $\mathrm{NA}$ \\
\hline WATER TEMP, FIELD MMT & (DEG. CENT.) & 16 & NA & 18 & & 13 & & 15 & $\mathrm{NR}$ & 0 \\
\hline ALKALINITY-HCO3 & $(M G / L)$ & 16 & 16 & 180 & & 120 & & 160 & NR & $\mathrm{NA}$ \\
\hline CONDUCTIVITY & (UMHOS/CM) & 16 & 16 & 350 & & 230 & & 300 & NR & NA \\
\hline DISSOLVED SOLIDS & (MG/L) & 16 & 16 & 200 & & 120 & & 170 & 500.000 & 0 \\
\hline $\mathrm{PH}$ & (PH UNITS) & 16 & NA & 8.2 & & 7.2 & & 7.9 & $6.5 / 8.5$ & 0 \\
\hline TOTAL ORGANIC CARBON & (MG/L) & 16 & 10 & 26 & & 1.0 & & 4.7 & NR & NA \\
\hline TOTAL SUSPENDED SOLIDS & (MG/L) & 16 & 12 & 34 & & 1.0 & & 9.7 & NR & $\mathrm{NA}$ \\
\hline TURBIDITY & (NTU) & 16 & 16 & 130 & & 1.0 & & 15 & 1.000 & 14 \\
\hline GROSS ALPHA & $(\mathrm{PCT} / \mathrm{L})$ & 16 & 16 & 3.4 & & -5.3 & & -0.10 & 15.000 & 0 \\
\hline GROSS BETA & (PCI/L) & 16 & 16 & 5.6 & & -5.1 & & 0.50 & 50.000 & 0 \\
\hline $1,1,1$-TRICHLOROETHANE & (UG/L) & 16 & 3 & 1.0 & $\mathrm{~J}$ & 1.0 & $\mathrm{~J}$ & 1.0 & 200.000 & 0 \\
\hline 4-METHYL-2-PENTANONE & (UG/L) & 16 & 2 & 4.0 & BJ & 1.0 & BJ & 2.5 & NR & NA \\
\hline ACETONE & (UG/L) & 16 & 1 & 4.0 & JB & 4.0 & $\mathrm{JB}$ & 4.0 & $\mathrm{NR}$ & $\mathrm{NA}$ \\
\hline METHYLENE CHLORIDE & (UG/L) & 16 & 3 & 2.0 & BJ & 0.70 & JB & 1.2 & NR & NA \\
\hline
\end{tabular}


Table 7.48. Constituents in groundwater at the Y-12 Plant site $H G R=C R$ area=East $C R$ Wasle Pile

\begin{tabular}{|c|c|c|c|c|c|c|c|c|c|c|}
\hline \multicolumn{4}{|c|}{ VARIABLE } & \multirow{2}{*}{$\begin{array}{c}\begin{array}{c}* \\
\text { SAMPLES }\end{array} \\
16\end{array}$} & \multirow{2}{*}{$\frac{\|}{\text { DETECTED }}$} & \multirow{2}{*}{$\begin{array}{c}\text { MAXIMUM } \\
\text { DETECTED } \\
\text { MMT. } \\
16\end{array}$} & \multirow{2}{*}{$\begin{array}{c}\text { MINIMUM } \\
\begin{array}{c}\text { DETECTED } \\
\text { MMT! }\end{array} \\
2.4\end{array}$} & \multirow{2}{*}{$\begin{array}{l}\text { AVERAGE } \\
\text { DETECTED } \\
\text { MMTS. } \\
7.8\end{array}$} & \multirow{2}{*}{$\begin{array}{c}\text { REFERENCE } \\
\text { VALUE } \\
250.000\end{array}$} & \multirow{2}{*}{$\begin{array}{l}\# \text { MMTS. > } \\
\text { REFERENCE } \\
0\end{array}$} \\
\hline CHLORIDE & & & (MG/L) & & & & & & & \\
\hline FLUORIDE & & & (MG/L) & 16 & 1 & 0.10 & 0.10 & 0.10 & 2.000 & 0 \\
\hline NITRATE NI & GEN & & $(M G / L)$ & 16 & 16 & 3.1 & 0.27 & 1.0 & 10.000 & 0 \\
\hline SULFATE & & & (MG/L) & 16 & 15 & 4.5 & 1.0 & 3.2 & 250.000 & 0 \\
\hline ALUMINUM & & ICAP & $(M G / L)$ & 16 & 9 & 0.12 & 0.023 & 0.052 & 0.200 & 0 \\
\hline ALUMINUM & FILTERED & ICAP & $(M G / L)$ & 16 & 9 & 0.12 & 0.021 & 0.044 & 0.200 & 0 \\
\hline BARIUM & & ICAP & (MG/L) & 16 & 16 & 0.22 & 0.0095 & 0.086 & 1.000 & 0 \\
\hline BARIUM & FILTERED & ICAP & (MG/L) & 16 & 16 & 0.21 & 0.0094 & 0.084 & 1.000 & 0 \\
\hline BERYLLIUM & & ICAP & (MG/L) & 16 & 2 & 0.0008 & 0.0003 & 0.0005 & $0.004 * *$ & 0 \\
\hline BERYLLIUM & FILTERED & ICAP & (MG/L) & 16 & 4 & 0.0008 & 0.0003 & 0.0005 & $0.004 \star \star$ & 0 \\
\hline BORON & & ICAP & $(M G / L)$ & 16 & 15 & 0.034 & 0.0086 & 0.021 & NR & NA \\
\hline BORON & FILTERED & ICAP & (MG/L) & 16 & 14 & 0.029 & 0.0056 & 0.019 & NR & NA \\
\hline CALCIUM & & ICAP & (MG/L) & 16 & 16 & 56 & 44 & 50 & NR & NA \\
\hline CALCIUM & FILTERED & ICAP & (MG/L) & 16 & 16 & 56 & 44 & 50 & NR & NA \\
\hline CHROMIUM & & ICAP & $(M G / L)$ & 16 & 1 & 0.025 & 0.025 & 0.025 & 0.050 & 0 \\
\hline COBALT & & ICAP & (MG/L) & 16 & 1 & 0.0055 & 0.0055 & 0.0055 & NR & NA \\
\hline COPPER & & ICAP & $(M G / L)$ & 16 & 1 & 0.0082 & 0.0082 & 0.0082 & 1.000 & 0 \\
\hline COPPER & FILTERED & ICAP & (MG/L) & 16 & 1 & 0.0051 & 0.0051 & 0.0051 & 1.000 & 0 \\
\hline IRON & & ICAP & $(M G / L)$ & 16 & 16 & 7.0 & 0.0072 & 1.2 & 0.300 & 4 \\
\hline IRON & FILTERED & ICAP & $(M G / L)$ & 16 & 6 & 0.049 & 0.014 & 0.024 & 0.300 & 0 \\
\hline MAGNESIUM & & ICAP & $(M G / L)$ & 16 & 16 & 32 & 26 & 29 & NR & NA \\
\hline MAGNESIUM & FILTERED & ICAP & (MG/L) & 16 & 16 & 32 & 26 & 29 & NR & NA \\
\hline MANGANESE & & ICAP & $(M G / L)$ & 16 & 8 & 0.029 & 0.0017 & 0.012 & 0.050 & 0 \\
\hline MANGANESE & FILTERED & ICAP & $(M G / L)$ & 16 & 8 & 0.030 & 0.0010 & 0.010 & 0.050 & 0 \\
\hline NICKEL & & ICAP & $(M G / L)$ & 16 & 1 & 0.015 & 0.015 & 0.015 & $0.100^{\star \star}$ & 0 \\
\hline POTASSIUM & & ICAP & $(M G / L)$ & 16 & 16 & 1.6 & 0.61 & 1.1 & NR & NA \\
\hline POTASSIUM & FILTERED & ICAP & (MG/L) & 16 & 14 & 1.8 & 0.69 & 1.2 & NR & $\mathrm{NA}$ \\
\hline SODIUM & & ICAP & (MG/L) & 16 & 16 & 4.6 & 1.3 & 3.3 & NR & $\mathrm{NA}$ \\
\hline SODIUM & FILTERED & ICAP & $(M G / L)$ & 16 & 16 & 4.6 & 1.4 & 3.3 & NR & NA \\
\hline
\end{tabular}


Table 7.48 (continued)

\begin{tabular}{|c|c|c|c|c|c|c|c|c|c|}
\hline VARIABLE & $\begin{array}{c}\# \\
\text { SAMPLES }\end{array}$ & $\begin{array}{c}* \\
\text { DETECTED }\end{array}$ & $\begin{array}{c}\text { MAXIMUM } \\
\text { DETECTED } \\
\text { MMT. }\end{array}$ & & $\begin{array}{l}\text { MINIMUM } \\
\text { DETECTED } \\
\text { MMT. }\end{array}$ & & $\begin{array}{l}\text { AVERAGE } \\
\text { DETECTED } \\
\text { MMTS. }\end{array}$ & $\begin{array}{l}\text { REFERENCE } \\
\text { VALUE }\end{array}$ & $\begin{array}{l}\text { \# MMTS. > } \\
\text { REFERENCE }\end{array}$ \\
\hline ICAP (MG/L) & 16 & 16 & 0.024 & & 0.015 & & 0.018 & NR & NA \\
\hline FILTERED ICAP (MG/L) & 16 & 16 & 0.023 & & 0.015 & & 0.018 & NR & NA \\
\hline URANIUM FILT. FLUORIMETRIC (MG/L) & 16 & 3 & 0.0010 & & 0.0010 & & 0.0010 & 13.000 & 0 \\
\hline ICAP (MG/L) & 16 & 16 & 0.081 & & 0.0071 & & 0.019 & 5.000 & 0 \\
\hline FILTERED ICAP (MG/L) & 16 & 16 & 0.026 & & 0.0065 & & 0.013 & 5.000 & 0 \\
\hline CONDUCTIVITY, FIELD MMT (UMHOS/CM) & 16 & NA & 470 & & 370 & & 420 & NR & NA \\
\hline DISSOLVED OXYGEN, FIELD MMT. (PPM) & 16 & NA & 11 & & 5.2 & & 7.7 & NR & $\mathrm{NA}$ \\
\hline PH, FIELD MMT. & 16 & NA & 8.0 & & 7.3 & & 7.6 & $6.5 / 8.5$ & 0 \\
\hline REDOX, FIELD MMT. & 16 & NA & 270 & & 35 & & 180 & NR & NA \\
\hline WATER TEMP, FIELD MMT (DEG. CENT.) & 16 & $\mathrm{NA}$ & 20 & & 12 & & 15 & NR & 0 \\
\hline ALKALINITY-HCO3 & 16 & 16 & 250 & & 210 & & 230 & NR & NA \\
\hline CONDUCTIVITY & 16 & 16 & 500 & & 410 & & 460 & NR & NA \\
\hline DISSOLVED SOLIDS & 16 & 16 & 290 & & 220 & & 250 & 500.000 & 0 \\
\hline (PH UNITS) & 16 & NA & 7.9 & & 7.5 & & 7.6 & $6.5 / 8.5$ & 0 \\
\hline TOTAL SUSPENDED SOLIDS & 16 & 9 & 15 & & 1.0 & & 5.8 & NR & NA \\
\hline TURBIDITY & 16 & 16 & 27 & & 0.40 & & 5.7 & 1.000 & 8 \\
\hline GRCSS ALPHA & 16 & 16 & 5.2 & & -1.9 & & 0.48 & 15.000 & 0 \\
\hline GROSS BETA & 16 & 16 & 14 & & -8.1 & & 0.68 & 50.000 & 0 \\
\hline 4-METHYL-2-PENTANONE & 16 & 3 & 2.0 & $\mathrm{BJ}$ & 2.0 & BJ & 2.0 & NR & NA \\
\hline ACETONE & 16 & 2 & 4.0 & $\mathrm{BJ}$ & 1.0 & $\mathrm{BJ}$ & 2.5 & NR & NA \\
\hline CHLOROFORM & 16 & 1 & 0.70 & $\mathbf{J}$ & 0.70 & $\mathbf{J}$ & 0.70 & 100.000 & 0 \\
\hline METHYLENE CHLORIDE & 16 & 2 & 1.0 & $\mathrm{~J}$ & 1.0 & $\mathbf{J}$ & 1.0 & NR & $\mathrm{NA}$ \\
\hline
\end{tabular}


Table 7.49. Constituents In groundwater at the Y-12 Plant site $H G R=C R$ area=Kerr Hollow Quarry

\begin{tabular}{|c|c|c|c|c|c|c|c|c|c|c|}
\hline & VARIABLE & & & $\begin{array}{c}\# \\
\text { SAMPLES }\end{array}$ & $\begin{array}{c}\# \\
\text { DETECTED }\end{array}$ & $\begin{array}{c}\text { MAXIMUM } \\
\text { DETECTED } \\
\text { MMT. }\end{array}$ & $\begin{array}{c}\text { MINIMUM } \\
\text { DETECTED } \\
\text { MMT. }\end{array}$ & $\begin{array}{l}\text { AVERAGE } \\
\text { DETECTED } \\
\text { MMTS. }\end{array}$ & $\begin{array}{c}\text { REFERENCE } \\
\text { VALUE }\end{array}$ & $\begin{array}{l}\text { \# MMTS. > } \\
\text { REFERENCE }\end{array}$ \\
\hline CHLORIDE & & & (MG/L) & 28 & 28 & 13 & 1.0 & 4.8 & 250.000 & 0 \\
\hline FLUORIDE & & & $(M G / L)$ & 28 & 23 & 3.4 & 0.10 & 1.6 & 2.000 & 11 \\
\hline NITRATE NIT & OGEN & & $(M G / L)$ & 28 & 16 & 1.4 & 0.30 & 0.73 & 10.000 & 0 \\
\hline SULFATE & & & $(M G / L)$ & 28 & 28 & 71 & 2.9 & 21 & 250.000 & 0 \\
\hline ALUMINUM & & ICAP & (MG/L) & 28 & 22 & 2.3 & 0.020 & 0.33 & 0.200 & 7 \\
\hline ALUMINUM & FILTERED & ICAP & $(M G / L)$ & 28 & 13 & 0.046 & 0.022 & 0.032 & 0.200 & 0 \\
\hline BARIUM & & ICAP & (MG/L) & 28 & 28 & 0.49 & 0.022 & 0.13 & 1.000 & 0 \\
\hline BARIUM & FILTERED & ICAP & (MG/L) & 28 & 28 & 0.47 & 0.022 & 0.12 & 1.000 & 0 \\
\hline BORON & & ICAP & (MG/L) & 28 & 28 & 1.0 & 0.014 & 0.26 & NR & $\mathrm{NA}$ \\
\hline BORON & FILTERED & ICAP & (MG/L) & 28 & 28 & 1.0 & 0.0089 & 0.27 & NR & NA \\
\hline CALCIUM & & ICAP & $(M G / L)$ & 28 & 28 & 61 & 27 & 40 & NR & NA \\
\hline CALCIUM & FILTERED & ICAP & $(M G / L)$ & 28 & 28 & 50 & 27 & 38 & NR & NA \\
\hline CHROMIUM & & AAS & (MG/L) & 28 & 1 & 0.016 & 0.016 & 0.016 & 0.050 & 0 \\
\hline CHROMIUM & & ICAP & (MG/L) & 28 & 1 & 0.024 & 0.024 & 0.024 & 0.050 & 0 \\
\hline COPPER & & ICAP & $(\mathrm{MG} / \mathrm{L})$ & 28 & 11 & 0.021 & 0.0042 & 0.0077 & 1.000 & 0 \\
\hline COPPER & FILTERED & ICAP & $(M G / L)$ & 28 & 3 & 0.0085 & 0.0043 & 0.0057 & 1.000 & 0 \\
\hline IRON & & ICAP & (MG/L) & 28 & 26 & 64 & 0.0052 & 4.3 & 0.300 & 17 \\
\hline IRON & FILTERED & ICAP & (MG/L) & 28 & 12 & 1.8 & 0.0055 & 0.19 & 0.300 & 1 \\
\hline LEAD & & AAS & (MG/L) & 28 & 5 & 0.035 & 0.0042 & 0.017 & 0.050 & 0 \\
\hline MAGNESIUM & & ICAP & $(M G / L)$ & 28 & 28 & 44 & 14 & 25 & NR & NA \\
\hline MAGNESIUM & FILTERED & ICAP & $(M G / L)$ & 28 & 28 & 35 & 14 & 25 & NR & NA \\
\hline MANGANESE & & ICAP & (MG/L) & 28 & 23 & 0.31 & 0.0016 & 0.062 & 0.050 & 5. \\
\hline MANGANESE & FILTERED & ICAP & $(M G / L)$ & 28 & 20 & 0.071 & 0.0012 & 0.023 & 0.050 & 3 \\
\hline MOLYBDENUM & & ICAP & $(M G / L)$ & 28 & 5 & 0.020 & 0.010 & 0.016 & NR & NA \\
\hline MOLYBDENUM & FILTERED & ICAP & (MG/L) & 28 & 4 & 0.016 & 0.011 & 0.014 & NR & NA \\
\hline NICKEL & & ICAP & $(M G / L)$ & 28 & 3 & 0.19 & 0.011 & 0.071 & $0.100 * *$ & 1 \\
\hline NICKEL & FILTERED & ICAP & (MG/L) & 28 & 1 & 0.013 & 0.013 & 0.013 & $0.100 * *$ & 0 \\
\hline POTASSIUM & & ICAP & $(M G / L)$ & 28 & 28 & 18 & 0.89 & 8.0 & NR & NA \\
\hline POTASSIUM & FILTERED & ICAP & (MG/L) & 28 & 28 & 18 & 0,85 & 7.9 & NR & NA \\
\hline
\end{tabular}


Table 7.49 (continued)

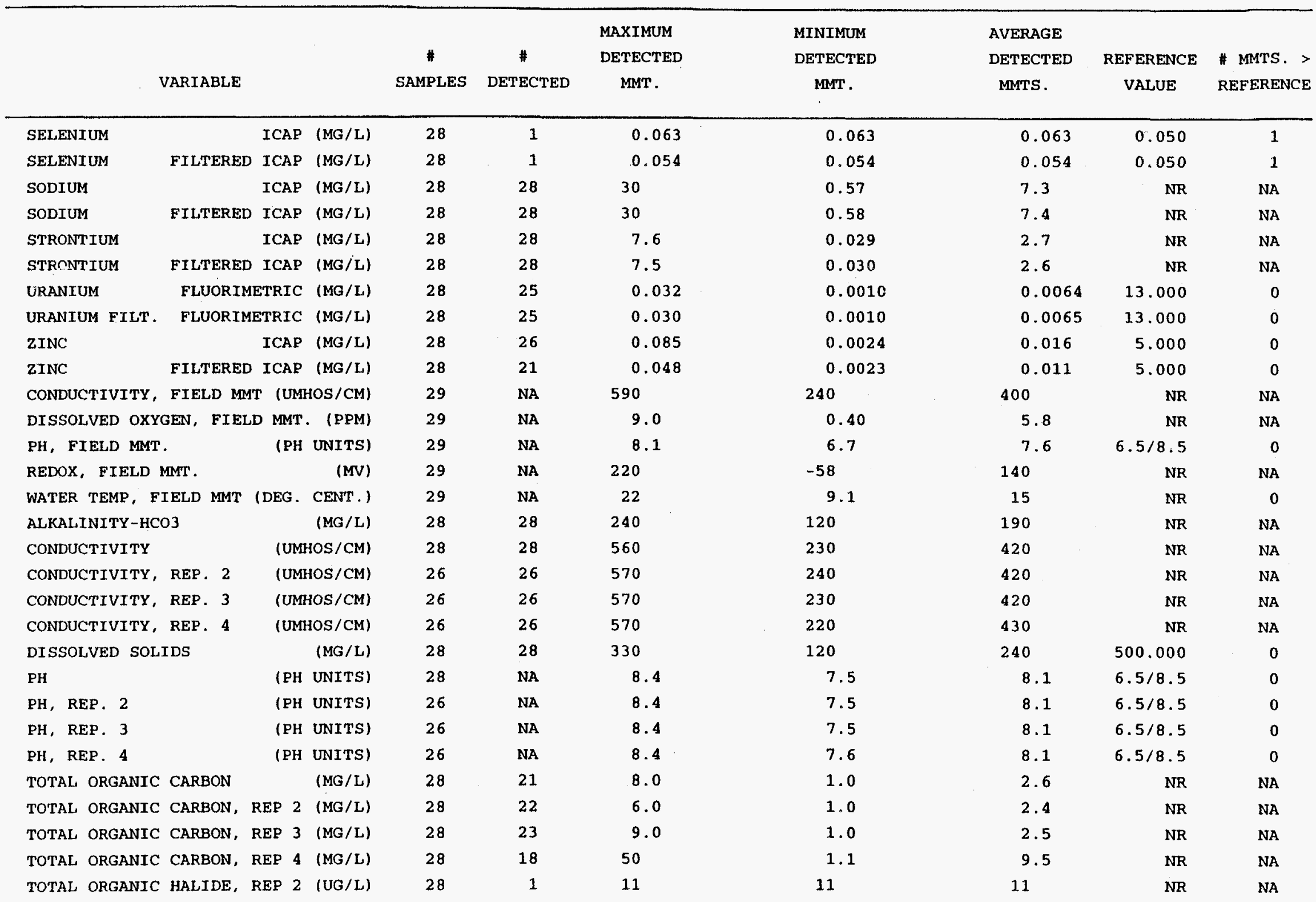

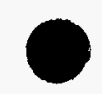


Table 7.49 (continued)

\begin{tabular}{|c|c|c|c|c|c|c|c|c|c|c|}
\hline VARIABLE & & $\begin{array}{c}\# \\
\text { SAMPLES }\end{array}$ & $\begin{array}{c}\# \\
\text { DETECTED }\end{array}$ & $\begin{array}{c}\text { MAXIMUM } \\
\text { DETECTED } \\
\text { MMT. }\end{array}$ & & $\begin{array}{c}\text { MINIMUM } \\
\text { DETECTED } \\
\text { MMT . }\end{array}$ & & $\begin{array}{l}\text { AVERAGE } \\
\text { DETECTED } \\
\text { MMTS. }\end{array}$ & $\begin{array}{c}\text { REFERENCE } \\
\text { VALUE }\end{array}$ & $\begin{array}{l}\text { \# MMTS. > } \\
\text { REFERENCE }\end{array}$ \\
\hline TOTAL ORGANIC HALIDE, REP 3 & $3(U G / L)$ & 28 & 1 & 11 & & 11 & & 11 & NR & NA \\
\hline TOTAL ORGANIC HALIDE, REP 4 & 4 (UG/L) & 28 & 1 & 11 & & 11 & & 11 & NR & NA \\
\hline TOTAL SUSPENDED SOLIDS & $(M G / L)$ & 28 & 23 & 2000 & & 1.0 & & 110 & NR & NA \\
\hline TURBIDITX & (NTU) & 28 & 28 & 800 & & 0.40 & & 67 & 1.000 & 24 \\
\hline GROSS ALPHA & $(\mathrm{PCI} / L)$ & 28 & 28 & 15 & & -0.80 & & 5.3 & 15.000 & 0 \\
\hline GROSS BETA & $(\mathrm{PCI} / \mathrm{L})$ & 28 & 28 & 23 & & -4.9 & & 9.6 & 50.000 & 0 \\
\hline 2-BUTANONE & $(U G / L)$ & 28 & 7 & 10 & JB & 7.0 & But & 8.9 & NR & NA \\
\hline 4-METHYL-2-PENTANONE & $(U G / L)$ & 28 & 2 & 2.0 & $\mathrm{BJ}$ & 1.0 & BJ & 1.5 & NR & NA \\
\hline ACETONE & (UG/L) & 28 & 3 & 10 & B & 3.0 & $\mathrm{BJ}$ & 6.3 & NR & NA \\
\hline BENZENE & $(U G / L)$ & 28 & 3 & 1.0 & JB & 1.0 & JB & 1.0 & 5.000 & 0 \\
\hline CARBON TETRACHLORIDE & (UG/L) & 28 & 3 & 3.0 & $J$ & 0.80 & $\mathfrak{J}$ & 2.3 & 5.000 & 0 \\
\hline CHLOROFORM & $(U G / L)$ & 28 & 4 & 4.0 & $\mathfrak{J}$ & 0.70 & $\mathrm{~J}$ & 1.6 & 100.000 & 0 \\
\hline METHYLENE CHLORIDE & (UG/L) & 2.8 & 6 & 2.0 & $\mathrm{BJ}$ & 1.0 & $J B$ & 1.2 & NR & NA \\
\hline TRICHLOROETHENE & $(U G / L)$ & 28 & 1 & 1.0 & $\mathrm{~J}$ & 1.0 & $\mathfrak{J}$ & 1.0 & 5.000 & 0 \\
\hline
\end{tabular}


Table 7.50. Constituents in groundwater at the Y-12 Plant slte HGR=CR area=Rogers Quarry

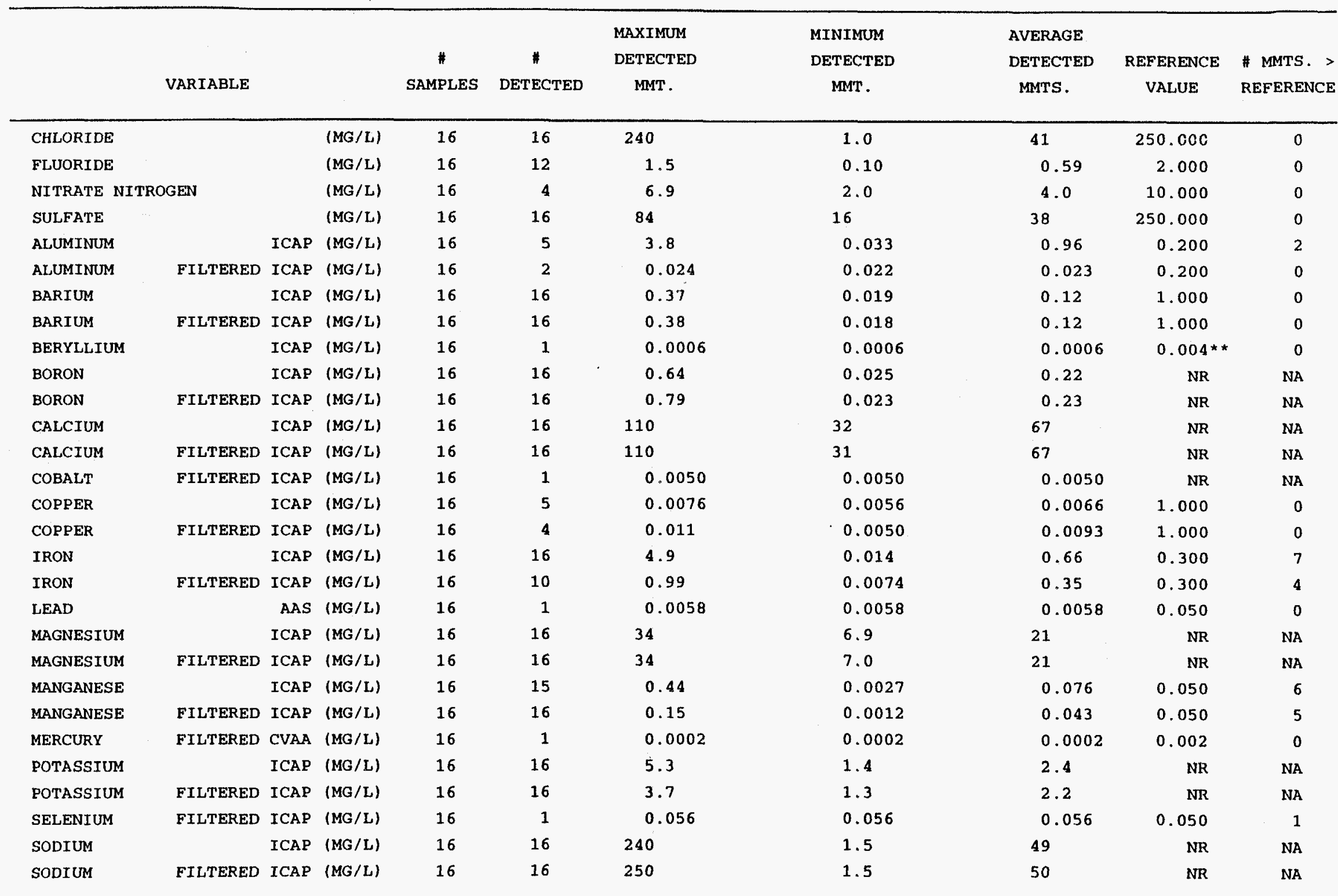


Table 7.50 (continued)

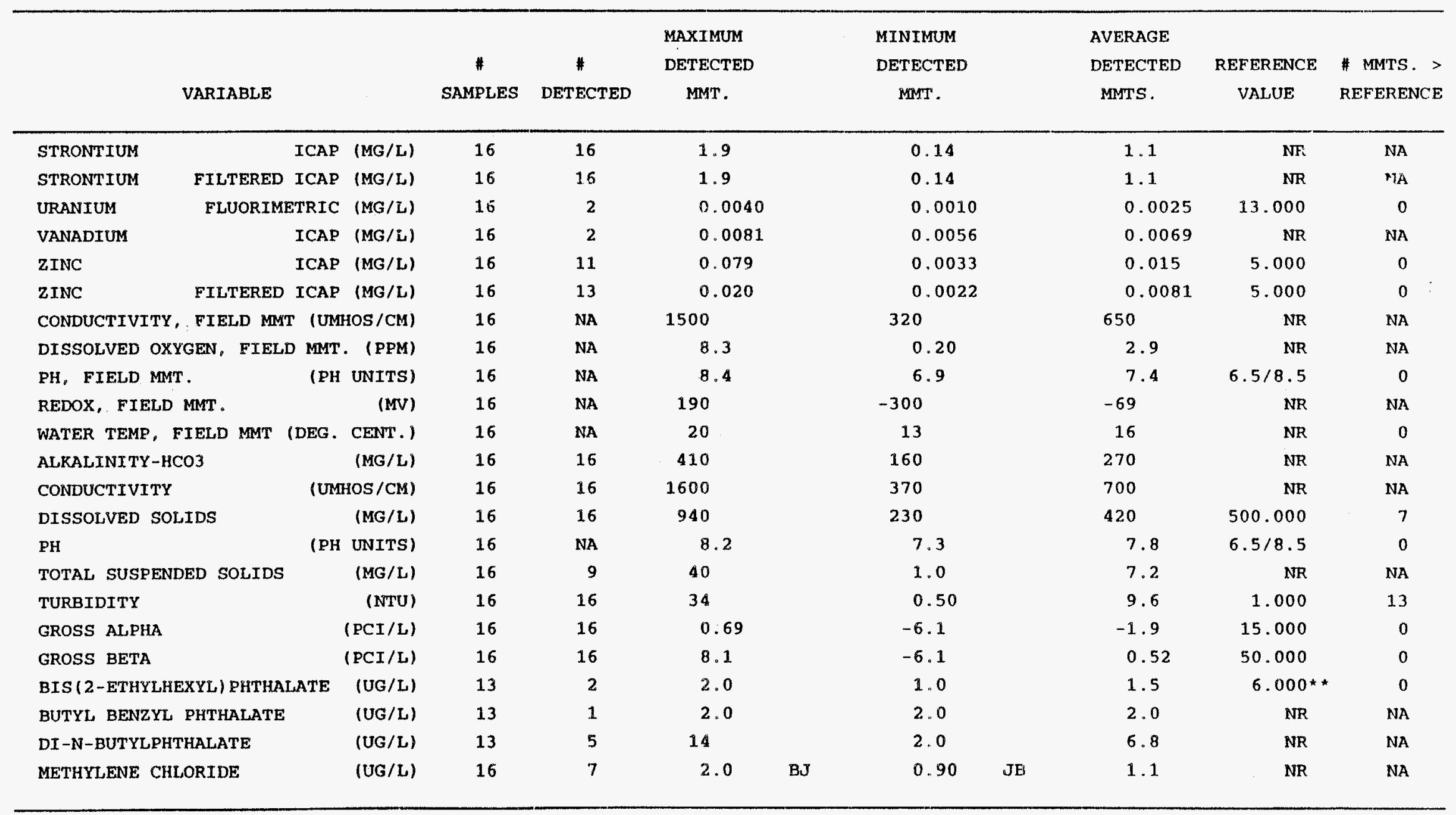


Table 7.51. Constituents in groundwater at the Y-12 Plant site

$H G R=C R$ area $=$ Securily Pits

\begin{tabular}{|c|c|c|c|c|c|c|c|c|c|c|}
\hline \multicolumn{4}{|c|}{ VARIABLE } & \multirow{2}{*}{$\begin{array}{c}\$ \\
\text { SAMPLES } \\
41\end{array}$} & \multirow{2}{*}{$\frac{\|}{\text { DETECTED }}$} & \multirow{2}{*}{$\begin{array}{c}\text { MAXIMUM } \\
\begin{array}{c}\text { DETECTED } \\
\text { MMT. }\end{array} \\
3.0\end{array}$} & \multirow{2}{*}{$\begin{array}{c}\text { MINIMUM } \\
\text { DETECTED } \\
\text { MMT }\end{array}$} & \multirow{2}{*}{$\begin{array}{l}\text { AVERAGE } \\
\text { DETËCTED } \\
\text { MMTS. } \\
2.1\end{array}$} & \multirow{2}{*}{$\begin{array}{c}\begin{array}{c}\text { REFERENCE } \\
\text { VALUE }\end{array} \\
250.000\end{array}$} & \multirow{2}{*}{$\begin{array}{c}\text { \# MMTS. > } \\
\text { REFERENCE } \\
0\end{array}$} \\
\hline CHLORIDE & & & $(M G / L)$ & & & & & & & \\
\hline NITRATE NI & GEN & & (MG/L) & 41 & 20 & 3.1 & 0.24 & 1.1 & 10.000 & $\mathbf{0}$ \\
\hline SULFATE & & & $(M G / L)$ & 41 & 38 & 13 & 1.0 & 5.2 & 250.000 & 0 \\
\hline ALUMINUM & & ICAP & $(M G / L)$ & 41 & 21 & 4.5 & 0.020 & 0.28 & 0.200 & 4 \\
\hline ALUMINUM & FILTERED & ICAP & $(M G / L)$ & 41 & 17 & 0.041 & 0.021 & 0.027 & 0.200 & 0 \\
\hline ARSENIC & & ICAP & $(M G / L)$ & 41 & 2 & 0.061 & 0.058 & 0.060 & 0.050 & 2 \\
\hline BARIUM & & ICAP & (MG/L) & 41 & 41 & 0.16 & 0.0074 & 0.034 & 1.000 & 0 \\
\hline BARIUM & FILTERED & ICAP & (MG/L) & 41 & 41 & 0.11 & 0.0076 & 0.031 & 1.000 & 0 \\
\hline BERYLLIUM & & ICAP & $(M G / L)$ & 41 & 4 & 0.0005 & 0.0003 & 0.0004 & $0.004 * *$ & 0 \\
\hline BERYLLIUM & FILTERED & ICAP & (MG/L) & 41 & 1 & 0.0004 & 0.0004 & 0.0004 & $0.004 * *$ & 0 \\
\hline BORON & & ICAP & $(M G / L)$ & 41 & 40 & 0.15 & 0.0045 & 0.046 & $\mathrm{NR}$ & NA \\
\hline BORON & FILTERED & ICAP & (MG/L) & 41 & 40 & 0.19 & 0.0053 & 0.041 & $\mathrm{NR}$ & NA \\
\hline CADMIUM & & ICAP & (MG/L) & 41 & 1 & 0.0034 & 0.0034 & 0.0034 & 0.005 & 0 \\
\hline CALCIUM & & ICAP & $(M G / L)$ & 41 & 41 & 89 & 27 & 41 & NR & NA \\
\hline CALCIUM & FILTERED & ICAP & (MG/L) & 41 & 41 & 59 & 28 & 39 & NR & NA \\
\hline CHROMIUM & & ICAP & (MG/L) & 41 & 1 & 0.046 & 0.046 & 0.046 & 0.050 & 0 \\
\hline COPPER & & ICAP & $(M G / L)$ & 41 & 7 & 0.062 & 0.0049 & 0.014 & 1.000 & 0 \\
\hline COPPER & FILTERED & ICAP & (MG/L) & 41 & 5 & 0.0065 & 0.0041 & 0.0049 & 1.000 & 0 \\
\hline IRON & & ICAP & (MG/L) & 41 & 35 & 7.9 & 0.0058 & 1.1 & 0.300 & 12 \\
\hline IRON & FILTERED & ICAP & (MG/L) & 41 & 11 & 1.0 & 0.0063 & 0.14 & 0.300 & 2 \\
\hline MAGNESIUM & & ICAP & (MG/L) & 41 & 41 & 37 & 16 & 23 & NR & $\mathrm{NA}$ \\
\hline MAGNESIUM & FILTERED & ICAP & $(M G / L)$ & 41 & 41 & 35 & 16 & 23 & NR & NA \\
\hline MANGANESE & & ICAP & $(M G / L)$ & 41 & 31 & 0.17 & 0.0010 & 0.021 & 0.050 & 4 \\
\hline MANGANESE & FILTERED & ICAP & (MG/L) & 41 & 26 & 0.10 & 0.0011 & 0.011 & 0.050 & 2 \\
\hline NICKEL & & ICAP & $(M G / L)$ & 41 & 2 & 0.043 & 0.022 & 0.033 & $0.100 * *$ & 0 \\
\hline NICKEL & FILTERED & ICAP & (MG/L) & 41 & 1 & 0.016 & 0.016 & 0.016 & $0.100 \star \star$ & 0 \\
\hline POTASSIUM & & ICAP & (MG/L) & 41 & 33 & 4.4 & 0.66 & 1.6 & $\mathrm{NR}$ & NA \\
\hline POTASSIUM & FILTERED & ICAP & (MG/L) & 41 & 34 & 4.5 & 0.62 & 1.6 & NR & NA \\
\hline SELENIUM & & ICAP & $(M G / L)$ & 41 & 1 & 0.052 & 0.052 & 0.052 & 0.050 & 1 \\
\hline
\end{tabular}


Table 7.51 (continued)

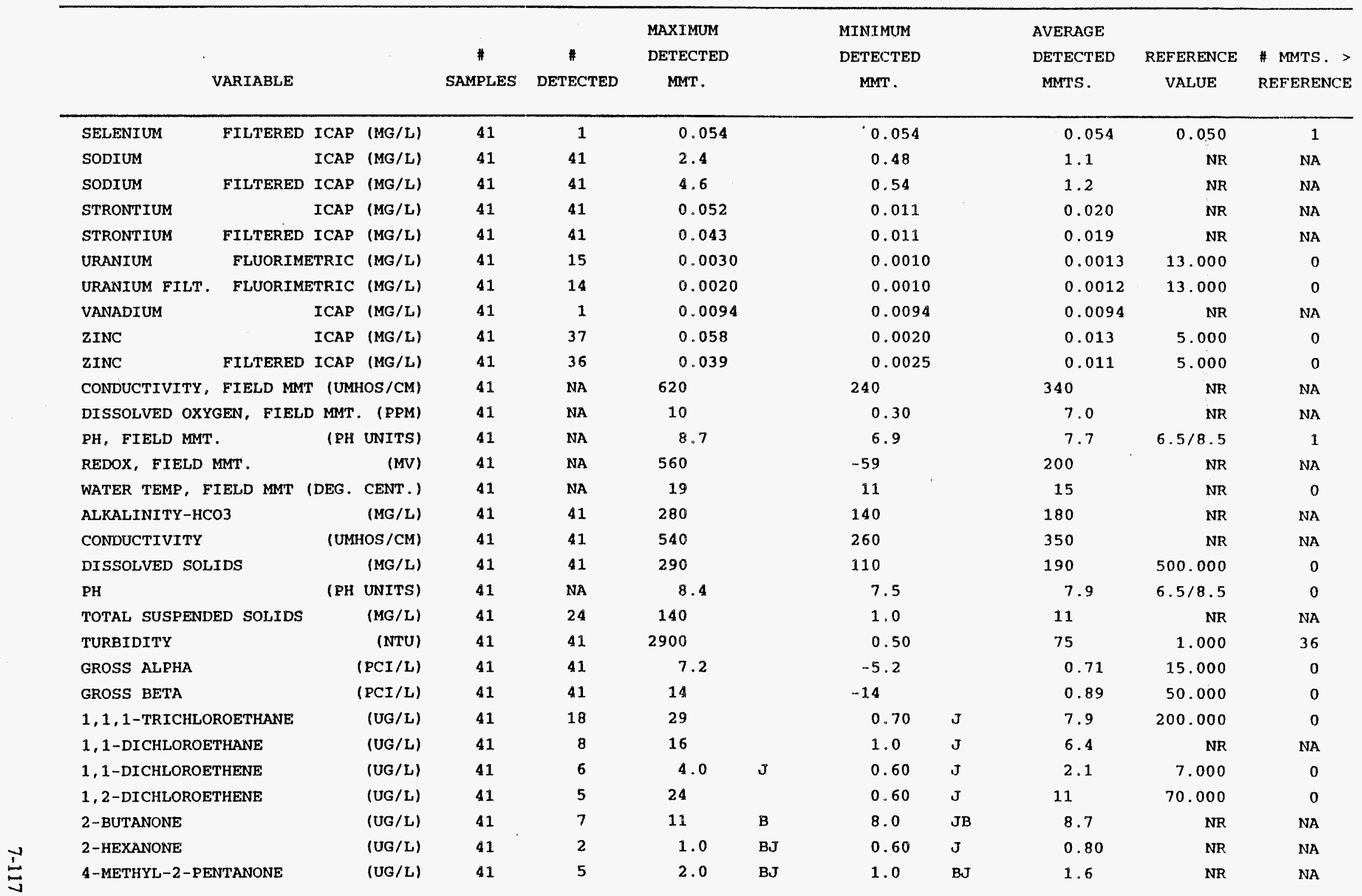


Table 7.51 (contlnued)

\begin{tabular}{|c|c|c|c|c|c|c|c|c|c|c|}
\hline VARIABLE & & $\begin{array}{c}\# \\
\text { SAMPLES }\end{array}$ & $\begin{array}{c}\# \\
\text { DETECTED }\end{array}$ & $\begin{array}{c}\text { MAXIMUM } \\
\text { DETECTED } \\
\text { MMT. }\end{array}$ & & $\begin{array}{c}\text { MINIMUM } \\
\text { DETECTED } \\
\text { MMT. }\end{array}$ & & $\begin{array}{l}\text { AVERAGE } \\
\text { DETECTED } \\
\text { MMTS. }\end{array}$ & $\begin{array}{l}\text { REFERENCE } \\
\text { VALUE }\end{array}$ & $\begin{array}{l}\# \text { MMTS. > } \\
\text { REFERENCE }\end{array}$ \\
\hline ACETONE & (UG/L) & 41 & 7 & 4.0 & BJ & 2.0 & BJ & 3.1 & NR & NA \\
\hline CARBON TETRACHLORIDE & $(\mathrm{UG} / \mathrm{L})$ & 41 & 1 & 0.20 & $\mathrm{~J}$ & 0.20 & $\mathrm{~J}$ & 0.20 & 5.000 & 0 \\
\hline METHYLENE CHLORIDE & $(\mathrm{UG} / \mathrm{L})$ & 41 & 11 & 4.0 & JB & 0.50 & JB & 1.9 & NR & NA \\
\hline TETRACHLOROETHENE & $(\mathrm{UG} / \mathrm{L})$ & 41 & 12 & 27 & & 1.0 & $\mathrm{~J}$ & 9.3 & 5.000 & 7 \\
\hline TRICHLOROETHENE & (UG/L) & 41 & 2 & 3.0 & $\mathrm{~J}$ & 1.0 & $\mathrm{~J}$ & 2.0 & 5.000 & 0 \\
\hline
\end{tabular}


Table 7.52. Constituents in groundwater at the $Y-12$ Plant slte

$H G R=C R$ area $=$ Sediment Disposal Basin

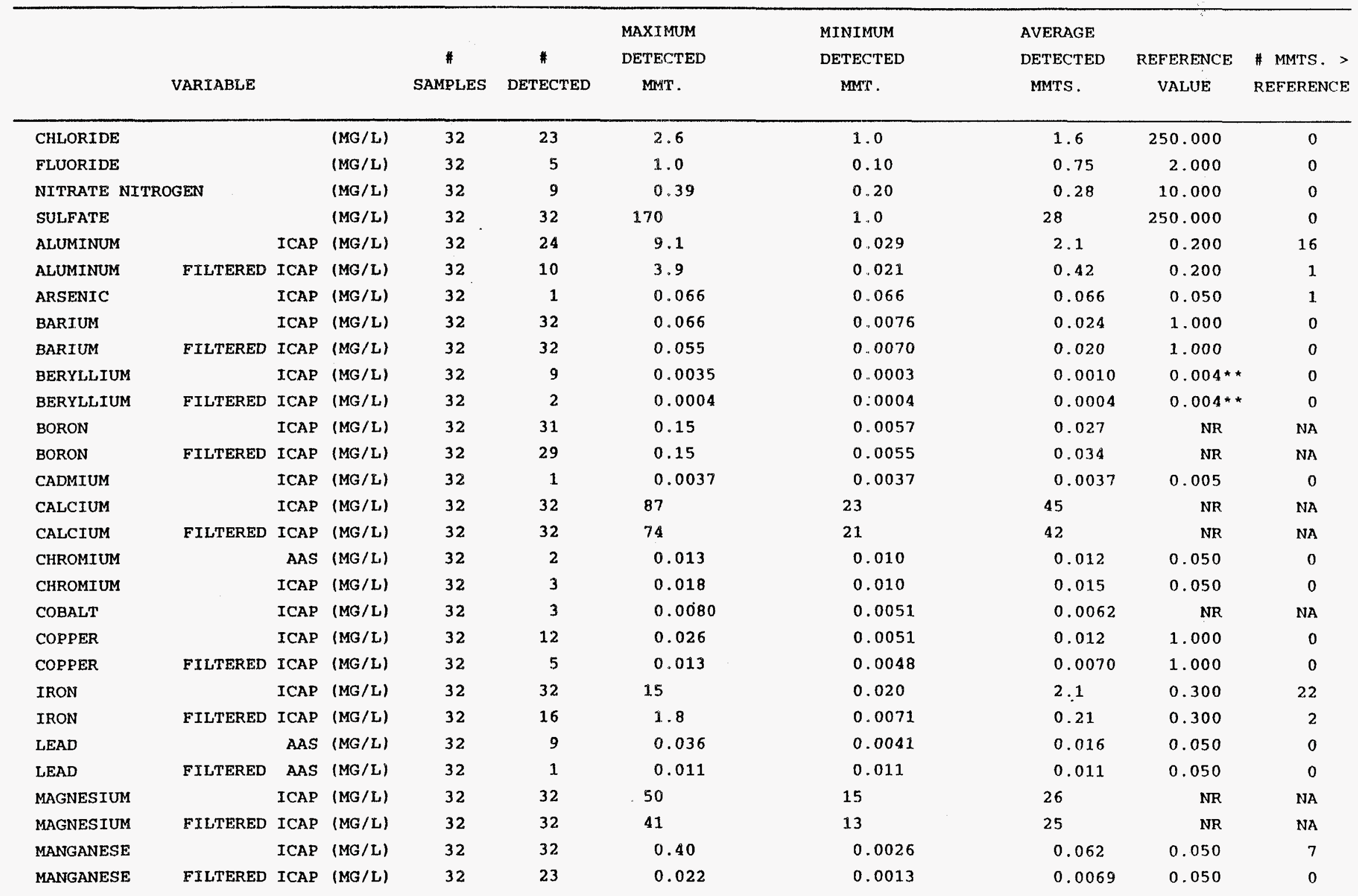


Table 7.52 (contlnued)

\begin{tabular}{|c|c|c|c|c|c|c|c|c|}
\hline VARIABLE & & $\begin{array}{c}\# \\
\text { SAMPLES }\end{array}$ & $\begin{array}{c}* \\
\text { DETECTED }\end{array}$ & $\begin{array}{c}\text { MAXIMUM } \\
\text { DETECTED } \\
\text { MMT. }\end{array}$ & $\begin{array}{l}\text { MINIMUM } \\
\text { DETECTED } \\
\text { MMT. }\end{array}$ & $\begin{array}{l}\text { AVERAGE } \\
\text { DETECTED } \\
\text { MMTS. }\end{array}$ & $\begin{array}{c}\text { REFERENCE } \\
\text { VALUE }\end{array}$ & $\begin{array}{l}\text { \# MMTS. > } \\
\text { REFERENCE }\end{array}$ \\
\hline MERCURY & CVAA (MG/L) & 32 & 1 & 0.0002 & 0.0002 & 0.0002 & 0.002 & 0 \\
\hline NICKEL & ICAP (MG/L) & 32 & 9 & 0.029 & 0.011 & 0.020 & $0.100 * \star$ & 0 \\
\hline FILTERED & ICAP $(M G / L)$ & 32 & 1 & 0.011 & 0.011 & 0.011 & $0.100 * \star$ & 0 \\
\hline POTASSIUM & ICAP (MG/L) & 32 & 32 & 9.6 & 0.79 & 3.0 & NR & NA \\
\hline POTASSIUM & ICAP (MG/L) & 32 & 32 & 10 & 0.82 & 3.0 & NR & NA \\
\hline SELENIUM & ICAP $(M G / L)$ & 32 & 1 & 0.051 & 0.051 & 0.051 & 0.050 & 1 \\
\hline FILTERED & ICAP (MG/L) & 32 & 1 & 0.064 & 0.064 & 0.064 & 0.050 & 1 \\
\hline SODIUM & ICAP (MG/L) & 32 & 32 & 7.6 & 0.50 & 2.2 & NR & NA \\
\hline FILTERED & ICAP (MG/L) & 32 & 32 & 7.6 & 0.52 & 2.2 & NR & NA \\
\hline STRONTIUM & $\operatorname{ICAP}(M G / L)$ & 32 & 32 & 3.1 & 0.018 & 0.40 & NR & NA \\
\hline STRONTIUM & ICAP (MG/L) & 32 & 32 & 3.1 & 0.016 & 0.39 & NR & NA \\
\hline FLUORIME & ETRIC (MG/L) & 32 & 23 & 0.0070 & 0.0010 & 0.0017 & 13.000 & 0 \\
\hline URANIUM FILT. FLUORIME & ETRIC (MG/L) & 32 & 18 & 0.0040 & 0.0010 & 0.0016 & 13.000 & 0 \\
\hline VANADIUM & ICAP (MG/L) & 32 & 6 & 0.033 & 0.0054 & 0.017 & NR & NA \\
\hline ZINC & ICAP (MG/L) & 32 & 28 & 0.23 & 0.0023 & 0.041 & 5.000 & 0 \\
\hline FILTERED & ICAP (MG/L) & 32 & 23 & 0.069 & 0.0023 & 0.016 & 5.000 & 0 \\
\hline CONDUCTIVITY, FIELD MMT & T (UMHOS/CM) & 34 & NA & 640 & 220 & 380 & NR & NA \\
\hline DISSOLVED OXYGEN， FIELD & D MMT, (PPM) & 34 & NA & 9.9 & 0.10 & 5.9 & NR & NA \\
\hline PH, FIELD MMT. & (PH UNITS) & 34 & NA & 9.0 & 7.1 & 7.8 & $6.5 / 8.5$ & 2 \\
\hline REDOX, FIELD MMT. & $(M V)$ & 34 & NA & 250 & -170 & 120 & NR & NA \\
\hline WATER TEMP, FIELD MMT & (DEG. CENT.) & 34 & NA & 20 & 9.3 & 15 & NR & 0 \\
\hline ALKALINITY-CO3 & (MG/L) & 32 & 7 & 8.0 & 2.0 & 3.7 & NR & NA \\
\hline ALKALINITY-HCO3 & $(M G / L)$ & 32 & 32 & 360 & 100 & 190 & NR & NA \\
\hline CONDUCTIVITY & (UMHOS/CM) & 34 & 34 & 660 & 230 & 400 & NR & NA \\
\hline CONDUCTIVITY, REP. 2 & (UMHOS/CM) & 32 & 32 & 650 & 230 & 390 & NR & NA \\
\hline CONDUCTIVITY, REP. 3 & (UMHOS/CM) & 32 & 32 & 660 & 230 & 390 & NR & NA \\
\hline CONDUCTIVITY, REP. 4 & (UMHOS/CM) & 32 & 32 & 650 & 230 & 390 & NR & NA \\
\hline DISSOLVED SOLIDS & $(M G / L)$ & 32 & 32 & 480 & 140 & 250 & 500.000 & 0 \\
\hline PH & (PH UNITS) & 34 & $\mathrm{NA}$ & 8.7 & 7.7 & 8.1 & $6.5 / 8.5$ & 3 \\
\hline
\end{tabular}


Table 7.52 (continued)

\begin{tabular}{|c|c|c|c|c|c|c|c|c|c|c|}
\hline VARIABLE & & $\begin{array}{c}\# \\
\text { SAMPLES }\end{array}$ & $\begin{array}{c}\# \\
\text { DETECTED }\end{array}$ & $\begin{array}{c}\text { MAXIMUM } \\
\text { DETECTED } \\
\text { MMT. }\end{array}$ & & $\begin{array}{l}\text { MINIMUM } \\
\text { DETECTED } \\
\text { MMT. }\end{array}$ & & $\begin{array}{l}\text { AVERAGE } \\
\text { DETECTED } \\
\text { MMTS. }\end{array}$ & $\begin{array}{c}\text { REFERENCE } \\
\text { VALUE }\end{array}$ & $\begin{array}{l}\text { \# MMTS. > } \\
\text { REFERENCE }\end{array}$ \\
\hline PH, REP. 2 & UNITS) & 32 & NA & 8.7 & & 7.6 & & 8.1 & $6.5 / 8.5$ & 3 \\
\hline PH, REP. 3 & UNITS) & 32 & NA & 8.6 & & 7.6 & & 8.1 & $6.5 / 8.5$ & 2 \\
\hline EH, REP. 4 & (UNITS) & 32 & NA & 8.7 & & 7.5 & & 8.1 & $6.5 / 8.5$ & 3 \\
\hline TOTAL ORGANIC CARBON & $(M G / L)$ & 32 & 20 & 11 & & 1.0 & & 2.5 & NR & NA \\
\hline TOTAL ORGANIC CARBON, REP 2 & (MG/L) & 32 & 22 & 8.0 & & 1.0 & & 2.1 & NR & NA \\
\hline TOTAL ORGANIC CARBON, REP 3 & (MG/L) & 32 & 22 & 11 & & 1. 0 & & 3.0 & NR & NA \\
\hline TOTAL ORGANIC CARBON, REP 4 & (MG/L) & 32 & 18 & 35 & & 1.0 & & 4.2 & NR & NA \\
\hline TOTAL ORGANIC HALIDE & (UG/L) & 32 & 2 & 12 & & 10 & & 11 & NR & NA \\
\hline TOTAL ORGANIC HALIDE, REP 2 & $(U G / L)$ & 32 & 2 & 13 & & 12 & & 13 & NR & NA \\
\hline TOTAL ORGANIC HALIDE, REP 3 & (UG/L) & 32 & 1 & 11 & & 11 & & 11 & NR & NA \\
\hline TOTAL ORGANIC HALIDE, REP 4 & (UG/L) & 32 & 3 & 16 & & 1.1. & & 14 & NR & NA \\
\hline TOTAL SUSPENDED SOLIDS & $(M G / L)$ & 32 & 28 & 590 & & 1. 0 & & 86 & NR & NA \\
\hline TURBIDITY & (NTU) & 32 & 32 & 370 & & 1.5 & & 43 & 1.000 & 32 \\
\hline GROSS ALPHA & $(\mathrm{PCI} / \mathrm{L})$ & 32 & 32 & 19 & & -3.2 & & 3.6 & 15.000 & 3 \\
\hline GROSS BETA & (PCI/L) & 32 & 32 & 22 & & -4.9 & & 2.5 & 50.000 & 0 \\
\hline 2-BUTANONE & (UG/L) & 32 & 1 & 9.0 & BJ & 9.0 & $\mathrm{BJ}$ & 9.0 & NR & NA \\
\hline 4-METHYL-2-PENTANONE & (UG/L) & 32 & 1 & 2.0 & $\mathrm{BJ}$ & 2.0 & $\mathrm{BJ}$ & 2.0 & NR & NA \\
\hline ACETONE & $(U G / L)$ & 32 & 1 & 4.0 & $\mathrm{BJ}$ & 4.0 & $\mathrm{BJ}$ & 4.0 & NR & NA \\
\hline BIS (2-ETHYLHEXYL) PHTHALATE & $(U G / L)$ & 32 & 10 & 11. & & 0.70 & & 3.0 & $6.000 * *$ & 1 \\
\hline BUTYL BENZYL PHTHALATE & (UG/L) & 32 & 1 & 0.60 & & 0.60 & & 0.60 & $\mathrm{NR}$ & NA \\
\hline CARBON DISULFIDE & (UG/L) & 32 & 1 & 3.0 & $\mathrm{~J}$ & 3.0 & $\mathbf{J}$ & 3.0 & NR & NA \\
\hline DI -N-BUTYLPHTHALATE & $(U G / L)$ & 32 & 8 & 17 & & 0.30 & & 6.1 & NR & NA \\
\hline DI-N-OCTYLPHTHALATE & (UG/L) & 32 & 1 & 0.20 & & 0.20 & & 0.20 & NR & NA \\
\hline DIETHYLPHTHALATE & (UG/L) & 32 & 2 & 0.70 & & 0.50 & & 0.60 & NR & NA \\
\hline ISOPHORONE & (UG/L) & 32 & 1 & 2.0 & & 2.0 & & 2.0 & NR & NA \\
\hline METHYLENE CHLORIDE & (UG/L) & 32 & 11 & 1.0 & $J$ & 1.0 & J & 1.0 & NR & NA \\
\hline
\end{tabular}


Table 7.53. Constituents in groundwater at the Y-12 Plant site

$H G R=C R$ area $=$ United Nuclear Site

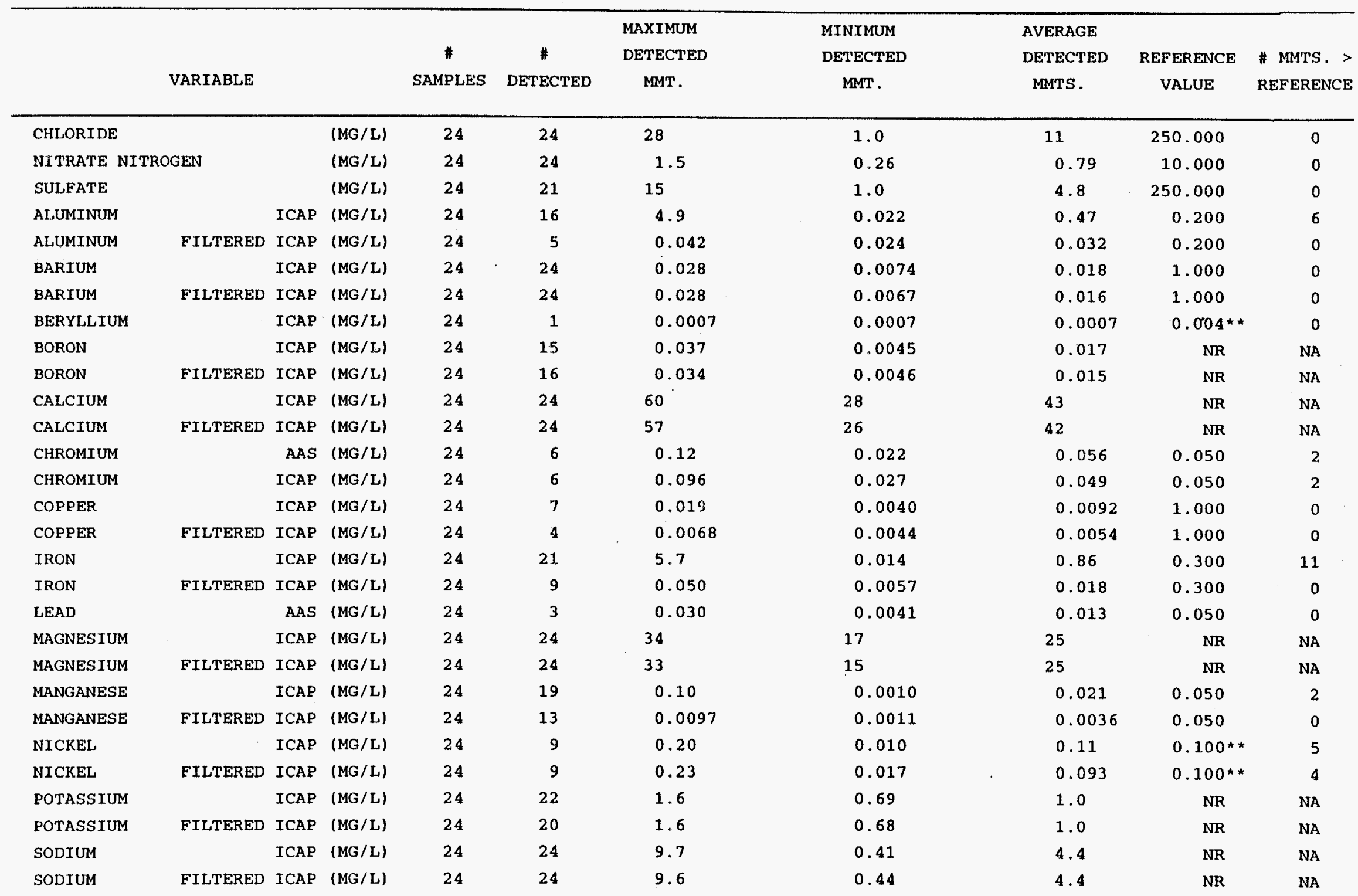

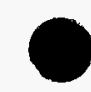


Table 7.53 (contlnued)

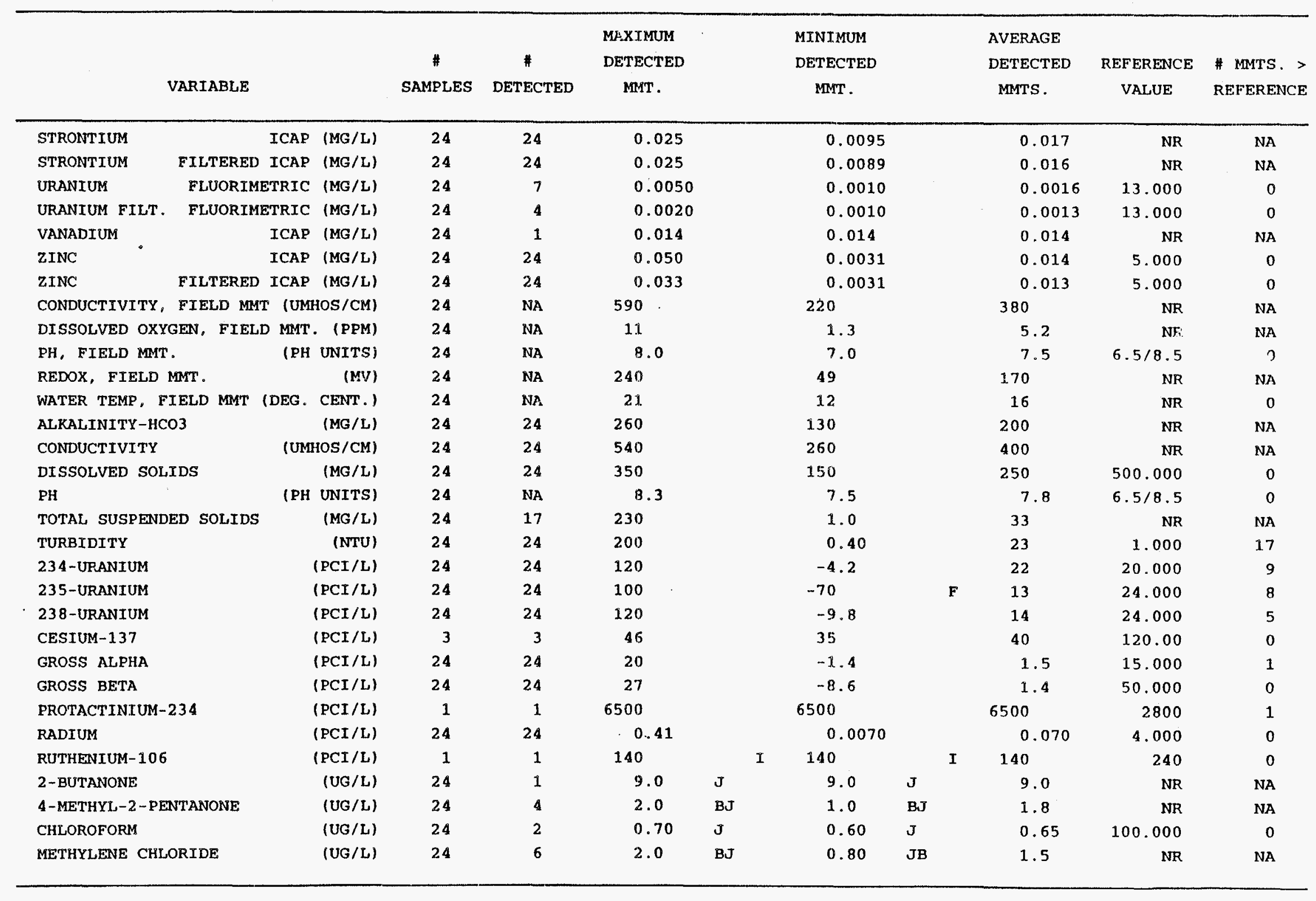


Table 7.54. Constituents in groundwater at the Y-12 Plant site

HGR=CH area=Industrial Landfill II

\begin{tabular}{|c|c|c|c|c|c|c|c|c|c|c|}
\hline \multicolumn{4}{|c|}{ VARIABLE } & \multirow{2}{*}{$\frac{\begin{array}{c}\# \\
\text { SAMPLES }\end{array}}{14}$} & \multirow{2}{*}{$\begin{array}{c}\# \\
\text { DETECTED } \\
14\end{array}$} & \multirow{2}{*}{$\begin{array}{c}\text { MAXIMUM } \\
\text { DETECTED } \\
\text { MMT } \\
32\end{array}$} & \multirow{2}{*}{$\begin{array}{c}\text { MINIMUM } \\
\text { DETECTED } \\
\text { MMT . } \\
1.0\end{array}$} & \multirow{2}{*}{$\begin{array}{c}\begin{array}{r}\text { AVERAGE } \\
\text { DETECTED } \\
\text { MMTS }\end{array} \\
9.2\end{array}$} & \multirow{2}{*}{$\begin{array}{c}\begin{array}{c}\text { REFERENCE } \\
\text { VALUE }\end{array} \\
\text { NR }\end{array}$} & \multirow{2}{*}{$\begin{array}{l}\text { \# MMTS. > } \\
\text { REFERENCE } \\
\text { NA }\end{array}$} \\
\hline CHLORIDE & & & (MG/L) & & & & & & & \\
\hline FLUORIDE & & & $(M G / L)$ & 14 & 7 & 1.8 & 0.10 & 1.5 & 4.000 & 0 \\
\hline NITRATE NI & OGEN & & (MG/L) & 14 & 3 & 0.38 & 0.20 & 0.30 & 10.000 & 0 \\
\hline SULFATE & & & (MG/L) & 14 & 14 & 12 & 1.2 & 7.2 & NR & NA \\
\hline ALUMINUM & & ICAP & $(M G / L)$ & 15 & 14 & 1.4 & 0.021 & 0.34 & NR & $\mathrm{NA}$ \\
\hline ALUMINUM & FILTERED & ICAP & $(M G / L)$ & 13 & 7 & 0.055 & 0.023 & 0.033 & NR & $\mathrm{NA}$ \\
\hline BARIUM & & ICAP & (MG /L) & 15 & 15 & 0.27 & 0.0094 & 0.14 & $2.000 * *$ & 0 \\
\hline BARIUM & FILTERED & ICAP & $(M G / L)$ & 13 & 13 & 0.26 & 0.0078 & 0.13 & $2.000 * *$ & 0 \\
\hline BERYLLIUM & FILTERED & ICAP & $(M G / L)$ & 13 & 1 & 0.0008 & 0.0008 & 0.0008 & $0.004^{* *}$ & 0 \\
\hline BORON & & ICAP & $(M G / L)$ & 15 & 14 & 0.038 & 0.0053 & 0.018 & NR & NA \\
\hline BORON & FILTERED & ICAP & $(M G / L)$ & 13 & 11 & 0.043 & 0.0042 & 0.020 & NR & NA \\
\hline CALCIUM & & ICAP & $(M G / L)$ & 15 & 15 & 54 & 20 & 33 & NR & NA \\
\hline CALCIUM & FILTERED & ICAP & $(M G / L)$ & 13 & 13 & 37 & 26 & 33 & NR & NA \\
\hline CHROMIUM & & AAS & $(M G / L)$ & 15 & 7 & 0.23 & 0.012 & 0.069 & NR & NA \\
\hline CHROMIUM & & ICAP & $(M G / L)$ & 15 & 7 & 0.16 & 0.016 & 0.055 & NR & NA \\
\hline CHROMIUM & FILTERED & AAS & $(M G / L)$ & 13 & 2 & 0.022 & 0.012 & 0.017 & NR & NA \\
\hline COBALT & & ICAP & (MG/L) & 15 & 1 & 0.0072 & 0.0072 & 0.0072 & NR & NA \\
\hline COBALT & FILTERED & ICAP & $(M G / L)$ & 13 & 3 & 0.0078 & 0.0063 & 0.0070 & NR & NA \\
\hline COPPER & & ICAP & $(M G / L)$ & 15 & 4 & 0.029 & 0.0060 & 0.013 & NR & $\mathrm{NA}$ \\
\hline COPPER & FILTERED & ICAP & $(M G / L)$ & 13 & 4 & 0.0079 & 0.0045 & 0.0063 & NR & $\mathrm{NA}$ \\
\hline IRON & & ICAP & $(M G / L)$ & 15 & 14 & 7.0 & 0.041 & 0.98 & $\mathrm{NR}$ & NA \\
\hline IRON & FILTERED & ICAP & $(M G / L)$ & 13 & 4 & 0.025 & 0.0070 & 0.012 & NR & $\mathrm{NA}$ \\
\hline LEAD & & AAS & (MG/L) & 15 & 1 & 0.014 & 0.014 & 0.014 & 0.050 & 0 \\
\hline MAGNESIUM & & ICAP & $(\mathrm{MG} / \mathrm{L})$ & 15 & 15 & 23 & 14 & 19 & $\mathrm{NR}$ & $\mathrm{NA}$ \\
\hline MAGNESIUM & FILTERED & ICAP & (MG/L) & 13 & 13 & 22 & 14 & 19 & NR & NA \\
\hline MANGANESE & & ICAP & (MG/L) & 15 & 14 & 0.11 & 0.0013 & 0.014 & $\mathrm{NR}$ & NA \\
\hline MANGANESE & FILTERED & ICAP & $(M G / L)$ & 13 & 8 & 0.0041 & 0.0013 & 0.0020 & NR & NA \\
\hline NICKEL & & ICAP & $(M G / L)$ & 15 & 5 & 0.26 & 0.049 & 0.12 & $0.100 * *$ & 2 \\
\hline NICKEL & FILTERED & ICAP & (MG/L) & 13 & 5 & 0.081 & 0.028 & 0.042 & $0.100 * *$ & 0 \\
\hline
\end{tabular}

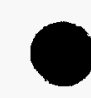


Table 7.54 (continued)

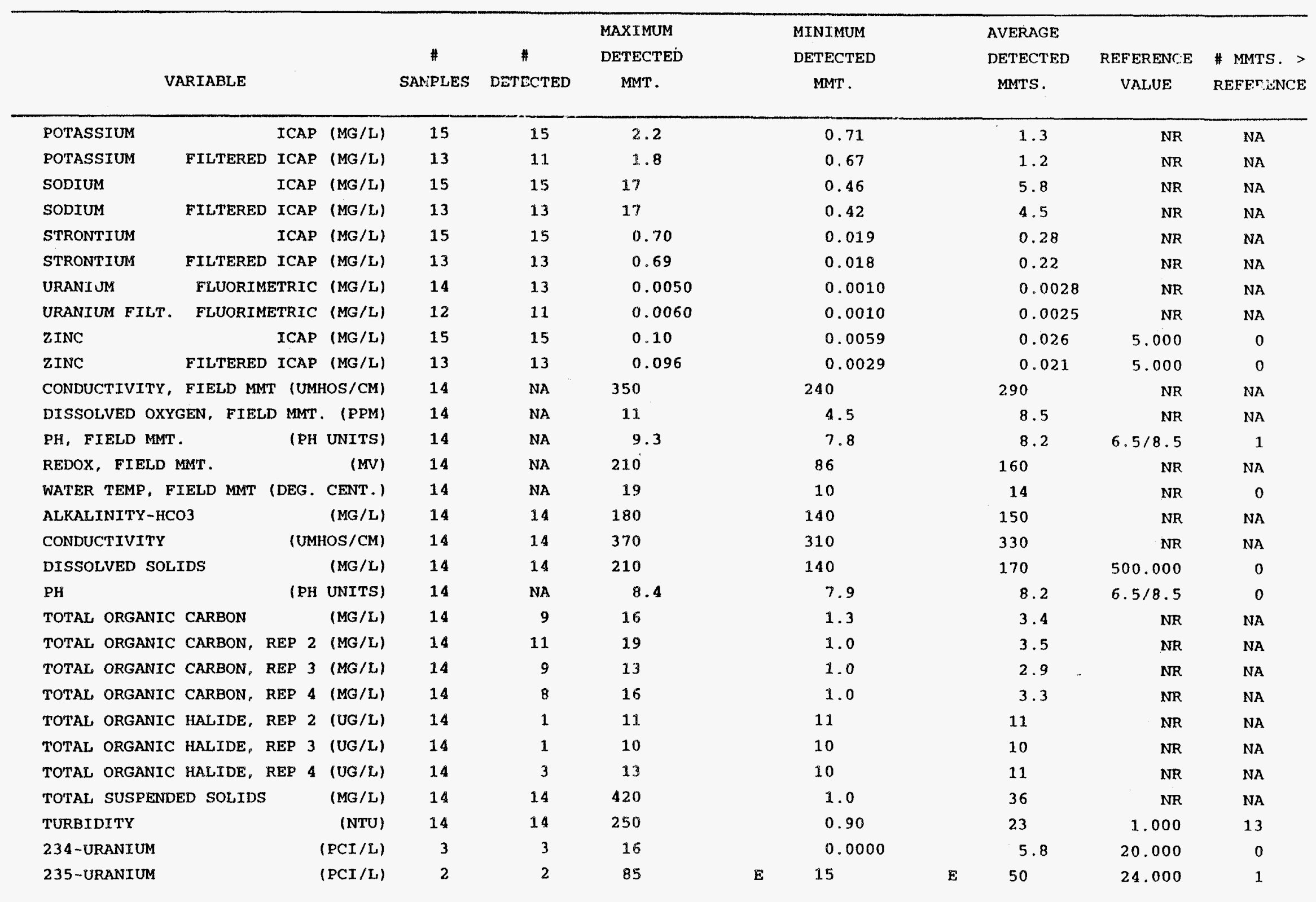


Table 7.54 (contlnued)

\begin{tabular}{|c|c|c|c|c|c|c|c|c|c|c|}
\hline VARIABLE & & $\begin{array}{c}\# \\
\text { SAMPLES }\end{array}$ & $\begin{array}{c}\# \\
\text { DETECTED }\end{array}$ & $\begin{array}{c}\text { MAXIMUM } \\
\text { DETECTED } \\
\text { MMT. }\end{array}$ & & $\begin{array}{c}\text { MINIMUM } \\
\text { DETECTED } \\
\text { MMT. }\end{array}$ & & $\begin{array}{l}\text { AVERAGE } \\
\text { DETECTED } \\
\text { MMTS. }\end{array}$ & $\begin{array}{c}\text { REFERENCE } \\
\text { VALUE }\end{array}$ & $\begin{array}{l}\# \text { MMTS. > } \\
\text { REFERENCE }\end{array}$ \\
\hline 238-URANIUM & $(\mathrm{PCI} / \mathrm{L})$ & 3 & 3 & 11 & & 0.0000 & & 4.0 & 24.000 & 0 \\
\hline GROSS ALPHA & $(\mathrm{PCI} / \mathrm{L})$ & 15 & 15 & 4.1 & & -3.5 & & 1.1 & 15.000 & 0 \\
\hline GROSS BETA & $(\mathrm{PCI} / \mathrm{L})$ & 15 & 15 & 5.2 & $\mathbf{s}$ & -13 & & 0.31 & 50.000 & 0 \\
\hline PROTACTINIUM-234 & $(\mathrm{PCI} / \mathrm{L})$ & 2 & 2 & 3100 & $\mathbf{E}$ & -740 & $\mathbf{F}$ & 1200 & NR & NA \\
\hline 1,2-DICHLOROETHANE & $(U G / L)$ & 14 & 2 & 2.0 & & 2.0 & & 2.0 & 5.000 & 0 \\
\hline 2-BUTANONE & (UG/L) & 14 & 2 & 12 & B & 8.0 & JB & 10 & NR & NA \\
\hline 4-METHYL-2-PENTANONE & (UG/L) & 14 & 3 & 2.0 & BJ & 1.0 & $\mathrm{BJ}$ & 1.7 & $\mathrm{NR}$ & NA \\
\hline ACETONE & (UG/L) & 14 & 2 & 6.0 & BJ & 1.0 & $\mathrm{BJ}$ & 3.5 & NR & NA \\
\hline METHYLENE CHLORIDE & (UG/L) & 14 & 5 & 48 & & 1.0 & JB & 20 & NR & $\mathrm{NA}$ \\
\hline
\end{tabular}


Table 7.55. Constituents in groundwater at the $\mathrm{Y}-12$ Plant site $H G R=C R$ area $=$ Industrial Landfiil III

\begin{tabular}{|c|c|c|c|c|c|c|c|c|c|c|}
\hline & VARIABLE & & & $\begin{array}{c}\# \\
\text { SAMPLES }\end{array}$ & $\begin{array}{c}* \\
\text { DETECTED }\end{array}$ & $\begin{array}{l}\text { MAXIMUM } \\
\text { DETECTED } \\
\text { MMT . }\end{array}$ & $\begin{array}{l}\text { MINIMUM } \\
\text { DETECTED } \\
\text { MMT. }\end{array}$ & $\begin{array}{l}\text { AVERAGE } \\
\text { DETECTED } \\
\text { MMTS. }\end{array}$ & $\begin{array}{c}\text { REFERENCE } \\
\text { VALUE }\end{array}$ & $\begin{array}{l}\text { \# MMTS. > } \\
\text { REFERENCE }\end{array}$ \\
\hline \multicolumn{3}{|l|}{ CHLORIDE } & $(M G / L)$ & 29 & 24 & 160 & 1.0 & 27 & 250.000 & 0 \\
\hline \multicolumn{3}{|l|}{ FLUORIDE } & $(M G / L)$ & 29 & 2 & 0.10 & 0.10 & 0.10 & 2.000 & 0 \\
\hline \multicolumn{3}{|c|}{ NITRATE NITROGEN } & (MG/L) & 29 & 16 & 0.89 & 0.20 & 0.44 & 10.000 & 0 \\
\hline \multicolumn{3}{|l|}{ SULFATE } & (MG/L) & 29 & 29 & 26 & 1.5 & 8.8 & 250.000 & 0 \\
\hline \multicolumn{2}{|l|}{ ALUMINUM } & ICAP & $(M G / L)$ & 29 & 23 & 140 & 0.023 & 7.6 & 0.200 & 12 \\
\hline ALUMINUM & FILTERED & ICAP & $(\mathrm{MG} / \mathrm{L})$ & 28 & 14 & 0.22 & 0.024 & 0.071 & 0.200 & 1 \\
\hline \multicolumn{2}{|l|}{ ARSENIC } & ICAP & (MG/L) & 29 & 1 & 0.69 & 0.69 & 0.69 & 0.050 & 1 \\
\hline ARSENIC & FILTERED & ICAP & (MG/L) & 28 & 1 & 0.054 & 0.054 & 0.054 & 0.050 & 1 \\
\hline \multicolumn{2}{|l|}{ BARIUM } & ICAP & $(M G / L)$ & 29 & 29 & 0.41 & 0.0076 & 0.032 & 1.000 & 0 \\
\hline BARIUM & FILTERED & ICAP & (MG/L) & 28 & 28 & 0.044 & 0.0078 & 0.017 & 1.000 & 0 \\
\hline \multicolumn{2}{|l|}{ BERYLLIUM } & ICAP & (MG/L) & 29 & 7 & 0.029 & 0.0006 & 0.0058 & $0.004^{*}$ * & 2 \\
\hline BERYLLIUM & FILTERED & ICAP & $(M G / L)$ & 28 & 1 & 0.0005 & 0.0005 & 0.0005 & $0.004 * \star$ & 0 \\
\hline \multicolumn{2}{|l|}{ BORON } & ICAP & (MG/L) & 29 & 28 & 0.30 & 0.0047 & 0.033 & NR & NA \\
\hline BORON & FILTERED & ICAP & $(M G / L)$ & 28 & 26 & 0.12 & 0.0047 & 0.023 & NR & NA \\
\hline \multicolumn{2}{|l|}{ CADMIUM } & AAS & $(M G / L)$ & 29 & 1 & 0.011 & 0.011 & 0.011 & 0.005 & 1 \\
\hline \multicolumn{2}{|l|}{ CADMIUM } & ICAP & (MG/L) & 29 & 5 & 0.022 & 0.0031 & 0.0073 & 0.005 & 1 \\
\hline \multicolumn{2}{|l|}{ CALCIUM } & ICAP & (MG/L) & 29 & 29 & 84 & 27 & 44 & NR & NA \\
\hline CALCIUM & FILTERED & ICAP & $(M G / L)$ & 28 & 28 & 69 & 27 & 39 & NR & NA \\
\hline \multicolumn{2}{|l|}{ CHROMIUM } & AAS & (MG/L) & 29 & 8 & 58 & 0.010 & 7.4 & 0.050 & 5 \\
\hline \multicolumn{2}{|l|}{ CHROMIUM } & ICAP & (MG/L) & 29 & 8 & 44 & 0.013 & 5.6 & 0.050 & 6 \\
\hline CHROMIUM & FILTERED & AAS & (MG/L) & 28 & 1. & 0.042 & 0.042 & 0.042 & 0.050 & 0 \\
\hline \multicolumn{2}{|l|}{ COBALT } & ICAP & $(M G / L)$ & 29 & 5 & 0.39 & 0.0061 & 0.097 & $\mathrm{NR}$ & NA \\
\hline COBALT & FILTERED & ICAP & (MG/L) & 28 & 2 & 0.073 & 0.024 & 0.049 & NR & NA \\
\hline \multicolumn{2}{|l|}{ COPPER } & ICAP & (MG/L) & 29 & 12 & 1.1 & 0.0042 & 0.10 & 1.000 & 1 \\
\hline COPPER & FILTERED & ICAP & (MG/L) & 28 & 1 & 0.010 & 0.010 & 0.010 & 1.000 & 0 \\
\hline \multicolumn{2}{|l|}{ IRON } & ICAP & $(M G / L)$ & 29 & 29 & 470 & 0.0073 & 19 & 0.300 & 15 \\
\hline IRON & FILTERED & ICAP & $(\mathrm{MG} / \mathrm{L})$ & 28 & 14 & 8.6 & 0.0072 & 0.71 & 0.300 & 3 \\
\hline \multicolumn{2}{|l|}{ LEAD } & AAS & (MG/L) & 29 & 12 & 1.7 & 0.0045 & 0.16 & 0.050 & 3 \\
\hline \multicolumn{2}{|l|}{ LEAD } & ICAP & $(M G / L)$ & 15 & 1 & 1.4 & 1.4 & 1.4 & 0.050 & 1 \\
\hline
\end{tabular}


Table 7.55 (continued)

\begin{tabular}{|c|c|c|c|c|c|c|c|c|}
\hline VARIABLE & & $\begin{array}{c}\# \\
\text { SAMPLES }\end{array}$ & $\begin{array}{c}\# \\
\text { DETECTED }\end{array}$ & $\begin{array}{c}\text { MAXIMUM } \\
\text { DETECTED } \\
\text { MMT. }\end{array}$ & $\begin{array}{l}\text { MINIMUM } \\
\text { DETECTED } \\
\text { MMT. }\end{array}$ & $\begin{array}{l}\text { AVERAGE } \\
\text { DETECTED } \\
\text { MMTS. }\end{array}$ & $\begin{array}{c}\text { REFERENCE } \\
\text { VALUE }\end{array}$ & $\begin{array}{l}\text { \# MMTS. > } \\
\text { REFERENCE }\end{array}$ \\
\hline MAGNESIUM & ICAP (MG/L) & 29 & 29 & 59 & 17 & 26 & NR & NA \\
\hline MAGNESIUM & ICAP (MG/L) & 28 & 28 & 33 & 16 & 23 & NR & NA \\
\hline MANGANESE & ICAP (MG/L) & 29 & 24 & 11 & 0.0013 & 0.58 & 0.050 & 10 \\
\hline MANGANESE & ICAP $(M G / L)$ & 28 & 18 & 1.2 & 0.0011 & 0.14 & 0.050 & 4 \\
\hline MERCURY & CVAA (MG/L) & 29 & 1 & 0.0030 & 0.0030 & 0.0030 & 0.002 & 1 \\
\hline MOLYBDENUM & ICAP (MG/L) & 29 & 3 & 0.67 & 0.011 & 0.23 & NR & NA \\
\hline MOLYBDENUM & ICAP (MG/L) & 28 & 4 & 0.11 & 0.011 & 0.037 & NR & NA \\
\hline NICKEL, & ICAP (MG/L) & 29 & 10 & 5.8 & 0.012 & 1.0 & $0.100^{* *}$ & 5 \\
\hline FILTERED & ICAP (MG/L) & 28 & 5 & 3.4 & 0.013 & 0.96 & $0.100^{* *}$ & 4 \\
\hline POTASSIUM & ICAP $(M G / L)$ & 29 & 22 & 16 & 0.61 & 2.5 & NR & NA \\
\hline POTASSIUM & ICAP (MG/L) & 28 & 21 & 5.9 & 0.61 & 1.7 & NR & NA. \\
\hline SILVER & ICAP (MG/L) & 29 & 1 & 0.0064 & 0.0064 & 0.0064 & 0.100 & 0 \\
\hline SODIUM & ICAP (MG/L) & 29 & 29 & 11 & 0.43 & 2.5 & NR & NA \\
\hline FILTERED & $\operatorname{ICAP}(\mathrm{MG} / \mathrm{L})$ & 28 & 28 & 11 & 0.37 & 2.2 & NR & NA \\
\hline STRONTIUM & ICAP (MG/L) & 29 & 29 & 0.13 & 0.012 & 0.036 & . NR & NA \\
\hline STRONTIUM & ICAP $(M G / L)$ & 28 & 28 & 0.13 & 0.011 & 0.032 & NR & $\mathrm{NA}$ \\
\hline FLUORIME & QTRIC (MG/L) & 29 & 12 & 0.0040 & 0.0010 & 0.0016 & 13.000 & 0 \\
\hline URANIUM FILT. FLUORIME & ETRIC (MG/L) & 28 & 12 & 0.0020 & 0.0010 & 0.0013 & 13.000 & 0 \\
\hline VANADIUM & ICAP (MG/L) & 29 & 9 & 1.1 & 0.0055 & 0.14 & NR & NA \\
\hline ZINC & ICAP (MG/L) & 29 & 26 & 3.8 & 0.0022 & 0.19 & 5.000 & 0 \\
\hline FILTERED & ICAP (MG/L) & 28 & 21 & 0.047 & 0.0038 & 0.012 & 5.000 & 0 \\
\hline CONDUCTIVITY, FIELD MMT & (UMHOS/CM) & 29 & NA & 620 & 250 & 370 & NR & NA \\
\hline DISSOLVED OXYGEN, FIELD & MMT. (PPM) & 29 & NA & 8.4 & 1.9 & 5.3 & NR & NA \\
\hline PH, FIELD MMT. & (PH UNITS) & 29 & NA & 8.4 & 7.2 & 7.7 & $6.5 / 8.5$ & 0 \\
\hline REDOX, FIELD MMT. & (MV) & 29 & NA & 240 & -220 & 120 & $\mathrm{NR}$ & NA \\
\hline WATER TEMP, FIELD MMT & (DEG. CENT.) & 29 & NA & 18 & 13 & 16 & NR & 0 \\
\hline ALKALINITY-HCO3 & (MG/L) & 29 & 29 & 240 & 86 & 170 & NR & NA \\
\hline CHEMICAL OXYGEN DEMAND & $(M G / L)$ & 29 & 2 & 7.0 & 7.0 & 7.0 & NR & NA \\
\hline CONDUCTIVITY & (UMHOS/CM) & 29 & 29 & 750 & 270 & 400 & NR & NA \\
\hline
\end{tabular}


Table 7.56. Constituents In groundwater at the Y-12 Plant site $\mathrm{HGA}=\mathrm{CR}$ area=Industrial Landlill IV

\begin{tabular}{|c|c|c|c|c|c|c|c|c|c|c|}
\hline \multicolumn{4}{|c|}{ VARIABLE } & \multirow{2}{*}{$\begin{array}{c}\# \\
\text { SAMPLES } \\
20\end{array}$} & \multirow{2}{*}{$\begin{array}{c}\# \\
\text { DETECTED } \\
20\end{array}$} & \multirow{2}{*}{$\begin{array}{c}\text { MAXIMUM } \\
\text { DETECTED } \\
\text { MMT. } \\
2.8\end{array}$} & \multirow{2}{*}{$\begin{array}{c}\text { MINIMUM } \\
\text { DETECTED } \\
\text { MMT. }\end{array}$} & \multirow{2}{*}{$\begin{array}{c}\begin{array}{r}\text { AVERAGE } \\
\text { DETECTED } \\
\text { MMTS }\end{array} \\
1.6\end{array}$} & \multirow{2}{*}{$\begin{array}{c}\text { REFERENCE } \\
\text { VALUE }\end{array}$} & \multirow{2}{*}{$\begin{array}{l}\text { \# MMTS. > } \\
\text { REFERENCE } \\
\text { NA }\end{array}$} \\
\hline CHLORIDE & & & (MG/L) & & & & & & & \\
\hline NITRATE NI & DGEN & & (MG/L) & 20 & 14 & 0.55 & 0.20 & 0.39 & 10.000 & 0 \\
\hline SULFATE & & & (MG/L) & 20 & 14 & 13 & 1.0 & 4.6 & NR & NA \\
\hline ALUMINUM & & ICAP & (MG/L) & 20 & 19 & 1.0 & 0.022 & 0.34 & NR & NA \\
\hline ALUMINUM & FILTERED & ICAP & $(M G / L)$ & 20 & 5 & 0.097 & 0.020 & 0.040 & NR & NA \\
\hline BARIUM & & ICAP & (MG/L) & 20 & 20 & 0.029 & 0.0070 & 0.014 & $2.000 * \star$ & 0 \\
\hline BARIUM & FILTERED & ICAP & (MG/L) & 20 & 20 & 0.027 & 0.0058 & 0.013 & $2.000^{\star \star}$ & 0 \\
\hline BERYLLIUM & & ICAP & (MG/L) & 20 & 6 & 0.0011 & 0.0004 & 0.0006 & $0.004 * *$ & 0 \\
\hline BERYLLIUM & FILTERED & ICAP & (MG/L) & 20 & 3 & 0.0008 & 0.0004 & 0.0006 & $0.004 * \star$ & 0 \\
\hline BORON & & ICAP & (MG/L) & 20 & 18 & 0.15 & 0.0046 & 0.044 & NR & NA \\
\hline BORON & FILTERED & ICAP & $(M G / L)$ & 20 & 16 & 0.15 & 0.0041 & 0.044 & NR & NA \\
\hline CALCIUM & & ICAP & $(M G / L)$ & 20 & 20 & 75 & 24 & 36 & NR & NA \\
\hline CALCIUM & FILTERED & ICAP & (MG/L) & 20 & 20 & 61 & 25 & 32 & NR & NA \\
\hline COBALT & & ICAP & (MG/L) & 20 & 3 & 0.0084 & 0.0057 & 0.0067 & NR & NA \\
\hline COBALT & FILTERED & ICAP & (MG/L) & 20 & 3 & 0.0085 & 0.0050 & 0.0062 & NR & NA \\
\hline COPPER & & ICAP & (MG/L) & 20 & 10 & 0.025 & 0.0044 & 0.0097 & NR & NA \\
\hline COPPER & FILTERED & ICAP & (MG/L) & 20 & 3 & 0.0049 & 0.0043 & 0.0047 & NR & NA \\
\hline IRON & & ICAP & $(M G / L)$ & 20 & 20 & 2.2 & 0.016 & 0.49 & NR & NA \\
\hline IRON & FILTERED & ICAP & (MG/L) & 20 & 4 & 0.12 & 0.0072 & 0.040 & NR & $\mathrm{NA}$ \\
\hline LEAD & & AAS & $(M G / L)$ & 20 & 3 & 0.027 & 0.0042 & 0.013 & 0.050 & 0 \\
\hline MAGNESIUM & & ICAP & (MG/L) & 20 & 20 & 44 & 14 & 22 & NR & NA \\
\hline MAGNESIUM & FILTERED & ICAP & (MG/L) & 20 & 20 & 36 & 15 & 20 & NR & NA \\
\hline MANGANESE & & ICAP & (MG/L) & 20 & 17 & 0.054 & 0.0032 & 0.017 & NR & NA \\
\hline MANGANESE & FILTERED & ICAP & (MG/L) & 20 & 9 & 0.016 & 0.0011 & 0.0036 & $\mathrm{NR}$ & NA \\
\hline NICKEL & & ICAP & (MG/L) & 20 & 1. & 0.033 & 0.033 & 0.033 & $0.100 * *$ & 0 \\
\hline NICKEL & FILTERED & ICAP & (MG/L) & 20 & 1 & 0.049 & 0.049 & 0.049 & $0.100 * *$ & 0 \\
\hline POTASSIUM & & ICAP & $(M G / L)$ & 20 & 18 & 4.5 & 0.61 & 1.5 & NR & NA \\
\hline POTASSIUM & FILTERED & ICAP & $(M G / L)$ & 20 & 12 & 4.4 & 0.63 & 1.9 & NR & NA \\
\hline SODIUM & & ICAP & (MG/L) & 20 & 20 & 5.0 & 0.51 & 1.6 & NR & NA \\
\hline
\end{tabular}


Table 7:56 (continued)

\begin{tabular}{|c|c|c|c|c|c|c|c|c|c|}
\hline VARIABLE & $\begin{array}{c}\# \\
\text { SAMPLES }\end{array}$ & $\begin{array}{c}\# \\
\text { DETECTED }\end{array}$ & $\begin{array}{c}\text { MAXIMUM } \\
\text { DETECTED } \\
\text { MMT }\end{array}$ & & $\begin{array}{c}\text { MINIMUM } \\
\text { DETECTED } \\
\text { MMT' }\end{array}$ & & $\begin{array}{l}\text { AVERAGE } \\
\text { DETECTED } \\
\text { MMTS. }\end{array}$ & $\begin{array}{c}\text { REFERENCE } \\
\text { VALUE }\end{array}$ & $\begin{array}{l}\text { \# MMTS. > } \\
\text { REFERFNCE }\end{array}$ \\
\hline FILTERED ICAP (MG/L) & 20 & 20 & 4.9 & & 0.44 & & 1.8 & NR & $\mathrm{NA}$ \\
\hline ICAP (MG/L) & 20 & 20 & 0.030 & & 0.0090 & & 0.014 & NR & $\mathrm{NA}$ \\
\hline FILTERED ICAP (MG/L) & 20 & 20 & 0.029 & & 0.0089 & & 0.014 & NR & NA \\
\hline FLUORIMETRIC (MG/L) & 20 & 1 & 0.0010 & & 0.0010 & & 0.0010 & NR & NA \\
\hline URANIUM FILT. FLUORIMETRIC (MG/L) & 20 & 1 & 0.0010 & & 0.0010 & & 0.0010 & NR & NA \\
\hline ICAP (MG/L) & 20 & .3 & 0.0077 & & 0.0050 & & 0.0061 & NR & NA \\
\hline ICAP (MG/L) & 20 & 20 & 0.11 & & 0.0040 & & 0.024 & 5.000 & 0 \\
\hline FILTERED ICAP (MG/L) & 20 & 20 & 0.041 & & 0.0040 & & 0.013 & 5.000 & 0 \\
\hline CONDUCTIVITY, FIELD MMT (UMHOS/CM) & 20 & NA & 470 & & 210 & & 300 & NR & NA \\
\hline DISSOLVED OXYGEN, FIELD MMT. (PPM) & 20 & NA & 11 & & 4.9 & & 7.4 & NR & NA \\
\hline $\mathrm{PH}_{\nabla}$ FIELD MMT. & 20 & NA & 8.2 & & 6.8 & & 7.7 & $6.5 / 8.5$ & 0 \\
\hline REDOX, FIELD MMT. & 20 & NA & 250 & & 130 & & 200 & NR & NA \\
\hline WATER TEMP, FIELD MMT (DEG. CENT.) & 20 & NA & 17 & & 13 & & 15 & NR & 0 \\
\hline ALKALINITY-HCO3 & 20 & 20 & 260 & & 130 & & 160 & NR & $\mathrm{NA}$ \\
\hline CONDUCTIVITY & 20 & 20 & 500 & & 240 & & 320 & NR & NA \\
\hline DISSOLVED SOLIDS & 20 & 20 & 270 & & 100 & & 170 & 500.000 & 0 \\
\hline (PH UNITS) & 20 & NA & 8.3 & & 7.3 & & 7.9 & $6.5 / 8.5$ & 0 \\
\hline TOTAL ORGANIC CARBON & 20 & 9 & 5.0 & & 1.0 & & 2.4 & NR & NA \\
\hline TOTAL ORGANIC CARBON, REP 2 (MG/L) & 20 & 9 & 3.6 & & 1.0 & & 1.8 & NR & NA \\
\hline TOTAL ORGANIC CARBON, REP 3 (MG/L) & 20 & 8 & 3.0 & & 1.0 & & 1.9 & NR & NA \\
\hline TOTAL ORGANIC CARBON, REP 4 (MG/L) & 20 & 9 & 4.0 & & 1.0 & & 1.7 & NR & NA \\
\hline TOTAL ORGANIC HALIDE, REP 3 (UG/L) & 20 & 1. & 11 & & 11 & & 11 & NR & NA \\
\hline TO'TAL SUSPENDED SOLIDS & 20 & 16 & 250 & & 1.0 & & 35 & NR & NA \\
\hline TURBIDITY & 20 & 20 & 110 & & 1.0 & & 16 & 1.000 & 19 \\
\hline GROSS ALPHA & 20 & 20 & .5 .7 & & -2.7 & & -0.12 & 15.000 & 0 \\
\hline GROSS BETA & 20 & 20 & 9.4 & & -12 & & -1.2 & 50.000 & 0 \\
\hline $1,1,1$-TRICHLOROETHANE & 20 & 4 & 4.0 & $J$ & 2.0 & $\pi$ & 3.0 & 200.000 & 0 \\
\hline 2-BUTANONE & 20 & 2 & 13 & $\mathrm{~B}$ & 13 & B & 13 & NR & NA \\
\hline 4-METHYL-2 - PENTANONE & 20 & 6 & 2.0 & $\mathrm{BJ}$ & 1.0 & $\mathrm{BJ}$ & 1.8 & $\mathrm{NR}$ & NA \\
\hline ACETONE & 20 & 7 & 6.0 & JB & 2.0 & $\mathrm{Bu}$ & 4.0 & $\mathrm{NR}$ & NA \\
\hline METHYLENE CHLORIDE & 20 & 7 & 3.0 & $\mathrm{BJ}$ & 1.0 & JB & 1.6 & NR & NA \\
\hline
\end{tabular}


Table 7.57. Constlituents in groundwater at the $\mathrm{Y}-12$ Plant site

$H G R=C R$ area=Industrial Landfill $V$

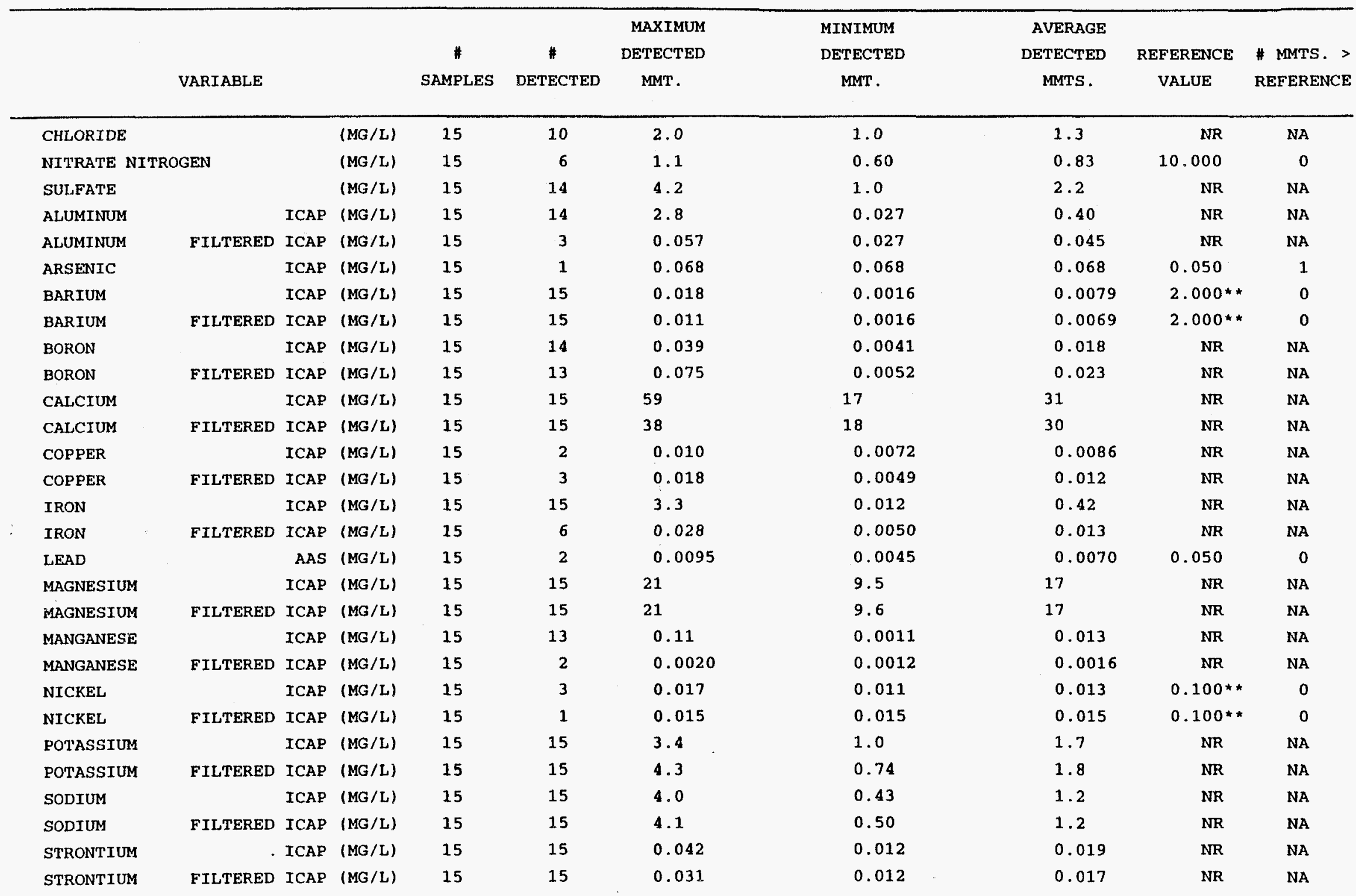


Table 7.57 (continued)

\begin{tabular}{|c|c|c|c|c|c|c|c|c|c|c|}
\hline VARIABLE & & $\begin{array}{c}\# \\
\text { SAMPLES }\end{array}$ & $\begin{array}{c}\# \\
\text { DETECTED }\end{array}$ & $\begin{array}{l}\text { MAXIMUM } \\
\text { DETECTED } \\
\text { MMT. }\end{array}$ & & $\begin{array}{c}\text { MINIMUM } \\
\text { DETECTED } \\
\text { MMT. }\end{array}$ & & $\begin{array}{l}\text { AVERAGE } \\
\text { DETECTED } \\
\text { MMTS. }\end{array}$ & $\begin{array}{c}\text { REFERENCE } \\
\text { VALUE }\end{array}$ & $\begin{array}{l}\text { \# MMTS. > } \\
\text { REFERENCE }\end{array}$ \\
\hline FLUORIMETRIC & (MG/L) & 15 & 2 & 0.0010 & & 0.0010 & & 0.0010 & NR & NA. \\
\hline URANIUM FILT. FLUORIMETRIC & (MG /L) & 15 & 3 & 0.0010 & & 0.0010 & & 0.0010 & NR & NA \\
\hline ICAP & $(M G / L)$ & 15 & 15 & 0.034 & & 0.0043 & & 0.012 & 5.000 & 0 \\
\hline FILTERED ICAP & (MG/L) & 15 & 11 & 0.032 & & 0.0039 & & 0.014 & 5.000 & 0 \\
\hline CONDUCTIVITY, FIELD MMT (UM & Hos (CM) & 19 & NA & 300 & & 150 & & 250 & NR & NA \\
\hline DISSOLVED OXYGEN, FIELD MMT & - (PPM) & 19 & NA & 12 & & 5.9 & & 8.9 & NR & NA \\
\hline PH, FIELD MMT. & UNITS) & 19 & NA & 9.3 & & 7.4 & & 8.0 & $6.5 / 8.5$ & 3 \\
\hline REDOX, FIELD MMT. & (MV) & 19 & NA & 280 & & 29 & & 190 & NR & NA \\
\hline WATER TEMP, FIELD MMT (DEG. & CENT.) & 19 & NA & 19 & & 13 & & 16 & NR & 0 \\
\hline ALKALINITY-CO3 & (MG/L) & 15 & 1 & 16 & & 16 & & 16 & NR & $\mathrm{NA}$ \\
\hline ALKALINITY-HCO3 & (MG/L) & 15 & 15 & 170 & & 73 & & 140 & NR & NA \\
\hline CONDUCTIVITY & HOS/CM) & 15 & 15 & 910 & & 180 & & 310 & NR & NA \\
\hline DISSOLVED SOLIDS & (MG/L) & 15 & 15 & 200 & & 78 & & 150 & 500.000 & 0 \\
\hline PH & UNITS) & 15 & NA & 9.1 & & 7.8 & & 8.2 & $6.5 / 8.5$ & 2 \\
\hline TOTAL ORGANIC CARBON & (MG/L) & 15 & 10 & 12 & & 1.0 & & 4.0 & NR & NA \\
\hline TOTAL ORGANIC CARBON, REP 2 & (MG/L) & 15 & 9 & 13 & & 1.0 & & 3.7 & $\mathrm{NR}$ & NA \\
\hline TOTAL ORGANIC CARBON, REP 3 & (MG/L) & 15 & 10 & 13 & & 1.0 & & 3.5 & NR & NA \\
\hline TOTAL ORGANIC CARBON, REP 4 & (MG/L) & 15 & 8 & 14 & & 1.0 & & 3.4 & NR & NA \\
\hline TOTAL SUSPENDED SOLIDS & (MG/L) & 15 & 10 & 100 & & 1.0 & & 16 & NR & NA \\
\hline TURBIDITY & (NTU) & 15 & 15 & 58 & & 1.5 & & 10 & 1.000 & 15 \\
\hline 234-URANIUM & (PCI/L) & 15 & 15 & 56 & & -0.81 & & 9.5 & 20.000 & 1 \\
\hline 235-URANIUM & $(\mathrm{PCI} / \mathrm{L})$ & 15 & 15 & 47 & $\mathbf{E}$ & -37 & $F$ & 12 & 24.000 & 3 \\
\hline 237-NEPTUNIUM & (PCI/L) & 1 & 1 & 160 & & 160 & & 160 & 1.000 & 1 \\
\hline 238-URANIUM & (PCI/L) & 15 & 15 & 17 & & 0.0000 & $\star$ & 4.1 & 24.000 & 0 \\
\hline CESIUM- 137 & (PCI/L) & 15 & 15 & 35 & $\mathbf{E}$ & -21 & $\mathbf{F}$ & 3.6 & 120 & 0 \\
\hline GROSS ALPHA & $(\mathrm{PCI} / \mathrm{L})$ & 15 & 15 & 1.4 & & -5.1 & & -0.97 & 15.000 & 0 \\
\hline GROSS BETA & (PCI/L) & 15 & 15 & 4.6 & & -5.3 & & -1.6 & 50.000 & 0 \\
\hline POTASSIUM-40 & $(\mathrm{PCI} / \mathrm{L})$ & 1 & 1 & 570 & $\mathrm{I}$ & 570 & I & 570 & NR & NA \\
\hline PROTACTINIUM-234 & (PCI/L) & 15 & 15 & 8400 & & -2200 & $\mathbf{F}$ & 2800 & 2800 & 6 \\
\hline THORIUM-228 & $(\mathrm{PCI} / \mathrm{L})$ & 2 & 2 & 1800 & & 1400 & & 1600 & 16.000 & 2 \\
\hline
\end{tabular}


Table 7.57 (continued)

\begin{tabular}{|c|c|c|c|c|c|c|c|c|c|c|}
\hline VARIABLE & & $\begin{array}{c}\# \\
\text { SAMPLES }\end{array}$ & $\begin{array}{c}\# \\
\text { DETECTED }\end{array}$ & $\begin{array}{l}\text { MAXIMUM } \\
\text { DETECTED } \\
\text { MMT. }\end{array}$ & & $\begin{array}{l}\text { MINIMUM } \\
\text { DETECTED } \\
\text { MMT. }\end{array}$ & & $\begin{array}{l}\text { AVERAGE } \\
\text { DETECTED } \\
\text { MMTS. }\end{array}$ & $\begin{array}{c}\text { REFERENCE } \\
\text { VALUE }\end{array}$ & $\begin{array}{l}\text { \# MMTS. > } \\
\text { REFERENCE }\end{array}$ \\
\hline $1,1,1$-TRICHLOROETHANE & (UG/L) & 15 & 3 & 2.0 & $\mathrm{~J}$ & 2.0 & $\mathrm{~J}$ & 2.0 & 200.000 & 0 \\
\hline 2-BUTANONE & (UG/L) & 15 & 2 & 8.0 & B & 4.0 & $\mathrm{BJ}$ & 6.0 & NR & NA \\
\hline 4-METHYL-2-PENTANONE & $(U G / L)$ & 15 & 2 & 1.0 & BJ & 1.0 & $\mathrm{BJ}$ & 1.0 & NR & NA \\
\hline ACETONE & (UG/L) & 15 & 2 & 7.0 & $J$ & 3.0 & JB & 5.0 & NR & NA \\
\hline CARBON DISULFIDE & (UG/L) & 15 & 1 & 1.0 & $\mathrm{~J}$ & 1.0 & $\mathbf{J}$ & 1.0 & NR & NA \\
\hline METHYLENE CHLORIDE & (UG/L) & 15 & 3 & 1.0 & JB & 0.90 & JB & 0.97 & NR & NA \\
\hline TRICHLOROETHENE & (UG/L) & 15 & 1 & 3.0 & $\mathfrak{J}$ & 3.0 & $\mathbf{J}$ & 3.0 & 5.000 & 0 \\
\hline
\end{tabular}


Table 7.58. Constlituents in groundwater at the Y-12 Plant site

HGR $=C R$ area $=$ Const. Debris Landfill VI

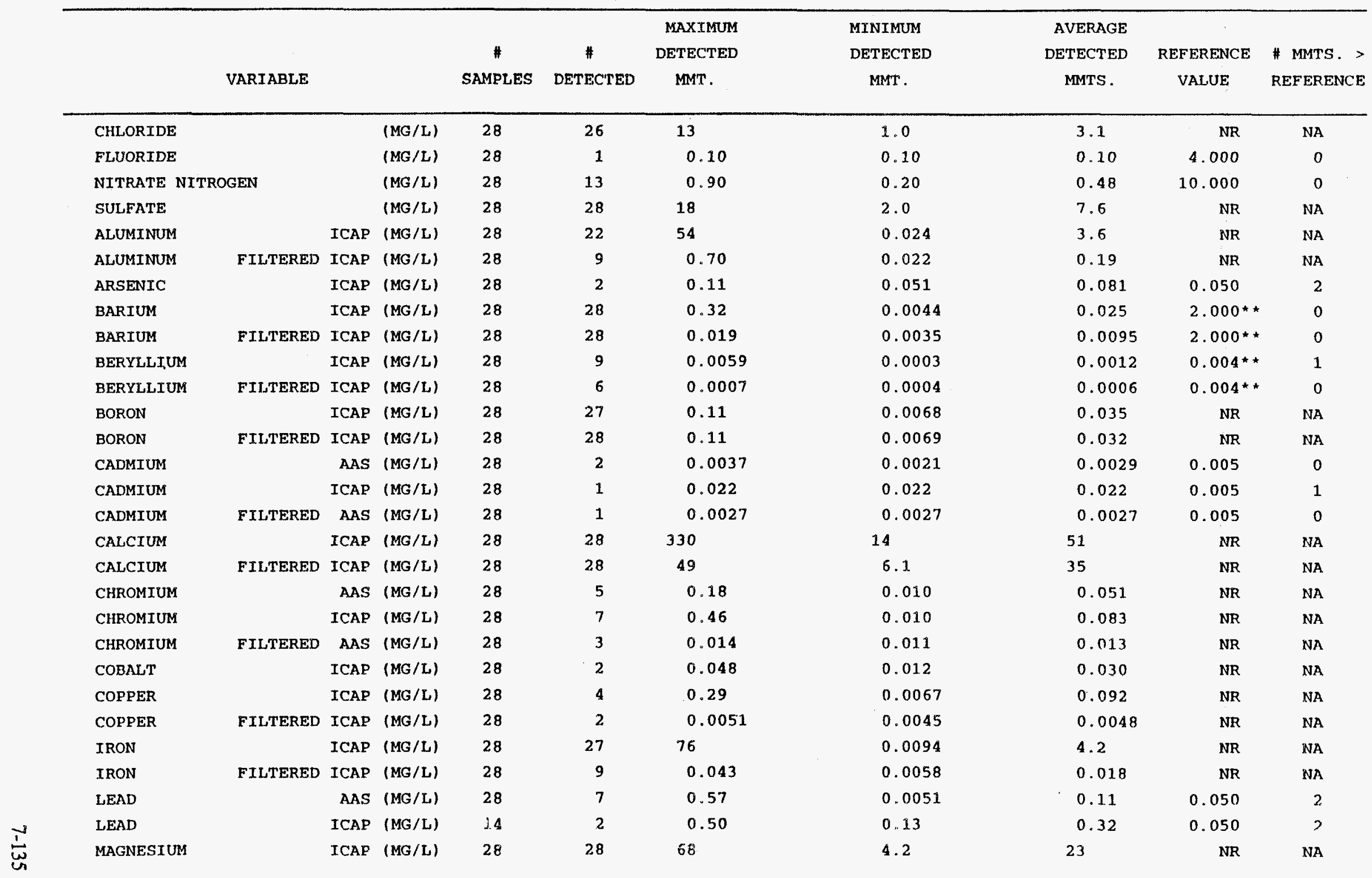


Table 7.58 (continued)

\begin{tabular}{|c|c|c|c|c|c|c|c|c|c|}
\hline VARIABLE & & & $\begin{array}{c}\# \\
\text { SAMPLES }\end{array}$ & $\begin{array}{c}\# \\
\text { DETECTED }\end{array}$ & $\begin{array}{c}\text { MAXIMUM } \\
\text { DETECTED } \\
\text { MMT. }\end{array}$ & $\begin{array}{l}\text { MINIMUM } \\
\text { DETECTED } \\
\text { MMT. }\end{array}$ & $\begin{array}{l}\text { AVERAGE } \\
\text { DETECTED } \\
\text { MMTS. }\end{array}$ & $\begin{array}{c}\text { REFERENCE } \\
\text { VALUE }\end{array}$ & $\begin{array}{l}\text { \# MMTS. > } \\
\text { REFERENCE }\end{array}$ \\
\hline MAGNESIUM & ICAP & (MG/L) & 28 & 28 & 28 & 0.023 & 18 & NR & NA \\
\hline MANGANESE & ICAP & $(M G / L)$ & 28 & 19 & 1.8 & 0.0010 & 0.15 & NR & NA \\
\hline MANGANESE & ICAP & (MG/L) & 28 & 7 & 0.0066 & 0.0017 & 0.0026 & NR & NA \\
\hline MERCURY & CVAA & $(M G / L)$ & 28 & 1 & 0.0005 & 0.0005 & 0.0005 & 0.002 & 0 \\
\hline MOLYBDENUM & ICAP & $(M G / L)$ & 28 & 6 & 0.061 & 0.027 & 0.039 & NR & NA \\
\hline MOLYBDENUM & ICAP & $(M G / L)$ & 28 & 7 & 0.037 & 0.010 & 0.029 & NR & NA \\
\hline NICKEL & ICAP & (MG/L) & 28 & 8 & 0.28 & 0.011 & 0.050 & $0.100 \star \star$ & 1 \\
\hline FILTERED & ICAP & $(M G / L)$ & 28 & 3 & 0.016 & 0.010 & 0.014 & $0.100 \star \star$ & 0 \\
\hline POTASSIUM & ICAP & (MG/L) & 28 & 25 & 26 & 0.62 & 4.4 & NR & $\mathrm{NA}$ \\
\hline POTASSIUM & ICAP & $(M G / L)$ & 28 & 26 & 23 & 0.64 & 3.8 & NR & NA \\
\hline FILTERED & ICAP & $(M G / L)$ & 28 & 1 & 0.061 & 0.061 & 0.061 & NR & NA \\
\hline SODIUM & ICAP & (MG/L) & 28 & 28 & 8.0 & 0.41 & 2.5 & NR & NA \\
\hline FILTERED & ICAP & $(\mathrm{MG} / \mathrm{L})$ & 28 & 28 & 7.6 & 0.44 & 2.5 & NR & NA \\
\hline STRONTIUM & ICAP & (MG/L) & 28 & 28 & 0.37 & 0.015 & 0.042 & NR & NA \\
\hline STRONTIUM & ICAP & (MG /L) & 28 & 28 & 0.054 & 0.015 & 0.026 & NR & NA \\
\hline FLUORIME & ETRIC & $(M G / L)$ & 28 & 12 & 0.0040 & 0.0010 & 0.0019 & NR & NA \\
\hline URANIUM FILT. FLUORIME & ETRIC & $(M G / L)$ & 28 & 7 & 0.0030 & 0.0010 & 0.0019 & NR & $\mathrm{NA}$ \\
\hline VANADIUM & ICAP & $(M G / L)$ & 28 & 6 & 0.15 & 0.0054 & 0.039 & NR & NA \\
\hline FILTERED & ICAP & $(\mathrm{MG} / \mathrm{L})$ & 28 & 3 & 0.014 & 0.0055 & 0.011 & NR & NA \\
\hline ZINC & ICAP & $(M G / L)$ & 28 & 28 & 0.31 & 0.0076 & 0.048 & 5.000 & 0 \\
\hline FILTERED & ICAP & $(M G / L)$ & 28 & 24 & 0.060 & 0.0038 & 0.021 & 5.000 & 0 \\
\hline CONDUCTIVITY, FIELD MMT & T IUMH & $\operatorname{los} / \mathrm{CM})$ & 28 & NA & 430 & 190 & 310 & NR & NA \\
\hline DISSOLVED OXYGEN, FIELD & D MMT. & $(\mathrm{PPM})$ & 28 & NA & 8.5 & 1.7 & 6.1 & NR & NA \\
\hline PH， FIELD MMT. & (PH & UNITS) & 28 & NA & 11 & 6.4 & 8.0 & $6.5 / 8.5$ & 5 \\
\hline REDOX，FIELD MMT . & & $(M V)$ & 28 & NA & 250 & 39 & 180 & NR & NA. \\
\hline WATER TEMP, FIELD MMT & IDEG & CENT.) & 28 & NA & 19 & 11 & 15 & NR & 0 \\
\hline ALKALINITY-CO3 & & $(M G / L)$ & 28 & 5 & 54 & 4.0 & 30 & NR & NA \\
\hline ALKALINITY-HCO3 & & (MG/L) & 28 & 24 & 230 & 100 & 180 & $\mathrm{NR}$ & NA \\
\hline CONDUCTIVITY & IUMH & $\operatorname{loS} / \mathrm{CM})$ & 28 & 28 & 640 & 120 & 330 & NR & NA \\
\hline DISSOLVED SOLIDS & & $(M G / L)$ & 28 & 28 & 270 & 78 & 170 & 500.000 & 0 \\
\hline
\end{tabular}


Table 7.58 (continued)

\begin{tabular}{|c|c|c|c|c|c|c|c|c|c|c|c|}
\hline VARIABLE & & $\begin{array}{c}\# \\
\text { SAMPLES }\end{array}$ & $\begin{array}{c}\# \\
\text { DETECTED }\end{array}$ & $\begin{array}{l}\text { MAXIMUM } \\
\text { DETECTED } \\
\text { MMT. }\end{array}$ & & $\begin{array}{l}\text { MINIMUM } \\
\text { DETECTED } \\
\text { MMT. }\end{array}$ & & & $\begin{array}{l}\text { AVERAGE } \\
\text { DETECTED } \\
\text { MMTS. }\end{array}$ & $\begin{array}{c}\text { REFERENCE } \\
\text { VALUE }\end{array}$ & $\begin{array}{l}\text { \# MMTS. > } \\
\text { REFERENCE }\end{array}$ \\
\hline ( $\mathrm{PH}$ & UNITS) & 28 & NA & 83 & & 6.4 & & & 11 & $6.5 / 8.5$ & 6 \\
\hline TOTAL ORGANIC CARBON & $(M G / L)$ & 28 & 16 & 7.0 & & 1.0 & & & 1.9 & NR & NA \\
\hline TOTAL ORGANIC CARBON, REP 2 & $(M G / L)$ & 28 & 17 & 3.0 & & 1.0 & & & 1.7 & NR & NA \\
\hline TOTAL ORGANIC CARBON, REP 3 & $(\mathrm{MG} / \mathrm{L})$ & 28 & 19 & 3.0 & & 1.0 & & & 1.5 & NR & NA \\
\hline TOTAL ORGANIC CARBON, REP 4 & $(M G / L)$ & 28 & 18 & 35 & & 1.0 & & & 3.9 & NR & NA \\
\hline TOTAL SUSPENDED SOLIDS & $(M G / L)$ & 28 & 2.5 & 2300 & & 1.0 & & & 130 & NR & NA \\
\hline TURBIDITY & (NTU) & 28 & 28 & 900 & & 1.0 & & & 48 & 1.000 & 26 \\
\hline 234-URANIUM & $(\mathrm{PCI} / \mathrm{L})$ & 21 & 21 & 22 & & -7.6 & & & 6.3 & 20.000 & 2 \\
\hline 235-URANIUM & $(\mathrm{PCI} / \mathrm{L})$ & 21 & 21 & 54 & $\mathbf{E}$ & -31 & & $\mathbf{F}$ & 10 & 24.000 & 5 \\
\hline 238-URANIUM & $(P C I / L)$ & 21 & 21 & 31 & & -5.5 & & & 5.4 & 24.000 & 2 \\
\hline CESIUM-137 & (PCI/L) & 21 & 21 & 64 & & -46 & & G & 1.3 & 120 & 0 \\
\hline GROSS ALPHA & $(\mathrm{PCI} / \mathrm{L})$ & 28 & 28 & 14 & & -4.3 & & & 0.83 & 15.000 & 0 \\
\hline GROSS BETA & $(\mathrm{PCI} / \mathrm{L})$ & 28 & 28 & 18 & & -12 & & & 0.64 & 50.000 & 0 \\
\hline POTASSIUM-40 & $(\mathrm{PCI} / \mathrm{L})$ & 3 & 3 & 420 & I & 280 & & $\mathbf{I}$ & 370 & 280 & NA. \\
\hline PROTACTINIUM-234 & $(\mathrm{PCI} / \mathrm{L})$ & 21 & 21 & 8100 & & 210 & & EH & 3200 & 2800 & 10 \\
\hline RUTHENIUM-106 & $(\mathrm{PCI} / \mathrm{L})$ & 3 & 3 & 170 & I & 120 & & $\mathrm{I}$ & 140 & 240.00 & 0 \\
\hline 2-BUTANONE & (UG/L) & 28 & 3 & 10 & B & 4.0 & BU & & 7.7 & $\mathrm{NR}$ & NA \\
\hline 4-METHYL-2-PENTANONE & (UG/L) & 28 & 2 & 3.0 & BJ & 3.0 & $\mathrm{BJ}$ & & 3.0 & NR & NA \\
\hline ACETONE & (UG/L) & 28 & 2 & 3.0 & $\mathrm{BJ}$ & 3.0 & $\mathrm{BJ}$ & & 3.0 & NR & NA \\
\hline METHXLENE CHLORIDE & $(O G / L)$ & 28 & 8 & 2.0 & $\mathrm{BJ}$ & 1.0 & $\mathrm{JB}$ & & 1.1 & NR & NA \\
\hline
\end{tabular}


Table 7.59. Constituents in groundwater at the $Y-12$ Plant site $H G R=C F$ area=Const. Debris Landfill VII

\begin{tabular}{|c|c|c|c|c|c|c|c|c|c|c|}
\hline \multicolumn{4}{|c|}{ VARIABLE } & \multirow{2}{*}{$\begin{array}{c}\# \\
\text { SAMPLES } \\
12\end{array}$} & \multirow{2}{*}{$\frac{\begin{array}{l}\# \\
\text { DETECTED }\end{array}}{7}$} & \multirow{2}{*}{$\begin{array}{l}\text { MAXIMUM } \\
\text { DETECTED } \\
\text { MMT. }\end{array}$} & \multirow{2}{*}{$\begin{array}{c}\begin{array}{c}\text { MINIMUM } \\
\text { DETECTED } \\
\text { MMT }\end{array} \\
1.0\end{array}$} & \multirow{2}{*}{$\begin{array}{c}\text { AVERAGE } \\
\text { DETECTED } \\
\text { MMTS. }\end{array}$} & \multirow{2}{*}{$\begin{array}{c}\text { REFERENCE } \\
\text { VALUE } \\
\text { NR }\end{array}$} & \multirow{2}{*}{$\begin{array}{c}\text { \# MMTS. > } \\
\text { REFERENCE } \\
\text { NA }\end{array}$} \\
\hline CHLORIDE & & & (MG/L) & & & & & & & \\
\hline FLUORIDE & & & (MG/L) & 12 & 1 & 0.20 & 0.20 & 0.20 & 4.000 & 0 \\
\hline NITRATE NI & OGEN & & (MG/L) & 12 & 8 & 0.69 & 0.20 & 0.46 & 10.000 & 0 \\
\hline SULFATE & & & (MG/L) & 12 & 11 & 9.0 & 1.0 & 2.8 & NR & NA \\
\hline ALUMINUM & & ICAP & (MG /L) & 12 & 8 & 3.8 & 0.021 & 0.66 & NR & NA \\
\hline ALUMINUM & FILTERED & ICAP & (MG/L) & 12 & 4 & 0.043 & 0.023 & 0.033 & NR & NA \\
\hline BARIUM & & ICAP & (MG /L) & 12 & 12 & 0.24 & 0.0082 & 0.066 & $2.000 * \star$ & 0 \\
\hline BARIUM & FILTERED & ICAP & (MG/L) & 12 & 12 & 0.24 & 0.0080 & 0.064 & $2.000^{\star *}$ & 0 \\
\hline BERYLLIUM & & ICAP & (MG/L) & 12 & 1 & 0.0006 & .0 .0006 & 0.0006 & $0.004 * \star$ & 0 \\
\hline BORON & & ICAP & (MG/L) & 12 & 11 & 0.063 & 0.0059 & 0.023 & $\mathrm{NR}$ & NA \\
\hline BORON & FILTERED & ICAP & (MG/L) & 12 & 12 & 0.063 & 0.0081 & 0.020 & NR & NA \\
\hline CALCIUM & & ICAP & (MG/L) & 12 & 12 & 40 & 24 & 33 & NR & NA \\
\hline CALCIUM & FILTERED & ICAP & (MG/L) & 12 & 12 & 39 & 24 & 33 & $\mathrm{NR}$ & NA \\
\hline COPPER & & ICAP & (MG/L) & 12 & 2 & 0.014 & 0.0064 & 0.010 & $\mathrm{NR}$ & NA \\
\hline COPPER & FILTERED & ICAP & (MG/L) & 12 & 1 & 0.0050 & 0.0050 & 0.0050 & NR & NA \\
\hline IRON & & ICAP & (MG/L) & 12 & 11 & 3.9 & 0.014 & 0.54 & NR & NA \\
\hline IRON & FILTERED & ICAP & (MG/L) & 12 & 1 & 0.18 & 0.18 & 0.18 & NR & NA \\
\hline LEAD & & AAS & (MG/L) & 12 & 1 & 0.0041 & 0.0041 & 0.0041 & 0.050 & 0 \\
\hline MAGNESIUM & & ICAP & (MG/L) & 12 & 12 & 22 & 13 & 16 & NR & NA \\
\hline MAGNESIUM & FILTERED & ICAP & (MG/L) & 12 & 12 & 21 & 13 & 16 & NR & NA \\
\hline MANGANESE & & ICAP & (MG/L) & 12 & 7 & 0.096 & 0.0010 & 0.022 & NR & NA \\
\hline MANGANESE & FILTERED & ICAP & (MG/L) & 12 & 3 & 0.0023 & 0.0010 & 0.0017 & NR & NA \\
\hline NICKEL & & ICAP & (MG/L) & 12 & 3 & 0.029 & 0.017 & 0.021 & $0.100 * \star$ & 0 \\
\hline NICKEL & FILTERED & ICAP & (MG/L) & 12 & 3 & 0.029 & 0.012 & 0.020 & $0.100 * *$ & 0 \\
\hline POTASSIUM & & ICAP & $(M G / L)$ & 12 & 11 & 3.4 & 0.95 & 1.6 & NR & $\mathrm{NA}$ \\
\hline POTASSIUM & FILTERED & ICAP & (MG/L) & 12 & 10 & 3.9 & 0.96 & 1.6 & NR & NA \\
\hline SODIUM & & ICAP & (MG/L) & 12 & 12 & 0.87 & 0.44 & 0.64 & NR & NA \\
\hline SODIUM & FILTERED & ICAP & (MG/L) & 12 & 12 & $0.76^{\circ}$ & 0.45 & 0.63 & NR & NA \\
\hline STRONTIUM & & ICAP & $(M G / L)$ & 12 & 12 & 0.024 & 0.016 & 0.019 & NR & $\mathrm{NA}$ \\
\hline
\end{tabular}

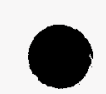


Table 7.59 (continued)

\begin{tabular}{|c|c|c|c|c|c|c|c|c|c|}
\hline VARIABLE & $\begin{array}{c}\# \\
\text { SAMPLES }\end{array}$ & $\begin{array}{c}\# \\
\text { DETECTED }\end{array}$ & $\begin{array}{l}\text { MAXIMUM } \\
\text { DETECTED } \\
\text { MMT. }\end{array}$ & & $\begin{array}{l}\text { MINIMUM } \\
\text { DETECTED } \\
\text { MMT. }\end{array}$ & & $\begin{array}{l}\text { AVERAGE } \\
\text { DETECTED } \\
\text { MMTS. }\end{array}$ & $\begin{array}{l}\text { REFERENCE } \\
\text { VALUE }\end{array}$ & $\begin{array}{l}\text { \# MMTS . > } \\
\text { REFERENCE }\end{array}$ \\
\hline FILTERED JCAP (MG/L) & 12 & 12 & 0.024 & & 0.016 & & 0.019 & NR & $\mathrm{NA}$ \\
\hline FLUORIMETRIC (MG/L) & 12 & 1 & 0.0010 & & 0.0010 & & 0.0010 & NR & NA \\
\hline URANIUM FILT. FLUORIMETRIC (MG/L) & 12 & 2 & 0.0010 & & 0.0010 & & 0.0010 & NR & NA \\
\hline ICAP (MG/L) & 12 & 1 & 0.0070 & & 0.0070 & & 0.0070 & NR & NA \\
\hline ICAP (MG/L) & 12 & 12 & 0.055 & & 0.0069 & & 0.019 & 5.000 & 0 \\
\hline FILTERED ICAP (MG/L) & 12 & 9 & 0.049 & & 0.0030 & & 0.019 & 5.000 & 0 \\
\hline CONDUCTIVITY, FIELD MMT (UMHOS/CM) & 15 & NA & 310 & & 230 & & 260 & NR & INA \\
\hline DISSOLVED OXYGEN, FIELD MMT. (PPM) & 15 & NA & 8.8 & & 5.8 & & 7.2 & NR & NA \\
\hline PH, FIELD MMT. $\quad$ (PH UNITS) & 15 & NA & 7.9 & & 7.1 & & 7.5 & $6.5 / 8.5$ & 0 \\
\hline REDOX, FIELD MMT. & 15 & NA & 230 & & 140 & & 190 & NR & NA \\
\hline WATER TEMP, FIELD MMT (DEG. CENT.) & 15 & NA & 17 & & 13 & & 15 & NR & 0 \\
\hline ALKALINITY-HCO3 & 12 & 12 & 180 & & 120 & & 150 & NR & NA \\
\hline CONDUCTIVITY & 12 & 12 & 340 & & 240 & & 280 & NR & NA \\
\hline DISSOLVED SOLIDS & 12 & 12 & 240 & & 140 & & 170 & 500.000 & 0 \\
\hline (PH UNITS) & 12 & NA & 8.2 & & 7.6 & & 7.9 & $6.5 / 8.5$ & 0 \\
\hline TOT. PETROLEUM HYDROCARBONS (MG/L) & 12 & 1 & 0.0070 & & 0.0070 & & 0.0070 & 1.000 & 0 \\
\hline TOTAL ORGANIC CARBON & 12 & 6 & 2.2 & & 1.0 & & 1.5 & NR & NA \\
\hline TOTAL ORGANIC CARBON, REP 2 (MG/L) & 12 & 7 & 2.0 & & 1. 0 & & 1.3 & NR & NA \\
\hline TOTAL, ORGANIC CARBON, REP 3 (MG/L) & 12 & 4 & 28 & & 1.1 & & 8.3 & NR & NA \\
\hline TOTAL ORGANIC CARBON, REP 4 (MG/L) & 12 & 6 & 4.0 & & 1.0 & & 1.6 & NR & NA \\
\hline TOTAL SUSPENDED SOLIDS $\quad$ (MG/L) & 12 & 9 & 40 & & 1.0 & & 8.4 & NR & NA \\
\hline TURBIDITY & 12 & 12 & 95 & & 0.90 & & 12 & 1.000 & 10 \\
\hline 234-URANIUM & 12 & 12 & 34 & & -7.4 & & 9.1 & 20.000 & 3 \\
\hline 235-URANIUM & 12 & 12 & 62 & & -15 & $\mathbf{F}$ & 23 & 24.000 & 4 \\
\hline 237-NEPTUNIUM & 1 & 1 & 91 & I & 91 & I & 91 & 1.000 & 1 \\
\hline 238-URANIUM & 12 & 12 & 40 & & 0.0000 & & 6.4 & 24.000 & 1 \\
\hline CESIUM-137 & 12 & 12 & 23 & $\mathbf{E}$ & -20 & $\mathbf{F}$ & -2.8 & 120.00 & 0 \\
\hline GROSS ALPHA & 12 & 12 & 1.2 & & -4.0 & & -1.2 & 15.000 & 0 \\
\hline GROSS BETA & 12 & 12 & 2.1 & & -6.0 & & -1.4 & 50.000 & 0 \\
\hline POTASSIUM-40 & 2 & 2 & 330 & $I$ & 270 & I & 300 & 280 & NA \\
\hline
\end{tabular}


Table 7.59 (continued)

\begin{tabular}{|c|c|c|c|c|c|c|c|c|c|c|c|c|}
\hline VARIABLE & & $\begin{array}{c}\# \\
\text { SAMPLES }\end{array}$ & $\begin{array}{c}* \\
\text { DETECTED }\end{array}$ & $\begin{array}{c}\text { MAXIMUM } \\
\text { DETECTED } \\
\text { MMT. }\end{array}$ & & & $\begin{array}{c}\text { MINIMUM } \\
\text { DETECTED } \\
\text { MMT }\end{array}$ & & & $\begin{array}{c}\text { AVERAGE } \\
\text { DETECTED } \\
\text { MMTS. }\end{array}$ & $\begin{array}{c}\text { REFERENCE } \\
\text { VALUE }\end{array}$ & $\begin{array}{l}\# \text { MMTS. > } \\
\text { REFERENCE }\end{array}$ \\
\hline PROTACTINIUM-234 & (PCI/L) & 12 & 12 & 8000 & & $\mathbf{I}$ & -1500 & & $\mathbf{F}$ & 3600 & 2800 & 9 \\
\hline THORIUM-228 & $(\mathrm{PCI} / \mathrm{L})$ & 1 & 1 & 980 . & & $\mathbf{I}$ & 980 & & I & 980 & 16.000 & 1 \\
\hline 2-BUTANONE & $(\mathrm{UG} / \mathrm{L})$ & 12 & 5 & 14 & B & & 3.0 & $\mathrm{BJ}$ & & 9.2 & $\mathrm{NR}$ & NA \\
\hline 4-METHYL-2-PENTANONE & (UG/L) & 12 & 5 & 2.0 & BJ & & 1.0 & JB & & 1.8 & NR & $\mathrm{NA}$ \\
\hline ACETONE & (UG/L) & 12 & 5 & 17 & & & 4.0 & JB & & 7.4 & $\mathrm{NR}$ & NA \\
\hline BENZENE & (UG/L) & 12 & 2 & 1.0 & JB & & 0.80 & JB & & 0.90 & 5.000 & 0 \\
\hline METHYLENE CHLORIDE & (UG/L) & 12 & 4 & 2.0 & $\mathrm{BJ}$ & & 2.0 & BJ & & 2.0 & NR & NA \\
\hline TRICHLOROETHENE & $(U G / L)$ & 12 & 1 & 7.0 & & & 7.0 & & & 7.0 & 5.000 & 1 \\
\hline
\end{tabular}


Table 7.60. Constituents in Waste Area Grouping (WAG) 1 groundwater at ORNL,

May 19-June 25, 1993

\begin{tabular}{|c|c|c|c|c|c|c|}
\hline Parameter & $\begin{array}{l}N \text { det } / \\
N \text { total }\end{array}$ & $\operatorname{Max}$ & $\operatorname{Min}^{*}$ & $A V^{b}$ & $\begin{array}{l}\text { Reference } \\
\text { value }\end{array}$ & $\begin{array}{l}\text { Number of val } \\
\text { exceeding } \\
\text { reference lref }\end{array}$ \\
\hline \multicolumn{7}{|c|}{ Downgradient Wells } \\
\hline \multicolumn{7}{|l|}{ Anions, unfiltered (mg/L) } \\
\hline Bronide & $1 / 23$ & 1.1 & $<1.0$ & -1.0 & $\mathrm{~d}$ & [d] \\
\hline Chloride & $20 / 23$ & 110 & $<2.0$ & -18 & 250 & $0[3]$ \\
\hline Fluoride & $7 / 23$ & 3.2 & $<0.10$ & -0.41 & 4 & $0[2]$ \\
\hline Nitrate & $2 / 23$ & 10 & $<I .0$ & -1.5 & 10 & $0[2]$ \\
\hline Sulfate, as so, & $19 / 23$ & 120 & $<10$ & -34 & 250 & $0[3]$ \\
\hline \multicolumn{7}{|c|}{ Base neutral/acid extractable organics, unfiltered $(\mu g / L)$} \\
\hline Benzyl butyl phthalate & $6 / 23$ & US.0 & JB1.0 & -4.1 & a & [a] \\
\hline Bis (2-ethylhexyI) phthalate & $12 / 23$ & 847 & $J 82.0$ & -11 & $a$ & [a] \\
\hline Diethyl phthalate & $5 / 23$ & 5.0 & JB2.0 & -4.6 & a & [0] \\
\hline \multicolumn{7}{|l|}{ Field measurements, unfiltered } \\
\hline Conauctiviey $(\bar{m} \mathbf{s} / \mathrm{cm})$ & $23 / 23$ & 1.3 & 0.32 & 0.67 & d & [a] \\
\hline Dissolved oxygen (mg/L) & $23 / 23$ & 13 & 9.5 & 11 & $a$ & [a] \\
\hline Redox (mV) & $23 / 23$ & 630 & 110 & 350 & a & [a] \\
\hline Temperature $\left({ }^{\circ} \mathrm{C}\right\rangle$ & $23 / 23$ & 22 & 13 & 16 & 30.5 & $0[1]$ \\
\hline Turbidity (JTU) & $23 / 23$ & 2,100 & 2.1 & 230 & 1 & $23[2]$ \\
\hline $\mathrm{pH}$ (SU) & $23 / 23$ & 9.1 & 6.8 & 7.3 & $(5.5,8.5)$ & $I[3]$ \\
\hline \multicolumn{7}{|l|}{ Metals, unfilrered (mg/L) } \\
\hline Aluminum, total & $10 / 23$ & 3.0 & $<0.050$ & -0.50 & 0.2 & $7[3]$ \\
\hline Antimony, total. & $2 / 23$ & 0.0070 & $<0.0050$ & -0.0051 & $d$ & [a] \\
\hline Arsenic, rotal & $1 / 23$ & 0.011 & $<0.020$ & -0.010 & 0.05 & $0[1]$ \\
\hline Baríum, total & $23 / 23$ & 0.67 & 0.012 & 0.13 & 2 & $0[2]$ \\
\hline Boron, total & $9 / 23$ & 1.0 & $<0.080$ & -0.19 & $a$ & [0] \\
\hline Calcium, total & $23 / 23$ & 150 & 1.1 & 82 & $a$ & [a] \\
\hline Chromium, total & $5 / 23$ & 0.0086 & $<0.0040$ & -0.0046 & 0.05 & $0[1]$ \\
\hline Iron, toisal & $19 / 23$ & 11 & $<0.050$ & -2.1 & 0.3 & $14[3]$ \\
\hline Magnesium, total & $23 / 23$ & 98 & 0.50 & 19 & d & [d] \\
\hline Manganese, total & $21 / 23$ & 5.7 & $<0.0010$ & -1.0 & 0.05 & $11[3]$ \\
\hline Mercury, total & $1 / 23$ & 0.000060 & $<0.000050$ & -0.000050 & 0.002 & $0[1]$ \\
\hline Nickel, total & $4 / 23$ & 0.024 & $<0.010$ & -0.011 & a & [d] \\
\hline Potassium, total & $12 / 23$ & 5.8 & $<1.0$ & -1.7 & d & [d] \\
\hline Silicon, total & $23 / 23$ & 12 & 3.2 & 5.7 & d & [a] \\
\hline Sodium, toral & $23 / 23$ & 280 & 2.0 & 37 & $d$ & [d] \\
\hline Vanadium, total & $7 / 23$ & 0.0049 & $<0.0020$ & -0.0024 & d & [d] \\
\hline zine, total & $9 / 23$ & 0.052 & $<0.0050$ & -0.0098 & 5 & $0[3]$ \\
\hline \multicolumn{7}{|l|}{ Others, unfiltered } \\
\hline A.lkalinity (mg/L) & $23 / 23$ & 470 & 180 & 310 & d & [d] \\
\hline Total organic carbon (mg/L) & $23 / 23$ & 3.4 & 0.50 & 1.4 & a & [d] \\
\hline Total organic halides $(\mu g / L)$ & $14 / 23$ & 120 & $<5.0$ & -19 & a & [a] \\
\hline Total suspended solids (mg/L) & $11 / 23$ & 130 & $<5.0$ & -14 & $a$ & [a] \\
\hline \multicolumn{7}{|l|}{ Radionuclides, filtered $(p C i / L)^{\circ}$} \\
\hline Co-60 & $4 / 23$ & $12 *$ & -1.6 & $2.2 \%$ & 200 & $O[4]$ \\
\hline Cs -137 & $1 / 23$ & 3.8 & -4.6 & 0.38 & 120 & $0[4]$ \\
\hline Gross alpha & $9 / 23$ & $200 *$ & -0.62 & 10 & 15 & $1[2]$ \\
\hline Gross beta & $15 / 23$ & $11,000^{*}$ & -0.81 & 490 & 50 & $3[2]$ \\
\hline$H-3$ & $22 / 23$ & $15.000 *$ & 160 & $3,000 *$ & 20.000 & $0[2]$ \\
\hline$T h-230$ & $1 / 1$ & $6.8^{*}$ & $6.8^{*}$ & 6.8 & 12 & $0[4]$ \\
\hline Th-232 & $1 / 1$ & $0.57 *$ & $0.57 *$ & 0.57 & 2 & $0[4]$ \\
\hline Total rad $\mathrm{Sr}$ & $10 / 23$ & $7.600 *$ & -0.027 & 340 & 8 & $5[2]$ \\
\hline$U-234$ & $1 / 1$ & $180 *$ & $180^{*}$ & 180 & 20 & $I[4]$ \\
\hline U-235 & $1 / 1$ & $5.4^{\star}$ & $5.4^{*}$ & 5.4 & 24 & $0[4]$ \\
\hline$U-238$ & $1 / 1$ & $30 *$ & $30^{*}$ & 30 & 24 & $1[4]$ \\
\hline \multicolumn{7}{|l|}{ Radionuclides, unfiltered (pCi/L)" } \\
\hline Co-60 & $3 / 23$ & $16 \star$ & -5.4 & 1.3 & 200 & $0[4]$ \\
\hline Cs -137 & $2 / 23$ & $5.9 *$ & -2.2 & $1.6^{*}$ & 120 & $0[4]$ \\
\hline
\end{tabular}


Table 7.60 (continued)

\begin{tabular}{|c|c|c|c|c|c|c|}
\hline Parameter & $\begin{array}{l}\mathbf{N} \text { det } \\
\mathrm{N} \text { total }\end{array}$ & $\operatorname{Max}$ & Min* & $A v^{b}$ & $\begin{array}{l}\text { Reference } \\
\text { value }\end{array}$ & $\begin{array}{l}\text { Number of values } \\
\text { exceeding } \\
\text { reference [ref]c }\end{array}$ \\
\hline \multicolumn{7}{|c|}{ Radionuclides, unfiltered $(\mathrm{pC} \mathrm{L} / \mathrm{L})^{\bullet}$} \\
\hline Gross alphe & $7 / 23$ & $490 *$ & -1.7 & 23 & 15 & $1\{2\}$ \\
\hline Gross beta & $21 / 23$ & $14,000 *$ & 1.1 & 650 & 50 & $3[2]$ \\
\hline $\mathrm{H}-3$ & $20 / 23$ & $17,000 *$ & -160 & $3,000 *$ & 20,000 & $0[2]$ \\
\hline Th-228 & $1 / 1$ & $1.1 *$ & $1.1 *$ & 1.1 & 16 & $0[4]$ \\
\hline Th-230 & $1 / 1$ & $7.8 *$ & $7.8 *$ & 7.8 & 12 & $0[4]$ \\
\hline Th-232 & $1 / 1$ & $1.0 *$ & $1.0^{*}$ & 1.0 & 2 & $0[4]$ \\
\hline Total rad Sr & $10 / 23$ & $7,000 *$ & -1.1 & 310 & 8 & $5[2]$ \\
\hline$U-234$ & $1 / 1$ & $410^{*}$ & $410^{\star}$ & 410 & 20 & $1[4]$ \\
\hline$U-235$ & $1 / 1$ & $6.2 *$ & $6.2 *$ & 6.2 & 24 & $O[4]$ \\
\hline$U-238$ & $1 / 1$ & $11 *$ & $11 *$ & 11 & 24 & $0[4]$ \\
\hline \multicolumn{7}{|c|}{ Volatile organics, unfiltered $(\mu \mathrm{g} / \mathrm{L})$} \\
\hline 1,1,1-Trichloroethane & $1 / 23$ & 16 & U5.0 & -5.5 & 200 & $0[1]$ \\
\hline 1.2-Dichloroethene & $1 / 23$ & 7.4 & U5.0 & -5.1 & 70 & $0[2]$ \\
\hline Carbon disulfide & $3 / 23$ & U5.0 & $\mathrm{J} 1.4$ & -4.6 & $d$ & [d] \\
\hline Chloroform & $2 / 23$ & 15 & U5. 0 & -5.5 & 100 & $0[2]$ \\
\hline Trichloroethene & $1 / 23$ & U5.0 & 34.4 & -5.0 & 5 & $0[1]$ \\
\hline \multicolumn{7}{|c|}{ Upgradient Wells } \\
\hline \multicolumn{7}{|l|}{ Anions, unfiltered (mg/L) } \\
\hline Chloride & $2 / 3$ & 13 & $<2.0$ & -6.7 & 250 & $0[3]$ \\
\hline Fluoride & $1 / 3$ & 1.2 & $<0.10$ & -0.47 & 4 & $0[2]$ \\
\hline Nitrate & $1 / 3$ & 5.4 & $<1.0$ & -2.5 & 10 & $O[2]$ \\
\hline Sulfate, as so, & $3 / 3$ & 34 & 13 & 25 & 250 & $O[3]$ \\
\hline \multicolumn{7}{|c|}{ Base neutral/acid extractable organics, unfiltered $\langle\mu g / L\rangle$} \\
\hline 4-Methylphenol & $1 / 3$ & U5. 0 & $\mathrm{J3} .0$ & -4.3 & $a$ & [d] \\
\hline \multicolumn{7}{|l|}{ Field measurements, unfiltered } \\
\hline Conductivity $(\mathrm{ms} / \mathrm{cm})$ & $3 / 3$ & 0.77 & 0.59 & 0.67 & a & [d] \\
\hline Dissolved oxygen (mg/L) & $3 / 3$ & 10 & 7.5 & 9.1 & a & [d] \\
\hline Redox $(\mathrm{mV})$ & $3 / 3$ & 670 & 290 & 430 & a & [d] \\
\hline Temperature $\left({ }^{\circ} \mathrm{C}\right)$ & $3 / 3$ & 16 & 15 & 15 & 30.5 & $0[1]$ \\
\hline Turbiaity (JTU) & $3 / 3$ & 340 & 8.4 & 120 & 1 & $3[2]$ \\
\hline $\mathrm{pH} \quad(\mathrm{SU})$ & $3 / 3$ & 8.1 & 7.1 & 7.5 & $(6.5,8.5)$ & $0\{3\}$ \\
\hline \multicolumn{7}{|l|}{ Metals, unfiltered (mg/L) } \\
\hline Aluminum, total & $1 / 3$ & 4.2 & $<0.050$ & -1.4 & 0.2 & $1[3]$ \\
\hline Barium, total & $3 / 3$ & 0.21 & 0.080 & 0.15 & 2 & $0[2]$ \\
\hline Boron, total & $1 / 3$ & 0.25 & $<0.080$ & -0.14 & $a$ & [d] \\
\hline Calcium, total & $3 / 3$ & 110 & 35 & 83 & $d$ & [a] \\
\hline Chromium, total & $1 / 3$ & 0.0063 & $<0.0040$ & -0.0048 & 0.05 & $0[1]$ \\
\hline Iron, total & $2 / 3$ & 5.5 & $<0.050$ & -1.9 & 0.3 & $1[3]$ \\
\hline Magnesium, total & $3 / 3$ & 28 & 10 & 21 & a & [d] \\
\hline Manganese, total & $3 / 3$ & 0.17 & 0.0060 & 0.061 & 0.05 & $1[3]$ \\
\hline Nickel, total & $1 / 3$ & 0.024 & $<0.010$ & -0.015 & d & [d] \\
\hline Selenium, tatal & $1 / 3$ & $<0.0050$ & 0.0040 & -0.0047 & 0.01 & $0\{1\}$ \\
\hline Silicon, total & $3 / 3$ & 9.5 & 6.0 & 7.7 & d & [d] \\
\hline Sodium, total & $3 / 3$ & 37 & 5.3 & 19 & $d$ & [d] \\
\hline Vanadium, total & $1 / 3$ & 0.0082 & $<0.0020$ & -0.0041 & d & [d] \\
\hline zine, rotal & $1 / 3$ & 0.0054 & $<0.0050$ & -0.0051 & 5 & $0[3]$ \\
\hline \multicolumn{7}{|l|}{ others, unfiltered } \\
\hline Alkalinity (mg/L) & $3 / 3$ & 300 & 240 & 280 & d & [d] \\
\hline Tocal organic carbon (mg/L) & $3 / 3$ & 1.2 & 0.50 & 0.84 & d & [d] \\
\hline Total organic halides $(\mu \mathrm{g} / \mathrm{L})$ & $1 / 3$ & $<7.3$ & $<5.0$ & -5.8 & d & [d] \\
\hline Total suspended solids (mg/L) & $2 / 3$ & 170 & $<5.0$ & -64 & a & [d] \\
\hline \multicolumn{7}{|l|}{ Radionuclides, filtered $(\mathrm{pCi} / \mathrm{L})^{*}$} \\
\hline Gross beta & $2 / 3$ & $4.9 *$ & 1.4 & $3.2 *$ & 50 & $O[2]$ \\
\hline $\mathrm{H}-3$ & $3 / 3$ & $3,000 *$ & $840^{*}$ & $1,900 *$ & 20.000 & $O[2]$ \\
\hline
\end{tabular}




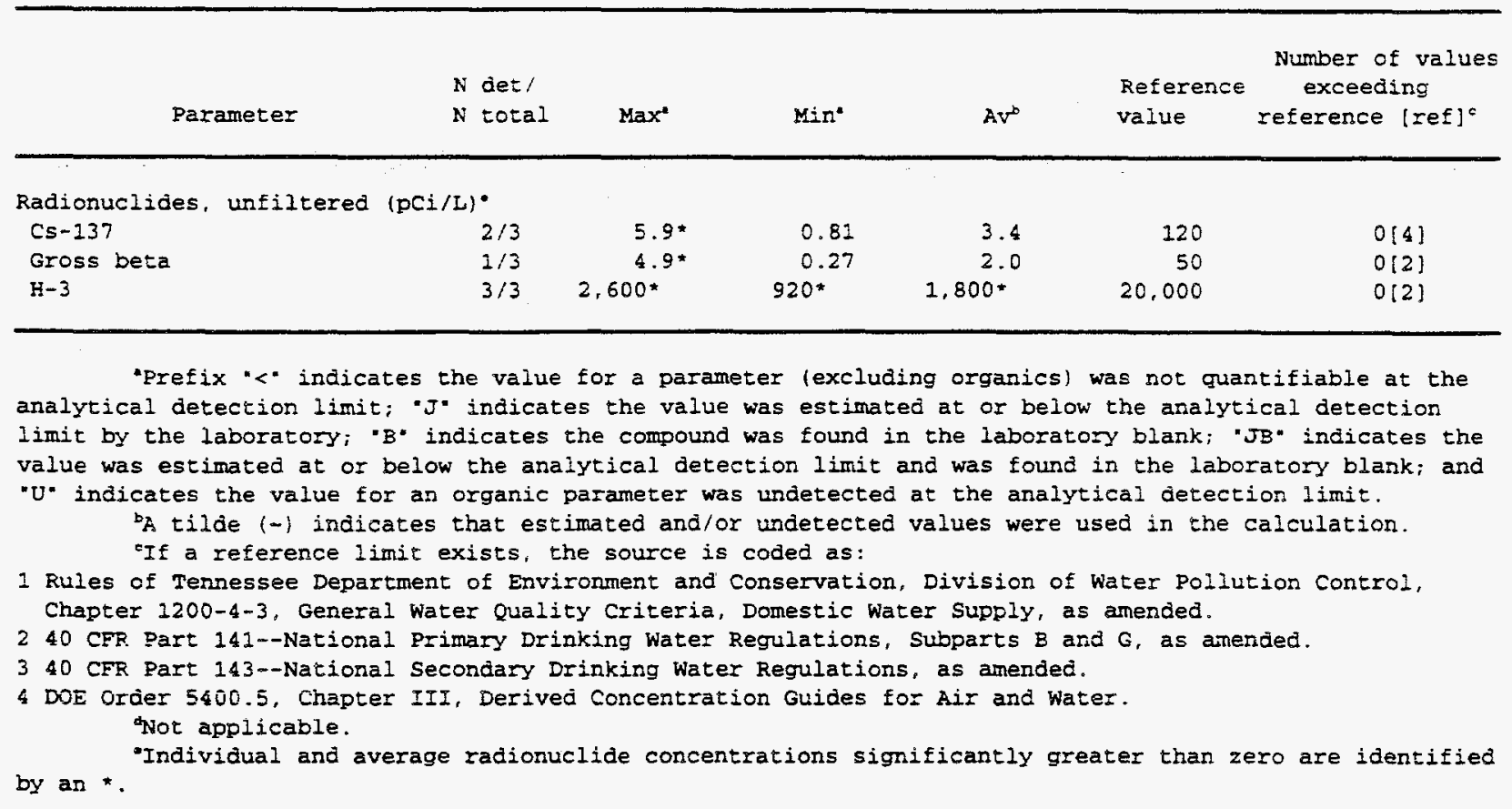


Table 7.61. Constituents in Waste Area Grouping (WAG) 2 groundwater at ORNL, January 25-February 12, 1993

\begin{tabular}{|c|c|c|c|c|c|c|}
\hline Parameter & $\begin{array}{l}\mathbf{N} \text { det } / \\
\mathbf{N} \text { total }\end{array}$ & $\operatorname{Max}$ & Min* & $A v^{2}$ & $\begin{array}{l}\text { Reference } \\
\text { value }\end{array}$ & $\begin{array}{l}\text { Number of values } \\
\text { exceeding } \\
\text { reference [ref]" }\end{array}$ \\
\hline \multicolumn{7}{|c|}{ Downgradient wells } \\
\hline \multicolumn{7}{|l|}{ Anions, unfiltered (mg/L) } \\
\hline Chloride & $4 / 8$ & 22 & $<2.0$ & -6.3 & 250 & $0[3]$ \\
\hline Fluoride & $2 / 8$ & 2.0 & $<0.10$ & -0.35 & 4 & $O[2]$ \\
\hline Phosphate & $6 / 8$ & 1.4 & $<1.0$ & -1.1 & $d$ & [d] \\
\hline Sulfate, as so. & $4 / 8$ & 37 & $<10$ & -16 & 250 & $0[3]$ \\
\hline \multicolumn{7}{|c|}{ Base neutral/acid extractable organics, unfiltered ( $\mu g / I$ ) } \\
\hline Benzyl buty I phthalate & $3 / 8$ & B10 & 34.0 & -9.0 & $d$ & [a] \\
\hline Bis (2-ethylhexyl) phthalate & $4 / 8$ & $\mathrm{U10}$ & B6.0 & -8.4 & $a$ & [a] \\
\hline \multicolumn{7}{|l|}{ Field measurements, unfiltered } \\
\hline Conductivity $(\mathrm{ms} / \mathrm{cm})$ & $8 / 8$ & 0.85 & 0.24 & 0.56 & d & [a] \\
\hline Dissolved oxygen (mg/L) & $8 / 8$ & 11 & 8.2 & 9.2 & $d$ & [d] \\
\hline Redox (mV) & $8 / 8$ & 510 & 220 & 420 & $d$ & [a] \\
\hline Temperature $\left({ }^{\circ} \mathrm{C}\right)$ & $8 / 8$ & 17 & 14 & 15 & 30.5 & $0[1]$ \\
\hline Turbidity (JTU) & $8 / 8$ & 21 & 4.2 & 19 & 1 & $8[2]$ \\
\hline $\mathrm{pH}$ (SU) & $8 / 8$ & 9.8 & 6.8 & 7.6 & $(6.5,8.5)$ & $2\{3\}$ \\
\hline \multicolumn{7}{|l|}{ Metals, filtered (mg/L) } \\
\hline Arsenic & $2 / 8$ & 0.030 & $<0.010$ & -0.013 & 0.05 & $0[1]$ \\
\hline Barium & $8 / 8$ & 0.94 & 0.028 & 0.28 & 2 & $0[2]$ \\
\hline Boron & $3 / 8$ & 1.1 & $<0.080$ & -0.23 & $d$ & [d] \\
\hline Calcium & $8 / 8$ & 130 & 0.61 & 65 & $d$ & [a] \\
\hline Iron & $4 / 8$ & 20 & $<0.050$ & -4.4 & 0.3 & $3[3]$ \\
\hline Magnesium & $8 / 8$ & 20 & 0.17 & 8.8 & $d$ & [d] \\
\hline Manganese & $8 / 8$ & 0.98 & 0.0033 & 0.20 & 0.05 & $4[3]$ \\
\hline Nickel & $1 / 8$ & 0.036 & $<0.010$ & -0.013 & d & [d] \\
\hline Potassium & $7 / 8$ & 2.4 & $<1.0$ & -1.6 & $d$ & [a] \\
\hline Silicon & $8 / 8$ & 10 & 3.8 & 7.1 & $d$ & {$[a]$} \\
\hline Sodium & $8 / 8$ & 220 & 4.0 & 55 & $d$ & [d] \\
\hline Vanadium & $3 / 8$ & 0.022 & $<0.0020$ & -0.0046 & $d$ & [a] \\
\hline Zinc & $4 / 8$ & 0.0078 & $<0.0050$ & -0.0057 & 5 & $0[3]$ \\
\hline \multicolumn{7}{|l|}{ Metals, unfiltered (mg/L) } \\
\hline Aluminum, total & $3 / 8$ & 1.9 & $<0.050$ & -0.47 & 0.2 & $2[3]$ \\
\hline Arsenic, total & $1 / 8$ & 0.036 & $<0.010$ & -0.013 & 0.05 & $O[1]$ \\
\hline Barium, cotal & $8 / 8$ & 0.86 & 0.046 & 0.28 & 2 & $O[2]$ \\
\hline Boron, total & $3 / 8$ & 1.1 & $<0.080$ & -0.22 & d & (d) \\
\hline Calcium, total & $8 / 8$ & 130 & 1.2 & 62 & $d$ & {$[d]$} \\
\hline Chromiun, total & $3 / 8$ & 0.91 & $<0.0040$ & -0.12 & 0.05 & $1\{1\}$ \\
\hline Cobalt, total & $1 / 8$ & 0.0084 & $<0.0040$ & -0.0046 & $a$ & {$[d]$} \\
\hline Iron, total & $8 / 8$ & 20 & 0.053 & 6.9 & 0.3 & $6[3]$ \\
\hline Magnesium, cotal & $8 / 8$ & 18 & 0.32 & 8.4 & $a$ & [a] \\
\hline Manganese, total & $8 / 8$ & 0.94 & 0.0047 & 0.20 & 0.05 & $5[3]$ \\
\hline Nickel, total & $2 / 8$ & 0.085 & $<0.010$ & -0.019 & $\mathrm{~d}$ & [d] \\
\hline Potassium, total & $6 / 8$ & 2.2 & $<1.0$ & -1.6 & $d$ & [d] \\
\hline Selenium, total & $1 / 8$ & 0.0050 & $<0.0050$ & -0.0050 & 0.01 & $0\lfloor 1\rfloor$ \\
\hline Silicon, total & $8 / 8$ & 12 & 3.8 & 7.8 & d & [d] \\
\hline Sodium, sotal & $8 / 8$ & 210 & 3.9 & 52 & $a$ & [d] \\
\hline vanadium, total & $2 / 8$ & 0.022 & $<0.0020$ & -0.0045 & $d$ & [d] \\
\hline zinc, total & $7 / 8$ & 0.027 & $<0.0050$ & -0.014 & 5 & $0[3]$ \\
\hline \multicolumn{7}{|l|}{ Others, filtered: } \\
\hline Alkalinity $(\mathrm{mg} / \mathrm{t})$ & $8 / 8$ & 470 & 110 & 300 & d & [d] \\
\hline Total dissolved solids (mg/L) & $8 / 8$ & 570 & 140 & 360 & 500 & $1[1]$ \\
\hline \multicolumn{7}{|l|}{ others, unfiltered } \\
\hline Alkalinity (mg/L) & $8 / 8$ & 480 & 110 & 300 & $d$ & [d] \\
\hline Ammonia $(m g / L)$ & $8 / 8$ & 9.5 & 0.070 & 1.3 & $d$ & [d] \\
\hline Total organic carbon (mg/L) & $8 / 8$ & 4.9 & 0.55 & 1.5 & d & [d] \\
\hline
\end{tabular}




\begin{tabular}{|c|c|c|c|c|c|c|}
\hline Parameter & $\begin{array}{l}\text { N det/ } \\
N \text { total }\end{array}$ & $\operatorname{Max}$ & Min* & $A v^{2}$ & $\begin{array}{l}\text { Reference } \\
\text { value } r\end{array}$ & $\begin{array}{l}\text { Number of vaiues } \\
\text { exceeding } \\
\text { reference [ref }{ }^{c}\end{array}$ \\
\hline \multicolumn{7}{|l|}{ Others, unfiltered } \\
\hline Toral organic halides ( $\mu g / L)$ & $1 / 8$ & $<5.3$ & $<5.0$ & -5.0 & d & [d] \\
\hline Total suspenced solids (mg/L) & $4 / 8$ & 100 & $<5.0$ & -28 & $\mathrm{~d}$ & [a] \\
\hline \multicolumn{7}{|l|}{ Radionuclides, filtered $(\mathrm{pCi} / \mathrm{L})$} \\
\hline Co-60 & $1 / 8$ & $8.6^{*}$ & 0.27 & $2.4 *$ & 200 & $0[4]$ \\
\hline Gross alpina & $4 / 8$ & $18^{*}$ & 0.41 & $4.5^{\star}$ & 15 & $1[2]$ \\
\hline Gross beta & $7 / 8$ & $1,500 *$ & 1.6 & 200 & 50 & $1[2]$ \\
\hline $\mathrm{H}-3$ & $7 / 8$ & $190,000^{*}$ & -430 & $50,000 *$ & 20,000 & $3[2]$ \\
\hline Total rad Sr & $4 / 8$ & $840^{*}$ & 0.38 & 110 & 8 & $2[2]$ \\
\hline \multicolumn{7}{|l|}{ Radionuclides, unfiltered $(p C i / L)^{*}$} \\
\hline co-60 & $1 / 8$ & $5.4^{\star}$ & -0.27 & $1.7^{*}$ & 200 & $0[4]$ \\
\hline Gross alpha & $7 / 8$ & $10 *$ & -0.27 & $5.2^{*}$ & 15 & $0[2]$ \\
\hline Gross beta & $8 / 8$ & $1,400 \%$ & $4.9 *$ & 190 & 50 & $1\{2\}$ \\
\hline$H-3$ & $7 / 8$ & $190,000^{*}$ & -140 & 50.000 & 20,000 & $3[2]$ \\
\hline Total rad $S r$ & $3 / 8$ & $840^{*}$ & -0.41 & 110 & 8 & $1[2]$ \\
\hline \multicolumn{7}{|c|}{ Upgradient Wells } \\
\hline \multicolumn{7}{|l|}{ Anions, unfiltered (mg/L) } \\
\hline Chloride & $5 / 12$ & 4.5 & $<2.0$ & -2.6 & 250 & $0[3]$ \\
\hline Fluoride & $6 / 12$ & 0.30 & $<0.10$ & -0.26 & 4 & $0[2]$ \\
\hline Nitrare & $2 / 12$ & 11 & $<1.0$ & -1.8 & 10 & $1[2]$ \\
\hline Phosphate & $5 / 12$ & 1.3 & $<1.0$ & -1.1 & $a$ & [d] \\
\hline Sulfare, as so, & $9 / 12$ & 97 & $<10$ & -36 & 250 & $0[3]$ \\
\hline \multicolumn{7}{|c|}{ Base neutral/acid extractable organics, unfiltered $(\mu \mathrm{g} / \mathrm{L})$} \\
\hline Benzyl butyl phthalate & $5 / 12$ & U10 & 82.0 & -6.8 & d & (d) \\
\hline Bis (2-ethylhexyl) phthalate & $3 / 12$ & U10 & 83.0 & -8.7 & d & [d] \\
\hline Diethyl phthalate & $1 / 12$ & U10 & $\mathrm{J} 4.0$ & -9.5 & $a$ & [d] \\
\hline \multicolumn{7}{|l|}{ Field measurements, unfiltered } \\
\hline Conductivity $(\mathrm{mS} / \mathrm{cm})$ & $12 / 12$ & 0.70 & 0.27 & 0.53 & d & [d] \\
\hline Dissolved oxygen $(\mathrm{mg} / \mathrm{L})$ & $12 / 12$ & 11 & 7.7 & 9.8 & d & [d] \\
\hline Redox (mV) & $12 / 12$ & 630 & 320 & 500 & a & la: \\
\hline Temperature $\left({ }^{\circ} \mathrm{C}\right)$ & $12 / 12$ & 14 & 11 & 13 & 30.5 & $0[1]$ \\
\hline Turbiaity (JTU) & $12 / 12$ & 110 & 5.3 & 25 . & 1 & $12\lceil 2\rfloor$ \\
\hline $\mathrm{pH} \quad(\mathrm{SU})$ & $12 / 12$ & 9.4 & 6.6 & 7.6 & $(6.5,8.5)$ & $1(3)$ \\
\hline \multicolumn{7}{|l|}{ Metals, filtered (mg/L) } \\
\hline Aluminum & $1 / 12$ & 0.052 & $<0.050$ & -0.050 & 0.2 & $0[3]$ \\
\hline Antimony & $3 / 12$ & 0.0070 & $<0.0050$ & -0.0054 & a & [d] \\
\hline Barium & $12 / 12$ & 0.51 & 0.041 & 0.18 & 2 & $0[2]$ \\
\hline Boron & $7 / 12$ & 0.88 & $<0.080$ & -0.21 & $d$ & [d] \\
\hline Calcium & $12 / 12$ & 130 & 0.93 & 59 & $d$ & [d] \\
\hline Cobalt & $2 / 12$ & 0.021 & $<0.0040$ & -0.0056 & $d$ & {$[a]$} \\
\hline Iron & $5 / 12$ & 3.8 & $<0.050$ & -0.40 & 0.3 & $2[3]$ \\
\hline Magnesium & $12 / 12$ & 28 & 0.27 & 13 & a & [a] \\
\hline Manganese & $12 / 12$ & 11 & 0.0029 & 1.1 & 0.05 & $5[3]$ \\
\hline Nickel & $2 / 12$ & 0.022 & $<0.010$ & -0.012 & a & [d] \\
\hline Potassium & $11 / 12$ & 7.1 & $<1.0$ & -2.7 & a & [a] \\
\hline silicon & $12 / 12$ & 10 & 3.0 & 7.1 & $\mathrm{a}$ & [d] \\
\hline Sodium & $12 / 12$ & 190 & 4.7 & 48 & $a$ & [a] \\
\hline Vanadium & $6 / 12$ & 0.0032 & $<0.0020$ & -0.0023 & $a$ & [a] \\
\hline zinc & $5 / 12$ & 0.014 & $<0.0050$ & -0.0068 & 5 & $0[3]$ \\
\hline \multicolumn{7}{|l|}{ Metals, unfiltered (mg/L) } \\
\hline Aluminum, totál & $5 / 12$ & 0.17 & $<0.050$ & -0.072 & 0.2 & $0[3]$ \\
\hline Barium, total & $12 / 12$ & 0.50 & 0.042 & 0.19 & 2 & $0[2]$ \\
\hline Boron, total & $7 / 12$ & 0.87 & $<0.080$ & -0.22 & 0 & {$[a]$} \\
\hline Calcium, cotal & $12 / 12$ & 110 & 0.91 & 55 & a & [d] \\
\hline Cobalt, rotal & $2 / 12$ & 0.018 & $<0.0040$ & -0.0054 & $\mathrm{a}$ & [d] \\
\hline Iron, total & $9 / 12$ & 3.9 & $<0.050$ & -0.49 & 0.3 & $3[3]$ \\
\hline
\end{tabular}


Table 7.61 (continued)

\begin{tabular}{|c|c|c|c|c|c|c|}
\hline Parameter & $\begin{array}{l}\mathbf{N} \text { det/ } \\
\mathbf{N} \text { total }\end{array}$ & $\operatorname{Max}$ & Min* & $A v^{b}$ & $\begin{array}{l}\text { Reference } \\
\text { value }\end{array}$ & $\begin{array}{l}\text { Number of values } \\
\text { exceeding } \\
\text { reference [ref }]^{e}\end{array}$ \\
\hline \multicolumn{7}{|l|}{ Metals, unfiltered (mg/L) } \\
\hline Magnesiun, total & $12 / 12$ & 24 & 0.26 & 12 & d & [d] \\
\hline Manganese, total & $12 / 12$ & 10 & 0.0041 & 1.0 & 0.05 & $7[3]$ \\
\hline Nickel, total & $1 / 12$ & 0.023 & $<0.010$ & -0.011 & $\mathrm{~d}$ & [d] \\
\hline Potassium, total & $12 / 12$ & 7.3 & 1.0 & 2.8 & $d$ & [a] \\
\hline silicon, total & $12 / 12$ & 11 & 3.2 & 7.1 & $a$ & [a] \\
\hline Sodium, total & $12 / 12$ & 180 & 4.3 & 46 & $a$ & [a] \\
\hline Zine, total & $12 / 12$ & 0.16 & 0.0075 & 0.026 & 5 & $0[3]$ \\
\hline \multicolumn{7}{|l|}{ Others, filtered } \\
\hline Alkalinity (mg/L) & $12 / 12$ & 400 & 130 & 270 & $a$ & [a] \\
\hline Total dissolved solids (mg/L) & $12 / 12$ & 470 & 170 & 340 & 500 & $0[1]$ \\
\hline \multicolumn{7}{|l|}{ Others, unfiltered } \\
\hline Alkalinity (mg/L) & $12 / 12$ & 390 & 130 & 270 & d & [d] \\
\hline Armonia (mg/L) & $12 / 12$ & 0.45 & 0.10 & 0.18 & d & [d] \\
\hline Total organic carbon (mg/L) & $11 / 12$ & 2.0 & $<0.50$ & -1.0 & $\mathrm{~d}$ & [d] \\
\hline Total organic halides $(\mu \mathrm{g} / \mathrm{L})$ & $1 / 12$ & 9.5 & $<5.0$ & -5.4 & $\mathrm{~d}$ & [d] \\
\hline Total suspended solids (mg/L) & $4 / 12$ & 20 & $<5.0$ & -7.1 & $d$ & [d] \\
\hline \multicolumn{7}{|l|}{ Radionuclides, filtered $(p \subset i / L)^{\bullet}$} \\
\hline Co-60 & $2 / 12$ & $250 *$ & -5.7 & 21 & 200 & $1[4]$ \\
\hline Gross alpha & $11 / 12$ & $12 *$ & 0.97 & $4.2^{\star}$ & 15 & $0[2]$. \\
\hline Gross beta & $11 / 12$ & $700 *$ & 2.4 & 91 & 50 & $2[2]$ \\
\hline $\mathrm{H}-3$ & $11 / 12$ & $240,000 *$ & -300 & 24,000 & 20,000 & $2[2]$ \\
\hline Total rad sr & $4 / 12$ & $3.8^{*}$ & -0.73 & $1.1 *$ & 8 & $0[2]$ \\
\hline \multicolumn{7}{|l|}{ Radionuclides, unfiltered (DCi/L)• } \\
\hline $\operatorname{co}-60$ & $2 / 12$ & $270^{*}$ & -2.7 & 23 & 200 & $1[4]$ \\
\hline $\mathrm{Cs}-137$ & $2 / 12$ & $3.8 *$ & -5.1 & 0.023 & 120 & $0[4]$ \\
\hline Gross alpha & $10 / 12$ & $9.7 *$ & 0.30 & $2.9 *$ & 15 & $0[2]$ \\
\hline Gross beta & $12 / 12$ & $840 *$ & $5.4^{*}$ & 92 & 50 & $2[2]$ \\
\hline $\mathrm{H}-3$ & $11 / 12$ & $240,000 *$ & -540 & 24.000 & 20,000 & $2[2]$ \\
\hline Total rad Sr & $6 / 12$ & $5.9 *$ & 0.081 & $2.3 *$ & 8 & $0[2]$ \\
\hline \multicolumn{7}{|c|}{ Volatile organics, unfiltered $(\mu \mathrm{g} / \mathrm{L})$} \\
\hline Acetone & $1 / 12$ & 83 & u10 & -16 & $d$ & [d] \\
\hline Carbon disulfide & $1 / 12$ & 35 & U5.0 & -7.5 & $\mathrm{~d}$ & [d] \\
\hline
\end{tabular}

-Prefix "<" indicates the value for a parameter (excluding organies) was not quantifiable at the analytical detection limit; " $J$ " indicates the value was estimated at or below the analytical detection limit by the laboratory; ' $\mathrm{B}$ " indicates the compound was found in the laboratory blank; "JB" indicates the value was estimated at or below the analytical detection limit and was found in the laboratory blank; and ' $U$ " indicates the value for an organic parameter was undetected at the analytical detection limit.

B tilde ( $\sim$ ) indicates that estimated and/or undetected values were used in the calculation.

If a reference limit exists, the source is coded as:

I Rules of Tennessee Department of Environment and Conservation, Division of water Pollution Control,

Chapter 1200-4-3, General Water Quality Criteria, Domestic Water Supply, as amended.

240 CFR Part 141--National Primary Drinking water Regulations, Subparts B and G, as amended.

340 CFR Part 143--National Secondary Drinking Water Regulations, as amended.

4 DOE Order 5400.5, Chapter III, Derived Concentration Guides for Air and water.

Not applicable.

-Individual and average radionuclide concentrations significantly greater than zero are identified by an *. 
Table 7.62. Constituents in Waste Area Grouping (WAG) 3 groundwster at ORNL, October 22-November 12, 1993

\begin{tabular}{|c|c|c|c|c|c|c|}
\hline Parameter & $\begin{array}{l}\mathrm{N} \text { det/ } \\
\mathrm{N} \text { total }\end{array}$ & Max' & Miñ & $A V^{b}$ & $\begin{array}{l}\text { Reference } \\
\text { value }\end{array}$ & $\begin{array}{l}\text { Number of values } \\
\text { exceeding } \\
\text { reference [ref]c }\end{array}$ \\
\hline \multicolumn{7}{|c|}{ Downgradient wells } \\
\hline \multicolumn{7}{|l|}{ Anions, unfiltered (mg/L) } \\
\hline Chloride & $10 / 10$ & 330 & 2.1 & 73 & 250 & $2[3]$ \\
\hline Eluoride & $1 / 10$ & 0.50 & $<0.10$ & -0.14 & 4 & $0[2]$ \\
\hline Nitrate & $3 / 10$ & 2.2 & $<1.0$ & -1.1 & 10 & $0[2]$ \\
\hline Sulfate, as so, & $10 / 10$ & 80 & 3.0 & 35 & 250 & $0[3]$ \\
\hline \multicolumn{7}{|l|}{ Field measurements, unfiltered } \\
\hline Conductivity (mS/cm) & $10 / 20$ & 1.8 & 0.59 & 0.96 & a & [d] \\
\hline Dissolved oxygen $(\mathrm{mg} / \mathrm{L})$ & $10 / 10$ & 14 & 11 & 12 & d & (d) \\
\hline Temperature $\left({ }^{\circ} \mathrm{C}\right)$ & $10 / 10$ & 16 & 13 & 14 & 30.5 & $0[1]$ \\
\hline Turbidity (NTU) & $10 / 10$ & 15 & 0 & 3.6 & 1 & $7[2]$ \\
\hline $\mathrm{pH} \quad(\mathrm{SU})$ & $10 / 10$ & 8.0 & 6.6 & 7.2 & $(6.5,8.5)$ & $0[3]$ \\
\hline \multicolumn{7}{|l|}{ Metals, unfiltered $(\mathrm{mg} / \mathrm{L})$} \\
\hline Aluminum, cotal & $2 / 10$ & 0.58 & $<0.050$ & -0.10 & 0.2 & $1[3]$ \\
\hline Barium, total & $10 / 10$ & 0.79 & 0.043 & 0.20 & 2 & $0[2]$ \\
\hline Boron, total & $8 / 10$ & 1.1 & $<0.080$ & -0.31 & $\mathrm{~d}$ & [d] \\
\hline Calcium, total & $20 / 10$ & 160 & 42 & 110 & $d$ & [d] \\
\hline Chromium, total & $2 / 10$ & 0.0091 & $<0.0040$ & -0.0050 & 0.05 & $0[1]$ \\
\hline Copper, total & $1 / 10$ & 0.010 & $<0.0070$ & -0.0073 & 1.3 & $0[2]$ \\
\hline Iron, total & $7 / 10$ & 2.4 & $<0.050$ & -0.60 & 0.3 & $6[3]$ \\
\hline Magnesium, total & $10 / 10$ & 50 & 6.8 & 26 & $d$ & [d] \\
\hline Manganese, total & $10 / 10$ & 0.85 & 0.0014 & 0.17 & 0.05 & $4[3]$ \\
\hline Nickel, total & $2 / 10$ & 0.14 & $<0.010$ & -0.023 & d & [a] \\
\hline Potassium, cotal & $9 / 10$ & 12 & 1.8 & -3.6 & d & [a] \\
\hline silicon, total & $10 / 10$ & 8.3 & 4.1 & 6.0 & d & [d] \\
\hline Sodiun, total & $10 / 10$ & 200 & 5.4 & 37 & d & [d] \\
\hline Vanadium, total & $5 / 10$ & 0.0030 & $<0.0020$ & -0.0024 & a & [a] \\
\hline zinc, total & $8 / 10$ & 0.012 & $<0.0050$ & -0.0078 & 5 & 0131 \\
\hline \multicolumn{7}{|l|}{ Others, unfiltered } \\
\hline Alkalinity (mg/L) & $10 / 10$ & 440 & 240 & 330 & d & [d] \\
\hline Total organic carbon (mg/L) & $10 / 10$ & 6.4 & 0.50 & 1.9 & $a$ & [d] \\
\hline Total organic halides $(\mu \mathrm{g} / \mathrm{L})$ & $5 / 10$ & 77 & $<5.0$ & -21 & d & [d] \\
\hline Total suspended solids (mg/L) & $2 / 10$ & 13 & $<5.0$ & -5.9 & $d$ & [d] \\
\hline \multicolumn{7}{|l|}{ Radionuclides, filtered $(\mathrm{pCi} / \mathrm{L})^{\circ}$} \\
\hline $\operatorname{co}-60$ & $3 / 10$ & $4.6 *$ & -1.4 & $2.5^{*}$ & 200 & $0[\&]$ \\
\hline Cs -137 & $2 / 10$ & $6.8 *$ & -1.1 & $1.9 *$ & 120 & $0[4]$ \\
\hline Gross alpha & $5 / 10$ & 15* & -0.57 & $4.0 *$ & 15 & $0[2]$ \\
\hline Gross beta & $10 / 10$ & $1,300 *$ & $3.2^{\star}$ & 190 & 50 & $4[2]$ \\
\hline $\mathrm{H}-3$ & $9 / 10$ & $27,000 *$ & 160 & 3,600 & 20,000 & $1[2]$ \\
\hline Total rad $\mathrm{Sr}$ & $7 / 10$ & $730 *$ & 1.6 & 100 & 8 & $4[2]$ \\
\hline \multicolumn{7}{|l|}{ Radionuclides, unfiltered (pCi/L)* } \\
\hline Cs -137 & $1 / 10$ & $3.5 *$ & -1.9 & 0.89 & 120 & $0[4]$ \\
\hline Gross alpha & $7 / 10$ & $11 *$ & -0.70 & $3.4^{\star}$ & 15 & $0[2]$ \\
\hline Gross beta & $10 / 10$ & $2,600 *$ & $4.6 *$ & 230 & 50 & $4[2]$ \\
\hline $\mathrm{H}-3$ & $10 / 10$ & $30,000 *$ & $700 *$ & 4.000 & 20,000 & $1[2]$ \\
\hline Total rad SI & $6 / 10$ & $680^{*}$ & 0.59 & 97 & 8 & $4[2]$ \\
\hline \multicolumn{7}{|c|}{ Volatile organics, unfiltered $(\mu g / L)$} \\
\hline 1,2-Dichloroethene & $2 / 10$ & 16 & 05.0 & -6.2 & 70 & $0[2]$ \\
\hline Trichloroethene & $1 / 10$ & 11 & U5.0 & -5.6 & 5 & $1[1]$ \\
\hline \multicolumn{7}{|c|}{ Upgradient wells } \\
\hline \multicolumn{7}{|l|}{ Anions, unfiltered (mg/L) } \\
\hline Chloride & $3 / 3$ & 1.8 & 1.3 & 1.6 & 250 & $0[3]$ \\
\hline Sulfate, as so, & $3 / 3$ & 16 & 6.7 & 9.9 & 250 & $0[3]$ \\
\hline
\end{tabular}


Table 7.62 (continued)

\begin{tabular}{|c|c|c|c|c|c|c|}
\hline Parameter & $\begin{array}{l}\text { N det/ } \\
\text { N total }\end{array}$ & Max & Min* & $A v^{b}$ & $\begin{array}{l}\text { Reference } \\
\text { value }\end{array}$ & $\begin{array}{l}\text { Number of values } \\
\text { exceeding } \\
\text { reference [ref } \text { f }^{c}\end{array}$ \\
\hline \multicolumn{7}{|l|}{ Field measurements, unfiltered } \\
\hline Conductivity (ms/cm) & $3 / 3$ & 0.68 & 0.34 & 0.55 & d & [d] \\
\hline Dissolved oxygen $\langle\mathrm{mg} / \mathrm{L}\rangle$ & $3 / 3$ & 13 & 12 & 12 & $d$ & [d] \\
\hline Temperature $\left|{ }^{\circ} \mathrm{C}\right\rangle$ & $3 / 3$ & 15 & 13 & 14 & 30.5 & $0[2]$ \\
\hline Turbidity (NTU) & $3 / 3$ & 38 & 10 & 25 & 1 & $3[2]$ \\
\hline $\mathrm{pH}$ (SU) & $3 / 3$ & 7.2 & 6.5 & 7.0 & $(6.5,8.5)$ & $1[3]$ \\
\hline \multicolumn{7}{|l|}{ Metals, unfiltered (mg/L) } \\
\hline Aluminum, total & $2 / 3$ & 0.57 & $<0.050$ & -0.24 & 0.2 & $1[3]$ \\
\hline Barium, total & $3 / 3$ & 0.042 & 0.021 & 0.030 & 2 & $0[2]$ \\
\hline Calcium, total & $3 / 3$ & 220 & 59 & 93 & $\mathrm{~d}$ & [a] \\
\hline Iron, total & $3 / 3$ & 1.2 & 0.12 & 0.77 & 0.3 & $2[3]$ \\
\hline Magnesium, total & $3 / 3$ & 14 & 2.8 & 7.9 & $\mathrm{~d}$ & [d] \\
\hline Manganese, total & $3 / 3$ & 0.086 & 0.0020 & 0.055 & 0.05 & $2\{3\}$ \\
\hline Potassium, total & $2 / 3$ & $<2.0$ & 1.6 & -1.8 & $\mathrm{~d}$ & [d] \\
\hline Silicon, total & $3 / 3$ & 5.8 & 3.4 & 4.6 & d & [d] \\
\hline Sodium, total & $3 / 3$ & 2.7 & 2.0 & 2.3 & d & (d) \\
\hline Vanadium, total & $3 / 3$ & 0.0024 & 0.0023 & 0.0024 & d & [d] \\
\hline zinc, total & $3 / 3$ & 0.015 & 0.0053 & 0.010 & 5 & $0[3]$ \\
\hline \multicolumn{7}{|l|}{ Others, unfiltered } \\
\hline Alkalinity (mg/L) & $3 / 3$ & 370 & 150 & 280 & $a$ & [d] \\
\hline Total organic carbon (mg/L) & $3 / 3$ & 1.7 & 0.50 & 1.2 & $\mathrm{~d}$ & [d] \\
\hline Total suspended solids $\{\mathrm{mg} / \mathrm{L}\rangle$ & $2 / 3$ & 8.0 & $<5.0$ & -6.7 & d & [d] \\
\hline \multicolumn{7}{|l|}{ Radionuclides, filtered $(\mathrm{pCi} / L\rangle^{\bullet}$} \\
\hline Gross alpha & $2 / 3$ & $4.9 *$ & 0.89 & 2.8 & 15 & $0[2]$ \\
\hline Gross beca & $1 / 3$ & $10^{\bullet}$ & $2: 2$ & 5.0 & 50 & $O[2]$ \\
\hline $\mathrm{H}-3$ & $3 / 3$ & $1,200 *$ & $570 *$ & $910^{*}$ & 20,000 & $0[2]$ \\
\hline \multicolumn{7}{|l|}{ Radionuclices, unfiltered $(\mathrm{pCi} / \mathrm{L})^{*}$} \\
\hline Gross alpha & $1 / 3$ & $2.7 *$ & 0.78 & $1.7 *$ & 15 & $0[2]$ \\
\hline Gross beta & $1 / 3$ & $3.2 *$ & 0.84 & 1.7 & 50 & $0[2]$ \\
\hline$H-3$ & $3 / 3$ & $1,400^{*}$ & $730^{*}$ & $1.100 *$ & 20.000 & $0[2]$ \\
\hline
\end{tabular}

-Prefix "<" indicates the value for a parameter (excluding organics) was not quantifiable at the analytical detection limit; and " $U$ " indicates the value for an organic parameter was undetected at the analytical detection limit.

A tilde $(-)$ indicates that estimated and/or undetected values were used in the calculation.

If a reference limit exists, the source is coded as:

1 Rules of Tennessee Department of Environment and Conservation, Division of water Pollution

Control, Chapter 1200-4-3, General water Quality Criteria, Domestic Water Supply, as amended.

240 CFR Part 141--National Primary Drinking Water Regulations, Subparts $B$ and $G$, as amended.

340 CFR Part 143--National Secondary Drinking water Regulations, as amended.

4 DOE Order 5400.5. Chapter III, Derived Concentration Guides for Air and water.

"Not applicable.

-Individual and average radionuclide concentrations significantly greater than zero are identified by an *. 
Table 7.63. Constltuents In Waste Area Grouping (WAG) 5 groundwater at ORNL, Aprll 5-30, 1993

\begin{tabular}{|c|c|c|c|c|c|c|}
\hline Parameter & $\begin{array}{l}\mathrm{N} \text { det/ } \\
\mathrm{N} \text { total }\end{array}$ & $\operatorname{Max}^{\mathbf{n}}$ & Min" & $A v^{b}$ & $\begin{array}{l}\text { Reference } \\
\text { value }\end{array}$ & $\begin{array}{l}\text { Number of values } \\
\text { exceeding } \\
\text { reference [xef]c }\end{array}$ \\
\hline \multicolumn{7}{|c|}{ Downgradient Wells } \\
\hline \multicolumn{7}{|l|}{ Anions, unfiltered $(\mathrm{mg} / \mathrm{L})$} \\
\hline Chloride & $14 / 20$ & 27 & 0.10 & -6.6 & 250 & $0[3]$ \\
\hline Sulfate, as $\mathrm{so}_{4}$ & $11 / 20$ & 280 & $<10$ & -45 & 250 & $1(3)$ \\
\hline \multicolumn{7}{|c|}{ Base neutral/acid extractable organics, unfiltered $(\mu g / L)$} \\
\hline Bis(2-ethylhexyl) phthalate & $5 / 20$ & YB3. 100 & U10 & -170 & $\mathrm{~d}$ & \{d\} \\
\hline \multicolumn{7}{|l|}{ Field measurements, unfiltered } \\
\hline Conductivity $(\mathrm{ms} / \mathrm{cm})$ & $20 / 20$ & 1.3 & 0.46 & 0.86 & $\mathrm{~d}$ & [d] \\
\hline Dissolved oxygen (mg/L) & $20 / 20$ & 15 & 9.6 & 11 & $\mathrm{~d}$ & [d] \\
\hline Redox (mV) & $20 / 20$ & 670 & 320 & 470 & $\mathrm{~d}$ & (d) \\
\hline Temperature $\left({ }^{\circ} \mathrm{C}\right)$ & $20 / 20$ & 15 & 12 & 14 & 30.5 & $0[1]$ \\
\hline Turbidity (JTU) & $20 / 20$ & 490 & 1.0 & 56 & 1 & $19(2)$ \\
\hline $\mathrm{pH}(\mathrm{SU})$ & $20 / 20$ & 9.0 & 6.2 & 7.2 & $(6.5,8.5)$ & $2[3]$ \\
\hline \multicolumn{7}{|l|}{ Metals, filtered (mg/L) } \\
\hline Aluminum & $13 / 20$ & 0.49 & uo. 019 & $\sim 0.064$ & 0.2 & $17[3]$ \\
\hline Ant imony & $1 / 20$ & บo.019 & vo.0050 & -0.017 & $d$ & (d) \\
\hline Arsenic & $1 / 20$ & vo.0035 & บo.0015 & -0.0025 & 0.05 & $0[1]$ \\
\hline Barium & $20 / 20$ & 0.89 & B0. 019 & 0.27 & 2 & $0[2]$ \\
\hline Bery11ium & $4 / 20$ & vo.0020 & vo. 00030 & -0.00056 & $\mathrm{~d}$ & {$[d]$} \\
\hline Boron & $20 / 20$ & 1.5 & Bo. 031 & 0.19 & $\mathrm{~d}$ & [d] \\
\hline Calcium & $20 / 20$ & $180^{\circ}$ & B2. 4 & 96 & $\mathrm{~d}$ & [d] \\
\hline Copper & $6 / 20$ & vo.010 & v0.0027 & -0.0043 & 1.3 & $0[2]$ \\
\hline Iron & $19 / 20$ & 1.2 & B0.0089 & $\sim 0.30$ & 0.3 & $6[3]$ \\
\hline Lead & $16 / 20$ & 0.0051 & บo.0012 & $\sim 0.0026$ & 0.015 & $0[2]$ \\
\hline Magnesium & $20 / 20$ & 37 & Bo. 95 & 18 & $\mathrm{~d}$ & [d] \\
\hline Manganese & $20 / 20$ & 1.3 & B0.0010 & 0.21 & 0.05 & $12[3]$ \\
\hline Mercuxy & $1 / 20$ & บo. 00020 & บo.00010 & -0.00012 & 0.002 & $0[1]$ \\
\hline Nickel. & $5 / 20$ & B0. 028 & U0.0042 & -0.0078 & $d$ & [d] \\
\hline Potassium & $20 / 20$ & 8.2 & B0. 91 & 2.2 & $\mathrm{~d}$ & [d] \\
\hline silicon & $20 / 20$ & 1.4 & 1.8 & 8.4 & $\mathrm{~d}$ & [d] \\
\hline sodium & $20 / 20$ & 130 & B4. 1 & 22 & d & [d] \\
\hline Vanadium & $1 / 20$ & 00.010 & 00.0021 & $\sim 0.0033$ & d & [d] \\
\hline zine & $5 / 20$ & B0.018 & vo.0048 & $\sim 0.0065$ & 5 & $0[3]$ \\
\hline \multicolumn{7}{|l|}{ Metals, unfiltered $(\mathrm{mg} / \mathrm{L})$} \\
\hline Aluminum, total & $19 / 20$ & 1.9 & U0. 019 & $\sim 0.33$ & 0.2 & $15[3]$ \\
\hline Arsenic, total & $2 / 20$ & 00.0075 & บo. 0020 & -0.0029 & 0.05 & $0[1]$ \\
\hline Barium, total & $20 / 20$ & 0.98 & BO. 019 & 0.28 & 2 & $0[2]$ \\
\hline
\end{tabular}


Table 7.63 (continued)

\begin{tabular}{|c|c|c|c|c|c|c|}
\hline Parameter & $\begin{array}{l}\mathbf{N} \text { det/ } \\
\mathbf{N} \text { total }\end{array}$ & $\operatorname{Max}$ & $\operatorname{Min}^{*}$ & $A v^{b}$ & $\begin{array}{l}\text { Reference } \\
\text { value }\end{array}$ & $\begin{array}{l}\text { Number of values } \\
\text { exceeding } \\
\text { reference [ref }]^{c}\end{array}$ \\
\hline \multicolumn{7}{|l|}{ Metals, unfiltered $(\mathrm{mg} / \mathrm{L})$} \\
\hline Beryllium, total & $1 / 20$ & vo.0020 & vo .00030 & -0.00056 & d & [d] \\
\hline Boron, total & $20 / 20$ & 1.5 & $\mathrm{~B} 0.030$ & 0.19 & $\mathrm{~d}$ & [d] \\
\hline Calcium, total & $20 / 20$ & 180 & $\mathrm{~B} 2.8$ & 97 & d & [d] \\
\hline Chromium, total & $1 / 20$ & 0.015 & v0.0033 & $\sim 0.0049$ & 0.05 & $0[1]$ \\
\hline Copper, total & $8 / 20$ & vo.010 & 00.0027 & -0.0050 & 1.3 & $0[2]$ \\
\hline Iron, total & $20 / 20$ & 2.0 & B0.031 & 0.59 & 0.3 & $9[3]$ \\
\hline Lead, total & $14 / 20$ & 0.0049 & v0.0012 & -0.0024 & 0.015 & $0[2]$ \\
\hline Magnesium, total & $20 / 20$ & 36 & B1.1 & 18 & $\mathrm{~d}$ & [d] \\
\hline Manganese, total & $20 / 20$ & 1.3 & BO. 0035 & 0.24 & 0.05 & $12[3]$ \\
\hline Mercury, total & $5 / 20$ & 0.00042 & vo.00010 & $\sim 0.00014$ & 0.002 & $0[1]$ \\
\hline Nickel, total & $7 / 20$ & $\mathrm{~B} 0.022$ & vo.0042 & $\sim 0.0081$ & $\mathrm{~d}$ & [d] \\
\hline Potassium, total & $20 / 20$ & 8.1 & 80.92 & 2.3 & d & [d] \\
\hline Selenium, total & $1 / 20$ & vo.018 & vo.0020 & $\sim 0.0058$ & 0.01 & $4[1]$ \\
\hline silicon, total & $20 / 20$ & 14 & 1.9 & 8.7 & $\mathrm{~d}$ & [d] \\
\hline Sodium, total & $20 / 20$ & 130 & 84.2 & 22 & $\mathrm{~d}$ & [d] \\
\hline Thallium, total & $1 / 20$ & B0.013 & vo.0012 & $\sim 0.0034$ & d & [d] \\
\hline Vanadium, tota 1 & $2 / 20$ & vo.010 & บo.0021 & $\sim 0.0033$ & $\mathrm{~d}$ & [d] \\
\hline Zinc, total & $6 / 20$ & 0.050 & บo.0048 & $\sim 0.0085$ & 5 & $0[3]$ \\
\hline \multicolumn{7}{|l|}{ Others, filtered } \\
\hline Alkalinity (mg/L) & $20 / 20$ & 550 & 150 & 320 & $\mathrm{~d}$ & [d] \\
\hline Total dissolved solids (mg/L) & $20 / 20$ & 750 & 220 & 420 & 500 & $6[1]$ \\
\hline \multicolumn{7}{|l|}{ Others, unfiltered } \\
\hline Alkalinity (mg/L) & $20 / 20$ & 560 & 150 & 320 & $a$ & [d] \\
\hline Cyanide, total $(\mu \mathrm{g} / \mathrm{L})$ & $1 / 20$ & 0.000049 & vo. 0000020 & -0.000011 & $\mathrm{~d}$ & (d) \\
\hline Nitrogen, total kjeldahl (mg/L) & $16 / 20$ & 0.68 & vo. 010 & -0.26 & $\mathrm{~d}$ & [d] \\
\hline Phenolics, total recoverable (mg/L) & $2 / 20$ & 0.014 & $<0.0010$ & -0.0017 & d & [d] \\
\hline Total organic carbon (mg/L) & $19 / 20$ & 4.6 & $<0.50$ & $\sim 1.3$ & a & [d] \\
\hline Total organic halides $(\mu \mathrm{g} / \mathrm{L})$ & $5 / 20$ & 1,200 & $<5.0$ & $\sim 77$ & d & [d] \\
\hline Total suspended solids (mg/L) & $9 / 20$ & 440 & 2.0 & $\sim 48$ & $\mathrm{~d}$ & [d] \\
\hline \multicolumn{7}{|l|}{ Radionuclides, filtered $(\mathrm{pCi} / \mathrm{L})^{\circ}$} \\
\hline Am-241 & $7 / 20$ & $0.51 *$ & -0.19 & $0.071 *$ & 1.2 & $0[4]$ \\
\hline$c-14$ & $9 / 20$ & $2,700 *$ & -220 & $490^{\star}$ & 101 & $0[4]$ \\
\hline $\mathrm{Cm}-244$ & $7 / 20$ & $16^{*}$ & -0.15 & 1.6 & 2.4 & $2(4)$ \\
\hline $\mathrm{Co}-60$ & $3 / 20$ & 24 * & -4.1 & 2.4 & 200 & $0[4]$ \\
\hline $\mathrm{Cs}-1 \supset 7$ & $2 / 20$ & $8.4^{*}$ & -1.6 & $1.4^{*}$ & 120 & $0[4]$ \\
\hline Gruss alpha & $9 / 20$ & 27 * & -1.8 & $3.5^{\star}$ & 15 & $2[2]$ \\
\hline Gross beta & $14 / 20$ & $510^{*}$ & -0.16 & $71^{\star}$ & 50 & $3[2]$ \\
\hline $\mathrm{H}-3$ & $16 / 20$ & $270,000,000 *$ & $-3,500$ & $20,000,000$ & 20,000 & $13[2]$ \\
\hline
\end{tabular}


Table 7.63 (continued)

\begin{tabular}{|c|c|c|c|c|c|c|}
\hline Parameter & $\begin{array}{l}\mathrm{N} \text { det } / \\
\mathrm{N} \text { total }\end{array}$ & $\operatorname{Max}$ & $\operatorname{Min}$ & $A v^{b}$ & $\begin{array}{l}\text { Reference } \\
\text { value }\end{array}$ & $\begin{array}{l}\text { Number of values } \\
\text { exceeding } \\
\text { refexence [ref }]^{c}\end{array}$ \\
\hline \multicolumn{7}{|c|}{ Radionuclides, filtered $(p C i / L)^{*}$} \\
\hline $\mathrm{Pu}-238$ & $3 / 20$ & 0.54 & -0.068 & $0.091 *$ & 1.6 & $0[4]$ \\
\hline$p u-239$ & $2 / 20$ & $0.13^{\star}$ & -0.20 & -0.047 & 1.2 & $0[4]$ \\
\hline Total raf $\mathrm{Sr}$ & $8 / 20$ & $510^{*}$ & -1.1 & 41 & 8 & $5[2]$ \\
\hline$U-234$ & $19 / 20$ & $0.68 *$ & 0.035 & $0.42 *$ & 20 & $0[4]$ \\
\hline $\mathrm{U}-235$ & $8 / 20$ & $0.30^{*}$ & -0.10 & 0.089 * & 24 & $0[4]$ \\
\hline $\mathrm{U}-238$ & $11 / 20$ & $0.27 *$ & -0.027 & $0.12 *$ & 24 & $0[4]$ \\
\hline \multicolumn{7}{|c|}{ Radionuclides, unfiltered $(\mathrm{pCi} / \mathrm{L})^{\bullet}$} \\
\hline Am-241 & $5 / 20$ & $1.6^{*}$ & -0.22 & 0.12 & 1.2 & $1[4]$ \\
\hline $\mathrm{C}-14$ & $8 / 16$ & $2,500 \star$ & -270 & $520^{\star}$ & 101 & $0[4]$ \\
\hline $\mathrm{Cm}-244$ & $9 / 20$ & $18 *$ & -0.22 & 1.6 & 2.4 & $2[4]$ \\
\hline Co- 60 & $4 / 20$ & $32 *$ & -2.4 & $3.4^{\star}$ & 200 & $0[4]$ \\
\hline Cs -137 & $1 / 20$ & 5.1 & -4.1 & $0.88 *$ & 120 & $0[4]$ \\
\hline Gross alpha & $7 / 20$ & $25^{*}$ & -0.76 & $3.0^{\star}$ & 15 & $2[2]$ \\
\hline Gross beta & $14 / 20$ & $970 *$ & 0.46 & $100^{\star}$ & 50 & $4[2]$ \\
\hline $\mathrm{H}-3$ & $16 / 20$ & $270.000,000$ & $-3,800$ & $19,000,000$ & 20,000 & $13(2)$ \\
\hline $\mathrm{Pu}-238$ & $3 / 20$ & $0.57^{\star}$ & -0.16 & 0.055 & 1.6 & $0[4]$ \\
\hline Total rad $\mathrm{Sr}$ & $10 / 20$ & 540 & -0.59 & 42 & 8 & $5[2]$ \\
\hline $\mathrm{U}-234$ & $19 / 20$ & $1.4^{\star}$ & 0.024 & $0.58^{\star}$ & 20 & $0(4)$ \\
\hline $\mathrm{U}-235$ & $8 / 20$ & $0.32^{\star}$ & -0.030 & $0.10^{*}$ & 24 & $0[4]$ \\
\hline $\mathrm{U}-238$ & $13 / 20$ & $0.41 *$ & -0.01 .2 & $0.13^{*}$ & 24 & $0[4]$ \\
\hline \multicolumn{7}{|c|}{ Volatile organics, unfiltered $(\mu \mathrm{g} / \mathrm{L})$} \\
\hline 1,1,1-Trichloroethane & $1 / 20$ & U5.0 & $\mathrm{J} 1.0$ & -4.8 & 200 & $0[1]$ \\
\hline 1.1-Dichloroethane & $4 / 20$ & U5.0 & $\mathrm{J} 1.0$ & -4.5 & d & [d] \\
\hline 1.1-Dichloroethene & $1 / 20$ & 5.0 & U5.0 & -5.0 & 7 & $0[1]$ \\
\hline 1,2-Dichloroethene & $5 / 20$ & $Y 3,300$ & 32.0 & $\sim 180$ & 70 & $1[2]$ \\
\hline Acetone & $1 / 20$ & 12 & v10 & -10 & d & [d] \\
\hline Benzene & $1 / 20$ & 25 & U5. 0 & $\sim 6.0$ & 5 & $1[1]$ \\
\hline Methylene chloride & $2 / 20$ & JB5. 0 & U5. 0 & -5.0 & $\mathrm{~d}$ & [d] \\
\hline Tetrachloroethene & $1 / 20$ & U5.0 & $\mathrm{J1} .7$ & -4.8 & 5 & $0[2]$ \\
\hline Trichloroethene & $5 / 20$ & 38 & J2.0 & -7.6 & 5 & $3(1)$ \\
\hline Vinyl chloride & $4 / 20$ & $Y 2,700$ & $\mathrm{~J} 4.7$ & $\sim 150$ & 2 & $20\{1\}$ \\
\hline xylene, total & $1 / 20$ & U5.0 & $\mathrm{J} 2.0$ & $\sim 4.9$ & 10,000 & $0\lfloor 2]$ \\
\hline \multicolumn{7}{|c|}{ Upgradient Wells } \\
\hline \multicolumn{7}{|l|}{ Anions, unfiltered (mg/L) } \\
\hline Chloride & $1 / 2$ & $<2.0$ & 0.20 & -1.1 & 250 & $0[3]$ \\
\hline Phosphate & $1 / 2$ & $<3.0$ & 1.2 & -2.1 & d & [d] \\
\hline Sulfate, as $\mathrm{SO}_{4}$ & $2 / 2$ & 15 & . 15 & 15 & 250 & $0[3]$ \\
\hline
\end{tabular}


Table 7.63 (continued)

\begin{tabular}{|c|c|c|c|c|c|c|}
\hline Parameter & $\begin{array}{l}\mathrm{N} \text { det } / \\
\mathrm{N} \text { total }\end{array}$ & $\operatorname{Max}^{\mathbf{a}}$ & $\operatorname{Min}^{a}$ & $A v^{b}$ & $\begin{array}{l}\text { Reference } \\
\text { value }\end{array}$ & $\begin{array}{l}\text { Number of values } \\
\text { exceeding } \\
\text { reference }[\text { ref }]^{c}\end{array}$ \\
\hline \multicolumn{7}{|l|}{ Field measurements, unfiltered } \\
\hline Conductivity $(\mathrm{mS} / \mathrm{cm})$ & $2 / 2$ & 0.59 & 0.49 & 0.54 & d & [d] \\
\hline Dissolved oxygen (mg/L) & $2 / 2$ & 9.4 & 8.3 & 8.9 & d & [d] \\
\hline Redox (mV) & $2 / 2$ & 570 & 530 & 550 & d & [d] \\
\hline Temperature $\left({ }^{\circ} \mathrm{C}\right)$ & $2 / 2$ & 14 & 12 & 13 & 30.5 & $0[1]$ \\
\hline Turbidity (JTU) & $2 / 2$ & 11 & 10 & 11 & 1 & $2[2]$ \\
\hline $\mathrm{pH}$ (sU) & $2 / 2$ & 7.4 & 6.8 & 7.1 & $(6.5,8.5)$ & $0[3]$ \\
\hline \multicolumn{7}{|l|}{ Metals, filtered (mg/L) } \\
\hline Aluminum & $1 / 2$ & Bo.11 & vo.019 & $\sim 0.066$ & 0.2 & $1[3]$ \\
\hline Barium & $2 / 2$ & 80.14 & B0.14 & 0.14 & 2 & $0[2]$ \\
\hline Boron & $2 / 2$ & 80.029 & B0.022 & 0.025 & d & [a] \\
\hline Calcium & $2 / 2$ & 74 & 70 & 72 & d & [d] \\
\hline Iron & $1 / 2$ & в0.076 & vo.0084 & $\sim 0.042$ & 0.3 & $0[3]$ \\
\hline Lead & $2 / 2$ & 80.0026 & B0.0014 & 0.0020 & 0.015 & $0\{2\}$ \\
\hline Magnesium & $2 / 2$ & 12 & B2.8 & 7.3 & d & [d] \\
\hline Manganese & $2 / 2$ & 0.027 & B0.0030 & 0,015 & 0.05 & $0[3]$ \\
\hline Potassium & $2 / 2$ & B1.1 & B0. 52 & 0.83 & d & [d] \\
\hline silicon & $2 / 2$ & 12 & 7.5 & 9.8 & $\mathrm{~d}$ & [d] \\
\hline Sodium & $2 / 2$ & 7.4 & 5.9 & 6.6 & $\mathrm{~d}$ & [d] \\
\hline \multicolumn{7}{|l|}{ Metals, unfi1tered (mg/L) } \\
\hline Aluminum, total & $2 / 2$ & 0.41 & Bo. 052 & 0.23 & 0.2 & $1[3]$ \\
\hline Barium, total & $2 / 2$ & в0.15 & B0.14 & 0.14 & 2 & $0[2]$ \\
\hline Boron, total & $2 / 2$ & 80.046 & B0.025 & 0.035 & $\mathrm{~d}$ & [d] \\
\hline Calcium, total & $2 / 2$ & 73 & 72 & 73 & d & [d] \\
\hline Iron, total & $2 / 2$ & 0.57 & B0.018 & 0.29 & 0.3 & $1[3]$ \\
\hline Lead, total. & $2 / 2$ & 0.0044 & B0.0026 & 0.0035 & 0.015 & $0[2]$ \\
\hline Magnesium, total & $2 / 2$ & 12 & B3.0 & 7.2 & d & [d] \\
\hline Manganese, total & $2 / 2$ & 0.077 & $\mathrm{~B} 0.0048$ & 0.041 & 0.05 & $1[3]$ \\
\hline Nickel, total & $1 / 2$ & BO. 0045 & Uo 0.0042 & -0.0044 & a & [d] \\
\hline Potassium, total & $2 / 2$ & $\mathrm{~B} 1.2$ & Bo. 48 & 0.86 & $\mathrm{~d}$ & [d] \\
\hline Silicon, total & $2 / 2$ & 13 & 7.7 & 10 & d & [d] \\
\hline Sodium, total & $2 / 2$ & 7.3 & 6.1 & 6.7 & d & [d] \\
\hline \multicolumn{7}{|l|}{ Others, filtered } \\
\hline Alkalinity (mg/L) & $2 / 2$ & 230 & 210 & 220 & $\mathrm{~d}$ & [d] \\
\hline Total dissolved solids (mg/L) & $2 / 2$ & 270 & 240 & 260 & 500 & $0[1]$ \\
\hline \multicolumn{7}{|l|}{ Others, unfiltered } \\
\hline Alkalinity (mg/L) & $2 / 2$ & 230 & 210 & 220 & d & [d] \\
\hline Nitrogen, total kjeldahl (mg/L) & $2 / 2$ & 0.38 & 0.21 & 0.30 & $\mathrm{~d}$ & [d] \\
\hline
\end{tabular}


Table 7.63 (continued)

\begin{tabular}{|c|c|c|c|c|c|c|}
\hline Parameter & $\begin{array}{l}\mathrm{N} \text { det } / \\
\mathrm{N} \text { total }\end{array}$ & $\operatorname{Max}^{\wedge}$ & $\operatorname{Min}^{*}$ & $A v^{b}$ & $\begin{array}{l}\text { Reference } \\
\text { value }\end{array}$ & $\begin{array}{l}\text { Number of values } \\
\text { exceeding } \\
\text { reference [ref]c }\end{array}$ \\
\hline \multicolumn{7}{|l|}{ Others, unfiltered } \\
\hline Total organic carbon $(\mathrm{mg} / \mathrm{t})$ & $2 / 2$ & 0.73 & 0.73 & 0.73 & d & [d] \\
\hline Total organic halides $(\mu \mathrm{g} / \mathrm{L})$ & $1 / 2$ & $<5.3$ & $<5.0$ & -5.1 & d & [d] \\
\hline Total suspended solids (mg/L) & $1 / 2$ & 55 & $<5.0$ & $\sim 30$ & d & [d] \\
\hline \multicolumn{7}{|l|}{ Radionuclides, filtered $(\mathrm{pCi} / \mathrm{L})^{\bullet}$} \\
\hline$A m-241$ & $1 / 2$ & $0.070^{*}$ & 0.046 & 0.058 & 1.2 & $0[4]$ \\
\hline Co- 60 & $1 / 2$ & $5.7^{\star}$ & -2.4 & 1.6 & 200 & $0[4]$ \\
\hline Gross beta & $1 / 2$ & $4.6^{\star}$ & -0.054 & 2.3 & 50 & $0[2]$ \\
\hline $\mathrm{H}-3$ & $2 / 2$ & $1.400^{\star}$ & $1,200 *$ & $1.300 *$ & 20,000 & $0[2]$ \\
\hline Total rad Sr & $1 / 2$ & $1.2 *$ & -0.32 & 0.46 & 8 & $0[2]$ \\
\hline$u-234$ & $2 / 2$ & $0.70^{\star}$ & $0.59 *$ & $0.65^{*}$ & 20 & $0[4]$ \\
\hline$U-238$ & $1 / 2$ & $0.14 *$ & 0.070 & 0.11 & 24 & $0[4]$ \\
\hline \multicolumn{7}{|l|}{ Radionuclides, unfiltered $(\mathrm{pci} / \mathrm{L})$. } \\
\hline Gross alpha & $1 / 2$ & $1.4^{\star}$ & 0.89 & 1.1 & 15 & $0[2]$ \\
\hline $\mathbf{H}-3$ & $2 / 2$ & $1,100 *$ & $950^{\star}$ & $1,000^{*}$ & 20,000 & $0[2\}$ \\
\hline Total rad Sr & $1 / 2$ & $1.1 *$ & 0.27 & 0.68 & 8 & $0[2]$ \\
\hline$u-234$ & $2 / 2$ & 0.51 * & $0.38^{*}$ & $0.45^{\star}$ & 20 & $0[4]$ \\
\hline $\mathrm{u}-235$ & $1 / 2$ & $0.15^{\star}$ & 0.057 & 0.11 & 24 & $0[4]$ \\
\hline $\mathrm{u}-238$ & $1 / 2$ & $0.15^{\star}$ & 0.051 & 0.099 & 24 & $0[4]$ \\
\hline
\end{tabular}

"Prefix "<" indicates the value for a parameter (excluding metals and organics) was not quantifiable at the analytical detection 1imit; " $J$ " indicates the value was estimated at or below the analytical detection limit by the laboratory; "B" indicates the compound was found in the laboratory blank; "JB" indicates the value was estimated at or below the analytical detection limit. and was found in the laboratory blank; " $Y$ " indicates the value exceeded the calibration range and the sample was diluted and reanalyzed; and " $U$ " indicates the value for a parametex was undetected at the analytical detection 1 imit.

"A tilde ( -1 indicates that estimated and/or undetected values were used in the calculation.

'If a reference limit exists, the source is coded as:

1 Rules of Tennessee Department of Environment and Conservation, Division of Water Pollution Control, Chapter 1200-4-3, General Water Quality Criteria, Domestic Water Supply, as amended.

240 CFR Part 141--National Primary Drinking Water Regulations, Subparts $B$ and G, as amended.

340 CFR Paxt 143--National Secondary Drinking water Regulations, as amended.

4 DOE Order 5400.5. Chapter III, Derived Concentration Guides for Air and water.

dNot applicable.

- Individual and average radionuclide concentrations significantly greater than zero are identified by an $\star$ 
Table 7.64. Constituents in Waste Area Grouping (WAG) 6 groundwater at ORNL, February 18-March 29, May 6-14, August 9-September 24, and November 16-23, 1993

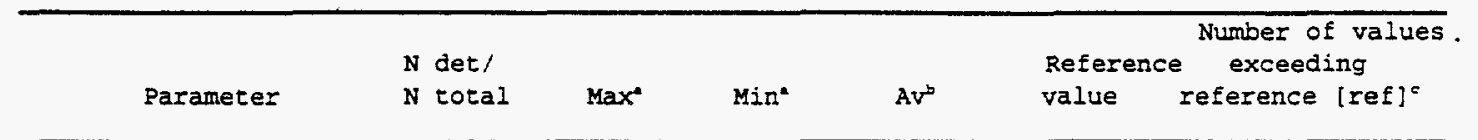

Downgradient Wells

\begin{tabular}{|c|c|c|c|c|c|c|}
\hline Conductivity (ms/cm) & $50 / 50$ & 1.3 & 0.020 & 0.59 & a & [d] \\
\hline Dissolved oxygen (mg/L) & $50 / 50$ & 18 & 2.1 & 11 & $d$ & [d] \\
\hline Redox (mV) & $42 / 42$ & 780 & 300 & 480 & a & [d] \\
\hline Temperature $\left.1^{\circ} \mathrm{C}\right\rangle$ & $50 / 50$ & 19 & 11 & 15 & 30.5 & $0[1]$ \\
\hline Turbidity (NTU) & $50 / 50$ & 550 & 0 & 37 & 1 & $38\{2\}$ \\
\hline $\mathrm{pH}$ (SU) & $50 / 50$ & 7.6 & 5.1 & 6.8 & $(6.5,8.5)$ & $8[3]$ \\
\hline \multicolumn{7}{|l|}{ Others, unfiltered } \\
\hline Alkalinity (mg/L) & $41 / 42$ & 500 & $<1.0$ & -240 & d & [d] \\
\hline \multicolumn{7}{|c|}{ Radionuclides, filtered (pCi/L)• } \\
\hline $\operatorname{co}-60$ & $14 / 50$ & $410^{*}$ & -7.8 & $26 *$ & 200 & $3[4]$ \\
\hline Cs -137 & $10 / 50$ & $8.9 *$ & -5.4 & $2.2 *$ & 120 & $0[4]$ \\
\hline Gross alpha & $14 / 50$ & $10 *$ & -0.46 & $1.6 *$ & 15 & $\{2\}$ \\
\hline Gross beta & $16 / 25$ & $260^{*}$ & -0.16 & $23 *$ & 50 & [1] \\
\hline $\mathrm{H}-3$ & $47 / 50$ & $2,300,000 *$ & 350 & $280.000^{*}$ & 80,000 & $21[4]$ \\
\hline Total rad Sr & $13 / 50$ & $4.9 *$ & -2.2 & i.1* & 40 & $0[4]$ \\
\hline \multicolumn{7}{|c|}{ volatile organics, unfiltered $(\mu \mathrm{g} / \mathrm{L})$} \\
\hline 1,1,1-Trichloroethane & $1 / 50$ & U5.0 & $\mathrm{J} 2.0$ & -4.9 & 200 & $0[1]$ \\
\hline 1.1-Dichloroethane & $1 / 50$ & U5. 0 & $\mathrm{~J} 2.2$ & -4.9 & d & [d] \\
\hline 1,2-Dichloroethane & $4 / 50$ & 11 & US. 0 & -5.2 & 5 & $3[1]$ \\
\hline 1,2-Dichloroethene & $7 / 50$ & 7.9 & 33.0 & -5.0 & 70 & $0[2]$ \\
\hline Acetone & $1 / 50$ & 35 & $\mathrm{U} 10$ & -11 & d & [d] \\
\hline Carbon disulfide & $2 / 50$ & 14 & $\mathrm{J3} .0$ & -5.1 & $d$ & [d] \\
\hline Carbon tetrachloride & $4 / 50$ & 34 & 05.0 & -7.1 & 5 & $4[1]$ \\
\hline Chloroform & $8 / 50$ & 39 & $\mathrm{J1.0}$ & -7.0 & 100 & $0[2]$ \\
\hline Methylene chloride & $1 / 50$ & US.0 & JB3.1 & -5.0 & d & [d] \\
\hline Tetrachloroethene & $1 / 50$ & $\mathrm{U} 5.0$ & 52.1 & -4.9 & 5 & $0[2]$ \\
\hline Trichloroethene & $7 / 50$ & $Y 160$ & $\mathrm{~J} 3.0$ & -17 & 5 & $4[1]$ \\
\hline \multicolumn{7}{|c|}{ Upgradient Wells } \\
\hline \multicolumn{7}{|c|}{ Field measurements, unfiltered } \\
\hline Conductivity (ms/cm) & $14 / 14$ & 1.1 & 0.010 & 0.50 & d & [d] \\
\hline Dissolved oxygen (mg/L) & $14 / 14$ & 15 & 8.0 & 11 & $d$ & [d] \\
\hline Redox (mv) & $14 / 14$ & 660 & 380 & 560 & $d$ & [d] \\
\hline Temperature $\left({ }^{\circ} \mathrm{C}\right\rangle$ & $14 / 14$ & 16 & 12 & 14 & 30.5 & $0[1]$ \\
\hline Turbidity (NIU) & $14 / 14$ & 190 & 2.0 & 49 & 1 & $14\{2\}$ \\
\hline $\mathrm{pH}$ (SU) & $14 / 14$ & 8.3 & 5.0 & 7.2 & $(6.5,8.5)$ & $2[3]$ \\
\hline \multicolumn{7}{|l|}{ others, unfiltered } \\
\hline Alkalinity (mg/L) & $14 / 14$ & 430 & 7.5 & 200 & d & [d] \\
\hline \multicolumn{7}{|c|}{ Radionuclides, Eiltered $(\mathrm{pCi} / \mathrm{L})^{*}$} \\
\hline $\mathrm{Co}-60$ & $1 / 14$ & $6.5 *$ & -2.2 & $1.4^{*}$ & 200 & $0[4]$ \\
\hline $\operatorname{cs}-137$ & $3 / 14$ & $13 *$ & -1.1 & $2.2 *$ & 120 & $0[4]$ \\
\hline Gross alpha & $6 / 14$ & $3.5^{*}$ & -0.73 & $1.1 *$ & $d$ & [d] \\
\hline Gross beta & $4 / 7$ & $9.2 *$ & 1.1 & $3.7 *$ & $d$ & [d] \\
\hline$H-3$ & $11 / 14$ & $3,200 *$ & 270 & $1,600 *$ & 80,000 & $O[4]$ \\
\hline Total rad Sr & $3 / 14$ & $3.8 *$ & -1.0 & 0.71 & 40 & $0[4]$ \\
\hline
\end{tabular}


Table 7.64 (continued)

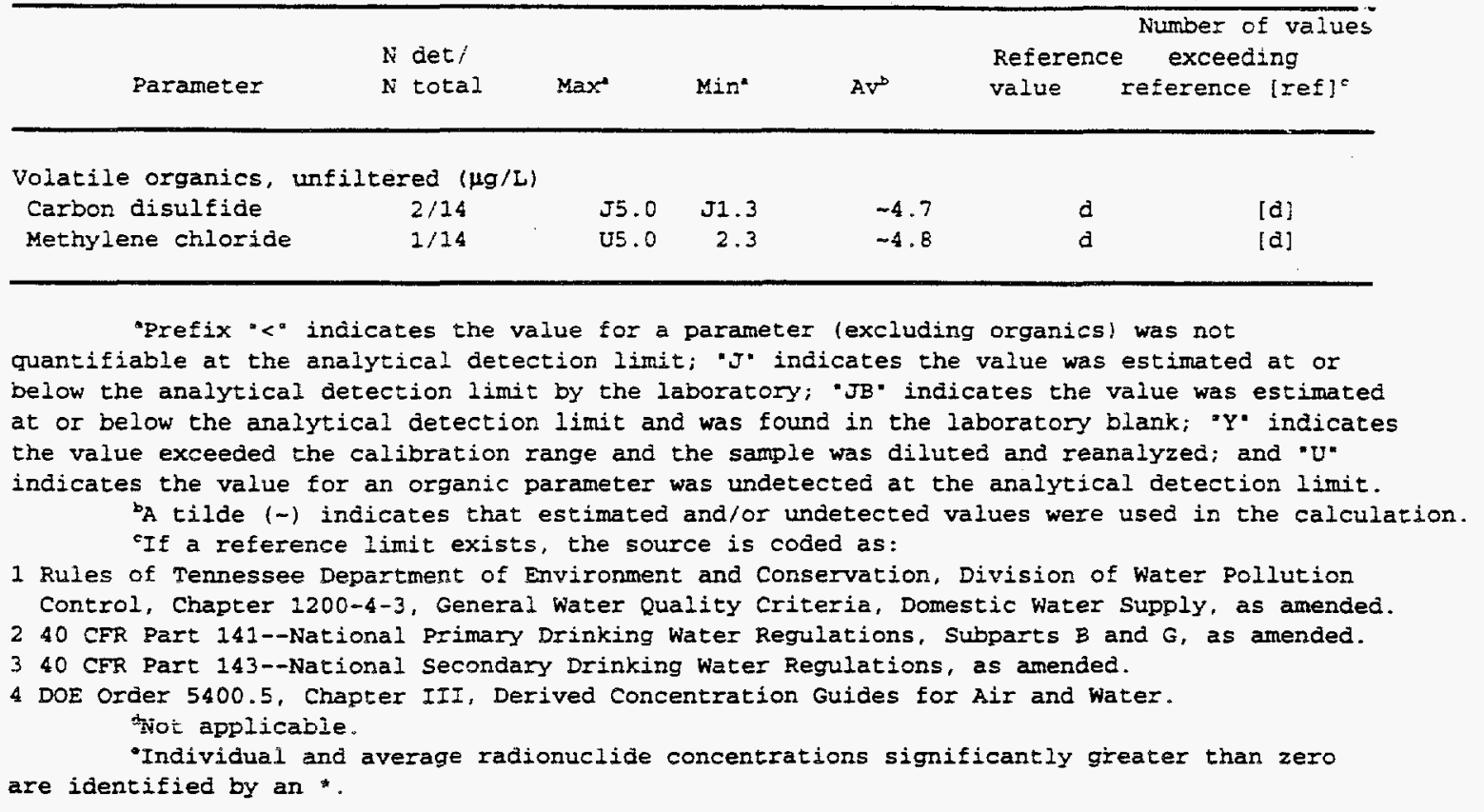


Table 7.65. Constituents in Waste Area Grouping (WAG) 7 groundwater at ORNL, July 14-August 13,1993

\begin{tabular}{|c|c|c|c|c|c|c|}
\hline Parameter & $\begin{array}{l}\mathrm{N} \text { det } / \\
\mathrm{N} \text { total }\end{array}$ & $\operatorname{Max}$ & Min* & $A v^{b}$ & $\begin{array}{l}\text { Reference } \\
\text { value }\end{array}$ & $\begin{array}{l}\text { Number of va } \\
\text { exceeding } \\
\text { reference tret }\end{array}$ \\
\hline \multicolumn{7}{|c|}{ Downgradient Wells } \\
\hline Anions, unfiltered (mg/L) & & & & & & \\
\hline Chloride & $9 / 13$ & 80 & $<2.0$ & -14 & 250 & $0[3]$ \\
\hline Fluoride & $3 / 13$ & 3.8 & $<0.10$ & -0.49 & 4 & $0[2]$ \\
\hline Nitrate & $4 / 13$ & 96 & $<1.0$ & -13 & 10 & $2\{2\}$ \\
\hline Sulfate, as so, & $13 / 13$ & 780 & 10 & -170 & 250 & $2[3]$ \\
\hline \multicolumn{7}{|c|}{ Base neutral/acid extractable organics, unfiltered $(\mu g / L)$} \\
\hline Benzyl butyl phthalate & $5 / 13$ & B6.0 & $\mathrm{JB1} .0$ & -4.2 & d & [a] \\
\hline Diethyl phthalate & $3 / 13$ & $\mathrm{U} 5.0$ & $\mathrm{JB} 2.0$ & -4.3 & $d$ & [d] \\
\hline \multicolumn{7}{|l|}{ Field measurements, unfiltered } \\
\hline Conductivity $(\mathrm{ms} / \mathrm{cm})$ & $13 / 13$ & 3.7 & 0.42 & 1.1 & d & [d] \\
\hline Dissolved oxygen $(\mathrm{mg} / \mathrm{L})$ & $13 / 13$ & 14 & 9.6 & 12 & d & [d] \\
\hline Redox (mV) & $13 / 13$ & 590 & 130 & 380 & d & [d] \\
\hline Temperature $\left({ }^{\circ} \mathrm{C}\right)$ & $13 / 13$ & 19 & 14 & 15 & 30.5 & $0[1]$ \\
\hline Turbidity (NTU) & $13 / 13$ & 320 & 5.0 & 100 & 1 & $13[2]$ \\
\hline $\mathrm{pH}$ (SU) & $13 / 13$ & 8.7 & 6.9 & 7.6 & $(6.5,8.5)$ & $2[3]$ \\
\hline \multicolumn{7}{|l|}{ Metals, unfiltered (mg/L) } \\
\hline Aluminum, total & $10 / 13$ & 7.5 & $<0.050$ & -1.1 & 0.2 & $7[3]$ \\
\hline Barium, total & $13 / 13$ & 0.21 & 0.027 & 0.11 & 2 & $0[2]$ \\
\hline Boron, total & $4 / 13$ & 0.42 & $<0.080$ & $\sim 0.15$ & $\mathrm{~d}$ & [d] \\
\hline Calcium, total & $13 / 13$ & 340 & 3.3 & 85 & $d$ & [d] \\
\hline Chromium, total & $2 / 13$ & 0.0090 & $<0.0040$ & -0.0047 & 0.05 & $0[1]$ \\
\hline Cobalt, total & $3 / 13$ & 0.030 & $<0.0040$ & -0.0076 & $a$ & [d] \\
\hline Copper, total & $1 / 13$ & 0.0081 & $<0.0070$ & -0.0071 & 1.3 & $0[2]$ \\
\hline Iron, total & $13 / 13$ & 14 & 0.077 & 1.8 & 0.3 & $9[3]$ \\
\hline Lead, total & $1 / 13$ & 0.025 & $<0.020$ & -0.020 & 0.015 & $13[2]$ \\
\hline Magnesium, total & $13 / 13$ & 200 & 0.90 & 35 & $\mathrm{~d}$ & [d] \\
\hline Manganese, total & $13 / 13$ & 1.3 & 0.0073 & 0.21 & 0.05 & $6[3]$ \\
\hline Mercury, total & $10 / 13$ & 0.00014 & $<0.000050$ & -0.00010 & 0.002 & $0[1]$ \\
\hline Nickel, total & $5 / 13$ & 0.36 & $<0.010$ & -0.048 & ¿ & [a] \\
\hline Potassium, total & $13 / 13$ & 8.0 & 1.0 & 3.0 & a & [a] \\
\hline Silicon, total & $13 / 13$ & 17 & 4.8 & 8.2 & d & [d] \\
\hline Sodium, total & $13 / 13$ & 620 & 3.6 & 120 & d & [d] \\
\hline Vanadium, total & $3 / 13$ & 0.010 & $<0.0020$ & -0.0029 & d & [d] \\
\hline Zine, total & $8 / 13$ & 0.028 & $<0.0050$ & -0.0087 & 5 & $0[3]$ \\
\hline \multicolumn{7}{|l|}{ Ochers, unfiltered } \\
\hline Alkalinity (mg/L) & $13 / 13$ & 660 & 70 & 300 & d & [a] \\
\hline Total organic carbon $(\mathrm{mg} / \mathrm{L})$ & $8 / 13$ & 2.5 & $<0.50$ & -0.92 & d & [d] \\
\hline Total arganic halides $(\mu \mathrm{g} / L)$ & $8 / 13$ & 64 & $<5.0$ & -16 & d & [a] \\
\hline Total suspended solids (mg/L) & $9 / 13$ & 360 & $<5.0$ & -51 & d & [d] \\
\hline \multicolumn{7}{|l|}{ Radionuclides, filtered $(\mathrm{pCi} / \mathrm{L})^{\bullet}$} \\
\hline Am-241 & $1 / 1$ & $0.22 *$ & $0.22 *$ & 0.22 & 1.2 & $0[4]$ \\
\hline $\mathrm{Co}-60$ & $5 / 13$ & $510^{*}$ & -1.6 & 44 & 200 & $1[4]$ \\
\hline Cs-137 & $4 / 13$ & $17^{*}$ & -2.4 & $3.7 *$ & 120 & $0[4]$ \\
\hline Gross alpha & $7 / 23$ & $160^{*}$ & -0.73 & 16 & 15 & $1[2]$ \\
\hline Gross beta & $11 / 13$ & $4,900 *$ & $1.1 *$ & 480 & 50 & $5[2]$ \\
\hline $\mathrm{H}-3$ & $10 / 13$ & $1,300,000 *$ & -380 & 170,000 & 20,000 & $4[2]$ \\
\hline Pu-239 & $1 / 1$ & $0.16 *$ & $0.16 *$ & 0.16 & 1.2 & $0[4]$ \\
\hline Th-228 & $1 / 1$ & $0.18 *$ & $0.18 *$ & 0.18 & 16 & $0[4]$ \\
\hline Th-230 & $1 / 1$ & $0.25 *$ & $0.25 *$ & 0.25 & 12 & $0[4]$ \\
\hline Total rad Sr & $4 / 13$ & $7.0 *$ & -0.11 & $1.4^{*}$ & 8 & $0[2]$ \\
\hline$v-234$ & $1 / 1$ & $51 *$ & 51* & 51 & 20 & $1[4]$ \\
\hline $\mathrm{U}-235$ & $1 / 1$ & $0.73 *$ & $0.73 *$ & 0.73 & 24 & $0[4]$ \\
\hline $\mathrm{U}-238$ & $1 / 1$ & $5.9 *$ & $5.9^{*}$ & 5.9 & 24 & $0[4]$ \\
\hline \multicolumn{7}{|l|}{ Radionuclides, unfiltered (pCi/L) } \\
\hline $\mathrm{Co}-60$ & $5 / 13$ & $490^{*}$ & -110 & 33 & 200 & $1[4]$ \\
\hline$c s-137$ & $6 / 13$ & 32 & -2.4 & $6.3^{*}$ & 120 & $0[4]$ \\
\hline Gross alpha & $7 / 12$ & $140^{*}$ & -2.7 & 13 & 15 & $1[2]$ \\
\hline Gross beta & $11 / 13$ & $5,900 *$ & 1.6 & 560 & 50 & $4[2]$ \\
\hline
\end{tabular}


Table 7.65 (continued)

\begin{tabular}{|c|c|c|c|c|c|c|}
\hline Parameter & $\begin{array}{l}\text { N det } \\
\text { N total }\end{array}$ & $\operatorname{Max}^{2}$ & Min: & $A v^{b}$ & $\begin{array}{l}\text { Reference } \\
\text { value }\end{array}$ & $\begin{array}{l}\text { Number of values } \\
\text { exceeding } \\
\text { reference [ref }]^{c}\end{array}$ \\
\hline \multicolumn{7}{|c|}{ Radionuclides, unfiltered $(\mathrm{pCi} / \mathrm{L})$} \\
\hline $\mathrm{H}-3$ & $9 / 23$ & $1,300,000 *$ & -570 & 170,000 & 20,000 & $4[2]$ \\
\hline Pu-238 & $1 / 1$ & $2.7 *$ & $2.7 *$ & 2.7 & 1.6 & $1[4]$ \\
\hline Th-228 & $1 / 1$ & $0.17 *$ & $0.17 *$ & 0.17 & 16 & $0[4]$ \\
\hline Th -230 & $1 / 1$ & $0.14 *$ & $0.14=$ & 0.14 & 12 & $0[4]$ \\
\hline Total rad Sr & $3 / 13$ & $5.4^{*}$ & -0.51 & $1.4 *$ & 8 & $0[2]$ \\
\hline $0-234$ & $1 / 1$ & $54^{\circ}$ & $54 *$ & 54 & 20 & $1[4]$ \\
\hline $\mathrm{u}-235$ & $1 / 1$ & $1.6 *$ & $1.6 *$ & 1.6 & 24 & $0[4]$ \\
\hline $\mathrm{U}-238$ & $1 / 1$ & $5.4^{*}$ & $5.4^{*}$ & 5.4 & 24 & $0[4]$ \\
\hline \multicolumn{7}{|c|}{ Volatile organics, unfiltered $(\mu g / L)$} \\
\hline Acetone & $1 / 13$ & $\mathrm{U} 10$ & 57.0 & -9.8 & a & [d] \\
\hline Carbon disulfide & $1 / 13$ & 11 & U5.0 & -5.5 & $\bar{c}$ & [a] \\
\hline Tetrachloroethene & $1 / 13$ & U5.0 & 22.0 & -4.8 & 5 & $0[2]$ \\
\hline Trichloroethene & $1 / 13$ & U5.0 & J2.0 & -4.8 & 5 & $0[1]$ \\
\hline \multicolumn{7}{|c|}{ Upgradient Wells } \\
\hline \multicolumn{7}{|l|}{ Anions, unfiltered (mg/L) } \\
\hline Chloride & $2 / 2$ & 3.5 & $<\mathrm{H} 2.0$ & -2.8 & 250 & $0[3]$ \\
\hline Fluoride & $1 / 2$ & 1.7 & $<0.10$ & $\sim 0.90$ & 4 & $0[2]$ \\
\hline Sulfate, as $\mathrm{SO}_{4}$ & $1 / 2$ & 80 & $<10$ & -45 & 250 & $0[3]$ \\
\hline \multicolumn{7}{|l|}{ Field measurements, unfiltered } \\
\hline Conductivity (ms/cm) & $2 / 2$ & 0.52 & 0.010 & 0.27 & $\dot{a}$ & [ฮ] \\
\hline Dissolved oxygen (mg/L) & $2 / 2$ & 13 & 11 & 12 & $d$ & [a] \\
\hline Redox $(\mathrm{mV})$ & $2 / 2$ & 440 & 390 & 520 & $a$ & [a] \\
\hline Temperature $\left({ }^{\circ} \mathrm{C}\right)$ & $2 / 2$ & 16 & 16 & 16 & 30.5 & $0[1]$ \\
\hline Turbidity (NTU) & $2 / 2$ & 140 & 51 & 93 & 1 & $2[2]$ \\
\hline $\mathrm{pH}(\mathrm{SU})$ & $2 / 2$ & 7.7 & 6.5 & 7.1 & $(6.5,8.5)$ & $1[3]$ \\
\hline \multicolumn{7}{|l|}{ Metals, unfiltered img/L) } \\
\hline Aluminum, cotal & $2 / 2$ & 2.1 & 0.064 & 1.1 & 0.2 & $2[3]$ \\
\hline Barium, total & $2 / 2$ & 0.056 & 0.052 & 0.054 & 2 & $0[2]$ \\
\hline Boron, total & $1 / 2$ & 0.098 & $<0.080$ & -0.089 & d & [a] \\
\hline Calcium, total & $2 / 2$ & 38 & 0.82 & 20 & ¿े & [व] \\
\hline Chromium, total & $1 / 2$ & 0.0069 & $<0.0080$ & -0.0055 & 0.05 & $0[1]$ \\
\hline Iron, total & $2 / 2$ & 2.8 & 0.17 & 1.5 & 0.3 & $1[3]$ \\
\hline Magnesium, total & $2 / 2$ & 8.0 & 1.6 & 4.8 & $a$ & [d] \\
\hline Manganese, total & $2 / 2$ & 0.19 & 0.035 & 0.26 & 0.05 & $1\{3\}$ \\
\hline Mercury, rotal & $2 / 2$ & Hо 0.00010 & H0.000091 & -0.000096 & 0.002 & $0[1]$ \\
\hline Potassium, Eotal & $2 / 2$ & 4.5 & 1.6 & 3.0 & $a$ & [d] \\
\hline silicon, total & $2 / 2$ & 11 & 10 & 11 & $a$ & [a] \\
\hline Sodium, total & $2 / 2$ & 65 & 3.4 & 34 & $a$ & [d] \\
\hline Vanadium, total & $1 / 2$ & 0.0034 & $<0.0020$ & -0.0027 & $a$ & [d] \\
\hline zinc, total & $1 / 2$ & 0.0099 & $<0.0050$ & -0.0075 & 5 & $0[3]$ \\
\hline \multicolumn{7}{|l|}{ others, unfiltered } \\
\hline Alkalinity (mg/L) & $2 / 2$ & 210 & 13 & 110 & d & (d) \\
\hline Total organic carbon $\{\mathrm{mg} / \mathrm{L}\rangle$ & $2 / 2$ & 0.50 & $<0.50$ & -0.50 & a & [d] \\
\hline Total organic halides $(\mu g / L)$ & $2 / 2$ & 33 & 22 & 27 & $a$ & [d] \\
\hline Total suspended solids $(\mathrm{mg} / \mathrm{L})$ & $1 / 2$ & 73 & $<5.0$ & -39 & a & [d] \\
\hline \multicolumn{7}{|l|}{ Radionuclides, filterec ( $\mathrm{pCi} / \mathrm{L})^{\bullet}$} \\
\hline $\mathrm{Cs}-137$ & $2 / 2$ & $9.5^{*}$ & $6.8 *$ & 8.1 & 120 & $0[4]$ \\
\hline Gross alpha & $2 / 2$ & $3.2 *$ & $2.5^{*}$ & $2.9 *$ & 15 & $0[2]$ \\
\hline Gross beta & $2 / 2$ & $7.6^{*}$ & $3.5^{*}$ & 5.5 & 50 & $0[2]$ \\
\hline $\mathrm{H}-3$ & $1 / 2$ & $3,800 *$ & 890 & 2,300 & 20,000 & $0[2]$ \\
\hline \multicolumn{7}{|c|}{ Radionuclides, unfiltered $(\mathrm{PC} / \mathrm{L})^{\circ}$} \\
\hline Cs-137. & $1 / 2$ & $35 *$ & 1.4 & 18 & 120 & $0[4]$ \\
\hline Gross alpha & $2 / 2$ & $4.1 *$ & $3.0 *$ & $3.5^{*}$ & 15 & $0[2]$ \\
\hline
\end{tabular}


Table 7.65 (continued)

\begin{tabular}{|c|c|c|c|c|c|c|}
\hline Parameter & $\begin{array}{l}N \text { det/ } \\
N \text { total }\end{array}$ & $\operatorname{Max}$ & Min" & $A v^{b}$ & $\begin{array}{l}\text { Reference } \\
\text { value }\end{array}$ & $\begin{array}{l}\text { Number of values } \\
\text { exceeding } \\
\text { reference }[\text { ref }]^{c}\end{array}$ \\
\hline \multicolumn{7}{|c|}{ Radionuclides, unfiltered (pci/L). } \\
\hline Gross beta & $2 / 2$ & $13 *$ & $5.9 *$ & 9.3 & 50 & $0[2]$ \\
\hline H-3 & $2 / 2$ & $16,000 *$ & $320 *$ & 8,000 & 20,000 & $0[2]$ \\
\hline Total rad $\mathrm{Sr}$ & $1 / 2$ & $1.5^{*}$ & 0.22 & 0.84 & 8 & $0[2]$ \\
\hline
\end{tabular}

"Prefix "<" indicates the value for a parameter (excluding organics) was not quantifiable at the

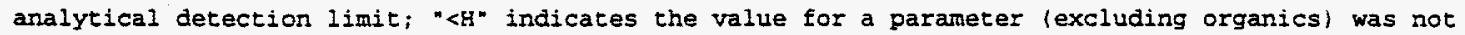
quantifiable at the analytical detection limit and that the holding time was exceeded by the laboratory; "J" indicates the value was estimated at or below the analytical detection limit by the laboratory;

"B" indicates the compound was found in the laboratory blank: "JB" indicates the vaiue was estimated at or below the analytical detection limit and was found in the laboratory blank; and "U" indicates the value for an organic parameter was undetected at the analytical detection limit.

A tilde ( - indicates that estimated and/or undetected values were used in the calculation.

'If a reference limit exists, the source is coded as:

1 Rules of Tennessee Department of Environment and Conservation, Division of water Pollution Control, Chapter 1200-4-3, General Water Quality Criteria, Domestic Water Supply, as amended.

240 CFR Part 141--National Primary Drinking Water Regulations, Subparts B and G, as amended.

340 CFR Part 143--National Secondary Drinking Water Regulations, as amended.

4 DOE Order 5400.5. Chapter III, Derived Concentration Guides for Air and Water. stot applicable.

- Individual and average radionuclide concentrations significantly greater than zero are identified by an * 
Table 7.66. Constituents in Waste Area Groupings (WAGs) $8 \& 9$ groundwater at ORNL, September 28-October 15, 1993

\begin{tabular}{|c|c|c|c|c|c|c|}
\hline Parameter & $\begin{array}{l}\mathrm{N} \text { det/ } \\
\mathrm{N} \text { total }\end{array}$ & $\operatorname{Max}{ }^{\circ}$ & Min" & $A v^{b}$ & $\begin{array}{l}\text { Reference } \\
\text { value } \quad\end{array}$ & $\begin{array}{l}\text { Number of va } \\
\text { exceeding } \\
\text { reference ire }\end{array}$ \\
\hline \multicolumn{7}{|c|}{ Downgradient Wells } \\
\hline \multicolumn{7}{|l|}{ Anions, unfiltered (mg/L) } \\
\hline Chioride & $9 / 9$ & 11 & 3.4 & 5.7 & 250 & $0[3]$ \\
\hline Fluozide & $6 / 9$ & 1.9 & $<0.10$ & -0.47 & $\varepsilon$ & $0[2]$ \\
\hline Nitrate & $2 / 9$ & 5.8 & $<1.0$ & -1.7 & 10 & $0[2]$ \\
\hline Sulfate, as so, & $9 / 9$ & 300 & 11 & 68 & 250 & $1[3]$ \\
\hline \multicolumn{7}{|c|}{ Base neutral/acid extractable organics, unfiltered ( $\mu g / L)$} \\
\hline Benzyl butyl phthalate & $3 / 9$ & B10 & JE2.0 & -5.1 & d & [a] \\
\hline Diethyl phthalate & $1 / 9$ & 05.0 & JB1.0 & -4.6 & d & [d] \\
\hline \multicolumn{7}{|l|}{ Field measurements, unfiltered } \\
\hline Conduetivity (mS/cm) & $9 / 9$ & 0.92 & 0.26 & 0.53 & d & [a] \\
\hline Dissolved oxygen (mg/L) & $9 / 9$ & 12 & 9.0 & 10 & d & [d] \\
\hline Redox (mV) & $8 / 8$ & 540 & 260 & 400 & d & [d] \\
\hline Temperature $\left({ }^{\circ} \mathrm{C}\right)$ & $9 / 9$ & 20 & 15 & 17 & 30.5 & $0[1]$ \\
\hline Turbiaity (NTU) & $9 / 9$ & 39 & 2.0 & 9.3 & 1 & $9[2]$ \\
\hline $\mathrm{pH} \quad(\mathrm{SU})$ & $9 / 9$ & 9.3 & 6.2 & 7.4 & $(6.5,8.5)$ & $2[3]$ \\
\hline \multicolumn{7}{|l|}{ Metals, unfiltered (mg/L) } \\
\hline Aluminum, total & $3 / 9$ & 2.0 & $<0.050$ & -0.32 & 0.2 & $2[3]$ \\
\hline Arsenic, total & $1 / 9$ & 0.013 & $<0.010$ & -0.010 & 0.05 & $0[1]$ \\
\hline Barium, total & $9 / 9$ & 0.28 & 0.023 & 0.095 & 2 & $0[2]$ \\
\hline Boron, total & $4 / 9$ & 0.70 & $<0.080$ & $\sim 0.17$ & $a$ & [a! \\
\hline Calcium, total & $9 / 9$ & 100 & 1.3 & 46 & ¿ & [a] \\
\hline Chromium, total & $1 / 9$ & 0.0054 & $<0.0040$ & -0.0042 & 0.05 & $0[1]$ \\
\hline Cobalt, total & $1 / 9$ & 0.016 & $<0.0040$ & -0.0053 & $d$ & [a] \\
\hline Iron, rozal & $8 / 9$ & 22 & $<0.050$ & -3.4 & 0.3 & $5[3]$ \\
\hline Magnesium, total & $9 / 9$ & 18 & 0.13 & 11 & $d$ & [d] \\
\hline Manganese, total & $9 / 9$ & 2.8 & 0.0024 & 0.61 & 0.05 & $6\{3\}$ \\
\hline Potassium, total & $9 / 9$ & 4.6 & 1.6 & 2.7 & a & [d] \\
\hline Silicon, total & $9 / 9$ & 18 & 2.6 & 7.6 & a & [d] \\
\hline Sodium, total & $9 / 9$ & 180 & 4.6 & 41 & d & [a] \\
\hline Vanadium, total & $3 / 9$ & 0.0036 & $<0.0020$ & -0.0024 & d & [a] \\
\hline zinc, total & $2 / 9$ & 0.015 & $<0.0050$ & -0.0063 & 5 & $0[3]$ \\
\hline \multicolumn{7}{|l|}{ Others, unfiltered } \\
\hline Alkalinity (mg/L) & $9 / 9$ & 350 & 85 & 230 & a & [a] \\
\hline Total organic carbon (mg/L) & $4 / 9$ & 1.6 & $<0.50$ & -0.67 & d & [d] \\
\hline Total organic halides $(\mu \mathrm{g} / \mathrm{L}\}$ & $3 / 9$ & 14 & $<5.0$ & -7.1 & d & [a] \\
\hline Total suspended solids $(\mathrm{mg} / L)$ & $2 / 9$ & 64 & $<5.0$ & -14 & d & [d] \\
\hline \multicolumn{7}{|l|}{ Radionuclides, filtered (pCi/L)* } \\
\hline co-60 & $2 / 9$ & $4.9 *$ & -1.6 & $2.0 *$ & 200 & $0[4]$ \\
\hline cs-137 & $1 / 9$ & $4.6^{*}$ & -0.81 & 0.86 & 120 & $0[4]$ \\
\hline Gross alpha & $5 / 9$ & $12 *$ & -0.35 & $3.4 *$ & 25 & $0[2]$ \\
\hline Gross beta & $8 / 9$ & $3,500^{*}$ & 0.81 & 650 & 50 & $3[2]$ \\
\hline $\mathrm{H}-3$ & $7 / 9$ & $65,000 *$ & 140 & 8,000 & 20,000 & $1[2]$ \\
\hline Total rad Sr & $5 / 9$ & $1,900 *$ & -0.84 & 340 & 8 & $3[2]$ \\
\hline \multicolumn{7}{|l|}{ Radionuclides, unfiltered (PCi/L)• } \\
\hline Co-60 & $2 / 9$ & $5.4 *$ & -0.54 & $2.1 *$ & 200 & $0[4]$ \\
\hline Gross alpha & $5 / 9$ & $5.7 *$ & 0.24 & $2.5 *$ & 15 & $0[2]$ \\
\hline Gross beta & $8 / 9$ & $3,800^{*}$ & 1.9 & 650 & 50 & $3[2]$ \\
\hline $\mathrm{H}-3$ & $8 / 9$ & $65,000 *$ & -27 & 8,000 & 20,000 & $2[2]$ \\
\hline Total rad SF & $4 / 9$ & $2,100 *$ & -0.38 & 350 & 8 & $3[2]$ \\
\hline \multicolumn{7}{|c|}{ Volatile organics, unfiltered ( $\mu \mathrm{g} / \mathrm{L})$} \\
\hline 1,2-Dichloroethere & $1 / 9$ & 11 & U5.0 & -5.7 & 70 & $0[2]$ \\
\hline Trichloroethene & $1 / 9$ & 6.3 & U5.0 & -5.1 & 5 & $1[1]$ \\
\hline Vinyl chloride & $1 / 9$ & 510 & 51.3 & -9.0 & 2 & $8[1\}$ \\
\hline
\end{tabular}


Table 7.66 (continued)

\begin{tabular}{|c|c|c|c|c|c|c|}
\hline Parameter & $\begin{array}{l}\mathrm{N} \text { det/ } \\
\mathrm{N} \text { total }\end{array}$ & $\operatorname{Max}$ & Mis* & $A v^{b}$ & $\begin{array}{l}\text { Reference } \\
\text { value }\end{array}$ & $\begin{array}{l}\text { Number of values } \\
\text { exceeding } \\
\text { reference }[\text { ref }]^{\circ}\end{array}$ \\
\hline \multicolumn{7}{|c|}{ Upgradient wells } \\
\hline \multicolumn{7}{|l|}{ Anions, unfiltered (mg/L) } \\
\hline Chloride & $2 / 2$ & 4.6 & 2.8 & 3.7 & 250 & $0[3]$ \\
\hline Fluoride & $1 / 2$ & 0.50 & $<0.10$ & -0.30 & 4 & $0[2]$ \\
\hline Sulfate, as $\mathrm{SO}_{4}$ & $2 / 2$ & 130 & 25 & 75 & 250 & $O[3]$ \\
\hline \multicolumn{7}{|l|}{ Field measurements, unfiltered } \\
\hline Conductivity (ms/cm) & $2 / 2$ & 0.54 & 0.39 & 0.46 & d & [d] \\
\hline Dissolved oxygen (mg/L) & $2 / 2$ & 11 & 11 & 11 & d & [d] \\
\hline Redox (mV) & $2 / 2$ & 450 & 310 & 380 & $\mathrm{~d}$ & [a] \\
\hline Temperature $\left({ }^{\circ} \mathrm{C}\right)$ & $2 / 2$ & 15 & 14 & 15 & 30.5 & $0\{1\}$ \\
\hline Turbidity (NTU) & $2 / 2$ & 12 & 10 & 11 & 1 & $2\lfloor 2\rceil$ \\
\hline $\mathrm{PH}$ (SU) & $2 / 2$ & 8.1 & 6.7 & 7.4 & $(6.5,8.5)$ & $0\{3\}$ \\
\hline \multicolumn{7}{|l|}{ Metals, unfiltered (mg/L) } \\
\hline Aluminum, total & $1 / 2$ & 2.2 & $<0.050$ & -1.1 & 0.2 & $1[3]$ \\
\hline Barium, total & $2 / 2$ & 0.084 & 0.045 & 0.065 & 2 & $0[2]$ \\
\hline Boron, total & $1 / 2$ & 0.31 & $<0.080$ & -0.20 & $\mathrm{~d}$ & [d] \\
\hline Calcium, total & $2 / 2$ & 59 & 15 & 37 & d & [a] \\
\hline Chromium, total & $1 / 2$ & 0.0045 & $<0.0040$ & -0.0043 & 0.05 & $0[1]$ \\
\hline Copper, total & $1 / 2$ & 0.012 & $<0.0070$ & -0.0095 & 1.3 & $0[2]$ \\
\hline Iron, total & $1 / 2$ & 9.7 & $<0.050$ & -4.9 & 0.3 & $1[3]$ \\
\hline Magnesium, total & $2 / 2$ & 19 & 3.3 & 11 & $d$ & [d] \\
\hline Manganese, total & $2 / 2$ & 3.1 & 0.041 & 1.6 & 0.05 & $1\{3\}$ \\
\hline Potassium, total & $2 / 2$ & 3.6 & 3.0 & 3.3 & a & [a] \\
\hline Silicon, total & $2 / 2$ & 17 & 5.6 & 11 & $d$ & [d] \\
\hline Sodium, total & $2 / 2$ & 59 & 10 & 35 & d & [d] \\
\hline Vanadium, total & $1 / 2$ & 0.0045 & $<0.0020$ & -0.0033 & a & [a] \\
\hline Zine, total & $1 / 2$ & 0.013 & $<0.0050$ & -0.0090 & 5 & $0[3]$ \\
\hline \multicolumn{7}{|l|}{ Others, unfiltered } \\
\hline Alkalinity $(\mathrm{mg} / \mathrm{L})$ & $2 / 2$ & 170 & 120 & 150 & d & [a] \\
\hline Total organic carbon (mg/L) & $1 / 2$ & $<0.50$ & $<0.50$ & -0.50 & a & [d] \\
\hline rotal suspended solids (mg/L) & $1 / 2$ & 69 & $<5.0$ & -37 & $a$ & (d) \\
\hline \multicolumn{7}{|l|}{ Radionuclides, filtered $(\mathrm{pCi} / \mathrm{L}\}^{\circ}$} \\
\hline Gross beta & $1 / 2$ & $5.4 *$ & 2.2 & 3.8 & 50 & $0[2]$ \\
\hline $\mathrm{H}-3$ & $2 / 2$ & $1,200 *$ & $700 *$ & 930 & 20,000 & $O[2]$ \\
\hline \multicolumn{7}{|l|}{ Radionuclides, unfiltered (PCi/L)" } \\
\hline Gross beta & $2 / 2$ & $6.5 *$ & 4.3* & 5.4 & 50 & $0[2]$ \\
\hline $\mathrm{E}-3$ & $2 / 2$ & $1,100^{\star}$ & $840^{*}$ & $950 *$ & 20,000 & $0[2]$ \\
\hline \multicolumn{7}{|c|}{ Volatile organics, unfiltered ( $\mu \mathrm{g} / \mathrm{L})$} \\
\hline Methylene chloride & $1 / 2$ & U5.0 & J3.6 & -4.3 & d & [a] \\
\hline
\end{tabular}

"Prefix "<" indicates the value for a parameter (excluding organics) was not quantifiable at the analytical detection limit; " $\mathrm{J}$ " indicates the value was estimated at or below the analytical by the laboratory; " $\mathrm{B}$ " indicates the compound was found in the laboratory blank; "JB" indicates the value was estimated at or below the analytical detection limit and was found in the laboratory blank; and " $U$ " indicates the value for an organic parameter was undetected at the analytical detection limit.

bA tilde (-) indicates that estimated and/or undetected values were used in the calculation. 'If a reference limit exists, the source is coded as:

1 Rules of Tennessee Department of Environment and Conservation. Division of Water pollution Control, Chapter 1200-4-3. General Water Quality Criteria, Domestic Water Supply, as amended.

240 CFR Part 141--National Primary. Drinking Water Regulations, Subparts $B$ and $G$, as amended.

340 CFR Part 143--National Secondary Drinking water Regulations, as amended.

4 DOE Order 5400.5. Chapter III, Derived Concentration Guides for Air and Water.

Not applicable.

-Individual and average radionuclide concentrations significantly greater than zero are identified by an * 
Table 7.67. Constituents in Waste Area Grouping (WAG) 11 groundwater at ORNL,

December 18, 1992-January 18, 1993

\begin{tabular}{|c|c|c|c|c|c|c|}
\hline Parameter & $\begin{array}{l}\text { N det/ } \\
N \text { total }\end{array}$ & $\operatorname{Max}$ & Min* & $A v^{b}$ & $\begin{array}{l}\text { Reference } \\
\text { value }\end{array}$ & $\begin{array}{l}\text { Number of val } \\
\text { exceeding } \\
\text { reference lref }\end{array}$ \\
\hline \multicolumn{7}{|c|}{ Downgradient Wells } \\
\hline \multicolumn{7}{|l|}{ Anions, unfiltered (mg/L) } \\
\hline Chloride & $3 / 5$ & 2.5 & $<2.0$ & -2.1 & 250 & $0[3]$ \\
\hline Fluoride & $2 / 5$ & 0.30 & $<0.10$ & -0.16 & 4 & $0[2]$ \\
\hline Nitrate & $2 / 5$ & 1.8 & $<1.0$ & -1.2 & 10 & $0[2]$ \\
\hline Phosphate & $1 / 5$ & 1.0 & $<1.0$ & -2.0 & $a$ & [d] \\
\hline Sulfate, as so, & $2 / 5$ & 12 & $<10$ & -11 & 250 & $0[3]$ \\
\hline \multicolumn{7}{|c|}{ Base neutral/acid extractable organics, unfiltered $(\mu \mathrm{g} / \mathrm{L})$} \\
\hline Benzyl butyl phthalate & $1 / 5$ & 010 & B1.0 & -8.2 & a & [d] \\
\hline \multicolumn{7}{|l|}{ Field measurements, unfiltered } \\
\hline Conductivity $(\mathrm{mS} / \mathrm{cm})$ & $5 / 5$ & 0.21 & 0.040 & 0.11 & $\mathrm{a}$ & [a] \\
\hline Dissolved oxygen (mg/L) & $5 / 5$ & 9.2 & 6.1 & 7.2 & $d$ & [व] \\
\hline Redox (mV) & $5 / 5$ & 690 & 580 & 630 & d & [a] \\
\hline Temperature $\left({ }^{\circ} \mathrm{C}\right)$ & $5 / 5$ & 14 & 13 & 13 & 30.5 & $0[1]$ \\
\hline Turbidity (JTU) & $5 / 5$ & 350 & 8.4 & 160 & 1 & $5[2]$ \\
\hline $\mathrm{pH}(\mathrm{SU})$ & $5 / 5$ & 9.1 & 6.9 & 7.7 & $(6.5,8.5)$ & $1[3]$ \\
\hline \multicolumn{7}{|l|}{ Metals, filtered (mg/L) } \\
\hline Antimony & $3 / 5$ & 0.0060 & $<0.0050$ & -0.0054 & $\mathrm{a}$ & [d] \\
\hline Barium & $5 / 5$ & 0.13 & 0.012 & 0.046 & 2 & $0[2]$ \\
\hline Calcium & $5 / 5$ & 56 & 10 & 34 & $\bar{a}$ & [d] \\
\hline Magnesium & $5 / 5$ & 18 & 1.1 & 8.8 & $a$ & [d] \\
\hline Manganese & $2 / 5$ & 0.0035 & $<0.0010$ & -0.0016 & 0.05 & $0[3]$ \\
\hline Mercury & $1 / 5$ & $<H 0.000050$ & $<0.000050$ & -0.000050 & 0.002 & $0[1]$ \\
\hline Potassium & $4 / 5$ & 3.5 & $<0.40$ & -2.1 & $\mathrm{~d}$ & [d] \\
\hline silicon & $5 / 5$ & 11 & 3.4 & 6.0 & a & [a] \\
\hline Sodium & $5 / 5$ & 11 & 0.74 & 2.9 & $d$ & [d] \\
\hline zinc & $1 / 5$ & 0.0056 & $<0.0050$ & -0.0051 & 5 & $0[3]$ \\
\hline \multicolumn{7}{|l|}{ Metals, unfiltered (mg/L) } \\
\hline Aluminum, total & $2 / 5$ & 1.2 & $<0.050$ & -0.51 & 0.2 & 2 [3] \\
\hline Barium, total & $5 / 5$ & 0.24 & 0.020 & 0.087 & 2 & $0[2]$ \\
\hline Calciun, total & $5 / 5$ & 48 & 9.7 & 30 & $a$ & [d] \\
\hline Iron, total & $2 / 5$ & 2.8 & $<0.050$ & -0.88 & 0.3 & $2[3]$ \\
\hline Magnesium, total & $5 / 5$ & 16 & 1.3 & 8.1 & $a$ & [d] \\
\hline Manganese, total & $4 / 5$ & 0.28 & $<0.0010$ & -0.069 & 0.05 & $2[3]$ \\
\hline Mercury, total & $1 / 5$ & $<\mathrm{HO} .000050$ & $<0.000050$ & -0.000050 & 0.002 & $0[1]$ \\
\hline Potassium, total & $5 / 5$ & 3.1 & 0.85 & 2.0 & $a$ & [d] \\
\hline silicon, total & $5 / 5$ & 10 & 4.5 & 6.0 & $a$ & [d] \\
\hline Sodium, total & $5 / 5$ & 9.5 & 0.58 & 2.6 & a & [a] \\
\hline vanadium, total & $2 / 5$ & 0.0024 & $<0.0020$ & -0.0021 & $a$ & [d] \\
\hline zine, total & $5 / 5$ & 0.016 & 0.0063 & 0.012 & 5 & $0[3]$ \\
\hline \multicolumn{7}{|l|}{ Others, Eiltered } \\
\hline Alkalinity (mg/L) & $5 / 5$ & 200 & 33 & 120 & $a$ & [d] \\
\hline Total dissolved solids (mg/L) & $5 / 5$ & 210 & 88 & 140 & 500 & $0[1]$ \\
\hline \multicolumn{7}{|l|}{ Others, unfiltered } \\
\hline Alkalinicy (mg/L) & $5 / 5$ & 190 & 35 & 110 & d & [d] \\
\hline Total organic carbon (mg/L) & $4 / 5$ & 0.71 & $<0.50$ & -0.59 & d & [d] \\
\hline Total organic halides $(\mu g / L)$ & $2 / 5$ & 29 & $<5.0$ & -12 & $\mathrm{~d}$ & [a] \\
\hline Total suspended solids (mg/L) & $2 / 5$ & 39 & $<5.0$ & -13 & d & [a] \\
\hline \multicolumn{7}{|l|}{ Radionuclides, filtered (pCi/L)" } \\
\hline Gross alpha & $4 / 5$ & $5.9 *$ & -0.38 & $2.5 *$ & 15 & $0[2]$ \\
\hline Gross beta & $5 / 5$ & $27 *$ & $8.4^{*}$ & $15^{*}$ & 50 & $0[2]$ \\
\hline$H-3$ & $1 / 5$ & $1,400 *$ & -220 & 160 & 20,000 & $0[2]$ \\
\hline Total rad Sr & $2 / 5$ & $3.8 *$ & 0 & $1.9 *$ & 8 & $0[2]$ \\
\hline
\end{tabular}


Table 7.67 (continued)

\begin{tabular}{|c|c|c|c|c|c|c|}
\hline Parameter & $\begin{array}{l}\text { det/ } \\
\text { total }\end{array}$ & $\operatorname{Max}$ & $\operatorname{Min}^{*}$ & $A v^{b}$ & $\begin{array}{l}\text { Reference } \\
\text { value } r\end{array}$ & $\begin{array}{l}\text { Number of values } \\
\text { exceeding } \\
\text { reference [ref]c }\end{array}$ \\
\hline \multicolumn{7}{|l|}{ Radionuclides, unfiltered $(\mathrm{pCi} / \mathrm{L})^{*}$} \\
\hline Co-60 & $3 / 5$ & $12 *$ & -8.4 & 2.4 & 200 & $0[4]$ \\
\hline $\mathrm{Cs}-137$ & $1 / 5$ & $2.2 *$ & -1.6 & 0.66 & 120 & $0[4]$ \\
\hline Gross alpha & $3 / 5$ & $5.4^{*}$ & 0.27 & $2.6 *$ & 15 & $0[2]$ \\
\hline Gross beta & $5 / 5$ & $35^{*}$ & $4.3 *$ & $18 *$ & 50 & $0\{2\}$ \\
\hline Total rad SI & $2 / 5$ & $3.5 *$ & -2.0 & 1.3 & 8 & $0[2]$ \\
\hline \multicolumn{7}{|l|}{ Volatile organics, unfiltered $(\mu \mathrm{g} / \mathrm{L})$} \\
\hline Trichloroethene & $2 / 5$ & 58 & $\mathrm{U} 5.0$ & -18 & 5 & $2[1]$ \\
\hline \multicolumn{7}{|c|}{ Upgradient Wells } \\
\hline \multicolumn{7}{|l|}{ Anions, unfiltered (mg/L) } \\
\hline Eluoride & $4 / 6$ & 0.20 & $<0.10$ & -0.12 & 4 & $O[2]$ \\
\hline Phosphate & $1 / 6$ & 2.0 & $<1.0$ & -1.2 & d & [d] \\
\hline \multicolumn{7}{|c|}{ Base neutral/acid extractable organics, unfiltered (Hg/L) } \\
\hline Diethyl phthalate & $2 / 6$ & B10 & B6. 0 & -9.3 & d & [d] \\
\hline \multicolumn{7}{|l|}{ Field measurements, unfiltered } \\
\hline Conductivity $(\mathrm{mS} / \mathrm{cm})$ & $6 / 6$ & 0.27 & 0.12 & 0.19 & $d$ & [d] \\
\hline Dissolved oxygen (mg/L) & $6 / 6$ & 9.5 & 8.0 & 8.8 & $d$ & [d] \\
\hline Redox (mV) & $6 / 6$ & 660 & 400 & 540 & $\mathrm{~d}$ & [d] \\
\hline Temperature $\left({ }^{\circ} \mathrm{C}\right)$ & $6 / 6$ & 14 & 12 & 14 & 30.5 & $O[1]$ \\
\hline Turbidity (JTU) & $6 / 6$ & 34 & 6.3 & 19 & 1 & $6[2]$ \\
\hline $\mathrm{pH}(\mathrm{SU})$ & $6 / 6$ & 9.2 & 7.3 & 8.0 & $\{6.5,8.5\}$ & $1[3]$ \\
\hline \multicolumn{7}{|l|}{ Metals, filtered (mg/L) } \\
\hline Barium & $6 / 6$ & 0.21 & 0.035 & 0.099 & 2 & $O[2]$ \\
\hline Calcium & $6 / 6$ & 82 & 27 & 63 & $d$ & [d] \\
\hline Iron & $2 / 6$ & 0.27 & $<0.050$ & -0.10 & 0.3 & $O[3]$ \\
\hline Magnesium & $6 / 6$ & 15 & 2.7 & 10 & $d$ & [d] \\
\hline Manganese & $2 / 6$ & 0.38 & $<0.0010$ & -0.088 & 0.05 & $2[3]$ \\
\hline Mercury & $1 / 6$ & 0.000050 & $<0.000050$ & -0.000050 & 0.002 & $O[1]$ \\
\hline Potassium & $6 / 6$ & 4.3 & 2.0 & 2.9 & d & [d] \\
\hline silicon & $6 / 6$ & 9.3 & 5.9 & 7.3 & $d$ & [d] \\
\hline Sodium & $6 / 6$ & 5.4 & 1.2 & 2.7 & $d$ & [a] \\
\hline zinc & $1 / 6$ & $0: 065$ & $<0.0050$ & -0.015 & 5 & $0[3]$ \\
\hline \multicolumn{7}{|l|}{ Metals, unfiltered (mg/L) } \\
\hline Aluminum, total & $3 / 6$ & 0.40 & $<0.050$ & -0.11 & 0.2 & $1[3]$ \\
\hline Barium, total & $6 / 6$ & 0.21 & 0.042 & 0.11 & 2 & $0[2]$ \\
\hline Calcium, total & $6 / 6$ & 77 & 26 & 57 & d & [d] \\
\hline Iron, total & $5 / 6$ & 0.34 & $<0.050$ & -0.16 & 0.3 & $1[3]$ \\
\hline Magnesium, total & $6 / 6$ & 15 & 2.5 & 10 & $d$ & [d] \\
\hline Manganese, total & $5 / 6$ & 0.36 & $<0.0010$ & -0.087 & 0.05 & $2[3]$ \\
\hline Potassium, total & $6 / 6$ & 4.1 & 1.8 & 2.6 & d & [d] \\
\hline Silicon, total & $6 / 6$ & 8.6 & 5.7 & 6.7 & $\mathrm{~d}$ & (d) \\
\hline Sodium, total & $6 / 6$ & 4.9 & 1.0 & 2.4 & $\mathrm{~d}$ & [d] \\
\hline zinc, total & $5 / 6$ & 0.0080 & $<0.0050$ & -0.0060 & 5 & $0[3]$ \\
\hline \multicolumn{7}{|l|}{ others, filtered } \\
\hline Alkalinity $(\mathrm{mg} / \mathrm{L})$ & $6 / 6$ & 250 & 130 & 190 & d & [d] \\
\hline Total dissolved solids (mg/L) & $6 / 6$ & 260 & 160 & 210 & 500 & $0[1]$ \\
\hline \multicolumn{7}{|l|}{ Others, unfiltered } \\
\hline Alkalinity (mg/L) & $6 / 6$ & 260 & 130 & 190 & $d$ & [d] \\
\hline Total organic carbon (mg/L) & $4 / 6$ & 0.83 & $<0.50$ & -0.63 & $d$ & [a] \\
\hline Total organic halides $(\mu \mathrm{g} / \mathrm{L})$ & $1 / 6$ & $<5.8$ & $<5.0$ & -5.1 & $d$ & [d] \\
\hline Total suspended solids (mg/L) & $1 / 6$ & 9.0 & $<5.0$ & -5.7 & $d$ & [d] \\
\hline \multicolumn{7}{|l|}{ Radionuclides, filtered $(\mathrm{pCi} / \mathrm{L})^{\bullet}$} \\
\hline $\operatorname{co-60}$ & $1 / 6$ & $4 \cdot 1 *$ & -6.8 & -1.1 & 200 & $0[4]$ \\
\hline Gross alphe & $3 / 6$ & $7.0^{*}$ & 0.59 & $2.4^{*}$ & 15 & $0[2]$ \\
\hline
\end{tabular}


Table 7.67 (continued)

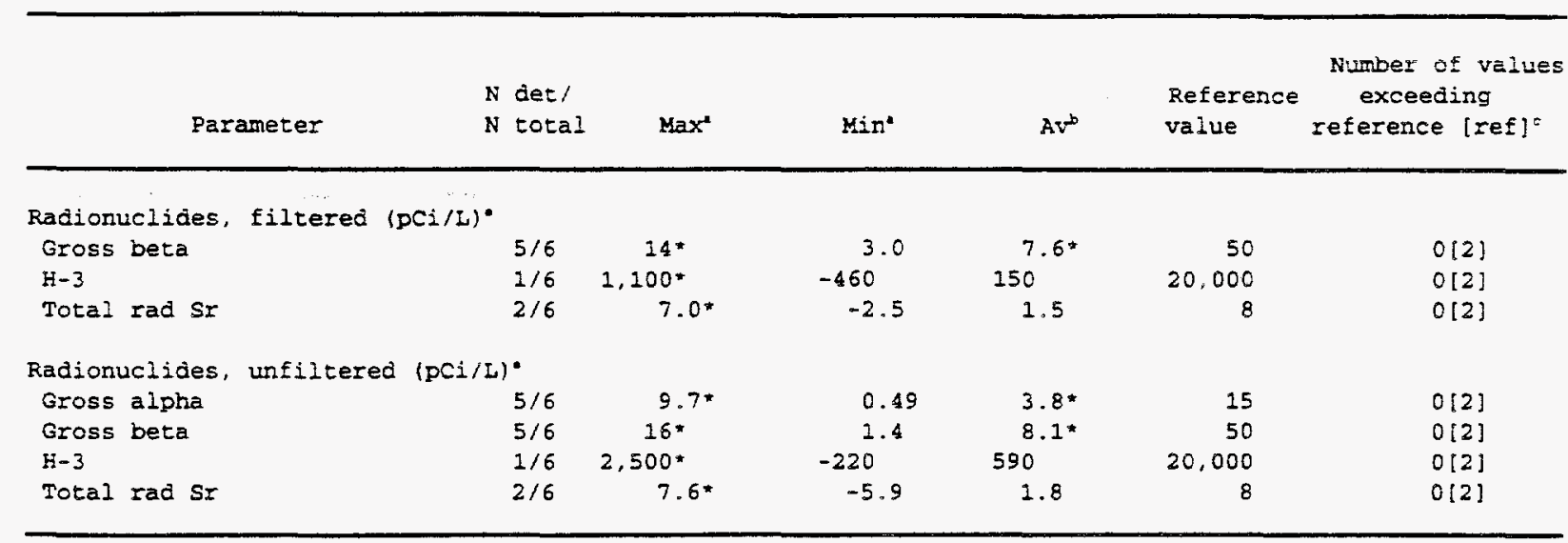

"Prefix '<" indicates the value for a parameter (excluding organics) was not quantifiable at the anaiytical detection limit; " $\angle H$ " indicates the value for a parameter excluding organics) was not guantifiable at the analytical detection limit and that the holding time was exceeded by the laboratory; ' $B$ " indicates the compound was found in the laboratory blank; " $<\mathrm{H}^{\circ}$ indicates the holding time was exceeded by the laboratory; and $" U$ " indicates the value for an organic parameter was undetected at the analytical aetection limit.

${ }_{B}$ tilde $(\sim)$ indicates that estimated and/or undetected values were used in the calculation.

'If a reference limit exists, the source is coded as:

1 Rules of Tennessee Department of Environment and Conservation, Division of Water Pollution Control, Chapter 1200-4-3. General Water Quality Criteria, Donestic Water Supply, as amended.

240 CFR Part 141--National Primary Drinking Water Regulations, Subparts $B$ and G. as amendea.

$340 \mathrm{CFR}$ Part 143--National Secondary Drinking Water Regulations, as amended.

4 DOE Order 5400.5. Chapter III, Derived Concentration Guides for Air and Water.

Not applicable.

-Incividual and average radionuclide concentrations significantly greater than zero are identified by an *. 
Table 7.68. Constituents in Waste Area Grouping (WAG) 17 groundwater at ORNL, June 29-July 9, 1993

\begin{tabular}{|c|c|c|c|c|c|c|}
\hline Parameter & $\begin{array}{l}\text { N det/ } \\
\text { N totaI }\end{array}$ & $\operatorname{Max}$ & $\operatorname{Min}^{2}$ & $A v^{b}$ & $\begin{array}{l}\text { Reference } \\
\text { value }\end{array}$ & $\begin{array}{l}\text { Number of values } \\
\text { exceeding } \\
\text { reference [ref]c }\end{array}$ \\
\hline \multicolumn{7}{|c|}{ Downgradient wells } \\
\hline \multicolumn{7}{|l|}{ Anions, unfiltered (mg/L) } \\
\hline Chloride & $4 / 4$ & 15 & 3.5 & 8.6 & 250 & $0[3]$ \\
\hline Flucride & $1 / 4$ & 0.30 & $<0.10$ & -0.15 & 4 & $0[2]$ \\
\hline Nitrate & $1 / 4$ & 3.5 & $<1.0$ & -1.6 & 10 & $0[2]$ \\
\hline Sulfate, as so, & $4 / 4$ & 50 & 30 & 41 & 250 & $0[3]$ \\
\hline \multicolumn{7}{|l|}{ Field measurements, unfiltered } \\
\hline Conductivity $(\mathrm{ms} / \mathrm{cm})$ & $4 / 4$ & 0.81 & 0.39 & 0.64 & $d$ & [a] \\
\hline Dissolved oxygen (mg/L) & $4 / 4$ & 13 & 10 & 11 & $d$ & [d] \\
\hline Redox (mV) & $4 / 4$ & 310 & 250 & 270 & $d$ & [a] \\
\hline Temperature $\left({ }^{\circ} \mathrm{C}\right)$ & $4 / 4$ & 19 & 15 & 17 & 30.5 & $0(1)$ \\
\hline Turbidity (JTU) & $4 / 4$ & 55 & 6.3 & 29 & 1 & $4[2]$ \\
\hline $\mathrm{pH} \quad(\mathrm{SU}\rangle$ & $4 / 4$ & 7.3 & 6.9 & 7.0 & $(6.5,8.5)$ & $0[3]$ \\
\hline \multicolumn{7}{|l|}{ Metals, unfiltered (mg/L) } \\
\hline Aluminum, total & $1 / 4$ & 0.98 & $<0.050$ & -0.28 & 0.2 & $1[3]$ \\
\hline Barium, total & $4 / 4$ & 0.16 & 0.031 & 0.092 & 2 & $0[2]$ \\
\hline Boron, total & $1 / 4$ & 0.13 & $<0.080$ & $\sim 0.093$ & $\mathrm{~d}$ & [d] \\
\hline Calcium, total & $4 / 4$ & 120 & 72 & 100 & $a$ & [d] \\
\hline Iron, total & $2 / 4$ & 0.98 & $<0.050$ & $\sim 0.32$ & 0.3 & $1[3]$ \\
\hline Magnesium, total & $4 / 4$ & 36 & 7.6 & 25 & $a$ & [d] \\
\hline Manganese, total & $4 / 4$ & 0.17 & 0.0086 & 0.058 & 0.05 & $1[3]$ \\
\hline Mercury, total & $4 / 4$ & 0.00014 & 0.000052 & 0.000077 & 0.002 & $0[1]$ \\
\hline Potassium, total & $2 / 4$ & 2.6 & $<1.0$ & -1.6 & d & [a] \\
\hline silicon, total & $4 / 4$ & 6.5 & 4.3 & 5.1 & $a$ & (d) \\
\hline Sodium, total & $4 / 4$ & 12 & 5.5 & 8.0 & a & [d] \\
\hline Vanadium, total & $1 / 4$ & 0.0029 & $<0.0020$ & -0.0022 & $d$ & [d] \\
\hline zinc, total & $2 / 4$ & 0.010 & $<0.0050$ & -0.0065 & 5 & $O[3]$ \\
\hline \multicolumn{7}{|l|}{ others, unfiltered } \\
\hline Alkalinity (mg/L) & $4 / 4$ & 440 & 190 & 330 & $d$ & [d] \\
\hline Total organic carbon (mg/L) & $4 / 4$ & 1.4 & 0.75 & 1.1 & d & [a] \\
\hline Total organic halices $(\mu \mathrm{g} / \mathrm{L})$ & $3 / 4$ & 11,000 & $<5.0$ & $-2,700$ & d & [d] \\
\hline Total suspended solids (mg/L) & $1 / 4$ & 26 & $<5.0$ & -10 & $a$ & {$[a]$} \\
\hline \multicolumn{7}{|l|}{ Radionuclides, filtered (pci/L)" } \\
\hline Co-60 & $1 / 4$ & $2.7 *$ & 1.1 & $1.9 *$ & 200 & $0[4]$ \\
\hline Gross beta & $4 / 4$ & $5.4 *$ & $3.8 *$ & $4.5^{*}$ & 50 & $0[2]$ \\
\hline $\mathrm{H}-3$ & $4 / 4$ & $5,900^{\star}$ & $2.300 *$ & $4.600 *$ & 20,000 & $0[2]$ \\
\hline Total rad SI & $1 / 4$ & $3.8 *$ & 0.027 & 1.3 & 8 & $0[2]$ \\
\hline \multicolumn{7}{|l|}{ Radionuclides, unfiltered (pCi/L) } \\
\hline Gross alpha & $1 / 4$ & $7.6 *$ & -0.73 & 2.0 & 15 & $0[2]$ \\
\hline Gross beta & $3 / 4$ & $8.4 *$ & -0.27 & $4.7^{*}$ & 50 & $0[2]$ \\
\hline $\mathrm{H}-3$ & $4 / 4$ & $5,900^{*}$ & $1,800 *$ & $4,300 *$ & 20,000 & $0[2]$ \\
\hline Total rad Sr & $2 / 4$ & $4.1 *$ & 1.9 & $2.7^{\star}$ & 8 & $0[2]$ \\
\hline \multicolumn{7}{|c|}{ Volatile organies, unfiltered $(\mu \mathrm{g} / \mathrm{L})$} \\
\hline 1,1,1-Trichloroethane & $1 / 4$ & Us.0 & J3.5 & -4.6 & 200 & $0\{1\}$ \\
\hline 1,1-Dichloroethane & $1 / 4$ & US.0 & $\mathrm{J} 2.0$ & -4.3 & $\mathrm{~d}$ & [a] \\
\hline 1,1-Dichloroethene & $1 / 4$ & 20 & U5. 0 & -8.8 & 7 & $1[1]$ \\
\hline 1.2-Dichloroethene & $2 / 4$ & Y3, 100 & $\mathrm{~J} 3.0$ & -780 & 70 & $1[2]$ \\
\hline Benzene & $1 / 4$ & 16 & U5.0 & -7.8 & 5 & $1[1]$ \\
\hline Chloroform & $1 / 4$ & $\mathrm{US} .0$ & J3.1 & -4.5 & 100 & $0[2]$ \\
\hline Tetrachloroethene & $1 / 4$ & 18 & 05.0 & -8.3 & 5 & $1[2]$ \\
\hline Trichloroethene & $2 / 4$ & $Y 9,100$ & U5.0 & $-2,300$ & 5 & $2[1]$ \\
\hline Vinyl chloride & $1 / 4$ & 95 & v10 & -31 & 2 & $4[1]$ \\
\hline
\end{tabular}


Table 7.68 (continued)

\begin{tabular}{|c|c|c|c|c|c|c|}
\hline Parameter & $\begin{array}{l}\mathrm{N} \text { det } / \\
\mathrm{N} \text { total }\end{array}$ & $\operatorname{Max}$ & $\operatorname{Min}^{*}$ & $A V^{E}$ & $\begin{array}{l}\text { Reference } \\
\text { value }\end{array}$ & $\begin{array}{l}\text { Nunber of } \\
\text { exceedin } \\
\text { zeference }\end{array}$ \\
\hline \multicolumn{7}{|c|}{ Upgradient wells } \\
\hline \multicolumn{7}{|l|}{ Anions, unfiltered (mg/L) } \\
\hline Chloride & $3 / 4$ & 11 & $<2.0$ & -4.9 & 250 & $0[3]$ \\
\hline Fluoride & $1 / 4$ & 0.20 & $<0.10$ & -0.13 & 4 & $0[2]$ \\
\hline \multicolumn{7}{|l|}{ Anions, unfiltered (mg/L) } \\
\hline Nitrate & $1 / 4$ & 2.0 & $<1.0$ & -1.3 & 10 & $0\{2\}$ \\
\hline Sulfate, as $\mathrm{SO}_{4}$ & $4 / 4$ & 62 & 15 & 36 & 250 & $0[3]$ \\
\hline \multicolumn{7}{|l|}{ Field measurements, unfiltered } \\
\hline Conductivity $(\mathrm{ms} / \mathrm{cm})$ & $4 / 4$ & 0.74 & 0.46 & 0.63 & a & [d] \\
\hline Dissolved oxygen (mg/L) & $4 / 4$ & 12 & 12 & 12 & a & [d] \\
\hline Redox (mV) & $4 / 4$ & 340 & 260 & 290 & d & [d] \\
\hline Temperature $\left({ }^{\circ} \mathrm{C}\right)$ & $4 / 4$ & 16 & 14 & 15 & 30.5 & $0[1]$ \\
\hline Turbiaity (JTU) & $4 / 4$ & 360 & 13 & 140 & 1 & $4[2]$ \\
\hline $\mathrm{pHi}$ (SU) & $4 / 4$ & 7.5 & 6.7 & 7.2 & $(6.5,8.5)$ & $0[3]$ \\
\hline \multicolumn{7}{|l|}{ Metais, unfiltered (mg/L) } \\
\hline Aluminum, total & $1 / 4$ & 0.50 & $<0.050$ & -0.16 & 0.2 & $1[3]$ \\
\hline Barium, total & $4 / 4$ & 0.11 & 0.034 & 0.076 & 2 & $0[2]$ \\
\hline Baron, total & $2 / 4$ & 0.16 & $<0.080$ & -0.099 & $d$ & [d] \\
\hline Calcium, total & $4 / 4$ & 160 & 46 & 100 & ¿ & [a] \\
\hline Iron, total & $3 / 4$ & 0.61 & $<0.050$ & -0.19 & 0.3 & $I[3]$ \\
\hline Magnesium, tatal & $4 / 4$ & 39 & 5.6 & 23 & d & {$[a]$} \\
\hline Manganese, total & $3 / 4$ & 0.017 & $<0.0010$ & -0.0065 & 0.05 & $0[3]$ \\
\hline Mercury, total & $2 / 4$ & 0.000067 & $<0.000050$ & -0.000059 & 0.002 & $0[1]$ \\
\hline Potassium, total & $3 / 4$ & 3.3 & $<1.0$ & -2.2 & a & [a] \\
\hline silicon, total & $4 / 4$ & 8.0 & 3.9 & 6.1 & a & [d] \\
\hline Sodium, Eotal & $4 / 4$ & 7.3 & 4.6 & 6.2 & $\nsubseteq$ & [d] \\
\hline Vanadium, total & $1 / 4$ & 0.0024 & $<0.0020$ & -0.0021 & $a$ & [d] \\
\hline zinc, total & $1 / 4$ & 0.0054 & $<0.0050$ & -0.0051 & 5 & $013 !$ \\
\hline \multicolumn{7}{|l|}{ Others, unfiltered } \\
\hline Alkalinity (mg/L) & $4 / 4$ & 390 & 260 & 330 & c! & [a] \\
\hline Total organic carbon (mg/L) & $4 / 4$ & 1.3 & 0.63 & 0.90 & d & [a] \\
\hline Total suspended solids (mg/L) & $1 / 4$ & 68 & $<5.0$ & -21 & $d$ & [a] \\
\hline \multicolumn{7}{|l|}{ Radionuclides, filtered (pci/L) } \\
\hline $\mathrm{Co}-60$ & $1 / 4$ & $5.4 *$ & -1.6 & 1.0 & 200 & $0[4]$ \\
\hline Cs -137 & $1 / 4$ & $5.4 *$ & -1.4 & 1.8 & 120 & $0[4]$ \\
\hline Gross alpha & $2 / 4$ & $3.8^{*}$ & 0.32 & $1.8 *$ & 25 & $0[2]$ \\
\hline Gross beta & $2 / 4$ & $7.6^{*}$ & 2.2 & $4.9 *$ & 50 & $0[2]$ \\
\hline H -3 & $4 / 4$ & $14.000^{*}$ & $3,200^{*}$ & $7,200 *$ & 20,000 & $0[2]$ \\
\hline \multicolumn{7}{|l|}{ Radionuclides, unfiltered $(\mathrm{pCi} / \mathrm{L})^{\bullet}$} \\
\hline co- 60 & $1 / 4$ & $4.9 *$ & -5.7 & -0.068 & 200 & $0[4]$ \\
\hline Gross beta & $3 / 4$ & $16 *$ & -0.57 & 9.4 & 50 & $0[2]$ \\
\hline $\mathrm{H}-3$ & $4 / 4$ & $23,000^{*}$ & $3,200^{*}$ & $7,200 *$ & 20,000 & $0[2]$ \\
\hline Total rad Sr & $1 / 4$ & $2.5 *$ & -0.81 & 0.73 & 8 & $0[2]$ \\
\hline
\end{tabular}


Table 7.68 (continued)

\begin{tabular}{|c|c|c|c|c|c|c|}
\hline Parameter & $\begin{array}{l}\mathbf{N} \text { det } / \\
\mathrm{N} \text { total }\end{array}$ & Max & Min" & $A v^{b}$ & $\begin{array}{l}\text { Reference } \\
\text { value }\end{array}$ & $\begin{array}{l}\text { Number of values } \\
\text { exceeding } \\
\text { reference [ref]c }\end{array}$ \\
\hline $\begin{array}{l}\text { Volatile organics, w } \\
\text { Carbon disulfide }\end{array}$ & $\begin{array}{r}(\mu g / L) \\
1 / 4\end{array}$ & U5. 0 & $\mathrm{JI.3}$ & -4.1 & d & [d] \\
\hline
\end{tabular}

"Prefix "<" indicates the value for a parameter (excluding organics) was not quantifiable at the analytical detection limit: " $J$ " indicates the value was estimated at or below the analytical detection limit by the laboratory; " $Y$ " indicates the value exceeded the calibration range and the sample was diluted and reanalyzed; and ' $U$ ' indicates the value for an organic parameter was undetected at the analytical detection limit.

'A tilde ( ) indicates that estimated and/or undetected values were used in the calculation.

eIf a reference limit exists, the source is coded as:

1 Ruies of Tennessee Department of Environment and Conservation, Division of Water Pollution Control. Chapter 1200-4-3, General water Quality Criteria, Domestic Water Supply, as amended.

240 CFR Part 141--National Primary Drinking water Regulations, Subparts $B$ and $G$, as amended.

340 CFR Part 143--National Secondary Drinking water Regulations, as amended.

4 DOE Order 5400.5. Chapter III, Derived Concentration Guides for Air and water.

Not applicable.

by an *.

-Individual and average radionuclide concentrations significantly greater than zero are identified 


\begin{tabular}{|c|c|c|c|c|c|}
\hline Parameter & $\begin{array}{l}N \text { det } / \\
N \text { toral }\end{array}$ & $\operatorname{Max}$ & $\operatorname{Min}^{*}$ & $A V^{b}$ & $\begin{array}{l}\text { Number of values } \\
\text { Reference exceeding } \\
\text { value reference [ref]c }\end{array}$ \\
\hline
\end{tabular}

Melton Valley Exit Pathway

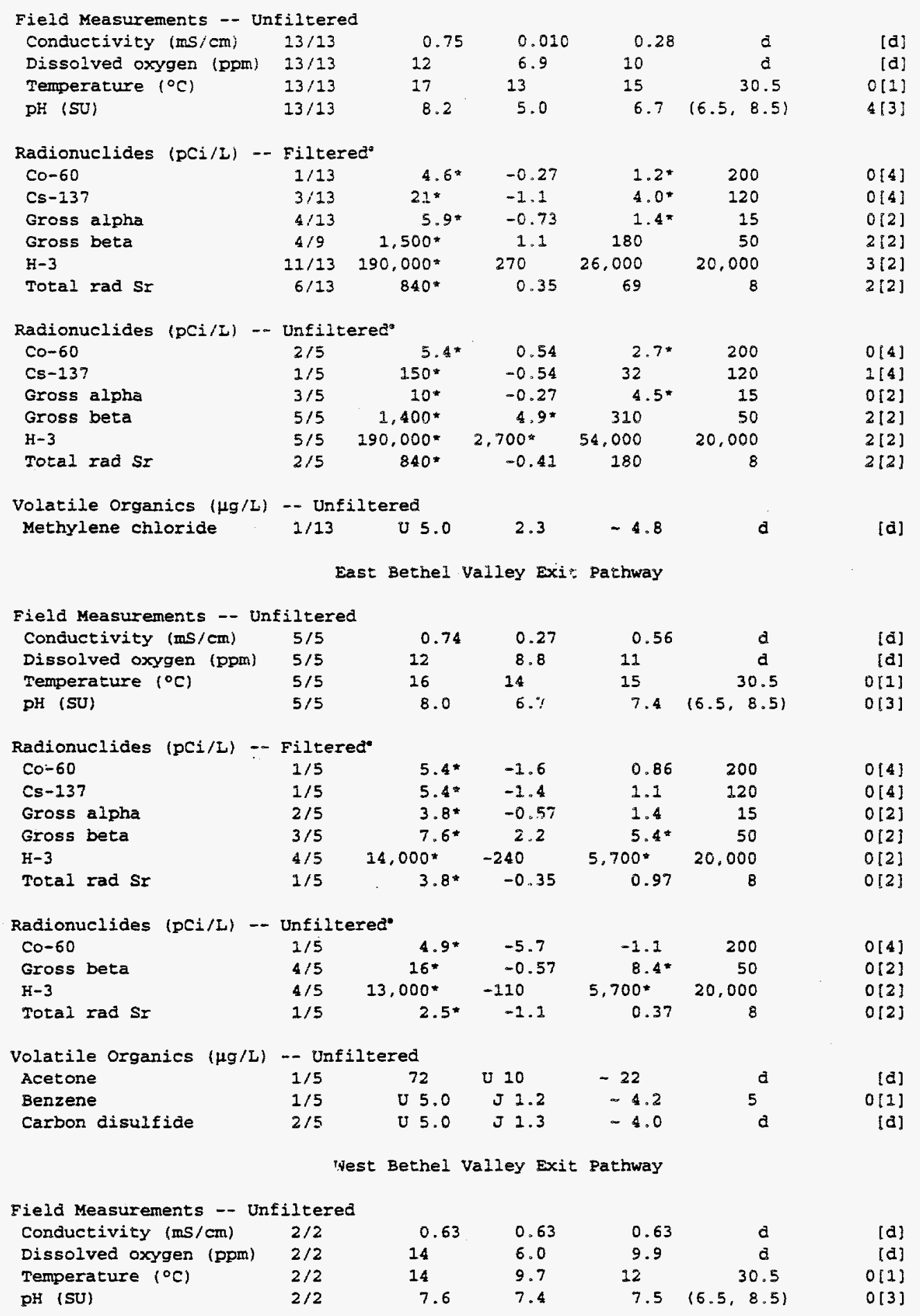


Table 7.69 (continued)

\begin{tabular}{|c|c|c|c|c|c|c|}
\hline Parameter & $\begin{array}{l}\text { N det/ } \\
\text { N total }\end{array}$ & $\operatorname{Max}$ & $\operatorname{Min}^{*}$ & $A v^{b}$ & $\begin{array}{l}\text { Reference } \\
\text { value }\end{array}$ & $\begin{array}{l}\text { Number of values } \\
\text { exceeding } \\
\text { reference }[r e f]^{c}\end{array}$ \\
\hline \multicolumn{7}{|c|}{ Radionuclides (DCi/L) -- Filcered" } \\
\hline $00-60$ & $1 / 2$ & $3.8^{*}$ & -0.27 & 1.8 & 200 & $O[4]$ \\
\hline $\mathrm{Cs}-137$ & $1 / 2$ & $3.8 *$ & 0.81 & 2.3 & 120 & $0[4]$ \\
\hline Gross beta & $2 / 2$ & $260 *$ & $3.2 *$ & 130 & 50 & $I[2]$ \\
\hline $\mathrm{H}-3$ & $2 / 2$ & $1,000^{*}$ & $920^{*}$ & 970* & 20,000 & $0[2]$ \\
\hline Total rad ST & $1 / 2$ & $120^{\star}$ & 5.1 & 61 & 8 & $1\{2\rfloor$ \\
\hline \multicolumn{7}{|c|}{ Radionuclides $(\mathrm{pCi} / L)$-- Unfiltered• } \\
\hline Gross alpha & $1 / 2$ & $1.6 *$ & 1.5 & $1.6 *$ & 15 & $0[2]$ \\
\hline Gross beta & $2 / 2$ & $260 *$ & $11 *$ & 140 & 50 & $1[2]$ \\
\hline $\mathrm{H}-3$ & $2 / 2$ & $1,400 *$ & $810^{*}$ & 1,100 & 20,000 & $0[2]$ \\
\hline \multirow[t]{2}{*}{ Total rad Sr } & $1 / 2$ & $130 *$ & 4.6 & 66 & 8 & $1[2]$ \\
\hline & \multicolumn{5}{|c|}{ White wing Scrapyard Exit Pathway } & \\
\hline \multicolumn{7}{|c|}{ Field Measurements -- Unfiltered } \\
\hline Conductivity (ms/cm) & $4 / 4$ & 0.45 & 0.040 & 0.21 & a & [d] \\
\hline Dissolved oxygen (ppm) & $4 / 4$ & 12 & 6.1 & 9.1 & d & [d] \\
\hline Temperature $\left({ }^{\circ} \mathrm{C}\right)$ & $4 / 4$ & 13 & 10 & 12 & 30.5 & $0[1]$ \\
\hline $\mathrm{pH}$ (SU) & $4 / 4$ & 8.2 & 6.9 & 7.6 & $(6.5,8.5)$ & $0[3]$ \\
\hline \multicolumn{7}{|c|}{ Radionuclides ( $\mathrm{pC} i / L)$-- Filtered" } \\
\hline Gross alpha & $3 / 4$ & $15^{*}$ & -0.38 & 4.6 & 15 & $1[2]$ \\
\hline Gross beta & $4 / 4$ & 21* & $8.4 *$ & 14* & 50 & $0[2]$ \\
\hline $\mathrm{H}-3$ & $2 / 4$ & $1,400=$ & -220 & 520 & 20,000 & $0\{2\}$ \\
\hline Total rad SI & $4 / 4$ & $7.0 *$ & $2.7 *$ & $4.6 *$ & 8 & $0[2]$ \\
\hline \multicolumn{7}{|c|}{ Radionuclides $(\mathrm{pCi} / L)$-- Unfiltered* } \\
\hline $\operatorname{co-} 60$ & $1 / 4$ & 3.2 & 0.27 & $2.2 *$ & 200 & $0[4]$ \\
\hline Cs -137 & $1 / 4$ & $2.2 *$ & -3.2 & 0.49 & 120 & $0[4]$ \\
\hline Gross alpha & $2 / 4$ & 17* & 0.27 & 5.1 & 15 & $1[2]$ \\
\hline Gross beta & $4 / 4$ & $27 *$ & $4.3 *$ & $16 *$ & 50 & $0[2]$ \\
\hline Total rad Sr & $2 / 4$ & $7.0^{*}$ & 0.054 & 2.5 & 8 & $0\{2\}$ \\
\hline \multicolumn{7}{|c|}{ Volatile Organics $(H g / L)$-- Unfiltered } \\
\hline Trichloroethene & $1 / 4$ & 17 & U 5.0 & -8.0 & 5 & $1[1]$ \\
\hline
\end{tabular}

'Prefix " $\mathrm{J}$ ' indicates the value was estimated at or below the analytical detection limit by the laboracory; and " $U$ " indicates the value for an ozganic parameter was underected at the analytical detection limit. calculation.

b tilde $(-)$ indicates that estimated and/or undetected values were used in the

'If a reference limit exists, the source is coded as:

1 Rules of Tennessee Department of Environment and Conservation, Division of Water Pollution Control, Chapter 1200-4-3, General Water Quality Criceria, Domestic Water Supply, as amended.

240 CFR Part 141--National Primary Drinking water Regulations, Subparts $B$ and G, as amended.

340 CFR Part 143--National Secondary Drinking water Regulations, as anended.

4 DOE Order 5400.5, Chapter III, Derived Concentration Guides for Air and Water.

Not applicable.

-Individual and average radionuclide concentrations significantly greater than zero are identified by an * 
Table 7.70. Constituents in the groundwater welis located at the K-1407 Operable Unit at the K-25 Site, 1993

\begin{tabular}{|c|c|c|c|c|c|c|}
\hline \multirow{2}{*}{ Anałyte } & \multirow{2}{*}{$\begin{array}{l}\text { Number } \\
\text { detected/ } \\
\text { number of } \\
\text { results }\end{array}$} & \multicolumn{3}{|c|}{ Detected results } & \multirow{2}{*}{$\begin{array}{l}\text { Reference } \\
\text { Value }^{s}\end{array}$} & \multirow{2}{*}{$\begin{array}{l}\text { Number } \\
\text { erceoding } \\
\text { reference } \\
\text { value }\end{array}$} \\
\hline & & Minimum ${ }^{3}$ & Average & Maximum $^{3}$ & & \\
\hline
\end{tabular}

Field mearurements

Conductivity ( $\mu \mathrm{mho} / \mathrm{cm})$

Conductivity - initial (umho/em)

Dissolved arygen (ppm)

Dissolved axygen - initial (ppm)

Redox (mv)

Redox - initial (mv)

Temperature (degrees $C$ )

Temperature - initial (degrees $C$ ) $\mathrm{pH}$ (std units)

$\mathrm{pH}$ - initial (std units)

\begin{tabular}{c|ccc}
$n$ & 32 & 128 & 876.53 \\
$n$ & 32 & 66 & 829.87 \\
$n / 32$ & 1.1 & 4.9 \\
$n / 32$ & 0.4 & 24906 \\
$n / 32$ & -95 & 74.281 \\
$n / 32$ & -120 & 64.688 \\
$n / 32$ & 10.4 & 15.728 \\
$n / 32$ & 8.9 & 16.097 \\
$n$ & 32 & 6 & 6.7406 \\
$n / 32$ & 5.3 & 6.5562
\end{tabular}

Discolved metais by ICP (moll)
Aluminum

Antimony

Barium

Beryllium

Cadmium

Calcium

Crromium

Cobalt

Copper

Iron

Lithium

Magnesium

Manganese

Molybdenum

Nickel

Niobium

Potassium

Silver

Sodium

Strontium

Thorium

Titanium

Vanadium

Zinc

Zirconium

Ajuminum

Antimony

Barium

Beryllium

Cadmium
$19 / 26$

$0 / 26$

$26 / 26$

$0 / 27$

$2 / 28$

$26 / 26$

$4 / 26$

$9 / 26$

$6 / 26$

$24 / 26$

$1 / 26$

$26 / 26$

$26 / 26$

$0 / 26$

$1 / 26$

$2 / 26$

$26 / 26$

$1 / 26$

$26 / 26$

$26 / 26$

$0 / 26$

$1 / 26$

$0 / 26$

$17 / 26$

$0 / 26$

0.02

0.018

0.0042

19

0.015

0.0056

0.0045

0.008

0.0056

3.3

0.001

0.01

0.0079

1.2

0.0068

1.5

0.032

0.0046

c 0.0026

Tocal' metals by ICP (mg/L)

$26 / 26$

$0 / 26$

$26 / 26$

$8 / 27$

$7 / 28$
0.03

0.017

0.0004

0.0035
0.0463

0.1328

0.0046

114

0.0333

0.0268

0.0066

4.5051

0.0056

17.023

8.2789

0.01

0.008

2.6038

0.0068

43.135

0.2358

0.0046

0.0092
1875

1834

8.2

250

229

24.6

22

7.6

7.4

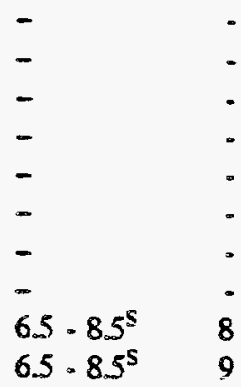

$\begin{array}{ll}6.5 \cdot 85^{5} & 8 \\ 6.5 \cdot 85^{5} & 9\end{array}$

Cadmium

\section{(}


Table 7.70 (continued)

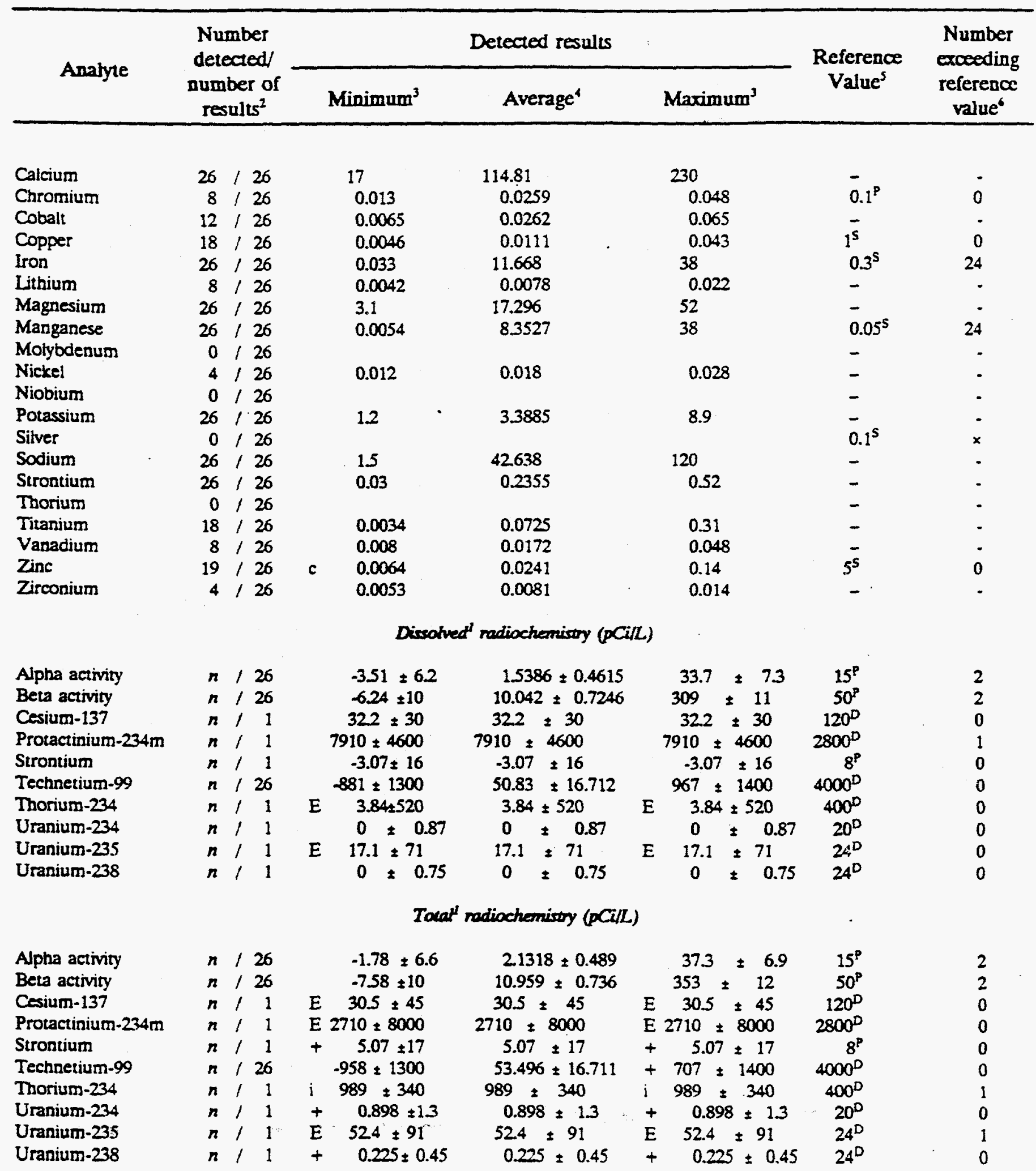


Table 7.70 (continued)

\begin{tabular}{|c|c|c|c|c|c|c|}
\hline \multirow{2}{*}{ Analyte } & \multirow{2}{*}{$\begin{array}{l}\text { Number } \\
\text { detected } \\
\text { number of } \\
\text { results }\end{array}$} & \multicolumn{3}{|c|}{ Detected results } & \multirow{2}{*}{$\begin{array}{c}\text { Reference } \\
\text { Value }^{5}\end{array}$} & \multirow{2}{*}{$\begin{array}{l}\text { Number } \\
\text { exceeding } \\
\text { reference } \\
\text { value }^{6}\end{array}$} \\
\hline & & Minimum $^{3}$ & Average & Maximum ${ }^{3}$ & & \\
\hline
\end{tabular}

1,2,4-Trichlorobenzene

1,2-Dichlorobenzene

1,2-Diphenylhydrazine

1,3-Dichlorobenzene

1,4-Dichlorobenzene

2,3,4,6-Tetrachlorophenol

2,4,5-Trichlorophenol

2,4,6-Trichlorophenol

2,4-Dichlorophenol

2,4-Dimethyiphenol

2,4-Dinitrophenol

2,4-Dinitrotoluene

2,6-Dinitrotoluene

2-Chioronaphthalene

2-Chlorophenol

2-Methylnaphthalene

2-Methylphenol

2-Nitroaniline

2-Nitrophenol

3,3'-Dichlorobenzidine

3-Nitroaniline

4,6-Dinitro-2-methylphenol

4-Bromophenyl-phenylether

4-Chloro-3-methylphenol

4-Chloroaniline

4-Chlorophenyl-phenylether

4-Methylphenol

4-Nitroaniline

4-Nitrophenol

Acenaphthene

Acenaphthylene

Aniline

Anthracene

Benzidine

Benzo(a)anthracene

Benzo(a)pyrene

Benzo(b)fluoranthene

Benzo(g,h,i)perylene

Benzo(k)fluoranthene

Benzoic acid

Benzyl alcohol

Butylbenzylphthalate

Chrysene

Di-n-butylphthalate

Di-n-octylphthalate

Dibenz(a,b)anturacene

Dibenzofuran

Semivalatile arguries (mglL)

$0 / 26$

$0 / 26$

$0 / 26$

$0 / 26$

$0 / 26$

$0 / 26$

$0 / 26$

$0 / 26$

$0 / 26$

$0 / 26$

$0 / 26$

$0 / 26$

$0 / 26$

$0 / 26$

$0 / 26$

$0 / 26$

$0 / 26$

$0 / 26$

$0 / 26$

$0 / 26$

$0 / 26$

$0 / 26$

$0 / 26$

$0 / 26$

$0 / 26$

$0 / 26$

$0 / 26$

$0 / 26$

$0 / 26$

$0 / 26$

$0 / 26$

$0 / 26$

$0 / 26$

$0 / 26$

$0 / 26$

$0 / 26$

$0 / 26$

$0 / 26$

$0 / 26$

$0 / 26$

$0 / 26$

$0 / 26$

$0 / 26$

$8 / 26$

$0 / 26$

$0 / 26$

$0 / 26$
J 0.2

1.475

JB 3

\begin{tabular}{|c|c|}
\hline $600^{\bar{p}}$ & \\
\hline- & \\
\hline $5^{\bar{s}}$ & \\
\hline - & \\
\hline - & \\
\hline - & \\
\hline - & \\
\hline - & \\
\hline- & \\
\hline - & \\
\hline - & \\
\hline - & \\
\hline 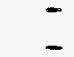 & \\
\hline - & \\
\hline- & \\
\hline$\overline{-}$ & \\
\hline - & \\
\hline - & \\
\hline - & \\
\hline - & \\
\hline - & \\
\hline - & \\
\hline - & \\
\hline- & \\
\hline- & \\
\hline- & \\
\hline - & \\
\hline- & \\
\hline - & \\
\hline- & \\
\hline- & \\
\hline - & \\
\hline I & \\
\hline - & \\
\hline - & \\
\hline$\overline{-}$ & \\
\hline
\end{tabular}


Table 7.70 (continued)

\begin{tabular}{|c|c|c|c|c|c|c|c|c|}
\hline \multirow{2}{*}{ Anatyte } & \multirow{2}{*}{$\begin{array}{l}\text { Number } \\
\text { detectedy } \\
\text { number of } \\
\text { results }\end{array}$} & \multicolumn{5}{|c|}{ Detected results } & \multirow{2}{*}{$\begin{array}{c}\text { Reference } \\
\text { Value }^{5}\end{array}$} & \multirow{2}{*}{$\begin{array}{c}\text { Number } \\
\text { erceeding } \\
\text { reference } \\
\text { value }\end{array}$} \\
\hline & & \multicolumn{2}{|c|}{ Minimum $^{3}$} & \multirow{2}{*}{$\frac{\text { Average }^{4}}{0.9857}$} & \multicolumn{2}{|c|}{ Maximum $^{3}$} & & \\
\hline Dietmylphthalate & $7 / 26$ & JB & 0.5 & & JB & 2 & - & - \\
\hline Dimethylphthaiate & $0 / 26$ & & & & & & - & - \\
\hline Fuoranthene & $0 / 26$ & & & & & & - & $\cdot$ \\
\hline Fluorene & $0 / 26$ & & & & & & - & - \\
\hline Hexachlorobenzene & $0 / 26$ & & & & & & - & - \\
\hline Hexachlorobutadiene & $0 / 26$ & & & . & & & - & - \\
\hline Hecachlorocyclopentadiene & $0 / 26$ & & & & & & - & - \\
\hline Hexachloroethane & $0 / 26$ & & & & & & - & - \\
\hline Indeno $(1,2,3-c d)$ pyrene & $0 / 26$ & & & & & & - & - \\
\hline Isophorone & $0 / 26$ & & & & & & - & - \\
\hline N-Nitroso-di-n-propylamine & $0 / 26$ & & & & & & - & $\cdot$ \\
\hline N-Nitrosodimethylamine & $0 / 26$ & & & & & & - & - \\
\hline N-Nitrosodiphenylamine & $0 / 26$ & & & & & & - & - \\
\hline Naphthalene & $0 / 26$ & $\because$ & & & & & - & - \\
\hline Nitrobenzene & $0 / 26$ & & & & & & - & - \\
\hline Peniachlorophenol & $1 / 26$ & $\mathrm{~J}$ & 1 & 1 & $\mathrm{~J}$ & 1 & $1^{P}$ & 0 \\
\hline Phenanthrene & $0 / 26$ & & & & & & - & - \\
\hline Phenol & $0 / 26$ & & & & & & - & - \\
\hline Pyrene & $0 / 26$ & & & & & & - & - \\
\hline Pyridine & $0 / 26$ & & & & & & - & - \\
\hline bis(2-Chloroethoxy)methane & $0 / 26$ & & & & & & - & - \\
\hline bis(2-Chjoroethyl)ether & $0 / 26$ & & . & & & & - & - \\
\hline bis(2-Chloroisopropyl)ether & $0 / 26$ & & & & & & - & - \\
\hline bis(2-Ethylhexyl)phthalate & $6 / 26$ & JB & 0.9 & 1.4833 & JB & 2 & - & $\cdot$ \\
\hline
\end{tabular}

1,4-Dicuane

1-Propanol,2-11-methylethox

2-Pentanol,4-methyl-

2-Pentanone, 4-hydroxy-4-methy

3-Penten-2-one, 4-methyl-

4-Octanol,4-methyl-

Cyclohexene

Sulfur (S8)

Tetrachloroethene

Toluene

Tributyl phosphate

Trichloroethene

Unknown

Unknown alcohol

Unknown alcohol/alkoxy compound

Semivalarile argarics, tentotively iderrified compounds (ng/L)

$\begin{array}{rlrlrclr}3 / & 3 & \text { J } & 8 & 22.667 & \text { J } & 50 \\ 1 / & 1 & \text { J } & 27 & 27 & \text { J } & 27 \\ 1 / & 1 & \text { J } & 21 & 21 & \text { J } & 21 \\ 12 / & 12 & \text { JAB } & 14 & 122.92 & \text { JAB } & 680 \\ 1 / & 1 & \text { A } & 28 & 28 & \text { A } & 28 \\ 1 / & 1 & \text { J } & 28 & 28 & \text { J } & 28 \\ 7 / & 7 & \text { JB } & 17 & 23.857 & \text { JB } & 33 \\ 2 / & 2 & \text { J } & 15 & 20.5 & \text { J } & 26 \\ 2 / & 2 & \text { J } & 63 & 131.5 & \text { J } & 200 \\ 1 / & 1 & \text { J } & 9 & 9 & \text { J } & 9 \\ 1 / & 1 & \text { J } & 10 & 10 & \text { J } & 10 \\ 3 / & 3 & \text { J } & 8 & 186 & \text { J } & 470 \\ 39 / & 39 & \text { J } & 9 & 28.205 & \text { J } & 100 \\ 8 & 8 & \text { J } & 10 & 25.625 & \text { J } & 51 \\ 9 & 9 & \text { J } & 8 & 29.333 & \text { J } & 51\end{array}$

Dirsolved" metals by spectochemisty (mg/L)

\begin{tabular}{|c|c|c|c|c|c|}
\hline Arsenic & $5 / 26$ & 0.005 & 0.0068 & 0.0084 & $0.05^{p}$ \\
\hline Lead & $0 / 27$ & & & & $0.015^{\mathrm{P}}$ \\
\hline Mercury & $1 / 26$ & 0.0002 & 0.0002 & 0.0002 & $0.002^{P}$ \\
\hline Selenium & $0 / 26$ & & & & $0.05^{p}$ \\
\hline Thallium & $0 / 26$ & & & & - \\
\hline
\end{tabular}


Table 7.70 (continued)

\begin{tabular}{|c|c|c|c|c|c|c|}
\hline \multirow{2}{*}{ Analyte } & \multirow{2}{*}{$\begin{array}{l}\text { Number } \\
\text { detected/ } \\
\text { number of } \\
\text { results }\end{array}$} & \multicolumn{3}{|c|}{ Detected results } & \multirow{2}{*}{$\begin{array}{c}\text { Reference } \\
\text { Value }^{s}\end{array}$} & \multirow{2}{*}{$\begin{array}{l}\text { Number } \\
\text { exceeding } \\
\text { reference } \\
\text { value }\end{array}$} \\
\hline & & Minimum $^{3}$ & Average & Marimum ${ }^{3}$ & & \\
\hline
\end{tabular}

$\begin{array}{lr:cccc}\text { Arsenic } & 10 & 26 & 0.0062 & 0.0101 & 0.016 \\ \text { Lead } & 8 / 27 & 0.0048 & 0.0191 & 0.081 \\ \text { Mercury } & 0 / 26 & & & \\ \text { Selenium } & 0 / 26 & & & \\ \text { Thallium } & 0 / 26 & & & \end{array}$

Volatiie arguris (kg/L)

1,1,1-Trichloroethane 1,1,2,2-Tetrachloroethane 1,1,2-Trichloroethane

1,1-Dichloroetbane

1,1-Dichloroethene

1,2-Dichloroethane

1,2-Dichloroethene (total)

1,2-Dichloropropane

2-Butanone

2-Hexanone

4-Methyl-2-pentanone

Acetone

Benzene

Bromodichloromethane

Bromoform

Bromomethane

Carbon disulfide

Carbon tetrachloride

Chlorobenzene

Chloroethane

Chloroform

Chloromethane

Dibromochloromethane

Ethylbenzene

Methylene chloride

Styrene

Tetrachloroethene

Toluene

Trichloroethene

Vinyl acetate

Vinyl chloride

Xylene (total)

cis-1,3-Dichloropropene

trans-1,3-Dichloropropene

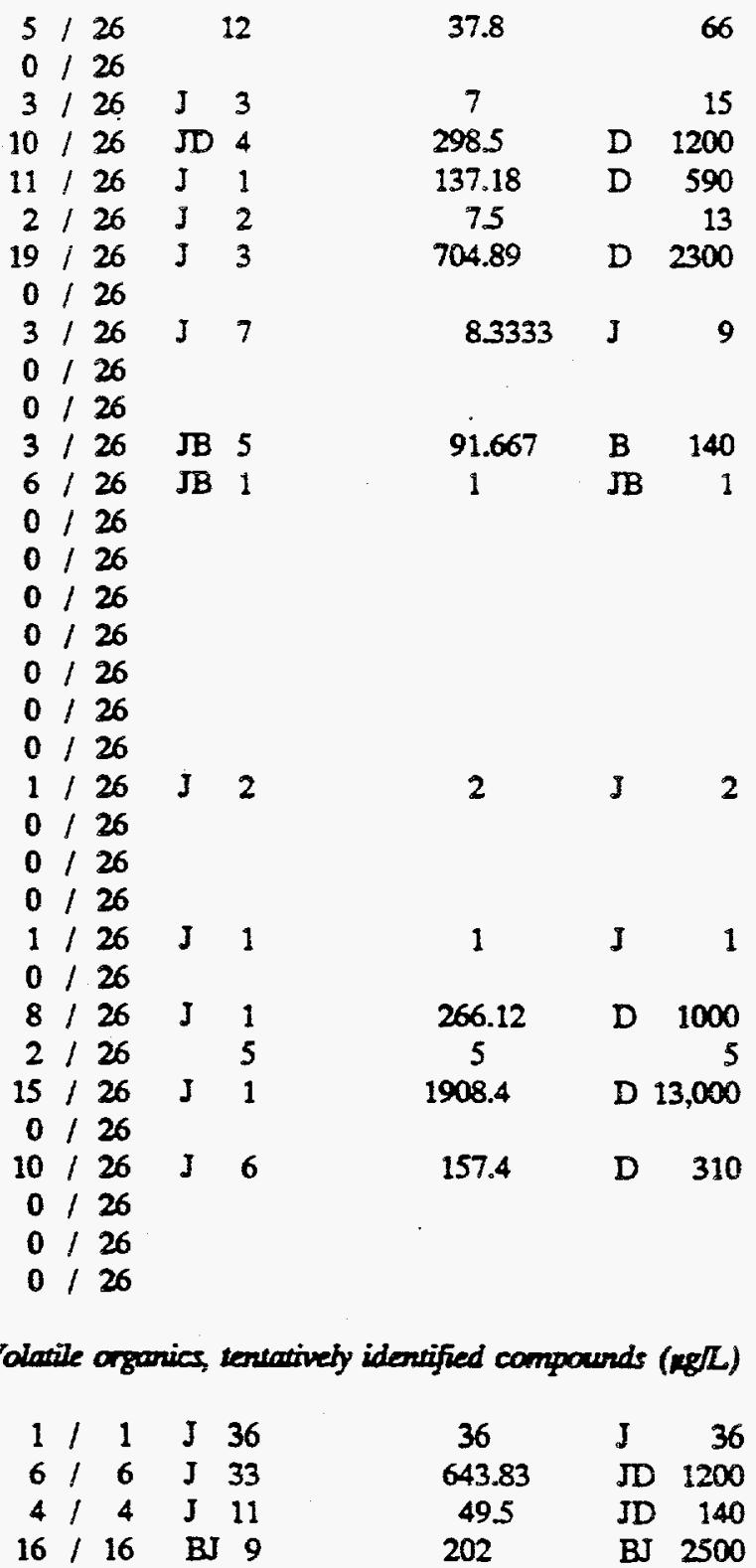

Chlorotrifluoroethene

Freon 113

Freon 123

Hexane
Tolal metais by spectochemidty (mg/)

.016

$0.05^{\mathrm{P}}$

$0.015^{\mathrm{P}}$

$0.002^{P}$

$0.05^{\mathrm{P}}$

$-$

value 6 
Table 7.70 (continued)

\begin{tabular}{|c|c|c|c|c|c|c|}
\hline \multirow{2}{*}{ Analyte } & \multirow{2}{*}{$\begin{array}{l}\text { Number } \\
\text { detected/ } \\
\text { number of } \\
\text { results }\end{array}$} & \multicolumn{3}{|c|}{ Detected results } & \multirow{2}{*}{$\begin{array}{l}\text { Reference } \\
\text { Value }^{5}\end{array}$} & \multirow{2}{*}{$\begin{array}{l}\text { Number } \\
\text { croeeding } \\
\text { reference } \\
\text { value" }\end{array}$} \\
\hline & & Minimum & Average & Maximum $^{3}$ & & \\
\hline Uniknown & $2 / 2$ & J 5 & 97.5 & 190 & - & - \\
\hline \multicolumn{7}{|c|}{ Discolved' wet chersisty $(\mathrm{mg} / \mathrm{L})$} \\
\hline Uranium fluorometric & $7 / 26$ & 0.001 & 0.0014 & 0.003 & $0.02^{\mathrm{P7}}$ & 0 \\
\hline \multicolumn{7}{|c|}{ Total' wet chemistry } \\
\hline Chloride $(\mathrm{mg} / \mathrm{L})$ & $26 / 26$ & 2 & 13274 & 478 & $250^{s}$ & 9 \\
\hline Conductivity ( $\mu \mathrm{mho} / \mathrm{cm})$ & $n / 104$ & 147 & 953.95 & 2050 & - & - \\
\hline Dissolved solids (mg/) & $26 / 26$ & 70 & 591.85 & 1224 & $500^{5}$ & 17 \\
\hline Fuoride $(\mathrm{mg} / \mathrm{L})$ & $6 / 26$ & 0.1 & 0.1333 & 0.2 & $2^{5}$ & 0 \\
\hline Nitrate brucine (mg/L) & $14 ! 26$ & 0.11 & 0.3471 & 1.5 & $10^{p}$ & 0 \\
\hline $\begin{array}{l}\text { fnetois (mg/L) } \\
\text { Sulfate }(\mathrm{mg} / \mathrm{L})\end{array}$ & $26 / 26$ & 3 & 76.038 & 390 & $250^{\bar{s}}$ & $\dot{2}$ \\
\hline Suspended solids (mg/L) & $26 / 26$ & 2 & 60.423 & 680 & - & - \\
\hline Total organic carbon (mg/L) & $31 / 103$ & 1.7 & 6.6839 & 23 & - & - \\
\hline Total organic halides $(\mu g / L)$ & $67 / 111$ & 11 & 22528 & 9484 & - & - \\
\hline Turbidiry (NTU) & $26 / 26$ & 1 & 46.112 & 240 & $1^{\mathrm{P}}$ & 25 \\
\hline Uranium fluorometric $(\mathrm{mg} / \mathrm{L})$ & $9 / 26$ & 0.001 & 0.0014 & 0.003 & $0.02^{P}$ & 0 \\
\hline pH (std units) & $n / 108$ & 6 & 6.8926 & 7.6 & $6.5 \cdot 8.5^{5}$ & 11 \\
\hline
\end{tabular}

Tocal $=$ unfiltered sample (soluable + suspended) and Disoolved $=$ Eitered sample (soluable only). ICP = inductively coupled plasma

2 Both the number of detected results and the total number of results include all duplicate and replicate measurements. No blanks, matrix spikes, equipment rinsate or other QANOC data are reported in this table. $n$ denotes not applicable.

The minimum and maximum detected results are listed with their laboratory analytical qualifiers.

A denotes aldol condensation product

B denotes parameter found in blank as well as sample

C denotes possible contamination

D denotes the compound identified at a secondary dilution factor

E denotes for radiochemistry parameters the result is less than the minimum detectable activity (MDA), confidence level is less than

95\%. Also denotes for organics the measurement exceded the instrument calibration range

i denotes a tentatively identified isotope from the radiochemistry laboratory

J denotes an estimated value (usualty below the detection limit)

+ denotes the duplicate control limits do not apply, duplicate and sample near the MDA

4 The average raciochemistry results and their associated limits of error were calculated from all of the results using optimally weighted mean and variance estimates assuming independent measurements with unequal errors, as documented in Radiorion Detection and Mearunement by Glenn F. Knoll, New York: John Wiley and Sons (1979), pp. 137-139. For the non-radiochemistry analytes the average listed is the unweighted arithmetic mean of all detected results. No analytical qualifiers are listed for the average.

If a reference value exists it originates from the following sources:

P 40 CFR Part 141 National Primary Drinking Water Regulations, Subpans B and G, as amended

S 40 CFR Part 143 National Secondary Drinking Water Regulations, as amended

D DOE Order 5400.5, Chapter III, Derived Concentration Guides (DCG) for Air and Water. Four percent of the DOE DCG represents the DOE eriterion of 4 mrem effective dose equivalent from ingestion of drinking water.

- denotes no reference value exists for this anatyle

6 The number of detected results exeeding the reference value is given

- denotes that since no reference value exists for this anatyte, the number exceeding is not applicable.

x denotes that no detected measurements were reported for this analyte so that no comparisons were made to the reference value.

7 Proposed drinking water standard. 
Table 7.71. Constituents in the Exit Pathway Wells at 'he K-25 Site, 1993

\begin{tabular}{|c|c|c|c|c|c|c|}
\hline \multirow{2}{*}{ Analyte } & \multirow{2}{*}{$\begin{array}{l}\text { Number } \\
\text { detected/ } \\
\text { number of } \\
\text { results }\end{array}$} & \multicolumn{3}{|c|}{ Detected results } & \multirow{2}{*}{$\begin{array}{l}\text { Reference } \\
\text { Value }^{5}\end{array}$} & \multirow{2}{*}{$\begin{array}{l}\text { Number } \\
\text { excoeding } \\
\text { reference } \\
\text { value }^{6}\end{array}$} \\
\hline & & Minimum ${ }^{3}$ & Average & Marimum $^{3}$ & & \\
\hline
\end{tabular}

Field meacorements

Conductivity ( $\mu \mathrm{mho} / \mathrm{cm}$ )

Conductivity - initial $(\mu \mathrm{mho} / \mathrm{cm})$

Dissolved axygen (ppm)

Dissolved coxygen - initial (ppm)

Redox (mv)

Redox - initial (mv)

Temperature (degrees $C$ )

Temperature - initial (degrees C)

$\mathrm{pH}$ (std units)

$\mathrm{pH}$ - initial (std units)

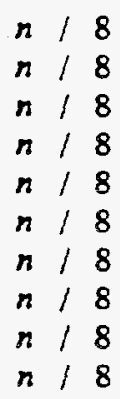

18

$n / 8$

$n / 8$

$n / 8$

$n / 8$

$n / 8$

$n / 8$

$n / 8$
176

195

0.4

0.4

$-153$

$-116$

10.3

11.7

6.3

6.3

411.87
422.62
2.25
1.3875
16.75
126.75
13.275
13.425
7.625
7.8875

Dissolved' nadiochemisty (PCi/L)

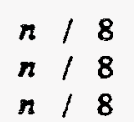

Alpha activity

Technetium-99

$$
\begin{array}{r}
-0.952 \pm 1.7 \\
1.79 \pm 2.9 \\
-1050 \pm 1300
\end{array}
$$

$0.8106 \pm 0.5255$
$5.9852 \pm 0.8637$
$-310.1 \pm 467.74$

656
708
4
26
212
229
16.2
15.4
9.2
9.7

$\begin{array}{ll}- & \\ = & \\ - & \\ - & \\ - & \\ - & \\ - & \\ 6.5 \cdot 8.5^{5} & 3 \\ 6.5 \cdot 8.5^{5} & 4\end{array}$

\section{Tocal' madiochemistry (PCin)}

Aipha activity

Beta activity

Technetium-99
$n / 8$
$-0.576 \pm 1.8$
$0.7077 \pm 0.5301$
$4.29 \pm 2.4$
$n / 8$
$0.0854 \pm 2.8$
$5.4102 \pm 0.8639+10.7 \div 3.2$
$n / 8+-1170 \pm 1300$
$.377 .1 \pm 467.74$
$536 \pm 1400$
$15^{\mathrm{P}}$
$50^{\mathrm{P}}$
$4000^{\mathrm{D}}$

$$
\begin{aligned}
& 0 \\
& 0 \\
& 0
\end{aligned}
$$

\section{Volatile argarias (mgll)}

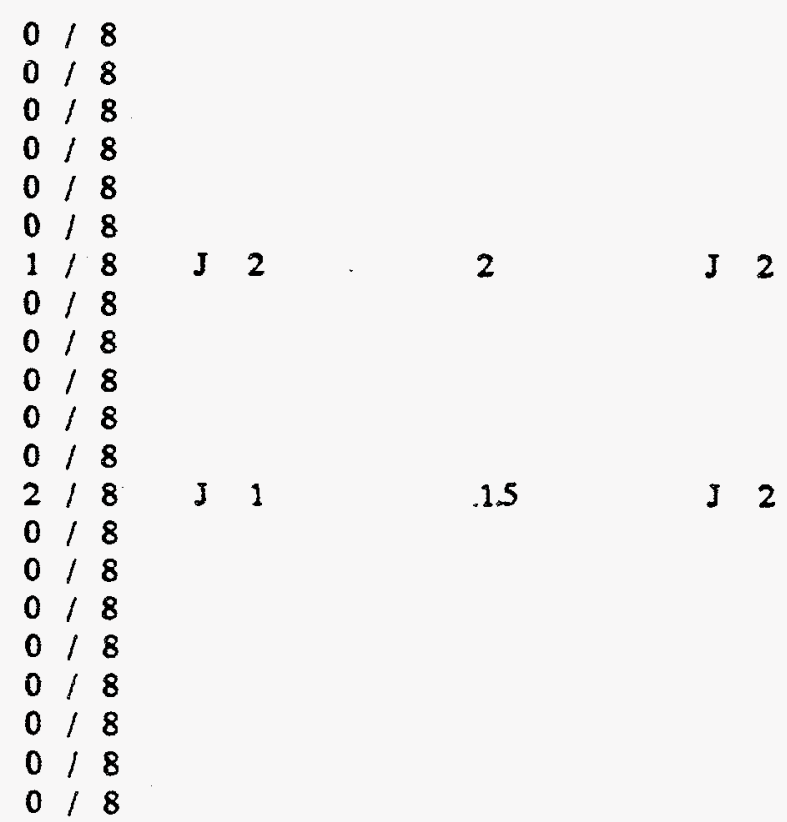

$0 / 8$

$0 / 8$

1,1,2,2-Tetrachioroethane

1,1,2-Trichloroethane

1,1-Dichloroethane

1,1-Dichloroethene

1,2-Dichloroethane

1,2-Dichloroethene (total)

1,2-Dichioropropane

2-Butanone

2-Hexanone

4-Methyl-2-pentanone

Acetone

Berzene

Bromodichloromethane

Bromoform

Bromomethane

Carbon disulfide

Carbon tetrachloride

Chlorobenzene

Chloroethane

Chloroform
$0 / 8$

$0 / 8$

$0 / 8$

$0 / 8$

$1 / 8$

$0 / 8$

$0 / 8$

$0 / 8$

$0 / 8$

$0 / 8$

\begin{tabular}{|c|c|}
\hline $200^{P}$ & $x$ \\
\hline- & . \\
\hline- & - \\
\hline$T^{P}$ & . \\
\hline $\begin{array}{l}7^{p} \\
5^{p}\end{array}$ & $x$ \\
\hline $70^{\mathrm{p}}$ & $\begin{array}{l}x \\
0\end{array}$ \\
\hline $5^{\mathrm{P}}$ & $x$ \\
\hline - & - \\
\hline - & - \\
\hline - & - \\
\hline $5^{\bar{P}}$ & $\dot{0}$ \\
\hline $100^{P}$ & $\begin{array}{l}0 \\
x\end{array}$ \\
\hline $100^{P}$ & $x$ \\
\hline - & 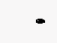 \\
\hline $5^{-\bar{p}}$ & $\mathbf{x}$ \\
\hline $100^{P}$ & $x$ \\
\hline $\begin{array}{l}200^{\mathrm{P}} \\
100^{\mathrm{P}}\end{array}$ & $x$ \\
\hline
\end{tabular}

$2 / 8$

$0 / 8$

$0 / 8$

$0 / 8$

$0 / 8$

$0 / 8$

$0 / 8$ 
Table 7.71 (continued)

\begin{tabular}{|c|c|c|c|c|c|c|}
\hline \multirow{2}{*}{ Anatyre } & \multirow{2}{*}{$\begin{array}{l}\text { Number } \\
\text { detected/ } \\
\text { number of } \\
\text { results }\end{array}$} & \multicolumn{3}{|c|}{ Detected results } & \multirow{2}{*}{$\begin{array}{c}\text { Reference } \\
\text { Value }^{5}\end{array}$} & \multirow{2}{*}{$\begin{array}{c}\text { Number } \\
\text { exceeding } \\
\text { reference } \\
\text { value }^{6}\end{array}$} \\
\hline & & Minimum $^{3}$ & Average $^{4}$ & Maximum $^{3}$ & & \\
\hline $\begin{array}{l}\text { Chloromethane } \\
\text { Dibromochloromethane } \\
\text { Ethylbenzene } \\
\text { Methylene chloride } \\
\text { Styrene } \\
\text { Tetrachloroethene } \\
\text { Toluene } \\
\text { Trichloroethene } \\
\text { Vinyl acetate } \\
\text { Vinyl chloride } \\
\text { Xylene (total) } \\
\text { cis-1,3-Dichloropropene } \\
\text { trans-1,3-Dichloropropene }\end{array}$ & 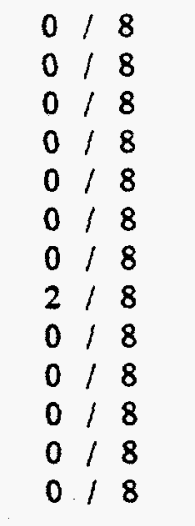 & 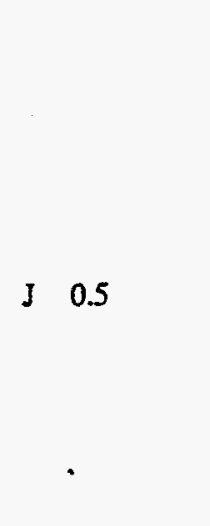 & 0.6 & J 0.7 & $\begin{array}{r}100^{\bar{P}} \\
700^{P} \\
\overline{-} \\
100^{\bar{P}} \\
5^{P} \\
1000^{P} \\
5^{P} \\
\overline{2^{P}} \\
10,000^{P} \\
- \\
-\end{array}$ & $\begin{array}{l}- \\
x \\
x \\
- \\
x \\
x \\
x \\
0 \\
: \\
x \\
x \\
:\end{array}$ \\
\hline \multicolumn{7}{|c|}{ Dissolved wet cherrissol $(\mathrm{mg} / \mathrm{L})$} \\
\hline Uranium fluorometric & \multicolumn{6}{|c|}{ Totarl' wet chemistry } \\
\hline $\begin{array}{l}\text { Conductivity ( } \mu \mathrm{mho} / \mathrm{cm}) \\
\text { Fluoride }(\mathrm{mg} / \mathrm{L}) \\
\text { Uranium luoorometric (mg/L) } \\
\text { pH (std units) }\end{array}$ & 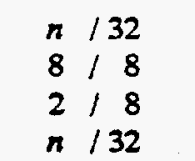 & $\begin{array}{l}207 \\
0.1 \\
0.001 \\
6.7\end{array}$ & $\begin{array}{r}513.62 \\
0.7125 \\
0.0025 \\
8.0812\end{array}$ & $\begin{array}{l}839 \\
1.8 \\
0.004 \\
9.5\end{array}$ & $\begin{array}{l}2^{\bar{S}} \\
0.02^{P} \\
6.5 \cdot 8.5^{5}\end{array}$ & $\begin{array}{r}\dot{0} \\
0 \\
12\end{array}$ \\
\hline
\end{tabular}

1 Total $=$ unfiltered sample (soluable + suspended) and Dissolved $=$ filtered sample (soluable only).

2 Both the number of detected results and the total number of results include all duplicate and replicate measurements. No blanks, matrix spikes, equipment rinsate or other QAVOC data are reported in this table. $n$ denotes not applicabie.

3 The minimum and maximum detected results are listed with their laboratory analytical qualifiers.

J denotes an extimated value (usually beiow the detection limit)

+ denotes the duplicate control limits do not apply; duplicate and sample near the minimum detectable activity (MDA).

4 The average radiochemistry results and their associated limits of error were calculated from all of the results using optimally weighted mean and variance estimates assuming independent measurements with unequal errors, as documented in Rediazion Desecrion and Mearurement by Glenn F. Knoll, New York: John Wiley and Sons (1979), pp. 137-139. For the non-radiochemistry anatytes the average listed is the unweighted arithmetic mean of all detected results. No anabytical qualifiers are listed for the average.

5 If a reference value exists it originates from the following sources:

P 40 CFR Part 141 National Primary Drinking Water Regulations. Subparts B and G, as amended

S 40 CFR Part 143 National Secondary Drinking Water Regulations, as amended

D DOE Order 5400.5, Chapter III, Derived Concentration Guides (DCG) for Air and Water. Four percent of the DOE DCG represents the DOE criterion of 4 mrem effective dose equivalent from ingestion of drinking water.

- denotes no reference value exists for this analyte

6 The number of detected results exceding the reference value is given.

- denotes that since no reference value exists for this anatyte, the number exceeding is not applicable.

$x$ denotes that no detected measurements were reponed for this anatyre so that no comparisons were made to the reference value. 
Table 7.72. Constituents in the groundwater wells located at the K-770 Operable Unit at the K-25 Site, 1993

\begin{tabular}{|c|c|c|c|c|c|c|}
\hline \multirow{2}{*}{ Anatyte } & \multirow{2}{*}{$\begin{array}{l}\text { Number } \\
\text { detected/ } \\
\text { number of } \\
\text { results }{ }^{2}\end{array}$} & \multicolumn{3}{|c|}{ Detected results } & \multirow{2}{*}{$\begin{array}{c}\text { Reference } \\
\text { Value }^{s}\end{array}$} & \multirow{2}{*}{$\begin{array}{c}\text { Number } \\
\text { exceeding } \\
\text { reference } \\
\text { value }\end{array}$} \\
\hline & & Minimum ${ }^{3}$ & Averaget & Marimum ${ }^{3}$ & & \\
\hline
\end{tabular}

Field mearoments

\begin{tabular}{|c|c|c|c|c|c|}
\hline Conductivity $(\mu \mathrm{mho} / \mathrm{cm})$ & $n / 34$ & 41 & 473.79 & 2230 & - \\
\hline Conductivity - initial $(\mu \mathrm{mho} / \mathrm{cm})$ & $n / 34$ & 53 & 528.79 & 2210 & - \\
\hline Dissoived crygen (ppm) & $n / 34$ & 0.9 & 5.1206 & 8.5 & - \\
\hline Dissolved axygen - initial (ppm) & $n / 34$ & 0.5 & 3.7853 & 8 & - \\
\hline Redoox (mv) & $n / 34$ & .58 & 195.26 & 430 & - \\
\hline Redax - initial (mv) & $n / 34$ & .52 & 174.79 & 438 & _- \\
\hline Temperature (degrees $C$ ) & $n / 34$ & 13.6 & 16.191 & 20.2 & - \\
\hline Temperature - initial (degrees C) & $n / 34$ & 13.8 & 16374 & 21 & - \\
\hline pH (std units) & $n / 34$ & 4.9 & 6.7824 & 11.5 & $6.5-8.5^{\mathrm{S}}$ \\
\hline pH - initial (std units) & $n \mid 34$ & 4.8 & 6.85 & 115 & $6.5-8.5^{\mathrm{s}}$ \\
\hline
\end{tabular}

Discalved metais by ICP (mg/L)

Aluminum
Antimony
Barium
Beryllium
Cadmium
Calcium
Caromium
Cobalt
Copper
Iron
Lead
Lithium
Magnesium
Manganese
Molybdenum
Nickel
Phosphorus
Potassium
Silver
Sodium
Strontium
Vanadium
Zinc

$7 / 16$

$0 / 16$

$16 / 16$

$0 / 16$

$3 / 16$

$16 / 16$

$2 / 16$

$0 / 16$

$2 / 16$

$11 / 16$

$0 / 8$

$0 / 8$

$16 / 16$

$15 ; 16$

$0 / 16$

$3 / 16$

0/8

$13 / 16$

$0 / 16$

$16 / 16$

$8 / 8$

$1 / 16$

$12 / 16$

\section{3 \\ 0.11 \\ 0.0067 \\ 73 \\ 0.016 \\ 0.007$$
9
$$

31

$\begin{array}{lcc}0.034 & 12433 & 31 \\ 0.0016 & 0.2408 & 1.5\end{array}$

$\begin{array}{ll}0.011 & 0.0137\end{array}$

0.72

3.7262

0.79

0.03

0.0067

0.0031

4.6619

0.0897

0.0067

0.0081

Towat metais by ICP (mg/L)

Aluminum
Antimony
Barium
Beryllium
Cadmium
Calcium
Chromium

$15 / 16$

0.04

24.267

0.04

$16 / 16$

$6 / 16$

$6 / 16$

$16 / 16$

$10 / 16$

0.0004

0.1478

0.0048

0.0057

4.1

0.0261

0.011

65.444

0.1918

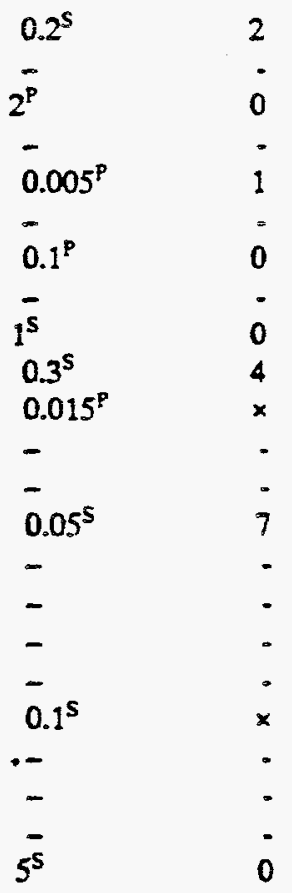


Table 7.72 (continued)

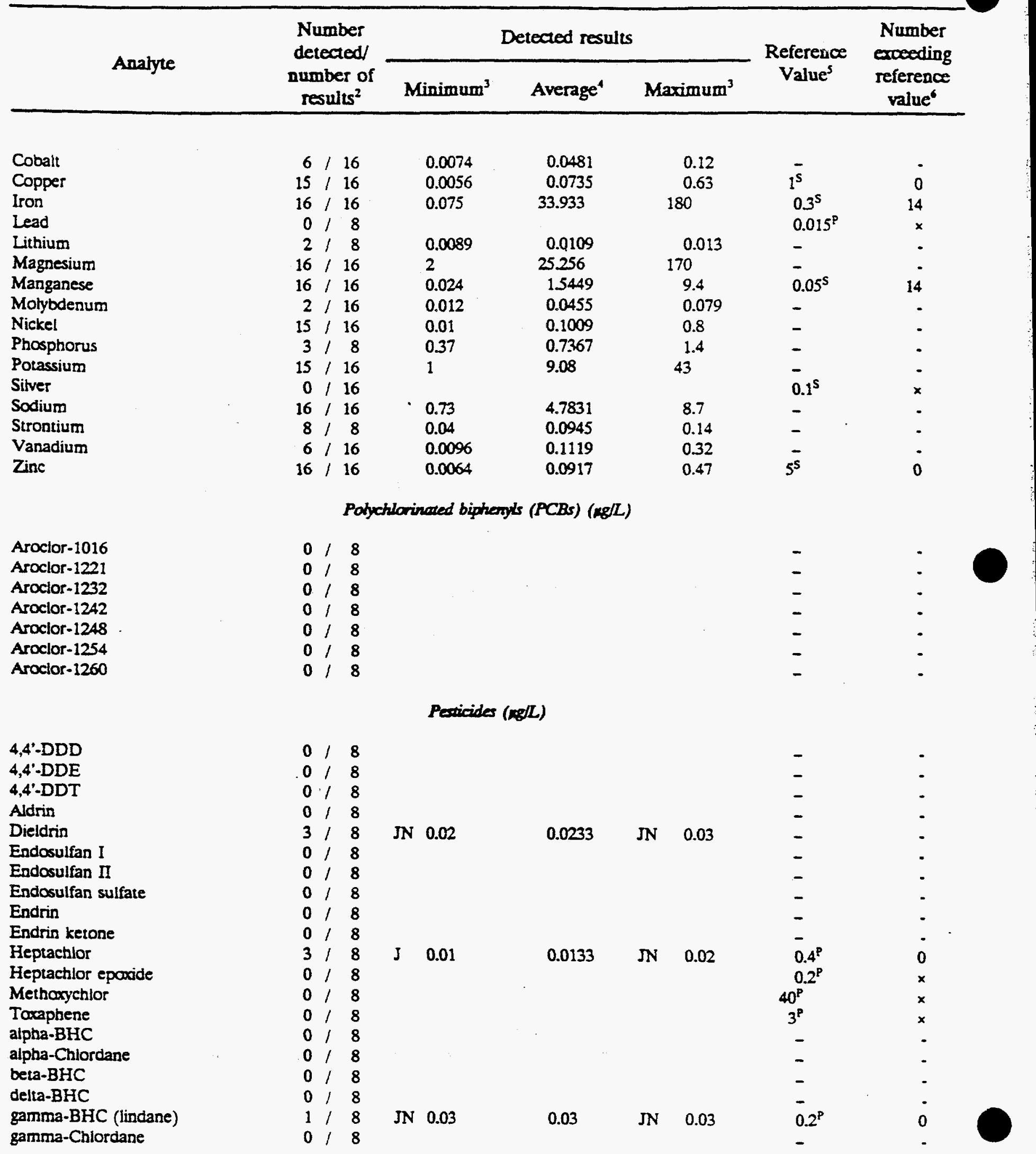


Table 7.72 (continued)

\begin{tabular}{|c|c|c|c|c|c|c|}
\hline \multirow{2}{*}{ Analyre } & \multirow{2}{*}{$\begin{array}{l}\text { Number } \\
\text { detected/ } \\
\text { number of } \\
\text { results }\end{array}$} & \multicolumn{3}{|c|}{ Detected resuits } & \multirow{2}{*}{$\begin{array}{l}\text { Reference } \\
\text { Value }^{5}\end{array}$} & \multirow{2}{*}{$\begin{array}{l}\text { Number } \\
\text { excoeding } \\
\text { reference } \\
\text { value }\end{array}$} \\
\hline & & Minimum $^{3}$ & Average $^{4}$ & Marimum ${ }^{3}$ & & \\
\hline
\end{tabular}

\section{Dissahed" radiochemingy (PCiL)}

$\begin{array}{lr:r}\text { Alpha activity } & n / 16 \\ \text { Beta activity } & n / 16 \\ \text { Neptunium-237 } & n / 8 \\ \text { Plutonium-238 } & n / 8 \\ \text { Plutonium-239 } & n / 8 \\ \text { Technetium-99 } & n / 16 \\ \text { Thorium-228 } & n / 8 \\ \text { Thorium-230 } & n / 8 \\ \text { Thorium-232 } & n / 8 \\ \text { Uranium-234 } & n / 8 \\ \text { Uranium-238 } & n / 8\end{array}$

Alpha activity

Beta activity

Neptunium-237

Plutonium-238

Plutonium-239

Technetium-99

Thorium-228

Thorium-230

Thorium-232

Uranium-234

Uranium-238 $n / 16$

n / 16

n! 8

$n / 8$

$n / 8$

$n / 16$

$n / 8$

n/8

$n / 8$

$n / 8$

$n / 8$
$-1.89 \pm 2.3$

$-233 \pm 3$

$.5 .51 \pm 12$

$-13.7 \pm 17$

$-6.87 \pm 15$

$-337 \pm 1300$

$-7.4 \pm 11$

$0 \pm 9.2$

$-4.9 \pm 11$

$-0.982 \pm 2.2$

$-2.7 \pm 11$

\section{Total' radiachemisy (pCill)}

$$
\begin{array}{r}
-2.28 \pm 23 \\
-1.82 \pm 3.1 \\
+-5.93 \pm 13 \\
+-18.5 \pm 21 \\
-6.87 \pm 15 \\
-55 \pm 52 \\
-7.4 \pm 11 \\
C \quad-7.4 \pm 12 \\
-4.93 \pm 11 \\
-1.03 \pm 23 \\
0 \pm 200
\end{array}
$$

$2.1001 \pm 0.5125$

$11.774 \pm 0.8623$

$-0.141 \pm 0.92$

$-0.08 \pm 1.1695$

$41.254 \pm 20.094$

$-0.526 \pm 22256$

$0.5697 \pm 1.5509$

$-0.311 \pm 1.5992$

$0.1043 \pm 0.9172$

$0.0765 \pm 0.8067$
$0.1291 \pm 1.3053$

$$
\begin{aligned}
& 37.6 \pm 5.4 \\
& 167 \pm 7.9 \\
& \text { s } 0 \pm 1.7 \pm 0.738 \pm 2.6 \\
& 0.738 \pm 1.5 \\
& 1680 \pm 1400 \\
& 71 \pm 27 \\
& C \quad 4.9 \pm 14 \\
& 2.65 \pm 12 \\
& 10.8 \pm 11 \\
& 1.45 \pm 2
\end{aligned}
$$

$\begin{array}{cc}15^{\mathrm{P}} & 1 \\ 50^{\mathrm{P}} & 4 \\ 1.2^{\mathrm{D}} & 0 \\ 1.6^{\mathrm{D}} & 0 \\ 1.2^{\mathrm{D}} & 0 \\ 4000^{\mathrm{D}} & 0 \\ 16^{\mathrm{D}} & 2 \\ 12^{\mathrm{D}} & 0 \\ 2^{\mathrm{D}} & 1 \\ 20^{\mathrm{D}} & 0 \\ 24^{\mathrm{D}} & 0\end{array}$

\section{Semivalatile argorics (moll)}

$$
\begin{aligned}
& 38 \pm 5.5 \\
& 336 \pm 5.3 \\
& 274 \pm 12 \\
&+2.05 \pm 3 \\
& 0.682 \pm 1.4 \\
& 1840 \pm 1400 \\
& 81.4 \pm 29 \\
& 37.1 \pm 20 \\
&+10.6 \pm 11 \\
& 72.4 \pm 140 \\
& 37.5 \pm 11
\end{aligned}
$$

$\begin{array}{cc}15^{\mathrm{P}} & 3 \\ 50^{\mathrm{P}} & 4 \\ 1.2^{\mathrm{D}} & 1 \\ 1.6^{\mathrm{D}} & 1 \\ 1.2^{\mathrm{D}} & 0 \\ 4000^{\mathrm{D}} & 0 \\ 16^{\mathrm{D}} & 2 \\ 12^{\mathrm{D}} & 2 \\ 2^{\mathrm{D}} & 1 \\ 20^{\mathrm{D}} & 2 \\ 24^{\mathrm{D}} & 1\end{array}$

1,2,4-Trichlorobenzene

1,2-Dichlorobenzene

1,2-Diphenythydrazine

1,3-Dichlorobenzene

1,4-Dichlorobenzene

2,3,4,6-Tetrachlorophenol

2,4,5-Trichlorophenol

2,4,6-Trichlorophenol

2,4-Dichlorophenol

2,4-Dimethyiphenol

2,4-Dinitrophenol

2,4-Dinitrotoluene

2,6-Dinitrotoluene

2-Chloronaphthalene

2-Chlorophenol

2-Methylnaphthalene

2-Methylphenol

2-Nitroaniline

2-Nitrophenol

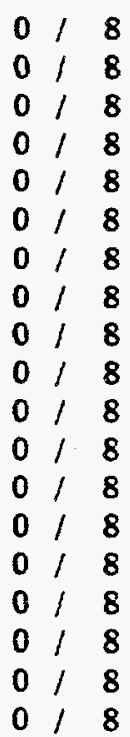


Table 7.72 (continued)

\begin{tabular}{|c|c|c|c|c|c|c|c|c|}
\hline \multirow{2}{*}{ Analyte } & \multirow{2}{*}{\multicolumn{2}{|c|}{$\begin{array}{l}\text { Number } \\
\text { detected/ } \\
\text { number of } \\
\text { results }{ }^{2}\end{array}$}} & \multicolumn{4}{|c|}{ Detected results } & \multirow{2}{*}{$\begin{array}{l}\text { Reference } \\
\text { Value }^{5}\end{array}$} & \multirow{2}{*}{$\begin{array}{l}\text { Number } \\
\text { exceding } \\
\text { reference } \\
\text { value }^{6}\end{array}$} \\
\hline & & & Minimum ${ }^{3}$ & Average & & rimum ${ }^{3}$ & & \\
\hline 3,3'-Dichlorobenzidine & 01 & 8 & & & & & - & . \\
\hline 3-Nitroaniline & 01 & 8 & & & & & - & - \\
\hline 4,6-Dinitro-2-methylphenol & 01 & 8 & & & & & - & - \\
\hline 4-Bromophenyl-phenylether & 01 & 8 & & & & & - & - \\
\hline 4-Chloro-3-methylphenol & 01 & 8 & & & & & - & - \\
\hline 4-Chloroaniline & 01 & 8 & & & & & - & - \\
\hline 4-Chlorophenyl-phenylether & 01 & 8 & & & & & - & - \\
\hline 4-Methylphenol & 01 & 8 & & & & & - & - \\
\hline 4-Nitroaniline & 01 & 8 & & & & & - & - \\
\hline 4-Nitrophenol & 01 & 8 & & & & & - & $\cdot$ \\
\hline Acenaphthene & 01 & 8 & & & & & - & - \\
\hline Acenaphthylene & 01 & 8 & & & & & - & - \\
\hline Aniline & 01 & 8 & • & & & & - & - \\
\hline Anthracene & 01 & 8 & & & & & - & - \\
\hline Benzidine & 01 & 8 & & & & & - & - \\
\hline Benzo(a)anthracene & 01 & 8 & & & & & - & $\cdot$ \\
\hline Benzo(a)pyrene & 01 & 8 & & & & & - & - \\
\hline Benzo(b)fluoranthene & 01 & 8 & & & & & - & - \\
\hline Benzo $(g, h, i)$ perylene & 01 & 8 & & & & & - & $\cdot$ \\
\hline Benzo(k)fluoranthene & 01 & 8 & & & & & - & - \\
\hline Benzoic acid & 01 & 8 & & & & & - & - \\
\hline Benzyl alcohol & 01 & 8 & & & & & - & - \\
\hline Butylbenzylphthaiate & 01 & 8 & & & & & - & - \\
\hline Chrysene & 01 & 8 & & & & & - & - \\
\hline Di-n-butyiphthalate & 71 & 8 & JB 2 & 4.2857 & $\mathrm{JB}$ & 8 & - & - \\
\hline Di-D-octylphthalate & 01 & 8 & & & & & - & - \\
\hline Dibenz(a,h)anthracene & 01 & 8 & & & & & - & - \\
\hline Dibenzofuran & 01 & 8 & & & & & - & - \\
\hline Diethylphthalate & $3 /$ & 8 & JB 0.6 & 1.2 & JB & 2 & - & - \\
\hline Dimethylphthalate & 01 & 8 & & & & & - & $\cdot$ \\
\hline Fluoranthene & 01 & 8 & & & & & - & - \\
\hline Fluorene & 01 & 8 & & & & & - & - \\
\hline Hexachlorobenzene & 01 & 8 & & & & & - & - \\
\hline Hexachlorobutadiene & 01 & 8 & & & & & - & - \\
\hline Hexachlorocyclopentadiene & 01 & 8 & & & & & - & - \\
\hline Hexachloroethane & 01 & 8 & & & & &,- & - \\
\hline Indeno(1,2,3-ed)pyrene & 01 & 8 & & & & & - & - \\
\hline Isophorone & 01 & 8 & & & & & - & $\cdot$ \\
\hline N-Nitroso-di-n-propylamine & 01 & 8 & & & & & - & $\cdot \cdot$ \\
\hline N-Nitrosodimethylamine & 01 & 8 & & & & & - & - \\
\hline N-Nitrosodiphenylamine & 01 & $8^{-}$ & & & & & - & - \\
\hline Naphthalene & 01 & 8 & & & & & - & - \\
\hline Nitrobenzene & 01 & 8 & & & & & - & - \\
\hline Pentachlorophenol & 01 & 8 & & & & & $1^{P}$ & $x$ \\
\hline Phenanthrene & 01 & 8 & & & & & - & - \\
\hline Phenol & 01 & 8 & & & & & - & - \\
\hline Pyrene & 01 & 8 & & & & & - & - \\
\hline Pyridine & 01 & 8 & & & & & - & - \\
\hline bis(2-Chlorocthoxy)methane & 01 & 8 & & & & & - & - \\
\hline
\end{tabular}


Table 7.72 (continued)

\begin{tabular}{l} 
Anahyte \\
\\
\hline \\
bis(2-Chloroethyl)ether \\
bis(2-Chloroisopropyl)ether \\
bis(2-Ethylhexyl)phthalate \\
\\
1,2-Benzenedicarbaxylic acid \\
1,2-Xylene \\
2-Pentanone, 4-hydroxy-4-methy \\
Dimethyl benzene \\
Ethylbenzene \\
Toluene \\
Unknown
\end{tabular}

Number

detected/ number of results ${ }^{2}$ $\begin{array}{ll}0 / & 8 \\ 0 / & 8 \\ 8 / & 8\end{array}$

Semivalatile agaries, tentatively identified compands (ng/L)

$\begin{array}{rrrrcclr}1 / & 1 & \mathrm{~J} & 8 & 8 & \mathrm{~J} & 8 \\ 1 & / & 1 & \mathrm{~J} & 35 & 35 & \mathrm{~J} & 35 \\ 7 & 7 & \mathrm{JAB} & 84 & 153.86 & \mathrm{JAB} & 230 \\ 5 / & 5 & \mathrm{JY} & 49 & 99.8 & \mathrm{JY} & 170 \\ 2 & 2 & \mathrm{~J} & 28 & 69 & \mathrm{~J} & 110 \\ 1 & 1 & \mathrm{~J} & 18 & 18 & \mathrm{~J} & 18 \\ 15 & 15 & \mathrm{~J} \cdot & 8 & 32.933 & \mathrm{~J} & 110\end{array}$

Discohed" metals by spectrochemisty (mg/L)

Arsenic
Lead
Mercury
Selenium
Thallium

Arsenic

Lead

Mercury

Selenium

Thallium $\begin{array}{l:l}0 & 16 \\ 0 / 16 \\ 0 / 16 \\ 1 / 16 \\ 0 / 16\end{array}$

0.0059

0.0059

0.0059

Talat metals by spectrocheming (ing/L)
0.0061

0.0049

0.0002

0.0067

$1 / 16$

$0 / 16$
0.0205

0.0505

0.0003

0.0067
0.039

0.2

0.0004

0.0067
Number

Reference Values erceeding

reference value 6

Volatite orgurie (ag/L)

$\begin{array}{llll}\text { 1,1,1-Trichloroethane } & 0 / 16 \\ \text { 1,1,2,2-Tetrachloroethane } & 0 / 16 \\ \text { 1,1,2-Trichloroethane } & 0 / 16 \\ \text { 1,1-Dichloroethane } & 0 / 16 \\ \text { 1,1-Dichloroethene } & 0 / 16 \\ \text { 1,2-Dichloroethane } & 0 / 16 \\ \text { 1,2-Dichloroethene (total) } & 0 / 16 \\ \text { 1,2-Dichloropropane } & 0 / 16 \\ \text { 2-Butanone } & 0 / 16 \\ \text { 2-Hexanone } & 0 / 16 \\ \text { 4-Methyl-2-pentanone } & 0 / 16 \\ \text { Acetone } & 1 / 16 \\ \text { Benzene } & 0 / 16 \\ \text { Bromodichloromethane } & 0 / 16 \\ \text { Bromoform } & 0 / 16 \\ \text { Bromomethane } & 0 / 16 \\ \text { Carbon disulfide } & 0 / 16\end{array}$

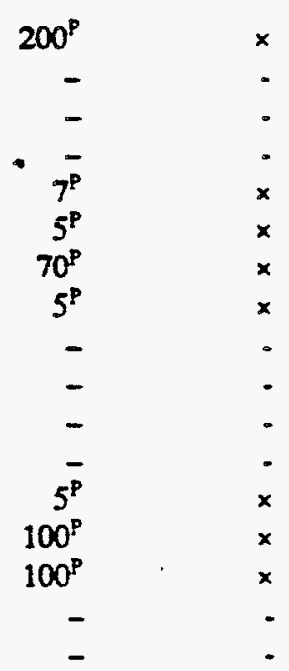


Table 7.72 (continued)

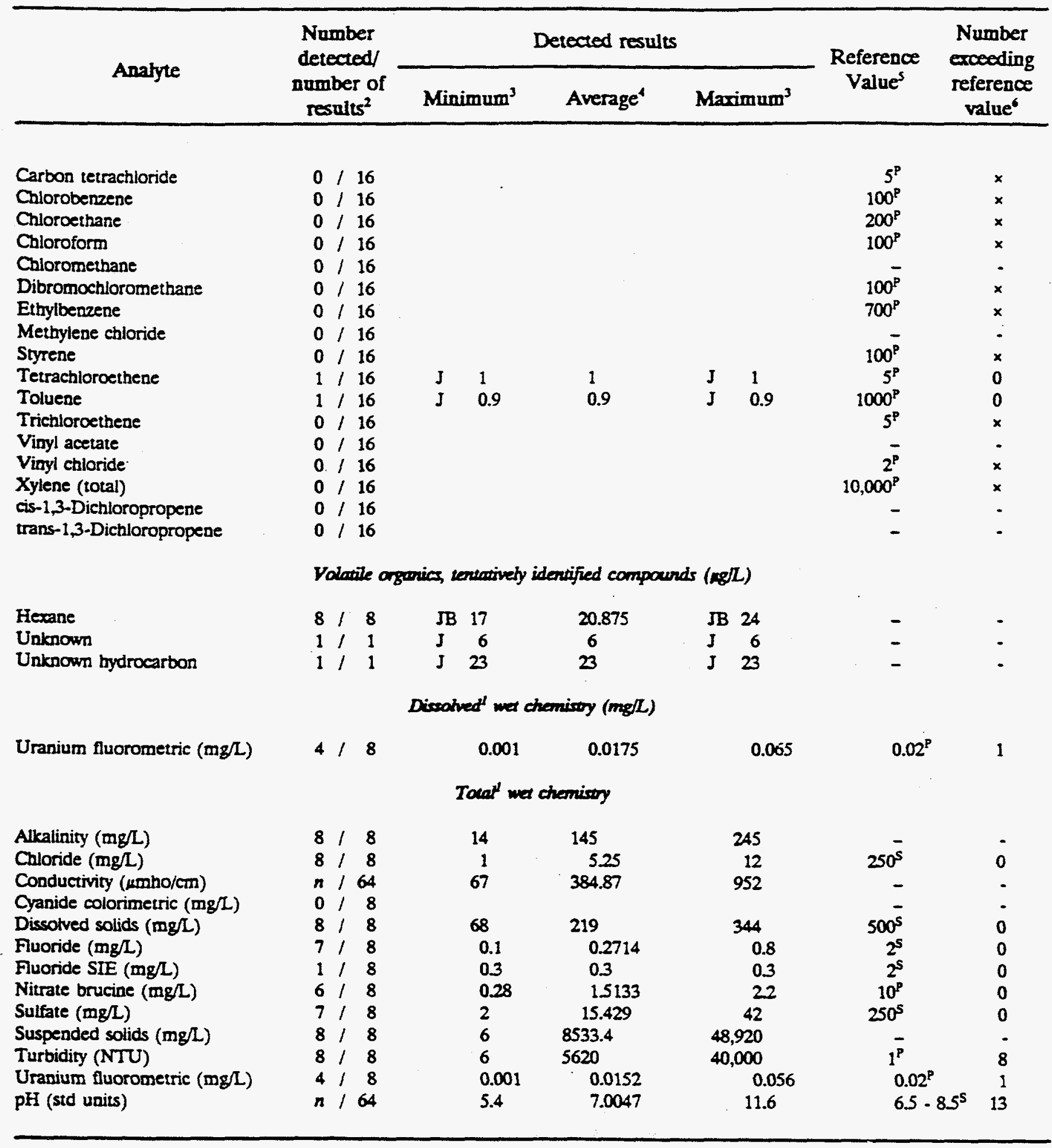

Toul $=$ unfiltered sampie (soiuable + suspended) and Disolved $=$ filtered sample (soluable onty). ICP = inductively coupled plasma

2 Both the number of detected results and the total number of results include all duplicate and replicate measurea ents. No blanks, matrix spikes, equipment rinsate or other QAVQC data are reponed in this table. $n$ denotes not applicable. 
Table 7.72 (continued)

\begin{tabular}{|c|c|c|c|c|c|c|c|}
\hline \multirow[b]{2}{*}{$\ldots$} & \multirow{2}{*}{ Anatyte } & \multirow{2}{*}{$\begin{array}{l}\text { Number } \\
\text { detected/ } \\
\text { number of } \\
\text { results }{ }^{2}\end{array}$} & \multicolumn{3}{|c|}{ Detected results } & \multirow{2}{*}{$\begin{array}{l}\text { Reference } \\
\text { Value }^{s}\end{array}$} & \multirow{2}{*}{$\begin{array}{l}\text { Number } \\
\text { ercoeding } \\
\text { reference } \\
\text { value }\end{array}$} \\
\hline & & & Minimum ${ }^{3}$ & Average & Maximum ${ }^{3}$ & & \\
\hline
\end{tabular}

3

The minimum and maximum detected results are listed with their laboratory anatytical qualifiers.

A denotes aldol condensation product

$B$ denotes parameter found in blank as well as sample

C denotes control analysis outside of control limits

$\mathrm{J}$ denotes an estimated value (usually below the detection limit)

$N$ denotes confirmed by second column; quantative results differed by more than $50 \%$ between columns.

s denotes the spike recovery is not within limits

$Y$ denotes indistinguishable isomer in tentatively identified compound (TIC)

+ denotes the duplicate control limits do not apply, duplicate and sample near the minimum detectable activity (MDA).

4 The average radiochemistry results and their associated limits of error were calculated from all of the results using optimally weighted mean and variance estimates assuming independent measurements with unequal errors, as documented in Radiation Detection and Measuremern by Gienn F. Knoll, New York: John Wiley and Sons (1979). pp. 137-139. For the non-radiochemistry anatyres the average listed is the unweighted arithmetic mean of all detected results. No analytical qualifiers are listed for the average.

If a reference value ecists it originates from the following sources:

P 40 CFR Pan 141 National Primary Drinking Water Regulations, Subparts B and G, as amended

S 40 CFR Part 143 National Secondary Drinking Water Regulations, as amended

D DOE Order 5400.5, Chapter III, Derived Concentration Guides (DCG) for Air and Water. Four percent of the DOE DCG represents the DOE criterion of 4 mrem effective dose equivalent from ingestion of drinking water.

- denotes no reference value exists for this analyte

The number of detected results exceding the reference value is given.

- denotes that since no reference value exists for this anatyre, the number exceeding is not applicable.

$x$ denotes that no detected measurements were reported for this analyte so that no comparisons were made to the reference value. 
Table 8.1. Number of measurements from external control programs, Y-12 Analytical Services Organization, CY 1993

\begin{tabular}{lcc}
\multicolumn{1}{c}{ Program } & $\begin{array}{c}\text { Total } \\
\text { measurements }\end{array}$ & $\begin{array}{c}\text { Measurements } \\
\text { in limits }\end{array}$ \\
\hline Proficiency Analytical Testing (PAT) & 120 & 120 \\
Proficiency Environmental Testing (PET) & 2368 & 2341 \\
EPA Discharge Monitoring Report (DMR) & 29 & 29 \\
EPA Drinking Water Certification & 70 & 65 \\
EPA Environmental Monitoring System & 46 & 27 \\
Laboratory (EMSL) & 36 & 30 \\
DOE Environmental Measurements & & \\
Laboratory (DOE-EML) & $a$ & 25 \\
EPA Contract Laboratory Program (CLP) & 25 & 2637 \\
Mixed Analyte Performance Evaluation & & \\
Program (MAPEP) & 2694 & \\
$\quad$ Total & & \\
\hline
\end{tabular}

"This program scores on additional criteria other than an "acceptable/unacceptable" evaluation of the measurement result. Y-12 Plant Analytical Services Organization score average for 1993; $77.12 \%$.

Table 8.2. ORNL Site Analytical Services Organization external performance evaluation programs, CY 1993

\begin{tabular}{lcccl}
\hline Program & $\begin{array}{c}\text { Total } \\
\text { measurements }\end{array}$ & $\begin{array}{c}\text { Measurements } \\
\text { in limits }\end{array}$ & $\begin{array}{c}\text { Percentage } \\
\text { acceptable }\end{array}$ & \multicolumn{1}{c}{$\begin{array}{c}\text { Program } \\
\text { numbers }\end{array}$} \\
\hline CLP & $a$ & $a$ & $a$ & OAL and IA 2Q93-1Q94 \\
DMR & 24 & 22 & 91.67 & DMR \#13 \\
PAT & 48 & 45 & 93.75 & Rounds 112-115 \\
PET & 1778 & 1763 & 99.16 & OAL and IA Jar.-Dec. 93 \\
WP $^{b}$ & 271 & 253 & 93.36 & WP030 and WP031 \\
WS $^{b}$ & 57 & 55 & 96.49 & WS032 \\
EML $^{\text {EMSL }}$ & 69 & 67 & 97.10 & EML 551 and 556 \\
\hline
\end{tabular}

"This program scores on additional criteria other than an "acceptable/unacceptable" evaluation of the measurement result. ORNL ASD average score for 1993: $81.8 \%$.

${ }^{b}$ WP: water pollution; WS: water supply.

In recalculating the total number of measurements and number of measurements in limits from the percentage acceptable, $16 / 22=72.7 \%$ was the closest value achievable. 
Table 8.3. Number of measurements from external control programs, K-25 Site Analytical Services Organization, CY 1993

\begin{tabular}{lcccc}
\hline Program & $\begin{array}{c}\text { Total } \\
\text { measurements }\end{array}$ & $\begin{array}{c}\text { Number } \\
\text { unacceptable }\end{array}$ & $\begin{array}{c}\text { Measurements } \\
\text { in limits }\end{array}$ & $\begin{array}{c}\text { Percentage } \\
\text { acceptable }\end{array}$ \\
\hline CLP $^{c}$ & 127 & 14 & 113 & 88.98 \\
DMR & 28 & 2 & 26 & 92.86 \\
ELPAT & 48 & 2 & 46 & 95.83 \\
EML-QAP & 41 & 6 & 35 & 85.37 \\
EMSL-LV & 31 & 11 & 20 & 64.52 \\
MAPEP & 27 & 0 & 27 & 100.00 \\
PAT & 112 & 0 & 112 & 100.00 \\
PET & 278 & 3 & 275 & 98.92 \\
WP & 180 & 13 & 167 & 92.78 \\
WS & 70 & 14 & 56 & 80.00 \\
Total & 942 & 65 & 877 & 93.1 \\
\hline
\end{tabular}

${ }^{a}$ Sources received for this program are based on weighted averages instead of "acceptable/unacceptable" ratios.

Environmental Measurements Laboratory-Quality Assessment Program.

This is a pilot program for which no acceptance limits have been established.

${ }^{d}$ Awaiting results for last evaluation study for 1993. 
Table A.1. Reference standards for water

\begin{tabular}{|c|c|c|c|c|c|c|c|}
\hline \multirow{3}{*}{ Parameter } & \multicolumn{5}{|c|}{ All parameters } & & \\
\hline & \multirow{2}{*}{$\begin{array}{c}\text { National } \\
\text { primary } \\
\text { drinking water }\end{array}$} & \multirow{2}{*}{$\begin{array}{c}\text { National } \\
\text { secondary } \\
\text { drinking water }\end{array}$} & \multirow{2}{*}{$\begin{array}{c}\text { Tennessee water } \\
\text { quality criteria- } \\
\text { domestic water } \\
\text { supplyc } \\
\end{array}$} & \multirow{2}{*}{$\begin{array}{c}\text { Tennessee water } \\
\text { quality criteria- } \\
\text { fish \& aquatic } \\
\text { life }^{c}\end{array}$} & \multirow{2}{*}{$\begin{array}{l}\text { Tennessee water } \\
\text { quality criteria- } \\
\text { recreation }\end{array}$} & \multicolumn{2}{|c|}{ Radionuclides only } \\
\hline & & & & & & $\begin{array}{c}4 \% \text { of DOE } \\
\text { DCG }^{d}\end{array}$ & DOE DCG \\
\hline \multicolumn{8}{|c|}{ Anions ( $\mathrm{mg} / \mathrm{L})$} \\
\hline Chloride & & 50 & & & & & \\
\hline Fluoride & 4.0 & 2.0 & & & & & \\
\hline Nitrate & 10 & & & & & & \\
\hline Nitrite & 1.0 & & & & & & \\
\hline Sulfate, as $\mathrm{SO}_{4}$ & & 50 & & & & & \\
\hline \multicolumn{8}{|c|}{ Base/neutral/acid exiractable organics $(\mu g / L)$} \\
\hline 1,2-Dichlorobenzene & 600 & & & & 17,000 & & \\
\hline 1,3-Dichlorobenzene & & & & & 2,600 & & \\
\hline 1,4-Dichlorobenzene & 75 & 5.0 & 75 & & 2,600 & & \\
\hline 2,4-Dinitrophenol & & & & & 1,400 & & \\
\hline 2,4-Dinitrotoluene & & & & & 42 & & \\
\hline 2,4,6-Trichlorophenol & & & & & 6.5 & & \\
\hline 2-Methyl-4,6-Dinitrophenol & & & & & 765 & & \\
\hline 3,4-Benzofluoranthene & & & & 。 & 0.3 & & \\
\hline Benzo(k)fluoranthene & & & & & 0.3 & & \\
\hline Acenaphthylene & & & & & 0.3 & & \\
\hline Anthracene & & & & & 0.03 & & \\
\hline Benzo(a)anthracene & & & & & 0.3 & & \\
\hline Benzo(a)pyrene & & & & & 0.3 & & \\
\hline bis-(2-chloroethyl)ether & & & & & 14 & & \\
\hline bis-(2-ethylhexyl)phthalate & & & & & 59 & & \\
\hline Di-n-butyl phthalate & & & & & 12,000 & & \\
\hline Diethyl phthalate & & & & & 120,000 & & \\
\hline Dimethyl phthalate & & & & & $2,900,000$ & & \\
\hline Fluoranthene & & & & & 54 & & \\
\hline Fluorene & & & & & 0.03 & & \\
\hline Hexachlorobenzene & & & & & 0.007 & & \\
\hline Hexachloroethane & & & & & 89 & & \\
\hline
\end{tabular}




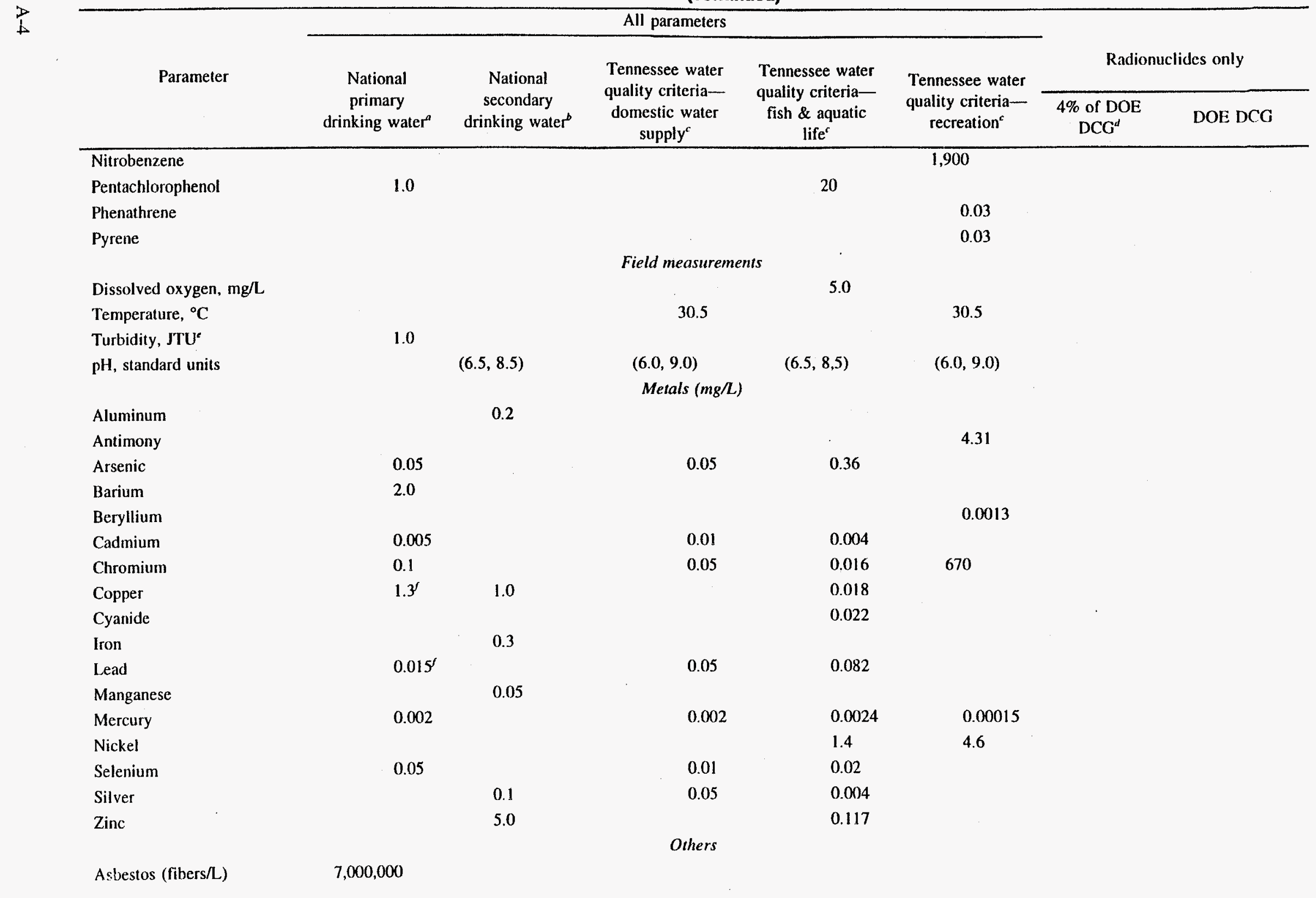


Table A.I (continued)

\begin{tabular}{|c|c|c|c|c|c|c|c|}
\hline \multirow{3}{*}{ Parameter } & \multicolumn{5}{|c|}{ All parameters } & & \\
\hline & \multirow{2}{*}{$\begin{array}{c}\text { National } \\
\text { primary } \\
\text { drinking water" }\end{array}$} & \multirow{2}{*}{$\begin{array}{c}\text { National } \\
\text { secondary } \\
\text { drinking water }\end{array}$} & \multirow{2}{*}{$\begin{array}{c}\text { Tennessee water } \\
\text { quality criteria- } \\
\text { domestic water } \\
\text { supplyc }\end{array}$} & \multirow{2}{*}{$\begin{array}{c}\text { Tennessee water } \\
\text { quality criteria- } \\
\text { fish \& aquatic } \\
\text { life }\end{array}$} & \multirow{2}{*}{$\begin{array}{l}\text { Tennessee water } \\
\text { quality criteria- } \\
\text { recreation" }\end{array}$} & \multicolumn{2}{|c|}{ Radionuclides only } \\
\hline & & & & & & $\begin{array}{c}4 \% \text { of DOE } \\
\text { DCG }^{d}\end{array}$ & DOE DCG \\
\hline Coliform Bacteria (ml) & 0.01 & & & & & & \\
\hline Color (color units) & & 15 & & & & & \\
\hline Cyanide $(\mathrm{mg} / \mathrm{L})$ & & & & 0.022 & & & \\
\hline Odor (T.O.N.) & & 3 & & & & & \\
\hline Total dissoiveu solids, mg/L & & 500 & & & & & \\
\hline \multicolumn{8}{|c|}{ Pesticides/herbicides/PCBs $(\mu \mathrm{g} / \mathrm{L})$} \\
\hline 2,3,7,8-TCDD (Dioxin) & & & & & 0.000001 & & \\
\hline 2,4-D & 70 & & & & & & \\
\hline 2,4,5-TP (Silvex) & 50 & & & & & & \\
\hline $4,4^{\prime}$-DDT & & & & 1.1 & 0.006 & & \\
\hline $4,4^{\prime}-\mathrm{DDE}$ & & & & & 0.006 & & \\
\hline $4,4^{\prime}-\mathrm{DDD}$ & & & & & 0.008 & & \\
\hline Alachlor & 2 & & & & & & \\
\hline Aldicarb sulfoxide & 4 & & & & & & \\
\hline Aldrin & & & & 3 & 0.014 & & \\
\hline Atrazine & 3 & & & & & & \\
\hline Carbofuran & 40 & & & & & & \\
\hline Chlordane & 2 & & & 2.4 & 0.006 & & \\
\hline Dalapon & 200 & & & & & & \\
\hline a-Endosulfan & & & & 0.22 & 2 & & \\
\hline b-Endosulfan & & & . & 0.22 & 2 & & \\
\hline Endrin & & & & 0.18 & & & \\
\hline Ethylene dibromide & 0.05 & & & & & & \\
\hline Heptachlor & 0.4 & & & 0.52 & 0.002 & & \\
\hline Heptachlor epoxide & 0.2 & & & 0.52 & 0.001 & & \\
\hline g-BHC (Lindane) & 0.2 & & & 2.0 & 0.63 & & \\
\hline Methoxychlor & 40 & & & & & & \\
\hline PCB- 1242 & & & & & 0.0005 & & \\
\hline PCB-1254 & & & & & 0.0005 & & \\
\hline
\end{tabular}


Table A.1 (continued)

b All parameters

\begin{tabular}{|c|c|c|c|c|c|c|c|}
\hline \multirow{3}{*}{ Parameter } & \multicolumn{5}{|c|}{ All parameters } & & \\
\hline & \multirow{2}{*}{$\begin{array}{c}\text { National } \\
\text { primary } \\
\text { drinking water }^{a}\end{array}$} & \multirow{2}{*}{$\begin{array}{c}\text { National } \\
\text { secondary } \\
\text { drinking water }\end{array}$} & \multirow{2}{*}{$\begin{array}{c}\text { Tennessee water } \\
\text { quality criteria- } \\
\text { domestic water } \\
\text { supply }\end{array}$} & \multirow{2}{*}{$\begin{array}{c}\text { Tennessee water } \\
\text { quality criteria- } \\
\text { fish \& aquatic } \\
\text { life }^{c}\end{array}$} & \multirow{2}{*}{$\begin{array}{c}\text { Tennessee water } \\
\text { quality criteria- } \\
\text { recreation }^{c}\end{array}$} & \multicolumn{2}{|c|}{ Radionuclides only } \\
\hline & & & & & & $\begin{array}{c}4 \% \text { of DOE } \\
\mathrm{DCG}^{d}\end{array}$ & DOE DCG \\
\hline PCB-1221 & & & & & 0.0005 & & \\
\hline PCB-1232 & & & & & 0.0005 & & \\
\hline PCB 1248 & & & & & 0.0005 & & \\
\hline PCB-1260 & & & & & 0.0005 & & \\
\hline PCB-1016 & & & & & 0.0005 & & . \\
\hline PCB, total & 0.5 & & & & 0.001 & & \\
\hline Toxaphene & 3.0 & & . & 0.73 & 0.008 & & \\
\hline \multicolumn{8}{|c|}{ Radionuclides $(p C i / L)^{8}$} \\
\hline$A m-241$ & & & & & & 1.2 & 30 \\
\hline Bi-214 & & & & & & 24,000 & 600,000 \\
\hline C. 14 & & & & & & 101 & 2,800 \\
\hline Cd-109 & & & & & & 400 & 10,000 \\
\hline $\mathrm{Ce}-143$ & & & & & & 1,200 & 30.000 \\
\hline $\mathrm{Co}-60$ & & & & & & 200 & 5,000 \\
\hline $\mathrm{Cr}-51$ & & & & & & 4,000 & 100,000 \\
\hline Cs -137 & & & & & & 120 & 3,000 \\
\hline Eu-155 & & & & r. & & 4,000 & 100,000 \\
\hline Gross alpha & 15 & & & & & & \\
\hline Gross beta & $50^{h}$ & & & & & & \\
\hline $\mathrm{H}-3$ & 20,000 & & & & & 80,000 & $2,000,000$ \\
\hline $\mid-13 !$ & & & & & & 120 & 3,000 \\
\hline $\mathrm{K}-40$ & & & & & & 280 & 7,000 \\
\hline $\mathrm{Np}-237$ & & & & & & 1.2 & 30 \\
\hline $\mathrm{Pa}-234 \mathrm{~m}$ & & & & & & 2,800 & 70,000 \\
\hline Pu-238 & & & & & & 1.6 & 40 \\
\hline Pu-239/240 & & & & & & 1.2 & 30 \\
\hline Ra-226 & 5.0 & & & & & 4 & 100 \\
\hline $\mathrm{Ra}-228$ & 5.0 & & & & & 4 & 100 \\
\hline$R u-106$ & & & & & & 240 & 6,000 \\
\hline
\end{tabular}

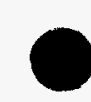




\section{Table A.1 (continued)}

\begin{tabular}{|c|c|c|c|c|c|c|c|}
\hline \multirow{3}{*}{ Parameter } & \multicolumn{5}{|c|}{ Aii parameters } & & \\
\hline & \multirow{2}{*}{$\begin{array}{c}\text { National } \\
\text { primary } \\
\text { drinking water }^{n}\end{array}$} & \multirow{2}{*}{$\begin{array}{c}\text { National } \\
\text { secondary } \\
\text { drinking water" }\end{array}$} & \multirow{2}{*}{$\begin{array}{l}\text { Tennessee water } \\
\text { quality criteria- } \\
\text { domestic water } \\
\text { supply }\end{array}$} & \multirow{2}{*}{$\begin{array}{c}\text { Tennessee water } \\
\text { quality criteria-- } \\
\text { fish \& aquatic } \\
\text { life }\end{array}$} & \multirow{2}{*}{$\begin{array}{c}\text { Tennessee water } \\
\text { quality criteria- } \\
\text { recreation }\end{array}$} & \multicolumn{2}{|c|}{ Radionuclides only } \\
\hline & & & & & & $\begin{array}{c}4 \% \text { of } \mathrm{DOE} \\
\mathrm{DCG}^{d}\end{array}$ & DOE DCG \\
\hline Tc-99 & & & & & & 4,000 & 100,000 \\
\hline Th-228 & & & & & & 16 & 400 \\
\hline Th-230 & & & & & & 12 & 300 \\
\hline Th-232 & & & & & & 2 & 50 \\
\hline Th-234 & & & & & & 400 & 10.000 \\
\hline Thorium, natural & & & & & & 2 & 50 \\
\hline Total rad Sr & 8.0 & & & & & 40 & 1,000 \\
\hline U-234 & & & & & & 20 & 500 \\
\hline $\mathrm{U}-235$ & & & & & & 24 & 600 \\
\hline U-238 & & & & & & 24 & 600 \\
\hline Uranium, natural & & & & & & 24 & 600 \\
\hline Uranium, total ${ }^{i}$ & & & & & & 20 & 500 \\
\hline \multicolumn{8}{|c|}{ Volatile organics $(\mu \mathrm{g} / \mathrm{L})$} \\
\hline 1,1,1-Trichloroethane & 200 & & 200 & & 170,000 & & \\
\hline 1,1-Dichloroethene & 7.0 & & 7.0 & & 32 & & \\
\hline 1,1,2-Trichloroctliane & & & & & 420 & & \\
\hline 1,1,2,2-Tetrachloroethane & & & & & 110 & & \\
\hline 1,2-Dichloroethane & 5.0 & & 5.0 & & 990 & & \\
\hline 1,2-Dichloroethene & 70 & & & & & & \\
\hline cis-1,2-Dichloroethene & 70 & & & & & & \\
\hline trans-1,2-Dichloroethene & 100 & & & & & & \\
\hline 1,2-Dichloropropane & 5.0 & & & & & & \\
\hline cis-1,3-Dichloropropane & & & & & 1,700 & & \\
\hline Irans-1,2-Dichloropropane & & & & & 1,700 & & \\
\hline Acrolein & & & & & 780 & & \\
\hline Acrylonitrile & & & & & 6.7 & & \\
\hline Benzene & 5.0 & & 5.0 & & 710 & & \\
\hline Bromodichloromethane & $100^{\prime}$ & & & & & & \\
\hline Bromoform & $100^{\prime}$ & . & & & 4,700 & & \\
\hline
\end{tabular}


Table A.1 (continued)

All parameters

\begin{tabular}{|c|c|c|c|c|c|c|c|}
\hline \multirow{2}{*}{ Parameter } & \multirow{2}{*}{$\begin{array}{c}\text { National } \\
\text { primary } \\
\text { drinking water }\end{array}$} & \multirow{2}{*}{$\begin{array}{c}\text { National } \\
\text { secondary } \\
\text { drinking water }\end{array}$} & \multirow{2}{*}{$\begin{array}{c}\text { Tennessee water } \\
\text { quality criteria- } \\
\text { domestic water } \\
\text { supplyc }\end{array}$} & \multirow{2}{*}{$\begin{array}{c}\text { Tennessee water } \\
\text { quality criteria- } \\
\text { fish \& aquatic } \\
\text { life }^{c}\end{array}$} & \multirow{2}{*}{$\begin{array}{c}\text { Tennessee water } \\
\text { quality criteria- } \\
\text { recreation }^{c}\end{array}$} & \multicolumn{2}{|c|}{ Radionuclides only } \\
\hline & & & & & & $\begin{array}{c}4 \% \text { of DOE } \\
\text { DCG }^{d}\end{array}$ & DOE DCG \\
\hline Carbon tetrachloride & 5.0 & & 5.0 & & 44 & & \\
\hline Chlorobenzene & 100 & & & & & & \\
\hline Chloroethane & 200 & & & & & & \\
\hline Chloroform & 100 & & & & 4,700 & & \\
\hline Dibromochloromethane & $100^{j}$ & & & & 4,700 & & \\
\hline Ethylbenzene & 700 & & & & 29,000 & & \\
\hline Methylene chloride & & & & & 16,000 & & \\
\hline Styrone & 100 & & & & & & \\
\hline Tetrachloroethene & 5.0 & & & & 88 & & \\
\hline Toluene & 1,000 & & & & 300,000 & & \\
\hline Total Trihalomethanes & 100 & & & & 100 & & \\
\hline Trichloroethene & 5.0 & & 5.0 & & 807 & & \\
\hline Vinyl chloride & 2.0 & & 2.0 & & 5,250 & & \\
\hline Xylene, total & 10,000 & & & & & & \\
\hline
\end{tabular}

Xylene, total

${ }^{a} 40$ CFR Part 141--National Primary Drinking Water Regulations, Subparts B and G, as amended.

${ }^{b} 40$ CFR Part 143--National Secondary Drinking Water Regulations, as amended.

'Rules of Tennessee Department of Environment and Conservation, Division of Water Pollution Control, Chapter 1200-4-3,

General Water Quality Criteria, as amended.

"DOE Order 5400.5. Chapter III, Derived Concentration Guides for Air and Water. Four percent of the DOE DCG to represent

the DOE criterion of 4 mrem effective dose equivalent from ingestion of drinking water.

"JTU an NTU are roughly equivalent in the range of 25 to $1000 \mathrm{JTU}$.

'Action level, which is applicable to community water systems and non-transient, non-community water systems.

'Only the radiounuclides that were sought at the Oak Ridge Reservation are listed.

"Regulatory guide for assessing compliance without further analysis.

'Minimum of uranium isotopes.

${ }^{j}$ Limit for total trihalomethanes (bromodichloromethane + bromoform + chloroform + dibromochloromethane). 
Table A.2. Air permits at the Y-12 Plant

\begin{tabular}{|c|c|c|c|}
\hline $\begin{array}{l}Y-12 \text { Piani } \\
\text { source number }\end{array}$ & $\begin{array}{c}\text { Emission source } \\
\text { reference } \\
\text { number }\end{array}$ & $\begin{array}{l}\text { Permit } \\
\text { number }\end{array}$ & Source description \\
\hline \multicolumn{4}{|c|}{ Pan 1. Operating permits at the Y-12 Plant } \\
\hline Fugitive emission source & $01-1020-89$ & $034295 P$ & Fugitive air emission at $Y-12$ Plant \\
\hline$Y-12-P$ lant $-A(00)$ & $01-0020-08$ & $035025 \mathrm{P}$ & Plantwide permit for fluorescent light crusher \\
\hline$Y-9201-1-A(01)$ & $01-0020-15$ & $730303 P$ & Welding booths \\
\hline$Y-9201-1-A(02)$ & $01-0020-15$ & $730303 P$ & Welding shop \\
\hline$Y-9201-1-A(04)$ & $01-0020-15$ & $730303 \mathrm{P}$ & Metal fabrication shop \\
\hline$Y-9201-1-A(05)$ & $01-0020-15$ & $730303 \mathrm{P}$ & Welding shop \\
\hline$Y-9201-1-A(15)$ & $01-0020-15$ & $730303 P$ & Metal fabrication shop \\
\hline$Y-9201-1-B(16)$ & $01-0020-59$ & $730310 \mathrm{P}$ & Tool grinding machines \\
\hline$Y-9201-1-B(18)$ & $01-0020-59$ & $730310 \mathrm{P}$ & Sand blaster exhaust \\
\hline$Y-9201-1-C(278)$ & $01-0020-17$ & $730304 \mathrm{P}$ & Graphitic carbon machining \\
\hline$Y-9201-1-C(279)$ & $01-0020-17$ & $730304 \mathrm{P}$ & Graphitic carbon machining \\
\hline$Y-9201-1-D(09)$ & $01-0020-59$ & $730310 \mathrm{P}$ & Fabrication shop \\
\hline$Y-9201-1-D(10)$ & $01-0020-59$ & $730310 \mathrm{P}$ & Fabrication shop \\
\hline$Y-9201-1-D(11)$ & $01-0020-59$ & $730310 \mathrm{P}$ & Fabrication shop \\
\hline$Y-9201-1-D(13)$ & $01-0020-59$ & $730310 \mathrm{P}$ & Metal grinders and milling machines \\
\hline$Y-9201-1-E(00)$ & $01-1020-92$ & $031880 \mathrm{P}$ & Lead machining operations \\
\hline $\mathrm{Y}-9201-2-\mathrm{B}(02)$ & $01-0020-43$ & $012887 \mathrm{P}$ & Acid wash station \\
\hline$Y-9201-3-A(01)$ & $01-0020-55$ & $013002 \mathrm{~F}$ & Diesel generator \\
\hline$Y-9201-A(264)$ & $01-1020-96$ & 032956P & Mercury flasking hood \\
\hline $\mathrm{Y}-9201-5-\mathrm{B}(071)$ & $01-0020-21$ & $730305 \mathrm{P}$ & Machining operations L5N hood exhaust \\
\hline $\mathrm{Y}-9201-5-\mathrm{B}(072)$ & $01-0020-21$ & $730305 P$ & Vacuum inlets LSE machining shop \\
\hline$Y-9201-5-B(03)$ & $01-0020-21$ & $730305 \mathrm{P}$ & Rubber-gel potting hood exhaust \\
\hline$Y-9201-5-B(073)$ & $01-0020-21$ & $730305 P$ & Palarite shop, machine exhaust \\
\hline$Y-9201-5-B(267)$ & $01-0020-21$ & $730305 P$ & Tool-grinding machines hood exhaust \\
\hline$Y-9201-5-B(277)$ & $01-0020-21$ & $730305 P$ & Cleaning hood, equipment service \\
\hline Y-9201-5-B(273) & $01-0020-21$ & $730305 \mathrm{P}$ & Electrochemical machine, stainiess steel \\
\hline$Y-9201-5-D(01)$ & $01-1020-44$ & $025902 \mathrm{P}$ & Hood \\
\hline$Y-9201-5-D(02)$ & $01-1020-44$ & $025902 \mathrm{P}$ & Film dryer exhaust fume hood \\
\hline$Y-9201-5-E(01)$ & $01-1020-70$ & $025983 \mathrm{P}$ & BeO hot press \\
\hline$Y-9201-5-E(02)$ & $01-1020-70$ & 025983P & A53 hot press house vacuum \\
\hline$Y-9201-5-E(08)$ & $01-1020-70$ & 025983P & Room exhaust \\
\hline$Y-9201-5-G(01)$ & $01-0020-44$ & $730308 \mathrm{P}$ & Arc melt \\
\hline$Y-9201-5-G(02)$ & $01-0020-44$ & $730308 \mathrm{P}$ & DeVilbiss hood \\
\hline$Y-9201-5-G(03)$ & $01-0020-44$ & $730308 \mathrm{P}$ & Nitric acid dip tanks \\
\hline$Y-9201-5-G(04)$ & $01-0020-44$ & $730308 \mathrm{P}$ & Acid pickling tanks \\
\hline$Y-9201-5-G(05)$ & $01-0020-44$ & $730308 \mathrm{P}$ & Abrasive saws \\
\hline$Y-9201-5-G(06)$ & $01-0020-44$ & 730308P & Scrap metal recycle \\
\hline$Y-9201-5-G(07)$ & $01-0020-44$ & $730308 \mathrm{P}$ & Vapor degreaser \\
\hline$Y-9201-5-H(01)$ & $01-0020-16$ & $026019 \mathrm{P}$ & Mixing process material \\
\hline$Y-9201 \cdots \bar{j}-\mathrm{H}(02)$ & $01-0020-16$ & $026019 \mathrm{P}$ & Setup and sample area \\
\hline $\mathrm{Y}-9201-5-\mathrm{H}(03)$ & $01-0020-16$ & $026019 \mathrm{P}$ & Vapor blaster \\
\hline $\mathrm{Y}-9201-5-\mathrm{H}(04)$ & $01-0020-16$ & 026019P & Nickel-plating tank exhaust \\
\hline$Y-9201-5-H(05)$ & $01-0020-16$ & $026019 \mathrm{P}$ & Material handling \\
\hline
\end{tabular}


Tabie A.2 (continued)

\begin{tabular}{|c|c|c|c|}
\hline $\begin{array}{l}\text { Y-12 Plant } \\
\text { source number }\end{array}$ & $\begin{array}{l}\text { Emission source } \\
\text { reference } \\
\text { number }\end{array}$ & $\begin{array}{l}\text { Permit } \\
\text { number }\end{array}$ & Source description \\
\hline$Y-9201-5-H(06)$ & $01-0020-16$ & $026019 \mathrm{P}$ & Material handling \\
\hline$Y-9201-5-H(07)$ & $01-0020-16$ & $026019 \mathrm{P}$ & Glove box and blending station \\
\hline$Y-9201-5-H(08)$ & $01-0020-16$ & $026019 \mathrm{P}$ & Inspection house vacuum \\
\hline$Y-9201-5 N-A(67)$ & $01-1020-18$ & $730314 \mathrm{P}$ & Machine shop exhaust \\
\hline$Y-9201-5 N-B(239)$ & $01-\infty 020-30$ & $030484 \mathrm{P}$ & Plating tanks and hoods \\
\hline$Y-9201-5 N-B(240)$ & $01-0020-30$ & $030484 \mathrm{P}$ & Plating tanks and hoods \\
\hline$Y-9201-5 N-B(241)$ & $01-0020-30$ & $030484 \mathrm{P}$ & Plating tanks and hoods \\
\hline$Y-9201-5 N-B(242)$ & $01-0020-30$ & $030484 \mathrm{P}$ & Incinerator \\
\hline$Y-9201-5 N-B(243)$ & $01-0020-30$ & $030484 \mathrm{P}$ & Grit blaster \\
\hline$Y-9201-5 N-B(244)$ & $01-0020-30$ & $030484 \mathrm{P}$ & Grit blaster and area exhaust \\
\hline$Y-9202-A-(20)$ & $01-0020-06$ & $031696 \mathrm{P}$ & Laboratory beryllium \\
\hline$Y-9202-A-(21)$ & $01-0020-06$ & 031696P & Laboratory \\
\hline$Y-9204-2-A(01)$ & $01-0020-46$ & $026107 \mathrm{P}$ & Storage tank \\
\hline$Y-9204-2-A(02)$ & $01-0020-46$ & 026107P & Storage tank \\
\hline$Y-9204-2-A(03)$ & $01-002046$ & $026107 \mathrm{P}$ & Storage tank \\
\hline$Y-9204-2-A(04)$ & $01-0020-46$ & 026107P & Storage tank \\
\hline$Y-9204-2-A(05)$ & $01-0020-46$ & $026107 \mathrm{P}$ & Storage tank \\
\hline$Y-9204-2-A(06)$ & $01-0020-46$ & $026107 \mathrm{P}$ & Storage tank \\
\hline$Y-9204-2-A(07)$ & $01-0020-46$ & $026107 \mathrm{P}$ & Storage tank \\
\hline$Y-9204-2 \cdot A(08)$ & $01-0020-46$ & $026107 \mathrm{P}$ & Storage tank \\
\hline $\mathrm{Y}-9204-2-\mathrm{A}(09)$ & $01-0020-46$ & $026107 \mathrm{P}$ & Storage $\tan k$ \\
\hline$Y-9204-2-A(10)$ & $01-0020-46$ & $026107 \mathrm{P}$ & Storage tank \\
\hline$Y-9204-2-A(11)$ & $01-0020-46$ & $026107 P$ & Storage tank \\
\hline$Y-9204-2-A(12)$ & $01-0020-46$ & 026107P & Storage tank \\
\hline$Y-9204-2-A(13)$ & $01-0020-46$ & $026107 \mathrm{P}$ & Storage tank \\
\hline Y-9204-2-B & $01-0020-45$ & $012889 \mathrm{P}$ & Storage tank \\
\hline$Y-9204-2-B(14)$ & $01-0020-71$ & $025954 \mathrm{P}$ & Reduction cell \\
\hline$Y-9204-2-B(15)$ & $01-0020-71$ & $025954 \mathrm{P}$ & Reduction cell \\
\hline$Y-9204-2-B(16)$ & $01-0020-71$ & $025954 \mathrm{P}$ & Reduction cell \\
\hline$Y-9204-2-B(17)$ & $01-0020-71$ & $025954 \mathrm{P}$ & Reduction cell \\
\hline$Y-5204-2-B(18)$ & $01-0020-71$ & $025954 \mathrm{P}$ & Caustic scrubber exhaust \\
\hline$Y-9204-2-B(19)$ & $01-0020-71$ & $025954 \mathrm{P}$ & Caustic scrubber exhaust \\
\hline$Y-9204-2-B(20)$ & $01-0020-71$ & $025954 \mathrm{P}$ & Storage area \\
\hline$Y-9204-2-B(21)$ & $01-0020-71$ & $025954 \mathrm{P}$ & Reduction cell \\
\hline $\mathrm{Y}-9204-2-\mathrm{B}(22)$ & $01-0020-71$ & $025954 \mathrm{P}$ & Reduction cell \\
\hline$Y-9204-2-B(23)$ & $01-0020-71$ & $025954 \mathrm{P}$ & Caustic scrubber exhaust \\
\hline$Y-9204-2-B(24)$ & $01-0020-71$ & $025954 \mathrm{P}$ & Caustic scrubber exhaust \\
\hline$Y \cdot 9204-2-B(25)$ & $01-0020-71$ & $025954 \mathrm{P}$ & Lithium metal wash station \\
\hline$Y-9204-2-B(26)$ & $01-0020-71$ & $025954 \mathrm{P}$ & Cleaning station \\
\hline$Y-9204-2-B(27)$ & $01-0020-71$ & $025954 \mathrm{P}$ & Lithium remeit oven \\
\hline$Y-9204-2-B(28)$ & $01-0020-71$ & $025954 \mathrm{P}$ & Reduction cell \\
\hline$Y-9204-2-C(29)$ & $01-1020-19$ & $025900 \mathrm{P}$ & Classified \\
\hline$Y-9204-2-C(30)$ & $01-1020-19$ & $025900 \mathrm{P}$ & Classified \\
\hline$Y-9204-2-C(31)$ & $01-1020-19$ & 025900P & Classified \\
\hline$Y-9204-2-C(32)$ & $01-1020-19$ & $025900 \mathrm{P}$ & Classified \\
\hline
\end{tabular}


Table A.2 (continued)

\begin{tabular}{|c|c|c|c|}
\hline $\begin{array}{c}\text { Y-12 Plant } \\
\text { source number }\end{array}$ & $\begin{array}{c}\text { Emission source } \\
\text { reference } \\
\text { number }\end{array}$ & $\begin{array}{l}\text { Permit } \\
\text { number }\end{array}$ & Source description \\
\hline$Y-9204-2-C(33)$ & $01-1020-19$ & $025900 \mathrm{P}$ & Classified \\
\hline$Y-9204-2-C(34)$ & $01-1020-19$ & $025900 \mathrm{P}$ & Classified \\
\hline$Y-9204-2-C(35)$ & $01-1020-19$ & $025900 \mathrm{P}$ & Classified \\
\hline$Y-9204-2-C(36)$ & $01-1020-19$ & $025900 \mathrm{P}$ & Classified \\
\hline$Y-9204-2-C(37)$ & $01-1020-19$ & $025900 \mathrm{P}$ & Classified \\
\hline$Y-9204-2-C(38)$ & $01-1020-19$ & $025900 \mathrm{P}$ & Classified \\
\hline$Y-9204-2-C(39)$ & $01-1020-19$ & $025900 \mathrm{P}$ & Classified \\
\hline$Y-9204-2-C(40)$ & $01-1020-19$ & $025900 \mathrm{P}$ & Classified \\
\hline$Y-9204-2-C(41)$ & $01-1020-19$ & $025900 \mathrm{P}$ & Classified \\
\hline$Y-9204-2-C(42)$ & $01-1020-19$ & $025900 \mathrm{P}$ & Classified \\
\hline$Y-9204-2-C(43)$ & $01-1020-19$ & $025900 \mathrm{P}$ & Classified \\
\hline$Y-9204-2-C(44)$ & $01-1020-19$ & $025900 \mathrm{P}$ & Classified \\
\hline$Y-9204-2-C(45)$ & $01-1020-19$ & $025900 \mathrm{P}$ & Classified \\
\hline$Y-9204-2-C(46)$ & $01-1020-19$ & $025900 \mathrm{P}$ & Classified \\
\hline$Y-9204-2-C(47)$ & $01-1020-19$ & $025900 \mathrm{P}$ & Classified \\
\hline$Y-9204-2-C(48)$ & $01.1020-19$ & $025900 \mathrm{P}$ & Classified \\
\hline$Y-9204-2-C(49)$ & $01-1020-19$ & $025900 \mathrm{P}$ & Classified \\
\hline$Y-9204-2-C(50)$ & $01-1020-19$ & $025900 \mathrm{P}$ & Classified \\
\hline$Y-9204-2 \cdot C(51)$ & $01-1020-19$ & $025900 \mathrm{P}$ & Classified \\
\hline$Y-9204-2-D(52)$ & $01-1020-57$ & $025967 \mathrm{P}$ & Storage tanks \\
\hline$Y-9204-2-D(53)$ & $01-1020-57$ & $025967 \mathrm{P}$ & Station \\
\hline$Y-9204-2-D(54)$ & $01-1020-57$ & $025967 \mathrm{P}$ & Salvage vats \\
\hline$Y-9204-2-D(55)$ & $01-1020-57$ & $025967 \mathrm{P}$ & Storage tank \\
\hline$Y-9204-2 \cdot D(56)$ & $01-1020-57$ & $025967 \mathrm{P}$ & Lithium chloride crystallizer \\
\hline$Y-9204-2-D(57)$ & $01-1020-57$ & $025967 \mathrm{P}$ & Lithium chloride crystallizer \\
\hline$Y-9204-2-D^{\prime}(58)$ & $01-1020-57$ & $025967 \mathrm{P}$ & Neutralizer \\
\hline$Y-9204-2-D(59)$ & $01-1020-57$ & $025967 \mathrm{P}$ & Three lab hoods \\
\hline$Y-9204-2-D(60)$ & $01-1020-57$ & $025967 \mathrm{P}$ & Process tank \\
\hline$Y-9204-2-D(61)$ & $01-1020-57$ & $025967 P$ & Lithium chloride crystallizer \\
\hline$Y-9204-2-D(62)$ & $01-1020-57$ & $025967 \mathrm{P}$ & Lithium hydroxide neutralizer \\
\hline$Y-9204-2-D(63)$ & $01-1020-57$ & $025967 \mathrm{P}$ & $\mathrm{HCl}$ head tanks \\
\hline$Y-9204-2-D(64)$ & $01-1020-57$ & 025967P & Process tanks \\
\hline$Y-9204-2-D(65)$ & $01-1020-57$ & $025967 \mathrm{P}$ & Process tank \\
\hline$Y-9204-2-D(66)$ & $01-1020-57$ & $025967 \mathrm{P}$ & Neutralizer \\
\hline$Y-9204-2-D(67)$ & $01-1020-57$ & 025967P & Neutralizer \\
\hline$Y-9204-2-E(68)$ & $01-1020-55$ & $730328 \mathrm{P}$ & Oven \\
\hline$Y-9204-2-E(69)$ & $01-1020-55$ & $730328 \mathrm{P}$ & Oven \\
\hline$Y-9204-2-E(70)$ & $01-1020-55$ & $730328 \mathrm{P}$ & Tungsten screener \\
\hline$Y-9204-2-E(71)$ & $01-1020-55$ & $730328 \mathrm{P}$ & Dry box vent \\
\hline$Y-9204-2-E(72)$ & $01-1020-55$ & $730328 \mathrm{P}$ & Glove boxes \\
\hline$Y-9204-2-E(73)$ & $01-1020-55$ & $730328 \mathrm{P}$ & Material handiing \\
\hline$Y-9204-2-E(74)$ & $01-1020-55$ & $730328 \mathrm{P}$ & Glove boxes \\
\hline$Y-9204-2-E(75)$ & $01-1020-55$ & $730328 \mathrm{P}$ & Outgassing/anneaiing owens \\
\hline$Y-9204-2-E(76)$ & $01-1020-55$ & $730328 \mathrm{P}$ & Material handing \\
\hline$Y-9204-2-E(77)$ & $01-1020-55$ & $730328 \mathrm{P}$ & Glove boxes \\
\hline
\end{tabular}


Table A.2 (continued)

\begin{tabular}{|c|c|c|c|}
\hline $\begin{array}{l}\text { Y-12 Plant } \\
\text { source number }\end{array}$ & $\begin{array}{l}\text { Emission source } \\
\text { reference } \\
\text { number }\end{array}$ & $\begin{array}{l}\text { Permit } \\
\text { number }\end{array}$ & Source description \\
\hline$Y-9204-2-E(78)$ & $01-1020-55$ & $730328 \mathrm{P}$ & Reactor unloading station \\
\hline$Y-9204-2-E(79)$ & $01-1020-55$ & $730328 \mathrm{P}$ & Reactor unloading station \\
\hline$Y-9204-2-E(80)$ & $01-1020-55$ & $730328 \mathrm{P}$ & Glove boxes \\
\hline$Y-9204-2 \cdot E(81)$ & $01-1020-55$ & $730328 \mathrm{P}$ & Vacuum pump \\
\hline$Y-9204-2-F$ & $01-0020-32$ & $012874 \mathrm{P}$ & Storage tank \\
\hline$Y-9204-2-F(082)$ & $01-0020-51$ & $025897 \mathrm{P}$ & Classified \\
\hline$Y-9204-2-F(083)$ & $01-0020-51$ & $025897 \mathrm{P}$ & Classified \\
\hline$Y-9204-2-F(084)$ & $01-0020-51$ & $025897 \mathrm{P}$ & Classified \\
\hline$Y-9204-2-F(085)$ & $01-0020-51$ & 025897P & Classified \\
\hline$Y-9204-2-F(086)$ & $01-0020-51$ & $025897 \mathrm{P}$ & Classified \\
\hline$Y-9204-2-F(087)$ & $01-0020-51$ & $025897 \mathrm{P}$ & Classified \\
\hline$Y-9204-2-G(088)$ & S01-1020-79 & $028350 \mathrm{P}$ & Inspection operation \\
\hline$Y-9204-2-G(089)$ & $501-1020-79$ & $028350 \mathrm{P}$ & Metalworking machine shop hood, B-2 \\
\hline$Y-9204-2-G(090)$ & S01-1020-79 & 028350P & Metalworking machine shop hood. B-2 \\
\hline $\mathrm{Y}-9204-2-\mathrm{H}(492)$ & S01-1020-42 & $025952 \mathrm{P}$ & Etching vats \\
\hline$Y-9204-2-H(493)$ & SO1-1020-42 & $025952 \mathrm{P}$ & Glue mixing \\
\hline$Y-9204-2 E-A(202)$ & $01-1020-91$ & $730938 \mathrm{P}$ & Positive lon Accelerator \\
\hline$Y-9204-2 E-A(436)$ & $01-0020-68$ & $730312 \mathrm{P}$ & Oven \\
\hline$Y-9204-2 E-A(439)$ & $01-0020-68$ & $730312 P$ & Hood exhaust \\
\hline$Y-9204-2 E-A(441)$ & $01-0020-68$ & $730312 \mathrm{P}$ & Hood \\
\hline$Y-9204-2 E-A(442)$ & $01-0020-68$ & $730312 \mathrm{P}$ & Hood \\
\hline$Y-9204-2 E-A(443)$ & $01-0020-68$ & $730312 \mathrm{P}$ & Degreaser \\
\hline$Y-9204-2 E-A(444)$ & $01-0020-68$ & $730312 P$ & Electropolishers \\
\hline Y-9204-2E-A(445) & $01-0020-68$ & $730312 \mathrm{P}$ & Surface coating \\
\hline$Y-9204-2 E-A(448)$ & $01-0020-68$ & $730312 \mathrm{P}$ & Glove box \\
\hline Y-9204-2E-B(12) & $01-1020-41$ & $025953 \mathrm{P}$ & $\mathrm{X}$-ray testing \\
\hline$Y-9204-2 E-B(14)$ & $01-1020-41$ & $025953 \mathrm{P}$ & Hoods \\
\hline Y-9204-2E-B(15) & $01-1020-41$ & $025953 \mathrm{P}$ & Hoods \\
\hline$Y-9204-2 E-C(12)$ & $01-1020-55$ & $730328 \mathrm{P}$ & Machine shop hood exhaust, B2E \\
\hline$Y-9204-2 E-C(13)$ & $01-1020-55$ & $730328 \mathrm{P}$ & Machine shop hood exhaust, specimen shop \\
\hline Y-9204-3-AJ-106 & $01-0020-89$ & $018208 \mathrm{P}$ & Roof exhaust stack \\
\hline$Y-9204-4-A(02)$ & $01-1020-56$ & $032416 \mathrm{P}$ & Wash tank \\
\hline$Y-9204-4-A(03)$ & $01-1020-56$ & $032416 \mathrm{P}$ & Quench tanks \\
\hline$Y-9204-4-A(04)$ & $01-1020-56$ & $032416 \mathrm{P}$ & 1,000 -ton press \\
\hline$Y-9204-4-A(05)$ & $01-1020-56$ & $032416 \mathrm{P}$ & 7,500 -ton press \\
\hline$Y-9204-4-A(06)$ & $01-1020-56$ & $032416 \mathrm{P}$ & Exhaust from press pit area \\
\hline$Y-9204-4-A(07)$ & $01-1020-56$ & $032416 \mathrm{P}$ & Plasma torch cutting machine \\
\hline$Y-9204-4-A(08)$ & $01-1020-56$ & $032416 \mathrm{P}$ & Vacuum quench fumace \\
\hline$Y-9204-4-A(09)$ & $01-1020-56$ & $032416 \mathrm{P}$ & Ingot cooler \\
\hline$Y-9204-4-A(10)$ & $01-1020-56$ & $032416 \mathrm{P}$ & Exhaust from lathe \\
\hline$Y-9204-4-A(11)$ & $01-1020-56$ & $032416 \mathrm{P}$ & Grinding facility \\
\hline$Y-9204-4-A(12)$ & $01-1020-56$ & $032416 \mathrm{P}$ & Dye penetrant \\
\hline$Y-9204-4-A(13)$ & $01-1020-56$ & $032416 \mathrm{P}$ & Salt baths \\
\hline$Y-92044-A(14)$ & $01-1020-56$ & $032416 \mathrm{P}$ & Quench tanks \\
\hline$Y-9204-4-A(15)$ & $01-1020-56$ & $032416 \mathrm{P}$ & Preheat furnace exhaust \\
\hline
\end{tabular}


Table A.2 (continued)

\begin{tabular}{|c|c|c|c|}
\hline $\begin{array}{l}\text { Y-12 Plant } \\
\text { source number }\end{array}$ & $\begin{array}{l}\text { Emission source } \\
\text { reference } \\
\text { number }\end{array}$ & $\begin{array}{l}\text { Permit } \\
\text { number }\end{array}$ & Sou ce description \\
\hline$Y-9204-4-A(17)$ & $01-1020-56$ & $032416 \mathrm{P}$ & Oven exhaust \\
\hline$Y-9204-4-A(18)$ & $01-1020-56$ & $032416 \mathrm{P}$ & Vacuum fumace giench chamber \\
\hline$Y-9204-A(19)$ & $01-1020-56$ & $032416 \mathrm{P}$ & 7.500 -ton press and 1.500 -ton press \\
\hline$Y-9204-4-A(88)$ & $01-1020-56$ & $032416 \mathrm{P}$ & Grit blast system \\
\hline$Y-9204-4-B(481)$ & $01-0020-72$ & $730313 P$ & Exhaust from machining operation \\
\hline$Y-9204-4-B(482)$ & $01-0020-72$ & $730313 P$ & Exhaust from hood, reclamation area \\
\hline$Y-9204-4-B(484)$ & $01-0020-72$ & $730313 P$ & Rolling mill, first floor assembly \\
\hline$Y-9204-4-B(485)$ & $01-0020-72$ & $730313 P$ & Exhaust from paint hood \\
\hline$Y-9204-4-B(486)$ & $01-0020-72$ & $730313 P$ & Filtering exhaust from paint booths \\
\hline $\mathrm{Y}-9204-4-\mathrm{B}(488)$ & $01-0020-72$ & $730313 \mathrm{P}$ & Laboratory hoods, first floct \\
\hline$Y-9204-4-B(489)$ & $01-0020-72$ & $730313 P$ & Laboratory hoods, reclamation area \\
\hline$Y-9204-4-B(490)$ & $01-0020-72$ & $730313 P$ & Assembly process, first floor \\
\hline$Y-9204-4-B(491)$ & $01-0020-72$ & $730313 \mathrm{P}$ & Assembly process, first floor \\
\hline$Y-9204-4-D(1)$ & $01-1020-35$ & $032584 \mathrm{P}$ & Product certification cleaning \\
\hline$Y-9204-4-E(258)$ & $01-0020-33$ & $030819 \mathrm{P}$ & Plating equipment \\
\hline$Y-9204-4-E(259)$ & s01-0020-33 & $025002 \mathrm{P}$ & Plating equipment \\
\hline$Y-9204-4-E(260)$ & S01-0020-33 & $025002 \mathrm{P}$ & Plating equipment \\
\hline$Y-92044-E(261)$ & S01-0020-33 & $025002 \mathrm{P}$ & Plating equipment \\
\hline$Y-9206-A(01)$ & $01-0020-48$ & $012892 \mathrm{P}$ & 8,500 -gal storage tank. tank farm \\
\hline$Y-9206-A(02)$ & $01-0020-48$ & $012892 \mathrm{P}$ & 12,800 -gal storage $\tan k, \tan k$ farm \\
\hline$Y-9206-A(03)$ & $01-0020-48$ & $012892 P$ & 10,000-gal storage tank, tank farm \\
\hline $\mathrm{Y}-9206-\mathrm{B}(013)$ & $01-0020-03$ & $731689 \mathrm{P}$ & South stack, incinerator \\
\hline $\mathrm{Y}-9206-\mathrm{B}(015)$ & $01-0020-03$ & $731689 \mathrm{P}$ & West stack \\
\hline$Y-9206-B(016)$ & $01-0020-03$ & $731689 \mathrm{P}$ & Dissolving hood \\
\hline$Y-9206-B(017)$ & $01-0020-03$ & $731689 \mathrm{P}$ & Steam cleaning hoods \\
\hline$Y-9206-B(115)$ & $01-0020-03$ & $731689 \mathrm{P}$ & Reduction fluid bed \\
\hline$Y-9206-B(135)$ & $01-0020-03$ & $731689 \mathrm{P}$ & Air emission control scrubber stack \\
\hline$Y-9206-B(136)$ & $01-0020-03$ & $731689 \mathrm{P}$ & Air emission control consolidated stack \\
\hline$Y-9206-B(208)$ & $01-0020-03$ & $731689 \mathrm{P}$ & Conversion fluid bed \\
\hline$Y-9206-B(209)$ & $01-0020-03$ & $731689 \mathrm{P}$ & HF purge vent \\
\hline $\mathrm{Y}-9206-\mathrm{B}(210)$ & $01-0020-03$ & $731689 \mathrm{P}$ & Chemical makeup area \\
\hline$Y-9206-B(211)$ & $01-0020-03$ & $731689 \mathrm{P}$ & Hoods 29 and 30 \\
\hline Y-9206-B(212) & $01-0020-03$ & $731689 P$ & Dry vacuum system \\
\hline$Y-9206-C(01)$ & $01-1020-24$ & $730316 \mathrm{P}$ & Classified \\
\hline$Y-9206-C(02)$ & $01-1020-24$ & $730316 \mathrm{P}$ & Classified \\
\hline Y-9206-E (NEW) & $01-1020-24$ & $730316 P$ & Classified \\
\hline$Y-9212-A(019)$ & $01-1020-72$ & $033581 P$ & Filter exhaust, denitrator, fluid bed, etc. \\
\hline$Y-9212-A(021)$ & $01-1020-72$ & $033581 P$ & Centrifuges, liquid pour-up station, etc. \\
\hline$Y .9212-A(022)$ & $01-1020-72$ & $033581 \mathrm{P}$ & Reduction salvage, crusher and hopper \\
\hline$Y-9212-A(024)$ & $01-1020-72$ & $033581 P$ & Caiciner and dry vacuum system enciosure \\
\hline$Y-9212-A(025)$ & $01-1020-72$ & $033581 \mathrm{~F}$ & Denitrator area and fluid bed room enclosure \\
\hline$Y-9212-A(027)$ & $01-1020-72$ & $033581 \mathrm{P}$ & D-wing, Rm 1010 hoods, Rms 26 and 29 \\
\hline$Y-9212-A(028)$ & $01-1020-72$ & $033581 \mathrm{P}$ & $\begin{array}{l}\text { Reduction, shear, and Rm 1010, enriched uranium } \\
\text { conversion facility }\end{array}$ \\
\hline$Y-9212-A(033)$ & $01-1020-72$ & $033581 P$ & Head house equipment and incinerator \\
\hline
\end{tabular}


Table A.2 (continued)

\begin{tabular}{|c|c|c|c|}
\hline $\begin{array}{l}\text { Y.12 Plant } \\
\text { source number }\end{array}$ & $\begin{array}{c}\text { Emission source } \\
\text { reference } \\
\text { number }\end{array}$ & $\begin{array}{l}\text { Permit } \\
\text { number }\end{array}$ & Source description \\
\hline$Y-9212-A(036)$ & $01-1020-72$ & $033581 \mathrm{P}$ & East scrubber ( $\mathrm{C}-1$ wing) exhaust \\
\hline$Y-9212-A(040)$ & $01-1020-72$ & $033581 \mathrm{P}$ & B-1 sampling lab hoods \\
\hline$Y-9212-A(042)$ & $01-1020-72$ & $033581 \mathrm{P}$ & Chloride removal system $/ C$ - 1 wing process exhaust \\
\hline$Y-9212-A(050)$ & $01-1020-72$ & $033581 \mathrm{P}$ & C-1 chip burner, enciosures. load hoods \\
\hline$Y-9212-A(111)$ & $01-1020-72$ & $033581 \mathrm{P}$ & Reduction fiuid beds \\
\hline $\mathrm{Y}-9212-\mathrm{A}(112)$ & $01-1020-72$ & 033581P & Conversion fluid beds \\
\hline $\mathrm{Y}-9212-\mathrm{A}(132)$ & $01-1020-72$ & $033581 P$ & Decontamination facility \\
\hline$Y-9212-A(430)$ & $01-1020-72$ & $033581 \mathrm{P}$ & HF dock cylinder/vaporizer purge vent \\
\hline$Y-9212-A(431)$ & $01-1020-72$ & $033581 \mathrm{P}$ & $\mathrm{N}_{2} \mathrm{O}_{4}$ cylinder purge vent \\
\hline Y-9212-A(432) & $01-1020-72$ & $033581 \mathrm{P}$ & Muffle fumaces (2) vent, $\operatorname{Rm} 29$ \\
\hline$Y-9212-A(500)$ & $01-1020-72$ & $033581 \mathrm{P}$ & Primary extraction vent \\
\hline Y-9212-A(501) & $01-1020-72$ & $033581 \mathrm{P}$ & Secondary extraction yent \\
\hline $\mathrm{Y}-9212-\mathrm{B}(01)$ & $01-0020-02$ & $730301 \mathrm{P}$ & $U$ metal drying and briquetting process \\
\hline$Y-9212-B(02)$ & $01-0020-02$ & $730301 P$ & Exhaust from chip washing and drying \\
\hline $\mathrm{Y}-9212-\mathrm{B}(03)$ & $01-0020-02$ & $730301 P$ & E-wing machine shop \\
\hline $\mathrm{Y}-9212-\mathrm{B}(04)$ & 01-0020-02 & $730301 P$ & $\mathrm{U}$ metal and $\mathrm{U}$ metal alloy casting \\
\hline$Y-9212-C(01)$ & $01-0020-05$ & $025984 \mathrm{P}$ & Drum receiving/sampling hood and glove box \\
\hline$Y-9212-C(02)$ & $01-0020-05$ & $025984 \mathrm{P}$ & Tube fumace/gas purge vents \\
\hline$Y-9212-C(03)$ & $01-0020-05$ & $025984 \mathrm{P}$ & Sampling hoods and safe bottles/Rm 1022 \\
\hline$Y-9212-C(04)$ & $01-0020-05$ & $025984 \mathrm{P}$ & Dry hoods/Rm 1021 \\
\hline $\mathrm{Y}-9212-\mathrm{C}(05)$ & $01-0020-05$ & $025984 \mathrm{P}$ & Dissolver tray hoods/Rm 1021 \\
\hline $\mathrm{Y}-9212-\mathrm{C}(06)$ & $01-0020-05$ & 025984P & Dissolver hood \\
\hline$Y-9212-C(07)$ & $01-0020-05$ & $025984 \mathrm{P}$ & Dissolver trays/scrubber \\
\hline$Y-9212 \cdot C(08)$ & $01-0020-05$ & $025984 \mathrm{P}$ & Shear and saw hood/Rm 1021 \\
\hline$Y-9212-C(09)$ & $01-0020-05$ & $025984 \mathrm{P}$ & Precipitation process \\
\hline$Y-9212-F(01)$ & $01-1020-49$ & $730321 \mathrm{P}$ & Two deburr benches, hood exhaust, A-wing \\
\hline$Y-9212-F(02)$ & $01-1020-49$ & $730321 \mathrm{P}$ & Two deburr benches, hood exhaust, A-wing \\
\hline$Y-9212-F(03)$ & $01-1020-49$ & $730321 \mathrm{P}$ & Machining, hood exhaust, A-wing \\
\hline$Y-9212-F(04)$ & $01-1020-49$ & $730321 P$ & Machining, hood exhaust, A-wing \\
\hline$Y-9212-F(05)$ & $01-1020-49$ & $730321 \mathrm{P}$ & Machining, hood exhaust, A-wing \\
\hline$Y-9212-G(01)$ & $01-1020-47$ & 028435P & Seal-peel pot \\
\hline $\mathrm{Y}-9215-\mathrm{A}(01)$ & $01-0020-37$ & $731839 \mathrm{P}$ & Machine shop hood exhaust, M-wing \\
\hline $\mathrm{Y}-9215-\mathrm{B}(02)$ & $01-0020-38$ & $012880 \mathrm{P}$ & Turco pretreat spray hood \\
\hline$Y-9215-B(1)$ & $01-1020-51$ & $732125 \mathrm{P}$ & O-wing metalworking operations \\
\hline $\mathrm{Y}-9215-\mathrm{B}(2)$ & $01-1020-51$ & $732125 \mathrm{P}$ & O-wing metalworking operations \\
\hline $\mathrm{Y}-9215-\mathrm{B}(4)$ & $01-1020-51$ & $732125 \mathrm{P}$ & O-wing metalworking operations \\
\hline $\mathrm{Y}-9215-\mathrm{B}(6)$ & $01-1020-51$ & $732125 \mathrm{P}$ & O-wing metalworking operations \\
\hline $\mathrm{Y}-9215-\mathrm{C}(02)$ & $01-1020-52$ & $025948 \mathrm{P}$ & Hydroform exhaust \\
\hline$Y-9215-C(03)$ & $01-1020-52$ & 730323P & Vapor biaster/metal cleaner \\
\hline$Y-9215-C(10)$ & $01-1020-52$ & $730323 \mathrm{P}$ & Nickel plating, metal working exhaust \\
\hline$Y-9215-C(11)$ & $01-1020-52$ & $730323 P$ & Exhaust \\
\hline$Y-9215-C(17)$ & $01-1020-52$ & $730323 \mathrm{P}$ & Rolling mill \\
\hline$Y-9215-C(19)$ & $01-1020-52$ & $730323 \mathrm{P}$ & Electric annealing oven \\
\hline$Y-9215-D(12)$ & $01-1020-53$ & $025966 \mathrm{P}$ & Rolling mill exhaust \\
\hline$Y-9215-D(13)$ & $01-1020-53$ & $025966 \mathrm{P}$ & Hood exhaust \\
\hline
\end{tabular}


Table A.2 (continued)

\begin{tabular}{|c|c|c|c|}
\hline $\begin{array}{c}\text { Y-12 Plant } \\
\text { source number }\end{array}$ & $\begin{array}{c}\text { Emission source } \\
\text { reference } \\
\text { number }\end{array}$ & $\begin{array}{l}\text { Permit } \\
\text { number }\end{array}$ & Source description \\
\hline$Y-9215-D(14)$ & $01-1020-53$ & $025966 \mathrm{P}$ & Exhaust from rolling mill \\
\hline$Y-9215-D(I S)$ & $01-1020-53$ & $025966 \mathrm{P}$ & Turret lathe and shear exhaust \\
\hline$Y-9215-E(6)$ & $01-1020-54$ & $025972 \mathrm{P}$ & Lab hood \\
\hline$Y-9215-E(7)$ & $01-1020-54$ & $025972 \mathrm{P}$ & Lab hoods \\
\hline$Y-9215-E(8)$ & $01 \cdot 1020-54$ & $025972 P$ & Lab hoods \\
\hline$Y-9401-2-A(205)$ & $01-0020-88$ & $730286 \mathrm{P}$ & Plating equipment \\
\hline$Y-9401-2-A(220)$ & $01-0020-88$ & $730286 \mathrm{P}$ & Plating equipment \\
\hline$Y-9401-2-A(221)$ & $01-0020-88$ & $730286 \mathrm{P}$ & Plating equipment \\
\hline$Y-9401-2-A(222)$ & $01-0020-88$ & $730286 \mathrm{P}$ & Plating equipment \\
\hline$Y-9401-2-A(223)$ & $01-0020-88$ & $730286 \mathrm{P}$ & Plating equipment \\
\hline$Y-9401-2-A(224)$ & $01-0020-88$ & $730286 \mathrm{P}$ & Plating equipment \\
\hline$Y-9401-2-A(225)$ & $01-0020-88$ & $730286 \mathrm{P}$ & Plating equipment \\
\hline$Y-9401-2-A(226)$ & $01-0020-88$ & $730286 \mathrm{P}$ & Plating equipment \\
\hline$Y-9401-2-A(227)$ & $01-0020-88$ & $730286 \mathrm{P}$ & Plating equipment \\
\hline$Y-9401-2-A(228)$ & $01-0020-88$ & $730286 \mathrm{P}$ & Plaring equipmen \\
\hline$Y-9401-2-A(229)$ & $01-0020-88$ & $730286 \mathrm{P}$ & Plating equipment \\
\hline$Y-9401-2-A(230)$ & $01-0020-88$ & $730286 \mathrm{P}$ & Plating equipment \\
\hline$Y-9401-2-A(231)$ & $01-0020-88$ & $730286 \mathrm{P}$ & Plating equipment \\
\hline$Y-9401-2-A(232)$ & $01-0020-88$ & $730286 \mathrm{P}$ & Plating equipment \\
\hline$Y-9401-2-A(233)$ & $01-0020-88$ & $730286 \mathrm{P}$ & Plating equipment \\
\hline$Y-9401-2-A(234)$ & $01-0020-88$ & $730286 \mathrm{P}$ & Plating equipment \\
\hline$Y-9401-2-A(235)$ & $01-0020 m 88$ & $730286 \mathrm{P}$ & Plating equipment \\
\hline$Y-9401-3-A$ & $01-1020-31$ & $029322 \mathrm{~F}$ & Coal-fired boiler \\
\hline$Y-9401-3-B(170)$ & $01-1020-31$ & $029322 \mathrm{~F}$ & Coal-fired boiler \\
\hline$Y-9401-3-C$ & $01-1020-31$ & $029322 \mathrm{~F}$ & Coal-fired boiler \\
\hline$Y-9401-3-D(171)$ & $01-1020-31$ & $029322 F$ & Coal-fired boiler \\
\hline$Y \cdot 9401-3-H(01)[9616-10]$ & $01-1020-62$ & $029280 \mathrm{P}$ & 20,000 -gal sulfuric acid storage tank \\
\hline $\mathrm{Y}-9.401-5-\mathrm{A}(01)$ & $01-0020-92$ & $026108 \mathrm{P}$ & Uranium chip oxidizer \\
\hline$Y-9404-11-A(1)$ & $01-1020-81$ & $028426 \mathrm{P}$ & Purisication plant \\
\hline$Y-9404-11-A(2)$ & $01-1020-81$ & $028426 \mathrm{P}$ & Purification plant \\
\hline$Y-9404-11-A(3)$ & $01-1020-81$ & $028426 \mathrm{P}$ & Purification plant \\
\hline$Y-9404-11-A(4)$ & $01-1020-81$ & $028426 \mathrm{P}$ & Purification plant \\
\hline$Y-9404-5-B(02)$ & $01-0020-25$ & $012866 \mathrm{P}$ & Spray room exhaust \\
\hline$Y-9404-5-B(03)$ & $01-0020-25$ & $012866 \mathrm{P}$ & Spray booth \\
\hline Y-9404-7-FUG-A(00) & $01-1020-89$ & 034295P & PCB drum storage facility \\
\hline$Y-9404-9 . C(03)$ & $01-0020-40$ & $012882 \mathrm{P}$ & PVC curing ovens \\
\hline$Y-9404-9-D(04)$ & $01-0020-40$ & $012882 P$ & PVC curing ovens \\
\hline$Y-9404-9-E(05)$ & $01-0020-40$ & $012882 \mathrm{P}$ & PVC curing ovens \\
\hline$Y-9616-7-A(459)$ & $01-1020-74$ & 033498P & West end treatment storage tank \\
\hline$Y-9616-7-A(460)$ & $01-1020-74$ & $033498 \mathrm{P}$ & West end treatment storage tank \\
\hline$Y-9616-7-A(461)$ & $01-1020-74$ & 033498P & West end treatment storage tank \\
\hline$Y-9616-7-A(462)$ & $01-1020-74$ & $033498 \mathrm{P}$ & West end treatment storage tank \\
\hline$Y-9616-7-A(463)$ & $01-1020-74$ & $033498 \mathrm{P}$ & West end treatment vent, reactor vessel \\
\hline Y-9616-7-A(464) & $01-1020-74$ & 033498P & West end treatment storage tank \\
\hline Y-9616-7-A(465) & $01-1020-74$ & $033498 \mathrm{P}$ & West end treatment vent, degasifier unit \\
\hline
\end{tabular}


Table A.2 (continued)

\begin{tabular}{|c|c|c|c|}
\hline $\begin{array}{l}\text { Y-12 Plant } \\
\text { source number }\end{array}$ & $\begin{array}{l}\text { Emission source } \\
\text { reference } \\
\text { number }\end{array}$ & $\begin{array}{l}\text { Permit } \\
\text { number }\end{array}$ & Source description \\
\hline$Y-9616-7-A(466)$ & $01-1020-74$ & C33498P & West end treatment storage tank \\
\hline$Y-9616-7-A(467)$ & $01-1020-74$ & $033498 \mathrm{P}$ & West end treatment storage tank \\
\hline$Y-9616-7-A(468)$ & $01-1020-74$ & 033498P & West end treatment storage tank \\
\hline$Y-9616-7-A(469)$ & $01-1020-74$ & 033498P & West end treatment vent, lime silo \\
\hline$Y-9616-7-A(470)$ & $01-1020-74$ & $033498 \mathrm{P}$ & West end treatment storage tank \\
\hline$Y-9616-7-A(1)$ & $01-1020-80$ & $031254 \mathrm{P}$ & Vent from air stripper \\
\hline$Y-9616-7-B(650)$ & $01-1020-74$ & 033498P & Biological treatment tanks \\
\hline$Y-9616-7-B(651)$ & $01-1020-74$ & 033498P & Biological treatment tanks \\
\hline$Y-9616-7-B(653)$ & $01-1020-74$ & 033498P & Biological treatment tanks \\
\hline$Y-9616-7-B(654)$ & $01.1020-74$ & 033498P & Biological treatment tanks \\
\hline$Y-9616-7-B(655)$ & $01-1020-74$ & 033498P & Biological treatment tanks \\
\hline$Y-9616-7-B(655)$ & $01-1020-74$ & $033498 \mathrm{P}$ & Biological treatment tanks \\
\hline$Y-9616-7-B(656)$ & $01-1020-74$ & 033498P & Solids storage tanks \\
\hline$Y-9616-7-B(657)$ & $01-1020-74$ & 033498P & Solids storage tanks \\
\hline$Y-9616-7-B(658)$ & $01-1020-74$ & 033498P & Solids storage tanks \\
\hline$Y-9616-7-B(659)$ & $01-1020-74$ & 033498P & Solids storage tanks \\
\hline$Y-9616-7-B(660)$ & $01-1020-74$ & 033498P & Solids storage tanks \\
\hline$Y-9616-7-B(661)$ & $01-1020-74$ & 033498P & Solids storage tanks \\
\hline$Y-9616-7-B(662)$ & $01-1020-74$ & 033498P & Solids storage tanks \\
\hline$Y-9620-2 A$ & $01-0020-50$ & $012894 \mathrm{P}$ & Storage tank \\
\hline$Y-9623-A(01)$ & $01-1020-25$ & $025970 \mathrm{P}$ & Vent from reactor vessel \\
\hline$Y-9623-A(02)$ & $01-1020-25$ & $025970 \mathrm{P}$ & Vent from eight tanks \\
\hline$Y-9623-A(03)$ & $01-1020-25$ & $025970 \mathrm{P}$ & Lab hood \\
\hline$Y-9623-A(04)$ & $01-1020-25$ & 025970P & Lime silo \\
\hline$Y-9623-A(05)$ & $01-1020-25$ & $025970 \mathrm{P}$ & Storage tank \\
\hline$Y-9623-A(06)$ & $01-1020-25$ & 025970P & Storage tank \\
\hline Y-9720-12-FUG-A(OO) & $01-1020-89$ & 034295P & Nonspecial nuclear material warehouse \\
\hline$Y-9720-19-A(01)$ & $01-0020-41$ & $012885 \mathrm{P}$ & Curing oven \\
\hline$Y-9720-19-C(01)$ & $01-0020-23$ & $012864 \mathrm{P}$ & Teflon sintering oven \\
\hline$Y-9720-19-D(03)$ & $01-0020-27$ & $012869 \mathrm{P}$ & Plastics spray booth \\
\hline Y-9720-20-A(01) & $01-1020-39$ & $025971 \mathrm{P}$ & Small maintenance shop, fabric filter \\
\hline$Y-9720-25-F U G-A(00)$ & $01-1020-89$ & 034295P & Classified waste storage facility \\
\hline Y-9720-28-FUG-A(00) & $01-1020-89$ & 034295P & Drum storage warehouse \\
\hline Y-9720-31-FUG-A-(00) & $01-1020-89$ & 034295P & RCRA and mixed waste storage and staging facility \\
\hline$Y-9720-32-A(201)$ & $01-0020-42$ & 032547P & Classified waste shredder \\
\hline$Y-9720-44-F U G-A(00)$ & $01-1020-89$ & $034295 \mathrm{P}$ & Low-level waste storage pad \\
\hline$Y-9720-5-A(130)$ & $01-1020-75$ & $031958 \mathrm{P}$ & Hood at $9720-5$ east end \\
\hline Y-9720-58-FUG-A(00) & $01-1020-89$ & $034295 \mathrm{P}$ & PCB and RCRA staging and storage facility \\
\hline$Y-9720-6-A(1)$ & $01-0020-26$ & $012867 \mathrm{P}$ & Paint spray booth \\
\hline$Y-9720-6-A(2)$ & $01-0020-26$ & $012867 \mathrm{P}$ & Paint spray booth \\
\hline$Y-9720-6-B(01)$ & $01-0020-75$ & $015154 \mathrm{P}$ & Wood working operation \\
\hline$Y-9720-6-B(03)$ & $01-0020-26$ & $012867 \mathrm{P}$ & Drying oven \\
\hline$Y-9720-6-E(01)$ & $01-\infty 020-83$ & $016548 \mathrm{P}$ & Clean room laboratory \\
\hline$Y-9720-60-F U G-A(00)$ & $01-1020-89$ & $034295 P$ & DARA solids siorage unit \\
\hline Y-9720-9-FUG-A(00) & $01-1020-89$ & $034295 \mathrm{P}$ & PCB and RCRA hazardous waste drum storage facility \\
\hline
\end{tabular}


Table A.2 (continued)

\begin{tabular}{|c|c|c|c|}
\hline $\begin{array}{l}\text { Y-12 Plant } \\
\text { source number }\end{array}$ & $\begin{array}{c}\text { Emission source } \\
\text { reference } \\
\text { number }\end{array}$ & $\begin{array}{l}\text { Permit } \\
\text { number }\end{array}$ & Source description \\
\hline$Y \cdot 9737-A(01)$ & $01-0020-22$ & $012863 \mathrm{P}$ & Oven \\
\hline $\mathrm{Y}-9738-\mathrm{A}(576)$ & $01-0020-14$ & $025975 \mathrm{P}$ & Sandblaster \\
\hline$Y-9738-A(577)$ & $01-0020-14$ & $025975 \mathrm{P}$ & Hood with fan \\
\hline Y-9738-A(578) & $01-0020-14$ & $025975 \mathrm{P}$ & Sand blaster \\
\hline$Y-9738-A(579)$ & $01-0020-14$ & $025975 \mathrm{P}$ & Hood with fan \\
\hline Y-9738-A(580) & $01-0020-14$ & $025975 \mathrm{P}$ & Hood with fan \\
\hline $\mathrm{Y}-9739-\mathrm{A}(01)$ & $01-1020-78$ & $028105 P$ & Print fold diazo blueprint copier/Rm 160 \\
\hline$Y-9739-B(02)$ & $01-1020-78$ & $028105 \mathrm{P}$ & Print fold diazo blueprint copier/Rm 174 \\
\hline$Y-9767-4-A(01)$ & $01-0020-35$ & $012877 \mathrm{P}$ & Chilled water circulating system \\
\hline$Y-9808-A$ & $01-0020-77$ & $015156 \mathrm{P}$ & Carpenter shop \\
\hline$Y-9808-A(01)$ & $01-1020-22$ & $026109 \mathrm{P}$ & Spray booth \\
\hline $\mathrm{Y}-9809 \cdot \mathrm{A}(01)$ & $01-0020-93$ & 025899P & Oxide storage vaults \\
\hline Y-9811-1-FUG-B(00) & $01-1020-89$ & $034295 \mathrm{P}$ & Waste oil/solvent drum storage facility (OD-8) \\
\hline$Y-9811-1-A(1)$ & $01-1020-95$ & $731997 \mathrm{P}$ & Waste oil/storage bulk storage facility (OD-7) \\
\hline$Y-9811-1-A(2)$ & $01-1020-95$ & $731997 \mathrm{P}$ & Waste oil/storage bulk storage facility (OD-7) \\
\hline$Y-9811-1-A(3)$ & $01-1020-95$ & $731997 \mathrm{P}$ & Waste oil/storage bulk storage facility (OD.7) \\
\hline$Y-9811-1-A(4)$ & $01-1020-95$ & $731997 \mathrm{P}$ & Waste oil/storage bulk storage facility (OD-7) \\
\hline$Y-981 \mathrm{i}-1-\mathrm{A}(5)$ & $01-1020-95$ & $731997 \mathrm{P}$ & Waste oil/storage bulk storage facility (OD-7) \\
\hline$Y-9811-1-A(6)$ & $01-1020-95$ & $731997 \mathrm{P}$ & Waste oil/storage bulk storage facility (OD-7) \\
\hline$Y-9811-1-A(7)$ & $01-1020-95$ & $731997 \mathrm{P}$ & Waste oil/storage bulk storage facility (OD-7) \\
\hline$Y-9811-6-A(1)$ & $01-1020-82$ & $029415 \mathrm{P}$ & Dry ash handling system \\
\hline$Y-9811-8-A(01)$ & $01-1020-63$ & $032988 \mathrm{P}$ & Waste oil/solvent storage facility (OD-9) \\
\hline$Y-9811-8-A(02)$ & $01-1020-63$ & $032988 \mathrm{P}$ & Waste oil/solvent storage facility (OD-9) \\
\hline$Y \cdot 9811-8-A(03)$ & $01-1020-63$ & $032988 \mathrm{P}$ & Waste oil/solvent storage facility (OD-9) \\
\hline$Y-9811-8-A(04)$ & $01-1020-63$ & $032988 \mathrm{P}$ & Waste oil/solvent storage facility (OD-9) \\
\hline$Y-S(811-\bar{s}-A(05)$ & $01-1020-63$ & 032988P & Waste oil/solvent storage facility (OD-9) \\
\hline $\mathrm{Y}-5811-\mathrm{B}(02)$ & $01-1020-45$ & $025903 \mathrm{P}$ & Incinerator \\
\hline$Y-9812-A-(287)$ & $01-1020-29$ & $033051 \mathrm{P}$ & 12,115-gal storage tank \\
\hline$Y-9812-A-(288)$ & $01.1020-29$ & $033051 \mathrm{P}$ & 12,133-gal storage tank \\
\hline$Y-9812-A-(289)$ & $01-1020-29$ & $033051 P$ & 4,876-gal storage tank \\
\hline$Y-9815-A(03)$ & $01-0020-11$ & $025895 \mathrm{P}$ & Vent from reactors \\
\hline$Y-9815-A(04)$ & $01-0020-11$ & $025895 \mathrm{P}$ & 12,000 -gal storage tank \\
\hline$Y-9815-A(05)$ & $01-0020-11$ & $025895 \mathrm{P}$ & 4,500 -gal storage tank \\
\hline$Y-9815-A(06)$ & $01-0020-11$ & $025895 \mathrm{P}$ & 4,400 -gal storage tank \\
\hline$Y-9815-A(07)$ & $01-0020-11$ & $025895 \mathrm{P}$ & $1,800-\mathrm{gal}$ storage tank \\
\hline$Y-9815-A(08)$ & $01-0020-11$ & $025895 \mathrm{P}$ & Two 2,200-gal storage tanks \\
\hline$Y-9818-A(01)$ & $01-0020-12$ & $025965 \mathrm{P}$ & Hot well seal tank \\
\hline $\mathrm{Y}-9818-\mathrm{A}(02)$ & $01-0020-12$ & $025965 \mathrm{P}$ & 11 storage tanks, nitric acid recovery \\
\hline$Y-9818-A(03)$ & $01-0020-12$ & $025965 \mathrm{P}$ & Two bioreactor tanks/ozonation tanks \\
\hline$Y-9818-A(04)$ & $01-0020-12$ & $025965 \mathrm{P}$ & Basement exhaust \\
\hline$Y-9818-A(05)$ & $01-0020-12$ & $025965 \mathrm{P}$ & Nitric acid supply line vent \\
\hline$Y-9818-A(06)$ & $0:-0020-12$ & $025965 \mathrm{P}$ & Ozone generator/area exhaust \\
\hline$Y-9818-A(07)$ & $01-0020-12$ & $025965 P$ & 10,000 -gal storage tank \\
\hline$Y-9818-A(08)$ & $01-0020-12$ & $025965 \mathrm{P}$ & 10,000-gal denitrification feed tank \\
\hline$Y-9818-A(09)$ & $01-0020-12$ & $025965 \mathrm{P}$ & 4,000 -gal nitrate receiving tank \\
\hline
\end{tabular}


Table A.2 (continued)

\begin{tabular}{|c|c|c|c|}
\hline $\begin{array}{l}\text { Y-12 Plant } \\
\text { source number }\end{array}$ & $\begin{array}{l}\text { Emission source } \\
\text { reference } \\
\text { number }\end{array}$ & $\begin{array}{l}\text { Permit } \\
\text { number }\end{array}$ & Source description \\
\hline$Y-9818-A(10)$ & $01-0020-12$ & $025965 \mathrm{P}$ & 10,000 -gal nitric acid waste tank \\
\hline$Y-9818-A(11)$ & $01-0020-12$ & $025965 \mathrm{P}$ & 10,000 -gal nitric acid waste tank \\
\hline$Y-9818-A(12)$ & $01-0020-12$ & $025965 \mathrm{P}$ & 10,000-gal nitric acid waste tank \\
\hline$Y \cdot 9828-6-F U G-A(00)$ & $01-1020-89$ & $034295 \mathrm{P}$ & Trash monitoring station \\
\hline$Y-9929-F(01)$ & M01-0020-39 & $012881 P$ & Open yard coal storage \\
\hline Y-9983-74-FUG-A(00) & $01-1020-89$ & $034295 \mathrm{P}$ & Old salvage yard \\
\hline Y-9998-A(01) & $01-0020-13$ & $025957 \mathrm{P}$ & Swaging machines \\
\hline$Y-9998-A(02)$ & $01-0020-13$ & $025957 \mathrm{P}$ & Swaging machines \\
\hline Y-9998-A(03) & $01-0020-13$ & $025957 \mathrm{P}$ & Furnaces \\
\hline$Y-9998-A(04)$ & $01-0020-13$ & $025957 \mathrm{P}$ & Nitric acid pickling tanks \\
\hline$Y-9998-A(05)$ & $01-0020-13$ & $025957 \mathrm{P}$ & Hood \\
\hline Y-9998-A(06) & $01-0020-13$ & $025957 \mathrm{P}$ & Foundry operations \\
\hline$Y-9998-B(1)$ & $01-1020-40$ & $026110 P$ & Machine shop \\
\hline Y-BCB-FUG-A(00) & $01-1020-89$ & $034295 \mathrm{P}$ & Bear Creek Burial Grounds \\
\hline Y-BCBG-NAK & $01-00020-00$ & 010002000 & Open burn for $\mathrm{NaK}$ \\
\hline Y-CSL-II-FUG-A(00) & $01-1020-89$ & 034295P & Y-12 Centralized Sanitary Landfill II \\
\hline Y-CWSF-FUG-A(00) & $01-1020-89$ & 034295P & Containerized Waste Storage Facility \\
\hline Y-IDY-FUG-A(00) & $01-1020-89$ & 034295P & Interim Drum Yard \\
\hline Y-IWF-FUG-A(00) & $01-1020-89$ & $034295 \mathrm{P}$ & Industrial Waste Landfill IV \\
\hline \multicolumn{4}{|c|}{ Part 1I. Construction permits at the Y-12 Plant } \\
\hline$Y-9201-1-A(01)$ & $01-0020-15$ & $730303 \mathrm{P}$ & Welding booths \\
\hline $\mathrm{Y}-9201-1-\mathrm{A}(02)$ & $01-0020-15$ & $730303 \mathrm{P}$ & Welding shop \\
\hline$Y-9201-1-A(04)$ & $01-0020-15$ & $730303 \mathrm{P}$ & Metal fabrication shop \\
\hline $\mathrm{Y}-9201-1-\mathrm{A}(05)$ & $01-0020-15$ & 730303P & Welding shop \\
\hline$Y-9201-1-A(15)$ & $01-0020-15$ & $730303 \mathrm{P}$ & Metal fabrication shop \\
\hline$Y-9201-1-B(16)$ & $01-0020-59$ & $730310 \mathrm{P}$ & Tool grinding machines \\
\hline$Y-9201-1-B(18)$ & $01-0020-59$ & $730310 \mathrm{P}$ & Sandblaster exhaust \\
\hline$Y-9201-1-C(278)$ & $01-0020-17$ & $730304 \mathrm{P}$ & Graphitic carbon machining \\
\hline$Y-9201-1-C(279)$ & $01-0020-17$ & $730304 \mathrm{P}$ & Graphitic carbon machining \\
\hline$Y-9201-1-D(09)$ & $01-0020-59$ & $730310 \mathrm{P}$ & Fabrication shop \\
\hline Y-9201-1-D(10) & $01-0020-59$ & $730310 \mathrm{P}$ & Fabrication shop \\
\hline$Y-9201-1-D(11)$ & $01-0020-59$ & $730310 \mathrm{P}$ & Fabrication shop \\
\hline$Y-9201-1-D(13)$ & $01-0020-59$ & $730310 \mathrm{P}$ & Metal grinders and milling machines \\
\hline$Y-9201-5-B(071)$ & $01-0020-21$ & $730305 \mathrm{P}$ & Machining operations $\mathrm{L} 5 \mathrm{~N}$ hood exhaust \\
\hline$Y-9201-5-B(072)$ & $01-0020-21$ & $730305 P$ & Vacuum inlets LSE machining shop \\
\hline $\mathrm{Y}-9201-5-\mathrm{B}(03)$ & $01-0020-21$ & $730305 \mathrm{P}$ & Rubber-gel potting hood exhaust \\
\hline$Y-9201-5-B(073)$ & $01-0020-21$ & $730305 \mathrm{P}$ & Palarite shop, machine exhaust \\
\hline$Y-9201-5-B(267)$ & $01-0020-21$ & $730305 \mathrm{P}$ & Tool grinding machines hood exhaust \\
\hline Y-9201-5-B(277) & $01-0020-21$ & $730305 \mathrm{P}$ & Cleaning hood, equipment service \\
\hline$Y-9201-5-B(273)$ & $01-0020-21$ & $730305 \mathrm{P}$ & Electrochemical machine, stainless steel \\
\hline$Y-9201-5-G(01)$ & $01-0020-44$ & $921689 \mathrm{P}$ & Arc melt \\
\hline$Y-9201-5-G(02)$ & $01-0020-44$ & $921689 \mathrm{P}$ & DeVilbiss hood \\
\hline$Y-9201-5-G(03)$ & $01-0020-44$ & $921689 \mathrm{P}$ & Nitric acid dip tanks \\
\hline$Y-9201-5-G(04)$ & $01-0020-4$ & $921689 \mathrm{P}$ & Acid pickling tanks \\
\hline$Y-9201-5-G(05)$ & $01-0020-44$ & $921689 \mathrm{P}$ & Abrasive saws \\
\hline
\end{tabular}


Tabie A.2 (continued)

\begin{tabular}{|c|c|c|c|}
\hline $\begin{array}{l}\text { Y-12 Plant } \\
\text { source number }\end{array}$ & $\begin{array}{c}\text { Emission source } \\
\text { reference } \\
\text { number }\end{array}$ & $\begin{array}{l}\text { Permit } \\
\text { number }\end{array}$ & Source description \\
\hline$Y-9201-5-G(06)$ & $01-0020-44$ & $921689 \mathrm{P}$ & Scrap metal recycle \\
\hline$Y-9201-5-G(07)$ & $01-0020-44$ & $921689 \mathrm{P}$ & Vapor degreaser \\
\hline$Y-9201-S N-A(67)$ & $01-1020-18$ & $730314 \mathrm{P}$ & Machine shop exhaust \\
\hline$Y-9202-A(162)$ & $01-1020-94$ & $931742 \mathrm{P}$ & Electrolytic deposition of uranium \\
\hline$Y-9203-B(108)$ & $01-1020-93$ & $931697 \mathrm{P}$ & Microanalytical lab \\
\hline$Y-9203-B(131)$ & $01-1020-93$ & $931697 \mathrm{P}$ & Microanalytical lab \\
\hline$Y-9203-B(137)$ & $01-1020-93$ & $931697 \mathrm{P}$ & Microanalytical lab \\
\hline$Y-9204-2-E(68)$ & $01-1020-55$ & $730328 \mathrm{P}$ & Oven \\
\hline$Y-9204-2-E(69)$ & $01-1020-55$ & $730328 \mathrm{P}$ & Oven \\
\hline$Y-9204-2-E(70)$ & $01-1020-55$ & $730328 \mathrm{P}$ & Tungsten screener \\
\hline$Y-9204-2-E(71)$ & $01-1020-55$ & $730328 \mathrm{P}$ & Dry box vent \\
\hline$Y-9204-2-E(72)$ & $01-1020-55$ & $730328 \mathrm{P}$ & Glove boxes \\
\hline$Y-9204-2-E(73)$ & $01-1020-55$ & $730328 \mathrm{P}$ & Material handling \\
\hline$Y-9204-2-E(74)$ & $01-1020-55$ & $730328 \mathrm{P}$ & Glove boxes \\
\hline$Y-9204-2-E(75)$ & $01-1020-55$ & $730328 \mathrm{P}$ & Outgassing/annealing ovens \\
\hline$Y-9204-2-E(76)$ & $01-1020-55$ & $730328 \mathrm{P}$ & Material handling \\
\hline$Y-9204-2-E(77)$ & $01-1020-55$ & $730328 \mathrm{P}$ & Glove boxes \\
\hline$Y-9204-2-E(78)$ & $01-1020-55$ & $730328 \mathrm{P}$ & Reactor unloading station \\
\hline$Y-9204-2-E(79)$ & $01-1020-55$ & $730328 \mathrm{P}$ & Reactor unloading station \\
\hline$Y-9204-2-E(80)$ & $01-1020-55$ & $730328 \mathrm{P}$ & Glove boxes \\
\hline$Y-9204-2-E(81)$ & $01-1020-55$ & $730328 \mathrm{P}$ & Vacuum pump \\
\hline$Y-9204-2 E-A(202)$ & $01-1020-91$ & $730938 \mathrm{P}$ & Positive ion accelerator \\
\hline$Y-9204-2 E-A(436)$ & $01-0020-68$ & $730312 \mathrm{P}$ & Oven \\
\hline$Y-9204-2 E-A(439)$ & $01-0020-68$ & $730312 \mathrm{P}$ & Hood exhaust \\
\hline$Y-9204-2 E-A(441)$ & $01-0020-68$ & $730312 \mathrm{P}$ & Hood \\
\hline$Y \cdot 9204-2 E-A(442)$ & $01-0020-68$ & $730312 \mathrm{P}$ & Hood \\
\hline$Y-9204-2 E-A(443)$ & $01-0020-68$ & $730312 \mathrm{P}$ & Degreaser \\
\hline$Y-9204-2 E-A(444)$ & $01-0020-68$ & $730312 \mathrm{p}$ & Electropolishers \\
\hline$Y-9204-2 E-A(445)$ & $01-0020-68$ & $730312 \mathrm{P}$ & Surface coating \\
\hline$Y-9204-2 E-A(448)$ & $01-0020-68$ & $730312 \mathrm{P}$ & Glove box \\
\hline$Y-9204-2 E-C(12)$ & $01-1020-55$ & $730328 \mathrm{P}$ & Machine shop hood exhaust, B2E \\
\hline Y-9204-2E-C(13) & $01-1020-55$ & $730328 \mathrm{P}$ & Machine shop hood exhaust, specimen shop \\
\hline$Y-9204-4-A(02)$ & $01-1020-56$ & $931629 \mathrm{P}$ & Wash tank \\
\hline$Y-9204-4-A(03)$ & $01-1020-56$ & $931629 \mathrm{P}$ & Quench tanks \\
\hline$Y-9204-4-A(04)$ & $01-1020-56$ & $931629 \mathrm{P}$ & 1.000-ton press \\
\hline$Y-9204-4-A(05)$ & $01-1020-56$ & $931629 \mathrm{P}$ & 7,500 -ton press \\
\hline$Y-9204-4-A(06)$ & $01-1020-56$ & $931629 \mathrm{P}$ & Exhaust from press pit area \\
\hline$Y-9204-4-A(07)$ & $01-1020-56$ & $931629 \mathrm{P}$ & Plasma torch cutting machine \\
\hline$Y-9204-4-A(08)$ & $01-1020-56$ & $931629 \mathrm{P}$ & Vacuum quench furnace \\
\hline$Y-9204-4-A(09)$ & $01-1020-56$ & $931629 \mathrm{P}$ & Ingot cooler \\
\hline$Y-9204-4-A(10)$ & $01-1020-56$ & $931629 \mathrm{P}$ & Exhaust from lathe \\
\hline$Y-9204-4-A(11)$ & $01-1020-56$ & $931629 \mathrm{P}$ & Grinding facility \\
\hline$Y-9204-4-A(12)$ & $01-1020-56$ & $931629 \mathrm{P}$ & Dye penetrant \\
\hline$Y-9204-4-A(13)$ & $01-1020-56$ & $931629 \mathrm{P}$ & Salt baths \\
\hline$Y-9204-4-A(14)$ & $01-1020-56$ & $931629 \mathrm{P}$ & Quench tanks \\
\hline
\end{tabular}


Table A.2 (continued)

\begin{tabular}{|c|c|c|c|}
\hline $\begin{array}{c}\text { Y-12 Plant } \\
\text { source number }\end{array}$ & $\begin{array}{c}\text { Emission source } \\
\text { reference } \\
\text { number }\end{array}$ & $\begin{array}{l}\text { Permit } \\
\text { number }\end{array}$ & Source description \\
\hline$Y-9204-4(15)$ & $01-1020-56$ & $931629 \mathrm{P}$ & Preheat fumace exhaust \\
\hline$Y-9204-4-A(17)$ & $01-1020-56$ & $931629 \mathrm{P}$ & Oven exhaust \\
\hline$Y-9204-4-A(18)$ & $01-1020-56$ & $931629 \mathrm{P}$ & Vacuum furnace quench chamber \\
\hline$Y-9204-4-A(19)$ & $01-1020-56$ & $931629 \mathrm{P}$ & 7,500 -ton press and 1,500 -ton press \\
\hline$Y-9204-4-A(88)$ & $01-1020-56$ & $931629 \mathrm{P}$ & Grit blast system \\
\hline$Y-9204-4-B(481)$ & $01-0020-72$ & $730313 P$ & Exhaust from machining operation \\
\hline$Y-9204-4-B(482)$ & $01-0020-72$ & $730313 \mathrm{P}$ & Exhaust from hood. reclamation area \\
\hline$Y-9204-4-B(484)$ & $01-0020-72$ & $730313 P$ & Rolling mill, first-floor assembly \\
\hline$Y-9204-4-B(485)$ & $01-0020-72$ & $730313 P$ & Exhaust from paint hood \\
\hline$Y-9204-4-B(486)$ & $01-0020-72$ & $730313 P$ & Filtering exhaust from paint booths \\
\hline$Y-9204-4-B(488)$ & $01-0020-72$ & $730313 \mathrm{P}$ & Laboratory hoods, first floor \\
\hline$Y-9204-4-B(489)$ & $01-0020-72$ & $730313 \mathrm{P}$ & Laboratory hoods, reclamation area \\
\hline$Y-9204-4-B(490)$ & $01-0020-72$ & $730313 \mathrm{P}$ & Assembly process, first floor \\
\hline$Y-9204-4-B(491)$ & $01-0020-72$ & $730313 \mathrm{P}$ & Assembly process, first floor \\
\hline$Y-92044-D(01)$ & $\dot{-}$ & $730317 \mathrm{P}$ & Exhaust hood \\
\hline$Y-9206-B(013)$ & $01-0020-03$ & $731689 \mathrm{P}$ & South stack, incinerator \\
\hline $\mathrm{Y}-9206-\mathrm{B}(015)$ & $01-0020-03$ & $731689 \mathrm{P}$ & West stack \\
\hline$Y \cdot 9206-B(016)$ & $01-\infty 020-03$ & $731689 \mathrm{P}$ & Dissolving hood \\
\hline$Y-9206-B(017)$ & $01-0020-03$ & $731689 \mathrm{P}$ & Steam cleaning hoods \\
\hline$Y-9206-B(115)$ & $01-0020-03$ & $731689 \mathrm{P}$ & Reduction fluid bed \\
\hline$Y-9206-B(135)$ & $01-0020-03$ & $731689 \mathrm{P}$ & Air emission control scrubber stack \\
\hline$Y-9206-B(136)$ & $01-0020-03$ & $731689 P$ & Air emission control consolidated stack \\
\hline$Y-9206-B(208)$ & $01-0020-03$ & $731689 \mathrm{P}$ & Conversion fluid bed \\
\hline$Y-9206-B(209)$ & $01-0020-03$ & $731689 \mathrm{P}$ & HF purge vent \\
\hline $\mathrm{Y}-9206-\mathrm{B}(210)$ & $01-0020-03$ & $731689 \mathrm{P}$ & Chemical makeup area \\
\hline$Y-9206-B(211)$ & $01-0020-03$ & $731689 P$ & Hoods 29 and 30 \\
\hline Y-9206-B(212) & $01-0020-03$ & $731689 P$ & Dry vacuum system \\
\hline$Y-9206-C(01)$ & $01-1020-24$ & $730316 \mathrm{P}$ & Classified \\
\hline$Y-9206-C(02)$ & $01-1020-24$ & $730316 \mathrm{P}$ & Classified \\
\hline Y-9206-E (NEW) & $01-1020-24$ & $730316 \mathrm{P}$ & Classified \\
\hline $\mathrm{Y}-9212-\mathrm{B}(01)$ & $01-0020-02$ & $730301 \mathrm{P}$ & $U$ metal drying and briquetting process \\
\hline$Y-9212-B(02)$ & $01-0020-02$ & $730301 \mathrm{P}$ & Exhaust from chip washing and drying \\
\hline$Y-9212-B(03)$ & $01-0020-02$ & $730301 P$ & E-wing machine shop \\
\hline$Y-9212-B(04)$ & $01-0020-02$ & $730301 \mathrm{P}$ & $U$ metal and $U$ metal alloy casting \\
\hline$Y-9212-F(01)$ & $01-1020-49$ & $730321 \mathrm{P}$ & Two deburr benches, hood exhaust, A-wing \\
\hline$Y-9212-F(02)$ & $01-1020-49$ & $730321 \mathrm{P}$ & Two deburr benches, hood exhaust, A-wing \\
\hline$Y-9212-F(03)$ & $01-1020-49$ & $730321 P$ & Machining, hood exhaust, A-wing \\
\hline$Y-9212-F(04)$ & $01-1020-49$ & $730321 P$ & Machining, hood exhaust, A-wing \\
\hline$Y-9212-F(05)$ & $01-1020-49$ & $730321 P$ & Machining, hood exhaust, A-wing \\
\hline$Y-9215-A(01)$ & $01-0020-37$ & $731839 \mathrm{P}$ & Machine shop hood exhaust, M-wing \\
\hline$Y-9215-B(1)$ & $01-1020-51$ & $732125 \mathrm{P}$ & O-wing metal working operations \\
\hline$Y-9215-B(2)$ & $01-1020-51$ & $732125 \mathrm{P}$ & O-wing metal working operations \\
\hline$Y-9215-B(4)$ & $01-1020-51$ & $732125 \mathrm{P}$ & O-wing metal working operations \\
\hline$Y-9215-B(6)$ & $01-1020-51$ & $732125 \mathrm{P}$ & O-wing metal working operations \\
\hline$Y-9215-C(03)$ & $01-1020-52$ & $730323 P$ & Vapor blaster/metal cleaner \\
\hline
\end{tabular}


Table A.2 (continued)

\begin{tabular}{|c|c|c|c|}
\hline $\begin{array}{l}\text { Y-12 Plant } \\
\text { source number }\end{array}$ & $\begin{array}{c}\text { Emission source } \\
\text { reference } \\
\text { number }\end{array}$ & $\begin{array}{l}\text { Permit } \\
\text { number }\end{array}$ & Source description \\
\hline$Y-9215-C(10)$ & $01-1020-52$ & $730323 \mathrm{P}$ & Nickel plating, metal working exhaust \\
\hline$Y-9215-C(11)$ & $01-1020-52$ & $730323 P$ & Exhaust \\
\hline$Y-9215-C(17)$ & $01-1020-52$ & $730323 P$ & Rolling mill \\
\hline$Y-9215-C(19)$ & $01-1020-52$ & $730323 \mathrm{P}$ & Electric annealing oven \\
\hline$Y-9401-2-A(205)$ & $01-0020-88$ & $730286 \mathrm{P}$ & Plating equipment \\
\hline$Y-9401-2-A(220)$ & $01-0020-88$ & $730286 \mathrm{P}$ & Plating equipment \\
\hline$Y-9401-2-A(221)$ & $01-0020-88$ & $730286 \mathrm{P}$ & Plating equipment \\
\hline$Y-9401-2-A(222)$ & $01-0020-88$ & $730286 \mathrm{P}$ & Plating equipment \\
\hline$Y-9401-2-A(223)$ & $01-0020-88$ & $730286 \mathrm{P}$ & Piating equipment \\
\hline$Y-9401-2-A(224)$ & $01-0020-88$ & $730286 \mathrm{P}$ & Plating equipment \\
\hline$Y-9401-2-A(225)$ & $01-0020-88$ & $730286 \mathrm{P}$ & Plating equipment \\
\hline$Y-9401-2-A(226)$ & $01-0020-88$ & $730286 \mathrm{P}$ & Plating equipment \\
\hline$Y-9401-2-A(227)$ & $01-0020-88$ & $730286 \mathrm{P}$ & Plating equipment \\
\hline$Y-9401-2-A(228)$ & $01-0020-88$ & $730286 \mathrm{P}$ & Plating equipment \\
\hline$Y-9401-2-A(229)$ & $01-0020-88$ & $730286 \mathrm{P}$ & Plating equipment \\
\hline$Y-9401-2-A(230)$ & $01-0020-88$ & $730286 \mathrm{P}$ & Plating equipment \\
\hline$Y \cdot 9401-2-A(231)$ & $01-0020-88$ & $730286 \mathrm{P}$ & Plating equipment \\
\hline$Y-9401-2-A(232)$ & $01-0020-88$ & $730286 \mathrm{P}$ & Plating equipment \\
\hline$Y-9401-2-A(233)$ & $01-0020-88$ & $730286 \mathrm{P}$ & Plating equipment \\
\hline$Y-9401-2-A(234)$ & $01-0020-88$ & $730286 \mathrm{P}$ & Plating equipment \\
\hline$Y-9401-2-A(235)$ & $01-0020-88$ & $730286 \mathrm{P}$ & Plating equipment \\
\hline$Y=9720-32-A(435)$ & $01-1020.99$ & 9332821 & Classified paper incinerator \\
\hline$Y-9811-1-A(1)$ & $01-1020-95$ & $731997 \mathrm{P}$ & Waste oil/storage bulk storage facility (OD-7) \\
\hline$Y-9811-1-A(2)$ & $01-1020-95$ & $731997 \mathrm{P}$ & Waste oil/storage bulk storage facility (OD-7) \\
\hline$Y-9811-1 \cdot A(3)$ & $01-1020-95$ & $731997 \mathrm{P}$ & Waste oil/storage bulk storage facility (OD-7) \\
\hline$Y-9811-1-A-(4)$ & $01-1020-95$ & $731997 \mathrm{P}$ & Waste oil/storage bulk storage facility (OD-7) \\
\hline$Y-3311-1-A-(5)$ & $01-1020-95$ & $731997 \mathrm{P}$ & Waste oil/storage bulk storage facility (OD-7) \\
\hline$Y-9811-1-A-(6)$ & $01-1020-95$ & $731997 \mathrm{P}$ & Wasie oil/storage bulk storage facility (OD-7) \\
\hline$Y-9811-1-A-(7)$ & $01-1020-95$ & $731997 \mathrm{P}$ & Waste oil/storage bulk storage facility (OD-7) \\
\hline
\end{tabular}


Table A.3. ORNL air permits

\begin{tabular}{|c|c|c|c|c|}
\hline $\begin{array}{l}\text { ORNL source } \\
\text { number }\end{array}$ & $\begin{array}{l}\text { Emission source } \\
\text { reference } \\
\text { number }\end{array}$ & $\begin{array}{l}\text { Permit } \\
\text { number }\end{array}$ & Source description & Permit type \\
\hline$X-2519-1 / 5$ & $73-0112-03,33,34$ & $030284 \mathrm{P}$ & Five boilers and ash system & Operation \\
\hline$X-2522-T I A$ & $73-0112-10$ & & No. 2 fuel oil storage tank & Application \\
\hline$X-2522-T L A$ & $73-0112-10$ & $024114 \mathrm{P}$ & No. 2 fuel oil storage tank & Operation \\
\hline$X-2525-01$ & $73-0112-14$ & $030835 \mathrm{P}$ & Degreaser (perchloroethylene) & Operation \\
\hline$X \cdot 2525-6$ & $73-0112-95$ & $027257 \mathrm{P}$ & Machine shop & Operation \\
\hline$X-2525-S V 11$ & $73-0112-49$ & & Electropiating shop & Application \\
\hline$X-2525-S \vee 11$ & $73-0112-49$ & $024151 \mathrm{P}$ & Electroplating shop & Operation \\
\hline$X-2525-S V 4$ & $73-0112-38$ & $031062 \mathrm{P}$ & Six wet and three dry grinders & Operation \\
\hline$X-2525-S \vee 8$ & $73-0112-62$ & & Spray booth and oven & Application \\
\hline $\mathrm{X}-2525-\mathrm{SV} 8$ & $73-0112-62$ & 024949P & Spray booth and oven & Operation \\
\hline$X-2547-01$ & $73-0112-27$ & $028439 \mathrm{P}$ & Spray booth & Operation \\
\hline$X-3039$ & & & Off-gas and hot cell ventilation & Application \\
\hline$X-3039$ & $73-0112-93$ & $035494 \mathrm{P}$ & Off-gas and hot cell ventilation & Operation \\
\hline $\mathrm{X}-3500-\mathrm{SV} 12$ & $73-0112-73$ & $036689 \mathrm{P}$ & Electric belt furnace & Operation \\
\hline$X-3502-01$ & $73-0112-05,06,07$ & $030881 \mathrm{P}$ & Spray booths $\# 1, \# 2$, and $\# 3$ & Operation \\
\hline $\mathrm{X}-3502-09$ & 73-0112-94 & $027194 \mathrm{P}$ & Hood-gluing & Operation \\
\hline$X-3502-1$ & $73-0112-05,06,07$ & & Manipulator boot shop & Application \\
\hline $\mathrm{X}-3502-\mathrm{SV} 1$ & $73-0112-39$ & $023808 \mathrm{P}$ & Oven, curing & Operation \\
\hline $\mathrm{X}-3502-\mathrm{SV} 2$ & $73-0112-40$ & $023807 \mathrm{P}$ & Oven, tempering & Operation \\
\hline$X-3502-S V 4$ & $73-0112-30$ & $036053 \mathrm{P}$ & Cyclone and carpenter shop & Operation \\
\hline $\mathrm{X}-3544-S V 1$ & $73-0112-70$ & $730468 \mathrm{P}$ & Process Waste Treatment Plant & Operation \\
\hline $\mathrm{X}-3587-5 V 1$ & $73-0112-56$ & 029830P & Printed circuit-board facility & Operation \\
\hline$X-3608-01$ & $73-0112-37$ & $730489 \mathrm{P}$ & NRWTP air stripper column & Operation \\
\hline $\mathrm{X}-4508-S V 8$ & $73-0112-61$ & $732645 \mathrm{P}$ & Acid etching process & Operation \\
\hline$X-4508-S V 9$ & & & Sand blaster & Application \\
\hline $\mathrm{X}-4508-S V 9$ & $73-0112-55$ & $024306 \mathrm{P}$ & Sand blaster & Operation \\
\hline$x-6010-00$ & $73-0112-85$ & $025282 \mathrm{P}$ & $\begin{array}{l}\text { Oak Ridge Electron Linear } \\
\text { Accelerator }\end{array}$ & Operation \\
\hline$X-7002-05$ & $73-0112-08$ & & Paint spray booth & Application \\
\hline$X-7002-05$ & $73-0112-08$ & $030980 \mathrm{P}$ & Spray booth & Operation \\
\hline$X-7005-00$ & $73-0112-45$ & $037516 \mathrm{P}$ & Lead shop-machining operations & Operation \\
\hline$x-7005-3 / 7$ & & & Five lead-melting furnaces & Application \\
\hline$X-7007-1 / 2$ & $73-0112-09$ & $030824 \mathrm{P}$ & Spray booth and cleaning booth & Operation \\
\hline$X-7007-1 / 2 / 3$ & $73-0112-09$ & & Spray booth and cleaning booth & Application \\
\hline $\mathrm{X}-7021-00$ & & & Sandblaster & Application \\
\hline$x-7021-00$ & $73-0112-58$ & D24307P & Grinding shop & Operation \\
\hline $\mathrm{X}-7057-\mathrm{SV} 1$ & $73-0112-76$ & $030101 \mathrm{P}$ & Sand blaster & Operation \\
\hline $\mathrm{X}-7069-\mathrm{T} 1$ & $73-0112-60$ NSPS & $730836 \mathrm{P}$ & Gasoline storage tank & Operation \\
\hline$X-7600-01$ & $73-0112-20$ & $017930 \mathrm{P}$ & Nuclear fuel reprocessing & Operation \\
\hline$X-7602-01$ & $73-0112-24$ & $027090 \mathrm{P}$ & Boiler, hot water & Operation \\
\hline$X-7603-01$ & & & Boiler & Application \\
\hline$X-7603-01$ & $73-0112-25$ & $035134 \mathrm{~F}$ & Steam boiler & Operation \\
\hline$X-7667-0$ & $73-0112-0067-5$ & $73-0112-0067-5$ & Chemical detonation facility & Open burning \\
\hline $\mathrm{X}-7830-\mathrm{SV} 1$ & 73.0112 .71 & $731010 \mathrm{P}$ & Liquid Waste Solidification Project & Operation \\
\hline
\end{tabular}


Table A.3 (continued)

\begin{tabular}{lllll}
\hline \multicolumn{1}{c}{$\begin{array}{c}\text { ORNL source } \\
\text { number }\end{array}$} & \multicolumn{1}{c}{$\begin{array}{c}\text { Emission source } \\
\text { reference } \\
\text { number }\end{array}$} & $\begin{array}{c}\text { Permit } \\
\text { number }\end{array}$ & Source description & Pemit type \\
\hline X-7911-00 & $73-0112-82$ & $034381 \mathrm{P}$ & HFIR, REDC 7920 and 7930 & Operation \\
X-7934-SV2 & $73-0112-53$ & Silver-recovery system & Application \\
X-7934-SV2 & $73-0112-53$ & $024912 \mathrm{P}$ & Silver-recovery system & Operation \\
X-7935-SV1 & $73-0112-78$ & $027393 \mathrm{P}$ & Equipment cleaning facility & Operation \\
X-FE & $73-0112-97$ & $029660 \mathrm{P}$ & Fugitive emission source & Operation \\
X-FLC & $73-0112-99$ & $034960 \mathrm{P}$ & Fluorescent lamp disposers & Operation \\
\hline
\end{tabular}


Table A.4. K-25 Site air permits

\begin{tabular}{|c|c|c|c|c|}
\hline $\begin{array}{l}\text { K-25 source } \\
\text { number }\end{array}$ & $\begin{array}{l}\text { Emission } \\
\text { source } \\
\text { reference } \\
\text { number }\end{array}$ & $\begin{array}{l}\text { Permit } \\
\text { number }\end{array}$ & Source description & Permit type \\
\hline $\mathrm{K} 1004 \mathrm{~L}$ & $73-0106-35$ & $012503 P$ & Main Vent of Development Facility & Operating \\
\hline K1004TWESTNOVEN & $73-0106-96$ & $024301 \mathrm{P}$ & Fiber and Polymer Matrix Composites Curing Oven & Operating \\
\hline K1004TSOUTHOVEN & $73-1106-01$ & $024304 \mathrm{P}$ & Fiber and Polymer Matrix Composites Curing Oven & Operating \\
\hline K1004THOOD & $73-1106-04$ & $024498 \mathrm{P}$ & $\begin{array}{l}\text { Hood Evacuates Fumes from Mixing Epoxy Resin and } \\
\text { Hardener }\end{array}$ & Operating \\
\hline K1004TWIND3 & $73-1106-28$ & $029901 \mathrm{P}$ & Fiber Winding Spools With Epoxy Dip & Operating \\
\hline K1004TWIND2 & $73-1106-28$ & $029901 P$ & Fiber Winding Spools With Epoxy Dip & Operating \\
\hline K1004TWIND4 & $73-1106-28$ & $029901 \mathrm{P}$ & Fiber Winding Spools With Epoxy Dip & Operating \\
\hline K1004TWIND1 & $73-1106-28$ & $029901 \mathrm{P}$ & Fiber Winding Spools With Epoxy Dip & Operating \\
\hline K1024FT1 & $73-0106-18$ & $025655 P$ & Filter Test Facility & Operating \\
\hline K1037AVLISLCDEV & $73-0106-69$ & 029897P & Expansion Lab C Spray Coating W Exhaust Filters & Operating \\
\hline K1037AVLISOOVEN & $73-0106-73$ & $029900 \mathrm{P}$ & Electric Oxidation Oven & Operating \\
\hline K1037AVLISEXLAB & $73-0106-68$ & $031404 \mathrm{P}$ & Materiais Test Unit (MTU) & Operating \\
\hline K1037AVLISEXIAB & $73-0106-68$ & $031404 \mathrm{P}$ & Vacuum System Vents & Operating \\
\hline K1037AVLISEXLAB & $73-0106-68$ & $031404 \mathrm{P}$ & Materials Handling Development Module (MHDM) & Operating \\
\hline K1037AVLISEXLAB & $73-0106-68$ & $031404 \mathrm{P}$ & Electron Beam One (EB-1) & Operating \\
\hline K1037AVLISLGB & $73-0106-77$ & $032345 P$ & Grit Blast Facility with Baghouse & Operating \\
\hline K1037AVLISQOVEN & $73-0106-79$ & $034645 P$ & Quincy Oven & Operating \\
\hline K1037AVLISGOVEN & $73-0106-80$ & $034646 \mathrm{P}$ & Grieve Oven TB-500 Electric & Operating \\
\hline K1037AVLISFURN & $73-0106-81$ & $034647 \mathrm{P}$ & Huppert Furnace & Operating \\
\hline K1037MLBH & $73-0106-84$ & 035867P & Mechanical Lab-Shaping Graphite and Metal Parts & Operating \\
\hline K1037AVLISSSB & $73.0106-85$ & $035868 \mathrm{P}$ & Small Sand Blaster & Operating \\
\hline K1037AVLISLAB & $73-1106-35$ & 932953P & $\begin{array}{l}\text { AVLIS Lab - metallothermic reduction unit, chlorinator, } \\
\text { and oxide cell }\end{array}$ & $\begin{array}{l}\text { Permit to } \\
\text { construct }\end{array}$ \\
\hline K1037AVLISPRODCON & $73-1106-36$ & $933170 \mathrm{P}$ & Products Conversion Demonstration & $\begin{array}{l}\text { Permit to } \\
\text { construct }\end{array}$ \\
\hline K1095PS1234 & $73-0106-14$ & $734461 P$ & $\begin{array}{l}\text { Paint Spray Operation, one Oven, two Spray Booths, and } \\
\text { one Silk Screen Degreaser }\end{array}$ & Operating \\
\hline K1098FSB 1 & $73-0106-13$ & $034231 P$ & Sand Blast Facility with Baghouse and Grit Recycle & Operating \\
\hline K1200SITF & $73-0106-61$ & $017338 \mathrm{P}$ & System Interface Test Facility seven Vacuum Pumps & Operating \\
\hline K1200CVTF & $73-0106-62$ & 017339P & Centrifuge Verification Test Facility ten Vacuum Pumps & Operating \\
\hline $\mathrm{K} 1200 \mathrm{~A} 123$ & $73-0106-56$ & $019608 \mathrm{P}$ & $\begin{array}{l}\text { Purge Evacuation, Feed, and Withdrawal } 13 \text { Vacuum } \\
\text { Pumps }\end{array}$ & Operating \\
\hline K1200NBAYOVEN & $73-0106-92$ & $024272 \mathrm{P}$ & $\begin{array}{l}\text { North Bay Oven Cures Fiber and Polymeric Matrix } \\
\text { Composites }\end{array}$ & Operating \\
\hline K1200FAE1 & $73-0106-86$ & $029192 \mathrm{P}$ & Isotope Separating Process & Operating \\
\hline K1200CENTERBAY & $73-0106-87$ & $732346 \mathrm{P}$ & $\begin{array}{l}\text { Two Hoods Vent Mixing Epoxy Resins, Coating Fibers, } \\
\text { Winding Fibers }\end{array}$ & Operating \\
\hline $\mathrm{K} 1202 \mathrm{ST} 1$ & $73-1106-20$ & 033203P & Tank Stores Waste Oils and Solvents for Incinerator & Operating \\
\hline K1202ST2 & $73-1106-41$ & 034392P & Tank Stores Waste Oils and Solvents for Incinerator & Operating \\
\hline K1401275029PL & 73-0106-38 & $012506 \mathrm{P}$ & Plastic Shop Curing Oven & Operating \\
\hline K1401121659 & $73-0106-09$ & 016306P & 1,1,1-Trichloroethane Degreaser & Operating \\
\hline $\mathrm{K} 1401 \mathrm{MSMC1}$ & $73-0106-32$ & $017337 \mathrm{P}$ & Motor Curing Oven & Operating \\
\hline K14010OOOVENNE2 & $73-0106-89$ & $028424 \mathrm{P}$ & $\begin{array}{l}\text { Electric Oven to Bake out Residual Organics from Metal } \\
\text { Parts }\end{array}$ & Operating \\
\hline
\end{tabular}


Table A.4 (continued)

\begin{tabular}{|c|c|c|c|c|}
\hline $\begin{array}{l}\text { K-25 source } \\
\text { number }\end{array}$ & $\begin{array}{l}\text { Emission } \\
\text { source } \\
\text { reference } \\
\text { number }\end{array}$ & $\begin{array}{l}\text { Permit } \\
\text { number }\end{array}$ & Source description & Permil type \\
\hline K1401JIGANDFIXT & $73-0106-71$ & $029898 \mathrm{P}$ & $\begin{array}{l}\text { Vacuum Exhaust for Parts Fabrication in the Jig and } \\
\text { Fixture Shop }\end{array}$ & Operating \\
\hline K1401PLS1,4,6 & $73-0106-72$ & 029899P & $\begin{array}{l}\text { Ovens } 1,4 \text {, and } 6 \text { Used for Curing Plastic Parts in the } \\
\text { Plastic Shop }\end{array}$ & Operating \\
\hline K1401CARPENTERSHOP & $73-1106-40$ & $032930 P$ & $\begin{array}{l}\text { Miscellaneous wood and acrylic plastic working. } \\
\text { operations with cycione control }\end{array}$ & Operating \\
\hline K1401HCLE & $73-0106-28$ & $035840 \mathrm{P}$ & Hydrochloric Acid Tank & Operating \\
\hline K25BULBCRUSHER & $73-1106-43$ & $934193 P$ & Flourescent Lamp Disposers with Fabric/Carbon Filters & Operaring \\
\hline K1414RG & $73-0106-28$ & $016312 \mathrm{P}$ & Gasoline Storage Tank & Operating \\
\hline K1414UNLGAS & $73-1106-39$ & $035063 \mathrm{P}$ & $\begin{array}{l}20,000 \text { Gal Unleaded Gasoline Underground Storage } \\
\text { Tank }\end{array}$ & Operating \\
\hline K1414UG & $73-0106-28$ & $037113 P$ & Methanol, unleaded gasoline storage tank & Operating \\
\hline $\mathrm{K} 1200 \mathrm{CPL} 1$ & $73-0106-58$ & $017051 \mathrm{P}$ & $\begin{array}{l}\text { Vent for aqueous spray chamber, ultrasonic cleaner, } \\
\text { solvent }\end{array}$ & Operating \\
\hline $\mathrm{K} 1200 \mathrm{CPL}$ & $73-0106-54$ & $017055 \mathrm{P}$ & Aqueous spray, ultrasonic cleaner, solvent degreaser & Operating \\
\hline K1420PHLLIPSVA & $73-0106-70$ & 023798P & Phillips Vapor Degreaser Perchloroethylene & Operating \\
\hline K1420DISASSEMBL & $73-0106-74$ & $032344 \mathrm{P}$ & Disassembly Stand for Dismantling Parts & Operating \\
\hline $\mathrm{K} 1420 \mathrm{~A} 1$ & $73-0106-82$ & 034619P & Flammabie Materials Storage Tank & Operating \\
\hline K1425WOSC & $73-0106-11$ & 029895P & Waste Oil and Solvent Storage Tanks & Operating \\
\hline K1425WOSA & $73-0106-11$ & $029895 \mathrm{P}$ & Waste Oil and Solvent Storage Tanks & Operating \\
\hline K1425WOSD & $73-0106-11$ & $029895 \mathrm{P}$ & Waste Oil and Solvent Storage Tanks & Operating \\
\hline K1425WOSB & $73-0106-11$ & $029895 \mathrm{P}$ & Waste Oil and Solvent Storage Tanks & Operating \\
\hline K1435TSCAINCIN & $73-0106-78$ & 032449I & TSCA Incinerator & Operating \\
\hline K1435CTANKFARM & 73-0106-75 & $024105 P$ & Tank Farm for Hazardous Liquid Wastes & Operating \\
\hline K15012720FO & $73-0106-28$ & $016312 P$ & K-1501 613,000-gal fuel oil tank & Operating \\
\hline $\mathrm{K} 15012810 \mathrm{FO}$ & $73-0106-28$ & $016312 P$ & K-1501 15,228 -gal fuel oil tank & Operating \\
\hline K1501BOILER4 & $73-0106-04$ & $029902 \mathrm{~F}$ & Natural Gas Boiler & Operating \\
\hline K1501BOILER7 & $73-0106-07$ & $029902 \mathrm{~F}$ & Gas/Oil Boiler & Operating \\
\hline K1501SULFACID & $73-0106-28$ & 035840P & Sulfuric Acid Storage Tank & Operaung \\
\hline K1501BOILER8 & $73-0106-12$ & $937114 \mathrm{~F}$ & Gas/Oil Boiler & $\begin{array}{l}\text { Permit to } \\
\text { ccinstruct }\end{array}$ \\
\hline K1SO1BOILER 9 & $73-0106-12$ & $937114 \mathrm{~F}$ & Gas/Oil Boiler & $\begin{array}{l}\text { Permit to } \\
\text { construst }\end{array}$ \\
\hline K1600TTFL & $73-0106-59$ & $017053 P$ & Development Lab with two Hoods and one Small Oven & Operating \\
\hline K1652FECS & $73-1106-42$ & $733774 \mathrm{P}$ & Fire extinquisher charging station & Operating \\
\hline $\mathrm{K}-25-\mathrm{B}-1$ & $73-0106-19$ & $016309 \mathrm{P}$ & Heat Exchange Medium Freon for Plant & Operating \\
\hline K291 & $73-0106-63$ & $015097 \mathrm{P}$ & Wet Air Evacuation System & Operating \\
\hline $\mathrm{K} 4029 \mathrm{PC}$ & $73-0106-42$ & $012660 \mathrm{P}$ & Gaseous Diffusion Purge Cascade & Operating \\
\hline K602WAP & $73-0106-93$ & 024297P & Evacuation Wet Air Pumps or Air Jets & Operating \\
\hline K60212543LO & $73-0106-23$ & $016310 \mathrm{P}$ & Lube Oil Tank & Operating \\
\hline K60222540LO & $73-0106-23$ & $016310 \mathrm{P}$ & Lube Oil Tank & Operating \\
\hline $\mathrm{K} 60232542 \mathrm{LO}$ & $73-0106-23$ & $016310 \mathrm{P}$ & Lube Oil Tank & Operating \\
\hline K60242541LO & $73-0106-23$ & $016310 \mathrm{P}$ & Lube Oil Tank & Operating \\
\hline K60252545LO & $73-0106-23$ & $016310 \mathrm{P}$ & Lube Oil Tank & Operating \\
\hline K60262544LO & $73-0106-23$ & $016310 \mathrm{P}$ & Lube Oil Tank & Operating \\
\hline K704316MO & $73-0106-24$ & $034218 \mathrm{P}$ & Mineral Oil Tank & Operating \\
\hline
\end{tabular}


Table A.4 (continued)

\begin{tabular}{|c|c|c|c|c|}
\hline $\begin{array}{c}\text { K-25 source } \\
\text { number }\end{array}$ & $\begin{array}{l}\text { Emission } \\
\text { source } \\
\text { reference } \\
\text { number }\end{array}$ & $\begin{array}{l}\text { Permit } \\
\text { number }\end{array}$ & Source description & Permit type \\
\hline $\mathrm{K} 7322140 \mathrm{MO}$ & $73-0106-24$ & $034218 \mathrm{P}$ & Mineral Oil Tank & Operating \\
\hline $\mathrm{K}-7322135 \mathrm{MO}$ & $73-0106-24$ & $034218 \mathrm{P}$ & Mineral Oil Tank & Operating \\
\hline $\mathrm{K} 7622427 \mathrm{MO}$ & $73-0106-24$ & $034218 \mathrm{P}$ & Mineral Oil Tank & Operating \\
\hline $\mathrm{K} 7622428 \mathrm{MO}$ & $73-0106-24$ & $034218 \mathrm{P}$ & Mineral Oil Tank & Operating \\
\hline $\mathrm{K} 7922423 \mathrm{MO}$ & $73-0106-24$ & $034218 \mathrm{P}$ & Mineral Oil Tank & Operating \\
\hline K7922431MO & $73-0106-24$ & $034218 \mathrm{P}$ & Mineral Oil Tank & Operating \\
\hline K892LIMESILO & $73-1106-08$ & $025120 \mathrm{P}$ & Lime Silo & Operating \\
\hline K894SULFACID & $73-0106-28$ & $035840 \mathrm{P}$ & Sulfuric Acid Storage Tank & Operating \\
\hline K902JET & $73-0106-93$ & $024298 \mathrm{P}$ & Exhaust Jet & Operating \\
\hline K902WAP & $73-0106-93$ & $024298 \mathrm{P}$ & Evacuation Wet Air Pumps & Operating \\
\hline K90212310LO & $73-0106-23$ & $016310 \mathrm{P}$ & Lube Oil Tank & Operating \\
\hline K90212318LO & $73-0106-23$ & $016310 \mathrm{P}$ & Lube Oil Tank & Operating \\
\hline K90222321LO & $73-0106-23$ & $016310 \mathrm{P}$ & Lube Oil Tank & Operating \\
\hline K90222319LO & $73-0106-23$ & $016310 \mathrm{P}$ & Lube Oil Tank & Operating \\
\hline K90222320LO & $73-0106-23$ & $016310 \mathrm{P}$ & Lube Oil Tank & Operating \\
\hline K90222311LO & $73-0106-23$ & $016310 \mathrm{P}$ & Lube Oil Tank & Operating \\
\hline K90232312LO & $73-0106-23$ & $016310 \mathrm{P}$ & Lube Oil Tank & Operating \\
\hline K90232322LO & $73-0106-23$ & $016310 \mathrm{P}$ & Lube Oil Tank & Operating \\
\hline K90232323LO & $73-0106-23$ & $016310 \mathrm{P}$ & Lube Oil Tank & Operating \\
\hline K9023324470FREON & $73-0106-28$ & $035840 \mathrm{P}$ & Freon R-114 Storage Tank & Operating \\
\hline K9023324383 & $73-0106-28$ & $035840 \mathrm{P}$ & Freon Storage Tank & Operating \\
\hline K9023324469FREON & $73-0106-28$ & 035840P & Freon R-114 Storage Tank & Operating \\
\hline K90242325LO & $73-0106-23$ & $016310 \mathrm{P}$ & Lube Oil Tank & Operating \\
\hline K90242324LO & $73-0106-23$ & $016310 \mathrm{P}$ & Lube Oil Tank & Operating \\
\hline K90242313LO & $73-0106-23$ & $016310 \mathrm{P}$ & Lube Oil Tank & Operating \\
\hline $\mathrm{K} 90252314 \mathrm{LO}$ & $73-0106-23$ & $016310 \mathrm{P}$ & Lube Oil Tank & Operating \\
\hline K9C252378LO & $73-0106-23$ & $016310 \mathrm{P}$ & Lube Oil Tank & Operating \\
\hline K90252379LO & $73-0106-23$ & $016310 \mathrm{P}$ & Lube Oil Tank & Operating \\
\hline K90262381LO & $73-0106-23$ & $016310 \mathrm{P}$ & Lube Oil Tank & Operating \\
\hline K90262380LO & $73-0106-23$ & $016310 \mathrm{P}$ & Lube Oil Tank & Operating \\
\hline K90262315LO & $73-0106-23$ & 016310P & Lube Oil Tank & Operating \\
\hline K90272383LO & $73-0106-23$ & $016310 \mathrm{P}$ & Lube Oil Tank & Operating \\
\hline K90272316LO & $73-0106-23$ & $016310 P$ & Lube Oil Tank & Operating \\
\hline K90272382LO & $73-0106-23$ & $016310 P$ & Lube Oil Tank & Operating \\
\hline K90282384LO & $73-0106-23$ & $016310 \mathrm{P}$ & Lube Oil Tank & Operating \\
\hline K90282317LO & $73-0106-23$ & $016310 \mathrm{P}$ & Lube Oil Tank & Operating \\
\hline K90282385LO & $73-0106-23$ & 016310 & Lube Oil Tank & Operating \\
\hline
\end{tabular}

